\title{
ELECTRICAL CONTACTS-2009
}

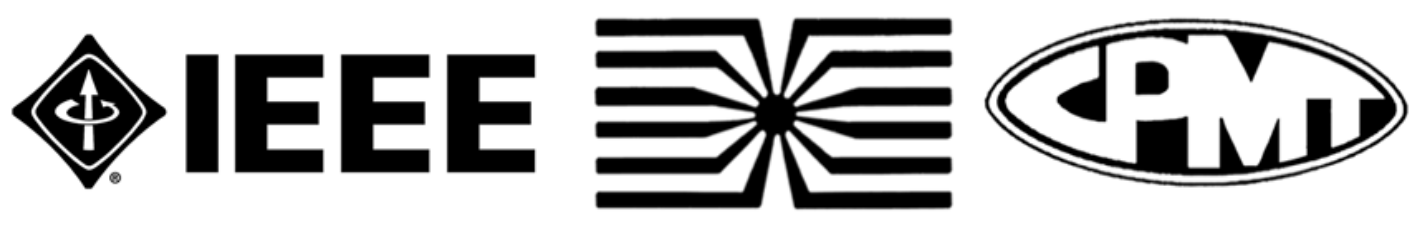

Proceedings of

\section{THE FIFTY-FIFTH \\ IEEE HOLM CONFERENCE ON ELECTRICAL CONTACTS}

Sponsored by the

Components, Packaging, and Manufacturing Technology Society of the Institute of Electrical and Electronics Engineers, Inc. 


\section{ELECTRICAL CONTACTS 2009}

\section{PROCEEDINGS OF THE $55^{\text {th }}$ IEEE HOLM CONFERENCE ON ELECTRICAL CONTACTS}

\section{PURPOSE}

To provide a forum for the presentation and discussion of the latest developments in the field of electric contacts, as well as, the application of recent advances in materials and processes in electrical, electronic and telecommunications equipment.

\section{FOR WHOM}

Practicing designers, engineers, physicists, and research scientists-those new to the field and those experienced. The 2009 IEEE Holm Conference will include excellent papers authored by some of the outstanding technical people in this field. The international contributors come from the USA, Austria, Canada, China, France, Germany, Japan, Spain, Switzerland, Taiwan, and United Kingdom. These papers will provide the attendees with up-to-date information on a wide range of subjects that makes this conference so attractive to the practicing engineer.

Additionally, the 2009 IEEE Holm Conference will make it possible for any attendee to discuss personally, with any author, either additional details concerning the work presented by the author at the conference or any subject related to the author's field of expertise.

\section{BACKGROUND}

The Holm Conference began in 1953 as a forum for the discussion of electrical contact phenomena and related fields. In 1968, the conference was named the Holm Conference in honor of Dr. Ragnar Holm. Dr. Holm, whose contributions to the field of electrical contacts spanned 50 years and forms the foundation of the electrical contacts field, was the inspiration and guide of the Conference from its inception until his death is 1970.

In addition to the Annual Conference, the Conference Organization regularly conducts an intensive course on contacts and participates in the biannual International Conference on Electrical Contacts.

Abstracting is permitted with credit to the source. Libraries are permitted to photocopy beyond the limit of U.S. copyright law, for private use of patrons, those articles in this volume that carry a code at the bottom of the first page, provided the per-copy fee indicated in the code is paid through the Copyright Clearance Center, 222 Rosewood Drive, Danvers, MA 01923. For other copying, reprint, or republication permission, write to the IEEE Copyrights Manager, IEEE Operations Center, 445 Hoes Lane, Piscataway, NJ 08855-1331. All rights reserved. Copyright $(2009$ by The Institute of Electrical and Electronics Engineers, Inc.

$14-16$ September 2009

The Renaissance Vancouver Hotel Harbourside

Vancouver, British Colombia, Canada

The Institute of Electric and Electronics Engineers, Inc.

CPMT

IEEE Catalog Number: CFP09HLM-CDR

ISBN Casebound: 978-1-4244-3613-2

Library of Congress: $\quad 88-645722$ 


\title{
$55^{\text {th }}$ IEEE Holm Conference on Electrical Contacts Contributors
}

\author{
Gold Level Plus \\ Checon Corporation \\ Heraeus \\ Tyco Electronics \\ Silver Level Plus \\ AMI DODUCO \\ Chugai USA, LLC \\ Deringer Ney, Inc. \\ Eaton Corporation \\ Umicore Technical Materials \\ Silver Level \\ Research and Engineering Society for \\ Electromechanical Components and \\ Contact Technology in Japan, Mie University \\ Bronze Level \\ Brainin \\ Rockwell Automation

\section{WITH COOPERATION FROM}

Air Force Institute of Technology

University of Maryland

AMI DODUCO

Amphenol TCS

Auburn University

AutoNetworks Technologies, Ltd.

Beijing University of Posts and Telecommunications

C\&K Components

Checon Corporation

Chitose Institute of Science \& Technology

Chugai USA, LLC

Delphi Packard Electric Systems

Deringer Ney, Inc.

Dietmar Gentsch, ABB AG

Eaton Corporation

EDF R\&D

FITEC Corp.

FormFactor, Inc.

Grenoble Electrical Engineering Lab

Guilin Aerospace Electronics Ltd.

Harbin Institute of Technology

Hebei University of Technology

Hydro Quebec IREQ

Institute of Power Transmission High Voltage Technology

Ishinomaki Senshu University

Kaneka Corporation

Laboratoire de Génie Electrique de Paris

Léti-MINATEC

Massachusetts Institute of Technology
MB Interface

Mie University

Molex, Inc.

Moog Components Group

Nippon Institute of Technology

Nokia Corporation

NSWCCD

PEM

RD Malucci Consulting

Rockwell Automation/Allen-Bradley

Schleifring und Apparatebau GmbH

Schneider Electric Industries

Shaan'Xi Sirui Industries Co.,LTD

Shizuoka University

Siemens AG

State Key Laboratory of Electrical Insulation Power Equipment

Supelec

The Furukawa Electric Co., Ltd.

Tongji University

TOSHIBA Corporation

Tver State University of Technology

Tyco Electronics

Umicore AG \& Co. KG

University of Applied Sciences

University of Rennes 1

University of Southampton

University of Technology Braunschweig

Xi'an Jiaotong University 


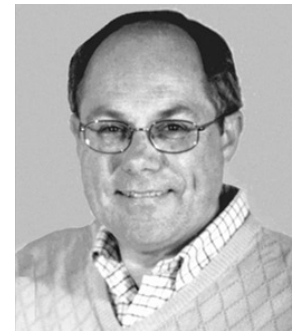

Chairman

Dr. Ed Smith III

Deringer Ney, Inc.

Bloomfield, CT

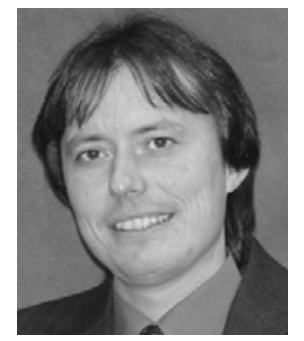

Vice Chairman

Dr. Thomas J. Schoepf

Eaton Corporation

Milwaukee, WI

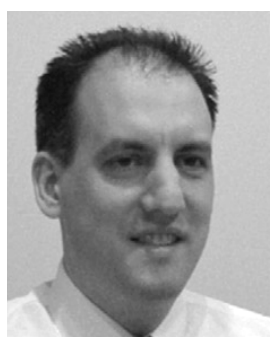

Technical Program Chairman

Dr. Brett Rickett

Molex, Inc.

Lisle, IL

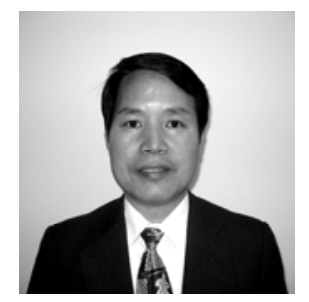

Technical Program Vice Chairman Mr. Z.K. Chen

Chugai USA, LLC

Waukegan, IL

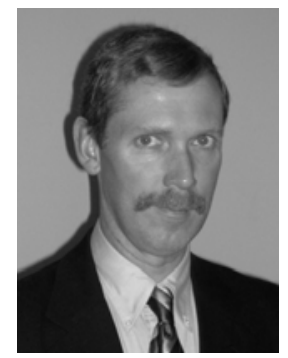

Finance Chairman

Mr. Henry Czajkowski

Rockwell Automation

Milwaukee, WI

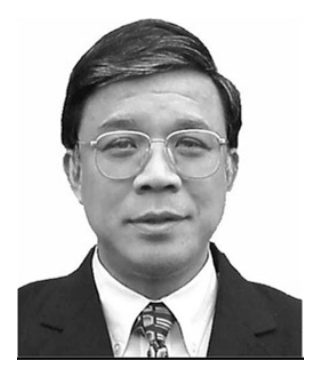

Publicity Chairman

Dr. Chi Leung

AMI DODUCO

Export, PA

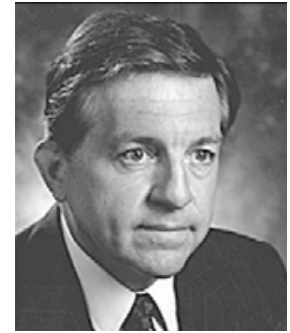

TC1-Chairman

Mr. Gerald J. Witter

Chugai USA, Inc.

Waukegan, IL

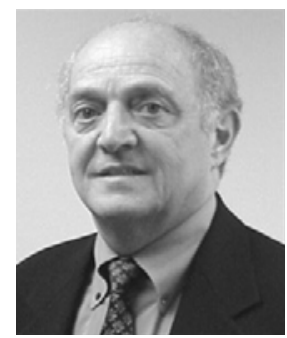

TC1-Vice Chairman

Dr. Robert Malucci

R.D. Malucci Consulting

Naperville, IL

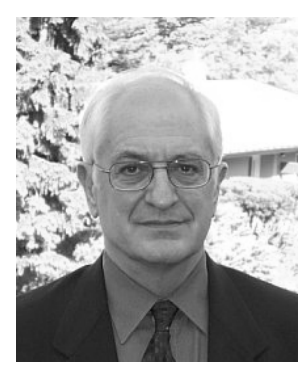

Course Director / International Rep. Dr. Paul G. Slade

Consultant

Ithaca, NY

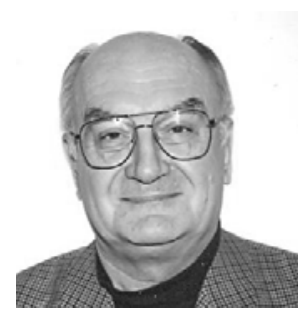

CPMT - Associate Editor

Dr. Milenko Braunovic

MB Interface

Montreal, QC, Canada

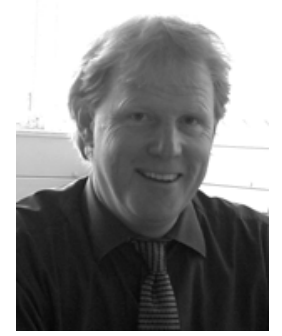

CPMT - Associate Editor Dr. John McBride University of Southampton Southampton, UK 


\section{IEEE HOLM CONFERENCE OFFICERS}

Chairman

Ed Smith, III

Deringer Ney, Inc.

Vice Chairman

Thomas J. Schoepf

Eaton Corporation

Finance Chairman

Henry Czajkowski

Rockwell Automation
Technical Program Chairman

Brett Rickett

Molex, Inc.

Technical Program Vice Chairman

Z.K. Chen

Chugai USA, LLC

Publicity Chairman

Chi Leung

AMI Doduco
CPMT Technical Committee 1 Electrical Contacts Chairman Gerald Witter

Chugai USA, LLC

Intensive Course Director, and US Representative for the International Conference

Paul Slade

Consultant

CPMT Technical Committee 1 Electrical Contacts Vice Chairman Robert Malucci

R.D. Malucci Consulting

\section{IEEE HOLM TECHNICAL PROGRAM COMMITTEE}

Chair

Brett Rickett

Molex, Inc.

Vice Chair

Z.K. Chen

Chugai USA, LLC

Bill Balme

Checon

Milenko Braunovic

MB Interface

Bella Chudnovsky

Stephen Cole

Moog Components Group
Henry Czajkowski

Rockwell Automation

George Drew

Delphi Packard Electric Systems

George T. Flowers

Auburn University

Daniel Gagnon

Hydro Quebec IREQ

Chi Leung

AMI Doduco

Rod Martens

FormFactor, Inc.
Richard Moore

C\&K Components

John J. Shea

Eaton Corporation

Ed Smith III

Deringer Ney, Inc.

Philip Wingert

Gerald J. Witter

Chugai USA, LLC

Xin Zhou

Eaton Corporation

2009 AWARDS COMMITTEE

Paul Slade, Chair

Consultant
Noureddine Ben Jemaa

University of Rennes 1
John McBride

University of Southampton

\section{PRIZE PAPER AWARD COMMITTEE}

Daniel Gagnon

Hydro Quebec IREQ
Eric Streicher

AMI Doduco
George T. Flowers Auburn University

\section{8 - 2009 ASSOCIATE EDITORS - CPMT}

John McBride

University of Southampton
Thomas J. Schoepf

Eaton Corporation
Milenko Braunovic

MB Interface 
TABLE OF CONTENTS

Foreword

Morton Antler Lecture xvi

Erle Shobert Prize Paper xvii

\title{
Arc Fault/Safety
}

Chair: H. Czajkowski

Co-Chair: G. Witter

1.1

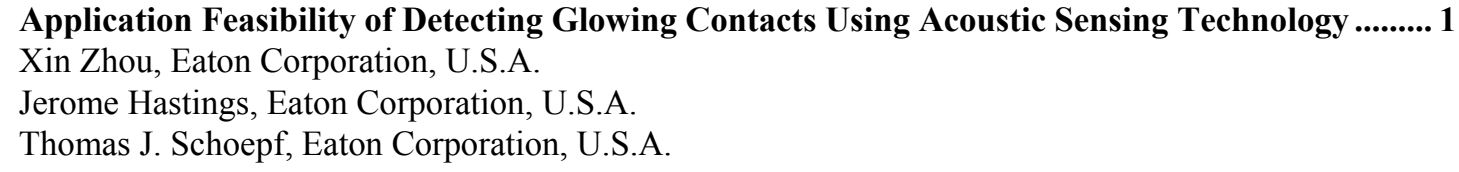

1.2

\author{
Dongwei Li, Xi'an Jiaotong University, China \\ Zhengxiang Song, Xi'an Jiaotong University, China \\ Jianhua Wang, Xi'an Jiaotong University, China \\ Yingsan Geng, Xi'an Jiaotong University, China \\ Huilin Chen, Xi'an Jiaotong University, China \\ Li Yu, Xi'an Jiaotong University, China \\ Bo Liu, Xi'an Jiaotong University, China
}

A Method for Residential Series Arc Fault Detection and Identification

7

1.3

Artificial Low Current Arc Fault for Pattern Recognition in Low Voltage Switchgear

Peter Müller, Institute of Power Transmission and High Voltage Technology (IEH), Germany

Stefan Tenbohlen, Institute of Power Transmission and High Voltage Technology (IEH), Germany

Reinhard Maier, Siemens AG, Germany

Michael Anheuser, Siemens AG, Germany

1.4

Electrical Arc Characterization For Ac-Arc Fault Applications.

E. Carvou, Université de Rennes 1, France

N. Ben Jemaa, Université de Rennes 1, France

S. Tian, Schneider Electric, France

Z. Belhaja, Schneider Electric, France

B. Jusselin, Schneider Electric, France

Fundamentals

Chair: M. Braunovic

Co-Chair: R. Martens

2.1

A Simplified Model of Multiscale Electrical Contact Resistance and Comparison to Existing Closed Form Models

Robert L. Jackson, Auburn University, United States

Robert D. Malucci, RD Malucci Consulting, United States

Santosh Angadi, Auburn University, United States

J. Robert Polchow, Auburn University, United States 
Mechanical and Electrical Characteristics of the Electromagnetic Relay under Vibration and Shock Environment

Wanbin Ren, Harbin Inst. of Tech., China

Huimin Liang, Harbin Inst. of Tech., China

Yinghua Chen, Harbin Inst. of Tech., China

Li Cui, Guilin Aerospace Electronics Ltd., China

Lizhong Wang, Guilin Aerospace Electronics Ltd., China

Yunzhi Kang, Harbin Inst. of Tech., China

\section{Arc Interruption I}

Chair: X. Zhou

Co-Chair: Z.K. Chen

Peter Zeller, University of Applied Sciences Upper Austria, Austria

Noboru Wakatsuki, Ishinomaki Senshu University, Japan

Nobuo Takatsu, Ishinomaki Senshu University, Japan

Toshiteru Maeda, Ishinomaki Senshu University, Japan

Takayuki Kudo, Ishinomaki Senshu University, Japan

\section{Sliding Contacts}

Chair: K. Sawa

Co-Chair: S. Cole

4.1

Liquid Additives to Improve Conductivity in Electric Contacts

W. A. Lynch, Raven LLC, United States

N.A. Sondergaard, NSWCCD, United States

Analysis of Peculiar Brush V-I Characteristics with Steel Ring by Improved Holm's psi-curly theta Theory in Electric Contact

Makoto Takanezawa, Nippon Institute of Technology, Japan

Noboru Morita, Nippon Institute of Technology, Japan

Takahiro Ueno, Nippon Institute of Technology, Japan

Toru Otaka, TOSHIBA Corporation, Japan

Daisuke Hiramatsu, TOSHIBA Corporation, Japan 
G. S. Erwin, NSWCCD, United States

N. A. Sondergaard, NSWCCD, United States

W. A. Lynch, Raven LLC, United States

M. J. Roberts, NSWCCD, United States

R. P. Reitz, NSWCCD, United States

N. A. Sondergaard, NSWCCD, United States

\section{Connector Design/Modeling}

Chair: B. Malucci

Co-Chair: G. Flowers

5.1

5.2
3-D Simulation of Open-Barrel Crimping Process: Study of the Effect of Serrations Dmitry V. Zhmurkin, Tyco Electronics, USA

Use of Modeling Techniques to Optimize the Electrical Performance of a Novel RF Connector Design

Sean P. McCarthy, Tyco Electronics Corporations, USA

Coupled Thermal Electrical Finite Element Analysis of Power Contacts Used in High Speed Differential Connectors

Trent K. Do, Amphenol-TCS, United States

Tom Cohen, Amphenol TCS, United States

Fretting

Chair: J. McBride

Co-Chair: E. Smith

6.1

Modeling and Analysis of a Connector System for the Prediction of Vibration-induced Fretting Degradation.

Chen Chen, Auburn University, United States

George T. Flowers, Auburn University, United States

Michael Bozack, Auburn University, United States

Jeffrey Suhling, Auburn University, United States

Yasuyuki Ishimaru, FITEC Corporation, Japan

Keiji Mashimo, The Furukawa Electric co., ltd., Japan

Kyota Susai, The Furukawa Electric co., Itd., Japan

$\mathrm{Fu}$ Yingchao, TongJi University, China

Wang Anlin, TongJi University, China 


\section{Materials/Surface Finishing I}

Chair: R. Timsit

Co-Chair: R. Martens

S. Noël, Supeléc, France

D. Alamarguy, Supeléc, France

S. Correia, Supeléc, France

P. Gendre, PEM, France

7.2

Deformation of Crystal Structure and Distribution of Mechanical Stress in

Tin-Plated Layer under Contact Loading

Terutaka Tamai, Mie University, Japan

Shigeru Sawada, Mie University, Japan

Yasuhiro Hattori, AutoNetworks Technology, Ltd., Japan

Yuuya Nabeta, Mie University, Japan

Yasushi Saitoh, Mie University, Japan

Shigeru Sawada, Mie University, Japan

Yasuhiro Hattori, AutoNetworks Technologies, Ltd., Japan

Terutaka Tamai, Mie University, Japan

\section{Materials/Arc Interruption}

Chair: C. Leung

Co-Chair: H. Czajkowski

Jutta Röhberg, AMI DODUCO GmbH, Germany

Thomas Honig, AMI DODUCO GmbH, Germany

Norbert Witulski, AMI DODUCO GmbH, Germany

Michael Finkbeiner, AMI DODUCO GmbH, Germany

Volker Behrens, AMI DODUCO GmbH, Germany 
$\mathrm{Li} \mathrm{Yu}$, State Key Laboratory of Electrical Insulation and Power Equipment, China Yingsan Geng, State Key Laboratory of Electrical Insulation and Power Equipment, China Qian Li, State Key Laboratory of Electrical Insulation and Power Equipment, China Jianhua Wang, State Key Laboratory of Electrical Insulation and Power Equipment, China Zhiyuan Liu, State Key Laboratory of Electrical Insulation and Power Equipment, China Dongwei Li, State Key Laboratory of Electrical Insulation and Power Equipment, China Wenbin Wang, Shaan'Xi Sirui Industries Co.,LTD, China Xiaojun Wang, Shaan'Xi Sirui Industries Co.,LTD, China

\section{Connector Reliability}

Chair: G. Flowers

Co-Chair: D. Gagnon

Karl Ring, Fraunhofer Institute for Reliability and Microintegration, Germany

Thomas Schreier-Alt, Fraunhofer Institute for Reliability and Microintegration, Germany 


\section{Arc Interruption II}

Chair: P. Slade

Co-Chair: T. Schoepf

10.1

Modelling of the Interruption Process in $\mathrm{SF}_{6}$ High Voltage Circuit Breaker and Preliminary Study of the Arc Characteristics for Different Gases.

Jian Zhang, Xi'an Jiaotong University, China

Shenli Jia, Xi'an Jiaotong University, China

Xingwen Li, Xi'an Jiaotong University, China

Pengwei Liu, Xi'an Jiaotong University, China

Zongquin Shi, Xi'an Jiaotong University, China

Lijun Wang, Xi'an Jiaotong University, China

10.2

Research on Contact Force Pairing of MCCB with Double Repulsive Contact Structure

Liang Ji, Xi'an Jiaotong University, China

Degui Chen, Xi'an Jiaotong University, China

Yingyi Liu, Xi'an Jiatong University, China

Weixiong Tong, Xi'an Jiaotong University, China

10.3

Rotational Drive of Break Arcs Using a Permanent Magnet Embedded in an Electrical Contact .

Junya Sekikawa, Shizuoka University, Japan

Takayoshi Kubono, Shizuoka University, Japan

10.4 Experimental Study on Arcing Process and Contact Erosion under

Transverse Magnetic Field.

Guofu Zhai, Harbin Inst. of Tech., China

Xue Zhou, Harbin Inst.of Tech., China

Xiaodong Zhao, Harbin Inst. of Tech., China

Chengyan Liu, Guilin Aerospace Electronics Ltd., China

Fubiao Luo, Guilin Aerospace Electronics Ltd., China

\section{Arc Interruption III}

Chair: J. Witter

Co-Chair: B. Balme

11.1

An Experimental Study on Influences of Silicon-Type and Non-Silicone-Type Polymeric Cured Materials on Contact Resistance Characteristics of Relay Contacts.

Makoto Hasegawa, Chitose Institute of Science \& Technology, Japan

Yoshiyuki Kohno, Kaneka Corporation, Japan

Hiroshi Ando, Kaneka Corporation, Japan

11.2 Optical Investigations of High-Current Vacuum Arc Behavior on Spiral-Shaped and Cup-Shaped RMF-Contacts

Christian Wolf, University of Technology Braunschweig, Germany

Michael Kurrat, University of Technology Braunschweig, Germany

Manfred Lindmayer, University of Technology Braunschweig, Germany

Ernst-Dieter Wilkening, University of Technology Braunschweig, Germany

Dietmar Gentsch, ABB AG, Germany 


\title{
MEMS
}

Chair: T. Schoepf

Co-Chair: B. Malucci

J.W. McBride, University of Southampton, United Kingdom

E.M. Yunus, University of Southampton, United Kingdom

S.M. Spearing, University of Southampton, United Kingdom

12.2

\author{
Maxime Vincent, Schneider Electric Industries, France \\ Laurent Chiesi, Schneider Electric Industries, France \\ Patrick Rousset, Schneider Electric Industries, France \\ Christophe Lapiere, Schneider Electric Industries, France \\ Christophe Poulain, CEA-Léti, Minatec, France \\ Laurent Carbone, Schneider Electric Industries, France \\ Frédéric Houze, Laboratoire de Génie Electrique de Paris, France \\ Jérôme Delamare, Grenoble Electrical Engineering Lab, France
}

An Original Apparatus for Endurance Testing of MEMS Electrical Contact Materials...

.285

12.3 Experimental Validation of a 2-D Constriction Resistance Model at the Microscale

Lise Bilhaut, CEA-LETI MINATEC, France

Christophe Poulain, CEA-LETI MINATEC, France

Romain Anciant, CEA-LETI MINATEC, France

Laurent Duraffourg, CEA-LETI MINATEC , France

12.4 Improved Micro-Contact Resistance Model that Considers Material Deformation, Electron Transport and Thin Film Characteristics

Ronald A. Coutu, Jr., Air Force Institute of Technology, United States

John W. McBride, University of Southampton, United Kingdom

LaVern A. Starman, Air Force Institute of Technology, United States

Contact Resistance in Flat Thin Films

M. B. Read, Massachusetts Institute of Technology, United States

J. H. Lang, Massachusetts Institute of Technology, United States

A. H. Slocum, Massachusetts Institute of Technology, United States

R. Martens, FormFactor Inc., United States

\section{Materials/Surface Finish II}

Chair: J. Zhang

Co-Chair: B. Rickett 
Tetsuya Ito, AutoNetworks Technologies, Ltd., Japan

Yoshiyuki Nomura, AutoNetworks Technologies, Ltd., Japan

Yasuhiro Hattori, AutoNetworks Technologies, Ltd., Japan

13.3 Investigation on Mechanism of Creep Corrosion of Immersion Silver Finished Printed Circuit Board by Clay Tests

Yilin Zhou, Beijing University of Posts and Telecommunications, China

Michael Pecht, University of Maryland, College Park, United States

13.4

Electrical Resistance of Connectors with Different Contact Finishes under

Low Level Conditions.

J. Song, University of Applied Sciences, Germany

H. Schmidt, Tyco Electronics AMP GmbH, Germany

Author Index follows page 338 


\section{FOREWORD}

Welcome to the $55^{\text {th }}$ IEEE Holm Conference. For all of us, 2009 has been a very trying year. The global financial crisis has taken its toll on our customers, our businesses and our personal psyche. Many of colleagues are still suffering the effects of cut backs and job losses. The scale and speed of this crisis is something that two years ago would have been considered science fiction. And yet, through all of the turmoil and panic, our community has put together a very impressive technical program for the 2009 Conference. We have 51 papers covering three days dealing with both theoretical and practical considerations and investigations discussing devices operating at both the nanoamp as well as kiloamp ranges. Considering last year's 53 papers were the most papers ever presented in a non ICEC co-sponsored Conference, this years' 51 papers is truly a monumental accomplishment. The credit for this achievement belongs to the strong heritage of technical excellence fostered by the Holm Conference and most importantly, to a passionate group of scientists and engineers who continue to advance our technical understanding of electrical contact phenomenon. I would like to personally thank everyone who has helped us put this program togetherincluding, but not limited to, the authors, the reviewers, the Holm Technical Program committee, the Antler awards committee, our conference sponsors and the IEEE staff. A special thanks goes to Brett and ZK for great stewardship of the TPC and all its activities. I would also like to thank Alicia Zupeck from IEEE Meeting \& Conference Management for keeping us on track.

This year, we are also fortunate to have Professor Frank Mücklich of the Materials Engineering Center Saarland (MECS) at Saarbrücken University as the Morton Antler award recipient. Professor Mücklich will discuss an exciting new technology, FIB-Nanotomography, used to re-create 3D microstructural images of a contact surface. This analytical tool should provide us with a much better fundamental understanding of the relationship between microstructure and contact performance.

This year marks the first time Vancouver has been the host city for our Conference and I hope that everyone gets a chance to enjoy this wonderful city. Vancouver is known for its spectacular panoramic views of both the Pacific Ocean and the Canadian Rockies. It also offers a great variety of wonderful restaurants, beautiful gardens, and interesting museums. In addition to being the Host City for the 2010 Winter Olympics, it was recently named one of the top travel destinations in North America. So I encourage all the conference attendees to enjoy the city (after Conference hours, of course).

Next year, the IEEE Holm Committee and the International Conference on Electrical Contacts (ICEC) Advisory Council will co-sponsor the $56^{\text {th }}$ Holm Conference and the $25^{\text {th }}$ ICEC Conference which will be held in Charleston, SC. By then, hopefully the worst of this crisis will be past and we can all enjoy some "Good Old Fashioned Southern Hospitality."

Thanks,

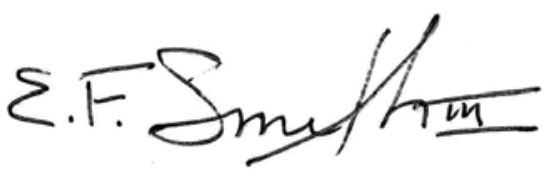

Ed Smith

Chairman, $55^{\text {th }}$ IEEE Holm Conference 


\section{MORTON ANTLER LECTURE}

The Morton Antler Lecture is an annual lecture given at the IEEE Holm Conference on a topic of special interest to the electrical contact community. This lecture series was established in honor of Dr. Morton Antler, a long time member of the Holm Steering Committee and participant in the Holm Conference. Dr. Antler was a distinguished scientist and lecturer in the fields of electrical contacts, tribology, corrosion, and electrodeposition. A list of past awardees is as follows:

$\begin{array}{ll}2008 & \text { T.J. Schoepf } \\ 2007 & \text { J.V.R. Heberlein } \\ 2006 & \text { W. Johler } \\ 2005 & \text { J. A. Wafer } \\ 2004 & \text { R. S. Mroczkowski } \\ 2003 & \text { N. L. Traub } \\ 2002 & \text { W. H. Abbott }\end{array}$

2009 Morton Antler Lecture

Nanotomography of Electrical Contacts - New Insights by High Resolution 3D Analysis of Local Material Degradation

Frank Mücklich, Head of Chair for Functional Materials, Saarland University and Chairman (CEO) of the Steinbeis Research Center MECS (Material Engineering Center Saarland), Saarbruecken, Germany

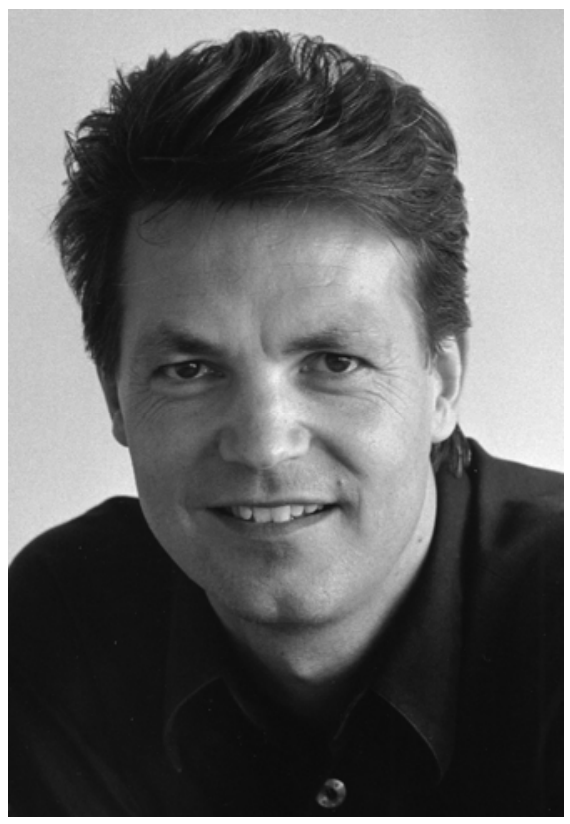

Frank Mücklich was born in Dresden in 1959 and studied Physical Metallurgy at the Bergakademie Freiberg. From 1990-95 he was Research Group Leader at the Max Planck Institute for Metals Research in Stuttgart. From there, he answered a call to create the Chair of Functional Materials at Saarland University in 1995. He headed the German Technical Committee for Materialography (1998-2001) and the Advisory Committee of the German Society for Materials Science and Engineering (DGM) (2005-2008). Presently, he is Board member of the DGM. Mücklich is, among other things, Head Referee for Materials Research in the "Feodor Lynen" Excellence Program of the Alexander von Humboldt Foundation. He heads the European School of Materials in Saarbruecken and is the European coordinator of the elite EU Materials Research Degree Program. For his work in the field of research, he was awarded the Masing Memorial Award (1995), the Alfried Krupp Award (1998), the Werner Koester Prize (2006), and the Roland Mitsche Prize (2008). His scientific interests are: new methods for 3D analysis of materials microstructures in the micro, nano and atomic scale; surface functionalization in the scale of microstructures by advanced Laser patterning techniques as well as new materials for electrical application. To strengthen his cooperation with industry, Prof. Mücklich got extensive funding of the Saarland government in 2009 and founded the "Materials Engineering Center Saarland (MECS)", a research center of the Steinbeis Foundation which is located on the Saarbrücken University Campus. 


\section{ERLE SHOBERT PRIZE PAPER}

The Holm Conference Prize Paper Award was established in 1970. At that time, the Conference Steering Committee recognized that at each Conference there was at least one paper that stood out from the others in its technical content and quality of presentation. Therefore, the Prize Paper Award Committee was established. The Committee's purpose is to review each paper, listen to each presentation and then judge which paper should receive the Prize Paper Award. The award is presented to the authors of the Prize Paper at the following year's Holm Conference.

\section{IEEE Erle Shobert Prize Paper}

\section{Influence of Grafting Properties of Organic Thin Films for Low Level Electrical Contacts Protection}

Sophie Noël, LGEP/Supelec, France; David Alamarguy, LGEP/Supelec, France; Alessendro Benedetto, CEA, France; Pascal Viel, CEA, France; and Mirela Balog, CIMMA/CNRS, France

The Previous Awardees have been:

Basic Investigations for Switching of RF Signals W. Johler

Glowing Contact Physics

J. J. Shea

Pore Corrosion Model for Gold Plated Copper Contacts

A.C. Sun, H.K. Moffat, D.G. Enos, and C.S. Glauner

Electrical Conduction through Small Contact Spots

R. Timsit

A Novel Concept for Fault Current Tolerable Contactors

X. Zhou and M. Little

Gassing Arc Chamber Wall Material Effect on Post Current-Zero Recovery Voltage Breakdown J. J. Shea

3D-Simulation of Arc Motion Between Arc Runners Including the Influence of Ferromagnetic Material M. Lindmayer and M. Springstubbe

Contact Current Distortion Due to Tunnel Effect

E. Takano

Make Arc Erosion and Welding in the Automotive Area

L. Morin, N. Ben Jemaa, D. Jeannot, J. Pinard, and L. Nedelec

Contact Physics of Gold MicroContacts for MEM Switches

D. Hyman and M. Mehregany

Intermittence Detection in Fretting Corrosion Studies of Electrical Contacts

S.R. Murrell and S.L. McCarthy

Surface Film Resistivity of $\mathrm{Cu}$ and $\mathrm{Cu}$-Alloy Crosswires

D. Kuhlmann-Wilsdorf and A. M. Rijke

Connector Contacts: Corrosion Inhibiting Surface Treatment for Gold Plated Finishes

W. H. Abbott and M. Antler

Effects of Wipe on Contact Resistance of Aged Surfaces

B. Malucci

Model Predictions of ARC Cathode Erosion Rate Dependance on Plasma Gas and on Cathode Material

X. Zhou, J. Heberlein and E. Pfender

Effect of Axial Magnetic Field Upon the Development of the Vacuum Arc Between Opening Electric Contacts M.B. Schulman, P.G. Slade and J. Heberlein

Enhanced Sulfur Segregation in Plastically Deformed OFHC Cu and the Effect of Surface Segregated Sulfur on Electric Contact Resistance

R. Kothari and R. Vook 
1988 A New Rapid Screening Method for Silicones by Size Exclusion Chromotography A.J. Muller and R.L. Opila

The Development and Performance Characteristics of Mixed Flowing Gas Test Environments W.H. Abbott

1986 In Situ Examination of Segregation and Wear Processes of Precious Metal Electrical Contact Alloys

L.E. Pope and D.E. Peebles

1985 Comparison of Reignition Properties of Several Ag/W, Ag/WC and Ag/Mo Electrical Contact Materials

C.H. Leung, H.J. Kim and P.C. Wingert

1984 Titanium Nitride: A Potential Ceramic Low Energy Contact Material

J. Nickerson, C. Ernsberger, and A.E. Miller

1983 The Vacuum Interrupter Contact

P.G. Slade

Sliding Wear of Inlay Clad Metals and Electrodeposited Cobalt-Gold

M. Antler and E.T. Ratliff

1981 Cathode Spot Mechanism in the Transition from a Vacuum Metal Vapor Arc to a Nitrogen Arc-Erosion and Surface Contaminations Arced Copper Cathodes

D.R. Porto and C.W. Kimblin

1980 The Elucidation of Frictional Polymer Structure Using Fourier Transform, Infrared Spectroscopy and Pyrolysis Gas Chromatography/Mass Spectroscopy

L. Seebles and B.T. Reagor

Effect of Alkali Additives on the Reignition Voltage of Ag/CdO

M. Lindmayer

1978 Wear of Gold Electrodeposits: Effect of Substrate and of Nickel Underplate M. Antler and M.H. Drozdowicz

High Current Brushes I: Effect of Brush and Ring Materials

J.L. Johnson and L.E. Moberly

1975 Silicon Oils on Electrical Contacts*b2Effects, Sources and Countermeasures

C.A. Russell and N.M. Kitchen

1974

A Correlation of Material Toughness, Thermnal Shock, Resistance, and Microstructure of High Tungsten, Silver-Tungsten Composite Materials G.J. Witter and W.R. Warke

1973 The Use of Ceramic Matrices Infiltrated with Silver for Electric Contact Applications

P.G. Slade, R. Kossowsky, R.G. Aspden and R.J. Bratton

1972 Tribological Properties of Gold for Electric Contacts M. Antler

1971

The Effect of Electrode Material on the Initial Expansion of an Arc in Vacuum P.G. Slade and M.F. Hoyaux

1970 Scanning Electron Microscopy of Contact Surfaces Before and After Arcing M.J. Vasile and B.W. Kammlott 


\title{
Application Feasibility of Detecting Glowing Contacts Using Acoustic Sensing Technology
}

\author{
Xin Zhou, Senior Member, IEEE, Jerome Hastings, and Thomas J. Schoepf, Senior Member, IEEE \\ Eaton Corporation, U.S.A
}

\begin{abstract}
:
Glowing contacts may be precursors to arc faults and could lead to fire hazards even before arc faults occur. As of today there is no cost effective technology available to detect overheated or glowing contacts.

Experiments have been conducted in order to investigate both the acoustic characteristics associated with glowing contacts and the feasibility of their detection under various conditions by employing acoustic sensing technology. Experimental results show that the propagation or attenuation of the acoustic signal through electrical circuits is significantly influenced by size and length of electric wires and types of electric connections. The results also further demonstrate that the acoustic signal can propagate effectively through large cable conductors and busbars, hence enable glowing contacts detection for industrial applications. However, the attenuation of acoustic signal through electrical circuit could be a potential limitation for residential applications.
\end{abstract}

\section{Key words:}

Glowing contacts, acoustic sensing, arc fault, electrical contacts.

\section{Introduction}

Glowing contacts are considered to be one of the potential causes for residential arc faults and fire hazards [1]-[4]. Meese et al [1] showed that loose current-carrying $\mathrm{Cu}-$ connections subjected to mechanical vibrations could cause arcing or fretting. This in turn induces corrosion at contact interfaces to form copper oxide, $\mathrm{Cu}_{2} \mathrm{O}$. The high resistance of $\mathrm{Cu}_{2} \mathrm{O}$ may lead to overheating of the electrical contact due to Joule's heating, hence generate a glowing contact.

To date, no economical technology is available that can detect glowing contacts before they develop into arcing faults or fire hazards. Due to relatively low voltage drops across glowing contacts (usually lower than $1 \mathrm{Vrms}$,) it is very difficult to detect glowing contacts based on the characteristics of current and voltage waveforms. Commonly used methods to detect any glowing contacts are either using infrared sensing technology or manual examination of electric connections. Methods based on time or frequency domain reflectrometry (TDR or FDR) have been investigated to detect loose connections and the associated contact resistance change [11]. The published research focuses on automotive applications and shows that the technology has a potential to provide a resolution of $50 \mathrm{~m} \Omega$ based on the measurement of reflection coefficient of a high frequency pulse. This will allow monitoring degradation of electrical connectors. However, the technology not only requires high frequency transmitters and receivers, but also needs quite sophisticated data processing. The employed high frequency range may also limit the detection ability in the presence of long electric wires and circuit impedance due to the attenuation of the high frequency signals. Meanwhile, acoustic sensing technology has been widely used for structural integrity diagnostics and prognostics. Feasibility studies were carried out to use acoustic sensing technology for electric wire integrity monitoring [6], [7]. The studies concluded that the aging of wire insulation could be detected by monitoring the phase spectrum of the acoustic signals propagating through the electric wire. Acoustic sensing technology is also used to detect corona discharges inside power station transformers. Acoustic sensing technology has been applied to detect series arc faults under industrial conditions by Ref. [5]. The technology showed the ability to detect arcing events. The speed of detection, however, might be too slow in order to mitigate potential arc faults. Unlike arc faults, glowing contacts usually take a relatively long time to form. Glowing contacts, if detected in their early stage, do not require as immediate mitigation as arc faults. Therefore, the relatively slow acoustic sensing technology may be considered for glowing contact detection.

In this paper, investigations on acoustic signal characteristics and propagation through electric wires induced by glowing contacts are reported. The acoustic attenuation as a function of electric wire length, wire size, various electric connections and acoustic signal frequency has been investigated.

\section{Experimental Setup}

Two experimental fixtures have been employed to investigate both the acoustic noise generated by glowing contacts and the acoustic attenuation characteristics of typical residential and industrial wiring and connections. The experiments are described in the following sections. Detailed information on the experimental setup can be found in reference [8].

\subsection{Glowing contact experimental setup}

The test setup, which was used to create glowing contacts, is schematically shown in Fig. 1. AWG \#12 solid Cu-wires and AWG \#00 stranded $\mathrm{Cu}$-wires were used in all experiments. Glowing contacts were generated between two opposing $\mathrm{Cu}-$ wires as described in previous work [4] and [5], of which one 
$\mathrm{Cu}$-wire was connected to a $115 \mathrm{VAC}$ power supply and the other one was wired to the neutral potential. Both the current through the glowing contact and the voltage across the opposing $\mathrm{Cu}$-wires were recorded using a National Instrument data acquisition system with a sampling rate of $100 \mathrm{kHz}$. Various electrical loads were used.

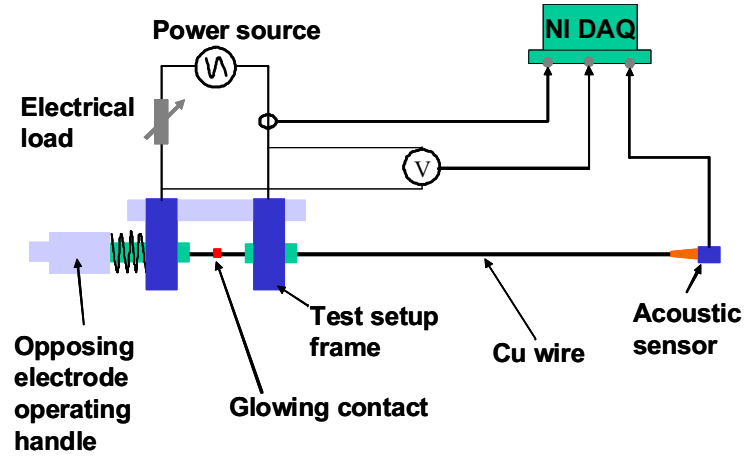

Fig. 1 Schematic layout of acoustic characterization for glowing contacts

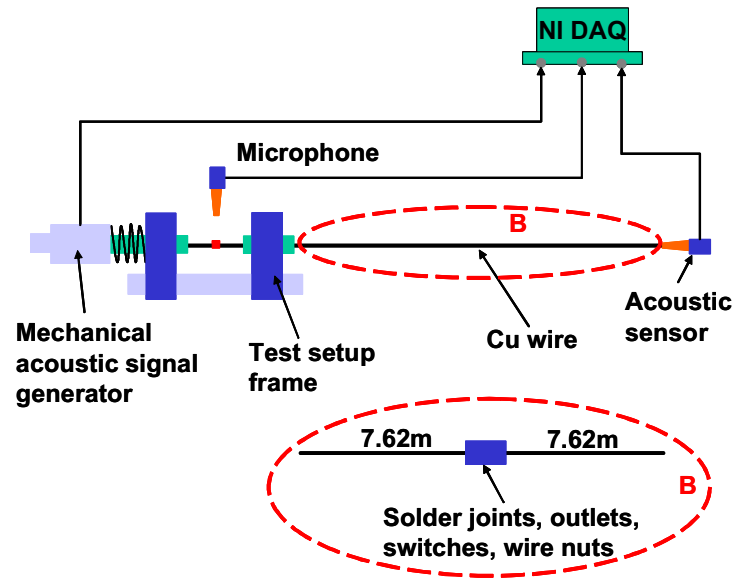

Fig. 2 Schematic layout of acoustic attenuation characterization test setup

To record the acoustic signal at the ends of the $\mathrm{Cu}$-wire, two different piezoelectric acoustic sensors were used. The acoustic signal was guided by an acoustic adapter from the cross section end of the wire to the piezoelectric sensor.

\subsection{Acoustic signal attenuation experimental setup}

Figure 2 shows the schematic layout of the experimental setup. Table 1 lists different electric wire sizes (AWG) and lengths investigated.
Table 1 Different electric wire size and length investigated

\begin{tabular}{|c|c|c|}
\hline & Type & Length $(\mathrm{m})$ \\
\hline AWG \#12 & solid & $7.62-40$ \\
\hline AWG \#00 & stranded & $7.62-45.72$ \\
\hline
\end{tabular}

First, acoustic attenuations through electric wires were measured. The lengths of these electric wires varied from $7.62 \mathrm{~m}$ up to $45.72 \mathrm{~m}$. Then acoustic attenuations through different electric connections such as commonly used in residential and industrial applications and depicted in Fig. 8 were measured. A $7.62 \mathrm{~m}$-long electric wire was attached to each side of the electric connection. The acoustic attenuation value of a $15.24 \mathrm{~m}$-long electric wire served as a reference for comparison purposes.

For this set of experiments, a mechanical acoustic signal generator as shown in Fig. 2 was employed to eliminate variations of the acoustic signals generated by glowing contacts. One of the opposing wires was attached to the acoustic signal generator. It provided a mechanical impact at one end of the other electric wire. The time delay between the microphone pick-up adjacent to the impact end of the electric wire and the piezoelectric sensor at the other end of the wire allowed estimation of acoustic propagation speed in electric wires and cables. A more detailed description on measuring acoustic attenuation through electric wires and cable was reported by the authors in previous work [8].

\section{Results and Discussions}

\subsection{Glowing contact and its associated acoustic signal}

The formation of glowing contacts can be categorized in three different modes such as shown in Fig. 3 and was reported in Ref. [4]. Mode I typically occurs at currents of 5 Amps or higher. Due to Joule heating and the instability of the molten $\mathrm{Cu}_{2} \mathrm{O}$, the glowing contact would momentarily rupture into an arc, and then re-form a glowing contact. Strong acoustic signals are generated by both arcing and glowing contacts. Mode II is characterized by moving glowing filaments. This typically occurs at currents below 5 Amps. In this mode, a constricted moving bright spot and glowing filament bridging the two opposing $\mathrm{Cu}$-wires can be observed. In this case, periodic acoustic signals can be observed. Mode III is the completely molten state. There is no constricted moving bright spot, no glowing filament and no arcing. This mode typically occurs after Mode I or Mode II has formed with sufficient $\mathrm{Cu}_{2} \mathrm{O}$ in the contact interface. Usually very weak or no acoustic signals can be detected.

Figure 4 depicts a glowing contact in Mode I formed at the interface between an AWG \#12 Cu-wire and a $\mathrm{Cu}$-connector. The voltage trace indicates that the intermittent arcing occurred almost at the peak of the current. The strong acoustic signal appeared at both arcing and non-arcing periods. 


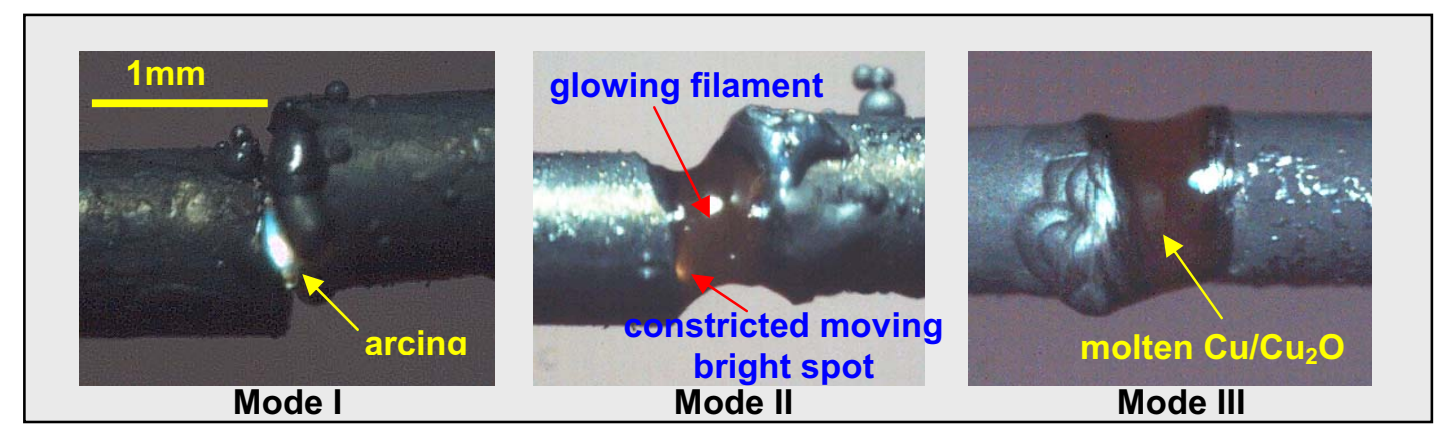

Fig. 3 High-speed images of Mode I: a Cu glowing contact in arcing/glowing mode at 7.5A $\mathrm{A}_{\text {rms; }}$ Mode II: a Cu glowing contact in moving glowing contacts mode at $3.5 \mathrm{~A}_{\mathrm{rms}}$; Mode III: a glowing contact in pure glowing mode at $7.5 \mathrm{~A}_{\mathrm{rms}}$, [4]
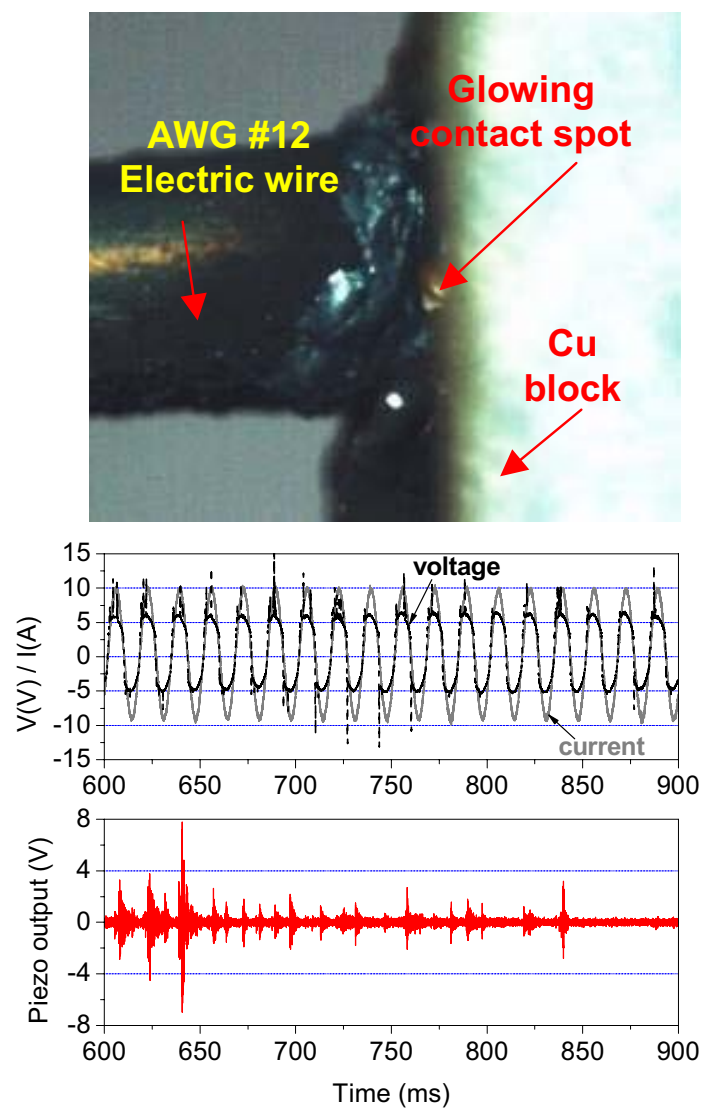

Fig. 4 Glowing contact of Cu-wires in Mode I at 7.5 Arms, and its V-, I-, and acoustic waveforms

The acoustic signal generated by both arcing faults and glowing contacts in an electrical circuit with a dimmer load is graphed in Figure 5. The microphone located adjacent to the opposing electrodes picked up the airborne acoustic signal when arcing occurred. However, there was no airborne

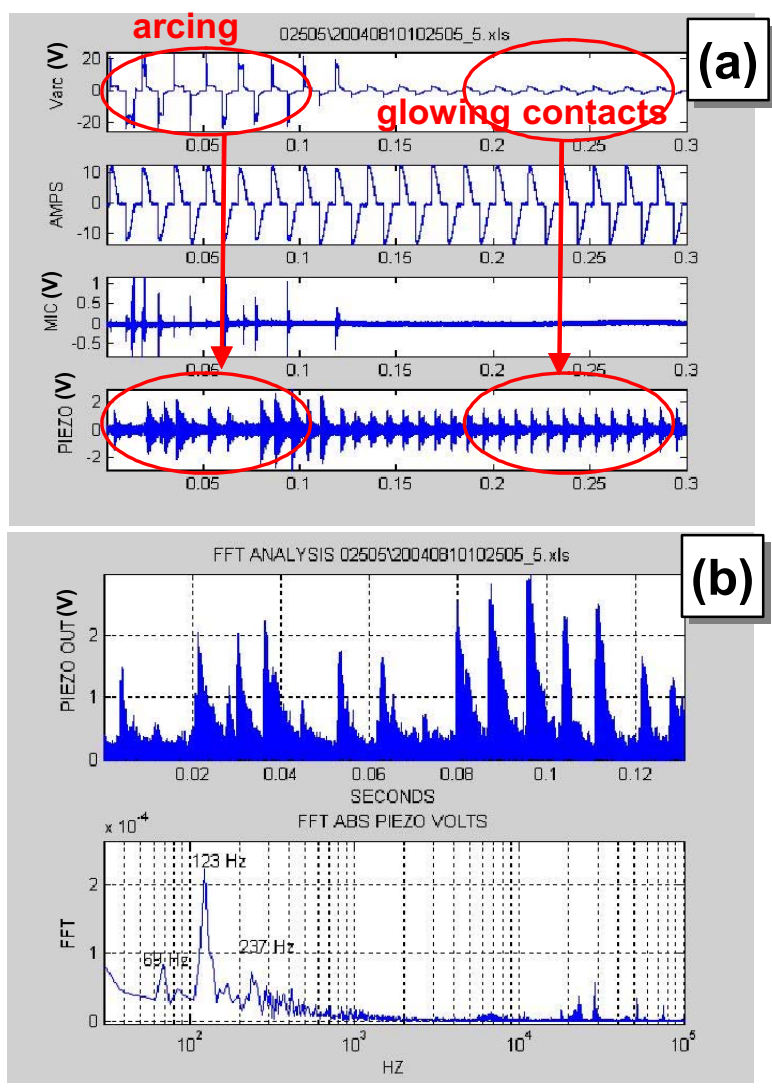

Fig. 5 (a) V-, I-, and acoustic signals recorded by both, the microphone and the piezoelectric sensor, in a dimmer circuit;

(b) Respective acoustic frequency spectrum from the piezoelectric sensor

acoustic signal recorded when glowing contacts formed. Meanwhile, the piezoelectric sensor could still detect the acoustic signal generated by the glowing contacts. Fig. $5 \mathrm{~b}$ indicates that the acoustic signal has strong frequency content around $120 \mathrm{~Hz}$, its harmonics and sub-harmonics. This 
characteristic is one of the key features used for acoustic detection of glowing contacts [9].

Based on the experimental results, the acoustic signals induced by glowing contacts could be caused either by intermittent arcing impact in glowing contact Mode $I$ or by contraction and expansion of the molten $\mathrm{Cu}_{2} \mathrm{O}$ in the contact interface due to the AC Joule heating in Modes II and III. The mechanisms for generating acoustic signals by glowing contacts are not fully understood and would need further investigation.

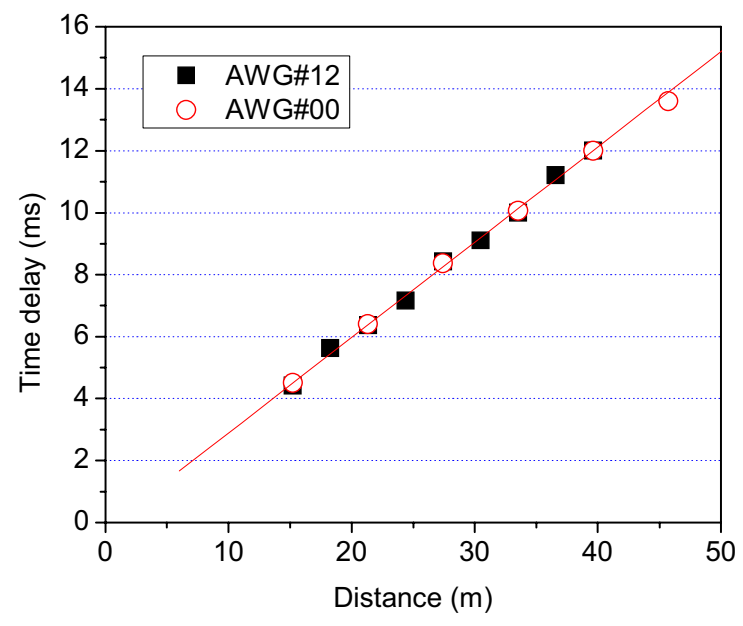

Fig. 6 Propagation time as a function of distance through the AWG \#12 wire and AWG \#00 stranded cable

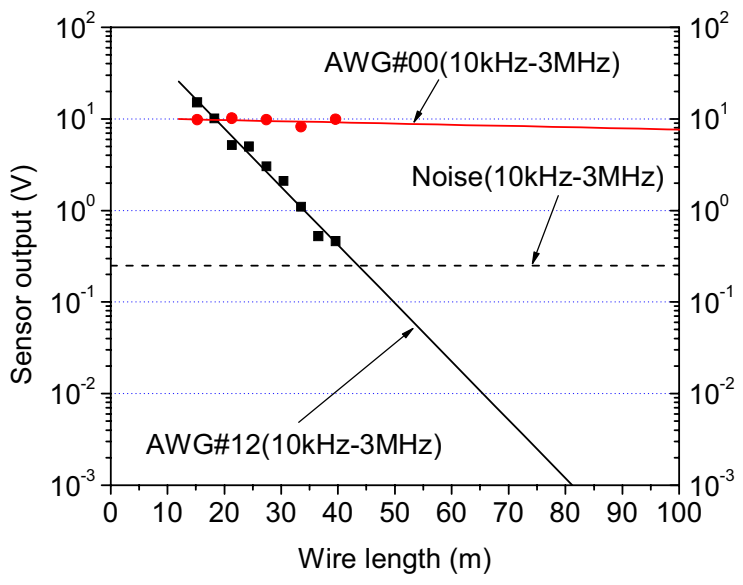

Fig. 7 Intensities of acoustic signals and sensor baseline noise as a function of wire length and size

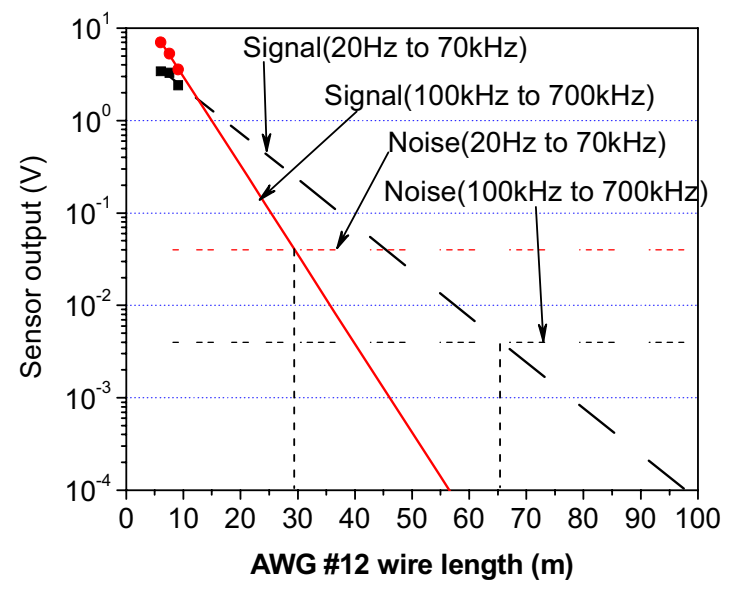

Fig. 8 Intensities of acoustic signals and sensor baseline noise as a function of wire length and frequency
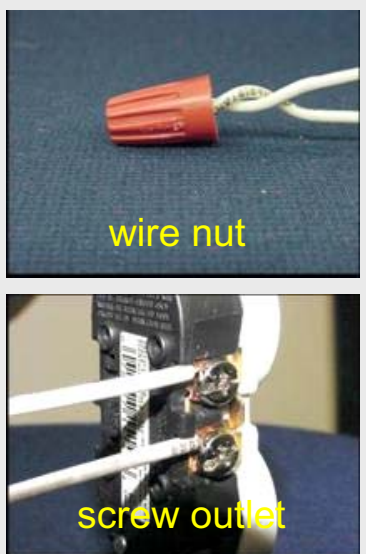
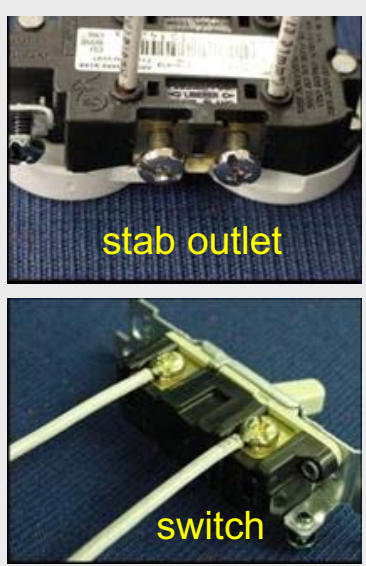
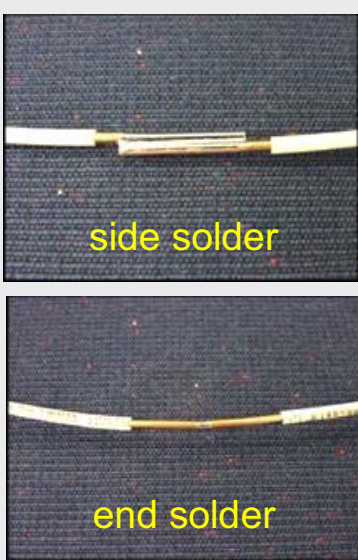

Fig. 9 Photos of various electric connections for AWG\#12 solid Cu-wires 


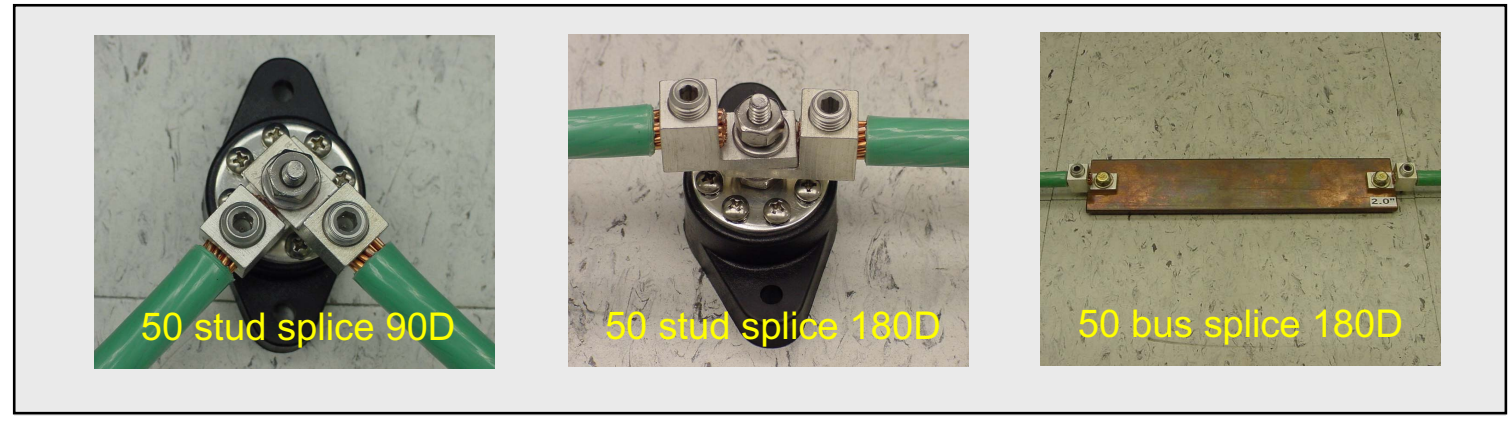

Fig. 10 Splice connections for AWG\#00 stranded Cu-wires

\subsection{Characterization of acoustic signals through $\mathrm{Cu}$ - wires}

Acoustic propagation speed, attenuation rate and the effect of frequency on attenuation through $\mathrm{Cu}$-wires as well as various connectors are reported in this section.

The average speed of acoustic signals through both AWG \#12 solid $\mathrm{Cu}$-wires and $\mathrm{AWG} \# 00$ stranded $\mathrm{Cu}$-wires can be derived from Fig. 6. The derived results show that acoustic signal has a propagation speed at $3331 \mathrm{~m} / \mathrm{sec}$. This agrees relatively well with the acoustic propagation speed in copper of $3700 \mathrm{~m} / \mathrm{s}$ as published by other researchers [10]. It shows that the acoustic propagation speed is not affected by the frequency of the acoustic signal. At this speed, it will take about $9 \mathrm{~ms}$ for the acoustic signal to reach the acoustic sensor when a glowing contact or an arc fault occurs $30 \mathrm{~m}$ away from the sensor. With current technology it will take more than $9 \mathrm{~ms}$ for a detection algorithm to confirm a glowing contact or an arc fault after their initiation. This is a major challenge that prevents this technology from being applied for arc fault detection today.

Figure 8 shows the acoustic attenuation through both AWG \#12 solid and AWG \#00 stranded $\mathrm{Cu}$-wires employing the piezoelectric sensor with a bandpass filter from $10 \mathrm{kHz}$ to 3 $\mathrm{MHz}$. Significant acoustic attenuation through AWG \#12 Cuwires was observed, while there was minimal acoustic attenuation through AWG $\# 00 \mathrm{Cu}$-wires. The acoustic signal can only propagate up to about $45 \mathrm{~m}$ through an AWG \#12 electric wire before the signal to noise ratio reaches 1 . The acoustic signal attenuation as a function of both wire length and acoustic frequency components is shown in Fig. 8. Acoustic signal intensities in two groups of frequencies were recorded for $6 \mathrm{~m}-, 7.6 \mathrm{~m}$ - and $9.1 \mathrm{~m}$-long AWG \#12 wires. Extrapolations of curve-fittings of the data provide acoustic signal attenuations and approximate distances that the acoustic signal can travel. The $20 \mathrm{kHz}-70 \mathrm{kHz}$ acoustic component has an attenuation rate of $0.99 \mathrm{~dB} / \mathrm{m}$, and the $100 \mathrm{kHz}-700 \mathrm{kHz}$ component one of $1.92 \mathrm{~dB} / \mathrm{m}$. Whereas the $20 \mathrm{kHz}-70 \mathrm{kHz}$ component can propagate up to $65 \mathrm{~m}$, the $100 \mathrm{kHz}-700 \mathrm{kHz}$ component can only propagate up to $30 \mathrm{~m}$ before the signal to noise ratio equals value 1 . This indicates that high frequency acoustic components have a much higher attenuation rate than low frequency components. Therefore, monitoring acoustic signals at low frequencies may be most effective for detecting acoustic signals generated by glowing contacts.

\subsection{Attenuation of acoustic signals through electric connections}

For the investigations on acoustic signal attenuation through different electric connections such as commonly used for both residential and industrial applications in North America as depicted in Figures 9 and 10, the test setup shown in Fig. 2 was employed. The statistical variation of measurements with the piezoelectric sensor falls within the range of $\pm 1.3 \mathrm{~V}( \pm 2.5 \mathrm{~dB}$.

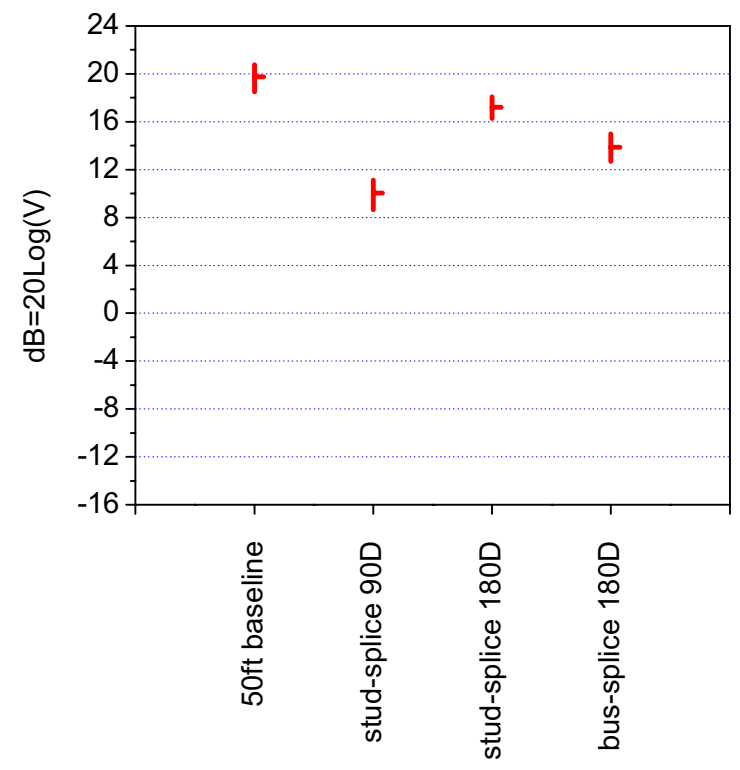

Fig. 11 Acoustic intensities in reference to baseline acoustic noise for various electric connections with AWG\#00 Cuwires 


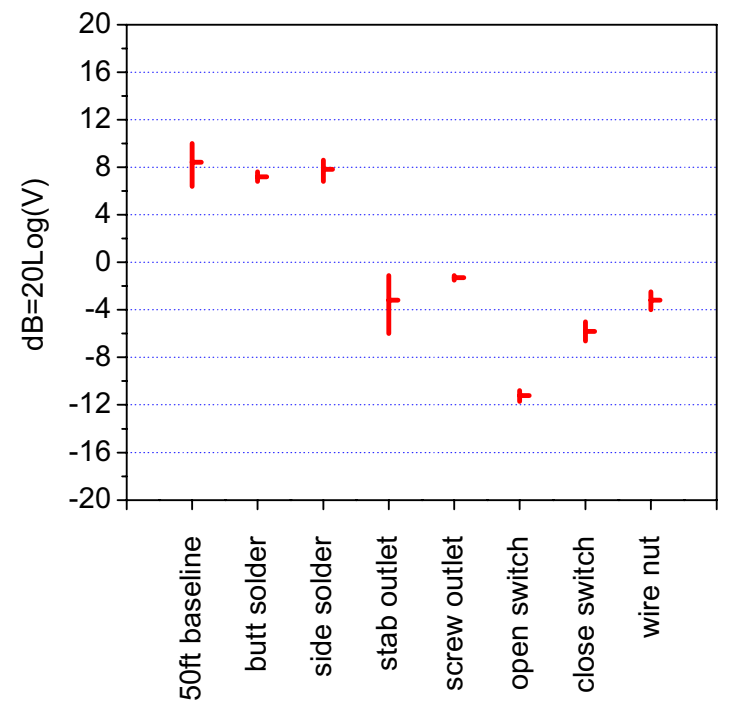

Fig. 12 Acoustic intensities in reference to baseline acoustic noise for various electric connections with AWG\#12 Cuwires

Figure 11 shows the acoustic attenuation through three different electric connections with AWG $\# 00$ wires. The stud splice connection with 90 degree turn has the highest attenuation at about $10 \mathrm{~dB}$, whereas the straight stud splice connection has only about $2 \mathrm{~dB}$ attenuation. The bus splice connection has about $6 \mathrm{~dB}$ attenuation. Soldered connections mating AWG \#12 wires yielded the least acoustic attenuation at around $1 \mathrm{~dB}$, followed by screw connection with attenuation at about $10 \mathrm{~dB}$, as indicated in Fig. 12. Wire nuts and stab connections caused similar acoustic attenuation at about $13 \mathrm{~dB}$, and about $15 \mathrm{~dB}$ attenuation could be measured for a closed switch. An open switch produced the highest attenuation at about $20 \mathrm{~dB}$ as expected.

\section{Conclusions}

Investigations on acoustic sensing of glowing contacts were carried out for both residential and industrial applications under the consideration of acoustic attenuation as a function of electric wire size, length and various electric connections. The results lead to the following conclusions:

a) Glowing contacts generate acoustic signals that can propagate along $\mathrm{Cu}$-wires. For $\mathrm{AC}$ residential applications, the acoustic signal is modulated by the power frequency, its harmonics and sub-harmonics. This characteristic may be used for the detection of glowing contacts.

b) High frequency acoustic components have a much higher attenuation rate than low frequency components. Hence, monitoring acoustic signals at low frequencies may be most effective for detecting acoustic signals generated by glowing contacts. c) Electric connection causes acoustic attenuation. The soldered connections yielded lowest attenuation of about $1 \mathrm{~dB}$. Stab, screw and wire nut connections produced high attenuation of 10-15 dB. Hence, in electrical installations where such high attenuation connector types are common such as residential applications, the acoustic sensing technology might turn out not to be feasible.

d) Considering the strong acoustic attenuation through thin $\mathrm{Cu}$-wires such as AWG \#12, attenuation of the acoustic signal could be a potential limitation for employing acoustic sensing technology to detect glowing contacts in residential applications. However, acoustic sensing technology could be an effective means for glowing contacts detection in industrial applications due to the minimal attenuation of acoustic signal through large $\mathrm{Cu}-$ wires and busbars such as AWG \#00.

\section{Acknowledgments}

The authors wish to thank Mr. Dale Gass for performing part of the evaluation tests, Mr. Robert Yanniello, director of engineering of Eaton's PDO-A, and Mr. William Beatty, director of engineering of Eaton's LVCD, for their helpful discussions, comments and supports.

\section{References}

[1] W.J. Meese and R.W.. Beausoliel, "Exploratory Study of Glowing Electrical Connections," NBS Building Science Series 103, October 1977.

[2] J. Sletbak, R. Kristensen, H. Sundklakk, G. Navik, and M. Runde, "Glowing Contact Area in Loose Copper Wire Connections," IEEE Transactions on CHMT, Vol. 15, No. 3, pp. 244-248, 1992.

[3] J. Shea, "Conditions for Series Arcing Phenomena in PVC Wiring," 51th IEEE Holm Conference on Electrical Contacts, pp. 167-175, September 2005.

[4] X. Zhou and J. Shea, "Characterization of Glowing Contact Using Optical Emission Spectroscopy" 53rd IEEE Holm Conference on Electrical Contacts, pp. 167-175, September 2007.

[5] C.S. Maroni, R. Cittadini, Y. Cadoux, and M. Serpinet, "Series Arc Detection in Low Voltage Distribution Switchboard Using Spectral Analysis," International Symposium on Signal processing and its Applications, Kuala Lumpur, Malaysia, August 2001

[6] E.I. Madraras and R.F. Anastasi, "Evaluating Thermally Damaged Polyamide Insulated Wiring (MIL-W-81381) with Ultrasound," NASA Technical Report Doc ID 20030014498, 2002.

[7] R.F. Anastasi and E.I. Madraras, "Aging Wire Insulation Assessment by Phase Spectrum Examination of Ultrasonic Guided Waves," SPIE 8th international Symposium on NDE for Health Monitoring and Diagnostics, Conference 5046, pp. 29, San Diego, CA, March 2003.

[8] X. Zhou, J. Hastings and Th. Schoepf, "Detection of Glowing Contacts Employing Acoustic Sensing Technology," 24 $4^{\text {th }}$ International Conference on Electrical Contacts, Saint Malo, France, June 2008

[9] X. Zhou, J. Hastings and J. Zuercher, "Electrical switching apparatus and method including fault detection employing acoustic signature," US patent 7,148,696, 2006.

[10] The Science and Applications of Acoustics, $2^{\text {nd }}$ Edition, by D.R. Raichel, Springer, 2006.

[11] F. Loete, S. Noel, M. Olivas Carrion and F. Auzanneau, "Feasibility of the Detection of Vibration Induced Faults in Connectors by Reflectometry," $24^{\text {th }}$ International Conference on Electrical Contacts, pp. 440-443, June 2008 


\title{
A Method for Residential Series Arc Fault Detection and Identification
}

\author{
Dongwei Li, Zhengxiang Song, Jianhua Wang, Yingsan Geng, Huilin Chen, Li Yu, Bo Liu \\ State Key Laboratory of Electrical Insulation \& Power Equipment \\ School of Electrical Engineering, Xi'an Jiaotong University \\ Xi'an, P.R.China \\ E-mail: lidw84@gmail.com
}

\begin{abstract}
Because of electrical problems such as aging cables and loose connections, arc faults occur. Generating high temperature and discharging molten metal, arc faults finally lead to electrical fires. Every year such fires bring great loss and damage. It is identified that conventional protecting technique is unable to break a circuit in the situation when a brief arc fault occurs and the arc current is below thermal or instantaneous trip levels. The paper describes how experiments on series arc faults (defined in UL1699) are performed. Then waveforms of arc faults in circuits with purely resistive loads are contrasted to those with inductive loads of different power factors. They are different especially on the 'shoulders' and the rising edges followed, which are analyzed both in time and frequency domain. Similar waveforms of normal working household appliances like air conditioners and computers, which have special characteristics, are also studied in the paper. Although the waveforms of computers are not easily distinguished from those of arc faults, it is found that the harmonic components of them are different. A method combining DFT and DWT, which is relatively simple, reliable and easily performed in Arc Fault Circuit Interrupters, is proposed to identify hazardous arc from normal waveforms.
\end{abstract}

Keywords-Arc fault, series arc, residential circuit breaker, DFT, DWT, UL1699

\section{INTRODUCTION}

During a typical year, home electrical problems account for 67,800 fires, 485 deaths, and $\$ 868$ million in property losses [1]. Electrical fire accidents have become a very important part of fire accidents. Conventional circuit breakers and fuses play an effective role in protecting residential electrical circuits from over-current and short-circuit conditions. But they cannot prevent fire accidents caused by arc faults with relatively low current.

Defined by UL1699, arcing is "a luminous discharge of electricity across an insulating medium, usually accompanied by the partial volatilization of the electrodes" and arcing fault is "an unintentional arcing condition in a circuit" [2]. This distinguishes arcing fault from other arc created by some normal loads such as air-conditions and drills. Arc faults in residential circuits can be divided into two categories: series and parallel. In a 120VAC circuit, fires can happen in circuits with a current as little as $200 \mathrm{~mA}$ [3]. According to Paschen's Law, gas pressure and gap distance between the electrodes are two of the most important factors in relation to the breakdown voltage to generate an arc. It has been shown that other factors such as decomposing and charring of wire insulation, combustible gases generated will also help sustain an arc to cause a fire [4]. And it was found that the probability of ignition at $240 \mathrm{~V}_{\mathrm{rms}}$ was significantly greater than that at $120 \mathrm{~V}_{\mathrm{rms}}$ at the same corresponding normal current value [5].

ARC-FAULT CIRCUIT-INTERRUPTER (AFCI) is defined as "a device intended to mitigate the effects of arcing faults by functioning to deenergize the circuit when an arc-fault is detected" [2]. Branch/Feeder AFCIs have been introduced to the market for years and were previously required to protect the bedroom; the 2008 National Electric Code (NEC) now requires Combination AFCIs to be installed in additional areas of the home, including dining rooms, living rooms, and other habitable areas [6]. Branch/Feeder and Combination AFCIs must meet the same requirements for high current arc detection: the Point Contact Arc Test and the Carbonized Path Arc Interruption Test in UL1699. However, the Combination AFCIs must also meet the requirements of the Carbonized Path Arc Clearing Time Test. Combination AFCIs can provide series arc protection and therefore mitigate the effect of low current arcs not only in the installed wiring but also in the two wire cord sets and power-supply cords connected to the outlets [7].

In recent years, researchers have developed various systems and experiments to study the behavior and nature of arc faults [3][4][5][8][9]. D.Kolker, S.Campolo and N.DiSalvo studied the role of temperature rating of the insulation in affecting ignition and the possibility of ignition in different conditions [5]. John J. Shea exhibited how the overheated PVC insulation decomposes and produces ignitable gases to promote fire happening [4]. And he also set up experiment to prove that the probability of ignition at $240 \mathrm{~V}_{\mathrm{rms}}$ is higher than $120 \mathrm{~V}_{\mathrm{rms}}$ [8]. Xin Zhou and John J. Shea attempted to identify the mechanism that creates the moving glowing filament with attached constricted moving bright spot [9]. Carlos E. Restrepo put forward a method based on AC load current information at $60 \mathrm{~Hz}$ and the broadband RF content that series arc faults produce, a relatively simple method for detecting parallel arc faults was also shown [3]. Although so much research was performed on arc faults detection, there hasn't been a relatively simple and reliable method to detect series arc faults. Most of the current research focused on how arc faults take place and conditions that lead to a fire, which is helpful but not a direct solution for the series arc faults problem. 
Some previous investigations showed that arc faults detection needs to be accurate in distinguishing arc faults from normal loads operation such as air-conditions, vacuum cleaners, washing machines and switches [3][10], which is in accord with Unwanted Tripping Tests in UL1699 [2]. The current of series arc faults, usually below $30 \mathrm{~A}$, is in the same range of normal residential loads'. No nuisance trip is allowed to happen in arc faults detection. So arc faults detection circuit is supposed to be intelligent to recognize different kinds of arc faults. As it is difficult to predict where arc faults will take place, an economic way is to detect arc faults through $\mathrm{AC}$ wires. Another requirement for arc faults detection is being actualized simply so that it can be achieved easily and reliably.

To meet with those requirements, a method combining DFT and DWT is presented in the following sections. As the main problem is to identify arc faults from normal domestic appliance operations, the method is performed on both arc faults and normal domestic appliance loads to show its discriminating capability. The arc faults mentioned previously are in the circuit with purely resistive loads. Arc faults in circuits with inductive loads of different power factors are also shown in this paper.

\section{EXPERIMENT}

\section{A. Series Arc Faults in Circuits with Resistive and Inductive Loads}

The experimental setup was used to obtain series arcing voltage and current data for the $220 \mathrm{~V}_{\mathrm{rms}}, 50 \mathrm{~Hz}$ electrical system. There are many ways to create arc faults, such as carbonized path and point contact by cutting [2]. To get the waveforms of a long lasting and continuous arc fault, an arc generator was chosen as the arc faults producing device.

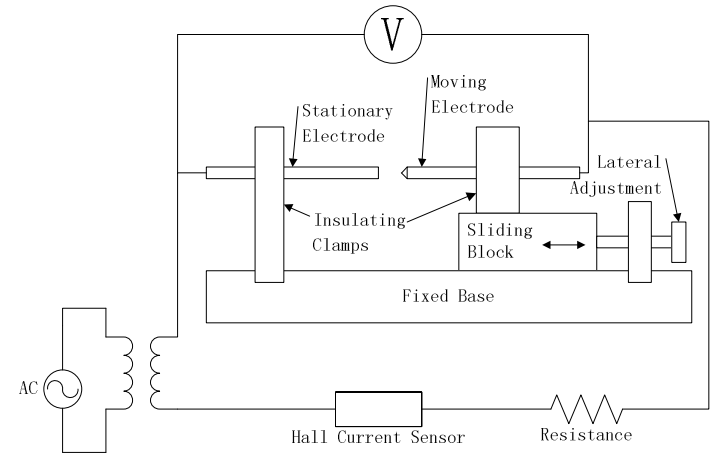

Figure 1. Schematic of experimental setup used to capture waveforms of arc faults current and voltage.

The schematic of experiment is shown in Fig.1. A 100A thermal-magnetic (T-M) breaker was connected to a $5 \mathrm{kVA}$, $50 \mathrm{~Hz}$ transformer with available short-circuit current limited to $20 \mathrm{~A}_{\mathrm{rms}} \pm 10 \%$ at $220 \mathrm{~V}_{\mathrm{rms}}$, that was wired to the stationary electrode of the arc generator. The arc generator consisted of a stationary electrode and a moving electrode as specified in the UL1699 standard [2]. The moving electrode was connected through a variable resistive load to the opposite polarity terminal. A Hall Current Sensor (CSM020GT with sensitivity of 20A/V) and a Voltage Probe (Tektronix model P2200) were used in conjunction with a Tektronix oscilloscope (models TDS 2014) to acquire waveforms of arc faults current and voltage. The main function capabilities of the oscilloscope were used to record waveforms and save data of arc current and voltage.

Both electrodes were $8 \mathrm{~mm}$ diameter copper rods, which better emulated arc faults happening in the residential electrical system. With the electrodes touching each other, the circuit was closed. Then the electrodes were separated slowly using the lateral adjustment until arcing occurred. Usually at first the arc would extinguish when the electrodes were separated to a little distance. After the moving electrode had been slid back and forth for several times, steady arc faults could be produced. In the test circuit with inductive loads, a variable inductive load needed to be added in series with the variable resistance. The experimental parameters are listed in Table 1 below.

TABLE I. PARAMETERS FOR SERIES ARC FAULTS EXPERIMENT

\begin{tabular}{|c|c|c|c|}
\hline $\boldsymbol{U}_{\boldsymbol{r m s}}(\boldsymbol{V}) / \boldsymbol{f}(\boldsymbol{H z})$ & $\boldsymbol{R}(\boldsymbol{\Omega})$ & $\boldsymbol{L}(H)$ & Power Factor \\
\hline $220 / 50$ & 44 & 0 & 1 \\
\hline $220 / 50$ & 4.2 & 0.059 & 0.22 \\
\hline $220 / 50$ & 11.5 & 0.059 & 0.52 \\
\hline $220 / 50$ & 17.7 & 0.045 & 0.78 \\
\hline
\end{tabular}

\section{B. DFT and DWT Analysis Experiments}

The discrete Fourier transform (DFT) is one of the specific forms of Fourier analysis. It transforms signals from the original function (which is often a function in the time domain) to another, which is called the frequency domain representation. The sequence of $\mathrm{N}$ complex numbers $\mathrm{x}[0], \mathrm{x}[1]$, $\ldots, \mathrm{x}[\mathrm{N}-1]$ is transformed into the sequence of $\mathrm{N}$ complex numbers $\mathrm{X}[0], \mathrm{X}[1], \ldots, \mathrm{X}[\mathrm{N}-1]$ by the DFT according to the formula[11]:

$$
X[k]=\sum_{n=0}^{N-1} x[n] e^{-j \frac{2 \pi k n}{N}} \quad \mathrm{k}=0, \ldots, \mathrm{N}-1
$$

Where $e^{-\frac{2 \pi j}{N}}$ is a primitive N'th root of unity, the complex number $\mathrm{X}[\mathrm{k}]$ represents the amplitude and phase of the different sinusoidal components of the input "signal" $x[n]$.

DFT requires a discrete input function whose non-zero values have a finite duration. In general, almost all arc faults take place intermittently. It is possible for the current in the circuit to be normal in a few periods during arc faults occurrence. And it is also very likely that the circuit breaks for several periods during the "arc faults time", and surely the current is zero. So arc current and voltage waveforms are impossible to have periods. A typical period, during which arc faults occur, is analyzed by DFT. Firstly characteristic of arc faults in frequency domain is discovered in a typical period, and then it is easy to deal with those untypical periods. For example, numbers of arc faults during a short time, such as eight periods, are counted and a threshold number should be made by the circuit breaker to decide whether to cut off the circuit or not.

\footnotetext{
This paper is supported by Program for New Century Excellent Talents in University (NCET -07-0675)
} 
A discrete wavelet transform (DWT) is any wavelet transform for which the wavelets are discretely sampled. The wavelet transform is a powerful mathematical tool to analyze a non-stationary signal (e.g. power system transient signals) as it gives a good localization in time at high frequencies and good localization in frequency at low frequencies [12]. The advantage of the transform is that the band of analysis can be fine adjusted so that high frequency components and low frequency components can be detected precisely [13]. The DWT is defined as [11]:

$$
D W T[m, k]=\frac{1}{\sqrt{a_{0}^{m}}} \sum_{n} x[n] g\left[\frac{k-n a_{0}^{m}}{a_{0}^{m}}\right]
$$

In this paper, simulation analysis was conducted by MATLAB (edition R2009a). Through simulation comparison, it was found that the wavelet db1 (Haar wavelet) had good effect in decomposing and reconstructing analyzed signal, so $\mathrm{db} 1$ was adopted as the basic function of Wavelet Analysis in this paper. Haar wavelet transform was used to pair up waveforms of arc faults current to discriminate the special scales in this paper. It made up the disadvantages of DFT in location information to enrich the arc faults detection method.

The Haar wavelet's mother wavelet function $\psi(t)$ can be described as [14]:

$$
\psi(t)= \begin{cases}1 & 0 \leq t<1 / 2, \\ -1 & 1 / 2 \leq t<1, \\ 0 & \text { otherwise. }\end{cases}
$$

\section{RESULTS AND DISCUSSION}

\section{A. Time Domain Results}

As it is mentioned before, the difficulty of detecting series arc faults is that its current value lies in the same range of normal loads. The method attempted to identify arc faults in the frequency domain. In Fig.2 (a), CH1 in blue traced the waveform of voltage between the two electrodes and $\mathrm{CH} 2$ in yellow traced the current waveform, that were the typical traces of an arc fault. The flat parts in the current trace are called "shoulders". Immediately after every "shoulder" the current rose up onto a stage value, during which breaking down across the gap of $\mathrm{Cu}$ electrodes happened. And there was a corresponding voltage drop at the same time. $\mathrm{CH} 1$ in yellow traced the current waveform in Fig.2 (b)(c) and $\mathrm{CH} 2$ in blue traced the voltage waveform in Fig.2 (b). The current waveform in Fig.2 (b) looked much like that in Fig.2 (a). The peak current value would be in the same range of that of arc faults if more computers were connected. So it was hard to discriminate them in the time domain. Fig.2 (c) shows that the current waveforms were more like sine wave during the starting process of the air conditioner, although there were 9-10 periods of high magnitude which was almost five times value of normal working current. Air conditioners are representative household appliances of those having electro motors, such as electric drills, vacuum cleaners and refrigerators.

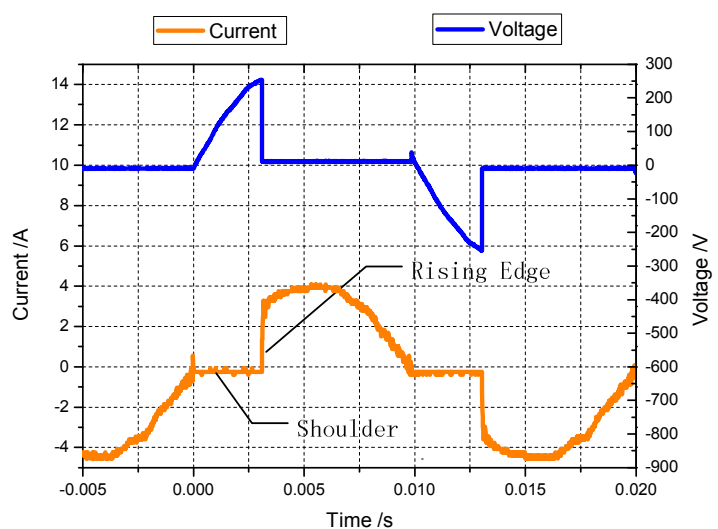

a). Arc faults with a peak current of $5 \mathrm{~A}$

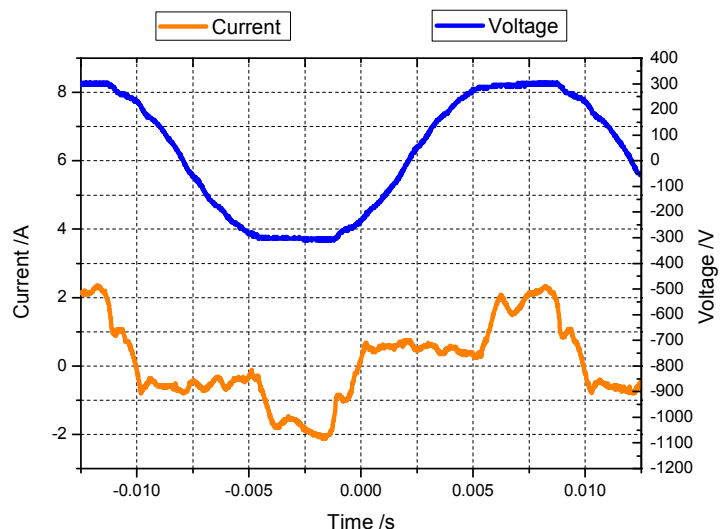

b). Waveforms of four computers

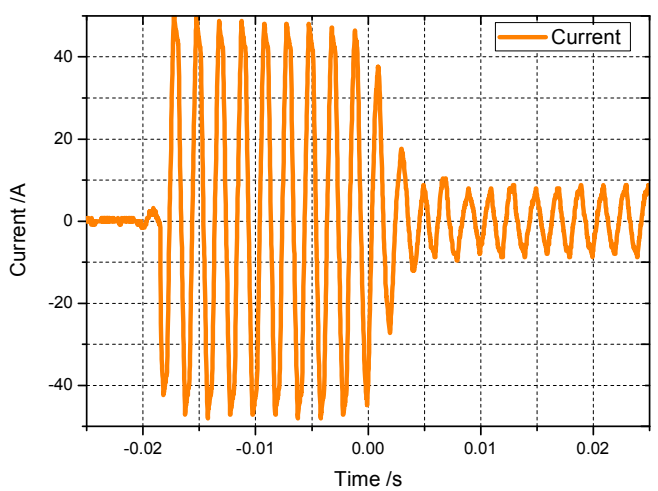

c). Starting process of an air conditioner

Figure 2. Current and voltage waveforms of three different loads: a) Series arc faults in the circuit with purely resistant loads b) four sets of computers and CRT displays c) a 4000W MD air conditioner

Usually in residential and industrial electrical systems, arc faults happen in circuits with inductive loads. For example, common loads such as televisions, refrigerators, electronic energy saving lamps and induction cookers have about 0.7-0.9 power factors. This investigation attempted to depict how the inductive loads of different power factors influenced arc faults 
and how to detect these arc faults. In Fig.3, $\mathrm{CH} 2$ in blue traced the waveform of power supply voltage and $\mathrm{CH} 1$ in yellow traced the current waveform of the circuit. As the power factor increased, "shoulders" tended to be flatter and remained more persistent. This was because the voltage was relatively lower in the "shoulder" time when the power factor was higher.

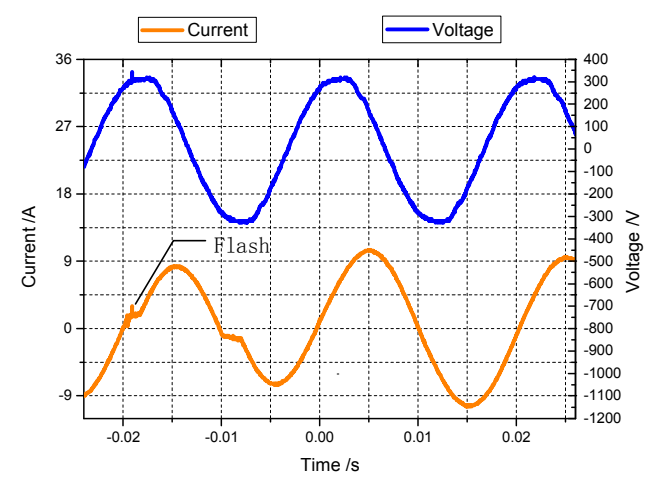

a). $P F=0.22$

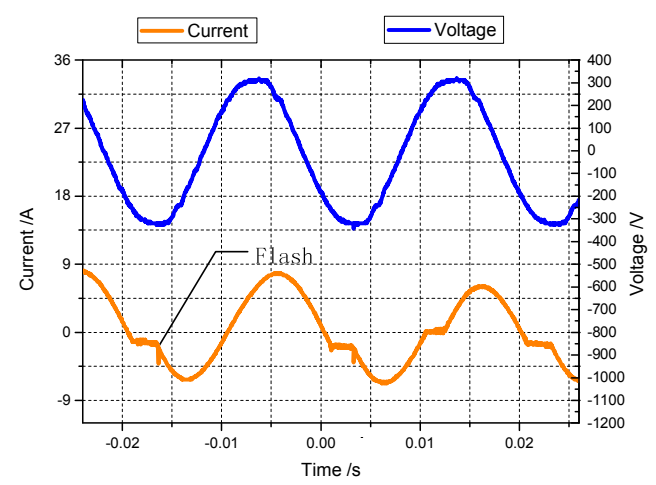

b). $P F=0.52$

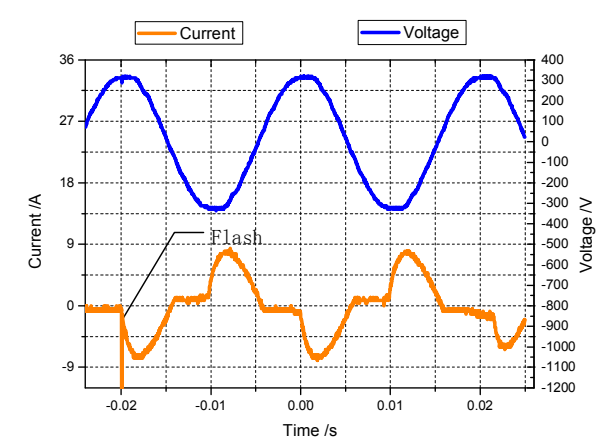

c). $P F=0.78$

Figure 3. Current and voltage waveforms of arc faults in circuits with different power factors (PFs) inductive loads

Fig.3 (a) shows that the power supply voltage was nearly at the peak point in the "shoulder" time. So it was easy for the gap between electrodes to be broken down by such a high voltage.
Then the "shoulders" were not so flat and persisted a relatively short time. Another reason is that the inductance in the circuit tended to keep the current not to break off. It is obvious that rising rate of arc current' edges in inductive circuit in Fig.3 was smaller than that in purely resistive circuit in Fig.2 (a).

\section{B. Frequency Analysis}

Fig.4 shows that the relative magnitude of the harmonic component of computers' current compared to the fundamental component $(50 \mathrm{~Hz})$ was rather high; especially the third harmonic component $(150 \mathrm{~Hz})$ reached nearly 0.6 of the fundamental component. While the relative magnitudes of the harmonic component of the air conditioner and arc faults, including those in inductive circuit, were not so distinct. This was a great difference between current of arc faults and the computer load. The third harmonic component $(150 \mathrm{~Hz})$ value of arc faults in purely resistive circuit was nearly 0.25 of the fundamental component value. And the relative magnitudes of the third harmonic component $(150 \mathrm{~Hz})$ of the air conditioner (Fig.4 (c)) and arc faults in inductive circuit (Fig.4 (d)(e)(f)) were respectively $0.06,0.03,0.13$ and 0.16 . So it was difficult to distinguish arc faults from air conditioner loads by DFT analysis results, especially in a circuit with inductive loads. But it works in identifying the computer load. It was the inductance in the circuit that filtered or reduced the harmonic component of arc faults current.

DWT analyzes signals in various scales without losing location information. To keep the same sampling frequency as arc faults in inductive circuit, the first two and a half periods of the starting process of air conditioners (Fig.6) were analyzed here. Fig.5 shows that the high-frequency detail sequence d 2 of arc faults' current in a purely resistive circuit (Fig.5 (a)) was significantly different from the high-frequency detail sequence d1 of air conditioners' current (Fig.5 (c)). They were chosen because the sampling frequency of arc faults in purely resistive circuit was twice as that of air conditioners in this paper. The $\mathrm{d} 2$ relative magnitude of arc faults current's rising edge in a purely resistive circuit compared to the original peak current was $0.25(0.05 / 0.2)$, while the d1 relative magnitude of the air conditioners current was under $0.05(0.1 / 2)$. A threshold could be set on the relative magnitude level to distinguish them. That was the way to differentiate purely resistive arc faults from air conditioner. But for arc faults in inductive circuits, other characteristics should be used for the detection. It was found that arc faults occurred commonly with "flash" in the current waveforms. And it was possible to detect arc faults in low PF circuits by "flash points" in the high-frequency detail sequence d1 as shown in Fig.5 (d) (e) (f). Although a "flash point" was also found in the starting process of the air conditioner, it seldom happened and the relative magnitude was also quite low.

Fig.7 shows the flowchart of detecting arc faults process. The program was written in MATLAB and the wavelets used were those implemented in MATLAB. 


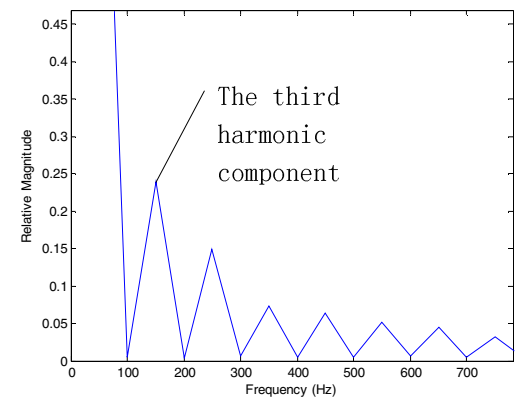

a ).

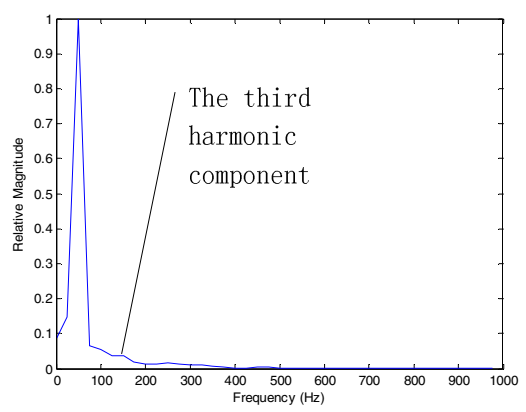

d).

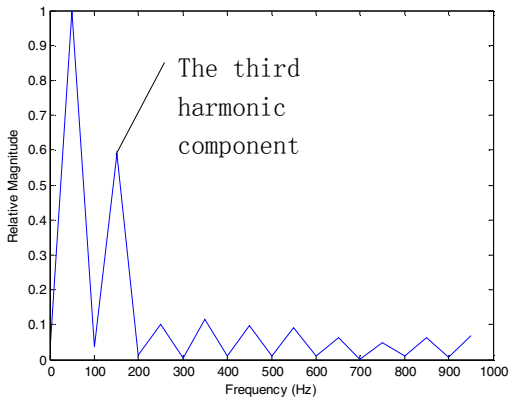

b).

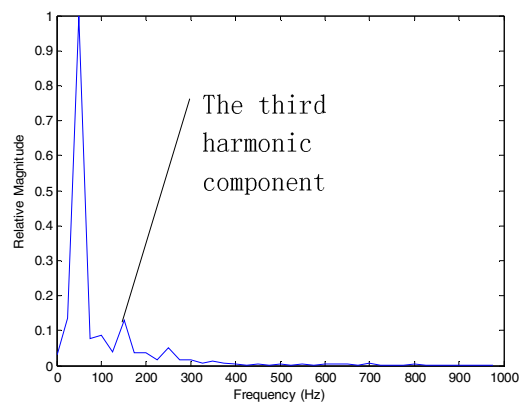

e).

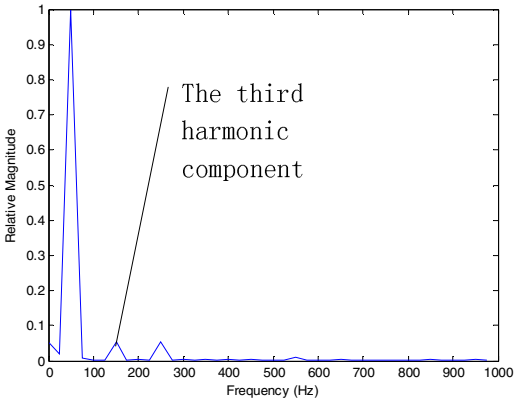

c).

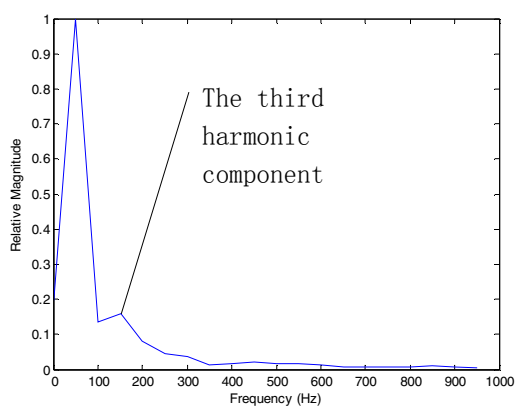

f).

Figure 4. Analysis results of different loads current with DFT: (a). Series arc faults in the circuit with purely resistant loads, (b). four sets of computers and CRT displays, (c). a 4000W MD air conditioner, (d). (e). (f). arc faults in circuits with different power factors ( $\mathrm{PF}=0.22,0.52,0.78$ respectively) inductive loads

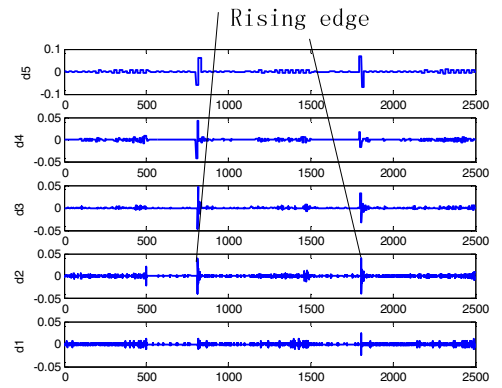

a ).

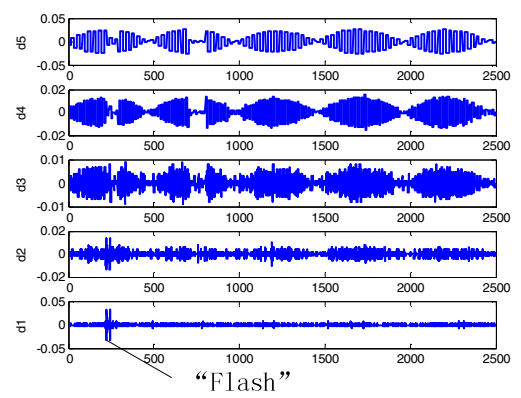

d).

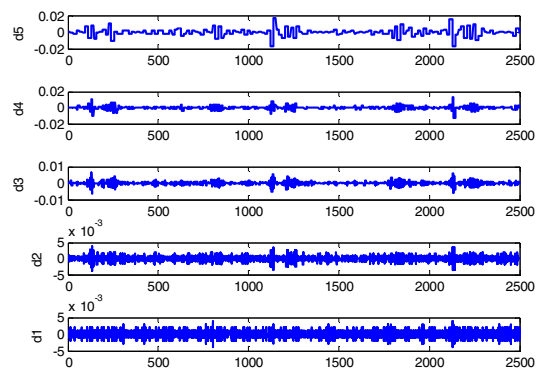

b).

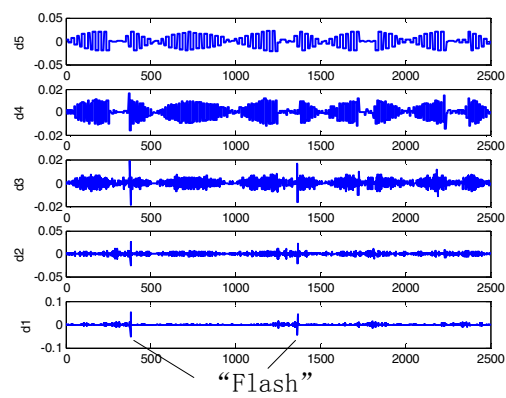

e ).

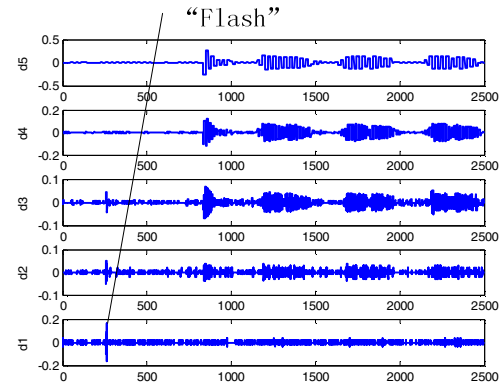

c).

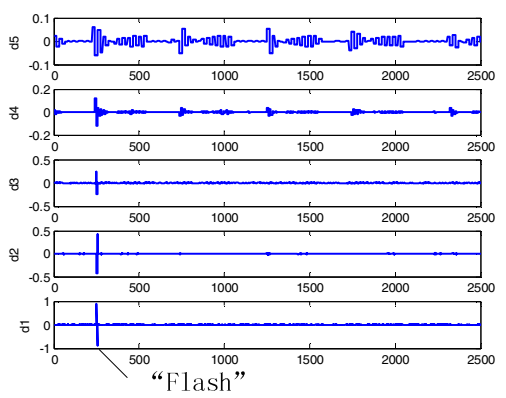

f).

Figure 5. Analysis results of different loads current waveforms with DWT: (a). Series arc faults in the circuit with purely resistant loads, (b). four sets of computers and CRT displays, (c). a 4000W MD air conditioner, (d). (e). (f). arc faults in circuits with different power factors ( $\mathrm{PF}=0.22,0.52,0.78$ respectively) inductive loads 


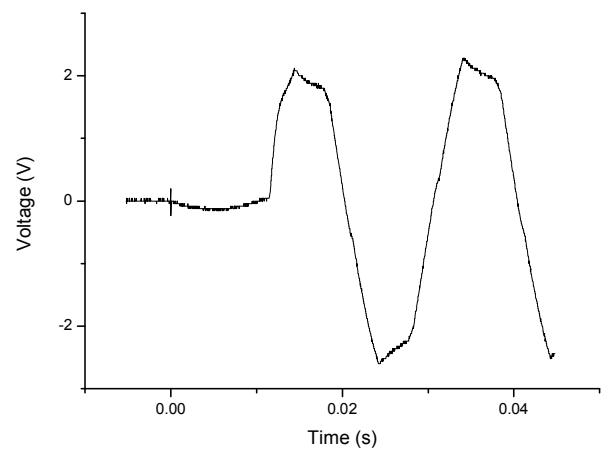

Figure 6. Current waveform of first two and a half periods of the starting process of air conditioners

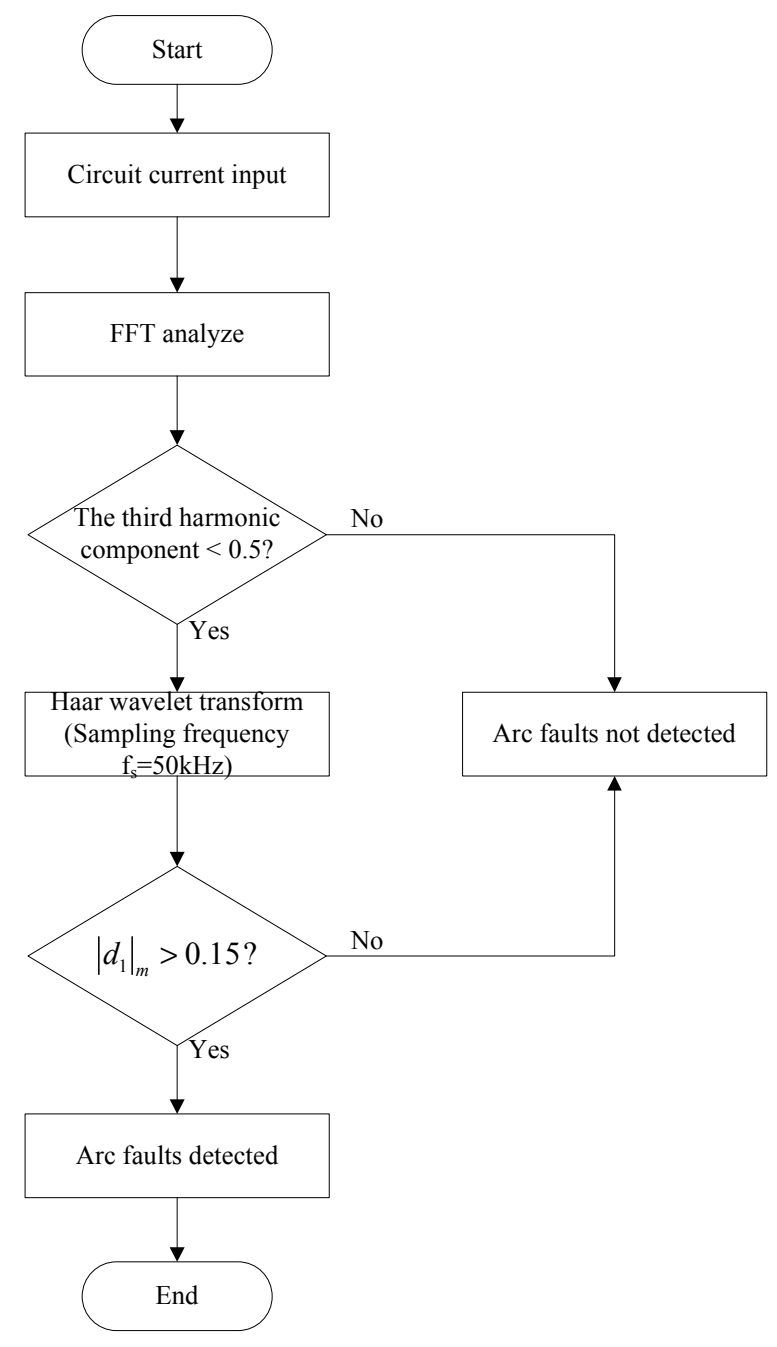

Figure 7. Flowchart of detecting arc faults process

\section{CONCLUSIONS}

This paper focused on series arc faults detection. A new method for the identification of residential series arc fault based on MATLAB was presented. The current waveforms of arc faults in circuits with purely resistive loads and inductive loads of different PFs were shown in this paper. It was found that arc faults have unique features in their curves of current. As described in this paper, firstly various arc faults can be distinguished from the computer loads' performances by the third harmonic component in the DFT analysis results of circuits current. Then by the results of DWT analysis, various arc faults can be differentiated from air conditioners' performances as described in the last section. It has been found that the proposed technique gives satisfactory results in detecting faults. It was also found that arc faults were often accompanied by "flash" in current traces. More investigations may be needed to study on this phenomenon.

However, one difficult point of this paper is the optimum selection of wavelet function, which is accomplished by comparison of multiple simulation results in this paper. How to select an optimum wavelet function for a special signal accurately and quickly is a problem worthy of further research.

This study must be regarded as an initial attempt to identify series arc faults in circuits with loads of different PFs. Further investigation is needed to prove that this method can be applied to residential electrical systems with more kinds of loads. With some perfection work, this method can be applied on present circuit protective devices.

\section{ACKNOWLEDGMENT}

The authors would like to thank Mr. Xuandong Wang for devices providing and Dr. Xiaoshe Zhai for support on this study.

\section{REFERENCES}

[1] USFA, 2008. Electrical Fire Safety: A Factsheet on Home Electrical Fire Prevention. United States Fire Administration, http://www.usfa.dhs.gov/citizens/all_citizens/home_fire_prev/electrical. shtm

[2] UL Standard for Safety for Arc-Fault Circuit-Interrupters (UL 1699), April 7, 2006.

[3] Restrepo, C.E, "Arc Fault Detection and Discrimination Methods," 53rd IEEE holm conference on Electrical Contacts, pp.115 - 122, Sept. 2007

[4] J.J. Shea, "Conditions for Series Arcing Phenomena in PVC Wiring," IEEE Transactions on CPT, vol. 30, no. 3, pp. 532-539, Sept. 2007.

[5] D. Kolker, S.Campolo, N. DiSalvo, "A study of Time/Current Characteristics of the Ignition Processes in Cellulosic Material Caused by Electrical Arcing for Application in 240V Arc-Fault Circuit Interrupters," 53rd IEEE Holm Conference Proceedings on Electrical Contacts, Pittsburgh, PA. pp. 105-114, Sept.2007.

[6] 2008 NEC $®$ AFCI Code Language, National Electrical Manufacturers Association (NEMA), 2008 http://www.afcisafety.org/codes.html

[7] Eaton White Paper 2008, "Branch/Feeder \& Combination AFCIs Differences", $\underline{\mathrm{http}: / / \text { www.eaton.com/ecm/idcplg?IdcService }=\text { GET FILE\&dID }=14046}$ $\underline{5}$

[8] J.J. Shea, "Comparing 240 Vrms to 120 Vrms Series Arcing Faults in Residential Wire," 54rd IEEE Holm Conference Proceedings on Electrical Contacts, Orlando, Florida. pp. 218 - 224, 27-29 Oct. 2008.

[9] Xin Zhou, J.J. Shea, "Characterization of Glowing Contacts using Optical Emission Spectroscopy," 53rd IEEE Holm Conference Proceedings on Electrical Contacts, Pittsburgh, PA. pp. 98-104, Sept.2007.

[10] Gregory, G. and Scott, G., "The Arc-Fault Circuit Interrupter: An 
Emerging Product," IEEE Transactions on Industry Applications, Vol. 34, No. 5, pp. 928-933, September/October 1998.

[11] Robertson D.C., Camps O. I., Mayer J.S., "Wavelets and electromagnetic power system transients", IEEE Transactions on Power Delivery, Vol. 11, No. 2, pp.924-930, April 1996.

[12] Megahed A.I., Monem Moussa A., Elrefaie H.B., Marghany Y.M.,, "Selection of a suitable mother wavelet for analyzing power system fault transients," 2008 IEEE Power and Energy Society General Meeting Conversion and Delivery of Electrical Energy in the 21st Century, Pittsburgh, PA, pp. $1-7$, July 2008.
[13] P. Makming, S. Bunjongiit, A. Kunakorn, S. Jiriwibhakorn and M.Kando, "Fault diagnosis in transmission lines using wavelet transforms," IEEE Transmission and Distribution Conference, Yokohama, pp. 2246-2250, Japan, October 2002.

[14] Jiang JA, Fan PL, Chen CS, et al. "A fault detection and faulted-phase selection approach for transmission lines with Haar wavelet transform," IEEE PES Transmission and Distribution Conference and Exposition, VOLS 1-3, Dallas, TX, pp. 285-289 SEP, 2003 


\section{Artificial Low Current Arc Fault for Pattern Recognition in Low Voltage Switchgear}

\author{
Peter Müller \\ Institute of Power Transmission and High Voltage \\ Technology (IEH) \\ Stuttgart, Germany \\ peter.mueller@ieh.uni-stuttgart.de \\ stefan.tenbohlen@,ieh.uni-stuttgart.de
}

\author{
Reinhard Maier \\ Michael Anheuser \\ Siemens AG, Germany \\ reinhard.maier@siemens.com \\ michael.anheuser@siemens.com
}

\begin{abstract}
Low voltage switchgear is usually protected against over-current and short-circuit by fuses and circuit-breakers. However, protection devices are not sensitive enough to detect low current arc faults, which may arise for example by slow loosening of screw or rivet connections in an electrical busbar [11]. Here, the current is within the range of the rated current and limited by the connected load, known as serial arcs. Parallel low current arcs between two phases or phase and ground are also possible and may be initiated by contamination. Both types of failures result in a local heating-up of the busbar material and insulation material and can cause local carbonization and low current arcing. After a certain time this process can result in a short circuit and may damage the whole switchgear. To test the reliability of arc fault protection devices, usually an arc is generated by a short circuit between 2 or more phases. However, it is difficult to test other faults than a very low resistive short circuit with usually high arc current. In this paper, a method will be demonstrated and discussed, how to ignite an arc with a relatively low arc current which is burning for at least $80 \mathrm{~ms}$. By using resistors to limit the arc current and due to special devices to ignite the arc, it is possible to generate arcs up to $1200 \mathrm{~A}$ peak to peak current in a very reliable, reproducible and save way. Using this method, arc fault detection devices, which are able to detect arc faults within the rated current range of the switchgear, can be tested.
\end{abstract}

Keywords- arc fault, switchgear, arc ignition, low voltage, pattern recognition

\section{INTRODUCTION}

Arc faults can be one of the most hazardous events for switchgear, no matter if low voltage, medium voltage or high voltage level. Not only the power supply can be interrupted; also the hot gases and toxic fumes can endanger operating personal. Moreover, switchgear in which an arc fault has occurred cannot be put into service offhand. Depending on the strength and duration of the arc only one field or the entire substation can be affected. It may also happen, that because of the strong heat the entire substation is irreparably damaged and has to be replaced completely. It is thus not only the cost for the replacement of the switchgear, but especially the failure of the power supply can cause significant costs. In particular, because of the increased demand for security of supply in low voltage range, new efforts to improve the detection of arc faults are required.
In recent years, because of shorter opening times of switches and new approaches to detect short circuit, safety against high current failures could be improved further. However, a field which is currently rarely dealt with is the early detection of arcing and especially the detection of low current arc faults. To prevent major fault events it is essential to detect these initial low current fault events reliably to avoid possible further damage to the switchgear.

Since failure statistics only show arc accidents in switchgear with injury to persons, it is not clear how many arc fault events occur with damage only to the switchgear [13]. . It is not possible to clarify, whether some high current arcs, such as short circuit arc faults, started as a low current arc fault. However it is mostly accepted that low current arc faults can exist in switchgear due to contact interruption in the busbar or due to contamination of insulation material $[14,15]$. In the following text, low current arc faults will be called arcs with arc current in the range of rated current $\mathrm{I}_{\mathrm{N}}$ of the switchgear. Since traditional protection devices such as fuses and circuitbreakers are able to carry rated current by nature, they will not detect such faults. Because switchgear can be configured for various ranges of rated current, in this paper the discussed arcs are in the range of $40-400 \mathrm{~A}_{\mathrm{RMS}}$.

\section{APPEARANCE OF ARC FAULTS}

In general, in a substation two different types of arc fault can appear: Serial arcs and parallel arcs. They differ very much in type of origin and effect on the switchgear.

\section{A. Parallel arc fault}

Parallel arcs can appear between two phases, phase to ground or phase to neutral of the switchgear. They are mostly a result of short-circuit and therefore carry a high current in the range of kilo ampere. For example, they can be caused by an object, which is making a connection between two different potentials in the switchgear. Parallel arcs discussed in this paper may have other reasons, for example contamination and much lower amplitudes and may therefore not be compared to short circuit arc flash. Arc flash tests, maintained by switchgear manufacturers are usually realized by conducting a piece of copper wire between two or three phases. After energizing, the 
wire evaporates and forms plasma, which is able to conduct the arc current. Due to the very high current during shortcircuit, a plasma channel between the two potentials can be established. Evaporated, hot copper boosts this effect, so the arc can easily reignite after current zero. The arc burns until it is switched off, or the distance between the two arc points is too large and the driving voltage is no longer able to maintain the arc. Distance between the arc points can increase because of traveling arc or because more and more copper is evaporated. Low current parallel arc fault differ from shortcircuit arcs, because of their current, which is far below the range of short circuit current. Because of that, it is not very common for low current arc to re-ignite after every current zero crossing. To be able to check the performance of any arc fault detection device, it is necessary to generate realistic low current arc faults. Therefore, arcs which are burning over several half cycles are required. Only this way all characteristics of an arc can be detected reliably. The experiments will discuss this in more detail.

\section{B. Serial arc fault}

Serial arc faults are caused by a contact interruption within a cable or busbar of only one phase. At first this kind of failure does not affect the other phases. One reason may be a loosening screw connection within a busbar or a broken cable connection as shown in [5], for example. Currently, such failures are mainly detected by thermography. Infrared cameras can show hot-spots at connections, which may indicate a bad contact within the busbar or cable. At a loosening screw connection, the good electrical contact within the busbar is partially lost. Because the cross-section for the current is reduced at this point, is comes to a local heating due to the increased resistance. Seen over a longer period, this may lead to increased local oxidation, due to the continued heating, as also mentioned in [12]. In the end, the locally heated screw connection may start melting off, which can form a gap. The current then has to bridge the gap by an arc. Increased heating, also by the arc and molten materials will soon increase the gap and the arc. The arc can also extend to other phases. For example, hot gases can damage the insulation material and decrease the insulation performance between the phases. Also thermal arc convection can ionize the gap between the phases. In the end, a serial arc can then be the beginning of a parallel arc. The arc current of serial arcs is limited by the connected load. It may therefore be by definition not larger than the rated current of the switchgear. Therefore, conventional fuses and circuit breakers wouldn't detect a serial arc. Since these kind of arcs may develop very slowly and may burn over a long time till detection, they are a huge problem for reliable power distribution.

\section{GENERATION OF ELECTRIC ARCS}

To develop an effective arc fault protection device, it is necessary to recognize all the relevant characteristics of an electric arc fault. For high current, short circuit similar arcs, there is extensive data available. Also arcing at higher voltage levels is very well understood. This is not the case for arcing at low voltage and with limited current. Due to the low voltage it is under normal conditions not very easy to ignite an arc. There are either very small distances required, or a material, which can be used to ignite an arc and bridges the initial distance between the electrodes. This method is used for short-circuit tests in low-voltage switchgear. In section V. it will be shown why the same method cannot be applied completely to the generation of low voltage arc fault. In this work, the option of igniting an electrical arc with the help of metal and carbon film resistors is analyzed. Furthermore, it will be discussed, how realistic the generated arcs are compared to real world arc faults in service.

\section{A. Conditions for a reproducible arc}

As mentioned above, an arc should be generated where the properties of a low-voltage electric arc could be investigated. It should be possible to produce arcs with a current of $40-$ $400 \mathrm{~A}_{\mathrm{RMS}}$. The lower limit is in the range of current through residential branch circuit [4] and the upper limit can appear in industrial switchgear. Since average minimum distances in low voltage switchgear are $20 \mathrm{~mm}$ between two different potentials, the gap distance for an electrical arc should be equal to this distance. The arcs should be reproducible as often as required, apart from the usual scattering of ignition processes. To capture all the relevant characteristics, the arc should at least be burning for $80 \mathrm{~ms}$. With this, it is possible to also observe variations and gradual change in the arc current. It is important to make sure that the ignition of the arc and its gap distance are as realistic as possible.

\section{B. Ignition conditions for an electric arc}

For an electric arc in air, Paschen's law (1) can be used.

$$
\mathrm{U}_{\mathrm{d}}=B \cdot \frac{p \cdot d}{\ln \left(\frac{A}{k} \cdot p \cdot d\right)}=\mathrm{f}(\mathrm{p} \cdot \mathrm{d})
$$

To ignite an arc in a low voltage AC network, very low values for $p \cdot d$ are necessary. For air, according to [1] $U_{d, m i n}$ is $327 \mathrm{~V}$ for a $(\mathrm{p} \cdot \mathrm{d}) \mathrm{min}$ of $0.76 \mathrm{~Pa} \cdot \mathrm{m}$. Therefore, in an atmospheric pressure of $101325 \mathrm{~Pa}$ at sea level, a distance of $7.5 \mu \mathrm{m}$ is necessary. However, such small distances do not occur in substation insulation under normal conditions. So it is not possible to ignite an arc in a homogeneous field with 20 $\mathrm{mm}$ gap distance at a voltage of $400 \mathrm{~V}$.

On the one hand, it is possible to generate a highly inhomogeneous field, for example with a needle-to-plate arrangement of electrodes. However, to get field strength high enough to reach the breakdown voltage, a very thin needle and also substantially shorter clearance have to be chosen. Since these conditions occur not very often in substation and also a thin needle would not survive high current, this method of arc generation was not further investigated.

On the other hand, an arc can be ignited on the surface of an auxiliary object attached to the busbars of the switchgear and then burn over a larger distance. A gap of $20 \mathrm{~mm}$ was selected, which is roughly equivalent to the minimum distance in low voltage switchgear. By inserting an auxiliary object into the air gap, the arc will be ignited. 
Another possibility, as shown in $[9,10]$, is to „pull“ an electrical arc. This is often applied to generate DC arcs. Therefore, a pike-to-pike configuration is chosen. At first, the two pikes touch each other. After applying voltage, the two pikes are slowly separated. This interrupts the initial contact and the resulting gap must be bridged by an arc. Due to the constantly increasing gap distance the current shape shows much scatter resulting in a poor reproducibility which makes the evaluation more difficult. While "pulling" an arc over the air gap of $20 \mathrm{~mm}$, it is also possible, that the arc extinguishes before reaching full length and then the process has to be started again. Since this dynamic movement does not occur in a substation and is more common to investigate switch arcs, further observation was skipped.

\section{Characteristics of the AC arc}

The AC arc has in contrast to the DC arc the special characteristic of natural zero crossings. It may temporarily extinguish and, depending on the ignition conditions, reignite when the voltage increases again. This results in two important characteristics as also described in [2,3, and 7]:

- After current zero crossing, the arc expires because of the low voltage difference between the electrodes. If the voltage difference drops below a certain level, which differs for each test setup, the arc can't be maintained. The arc may reignite after current zero crossing, if the required ignition voltage is reached. Till reaching this re-ignition voltage no (relevant) current flows through the spark gap. There is a short dead time, which forms a shoulder in the sinusoidal current (see point 1 in figure 1).

- After reaching re-ignition voltage, the dielectric strength of the spark gap breaks down abruptly and is then able to carry the arc current. This rapid breakdown causes a rapid increase in current (see point 2 in fig.1). The resulting steep current edge is characteristically for spark gaps, which lose their insulation ability in the short period between arc extinguishing and re-ignition. Short current edges may also appear shortly before current zero. The voltage is then below the burning voltage of the arc. Properties shown in figure 1 are valid for all $\mathrm{AC}$ arc. However, properties marked with point 1 and 2 may blur or even disappear for high current arcs. This happens if the plasma is still very hot after current zero crossing, so the arc can reignite immediately. The mentioned properties mainly occur in low voltage switchgear during low current arc fault and for high-impedance fault, as also discussed in [8].

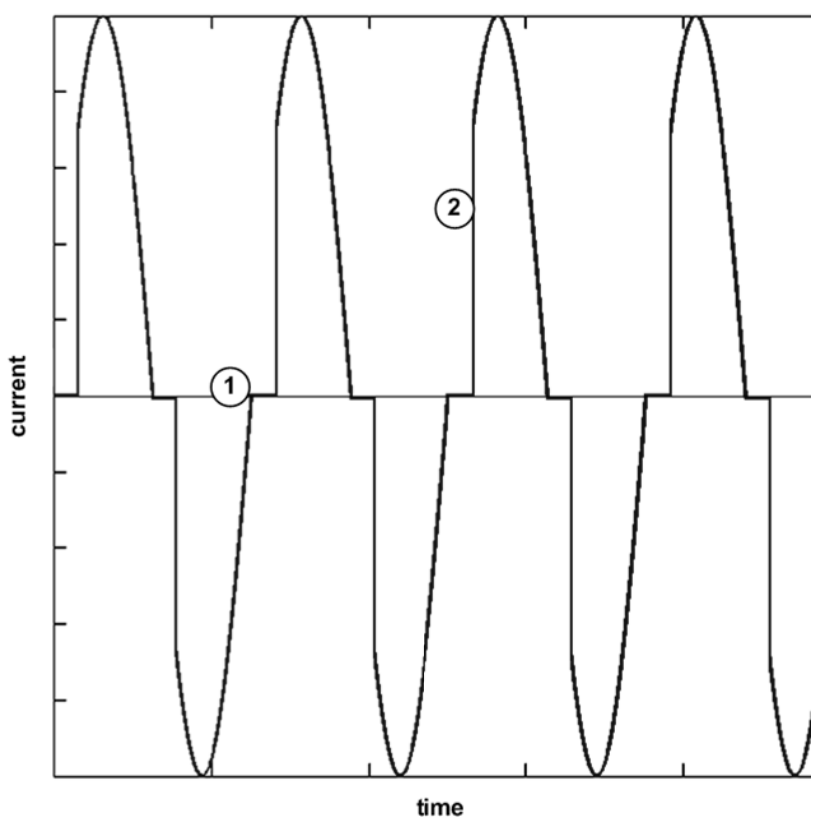

Figure 1: Typical AC current signal with current interruption (1) and reignition of the arc with steep current edge (2)

\section{TEST SETUP FOR LOW CURRENT ARC FAULTS}

A test setup with two essential features is required. First, it has to limit the current to values below the rated current of the switchgear. Second, it must be able to ignite several arcs under almost the same conditions to obtain comparable results. The test setup in Fig. 2 is additionally equipped with a circuit breaker and a fuse. The circuit is supplied with a three-phase cable to the laboratory network. This allows investigation of different combinations, as phase to phase or phase to ground arcs. With the main switch of the power supply, it is possible to turn electric power supply on and off. A resistor limits the current through the arc. It can be varied according to the required arc current amplitude. The spark gap consists of two copper bars with a gap distance of $20 \mathrm{~mm}$ (see also fig. 3). First current measurements were done with a Pearson probe. In the later course, the current was measured with a current clamp with a range of $1200 \mathrm{~A}$ and a bandwidth of $300 \mathrm{kHz}$. The data obtained were recorded with an oscilloscope and processed with MATLAB.

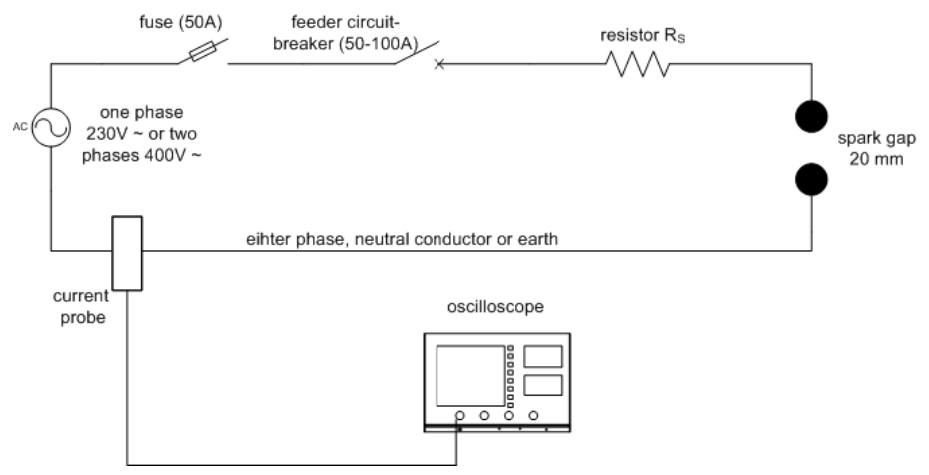

Figure 2: Test setup for generation of arcs. Current is limited by a resistor 


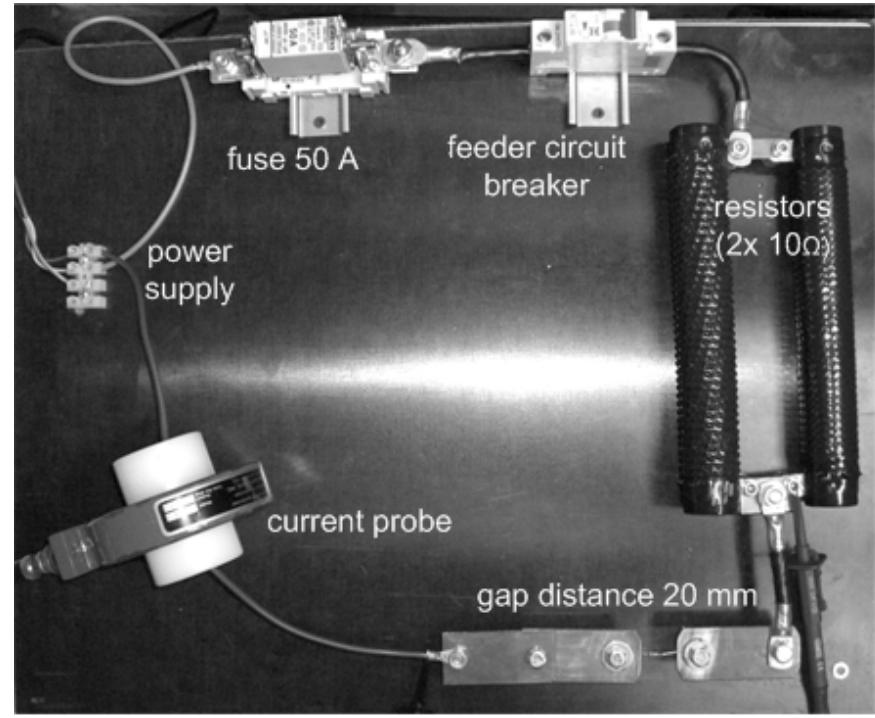

Figure 3: Test setup for an arc with current limited to $40 \mathrm{~A}$ by 2 parallel wire wound resistor. Current measurement is done by Pearson-probe. For higher arc current, the resistor was replaced and current measurement was done by a clamp-meter, maximum range $1200 \mathrm{~A}$

\section{SHORT CIRCUIT TESTS WITH A COPPER WIRE}

First tests were carried out by a thin wire between two busbar conductors in serials with a resistor to limit the current. It showed, that by limiting the current to $40 \mathrm{~A}$ it was not possible to get an arc burning for at least several $50 \mathrm{~Hz}$ cycles. For thin copper wires, the wire already burned through during the first half-wave and the arc extinguished after the first current zero crossing as shown in fig. 4.

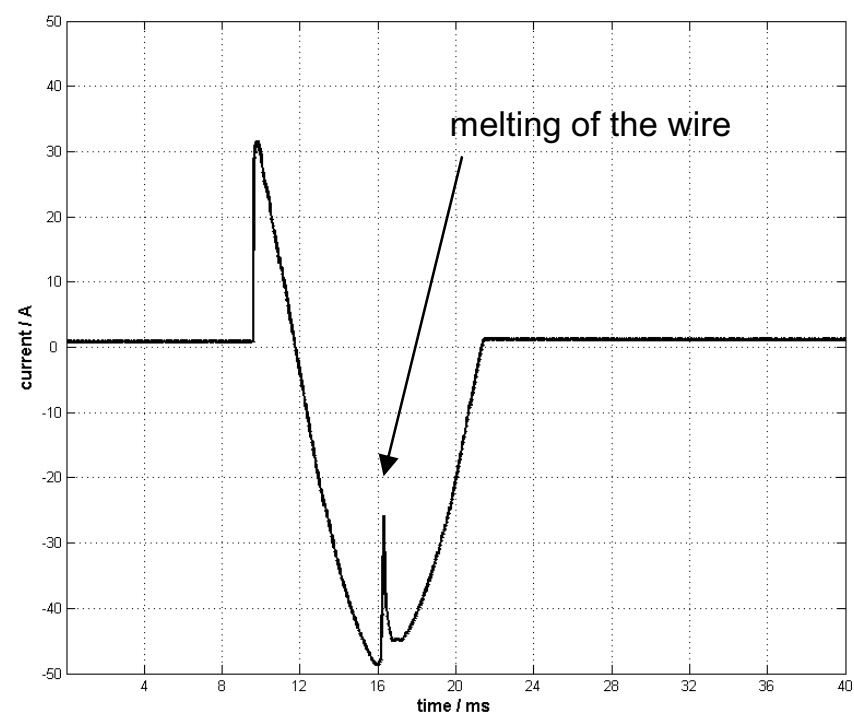

Figure 4: Current profile during an arc, ignited by a thin copper wire. The arc extinguishes after the first zero crossing [measured]

There were not enough heat and ionized gases produced by the thin wire. Re-ignition was not possible. Thicker wires have a better ability to carry the current and do not burn through that fast. Yet they were also not able to ignite an arc. Since the thicker copper wire has almost no resistance, it does not heat up that much and could not generate an arc. It simply did not burn through. Also the circuit breaker cut the circuit because of the high current during the first half-wave. As a result it showed that it is necessary to have a certain resistivity while bridging the spark gap. This helps to start heating up the gap bridging material and will then ignite an arc.

\section{CARBON AND METAL FILM RESISTOR IGNITING THE ARC}

Ignition of a low current arc by using low resistive copper wires between the electrodes was not possible. The wire crosssection was either too small to produce enough heat and gases out of molten copper to reignite the arc after current zero, or the wire was too thick and its resistivity too small to heat up the wire at all, before the circuit breaker interrupted the test.

To have enough heat for the re-ignition of the arc, it is necessary to get more plasma into the gap between the copper electrodes. This was done by burning off (overloading) a metal film or carbon film resistor.

It turned out that a resistor (nominal rating $0.25 \mathrm{~W}$ ) as in fig. 6 with $1 \mathrm{k} \Omega$ is able to stand the applied current shortly after closing the circuit. But since the resistor is designed for very low constant load it is strongly heating up already after few milliseconds. After some half cycles (depending on performance of the resistor) the resistor starts to burn through. This first shows in small current spikes in the peak region as shown in fig. 5. Until that time the resistor was able to limit the current according to its nominal value (for $1 \mathrm{k} \Omega$ plus serial resistor and $\mathrm{U}_{\mathrm{N}}=230 \mathrm{~V}, \mathrm{I}_{\text {eff }}$ ca $230 \mathrm{~mA}$ ). Till the time of total failure of the resistor and arc ignition already hot combustible gases are generated. This is caused by constantly heating the resistance material inside and outside the resistor. After igniting completely, all characteristics of an $\mathrm{AC}$ arc are present. The arc extinguishes during current zero crossing and re-ignites several hundred microseconds later. These characteristic AC arc patterns also became clear while recording the arc with a high speed camera as seen in fig. 7. It shows the test setup with the two copper plates as electrodes in the middle. Because of the extreme brightness of the arc plasma the camera is overexposed and shows the arc area in different grayscales. This way it is possible to see the arc and also the surrounding combustion gases. 


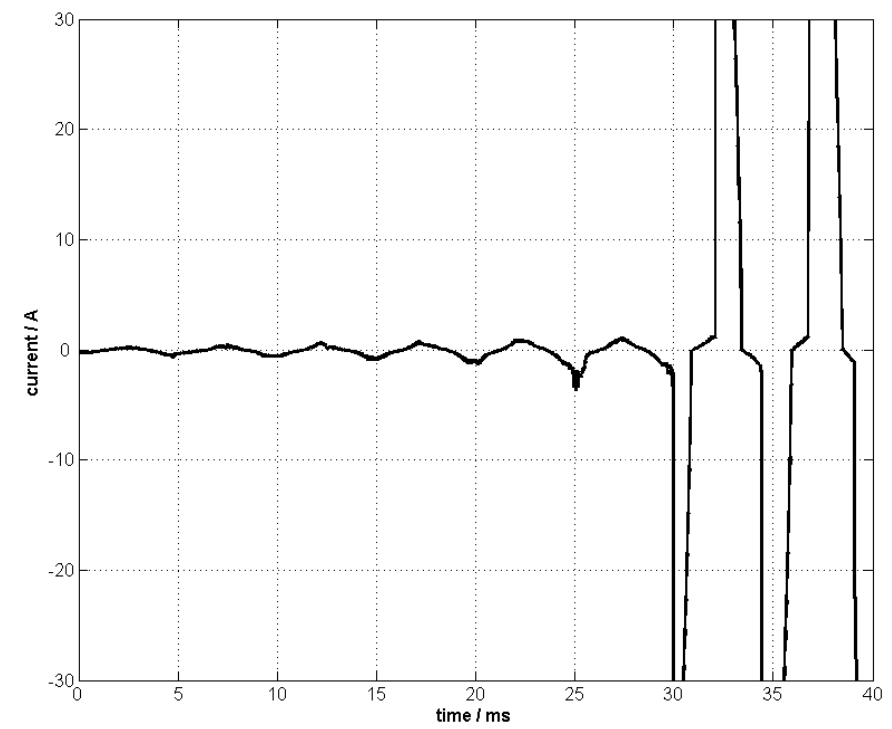

Figure 5: Beginning of burning through of a metal film resistor. Small current spikes can be seen during peak value and finally the resistor fails to isolate the voltage [measured]

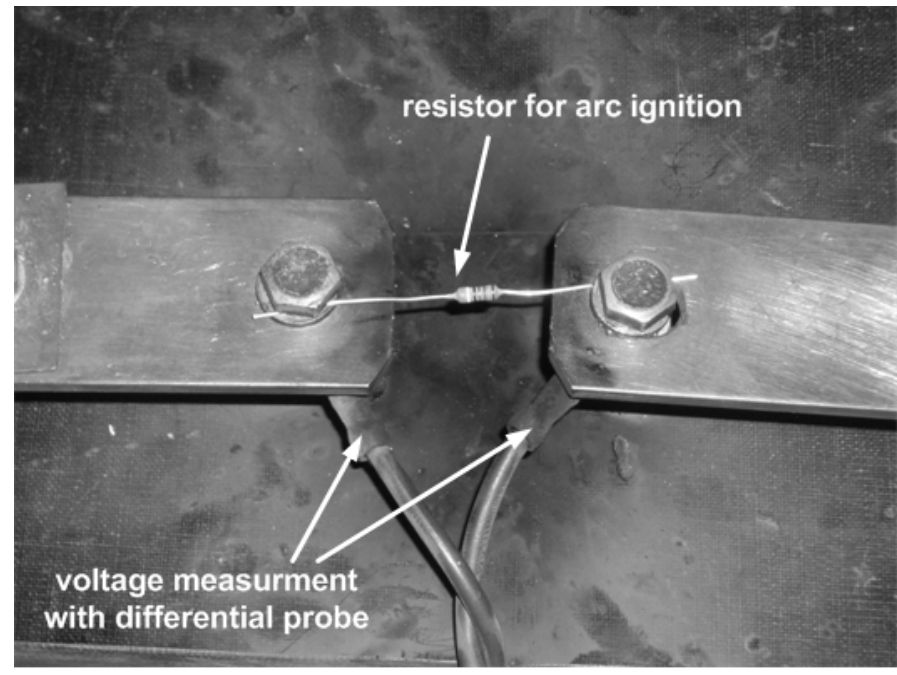

Figure 6: With a metal film resistor between the copper electrodes (gap distance $20 \mathrm{~mm}$ ) it is possible to ignite an arc

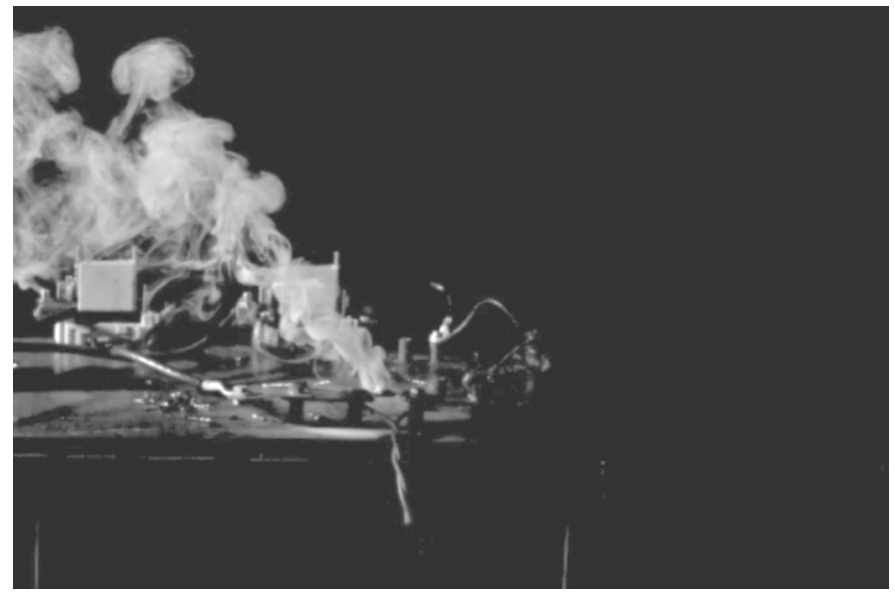

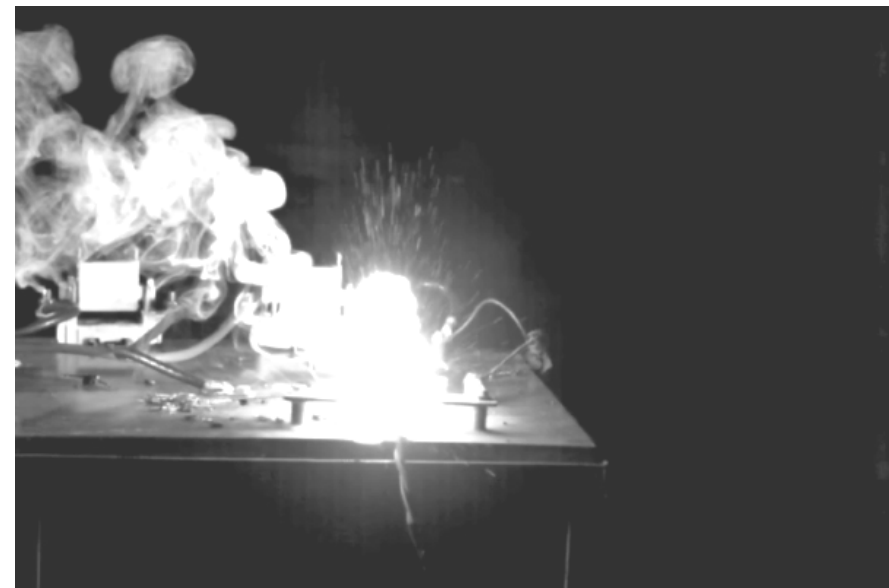

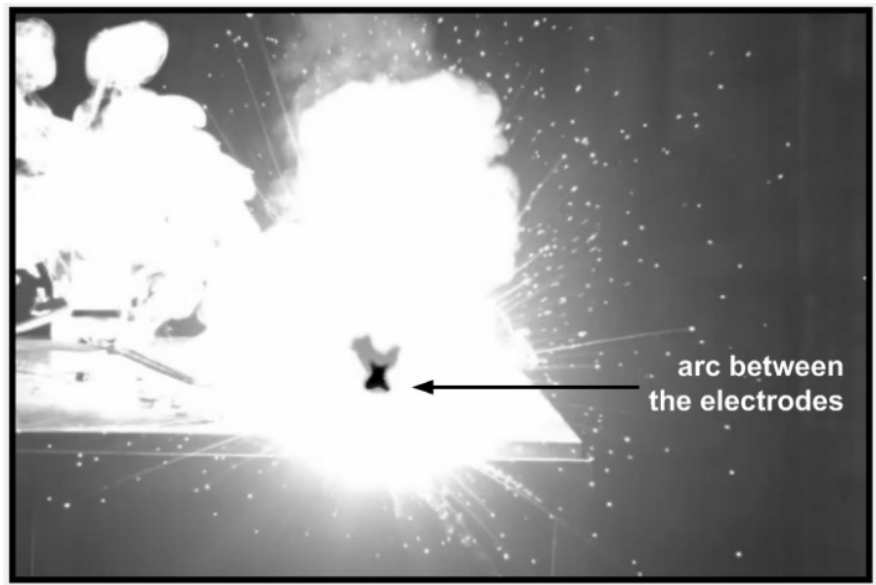

Figure 7: 400A AC arc recorded with a high-speed camera. The black area in the middle is the actual arc (camera overexposed because of the brightness of the plasma). Clearly visible is also the smoke at the beginning, which indicates heating up of the resistor

\section{CHARCTERISTIC PATTERNS IN LOW CURRENT AC ARCS}

Arc ignition typically takes place during the voltage rise or in the peak region. The resistor can't isolate the rising voltage anymore. Due to this, local punctures on the surface occur, which then are able to bridge the whole resistor. Current rises rapidly to its maximum, limited by the serial resistor (see fig. 8). Maximum amplitude is calculated by $I=U_{\max } / R_{S}$. To test the influence of arc current on $\mathrm{AC}$ arc patterns, the serial resistance was reduced gradually from $5 \Omega$ to $1 \Omega$. Hence there is an arc current range from $40-400 \mathrm{~A}_{\mathrm{RMS}}$. Also tests were done to analyze whether there is a difference in ignition between phase and ground or phase to phase. Results showed that besides the increased current due to higher voltage (400 $\mathrm{V}_{\mathrm{RMS}}$ ) for phase to phase arcs there is no big difference compared to phase to ground. The characteristic patterns of AC arcs remain. It is striking that with increased arc current time between current zero and re-ignition decreases. This is because of higher current which enables the plasma channel between the electrodes to contain much more heat. As a result the arc is able to reignite much earlier with at lower ignition voltage [6]. This creates a more flat and shorter rising edge of current as shown in fig. 9. 
Depending on the applied resistor, the arc burns stable over several half cycles. For lower current (40 A) a resistor of 1-2 $\mathrm{k} \Omega$ and nominal rating of $0,5 \mathrm{~W}$ is enough to have a stable arc, burning for up to $80 \mathrm{~ms}$. Increasing the current over the arc by reducing the series resistor results in a much lower burning time for these kind of resistors. The arc extinguishes after 1 -2half cycles. The higher current results in a single hot spot inside the resistor, which interrupts the current flow before an arc can be established. To get long burning arcs with even higher current, several resistors have to be conducted in parallel. Thus increasing the nominal rating of the resistors and makes it possible for the spark gap to carry higher current. The resulting arc takes over to carry the higher current and enables as a result to ignite arcs with up to $400 \mathrm{~A}_{\mathrm{RMS}}$ in the applied test setup. Arcs with even higher current are possible if the value of the serial resistor is decreased.

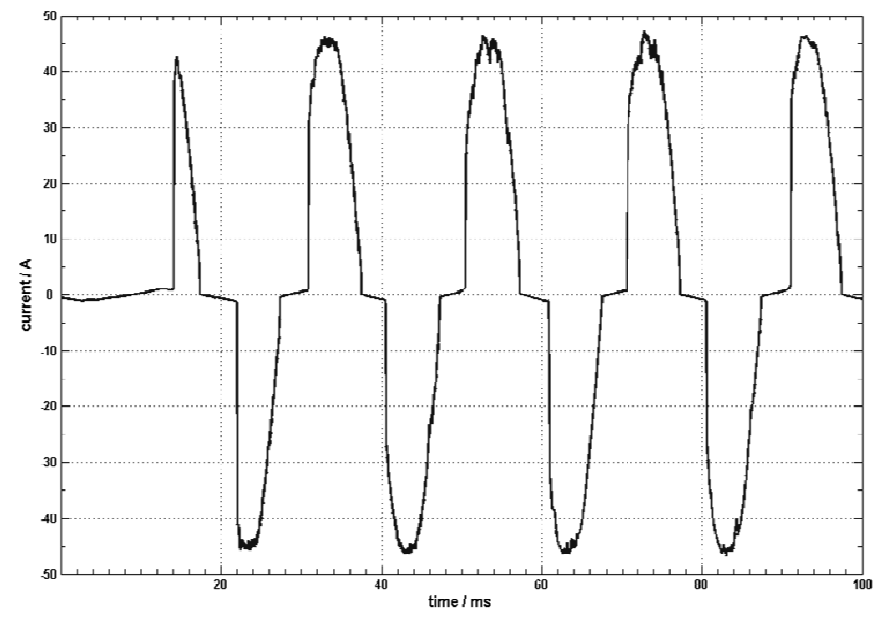

Figure 8: Measured arc current, arc ignited by a metal film resistor. Current is limited by $5 \mathrm{Ohm}$ resistor. Ignition between phase and ground [measured]

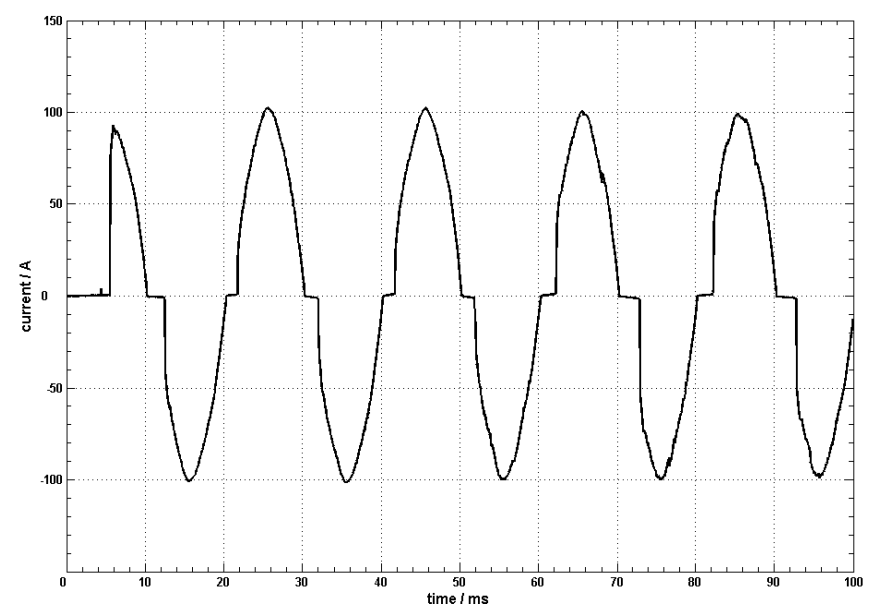

Figure 9: Current profile of an arc between two phases [measured]

After 80 to $100 \mathrm{~ms}$ the arc self-extinguishes (see Fig. 10). This is done mostly by the two wire leads of the resistor, which melt down and become shorter and shorter. As a result, the gap between the electrodes gets larger and larger. Furthermore, the hot gases, generated by burning the resistor, cool down more and more and the re-ignition of the arc is stopped. In the end, the arc is not able to bridge the growing gap anymore. At one point, the arc won't reignite after a current zero crossing. The system is considered as very save and stable. For the arc it is not possible to burn unregulated and it also self-extinguishes. The igniting process starts after applying voltage and stops after a few hundred milliseconds autonomously.

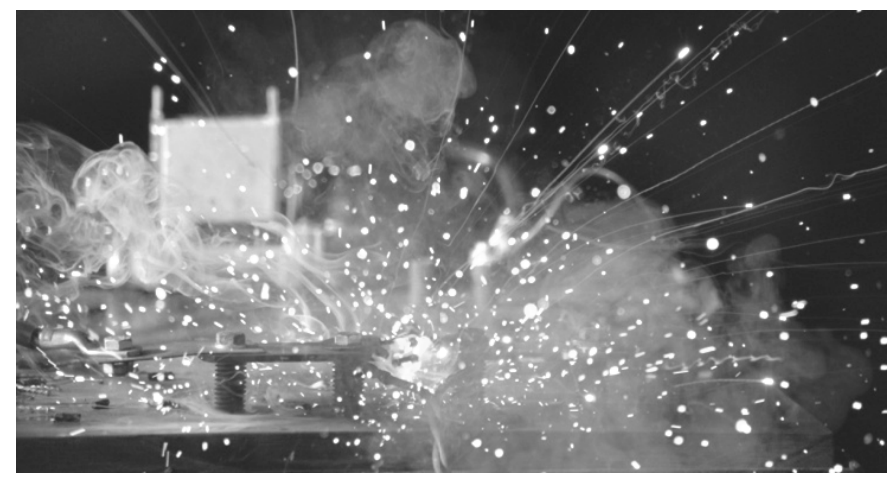

Figure 10: Arc gap shortly after the extinction of the arc

\section{CONCLUTIONS}

Stable burning and controllable arcs are difficult to generate, in particular for arc currents below $1000 \mathrm{~A}$. However, they are essential for testing arc fault detecting devices, especially for those detecting low current arc faults. With the method described here, it is possible to create realistic low current AC arcs with the help of commercially available leaded resistors. The current amplitude of generated arcs are mainly determined by serial resistors to limit the arc current to values below the rated current of the switchgear. This is why the arc is not detected by existing fuses and circuit breakers. To the identifier, an arc may look like a variable load [2].

- It was shown that stable burning arcs can be generated by burning metal film and carbon film resistors between the busbars.

- Measured current profiles show all characteristics of low current $\mathrm{AC}$ arcs. These are in particular the extinguishing of the arc after current zero and the steep current rise during reignition of the arc. Breakdown voltage depends strongly on the remaining charge carriers in the plasma channel (i.e. due to arc heating and evaporated copper). These characteristics may differ for highly inductive electrical systems or circuits if the resistivity of the arc is not dominant in the circuit compared to its inductivity.

- It was found, that time to re-ignition strongly depends on the amplitude of the arc current. The higher the current, the shorter the time to re-ignition after current zero crossing.

- This also reduces the step amplitude of the current rise during reignition since the reignition voltage is much lower for high current amplitudes. These characteristics don't show, or only very weak, for high current arc faults like a short circuit. 


\section{REFERENCES}

[1] S. C. Brown, "Introduction to Electrical Discharges in Gases", John Wiley 1966.

[2] J. B. Beck and D. C. Nemir, "Arc fault detection through model reference estimation" SAE International, 2006.

[3] T. Gammon and J. Matthews "Arcing-fault models for low-voltage Power Systems" IEEE, 2000.

[4] C. E. Restrepo "Arc fault detection and discrimination methods", $53^{\text {rd }}$ HOLM Conference, Pittsburgh PA, 2007.

[5] J.J. Shea, "Comparing $240 \mathrm{~V}_{\mathrm{RMS}}$ to $120 \mathrm{~V}_{\mathrm{RMS}}$ series arcing faults in residential wire", IEEE 2008

[6] W. Rieder, "Plasma and arc", Vieweg Verlag, Germany, 1967

[7] G. D. Gregory and G. W. Scott, "The arc-fault circuit interrupter: An emerging produft", IEEE Transactions on Industry Applications, Vol. 34, No. 5, 1998

[8] A. F. Sultan, G. W. Swift, D. J. Fedirchuk, "Detecting arcing downedwires using fault current flicker and half-cycle asymmetry", IEEE Transactions on Power Delivery, Vol. 9, No. 1, 1994
[9] X. Zhou, J. Hastings, T. J. Schoepf, "Detection of glowing contacts employing acoustic sensing technology", ICEC 2008

[10] C. Strobl and P. Meckler, "Basic experiments for detectiong arc faults in dc aircraft networks", ICEC 2008

[11] C. S. Maroni, R. Cittadini, Y. Cadoux, M. Serpinet, "Series arc detection in low voltage distribution switchboard using spectral analysis" ISSPA Kuala Lumpur, Malaysia, 2001

[12] J. Sletbak, R. Kristensen, H. Sundklakk, G. Navik, M. Runde, "Glowing contact areas in loose copper wire connections", IEEE Transactions on Components, Hybrids and Manufacturing Technology, Vol. 15, No.3, Junge 1992

[13] A. Schumacher, H. Schmolke, "Störlichtbogen - nicht zu vermeiden aber beherrschbar", ETZ Volume 7, Germany, 2005

[14] D. Brechtken, "Konzept eines präventiven Störlichtbogenschutzes" EPElektropraktiker, Berlin 56/4, p.306-310, 2002

[15] P. L. Koenen, H. Schäfer, "Störlichtbogenschutz in der Niederspannung - eine Herausforderung in der Schutztechnik", Klöckner-Moeller, VER $27-869,12 / 97$, Bd. $1-6$ 


\title{
Electrical Arc Characterization For Ac-Arc Fault Applications
}

\author{
E. Carvou, N. Ben Jemaa \\ (erwann.carvou@univ-rennesl.fr, tel. +33 (0)2 23236580 ,fax : +33 (0)2 232369 57), \\ Université de Rennes 1, IPR UMR CNRS 6251, Bat. 11B Campus de Beaulieu, 35042 RENNES, France \\ S. Tian, Z. Belhaja, B. Jusselin \\ Schneider Electric, Innovation Division, 38TEC/T3 Plant, 37 Quai Paul-Louis Merlin 38000 GRENOBLE
}

\begin{abstract}
Summary - The improvement of security in relation to hazardous disconnections and deteriorated connections requires a deeper knowledge of the characteristics of the ensuing arcs and their subsequent damage to the contact materials, insulation and wiring. Numerous studies have been performed on arcs for the DC power supplies used in telecommunications, and more recently in automotive fields. Furthermore, there is an important demand in the sense of safety and reliability in the areas of domestic applications, i.e. in the domain of alternative current. The purpose of this study is to extend our knowledge of AC-arc characteristics and consequences (e.g. risk of fire ignition).

The electrical and physical characteristics of arcs that can appear in carbonized paths in domestic wires have been studied for alternative currents with the objective of comparing European and US electric loads on their characteristics.

The arcs are produced by separating electrodes made with graphite, in order to reproduce the carbonized path environment. A very low separation speed $(50 \mu \mathrm{m} / \mathrm{s})$ allows arcs to reinitiate, as in arc fault in wires. Arc voltage and current are measured and arc characteristics (voltage and current level, arc duration, power and energy) are defined at each cycle. The main result shows that, because of the constancy of arc voltage, the electrical energy consumption at each cycle does not depend on the voltage supply level. However, a large voltage make more probable the arc re-ignition, thus making higher, the total amount of consumed energy, with consequences for fire ignition.
\end{abstract}

Key words - Alternative current, arc, domestic security, AFCI.

\section{INTRODUCTION}

The generalization of safety devices used to prevent home fires associated with arcs in weakened electrical links has resulted in an increase in work related to this area [1-7]. One example is the series arcing faults objectives of the standard test UL 1699. In this test, a wire is prepared so as to weaken the insulation between the conductor strands. Current between the strands, on carbonized path, leads to the apparition of arcs and to burning of the wire. Thus, in this work, we have chosen to study only the arcs and the effect of voltage and current on their characteristics. In addition, to simplifying the analysis, we chose to represent them by breaking arcs. Indeed, even if the physical environment differs, the electrical characteristics are sufficiently close to the series arcing faults to obtain some useful results. These arcs have several peculiarities; they occur in an environment consisting of carbon (the remains of cables PVC) and can re-ignite at each alternating current, despite low frequencies $(50$ or $60 \mathrm{~Hz})$. This is achieved by using graphite electrodes and the use of a very small electrode separation speed. Furthermore, by using a variable gap we can study the study the effect of this gap on the arcs.

Finally, the purpose of this study is to extend our knowledge of AC-arc characteristics in order to contribute to development of arc fire protection device in Europe.

Following a brief review of the test apparatus and procedures, we relate in the first part, the characteristics of $220 \mathrm{Vrms}-50 \mathrm{~Hz}$ arcs. The second part is dedicated to a comparison of previous arcs with $110 \mathrm{Vrms}-60 \mathrm{~Hz}$.

\section{EXPERIMENTAL SETUP AND PROCEDURE}

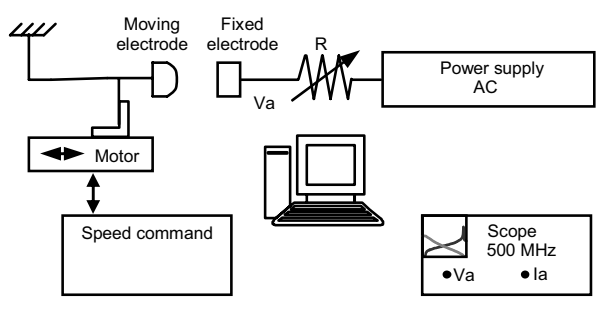

Fig. 1 Schematic of the experimental test apparatus.

The experimental apparatus (fig. 1) is a fully automated device, developed by adaptation from the DC apparatus described in previous papers ([8]). It is modified to perform contact breaking with alternative current with $220 \mathrm{Vrms}$ or $110 \mathrm{Vrms}$ and a current from 0.5 to 6 Arms. It is mainly composed of an electrical part (power supply, variable resistance) and a mechanical part using a stepping motor coupled with a sliding plate, which ensures the separation between the fixed and the moving contact, with a controlled constant speed of $50 \mu / \mathrm{s}$.

The main measuring instrument is a digital oscilloscope which samples and stores the voltage characteristics of the arc during the break, i.e. $\mathrm{Va}(\mathrm{t})$ and $\mathrm{Ia}(\mathrm{t})$. GPIB buses control the whole device (power supply, motor and oscilloscope). A personal computer, equipped with Testpoint software, ensures the experimental process and data collection.

The contacts are made from graphite. The moving electrode has a hemispherical shape of $4 \mathrm{~mm}$ diameter while the fixed electrode is plane. 


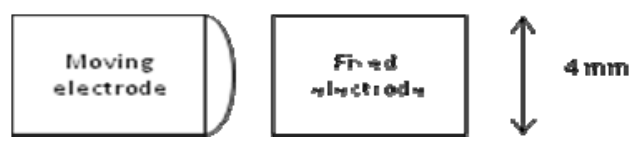

Table 1: Test parameters.

\begin{tabular}{|c|c|}
\hline $\begin{array}{c}\text { Contact } \\
\text { material }\end{array}$ & $\mathrm{C}$ \\
\hline Loads & $\begin{array}{c}220-110 \mathrm{Vrms}, 0,5-6 \mathrm{Arms}, \\
\text { resistive }\end{array}$ \\
\hline $\begin{array}{c}\text { Disconnection } \\
\text { speed }\end{array}$ & $50 \mu / \mathrm{s}$ \\
\hline
\end{tabular}

Typical arc voltages and currents are shown in figure 2 for $220 \mathrm{~V}$ and 6A. Parameters are extracted using a home-made program (with TestPoint software) during the entire experimental cycle, i.e. from separation of electrodes to the last arc. The extracted parameters at each half-cycle are:

Vmax:

The maximum voltage at the arc terminals on each half-cycle. This voltage is used to determine the variation of the breakdown voltage and, if this voltage is equal to the supply voltage, if an arc does not occur.

Vplateau: $\quad$ The voltage at the quarter-cycle $\left(\varphi=90^{\circ}\right)$, that corresponds to the arc voltage (if arc occurs).

Imax:

The maximum of the current on half-cycle. A value of zero indicates an open circuit.

Energy: The black area presents the energy consumed by arc at each half-cycle. The curve envelop is instantaneous power which is calculated with measured voltage and current product.

Arc duration: Duration when the arc electrical power is different than zero.

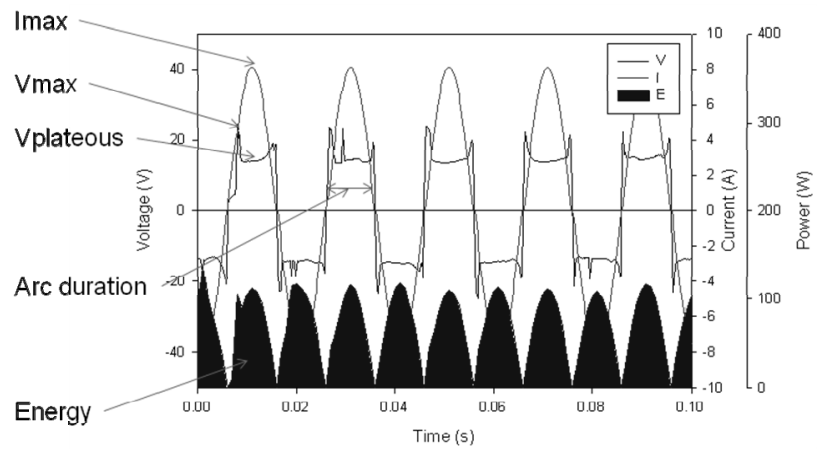

Fig. 2 Typical arc voltage and current.

\section{ARCS FOR 220VRMS $-50 \mathrm{~Hz}$}

The tests presented below were performed at $220 \mathrm{~V}$ (and $50 \mathrm{~Hz}$ ) for currents ranging from 0.5 to $6 \mathrm{~A}$. Fig. 3 shows the superposition of different voltage measurements for these currents. The voltage curve shape is close to the series arcing faults voltages as see on ref. [7]. Apart from the fact that the reignition of the arc is well ensured by very low electrode separation speed, two features are to be noticed. Firstly, the number of arcs, or the time during which the arcs are ignited, increases with the current. Secondly, curves are superposed, so the voltage does not depend on the current level by Ohm's law.

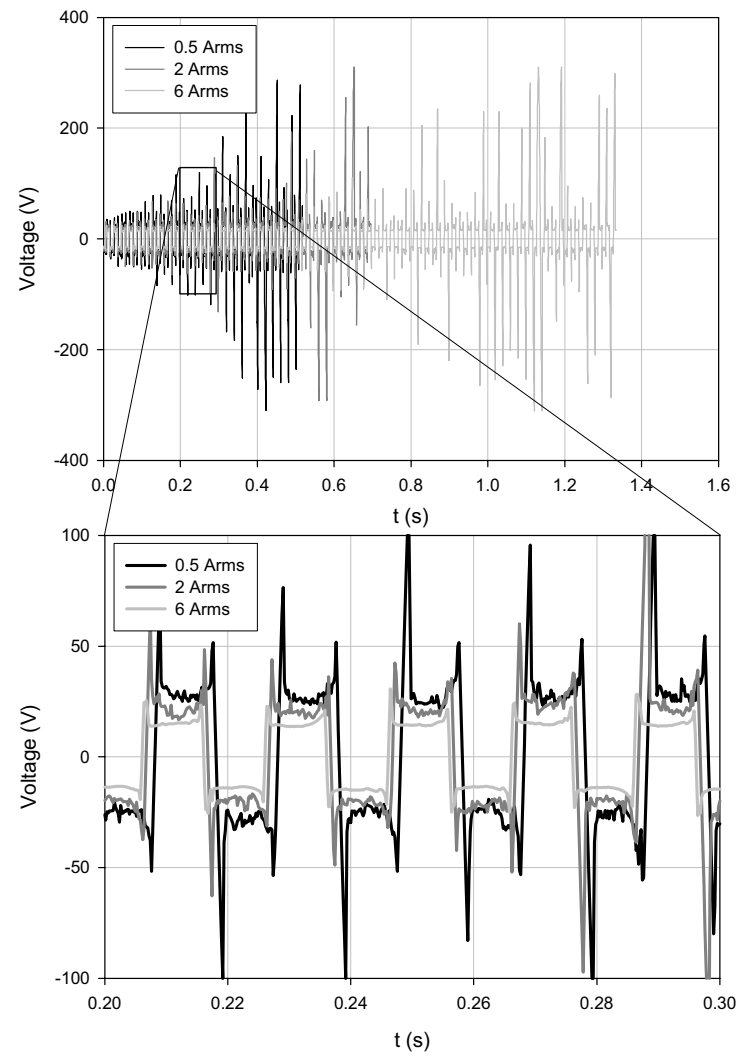

Fig. 3 Voltage for 220 Vrms and 0.5 to 6 Arms

\section{A. Maximum voltage}

Fig. 4 shows the evolution of the maximum voltage (Vmax) during the experiment, which is equivalent to the breakdown voltage. It can be noted that Vmax is low at the beginning of the experiment, whatever the current. However, the higher the current, the later the voltage fluctuations appear, fluctuation announcing the final extinction of the arcs. Consequently, high currents make experiment cycle longer.

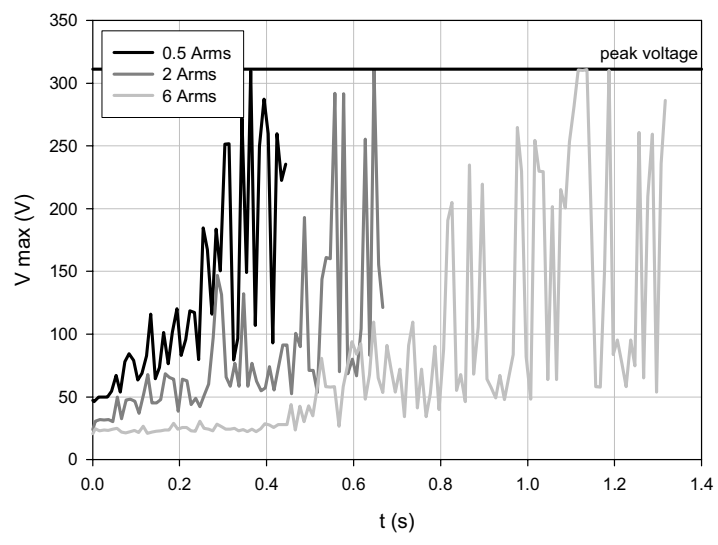

Fig. 4 Evolution of maximum voltage for $220 \mathrm{Vrms}$. 


\section{B. Plateau voltage}

Fig. 5 presents the voltage measured at the quarter-cycle $\left(\varphi=90^{\circ}\right)$. Apart from when arc extinguishes, this voltage is close to $20 \mathrm{~V}$, which corresponds to the arc voltage. This voltage is constant during the arc (see fig. 2). That means that between breakdown voltage and arc extinction, the voltage is constant and close to the arc ignition voltage value. Furthermore, the arc voltage presents a slight increase with increasing spacing of the electrodes. It is still around $20 \mathrm{~V}$, the voltage characteristic of carbon arc ignition [9]. Furthermore, like for DC arcs, a same spacing electrodes leads to lower arc voltage when the current increases.

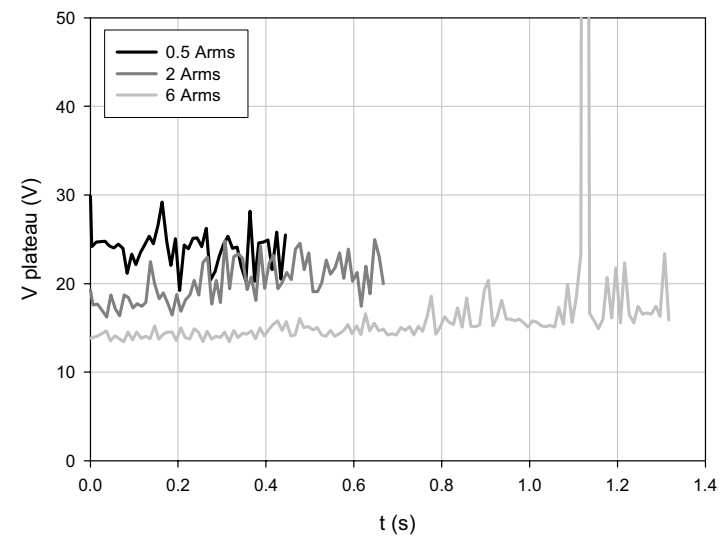

Fig. 5 Evolution of plateau voltage for $220 \mathrm{Vrms}$.

\section{Maximum current}

One consequence of the constancy of the plateau voltage is the constancy of the maximum current value as see in fig. 6 .

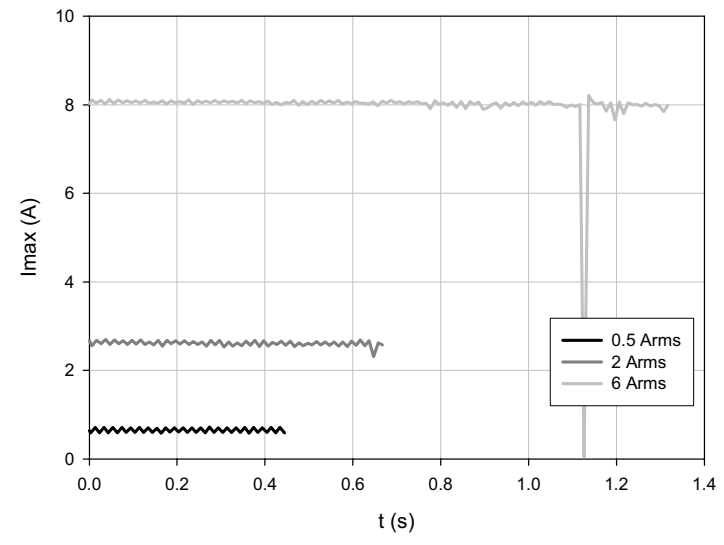

Fig. 6 Evolution of maximum current for $220 \mathrm{Vrms}$.

\section{Arc duration}

The variation of arc duration during the experiment for different currents is presented in fig. 7. First of all, the arc durations are close to the maximum value, i.e. the half period $(10 \mathrm{~ms}$ for $50 \mathrm{~Hz})$. Furthermore, the current has a slight effect on arc duration, due to the increase of Vmax. The period is too short to reveal the effect of current on arc duration, as in the $\mathrm{DC}$ arc.

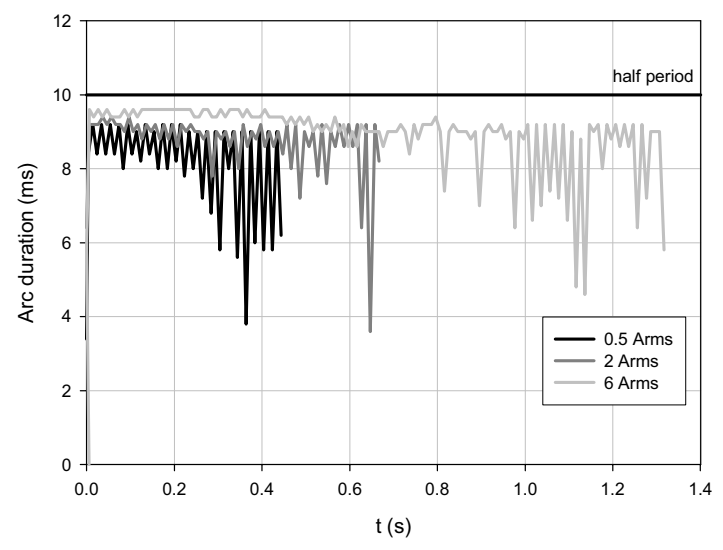

Fig. 7 Evolution of arc duration for 220Vrms.

\section{E. Energy}

Regarding the energy consumed in each arc (fig. 8), it is highly dependent on the current and slightly with time. Indeed, because the voltage variation (except fluctuations) does not depend on the value of the current, (see fig. 5) only the later has an effect. The result of the sum of these energies (fig. 9) is in this case obvious. A high current for each cycle causes a large energy consumption (resulting in a pronounced slope of the curve in fig. 9). Furthermore, a large current leads to more arcs so the total energy consumed by the system is much greater.

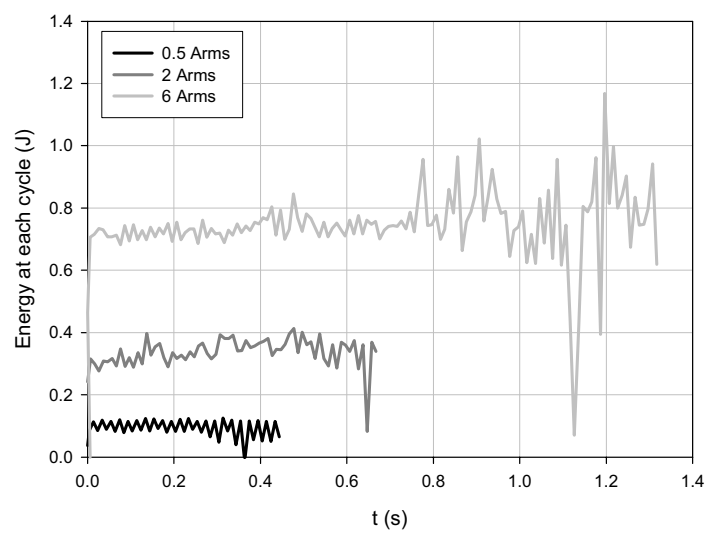

Fig. 8 Evolution of energy for 220Vrms.

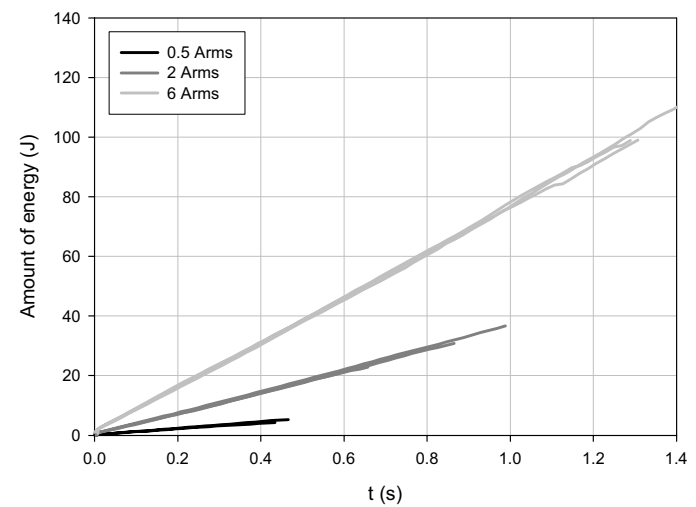

Fig. 9 Evolution of energy for 220Vrms. 


\section{COMPARISON BETWEEN 220 - 110 VRMS}

To determine differences in the behavior of the series arcing faults between the European and U.S. electrical networks, we should study the effect of voltage on arc characteristics. Fig. 10 shows the superposition of voltages measurements for arcs at $220 \mathrm{Vrms} / 50 \mathrm{~Hz}$ and $110 \mathrm{Vrms} / 60 \mathrm{~Hz}$ (6Arms in both cases). Two characteristics appear. First, the voltages are relatively similar. Moreover, arcs at $220 \mathrm{~V}$ re-ignite for a longer time than for $110 \mathrm{~V}$.

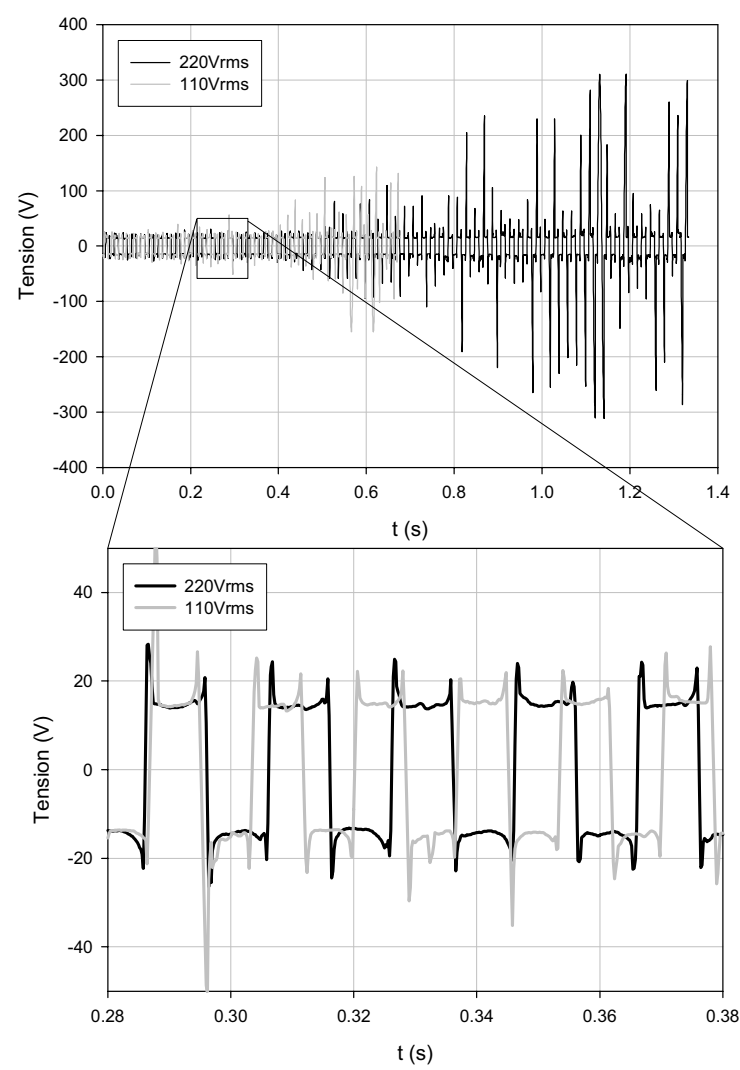

Fig. 10 Voltage traces for $220 \mathrm{Vrms}$ and $110 \mathrm{Vrms}$ at 6 Arms.

\section{A. Maximum voltage}

Fig. 11 presents the variation of maximum voltage. For both voltage levels, values are the same at the beginning of the experiment (around $20 \mathrm{~V}$, which corresponds to the carbon arc voltage). Similarly, in both cases, large fluctuations appear after $0.4 \mathrm{~s}$. However, reaching value close to $155 \mathrm{~V}$ (the peak voltage at $110 \mathrm{Vrms}$ ), leads to the extinction of the $110 \mathrm{~V}$ arcs. For $220 \mathrm{~V}$, this extinction voltage is $311 \mathrm{~V}$, value reached for the largest electrodes spacing and so for a greater time. This could explain the large number of arcs for $220 \mathrm{~V}$.

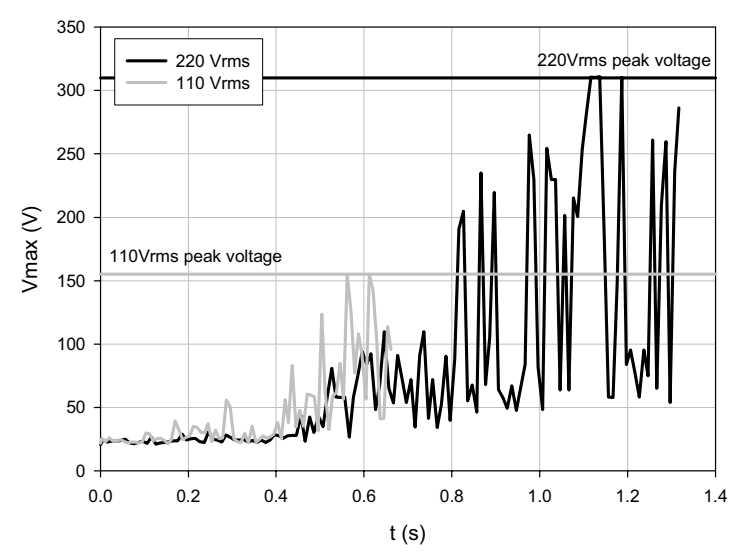

Fig. 11 Evolution of maximum voltage for 220 and $110 \mathrm{Vrms}$.

\section{B. Plateau voltage}

For both voltage (220 and 110), plateau voltages are similar and close to the carbon arc voltage. The independence of arc voltage with supply voltage is an important point to notice, particularly for its consequences on energy.

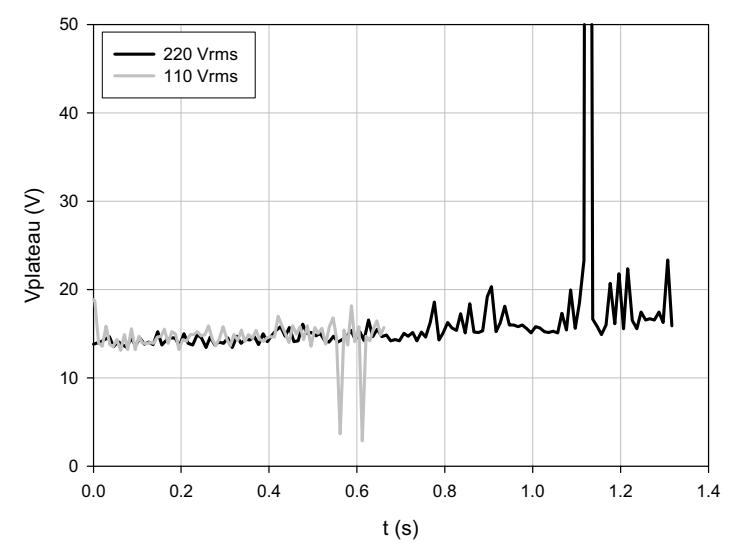

Fig. 12 Evolution of plateau voltage for 220 and 110 Vrms at 6Arms.

\section{Maximum current}

Regarding the current, the only difference comes from the voltage drop due to arcing that leads to a greater decrease for the case of $110 \mathrm{~V}$. In both cases, because of the constancy of the plateau voltage, the maximum current remains constant during the whole experiment. 


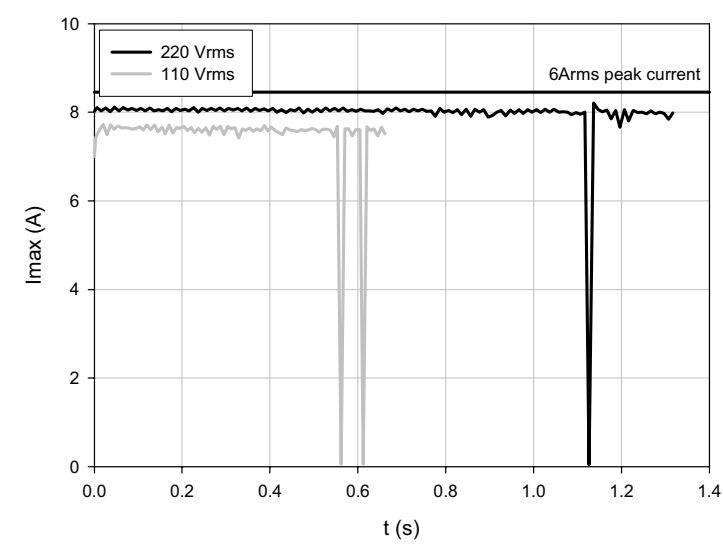

Fig. 13 Evolution of maximum current for 220 and 110Vrms.

\section{Arc duration}

Both for the two voltages, the arc duration reaches the maximal value, i.e. $10 \mathrm{~ms}$ for $50 \mathrm{~Hz}$ and $8.3 \mathrm{~ms}$ for $60 \mathrm{~Hz}$. Indeed, this difference has an effect on consumed energy, as shown in the following section.

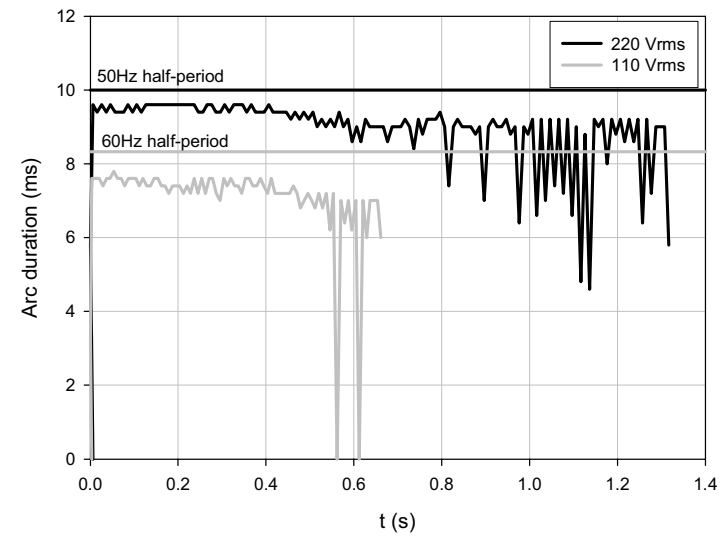

Fig. 14 Evolution of arc duration for 220 and 110Vrms.

\section{E. Energy}

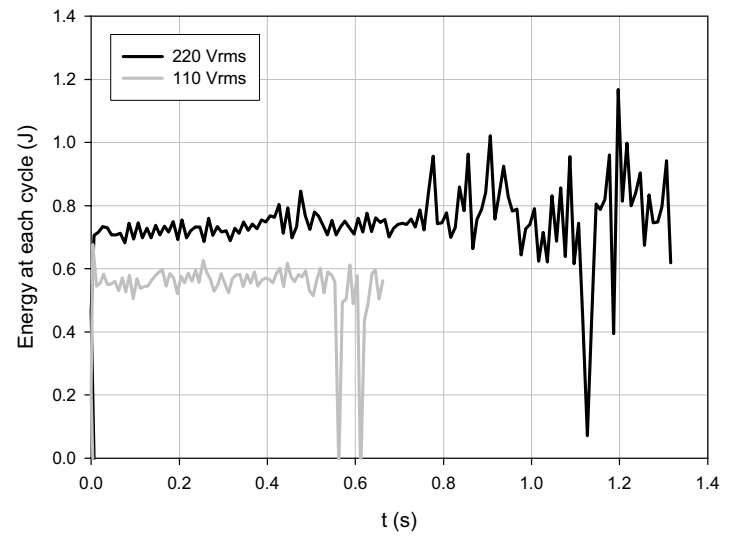

Fig. 15 Evolution of energy for 220 and 110 Vrms.
The consequence of the difference in Imax is that the energy consumed by the arcs is slightly lower at $110 \mathrm{~V}$ than at $220 \mathrm{~V}$ (see fig. 15). This, adds to the fact that at $60 \mathrm{~Hz}$, the arcs being shorter, (see fig. 14) have as a consequence regarding the energy consumed over time (fig. 16), a value for $110 \mathrm{~V}$ that is lower than for $220 \mathrm{~V}$. Furthermore, $220 \mathrm{~V}$ voltage makes easier the ignition of arcs so leads to products more arcs. Thus, the consumed energy is greater (100J compared with $40 \mathrm{~J})$. This is in line with fire ignition by arc fault in domestic wires (see [7]).

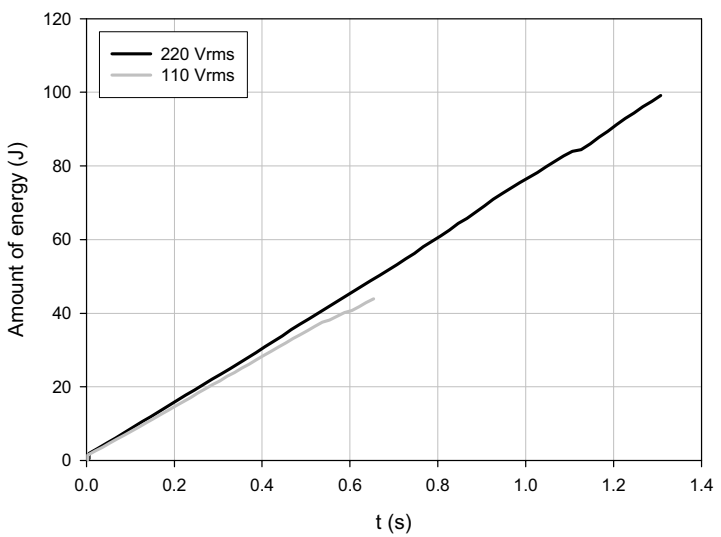

Fig. 16 Evolution of energy for 220 and 110 Vrms.

\section{CONCLUSIONS}

This work has presented the characteristics of breaking arcs as well as series arc faults and their variations in terms of current and voltage. Indeed, the phenomenon that leads to fires by arcing defects in electrical installation requires a good understanding of different causes of arcs. This work has been focused on the study of various parameters such as maximum voltage, arc voltage, arc duration or electrical energy consumed, and comparison between European and U.S. networks. The main result is that for these two different voltages, arcs have similar characteristics for same current and equivalent energy dissipated. However, $220 \mathrm{~V}$, due to its higher voltage, causes higher possibility of breakdown, which leads in our experiments to a larger number of arcs, thus to a larger energy consumption. This is a possible explanation for the greater risk of fire in the $220 \mathrm{~V}$ network compared to $110 \mathrm{~V}$ for similar current.

The future of this work will improve understanding of the arc effect on wire insulator and arc mechanism which leads fire in wire. We expected that this knowledge will improve electrical fire protection in domestic area.

\section{AKNOWLEDGMENTS}

The Authors thank Professor B. Mitchell for critical reading of the manuscript. 


\section{References}

[1] J. Swingler, J. W. McBride, "The net zero erosion phenomena on opening contacts with ac loading", Proc. IEEE Holm Conf. on EC, pp. 238-245, 1997.

[2] T. S. Davies, H. Nouri, J. Head, «A comparative assessment of different silver based contact materials under inductive and capacitive loads, Proc. ICEC p. 445, 1998.

[3] H. Nouri, T. S. Davies, J. Head, «Influence of AC interruption points on AC arc erosion of silver based contact materials », Proc. IEEE Holm Conf. on EC, pp. 207-213, 1998.

[4] C. E. Restrepo, "Arc fault detection and discrimination methods", Proc. IEEE Holm Conf. on EC, pp. 115-122, 2007.

[5] D. Kolker, S. Campolo, N. DiSalvo, "A study of time/current characteristics of the ignition processes in cellulosic material caused by electrical arcing for application in $240 \mathrm{v}$ arc-fault circuit interrupters" Proc. IEEE Holm Conf. on EC, pp. 105-114, 2007.

[6] E. Carvou, N. Ben Jemaa, "Experimental Studies on Arc Duration in Ac Voltage Supply" Proc. of the 24th International Conference on Electrical Contacts; St Malo (France), pp 7-12, 2008.

[7] J. Shea, "Comparing 240 Vrms to 120 Vrms Series Arcing Faults in Residential Wire", Proc. IEEE Holm Conf. on EC, pp. 218-224, 2008.

[8] E. Carvou, N. Ben Jemaa, "Electrical arc study in the range of 14$112 \mathrm{vdc}$ for automotive power contacts" Proc. of the 23rd International Conference on Electrical Contacts; Sendai (Japan), pp 28-33, 2006.

[9] R. Holm "Electric Contacts Theory and Application", Springer, fourth Edition, 2000.

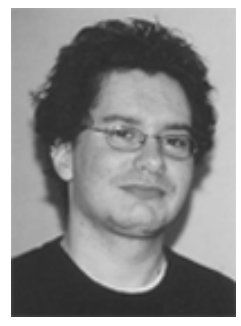

Erwann CARVOU received the PH.D. degree in Electronics from the University of Rennes in 2003.

He currently works on electrical contacts for automotive, power and domestic area. This research concerned the conduction and effect of arc disconnection.

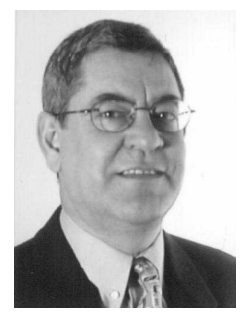

N. BENJEMAA (IEEE Member for 11 years NO 01629807) received the Doctorat és-sciences in physics from the University of Rennes I, France, in 1985. He has 25 years of research covering the physics and degradation encountered in electrical contacts. This research has been mainly concerned with low and medium electrical levels and has covered arc parameters and contact resistance. This work has been published in more than 80 papers mainly in ICEC, Holm, NARMS, IEEE journals and used in the telecommunication and automobile fields. He is currently professor of physics and electronics at the University of Rennes I where he directs and manages the electrical contacts group research.

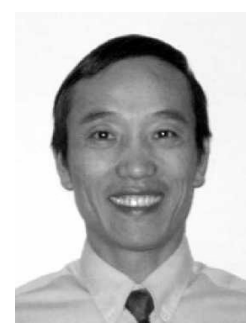

Simon TIAN received the Doctorat éssciences in physics from the University of Paris VI, in 1987. He joined Schneider Electric in 1991 and has worked in different research and development fields. He is currently the technical manager for Earth leakage and arc fault protection within Schneider.

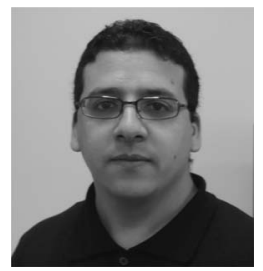

Zakaria BELHAJA Received his M.E. degree in electrical engineering from INSA Lyon (France) in 2007.

$\mathrm{He}$ is a Mechatronic Design Engineer with Schneider Electric since 2007. He currently works on the development of the arc fault protection.

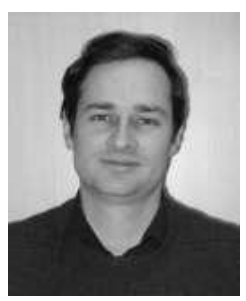

Benoit JUSSELIN

Received his M.E. degree in electrical engineering from ESMESudria France. He joined Schneider Electric in 1996 and he is currently the Electrical contact team manager. 


\title{
A Simplified Model of Multiscale Electrical Contact Resistance and Comparison to Existing Closed Form Models
}

\author{
Robert L. Jackson* \\ Robert D. Malucci ${ }^{+}$ \\ Santosh Angadi* \\ J. Robert Polchow ${ }^{*}$ \\ ${ }^{*}$ Center for Advanced Vehicle Electronics \\ Department of Mechanical Engineering \\ Auburn University, Auburn, AL 36849-5341 \\ ${ }^{+}$RD Malucci Consulting \\ Naperville, Illinois
}

\begin{abstract}
The prediction of the electrical contact resistance due to the contact of rough surfaces is important for electrical connectors, relays, circuit breakers, compliant pins, and many other applications. When modeling the contact between surfaces it is important to consider the multiple scales of roughness that exist. Many recent rough surface contact models exist in the literature, but can be difficult to implement. The current work derives and presents a simplified closed-form multiscale model of electrical contact resistance. The results are then compared to other existing closed form models of rough surface contact. The comparison shows that some existing models are in relatively close agreement with the multiscale model results which adds validity to both.
\end{abstract}

Keywords: contact resistance, electrical resistance, roughness, surface contact

\section{Nomenclature}

$A_{i} \quad$ area of contact for a particular scale

$A_{n} \quad$ nominal contact area

$a$ radius of the asperity area of contact

$d \quad$ separation of mean asperity height

D macroscale contact diameter

E elastic modulus

$E^{\prime}$ equivalent elastic modulus: $\left(\left(1-v_{1}^{2}\right) / E_{1}+\left(1-v_{2}^{2}\right) / E_{2}\right)^{-1}$

$F \quad$ contact force

$f \quad$ spatial frequency (reciprocal of wavelength)

$H \quad$ surface hardness $\left(H \approx 2.84 S_{y}\right)$

$L \quad$ scan length

$M \quad$ spectral moment of the surface

$N \quad$ total number of asperities $n \quad$ number of contact spots (asperities in contact)

$P \quad$ contact force

$p^{*} \quad$ average pressure for complete contact

$R \quad$ electrical contact resistance

$S_{y} \quad$ yield strength

$z \quad$ height of asperity measured from the mean of asperity heights

Greek Symbols

$\eta \quad$ areal density of asperities

$\sigma \quad$ standard deviation of surface heights

$\lambda \quad$ asperity wavelength

$\rho \quad$ electrical resistivity of surface material

$\Delta \quad$ asperity amplitude

$\Phi$ distribution function of asperity heights

$\Psi \quad$ alleviation factor

$\omega$ interference between hemisphere and surface

$v \quad$ Poisson's ratio

Subscripts

c critical value at onset of plastic deformation

$i \quad$ frequency level or scale

\section{BACKGROUND}

There are many different methods to model the contact of rough surfaces including statistical [1], fractal [2], and multiscale models [3-6]. The fractal mathematics based methods were derived to account for different scales of surface features not accounted for by the statistical models. The multiscale models were developed to alleviate the assumption of self-affinity imposed by fractal mathematics and also improve how the mechanics are considered. This work uses a Fourier transform to convert the data into a series of stacked sinusoids (see Fig. 1). Also, the surface characteristics necessary to obtain convergence of the iterative multiscale scheme are examined. 


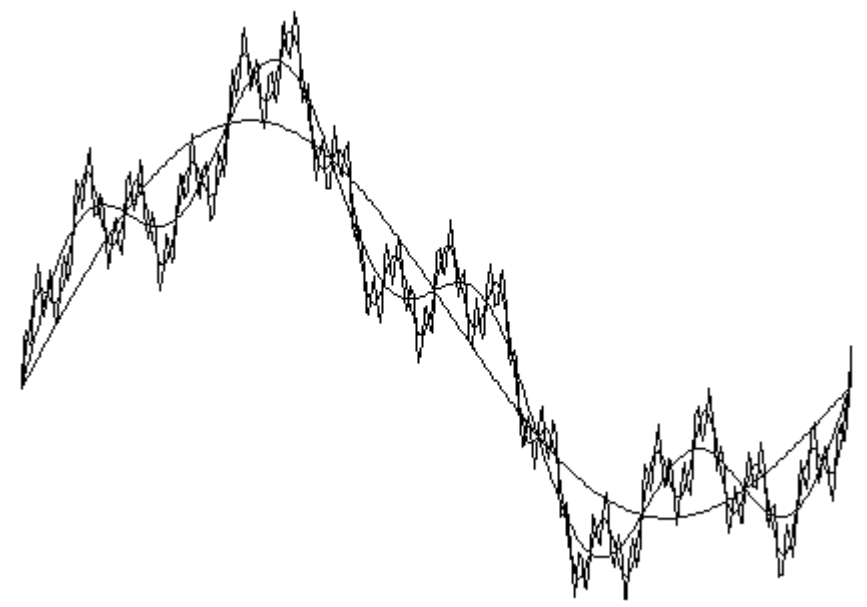

Fig. 1: Schematic depicting the decomposition of a surface into superimposed sine waves. Each line represents a different scale of roughness.

However, many of these models require complex numerical methods in order to predict the real area of contact and then the electrical contact resistance (ECR). There are some closed-form models that are available and widely used in the field. To predict the real area of contact, probably the most simple equation assumes that the real contact pressure is equal to Tabor's hardness [7]:

$$
A=\frac{F}{H}=\frac{F}{2.84 S_{y}}
$$

Others later found that the real contact pressure was often higher than the predicted hardness and therefore refined Eq. (1) further [8]. In an equation form that can be easily used this is given as [9]:

$$
A=\frac{F}{H}\left(1+\frac{F}{A_{n} H}\right)^{-1}=\frac{F}{2.84 S_{y}}\left(1+\frac{F}{A_{n} 2.84 S_{y}}\right)^{-1}
$$

Equation (2) predicts that as surfaces come into more complete contact, that the real contact pressure increases. The rough surface models predictions of the real area of contact are then used to predict contact resistance. Holm [10] gives a simple formula to calculate the electrical resistance due to asperity contact.

$$
R_{a s p}=\frac{\rho_{1}+\rho_{2}}{4 a}=\frac{\rho}{2 a}
$$

where $R$ refers to the resistance value, $a$ to the radius of contact, and $\rho$ to the electrical resistivity of the respective surfaces. If the surfaces are made of identical materials then the equations reduce to the right side of Eq. (3). However, this equation is only good for a single asperity. Holm [10] and Greenwood [11] gave very similar equations to predict the ECR between rough surfaces:

$$
R=\frac{\rho}{2 n a}+\frac{\rho}{D}
$$

where $n$ is the number of asperity contacts in the diameter of contact $D$. The second term could be categorized as resistance due to bulk or macroscale contact and therefore the current work will concentrate on the first term in Eq. (4). In a previous work it was found that $n$ was typically between 100 and 1000, but was also dependent on load. Using the same methodology implemented by Malucci [12] and fitting an equation to the predictions, results in the following equation for predicting the number of asperities in contact:

$$
n=196 \cdot F\left(\frac{0.24 \cdot 10^{-6}}{\sigma}\right)^{2}\left(1+\frac{F}{A_{n} 2.84 S_{y}}\right)^{-1}
$$

It should be noted that in the surface model used to develop Eq. (5a), $n$ depends on the surface roughness parameter $\sigma$ and was normalized to surface 1 using the data in table 1 and Eq. 15. Assuming a random rectangular distribution of asperity sizes, the value of $n a$ can be calculated as [9]:

$$
n a=\left[\frac{3 n F}{\pi 2.84 S_{y}}\left(1+\frac{F}{A_{n} 2.84 S_{y}}\right)^{-1}\right]^{1 / 2}
$$

Equation (5b) can then be used in the first term in Eq. (4) to calculate the micro-scale multi-spot contact resistance. Greenwood and Williamson also derived a perfectly elastic statistical based model of electrical contact resistance (See Appendix). The 'fully plastic' model given by Eq. (4) and (5) along with the Greenwood and Williamson model given by Eq. (A10) will later be compared to the multiscale methods described next.

\section{Model \\ A.Simplified Multi-Scale Contact Model}

The full multiscale electrical contact model is described in [13]. The contact mechanics used in the multiscale model are derived by Jackson and Streator [5]. First, a fast Fourier transform is performed on the surface profile data. Then the resulting data is a summation of a series 
of sine and cosine waves. The complex forms of the sine and cosine terms at each frequency are combined using a complex conjugate to provide the amplitude of the waveform at each scale for further calculations. Each frequency is considered a scale or layer of asperities which are stacked iteratively upon each other. In equation form these relationships are given by:

$$
\begin{aligned}
& A=\left(\prod_{i=1}^{i_{\max }} \overline{A_{i}} \eta_{i}\right) A_{n} \\
& P=\overline{P_{i}} \eta_{i} A_{i-1}
\end{aligned}
$$

where $A$ is the real area of contact, $\eta$ is the areal asperity density, $P$ is the contact load, $A_{n}$ is the nominal contact area, and the subscript $i$ denotes a frequency level, with $i_{\max }$ denoting the highest frequency level considered.

In its full form, multiscale model of rough surface contact requires an iterative numerical algorithm to solve it, which can be difficult to implement for practicing engineers. Therefore the model can be simplified by making a few assumptions as shown in [14]. In that work it is shown that the real contact pressure $\left(P_{r}\right)$ predicted by the multiscale model is approximated by the following equation from [15]:

$$
\begin{gathered}
\left(P_{r}\right)_{\text {elastic }} \approx \sqrt{2} \pi E^{\prime} C B_{\max }=\frac{F}{A_{r}} \\
\left(P_{r}\right)_{\text {elastic-plastic }} \approx \sqrt{2} \pi E^{\prime} C B_{\max }\left(\frac{11}{\frac{12 \pi E^{\prime}}{\sqrt{2} S_{y} e^{2 v / 3}} C B_{\max }+7}\right)^{3 / 5}=\frac{F}{A_{r}}
\end{gathered}
$$

where $B_{\max }$ is the maximum ratio of the amplitude to the wavelength for the surface acquired from a fit to the Fourier Series of the surface. $C$ is a constant that when set to 0.8 , Eqs. (8 and 9) produce predictions that agree well with the full multiscale model. Equations (8 and 9) are derived from the pressures required to obtain complete contact between sinusoidal surfaces [15]. The current model decomposes the surface into a series of stacked sine waves and finds that the real contact pressure is only slightly smaller than the pressure required to flatten sine waves defined by $B_{\max }$.

Equation (8) should be used when the surface is believed to be deforming perfectly elastically, while Eq. (9) should be used when elastic perfectly-plastic deformation is expected. This can be determined from the critical amplitude that was found analytically in [15].

The predictions of the real contact pressure $\left(P_{r}\right)$ using Eqs. (8 and 9$)$ are compared to the predictions generated by the full multiscale contact model for three different surfaces (see Tables 1 and 2). It should be noted that Eqs. (8 and 9) are approximate and may not be accurate for all surfaces. This comparison is also only for small values of $A_{r} / A_{n}$, and well before the surface comes into complete contact. This is important, because as the surfaces come into complete contact, $P_{r}$ will have to increase to overcome the pressure required to obtain complete contact for the sinusoidal shaped asperities defined by $B_{\max }$. This will result in a trend similar to that predicted by [8] and Eq. (2).

Table 1: The geometrical properties of several surfaces.

\begin{tabular}{|l|l|l|l|}
\hline Surface & $\sigma(\mu \mathrm{m})$ & $\lambda_{\max }(\mu \mathrm{m})$ & $B_{\max }$ \\
\hline 1 & 0.24 & 16.00 & 0.000818 \\
\hline 2 & 0.34 & 17.39 & 0.00215 \\
\hline 3 & 1.05 & 25.00 & 0.00574 \\
\hline
\end{tabular}

Table 2: A comparison of the normalized real contact pressure calculated from various methods for small $A_{r} / A_{n}$.

\begin{tabular}{|l|l|l|l|l|}
\hline \multirow{2}{*}{ Surface } & \multicolumn{4}{|c|}{ Real Contact Pressure $\left(P_{r} / S_{y}\right)$} \\
\cline { 2 - 5 } & $\begin{array}{l}\text { Elastic } \\
\text { Multiscale } \\
\text { Model (Full) }\end{array}$ & $\begin{array}{l}\text { Elastic-Plastic } \\
\text { Multiscale } \\
\text { Model (Full) }\end{array}$ & Eq. (8) & Eq. (9) \\
\hline 1 & 2.96 & 1.96 & 2.90 & 1.98 \\
\hline 2 & 7.74 & 3.27 & 7.64 & 3.37 \\
\hline 3 & 19.9 & 4.66 & 20.4 & 5.32 \\
\hline
\end{tabular}

To account for this increase in $P_{r}$ as complete contact is approached, the following equation is proposed:

$$
\left(P_{r}\right)_{\text {eff }}=P_{r}\left(C B_{\max }\right)+\left[P_{r}\left(B_{\max }\right)-P_{r}\left(C B_{\max }\right)\right]\left(\frac{F}{A_{n} P_{r}\left(B_{\max }\right)}\right)^{\beta}
$$

where $\beta=2$ for elastic contact and $\beta=4$ for elastic-perfectly plastic contact, and $P_{r}$ can be predicted using either Eq (8) for perfectly elastic contact or Eq (9) for elastic-perfectly plastic contact. The predictions from Eq. (10) appear to compare fairly well to the full multiscale model as shown in Fig. 2 for the three surfaces described in Table 1. Then the real area of contact is predicted by

$$
A_{r}=\frac{F}{\left(P_{r}\right)_{e f f}}
$$

\section{B. Simplified Multiscale Contact Resistance Model}

The electrical contact resistance predicted by Eq. (3) is only good for a single asperity. In the case of both multiscale and statistical techniques, additional equations are required to calculate resistance for the entire surface. 

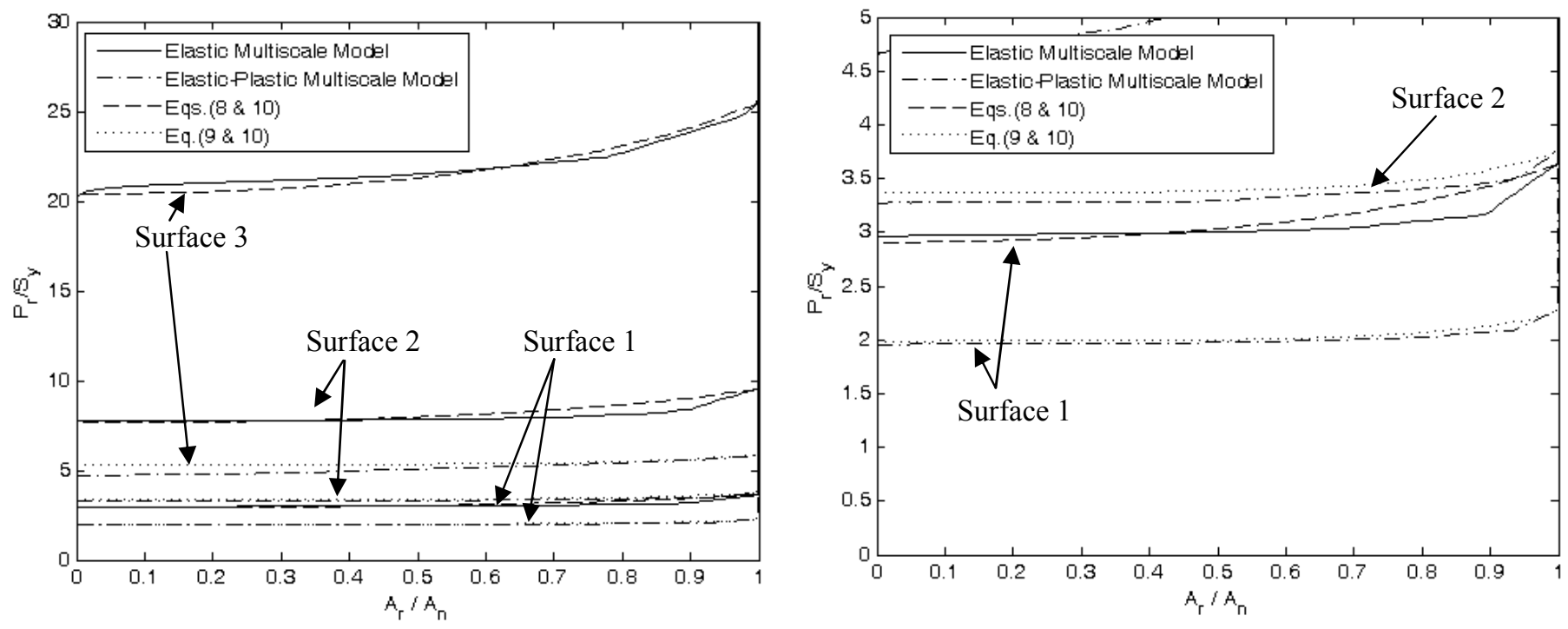

Figure 2: Comparison of pressure predicted by full multiscale models and the new simplified models.

In a previous work on multiscale electrical contact resistance, the entire procedure is outlined in detail [13]. It is assumed that each scale of asperity contacts can be modeled as an evenly spaced cluster of contacts with the same contact radius (the first term in Eq. (4). The simplified version of the alleviation factor offered by Cooper et al. [16] is also used between scales. The contact resistance from each scale is summed over all the possible iteration levels to find the total resistance for the entire surface in contact:

$$
R_{\text {total }}=\sum_{i=1}^{i_{\max }} R_{i} \eta_{i} A_{i}
$$

It is important to note that this technique calculates the resistance for each frequency level and then sums over all frequency levels to calculate the total. In order to simplify Eq. (12), the multiple scales of stacked asperities are now considered using a single cone shaped asperity concept. Consider the geometry of a single asperity in contact located on top of the stacks of larger scale asperities (see Fig. 3). If it is assumed that each of the smallest scale contacts is isolated, then the multispot contact resistance can be approximated by

$$
R=\frac{\rho}{2 a}\left(\frac{1}{n}\right)
$$

which is identical to the first term of Eq. (4). The main difference is how we find $a$ and $n$ from the multiscale model. The multiscale model does not use spherical contacts to consider asperities, therefore to find $a$, one must consider the predicted real contact area:

$$
a=\left(\frac{A_{r}}{\pi n}\right)^{1 / 2}
$$

Finally, the number of asperities in contact, $n$, as predicted by the multiscale model must be calculated. As each scale of asperities is included in the multiscale model, the real area of contact is reduced. This also results in the real contact pressure increasing. The pressure will increase until the maximum value of $B$ in the surfaces' Fourier Series is reached $\left(B_{\max }\right)$. All scales smaller than $\lambda_{\max }$ will flatten out and reduce the contact area further. Since there are two asperity peaks in contact for one square wavelength area of the sinusoidal surface:

$$
n=2 \frac{A_{r}}{\lambda_{\max }^{2}}
$$

Enlarged view of smallest asperity in contact

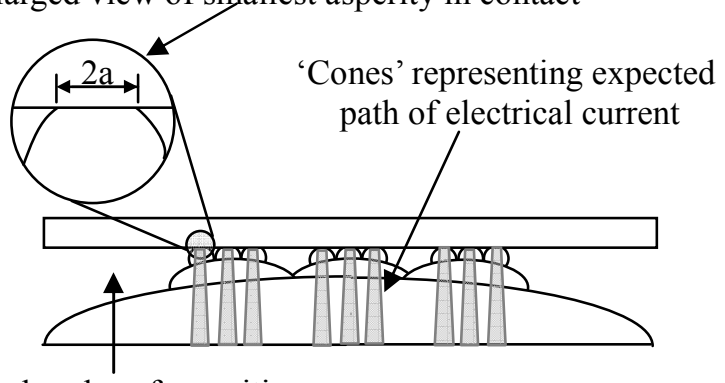

Stacked scales of asperities

Figure 3: Schematic of a simplified multiscale perspective of contact resistance. 


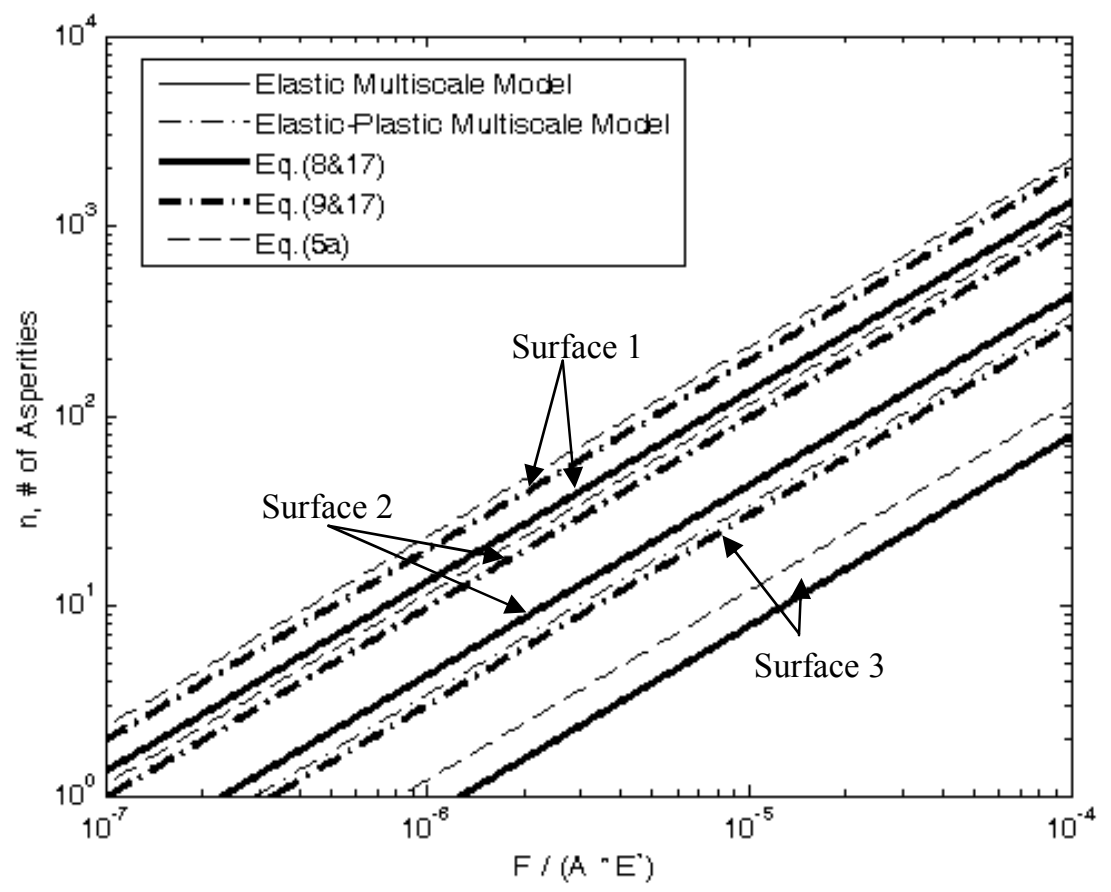

Figure 4: The number of asperities in contact as a function of load as predicted by the models.

The predictions of Eq. (15) are then compared for the simplified multiscale models given by Eqs. (8) and (9) and compared to the predictions of the full multiscale rough surface contact model (see Fig. 4). As shown in Fig. 3, the simplified models and the full multiscale models agree very well. In addition, some general observations can be made. First, when elasto-plastic asperity deformation is considered, more asperities are expected to be in contact than the perfectly elastic case. Second, the rougher surfaces tend to result in less asperities in contact at the same load. Finally, the trends shown in Fig. (4) are very similar to trends shown by Malucci [12] in Fig. 2(a) in that paper.

Substituting Eq. (15) into Eq. (14) results in

$$
a=\frac{\lambda_{\max }}{\sqrt{2 \pi}}
$$

Then substituting Eqs. (16 and 15) into Eq. (13) results in

$$
R=\frac{\sqrt{2 \pi} \rho}{2}\left(\frac{\lambda_{\max }}{2 A_{r}}\right)
$$

The real area of contact, $A_{r}$, in Eq. (17) can be predicted by Eq. (11), resulting in

$$
R=\sqrt{\frac{\pi}{8}}\left(\frac{\rho \lambda_{\max }}{F}\right)\left(P_{r}\right)_{e f f}
$$

Finally, the prediction of $\left(P_{r}\right)_{e f f}$ from Eqs. (8-10) is substituted into Eq. (18) to predict the contact resistance. To clarify, Eqs. $(8,10$ and 18) are used to calculate contact resistance for multiscale surfaces in elastic contact while Eqs. $(9,10$ and 18) are used for surfaces in elasto-plastic contact.

\begin{tabular}{|c|c|}
\hline \multicolumn{2}{|c|}{ Table 3: Material Properties of Tin } \\
\hline$E=41.369 * 10^{9} \mathrm{~Pa}$ & $S_{y}=14 * 10^{6} \mathrm{~Pa}$ \\
\hline$v=0.36$ & $\rho_{L}=11.5 * 10^{-8} \Omega \cdot m$ \\
\hline
\end{tabular}

\section{MODEL PREDICTIONS}

This work assumes that the two surfaces in contact are tin and have the material properties shown in Table 3 for all the results.

The electrical contact resistance was calculated using the new simplified multiscale model using Eqs. (8, 10 and 18) for the elastic case and Eqs. (9, 10, and 18) for the elasticplastic case and compared to the predictions of the 'fully plastic' model given by Eq. (4) and (5) along with the Greenwood and Williamson model given by Eq. (A10). The comparisons are shown for three different surfaces (see Table 1) in Figs. 5, 6 and 7.

Figures 5a, 6a and 7a show the predictions of the elastic statistical model of Greenwood and Williamson[1], the fully plastic model given by Malucci[12], and the elastic and elastic-plastic multiscale models (full and simplified versions) 
over a wide range of contact forces. Since some of the predictions are in very close agreement and indistinguishable

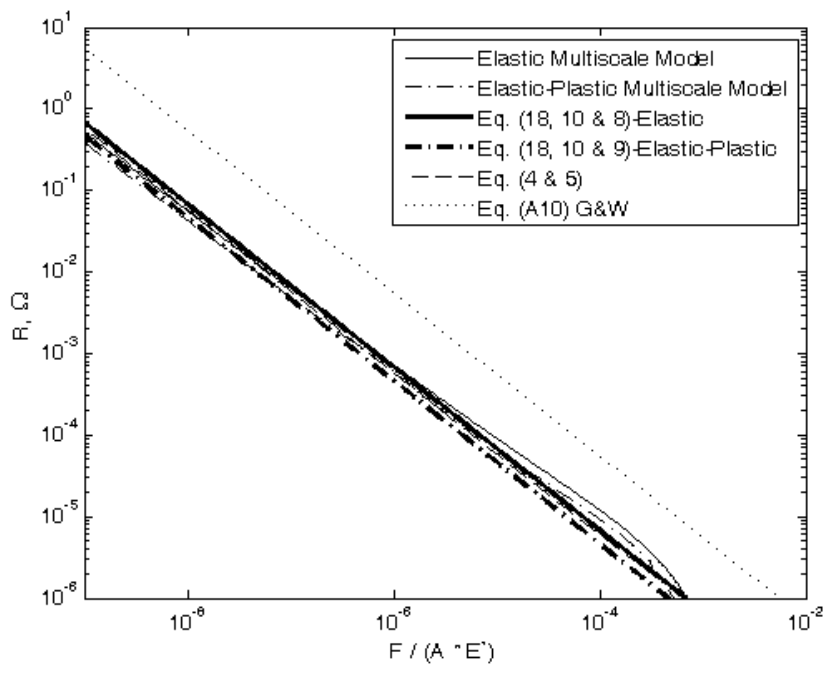

(a) in the plots, views over a smaller scale are shown in Figs. 5b, $6 \mathrm{~b}$, and $7 \mathrm{~b}$, so that the differences can be recognized.

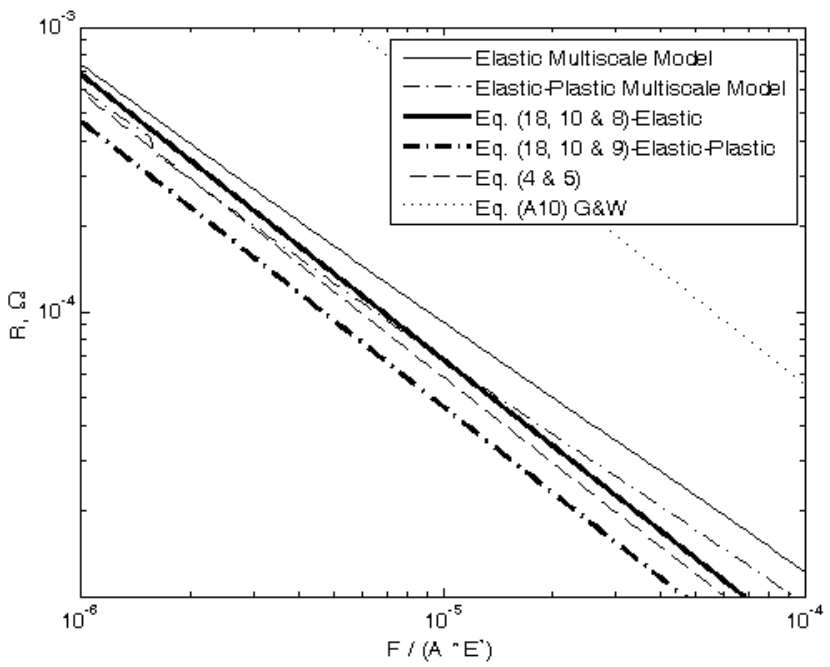

(b)

Figure 5: The electrical contact resistance for Surface 1 as predicted by several models.

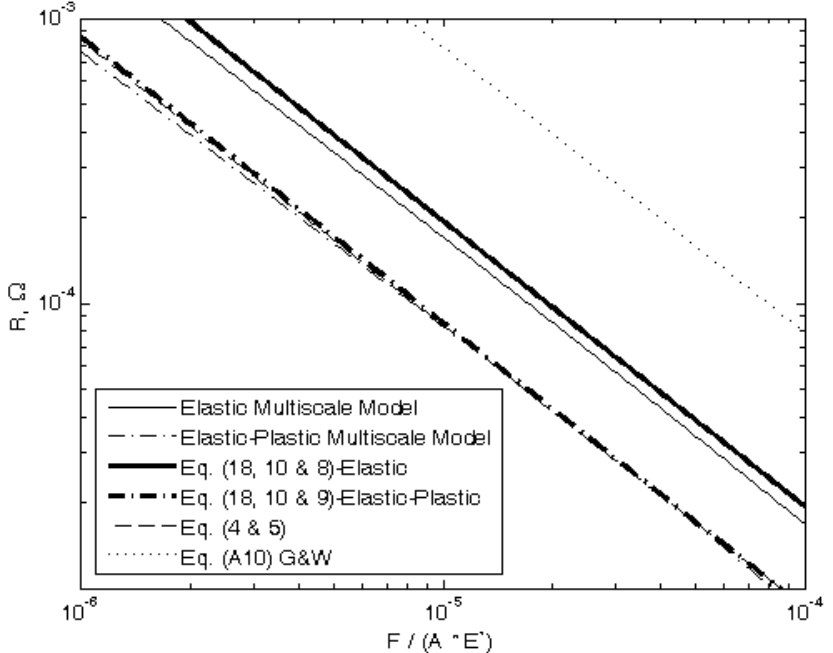

(a)

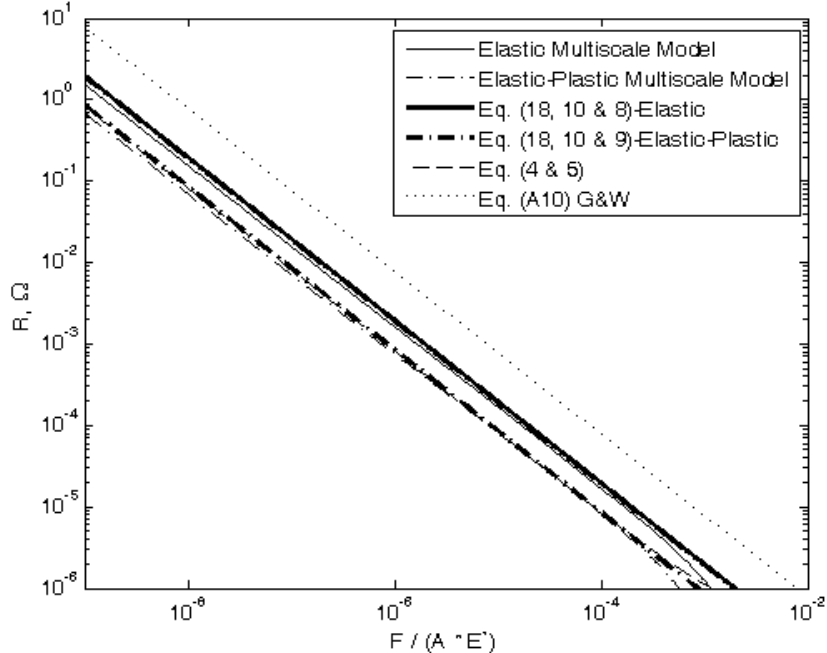

(b)

Figure 6: The electrical contact resistance for Surface 2 as predicted by several models.

As shown in Figs. 5-7, it appears that the full elastic multiscale model and the simplified elastic multiscale model make predictions of electrical contact resistance that falls in between the statistical perfectly elastic model (Eq. (A10)) and the fully plastic model given by Eqs (4 and 5). All the models appear to follow the same trend of decreasing resistance with load and also have similar slopes. The elastic-plastic multiscale models appear to usually predict lower contact resistances than the fully plastic model (Eqs. (4 and 5)). The elastic and elastic-plastic simplified multiscale models also agree well with the full multiscale models for surfaces 1 and 2 (see Figs. 5 and 6), but not as well for surface 3 which is the roughest (see Fig. 7). Still, the simplified multiscale models agree better with the full multiscale models than the other models, except for in some cases where the model given by Malucci (Eqs. 4 and 5) agrees very well with the full elastoplastic multiscale results.

The electrical contact resistance predicted by the statistical perfectly elastic model is greater than the other two models probably because it does not account for plastic 
deformation. In addition, it does not account for interaction between adjacent asperities, while both the multiscale models

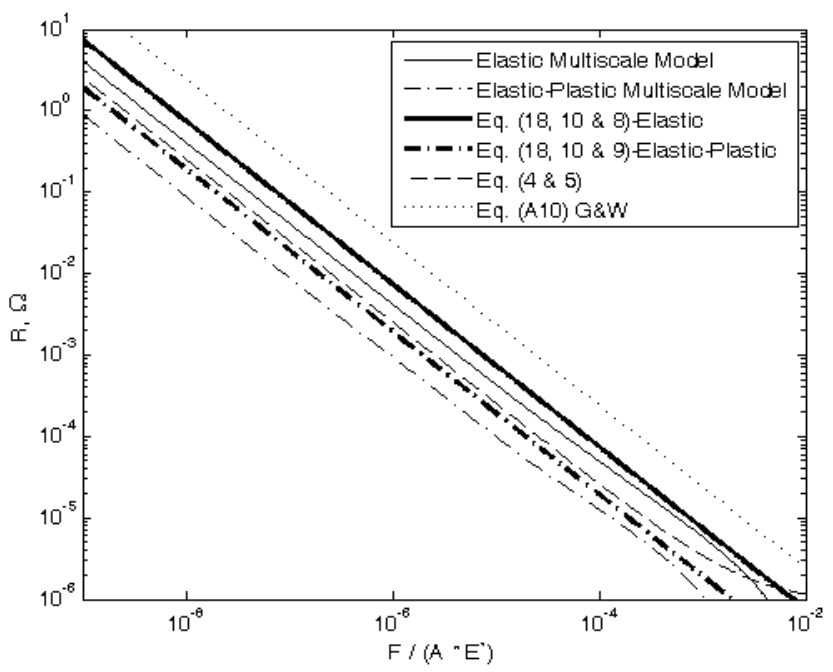

(a) and the fully plastic model do Eqs. (4 and 5).

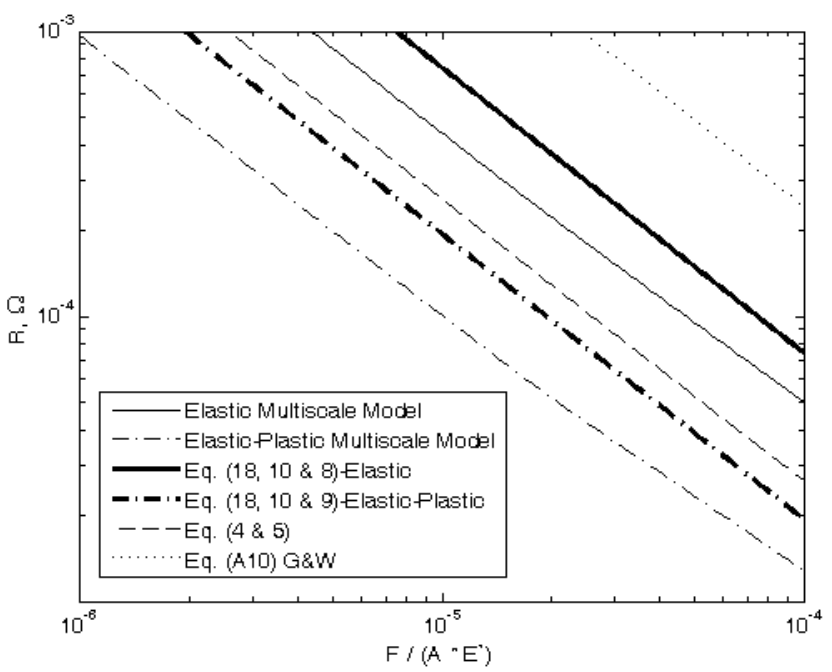

(b)

Figure 7: The electrical contact resistance for Surface 3 as predicted by several models.

Although the model presented by Malucci (Eqs. (4 and 5)) agrees well with the current multiscale methodology, there are reasons why there are some differences. The Malucci model is a fully plastic contact model, while the multiscale contact model considers both elastic and plastic deformation. The reason that the agreement is good might be because the asperities are usually heavily loaded and hence mostly undergoing plastic deformation. In addition, the current work only considers the first term of Eq. (4): Because the second term in Eq. (4) considers resistance from a larger scale of contact, the multiscale model should automatically consider it if surface data is used which captures the largest scale of the contact surface geometry. In the fully plastic model the second term must be calculated separately using the macroscopic contact shape and loading conditions.

Another reason for the differences between the three models is how the contact resistance is calculated from the asperity distribution and sizes. In the fully plastic model, the conducting asperity contacts are assumed to be distributed in size using a random rectangular distribution. The statistical perfectly elastic model considers asperities of a constant radius but with different contact radii, $a$, depending on their individual height and amount of loading. The simplified multiscale model is probably most similar to the fully plastic model since the number of asperities in the fully plastic model were normalized to surface 1 using the data in table 1 and equation 15. However, the full and simplified multiscale models calculate the number of asperities in contact differently than the other two models.
Since the model given by Malucci is in close agreement with the full and simplified multiscale models, both models can be considered more 'robust.' It is probably acceptable to use either of the models for making predictions of the electrical contact resistance. Still, one should use care in using these models due to the simplifications they make in modeling the surface geometry and contact mechanics.

\section{CONCLUSIONS}

This work has presented a new simplified method of calculating elastic-plastic electrical contact resistance based upon a multiscale model of rough surfaces built on stacked sinusoids. Results from this new technique have also been compared with the pre-existing elastic statistical methods and a fully plastic method presented by Malucci for predicting electrical contact resistance. The models all show the same trends, but the simplified multiscale models and the fully plastic model appear to be in reasonable agreement for some surfaces. In the future the authors plan to investigate and develop methods for including surface degradation in the models.

\section{ACKNOWLEDGMENTS}

Thanks to W. Everett Wilson for his assistance with the surface profile measurements and for some of his preliminary work on the subject. This work was supported partially by the Center for Advanced Vehicle Electronics, a National Science Foundation Industry/University Cooperative Research Center. 


\section{APPENDIX: Perfectly Elastic Statistical Model}

The Greenwood and Williamson model of rough surface contact assumes that the asperities follow a height distribution, $\Phi$, and all have a radius of curvature at their tips, $R$. The area and load are calculated using an integral of $\Phi$ and a function relating the asperity height, $z$ to the surface separation, $d$. All asperities in the range $z>d$ will be in contact with the rigid flat. The compression distance, $z-d$, is the interference of the rigid flat with the asperity peaks and is known as $\omega$ for the remainder of this work. The resulting integrals for electrical contact resistance and load are:

$$
\begin{array}{r}
\frac{1}{R}=\frac{2 A_{n} \eta \sqrt{\beta}}{\rho} \int_{\cdot d}^{\infty} \sqrt{z-d} \cdot \Phi(z) \cdot d z \\
F=A_{n} \eta \int_{\cdot d}^{\infty} \bar{F}(\omega) \cdot \Phi(z) \cdot d z
\end{array}
$$

For the perfectly elastic case, the values of $\bar{F}$ are acquired from the Hertz solutions given as:

$$
\bar{F}=\frac{4}{3} E^{\prime} \sqrt{R}(\omega)^{3 / 2}
$$

As this is a statistical method, it hinges upon obtaining statistical parameters that describe the surface.

$$
\begin{aligned}
& M_{2}=\frac{1}{N} \sum_{n=1}^{N}\left(\frac{d z}{d x}\right)_{n}^{2} \\
& M_{4}=\frac{1}{N} \sum_{n=1}^{N}\left(\frac{d^{2} z}{d x^{2}}\right)_{n}^{2}
\end{aligned}
$$

where $N$ is the total number of asperities on the surface and $z$ is the distance from the mean height of the surface to the asperity peak. The radius of curvature, $R$, and the areal asperity density, $\eta$, are then calculated by McCool [17] using spectral moments (Eqs. 26-27) about the surface.

$$
\begin{aligned}
& \eta=\left(\frac{M_{4}}{M_{2}}\right) \cdot\left(\frac{1}{6 \pi \sqrt{3}}\right) \\
& \beta=0.375 \cdot\left(\frac{\pi}{M_{4}}\right)^{0.5}
\end{aligned}
$$

Instead of a Gaussian distribution for the asperity heights, Greenwood and Williamson suggest using a exponential distribution so that the equations can be analytically solved. The resulting solutions found in Greenwood and Williamson are given as follows:

$$
\begin{aligned}
& \frac{1}{R}=\frac{\sqrt{\pi} A_{n} \eta \sqrt{\sigma \beta}}{\rho} e^{-d} \\
& F=E^{\prime} A_{n} \sqrt{\pi \sigma / \beta}(\eta \beta \sigma) e^{-d}
\end{aligned}
$$

Then substituting Eq. (A9) into Eq. (A8) results in:

$$
R=\frac{\rho \sigma E^{\prime}}{F}
$$

\section{REFERENCES}

[1] J. A. Greenwood and J. B. P. Williamson, "Contact of Nominally Flat Surfaces," Proc. R. Soc. Lond. A, vol. A295, pp. 300-319., 1966.

[2] A. Majumdar and B. Bhushan, "Fractal Model of Elasticplastic Contact Between Rough Surfaces," ASME J. of Tribol., vol. 113, pp. 1-11, 1991.

[3] M. Ciavarella, G. Demelio, J. R. Barber, and Y. H. Jang, "Linear elastic contact of the Weierstrass Profile," Proc. $R$. Soc. Lond. A, pp. 387-405, 2000.

[4] R. L. Jackson, S. H. Bhavnani, and T. P. Ferguson, "A Multi-scale Model of Thermal Contact Resistance between Rough Surfaces," ASME J. Heat Transfer, vol. 130, pp. 081301, 2008.

[5] R. L. Jackson and J. L. Streator, "A Multiscale Model for Contact between Rough Surfaces," Wear, vol. 261, pp. 1337-1347, 2006.

[6] B. N. J. Persson, "Elastoplastic contact between randomly rough surfaces," Physical Review Letters, vol. 87, pp. 116101, 2001.

[7] D. Tabor, The Hardness of Materials. Oxford: Clarendon Press, 1951.

[8] J. Pullen and J. B. P. Williamson, "On the Plastic Contact of Rough Surfaces," Proc. R. Soc. Lond. A, vol. 327, pp. 159-173, 1972.

[9] R. D. Mallucci and F. R. Ruffino, "Materials Considerations in Using Voltage Drop for Power Rating," presented at IEEE Holm Conference on Electrical Contacts, Pittsburgh, PA, 2007.

[10] R. Holm, Electric Contacts. New York: Springer, 1967.

[11] J. A. Greenwood, "Constriction Resistance and the Real Area of Contact," Brit. J. Appl. Phys., vol. 17, pp. 1621$1632,1966$.

[12] R. D. Malucci, "Multi-spot model showing the effects of nano-spot sizes," presented at IEEE Holm Conference on Electrical Contacts, 2005.

[13] W. E. Wilson, S. V. Angadi, and R. L. Jackson, "Electrical Contact Resistance Considering Multi-Scale Roughness," presented at IEEE Holm Conference on Electrical Contacts, Orlando, FL, 2008.

[14] R. L. Jackson, W. E. Wilson, and S. Angadi, "A Study of the Average Real Contact Pressure Between Rough Surfaces," presented at 2008 STLE/ASME International Joint Trib. Conference, Miami, FL, 2008.

[15] R. L. Jackson, V. Krithivasan, and W. E. Wilson, "The Pressure to Cause Complete Contact between Elastic Plastic Sinusoidal Surfaces," IMechE J. of Eng. Trib. - Part J., vol. 222, pp. 857-864, 2008.

[16] M. G. Cooper, B. B. Mikic, and M. M. Yovanovich, "Thermal Contact Conductance," Int. J. Heat Mass Transfer, vol. 12, pp. 279-300, 1969.

[17] J. I. McCool, "Comparison of Models for the Contact of Rough Surfaces," Wear, vol. 107, pp. 37-60, 1986. 


\section{A numerical method to predict the stick/slip zone of contacting, nonconforming, layered rough surfaces subjected to shear traction.}

\author{
Leidner M.; Schmidt H. \\ PE GAD EMA \\ Tyco Electronics AMP GmbH \\ Bensheim, Germany
}

\author{
Myers M. \\ Interconnection and Process Technology \\ Tyco Electronics \\ Harrisburg, USA
}

\begin{abstract}
It is known from experimental and field data that connector contacts may fail due to mechanical wear without being subjected to a sliding motion. Applying shear traction below the friction force results in a sliding annulus of the contact area since the individual a-spot tractions are limited by the friction coefficient of the contact material. Hence a constant displacement of the contacting a-spots is not possible. The paper proposes a numerical method based on a conjugate gradient method and Papkovich-Neuber potentials [1] to solve the contact problem for nonconforming, layered and arbitrary rough surfaces. The stick/slip zone as a function of the shear traction is calculated for $\mathrm{Au}, \mathrm{Ag}$ and $\mathrm{Sn}$ contact systems. The results are also visualized as $3 \mathrm{D}$ images.
\end{abstract}

Keywordst; stick/slip zone; electrical contact; conjugate gradient method; shear force

\section{INTRODUCTION}

It is known that connector contacts may degrade when subjected to a repetitive relative motion in the range of a micrometer. These motions can be caused by temperature cycles or vibrations. Depending on the magnitude of the relative displacement we have to distinguish between the so called partial slip and gross slip wear regimes. During partial slip, there is always a fraction of the initial contact area where the contacting a-spots remain connected. While in the gross slip wear regime the a-spots across the whole contact area are broken as relative motion occurs. It is obvious that gross slip reciprocating sliding results in more severe wear and fatigue, and depending on the contact finish, tribochemical corrosion. The transition from the partial to the gross slip wear regime depends on factors such as contact geometry, contact normal force, surface topography, surface finish, underplate and especially the friction coefficient. Extensive experimental research has been carried out in recent years [2][3][4][5] to measure the critical displacement associated with the transition from the partial slip to the gross slip sliding wear regime. A major problem found with these types of experiments was inherent test equipment compliance (C). Reference [2] shows this test equipment compliance typically ranges from 0.25 $\mu \mathrm{m} / \mathrm{N}$ to $1.5 \mu \mathrm{m} / \mathrm{N}$ (see also Figure 1 ). Assuming a compliance $\mathrm{C}$ of $1 \mu \mathrm{m} / \mathrm{N}$, at an applied shear force of $3 \mathrm{~N}$ a deformation of $\pm 3 \mu \mathrm{m}$ in the instrumentation would occur. According to reference [6] the compliance of two identical crossed cylinders can be estimated by:

$$
C_{C}=\frac{2 K}{3 \alpha}
$$

With $K=\frac{3}{8}\left(\frac{2(2-v)(1+v)}{E}\right)$ and $\alpha=\sqrt[3]{\frac{3}{2} \frac{\left(1-v^{2}\right) F_{N} r}{E}}$

Where $\alpha$ denotes the Hertzian contact radius, $r$ is the radius of the cylinders and $\mathrm{E}$ is the modulus of elasticity. Considering two phosphor bronze cylinders of $2 \mathrm{~mm}$ radius with $\mathrm{E}=120$ $\mathrm{GPa}, v=0.3$ subjected to a normal load of $\mathrm{F}_{\mathrm{N}}=5 \mathrm{~N}$ and a traction force of $\mathrm{F}_{\mathrm{T}}=3 \mathrm{~N}$ results in a compliance of $\mathrm{C}_{\mathrm{c}}=0.15 \mu \mathrm{m} / \mathrm{N}$ and a deformation of the contact interface of $\pm 0.45 \mu \mathrm{m}$.

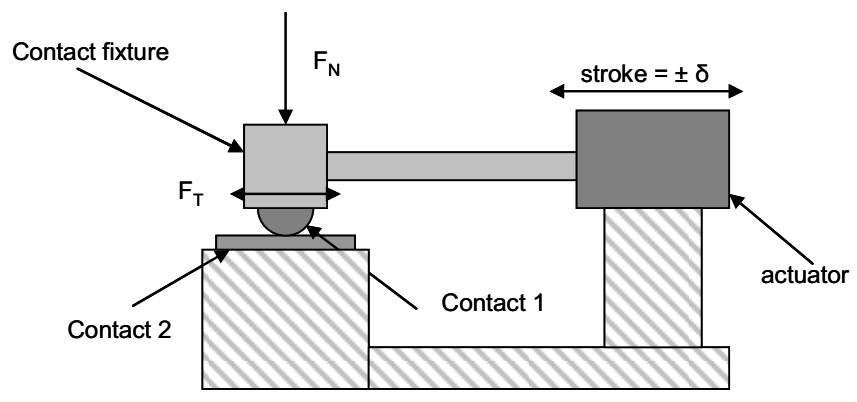

Figure 1: Schematic of an experimental test equipment example

In other words, the test set up described above having two contacts loaded to $5 \mathrm{~N}$, an interfacial static friction coefficient $\mu_{\text {static }}>0.6$, an actuator stroke $\pm 3.45 \mu \mathrm{m}$, and a test equipment compliance of $1 \mu \mathrm{m} / \mathrm{N}$ would not lead to gross slip. Another important point that needs to be illustrated is the width of the sliding annulus. With a Hertzian contact, the sliding of the contact area starts at the outer rim of the contact area and increases radially inward with increasing shear force. The width of the sliding annulus increases until only the center of the original contact area remains unbroken. That point is reached when $\mathrm{F}_{\mathrm{T}}=\mu \mathrm{F}_{\mathrm{N}}$. A further increase of the shear force will initiate sliding motion. Using the example of a Herzian contact with a radius of $\alpha=61 \mu \mathrm{m}$, this radius equals the maximum possible thickness of the sliding annulus. If a static friction coefficient $\mu_{\text {static }}=0.6$ is assumed, at a $F_{N}$ of $5 \mathrm{~N}$ the maximum traction force possible would be $3 \mathrm{~N}$. With an 
assumed test equipment compliance of $\mathrm{C}_{\mathrm{c}}=0.45 \mu \mathrm{m} / \mathrm{N}$, the maximum added deformation of the contact surface due to the shear force would be $0.45 \mu \mathrm{m}$. In other words, a relative motion of the test contacts in the range between $0 \mu \mathrm{m}$ and 0.45 $\mu \mathrm{m}$ results in an annulus width variation of $61 \mu \mathrm{m}$. Such an amplification ratio makes it impossible to determine the growth of the stick slip annulus as a function of relative contact motion experimentally. Especially when considering additional effects such as partial cold welding adhesion.

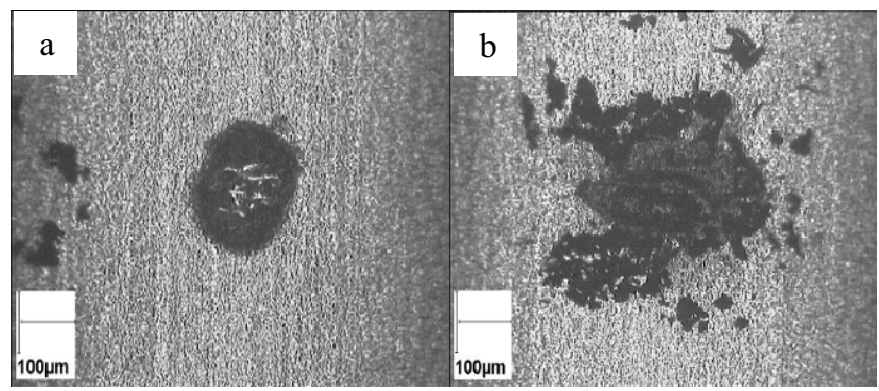

Figure 2: Wear scars of uncoated CuSn4 after $10^{6}$ cycles; a. displacment stroce $\pm 2 \mu \mathrm{m}$; b. displacment stroce $\pm 2.5 \mu \mathrm{m}$

The following algorithm has been developed in order to get a more detailed understanding of the influence factors making the difference between partial slip and gross slip. Enhancing this knowledge can be used to design more robust connectors for applications where shear force cannot be avoided.

\section{PROBLEM DESCRIPTION AND SOLUTION METHOD}

In the following simulations and discussion the two contacts will have symmetrical layer structures. Asymmetric contact pairs can be investigated as well, but require at some steps a parallel calculation and shall be omitted within this article. The nodes of the rectangular calculation grid shall be denoted by $(i, j)$ where $i$ and $j$ coincide with the $x$ - and $y-$ direction, respectively. $\Delta_{\mathrm{x}}$ and $\Delta_{\mathrm{y}}$ are the grid spacing. Two sets of modes shall be defined as follows:

$$
\begin{gathered}
I_{g} \text { with } \quad 0<i<N_{x} \wedge 0<j<N_{y} \\
I_{C} \quad \text { with } \quad(i, j) \in I_{g} \wedge \quad P_{i, j}>0
\end{gathered}
$$

Where $P_{i, j}$ is the point pressure due to pure normal load $F_{N}$.

$$
F_{N}=\Delta_{x} \Delta_{y} \sum_{(i, j)} P_{i, j}
$$

The shear force $\mathrm{F}_{\mathrm{T}}$ and the associated traction pressure distribution $\mathrm{T}_{\mathrm{i}, \mathrm{j}}$ is defined by:

$$
F_{T}=\Delta_{x} \Delta_{y} \sum_{(i, j)} T_{i, j}
$$

\section{A. Basic considerations}

Let us assume two identical spherical contacts with ideal smooth surfaces are pressed together under pure normal load (Hertz contact). Figure 3 illustrates a $\mathrm{z} / \mathrm{x}$-cross section through such a contact where the origin of $x$ coincides with the centerline. The left hand side illustrates the deflections due to a pure normal load $\mathrm{F}_{\mathrm{T}}=0$. At the center of the contact, the contact deformation is purely compressive. The deflections in $\pm \mathrm{x}-$ direction have equal magnitude but opposite direction. Now let us assume that an additional shear force $\mathrm{F}_{\mathrm{T}}$ is acting as indicated on the right hand side of Figure 3. If the contacting points (nodes $(\mathrm{i}, \mathrm{j}) \in \mathrm{I}_{\mathrm{C}}$ ) are bonded together ideally, the whole contact area would develop an additional deformation distribution with the following characteristic:

$$
\vec{u}_{i, j}^{\text {traction }}=e_{x} \cdot \text { const }^{(r)}
$$

Here $e_{x}$ denotes the unit vector in $\mathrm{x}$-direction and $\mathrm{r}$ indicates contacts 1 and 2, respectively. Since the shear force is acting in opposite direction with respect to contacts 1 and 2 , the constant factor in (6) has again the same magnitude but opposite algebraic sign. The traction distribution that creates such a deformation field has not the form:

$$
T_{i, j}=\mu_{\text {static }} \cdot P_{i, j}
$$

Consider a contact spot/point located in the center of the contact area. Such a contact point would be deflected by the deformation fields of its neighboring contact points. Therefore, each individual point has a potential stress field, long range effect, which has to be integrated. Conversely, a contact point located at the outer rim of the contact area only has neighbors in the direction of the contact center and hence will be deflected significantly less by the surrounding stress field. Therefore a contact point at the outer rim will experience a higher shear stress than a point inside the contact area in order to develop the same deflection in the x-direction. The resulting traction distribution has to meet the boundary condition in (5). In the 'real' world, contact points are not bonded with an infinite force. A contact point will slip when the shear pressure exceeds a level defined in (7).
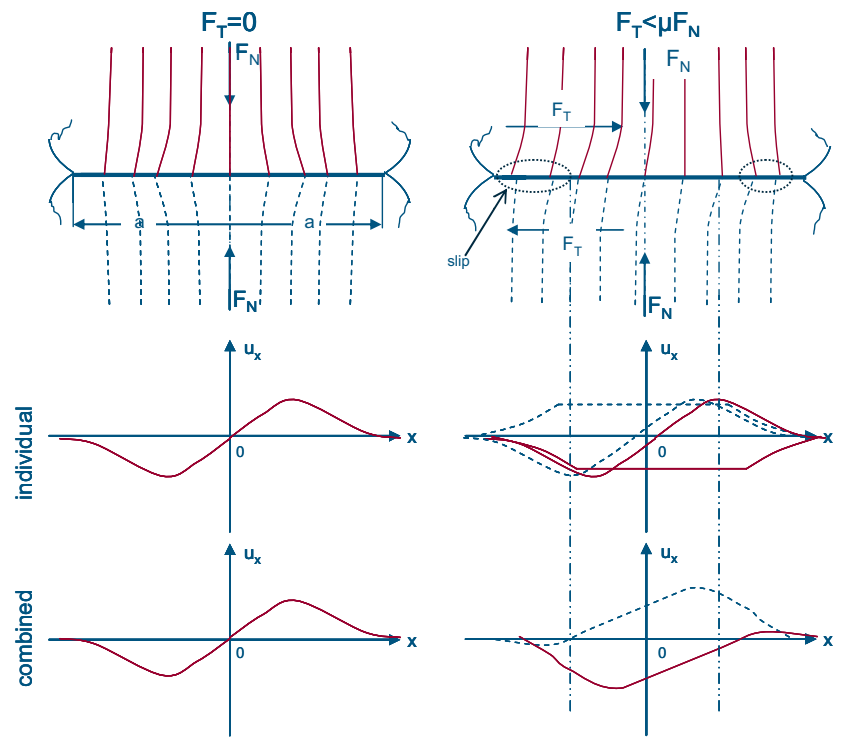

Figure 3: Superposition of the two deformation fields in $\mathrm{x}$-direction and creation of the sliding annulus (solid lines contact1, dashed lines contact 2)

Figure 3 also shows that the total displacement in x-direction can be described using two independent displacement fields a displacement field caused by the application of a pure normal load and a displacement field caused by the application of a pure shear force. In the following section an iterative algorithm is presented which is capable of predicting the shear 
stress distribution generated within two contacting nonconforming, layered and arbitrarily rough surfaces.

\section{B. Numerical algorithm}

The following algorithm is similar to the conjugate gradient method presented by [7]. After a contact interface is normally loaded followed by the application of a shear force, the resulting total displacement vector can be written as a superposition of two vectors.

$$
\vec{u}_{i, j}^{\text {total }}=\vec{u}_{i, j}^{\text {normal }}+\vec{u}_{i, j}^{\text {shear }}
$$

In the following analysis, it is assumed that the shear traction only has a component in the x-direction. Additional components in y-direction (e.g. torque) can be handled in a similar manner, but will be omitted for brevity.

First, an iterative calculation is done to calculate the normal pressure distribution $\mathrm{P}_{\mathrm{i}, \mathrm{j}}$ based on the algorithm shown in reference [1][8]. This algorithm calculates the stresses and displacements generated when two multi layer, rough, non conforming surfaces come into normal contact.

Next, a shear force is applied and the resulting displacement vector field $\vec{u}_{i, j}^{\text {normal }}$ is calculated through another iterative process. An initial traction distribution is generated using:

$$
T_{i, j}=0.1 \cdot \mu \cdot P_{i, j}
$$

Then an iterative loop calculation of the displacement vector field $\vec{u}_{i, j}^{\text {shear }}$ is initiated. Since this vector field has been defined as only having an $\mathrm{x}$-direction component, it can be represented using an abbreviated notation $u_{x i, j}^{\text {shear }}$.

$$
u_{x i, j}^{\text {shear }}=f_{u}(P, T)_{i, j} \text { with } \quad P=0
$$

$f_{u}(P, T)$ is a function with input parameters $P$ (normal pressure distribution) and $\mathrm{T}$ (shear traction distribution). The calculation algorithm used in this analysis to generate displacement and stress fields is based on a numerical solution derived from Papkovich Neuber potentials [1][8][9], though other approaches could be used.

The following algorithm is designed to derive a traction distribution leading to a constant displacement in response to the applied shear stress. Therefore, $\mathrm{P}$ has to be set to zero prior to the application of the shear stress. The components of the displacement vector generated as the pure normal load was applied must not be considered during this iteration and have to be added afterwards. Using the same algorithm in reference [1], the normalized "von Mises" Stress distribution $\sigma^{\text {Mises }}$ is:

$$
\sigma^{\text {Mises }}=\frac{\sqrt{\frac{1}{2}\left[\left(\sigma_{1}-\sigma_{2}\right)^{2}+\left(\sigma_{2}-\sigma_{3}\right)^{2}+\left(\sigma_{1}-\sigma_{3}\right)^{2}\right]}}{H^{\text {eff }}}
$$

$\mathrm{H}^{\text {eff }}$ denotes the hardness of the surface coating and $\sigma_{1.3}$ denotes the principal stresses. Here both the normal and shear load distributions have to be considered. The average value of the displacement is calculated by:

$$
\bar{u}_{x}=\frac{1}{N_{p}} \cdot \sum_{(i, j) \in I_{C}} u_{x_{i, j}} ; \quad T_{i, j}<\mu_{\text {load }} P_{i, j}
$$

Where $\mathrm{N}_{\mathrm{P}}$ denotes the number of contact spots satisfying the conditions of above formula: in a state up to and not beyond the point where contact points are 'broken'. Stated another way:

1. if a mesh point is a contact point $\rightarrow(i, j) \in I_{C}$

2. traction $\mathrm{T}_{\mathrm{i}, \mathrm{j}}$ is below a friction coefficient times $\mathrm{P}_{\mathrm{i}, \mathrm{j}}$

The value of the friction coefficient depends on the deformation response to the load regime. In case of $\sigma^{\text {Mises }}<1$ (elastic regime) $\mu_{\text {load }}$ is set to the static friction coefficient $\mu_{\text {static }}$. For $\sigma_{\text {Mises }}>1$ (plastic regime) $\mu_{\text {Load }}$ is a chosen value $>\mu_{\text {Static }}$.

$$
\begin{array}{ll}
\mu_{\text {Load }}=\mu_{\text {static }} ; & \sigma^{\text {Mises }}<1 \\
\mu_{\text {Load }}=\mu_{\text {plast }} ; & \sigma^{\text {Mises }}>1
\end{array}
$$

In a next step the displacement vector field $u_{x i, j}^{\text {shear }}$-values are shifted according to:

$$
u_{x i, j}^{\text {shear }} \leftarrow u_{x i, j}^{\text {shear }}-\bar{u}_{x}
$$

After calculating the quadratic sum:

$$
U=\sum_{(i, j) \in I_{C}} u_{x_{i, j}}^{\text {shear }}{ }^{2} ; T_{i, j}<\mu_{\text {Load }} P_{i, j}
$$

the conjugate directions can be calculated as follows:

$$
\begin{aligned}
& t_{i, j} \leftarrow u_{x i, j}^{\text {shear }}+\delta \cdot\left(U / U_{\text {old }}\right) \cdot t_{i, j} ; \quad(i, j) \in I_{C} \wedge T_{i, j}<\mu_{\text {Load }} P_{i, j} \\
& t_{i, j}=0 ; \quad(i, j) \notin I_{C}
\end{aligned}
$$

The auxiliary value $\delta$ in above formula is set in the initial loop to zero. Hence, defining a start distribution of the conjugate directions is not necessary. After the first calculation of the $t_{i, j}$, $\delta$ can be set to one and $U$ is stored into $U_{\text {old }}$. The $t_{i, j}$ distribution is the direction of steepest descent in which the pressure distribution has to be corrected. Next a residual distribution $r_{i, j}$ is calculated, based on the same numerical algorithm used in (10).

$$
r_{i, j}=f_{u}(P, t)_{i, j} \text { with } P=0
$$

The calculation of the mean value and subsequent value shift is done analogous to (12) and (14).

$$
\begin{gathered}
\bar{r}_{x}=\frac{1}{N_{p}} \cdot \sum_{(i, j) \in I_{P}} r_{i, j} ; \quad T_{i, j}<\mu_{\text {load }} P_{i, j} \\
r_{i, j} \leftarrow r_{i, j}-\bar{r}
\end{gathered}
$$

Now a scale factor has to be calculated in order to weight the $t_{i, j}$ direction distribution.

$$
\tau=\frac{\sum_{(i, j) \in I_{C}} u_{x_{i, j}}^{\text {shear }} t_{i, j}}{\sum_{(i, j) \in I_{C}} r_{i, j} t_{i, j}} ; \quad T_{i, j}<\mu_{\text {Load }} P_{i, j}
$$

The $\mu_{\text {Load }}$ conditions defined in (8) can then be applied to the traction distribution using the following conditional equations: 


$$
\begin{aligned}
& T_{i, j} \leftarrow T_{i, j}-\tau \cdot t_{i, j} ; \quad(i, j) \in I_{C} \wedge T_{i, j}<\mu_{\text {Load }} P_{i, j} \\
& T_{i, j} \leftarrow \mu_{\text {Load }} P_{i, j} \quad ; \quad(i, j) \in I_{C} \wedge T_{i, j}>\mu_{\text {Load }} P_{i, j} \\
& T_{i, j} \leftarrow 0 \quad ; \quad(i, j) \notin I_{C}
\end{aligned}
$$

Because the shear force is applied in the positive x-direction, the following condition can be used to accelerate the convergence of the algorithm:

$$
T_{i, j}=0 ; \text { if } T_{i, j}<0
$$

At this point the actual traction force, $\mathrm{F}_{\mathrm{T}}$ actual , can be calculated using equation (5) and a balance of the forces is maintained by the following:

$$
T_{i, j} \leftarrow\left(\frac{F_{T}}{F_{T}^{\text {actual }}}\right) \cdot T_{i, j}
$$

The actual error can be estimated through:

$$
\varepsilon^{\text {actual }}=\left|F_{T}^{\text {actual }}-F_{T}\right|
$$

If $\varepsilon^{\text {actual }}$ is above a preset error, the iteration starts again with the calculation of the displacement field $u_{x i, j}^{\text {shear }}$ according to (10). The number of iterations strongly depends on the accuracy chosen and the normal to shear force ratio. Typically the iteration converges after about 20 cycles.

\section{Estimation of the displacement hysteresis and adhesion forces}

After the convergence of the above described iteration scheme, the normal load, the shear traction, and the von Mises Stress distributions are known. Using this data the following estimation can be done:

1) When a contact interface is subjected to a shear force, regions where asperities are elastically $\left(\sigma^{\text {Mises }}<1\right)$ deformed and regions where the asperities are plastically $\left(\sigma^{\text {Mises }}>1\right)$ deformed will develop. Assuming the following occurs after release of the shear force:

- The elastically deformed asperities $\left(\sigma^{\text {Mises }}<1\right)$ will become point sources of residual load equal in magnitude and inverse in direction.

- The plastically deformed asperities $\left(\sigma^{\text {Mises }}>1\right)$, will not contribute to the residual pressure distribution.

The resulting pressure distribution can then be estimated as:

$$
\begin{aligned}
& T_{i, j}^{\text {residual }}=T_{i, j} ; \quad(i, j) \in I_{C} \wedge \sigma^{\text {Mises }}<1 \\
& T_{i, j}^{\text {residual }}=0 \quad ; \quad(i, j) \in I_{C} \wedge \sigma^{\text {Mises }}>1 \\
& T_{i, j}^{\text {residual }}=0 \quad ; \quad(i, j) \notin I_{C}
\end{aligned}
$$

Using $\mathrm{T}^{\text {Residual }}$ as input variable in (10) results in a virtual spring back of the deformed contact area. Hence:

$$
u^{h}=\max \left(f_{u}(0, T)_{i, j}-f_{u}\left(0, T^{\text {Re sidual }}\right)_{i, j}\right)
$$

is a measure of the deformation hysteresis.

2) By knowing the number of asperities in the plastic load regime, another assumption can be made. Since the contacting asperities are metallic (no films considered within this model), anywhere a point pressure is high enough to cause plastic deformation should lead to 'cold welding' adhesion at these contact spots. Assuming there are no other sources of adhesion (e.g. meniscus effects [10]), this leads to a maximum possible adhesion force on the order of:

$$
F^{\text {adhesion }}=\Delta_{x} \Delta_{y} N^{\text {plast }} Y_{\min }
$$

Where $\mathrm{N}^{\text {Plast }}$ denotes the number of plastic deformed contact spots within the contact interface and $Y_{\min }$ is the Yield strength of the contact material with the lowest strength. In reality, all environmentally exposed contact materials will develop surface films due to phenomena such as absorption or oxidation. Therefore only a fraction of the $\mathrm{N}^{\text {Plast }}$ will contribute to 'cold welding' adhesion. Further investigation on samples sputter cleaned and evaluated under vacuum are planned in order to prove these assumptions.

\section{Plausibility test of the algorithm}

In order to evaluate the algorithm results, a Sn finished sphere on sphere contacting geometry will be evaluated. The contact layer sequence is $3 \mu \mathrm{m}$ Sn over $1.27 \mu \mathrm{m} \mathrm{Ni}$ on a $\mathrm{CuSn} 4$ bronze substrate. The contact radii are $2.5 \mathrm{~mm}$. The normal force $\mathrm{F}_{\mathrm{N}}$ is set to $3 \mathrm{~N}$, the shear force in the $\mathrm{x}$-direction is set to $\mathrm{F}_{\mathrm{T}}=2 \mathrm{~N}$, and the friction coefficients are set to $\mu_{\text {static }}=$ 1 and $\mu_{\text {plast }}=1.2$, respectively. The surface topography is simulated according to [1] using a linear autocorrelation function with correlation lengths $\beta_{\mathrm{x}}=5$ and $\beta_{\mathrm{x}}=20$, skew $=0$, and kurtosis $=3$. The mesh size is $128 \times 128$ nodes, with a grid spacing of $\Delta_{\mathrm{x} / \mathrm{y}}=2.17 \mu \mathrm{m}$. Three different rms roughness values $\sigma$ are simulated.

The first simulation presented here uses an ideal smooth rms roughness of $\sigma=0$. Figure 4 illustrates the $\mathrm{x}$-direction component of the deformation field, $u_{x i, j}^{\text {shear }}$, generated by the application of a pure shear force. It can be clearly seen that the algorithm predicts a constant deformation $\left(u_{x}^{\text {shear }}=0.25 \mu \mathrm{m}\right)$ within a circular area. Figure 5 shows a 2 dimensional crosssectional plot of the normal pressure and shear distributions taken from the center of the contact area.

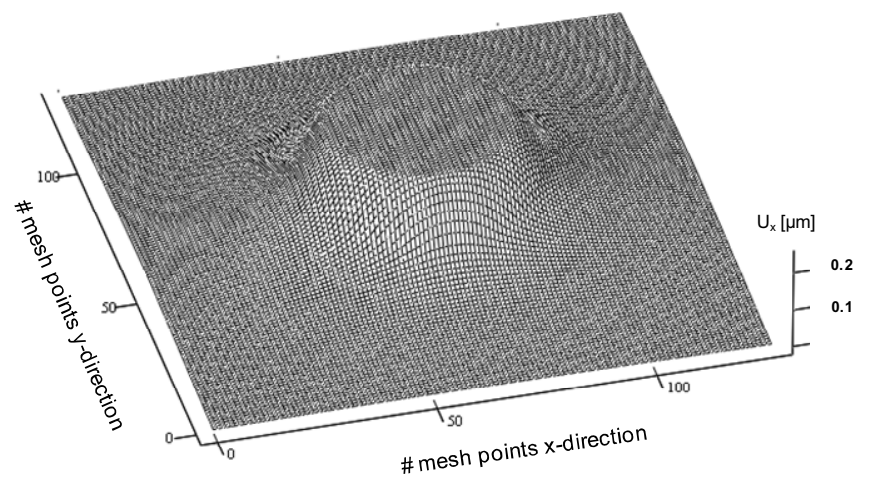

Figure 4: Deformation in x-direction Sn-contact, rms roughness $0 \mu \mathrm{m}$

The form of the curves shows the well known progression as derived by Johnson [11] for contacting smooth parallel cylinders. For a simulation where the rms roughness is set to $\sigma=0.1 \mu \mathrm{m}$, figure 6 shows that the deformation field, $u_{x}^{\text {shear }}$, now takes the form of a plateau. A plateau where there are areas of constant deformation ( $u_{x}^{\text {shear }}=0.25 \mu \mathrm{m}$ ) interspersed by 
areas of no contact $\left(u_{x}^{\text {shear }}<0.25 \mu \mathrm{m}\right)$. The center cut cross sectional 2D-plots of the normal load and shear distribution shown in figure 7 also have a more erratic appearance due to the rough surface topography used in this simulation. Similar to the $\sigma=0 \mu \mathrm{m}$ simulation results in figure 5 , the shear stress distribution still shows a tendency toward elevated values at the rim of the contact area and the maximum normal pressure distribution values are still within the middle region of the contact area. A further increase in surface roughness would result in a shear stress distribution where inner an outer contact area show comparable pressure levels.

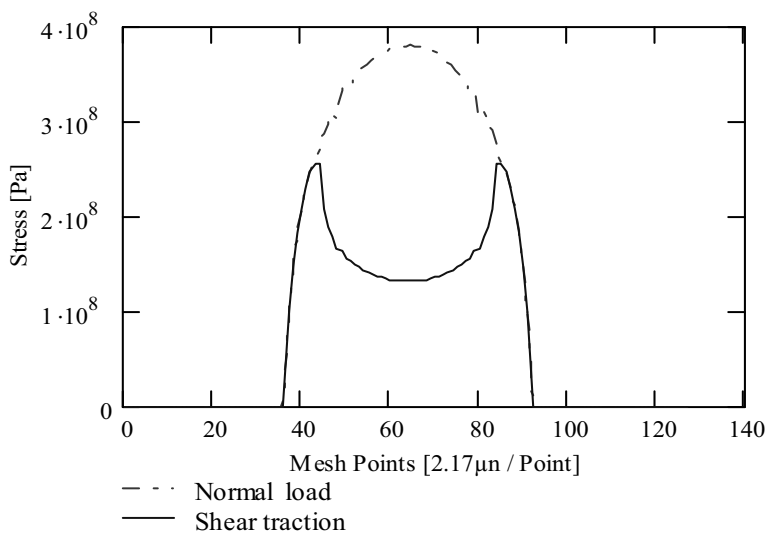

Figure 5: Pressure disribution due to shear and normal load; $\sigma=0$

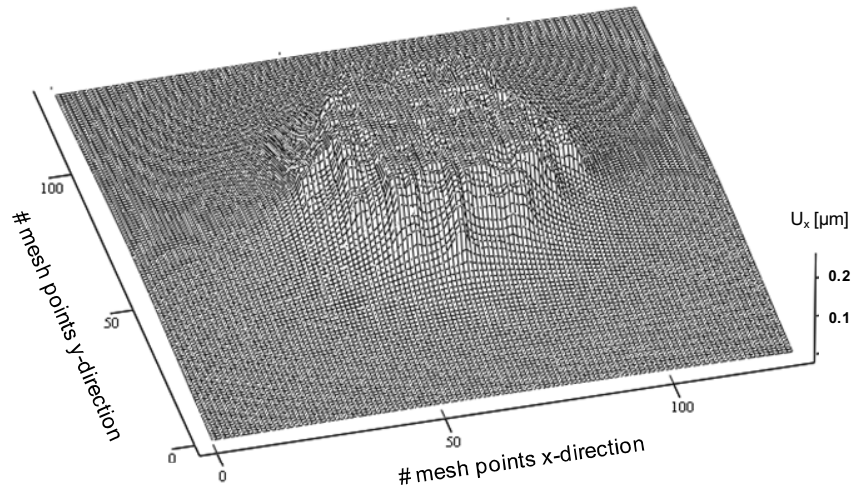

Figure 6: Deformation in $\mathrm{x}$-direction Sn-contact rms roughness $\sigma=0.1 \mu \mathrm{m}$

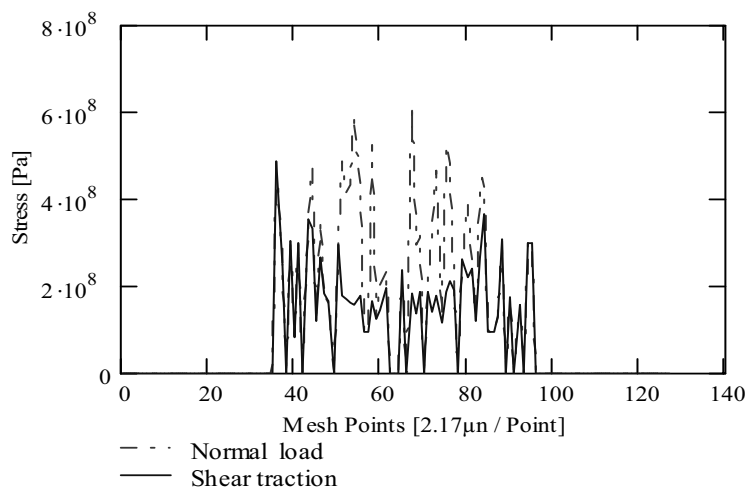

Figure 7: Pressure disribution due to shear and normal load; $\sigma=0.1 \mu \mathrm{m}$
Figure 8 shows a gray scale map of the stick/slip zone for contact topographies with 3 different rms roughness values. In case of an ideal smooth surface $(\sigma=0)$, the calculated sliding zone shows the expected annular ring morphology. The width of the sliding annulus is $\sim 13 \mu \mathrm{m}$. Here again it shall be emphasized that a displacement of $u_{x}^{\text {shear }}=0.25 \mu \mathrm{m}$ results in a sliding annulus $\sim 50$ times greater in width. The sliding annulus is still observable using a surface roughness of $\sigma=0.2$ $\mu \mathrm{m}$. At higher roughness values, the sliding area is not longer confined to the outer contact area. Sliding contact areas now develop also within the center of the contact area. These results agree with information reported in the literature $[2][3][4][5]$ as well as practical experience.

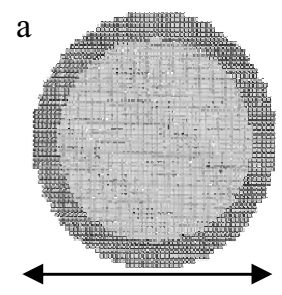

$120 \mu \mathrm{m}$

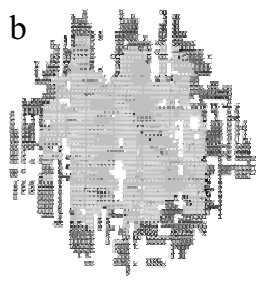

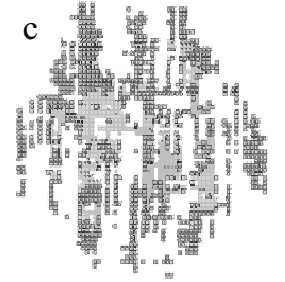

Figure 8: Stick/Slip area for increasing surface roughness; a.: $\sigma=0 ;$ b.: $\sigma=0.1 \mu \mathrm{m} ;$ c.: $\sigma=0.2 \mu \mathrm{m}$

Images from 'real' world contacts show similar wear patterns. Figure 9a shows an annular ring developed on a Sn plated contact in response to thermal cycle testing. The wear scar on the Au-contact seen in figure $9 \mathrm{~b}$ was obtained after vibration testing.

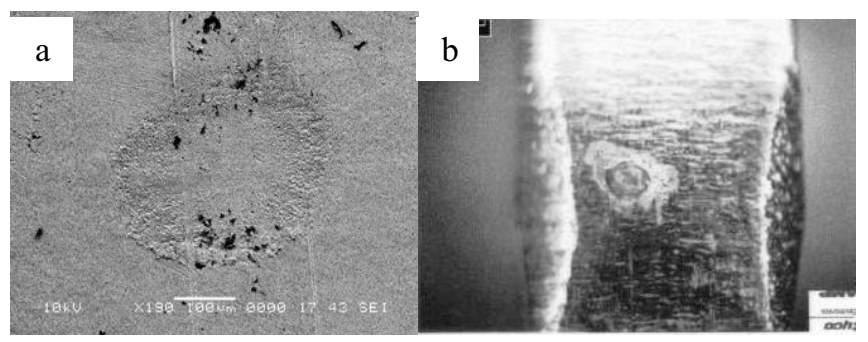

Figure 9: Annular ring pattern; a.: Sn-contact after temperature cycle test; b.: Au-contact after vibration test

\section{E. Difference between elastic/plastic and fully plastic contacts}

It is known that $\mathrm{Au}$ finished contacts will have a greater elastic response to stress than Sn finished contacts; especially when subjected to shear forces. Figure 10 compares the von Mises Stress distribution generated at the surface of an Au vs. a Sn crossed rod contact pair subjected to the same conditions. The topographies are calculated based on the parameters described in the previous section. The contact simulation parameters used are listed in table 1 . The normal force is set to $F_{N}=5 \mathrm{~N}$ and the shear force is set to $\mathrm{F}_{\mathrm{T}}=2 \mathrm{~N}$. It can be clearly seen that the $\mathrm{Sn}$ contact area responds plastically across the entire contact area. The Au-contact area shows a circular plastic zone at the outer rim of the contact area. This difference should also affect the hysteresis behavior according to (26). 
TABLE I. SIMULATION PARAMETERS

\begin{tabular}{|l|c|c|c|c|}
\hline \multirow{2}{*}{} & \multicolumn{3}{|c|}{ Contact material } & \\
\cline { 2 - 5 } & $\boldsymbol{S n}$ & $\boldsymbol{A g}$ & $\boldsymbol{A u}$ & \\
\hline Top coating & 3 & 5 & 1 & $\mu \mathrm{m}$ \\
\hline Ni-underplate & NA & NA & 3 & $\mu \mathrm{m}$ \\
\hline Base material & \multicolumn{3}{|c|}{$\mathrm{CuSn} 4$} & \\
\hline Roughness & \multicolumn{3}{|c}{0.1} & $\mu \mathrm{m}$ \\
\hline Contact cylinder radii & \multicolumn{3}{|c}{2.5} & $\mathrm{~mm}$ \\
\hline$\mu_{\text {static }}$ & 0.9 & 1 & 0.5 & Iput value \\
\hline$\mu_{\text {plast }}$ & 1.2 & 1.2 & 1.2 & Input value \\
\hline
\end{tabular}

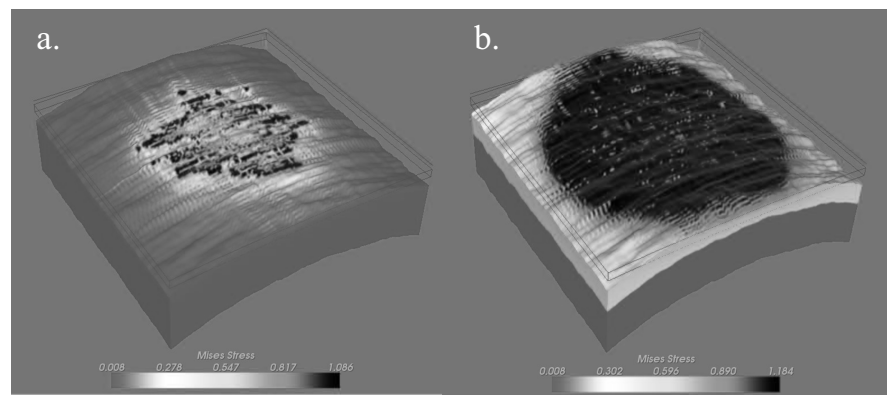

Figure 10: Von Mises Stress distribution; $\mathrm{F}_{\mathrm{N}}=5 \mathrm{~N} ; \mathrm{F}_{\mathrm{T}}=2 \mathrm{~N} \sigma=0.2 \mu \mathrm{m}$; a.: Au contact; b.: Sn-contact

Figure 11 illustrates the principal difference in the deformation hysteresis between elastic/plastic and fully plastic connector contacts. Au-contacts are nearly completely elastic under pure normal load. With increasing shear force, more and more plastic contact spots develop resulting in an increased hysteresis. Sn-contact surfaces plastically deform under pure normal load even before a shear force is applied. Therefore the deformation hysteresis response to the application of a shear force is nearly linear.

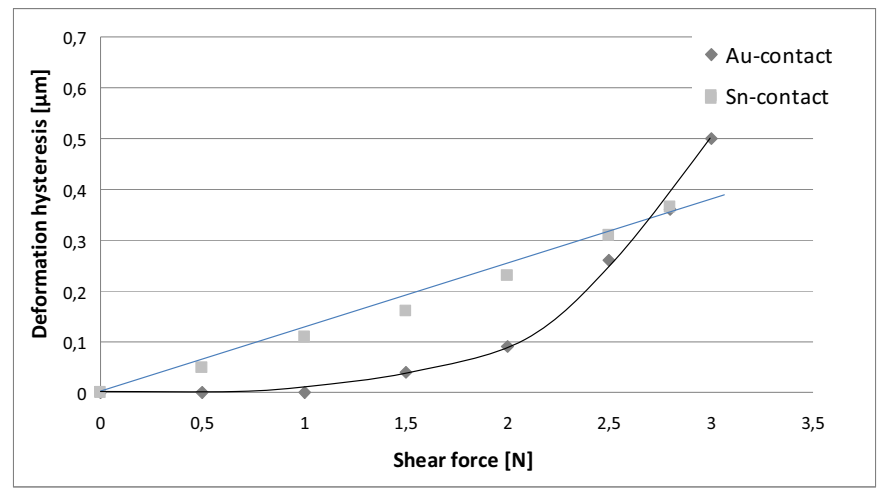

Figure 11: Simulatetd dislocation hysteresis; $F_{N}=5 N ; \sigma=0.2 \mu \mathrm{m}$

\section{CONCLUSIONS AND FURTHER WORK}

The present algorithm is capable of predicting the development of the stick/slip zone for nonconforming, layered, arbitrarily rough surfaces. Results are shown for smooth and rough surfaces. These results agree with previous theoretical work and practical experience. The difference between elastic and plastic contact response is also shown. Further work would involve further verifying and/or quantifying the results of this work, though this effort may be hindered by the inherent compliance of testing instrumentation. In order to evaluate cold welding adhesion effects and to validate (27), tests with in situ cleaned contacts under vacuum have to be carried out. Calculated maximum adhesion forces for the contacts systems listed in table 1 are shown in table 2.

TABLE II. MAXIMUM POSSIBLE ADHESION FORCE

\begin{tabular}{|l|c|c|c|c|}
\hline \multirow{2}{*}{} & \multicolumn{3}{|c|}{ Contact material } & \multirow{2}{*}{} \\
\cline { 2 - 4 } & $\boldsymbol{S n}$ & $\boldsymbol{A g}$ & $\boldsymbol{A u}$ & \\
\hline Maximum adhesion & 0.23 & 0.427 & 0.47 & \multirow{2}{*}{ N } \\
\hline
\end{tabular}

\section{REFERENCES}

[1] M. Leidner, M. Myers, H. Schmidt, H. Schlaak., "A New Simulation Approach to Characterizing the Mechanical and Electrical Qualities of a Connector Contact”, ICEC 2008 proceedings, June 2008, pp 165-170.

[2] Å. K. Rudolphi et al., Fretting Testing of Electrical contacts at Small Displacement Amplitudes - Experience from a BriteEuram Project, Proceedings of the 20th International Conference on Electrical Contacts (ICEC), June 19-23, 2000, Stockholm, Sweden, pp 471.

[3] L. Boyer and L. Tristani: A Model for the Contact Resistance Evolution, Proceedings of the 20th International Conference on Electrical Contacts (ICEC), June 19-23, 2000, Stockholm, Sweden, pp 229 - 234.

[4] S. Hannel, S. Fouvry, and Ph. Kapsa: Finite Element Modelling of the Fretting Transition as the Criterion for Electrical Performance, Proceedings of the 20th International Conference on Electrical Contacts (ICEC), June 19-23, 2000, Stockholm, Sweden, 445 - 450.

[5] S. Hannel, J.C. Abry, S. Fouvry, and Ph. Kapsa: Experimental Relationship Between the Electrical Performance and the Fretting Regime, Proceedings of the 20th International Conference on Electrical Contacts (ICEC), June 19-23, 2000, Stockholm, Sweden, pp 451 - 456.

[6] Midlin, R.D.; Compliance of elastic bodies in contact. Journal of Applied Physics 16 (1949), 259-268.

[7] Polonsky, I.A.; Kerr, L.M.: A New Numerical Method for Solving Rough Contact Problems Based On The Multil-Level Multi-Summation and Conjungate Gradient Technique. WEAR 231 (1999), 206 - 219.

[8] Myers, M.; Leidner, M.; Schmidt, H.; Schlaak, H.: 'Extension and Experimental Verification of a New 'First Contact' Method to Model Performance of Multilayer Contact Interfaces', Proceedings of the 54th HOLM conference, (2008), $66-73$.

[9] Liu, S.; Wang, Q.: "Studying Contatct Stress Fields Caused by Surface Tractions With a Discrete Convolution and Fast Fourier Transform Algorithm."'Transactions of the ASME 124 (2002), $36-45$

[10] Peng, W.; Bushan, B.: A numerical three dimensional model for the contact of layered elas-tic/plastic solids with rough surfaces by a variational principle. Journal of Tribology 123 (2001), 330 - 342.

[11] K. L. Johnson: "Contact Mechanics", Chapter 7, 1985, Cambridge University Press. 


\title{
Mechanical and Electrical Characteristics of the Electromagnetic Relay under Vibration and Shock environment
}

\author{
Wanbin REN ${ }^{1}$, Huimin $\mathrm{LIANG}^{1}$, Yinghua $\mathrm{CHEN}^{1}, \mathrm{Li} \mathrm{CUI}^{2}$, Lizhong $\mathrm{WANG}^{2}$, Yunzhi KANG ${ }^{1}$ \\ 1. School of Electrical Engineering and Automation, Harbin Institute of Technology \\ P.O.Box401 Harbin Inst. Of Tech., Harbin, 150001, China \\ 2. Guilin Aerospace Electronics Ltd., Guilin, 541002, China \\ Email: renwanbin@yahoo.com.cn
}

\begin{abstract}
The electromagnetic relay, which has mechanical structure and break/make contacts, is affected by vibration and shock environment greatly. Contact wear and failure will be result from serious mechanical conditions. The samples used in this study were relays consisting two spring sets connected with armature component. Simulation results for a variety of vibration levels, frequencies, and shock levels are presented. Thereafter main factors and threshold conditions which influencing contact reliability were determined by the modal analysis and the harmonic response analysis and transient response analysis. The vibration and shock characteristics of movable parts within relay were also obtained by using high speed camera PhantomV7.3 (10000fps), thus the mechanism of contact failure is revealed clearly. In addition, the characteristics of contact resistance were investigated when relay working in the mechanical environment. The results are valuable in modeling characteristics degradation and contact failure evaluation caused by mechanical environment for electromagnetic relay.
\end{abstract}

Key Words- Electromagnetic relay, sinusoidal vibration, shock, mechanical characteristics, electrical characteristics.

\section{INTRODUCTION}

$\mathrm{N}$ owadays, more and more electromagnetic relays per year are put into service in industrial automation and communication for system control, information transfer and circuit switch. Under mechanical environment such as vibration, shock and constant acceleration, spring system within electromagnetic relay component will oscillate periodically, meanwhile the electrical parameters (including contact resistance, pick up voltage and release voltage) and time parameters will fluctuate cyclically. Overload mechanical conditions may cause normally closed contacts break away. And contact fretting wear, component mechanical fatigue and fracture failure modes will be induced during long term vibration and shock condition.

The most vulnerable part within the electromagnetic relay is the spring system, when it faced to mechanical environment, so most of research work on relay anti-vibration analysis has been focus on this aspect. Chambega [1] analyzed the natural frequency of the spring group within the relay, and found that the release current increased when relay was exposed to the vibration condition. Ройзен [2] presented the analysis method for estimating the vibration reliability of spring system by taking the movable spring as fixed-hinged support beam structure. Zhai [3] studied the natural frequency and vibration reaction force of reed by using the analytical method and the finite element program ANSYS/LS-DYNA individually. In his research, the limit vibration acceleration of spring system, referring to the maximum of vibration acceleration which can keep the spring contact reliable, is presented. The results are valuable in determining the thresholds of vibration condition for electromagnetic relay. The other relative research on testing and analyzing performance for relay that working in the mechanical vibration environment were so few, except Flowers [4-7] investigated the electrical connector fretting behavior induced by vibration.

We have ever designed the vibration characteristics testing and analyzing system specialized for electromagnetic relay [8]. In this paper, simulation model of some commercial electromagnetic relay was built by using FEA software MSC.Patran/Nastran, subsequently modal analysis and harmonic analysis and transient analysis induced by vibration and shock were completed. Experiment results agreed with simulation results well, so the threshold conditions that keep the spring contact reliable was determined. At last the effect of vibration and shock condition on contact resistance of relay is also investigated.

\section{MODELING AND SIMULATION ANALYSIS}

\section{A. RELAY FEATURE}

The electromagnetic relay excited by AC-DC voltage is used widely in switching medium and heavy load in engineering application. The analyzed magnetic system of this type relay is mainly includes $L$-shape yoke, coil and straight armature. The clapper structure relay has optimal dynamic and steady-state performance by comparing different kinds of electromagnetic relay. The movable parts are the two spring groups riveted together with the armature, and the spring groups (conductors) are insulated with the armature by polymer materials. The armature rotates along the yoke end during operation, and the mechanical counterforce was provided by tension spring mainly. The coil rated voltage is $\mathrm{DC} 24 \mathrm{~V} / \mathrm{AC} 220 \mathrm{~V}$, and contact arrangement is $2 \mathrm{P}$ (two groups break and make contacts), contact rated load is $10 \mathrm{~A}$. The initial pressure between normally closed contacts is $0.38 \mathrm{~N}$. The photograph of relay (Type:LY2NJ made by Ormon 
Company) is shown in Figure 1(a). ' $X, Y, Z$ ' is refers to the vibration and shock direction.

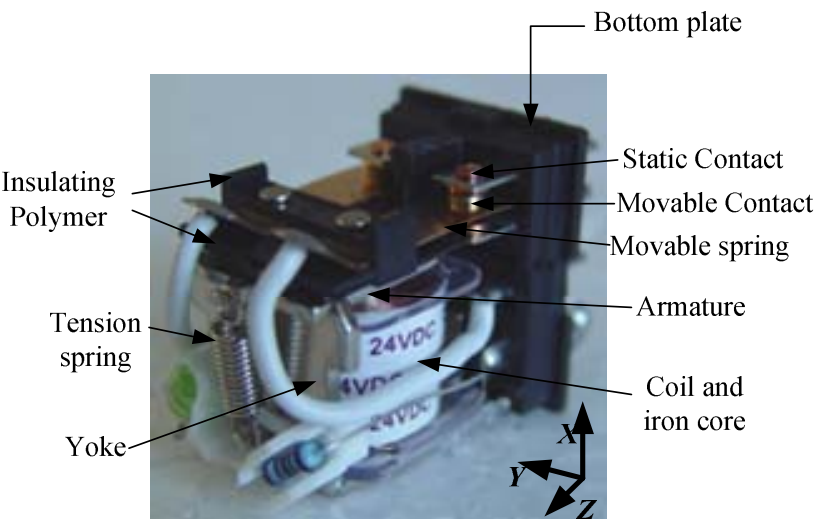

(a) Photograph

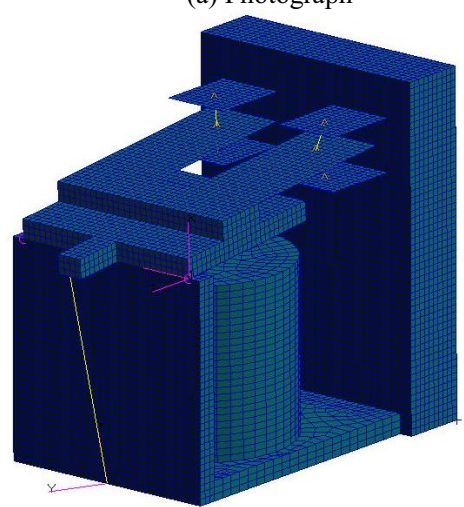

(b) Finite Element Model

Figure 1. The tested electromagnetic relay (without housing)

\section{B. MODELING BY FEM}

With the availability of advanced computing facilities and finite element codes, the feasibility of conducting virtual vibration and shock tests via finite element simulation may be realized. As known, structure dynamic analysis by using finite element method has been used in the world wide, for instance commercial software MSC.Patran/Nastran. Different element types including solid, shell, bar elements are used to model each parts and components of the relay (details see Table 1). The FEM model in MSC.Patran is shown in Figure 1(b).

Spring system: Because the ratio of length to thickness for the springs greater than 10, shell elements are used for modeling the static and movable spring. The terminals in the relay have radius that is much smaller than its length. As such, bar elements are used. Contacts are simplified as lumped mass points attached to the spring groups.

Electromagnetic system: Using solid elements gives the exact volume of the component and allows accurate clearances between different parts. The solid elements are used to model coil, iron core, yoke, armature and bottom plate. Their inclusion is to provide appropriate mass distribution and stiffness to the relay.

Contact Interaction: There are two kinds separable contact interactions of relay. One kind is that between the static spring and the movable spring of normally closed contacts group. The equivalent spring elements were introduced to connect the two group movable springs and normally closed static springs. The value of spring rigidity is gotten by using classical Hertz contact theory, and our ever research showed the equivalent spring element between the spring groups can show the dynamic response of relay accurately [9].

Point 3 and Point 2 (shown in Figure 2) is connected by an equivalent spring element, and the contact rigidity $k_{e}$ can be written as

$$
k_{e}=\frac{3}{2}\left(k^{2} F_{0}\right)^{1 / 3}
$$

in which $k=\frac{4 E^{*}}{3} R^{1 / 2}, F_{0}$-initial contact force,

$\frac{1}{E^{*}}=\frac{2\left(1-v^{2}\right)}{E}, E$-Young's modulus, $v$-Poisson's ratio, $R$-radius of contact curvature.

The tension spring modeled by bar element was also used to realize the connection between the armature and the yoke. The value of spring rigidity is $1300 \mathrm{~N} / \mathrm{m}$.

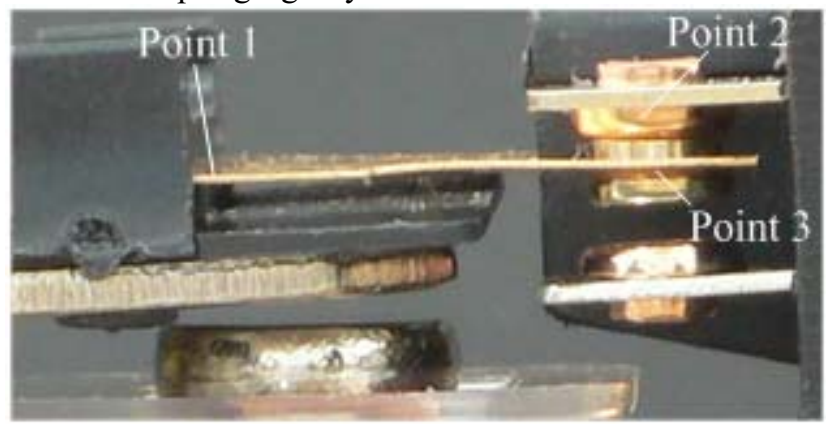

Figure 2. Photo of one spring-contact group

Table 1 Summary of the finite element model of relay

\begin{tabular}{|c|c|c|c|}
\hline $\begin{array}{c}\text { Components } \\
\text { modeled }\end{array}$ & $\begin{array}{c}\text { Element } \\
\text { type }\end{array}$ & $\begin{array}{c}\text { Element } \\
\text { numbers }\end{array}$ & Material \\
\hline Terminals & SHELL & 102 & Be-bronze \\
\hline Bottom plate & SOLID & 584 & Polymer \\
\hline Springs & SHELL & 1065 & Be-bronze \\
\hline Yoke & SOLID & 1060 & DT4E \\
\hline Armature & SHELL & 290 & DT4E \\
\hline Coil & SOLID & 616 & Copper \\
\hline Iron core & SOLID & 460 & DT4E \\
\hline $\begin{array}{c}\text { Tension } \\
\text { spring }\end{array}$ & BAR & 1 & Stainless-steel \\
\hline Contact & POINT & 6 & Copper \\
\hline $\begin{array}{c}\text { Equivalent } \\
\text { spring }\end{array}$ & BAR & 2 & \\
\hline
\end{tabular}

In the Nastran program, the material models are generally defined by the following parameters: density, Young's modulus and Poisson's ratio. These parameters allow 
experimental test data obtained to be converted into appropriate inputs for the program. Materials involved in the modeling of the relay are shown in Table 2 .

\begin{tabular}{l|l|l|l}
\multicolumn{4}{c}{ Table 2 Mechanical properties of involved materials } \\
\hline Material & $\begin{array}{l}\text { Density } \\
\left(\mathrm{kg} / \mathrm{m}^{3}\right)\end{array}$ & $\begin{array}{l}\text { Young's } \\
\text { modulus } \\
(\mathrm{GPa})\end{array}$ & $\begin{array}{l}\text { Poisson's } \\
\text { ratio }\end{array}$ \\
\hline Be-Bronze & 8250 & 135.3 & 0.3 \\
\hline DT4E & 7860 & 214.7 & 0.3 \\
\hline Copper & 8890 & 108 & 0.27 \\
\hline Stainless-steel & 7900 & 198 & 0.285 \\
\hline Polymer & 1400 & 0.94 & \\
\hline
\end{tabular}

\section{MODAL ANALYSIS AND HARMONIC ANALYSIS}

Normal mode analysis is the basis for many types of dynamic response analysis. These results including natural frequencies and normal mode shapes of the structure with damping neglected will respond to dynamic loading. The solution of the equation of motion for natural frequencies and normal modes requires a special reduced form of the equation of motion. If there is no damping and no applied loading, the equation of motion in matrix form reduced to

$$
[\boldsymbol{M}]\{\ddot{u}\}+[\boldsymbol{K}]\{u\}=0
$$

where $[\boldsymbol{M}]$ is mass matrix, $[\boldsymbol{K}]$ is stiffness matrix. Then the harmonic solution of the form can be expressed as

$$
\{u\}=\{\phi\} \sin 2 \pi f t
$$

where $\{\phi\}$ is the eigenvector or mode shape, $f$ is the natural frequency. Substitute Eq.(3) into Eq.(2), after simplifying becomes

$$
\left([\boldsymbol{K}]-4 \pi^{2} f^{2}[\boldsymbol{M}]\right)\{\phi\}=0
$$

Eq.(4) is called the eigenequation, which is a set of homogeneous algebraic equations for the components of the eigenvector and forms the basis for the eigenvalue problem. An eigenvector $\left\{\phi_{i}\right\}$ corresponds to each unique eigenvalue $f_{i}$. The number of eigenvalues and eigenvectors is equal to the number of degrees-of-freedom that have mass or the number of dynamic degrees-of-freedom.

Seven methods of real eigenvalue extraction are provided in MSC.Nastran software [10]. These methods are numerical approaches to solving for natural frequencies and mode shapes. The Lanczos method that overcomes the limitations and combines the best features of the other methods was chosen [10].

The first order normal mode shape of the relay is that the armature and movable spring group rotating along $\mathrm{X}-\mathrm{Z}$ plane and the corresponding natural frequency is $328 \mathrm{~Hz}$. The first normal mode shape is shown in Figure 3. As this mode shape is the most dangerous case for contacts of relay, which could cause the normally closed contacts break away directly. And other higher mode shapes of relay are not investigated in this paper.

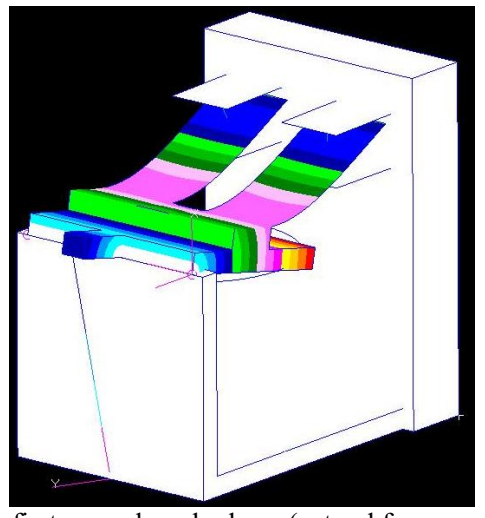

Figure 3. The first normal mode shape (natural frequency is $327.8 \mathrm{~Hz}$ )

In most cases the regulated sweep frequency vibration test is performed for the purpose of determining the effect on component parts of vibration. The typical constant vibration acceleration with varied excited frequency waveform was shown in Figure 4.

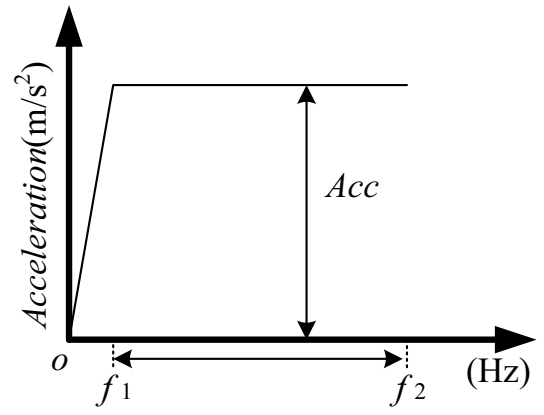

Figure 4. The sweep frequency vibration waveform

If the exciting sinusoidal displacement can be written as

$$
w=A \sin (2 \pi f t)
$$

in which $A$-vibration amplitude, $f$-vibration frequency.

Then vibration acceleration can be expressed by

$$
A c c=\ddot{w}=-4 A \pi^{2} f^{2} \sin (2 \pi f t)
$$

Keeping the vibration acceleration $A_{c c}$ constant when the vibration frequency is between $f_{1}(10 \mathrm{~Hz})$ and $f_{2}(800 \mathrm{~Hz})$, and adding the excitation at the bottom plate of relay, therefore the vibration response of relay in different direction is simulated. Considering the calculation efficiency, the scanning step size is set to $0.5 \mathrm{~Hz}$ within the frequency range of $[250 \mathrm{~Hz}, 450 \mathrm{~Hz}]$ nearby the resonance state, and in other cases larger step size is set to $20 \mathrm{~Hz}$.

The left end position of the movable spring (Marked by Point 1), which has the maximum displacement response obviously, is selected as the verification point for simulation. The static contact position (Marked by Point 2), which has the same displacement response with the exciting displacement, is taken as the reference point. And the movable contact position (Marked by Point 3) and the above static contact position, which reflect the contact reliability, are shown in the Figure 2. The displacement responses of these points in the mechanical vibration conditions are mainly investigated.

Firstly, we took Point 2 as the reference position, and the relative displacement amplitude of Point 1 with each assigned excited vibration frequency is shown in Figure 5. As seen in 
Figure 5 the peak value obtained by simulation is $109.54 \mu \mathrm{m}$, which is corresponding to the spring and armature group resonance state. The amplitude of their relative displacement is decreasing when the excited frequency is offset the natural frequency.

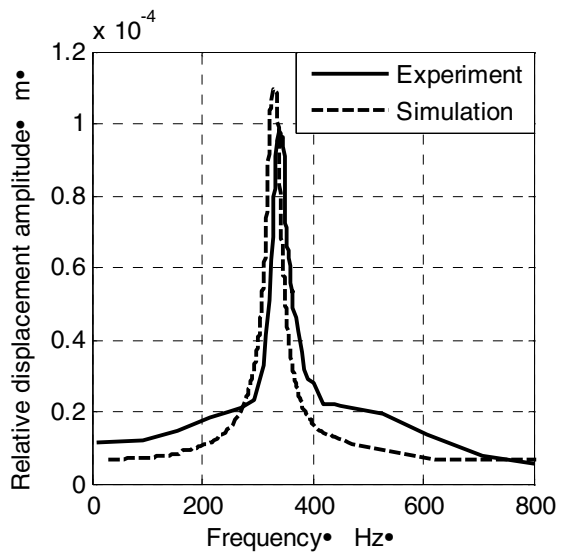

Figure 5. Comparisons between experiment and simulation (Acceleration is $49 \mathrm{~m} / \mathrm{s}^{2}$, vibration direction is along with " $\mathrm{X}$ " axis)

\section{SHOCK RESPONSE ANALYSIS}

Shock as another mechanical environment is conducted for the purpose of determining the suitability of component parts and subassemblies of electrical and electronic components when subjected to shocks such as those which may be expected as a result of rough handing and transportation. Generally the half-sine shock pulse waveforms are specified with tolerances. Shock pulse waveform used for simulation is shown in Figure 6, which is satisfied with relay test standards too. Shock response analysis belongs to time domain analysis, and direct integration method is applied for the iterative solution. Total calculation time is $18 \mathrm{~ms}$, three times of shock duration time. And the integral step is set to 40us.

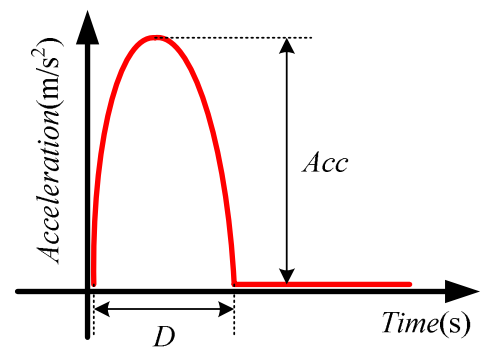

Figure 6. Shock pulse curve

$\boldsymbol{A}_{C C}$-amplitude of shock acceleration; $\boldsymbol{D}$-shock duration time $=6 \mathrm{~ms}$

\section{EXPERIMENTAL CONFIGURATION}

In order to verify the simulation method and obtained results, the electromagnetic relay was stick at the vibrator deck. While " $\mathrm{X}$ " direction is the most dangerous one, so we only present this case test. A schematic diagram of the experimental test setup is shown in Figure 7. The sample relay is excited by an electromagnetic vibrator driven by a power amplifier. The sinusoidal vibration and shock input is provided by a DSP (Digital signal Processing) unit. The coil current and contact voltage and current data is collected by designed monitor modular. Sweep frequency vibration $\left(10 \sim 1000 \mathrm{~Hz}\right.$, up to $\left.98 \mathrm{~m} / \mathrm{s}^{2}\right)$, shock vibration $\left(6 \mathrm{~ms}, 588 \mathrm{~m} / \mathrm{s}^{2}\right)$ can be realized by this system, and the resulting acceleration at the shaker head measured using an accelerometer. The dynamic procedures of spring group in the relay during vibration are recorded by using high speed camera "Phantom V7.3", which shooting speed is $10000 \mathrm{fps}$. The whole motion process of spring groups was recorded at every $5 \mathrm{~Hz}$ within the frequency range of $[250 \mathrm{~Hz}, 450 \mathrm{~Hz}]$ nearby the resonance state, and the vibration acceleration is $49 \mathrm{~m} / \mathrm{s}^{2}$. The motion is recorded at every $50 \mathrm{~Hz}$ during the nonresonant cases $(10 \mathrm{~Hz}$ to $250 \mathrm{~Hz}$ and $450 \mathrm{~Hz}$ to $800 \mathrm{~Hz}$ ).

Then the relative displacement amplitude between Point 1 and Point 2 could be calculated by pixels position in the recorded picture too. As seen in Figure 5 the curve peak value obtained by experiment is $99 \mu \mathrm{m}$ when excited frequency is equal to $339 \mathrm{~Hz}$, which can be taken as the resonant state. The simulation results are agreed with experiment results well, and the relative error is no less than $10 \%$.

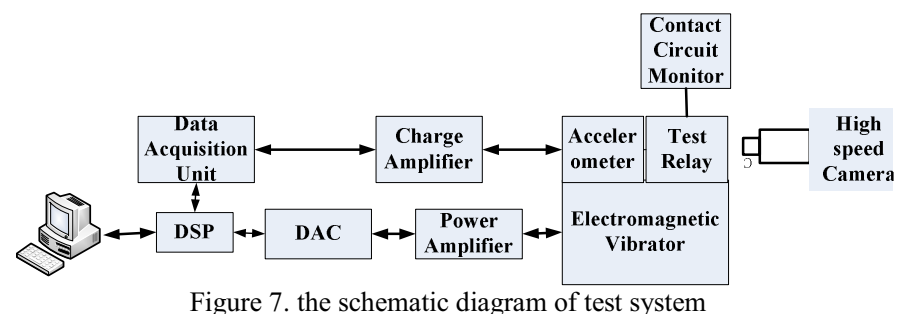

IV RESULTS AND DISSCUSSION

\section{A. LIMIT VIBRATION ACCELERATION}

The contact reliability of relay was determined by the normally closed contact force between Point 3 and Point 2. Basing on the expression of contact rigidity in Eq.(1), the variation of contact force $\Delta F$ could be calculated by two contact points' relative displacement $\Delta x$,

$$
\Delta F=k_{e} \Delta x=k_{e}\left(x_{p o \text { int } 3}-x_{p o \text { int } 2}\right)
$$

If the contact force response is greater than the initial force value, the contact break away failure mode could be judged. As seen in Figure 8(a) contact relative displacement response under sweep sinusoidal vibration in three directions are obtained. By Eq.(7), the contact force response is calculated and shown in Figure 8(b). " $\mathrm{X}$ " direction being accordance with the contact movement, is more dangerous than any other two directions. By contrasting the contact force with the initial force value $0.38 \mathrm{~N}$, contacts are sure to break away periodically when the vibration direction is same with the " $\mathrm{X}$ " direction, and the excited frequency is in the range of $[319 \mathrm{~Hz}, 335 \mathrm{~Hz}]$. The contact force response with different vibration acceleration is shown in Figure 9, we can see that contact force response value is increasing with the increment of vibration acceleration, and the frequency range which cause the contacts open is enlarging too. Thus the vibration acceleration threshold value, that is to say, the limit vibration acceleration, and corresponding excited frequency range must be existed. The relationship between the limit vibration acceleration and its corresponding excited frequency range is 
shown in Figure 10. The limit vibration acceleration has the minimum $42.68 \mathrm{~m} / \mathrm{s}^{2}$, when the excited frequency is equal to $328 \mathrm{~Hz}$. That is to say, the anti-vibration characteristic is the worst case when it is in the resonance. Meanwhile the limit vibration acceleration increases with the excited frequency bias from the resonance point. So the natural frequency and the limit vibration acceleration should be considered comprehensively to determine the anti-vibration performance of the relay.

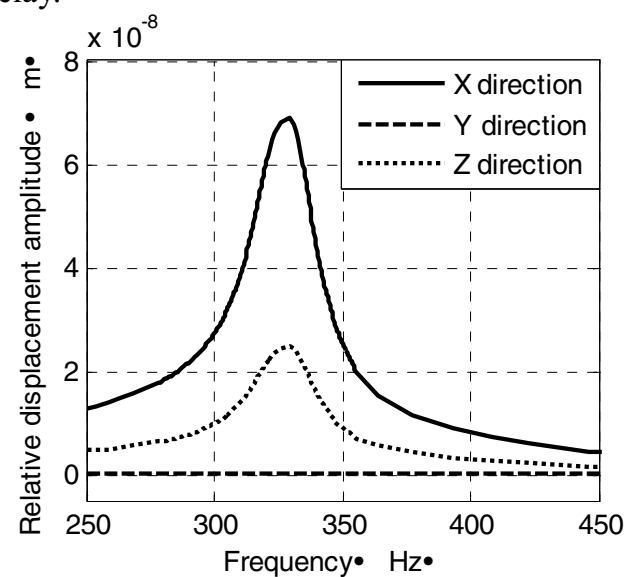

(a) contact relative displacement

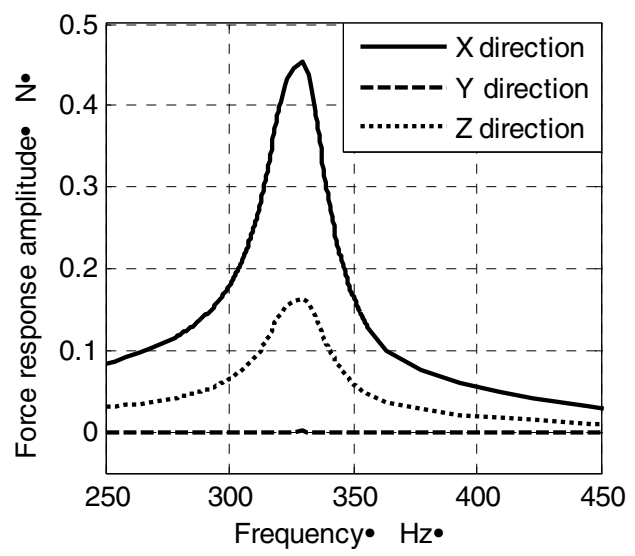

(b) contact force

Figure 8. Sweep sinusoidal vibration response with different direction (vibration acceleration is $50 \mathrm{~m} / \mathrm{s}^{2}$ )

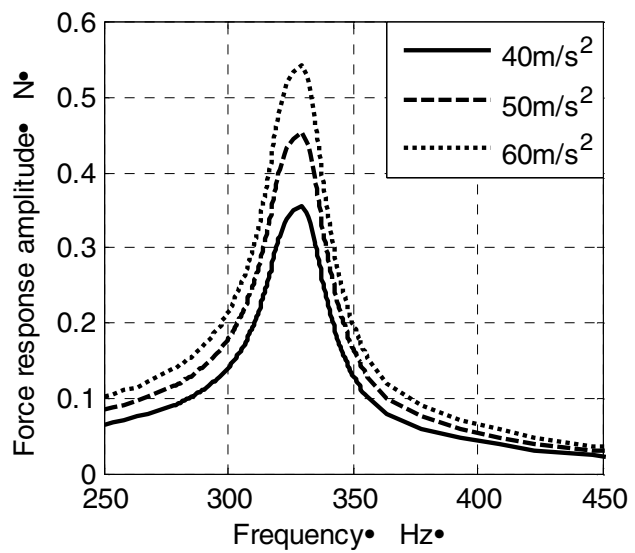

Figure 9. the contact force response with different vibration acceleration in " $\mathrm{X}$ " direction

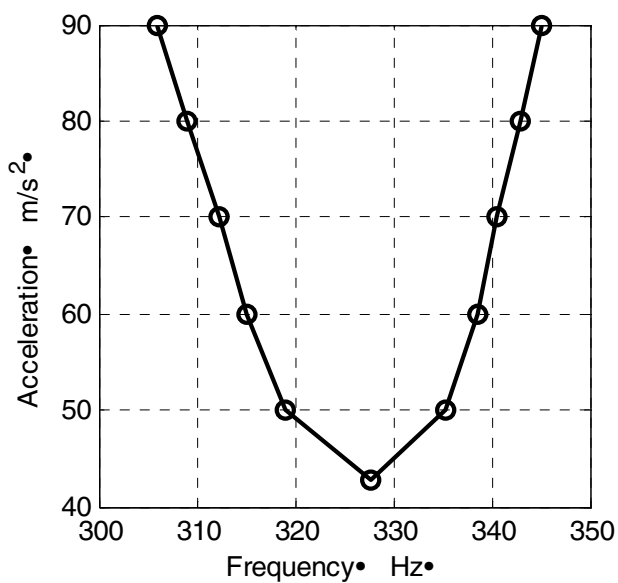

Figure 10. The relationship between limit vibration acceleration and excited frequency range

The tension spring provides not only the mechanical force, but also the fixed point of the armature group. The effect of rigidity of tension spring on mechanical characteristics of relay is investigated, which is shown in Figure 11 and Figure 12. The first order natural frequency of relay is elevated slightly with the increment of rigidity; meanwhile contact force response is reduced.

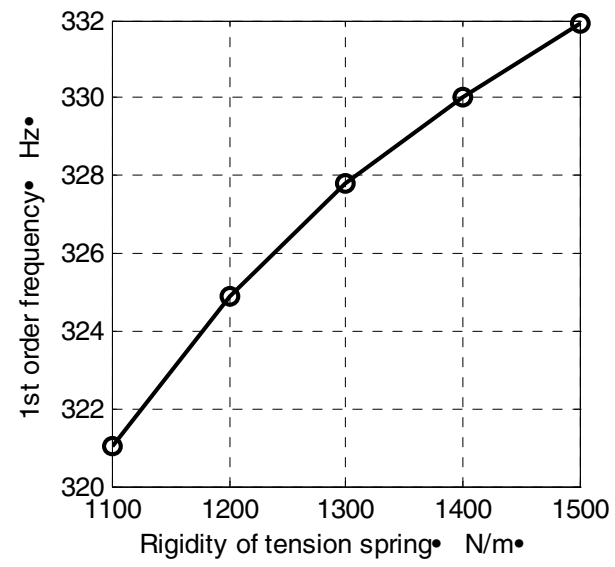

Figure 11. the effect of rigidity on natural frequency

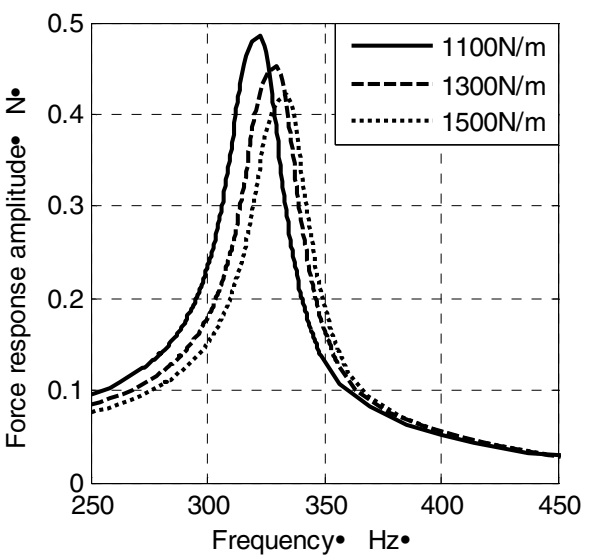

Figure 12. the effect of rigidity on force response 


\section{B. SHOCK}

As seen in Figure 13, contact force responses in three directions shock condition (refer to Figure 5) are analyzed individually. The " $\mathrm{X}$ " direction is more dangerous than any other two directions too, and the maximum force could be $0.085 \mathrm{~N}$, negative sign means the state of tension. Keep the shock duration time constant, contact force response is amplified with the increment of shock acceleration. As the acceleration reach up to $519.4 \mathrm{~m} / \mathrm{s}^{2}$, the tension force of contact is greater than the initial value, then contact failure (shown in Figure 14).

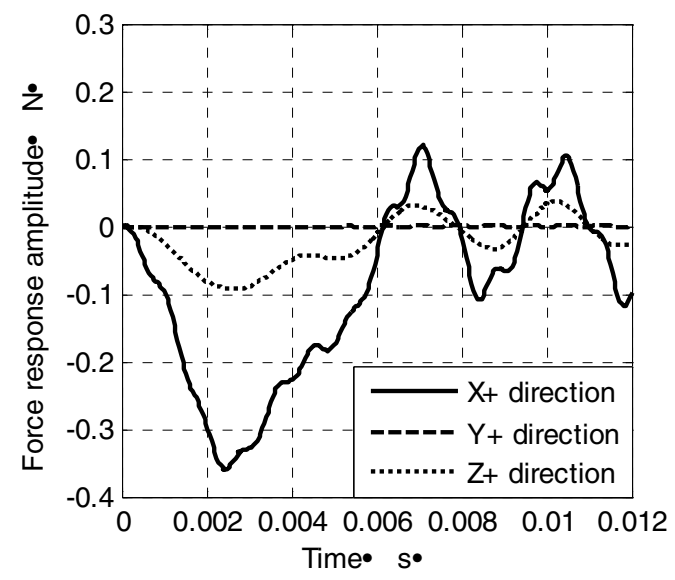

Figure 13. the shock responses in three directions $\left(A_{C C}=519.4 \mathrm{~m} / \mathrm{s}^{2}, D=6 \mathrm{~ms}\right)$

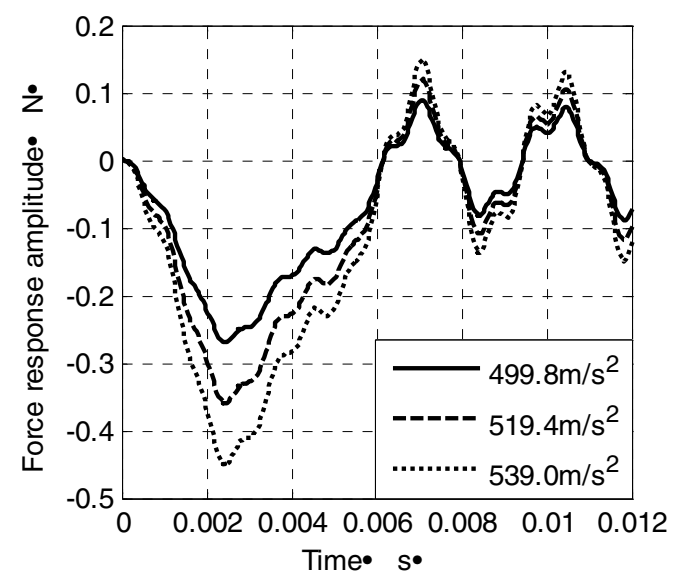

Figure 14. the shock response in " $\mathrm{X}$ " direction with varied $A_{C C}$

\section{Electrical characteristics}

The contact resistance of normally closed contact was measured according to the 4-wire method. Figure 15 shows the relationship between contact resistance and excited frequency vs. acceleration explicitly. The contact resistance fluctuated not obviously in small vibration acceleration condition, and the amplitude increased gradually with the higher vibration acceleration, and turned into infinite in the threshold region. That means contact break away failure. The exciting frequency region that caused contacts break away became wider when acceleration increased. Across the region, the contact resistance decreased gradually with the increment of excited frequency. We also calculated the variation contact resistance of relay by using the simulated contact force response, which can be expressed as below [11]

$$
R_{c}=\frac{\rho}{2} \sqrt{\frac{\pi \xi H_{B}}{F}}=\frac{\rho}{2} \sqrt{\frac{\pi \xi H_{B}}{F_{0}-|\Delta F|}}
$$

where $\rho$-electrical conductivity, $H_{B}$-hardness, $\xi-0.3$.

Figure 16 shows the effect of shock acceleration on the contact resistance dynamic response. As shown in Figure 15 and Figure 16, the change of contact resistance obtained by contact force response simulation under vibration and shock condition agree with test results well.

\section{CONCLUSION}

A study of mechanical characteristics and electrical characteristics of electromagnetic relay under different direction vibration and shock condition has been presented. This investigation aims to determine the contact failure mode of relay and contact reliability accurately. The current research examines the normal mode and the relationship between limit vibration acceleration and excited frequency range by modal analysis and sweep sinusoidal vibration simulation and shock simulation. A threshold level of excitation (vibration and shock condition) was identified, below which contact force response of relay was less than the initial value. The minimum limit vibration acceleration of the relay corresponds to the resonance frequency. Meanwhile, the vibration testing system for the electromagnetic relay was developed, and the motion of spring group recorded by using high speed camera agreed with simulation results well. The contact resistance of normally closed contact changed obviously with the increment of vibration acceleration, and became infinite between the threshold value regions. So to ensure the contact reliability of relay, the dangerous direction that accord with contact movement and limit vibration acceleration should be avoided. The effects of vibration environment on the operation characteristics of relay, such as pick-up voltage and release voltage and relative time parameters are being performed, and it will be published in the future.

\section{ACKNOWLEDGMENT}

The authors express their great thanks to the support of The National Natural Science Foundation of China (50777010), Special Research Fund for Scientific Innovation Talents of Harbin (2008RFQXG043) and China Postdoctoral Science Foundation (20070420854). 


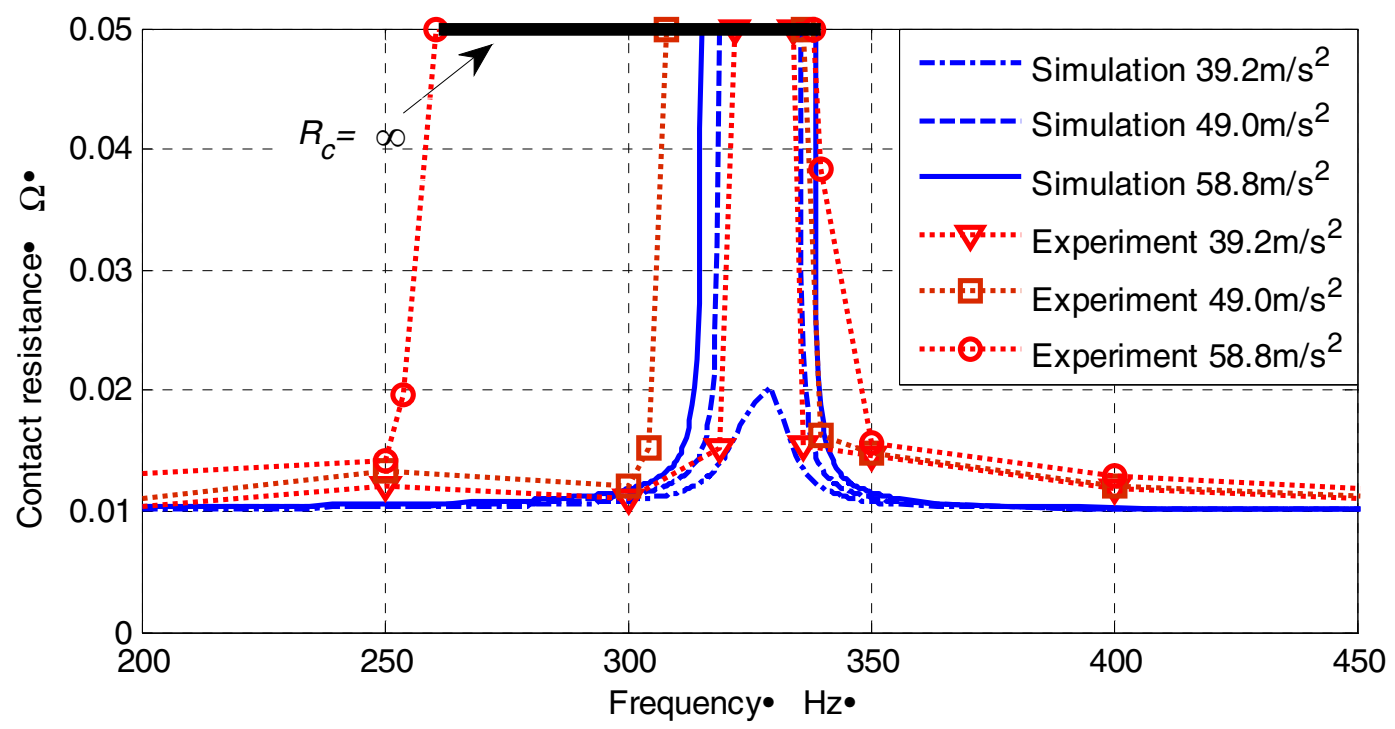

Figure 15. The relationship between contact resistance and excited frequency \& vibration acceleration

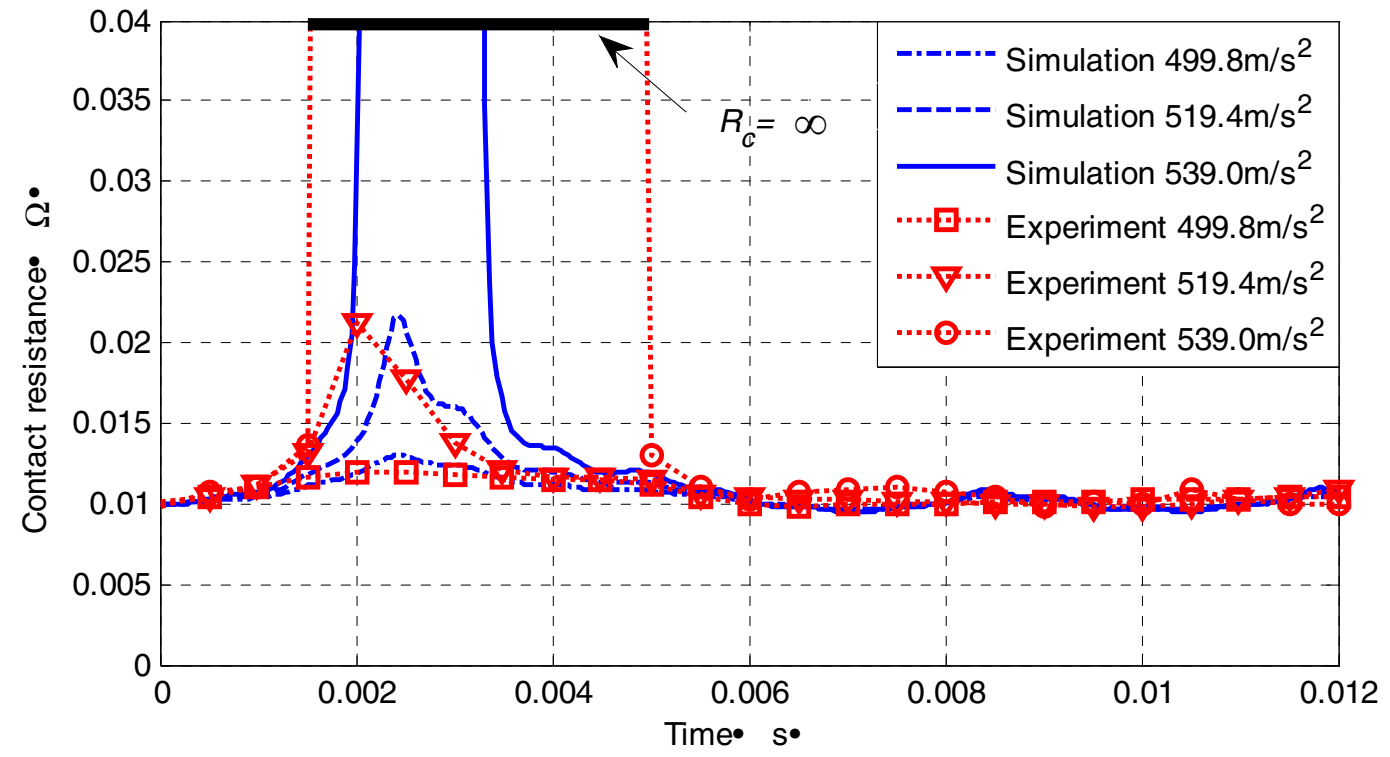

Figure 16. The relationship between contact resistance response $\&$ shock acceleration

Reference

[1] D.J. Chambega. Qualitative Analysis on the Effect of External Vibrations on the Performance of Relays, Proc.1996 4th IEEE AFRICON Conf. Part2, pp.1035-1039,1996.

[2] Ройзен. Miniature Sealed Electromagnetic Relay. Posts \& Telecom Press, China, 1979.

[3] Zhai Guofu, Ren Wanbin, Xu Feng. Research on the analytical method of contact vibration performance of reed relay. Proceedings of the 49th IEEE Holm Conference on Electrical Contacts. 2003,198-203.

[4] G. T. Flowers, F. Xie, M. Bozack, and R.D. Malucci. Vibration Thresholds for Fretting Corrosion in Electrical Connectors, Proceedings of the 48th IEEE Holm Conference on Electrical Connectors, 2002:133-139.

[5] G. T. Flowers, F. Xie, M. Bozack, R. Horvath, R. D. Malucci,, and B. Rickett. Vibration Testing of Fretting Corrosion in Electrical Connectors Subjected to Multi-Frequency and Random Spectral Profiles, Proceedings of the 49th IEEE Holm Conference on Electrical Contacts, 2003:45-50.
[6] G. T. Flowers, F. Xie, M. Bozack, X. Hai, B. Rickett and R. D. Malucci, A Study of the Physical Characteristics of Vibration Induced Fretting Corrosion, Proceedings of the 50th IEEE Holm Conference on Electrical Contacts. 2004:312-319.

[7] G. T. Flowers, F. Xie, M. Bozack, R. Horvath, B. Rickett, and R. D. Malucci. The Influence of Contact Interface Characteristics on Vibration-Induced Fretting Degradation, Proceedings of the 51st IEEE Holm Conference on Electrical Contacts, 2005: 82-88.

[8] Ren Wanbin, Kang Yunzhi, Chen Yinghua. The effect of external vibrations on the characteristics of the clapper relay. IEICE Technical Report EMD2008-11, 13-16.

[9] Ren Wanbin, Zhai Guofu, Cui Li. Contact vibration characteristic of electromagnetic relay. IEICE Transactions on Electronics, 2006,E89-C (8):1177-1181.

[10] MSC.Nastran Basic Dynamic Analysis User's Guide.

[11] Paul G. Slade. Electrical contacts principles and applications. Marcel Dekker,Inc. New York,USA,1999 


\title{
A Semi-analytical Model of Contact Resistance from Sinusoidal Asperity or Wavy Surface Contact
}

\author{
Robert L. Jackson \\ Center for Advanced Vehicle Electronics \\ Department of Mechanical Engineering \\ Auburn University, Auburn, AL 36849-5341
}

\begin{abstract}
All engineering surfaces have roughness that obstructs the flow of electrical current between to contacting surfaces. The peaks of roughness on the surface are modeled using the single asperity concept. In this work a 3-dimensional sinusoidal asperity is modeled as an electrical contact. A semi-analytical model of contact resistance based on pressure is obtained as a function of normal load using finite element results and analytical solutions. There is a significant difference between the sinusoidal contact model predictions and the spherical models typically used. These results could be used in rough surface contact models based on sinusoidal shaped asperities, such as recent multiscale stacked models.
\end{abstract}

Keywords: contact resistance, electrical resistance, roughness, surface contact

\section{INTRODUCTION}

Around a single asperity contact a 'flux tube' is theorized to exist from the contact surface to a depth into the material. The electrical current between the two surfaces must then pass through the flux tube and thus through the reduced cross-sectional area of the contact. The electrical current will constrict and then expand as it passes through the contact. In past studies the thermal and electrical resistance of the flux tube has been characterized where multiple functions are available in the literature if the flux tube is assumed to have a cylindrical geometry [1-9]. Although these models have proven to work reasonably well in some specific cases, [7] show that flux tubes can have many different geometries based on the geometry of the contacting asperities.

Although asperities have traditionally been modeled as spherical and thus having a circular interface, clearly, due to the usually complicated surface topography of typical surfaces, these asperity geometries can vary significantly. This makes the contact resistance problem very complex since the surface topography of a surface is very complicated and difficult to characterize precisely, especially during contact situations. Therefore, the current work examines the contact resistance for a sinusoidal or wavy surface in contact with a rigid flat. The author believes that this surface geometry is more realistic of actual surface asperity contact, especially at high loads which asperities are commonly exposed to.

The current analysis will examine the case of threedimensional elastic perfectly plastic sinusoidal contact by building on these previous works, using fundamental solid mechanics theory, and conducting a parametric study using the finite element method. The sinusoidal surface considered by the current work is described by

$$
h=\Delta \cdot\left(1-\cos \left(\frac{2 \cdot \pi \cdot x}{\lambda}\right) \cos \left(\frac{2 \cdot \pi \cdot y}{\lambda}\right)\right)
$$

and is shown Fig. 1, where $h$ is the height of the sinusoidal surface from its base. This is very similar to the surface used by Johnson et al. [10] and results in the same analytical equations for elastic contact (as will be discussed later). Subsequent work $[11,12]$ has also been performed to consider the elastic-plastic contact of sinusoidal surfaces using finite element modeling and the same surface geometry given by Eq. (1).

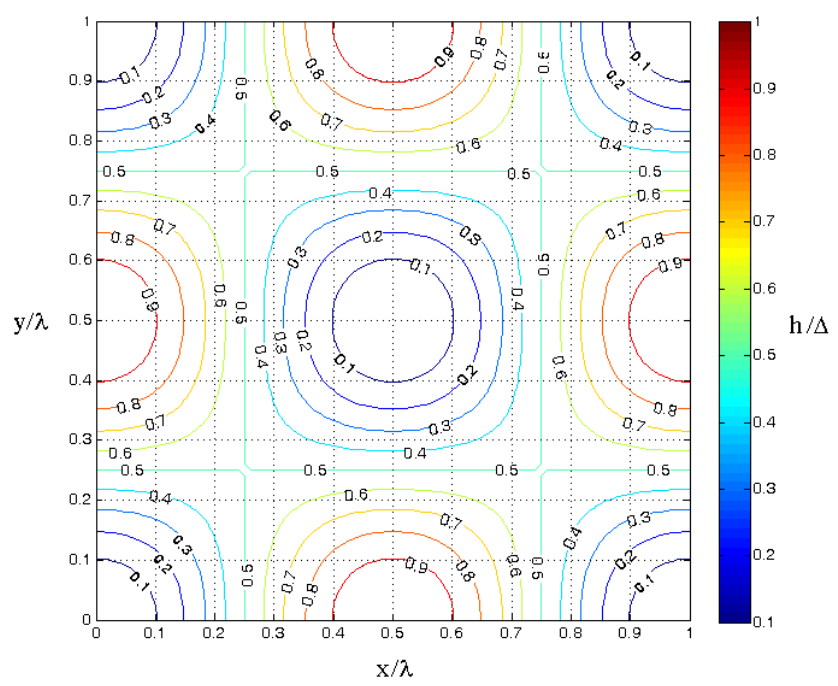

Figure 1: Topographical depiction of the sinusoidal surface considered in the current analysis. 


\section{METHODOLOGY}

\section{A. Model of Elastic 3-D Sinusoidal Contact}

For the same geometry given by Eq. (1), Johnson, Greenwood, and Higginson (JGH) [10] derived analytical asymptotic solutions to elastic 3-D sinusoidal contact. For low and high loads respectively, these equations are:

$$
\begin{aligned}
& \left(A_{J G H}\right)_{1}=\frac{\pi}{f^{2}}\left[\frac{3}{8 \pi} \frac{\bar{p}}{p^{*}}\right]^{2 / 3} \\
& \left(A_{J G H}\right)_{2}=\frac{1}{f^{2}}\left[1-\frac{3}{2 \pi}\left(1-\frac{\bar{p}}{p^{*}}\right)\right]
\end{aligned}
$$

where $p^{*}$ is the average pressure to cause complete contact between the surfaces and is given by [10] as:

$$
p^{*}=\sqrt{2} \pi E^{\prime} \Delta f
$$

Then based on numerical and experimental data provided by JGH and Krithivasan and Jackson [12], an equation was fit that bridged the gap between the solutions $[12,13]$ (see Fig. $2)$. The fit is given as:

$$
\begin{aligned}
& \text { For } \frac{\bar{p}}{p^{*}}<0.8 \\
& \qquad \begin{aligned}
A=\left(A_{J G H}\right)_{1}\left(1-\left[\frac{\bar{p}}{p^{*}}\right]^{1.51}\right)+\left(A_{J G H}\right)_{2}\left(\frac{\bar{p}}{p^{*}}\right)^{1.04}
\end{aligned}
\end{aligned}
$$

$$
\text { For } \begin{aligned}
\frac{\bar{p}}{p^{*}} & \geq 0.8 \\
A & =\left(A_{J G H}\right)_{2}
\end{aligned}
$$

Unlike the popular Hertz spherical contact model, this model considers the effect of interactions between adjacent asperities. This is because the sinusoidal model considers that the surface is periodic as shown by Eq. (1).

\begin{tabular}{|c|c|c|c|}
\hline \multicolumn{4}{|c|}{ Table 1: Nomenclature } \\
\hline & area of contact & $S_{v}$ & yield strength \\
\hline$A_{n}$ & nominal contact area & & cartesian coordinate on surface \\
\hline$a$ & radius of the area of contact & $\mathrm{y}$ & cartesian coordinate on surface \\
\hline & material dependant exponent & & \\
\hline$C$ & critical yield stress coefficient & Gre & ymbols \\
\hline$D$ & contact area factor & $\lambda$ & asperity wavelength \\
\hline$E$ & elastic modulus & & electrical resistivity of surface material \\
\hline$E^{\prime}$ & $E /\left(1-v^{2}\right)$ & & asperity amplitude \\
\hline$e_{y}$ & yield strength to elastic modulus ratio, $S_{y} / E^{\prime}$ & & $\begin{array}{l}\text { alleviation factor } \\
\text { interference between hemisphere and surface }\end{array}$ \\
\hline$F$ & contact force & $v$ & Poisson's ratio \\
\hline$f$ & spatial frequency (reciprocal of wavelength) & & \\
\hline$h$ & surface height & Sub. & \\
\hline $\bar{P}$ & individual asperity contact force & & elastic regime \\
\hline- & & & plastic regime \\
\hline$p$ & mean pressure & & critical value at onset of plastic deformation \\
\hline$p^{*}$ & average pressure of complete contact & & frequency level \\
\hline & electrical contact resistance & & $\begin{array}{l}\text { from Jackson and Green } \\
\text { asperity }\end{array}$ \\
\hline
\end{tabular}

\section{B. Electrical Contact Resistance}

The electrical contact resistance for a circular contact between two surfaces of the same material is given by Holm $[14]$ as

$$
R_{\text {circle }}=0.5 \frac{\rho}{a}
$$




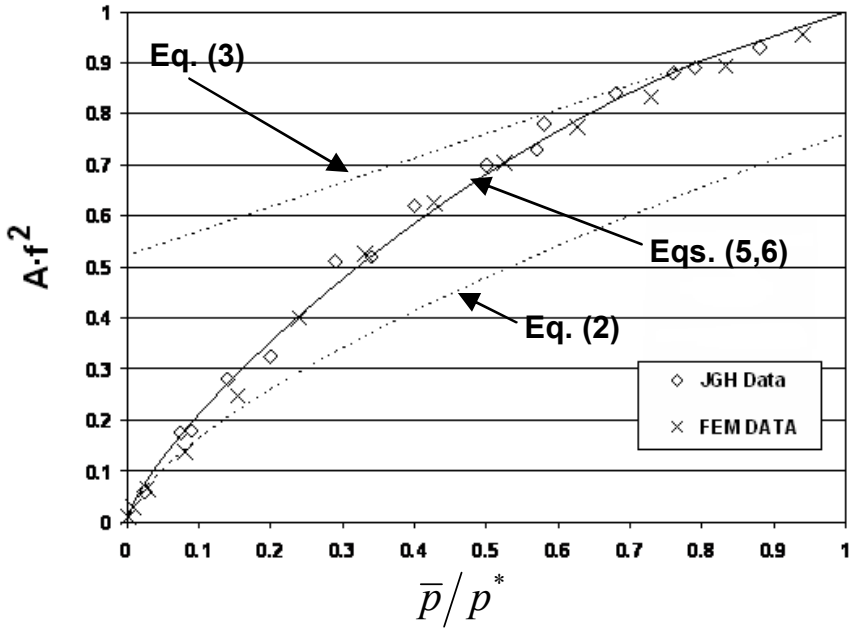

Figure 2: Comparison of elastic FEM results with JGH model and the fit provided by Jackson and Streator.

For isolated spherical contact, Eq. (7) is all that is needed to predict contact resistance. However, sinusoidal contact is different for several reasons. First, as the sinusoidal surface is loaded by a rigid flat surface and deforms, the contact evolves from an initial circular contact area (see Fig. 3 ), to a square like contact area (see Fig. 4). As the contact areas at the peaks grow, they also become large in relation to the entire surface area. Then the electrical current is less restricted because it can follow paths through contact areas that are in relatively close proximity. The alleviation factor [15] was developed to account for this and will be discussed further later.

Since the contact area is initially circular, for initial contact of sinusoidal surfaces, Eq. (7) can be used to predict contact resistance. However, the contact resistance must be modified to account for the change in shape.

When the contact area is half of the surface area $\left(A \cdot f^{2}=0.5\right)$ the contact areas become rectangular, or for the isotropic case, square in shape. One can visually observe this by truncating the surface at the average height of $h / \Delta=0.5$ (see Fig. 1 and 4). The contact resistance for a square contact area is given by as [16]:

$$
R_{\text {square }}=0.43 \frac{\rho}{b}
$$

Note that between the circle and square contact area resistances given by Eqs. ( 7 and 8 ) there is only a relatively small difference. Nonetheless, this may be significant in some engineering applications. One must also consider that there are two peaks on a sinusoidal surface for an area $\lambda x \lambda$ (see Figs. 1, 3 and 4). Therefore Eqs. (7 and 8) must be adjusted for this. Since a sinusoidal surface is periodic and contains many asperities, the resistance should also be normalized so that it can be adjusted for any size contact area.

$$
\begin{gathered}
R_{1}^{*}=\frac{R_{1} A_{n}}{\rho \lambda}=0.25 \frac{\lambda}{a} \\
R_{2}^{*}=\frac{R_{2} A_{n}}{\rho \lambda}=0.215 \frac{\lambda}{b}
\end{gathered}
$$

where $R_{1}^{*}$ is for initial contact of a sinusoidal surface, and $R_{2}^{*}$ is for near complete contact of sinusoidal surface (similar to Eqs. 2 and 3 for contact area). The contact radius, $a$, can also be determined from

$$
a=\sqrt{\frac{A}{2 \pi}}
$$

Likewise, the contact length, $b$, for a square contact is calculated by

$$
b=\sqrt{\frac{A}{2}}
$$

where $A$ is determined from Eqs. (5 and 6). Alternatively, but with less accuracy, the asymptotic solutions of Eqs. (2 and 3) might be substituted directly into Eqs, (11 and 12), respectively, resulting in:

$$
\begin{aligned}
& a=\frac{1}{\sqrt{2} f}\left[\frac{3}{8 \pi} \frac{\bar{p}}{p^{*}}\right]^{1 / 3} \\
& b=\frac{1}{\sqrt{2} f} \sqrt{1-\frac{3}{2 \pi}\left(1-\frac{\bar{p}}{p^{*}}\right)}
\end{aligned}
$$

From Johnson [17], the changing of the contact area from circular to square experimentally appears to happen at approximately $\frac{\bar{p}}{p^{*}}=0.34$ for the elastic case. This is also confirmed from FEM results from Krithivasan and Jackson [12] and analytical results from Johnson, Greenwood and Higginson [10], as shown in Fig. 2. However, these are for the perfectly elastic case and the elastic-perfectly plastic case might be slightly different. In addition, at this point the contact areas from neighboring peaks begin to touch and 
therefore the alleviation factor becomes very important. However, neglecting these points for now, but still considering that the contact becomes square at approximately $\frac{\bar{p}}{p^{*}}=0.34$, an approximate value of the contact resistance can be predicted by the following equations linking the above cases together.

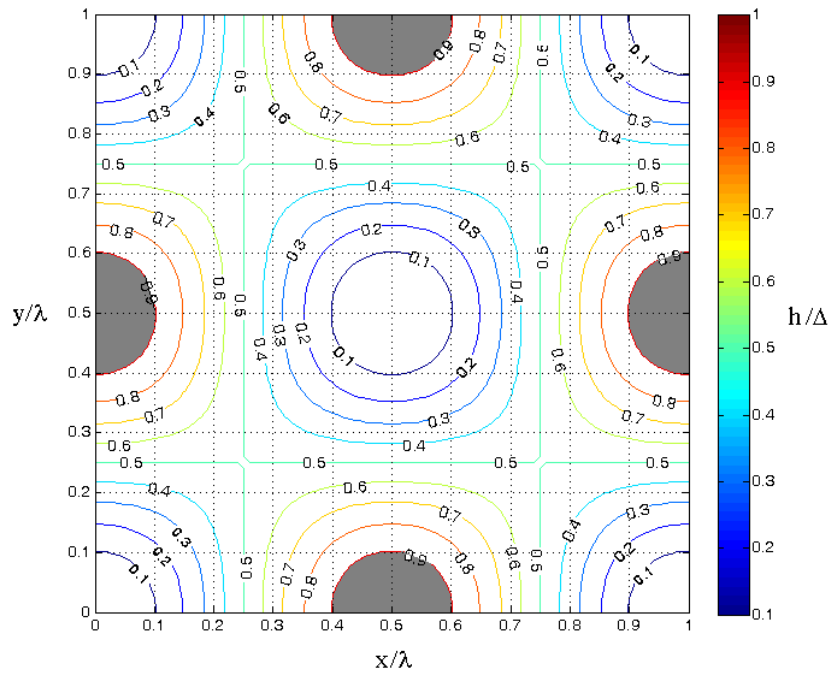

Figure 3: Sinusoidal surface with grey showing the circular shape of the peaks that are in initial contact.

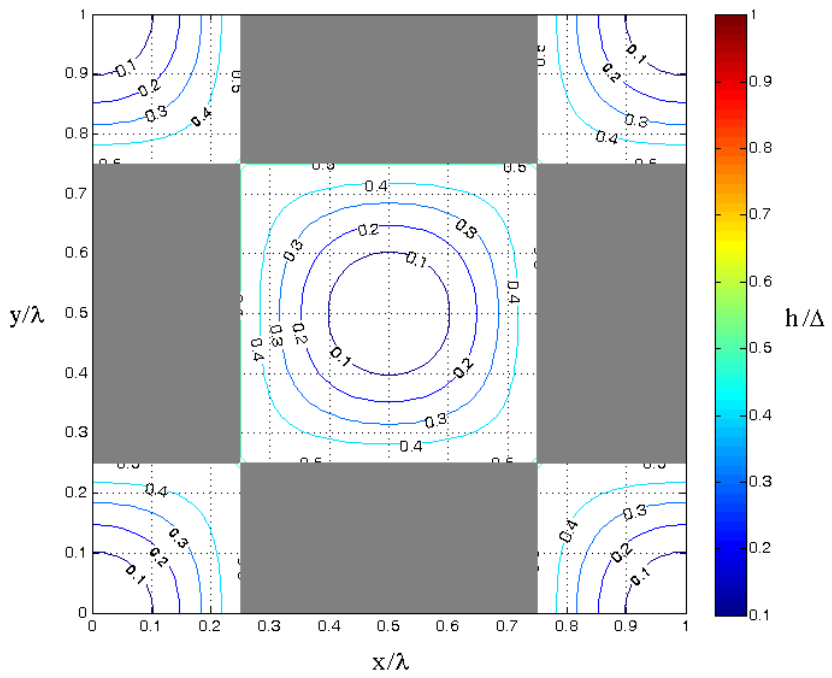

Figure 4: Sinusoidal surface with grey showing the square shape of the peaks that are in contact which fills half the surface area.
For $\frac{\bar{p}}{p^{*}} \leq 0.34$

$$
R^{*}=R_{1}^{*}\left(1-\frac{1}{0.34} \frac{\bar{p}}{p^{*}}\right)+R_{2}^{*}\left(\frac{1}{0.34} \frac{\bar{p}}{p^{*}}\right)
$$

For $\frac{\bar{p}}{p^{*}}>0.34$

$$
R^{*}=R_{2}^{*}
$$

The predictions of Eqs. (15 and 16) by using both possible predictions of $a$ and $b$ are shown in Fig. (5). Using Eqs. (11 and 12) directly calculated from Eqs. (5 and 6) is labeled as 'Full' and by using Eqs. (13 and 14) is labeled 'Approximation.' However, it should be emphasized that both equations are actually approximations and neither include the alleviation factor effect yet. Also shown are the asymptotic solutions given by Eqs. (9 and 10). The maximum percent error between the two methods is about $12.8 \%$, occurring at the 'transition' at $\frac{\bar{p}}{p^{*}}>0.34$. The average error between the two is $3.6 \%$. Therefore the full solution should probably be used, but the approximate solution may also be acceptable in some cases.

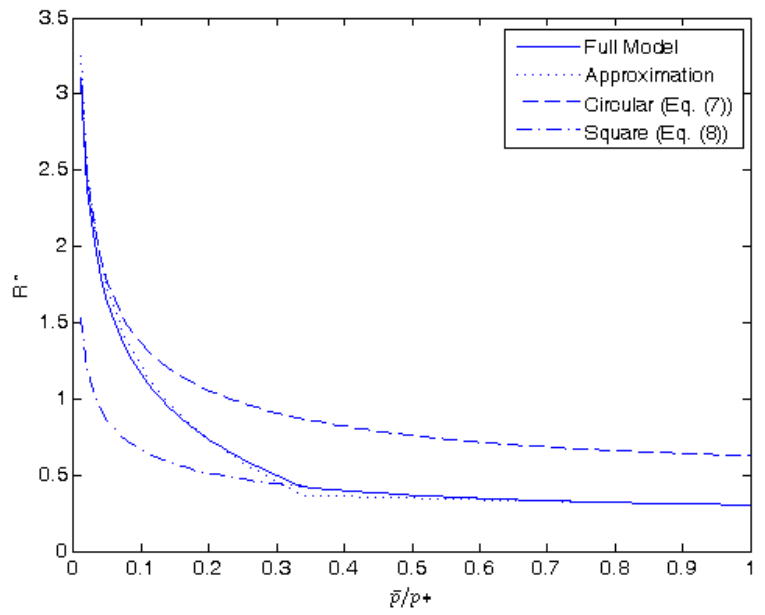

Figure 5: Comparison of approximate predictions of contact resistance between sinusoidal surfaces while neglecting the alleviation factor effect.

Next, the effect of the alleviation factor, or the increasing ratio of contact area to surface area needs to be considered in the above model. Though there are various 
ways to calculate this factor [15], the simplified version offered by Cooper et al. [18] is chosen for this work:

$$
\psi=\left(1-\sqrt{A / A_{n}}\right)^{1.5}
$$

where $A_{n}$ is set to $\lambda^{2}$. The alleviation factor is a factor widely used in thermal contact resistance (mathematically very similar to electrical contact resistance) that considers the effect contact spots have on each other when in close proximity. For instance, a surface could have contact spots that are in very close proximity and one could assume that they are in parallel to calculate the net contact resistance. However, this would assume that each contact acts independently, which they do not if they are close enough. Therefore the contact alleviation factor essentially causes the contact resistance to reduce to zero as the real contact area engulfs the entire contact region. This is also important because the rectangular contact areas meet and are no longer isolated after approximately half of the area is contact. The alleviation factor should help to consider this. Then the equations for predicting the contact resistance between sinusoidal surfaces in contact with a flat surface are:

$$
\text { For } \begin{aligned}
\frac{\bar{p}}{p^{*}} & \leq 0.34 \\
R^{*} & =\left[R_{1}^{*}\left(1-\frac{1}{0.34} \frac{\bar{p}}{p^{*}}\right)+R_{2}^{*}\left(\frac{1}{0.34} \frac{\bar{p}}{p^{*}}\right)\right] \Psi
\end{aligned}
$$

For $\frac{\bar{p}}{p^{*}}>0.34$

$$
R^{*}=R_{2}^{*} \Psi
$$

The results of using Eqs. (18 and 19) are shown in Fig. 6. As should be expected, the alleviation factor given by Eq. (17) causes the predicted electrical contact resistance to reduce to zero as complete contact is approached. Since Eqs. (15 and 16) do not correctly capture this trend, Eqs. (18 and 19) are more accurate and should be used when predicting contact resistance from a sinusoidal surface in contact with a flat surface.

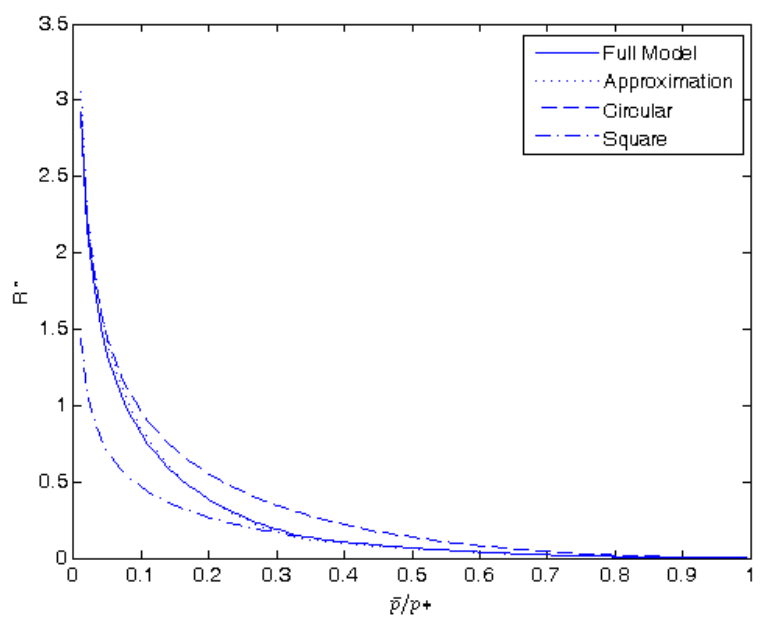

Figure 6: Comparison of approximate predictions of contact resistance between sinusoidal surfaces

\section{Elastic-Plastic Sinusoidal Contact}

Due concentrated contacts and the resulting high local pressures, many of the asperities on contacting rough surfaces undergo plastic deformation. Therefore an elasticplastic sinusoidal contact model is needed to consider this effect. The equations used in the current work to calculate the elastic-plastic contact are derived from the FEM results by Krithivasan and Jackson [12] and Jackson et al. [11]. The methodology is very similar to that of the perfectly elastic case with the exception of using a different set of formulas once a calculated critical pressure and area are reached. The critical load and area are given by:

$$
\begin{aligned}
& P_{c}=\frac{1}{6 \pi}\left(\frac{1}{\Delta f^{2} E^{\prime}}\right)^{2}\left(\frac{C}{2} \cdot S_{y}\right)^{3} \\
& A_{c}=\frac{2}{\pi}\left(\frac{C S_{y}}{8 \Delta f^{2} E^{\prime}}\right)^{2}
\end{aligned}
$$

where $C$ is given as

$$
C=1.295 \cdot \exp (0.736 v)
$$

At low loads, $P<P_{c}$, and consequently small areas of contact, it is acceptable to assume that any deformation of the asperities in contact will behave perfectly elastically. However, as load increases to the critical value, plastic deformation will begin to occur within the asperities. To evaluate the plastic deformation we replace Eq. (2) with: 


$$
\begin{aligned}
& A_{P}=2\left(A_{c}\right)^{\frac{1}{1+d}}\left(\frac{3 \bar{p}}{4 C S_{y}} \lambda^{2}\right)^{\frac{d}{1+d}} \\
& d=3.8 \cdot\left(\frac{E^{\prime}}{S_{y}} \cdot \frac{\Delta}{\lambda}\right)^{0.11}
\end{aligned}
$$

This replacement results in the following equation for contact area:

$$
A=\left(A_{P}\right)\left(1-\left[\frac{\bar{p}}{p_{P}^{*}}\right]^{1.51}\right)+\left(A_{J G H}\right)_{2}\left(\frac{\bar{p}}{p_{P}^{*}}\right)^{1.04}
$$

where the average pressure to obtain complete contact, $p^{*}$, is adjusted to consider plastic deformation as shown by the following equation for the average elastic-plastic pressure to cause complete contact:

$$
\begin{aligned}
& \frac{p_{P}^{*}}{p^{*}}=\left(\frac{11}{4 \cdot \Delta / \Delta c+7}\right)^{3 / 5} \\
& \Delta c=\frac{\sqrt{2} \cdot S_{y} \exp \left(\frac{2 v}{3}\right)}{3 \pi E^{\prime} f}
\end{aligned}
$$

\begin{tabular}{|c|c|}
\hline \multicolumn{2}{|c|}{ Table 2: $\quad$ Material Properties of Tin } \\
\hline$E=41.369 * 10^{9} \mathrm{~Pa}$ & $S_{y}=14 * 10^{6} \mathrm{~Pa}$ \\
\hline$v=0.36$ & $\rho_{L}=11.5 * 10^{-8} \Omega \cdot m$ \\
\hline
\end{tabular}

In addition, the point at which elastic-plastic sinusoidal contact becomes square $\left(\mathrm{A} / \lambda^{2}=0.5\right)$ is different from the perfectly elastic case. From Krithivasan and Jackson it is found to occur at approximately $\frac{\bar{p}}{p_{p}^{*}}>0.275$. Therefore, for elastic plastic contact of sinusoidal surfaces the contact resistance should be predicted by

For $\frac{\bar{p}}{p_{p}^{*}} \leq 0.275$

$$
R^{*}=\left[R_{1}^{*}\left(1-\frac{1}{0.34} \frac{\bar{p}}{p_{p}^{*}}\right)+R_{2}^{*}\left(\frac{1}{0.34} \frac{\bar{p}}{p_{p}^{*}}\right)\right] \Psi
$$

$$
\text { For } \begin{aligned}
\frac{\bar{p}}{p_{p}^{*}} & >0.34 \\
R^{*} & =R_{2}^{*} \Psi
\end{aligned}
$$

Also note that the values of $a$ and $b$ should be calculated from Eqs. (11 and 12) using $A$ from Eq. (13).

\section{MODEL COMPARISON}

The prediction of the contact resistance from elasticplastic sinusoidal contact (Eqs. 28 and 29) is shown in Fig. 7. The overall trend is generally similar to the elastic case, except that the contact resistance appears to decrease more rapidly as load is increased. This is the included plastic deformation allowing for the contact area to become larger.

Also shown in Fig. 7 is the predictions of an elastic plastic spherical contact model [19]. The details of this model are given in the Appendix. To make a reasonable comparison, it is also assumed that the two spherical asperity contacts for a surface area of $\lambda^{2}$. The radiuses of the spheres are also made to be the same as the radius of curvature of the sinusoidal surface at the tops of the peaks. The contact resistance for the spherical model is calculated using Eq. (7), because the contact area will always be circular. The spherical model predictions are then normalized using the same methodology used for the sinusoidal models.

As shown in Fig. 7, clearly the elastic-plastic sinusoidal and spherical models generate similar trends. However, the quantitative values of the predictions are significantly different. It is also interesting that the spherical model initially predicts more contact resistance than the sinusoidal case, but as the pressure increases the trend reverses and the sinusoidal model predict more contact resistance while the spherical contact model reaches zero at about $\frac{\bar{p}}{p_{p}^{*}}>0.5$. This is probably because interactions between adjacent asperities will cause the contact pressure to rise as complete contact is reached, and this mechanism is not properly considered in the spherical model, while it is in the sinusoidal model. 


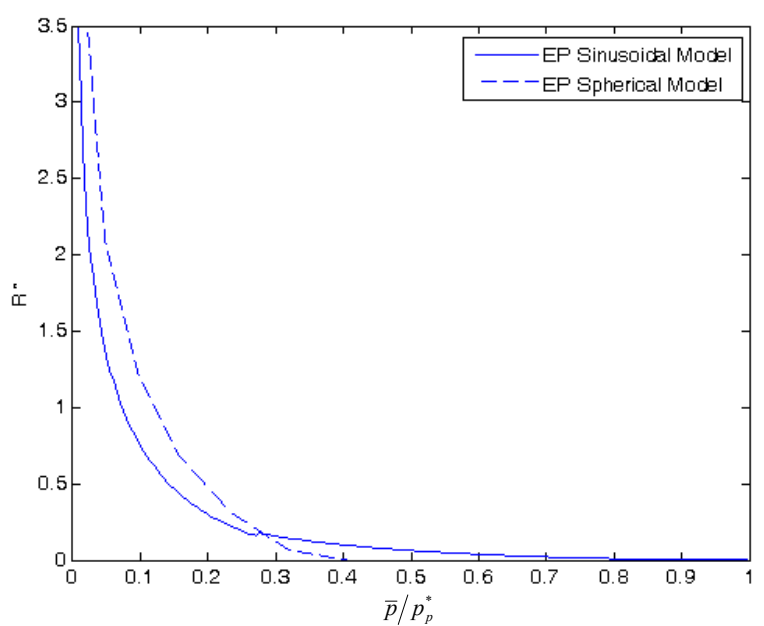

Fig. 7: A comparison of elastic-plastic sinusoidal and spherical asperity contact resistance models.

\section{CONCLUSIONS}

This work presents a new model for predicting the contact resistance of elastic and elastic-plastic sinusoidal asperities in contact. It is based on both previous analytical models and finite element results. These models could be used in rough surface contact models as a more realistic depiction of contacting asperities. In addition, the elasticplastic sinusoidal model is compared to an equivalent spherical model. The models appear to have the same qualitative trends but are significantly different quantitatively. The author would like to further confirm this work from experimental measurements and finite element simulations.

\section{APPENDICES}

\section{Elastic-Plastic Spherical Model}

As an alternative to elastic-plastic sinusoidal contact, elasticplastic spherical contact is often used model asperity contact in electrical contacts. The current work will use the finite element based model formulated by Jackson and Green [19]. The model employs a critical interference value given as follows:

$$
\omega_{c}=\left(\frac{\pi \cdot C \cdot S_{y}}{2 E^{\prime}}\right)^{2} \cdot R .
$$

For interference $\omega<1.9 \omega_{c}$, the plastic deformation is small and is considered to effectively be in the perfectly elastic regime and therefore coincides with Hertz contact. However, if $\omega \geq 1.9 \omega_{c}$ then the following equations from Jackson and Green must be used instead:

$$
\begin{aligned}
& A_{J G}=\pi R \omega\left(\frac{\omega}{\omega_{c}}\right)^{B} \\
& P_{J G}=P_{c}\left\{\begin{array}{l}
{\left[\exp \left(-\frac{1}{4}\left(\frac{\omega}{\omega_{c}}\right)^{5 / 12}\right)\right]\left(\frac{\omega}{\omega_{c}}\right)^{3 / 2}} \\
+\frac{4 H_{G}}{C S_{y}}\left[1-\exp \left(-\frac{1}{25}\left(\frac{\omega}{\omega_{c}}\right)^{5 / 9}\right)\right] \frac{\omega}{\omega_{c}}
\end{array}\right\} \\
& P_{c}=\frac{4}{3}\left(\frac{R}{E^{\prime}}\right)^{2}\left(\frac{C}{2} \pi S_{y}\right)^{3} \\
& B=0.14 \exp \left(23 e_{y}\right) \\
& e_{y}=\frac{S_{y}}{E^{\prime}} \\
& \frac{H_{G}}{S_{y}}=2.84-0.92\left(1-\cos \left(\pi \frac{a}{R}\right)\right)
\end{aligned}
$$

\section{REFERENCES}

[1] M. Bahrami, J. R. Culham, M. M. Yovanovich, and G. E. Schneider, "Review of thermal joint resistance models for non-conforming rough surfaces in a vacuum," presented at Proceedings of the ASME Summer Heat Transfer Conference, Niagara Falls, NY, USA, 2003.

[2] A. Bairi and N. Laraqi, "The thermal constriction resistance for an eccentric spot on a circular heat flux tube," Journal of Heat Transfer, vol. 126, pp. 652655, 2004.

[3] N. Laraqi, "Thermal constriction resistance of coated solids - static and sliding contacts," International Communications in Heat and Mass Transfer, vol. 26, pp. 299-309, 1999.

[4] N. Laraqi, "Thermal resistance for random contacts on the surface of a semi-infinite heat flux tube," Journal of Heat Transfer, vol. 125, pp. 532-535, 2003.

[5] K. J. Negus and M. M. Yovanovich, "Constriction Resistance of Circular Flux Tubes with Mixed Boundary Conditions by Linear Superposition of Neumann Solutions," presented at American Society of Mechanical Engineers (Paper), 1984. 
[6] K. J. Negus, M. M. Yovanovich, and J. V. Beck, "On the nondimensionalization of constriction resistance for semi-infinite heat flux tubes," Journal of Heat Transfer, Transactions ASME, vol. 111, pp. 804-807, 1989.

[7] A. A. Rostami, A. Y. Hassan, and P. C. Lim, "Parametric study of thermal constriction resistance," Heat and Mass Transfer/Waerme- und Stoffuebertragung, vol. 37, pp. 5-10, 2001.

[8] M. M. Yovanovich, J. R. Culham, and P. Teertstra, "Analytical modeling of spreading resistance in flux tubes, half spaces, and compound disks," IEEE Transactions on Components, Packaging, and Manufacturing Technology Part A, vol. 21, pp. 168176, 1998.

[9] A. F. Black, V. Singhal, and S. V. Garimella, "Analysis and prediction of constriction resistance for contact between rough engineering surfaces," Journal of Thermophysics and Heat Transfer, vol. 18, pp. 30-36, 2004.

[10] K. L. Johnson, Greenwood, J. A., and Higginson, J. G., "The Contact of Elastic Regular Wavy Surfaces," Int. J. Mech. Sci., vol. 27, pp. 383-396, 1985.

[11] R. L. Jackson, V. Krithivasan, and W. E. Wilson, "The Pressure to Cause Complete Contact between Elastic Plastic Sinusoidal Surfaces," IMechE J. of Eng. Trib. - Part J., vol. 222, pp. 857-864, 2008.

[12] V. Krithivasan and R. L. Jackson, "An Analysis of Three-Dimensional Elasto-Plastic Sinusoidal Contact," Trib. Letters, vol. 27, pp. 31-43, 2007.

[13] R. L. Jackson and J. L. Streator, "A Multiscale Model for Contact between Rough Surfaces," Wear, vol. 261, pp. 1337-1347, 2006.

[14] R. Holm, Electric Contacts. New York: Springer, 1967.

[15] C. V. Madhusudana, Thermal Contact Conductance. New York: Springer-Verlag, Inc., 1996.

[16] M. Nakamura, "Constriction Resistance of Conducting Spots by the Boundary Element Method," IEEE Trans. Comp., Hybrids Manufact. Tech., vol. 16, pp. 339-344, 1993.

[17] K. L. Johnson, Contact Mechanics: Cambridge University Press, 1985.

[18] M. G. Cooper, B. B. Mikic, and M. M. Yovanovich, "Thermal Contact Conductance," Int. J. Heat Mass Transfer, vol. 12, pp. 279-300, 1969.

[19] R. L. Jackson and I. Green, "A Finite Element Study of Elasto-Plastic Hemispherical Contact," ASME J. Tribol., vol. 127, pp. 343-354, 2005. 


\section{Switching Contact Bounce Reduction}

\author{
Bogdan O. Ciocirlan \\ Interconnection \& Process Technology \\ Tyco Electronics Corporation \\ Middletown, PA, US \\ bciocirlan@tycoelectronics.com
}

\author{
Hank Herrmann \\ Interconnection \& Process Technology \\ Tyco Electronics Corporation \\ Middletown, PA, US \\ hank.herrmann@tycoelectronics.com
}

\begin{abstract}
The effects of arcing in medium power relays and switches have been extensively reported in the literature. Repeated breaking and remaking of the electrical connection during bouncing at each contact closure generates multiple arcs that cause damage to the contacts, as well as other effects. Reduction of closure bounce is thus expected to alleviate these effects improving the life and switching energy capability of switching contacts. We propose a novel and cost-effective solution to the bounce problem that employs eccentric impact principles to enhance contact behavior during closure. The new concept was proven for a commercial electromechanical relay and showed $49 \%$ reduction in the number of bounces and $94 \%$ reduction in contact separation time during bouncing. Preliminary cycle testing was also performed to determine the effectiveness of the proposed method to improve the life of the contacts. Despite the significant reduction in bounce, only a marginal improvement in contact damage was realized. Several possible explanations have been proposed, but more work is required to identify the reason for the unexpected results.
\end{abstract}

Keywords-contact bounce, bounce reduction, eccentric impact.

\section{INTRODUCTION}

In typical constructions of electromechanical relays and switches, contact closure is accompanied by a series of impacts that cause the contacts to bounce until all of the kinetic energy is dissipated. Each bounce is a fast break and make sequence and resembles an opening operation in that the arcs are generated in the same fashion. If loading conditions permit, an arc is drawn as contacts separate that will cause contact erosion, increase the tendency for contact welding, and degrade the electrical performance. Also, the mechanical impact will plastically deform and fatigue the contact interface increasing the overall contact wear. Since multiple arcs and impacts are generated during contact bouncing, these effects may become significant and adversely affect the life and switching capacity of the device. In addition, special materials are required to withstand the damaging effects and achieve an acceptable service life.

Reducing contact bounce is a well known approach to mitigate contact closure effects. Some reduction in bounce can often be achieved by design [1]. Simple solutions like increasing the contact force or minimizing the kinetic energy of the moving contact through reducing the closing velocity and contact mass have typically been employed, but at the expense of overall performance. Increasing closing force will intensify the mechanical wear of the contacts. Decreasing closing velocity will affect the opening operation and reducing contact mass will limit the switching capacity of the device. If such performance tradeoffs are unacceptable, more sophisticated means of alleviating the effects of the bounce are required. Several techniques utilizing dynamic absorbers [2], energy dissipating contact support structures [3], or feedback control systems [4], among other means, have been reported. However, the additional components and provisions involved increase the complexity and the cost of the device, and constitute an impediment for their large-scale utilization. Therefore, a simple and yet effective solution to the bounce problem is needed.

Although the reduction in contact bounce is expected to increase the life of the contacts, predicting the extent of improvement is extremely difficult. Such predictors would require a known relationship between the amount of bounce and contact damage, which are not readily available. Moreover, the inherent variability of the bounce pattern would make such predictions almost impossible. The bounce pattern is strongly affected by current, pre-impact events, and arcing [5], [6], [7]. It is also influenced by the load type. For example, capacitive loads may have a different effect from the resistive or inductive ones due to the large in-rush currents. Even for a specific load and energy level, contact bounce is not expected to remain constant during operation. Work hardening and deformation of the contacts progressively reduce the ability of contact materials to dissipate the impact energy [8]. Also, the continuous change in the amount of wear modifies the contact interface geometry altering the impact process. Finally, manufacturing tolerances also introduce some degree of variability. Therefore, the effectiveness of any bounce reduction method has to be proven experimentally for each specific design and application requirements.

This paper has two objectives. The first objective is to propose a new method for reducing the closure bounce of electrical contacts. The proposed method employs the principles of eccentric impact to convert the rebound motion of the contacts after the initial impact into rolling or sliding motions that reduce contact separation. This is achieved through simply changing the relative orientation of the contacts. Since no additional parts or steps in the manufacturing process are required, the proposed method offers a very cost-effective solution to the bounce problem. The reduction in bounce was demonstrated for a generalpurpose relay with rivet-style contacts. 
The second objective is to report the results of preliminary cycle testing that were conducted to quantify the improvements in terms of contact life. Standard and modified relays incorporating the new concepts were tested for make-and-break and make-only conditions and the difference in the amount of contact damage was assessed at the end of each test.

The proposed bounce reduction technique is described in Section II. The experimental setup is explained in Section III. The proof of concept and preliminary cycle testing results are presented in Section IV. Section V contains a discussion about results and future directions. Conclusions are drawn in the last section.

\section{The Proposed Bounce Reduction PrinciPLE}

For button or rivet style switching contacts with one contact attached to a moving spring beam and the other stationary, existing constructions and treatments typically employ axially symmetric contacts aligned and driven along their centers of gravity, as shown in Figure 1(a). The reaction impulse due to the impact at the first contact closure is thus oriented along the normal to the point of contact and directed through the center of gravity of the moving contact. This orientation causes an unavoidable rebound that directly separates the contact surfaces. For a given design (moving beam stiffness, contact and support structure damping) and closure kinematics (velocity before closure, contact mass, amount of overtravel), the magnitude and duration of contact separation are determined by the amount of kinetic energy returned to the moving contact after the initial impact. Since the kinetic energy is a direct function of velocity, reducing or even eliminating contact separation would require a significant reduction of the separation velocity of the contacts.

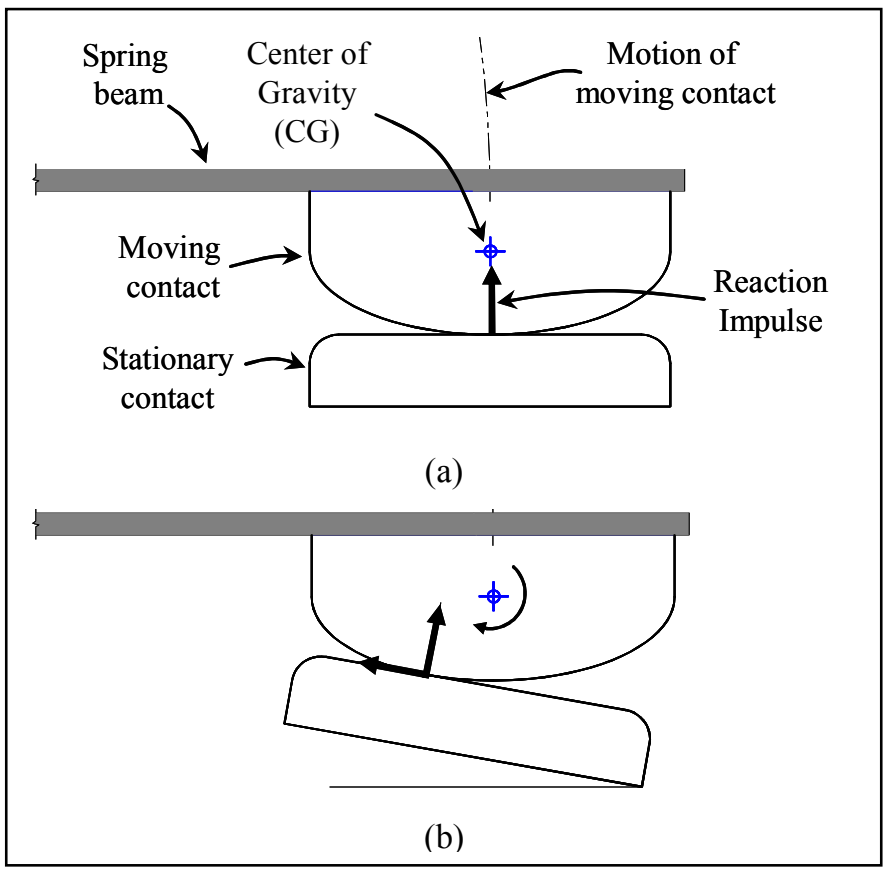

Figure 1. (a) Existing contact configuration; (b) Tilted stationary contact configuration.
In the proposed method, this reduction is achieved through modifying the relative orientation of the contacts such that the normal impulse during the impact is no longer aligned with the center of gravity of the moving contact, as illustrated in Figure 1(b). The proposed arrangement causes an eccentric impact that has the following benefits. First, along with the normal impulse, a tangential impulse is also generated at the point of contact due to the inherent friction present at the contact interface. Second, the moments of both normal and tangential impulses about the center of gravity instigate a rotational impulse that imposes on the moving contact a tendency to roll on the surface of the stationary contact. Finally, the separation velocity of the moving contact and, thus, the amplitude and duration of subsequent bounces is reduced due to the initiation of tangential and rotational motions.

The conversion from separation to rolling can also be accomplished through constructing an asymmetric moving contact, as shown in Figure 2(a). In this case, even a non-tilted stationary contact yields the desired result although the tilt arrangement can also be employed, as depicted in Figure 2(b). It was easier to create the tilted stationary contact condition shown in Figure 1(b) using the contacts of commercially available devices and, thus, only this configuration was pursued in this study. One potential drawback of the contact arrangement in Figure 1(b) is the fact that the electrical connection is established in areas with reduced mass of the moving contact compared to its center apex, which can make the contact susceptible of increased damage. This aspect will have to be taken into consideration when implementing the proposed concept into products.

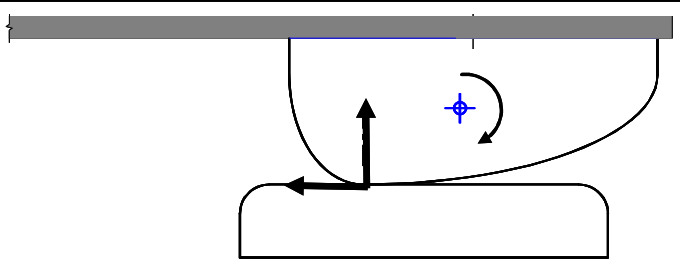

(a)

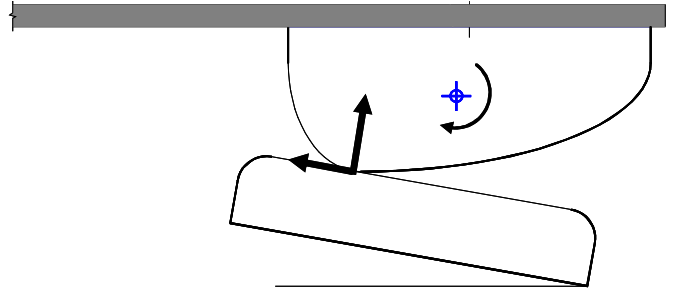

(b)

Figure 2. (a) Eccentric impact through asymmetric moving contact geometry; (b) Combined asymmetric moving and tilted stationary contacts.

\section{EXPERIMENTAL SETUP}

The general-purpose relay shown in Figure 3 was selected as a test object to investigate the eccentric impact concept. This relay is a one-pole, double-throw type and has rivet style contacts. The contacts are made out of copper with a layer of $\mathrm{AgCdO}$ on the surface and are separated by a $0.66 \mathrm{~mm}(0.026$ 
in.) gap in the open position. The relay was partially disassembled so that the parts could be mounted in the adjustable fixture shown in Figure 4(a). The coil, armature, and moving spring beam assembly was attached on an X-Y table and the normally open stationary contact was mounted on a separate structure that provided the means to varying its orientation and vertical position relative to the moving contact.

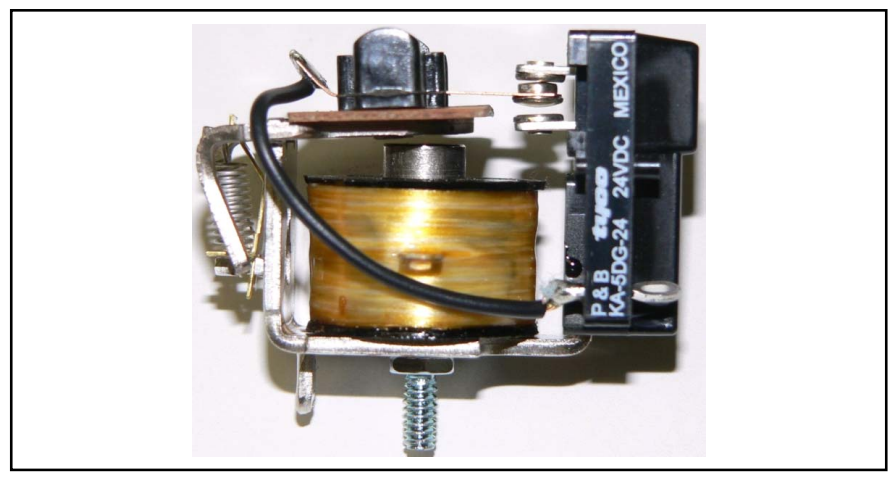

Figure 3. General purpose relay used in this study.

The three straight arrows in Figure 4(a) represent the $\mathrm{X}, \mathrm{Y}$ and $\mathrm{Z}$ micrometer adjustments. The $\mathrm{X}$ and $\mathrm{Y}$ adjustments of the $\mathrm{X}-\mathrm{Y}$ table are used to align the moving contact with the fixed contact. The $\mathrm{X}$ and $\mathrm{Y}$ axes are parallel and perpendicular to the direction of the spring beam, respectively. The $\mathrm{Z}$ adjustment sets the contact gap through fine-tuning the vertical position of the stationary contact. The arcing arrows in Figures 4(a) and (b) indicate the roll and pitch adjustments of the stationary contact, which tilt the stationary contact about the $\mathrm{X}$ and $\mathrm{Y}$ axes, respectively. The ovals in Figure 4(b) highlight the contacts and the adjustment mechanism that sets the armature travel limits.

The fixture was tested to determine whether the additional structures influence the dynamics of the bounce. The stationary contact was arranged in a non-tilted configuration and contact bounce voltage waveforms were captured for lowenergy levels (1VDC, 0.1A). These waveforms were compared to the contact bounce waveforms from five standard relays. It was found that the characteristics of both fixture and standard contacts listed in Table I were similar and within the expected variability limits.

TABLE I. BOUNCE CHARACTERISTICS OF STANDARD AND FIXTURE RELAYS

\begin{tabular}{|l|c|c|c|c|}
\hline \multirow{2}{*}{$\begin{array}{c}\text { 1VDC, } \\
\text { 0.1 A }\end{array}$} & \multicolumn{2}{|c|}{ Number of Bounces } & \multicolumn{2}{c|}{ Bounce Duration (msec) } \\
\cline { 2 - 5 } & Standard & Fixture & Standard & Fixture \\
\hline Average & 20 & 25 & 2.394 & 2.515 \\
\hline Min & 11 & 17 & 1.720 & 1.633 \\
\hline Max & 32 & 37 & 3.030 & 3.078 \\
\hline Range & 21 & 20 & 1.310 & 1.445 \\
\hline
\end{tabular}

Several circuits were developed to facilitate the testing of the proposed method. The electrical diagram of the test setup is depicted in Figure 5. The first circuit, which is denoted
"Control Box" in Figure 5, provides adjustable times for the relay to be ON and OFF, a bounceless coil voltage for stable triggering, and a voltage proportional to the coil current to indicate the armature closure. The second circuit, "Data Sequencer", produced the timing signals to trigger the data acquisition system so that the contact voltage was measured about $80 \mathrm{msec}$ after coil voltage application and about $200 \mathrm{msec}$ after the coil is de-energized. The third circuit, "FET Switch", is an electronic switch that can be used to perform make-only and break-only testing. It can switch currents greater than $100 \mathrm{~A}$ and floats electrically so it can be used on the high or low side of the system and with supplies of either polarity.

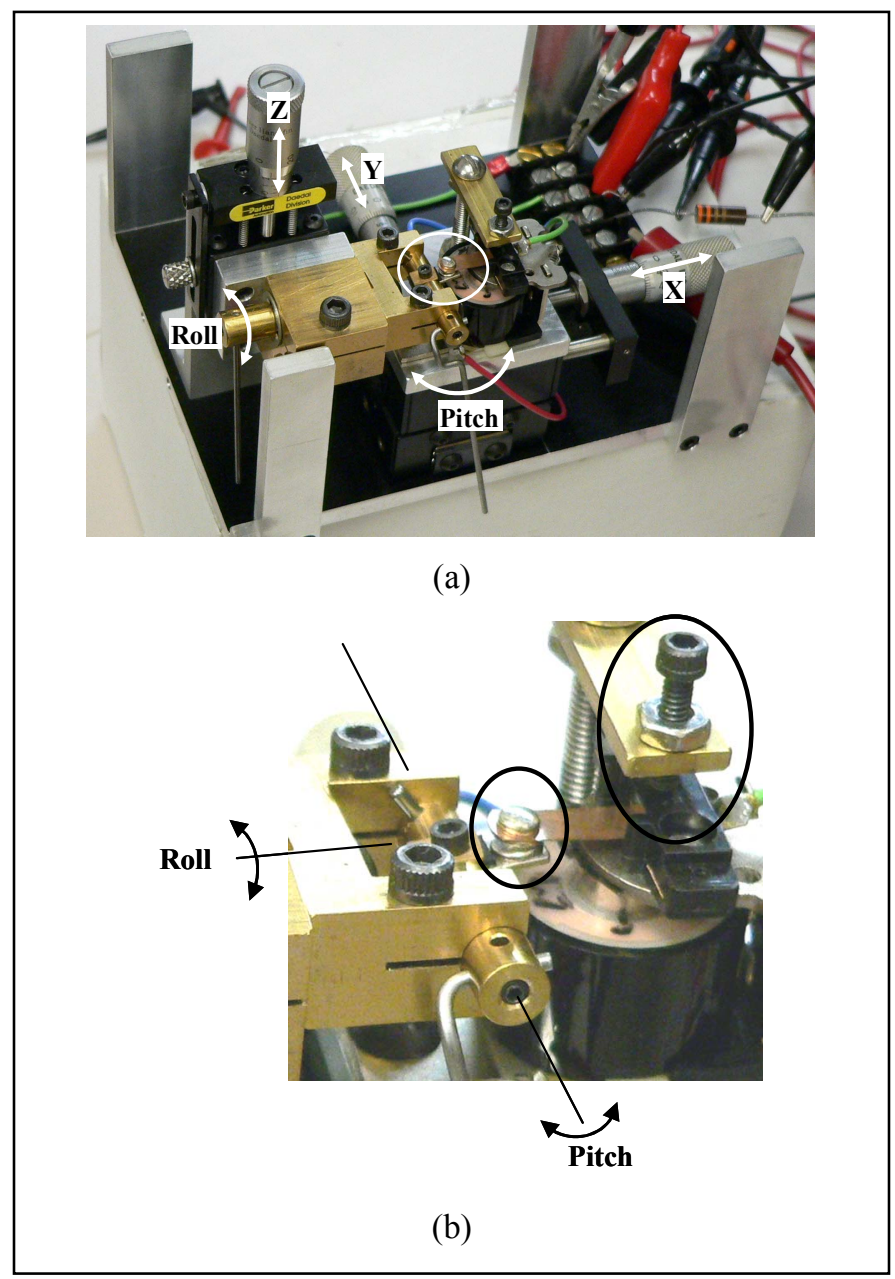

Figure 4. (a) Overall view of the relay test fixture; (b) Detail of contact and travel limit adjustment.

The effects of bouncing were studied for resistive loads. The load values were set through series and parallel combinations of individual isolated banks of non-inductive thick-film power resistors. The banks are mounted on watercooled plates and isolated to $1500 \mathrm{~V}$ peak. Each bank of resistors can dissipate $750 \mathrm{~W}$ when maintained at or below $25^{\circ} \mathrm{C}$.

Various combinations of pitch and roll tilt angles were tested at both low and high-energy levels. In the former case, enough voltage, 1 to $5 \mathrm{VDC}$, was used to assure breaking through films but not to induce significant arcing and the 
current was limited to $100 \mathrm{~mA}$. For the latter case, voltages up to $28 \mathrm{VDC}$ and a $10 \mathrm{~A}$ current were supplied. As a note, $28 \mathrm{~V}$ and $10 \mathrm{~A}$ is the $\mathrm{DC}$ rating of the selected relay.

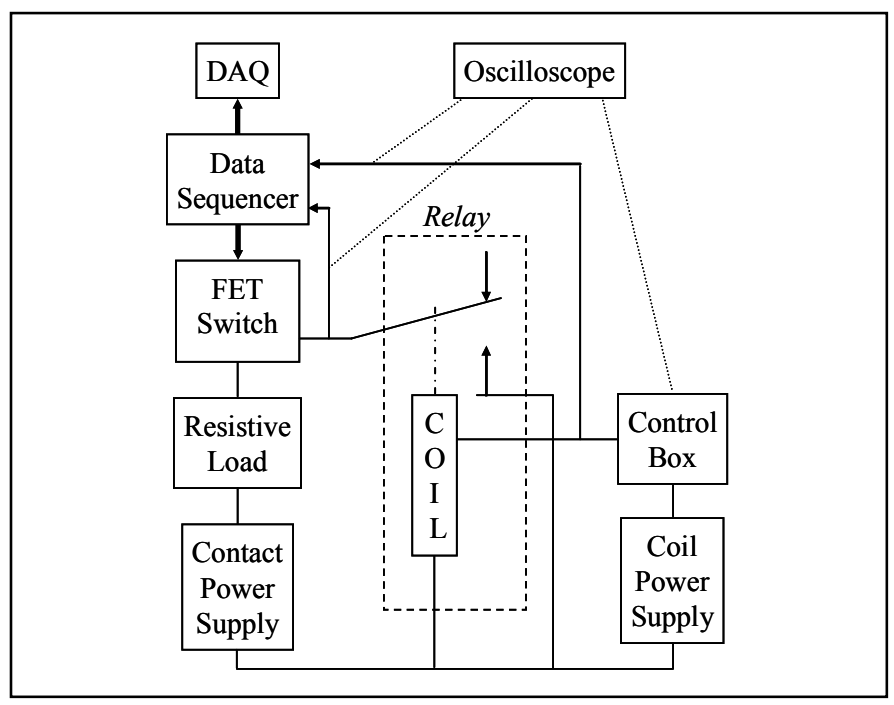

Figure 5. Test setup diagram.

\section{RESULTS}

\section{A. Contact Bounce Reduction}

As described in Section II, generating an eccentric impact will reduce contact separation by imposing a sliding or rolling action between the moving and stationary contacts. Tilting the stationary contact is a practical way of achieving these effects, especially when existing mass-produced devices are evaluated. Although minute contact separations during bounce may not be completely eliminated, they can be kept small through optimizing the tilt angle for a given contact geometry. An approximate analytical model of the planar eccentric impact was developed to determine if such an optimization is possible for the actual contact geometry of the selected relay, considering the fact that the curvature of the moving contact limits the maximum tilt angle to about $30^{\circ}$. Figure 6 shows the contact arrangement and the notations used in the model. The following assumptions were made. First, the planar approximation was considered suitable given the axialsymmetric geometry of the moving contact. Second, the contacts were modeled as rigid bodies to simplify the analysis. Contact time is assumed to be very short. Thus, the interactive forces are impulsive and all other finite forces negligible. Finally, only the contact rivet and the portion of the beam between the lower and upper parts of the rivet were modeled. This last simplification was considered acceptable since only a qualitative determination of contact tendencies after the impact is sought.

The equations of two-dimensional impact [9] were employed to calculate the normal, tangential, and rotational velocities of the moving contact immediately after the impact as a function of the tilt angle. For the contact configuration shown in Figure 6, the equations of motion at any time during the impact are

$$
\begin{gathered}
m\left(v_{n}-v_{n 0}\right)=P_{n} \\
m\left(v_{t}-v_{t 0}\right)=-P_{t} \\
I\left(\omega-\omega_{0}\right)=P_{n} d_{n}+P_{t} d_{t}
\end{gathered}
$$

where, $m$ and $I$ are the mass and the moment of inertia of the contact rivet, respectively; $v_{n}$ and $v_{t}$ are the normal and tangential components of the translational velocity of the $\mathrm{CG}$, respectively; $\omega$ is the rotational velocity with respect to the CG; $P_{n}$ and $P_{t}$ are the normal and tangential impulses, respectively; and $d_{n}$ and $d_{t}$ are the coordinates of the CG with respect to the point of contact, respectively. The subscript 0 was used to identify the velocities before impact. The location of the CG and the moment of inertia were calculated for the 3D geometry of the rivet.

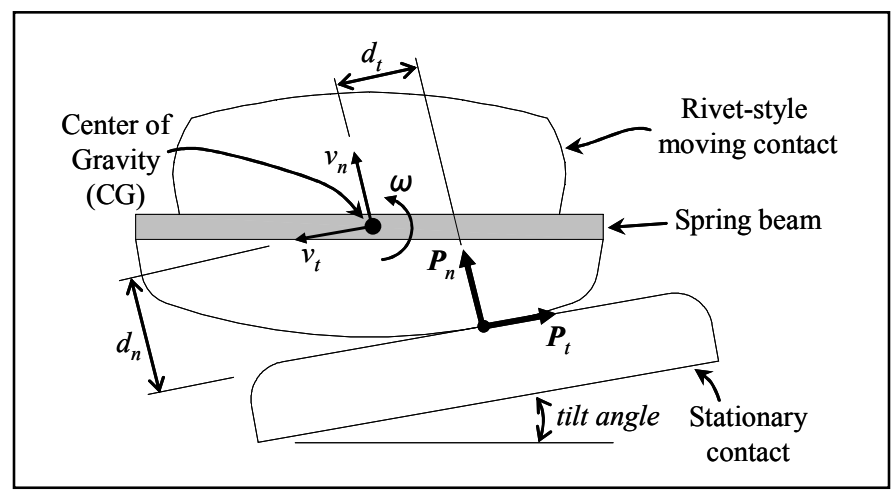

Figure 6. Tilted contact arrangement.

The solutions of the above equations in terms $v_{n}, v_{t}$, and $\omega$ at the end of the impact require the determination of the total impulse, which can be calculated by utilizing impact process diagrams. Depending upon the amount of friction, energy dissipation, which is accounted for through the utilization of the restitution coefficient, and tilt angle, several contact modes can occur, namely, sliding or rolling throughout the impact or only during each compression and restitution phases. Closedform expressions of these velocities were developed for each specific situation. However, due to the multitude of situations and the similarity of these expressions to those of the general case, which are available in [10], they will not be repeated here.

The impact equations were solved numerically for tilt angles from $0^{\circ}$, which is the standard contact arrangement, to the maximum of $30^{\circ}$. For illustration, the components of the CG velocity are plotted in Figure 7 versus the tilt angle for arbitrarily chosen restitution coefficient $e=0.3$ and friction coefficient $f=0.5$. Studying Figure 7, one can notice that $v_{n}$ crosses zero for a tilt angle of $17^{\circ}$ and becomes negative for larger angles meaning that that contact is given a downward motion immediately after the impact. That would suggest that, at least for a short time after the impact, the moving contact is either sliding or rolling instead of rebounding.

Table II lists the tilt angles that cause $v_{n}=0$ for other values of friction and restitution coefficients. Note that the chosen values of these parameters are purely hypothetical and are used for illustration purposes only, although some of them may be realistic. According to the results, it appears that there 
is a tilt angle that will maintain the contact for most of the combinations considered.

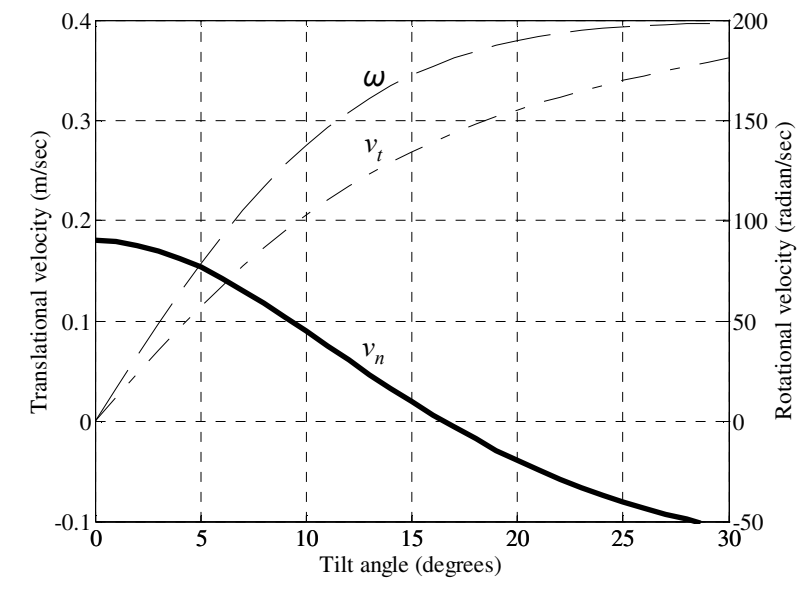

Figure 7. The components of CG velocity for various tilt angle for $e=0.3, f$ $=0.5$, and $v_{0}=0.6 \mathrm{~m} / \mathrm{sec}$.

After the impact, other factors come into play, such as the torsional resistance of the spring beam and the induced vibrations into the structure, that can limit the rolling/sliding motion of the contacts. Therefore, the predictions of this model should be treated as first order approximations of the effects of the contact tilt. The actual behavior of the contacts was investigated experimentally.
TABLE II. TILT ANGLES For ZERo ReBound CG VELOCITy

\begin{tabular}{|l|c|c|c|c|c|}
\hline & $f=0$ & $f=0.25$ & $f=0.5$ & $f=0.75$ & $f=1$ \\
\hline$e=0.7$ & 28 & 28 & & & \\
\hline$e=0.6$ & 23 & 24 & 28 & 28 & 28 \\
\hline$e=0.5$ & 19 & 21 & 24 & 24 & 24 \\
\hline$e=0.4$ & 16 & 18 & 20 & 20 & 20 \\
\hline$e=0.3$ & 13 & 15 & 17 & 17 & 17 \\
\hline$e=0.2$ & 10 & 13 & 13 & 13 & 13 \\
\hline$e=0.1$ & 7 & 9 & 9 & 9 & 9 \\
\hline
\end{tabular}

Several experiments were conducted to evaluate the influence of pitch and roll orientation of the stationary contact on the bounce behavior of the contacts (Figure 4 indicates the pitch and roll adjustments). Early investigations found that pitch tilt was less effective then the roll tilt and, thus, the pitch angle was set to $0^{\circ}$ for the rest of the experiments. Figure 8 shows the effect of increasing the roll tilt angle on the pattern of bounces. For $0^{\circ}$ tilt, only one bounce of about $0.35 \mathrm{msec}$ in duration occurs as shown in Figure 8(a). As the tilt angle increases, the duration of this bounce decreases, as shown in Figure 8(b). The one bounce is replaced by two short bounces for the tilt angle of $10^{\circ}$ in Figure 8(c), which become a single shorter bounce as the tilt angle is further increased, as in Figures 8(d) and (e).

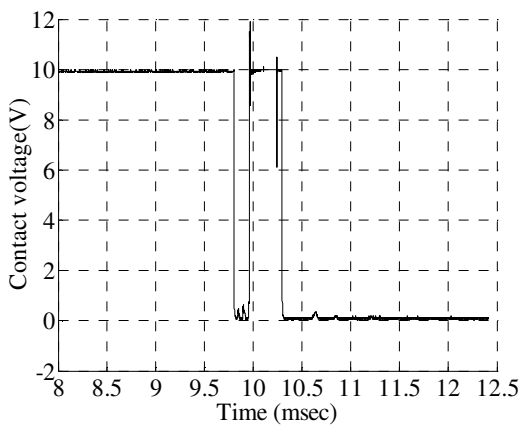

(a)

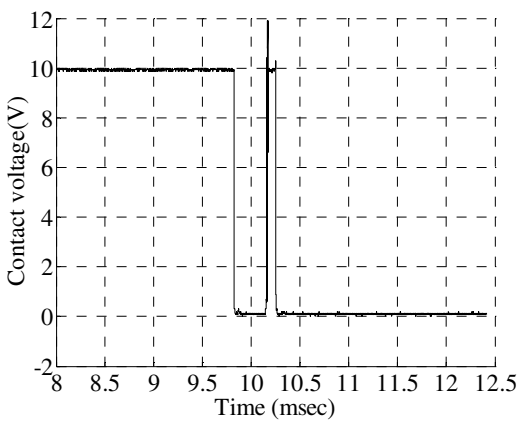

(d)

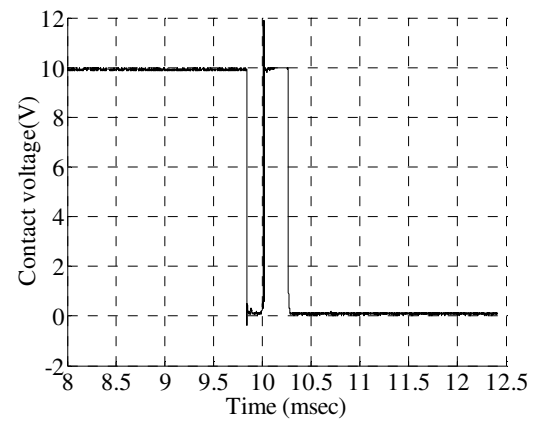

(b)

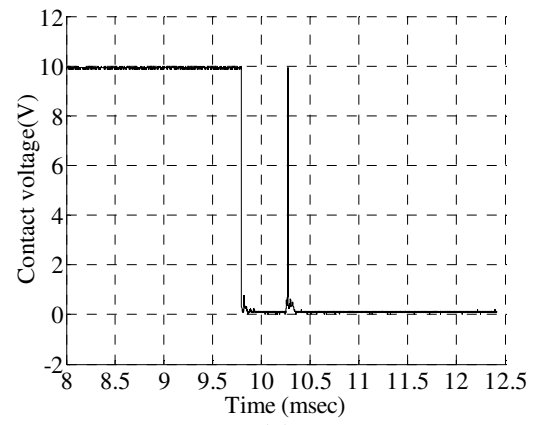

(e)

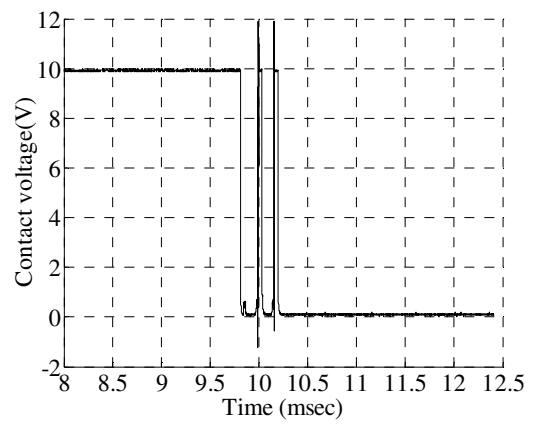

(c)

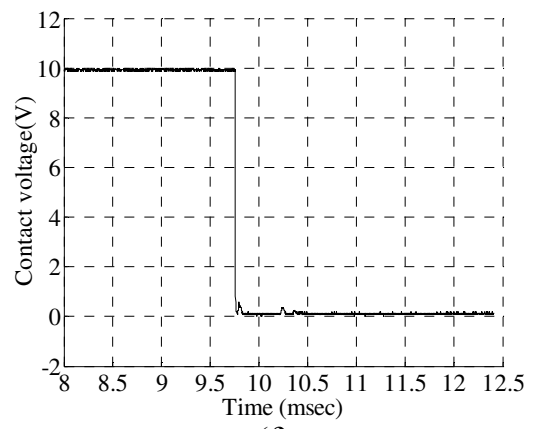

(f)

Figure 8. Contact voltage waveforms for $10 \mathrm{VDC}, 10 \mathrm{~A}$ showing the bounce patterns for various roll angles: (a) $0^{\circ}$ tilt; (b) $4^{\circ}$ tilt; (c) $10^{\circ}$ tilt; (d) $15^{\circ}$ tilt; (e) $17^{\circ}$ tilt; (f) $19^{\circ}$ tilt. 
Finally, the bounce is completely eliminated for the roll tilt angle of $19^{\circ}$ as shown in Figure 8(f). Therefore, increasing the roll angle of the stationary contact is accompanied by a gradual reduction and even the complete

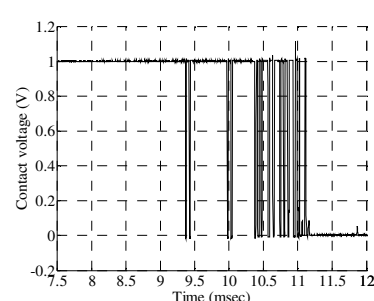

(a)

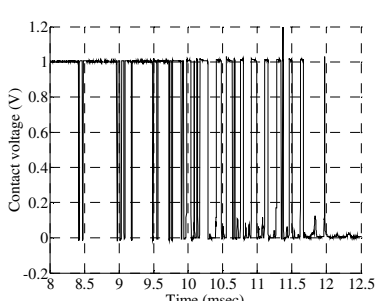

(b)

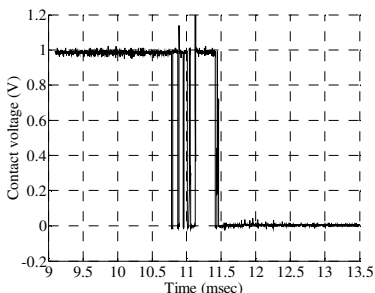

(c)

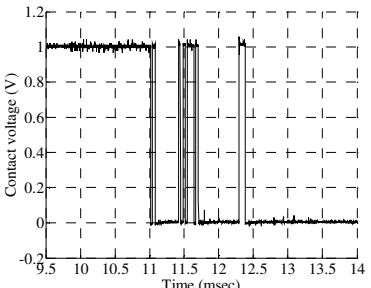

(d)

Figure 9. Contact bounce for 1VDC and 0.1A. (a) Standard relay "best" case; (b) Standard relay "worst" case"; (c) $19^{\circ}$ tilt contact arrangement "best" case; (d) $19^{\circ}$ tilt contact arrangement "worst" case.

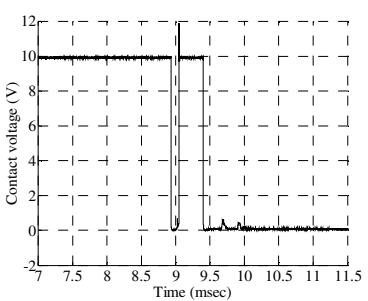

(a)

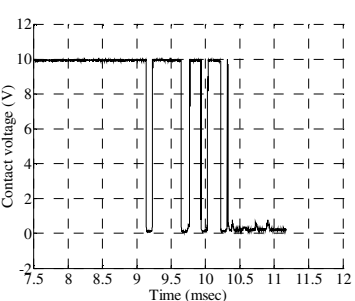

(b)

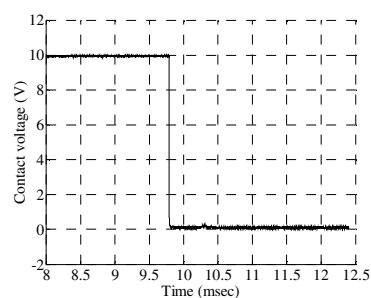

(c)

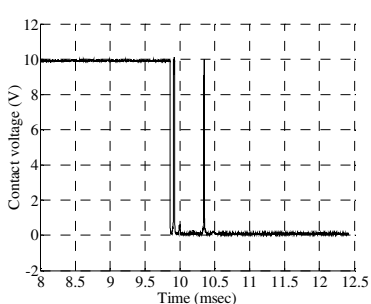

(d)

Figure 10. Contact bounce for 10VDC and 10A. (a) Standard relay "best" case; (b) Standard relay "worst" case"; (c) $19^{\circ}$ tilt contact arrangement "best" case; (d) $19^{\circ}$ tilt contact arrangement "worst" case.

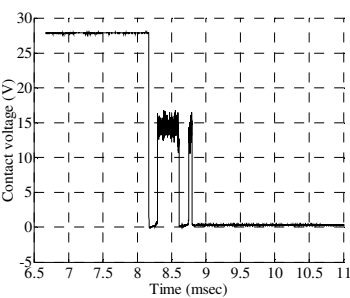

(a)

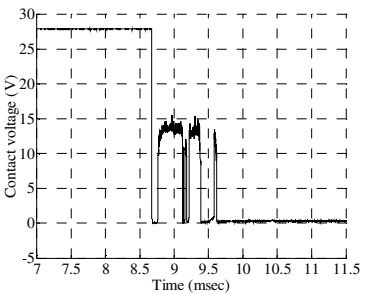

(b)

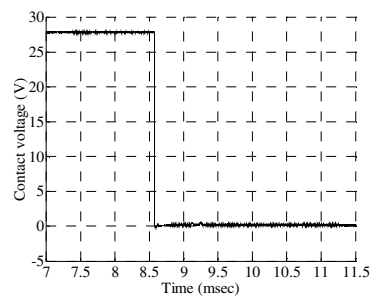

(c)

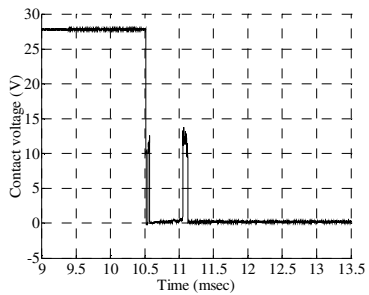

(d)

Figure 11. Contact bounce for 28VDC and 10A. (a) Standard relay "best" case; (b) Standard relay "worst" case"; (c) 19 tilt contact arrangement "best" case; (d) $19^{\circ}$ tilt contact arrangement "worst" case.

TABLE III. Improvements of The Proposed Contact Bounce Reduction Method.

\begin{tabular}{|c|c|c|c|c|c|c|c|c|c|c|c|c|}
\hline & \multicolumn{4}{|c|}{ 1VDC, 10A } & \multicolumn{4}{|c|}{ 10VDC, 10A } & \multicolumn{4}{|c|}{ 28VDC, 10A } \\
\hline & \multicolumn{2}{|c|}{ Number of Bounces } & \multicolumn{2}{|c|}{$\begin{array}{c}\text { Contact Separation } \\
\text { Time (msec) }\end{array}$} & \multicolumn{2}{|c|}{ Number of Bounces } & \multicolumn{2}{|c|}{$\begin{array}{c}\text { Contact Separation } \\
\text { Time (msec) }\end{array}$} & \multicolumn{2}{|c|}{ Number of Bounces } & \multicolumn{2}{|c|}{$\begin{array}{c}\text { Contact Separation } \\
\text { Time (msec) }\end{array}$} \\
\hline & Standard & $19^{\circ}$ tilt & Standard & $19^{\circ}$ tilt & Standard & $19^{\circ}$ tilt & Standard & $19^{\circ}$ tilt & Standard & $19^{\circ}$ tilt & Standard & $19^{\circ}$ tilt \\
\hline Average & 20.2 & 9.6 & 1.571 & 0.478 & 7.46 & 1.73 & 0.530 & 0.007 & 3.85 & 1.95 & 0.482 & 0.031 \\
\hline Min & 11 & 8 & 1.446 & 0.454 & 3 & 1 & 0.350 & 0.000 & 3 & 1 & 0.355 & 0.000 \\
\hline Max & 32 & 11 & 2.018 & 0.516 & 13 & 4 & 0.850 & 0.038 & 5 & 4 & 0.588 & 0.112 \\
\hline Range & 21 & 3 & 0.572 & 0.062 & 10 & 3 & 0.500 & 0.038 & 2 & 3 & 0.243 & 0.112 \\
\hline $\begin{array}{l}\text { Average } \\
\text { reduction }\end{array}$ & \multicolumn{2}{|c|}{$53 \%$} & \multicolumn{2}{|c|}{$70 \%$} & \multicolumn{2}{|c|}{$77 \%$} & \multicolumn{2}{|c|}{$99 \%$} & \multicolumn{2}{|c|}{$49 \%$} & \multicolumn{2}{|c|}{$94 \%$} \\
\hline
\end{tabular}


Several contact pairs with the stationary contact oriented at $19^{\circ}$ were tested at various energy levels. The behavior of the tilted contacts observed during the tests was compared to the behavior of the contacts from standard relays. The results are summarized in Figures 9-11. Both "best" and "worst" cases are provided to illustrate the extent of reduction that was accomplished.

Studying Figures 9-11, one can notice that the proposed method does significantly improve the bounce behavior of the contacts. Even the "worst" case of the tilt angle arrangement provides far better behavior compared to any standard relay. Table III lists specific improvements provided by the $19^{\circ}$ tilt angle fixture arrangement relative to standard relays. The calculations were based on 30 waveforms for each configuration. Note the substantial reduction in both the number of contact bounces and the total duration of contact separation time during bouncing, which should greatly reduce the arc energy and contact erosion.

\section{B. Cycle Testing}

A cycle test was conducted to estimate the reduction in the amount of contact wear that the bounce reduction concept can provide. A standard relay was also tested as a control sample. The condition of the contacts at the end of the test was compared visually. The load was resistive, the voltage was $10 \mathrm{VDC}$, and the current was 10A. The duration of the test was 24,000 make and break cycles. Both energy level and test duration were determined through preliminary investigations and determined that enough contact damage is generated without excessive burning. The total duration of each cycle was about 3.9 seconds out of which the contact close time was about 0.42 seconds. Contact polarity was chosen such that the stationary contact was the cathode and the moving contact was the anode. Electrical waveforms of the voltage across the closing contacts were collected at various stages of the test to monitor the consistency of closing behavior. Figures 12(a) and (c) show the contact voltage waveforms after 10,000 cycles for standard and fixture relays, respectively, and Figures 12(b) and (d) the contact voltage at the end of the test.

The bounce patterns of the fixture relay contacts for the two snapshots in Figures 12(c) and (d) are not significantly changed compared to the behavior at the beginning of the test, which is similar to the ones shown in Figures 10(c) and (d). Note the small disturbance in the voltage waveform around $11.25 \mathrm{msec}$ in Figure 12(d). It can be interpreted as a voltage drop along the molten bridge that is drawn between contacts. Occasionally, contact separation is large enough to break the bridge, as indicated by the larger spike in Figure 12(c). Another interpretation can be that the resolution of the oscilloscope, which was $1 \mu \mathrm{sec}$ in these tests, was not low enough to capture very short events. Additional investigation at higher time resolution indicated that both conditions randomly occurred.

Figure 13 shows the contacts after the completion of the 24,000 cycles. Studying the condition of the contacts, the following observations were made. First, the stationary contact, which was the cathode, gained material, and the moving contact, the anode, lost material. Second, the material transferred to the stationary contact of the fixture relay is more spread out compared to the standard relay and can be the result of the particular motions of the moving contact landing on a tilted surface. Finally, it appears that there is no substantial difference in the amount of material transferred between the two relay configurations in spite of the significantly reduced contact bounce achieved by the fixture relay. One explanation for the last finding could be that the arc stages that occur during the opening operation transferred considerably more material, thus masking any improvements that could actually have been accomplished through reduced closure bouncing.

A make-only cycle test was conducted to eliminate this possibility. The electronic switch circuit shown in Figure 5 was used to turn off the power applied to the load before the contacts break. The other general conditions employed in the previous test were the same. The condition of the stationary contacts of the standard and fixture relays at the end of the test is shown in two different views in Figures 14(a) and (b), and Figures 14(c) and (d), respectively. Note that the amounts of material buildup on the two contacts are marginally less than the amounts shown in Figures 13, thus suggesting that the bulk of material transfer occurred during contact make. Also, as for the first test, there was no significant improvement in the amount of buildup for the fixture arrangement compared to the standard relay.

Finally, another make-only cycle test was conducted for the rated $28 \mathrm{VDC}$ and 10A keeping the other test conditions unchanged. The contacts at the end of the test are shown in Figure 15.

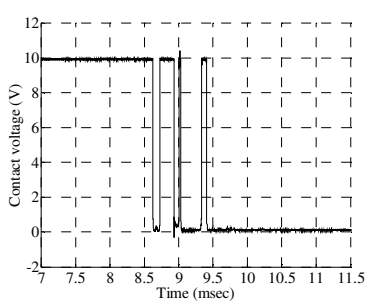

(a)

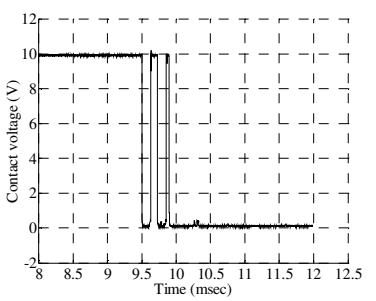

(b)

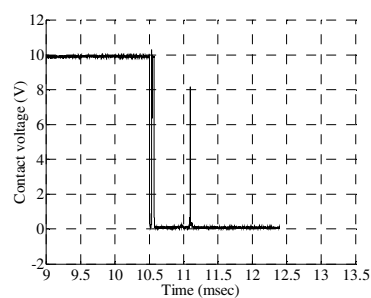

(c)

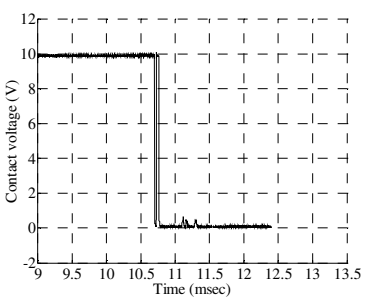

(d)

Figure 12. Contact voltage waveforms captured during the 24,000 cycle test, 10VDC, 10A. (a) Standard relay at 10,000 cycles; (b) Standard relay at 24,000 cycles; (c) $19^{\circ}$ tilt arrangement at 10,000 cycles; (d) $19^{\circ}$ tilt arrangement at 24,000 cycles. 


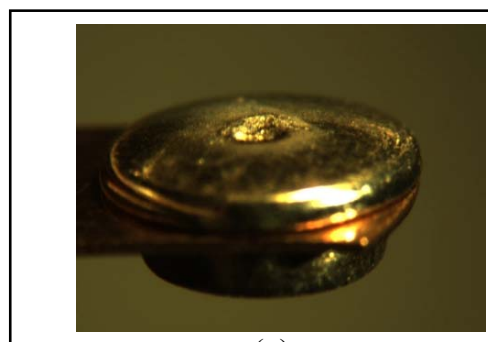

(a)

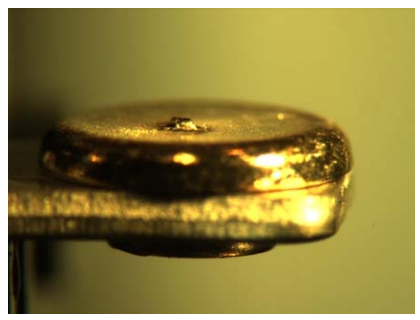

(b)

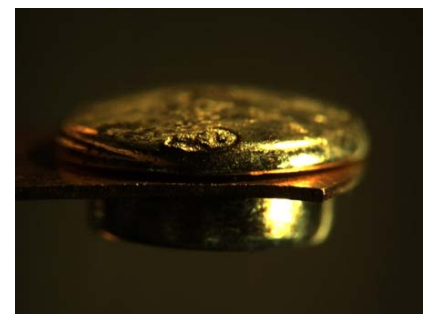

(c)

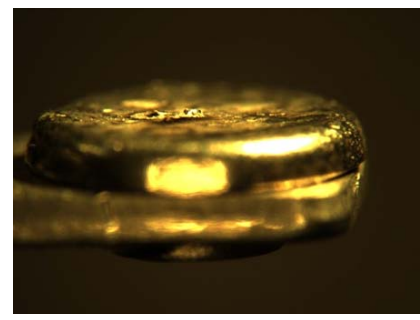

(d)

Figure 13. Contact condition at the end of the 10VDC, 10A make-and-break cycle test. (a) Standard moving contact; (b) Standard stationary contact; (c) $19^{\circ}$ tilt arrangement moving contact; (d) $19^{\circ}$ tilt arrangement stationary contact.

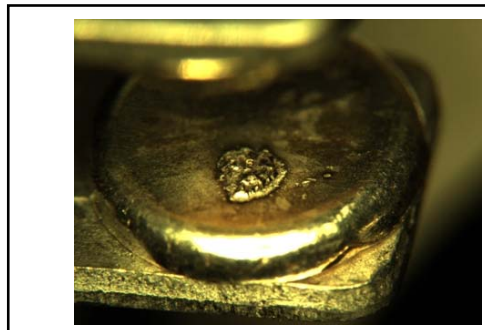

(a)

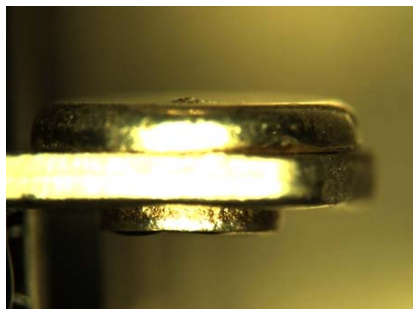

(b)

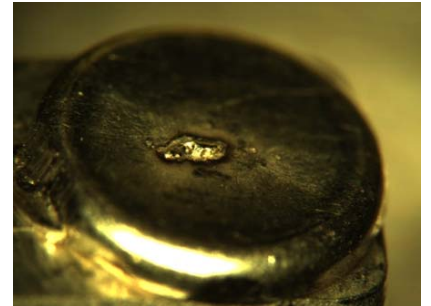

(c)

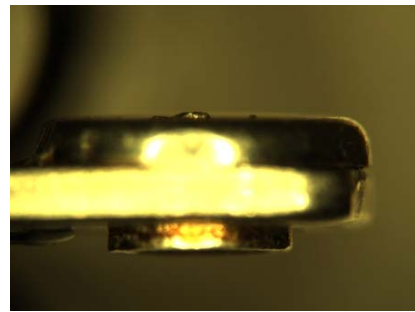

(d)

Figure 14. Stationary contact condition at the end of the 10VDC, 10A make-only cycle test. (a), (b) Standard relay; (c), (d) $19^{\circ}$ fixture arrangement.

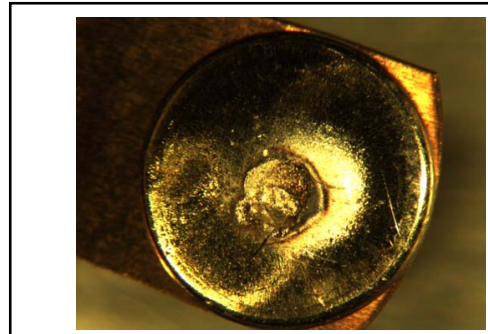

(a)

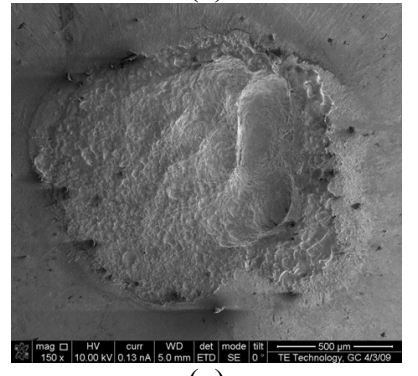

(e)

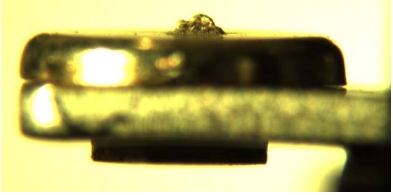

(b)

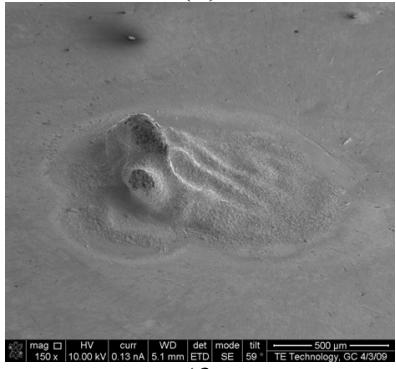

(f)

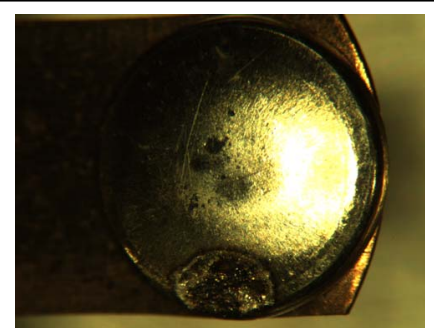

(c)

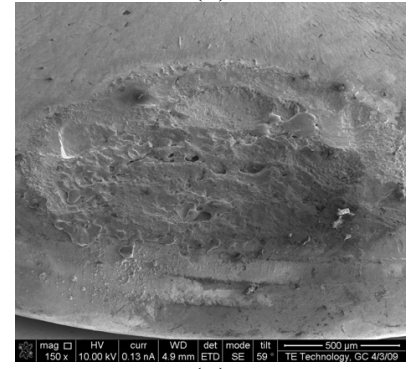

$(\mathrm{g})$

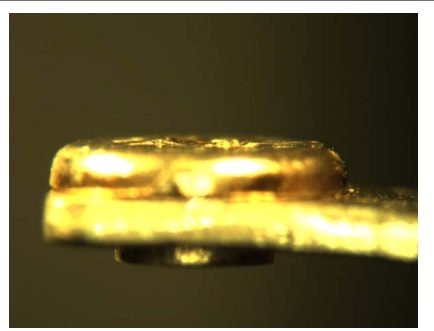

(d)

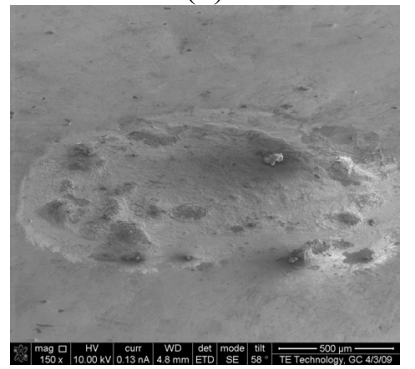

(h)

Figure 15. Contact condition at the end of the 28VDC, 10A make-only cycle test; (a),(e) Standard moving contact; (b),(f) Standard stationary contact; (c),(g) 19 ${ }^{\circ}$

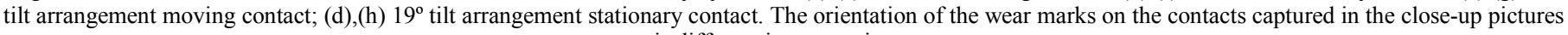
is different in some views.

Although it appears that the amount of material transfer onto the fixture stationary contact is significantly reduced, as shown in Figure 15(d), a careful examination of the moving contact condition shown in Figures 15(c) and (g) reveals that considerable damage has occurred. The fact that the material transfer on the stationary contact does not have the shape of a pip is again attributed to the pronounced wiping motion caused by the eccentric impact.

\section{DISCUSSION}

The findings of the preliminary cycle tests are somewhat surprising considering the significant improvement of key bounce parameters generated by the proposed method and indicated in Table III. These improvements were expected to produce a much more significant difference. This expectation was based on previous work that showed that the amount of contact erosion correlates with the arc energy 
dissipation [11]. Thus, it was reasonable to infer that reducing the number of contact bounces and the total duration of contact separation should reduce arcing time and the amount of damage to the contacts. However, cycle testing showed that, although the bounce was significantly reduced, the contact damage was only marginally improved.

Several potential explanations can be provided. First, it is known that several arc stages are developing as contacts separate during a bounce [12]. The material transfer is from the anode to the cathode in the beginning and at the end of the bounce when the contact gap is short and the arc is in the anodic stage [13]. If the bounce is long enough, the arc evolves into the cathodic stage and the direction of material transfer is progressively reversed resulting in cathode loss and anode gain. The transition from the anodic stage to the cathodic stage occurs when the arc reaches a critical length around $6 \mu \mathrm{m}$ [14], [15]. It is possible that the higher and longer bounces of the standard contact configuration are removing the additional material deposited in the more frequent, longer, and higher bouncing. The contacts would spend more time in the cathodic arc phase, which would not occur nearly as much with the substantially reduced bouncing of the improved configuration. Moreover, there is evidence that strong dynamic welds occur [16] if the bounce is short in amplitude $(<5 \mu \mathrm{m})$ and duration [17]. These high-strength welds are generated by the intense anode heating and rapid material transfer of the dominant anodic arcs. It may be that the shorter bounces of the improved configurations cause a large amount of material transfer due to the highly efficient anodic stage, which offsets the improvements of the reduced number of bounces.

Second, it may be that the material transfer is occurring during the molten bridge phase. That was thought to be the case up to about the mid 1940's, but has been accepted as not significant since the end of the 1950's, as mentioned in [12].

Finally, since the literature indicates that so many variables affect the material transfer process, it may be that these results are just a strange consequence of the specific conditions of this testing. Of the possible explanations, the first one seems most likely. More work is needed to understand what is really happening.

\section{CONCLUSIONS}

In this paper, a new and cost-effective means of reducing bounce has been presented. The configurations studied have shown significant achievements, especially for power applications. For the rated voltage and current and resistive loads, the improvements are:

- The number of bounces was reduced by $49 \%$.

- The bounce open time was reduced by $94 \%$.

Contrary to expectations, a significant improvement in material transfer was not realized. Several possible explanations have been proposed. However, more work is required to identify the reason for the unexpected results.

The advantages achievable for signal contacts with this means have not been investigated. The significant reduction in the number of transitions should certainly reduce the electrical noise generated by the electromechanical devices.

\section{ACKNOWLEDGMENT}

The authors would like to thank Steve Jarrett and Tvrtko Cizmek of Tyco Electronics Corporation for their continuous assistance that made this work possible.

\section{REFERENCES}

[1] J. W. McBride, "Low current switching," in Electrical Contacts: Principles and Applications, P. G. Slade, Ed. New York, NY: Marcel Dekker, 1999, pp. 573-624.

[2] L. Reiter, "Suppression of contact bounce by means of kinetic resonance absorption," in Proceedings of Holm Conference on Electrical Contacts, 1978, pp. 457-461.

[3] D. F. Hobart, "Defibrillator relay contact bounce reduction during low-energy defibrillation utilizing a kinetic energy absorbing polymer," in Proceedings of the $11^{\text {th }}$ Annual Northeast Bioengineering Conference, 1985, pp. 259-262.

[4] A. G. Espinosa, J. R. Riba, J. Cusido, J. A. Ortega, and L. Romeral, "Contact bounce elimination by means of a sensorless closed-loop current controller in dc core contactors," in Proceedings of the $54^{\text {th }}$ IEEE Holm Conference on Electrical Contacts, 2008, pp. 117-124.

[5] J. W. McBride, "Electrical contact bounce in medium-duty contacts," IEEE Transactions on Components, Hybrids, and Manufacturing, vol. 12, no. 1, pp. 82-90, March 1989.

[6] J. W. McBride, "An experimental investigation on contact bounce in medium duty contacts," IEEE Transactions on Components, Hybrids, and Manufacturing, vol. 14, no. 2, pp. 319-326, June 1991.

[7] J. W. McBride and S. M. Sharkh, "Electrical contact phenomena during impact," IEEE Transactions on Components, Hybrids, and Manufacturing, vol. 15, no. 2, pp. 184-192, April 1992.

[8] P. Barkan, "A study of the contact bounce phenomenon," IEEE Transactions on Power Apparatus and Systems, vol. PAS-86, no. 2, pp. 231-240, February 1967

[9] W. Goldsmith, Impact: The Theory and Physical Behavior of Colliding Solids, Edward Arnold Publishers Ltd., 1960

[10] Yu Wang and M. T. Mason, "Two-dimensional rigid-body collisions with friction," Journal of Applied Mechanics, vol. 59, pp. 635-642, September 1992.

[11] N. Ben Jamaa, "Experimental and theoretical investigations on break arc voltage, duration, energy, and erosion at low electrical level," in Proceedings of the $13^{\text {th }}$ International Conference on Electric Contacts, 1996, pp. 64-68.

[12] W. F. Rieder, "Low current arc modes of short length and time: a review," IEEE Transactions on Components and Packaging Technologies, vol. 23, no. 2, pp. 286-292, June 2000.

[13] L. Morin, N. Ben Jamaa, and D. Jeannot, "Make arc erosion and welding in the automotive area," IEEE Transactions on Components and Packaging Technologies, vol. 23, no. 1, pp. 240-246, March 2000.

[14] N. Ben Jamma, L. Morin, S. Benhenda, and L. Nedelec, "Anodic to cathodic arc transition according to break arc lengthening," IEEE Transactions on Components, Packaging, and Manufacturing Technology - Part A, vol. 21, no. 4, December 1998.

[15] N. Ben Jamaa and L. Morin, "Transition from the anodic arc phase to the cathodic metallic arc phase in vacuum at low DC electrical level," IEEE Transactions on Component and Packaging Technologies, vol. 25, no. 4, pp. 651-655, December 2002

[16] Z. Chen and G. Witter, "Dynamic welding of silver contacts under different mechanical bounce conditions," in Proceedings of the $19^{\text {th }}$ International Conference on Electrical Contacts, 1998, pp. 355-362.

[17] W. F. Rieder, "Short arc modes determining both contact welding and material transfer," IEEE Transactions on Components and Packaging Technologies, vol. 30, no. 1, March 2007. 


\section{A simple arc model for the simulation of the clearing time of drawn arcs with a commercial electronics simulation tool}

\author{
Prof (FH) Dr. Peter Zeller \\ University of Applied Sciences Upper Austria \\ Wels, Austria \\ peter.zeller@fh-wels.at
}

\begin{abstract}
If effects of DC interruption with electromechanical switches onto electrical circuits should be simulated a proper arc model has to be implemented into the simulation tool. Very complex models were introduced by various authors before. The lack of these models is the high computational effort required. Based on material properties and the most important governing effects this paper presents a way to find a very simple, proper arc model which can be implemented into any simulation tool. The arc was assumed as a straight cylindrically shaped column. With such an arc model an arc voltage gradient in good correlation to measured and published data was simulated. However the arc temperature computed by the model is too high. This could be caused by the very simple heat transfer model. Next steps should be the implementation of a more complex heat transfer model.
\end{abstract}

Keywords-Arc model, electronic circuit simulation, arc voltage gradient, arc structure.

\section{INTRODUCTION}

Under the aspect of utilization of renewable energy DC switching gains more attention as in the past. Typically photovoltaic (PV) module units are operated in a voltage range in between $50 \mathrm{~V}$ to $1000 \mathrm{~V}$ and a nominal current range in between $2 \mathrm{~A}$ to $20 \mathrm{~A}$. At battery supplied systems in electrical cars and off grid systems the typical voltage range is in between $12 \mathrm{~V}$ up to some $100 \mathrm{~V}$ and some Ampere up to some $100 \mathrm{~A}$. In all these systems loads and faults must be interrupted safely.

Since in PV systems a lot of electronics is applied (e.g. inverter systems for converting the DC-power into AC-power) the transient reaction onto a DC sub grid due to the switching operation as well as the interruption time of any fault is of highly interest for the design of the system. Simulation of switching operations helps to optimize the DC grid design. Any simulation of a switching operation requires a proper arc model which can be implemented into electronic circuit simulation software (ECSS).

It is the aim of this paper to introduce such a simple arc model for system simulation purpose. There are many arc models introduced in the past. Most of them are very complex and consider most of the governing physical effects in a very detailed way $[1,2]$. However the disadvantage of such models is the need of extremely high computational effort (numeric, finite element simulation). The simulation time for such models is inacceptable high for any implementation into ECSS what induced the author to develop a less complex model.

\section{THEORETICAL CONSIDERATIONS, ASSUMPTIONS}

\section{A. Electrical Circuit}

The equivalent circuit of considered DC circuits is given in Figure 1. A constant voltage source $U_{0}$ drives the current $i_{A}(t)$. The load and the line may be assumed as an ohmic/inductive load $(R$ and $L)$. The circuit is interrupted by the switch $S$. An arc will be drawn if the contacts of the switch are mechanically separated and a significant voltage $u_{A}(t)$ over the switch will be measured.

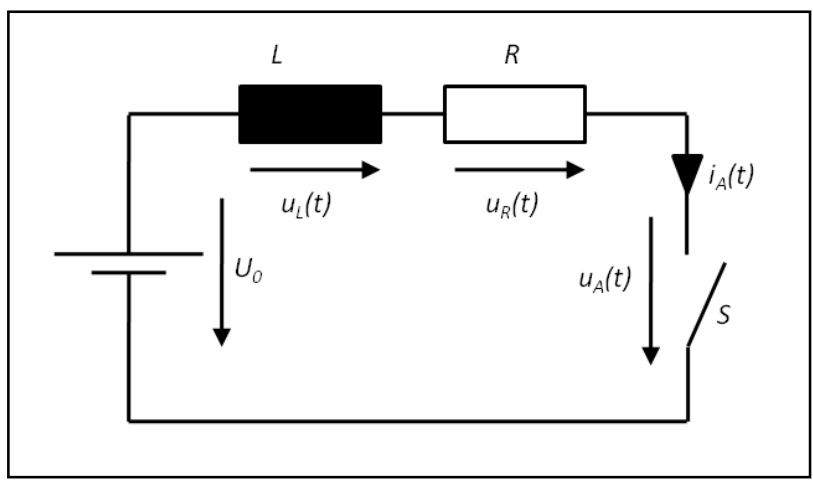

Figure 1. Equivalent DC circuit for simulation of switching operation.

The current may be computed if the differential equation (1) can be solved.

$$
U_{0}=L \dot{i}_{A}(t)+R i_{A}(t)+u_{A}\left(i_{A}(t)\right)
$$

The differential equation (1) can be solved by ECSS numerically if the voltage $u_{A}(t)$ is known. The voltage $u_{A}(t)$ is strongly influenced by the arc inside the switch. Hence a model which describes the arc voltage as a function of the arc current, by considering the changing boundary conditions in the switch 
has to be implemented. One significant boundary condition in the switch is the changing electrode distance.

\section{B. Electromechanical Switch}

The electromechanical switch is considered as two mechanically contacts which will be separated. The contact gap is a well known function of time. Simple relay switches should be considered. Consequently the arc is assumed, as

- not significantly interacting with the wall material,

- not magnetically blast and

- cylindrically shaped.

The missing function of the switching voltage is mainly influenced by

- cathode and anode drop,

- heat transfer effects,

- electrical and thermal properties of plasma,

- and gas flow effects.

The resistance and the inductivity of the current carrying parts inside the switch were neglected. If any significant resistance or inductivity should be considered it can be done by increasing the ohmic resistance $R$ and the inductivity $L$ in (1).

As a first approach to modeling only "short arcs" were considered and the effects of gas flow and arc motion were neglected. Also the sum of the anode and cathode drop was assumed as a constant voltage independent from current and time.

\section{Arc Model}

As described in section B the arc was assumed as cylindrically shaped with a length $l$ and a radius $r$ at any given time (see Figure 2).

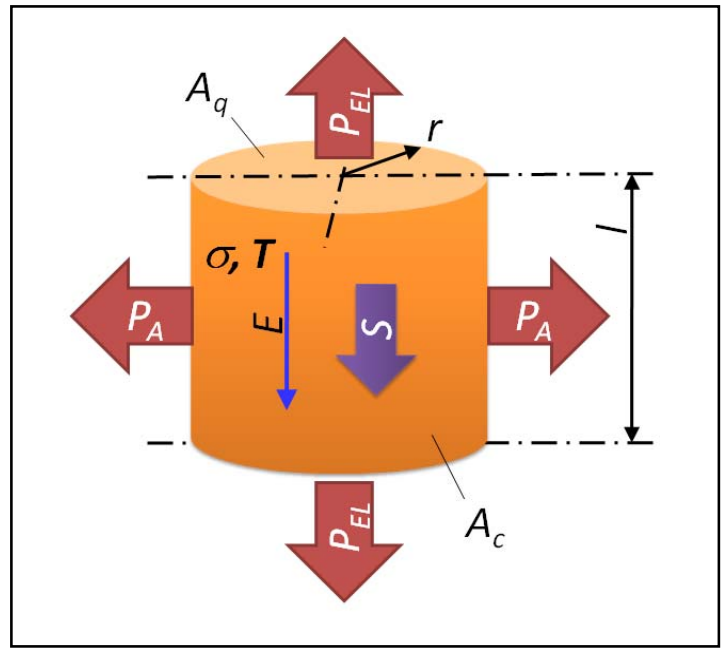

Figure 2. Arc model of the cylindrically shaped arc

The arc is characterized by it's temperature $T$ and the electrical conductivity $\sigma$. With the current density $S$ the electrical field strength $E$ inside the arc can be described by (2).

$$
E=\frac{S}{\sigma(T)}
$$

The arc voltage $u_{A}(t)$ can be computed by (3) where $U_{A K}$ represents the sum of the cathode and anode drop.

$$
u_{A}(t)=E(t) l+U_{A K}
$$

Heat is transferred to the electrodes $\left(P_{E L}\right)$ via the surfaces $A_{q}$ and to the ambient air $\left(P_{A}\right)$ via the $\operatorname{arc}$ surfaces $A_{c}$.

The specific electrical power losses (Joules losses; (per arc volume) are given by (4). In a thermal balanced state (no changing of the arc temperature) the Joules losses must be equal to the losses transferred via heat to the ambient air and electrodes according to (5) if heating and cooling of material is neglected. In (5) $V$ is the total arc volume.

$$
p_{V}=S E=\sigma(T) E^{2}=\frac{S^{2}}{\sigma(T)}
$$

The heat transfer losses are assumed in a first and simple approach by a linear term. The heat transfer via radiation is neglected.

$$
P_{A}+P_{E L}=\int_{V} p_{V} d V=\int_{V} \sigma E^{2} d V
$$

If there is any significant heat transfer via radiation, it has to be considered by the linear approach too. This will have two consequences:

- The qualitatively behavior of the model is only valid for a narrow arc temperature range which is valid if the arc temperature will not change significantly during the arc interruption.

- The coefficients for the linear approach will be higher than the coefficients for the heat transfer caused by both, conduction and convection, consequently the coefficients of the model may not be directly compared to coefficients yield by considering free convection and heat conduction.

Based on the assumptions above, the thermal heat transfer is described by (6) for the area $A_{c}$ and (7) for the two areas $A_{q}$, with the heat transfer coefficients $\alpha_{E L}$ considering axial heat transfer and $\alpha_{A}$ considering lateral heat transfer. The temperature $T_{0}$ was set to $293,15 \mathrm{~K}$ (room temperature).

$$
\begin{gathered}
P_{A}=A_{c} \alpha_{A}\left(T-T_{0}\right) \\
P_{E L}=2 A_{q} \alpha_{E L}\left(T-T_{0}\right)
\end{gathered}
$$


It has to be emphasized that the column constriction nearby the electrodes was neglected. Any consideration of this area would require a more dimensional model and more computational effort.

The order of the heat transfer coefficients may be estimated from literature data. [3] published stationary arc characteristics for different arc current values and electrode distances. [4] introduced the energy absorbed by the anode and cathode electrodes for different current values and electrode distances. Assuming an arc with a length of $1 \mathrm{~cm}$, from [4] the energy input into the electrodes may be yield according to Table I. For the further considerations the sum of the cathode and anode drop was assumed as $15 \mathrm{~V}$. The power losses caused by this voltage drop can be computed by multiplication of this characteristic voltage with the current. Subtracting this value from the total power losses into the electrodes, the power which is transferred from, the column into the electrodes can be computed according to [4]. Assuming that the constricted area of the electrodes is in the range of $0.5 \mathrm{~mm}$ and an average temperature of $10000 \mathrm{~K}$ the heat transfer coefficient can be calculated applying formula (7). By applying (6) and similar considerations the order of the heat transfer coefficient from the arc column to the ambient air can be estimated. Considering the data of [3], for an arc column of $1 \mathrm{~cm}$ length, a sum of anode and cathode of $15 \mathrm{~V}$, an arc diameter as $2 \mathrm{~mm}$ and an arc temperature of $10000 \mathrm{~K}$ the order of the heat transfer coefficient will result according to Table II.

TABLE I. EVAluation Of THE HEAT TRANSFER COEFFICIENT FOR THE HEAT TRANSFER INTO THE ELECTRODES BASED ON DATA FROM [4].

\begin{tabular}{|c|c|c|c|c|c|}
\hline \multirow[t]{2}{*}{$\begin{array}{c}\text { Arc } \\
\text { Current } \\
{[\mathrm{A}]}\end{array}$} & \multicolumn{2}{|c|}{$\begin{array}{c}\text { Transferred } \\
\text { Power } \\
\text { [W] }\end{array}$} & \multirow{2}{*}{$\begin{array}{c}\text { Power } \\
\text { caused by } \\
\text { anode } \\
\text { and } \\
\text { cathode } \\
\text { drop [W] }\end{array}$} & \multirow{2}{*}{$\begin{array}{c}\text { Power } \\
\text { transferred } \\
\text { from the } \\
\text { column into } \\
\text { the } \\
\text { electrodes } \\
\text { [W] }\end{array}$} & \multirow{2}{*}{$\begin{array}{c}\text { Heat } \\
\text { transfer } \\
\text { coefficient } \\
{\left[\mathbf{W} / \mathbf{m}^{2} \mathbf{K}\right]}\end{array}$} \\
\hline & $\begin{array}{c}\text { Cathode } \\
{[4]}\end{array}$ & $\begin{array}{c}\text { Anode } \\
{[4]}\end{array}$ & & & \\
\hline 10 & 194 & 255 & 150 & 300 & $47.110^{3}$ \\
\hline 20 & 340 & 441 & 300 & 482 & $75.710^{3}$ \\
\hline 30 & 479 & 620 & 450 & 650 & $10210^{3}$ \\
\hline
\end{tabular}

In order to find the arc voltage caused by a given operation point of the arc defined by the arc current, the balance (5) has to be fulfilled.

TABLE II. EVAluation OF THE HEAT TRANSFER COEFFICIENT FOR THE HEAT TRANSFER INTO THE AMBIENT AIR BASED ON DATA FROM [3].

\begin{tabular}{|c|c|c|c|c|}
\hline \multirow[t]{2}{*}{$\begin{array}{c}\text { Arc } \\
\text { Current } \\
{[\mathrm{A}]}\end{array}$} & \multicolumn{2}{|c|}{$\begin{array}{c}\text { Arc Voltage } \\
\text { according to [3] } \\
{[\mathrm{V}]}\end{array}$} & \multirow{2}{*}{$\begin{array}{l}\text { Power of } \\
\text { the arc } \\
\text { column } \\
{[W]}\end{array}$} & \multirow{2}{*}{$\begin{array}{c}\text { Heat } \\
\text { transfer } \\
\text { coefficient } \\
{\left[\mathbf{W} / \mathbf{m}^{2} \mathbf{K}\right]}\end{array}$} \\
\hline & $\begin{array}{c}\text { Total arc } \\
\text { voltage }\end{array}$ & $\begin{array}{l}\text { Column } \\
\text { arc } \\
\text { voltage }\end{array}$ & & \\
\hline 10 & 54.5 & 39.5 & 395 & 314 \\
\hline 20 & 46.4 & 31.4 & 627 & 499 \\
\hline 30 & 43.6 & 28.6 & 859 & 683 \\
\hline
\end{tabular}

Based on published conductivity data [5] and the data listed in Table II and Table III the heat balance can be found for any arc current for a specific temperature and arc radius.

Figure 3 shows the computation result for the variation of the temperature of an arc with constant boundary conditions according to Table III. The upper plot shows the electrical conductivity as a function of the temperature (data from [5]), the lower plot the electrically and thermally power losses. The electrically power losses decrease with increasing temperature. The thermal power losses increase with rising temperature. At the intersection of the power loss plots, the thermal equilibrium condition (5) is fulfilled. In case of Figure 3 the intersection is at a power of $408 \mathrm{~W}$ and a temperature of $6500 \mathrm{~K}$. Changing boundary conditions will result in a different equilibrium. In case of a larger arc radius, the electrical power losses will drop (since the current density drops) and the thermal losses will rise (because of the increased arc surface).

TABLE III. PARAMETER For THE SimUlation OF THE EQUATIONS (5) - (7)

\begin{tabular}{|l|c|c|c|}
\hline \multicolumn{1}{|c|}{ Parameter } & Symbol & Value & Unit \\
\hline Arc length & $L$ & $1 / 10$ & $\mathrm{~cm}$ \\
\hline $\begin{array}{l}\text { Heat transfer coefficient } \\
\text { into the electrodes }\end{array}$ & $\alpha_{E L}$ & $10010^{3}$ & $\mathrm{~W} / \mathrm{m}^{2} \mathrm{~K}$ \\
\hline $\begin{array}{l}\text { Heat transfer coefficient } \\
\text { into the ambient air }\end{array}$ & $\alpha_{A}$ & 200 & $\mathrm{~W} / \mathrm{m}^{2} \mathrm{~K}$ \\
\hline Arc current & $i_{A}$ & $10 / 20 / 30$ & $\mathrm{~A}$ \\
\hline
\end{tabular}

This will result in a lower arc temperature as indicated qualitatively by the second intersection in Figure 3. This is in agreement with published effects of wall stabilized arcs [6].
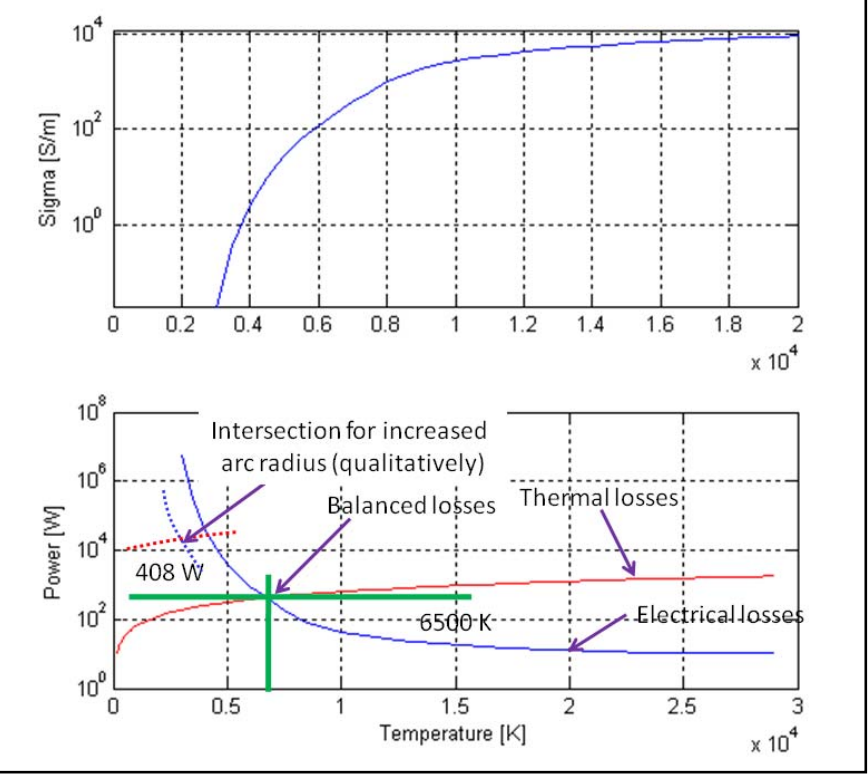

Figure 3. Electrical conductivity [5] (upper plot) and computed electrical and thermal power losses for an cylindrically shaped arc of $5 \mathrm{~mm}$ radius, $10 \mathrm{~mm}$ length and $30 \mathrm{~A}$.

Based on the results above for a given arc radius and arc current one point of thermal equilibrium exists which is 
defined by a specific temperature and a specific field strength of the arc column. A Change of the arc boundary conditions (e.g. geometry, arc current, heat transfer coefficients) will cause a change of the arc temperature and arc voltage gradient acc. to the equations (2) to (7). In Figure 4 all points of thermal equilibrium are plotted as the electrically field strength versus the arc temperature. It can be seen, that a certain minimum for the arc voltage gradient can be observed (in case of Figure 4 $35.6 \mathrm{~V} / \mathrm{cm}$ and $20660 \mathrm{~K}$ ). According to [6] the arc follows the principle of a minimum of energy which means the arc voltage gradient is set at a value where the change of the arc voltage gradient with the temperature is a minimum. In case of Figure 4 this is the highlighted minimum point. Hence the thermal equilibrium in combination with the minimum principle of [6] leads to the arc temperature and arc voltage gradient based on material properties only.

Table IV shows the computed electrical field strength compared to results derived from data in [3]. The maximum deviation from the arc voltage gradient values derived from [3] is $24 \%$. Considering the simplicity of the model introduced above, and the wide range of scatter of the real boundary conditions of a free burning arc (e.g. caused by unknown exact arc geometry due to eroded electrodes or bent arc due to electromagnetically force and gas flow effects) the arc models accuracy is sufficient enough.

For the implementation of this arc model into an ECSS the ECSS must be open for any implementation of extra source, since the model introduced above cannot be transformed into a simple, closed analytically formulation. Another aspect is the computational effort required for finding the thermal equilibrium iteratively.

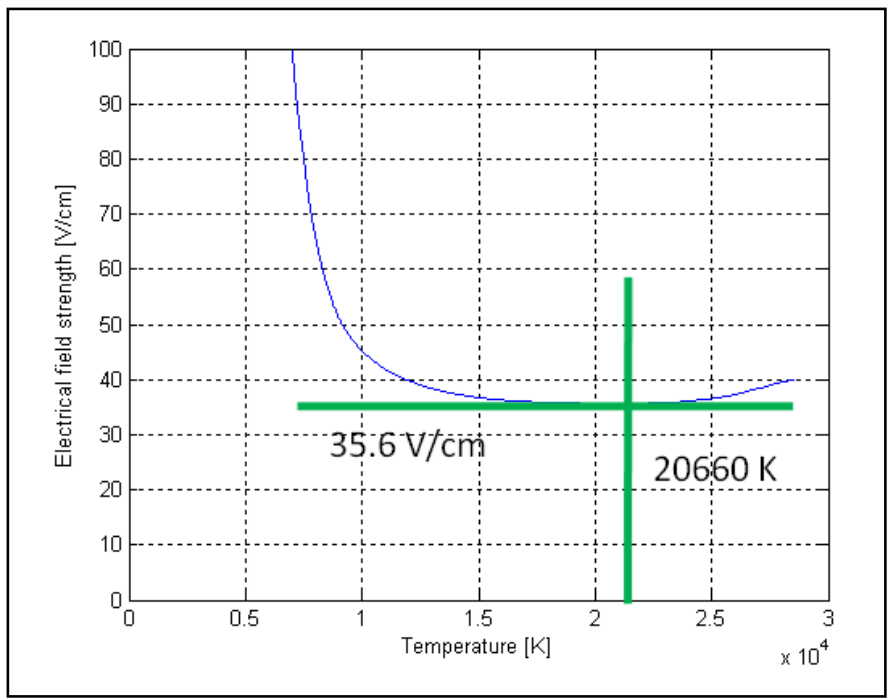

Figure 4. Points of thermal equilibrium for a $1 \mathrm{~cm}, 30 \mathrm{~A}$ arc plottet as the arc voltage gradient versus the arc temperature.

This simulation effort (simulation time) has to be invested for every time step and for every iteration step within this time step which will be done by the ECSS. Consequently the solver for the arc model may cause a huge extra computational effort. In order to reduce this effort, the thermal equilibrium can be solved in a wide current range and an empirical description for the simulation results derived. In such an approach the simulation effort for the arc model has to be invested only once for one operation point of the arc.

TABLE IV. SIMULATION RESUltS FOR A SET OF BOUNDARY CONDITIONS COMPARED TO DATA DERIVED FROM [3]

\begin{tabular}{|c|c|c|c|c|}
\hline $\begin{array}{c}\text { Arc } \\
\text { length } \\
\boldsymbol{l}[\mathbf{c m}]\end{array}$ & $\begin{array}{c}\text { Arc } \\
\text { Current } \\
\boldsymbol{i}_{\boldsymbol{A}}[\mathbf{A}]\end{array}$ & $\begin{array}{c}\text { Computed } \\
\text { Arc Voltage } \\
\text { Gradient } \boldsymbol{E} \\
{[\mathbf{V} / \mathbf{c m}]}\end{array}$ & $\begin{array}{c}\text { Arc Voltage } \\
\text { Gradient } \boldsymbol{E} \\
\text { derived from [3] } \\
\text { [V/cm] }\end{array}$ & Deviation \\
\hline 10 & 10 & 17,2 & 17.6 & $2 \%$ \\
\hline 10 & 20 & 15,4 & 13.7 & $-12 \%$ \\
\hline 10 & 30 & 14.6 & 11.9 & $-23 \%$ \\
\hline 1 & 10 & 37.4 & 39.5 & $6 \%$ \\
\hline 1 & 20 & 36.2 & 31.6 & $-15 \%$ \\
\hline 1 & 30 & 35.6 & 28.6 & $-24 \%$ \\
\hline
\end{tabular}

As an example the inverse influence of the arc length and the arc current onto the arc voltage gradient (the arc voltage gradient increases with decreasing current level and decreasing arc length) can be assumed as linearly. Applying equation (8) the length specific power losses of the arc column are inverse to the arc current and the power loses into the electrodes are constant. The empiric factors $K_{P C}$ considers the power losses of the arc column per length and $K_{P E L}$ the power losses into the electrodes. The values $I_{0}$ and $l_{0}$ are additional fitting parameter and necessary to avoid any infinitive results if the arc current and/or the electrode distance are zero. Since the power losses into the electrodes are assumed as constant and the electrical field strength is a length specific value, the losses into the electrodes have to be divided by the arc length.

$$
E=\frac{K_{P_{C}}}{i_{A}(t)-I_{0}}+\frac{K_{P_{E L}}}{l(t)-l_{0}}
$$

The arc model and the empiric Formula 8 introduced in this chapter are based on a thermal equilibrium neglecting heating and cooling of mass of the drawn arc in contrast to the heat balance introduced in [6] and by conclusions based on experimental results introduced in [7]. In order to find out whether the neglecting of this effect is valid experiments where performed.

\section{EXPERIMENTAL EQUIPMENT}

The experimental equipment consists of a model switch, a high speed camera and a power supply.

\section{A. Modelswitch and Optical Equipment}

The test equipment hardware is shown in Figure 5. It was equipped with a mass which was dropped down from a certain distance $d$. According to Newtons Law the velocity of the mass and consequently the opening speed of the moving contact depend on the distance $d$. After the mass hit the moving contact both where decelerated due to the compression of a spring. The 
length of the spring was adjusted in a way that a certain constant velocity was kept in the observation time interval.

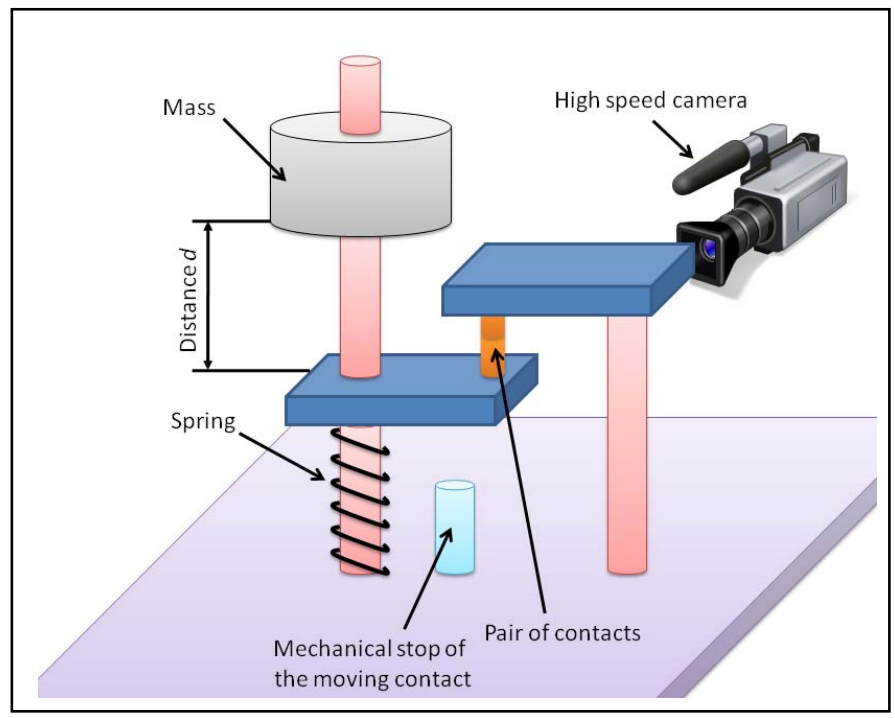

Figure 5. Mechanical equipment of the experiment.

A high speed camera was applied in order to record a high speed movie from the contact motion, the arc lenghtening and arc structure with a frame rate of 10000 frames per second.

\section{B. Electrical Equipment}

The electrical circuit was equivalent to the circuit introduced in Figure 1. The inductivity was set to $0 \mathrm{mH}$ and $53 \mathrm{mH}$. The resistance and the voltage $U_{0}$ where adjusted in a way that at closed contacts an arc current of $10 \mathrm{~A}, 20 \mathrm{~A}$ and 30 A was measured.

The voltage across the switch and the arc current where recorded with a digital oscilloscope and a sampling frequency of $100 \mathrm{kHz}$.

\section{Experimental Parameter}

The experimental parameters are summarized in Table V.

TABLE V. EXPERIMENTAL PARAMETERS

\begin{tabular}{|c|c|c|c|}
\hline $\begin{array}{c}\text { Arc } \\
\text { Current } \\
{[\mathbf{A}]}\end{array}$ & $\begin{array}{c}\text { Inductivity } \\
{[\mathbf{m H}]}\end{array}$ & $\begin{array}{c}\text { Falling } \\
\text { distance of } \\
\text { the mass } \\
{[\mathbf{c m}]}\end{array}$ & $\begin{array}{c}\text { Theoretical impact } \\
\text { velocity (computed, } \\
\text { acc. to Newtons Law) } \\
{[\mathbf{m} / \mathbf{s}]}\end{array}$ \\
\hline 10 & 0 & & \\
\hline 20 & 0 & \multirow{2}{*}{$1,2,5,10,20$} & $\begin{array}{c}0 \\
0.44,0.63,0.99,1.4 \\
1.98\end{array}$ \\
\cline { 1 - 2 } 30 & 0 & \\
\hline 10 & 53 & & \\
\hline 20 & 53 & & \\
\hline 30 & 53 & & \\
\hline
\end{tabular}

For each parameter combination one single experiment was carried out. The electrical contacts where made of brass screws with a metrical thread of $4 \mathrm{~mm}$.

\section{EXPERIMENTAL RESULTS}

\section{A. High Speed Films}

Figure 6 shows the high speed films from experiments with an ohmic circuit (inductivity is $0 \mathrm{mH}$ ), $5 \mathrm{~cm}$ falling distance and current levels of $10 \mathrm{~A}, 20 \mathrm{~A}$ and $30 \mathrm{~A}$.

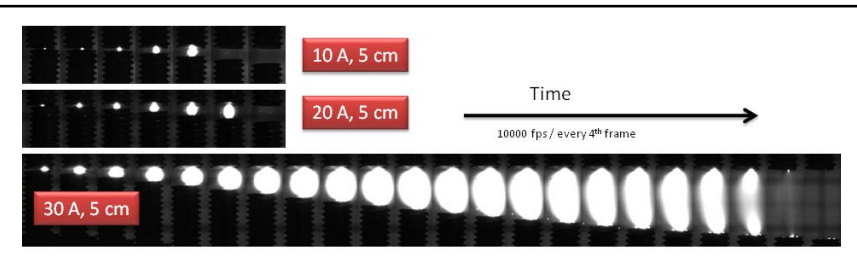

Figure 6. Influence of the arc current onto the arc structure.

According to Figure 3 the high speed films indicate an increasing arc width with increasing arc current and increasing arc length. The arc was cylindrically shaped, which is in good correlation to the assumptions on which the arc model is based on.

The influence of the variation of the falling distance is indicated by Figure 7 (no inductivity in the electrical circuit). The arc structure and the clearing distance of the arc where not influenced by the opening speed significantly. In Figure 8 the effect of an inductive load $(53 \mathrm{mH})$ is shown. It has to be emphasized, that only every $20^{\text {th }}$ frame is displayed in contrast to Figure 6 and Figure 7 where every $4^{\text {th }}$ frame is displayed. As indicated by Figure 8 the presence of the inductivity in the electrical network elongated the arc extinction time and distance which was already described by [7]. The reason for the extension of the clearing time is the magnetically energy stored in the inductivity [7].

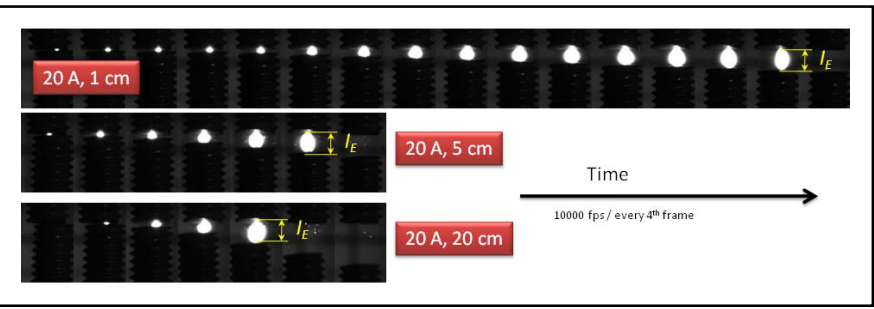

Figure 7. Influence of the opening speed (falling distance $d$ of the impact mass) onto the arc structure and clearing distance.

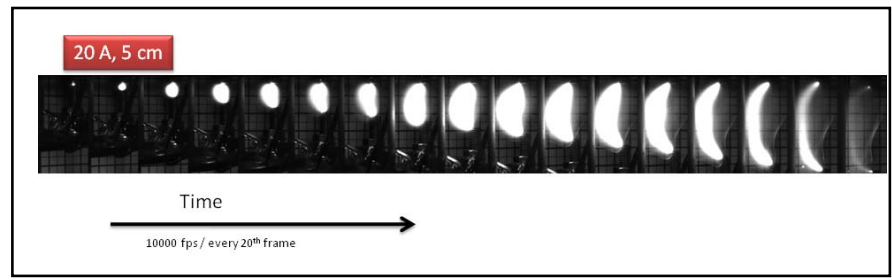

Figure 8. Extinction of the electrical arc at the presence of an inductivity $(53 \mathrm{mH})$.

For long arcing times the arc column may not be assumed as straight as indicated by the last frames of Figure 8. The straight arc column represents an instable equilibrium, a small deflection of the arc column from the straight shape leads to an 
electromagnetical force which acts into the direction of the deflection, thus the bending is strengthened $[8,9]$.

From all images of the high speed movies the arc length was processed from distance between the positions of the arc roots automatically.

\section{B. Electrical Measurement Results}

Figure 9 shows the current plots (upper diagram) and the voltage plots (lower diagram) of the experiments executed with $1 \mathrm{~cm}, 2 \mathrm{~cm}, 5 \mathrm{~cm}, 10 \mathrm{~cm}$ and $20 \mathrm{~cm}$ falling distance of the impact mass. The falling distances correspond to mean contact opening velocities of $34 \mathrm{~cm} / \mathrm{s}, 57 \mathrm{~cm} / \mathrm{s}, 78 \mathrm{~cm} / \mathrm{s}, 111 \mathrm{~cm} / \mathrm{s}$ and $170 \mathrm{~cm} / \mathrm{s}$. The experiments of figure 9 where executed with an ohmic load only.

It can be clearly seen, that at all experiments the contacts opened at the time $0 \mathrm{~ms}$. The voltage step of $12 \mathrm{~V}$ immediately after the beginning of the contact separation corresponds to the sum of the cathode and anode drop $U_{A K}$. Due to this extra voltage drop across the switch, the current dropped down from $19.5 \mathrm{~A}$ to $14 \mathrm{~A}$. When the arc voltage across the swhitch exceeded the voltage of the source $U_{0}$ current was interrupted (see Figure 9 "Voltage Level of the Source" and vertical lines which indicate the time point of arc extinction in all three plots). In contrast to the pure ohmic load Figure 10 represents the results yield with an ohmic / inductive load $(53 \mathrm{mH})$. The initial phase of the measured voltage corresponded to the results yield with an ohmic load only (observed voltage step after contact separation). In contrast to the pure ohmic circuit any inductivity in the circuit reacts against any change of the current according to Lorentz's law. Thus the current step does not appear immediately after the contact separation compared to the results with the ohmic load only. If the arc voltage exceeds the sum of the source voltage and the voltage caused by the inductivity the arc was extinguished.

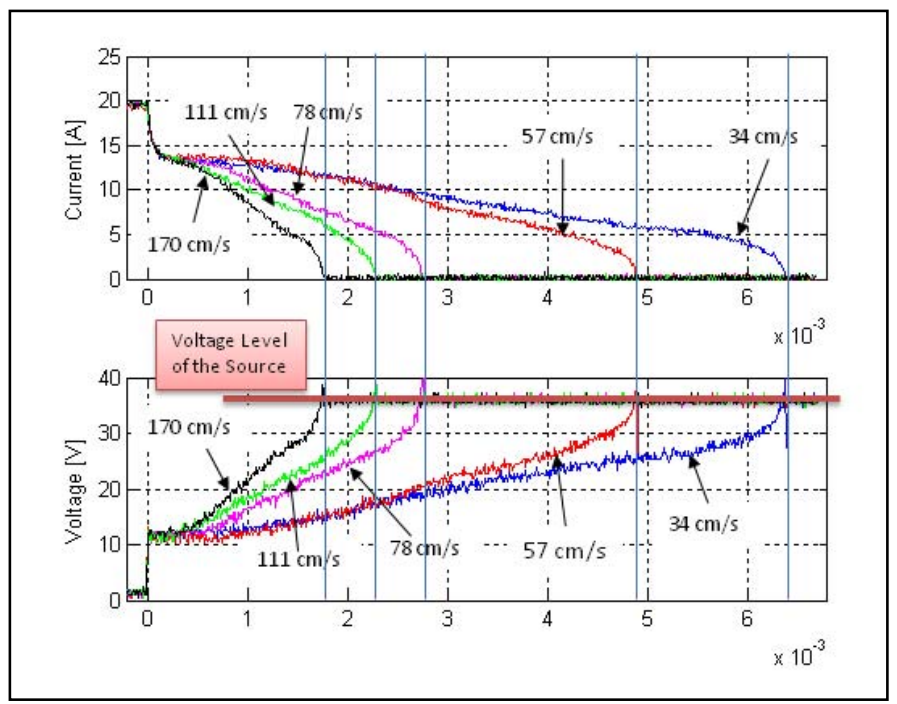

Figure 9. Current, and voltage plots with an ohmic load, an initial current of $20 \mathrm{~A}$, opening velocity corresponding to $1 \mathrm{~cm}, 2 \mathrm{~cm}, 5 \mathrm{~cm}, 10 \mathrm{~cm}$ and $20 \mathrm{~cm}$ falling distance.
It has to be emphasized, that the interruption can be observed at the same voltage level, independent from the opening speed!

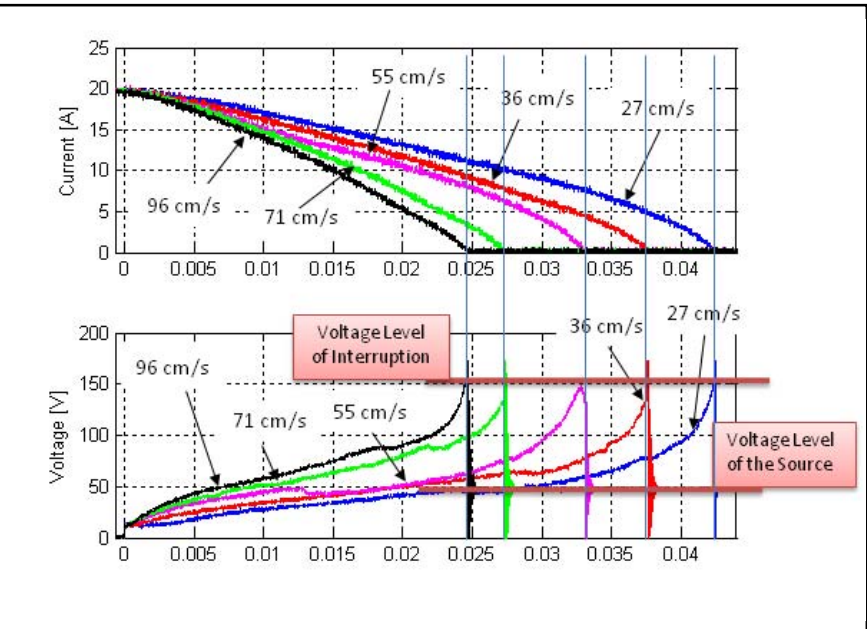

Figure 10. Current, and voltage plots for a contact opening at an ohmic inductive load $(53 \mathrm{mH})$, an initial current of $20 \mathrm{~A}$, with different opening velocity (corresponding to $1 \mathrm{~cm}, 2 \mathrm{~cm}, 5 \mathrm{~cm}, 10 \mathrm{~cm}$ and $20 \mathrm{~cm}$ falling distance).

This fact has to be considered with respect to the arc length plots shown in Figure 11 (corresponding to figure 10). At the experiments with the three highest opening speeds, the moving contact reached the mechanical stop before the arc could be extinguished. Consequently at the experiments carried out at $96 \mathrm{~cm} / \mathrm{s}, 71 \mathrm{~cm} / \mathrm{s}$ and $55 \mathrm{~cm} / \mathrm{s}$ there was only a slow contact motion at arc extinction.

Applying Equation (3) and taking into account the measured sum of the cathode and anode drop (12 V) the mean electrical field strength of the arc column may be computed.

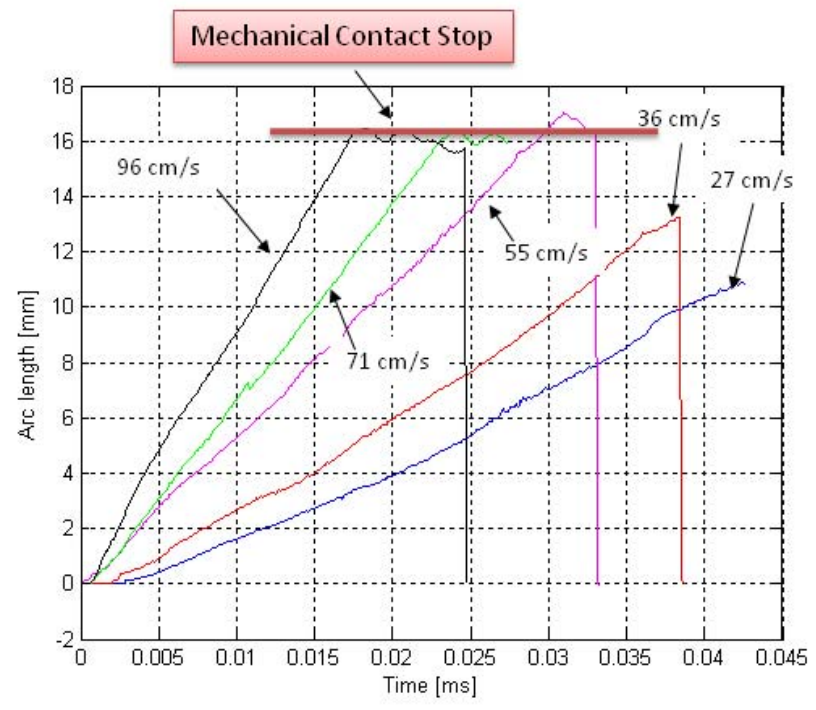

Figure 11. Development of the arc length via time. This figure is corresponding to figure 10 . 
The electrical field strength and the arc current may be plotted as a locus curve which is passed as a function of time and arc length respectively (see Figure 12).

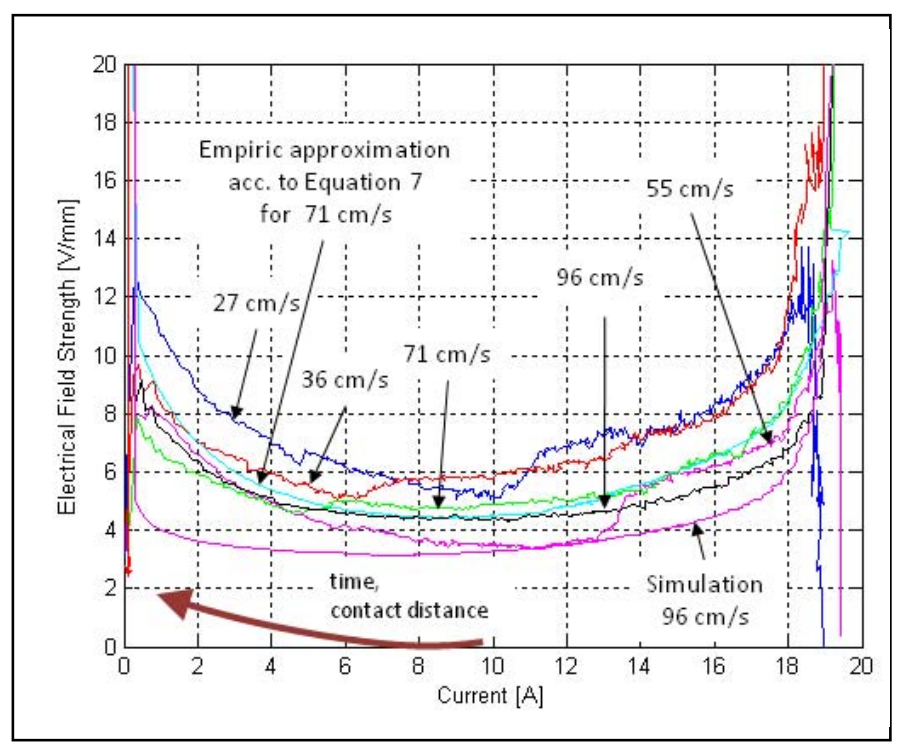

Figure 12. Locus of the field strenght for an ohmic-inductive circuit and $20 \mathrm{~A}$ initial current level.

No significant influence of the opening velocity onto the electrical field strength can be observed. The two experiments carried out with the lowest velocity $(27 \mathrm{~cm} / \mathrm{s}$ and $36 \mathrm{~cm} / \mathrm{s})$ show the highest electrical field strength which is in contrast to the effect that a high velocity leads to a high electrical field strength. During one single experiment the electrical field strength drops down from a high initial value (values between $8 \mathrm{~V} / \mathrm{mm}$ and $17.9 \mathrm{~V} / \mathrm{mm}$ ) at narrow electrode distances to a minimum (values between $3.4 \mathrm{~V} / \mathrm{mm}$ and $5.9 \mathrm{~V} / \mathrm{mm}$ ). From this minimum the electrical field strength rises again (values between $7.9 \mathrm{~V} / \mathrm{mm}$ and $13 \mathrm{~V} / \mathrm{mm}$ ) until the arc was extinguished. The electrical field strength was in good correlation with the values given in Table IV. Considering the minimum field strength of the results plotted in Figure 12 in the range between $3.4 \mathrm{~V} / \mathrm{mm}$ and $5.9 \mathrm{~V} / \mathrm{mm}$ and reading the electrode distance from the corresponding figure 10 and figure 11 (an electrode distance from $6.8 \mathrm{~mm}$ at the lowest opening velocity and $13 \mathrm{~mm}$ at the highest opening velocity can be found) the values at an electrode distance of $10 \mathrm{~mm}$ at $10 \mathrm{~A}$ from Table IV (3.9 V/mm bases on literature data [3] and $3.7 \mathrm{~V} / \mathrm{mm}$ from the arc model) are in a good agreement with the measured values. In Figure 12 the plot indicated by "Simulation $96 \mathrm{~cm} / \mathrm{s}$ " represents the electrical field strength yield by the simulation model.

The results show, that the values from the model yield too low. Applying the empiric Equation (8) (with the values $K_{P C}=20 \mathrm{~W} / \mathrm{Amm}, K_{P E L}=40 \mathrm{~W} / \mathrm{mm}^{2}, I_{0}=2 \mathrm{~A}$ and $l_{0}=3 \mathrm{~mm}$ ) satisfactorily agreement with the measured curves can be observed.

\section{Simulation Results}

A commercial simulation tool Micro Cap [10] was applied in order to simulate the electrical circuit Figure 1. The measured arc length, switching voltage and current where read from spread sheet tables into the simulation tool. The arc was modeled as described by Formula 7 and corresponding to the experiment displayed in Figure 12. The model parameter where the same as introduced in Chapter IV.

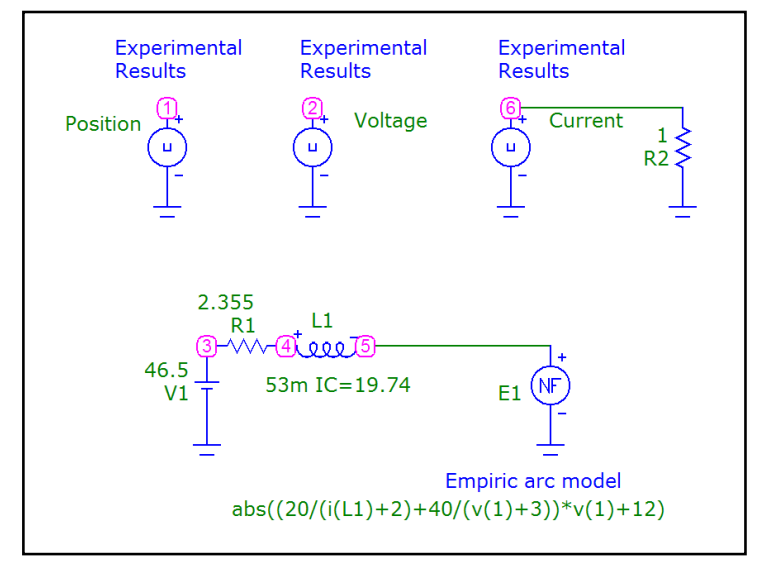

Figure 13. Simulation circuit and arc model.

The simulation circuit is shown in Figure 13 (corresponding to the circuit Figure 1 and the experiment).

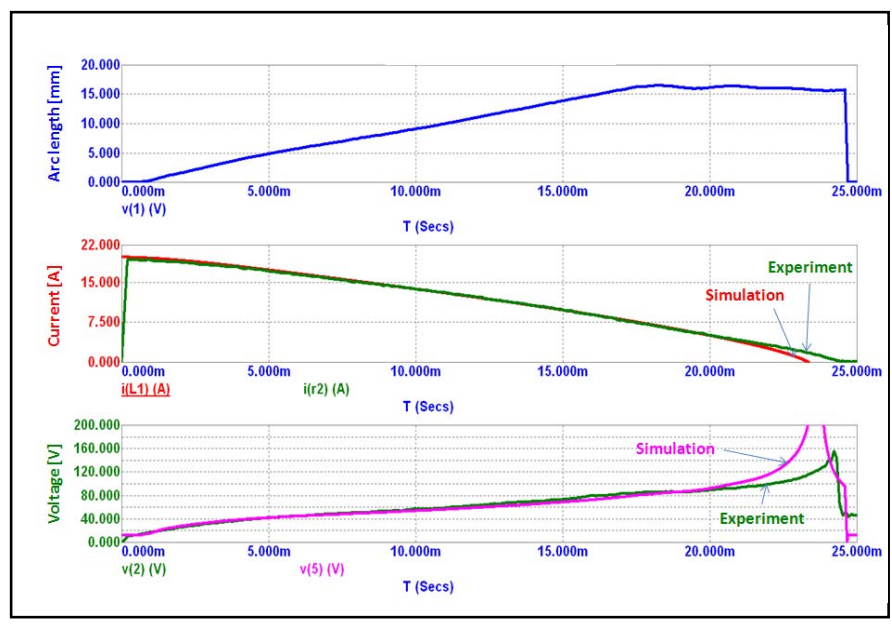

Figure 14. Simulation results versus experimental results for an initial arc current of $20 \mathrm{~A}$, an opening speed of $96 \mathrm{~cm} / \mathrm{s}$ and and ohmic inductive circuit corresponding to figure 14 and the results figure 10 to figure 14 .

In the lower part the arc model and its parameter are displayed (component "E1"). The parts indicated by "Experimental Results" are elements which read spread sheet tables into the simulation tool.

Figure 14 shows representative simulation results compared to the measured signals. From the experiment a clearing time of $24.4 \mathrm{~ms}$ is measured while the simulation yields a clearing time of $23.7 \mathrm{~ms}$ which is a deviation of $2.86 \%$. 


\section{DISCUSSION AND CONCLUSIONS}

The simplified arc model developed is in good correlation to measured and published data. However the neglecting of several conditions including thermal radiation and the lack of arc constriction at the electrodes holds the danger, that the simulation is valid in a small boundary range only.

The very high temperature in the operation point of the arc yield by the arc model (see Figure 4) is not in agreement with any published data of arc temperature values (in the considered boundary range). The very rough modeling of the heat transfer seems to be the reason for that result. The flat temperature curve at the minimum electrical field strength will require only a small change in the boundaries and heat transfer conditions in order to yield more reliable temperatures.

The arc models yield the electrical field strength too low. Considering that in the experiment the arc is not straight (as assumed for the arc model, see Figure 8) the disagreement between the measured and the computed electrical field strength may be caused by this. For the evaluation of the arc length the direct distance between the arc roots was processed from the high speed movie frames. In the case of Figure 8 the arc length is $10 \%$ longer compared to the direct distance between the arc roots (yield from manually geometrical analyses). Considering this fact, the measured arc field strength should be reduced by $10 \%$. However this does not cover the mismatch between the arc model and the measurement. The fact that the computed field strength seems to be generally lower than measured could mean too low simulated thermal power losses. A more detailed heat transfer model should be the next step of the improvement of the arc model.

Previous work [7] has shown a significant influence of the contact speed onto the electrical field strength of the arc. The results presented in this paper show no significant influence of the opening speed on the electrical field strength although the opening velocity of the experiments introduced in this paper is higher than the velocity measured in the previous experiments [7]. The experiments presented in this paper indicate clearly no significant influence of the contact speed onto the electrical field strength (see Figure 12). Although the contact speed was varied from $27 \mathrm{~cm} / \mathrm{s}$ up to $96 \mathrm{~cm} / \mathrm{s}$ (an increase by the factor of 3.6!) the electrical field strength shows no significant higher electrical field strength. The effect seems to be weaker than the effect of the rising field strength caused by the decreasing arc current. This means, that the modeling of the arc voltage gradient does not require an opening speed considering term.
However, considering the results of Figure 11 it can be seen, that at $96 \mathrm{~cm} / \mathrm{s}$ opening speed at $17 \mathrm{~ms}$ the contact motion stops due to the mechanical stop of the system. At $71 \mathrm{~cm} / \mathrm{s}$ contact speed this is the situation after $22 \mathrm{~ms}$ and at $55 \mathrm{~cm} / \mathrm{s}$ at $32 \mathrm{~ms}$. Looking to the measured voltage in the corresponding Figure 10 the slope of the correlated voltage plot decreases at this time points. It can be concluded that the effect of any increased arc voltage gradient is measured but is of negligible order in the considered parameter range. This effect may be considered, if the full energy balance (including the heating of the arc column) is implemented. Such an implementation will cause a huge extra effort, since the specific heat in nitrogen plasma is highly non linear.

The empiric model (which can easily be derived from some representative experiments only) is in a good agreement with the measured data. This leads to simulation results which are in good correlation to the measured data (see Figure 14). The simplicity of the empirical model and the closed analytical formulation require low implementation and computational effort. The range of application for the model described is limited to the experimentally verified range!

\section{REFERENCES}

[1] M. Lindmayer, J.Paulke, Arc motion and pressure formation in low voltage switchgear, IEEE Transactions on components, packaging and manufacturing Technology-Part A21,1988.

[2] G. Andre, Computation of the motion of magentically driven elctric arcs in simplieifeed arc chambers, PHD Thesis, Technical University of Vienna, 2000

[3] W. Rieder, Die Stabilität geshunteter Gleichstromlichtbögen, Elin-Z 7, 1955.

[4] W. Rieder, Leistungsbilanz der Elektroden und Charakteristiken frei brennender Niederstrombögen, Z. Phys. 146, 1956.

[5] W. Finkelnburg, H. Maecker, Elektrische Bögen und thermisches Plasma, Handbuch der Physik 22, Springer, Göttingen Heidelberg, 1956.

[6] W. Rieder, Plasma und Lichtbogen, Friedrich Vieweg \& Sohn, Braunschweig, 1967.

[7] D. Sallais, N. Ben Jemaa. E. Carvou, Minimization of arc extinction gap in the opening speed range of $1 \mathrm{~cm} / \mathrm{s}-1 \mathrm{~m} / \mathrm{s}$, proceedings of the $53^{\text {rd }}$ Internantional Holm Conference on Electrical Contatcs, 2007.

[8] G. Amft, Statistische Gesetzmäßigkeiten der Lichtbogenwanderung, Switching Arc Phenomena, Lodz, 1977

[9] P.Zeller, Experimentelle Untersuchung von Bewegung und Form magnetisch geblasener Lichtbögen unter besonderer Berücksichtigung der Strömungsverhältnisse in engen, länglichen Kammer, PHD Thesis, Technical University Vieann, 2000.

[10] Electronic Circuits Simulation Software, Micro Cap V.9, www.spectrum-soft.com, 20.4.2009 


\title{
Melting and Discharge Phenomena of Breaking Ag Contacts Using a Precisely Controlled Piezoelectric Actuator
}

\author{
Noboru Wakatsuki ${ }^{\dagger}$, Nobuo Takatsu Toshiteru Maeda and Takayuki Kudo \\ 'Ishinomaki Senshu University, Faculty of Science and Engineering, \\ Ishinomaki, Miyagi, 985-8580 Japan \\ e-mail: †wakatuki@isenshu-u.ac.jp
}

\begin{abstract}
Using a laminated ceramic piezoelectric actuator for a very slow $(4 \mu \mathrm{m} / \mathrm{s})$ precisely controlled make/break operation, contact voltage $\mathrm{Vc}$ and contact current Ic, residue of melt surface and discharge for breaking hinge relays with Ag contacting electrodes are measured. To control mechanical conditions, the movable electrode had a finely shaped convex surface (curvature radius: $0.5 \mathrm{~mm}$ ) and a flat fixed electrode. Make/break operation was attempted only once due to transformation of contact surface. Then, Vc and Ic were measured at 1.5 to $25 \mathrm{~V}$ and 0.1 to 5 A. Fusion spots and residue of electric discharge on the contact surface were quantitatively measured using a laser microscope. Even when Vc and Ic were larger than the minimum are voltage and minimum arc current, respectively, a continuous arc discharge occasionally did not occur. For the case of $\mathrm{Vc}=25 \mathrm{~V}$ and $\mathrm{Ic}=1 \mathrm{~A}$, electric discharge was spontaneously suppressed to less than 100 $\mu s$. The displacement of the movable electrode was estimated to be several nanometers for the discharging time interval. The residues of discharge were multiple damaged spots in the range of $100 \mu \mathrm{m}$ in radius, which had a black and/or white rectangular convex and concave shape.
\end{abstract}

Key words ; Opening contacts, Ag contacts, Melting 、 Discharge, Contact surface.

\section{Introduction}

Electromagnetic relays with large contact currents and high contact voltages inevitably cause molten metal and arc ignition, which decrease reliability and life [1]-[4]. The conventional model of breaking contact process is softening, melting, metal bridge formation, vaporization, arc ignition, and isolation as shown Fig.1. However, there is a lot of uncertainty about the process from softening to arc discharge. We propose a method for suppressing arc ignition in mechanical contact devices using a timely controlled transient current switch and a capacitor. [5]-[8]. Then, a rapid increase of contact voltage is suppressed and arc ignition is consequently suppressed. Therefore, the reproducibility of electric and mechanical measurements of breaking phenomena is improved [9]. Our measurements of melting phenomena at the last stage of breaking Ag contacts using the additional transient current circuit showed that the breaking phenomena after the contact voltage Vc exceeded the melting voltage Um did not depend on the mechanical condition controlled by a hinge spring [10]. We confirmed that it is spontaneous fusion of contacting metal. If the contact voltage is high enough to ignite electric discharge, fusion will be followed by discharge between contact electrodes. In this paper, electric discharge phenomena at the last stage of breaking electric contacts are studied using a simple conventional circuit of a DC power source and a load resistance without the additional transient current circuit. Our test sample was a hinge relay with an $\mathrm{Ag}$ contact. Using a laminated ceramic piezoelectric actuator for a very slow (4 $\mu \mathrm{m} / \mathrm{s}$ ) precisely controlled make/break operation, contact voltage Vc and contact current Ic, residue of melt surface were measured. To control mechanical contact conditions, the movable electrode had a finely shaped convex surface (curvature radius: $0.5 \mathrm{~mm}$ ) and the fixed electrode was flat. Make/break operation was attempted only once due to transformation of contact surface. Even when Vc and Ic were larger than the minimum arc voltage and minimum arc current, respectively, an arc discharge occasionally did not occur. For the case of $\mathrm{Vc}=25 \mathrm{~V}$ and $\mathrm{Ic}=1 \mathrm{~A}$, electric discharge was spontaneously suppressed to less than $100 \mu \mathrm{s}$. The contact gap or displacement of the movable electrode was several nanometers. The residues of discharge were multiple damaged spots in the range of $100 \mu \mathrm{m}$ in radius.

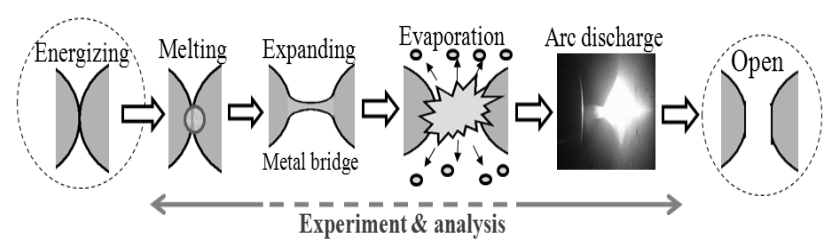

Fig.1 Conventional model of breaking contact procedure

\section{Experiment on breaking contact with super- low-speed piezoelectric actuator}

2-1 Measurement using piezoelectric linear actuator To study electric discharge following the fusion of contacts independent of mechanical motion, a super-low-speed breaking contact experiment was performed. A linear 
piezoelectric actuator was used for displacement controlled breaking contact. Figure 3 shows the experimental apparatus. Our test samples are conventional hinge relays. Using a laminated ceramic piezoelectric actuator for a very slow precisely controlled make/break operation, contact voltage Vc and contact current Ic, residue of melt surface and discharge for breaking relays with $\mathrm{Ag}$ contacting electrodes are measured. The displacement velocity is about $4 \mu \mathrm{m} / \mathrm{s}$ with a trapezoid wave driving voltage signal for one second for making and one second for breaking as shown in Fig.2(c). During the breaking operation, the movable contact electrode was measured by a laser displacement sensor and an irregular mechanical motion was not observed in the range of its measuring accuracy. To control contact mechanical conditions, the movable electrode had a finely shaped convex surface (curvature radius: $0.5 \mathrm{~mm}$ ) and the fixed electrode had a flat surface as shown in Fig.2 (b). Make/break operation was attempted only once due to transformation of contact surface. Then, $\mathrm{Vc}$ and Ic were measured at 1.5 to $25 \mathrm{~V}$ and 0.1 to $5 \mathrm{~A}$. Fusion spots and residue of electric discharge on the contact surface were quantitatively measured using a laser microscope.

\section{2-2 Contact current and voltage characteristics considered to be electric discharge}

Figure 3 shows the time response of contact voltage and current for a $25 \mathrm{~V}$ power source and a $1.2 \mathrm{~A}$ energizing current. After $\mathrm{Vc}=\mathrm{Um}, \mathrm{Vc}$ increases quickly to about half the voltage of the power source, $12 \mathrm{~V}$ and maintains the voltage until $\mathrm{Ic}=0$. Time interval from $\mathrm{Vc}=\mathrm{Um}$ to $\mathrm{Ic}=0$ is about 130 $\mu \mathrm{s}$. During this period, the actuator moves less than $1 \mathrm{~nm}$, judging from the velocity of the piezo actuator $(4 \mu \mathrm{m} / \mathrm{s} \times 130$ $\mu \mathrm{s})$. Then, a stable $120 \mu$ s response followed the initial response and spontaneously stopped. Ic falls to about half the amperes of the energizing current. It shows the typical short duration discharge phenomena. For the higher source voltage, the contentious arc discharge was ignited until the contact current was externally shut down. The responses will be discussed in the later chapter 3 of analytical rearrangement of measured data comparing with the fusion phenomena.

Figure 4 shows the three possible procedures of breaking contacts with super-low-speed piezoelectric actuator following melting phenomena; fusion, short duration discharge and nonstop arc discharge. As the short duration discharge with fusion was interested, the residues on the contact surface were carefully observed and analyzed.

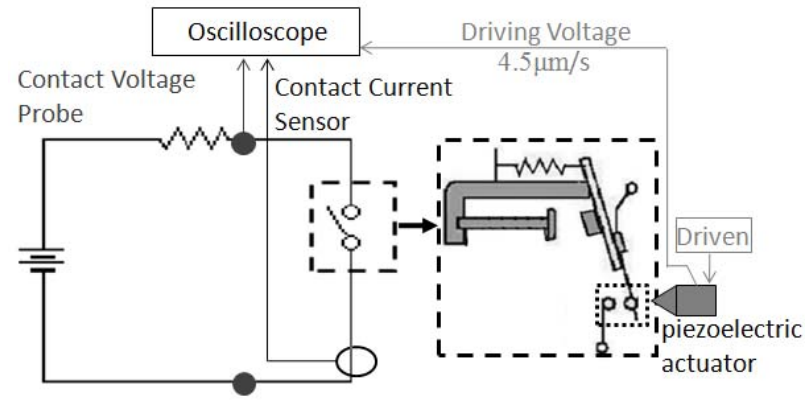

(a) Experimental apparatus using a super-lowspeed piezoelectric actuator.

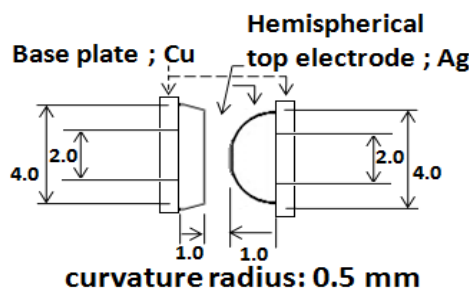

(b) Contact electrodes with a finely shaped convex surface

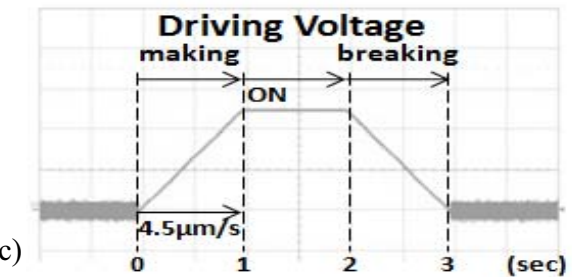

Fig.2 Experimental arrangement.

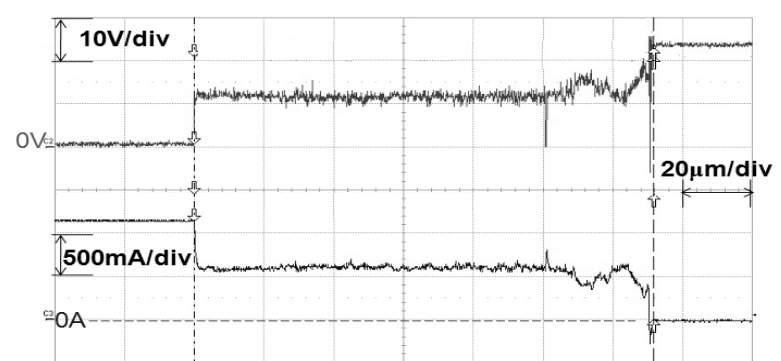

Fig.3 Responses of $\mathrm{Vc}$ and Ic for electric discharge with super-low speed mechanical motion ; $25 \mathrm{~V} 1.2 \mathrm{~A}$

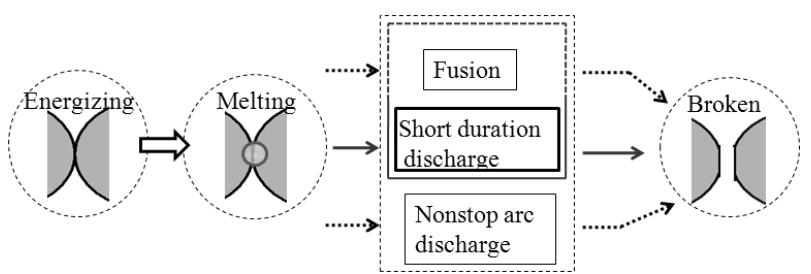

Fig.4. Procedure of breaking contacts with super-low-speed piezoelectric actuator

\section{2-3. Residues of Ag contact surface after electric discharge phenomenon}

We assumed that the phenomena shown in Fig.3 would be a transition from fusion to electric arc discharge. The residue of the Ag contact surface after electric arc discharge phenomenon was carefully observed with a laser microscope. Figure 5 shows an overview of the residue on the fixed electrode. A positive voltage was applied to the fixed electrode. Figure 5(a) shows an optical whole image of the fixed electrode, Fig.5(b) shows a photomicrograph of its contacted area including discharge residues. Figure 5(c) shows a three-dimensional image, and Fig.5 (d) shows a cross-section profile of the concave surface. The residues of discharge on the surface were multiple damaged spots in the range of $100 \mu \mathrm{m}$ in radius as shown in Fig.5(b). The residues have a rectangular shape. There lengths differ, but the width 
is within $16 \pm 5 \mu \mathrm{m}$. In Fig.5(c), height is expressed as monochrome density. Figure 5(d) shows the cross-section profile of the concave surface. The dotted line in Fig.5(b) corresponds to scanning for data. The scars in Fig.5(b) are devised into two types as shown in Figs. 6 and 7.
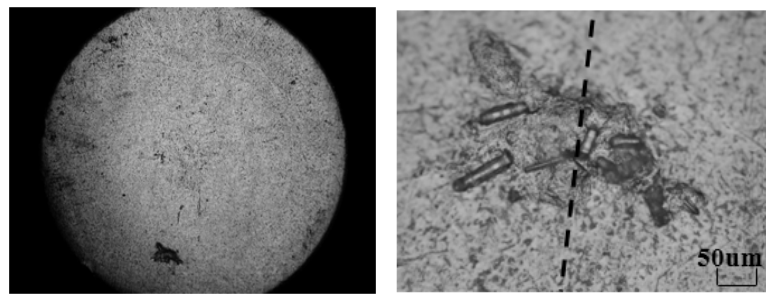

(a) Optical whole image

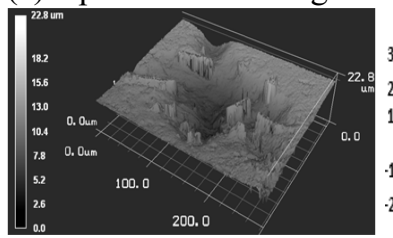

(b) Optical photomicrograph

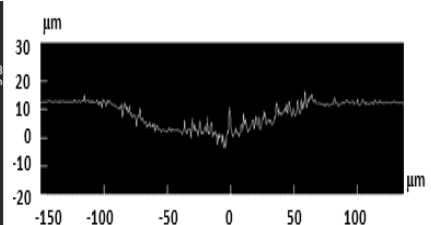

(c) Three-dimensional image (d) Cross-section profile

Fig.5 Photomicrograph of the discharge residues on the fixed electrode

Figure 6 shows the removed or concave residue. Figures 6 (a), (b) and (c) are the optical figure, the three-dimensional image, and the cross-section profile in the long direction, respectively. Top view is rectangular and depth gradually decreases from one end to the other.

Figure 7 shows the deposited or convex residue. Figs.7 (a), (b) and (c) are the optical figure, the three-dimensional image, and the cross-section profile in the long direction, respectively. Optical top view is similar to Fig. 6(a), but the three-dimensional image and the cross-section profile of the convex surface are quite opposite to those in Figs.6(b) and $6(\mathrm{c})$, respectively. The surface of the residue is smoother than that in Fig.6.
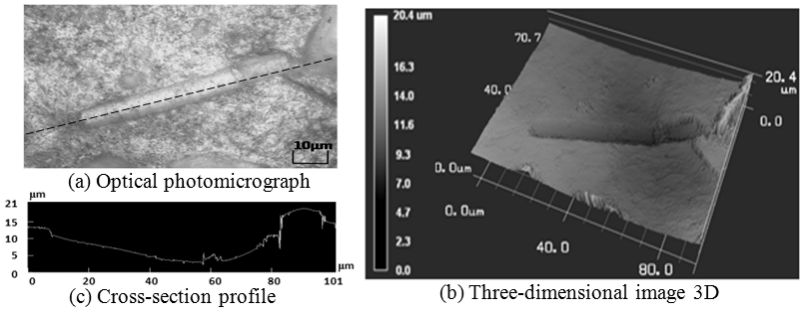

Fig. 6 Concave contact residue
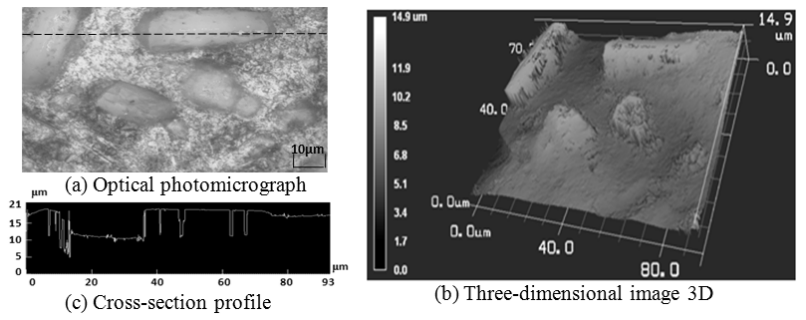

(b) Three-dimensional image 3D

Fig. 7 Convex contact residue

\section{Analytical rearrangement of measured data.}

Using a piezoelectric actuator for a very slow operation with finely shaped convex surface, the residues of concave and/or convex features were finely observed. It suggests that the residues on contact surface are due to the elastic/plastic deformation, the fusion and the discharge. Using a conventional electromagnetic hinge relay, $\mathrm{Vc}$ and Ic responses at $\mathrm{Vc}=\mathrm{Um}$ were precisely compared for the fusion and the discharge as shown in Fig.8(a) and Fig.8(b), respectively. Both responses show similar characteristics. The contact voltages $\mathrm{Vc}$ rise quickly at $\mathrm{Vc}=\mathrm{Um}$ and contact currents Ic smoothly fall down to the shut-down or to the discharge ignition, respectively. Figure 9 is an analytical illustration of measured residue data after electric discharge. The outermost circle at the center of the figure is an outline of the contact electrode. The second outermost circle
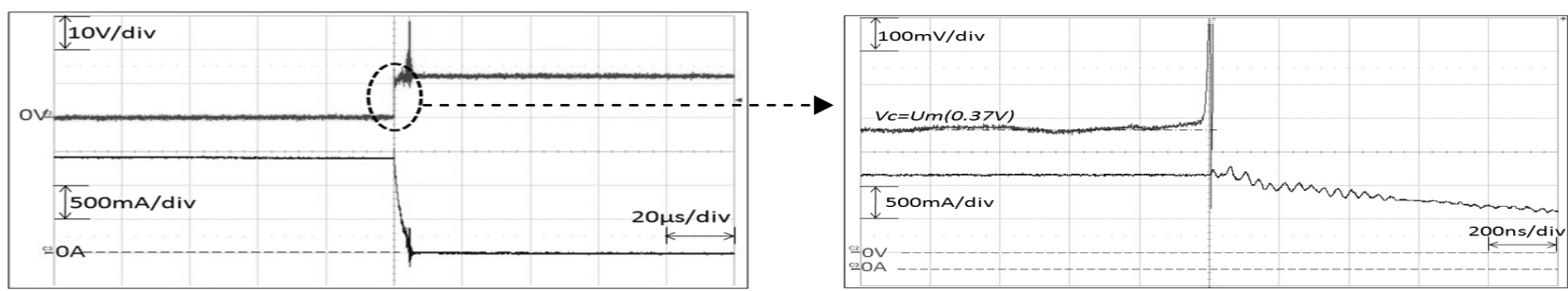

(a) Metal fusion ; $12.5 \mathrm{~V} 1.4 \mathrm{~A}$

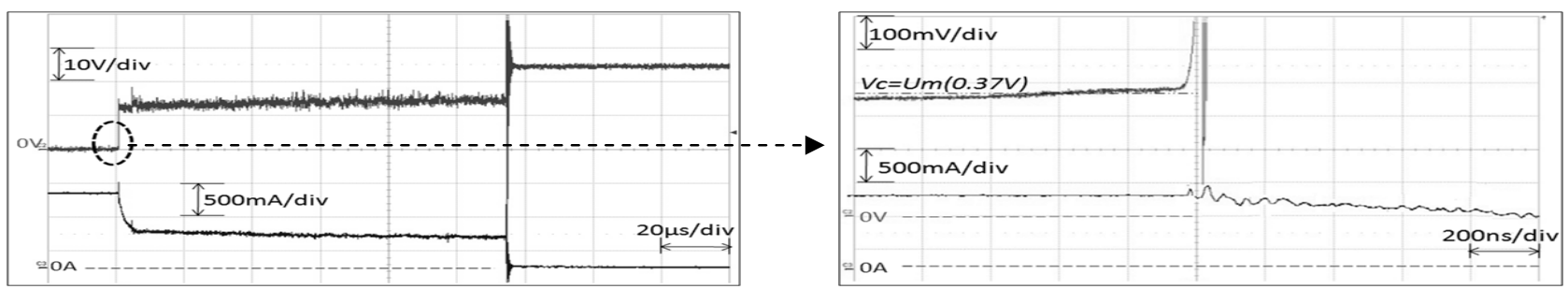

(b)Electric discharge; 25V 1A

Fig.8 Responses of Vc and Ic for metal fusion and electric discharge. 


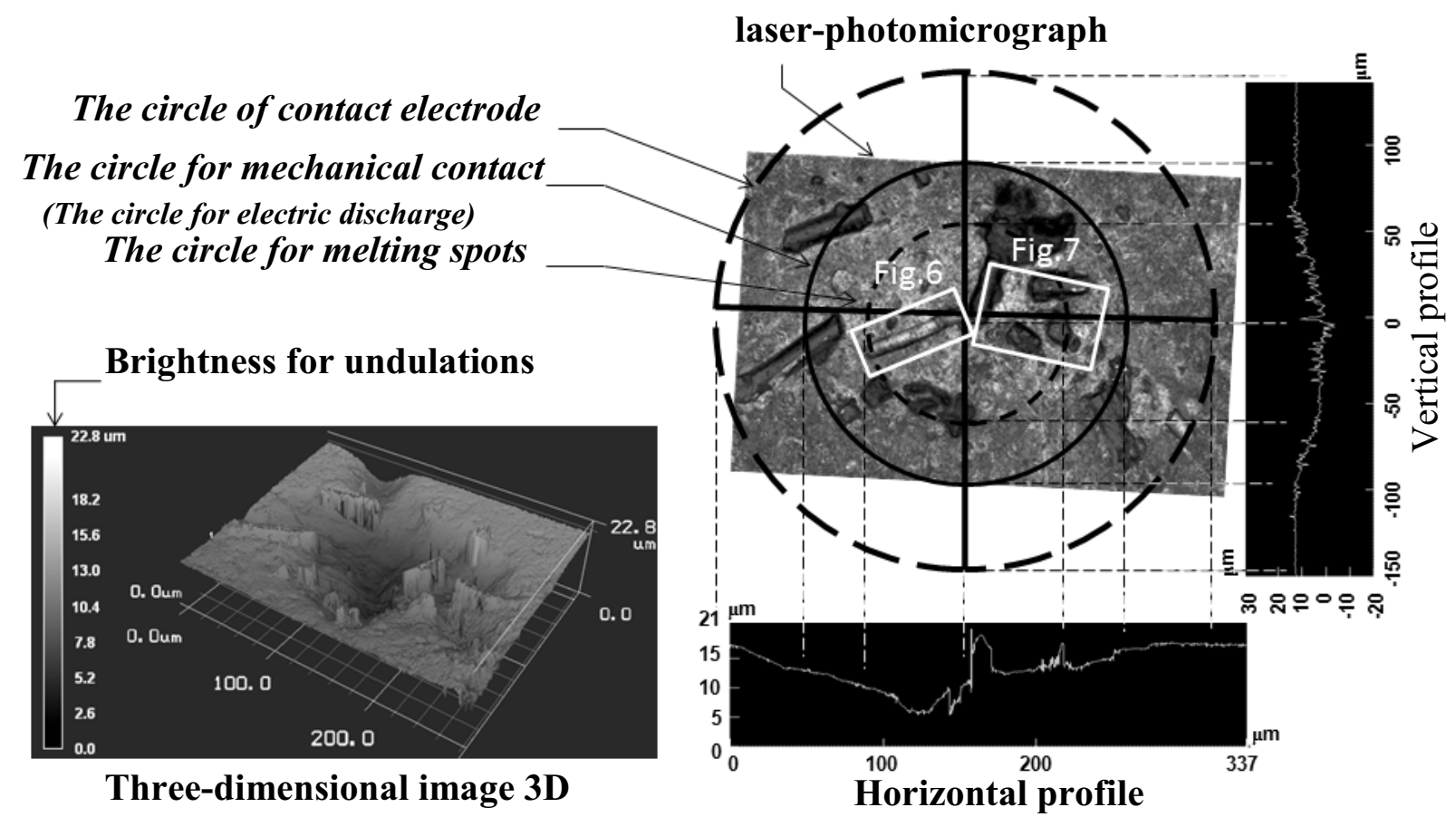

Fig.9 Analyrical rearrangement of measured data on contact surface.

shows the limit of mechanical contact due to contacting force. The inner circle shows the area where melting and electric discharge are estimated to occur. The residues of discharge shown in Fig.6 and 7 are located not only in the inner circle of about $100 \mu \mathrm{m}$ in radius but also out of the circle.

\section{Conclusion}

Using a laminated ceramic piezoelectric actuator for a very slow $(4 \mu \mathrm{m} / \mathrm{s})$ precisely controlled make/break operation, contact voltage $\mathrm{Vc}$ and contact current Ic, residue of melt surface, and discharge for breaking electromagnetic relays with $\mathrm{Ag}$ electrodes were measured. $25 \mathrm{~V} / 1$ A breaking operation showed a stable 130 micro-second electric discharge, which spontaneously stopped without mechanical dispatching. The residues of discharge on anode surface were multiple damaged spots in the range of $100 \mu \mathrm{m}$ in radius, which are classified into convex and concave, respectively. We estimate that the deep concave residue on the anode is due to the discharge of field-emission electrons and the convex residue is due to the cold-electron arc discharge assisted by field emission electrons. We will further investigate the formation process and physical significance of the residues.

\section{References}

[1] R.Holm, Electric Contacts: Theory and Application, 4th ed., Springer-Vela, pp.279-286, 1967

[2] S. Hoh, A. Oyama and S. Mitani, "The Parallel-type SparkQuenching Circuit at Relay Contacts Interrupting D.C. Circuit," J.IEEJ, pp.1331-1337, October 1956

[3] T.Takagi, Arc discharge phenomena in electric contacts, in Japanese, Corona Publishing, Tokyo, 1995

[4] P.G.Slade, Electrical Contact: Principles and Applications, MARCEL DEKKER, INC. New York pp.433-680,1999

[5] N.Wakatsuki and, Y. Yonezawa "Relay Contact of Multielectrodes with Timely Controlled Operation for Arc Discharge Suppression" Proceedings of the 50th IEEE Holm Conference on Electric Contacts p.474-479, Sep.2004

[6] Y. Yonezawa and N. Wakatsuki, "Relay contacts of multielectrodes with timely controlled operation," IEICE TRANS. ELECTRON., Vol.E87-C No.8 pp.1324-1332, Nov. 2004

[7] N.Wakatsuki and, Y.Akiba and, Y.Yonezawa "Timecoordinated Switching Relay for Arc Discharge Suppression," IEICE TRANS. ELECTRON., Vol.E88-C No.8 pp.1635-1640, Aug. 2005

[8] N.Wakatsuki, Y.Yonezawa and A.Yamamoto "Equivalent Circuit Analysis for Time-coordinated Non-arcing Operation of Reed Switches," IEICE TRANS. ELECTRON., Vol.E89-C No.8 pp.1182-1186, Aug. 2006

[9] N.Wakatsuki, H.Honnma "Breaking Contact Phenomena of Time-coordinated Non-arcing Relay"ICICE TRANS. ELECTRON., Vol.E91-C No.8 pp.1182-1186, Aug. 2008

[10] J. A. Greenwood,"Constriction Resistance and the real area of contact”, Brit. J. Appl. Phys., Vol.17, (1966). 


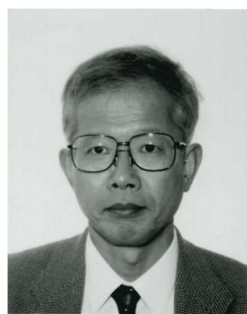

Noboru Wakatsuki was born in May 14, 1944 in Sendai, Japan. He earned a B.E., M.E., and $\mathrm{Ph} . \mathrm{D}$. in electrical engineering from Tohoku University in 1967, 1969, and 1972, respectively. In 1972 he joined Fujitsu Ltd., working on the research and development of electromechanical devices. Since 1995, he has been a Professor in the Faculty of Science and Engineering at Ishinomaki Senshu University. He is a fellow of IEICE (Japan) and a member of the Acoustic Society of Japan and IEEE.

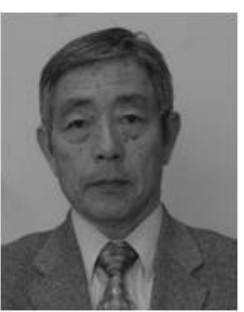

Nobuo Takatsu was born in 1943 in Niigata prefecture, Japan. He received a Ph.D. degree in mechanical engineering from Tohoku University in 1986. Since 1993 he has been a Professor in the Faculty of Science and Engineering at Ishinomaki Senshu University, and is engaged mainly in mechatronics.

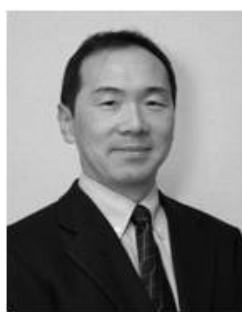

Toshiteru Maeda was born in 1961 inGifu pefecture, Japan. He received a Ph.D. degree in electrical engineering from Tohoku University in 1990. Since 2000 he has been a associate professor in the Faculty of Science and Engineering at Ishinomaki Senshu University, and is engaged mainly in physics.

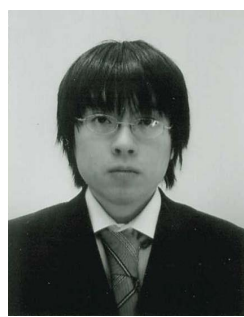

Takayuki Kudo was born in 1984 in Iwate, Japan. He is a graduate student in Science-andengineering graduate course of Ishinomaki Senshua University . He is engaged in the study of electric contact devices. 


\title{
Liquid Additives to Improve Conductivity in Electric Contacts
}

\author{
W. A. Lynch, Member, IEEE, and N. A. Sondergaard
}

\begin{abstract}
Electric contacts generally have high contact resistances because of the relatively small number of conducting contact spots and correspondingly small area through which to transfer current. Liquid metals have been used in the past to provide complete coverage in the contact region but generally require protective atmospheres to prevent corrosion of the liquid metal. Adding an atmospherically stable, non-consumable, conductive liquid additive to the electric contact could hypothetically increase the conductivity of the interface by increasing the contact area and, in the case of sliding contacts such as found in slip rings for example, may reduce wear. This paper presents the results of an initial investigation of several conducting aqueous solutions that could be used as conductivity enhancers for electric contacts. Cation solution pairs were formed using mixtures of the constituent single cation solutions. The technique is based on flowing electrolyte batteries; however energy is not stored in this application. Characteristics of the solutions containing cations of iron or copper in two different valance states, which serve as electron acceptors and electron donors, were evaluated without protective atmospheres. DC conductivity, $\mathrm{pH}$, and half-cell voltage of each solution were measured. The solution with the cation pair had significantly higher conductivity than that of a solution with only one cation valance state. Because of the relatively high redox half-cell potentials of the solutions used in this initial investigation, noble metal electrodes including gold and palladium were required. In future experiments additives based on other types of ions, which may be compatible with lower cost electrodes such as silver, carbon or copper, will be evaluated.
\end{abstract}

Index Terms - Additive, Current Collector, Electric Contact, Electrolyte, Electron Acceptor, Electron Donor, Electron Shuttle, Pourbaix Diagram, Slip Ring

\section{INTRODUCTION}

Electrical contact additives could have a wide range of applications including contactors, compliant electrical connectors, slip rings, rolling contacts, and advanced electromechanical machinery. For example, a corrosion inhibiting additive; BTA, [1] prevents copper corrosion by forming a polymeric complex on the metal surface. The key challenge is to have an additive that both protects the contact and remains electrically conductive. Meeting this challenge is further complicated if the electrical surfaces are moving

This work was supported by the Office of Naval Research

W. A. Lynch was most recently with Raven LLC, Brunswick Maine USA

N. A. Sondergaard is with NSWCCD-SSES, Philadelphia, PA 19112 USA relative to one another. Additives have been used to enhance the performance of many tribo-systems [2-4]. Both vapor phase and solid lubricants have been shown to improve sliding contact wear and continue to be fertile areas of exploration. These additives have been shown to reduce friction, but are often used in applications that do not require significant current transfer, and are generally thicker than natural water films generally present in slip rings $[5,6]$. The conductivity of graphite [7] is moderate, and it is applicable as a conductive solid lubricant [8] in a slip ring system.

We propose to compliment these other approaches and perhaps address wear between moving surfaces with novel aqueous liquid additives chosen by inherent electrochemical stability guidelines. As indicated in Table 1, aqueous electrolyte solutions [9] have electrical conductivity intermediate between water and metals. The proposed additives employ a novel non-consumable electron shuttle mechanism. The proposed additives consist of an aqueous solution containing electron acceptor and electron donor ions, which are generally cations of the same metal in two different valance states. Similar types of mixed valence solutions utilizing transition metal salts such as vanadium-based compounds have been used in flow batteries [10,11], however in such a battery the electrolyte is an energy storage mechanism rather than a conductivity enhancement mechanism and its net composition changes with state of charge. When a redox battery is charged the catholyte changes from vanadium(IV) to vanadium(V) and the anolyte changes from vanadium(III) to vanadium(II). In the contact additive application a similar material is used as an electron shuttle with no net change in composition. Marcel Pourbaix devised potential-pH "Pourbaix" diagrams [12] for the study of corrosion. These plots include $\mathrm{Eh}$ potential relative to a hydrogen electrode as the ordinate, and $\mathrm{pH}, 1 / \log \left(\mathrm{H}^{+}\right)$, as the abscissa. Contacts used for applications such as circuit breakers, relays, slip rings, and electrical machines such as automobile alternators all may be improved with the application of a conductive liquid additive. 
Table 1 Aqueous Solution and Metal Conductivity $\mathrm{mS} / \mathrm{cm}$

$\begin{array}{ll}\text { Distilled Water } & 0.000055 \\ \text { Saturated CO2 Solution } & 0.0011 \\ \text { Seawater } & 53 \\ 5 \% \mathrm{KOH} \text { Solution } & 178 \\ 2 \% \mathrm{HCl} \text { Solution } & 183 \\ 25 \% \mathrm{NaCl} \text { Solution } & 222 \\ \text { Graphite } & 1270000 \\ \text { NaK Eutectic Alloy } & 26300000 \\ \text { Gallium Indium Tin } & 27200000 \\ \text { Copper } & 584000000\end{array}$

Power loss mechanisms in electrical contacts include conduction, constriction, and film resistances. The constriction resistance described in [5] has a value of 8.8X10-3 ohms for a contact spot of $1 \mathrm{um}$ radius and is inversely proportional to this A-spot radius. The tunneling resistance of the water film described in [6] suggests that the film thickness is approximately 5 angstroms and has a resistivity of about $10^{-12}$ $\Omega \mathrm{m}^{2}$. The total resistance of a contact is the sum of the conduction, constriction, and film resistances. A metal fiber brush [13] used in a slip ring would have a large number of contact spots, therefore the main component of the contact voltage drop would be likely to be film resistance. Very small asperities of less than 1 um radius would cover less than $0.1 \%$ of the fiber tips. A liquid additive allows the entire fiber tip to carry transport current rather than a very small asperity thereby significantly increasing the effective interface area. Furthermore, the use of liquid additives could mitigate the often seen higher wear rate of positive anode brushes [14] by providing an ion shuttle mechanism to prevent dissolving of brush fibers in the aqueous solution formed by wet cover gas [15]. Additionally this concept could enable significantly lower wear rates because it facilitates operation of brushes with lubrication at lighter loads or lower power losses by providing lower conduction losses.

A moderately conductive additive can therefore possibly provide acceptable conductivity using an additive filled interface that is orders of magnitude thicker than the few molecule thickness required for water film that supports electron tunneling. The additive technique should also protect the rotor surface and fiber from possible damage due to arcing, electrochemical corrosion based wear resulting in electroplating, soluble byproduct formation or insoluble byproduct formation. The conductive liquid additive should decrease power losses due to friction and current conduction, therefore decrease slip ring operating temperature.

Description of the electrode additive concept, selection procedure, and preliminary test data on selected additive electrolytes are presented in this paper.

\section{LIQUID ADDITIVE CONCEPT}

A slip ring additive may consist a liquid metal mixture such as gallium indium tin [16], or a conductive electrolyte solution with brushes most likely made of compatible fibers. Metal fiber brushes such as used in a machine [17,18] may utilize a very thin interface solution generally consisting of water and $\mathrm{CO} 2$ that provides lubrication and prevents oxidation. More conductive liquid additives could reduce power losses. Multiple valence solutions are used to store energy in applications such as the vanadium redox flow battery as shown in Figure 1; however energy storage is not required in the present application, which is intended for conductivity enhancement, and may help reduce wear.

Research of copper corrosion by sulfuric acid deposited from $\mathrm{SO} 2$ in the atmosphere indicated that the corrosion rate would be increased at low $\mathrm{pH}$ because of metal ion solubility [19] as would be expected based on Pourbaix diagrams. Hypothetical positive brush wear mechanisms are illustrated in Figure $2 \mathrm{a}$ with a magnified view of the interface gap. The roles of the brush and rotor are reversed in the case of a negative brush. Wear could be caused by the copper fibers dissolving into solution with the copper metal forming copper cations that are water-soluble. These would travel through the solution and plate onto the rotor, thereby transporting current with the side effect of increased brush fiber wear rate. Insoluble byproducts such as oxides, sulfides, or hydroxides could also be formed depending on interface solution $\mathrm{pH}$. A thin protective semiconducting passivating layer limited by ion diffusion and electron tunneling may form or thicker non-protective brittle non uniform layers may accumulate and increase conduction voltage drop and wear off forming particulate debris. Such wear debris could increase friction and wear rates because of abrasion especially if it is harder than the brush fibers or slip ring running surfaces.

A multiple valence solution could hypothetically serve as a current shunt mechanism to prevent this type of wear if the electron donor reacts at a lower Eh than the contact metal therefore the contact metal is sufficiently noble to be immune to corrosion in the additive solution. To illustrate the concept, an example using vanadium $\mathrm{V}^{2+}$ and $\mathrm{V}^{3+}$ is shown in Figure $2 \mathrm{~b}$, including electrode half-cell reactions at the brush and rotor surfaces for a slip ring system with a positive brush. The two reactions are opposite, therefore there is no net reaction, and hence there should be no change in the composition of the solution when it is used in a current collector application. Liquid additives could also mitigate other wear mechanisms such as arcing due to current interruption by providing a conductive path or acting as a heat sink. A dual valence additive could contain metal cations such as $\mathrm{Fe}^{3+}$ or $\mathrm{Cu}^{2+}$ which serve as electron acceptors. Electron donor ions such as $\mathrm{Fe}^{2+}$ or $\mathrm{Cu}^{+}$would also be present; therefore no net electrochemical reaction occurs in the solution mixture within the contact interface. The electrode or contact metal must have sufficient nobility so that it is not corroded by the solution. 


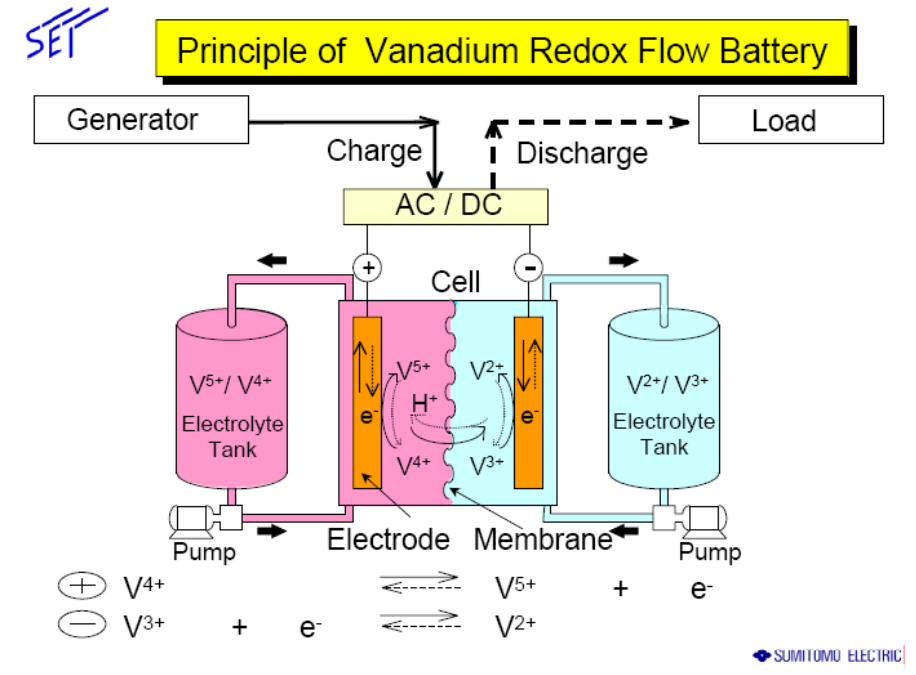

Figure 1 - Energy Storage Application using Two Sets of Dual Valence Solutions
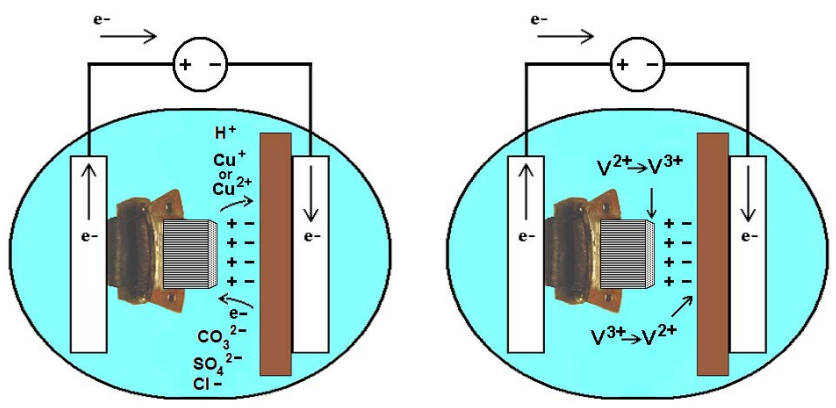

Figure 2a - Hypothetical Electrochemical Wear Mechanism

b - Proposed Conductive Ion Mitigation Method

Pourbaix diagrams are predominance area plots that include redox potential Eh on the ordinate axis generally relative to a standard hydrogen electrode where high values represent oxidizing conditions and low values represent reducing conditions and $\mathrm{pH}, \log$ of $1 /\left(\mathrm{H}^{+}\right)$, concentration on abscissa axis increasing from acid to alkaline. Pourbaix diagrams indicate which compounds of an element are most prevalent under a variety of conditions. The boundary locations are a function of concentration, and multiple concentrations may be included on a single diagram, however fixed concentration diagrams were used in this study. Other factors also affect the phase regions such as solution anions, temperature and pressure. A public domain software tool Medusa [20] may be used to draw these diagrams which may serve as a guide to estimate electrode stability for a variety of metals. This software exports files as bitmaps which may be modified and combined. These diagrams are useful to estimate relative stabilities of contact and solution combinations.
Equilibrium state boundary conditions that can be used as a guide to indicate electrode stability limits are based on the Nernst Equation, $\mathrm{E}=\mathrm{E}^{\mathrm{o}}-\mathrm{RT} / \mathrm{nF}(\ln (\mathrm{Q})$. Where $\mathrm{R}$ is the universal gas constant $8.3145 \mathrm{~J} / \mathrm{mol}$. K, $\mathrm{T}$ is the absolute temperature, $\mathrm{n}$ is the number of electrons in the reaction $\mathrm{F}$ is the Faraday constant $96485.34 \mathrm{C} / \mathrm{mol}$ and Q represents the activities of the product of the reduced side of the electrode reaction divided by those of the oxidized side which when expressed in terms of hydrogen activity is the $\mathrm{pH}$ of the solution. The diagonal dotted lines indicate the stability region of water. Hydrogen or oxygen gas evolution can occur outside these boundaries determined by a simplified Nernst equation $\mathrm{E}^{\mathrm{o}}-0.0591 \mathrm{pH}$, where $\mathrm{E}^{\mathrm{o}}=0$ for hydrogen and $\mathrm{E}^{\mathrm{o}}=1.229$ for oxygen.

Some electrode materials [21] including cadmium, tin, lead, zinc, and mercury exhibit significant overvoltage for hydrogen reduction in the form of visible bubbles as indicated in Table2. These materials can be used in solutions below the nominal limit for aqueous solutions with minimal hydrogen gas evolution. High hydrogen overvoltage materials such as lead and carbon, and bismuth provide minimal overvoltage with redox reactions in liquid dual valence solutions such as $\mathrm{Cr}(\mathrm{II}, \mathrm{III})$, therefore good activity and are therefore applicable in the anolyte half of a redox battery system. [22].

\section{TABLE 2 Electrode Hydrogen Bubble Overvoltage Volts}

$\begin{array}{llllll}\mathrm{Pt} & 0.00 & \mathrm{Ag} & -0.15 & \mathrm{Sn} & -0.53 \\ \mathrm{Pd} & 0.00 & \mathrm{Ni} & -0.21 & \mathrm{~Pb} & -0.64 \\ \mathrm{Au} & -0.02 & \mathrm{Cu} & -0.23 & \mathrm{Zn} & -0.70 \\ \mathrm{Fe} & -0.08 & \mathrm{Cd} & -0.48 & \mathrm{Hg} & -0.78\end{array}$

The electrode exchange current density $i_{0}$ is a more quantitative indicator of electrodes that are less likely to evolve hydrogen. This is the current at the standard hydrogen electrode Eh of 0 volts. The Tafel line, $i=i_{0} \exp (n / b)$ first reported by Julius Tafel in 1905 indicates that the electrode current increases by an order of magnitude for approximately each $120 \mathrm{mV}$ decrease in electrode voltage below Eh of $0 \mathrm{v}$. Values in the first two columns are averages based on researcher data summarized in [23] and the third column is projected based on the Tafel line. The metals are listed in order of standard reduction potentials. Electrode hydrogen or oxygen overvoltage can allow application of electric contact additives outside, but near aqueous solution boundaries. Table 3 indicates periodic table column IIB, IIIA, IVA and VA metal electrodes such as bismuth and indium used in low melting point lead free solder [24] should have low hydrogen gassing in addition to other high hydrogen overvoltage electrodes. 
TABLE 3 Electrode Exchange Current Density and Hydrogen Evolution Overvoltage

\begin{tabular}{|c|c|c|c|}
\hline Metal & Log $\mathrm{I}_{0}$ & Tafel slope $b$ & $\mathrm{~V}\left(1 \mathrm{~mA} / \mathrm{cm}^{2}\right)$ \\
\hline & $\mathrm{A} / \mathrm{cm}^{2}$ & $\mathrm{~m} /$ decade & Volts SHE \\
\hline $\mathrm{Au}$ & -5.7 & 106 & -0.29 \\
\hline $\mathrm{Pt}$ & -3.3 & 30 & -0.01 \\
\hline $\mathrm{Pd}$ & -2.4 & 98 & 0.06 \\
\hline $\mathrm{Ag}$ & -6.4 & 106 & -0.36 \\
\hline $\mathrm{Rh}$ & -2.5 & 68 & 0.03 \\
\hline $\mathrm{Hg}$ & -11.9 & 118 & -1.05 \\
\hline $\mathrm{Cu}$ & -7.4 & 113 & -0.50 \\
\hline $\mathrm{Bi}$ & -10.2 & 106 & -0.77 \\
\hline $\mathrm{Mo}$ & -6.5 & 94 & -0.33 \\
\hline $\mathrm{Pb}$ & -12.6 & 117 & -1.12 \\
\hline $\mathrm{Sn}$ & -9.2 & 117 & -0.72 \\
\hline $\mathrm{Ni}$ & -5.2 & 114 & -0.25 \\
\hline $\mathrm{In}$ & -10.9 & 129 & -1.02 \\
\hline $\mathrm{Cd}$ & -12.0 & 120 & -1.08 \\
\hline $\mathrm{Fe}$ & -5.8 & 118 & -0.33 \\
\hline $\mathrm{Zn}$ & -10.5 & 119 & -0.90 \\
\hline $\mathrm{Cr}$ & -6.4 & 117 & -0.40 \\
\hline $\mathrm{Ga}$ & -9.8 & 120 & -0.82 \\
\hline $\mathrm{V}$ & -6.2 & 182 & -0.58 \\
\hline $\mathrm{Ti}$ & -6.9 & 135 & -0.53 \\
\hline & & & \\
\hline
\end{tabular}

\section{Preliminary Additive Test Results}

Data from multiple Pourbaix diagrams may be superimposed in a multiple metal corrosion immunity diagram. This diagram includes corrosion immunity boundaries for various metal electrodes. The metal is stable in the region below the line plotted near the element label which is a boundary for a $1 \mathrm{mMol}$ solution of each metal.

Pourbaix diagrams shown Figures $3 a$ and $3 b$ indicate that $E h$ and $\mathrm{pH}$ conditions in an electrical contact additive are expected to be at a boundary highlighted as a bold red line between two soluble compounds of different valence states. Horizontal boundaries indicate reactions involving electrons only and these therefore can indicate likely additive mixtures if both species are water soluble. In this case FeII/FeIII is indicated in both plots as an additive, however ferric chloride is a corrosive low $\mathrm{pH}$ salt that is used as an etching solution.
Data from multiple Pourbaix diagrams of soluble dual valence solutions are superimposed on the metal immunity diagram in a combined diagram such as presented in Figure 4. These diagrams are useful as a tool suggestive of what ion pairs may be used with differing electrodes. Additive boundaries typically involving transition metal cations are plotted as bold horizontal and diagonal line segments. Diagonal lines indicate reactions involving both $\mathrm{pH}$ and electrons. These would probably be useful at very high or very low $\mathrm{pH}$ because significant amounts of acid $\mathrm{H}+$ or alkaline $\mathrm{OH}-$ are available to support reactions that involve water. The first solution evaluated and further discussed below, was iron sulfate which is suggested by the iron sulfate boundary in Figures $3 \mathrm{a}$ and 4 at about $\mathrm{pH}=1$ and $\mathrm{Eh}=0.7$. Copper and silver can form insoluble metal sulfides that could change contact resistance, friction and wear, indicated lack of corrosion immunity for most metals in the lower Eh palladium region near the middle of the diagram.

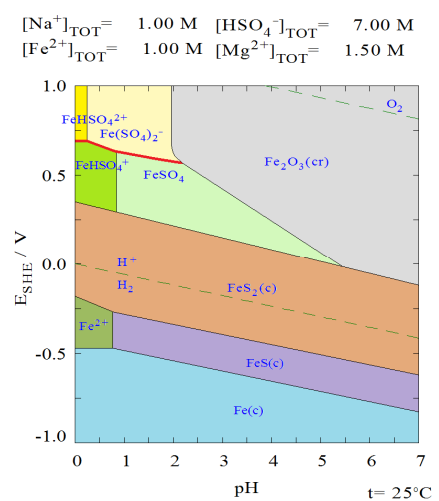

Figure 3a- Iron Sulfate Solution Moderate $\mathrm{pH} \mathrm{Fe}{ }^{2+} / \mathrm{Fe}^{3+}$ Boundary
$\left[\mathrm{Na}^{+}\right]_{\mathrm{TOT}}=2.00 \mathrm{M}\left[\mathrm{Cl}^{-}\right]_{\mathrm{TOT}}=10.00 \mathrm{M}$
$\left[\mathrm{Fe}^{2+}\right]_{\mathrm{TOT}}=1.00 \mathrm{M}\left[\mathrm{Mg}^{2+}\right]_{\mathrm{TOT}}=2.50 \mathrm{M}$

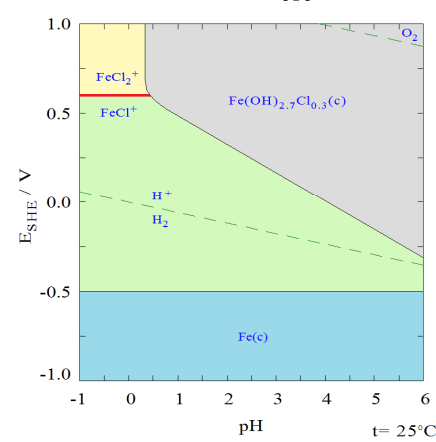

3b Iron Chloride Solution Low $\mathrm{pH} \mathrm{Fe}{ }^{2+} / \mathrm{Fe}^{3+}$ Boundary

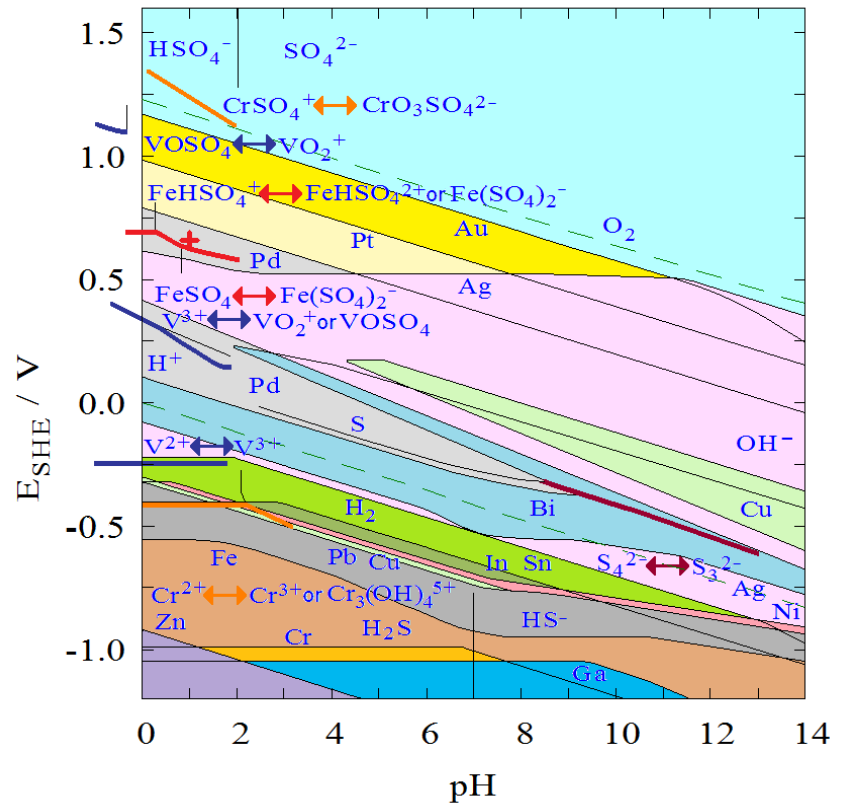

Figure 4 - Combined Sulfate Pourbaix Diagram Indicating Metal Stability Regions and Conditions in Additive Solutions

Electrodes $1 \mathrm{mM}$ Additive Cation $1 \mathrm{M} \mathrm{Mg}^{2+} 1 \mathrm{M} \mathrm{Na}^{+} 1 \mathrm{M} \mathrm{HSO}^{-} 6 \mathrm{M}$ 
Additive solutions generally include anions such as sulfate as components of the compounds as well as surplus anions and spectator cations to increase solubility and improve solution conductivity. Spectator ions with high solubility are selected for high concentration salt solutions. Vertical lines indicate reactions involving only $\mathrm{pH}$ change and are therefore conditions in buffer solutions.

The boundary for the vanadium battery anolyte solution is below the hydrogen evolution boundary, but this solution is applicable with high overvoltage electrodes. Some boundaries outside the left edge of the diagram such as a vanadium battery catholyte solution indicate very acidic conditions. The voltage of the vanadium redox flow battery is indicated as the difference between these vanadium solution boundaries, however less corrosive low Eh anolyte solutions are likely to be more suitable for application as contact additives. The two chromium solutions indicated by orange boundaries could also be applicable in a battery, however redox batteries have been developed that use the chromium (II/III) anolyte and iron (II/III) catholyte [25]. Moderate $\mathrm{pH}$ solutions offer increased safety, but are likely to have higher resistivity.

A dual valence conductive liquid additive solution is made from two solutions, for example; $\mathrm{Fe}(\mathrm{II} / \mathrm{III}) \mathrm{SO} 4$ is made by mixing solutions of iron(II) (Ferrous) sulfate and iron(III) (Ferric) sulfate. This is relatively easy to prepare because both compounds are highly water-soluble. It is corrosive to most metals as shown on Figure 4 at $\mathrm{pH}=1$ and $\mathrm{Eh}=0.7$ and therefore and is only compatible with noble metal electrodes.

Some anions facilitate metal complex ion formation in addition to causing shifts in metal corrosion immunity boundaries. Some of these compounds can be significantly more soluble than the original metal when a significant surplus amount of the salt is available. A copper solution with sulfate anions only includes cupric $\mathrm{Cu}(\mathrm{II})$ ions as a soluble species as indicated as the yellow area labeled $\mathrm{CuSO}_{4}$ in Figure 5a because cuprous $\mathrm{Cu}(\mathrm{I})$ compounds are generally not water soluble. When a significant amount of extra chloride is available the $\mathrm{Cu}(\mathrm{I})$ complex forms and this is very water soluble, so a large $\mathrm{Cu}(\mathrm{I})$ predominance area is indicated as the green area in Figure $5 \mathrm{~b}$. The boundary between these areas highlighted as the dark green line at about $\mathrm{pH}=2$ and $\mathrm{Eh}=0.5$ indicates the presence of a copper based additive in the chloride solution, but not in the sulfate solution.

This copper based additive and other possible additives are shown in Figure 6. Data from multiple Pourbaix diagrams of dual valence solutions are superimposed on the metal immunity diagram in this combined diagram with chloride anions in solution. The metal immunity regions and additive boundaries in this diagram are different from those in Figure4, therefore the anions in the solution also have a significant effect on electrical contacts and contact additives. Rhenium could be applicable as alkaline additive, but it is expensive.
The aqueous solutions for the present study were evaluated in an electrochemical conductivity cell as shown in Figure 7. A magnetic stirrer or rotating wiper is used to mix the fluid as might be representative of rotation in a slip ring application. The electrodes each have a surface area of approximately one square centimeter and are nominally separated by two centimeters of electrolyte solution.

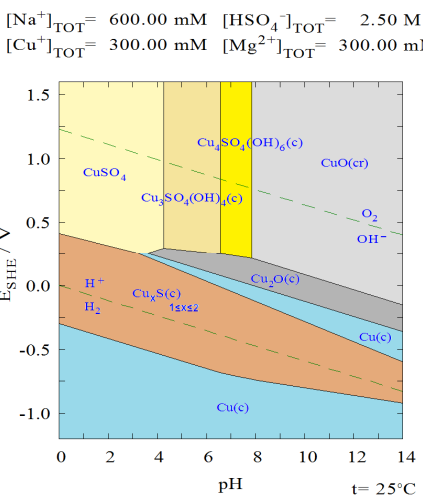

Figure 5a-Copper Sulfate Solution $\mathrm{Cu}$ (I) Predominance Area
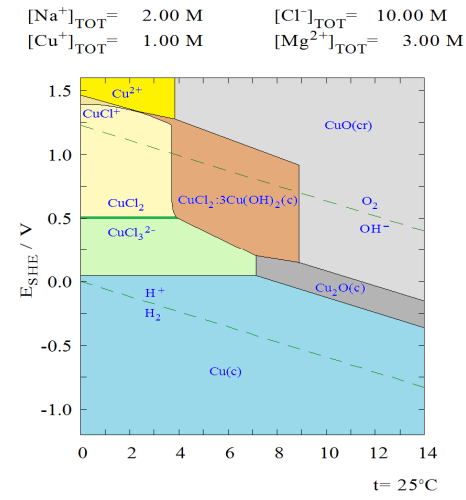

5b Copper Chloride Solution No Large Cu(I) Predominance Area

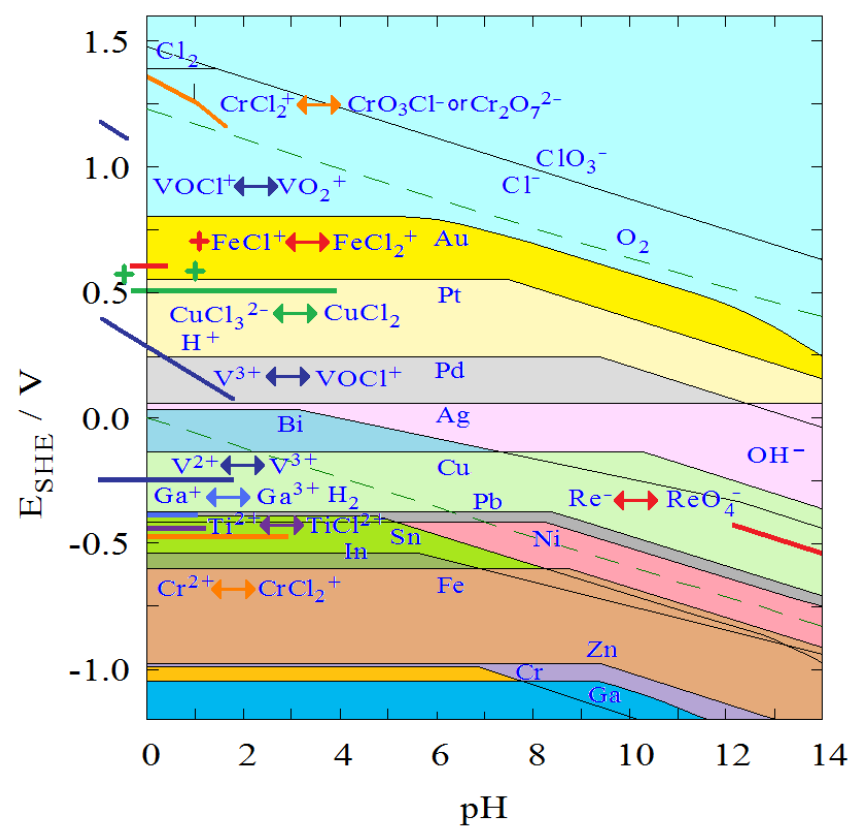

Figure 6 - Combined Chloride Pourbaix Diagram Indicating Metal Stability Regions and Conditions in Additive Solutions Electrodes 1mM Additive Cation $1 \mathrm{M} \mathrm{Mg}^{2+} 3 \mathrm{M} \mathrm{Na}^{+} \mathrm{NM} \mathrm{Cl}^{-} 10 \mathrm{M}$ 


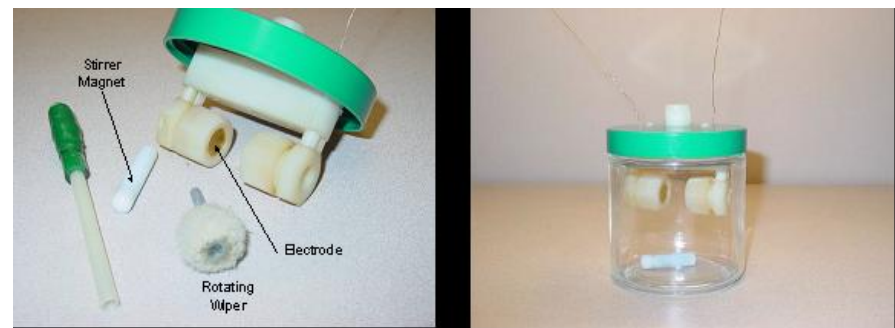

Figure 7 - Conductivity Cell Test Fixture Prototype

Preliminary studies were done with iron and copper compounds which are low cost and relatively non-toxic. The dual valence electrolyte concept has been verified using gold electrodes and relatively acidic oxidizing electrolyte solutions that are corrosive to most metals. The sulfate solution with two valence states, which provides both electron acceptor and electron donor ions is significantly more conductive than a single cation solution as shown in Figure 8. Silver electrodes corroded when used with these solutions as expected. Solutions including chloride anions provide higher conductivity, and solutions with $\mathrm{Cu}(\mathrm{I} / \mathrm{II})$ chloride were the most conductive tested as indicated in Table 3 and Figure 9.

Vanadium, chromium, gallium, and titanium additive boundaries below the nominal hydrogen stability limit indicate anolyte solutions that should be compatible with high hydrogen overvoltage electrodes such as lead, tin or bismuth but indium and zinc have a lower Eh than the additives, and probably would not be suitable for this application. High hydrogen overvoltage electrode materials may be plated over base metals such as copper or nickel.

Iron Sulfate Additive Test

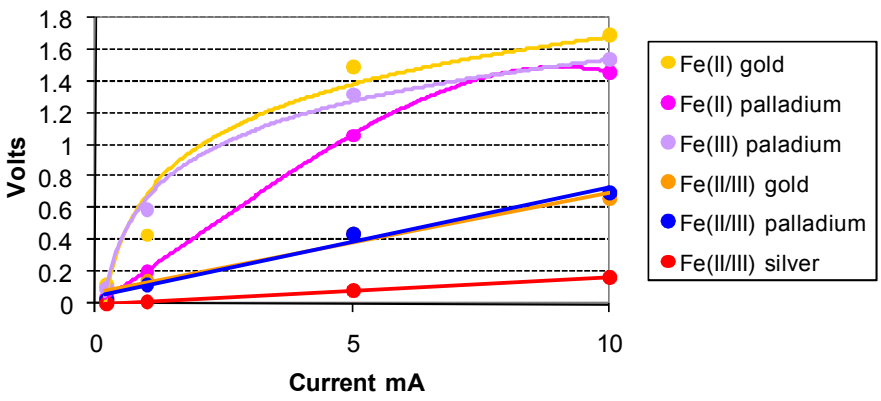

Figure 8 - Conductivity of Single Valence and Dual Valence Iron Sulfate Solutions

Table 3 Dual Valence Additive Solution Characteristics

$\begin{array}{llcccc}\text { Compound } & \text { Solvent } & \begin{array}{c}\text { Solutes } \\ \text { Mole/Lit }\end{array} & \begin{array}{c}\text { Solution } \\ \mathrm{pH}\end{array} & \begin{array}{c}\mathrm{E} \\ \mathrm{mV}\end{array} & \begin{array}{c}\text { Conductivity } \\ \mathrm{mS} / \mathrm{cm}\end{array} \\ \mathrm{Fe}(\mathrm{II}, \mathrm{III}) \text { Sulfate } & \mathrm{Dil} \mathrm{H}_{2} \mathrm{SO}_{4} & 1.00 & 1.00 & 660 & 28 \\ \mathrm{Fe}(\mathrm{II}, \mathrm{III}) \text { Chloride } & \text { Water } & 1.00 & 1.08 & 709 & 84 \\ \mathrm{Fe}(\mathrm{II}, \mathrm{III}) \text { Chloride } & \text { Water } & 2.00 & 0.61 & 711 & 265 \\ \mathrm{Fe}(\mathrm{II}, \mathrm{III}) \text { Chloride } & \text { Water } & 3.00 & 0.23 & 713 & 212 \\ \mathrm{Cu}(\mathrm{I}, \mathrm{II}) \text { Chloride } & 1 \mathrm{M} \mathrm{HCl} & 0.10 & 0.09 & 465 & 37 \\ \mathrm{Cu}(\mathrm{I}, \mathrm{II}) \text { Chloride } & 2.5 \mathrm{M} \mathrm{HCl} & 0.50 & -0.18 & 480 & 248 \\ \mathrm{Cu}(\mathrm{I}, \mathrm{II}) \text { Chloride } & 5 \mathrm{M} \mathrm{HCl} & 1.00 & -0.48 & 570 & 1221 \\ \mathrm{Cu}(\mathrm{I}, \mathrm{II}) \text { Chloride } & \mathrm{Sat} \mathrm{NaCl} & 1.00 & 1.00 & 592 & 353\end{array}$

Iron and Copper Aqueous Solutions With Gold Electrodes

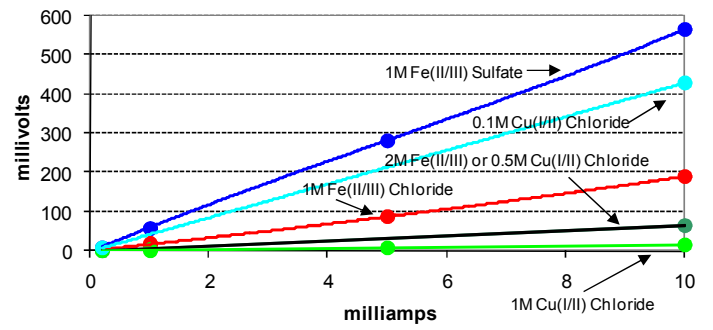

Figure 9 - Conductivity of Dual Valence Iron and Copper Electrolyte Additive Solution

Excess chloride is required to dissolve $\mathrm{CuCl}$ as a soluble $\mathrm{Cu}(\mathrm{I})$ cuprous complex however most of the hydrochloric acid $\mathrm{HCl}$ may be replaced with salt $\mathrm{NaCl}$. The conductivity of the less acidic solution is lower as indicated in Table 3. The characteristics of the solutions tested are plotted as ++ near expected additive boundaries in the upper left area of the Pourbaix diagrams in Figures 4 and 6. The projected solution condition in the sulfate solution is very close to the experimental result. The sulfate and chloride electrolyte solutions are more conductive at higher temperature as indicated in Figure 10.

1 Molar Solution Voltage with $\mathrm{I}=10 \mathrm{~mA} 2 \mathrm{~cm} 1.26 \mathrm{~cm}^{\wedge} 2 \mathrm{pH}=1.0$

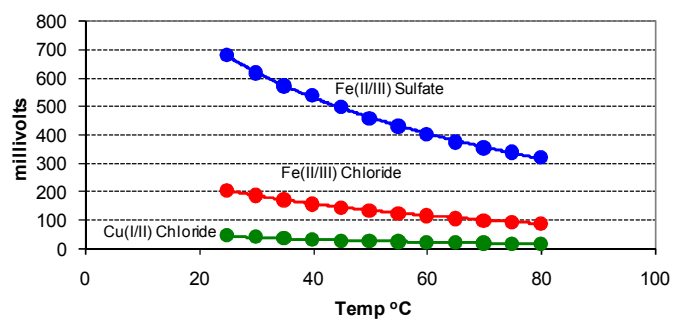

Figure 10 - Dual Valence Solution Conductivity as Functions of Temperature

\section{DISCUSSION}

An ammonium form of the copper solution may be applicable at moderate $\mathrm{pH}$ and lower redox potential, and therefore may be compatible with less costly metals. Ammonia forms complexes with both $\mathrm{Cu}(\mathrm{I})$ and $\mathrm{Cu}(\mathrm{II})$ as indicated by the green boundary in Figure 11a. This moderately alkaline additive includes a buffer at a $\mathrm{pH}$ of approximately 10.5 . Boundary plots for other possible similar additives are included to the right of the diagram. Ammonium compounds like ferric chloride and cupric chloride are used as copper etchant [26], however the lower Eh of these alkaline solutions should be compatible with silver electrodes. Various forms of copper ammonia complex could be practical electrical contact additives that should be compatible with silver contacts or slip rings. The carbon $\mathrm{CO} 2$ boundary in the center of the diagram indicates a possible operating condition for a carbon brush, but carbon and $\mathrm{CO} 2$ have low solubility. The ammonium carbonate salt is applicable in alkaline solutions, but is not practical for acid solutions because of low $\mathrm{CO} 2$ gas solubility. 
Ammonium chloride could be used in both acid and base solutions, and should form either ammonia complexes or chloride complexes with copper as indicated in Figure 11b. This would be compatible with silver or platinum electrodes depending on solution $\mathrm{pH}$. Ammonium chloride can be a component of iron(II/III) and vanadium(III/IV) additives with noble metal electrodes. A soluble additive with tin(IV) would be very acidic, but tin chloride metal additive may be useful. Copper tin and nickel additive compounds including solid metals are included on this diagram. These could be used with $\mathrm{AC}$ systems because plating on electrodes should be balanced with no net change. Phosphate solutions should be less corrosive than other electrolytes and could be used as additives as indicated in figure 12 .

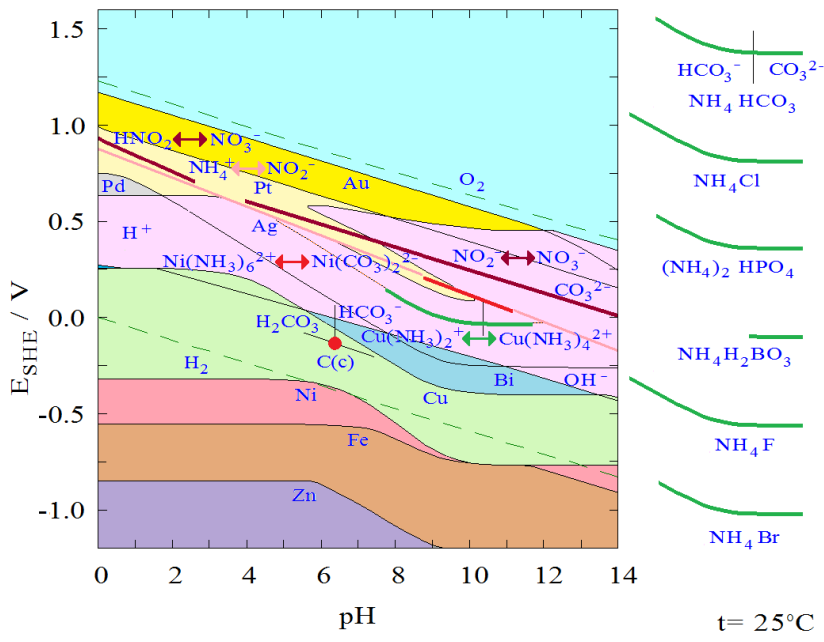

Figure 11a - Combined Ammonium Carbonate Pourbaix Diagram Indicating Metal Stability Regions and Conditions in Additive Solutions and Boundary Plots for Other $\mathrm{CuNH}_{4}$ Compounds Additive Cation 1M Electrodes $1 \mathrm{mM} \quad \mathrm{NH}_{4}{ }^{+} 9 \mathrm{M} \quad \mathrm{HCO}_{3}{ }^{-} 10 \mathrm{M}$

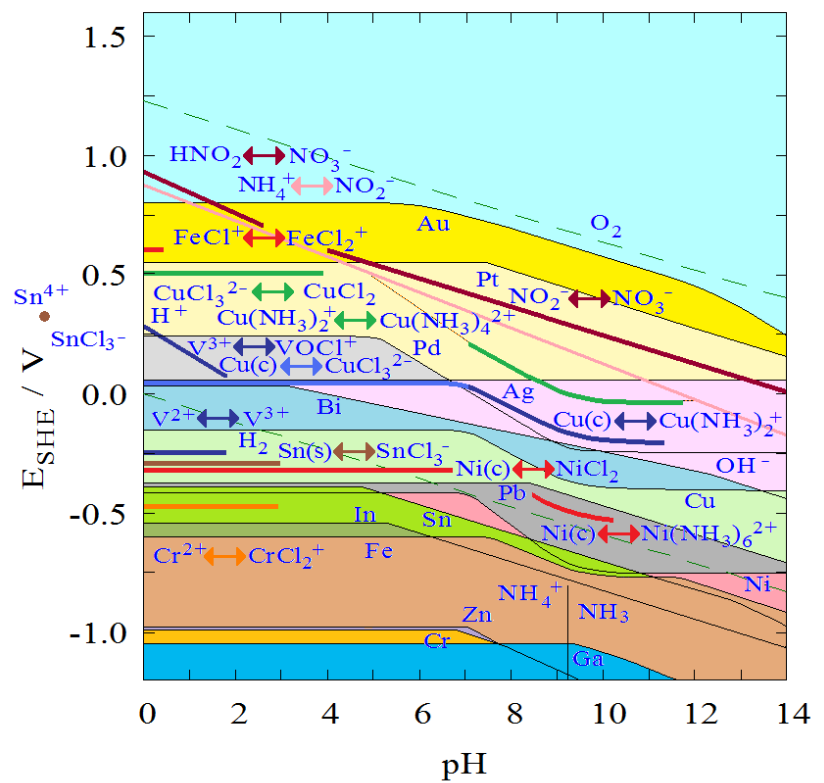

Figure 11b-Combined Ammonium Chloride Pourbaix Diagram Additive Cation 1M Electrodes $1 \mathrm{mM} \quad \mathrm{NH}_{4}^{+} 9 \mathrm{M} \quad \mathrm{Cl}^{-} 10 \mathrm{M}$

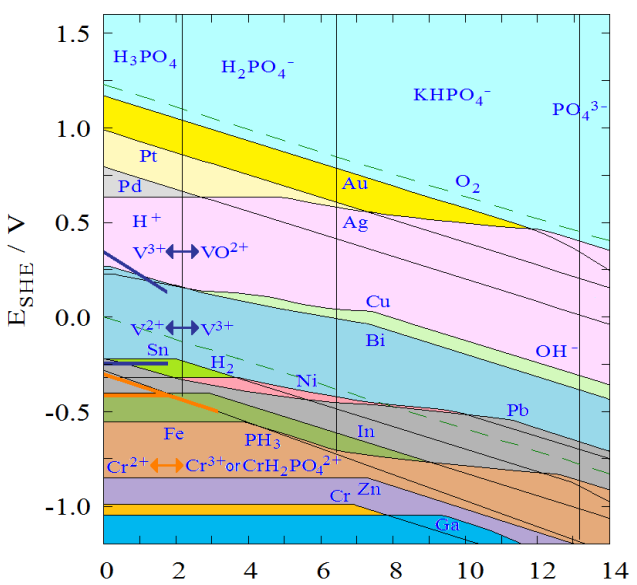

Figure 12-Combined Potassium Phosphate Pourbaix Diagram Additive Cation 1M Electrodes $1 \mathrm{mM} \quad \mathrm{K}^{+} 7 \mathrm{M} \quad \mathrm{PO}_{4}^{-3} 10 \mathrm{M}$

Figures 13 and 14 indicate that fluoride forms complexes with iron and vanadium and this results in additive boundaries at moderate Eh and $\mathrm{pH}$. These may be well suited for buffered moderate redox level dual valence additives that should be applicable to copper and silver contacts.
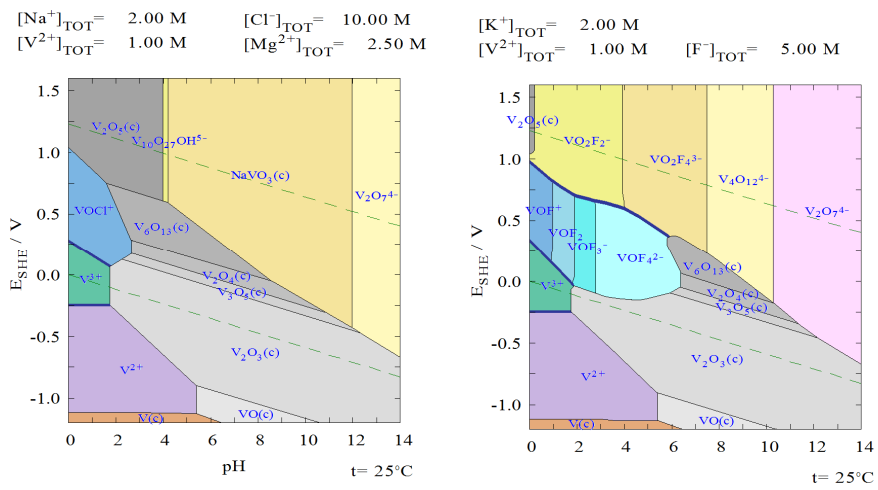

Figure 13a- Vanadium Chloride 13b Vanadium Fluoride Solution Small Soluble V(IV) Area Large Soluble V(IV) Predominance Area

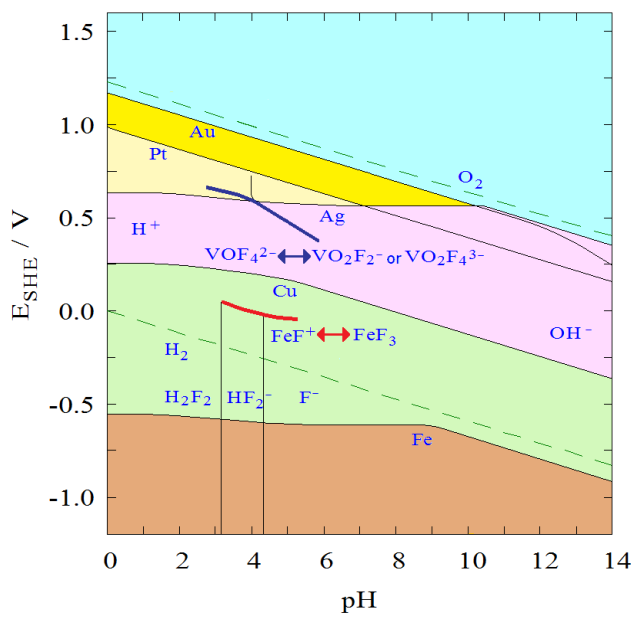

Figure 14 - Combined Potassium Fluoride Pourbaix Diagram Additive Cation 1M Electrodes 1mM $\quad \mathrm{K}^{+} 2 \mathrm{M} \quad \mathrm{F}^{-} \mathbf{5 M}$ 
Dual valence additives based on anions may also be applicable to enhancement of electric contacts. These include polysulfide shown in Figure 4. This alkaline additive is used in a flow battery [27] and should be compatible with a bismuth electrode. In Figure 11a red and brown boundaries above the copper complex boundary indicate possible $\mathrm{NH}_{4}^{+}, \mathrm{NO}^{-}$, and NO3 $^{-}$based additives.

Halogens are shown in Figure 15. An iodide based additive could utilize $1 / 3$ valence $I^{3-}$ as an electron acceptor. It should be applicable over a wide range of $\mathrm{pH}$, but it is used as a gold etching solution [28] that forms soluble complexes with noble metals especially group IB elements, however it should be compatible with platinum electrodes. Other halides form similar compounds that have also been used in flow batteries [29] but these are unlikely to be applicable to electrical contacts because of high Eh oxidizing potential.

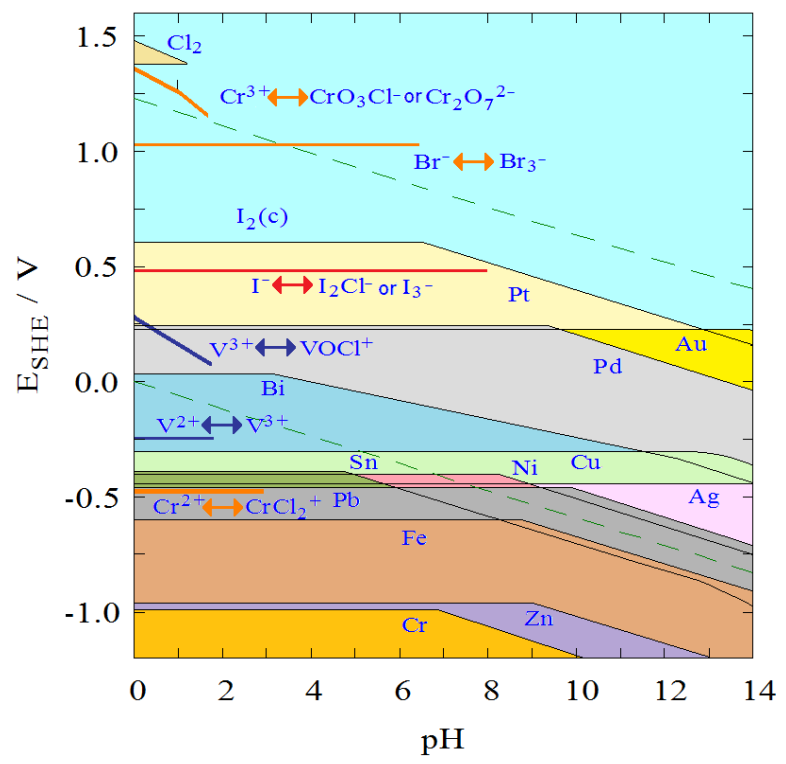

Figure 15 - Combined Chloride Iodide Pourbaix Diagram Additive Cation 1M Electrodes $1 \mathrm{mM} \quad \mathrm{Mg}^{2+} 9 \mathrm{M} \quad \mathrm{Cl}^{-} 9 \mathrm{M} \quad \mathrm{I}^{-} 10 \mathrm{M}$

\section{CONCLUSION AND FUTURE WORK}

In this paper we have presented the concept of dual valence liquid additives which can improve the conductivity and therefore potentially the performance of many electric contact systems. We have presented the approach to choosing suitable ion pairs for several electrodes based on where they fall on the relevant Pourbaix Diagram, such that solutions of these ions will not corrode the electrodes. Furthermore we have presented preliminary data which demonstrates the concept.

Conductivity of other additive solutions and their compatibility with metals suitable for fiber brushes would be verified in future experiments. As in the first series of experiments various types of additive solutions would be prepared. Future research could include more detailed study of iron and copper chloride based additives as well as study of other additive types. Solutions would contain electron donors, acceptors, and salts that provide high conductivity at moderate $\mathrm{pH}$ levels. Other additives are likely to be less corrosive, but some are somewhat more difficult to prepare. Addition of complexing salts or small amounts of acid or alkali may be required to dissolve the compounds in aqueous solution.

DC and AC conductivity would be measured for potential electrical contact additive compounds as a function of electron donor and acceptor ion concentration, $\mathrm{pH}$, temperature, spectator or complexing ion concentration, electrode separation, and solution flow rate. Sensors would include meters for DC current and voltage measurement as well as an $\mathrm{AC}$ impedance probe, a temperature probe, a $\mathrm{pH}$ probe, and a standard electrode probe. These tests would be done using a conductivity cell such as in previous experiments or alternatively using a more sophisticated apparatus including an electrolyte pump and continuously variable electrode separation that provides independent measurements of bulk solution impedance and boundary layer impedance.

Liquid additives could be tested in a variety of devices such as a slip ring evaluation fixture shown in Figure 16. These fixtures are suitable to study characteristics of small sliding contacts such as brushes. The aqueous additives can be compared with liquid metals and other techniques for wear mitigation. The fixture would be operated over a range of speeds and contact transport currents to produce a test data set. Measurements include voltage drop, torque, temperature, wear, additive $\mathrm{pH}$, and additive Eh. Test data sets are run under baseline conditions and using each additive. The additive solution may be dripped on to the current collector while the test fixture is operated, or it may be contained in a sealed liquid filled current collector region within the fixture.

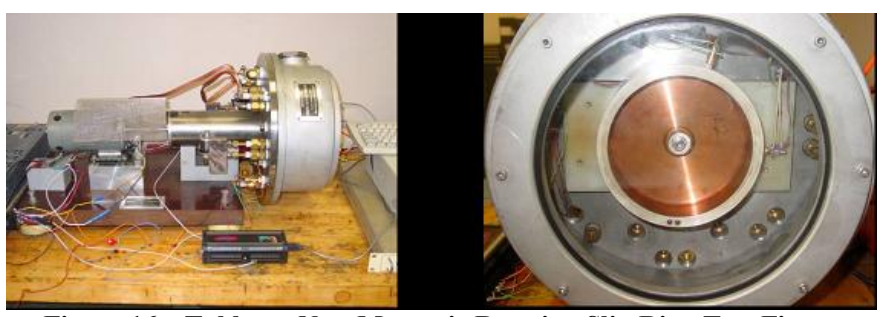

Figure 16 - Tabletop Non-Magnetic Rotating Slip Ring Test Fixture

Aqueous additive solutions should require little or no control of the environment or atmosphere within the machine. A wet $\mathrm{CO} 2$ environment is generally used for copper current collectors, but this may not be required if brushes contain more noble metals or are completely liquid immersed. These solutions are not as conductive as liquid metals, but they may be easier to apply. Further study is required to determine if this type of additive is suitable for application in equipment that uses dry contacts or as an enabling technology for new applications. Other applications for electrical current collectors include electric launchers, moving contacts for rail transit vehicles, and power contactors. 


\section{ACKNOWLEDGMENT}

The Authors wish to thank the Office of Naval Research for their continued generous support on this effort, in particular Program Officers Elaine Martin, Stephen Schreppler, Lynn Petersen and most recently Harold Coombe.

\section{REFERENCES}

1. Cortec

BTAhttp://www.cortecvci.com/Products/Chemicals/chemicals.html

2. I.L. Singer, Th. Le Mogne, Ch. Donnet, and J.M. Martin, "In situ analysis of the tribochemical films formed by SiC sliding against Mo in partial pressures of $\mathrm{SO}_{2}, \mathrm{O}_{2}$, and $\mathrm{H}_{2} \mathrm{~S}$ gases", J. Vac. Sci. Technol., Vol 14, pp. 38-45, 1996.

3. I.L. Singer, "Mechanics and Chemistry of Solids in Sliding Contact", Langmuir Vol. 12, pp.4486-4491, 1996.

4. I.L. Singer, S.D. Dvorak, K.J. Wahl and T.W. Scharf, "Role of Third Bodies in Friction and Wear of Protective Coatings", J Vac Sci Technol A, Vol. 21, No.5, pp.232-240, 2003.

5. Timsit, Roland, Edited by Paul G. Slade, "Electrical Contacts Principles and Applications", New York, Marcel Dekker, pp. 2-4.

6. Doris Kuhlmann-Wilsdorf, , Edited by Paul G. Slade "Electrical Contacts Principles and Applications", New York, Marcel Dekker, pp. 954-955.

7. Material Properties; Conductivity

http://www.ndt-ed.org/GeneralResources/MaterialProperties/ET/ET matlprop_Misc Matls.htm

8. J.A. Bares, N. Argibay, P.L. Dickrell, G.R. Bourne, D.L. Burris, J.C. Ziegert, W.G. Sawyer. "In situ graphite lubrication of metallic sliding electrical contacts", Wear (2009), doi:10.1016/j.wear.2009.03.024

9. David R. Lide, "CRC Handbook of Chemistry and Physics $87^{\text {th }}$ Edition ", CRC Press, 2006.

10. Maria Skyllas-Kazacos "Recent Progress with the UNSW Vanadium Battery" http://www.vrb.unsw.edu.au/

11. Vanadium Redox Flow Battery

http://www.electricitystorage.org/pubs/2001/IEEE_PES_Summer2001/Miyake.pdf

12. Pourbaix, Marcel, Atlas of Electrochemical Equilibria in Aqueous Solutions, Oxford, NY, Pergamon Press, 1966, second edition, Houston TX, National Association of Corrosion Engineers, 1974.

13. http://www.polysci.com/docs/fiberbrush.pdf

14. P.Reichner," Metallic Brushes for Extreme High Current Applications", Electrical Contacts - 1979, (III. Inst. Tech., Chicago, IL) pp.191-197.

15. P.Reichner and V.B. Doshi "A Homopolar Motor for the Demonstration of New High Current Brushes”, Wear, 78 (1982) pp. 69-79.

16. R. Gaines Burton and Ralph A. Burton, "Gallium Alloy as Lubricant for High Current Density Brushes", IEEE Transactions on Components, Hybrids and Manufacturing Technology, Vol. 11, No.1, March 1988

17. Lynn J. Petersen, Damian Urciuoli, Mehren Alam, Thomas H. Fikse, Laura D. Stubbs, William A. Lynch, Neal A. Sondergaard, Doris Kuhlmann Wilsdorf, John T. Moore, Robert B. Nelson, Matthew S. Bednar, Wallace M. Elger, Richard W. Johnson, Richard J. Martin, and Michael Heiberger, "A Study of the Magnetic Field Effects upon Metal Fiber Current Collectors in a High Critical Temperature Superconducting Homopolar Motor", Third Naval Symposium on Electric Machines, Philadelphia, PA, Dec 2000.

18. P.Reichner, "High Current Tests of Metal Fiber Brushes", Electrical Contacts - 1980, (Ill. Inst. Tech., Chicago, IL), 1980, pp.73-76.

19. J. H. Payer, "Corrosion Processes in the Development of Thin Tarnish Films", Proceedings of the 36th IEEE Holm Conference on Electric Contacts, Montreal Canada, pp203-211, 1990.
20. Ignasi Puigdomenech "MEDUSA: (Make Equilibrium Diagrams Using Sophisticated Algorithms) program and Hydra: Hydro-chemical Equilibrium Constant Database", Inorganic Chemistry, Royal Institute of Technology, Stockholm Sweden, November 2008 http://w1.156.telia.com/ u15651596/ http://www.kemi.kth.se/medusa/

21. Samuel Glasstone “An Introduction to Electrochemistry” pg 443 READ BOOKS, 2008

22. J. Ginger, K. Cahill "Advanced Screening of Electrode Couples" http://ntrs.nasa.gov/archive/nasa/casi.ntrs.nasa.gov/19800014289_1980014289.pdf

23. Edited by Allen J. Bard "Encyclopedia of the Electrochemistry of the Elements" Marcel Dekker Inc, 1982

24. http://www.indium.com/products/alloy_sorted_by_temperature.pdf

25. Vinod Jalan, Herbert Stark, Jose Giner "Requirements for Optimization of Electrodes and Electrolyte for the Iron/Chromium Redox Flow Cell" DOE/NASA/0097-80/1 NAS 1.26:165218

http://ntrs.nasa.gov/archive/nasa/casi.ntrs.nasa.gov/19820017764_1982017764.pdf

26. Clyde F. Coombs "Printed Circuits Handbook" Chapter 34, McGrawHill Professional, 2007

27. Polysulfide Flow Battery http://www.electricitystorage.org/tech/photo_psb1.htm

28. Gold Etch http://www.transene.com/au_etchant.html

29. Zinc Bromine Battery

http://electricitystorage.org/tech/technologies_technologies_znbr.htm

\section{BIOGRAPHIES}

Dr. William A. Lynch was most recently at Raven Technology and has 20 years of experimentalist R\&D experience in the areas of Current Collector/Brush Technology, Energy Storage Device Characterization, and Power Electronic Electric Machinery Drive Systems. His graduate degree research focused on development of an Electric Vehicle Battery Evaluation Laboratory Facility and Photovoltaic System Design. He received his BSc, MSc and Doctorate degrees in Electrical Engineering from the University of Massachusetts Lowell in 1987, 1991, and 1997. He participated in Solar Race events including Sunrayce, the World Solar Challenge, and the American Tour De Sol. He is a member of IEEE. He has co-authored 24 research papers and DON reports and is a co-inventor on 9 U.S. Navy patents.

Dr. Neal Sondergaard is a senior scientist at NSWCCD with 29 years experience in $\mathrm{R}+\mathrm{D}$ including sliding electric contacts(brushes), Tribology, MHD, superconductivity and electric power production, generation, conversion, machinery and distribution and Propulsion System Modeling. He received his B.A. in Chemistry from Marist College, his M.Sc. and Ph.D. in Physical Chemistry from Brown University and was a Post Doctoral Fellow at the Johns Hopkins University. He is a member of Sigma Xi Scientific Research Society, and ASNE American Society of Naval Engineers. He has co-authored 75 research papers and DON reports and has 9 patents. 


\section{Analysis of peculiar Brush V-I Characteristics}

\section{with Steel Rings by improved Holm's $\psi-\vartheta$ theory in Electric Contact}

\author{
Makoto Takanezawa, Noboru Morita, Takahiro Ueno \\ Nippon Institute of Technology \\ 4-1 Gakuendai, Miyashiro, Saitama 345-8501 JAPAN \\ m.takanezawa@gmail.com \\ morita@nit.ac.jp \\ ueno@nit.ac.jp
}

\begin{abstract}
The brush gear reliability for turbine generator, is more and more important in recent days. In this research, in case of steel ring, peculiar brush V-I characteristic has been found. It is clarified that figure of the peculiar brush V-I characteristic is "saturated" while the figure for copper ring is almost "linear".

Then, this research pays attention to the fact that softening temperature of steel is far higher than that of copper where mechanical strength decreases at the softening temperature. I research temperature of contact point where the ring and the brush come in contact. Based on the contact point temperature analysis, reason for the peculiar saturation characteristic is clarified.

Two theories by R.Holm were known as to electric contact. One is relation between contact resistance and contact spot radius. Another is relation between contact voltage drop and contact temperature rise. In the former theory, current constriction toward the contact point is assumed. On other hand, in the latter theory, Cylindrical Column model of contact part is assumed.

In this study, Two theories by R.Holm are integrated and $\psi-\vartheta$ theory is improved, considering electric circuit theory and heat conduction theory.

In this paper, it can be concluded that peculiar saturation of the V-I characteristic is caused by the higher softening temperature of the steel ring. These means, operating temperature of steel ring contact point is higher than that of copper, in ordinary operation range. The higher contact temperature may cause ring failure of roughened surface. Therefore careful maintenance is required for the steel ring.
\end{abstract}

Keywords- Peculiar brush V-I characteristics, Steel ring, Copper ring, Softening temperature, Contact point temperature, Plastic deformation, Contact voltage drop

\section{TECHNICAL BACKGROUND}

Brush \& collector ring sliding contact phenomena is very important for reliabilities of turbine generators in thermal \& nuclear power plant and for reliabilities of large AC motors.

\author{
Toru Otaka, Daisuke Hiramatsu \\ TOSHIBA Corporation \\ 2-4, Suehiro-cho, Tsurumi-ku, Yokohama, 230-0045 \\ JAPAN \\ toru.otaka@toshiba.co.jp \\ daisuke.hiramatsu@toshiba.co.jp
}

Because magnetic field currents are fed through the brush $\&$ collector ring systems from stationary side to rotational side of those machines.

On those machines, Steel Collector Rings are applied due to the mechanical strength for High Speed Rotation. However little has been disclosed or reported about the electrical \& mechanical sliding performance in case of brush \& steel collector ring system, while much has been disclosed or reported in case of brush \& copper ring / commutator system.

In our previous Paper ${ }^{1)}$, we reported that peculiar \& saturated brush contact V-I characteristics can be found in case of steel collector rings, while almost linear brush contact V-I characteristics can be found in case of copper rings.

In fig.-1 shows the V-I Characteristic features for both steel ring and copper ring.

As is shown in Fig.-1, V-I characteristic for carbon brush with copper ring in vacuum atmosphere, is linear, while V-I characteristic in air, is almost linear with a little saturation tendency, due to so-called "Film Resistance". The saturation tendency is increased according to the film thickness increase.

On the other hand, in case of steel ring, brush contact voltage drop is kept almost constant for all range of brush current.

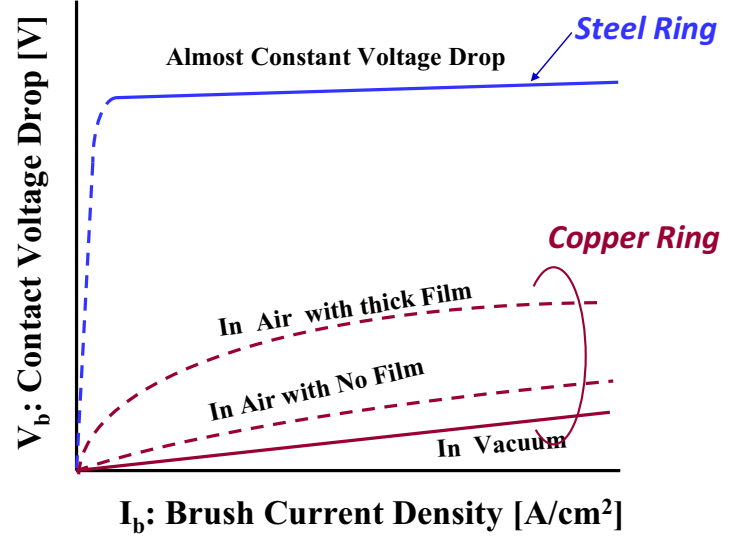

Fig.-1 V-I Characteristic Features for Steel ring \& Copper Ring 
As is well known, the brush contact drop consists from the drop caused by brush bulk resistance and the drop caused by constriction resistance.

In this paper, the technical term "contact drop" is to be a contact drop where the voltage drop caused by bulk resistance is subtracted from measured contact drop.

According to the R. Holm's text book, constriction resistance is defined as,

$$
\boldsymbol{R}_{c}=\frac{\rho}{4 \boldsymbol{a}}[\Omega]
$$

where $\boldsymbol{R}_{c}$ : Constriction Resistance $[\Omega]$

$$
\begin{aligned}
& \rho: \text { Specific Resistance for the Contact Material } \\
& \quad[\Omega-\mathrm{m}] \\
& \text { a }: \text { a-spot Radius }[\mathrm{m}]
\end{aligned}
$$

Referring to three point contact theory, three parallel current paths are made for one brush, resulting

$\frac{\boldsymbol{V}_{b}}{\boldsymbol{I}_{b}}=\frac{\boldsymbol{R}_{c}}{3}=\frac{\rho}{12 \boldsymbol{a}}[\Omega]$

where $V_{c}$ : the Brush Contact Voltage Drop excluding the Voltage Drop caused by the Bulk Resistance [V] $I_{b}$ : the Brush Current [A]

From expression (2), a-spot radius can be obtained by measured V-I characteristics.

The specific resistance of carbon is far higher than that of copper or steel. Therefore, for a-spot radius calculations, the voltage drop caused by constriction resistance in copper or steel ring, can be ignored.

Fig.-2 shows the constriction resistance model by R.Holm.

Fig.-3 shows brush contact voltage drop and the a-spot radius, as a function of brush current density for 3 cases of (a), (b) and (c).

The case (a) is a result of natural graphite brush and copper ring operation, in $0.02 \mathrm{~Pa}$ Vacuum atmosphere.

In this case, V-I characteristic is almost linear, resulting almost constant a-spot radius, for all range of the brush current density.

The case (b) is a result of electric graphite brush and copper ring operation. In this case, V-I characteristic is a little saturated, resulting a-spot radius increases with brush current, while a-spot radius is kept $15 \mu \mathrm{m}$ around zero brush current.

The case (c) is a result of the same electric graphite brush and steel ring operation. In this case, a-spot radius is almost linear, while the brush contact voltage drop is almost constant.

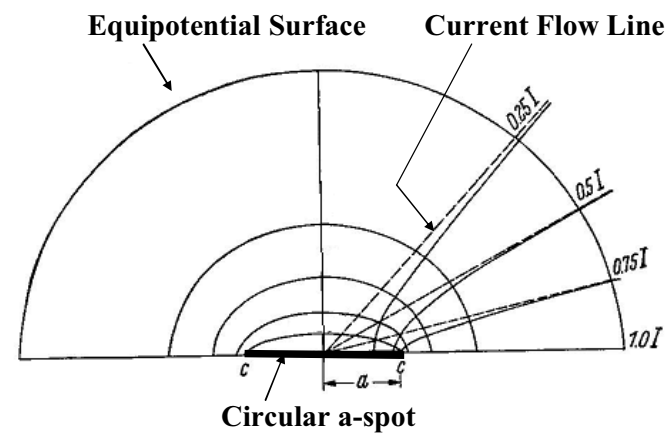

Fig.-2 Constriction Resistance Model by R.Holm

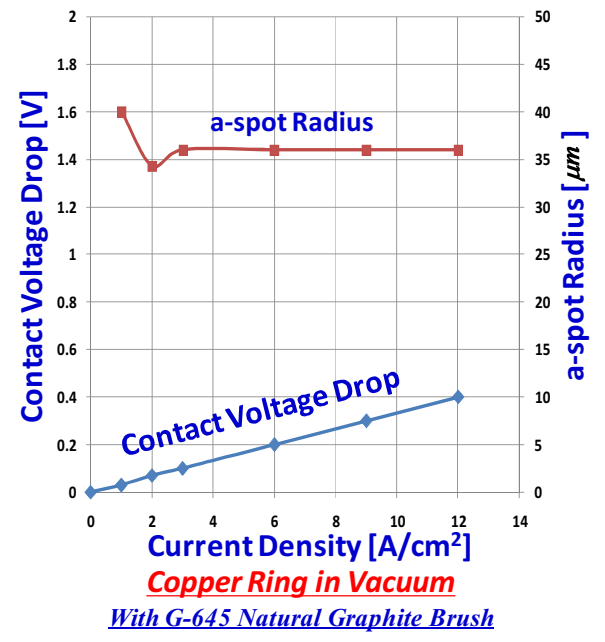

(a) Natural Graphite Brush and Copper Ring Operation, in $0.02 \mathrm{~Pa}$ Vacuum.

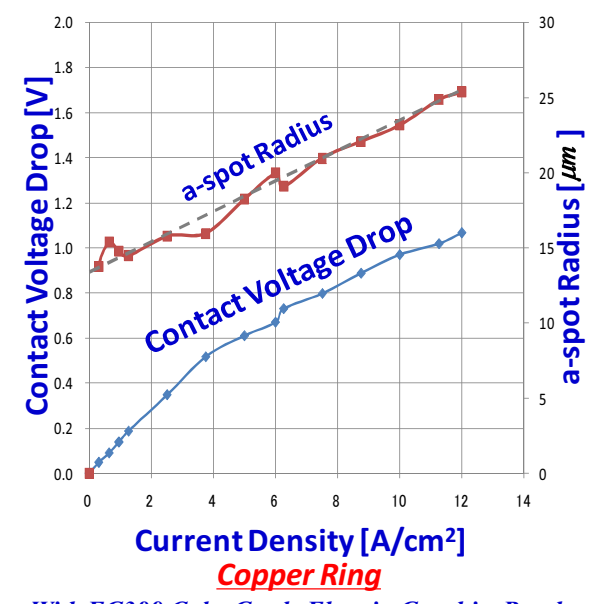

With EG389 Coke Grade Electric Graphite Brush

(b) Electric Graphite Brush and Copper Ring Operation, in Air.

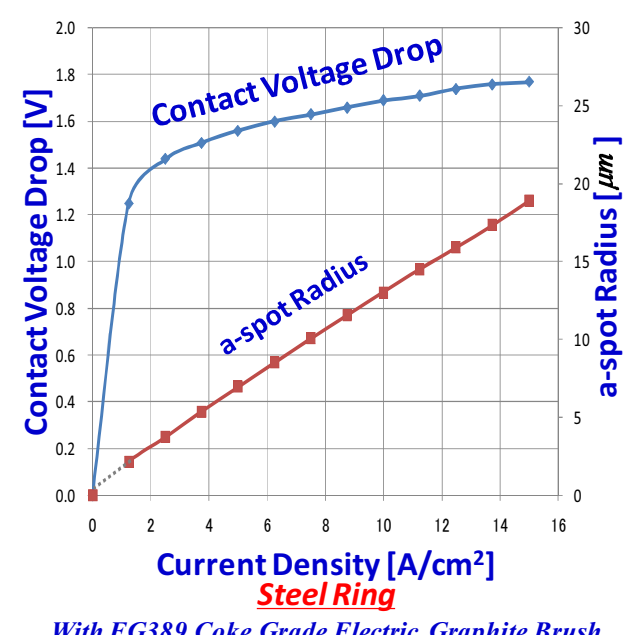

With EG389 Coke Grade Electric Graphite Brush

(c) Electric Graphite Brush and Steel Ring Operation, in Air.

Fig.-3 Contact Voltage Drop and a-spot Radius as a function of Brush Current Density. 


\section{INTEGRATION OF $\psi-\vartheta$ THEORY AND CONSTRICTION RESISTANCE CONCEPT}

As is described in the previous section, V-I characteristic at carbon / copper contact in vacuum, is linear, resulting almost constant a-spot contact area is kept for all range of brush current density shown in the figure. In this case, the aspot area, namely real contact area where brush current, is considered to be determined by mechanical origin.

On the other hand, V-I characteristic at carbon / steel contact in air, is heavily saturated, resulting a-spot contact area is linearly increased from according to the current density increase. Especially as is shown in Fig.-3(c), a-spot radius seems to become "zero" with brush current "zero".

Dr.R.Holm describes in his text book, "a-spot is able to carry current at a contact voltage below the "melting voltage" but above the "softening voltage".

According to this idea, such a hypothesis as "Some minimum brush current will be necessary for a-spot to carry current, in order to soften a-spot material and softened area will be increased with brush current increase.

As is well known, steel is elastic in room temperature and above several hundreds degree centigrade, state transition may occur resulting steel become plastic and "soft".

Based on these consideration, the carbon / steel contact point temperature study has been started.

\subsection{Constriction Resistance and Contact Column Model}

The constriction resistance theory only clarify the relation between a-spot radius dimension and the constriction resistance value.

However a-spot Temperature and temperature distribution or temperature gradient around the contact point, cannot be obtained by the constriction resistance theory.
On the other hand, $\psi-\vartheta$ theory only clarifies the relation between contact voltage drop distribution and temperature rise distribution.

However the contact column dimensions, such contact column radius, contact column cross section area and column length are not defined in the theory.

According to the $\psi-\vartheta$ theory, maximum temperature rise value has to be supposed, for the first time, then voltage drop distribution, so called $\psi, V$ and $U$ can be calculated.

However, in order to obtain contact temperature rise directly from measured V-I Characteristics, only sum of $\psi$, $V$ and $U$,namely $\psi+V+U$ is given.

Therefore in this paper, it is assumed that constriction resistance model shown in Fig.-1 can be entirely mapped on column model, as is illustrated in Fig.-5.

In this model, column radius shall be same as a-spot radius " $a$ ", which can be introduced by expression (2) in previous page based on R.Holm's constriction resistance, and the column length " $L$ " shall be $(\pi / 4) \cdot a$ so that the column resistance value may be same as the constriction resistance value $\boldsymbol{R}_{c}=\rho / 4 \boldsymbol{a}$.

Here, the current constriction and equipotential surface introduced by R.Holm shown in fig.-1, has been also confirmed by FEM analysis.

In fig.-6, shows an example of FEM analysis results. In fig.6 also, estimated current flow line is added.

As a result, it is considered that FEM result and R.Holm's illustration agree well each other.

It is considered that as to equipotantial line, isotherm line, current flow and heat flow, "constriction resistance model" is mapped on the "column model"

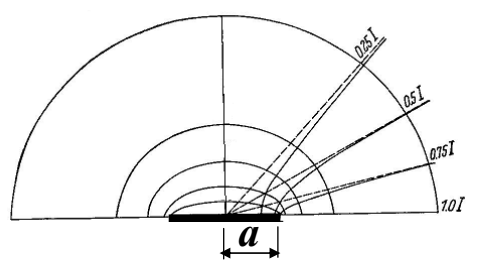

Current Constriction \& Equipotential Surfaces by $\mathrm{R}$. Holm

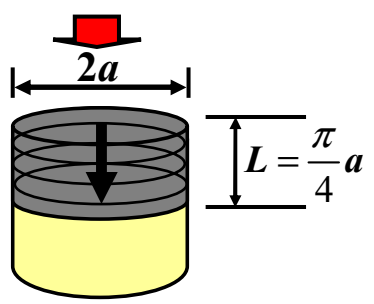

Here, it is assumed that

Current Constriction

\& Equipotential Surfaces can be mapped on Column Model

Fig.-4 Contact Column Model considering R.Holm's Constriction Resistance

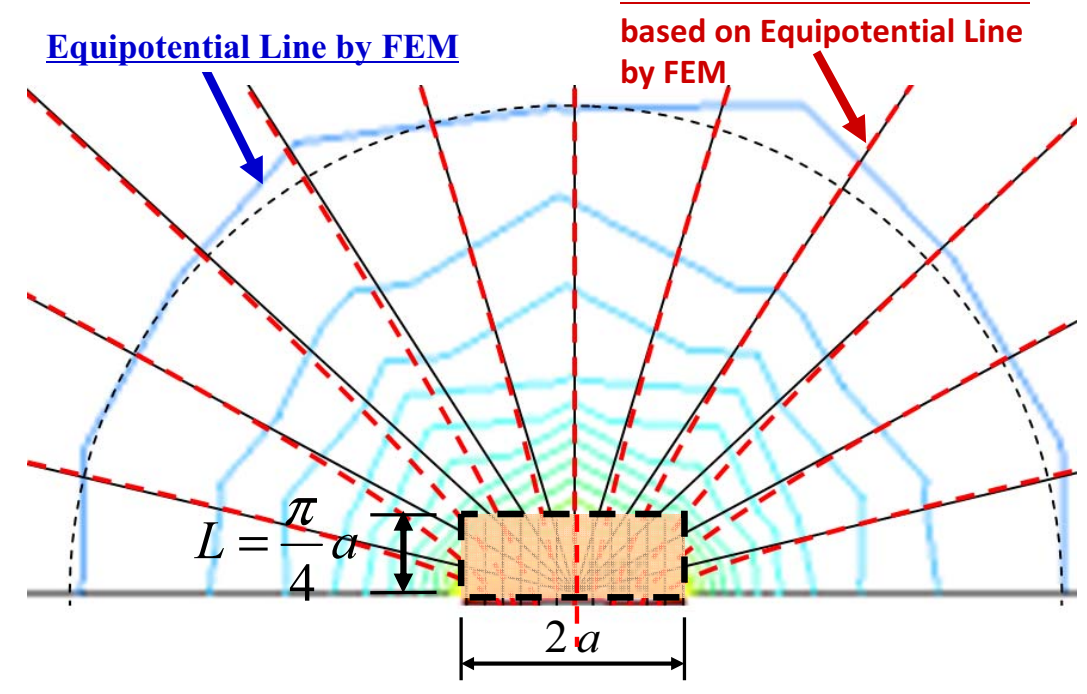

Fig.-5 Equipotentioal Lines by FEM and Estimated Current Flow Lines, with the Contact Column Model considering R.Holm's Constriction Resistance 
2.2 Contact Point Temperature Calculation based on measured V-I Characteristic

In this clause, how to calculate the contact point temperature and the maximum temperature at maximum temperature point in carbon brush, directly from measured V-I characteristic, is illustrated.

Calculated results by following procedures are referred to explain the peculiarly saturated V-I characteristics in case of steel ring.

The calculation procedure is consisting from following 7 steps.

(a) Contact voltage drop is obtained by subtracting brush bulk resistance voltage drop from measured voltage drop

(b) Determination of column radius and column length based on the contact voltage drop (a) and column current value is assumed one third of brush current.

(c) Assumption of maximum temperature point location

(d) Temperature gradient calculation between the maximum temperature point and brush side end of the column

(e) Temperature gradient calculation between the maximum temperature point and ring side end of the column

(f) The assumed maximum temperature point is modified, for example, the point is shifted by $1 \%$ of the column length, if the temperature gradient by (c) and the temperature gradient by (d), do not agree with each other. The process is repeated until the agreement is obtained.

(g) Contact voltage drop calculations, based on the contact column current and contact column resistances, for confirmation.

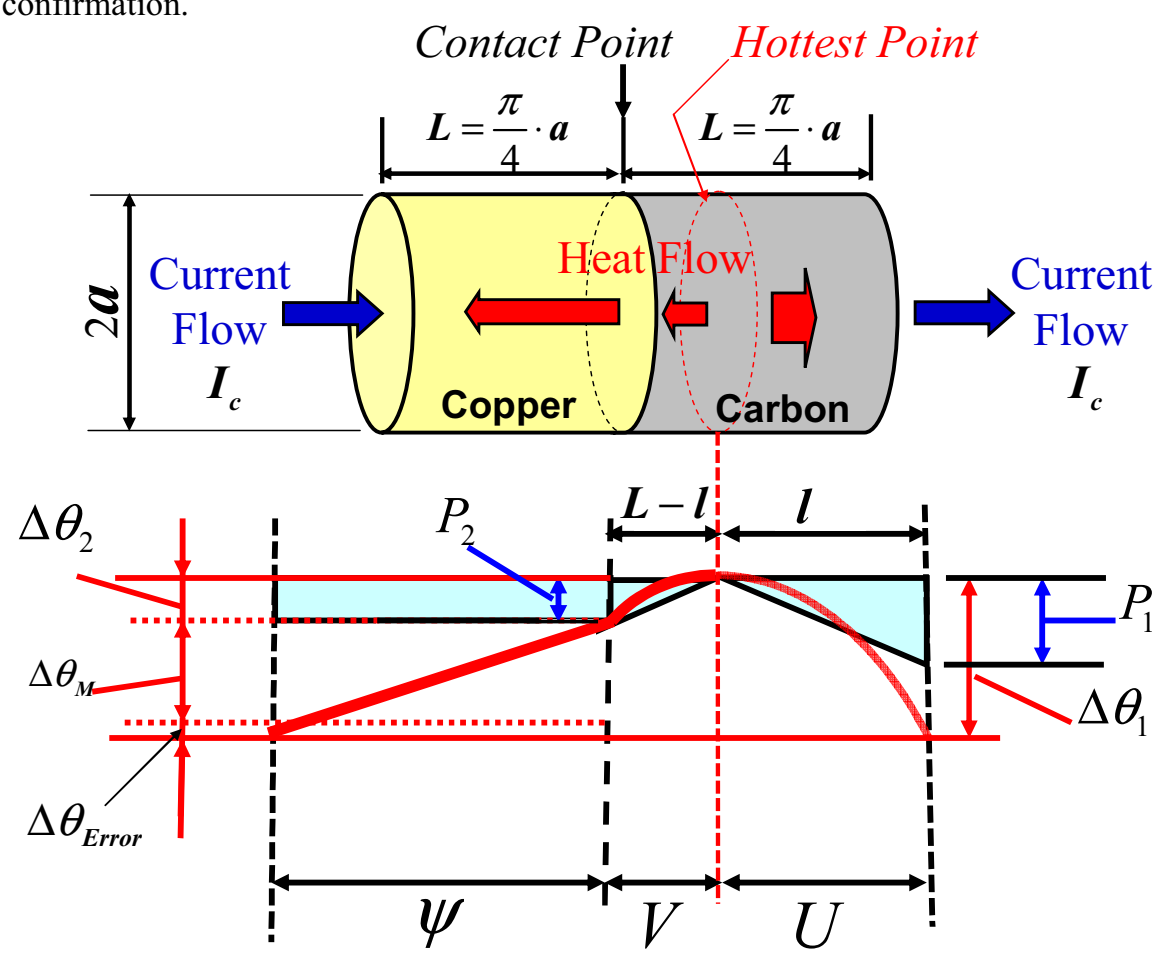

For the step (d), the temperature gradient $\Delta \theta_{1}$ can be calculated by

$$
\Delta \theta_{1}=\frac{\rho_{\text {brush }} \cdot I^{2}}{\lambda_{\text {brush }} \cdot S^{2}} \int_{0}^{l} x d x=\frac{\rho_{\text {brush }} \cdot I^{2} \cdot l^{2}}{2 \cdot \lambda_{\text {brush }} \cdot S^{2}}
$$

where

$\Delta \theta_{1}$ : Temperature gradient between maximum temp. point and the brush side end of the column $\rho_{\text {bursh }}$ : Electrical Resistivity for the brush

$\lambda_{\text {brush }}$ : Heat Conductivity for the brush

I : Contact column current

$\boldsymbol{S}$ : Contact column cross section area, namely $\boldsymbol{S}=\pi \cdot \boldsymbol{a}^{2}$

$l$ : Distance between maximum temperature point and brush side end of the column

$\boldsymbol{x}$ : Displacement in column axis direction

For the step (e) the temperature gradient $\Delta \theta_{2}$ and $\Delta \theta_{M}$ can be calculated by

$\Delta \theta_{2}=\frac{\rho_{\text {brush }} \cdot I^{2}}{\lambda_{\text {brush }} \cdot S^{2}} \int_{0}^{L-l} x d x=\frac{\rho_{\text {brush }} \cdot I^{2} \cdot(L-l)^{2}}{2 \cdot \lambda_{\text {brush }} \cdot S^{2}}$

where

$\Delta \theta_{2}$ : Temperature gradient between maximum temp. point and the contact point to the ring

$\boldsymbol{L} \quad$ : The column length. Namely $: \frac{\pi}{4} \cdot \boldsymbol{a}$

$\Delta \theta_{M}=\frac{\boldsymbol{L}}{\lambda_{\text {ring }} \cdot \boldsymbol{S}} \cdot \boldsymbol{P}_{2} \quad$ where heat flow $\boldsymbol{P}_{2}=\boldsymbol{I}^{2} \cdot \rho \cdot \frac{\boldsymbol{L}-\boldsymbol{l}}{\boldsymbol{S}}$

$\lambda_{\text {ring }}$ : Heat conductivity

Fig.-6 Temperature Gradient \& Contact Voltage Drop Calculation Scheme 


\section{CAlculation Results For the CONTACT Point TEMPERATURE}

This chapter describes calculated results by the modified $\psi-\vartheta$ theory, concerning with contact point temperature, maximum temperature and contact voltage drop.

The following results are in case of steel collector ring and copper ring, with world defact standard \& high resistance brush SA45 for large DC machines and typical low resistance brush EG389 for large AC machines.

In addition, the results are calculated for brush current densities between 0 and $15 \mathrm{~A} / \mathrm{cm}^{2}$.

In Fig.7 shows comparisons with the high resistance brush SA45 and low resistance brush EG389, for steel collector ring.

The results for the contact point temperature, maximum temperature can be shown for the brush current density up to $15 \mathrm{~A} / \mathrm{cm}^{2}$, in relation to the contact voltage drop saturation.

As is shown in Fig.7, even though brush contact voltage drop of the high resistance brush SA45 is higher than that of low resistance brush EG389, the contact point temperature and maximum temperature for the high resistance brush SA45 are lower than those for the low resistance brush EG389.

The contact point temperature rises for the low resistance brush EG389 are around 1,500 degree Centigrade, while those for high resistance brush SA45 are around 500 degree centigrade.

In case of the low resistance brush EG389, the contact point temperature exceeds well known "curie point temperature" 780 degree centigrade at $1 \mathrm{~A} / \mathrm{cm}^{2}$ of brush current density. Besides, the contact point temperature exceeds melting point temperature 1,540 degree centigrade at over $10 \mathrm{~A} / \mathrm{cm}^{2}$ of brush current density.

In case of high resistance brush SA45, the contact point temperature rise only exceeds so called softening temperature of 500 degree centigrade for all brush current density range.

In Fig. 8 shows comparisons with the steel ring \& the copper ring, for low resistance brush EG389.

As is shown in Fig.-8, in case of copper ring, the results of contact voltage drop are low, typically less than $1 \mathrm{~V}$ and almost linear V-I characteristic can be shown.

The max temperature rises for copper ring are around so called softening temperature of 200 degree Centigrade, and the contact point temperature rises for cooper ring are far less than the softening point temperature.

In Table. 2 summarizes the results for steel ring \& copper ring and for low resistance brush EG389 Brush \& high resistance brush SA45 Brush.

Table.1 Calculation Material Constant of Various Materials Grade

\begin{tabular}{|c||c|c|c|}
\hline Material Grade & $\begin{array}{c}\text { Electrical Resistivity } \\
{[\mu \Omega \cdot m]}\end{array}$ & $\begin{array}{c}\text { Heat Conductivity } \\
{[\mathrm{W} / \mathrm{m} \cdot \mathrm{K}]}\end{array}$ & Reference \\
\hline \hline Copper Ring & 0.017 & 380 & $\begin{array}{c}\text { About copper } \\
\text { which assumed commutator }\end{array}$ \\
\hline Steel Ring & 0.13 & 35 & $\begin{array}{c}\text { About steel material } \\
\text { which assumed steel ring }\end{array}$ \\
\hline $\begin{array}{c}\text { Coke } \\
\text { EG389 Brush }\end{array}$ & 17 & 20 & $\begin{array}{c}\text { About coke grade } \\
\text { electric graphite brush } \\
\text { EG389 of A.C Motor }\end{array}$ \\
\hline $\begin{array}{c}\text { Soot } \\
\text { SA45 Brush }\end{array}$ & 64 & 20 & $\begin{array}{c}\text { About soot grade } \\
\text { electric graphite brush } \\
\text { SA45 of D.C. Motor }\end{array}$ \\
\hline
\end{tabular}

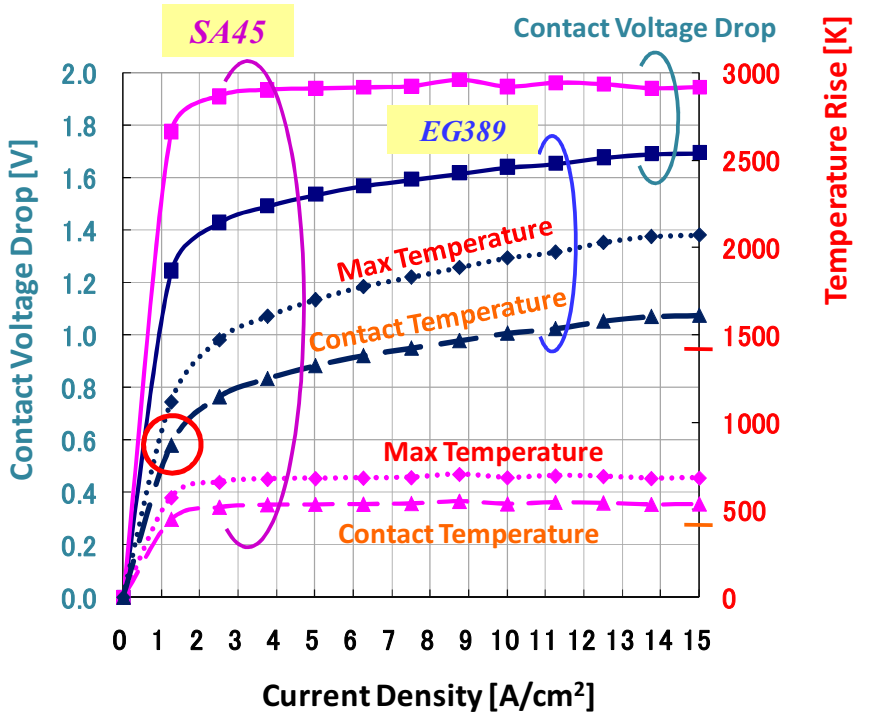

Fig.-7 Results of the measured Contact Voltage Drop and calculated Contact Point Temperature in comparison with high resistance brush SA45 \& low resistance brush EG389 for the Steel Ring

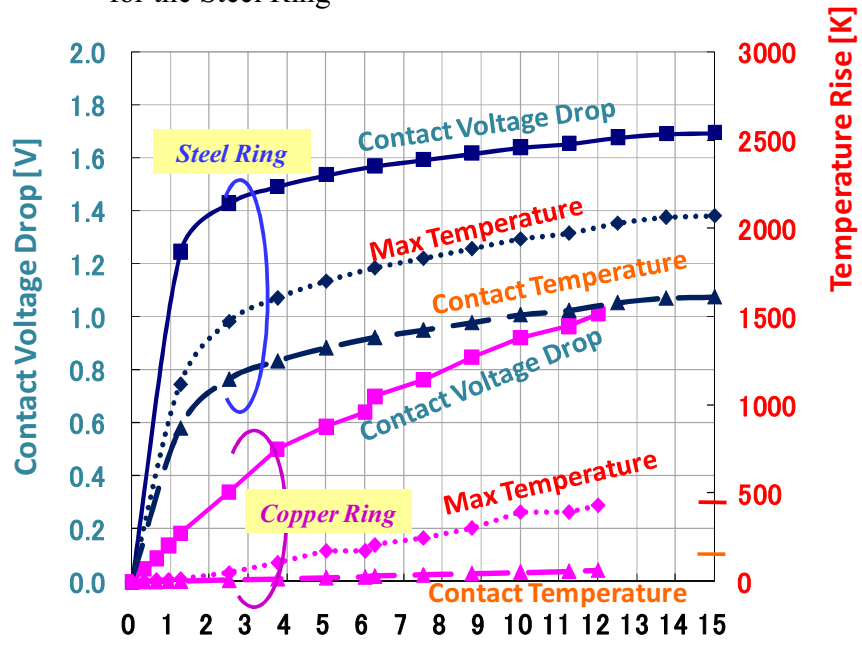

\section{Current Density [A/ $\left.\mathrm{cm}^{2}\right]$}

Fig.-8 Results of the measured Contact Voltage Drop and calculated Contact Point Temperature in comparison with Steel Ring \& Copper Ring for low resistance brush EG389

Table.2 Summary of Results for Steel ring \& Copper Ring and for low resistance brush EG389 \& high resistance brush SA45

\begin{tabular}{|c|c|c|}
\hline & $\begin{array}{c}\text { EG389-Brush } \\
16 \mu \Omega \cdot m\end{array}$ & $\begin{array}{l}\text { SA45-Brush } \\
64 \mu \Omega \cdot m\end{array}$ \\
\hline $\begin{array}{l}\text { Steel } \\
\text { Ring }\end{array}$ & $\begin{array}{c}\text { Contact Voltage Drop } \\
\mathbf{1 . 6 5}[\mathbf{V}] \\
\text { Contact Temperature } \\
\left.\left.\mathbf{1 6 0 0}\right|^{\circ} \mathrm{C}\right]\end{array}$ & 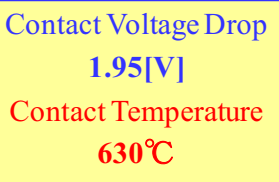 \\
\hline $\begin{array}{l}\text { Copper } \\
\text { Ring }\end{array}$ & $\begin{array}{c}\text { Contact Voltage Drop } \\
0.92[\mathbf{V}] \\
\text { Contact Temperature } \\
155\left[{ }^{\circ} \mathrm{C}\right]\end{array}$ & \\
\hline
\end{tabular}




\section{CONSIDERATION FOR THE RESULTS}

According to the long years of brush maintenance experience, fine threads, which are found on the brush sliding surface, are considered to be brush current conduction "trace".

If it is true, the trace width or the trace depth will have to be associating with the a-spot size.

Therefore, the fine thread dimensions have been measured by 3D scanning laser microscope.

Fig. -9 and 10 shows brush sliding surface photograph for EG389 and SA45 respectively, after brush seating operation with $5 \mathrm{~A}$.

In table. 3 and 4 , the fine thread dimensions, namely the trace width and the trace depth, which have been introduced by $3 \mathrm{D}$ scanning laser microscope analysis for fig.-9 and 10 , are compared with the calculated a-spot radius and the calculated maximum temperature point depth.

In case of the low resistance brush EG389, the calculated a-spot radius is $11.33 \mu \mathrm{m}$, while the estimated trace width by 3D scanning laser microscope is $11.27 \mu \mathrm{m}$ as 10 times averaging data.

In addition, the calculated maximum temperature point depth is $2.85 \mu \mathrm{m}$, while the estimated trace depth by 3D scanning laser microscope is $3.25 \mu \mathrm{m}$ for the brush EG389.

Therefore, the calculated a-spot radius agrees well with the estimated trace width, and the calculated maximum temperature point depth agrees well with the estimated trace depth.

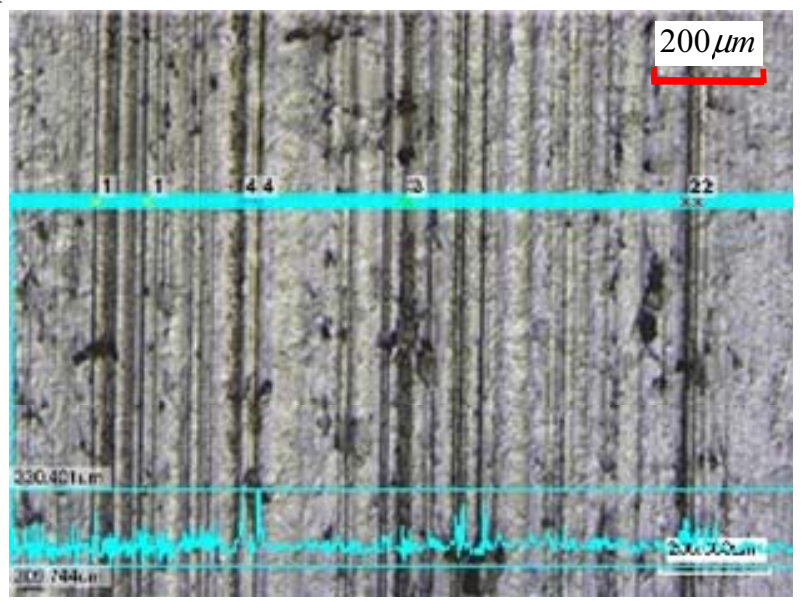

Fig.-9 EG389Brush Sliding Surface Photograph after Seating Operation with $5 \mathrm{~A}$

Table.3 Analysis Evaluation of Calculated Value \& 3D Scanning Laser Microscope with a-spot Radius and Max Temperature Point Depth by EG389 Brush

\begin{tabular}{|c|c|c|c|c|}
\hline \multirow{2}{*}{$\begin{array}{l}\text { Current } \\
\text { Density } \\
{\left[\mathrm{A} / \mathrm{cm}^{2}\right]}\end{array}$} & \multicolumn{2}{|c|}{ Calculated Value } & \multicolumn{2}{|c|}{$\begin{array}{c}\text { 3D Scanning } \\
\text { Laser Microscope }\end{array}$} \\
\hline & $\begin{array}{c}\text { a-spot } \\
\text { Radius } \\
{[\mu m]}\end{array}$ & $\begin{array}{c}\text { Max } \\
\text { Temperature } \\
\text { Point Depth } \\
{[\mu \mathrm{m}]} \\
\end{array}$ & $\begin{array}{c}\text { a-spot } \\
\text { Radius } \\
{[\mu m]}\end{array}$ & $\begin{array}{c}\text { Max } \\
\text { Temperature } \\
\text { Point Depth } \\
{[\mu \mathrm{m}]} \\
\end{array}$ \\
\hline 5.00 & 11.33 & 2.85 & 11.27 & 3.25 \\
\hline
\end{tabular}

In case of the high resistance brush SA45, the calculated aspot radius is $42.67 \mu \mathrm{m}$, while the estimated trace width by 3D scanning laser microscope is $45.08 \mu \mathrm{m}$ as 10 times averaging data.

In addition, the calculated maximum temperature point depth is $10.72 \mu \mathrm{m}$, while the estimated trace depth by 3D scanning laser microscope is $8.12 \mu \mathrm{m}$ for the brush SA45.

Therefore, the calculated a-spot radius agrees well with the estimated trace width, and the calculated maximum temperature point depth agrees well with the estimated trace depth.

It is considered that the width and the depth of the current trace found on the brush sliding contact surface, are in association with the a-spot radius and the maximum temperature point depth.

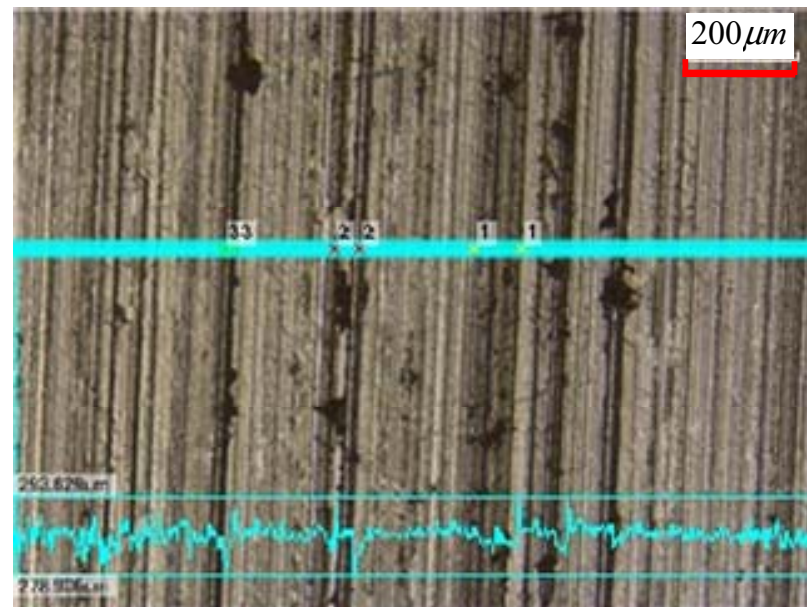

Fig.-10 SA45Brush Sliding Surface Photograph after Seating Operation with $5 \mathrm{~A}$

Table.4 Analysis Evaluation of Calculated Value \& 3D Scanning Laser Microscope with a-spot Radius and Max Temperature Point Depth by SA45 Brush

\begin{tabular}{|c||c|c||c|c|}
\hline \multirow{2}{*}{$\begin{array}{c}\text { Current } \\
\begin{array}{c}\text { Density } \\
{\left[\mathrm{A} / \mathrm{cm}^{2}\right]}\end{array}\end{array}$} & \multicolumn{2}{c||}{ Calculated Value } & \multicolumn{2}{c|}{$\begin{array}{c}\text { 3D Scanning } \\
\text { Laser Microscope }\end{array}$} \\
\cline { 2 - 5 } & $\begin{array}{c}\text { Radius } \\
{[\mu \mathrm{m}]}\end{array}$ & $\begin{array}{c}\text { Temperature } \\
\text { Point Depth } \\
{[\mu \mathrm{m}]}\end{array}$ & $\begin{array}{c}\text { a-spot } \\
\text { Radius } \\
{[\mu \mathrm{m}]}\end{array}$ & $\begin{array}{c}\text { Max } \\
\begin{array}{c}\text { Temperature } \\
\text { Point Depth } \\
{[\mu \mathrm{m}]}\end{array}\end{array}$ \\
\hline \hline 5.00 & 42.67 & 10.72 & 45.08 & 8.12 \\
\hline
\end{tabular}

Fig.11 shows a-spot radius expansion mechanism with a-spot current increase, in case of steel.

As is well known, in case of steel ring, material is elastic at less than the softening temperature of 500 degree centigrade.

It is considered that no stable contact can be existent or very high resistance between brush $\&$ ring can be existent, as far as the ring material is elastic.

As Dr. R.Holm implies in his text book, such as "More than softening voltage and less than melting voltage", the ring 
material has to be plastic for stable contact, even if the a-spot current is small.

That is why the contact point temperatures have to be higher than the softening temperature of steel, even if the aspot current is small.

Once, the contact area is established by softening of contact material "steel", the contact area is to be expanded by the softening area expansion caused by joule heat increase with a-spot current increase.

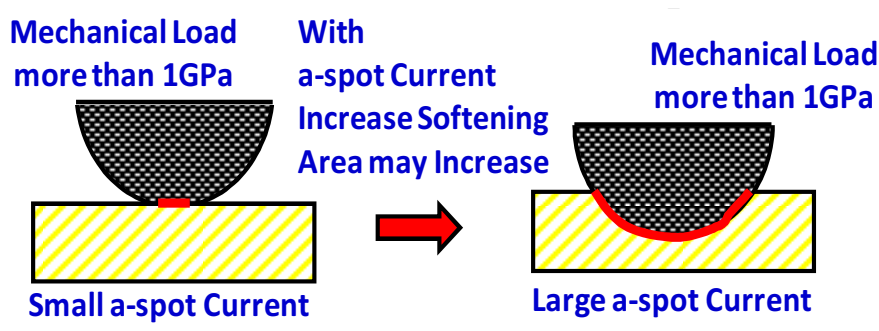

Fig.11 Consideration of Steel Ring in Contact Point stability

\section{CONCLUSION}

In this paper, following conclusions can be introduced, in association with the peculiarly saturated V-I characteristics for steel ring and almost linear V-I characteristics for copper ring.

\section{1) Features of the steel ring contact point}

The contact point temperature is to be more than softening temperature of ring side material, in order to make the contact material "steel" plastic and to obtain enough contact area for stable contact.

The contact area is to be expanded by the softening area increase with a-spot current joule heat increase, resulting peculiarly or heavily saturated V-I characteristics.

\section{2) Features of the copper ring contact point}

In case of copper, even at room temperature, the material is plastic. In this case, contact area for current conduction or aspot radius is considered to be determined almost by mechanical load, resulting almost linear V-I characteristics.

\section{3) Verification of improved $\psi-\vartheta$ theory}

For introducing above consideration, the improved $\psi-\vartheta$ theory has been introduced. It is considered that the improved $\psi-\vartheta$ theory can be verified by the agreement with the a-spot radius and the current trace width estimated by $3 \mathrm{D}$ scanning laser microscope for the threads on brush sliding surface.

\section{REFERENCES}

[1] N.Morita et. Al.52 ${ }^{\text {nd }}$ IEEE Holm Conference, 2006

[2] R.Holm: Electric Contact, Springer, pp7-26, 1967

[3] R.Holm: Electric Contact, Springer, pp60-86, 1967

[4] R.Holm: Electric Contact, Springer, pp.135-152, 1967

[5] Paul G.Slade:Electrical Contact, Marcel Dekker, pp75-83,1999
Makoto Takanezawa received his B.E.and M.E. degree in

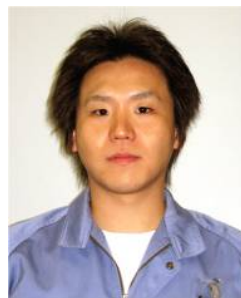
Electro Electrical Engineering of Nippon Institute of Technology, in 2005 and 2008 respectively. He is now a graduate school of Nippon Institute of Technology doctor couse. Now, he is engaged in a research about the issue of vibration by the electromechanical coupled system. $\mathrm{He}$ is a member of I.E.E. of Japan.

Takahiro Ueno received his B.E. and M.E. degree in

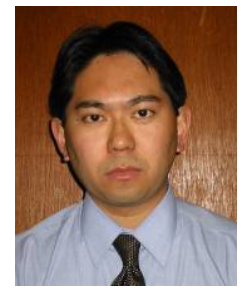
Electrical Engineering of Nippon Institute of Technology, Saitama, Japan, in 1992 and 1994 respectively, and Ph.D. degree from Electrical Engineering Department of Keio University, Yokohama, Japan, in 2002. Now he is a lecturer at Electrical Laboratory Center of Nippon Institute of Technology. His main filed of interest is a atmospheric influence of brush sliding contact. He is a member of I.E.E.E. and I.E.E. \& I.E.I.C.E. of Japan.

Noboru Morita received his B.E.,M.E. and Ph.D degree in

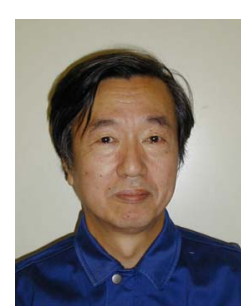
Electrical Engineering of Keio University, in 1970,1972 and 1986 respectively. He is now a professor of Nippon Institute of Technology. He has been an large dc machine designer for 24 years at TOSHIBA Corporation. He has been involved in the field of dc machine commutation analysis and carbon brush application technology for about 30 years. He is now a chairman of dc machine technical committee of I.E.E. of Japan. He is also a chairman of Carbon Brush Committee of 117-D-J.S.P.S of Japan.

Toru Otaka received his B.E., M.E. degrees in Electrical

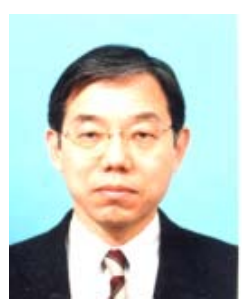
Engineering from Waseda University, Tokyo, Japan, in 1975, 1977 respectively. $\mathrm{He}$ is now a Chief Engineer of Generator Department at Keihin Product Operation of TOSHIBA Corporation. He is also a Counsel Member in Rotating Machine Technical Committee of I.E.E. of Japan.

Daisuke Hiramatsu received an M.E.degree in electrical

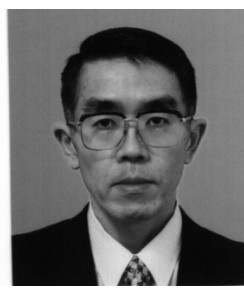
engineering from Keio University in 1980 and joined Toshiba Corp. He was engaged in the design of synchronous generators and motors. He is a Professional Engineer authorized by the Japanese Government and holds a Ph.D. degree in electrical engineering. 


\title{
Electromechanics of a Collection of Current Carrying Cantilevered Fibers Sliding against a Plane Moving Surface in a Three Dimensional Magnetic Field
}

\author{
G. S. Erwin and N. A. Sondergaard
}

\begin{abstract}
This paper builds on a previously developed model which describes the electromechanical behavior of a single, cantilevered current carrying fiber with arbitrary physical orientation relative to a moving surface in a three dimensional arbitrary magnetic field. In the present paper we extend that model to a collection of similar fibers spatially separated along the moving surface. The collection of fibers carries a transport current and an external mechanical force is applied to the sum of the fibers to keep their free ends in electrical contact with the moving surface. The contact resistance is an inverse function of the load and depends on whether the contact spots are elastic or plastic. A frictional force on the fibers in the direction of motion results. Furthermore, since the current carrying fibers are in the presence of a 3 component arbitrary magnetic field, a distributed Lorentz force along the length of an individual fiber exists which distorts the shape of the fibers and changes the individual contact load and contact resistance while keeping the sum of the loads the same. Furthermore, since the moving surface is cutting net flux, there exist different potentials at different fibers and circulating currents between these fibers result. These circulating currents are of different magnitudes and in opposite directions in individual fibers thus adding or subtracting from the transport current while keeping the total transport current the same. As a result of the circulating currents, the Lorentz forces are modified and the individual contact loads and resistances change while keeping the total mechanical load on and total transport current through all the fibers constant. This leads to a distorted current and mechanical load profile in the collection of fibers. For a given constant magnetic field and direction of moving surface, the direction of transport current results in differing distributions of current and mechanical load profile. Examples are presented.
\end{abstract}

\section{INTRODUCTION}

In a previous internal investigation ${ }^{1}$ we derived the formalism for analyzing the electromechanics of a single, current carrying fiber sliding against a plane in the presence of a large magnetic field of arbitrary orientation. In that study, which incorporated results found in previous studies ${ }^{2-7}$, the current and the mechanical contact force at the fiber tip-slider interface are specified and do not change but the fiber itself, changes shape, deflects and the applied electric potential necessary to drive the current varies with conditions such as slider speed and differing magnetic field conditions. We

This work was sponsored by the ONR

G.S. Erwin is with NSWCCD, USA

N. A. Sondergaard is with NSWCCD, USA discovered a bifurcation in the solution space of such fibers which leads to a history dependant equilibrium condition.

In the present paper we have extended that investigation to include a collection of fibers which carry a constant total transport current and are held against the slider by a constant total force. The currents and tip forces of the individual fibers are allowed to vary, but the sum over the fibers remains constant. The purpose of this model is to generate base-state results for comparison to the results from future models which will include effects that are neglected here. As examples, (1) the present model assumes that there is no wear, while a future model will include the change in length of each fiber due to the wear at its tip, and (2) the present model uses a simple variation of the contact resistance with the normal force between the fiber tip and the rotor surface, while a future model will include variations of the contact resistance with a number of other variables. This initial model uses the single fiber model which is presented in Reference 1. The combination of the present brush model with those future single-fiber models will generate other extensions of the present basic brush model.

\section{Assumptions}

We assume that the slider (e.g. a rotor) and fixed (e.g. stator) surfaces are planar and are parallel to each other. For the single-fiber model, we assumed that the rotor surface is effectively planar over the distance traveled by one fiber tip for all operational conditions. In the present study we assume that the rotor surface is planar over the length and breadth of the brush, which is a somewhat stronger assumption. Figure 1a, from reference 1, shows a free body diagram of the forces acting on an arbitrary cross-section of a single fiber and the fiber tip. Figure $1 \mathrm{~b}$ presents simple graphical representation of the collection of fibers and the rotor-stator system of the present study. Finally, Figure 1c depicts the deformation of two sample fibers located at differing positions in the row of fibers, the distortion resulting from carrying differing currents, both magnitude and direction, in the applied magnetic field .

For the brush model, we use a Cartesian coordinate system with the $y$ axis perpendicular to the rotor and stator surfaces, with the $x$ axis in the direction of the rotor velocity, i.e., in the azimuthal direction, and with the origin at the center of the base of brush at the stator surface. We assume that the 
undeformed brush has a rectangular cross section. For the base of the brush at the stator surface, $-L_{x} \leq x \leq L_{x},-L_{z} \leq z \leq L_{z}$, where $2 L_{x}$ is the azimuthal dimension of the brush and $2 L_{z}$ is the axial dimension of the brush for a radially oriented brush or the radial dimension for an axially oriented brush.
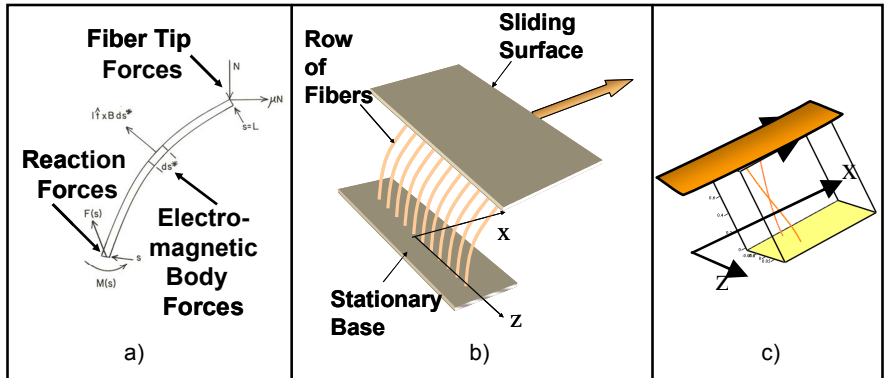

Figure 1 a)Tip and distributed EM Forces on Individual Fiber from Ref. 1. b) Row of fibers attached to fixed base with tips contacting sliding surface c) Example of two fibers displaying deformations resulting from transporting differing currents in the applied magnetic field.

The single-fiber model uses a local Cartesian coordinate system for each fiber with the origin at the root of the fiber at the stator surface. Since the single-fiber model is used in the present brush model, we need the relationships between the brush coordinates and the local fiber coordinates. If the root of a given fiber is located at $\left(x_{0}, 0, z_{0}\right)$ in the brush coordinate system, then

$$
x_{\text {brush }}=x_{0}+x_{\text {fiber }}, \quad y_{\text {brush }}=y_{\text {fiber }}, \quad z_{\text {brush }}=z_{0}+z_{\text {fiber }} \text {, }
$$
where $x_{\text {brush }}, y_{\text {brush }}, z_{\text {brush }}$ are the brush coordinates for the centerline of this fiber, while $x_{\text {fiber }}, y_{\text {fiber }}, z_{\text {fiber }}$ are the local coordinates for this fiber in the single-fiber model. Through all of this paper except the Results section, $x, y, z$ will denote the brush coordinates. In the Results section, all of the results for the entire brush involve the dimensional brush coordinates $x, y$, $z$, while all of the results for a single fiber involve the dimensionless, local fiber coordinates $x, y, z$, normalized by the length $L$ of the fiber.

We assume that all the fibers in the brush have the same length $L$, the same diameter $d$, the same elastic modulus $E$, and the same electrical resistivity, $\rho_{e}$. We also assume that all the undeformed fibers are parallel. In other words, all fibers have the same values of $\theta_{0}$ and $\alpha_{0}$, where $\theta_{0}$ is the angle between the undeformed fiber and $\hat{\mathbf{x}}$, while $\alpha_{0}$ is the angle between the projection of the undeformed fiber onto the $y-z$ plane and $\hat{\mathbf{y}}$. Here $\hat{\mathbf{x}}, \hat{\mathbf{y}}, \hat{\mathbf{z}}$ are unit vectors for both sets of Cartesian coordinates.

We assume that the bending stresses in all the fibers are less than the yield stress, so that all deformations are elastic. We assume that the fibers do not touch each other. The first consequence of this assumption is that there are no contact forces between fibers. (A subsequent model, to be published later, incorporates fiber-fiber interactions and shows that although contact and forces can exist between some of the tips of the fibers, the overall effect on contact resistance and friction is minimal). The second consequence is that there is no direct flow of electric current between fibers, so that the electric current $I$ through a given fiber is the same over the entire length of this fiber. We assume that the magnetic field, $\mathbf{B}=B_{x} \hat{\mathbf{x}}+B_{y} \hat{\mathbf{y}}+B_{z} \hat{\mathbf{z}}$, is uniform over the entire volume occupied by the brush. In the single-fiber model, we assumed that $\mathbf{B}$ is uniform over the length of each fiber, so that our brush assumption is stronger. However, it would be relatively easy to modify the present brush model to treat a magnetic field which varies with either $x$ or $z$ across the brush, but which is effectively uniform over the length of each fiber. A good example would be the variation of the azimuthal component of the magnetic field $B_{x}$ with $z$ due to the electric current through the brush.

We assume that the only forces on each fiber are the tip force, $\mu N \hat{\mathbf{x}}-N \hat{\mathbf{y}}$, and the distributed electromagnetic body force per unit of fiber length, $(\hat{I}) \times \mathbf{B}$. Here $\mu$ is the coefficient of sliding friction, $N$ is the normal force between the fiber tip and the rotor surface, and $\hat{\mathbf{t}}$ is the local unit vector which is tangent to the centerline of a fiber at each point along the fiber from its root at the stator surface to its tip at the rotor surface. We also assume that the tips of the fibers remain in contact with the rotor surface at all times and are held in place by a spring force applied to the base of the brush.

Other assumptions are presented in the problem formulation.

\section{ELECTRICAL RESISTANCES}

We assume that the rotor and stator are perfect electrical conductors, i.e., their electrical resistivities are effectively zero. Since the stator does not move, the electric potential function $\phi$ is uniform in the stator. We use the stator voltage as the datum, so that $\phi=0$ in the stator. Since the rotor moves with a constant uniform velocity $V_{x}$ and since there is a uniform magnetic field, there is a linear variation of $\phi$ in the rotor. At the rotor surface, $\phi=\phi_{0}+V_{x} B_{y} z$, where $\phi_{0}$ is the voltage difference across the center of the brush. The value of $\phi_{0}$ is determined by the condition that the sum of the electric currents through all of the fibers must equal the specified transport current, $I_{\text {trans }}$.

The electrical resistance of each fiber is in series with the contact resistance for the gap between the fiber tip and the rotor surface. The resistance of the fiber is $\rho_{e} L / \pi R^{2}$, where $R=d / 2$ is the radius of each fiber. Reference 2 presented a formula for the contact resistance of the entire brush times its cross sectional area,

$$
R_{c} A_{b}=\frac{3.4 \times 10^{-9}\left[\Omega m^{2}\right]}{K^{2} f \beta^{2 / 3}} .
$$


Here $\beta=p / p_{\text {trans }}$, where $p$ is the normal force per unit area across the interface between the brush and the rotor surface, while $p_{\text {trans }}$ is the value of $p$ for the elastic-plastic transition of the contact point on each fiber tip. Equation (2) assumes that the contact spot is elastic, $\beta<1$. Reference 2 defined $f$ as the fiber volume fraction. However, it was assumed that the undeformed fibers are perpendicular to the rotor surface, i.e., $\theta_{0}=\pi / 2, \alpha_{0}=0$, while we also want to treat undeformed fibers with other orientations. The only role of $f$ is the determination of the number of fiber contacts per unit area of the rotor surface, $n^{*}$. Reference 2 used the formula $n^{*}=f / \pi R^{2}$, while the formula which is valid for all undeformed-fiber orientations is

$$
n^{*}=\frac{v_{f} \sin \theta_{0} \cos \alpha_{0}}{\pi R^{2}},
$$

where we use $v_{f}$ to denote the fiber volume fraction. Thus we substitute $f=v_{f} \sin \theta_{0} \cos \alpha_{0}$ into the formulas of Reference 2. Reference 2 presented the formula

$$
K^{2}=1+7[\mu m] /\left(\beta^{2 / 3} d\right)
$$

as a correction which we set $\mathrm{K}^{2}=1$ to avoid finite resistance when $\beta$ (load) is 0 . Therefore the equation we use for the brush contact resistance times brush area is

$$
R_{c} A_{b}=\frac{3.4 \times 10^{-9}\left[\Omega m^{2}\right]}{v_{f} \sin \theta_{0} \cos \alpha_{0} \beta^{2 / 3}} .
$$

Reference 2 gave the value $p_{\text {trans }}=1.5 \times 10^{5} f[\mathrm{~Pa}]$ for copper fibers, and we use this value with $f=v_{f} \sin \theta_{0} \cos \alpha_{0}$.

Reference 2 treated brushes with uniform values of $\beta$ and uniform electric current distributions. Here the voltage variation $V_{x} B_{y} z$ leads to a very nonuniform electric current distribution, and then the electromagnetic body forces lead to a very nonuniform distribution of $\beta$ over the rotor-brush interface. Therefore we want the contact resistance for a single fiber rather than $R_{c} A_{b}$, and we want the normal tip force $N_{\text {trans }}$ for the elastic-plastic transition of the contact spot rather than $p_{\text {trans }}$. The contact resistance for a single fiber is given by $\left(R_{c} A_{b}\right) n^{*}$, where $n^{*}$ is given by equation (3), while $N_{\text {trans }}=p_{\text {trans }} / n^{*}$. Therefore the total resistance for a single fiber is

$$
R_{f}=\frac{1}{\pi R^{2}}\left[\left(3.4 \times 10^{-9}\right) \beta^{-2 / 3}+\rho_{e} L\right] .
$$

The electric current through each fiber from the stator to the rotor is given by

$$
I=-\frac{\pi R^{2}\left(\phi_{0}+V_{x} B_{y} z\right)}{\left[\left(3.4 \times 10^{-9}\right) \beta^{-2 / 3}+\rho_{e} L\right]},
$$

where $z$ is evaluated at the location of each fiber. For the single-fiber model, $I$ appears in the dimensionless parameters,

$$
\beta_{x}=\frac{I B_{x} L^{3}}{E I^{\prime}}, \quad \beta_{y}=\frac{I B_{y} L^{3}}{E I^{\prime}}, \quad \beta_{z}=\frac{I B_{z} L^{3}}{E I^{\prime}},
$$

where $I^{\prime}=\pi R^{4} / 4$ is the second moment of area of the fiber cross section. For copper, $N_{\text {trans }}=\left(1.5 \times 10^{5}\right) \pi R^{2}[N]$. In the single-fiber model, the normal force $N$ appears in the dimensionless parameter $\quad v=N L^{2} / E I^{\prime}, \quad$ so $v_{\text {trans }}=\left(1.5 \times 10^{5}\right) \pi R^{2} L^{2} / E I^{\prime}$ and $\beta=v / \nu_{\text {trans }}$. Note $\beta_{\mathrm{x}}, \beta_{\mathrm{y},} \beta_{\mathrm{z}}$, the components of the Lorentz force to fiber stiffness force are different from the unsubscripted $\beta$, which is the contact force to the elastic-plastic yield force.

\section{ApProACH}

For the present basic brush, the only variation between fibers arises from the fact that the electric current through each fiber is a function of $z$, as indicated by equation (7). In other words, if $B_{y}=0$, then there is no variation between fibers and $\beta$ is uniform over the brush-rotor interface. We are only interested in cases with $B_{y} \neq 0$, so that there is a significant eddy current driven by the voltage variation $V_{x} B_{y} z$. There is no variation in $x$. At any specific value of $z$, the entire row of fibers for $-L_{x} \leq x \leq L_{x}$ behaves in the same way, while an adjacent row of fibers at another value of $z$ may behave in a very different way from the first row. We divide $-L_{z} \leq z \leq L_{z}$ into $N Z$ equal intervals with $\Delta z=2 L_{z} / N Z$. We can insure that each interval contains only one row of fibers by setting $N Z$ equal to the number of rows of fibers across the $z$ dimension of the brush. However this would be computationally wasteful if we can obtain accurate results with two or three rows of fibers in each $z$ interval. We assume that all rows of fibers in one interval have the same values of $v, \beta_{x}, \beta_{y}, \beta_{z}$ and the same threedimensional deformations. Since the only variation between fibers arises from the $z$ variation of $I$ in equation (7), we evaluate equation (7) at the midpoint of each interval,

$$
z_{K}=-L_{z}+\left(K-\frac{1}{2}\right) \Delta z, \quad \text { for } K=1 \text { to } N Z \text {. }
$$

We assume that what we are calling the stator is mounted on a spring, (1) which keeps the stator surface parallel to the rotor surface, (2) which allows motion of the stator surface in the $y$ direction, and (3) which produces a specified normal force between the brush and the rotor surface. We represent the specified force by the average value of $\beta$, namely $\bar{\beta}$. With the $z$ variation of $I$, the electromagnetic body forces push the tips of some fibers against the rotor surface and pull others away from the rotor surface, leading to a $z$ variation of $v$. The dimensionless normal force on the tip of each fiber in the $\Delta z$ centered at $z_{K}$ is $v_{K}$. The specified force produced by the spring supporting the stator is represented by the condition

$$
\sum_{K=1}^{N Z} v_{K}=N Z \times \bar{\beta} \times v_{\text {trans }} .
$$

We apply the single-fiber model for the fibers in each of the $N Z$ intervals. For each interval, the inputs to the single-fiber model 
are $v_{K}, \beta_{x K}, \beta_{y K}, \beta_{z K}, \theta_{0}, \alpha_{0}$ and the outputs are $x_{K}(s), y_{K}(s), z_{K}(s)$. Here $x_{K}, y_{K}, z_{K}$ are the local coordinates normalized by $L$, for each fiber in the $K$-th interval, while $s$ is the dimensionless distance along the fiber from its root fixed into the stator at $s=0$ to its tip sliding along the rotor surface at $s=1$. With $y_{t K}=y_{K}(1)$ as the dimensionless $y$ coordinate at the tip of each fiber in the $K$-th interval, the condition that the rotor and stator surfaces remain parallel is

$$
y_{t K}=y_{t 0}, \text { for } K=1 \text { to } N Z \text {, }
$$

where $y_{t 0}$ is an unknown constant which must be determined as part of the solution.

The electric current through each fiber in the $K$-th interval is given by equation (7) with $z=z_{K}$ and with $\beta=\beta_{K}=v_{K} / v_{\text {trans }}$. The electric current per unit area of the brush-rotor interface is $I n *$, where $n^{*}$ is given by equation (3). Therefore the relationship between $I_{\text {trans }}$ and $\phi_{0}$ is

$$
I_{\text {trans }}=-2 L_{x}(\Delta z) v_{f} \sin \theta_{0} \cos \alpha_{0} \sum_{K=1}^{N Z} \frac{\left(\phi_{0}+V_{x} B_{y} z_{K}\right)}{\left[\left(3.4 \times 10^{-9}\right) \beta_{K}^{-2 / 3}+\rho_{e} L\right]} \text {. }
$$

The determination of the $N Z$ values of $\beta_{K}=v_{K} / \nu_{\text {trans }}$ requires an iterative solution, and the value of $\phi_{0}$ must be determined for each iteration from the equations

$$
\begin{aligned}
& Q_{1}=\sum_{K=1}^{N Z}\left[\left(3.4 \times 10^{-9}\right) \beta_{K}^{-2 / 3}+\rho_{e} L\right]^{-1}, \\
& Q_{2}=\sum_{K=1}^{N Z} z_{K}\left[\left(3.4 \times 10^{-9}\right) \beta_{K}^{-2 / 3}+\rho_{e} L\right]^{-1}, \\
& \phi_{0}=-\frac{1}{Q_{1}}\left[V_{x} B_{y} Q_{2}+\frac{I_{\text {trans }}}{2 L_{x}(\Delta z) v_{f} \sin \theta_{0} \cos \alpha_{0}}\right] .
\end{aligned}
$$

Each iteration begins with estimates of the values of $v_{K}$ for $K=1$ to $N Z$, which satisfy equation (10). First, $\beta_{K}=v_{K} / v_{\text {trans }}$. Second, equations (13) determine $\phi_{0}$. Third, equation (7) with $z=z_{K}$ and $\beta=\beta_{K}$ gives the electric current $I_{K}$ through each fiber in the $K$-th interval, so that equations (8) give the values of $\beta_{x K}, \beta_{y K}, \beta_{z K}$. Fourth, the single-fiber model is applied for each of the $N Z$ intervals. The inputs are $v_{K}, \beta_{x K}, \beta_{y K}, \beta_{z K}, \theta_{0}, \alpha_{0}$ and the outputs are $x_{K}(s), y_{K}(s), z_{K}(s)$. The single-fiber model also requires an iterative solution, so that there are $N Z$ single-fiber iterative solutions inside each brush iteration. For the iterative solution, the only output of interest is $y_{t K}=y_{K}(1)$. In general, the solution for each iteration is not the correct solution because the values of $y_{t K}$ for $K=1$ to $N Z$ are not the same, so that the $N Z$ conditions in equation (11) are not satisfied. The final steps in a brush iteration are the determination of improved estimates of the values of $v_{K}$ for $K=1$ to $N Z$, which satisfy equation (10). Here improved means that the next iteration using these values of $v_{K}$ leads to a smaller value of the error defined by Error $=\sum_{K=1}^{N Z}\left|y_{t K}-y_{t 0}\right|$.

In order to determine $y_{t 0}$ and the improved estimates of $v_{K}$, we assume a linear relationship between the correction to $v_{K}$ and the error $\left(y_{t 0}-y_{t K}\right)$ for the $K$-th interval,

$$
v_{K}^{(\text {next })}=v_{K}+\kappa\left(y_{t 0}-y_{t K}\right),
$$

where $\kappa$ is a constant. Since we want $\boldsymbol{v}_{K}^{(\text {next })}$ to satisfy equation (10), we introduce equation (15) into equation (10) and solve for $y_{t 0}$,

$$
y_{t 0}=\frac{1}{N Z} \sum_{K=1}^{N Z} y_{t K}+\frac{1}{\kappa}\left[\bar{\beta} \times v_{\text {trans }}-\frac{1}{N Z} \sum_{K=1}^{N Z} v_{K}\right] \text {. }
$$

If the values of $v_{K}$ satisfy equation (10), then $y_{t 0}$ is simply the average of the values of $y_{t K}$. The only reason the values of $v_{K}$ for a given iteration would not satisfy equation (10) is that any negative value of $v_{K}$ must be replaced by a small positive value, e.g., $10^{-14}$, in order to compute the values of $\beta_{K}^{-2 / 3}$. We use equation (16) in order to insure that $v_{K}$ satisfy equation (10) in the iteration after one where a negative value of $v_{K}$ occurred. Once $y_{t 0}$ has been determined, the next set of estimates of $v_{K}$ is given by equation (15). The numerical results to date have indicated that equation (15) may overestimate the correction to $v_{K}$ when the fibers are severely loaded and the deformations are large. This overestimation leads to oscillations in the values of $v_{K}$ and $y_{t 0}$ with successive iterations. Therefore we introduce a relaxation factor $w$, so that $\quad v_{K}^{(\text {next })}=v_{K}+w \kappa\left[y_{t 0}-y_{t K}\right]$.

For small deformations, $w=1$ leads to monotonic convergence of the brush iterations. For the large deformations discussed in the Results section, we found that $w=0.3$ eliminated oscillations between successive iterations and always led to a converged solution. The constant $\kappa$ is an estimate of $\Delta v / \Delta y_{t}$. For one fiber configuration, an increase in $v$ of one led to a decrease of $y_{t}$ of -0.016 , so that $\kappa=-62.5$.

The total Joulean heating in the brush is given by

$$
2 L_{x}(\Delta z) \sum_{K=1}^{N Z}\left(\phi_{0}+V_{x} B_{y} z_{K}\right)^{2}\left[\left(3.4 \times 10^{-9}\right) \beta_{K}^{-2 / 3}+\rho_{e} L\right]^{-1} \text {. }
$$

\section{SOME Typical Results}

One case is discussed here in order to illustrate the capability of the brush model. The fibers are copper with $\rho_{e}=1.7 \times 10^{-8} \Omega-m$ and $E=1.2 \times 10^{11} \mathrm{~Pa}$. The dimensions are $R=25 \mu \mathrm{m}, \quad L=1.27 \mathrm{~cm}, \quad L_{x}=1.0 \mathrm{~cm}$, and $L_{z}=0.5 \mathrm{~cm}$. The undeformed fibers are perpendicular to the rotor and stator 
surfaces, i.e., $\theta_{0}=\pi / 2 \mathrm{rad}$ and $\alpha_{0}=0$, the fiber volume fraction $v_{f}=0.17$, and the coefficient of sliding friction $\mu=0.32$. We treat a brush with $V_{x}=25 \mathrm{~m} / \mathrm{s}, B_{x}=0, B_{y}=1.1 T, B_{z}=0.7 T, \bar{\beta}=0.63 \quad$ and various values of the transport current, $I_{\text {trans }}$. Experience with the single-fiber model indicates that a history dependence is possible, so we follow a specific history. Throughout the history, $V_{x}=25 \mathrm{~m} / \mathrm{s}$ and $B_{x}=0$. We incrementally increase $B_{y}, B_{z}$ and $\bar{\beta}$ with $I_{\text {trans }}=0$ :

(a) $B_{y}=B_{z}=0.1 k, \bar{\beta}=0.09 k, k=1$ to 7 and then (b) $B_{z}=0.7 T, \bar{\beta}=0.63, B_{y}=0.7+0.1 k, k=1$ to 4 . For our base state, $B_{y}=1.1 T, B_{z}=0.7 T, \bar{\beta}=0.63, I_{\text {trans }}=0$. From our base state, we vary $I_{\text {trans }}$ with $B_{y}=1.1 T, B_{z}=0.7 T, \bar{\beta}=0.63$. We first increase $I_{\text {trans }}$ from 0 to $350 \mathrm{~A}$. We then decrease $I_{\text {trans }}$ from $350 \mathrm{~A}$ to $-350 \mathrm{~A}$. Finally we increase $I_{\text {trans }}$ from $-350 \mathrm{~A}$ to 0 . For all these calculations $N Z=40$ and $\kappa=-30$. The relaxation factor $w$ is decreased from 1.0 as $B_{y}, B_{z}, \bar{\beta}$ are incrementally increased from 0 , and $w=0.3$ for all the calculations for $B_{y}=1.1 T, B_{z}=0.7 T, \bar{\beta}=0.63$. The iterative single-fiber model with backward integration converges rapidly, generally reaching a relative error of $10^{-12}$ in 5-10 iterations. The number of brush iterations to achieve a relative error of $10^{-8}$ varies greatly. For the case considered here, a $10 \mathrm{~A}$ change in $I_{\text {trans }}$ may lead to abrupt changes in the values of $v_{K}$ and in the deformed shapes of some fibers, leading to significant changes for all the fibers through equation (10). For such abrupt changes, as many as 700 brush iterations were required to achieve convergence to eight significant figures. The number of integrations for single fibers is equal to the number of brush iterations times $N Z$ times the number of single-fiber iterations. However 700 brush iterations with $N Z=40$ takes several seconds on a standard PC. The present scheme for the determination of improved values of $v_{K}$ at the end of each brush iteration is a very simple scheme. A Newton-Raphson scheme with $v_{K}^{(\text {next })}=v_{K}+\Delta v_{K}$ and with the solution of linear equations for $\Delta v_{K}$ might reduce the amount of computer time needed to achieve a given relative error.

First we consider the results for our base state with $I_{\text {trans }}=0$. For this case, $\varphi_{0}=-58.16 \mathrm{~m} V, \mathrm{y}_{\mathrm{t} 0}=0.9937$ and the Joulean heating is $30.355 \mathrm{~W}$. This case involves only the eddy current, which is the local circulation of electric current through the brush, stator and rotor, driven by the voltage variation $V_{x} B_{y} z$. There is an electric current from the stator to the rotor through the fibers near $z=-L_{z}$ and an equal electric current from the rotor to the stator through the fibers near $z=L_{z}$. The frictional forces $\mu \nu_{K}$ pull all the fibers in the $+x$ direction. For the fibers near $z=-L_{z}$ with $I_{K}>0$, the electromagnetic body force due to $B_{z}$ pushes the fibers further into the $+x$ direction, thus bending them away from the rotor face and reducing the local values of $\beta_{K}$. For the fibers near $z=L_{z}$ with $I_{K}<0$, the electromagnetic body force due to $B_{z}$ pushes the fibers in the $-x$ direction, pushing them toward and beyond $x=0$. These fibers are shoved into the rotor surface, increasing the local values of $\beta_{K}$. The plot of $\beta$ versus $z$ for this case is presented in Figure 2.

Here $0.073 \leq \beta \leq 4.72$. Clearly there is a large deviation from $\bar{\beta}=0.63$. The voltage variation is linear in $z$, but the contact resistances for the fibers near $z=-L_{z}$ with small values of $\beta_{K}$ are much larger than those for the fibers near $z=L_{z}$ with much larger values of $\beta_{K}$, so that the electric current distribution across the brush is far from linear. The plot of $j_{y}$, which is the electric current per unit area of the brush-rotor interface, is also shown in Figure 2. Here $-9.06 \mathrm{MA} / \mathrm{m}^{2} \leq j_{y} \leq 1.66 \mathrm{MA} / \mathrm{m}^{2}$. The much smaller contact resistance near $z=L_{z}$ due to the large local values of $\beta_{K}$ allows much more local electric current, and $\phi_{0}=-58.16 \mathrm{mV}$ is needed to drive enough current from the stator to the rotor over the region with much larger contact resistance so that $I_{\text {trans }}=0$.
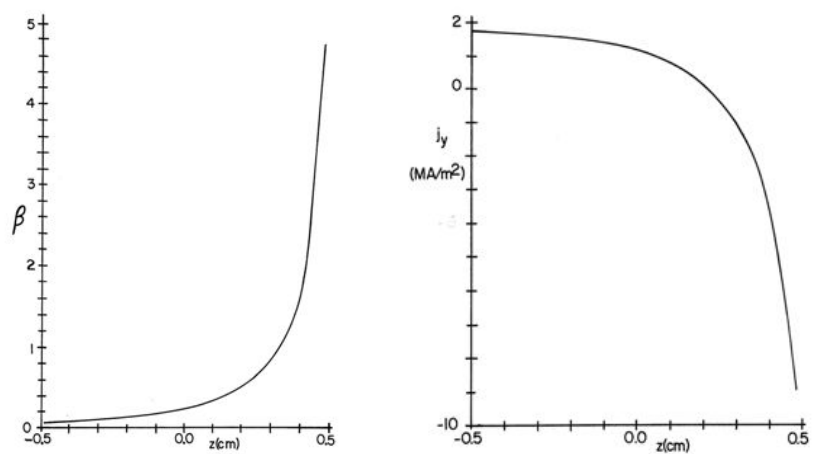

Figure 2. Results for $I_{\text {trans }}=0: \beta$ and $^{j_{y}}$ versus $z$.

The $x$ and $z$ coordinates of the tips of the $N Z$ "fibers" are plotted in Figure 3. We refer to all the fibers in the $\Delta z$ centered at $z_{K}$ as the $K$-th fiber. For all plots of $x_{t}, z_{t}$, $x_{t}=L x(1)$ and $z_{t}=z_{K}+L z(1)$, where $x(s), z(s)$ are the dimensionless local fiber coordinates. The fibers for $K=1$ to 29 all have essentially the same values of $x_{t}$. These fibers all have $I_{K}>0$, so that their electromagnetic body forces due to $B_{z}$ push them in the $+x$ direction. As $K$ increases from 1 to 29 , $I_{K}$ and the electromagnetic body forces due to $B_{z}$ decrease, but $\beta_{K}$ and the frictional forces in the $+x$ direction, $\mu \beta_{K} v_{\text {trans }}$ increase. The decrease in the electromagnetic body force is 
balanced by the increase in the frictional force, leading to essentially the same values of $x_{t}$ for $K=1$ to 29 . For $K=30$ to $40, I_{K}<0$, so that the electromagnetic body forces due to $B_{z}$ are in the $-x$ direction, and become very large as $K$ approaches 40 and $j_{y}$ approaches $-9.06 \mathrm{MA} / \mathrm{m}^{2}$.

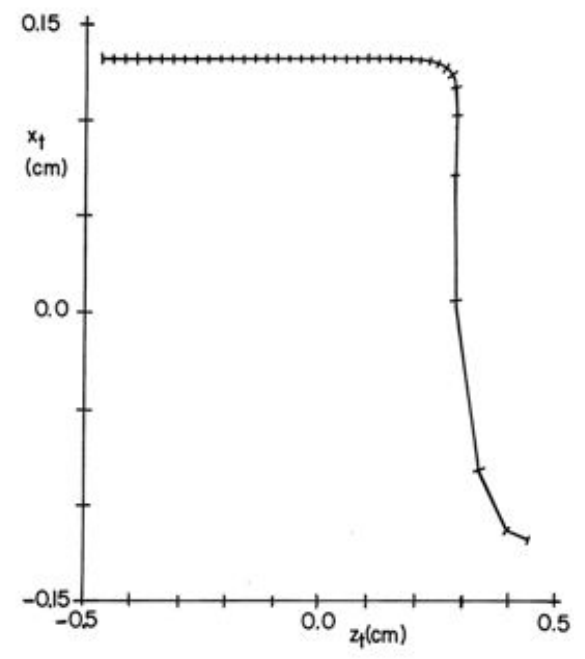

Figure 3. Results for $I_{\text {trans }}=0: x$ and $\mathrm{z}$ coordinates of the fiber tips.

As $K$ increases from 30, frictional forces in the $+x$ direction, $\mu \beta_{K} v_{\text {trans }}$, also increase. However, the electromagnetic body forces in the $-x$ direction have much larger increases, so that $x_{t}$ decreases and $x_{t}<0$ for $K=38$ to 40 . The values of $z_{t}$ in Figure 3 are the result of the electromagnetic body forces due to $B_{y}=1.1 T$. The sign of the electromagnetic body force in the $z$ direction due to $B_{y}$ depends on the signs of both $x_{t}$ and $I_{K}$. If $x_{t}>0$ and $I_{K}>0$, then the force is in the $+z$ direction, pushing all the fibers for $K=1$ to 29 in the $+z$ direction. For $K=30$ to $37, x_{t}>0$ and $I_{K}<0$, so the electromagnetic body forces due to $B_{y}$ push the fibers in the $-z$ direction. Figure 3 indicates that the fibers for $K=35$ and 36 have almost equal values of $z_{t}$. Therefore the tips of the fibers in these two rows are touching. Fiber-fiber contact forces are neglected here, but will be included in a future brush model. For $K=38$ to $40, \quad x_{t}<0$ and $I_{K}<0$, so that the electromagnetic body forces due to $B_{y}$ push these fibers in the $+z$ direction. Therefore these rows are more spread apart than those near $K=35$.

Next we increase $I_{\text {trans }}$ from 0 to $350 \mathrm{~A}$. Increments of $10 \mathrm{~A}$ and $50 \mathrm{~A}$ led to exactly the same results. For $I_{\text {trans }}=350 \mathrm{~A}, \phi_{0}=-91.0 \mathrm{mV}, y_{t 0}=0.9704$ and the Joulean heating is $58.82 \mathrm{~W}$. The plot of $\beta$ versus $z$ is presented in Figure 4.
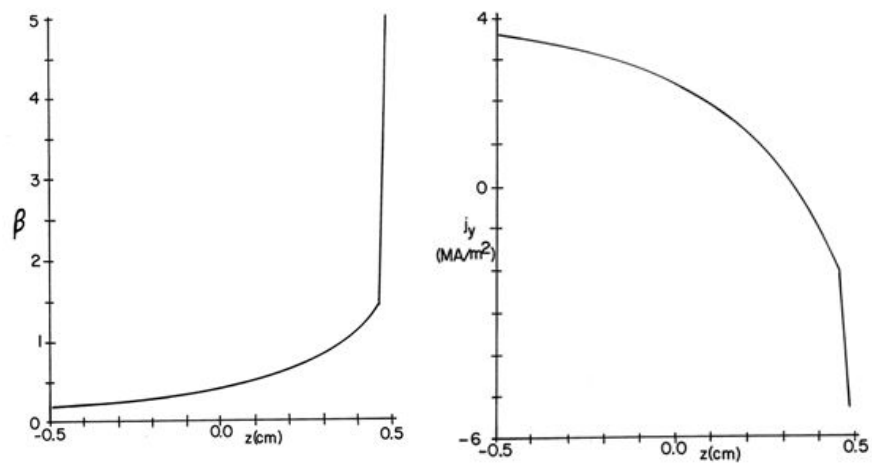

Figure 4. Results for: $I_{\text {trans }}=350 \mathrm{~A}, \beta$ and jy versus $\mathrm{z}$.

Here $0.186 \leq \beta \leq 5.02$. Compared to $I_{\text {trans }}=0$, the values of $\beta$ are more uniform, except for $\beta_{40}=5.02$. Clearly the $K=40$ fiber is behaving differently from the other 39 fibers. The plot of $j_{y}$ versus $z$ is also presented in Figure 4. Here, $-5.32 \mathrm{MA} / \mathrm{m}^{2} \leq j_{y} \leq 3.6 \mathrm{MA} / \mathrm{m}^{2}$. For $K=1$ to $33, I_{K}>0$ and for $K=34$ to $40, I_{K}<0$. Even with a transport current of $350 \mathrm{~A}$ from the stator to the rotor, the eddy current is strong enough to drive electric current from the rotor to the stator over roughly one-sixth of the brush. The values of $x_{t}, z_{t}$ are plotted

in Figure 5. As $K$ increases from 1 to 33, $x_{t}$ actually increases slightly, indicating that the increases in the frictional forces are slightly larger than the decreases in the electromagnetic body forces due to $B_{z}$. For $K=34$ to $40, I_{K}<0$, so that the electromagnetic body forces due to $B_{z}$ are in the $-x$ direction. However $x_{t}>0$ for $K=34$ to 39 , and $x_{t}$ is only negative for $K$ $=40$. This large change between $K=39$ and 40 is illustrated by the plots of the local dimensionless fiber coordinates, $y(s)$ versus $x(s)$, for $K=1,39$ and 40, which are also presented in Figure 5. Again we note that the $x_{t}, z_{t}$ in Figure 5 are dimensional brush coordinates, while the $x, y$ in Figure 5 are dimensionless fiber coordinates. 

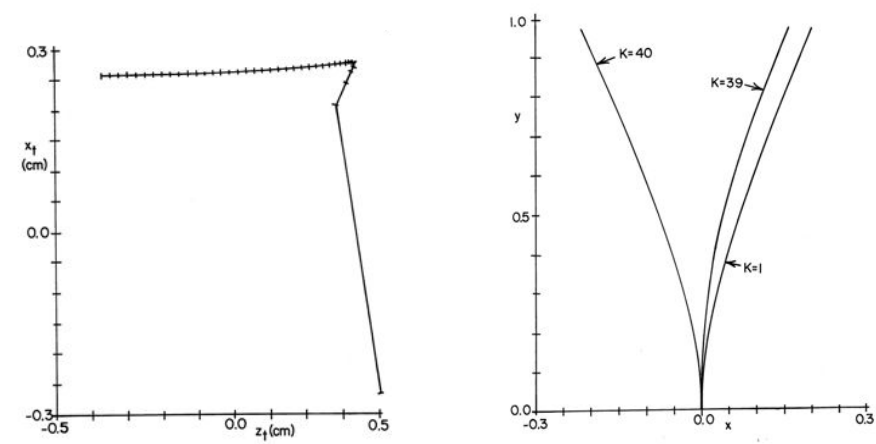

Figure 5. Results for $I_{\text {trans }}=350 \mathrm{~A} x$ and $\mathrm{z}$ coordinates of the fiber tips and plots of the dimensionless $x$ and $y$ coordinates along the deformed fibers for $K=1, K=39$ and $K=40$.

The transport current from the stator to the rotor interacts with $B_{z}$ to produce electromagnetic body forces in the $+x$ direction, increasing the values of $x_{t}$ for all the fibers, compared with the values for $I_{\text {trans }}=0$. For $K=39, j_{y}=-2.2 M A / \mathrm{m}^{2}$ with $I_{\text {trans }}=350 \mathrm{~A}$, so that the electromagnetic body forces due to $B_{z}$ are not large enough to overwhelm the frictional forces, and $x_{t}>0$. Only for $K=40$, with $j_{y}=-5.3 \mathrm{MA} / \mathrm{m}^{2}$, are the electromagnetic body forces due to $B_{z}$ large enough to lead to $x_{t}<0$.

For $K=34$ to $39, I_{K}<0$ and $x_{t}>0$, so that the electromagnetic body forces due to $B_{y}$ are in the $-z$ direction. The magnitudes of these body forces in the $-z$ direction increase greatly as $K$ increases from 34 to 39 , as $j_{y}$ decreases from 0 to $-2.2 \mathrm{MA} / \mathrm{m}^{2}$, and as $x_{t}$ only decreases from $0.27 \mathrm{~cm}$ to $0.20 \mathrm{~cm}$. With these body forces, the present model, which ignores fiber-fiber contact forces, predicts that the fibers pass through each other, e.g., $z_{t}$ for $K=39$ is less than $z_{t}$ for $K=$ 38. Of course, this is impossible, but the results clearly show that several rows of fibers are jammed together by the electromagnetic body forces due to $B_{y}$.

Next we decrease $I_{\text {trans }}$ from $350 \mathrm{~A}$ to 0 . After every incremental decrease in $I_{\text {trans }}$, we obtained exactly the same results that we obtained with increasing $I_{\text {trans }}$. Therefore there is no history dependence or hysteresis for $I_{\text {trans }}>0$.

Next we decrease $I_{\text {trans }}$ from to $-350 \mathrm{~A}$. With each $10 \mathrm{~A}$ decrease in $I_{\text {trans }}$, another fiber snaps from a deformation with a large value of $\beta$ and a double curvature to a deformation with a much smaller value of $\beta$ and with single curvature: the $K=$ 39 fiber snaps back as $I_{\text {trans }}$ is decreased from $-160 \mathrm{~A}$ to $-170 \mathrm{~A}$, the $K=38$ fiber snaps back as $I_{\text {trans }}$ is decreased from $-170 \mathrm{~A}$ to $-180 A$, etc.
For $I_{\text {trans }}=-350 \mathrm{~A}, \phi_{0}=+93.86 \mathrm{mV}, y_{t 0}=0.9979$ and the Joulean heating is $36.62 \mathrm{~W}$. The values of $x_{t}, z_{t}$ for $I_{\text {trans }}=$ $350 \mathrm{~A}$ are plotted in Figure 6.

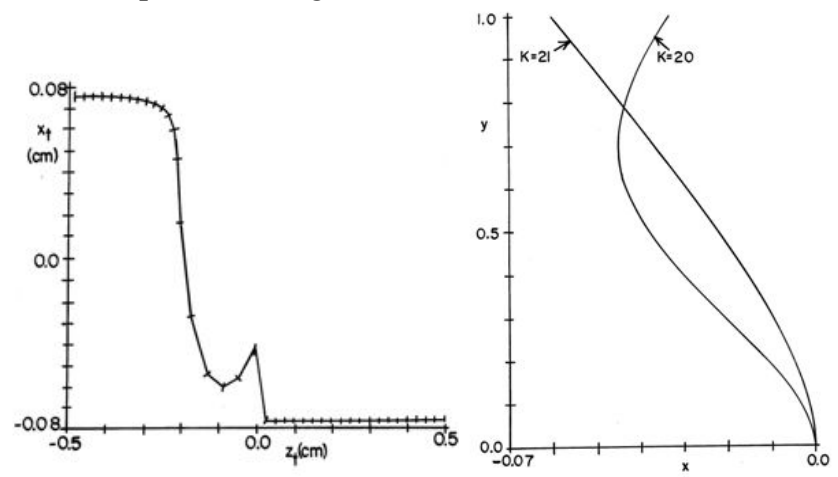

Figure 6. Results for $I_{\text {trans }}=-350 \mathrm{~A}$ with $I_{\text {trans }}$ decreasing: $x$ and $z$ coordinates of the fiber tips and plots of the dimensionless $x$ and $y$ coordinates along the deformed fibers for $K=20$ and $K=21$.
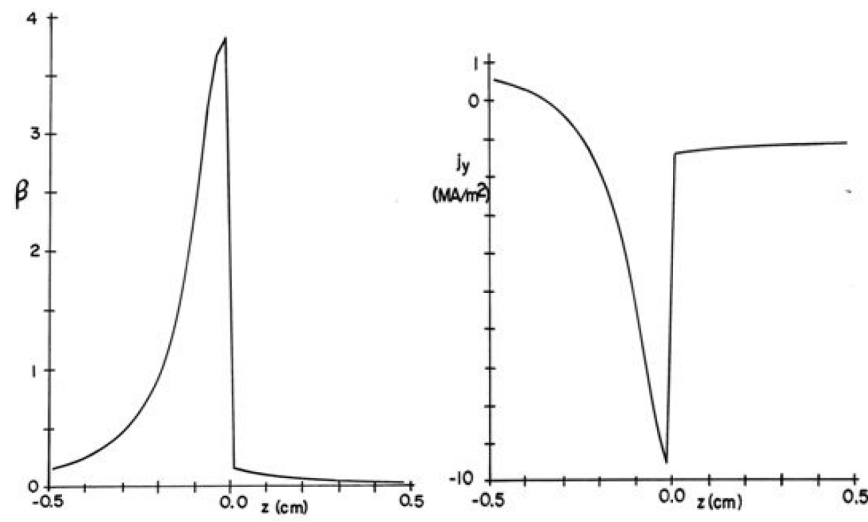

Figure 7. Results for $I_{\text {trans }}=-\mathbf{3 5 0 A}$ with $I_{\text {trans }}$ decreasing: $\beta$ and $j_{\mathrm{y}}$ versus $z$.

All of the fibers for $K=21$ to 40 have snapped back, and $x_{t}=-0.077$ for all of these fibers. The values of $y$ versus $x$ for $K=20$ and $K=21$ are plotted in Figure 6 also. The $K=21$ fiber snapped back when $I_{\text {trans }}$ was reduced from $-340 A$ to $350 A$, while the $K=20$ fiber now has the maximum value of $\beta$ and has the deformation with double curvature. The values of $\beta$ versus $z$ are plotted in Figure 7. Here, $0.032 \leq \beta \leq 3.83$, where the maximum value of $\beta$ occurs at $K=20$. The values of $j_{y}$ versus $z$ are plotted in Figure 7 also.

Here

$$
-9.58 \mathrm{MA} / \mathrm{m}^{2} \leq j_{y} \leq 0.583 \mathrm{MA} / \mathrm{m}^{2}
$$

$-0.5 \mathrm{~cm} \leq z \leq-0.34 \mathrm{~cm}$, there is a total current of $10 \mathrm{~A}$ from the stator to the rotor, for $-0.34 \mathrm{~cm} \leq z \leq 0$, there is a total current of $250 \mathrm{~A}$ from the rotor to the stator, and for $0 \leq z \leq 0.5 \mathrm{~cm}$, there is a total current of $110 \mathrm{~A}$ from the rotor to the stator. The values of $\beta$ in Figure 7 illustrate the extreme nonuniformity of the contact resistance over the brush-rotor interface, and the values of $j_{y}$ in Figure 7 illustrate the effect of the nonuniform contact resistance on the distributions of the eddy and transport currents. 
Next we increase $I_{\text {trans }}$ back from $-350 A$ to 0 . As $I_{\text {trans }}$ was decreased from $-150 A$ to $-350 A$, the fibers for $K=21$ to 40 snapped back. As we increase $I_{\text {trans }}$ from $-350 A$, so that the electromagnetic body forces in the $-x$ direction due to $B_{z}$ decrease in magnitude, we do not expect each fiber to reverse the abrupt change in deformation at the same value of $I_{\text {trans }}$. Experience with the single-fiber model indicates that there is some hysteresis associated with these abrupt changes in fiber deformation. We increased $I_{\text {trans }}$ from $-350 \mathrm{~A}$ with increments of $10 \mathrm{~A}$. With $I_{\text {trans }}$ increasing, the $K=21$ fiber is the first to be pulled abruptly forward to a deformation with double curvature, and there is a large increase in $\beta_{21}$. Before the abrupt change, the fiber is bent into the $-x$ direction and has a small value of $\beta$. The abrupt change occurs because the fiber tip digs into the rotor surface which is moving in the $+x$ direction. This leads to abrupt increases in $\beta$ and in the frictional force in the $+x$ direction at the tip. It also leads to an abrupt decrease in the contact resistance, so that the distributed electromagnetic body force in the $-x$ direction due to $B_{z}$ also has a dramatic increase in magnitude. The competition between the two large forces leads to a deformation with double curvature. As we increase $I_{\text {trans }}$, successive fibers are snagged by the moving rotor surface and pulled into deformations with double curvature at intervals of 17-18A, while they had snapped back at intervals of $10 \mathrm{~A}$ when $I_{\text {trans }}$ was decreasing.

When we increased $I_{\text {trans }}$ from $-350 A$ to $-150 A$, the $K=21$ to 32 fibers were snagged by the rotor surface, leading to larger values of $\beta$. With $I_{\text {trans }}$ varying back and forth between $\pm 350 \mathrm{~A}$, there is a hysteresis for $I_{\text {trans }}<0$, but there is none for $I_{\text {trans }}>0$. This hysteresis is the result of successive fibers snapping backward with abrupt decreases in $\beta$ as $I_{\text {trans }}$ is decreased from $-150 A$ to $-350 A$, and of successive fibers being snagged by the moving rotor surface with abrupt increases in $\beta$ as $I_{\text {trans }}$ is increased from $-350 \mathrm{~A}$ to 0 .

The values of $\phi_{0}$ versus $I_{\text {trans }}$ are plotted in Figure 8.

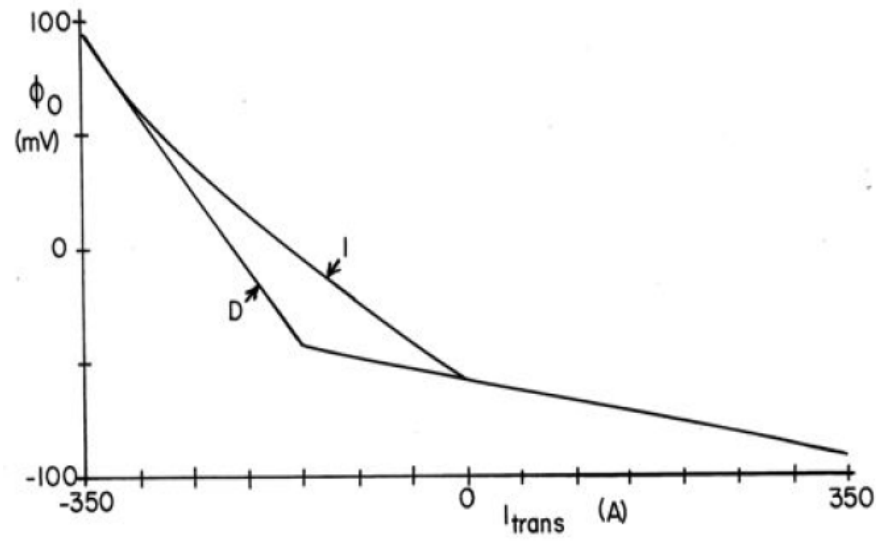

Figure 8. Electric Potential $\varphi_{0}$ versus Transport Current for increasing and decreasing $I_{\text {trans. }}$.

For $I_{\text {trans }}>0$, this plot is essentially a straight line. For $I_{\text {trans }}<0$ with $I_{\text {trans }}$ decreasing, this plot is composed of two straight lines with an abrupt change of slope at $I_{\text {trans }}=-150 \mathrm{~A}$. For $I_{\text {trans }}<0$ with $I_{\text {trans }}$ increasing, this plot is a curve with an abrupt change of slope at $I_{\text {trans }}=0$. The values of the Joulean heating versus $I_{\text {trans }}$ are plotted in Figure 9. The values of the Joulean heating are $30.355 \mathrm{~W}$ for $I_{\text {trans }}=0,58.825 \mathrm{~W}$ for $I_{\text {trans }}=350 \mathrm{~A}$, and $36.617 \mathrm{~W}$ for $I_{\text {trans }}=-350 \mathrm{~A}$. For $I_{\text {trans }}$ decreasing, the minimum Joulean heating is $26.39 \mathrm{~W}$ for $I_{\text {trans }}$ $=-190 \mathrm{~A}$, and for $I_{\text {trans }}$ increasing, the minimum Joulean heating is $27.68 \mathrm{~W}$ for $I_{\text {trans }}=-120 \mathrm{~A}$.

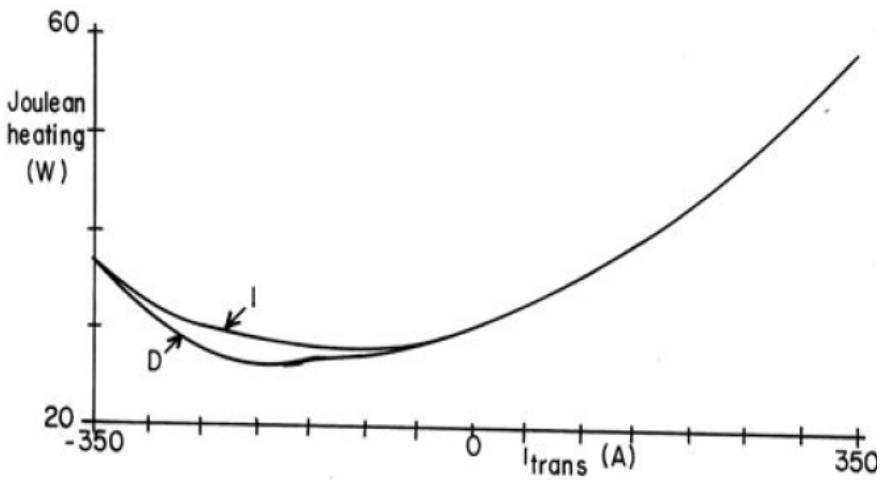

Figure 9. Joulean Heating versus Transport Current for increasing and decreasing $\mathbf{I}_{\text {trans. }}$ 


\section{CONCLUSIONS}

In a previous study we derived the formalism for analyzing the electromechanics of a single, current carrying fiber sliding against a plane in the presence of a large magnetic field of arbitrary orientation. In that study, the current and the mechanical contact force at the fiber tip-slider interface are specified and do not change but the fiber itself, changes shape, deflects and the applied electric potential necessary to drive the current varies with conditions such as slider speed and differing magnetic field conditions. We discovered a bifurcation in the solution space of such fibers which leads to a history dependant equilibrium condition.

In the present paper we have extended that investigation to include a collection of fibers that carry a constant total transport current and are held against the slider by a constant total force. The currents and tip forces of the individual fibers are allowed to vary but the sum of the electrical currents and forces over all of the fibers remains constant. First we find tremendous variation of fiber tip contact forces and currents among the collection of fibers even though the average load and total transport current remain constant. We find that the hysteretic behavior seen in the single fiber investigation carries over to the collection of fibers with additional phenomena occurring. Because the slider cuts flux, a potential gradient exists across the slider face and therefore between the fibers of the collection and circulating currents between the fibers result. These currents are in opposite directions on opposite sides of the collection and we have oppositely directed Lorentz forces on the collection causing individual fibers to move either with rotation, against rotation or perpendicular to rotation. These effects are altered by the direction of the transport current and hysteresis is seen in both the Joulean heating and the contact potential necessary to drive the transport current.

It is known that wear in fiber brushes is a highly non-linear function of the normal load ${ }^{2}$ and many previous studies $8,9,10,11$ report that wear depends on the direction of current. The present investigation will be extended in a future study by incorporating both fiber-fiber interactions and the effects of wear. In that study we may expect that the individual fibers carrying the most mechanical load and current will wear the fastest but the overall behavior of the collection may not be so simple because of nonlinear and hysteretic effects.

\section{ACKNOWLEDGMENT}

The Authors wish to thank the Office of Naval Research for their continued generous support on this effort, in particular Program Officers Elaine Martin, Stephen Schreppler, Lynn Petersen and most recently Harold Coombe. The authors also wish to acknowledge the pioneering contributions of John Walker; Professor Emeritus at U of ILL, without whose effort, this work would not be possible.

\section{REFERENCES}

1. J.S. Walker, G.S. Erwin and N.A. Sondergaard, "Modeling of Metal Fiber Brushes Part I: The Electromechanics of Single Fibers", NSWCCD-98TR-2009-02, 2009.
2. D. Kuhlmann-Wilsdorf, " Electric Fiber Brushes-Theory and Observations. ", IEEE Transactions on Components Hybrids, and Packaging and Manufacturing Technology ,Part A, Vol.19, No. 3, pp. 360-375, 1996.

3. P.G. Slade (Ed.), "Electrical Contacts Principles and Applications", Marcel Decker, New York, 1999

4. L. Boyer and G. Fornet, "Mechanical and Electrical Behavior of Fibre Brushes”, Wear,_Vol. 89, pp245-253, 1983.

5. M.N. Alexakis, J.S. Walker, L.J. Petersen and N. Sondergaard,

"Electromechanical effects on Metal Fiber Brushes, "Proceedings of the Third International Symposium of All Electric Ship Civil or Military”, Paris, France, pp.396-399, 2000.

6. Marika Nick Alexakis, "Modeling of Electromechanical Effects on Metal Fiber Brushes", Thesis for the Master of Science in Mechanical Engineering, University of Illinois at Urbana-Champaign, 2001

7. W.H. Lupton and P. Reichner, "Ultrahigh Speed Fiber Brush Design and Tests, ” Wear, Vol. 78, (1982) pp. 139-149

8. L. Boyer, S. Noel and J.P. Chabrerie, "Electrochemically Activated Wear of Metal Fibre Brushes”, Wear, 116(1987)43-57

9. P. Reichner, "High Current Tests of Metal Fiber Brushes", Electrical Contacts - 1980, (Ill. Inst. Tech., Chicago, IL), 1980, pp.73-76

10. J.A. Bares, N. Argibay, N. Mauntler, G.J. Dudder, S.S. Perry, G.R. Bourne, W.G. Sawyer. "High current density copper-on-copper sliding electrical contacts at low sliding velocities", Wear, 267, (2009), pp. 417-424.

11. N. Argibay, J.A. Bares, W.G. Sawyer. "Asymmetric wear behavior of self-mated copper fiber brush on disk sliding electrical contacts in a humid carbon dioxide environment", Wear (in review), (2009)

\section{BIOGRAPHIES}

Gary Erwin is a Mechanical Engineer with the Energy Conversion R\&D Branch of the Carderock Division of the Naval Surface Warfare Center. He has worked for the Naval Surface Warfare Center for 20 years in areas including piping analysis, experimental stress analysis, shock and vibration analysis, and heat transfer. He holds a B.S. in Mechanical Engineering from the University of Rochester and an M.S. in Mechanical Engineering from Drexel University.

Dr. Neal Sondergaard is a senior scientist at NSWCCD with 29 years experience in $\mathrm{R}+\mathrm{D}$ including sliding electric contacts(brushes), Tribology, MHD, superconductivity and electric power production, generation, conversion, machinery and distribution and Propulsion System Modeling. He received his B.A. in Chemistry from Marist College, his M.Sc. and Ph.D. in Physical Chemistry from Brown University and was a Post Doctoral Fellow at the Johns Hopkins University. He is a member of Sigma Xi and ASNE American Society of Naval Architects. 


\title{
Initial Experimental Studies of Metal Fiber Brushes in a Magnetic Field
}

\author{
W. A. Lynch, Member, IEEE, M. J. Roberts, R. P. Reitz, N. A. Sondergaard
}

\begin{abstract}
Metal fiber brushes were evaluated in a magnetic test fixture with variable speed, transport current and magnetic flux density. Silver alloy fiber brushes with a cross sectional area of 8.4 square millimeters were operated at a current of up to 90 amps, with a current density of 1070 amps per square centimeter. The brushes were operated in humidified air, at speeds of up to 15.5 meters per second and a flux density of up to about 0.6 Tesla. Significant differences between anode and cathode brush impedance characteristics were observed. The current-voltage curves of the cathode brush was approximately linear with current indicating constant contact resistance while the anode generally had higher impedance and in some cases its characteristic was non-linear with a diode like behavior. The magnetically induced circulating current losses were insignificant because of the small brush geometry and moderate magnetic flux density. Non-dimensional wear rates were on the order of 5E-11. Although operating temperatures in the brushes reached over $120^{\circ} \mathrm{C}$ for the higher speeds and currents, thermal runaway was not observed at temperatures up to $120^{\circ} \mathrm{C}$.
\end{abstract}

Index Terms - Brush, Conduction Power Loss, Friction, Magnet, Metal Fiber, Silver Alloy, Sliding Electric Contact, Slip Ring, Test Fixture

\section{INTRODUCTION}

Metal fiber brushes were first used in the late $19^{\text {th }}$ Century and were further developed by researchers at the University of Virginia [1,2], Westinghouse [3] and University of Paris [4,5] in the early 1980's. Metal fiber brushes have a lower effective conductivity compared with liquid metals principally because the liquid metal provides $100 \%$ coverage, but are significantly superior to monolithic carbon brushes because fiber brushes have thousands of contact spots compared to order 10 for monolithics. Furthermore, metal fiber brushes are mechanically lightly loaded and achieve additional benefit of low wear and low frictional loss. They operate with moisture present on the slip ring surface[6] but an environment with little oxygen present is generally required to minimize corrosion and wear. In the 1980s superconducting homopolar motor based drive systems were developed at NSWC in Annapolis[7]. Copper fiber brushes were more recently studied in the HiTC homopolar motor[8] at NSWCCD Philadelphia. This work was done to support the development of the General Atomics Homopolar Motor [9]. Commercial versions of metal fiber brushes are available from manufacturers including Moog[10], Hypercon[11], Sohre[12], and SSI[13].

This work was supported by the Office of Naval Research

W. A. Lynch was most recently with Raven LLC, Brunswick, Maine USA

M. J. Roberts is with NSWCCD-SSES, Philadelphia, PA 19112 USA

R. P. Reitz is retired from NSWCCD-SSES, Philadelphia, PA 19112 USA

N. A. Sondergaard is with NSWCCD-SSES, Philadelphia, PA 19112 USA
Although most of the fiber brush studies mentioned above used copper and copper alloy fibers, silver fiber brushes were also investigated but not generally in magnetic fields. For the present investigation we wished to explore silver brushes in a magnetic field and we used commercially available silver alloy fiber brushes manufactured by Moog shown in Figure 1. These brushes are capable of operation with oxygen present at the interface and at relatively high current densities. Initial experiments on the operation of $\mathrm{Ag}$ alloy brushes in magnetic field and with no cover gas as a function of speed and current are the subject of this paper. Theory relating to brushes and other electrical contracts was initially developed by [14] and the analytical model described in a report and anther paper in this conference $[15,16]$ may be applicable to these brushes, which have a uniform geometry and structure.

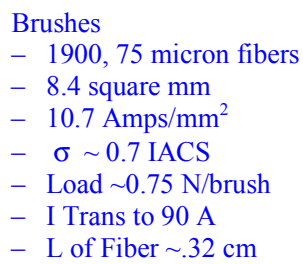

I Silver Alloy Fiber Brushes and Characteristics

\section{EXPERIMENTS WITH LOW MAGNETIC FIELD}

A magnetic test fixture was set up at NSWC Philadelphia. It was initially configured as shown in Figures 2 and 3 with no atmosphere control in the brush region and no cooling for the magnet. The rig consists of an iron rotor with two copper alloy slip rings, hard plated with silver providing separate tracts for positive and negative brushes and rotated by an external drive motor. Magnetic field is provided by a solenoid through which the rotor passes. The solenoid magnet produces magnetic fields in excess of 0.2 Tesla without cooling and 0.5 Tesla with cooling radially at the sliprings. In order to measure the contact drop between the brushes and the slipring in the presence of a magnetic field, two additional small brushes per slipring were installed on pickup wires which ride on either side of the main test brush, thus canceling the induced EMF. The location of the pickup wires are adjusted slightly to provide a zero millivolt signal with the magnetic field applied and zero transport current. Holes were made in the test fixture flange to provide viewing ports, shown in Figure 4, to observe the brushes. 


\section{Validation Test Fixture}

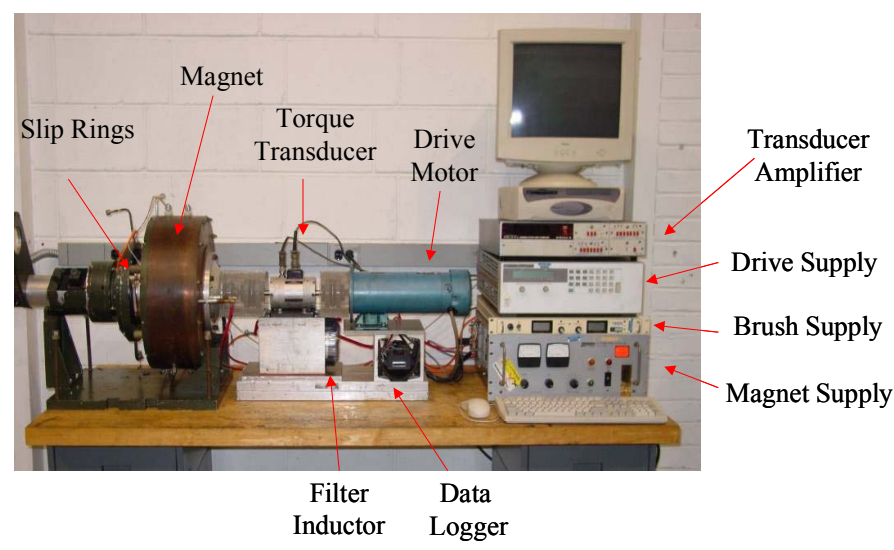

Figure 2 - Magnetic Brush Test Fixture

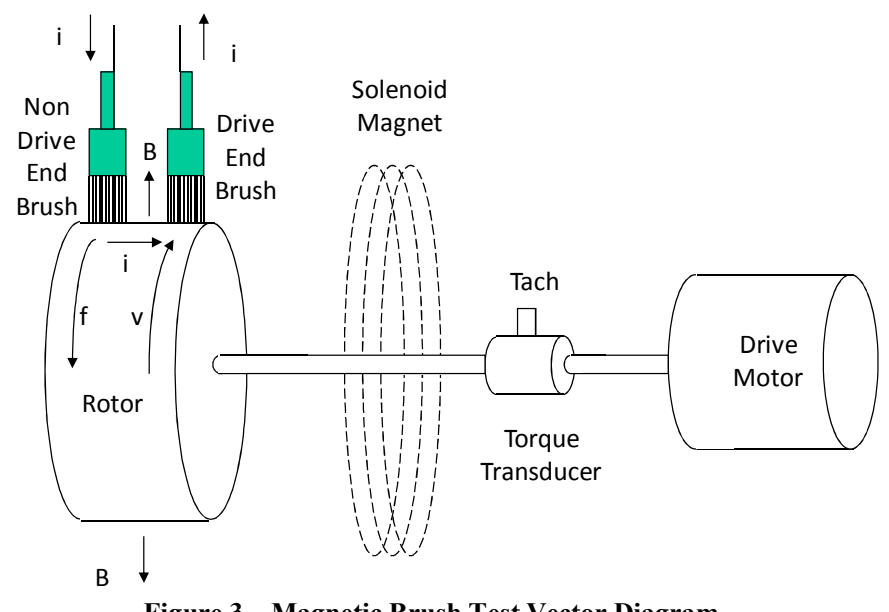

Figure 3 - Magnetic Brush Test Vector Diagram

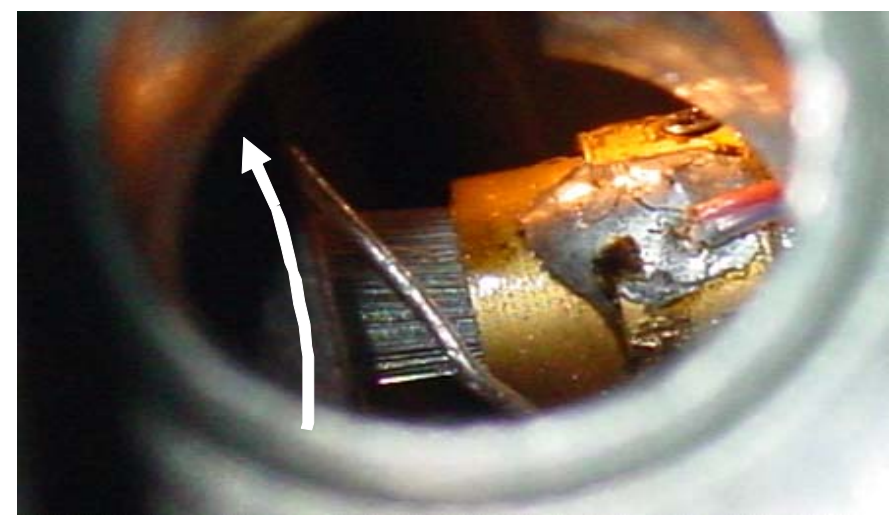

Figure 4 - Current Transport Brush from Viewing Port
Connected to the test fixture shown in Figure 5 are transport current leads, smaller voltage measurement leads and thermocouples soldered on each brush in order to provide temperature data. Pick-up brushes shown in Figure 6 were employed to average the voltage measurements across the brushes. These provided much more stable data, due to the multiple contact spots compared to the single wire pick-up initially used. Some pick-up brush fibers shown in Figure 7 were attached to a thermocouple so that the rotor surface temperature could be measured. The pick-up brush fibers were also used for a neutral analog ground connection for the data acquisition system. A thin layer of gold wand plating was applied over the silver plating. This wore off quickly so that the current collector brush and pickup brush wear tracks were clearly visible. Initial tests were run in ambient air.

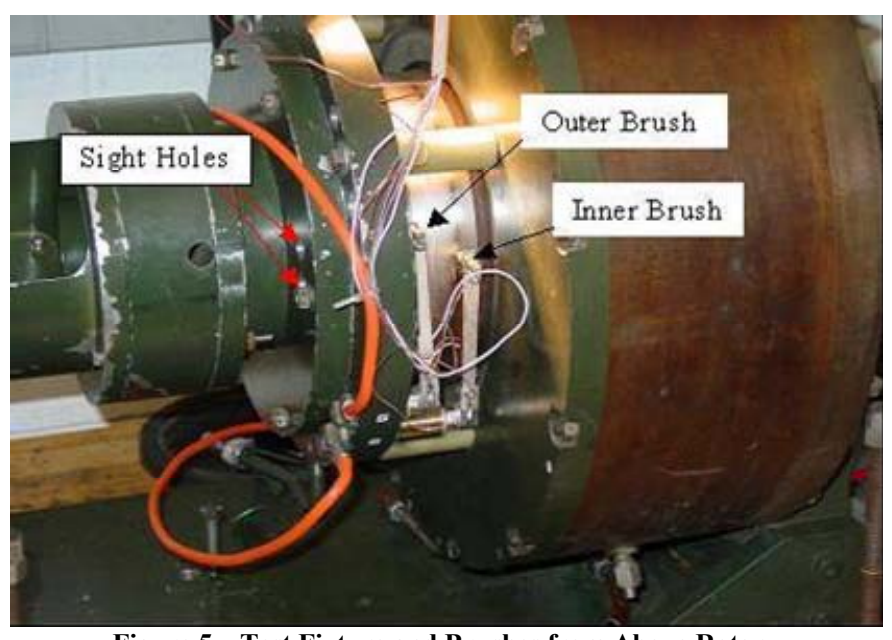

Figure 5 - Test Fixture and Brushes from Above Rotor

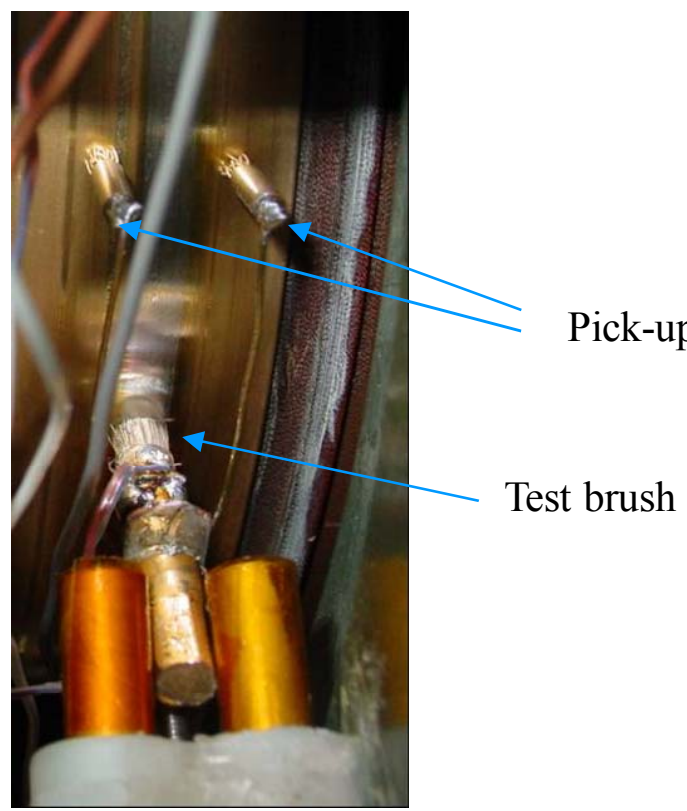

Figure 6- Transport and Pickup Brushes from Above Rotor 


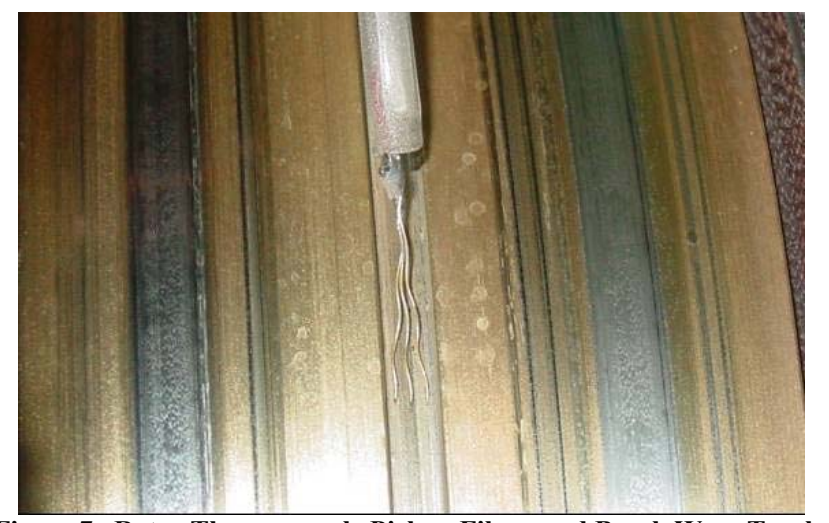

Figure 7- Rotor Thermocouple Pickup Fibers and Brush Wear Tracks

Initial tests were run without the magnet to determine the impedance characteristics of the brushes. As in [17] significant differences in voltage drop and friction of anode and cathode brushes were observed as indicated in Figure 8a. The positive anode brush had an average resistance of about $1 \mathrm{~m} \Omega$, but the resistance of the negative cathode brush was only about 0.5 $\mathrm{m} \Omega$. The temperature data plot for the same test shown in Figure $8 \mathrm{~b}$ indicates that the brush temperature was stable at $120 \mathrm{C}$ and $60 \mathrm{amps}$ but unstable at $130 \mathrm{C}$ and $90 \mathrm{amps}$ transport current. The thermal coefficients of the anode and cathode brushes were nearly the same and approximately $3.8^{\circ} \mathrm{C} / \mathrm{Watt}$.

\section{Brush Voltage Drop}

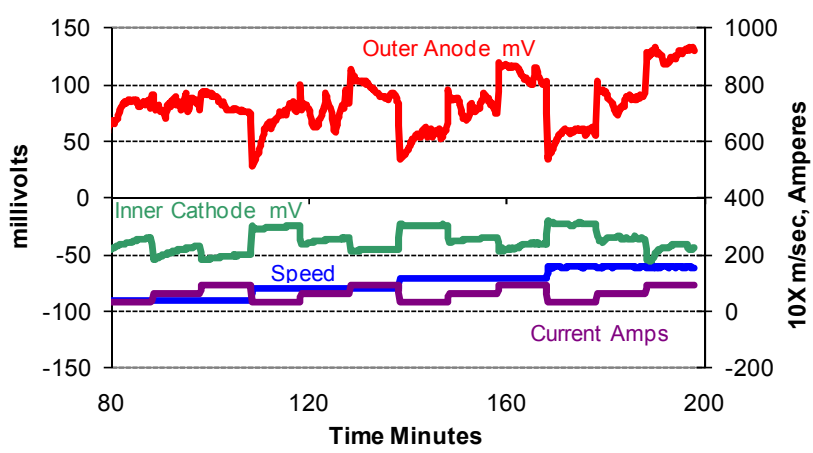

Figure 8a - Slip Ring Voltage at

Various Speeds and Currents

Brush Temperatures

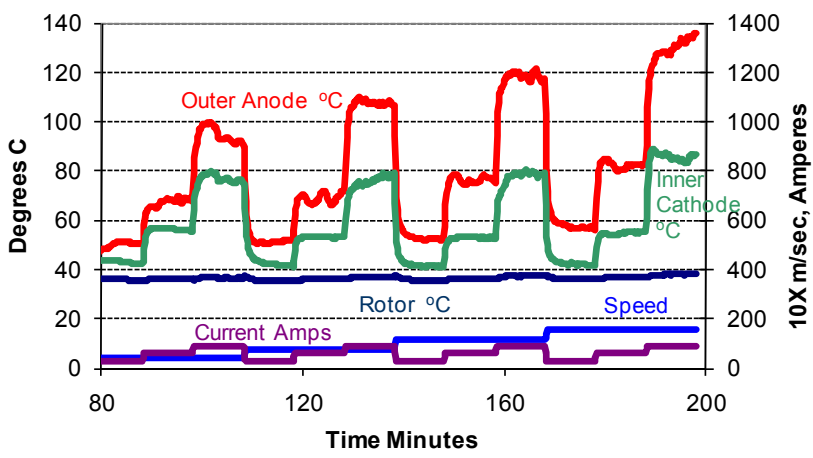

Figure 8b - Slip Ring Temperature at Various Speeds and Currents
The test fixture was operated both with brushes in normal operating position, and with the brushes lifted off the rotor, both individually and as a pair to measure torque and temperatures resulting from brush friction as shown in Figures 9 and 10, so that the torque resulting from brush friction can be determined by subtraction of the fixture torque from the total torque with brushes. The larger fixture torque that varies as a function of magnet solenoid current makes estimation of brush torque from total torque measurement difficult. Minimal torque associated with the brushes was observed at low field levels as shown in Figure 11.

$$
\begin{aligned}
& \text { No } \\
& \text { Brush Both }
\end{aligned}
$$

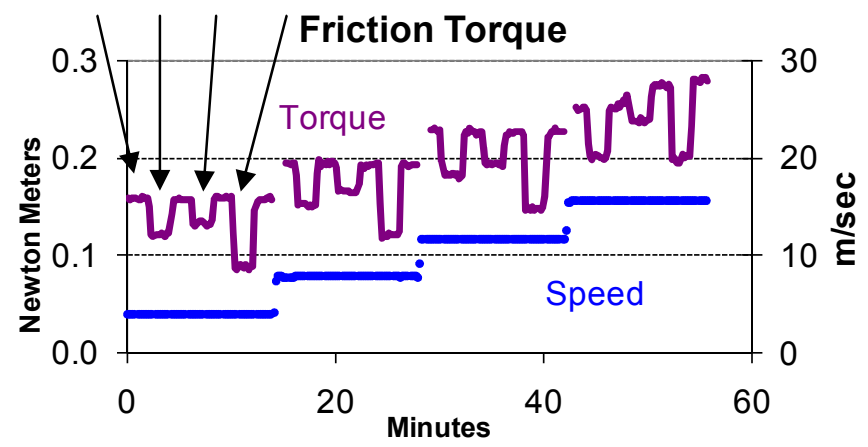

Figure 9 - Measured Torque due to Friction (outer anode)

\section{Brush and Rotor Temperatures}

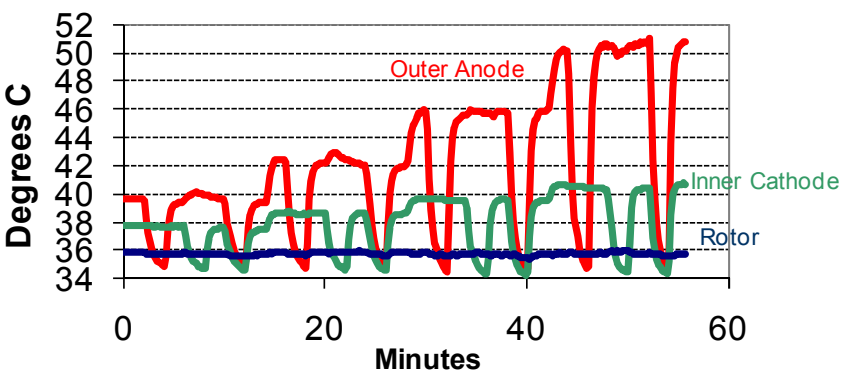

Figure 10 - Temperatures with No Transport Current

Torque

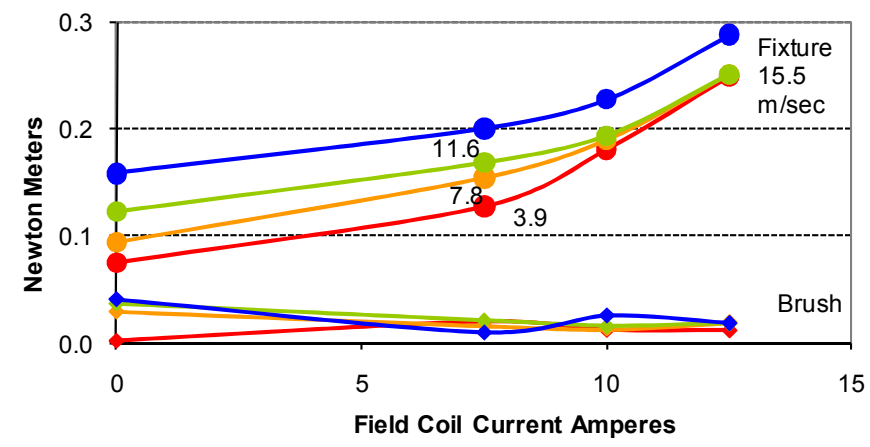

Figure 11 - Fixture and Average Brush Torque at Various Speed 


\section{EXPERIMENTS WITH HIGHER MAGNETIC FIELD}

The magnetic test fixture was modified to provide a higher magnetic field and humidity control. A cooling system was installed so that the test fixture magnet could be operated at a higher current of up to 45 amps. This system includes a mineral oil cooling loop for the magnet, oil to water heat exchanger, water to air heat exchanger and associated pumps. A cover was installed so that the atmosphere composition, humidity, and temperature in the brush region could be controlled. The fixture was operated using humidified air, but other atmospheres (e.g. carbon dioxide, nitrogen, helium, or argon) are possible. The cover is easily opened as shown in Figure 12 in order to closely inspect or service the brushes.

A bubbler shown in Figure 13 is used to maintain a controllable level of humidity. It includes two power supplies to regulate water heater temperature and vapor temperature for humidity measurement and a small bellows pumps to circulate humidified air. The system was designed to maintain the vapor temperature at approximately $5^{\circ} \mathrm{C}$ above the rotor using a second power supply and resistor to heat the vapor from the bubbler to maintain the water film. This was difficult in some cases because of the thermal time constant of the water reservoir and heater system, and because a closed loop controller was not included in the test fixture. A closed loop control system and higher wattage heater resistors could improve the dynamics of the cover gas control system.

The brushes were operated at various speeds and currents, however the electrical conduction appears to be the dominant loss mechanism as indicated by temperature plots in Figure 14. When operated without the magnet, the I/V characteristic of the cathode brush was relatively linear, however the voltage drop of the anode brush was higher and its I/V characteristic was nonlinear resembling a diode characteristic similar to that of an electrode in a methanol fuel cell [18]. These characteristics were observed regardless of which brush was operated as the anode as plotted in Figures 15a and 15b. Researchers at the University of Florida observed differences in resistance of copper fiber anode and cathode brushes, [19] however the negative cathode brush had higher resistance.

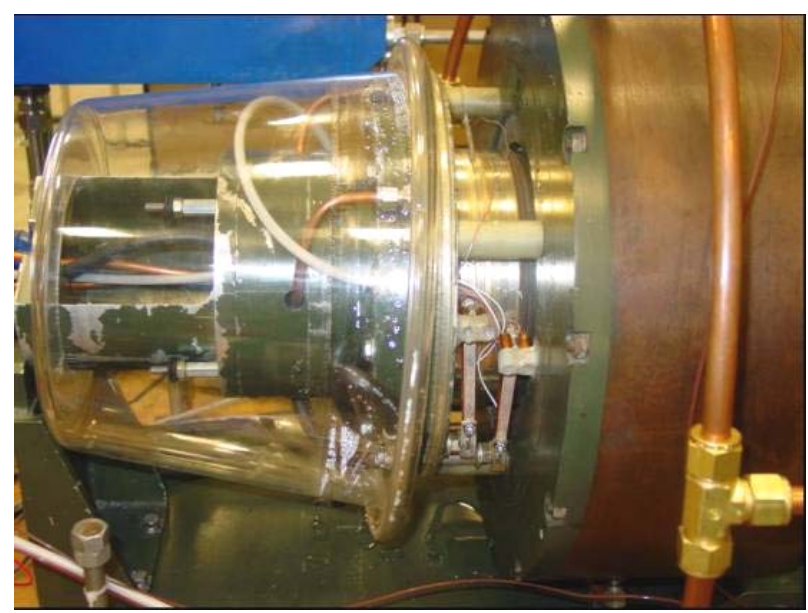

Figure 12 - Brush Region Environment Cover (open)

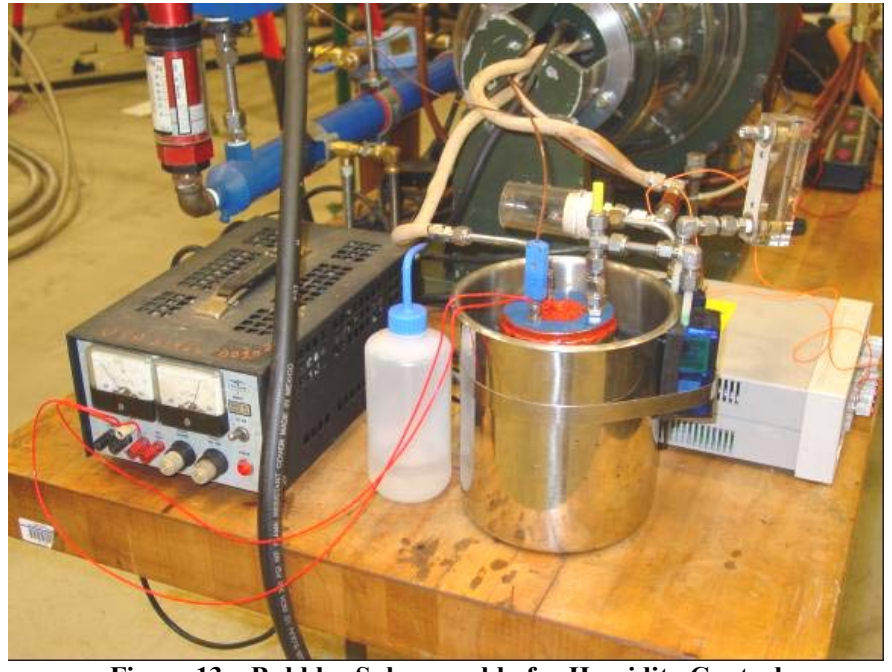

Figure 13 - Bubbler Sub-assembly for Humidity Control

\section{Rotor and Brush Temperatures}

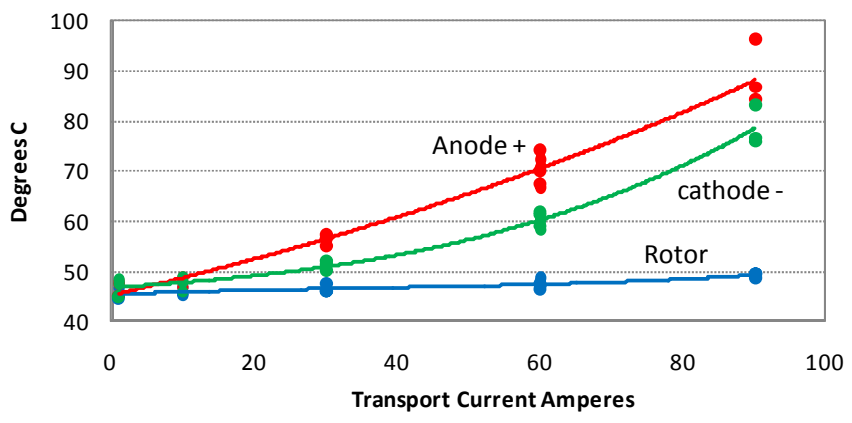

Figure 14 - Temperatures Without Magnetic Field in Humidified Atmosphere

\section{Brush Voltage}

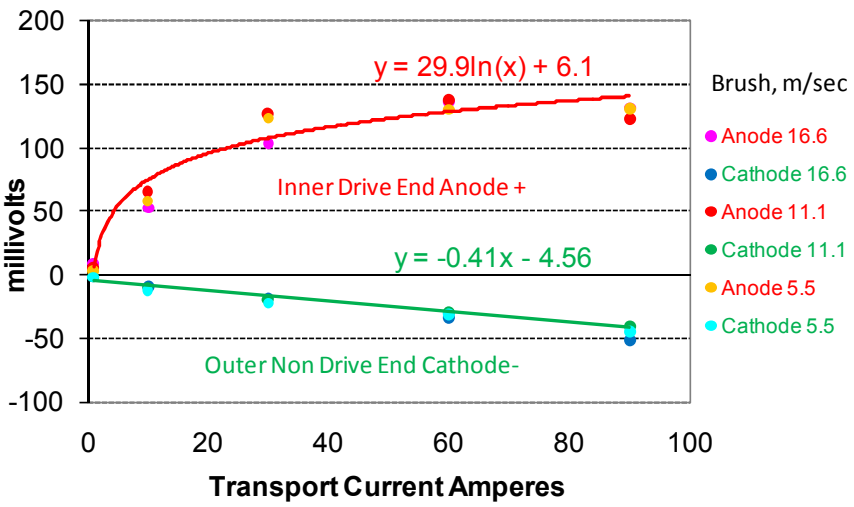

Figure 15a - Non-Magnetic Test with Humidified Atmosphere 


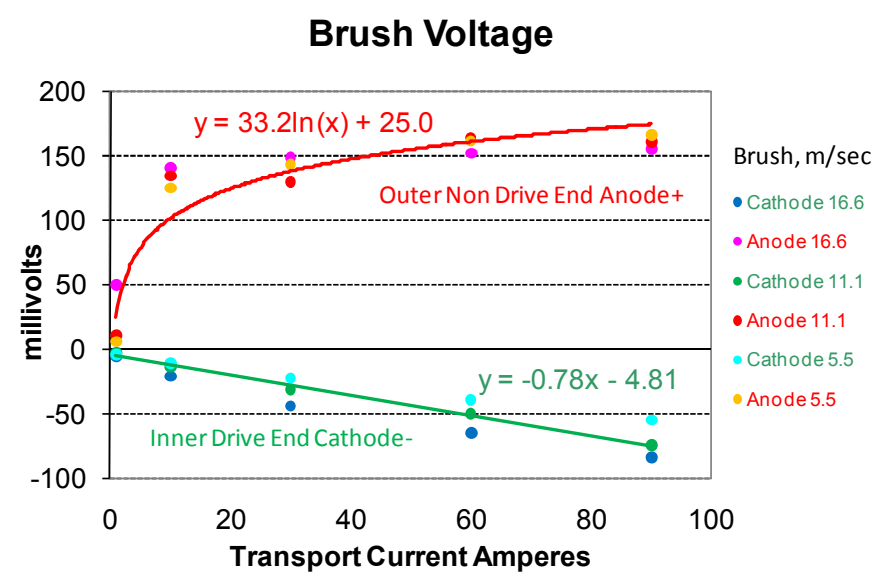

Figure 15b - Non-Magnetic Test with Humidified Atmosphere

In Table 1 the test conditions and results of copper and silver brushes run in humidified $\mathrm{CO} 2$ previously reported [20] are compared to the silver alloy brushes evaluated humidified ambient air in this study. The brush evaluation times for the previously reported copper brush study and this silver alloy brush test were 500 hours and 424 hours respectively. Also included in this study and Table 1 is a silver alloy brush test that was run without added humidity for 185 hours. The atmosphere was different, however other test conditions including load, speed, and transport current were similar. Our test fixture did not have a continuous wear sensor, so non dimensional wear was determined by measuring brushes before and after the test. The friction of the silver alloy brushes was much lower than the silver brushes, however the voltage drop across the alloy slip ring was much higher. These differences could result from differences in brush material characteristics as well as differences in the tribological films on the electrode surfaces that form under different atmospheres.

Table 1 Comparison of Previous Copper and Silver Brush Test Results [14] and This Silver Alloy Brush Study

$\begin{array}{lllllccccc}\text { Material } & \sigma & \text { Load } & \text { Current } & \text { Speed } & \text { + Brush } & \text { - Brush } & \text { + Brush } & \text { - Brush } & \text { Wear } \\ & \% \text { IACS } & \mathrm{N} / \mathrm{mm}^{2} & \mathrm{~A} / \mathrm{mm}^{2} & \mathrm{~m} / \mathrm{Sec} & \mu \text { friction } & \mu \text { friction } & \mathrm{mV} & \mathrm{mV} & \\ \mathrm{Cu} & 101 & 0.066 & 12.4 & 12.1 & 0.30 & 0.48 & 39 & 22 & 2.9 \mathrm{E}-11 \\ \mathrm{Ag} & 107 & 0.066 & 12.4 & 12.1 & 1.4 & 1.4 & 6 & 7 & \\ \text { Alloy Amb } & 70 & 0.088 & 10.7 & 11.6 & 0.64 & 0.52 & 96 & 46 & 1.5 \mathrm{E}-10 \\ \text { Alloy Wet } & 70 & 0.088 & 10.7 & 11.1 & 0.4 & 0.4 & 144 & 58 & 3.5 \mathrm{E}-11\end{array}$

\section{Magnetic Brush Test Results}

The flux density was estimated by simulation. Measured values matched closely with the output of the model simulation as shown in Figures 16 and 17. The brush and rotor temperatures were affected by the magnet current as shown in Figure 18. Magnetic losses such as circulating currents were difficult to measure, and appeared to be at least an order of magnitude lower than conduction and friction losses. Circulating currents were expected to be relatively low because of the small brush diameter and the modest flux density. Additional torque and heat associated with the magnet also compounded the difficulty of measuring the relatively small magnetic brush power losses. Brush voltage drops shown in Figures 19 were not a strong function of magnetic field coil current, and increased slightly with rotational speed.

\section{Magnet Flux Density}

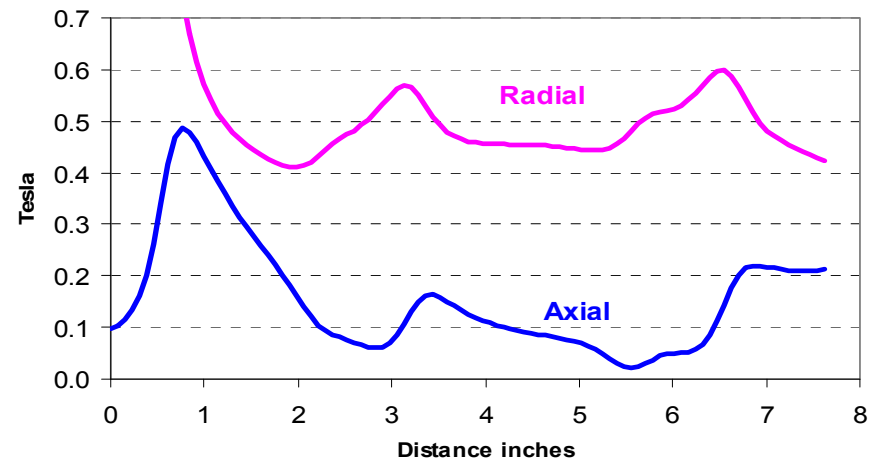

Figure 16 - Simulated Flux Density (45 amps excitation) at Distance from Magnet from Non-Drive End to Drive End

\section{Radial Magnet Flux Density}

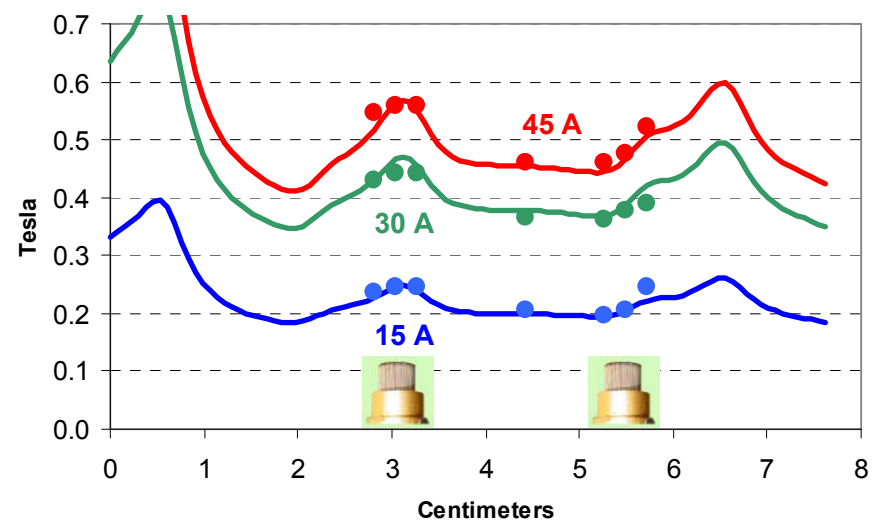

Figure 17 - Simulated and Measured Radial Flux Density 


\section{Rotor and Brush Temperatures}

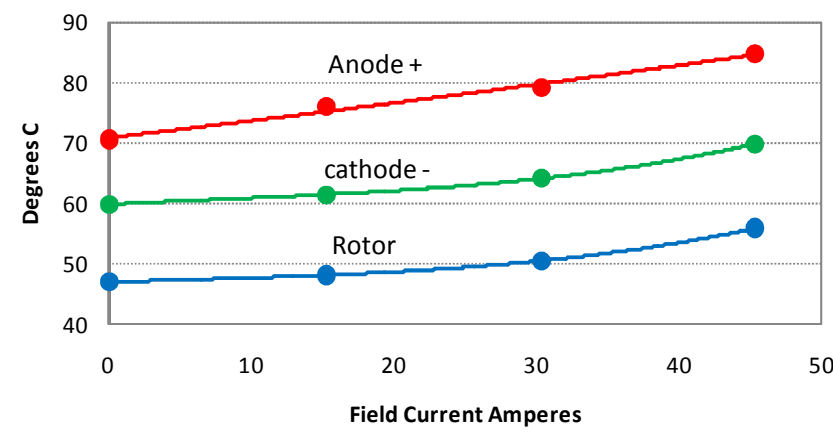

Figure 18 - Temperatures as a Function of Field Current at 60 Amperes Brush Transport Current

Figure 19 - Brush Voltage Drop at $I_{t}=60$ Amperes

\section{Brush Voltage}

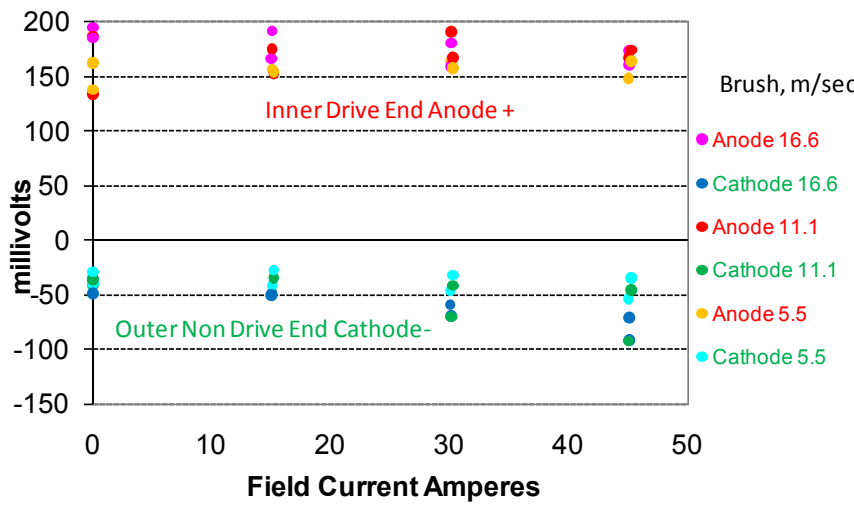

\section{DISCUSSION}

In previous studies, high current metal fiber brushes have been run in a humidified environment to provide lubrication on the surface using an inert gas such as $\mathrm{CO} 2$ to preclude oxygen. In the present study the silver alloy brushes were operated in ambient air and humidified air without the need to preclude oxygen. The relatively high nobility of silver could help prevent corrosion.

Pourbaix diagrams [21] are material stability diagrams often used to study corrosion. These plots include Eh potential relative to a hydrogen electrode as the ordinate, and $\mathrm{pH}$ $1 / \log (\mathrm{H}+)$ as the abscissa. These are useful for brush material selection, because the interface between the brush and the slip ring contains aqueous solution on the surface. These predominance area diagrams indicate which compounds of an element are most prevalent under a variety of conditions. A public domain software tool Medusa [22] was used to draw these diagrams which may serve as a guide to estimate electrode stability for a variety of metals.
Pourbaix diagrams were plotted for copper and silver electrodes in a solution that approximates the composition of tap water based on EPA MCL Secondary Standards [23] to indicate possible corrosion byproducts, however contaminants in typical tap water would be lower [24]. The solution in a machine could be much less concentrated than this if distilled water is used, but may contain small quantities of such salts, which may be contaminants, or possibly serve as additives. The diagrams include diagonal downward sloping dotted lines that indicate the nominal limits of aqueous solution, metal compound predominance areas, and solution buffer boundaries. The metal predominance or area within aqueous solution limits for copper shown in Figure 20a is much smaller than that for silver shown in Figure 20b, therefore the diagrams indicate that silver is a more noble metal with greater corrosion immunity over a much wider Eh range. Soluble and insoluble carbonates can form on copper and silver contacts in a relatively concentrated carbonate solution as indicated in Figures $22 \mathrm{a}$ and $22 \mathrm{~b}$, and these compounds may be useful as lubricants, however they have been reported to decompose or become desorbed from the electrode at $250^{\circ} \mathrm{C}$ [25]. The Pourbaix diagram of Figure 21a indicates that precious metals such as gold applicable in more concentrated electrolytes such as salt water are nobler than silver unlike base metals such as nickel as shown in Figure 21b.

Predominance areas directly adjoining the metal indicate compounds likely to form on contacts. Cuprous chloride which is soluble in excess chloride solution indicates possible conditions under which copper fibers may dissolve; however none to the corrosion byproducts associated with silver are significantly water soluble. Silver carbonate and silver(I) oxide may form if no chloride is present, but insoluble silver(II) and copper(II) compounds should not form directly on the metal surfaces.

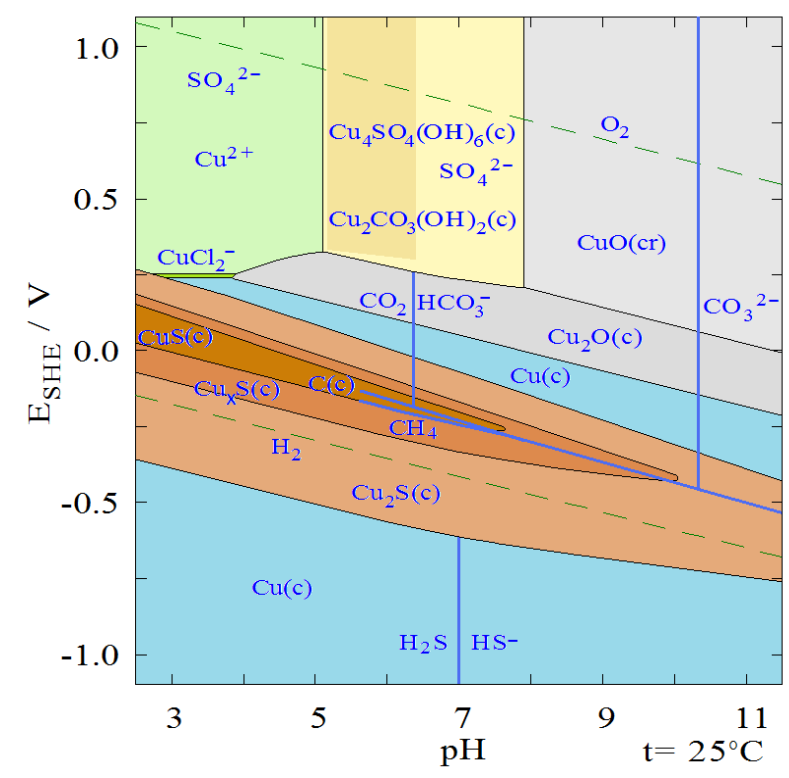

Figure 20a Pourbaix Diagram for Copper in Tap Water Equiv Solution

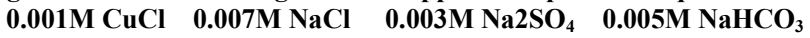




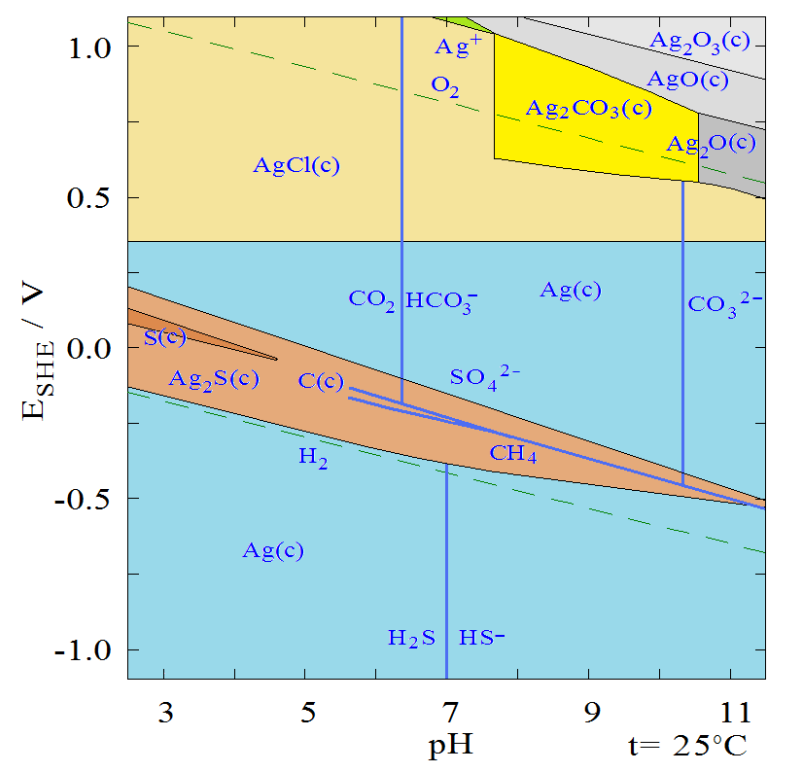

Figure 20b Pourbaix Diagram for Silver in Tap Water Equiv Solution

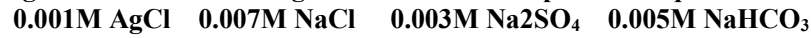

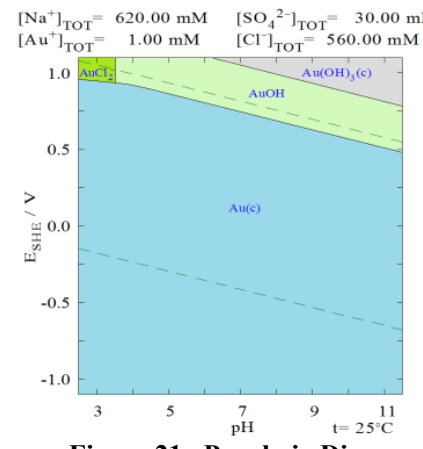

Figure 21a Pourbaix Diagram for Gold in Salt Water

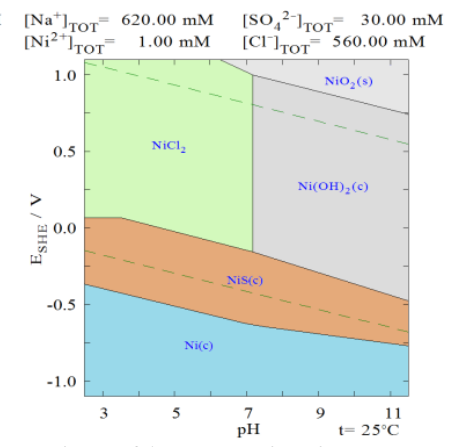

Figure 21b Pourbaix Diagram for Nickel in Salt Water

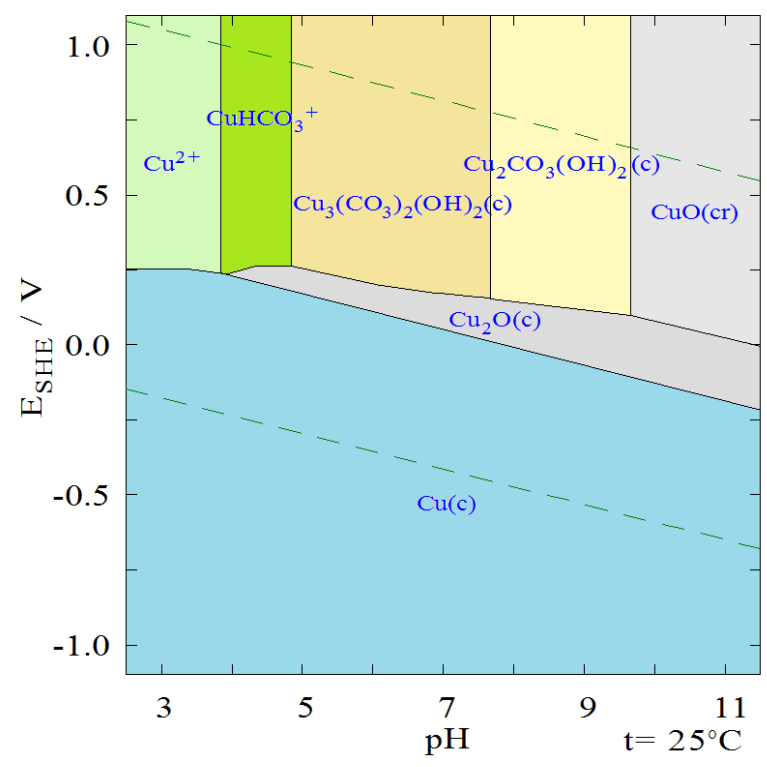

Figure 22a Pourbaix Diagram for Copper in Bicarbonate Solution $\begin{array}{ll}0.001 \mathrm{M} \mathrm{CuHCO}_{3} & 0.7 \mathrm{M} \mathrm{NaHCO}_{3}\end{array}$

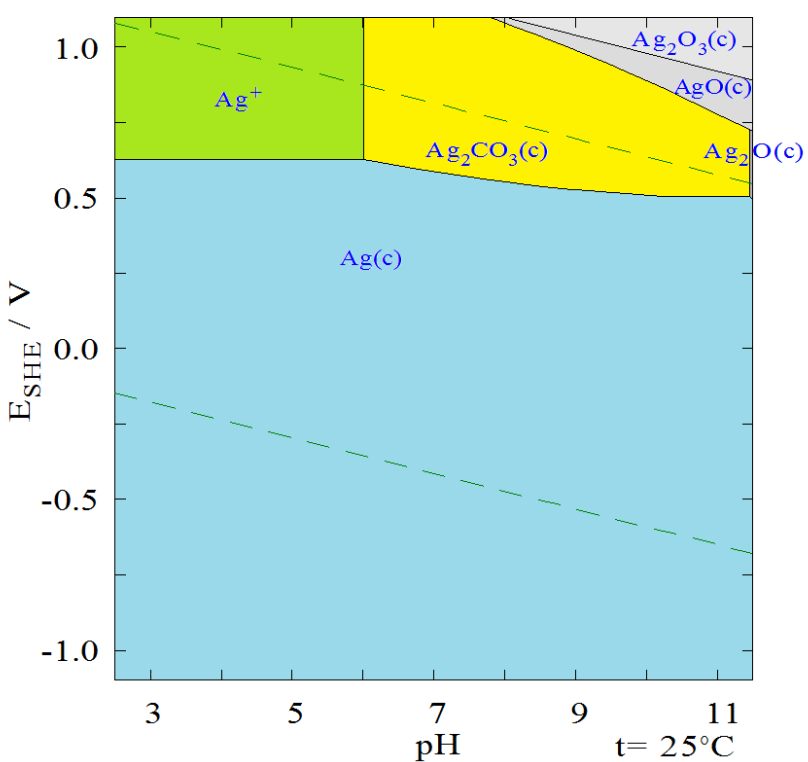

Figure 22b Pourbaix Diagram for Silver in Bicarbonate Solution $0.001 \mathrm{M} \mathrm{AgHCO}_{3} \quad 0.7 \mathrm{M} \mathrm{NaHCO}_{3}$

Table 2 includes characteristics of corrosion byproducts found in chemical data [26] that may form on contacts possibly causing a variety of effects ranging from beneficial lubrication to accelerated wear or contact passivation. Sulfides have lower resistivity and hardness, therefore sulfur compounds in small quantities may be useful as current collector additives. In some cases such materials can serve as lubricating third bodies with moderate conductivity [27]. Copper salts are more soluble than silver salts, therefore small amounts of sulfur or chloride contaminants in the brush atmosphere or water used for increased humidity would be likely to cause more corrosion of copper than silver. Research of copper corrosion by sulfuric acid deposited from $\mathrm{SO} 2$ in the atmosphere indicated that the corrosion rate would be increased at low $\mathrm{pH}$ because of metal ion solubility [28] as would be expected based on Pourbaix diagrams.

In prior experiments [20] silver brushes had lower resistivity and higher friction, however copper brushes had lower friction and a higher conduction voltage drop possibly because of corrosion byproducts. The silver alloy brush had operating characteristics similar to a copper brush; however it was run in air. Silver is a relatively noble metal as indicated in the Pourbaix diagrams and in an aqueous environment without oxygen the Eh may not be high enough for any corrosion, therefore very low electrical resistance and very high friction may result from metal on metal contact. Oxides are relatively hard and resistive, therefore these materials may cause increased conduction losses and higher wear rates because of abrasion and higher contact temperatures. The uncontrolled air atmosphere used in the current research would have contained $\mathrm{CO} 2$ and probably other trace substances such as sulfides which could have caused some of the difference between this and prior studies. 
Table 2 Possible Corrosion Byproduct Characteristics

\begin{tabular}{|c|c|c|c|c|c|c|c|c|}
\hline \multirow[b]{2}{*}{ Composition } & \multirow{2}{*}{$\begin{array}{l}\text { Metal } \\
\text { Compound }\end{array}$} & \multirow[b]{2}{*}{ Mineral } & \multicolumn{6}{|c|}{ Resistivity $\mathrm{m} \Omega \mathrm{cm}$ Hardness $\mathrm{mp}$ or $\mathrm{d}$ Density Solubility } \\
\hline & & & Minimum & Maximum & Mohs & Deg C & g/cc & $\mathrm{g} / 100 \mathrm{~mL}$ \\
\hline $\mathrm{Cu}$ & Copper & & 0.0017 & & 3.0 & 1085 & 8.96 & \\
\hline $\mathrm{Cu}_{2} \mathrm{O}$ & Copper(I) Oxide & Cuprite & 1000000 & 5000000 & 3.8 & 1244 & 6.00 & 0.00015 \\
\hline $\mathrm{Cu}_{2} \mathrm{~S}$ & Copper(I) Sulfide & Chalcocite & 8 & 10 & 2.8 & 1129 & 5.60 & Insoluble \\
\hline CuCl & Copper(I) Chloride & Nantokite & & & 2.5 & 423 & 4.14 & 0.0047 \\
\hline $\mathrm{Cu}_{2} \mathrm{O}$ & Copper(II) Oxide & Tenorite & & & & 1227 & 6.31 & Insoluble \\
\hline $\mathrm{Cu}\left(\mathrm{HCO}_{3}\right)_{2}$ & Copper(II) Carbonate & & & & & & & \\
\hline $\mathrm{Cu}_{2} \mathrm{CO}_{3}(\mathrm{OH})_{2}$ & Copper(II) Carbonate & Azurite & & & 3.8 & & 3.77 & \\
\hline $\mathrm{Cu}_{3}\left(\mathrm{CO}_{3}\right)_{2}(\mathrm{OH})_{2}$ & Copper(II) Carbonate & Malachite & & & 3.8 & 200 & 4.05 & Insoluble \\
\hline $\mathrm{CuCl}_{2}$ & Copper(II) Chloride & Eriochalcite & & & 2.5 & 598 & 3.40 & $\begin{array}{l}75.7 \\
\end{array}$ \\
\hline $\mathrm{CuSO}_{4}$ & Copper(II) Sulfate & Chalcocyanite & & & 3.5 & 560 & 3.60 & 22 \\
\hline & Silver & & 0.0016 & & 2.5 & 962 & 10.50 & \\
\hline $\mathrm{Ag}_{2} \mathrm{CO}_{3}$ & Silver(I) Carbonate & & & & & 218 & 6.08 & 0.0033 \\
\hline $\mathrm{Ag}_{2} \mathrm{O}$ & Silver(I) Oxide & & & & & 280 & 7.14 & 0.00002 \\
\hline $\mathrm{Ag}_{2} \mathrm{~S}$ & Silver(I) Sulfide & Argentite & 150 & 200 & 2.3 & 845 & 7.23 & 0.000014 \\
\hline $\begin{array}{l}\mathrm{AgCl} \\
\mathrm{Ag}_{2} \mathrm{SO}_{4}\end{array}$ & $\begin{array}{l}\text { Silver(I) Chloride } \\
\text { Silver(I) Sulfate }\end{array}$ & Cerargyrite & & & 2.5 & $\begin{array}{l}455 \\
660\end{array}$ & $\begin{array}{l}5.56 \\
5.45\end{array}$ & $\begin{array}{c}0.00016 \\
0.84\end{array}$ \\
\hline $\mathrm{Au}$ & Gold & & 0.0022 & & 2.5 & 1064 & 19.30 & \\
\hline $\begin{array}{l}\mathrm{AuCl} \\
\mathrm{AuCl}_{3}\end{array}$ & $\begin{array}{l}\text { Gold(I) Chloride } \\
\text { Gold(III) Chloride }\end{array}$ & & & & & $\begin{array}{l}289 \\
160\end{array}$ & $\begin{array}{l}7.60 \\
4.70\end{array}$ & $\begin{array}{c}0.000031 \\
68\end{array}$ \\
\hline $\mathrm{Ni}$ & Nickel & & 0.0071 & & 4.0 & 1455 & 8.90 & \\
\hline $\mathrm{NiOH}_{2}$ & Nickel(II) Hydroxide & Theophrastite & & & 3.5 & 230 & 4.10 & 0.00015 \\
\hline NiS & Nickel(II) Sulfide & & 0.02 & 0.04 & 3.3 & 797 & 5.50 & Insoluble \\
\hline $\mathrm{NiCl}_{2}$ & Nickel(II) Chloride & Nickelbischofite & & & 1.5 & 1031 & 3.51 & 67.5 \\
\hline NiS & Nickel(II) Sulfate & & & & & 840 & 4.01 & 40.4 \\
\hline
\end{tabular}

Techniques for choosing liquid additives are discussed in another paper in this conference. [29]. The pure metals are relatively soft, however alloys are harder, for example 14 karat gold [30] which is an alloy of gold, silver, and copper has significantly greater hardness than pure gold, and jewelry may include nickel or platinum to increase hardness, therefore such materials may be applicable to electrical contacts or metal fiber brushes.

\section{CONClusion And Future Work}

Silver metal fiber brushes were evaluated in a magnetic test fixture with variable speed, transport current and magnetic flux density. Silver alloy fiber brushes with a cross sectional area of 8.4 square millimeters were operated at a current of up to 90 amps, current density of 1070 amps per square centimeter. The brushes were operated in humidified air, at speeds of up to 15.5 meters per second and a flux density of up to about 0.6 Tesla. Significant differences between anode and cathode brush impedance characteristics were observed. The currentvoltage curves of the cathode brush were approximately linear with current indicating constant contact resistance while the anode had higher impedance and its characteristic was generally more non-linear with a diode like behavior. The magnetically induced circulating current losses were insignificant because of the small brush geometry and moderate magnetic flux density. Non-dimensional wear rates were on the order of $5 \mathrm{E}-11$. The brushes appeared to be thermally stable at temperatures up to $120^{\circ} \mathrm{C}$.

In this initial study, both the magnetic field and its effects on brush performance were limited. Further investigation of the effects of increased magnetic field on Ag Fiber brushes will require another apparatus such as the HiTC motor which can produce fields up to about $1 \mathrm{~T}$ in the brush region.

Continued work to study non-magnetic brush characteristics and to develop techniques for wear mitigation could be accomplished using smaller non-magnetic brush test fixtures. Brush wear mitigation methods may include brush region atmosphere control, or some form of additive.
A likely application for this technology is in Faraday homopolar machines. Smaller machines may utilize normally conducting field coils, high temperature superconducting field coils or a permanent magnet field in order to reduce cryogenic cooling requirements. Depending on application requirements, tradeoffs (e.g. between efficiency and power density) would drive the final configuration choices but all would enjoy the advantages of Faraday machines. Such advantages include a pure DC current to match a variety of alternative DC energy sources such as solar or fuel cells and simplified power electronic motor drive requirements.

\section{ACKNOWLEDGMENT}

The Authors wish to thank the Office of Naval Research for their continued generous support on this effort, in particular Program Officers Elaine Martin, Stephen Schreppler, Lynn Petersen and most recently Harold Coombe. The authors would also like to thank Mr. Barry Witherspoon and Norris Lewis of Moog Corporation for providing samples of silver brushes used in these preliminary studies, and Nils Nelson for test data collection support.

\section{REFERENCES}

1. D. Kuhlmann-Wilsdorf, " Electric Fiber Brushes-Theory and Observations.", IEEE Transactions on Components Hybrids, and Packaging and Manufacturing Technology ,Part A, Vol.19, No. 3, pp. 360-375, 1996.

2. D. Kuhlmann-Wilsdorf, "Metal Fiber Brushes", (Chapter 20 in "Electrical Contacts: Principles and Applications", Edited. By Paul G. Slade, Marcel Dekker, NY), 1999, pp.943-1017.

3. Philip Reichner, "Pressure-Wear Theory for Sliding Electrical Contacts" IEEE Transactions on Components, Hybrids and Manufacturing Technology, Vol CHMT4 ,No. 1, pp. 45-51, March 1981.

4. L. Boyer and J.P. Chabrerie, "Current Collection in a Homopolar Machine using Metallic Fiber Brushes", Proceedings of the 1983 Current Collector Conference, Annapolis, Md Oct 1983, pp.118-139

5. L. Boyer, J.P. Chabrerie and J. Saint-Michel, "Low wear metallic fiber brushes", Wear, 78 (1982) pp. 59-68.

6. Timsit, Roland, Edited by Paul G. Slade, "Electrical Contacts Principles and Applications", New York, Marcel Dekker, pp. 2-4.

7. T.J. Doyle and H.O. Stevens "Development and Demonstration of a $300 \mathrm{~kW}$ Advanced DC Ship Drive System”, Trans. I. Mar. E(c), Vol. 976. Conf. 3, Paper 7, pp. 59-65 (1985).

8. Lynn J. Petersen, Damian Urciuoli, Mehren Alam, Thomas H. Fikse, Laura D. Stubbs, William A. Lynch, Neal A. Sondergaard, Doris Kuhlmann Wilsdorf, John T. Moore, Robert B. Nelson, Matthew S. Bednar, Wallace M. Elger, Richard W. Johnson, Richard J. Martin, and Michael Heiberger "A Study of the Magnetic Field Effects upon Metal Fiber Current Collectors in a High Critical Temperature Superconducting Homopolar Motor", Third Naval Symposium on Electric Machines, Philadelphia, PA, Dec 2000.

9. M. Heiberger, M. R. Reed, W. P. Creedon, B. J. O'Hea, "Superconducting DC Homopolar motors For Ship Propulsion" AEC 2000 Conference Paper, Paris Oct, 2000. http://www.ga.com/atg/EMS/homopolar.php http://atg.ga.com/EMS/defense/dc-motor/index.php

10. http://www.polysci.com/docs/fiberbrush.pdf 
11. http://www.hipercon-llc.com/

12. http://www.sohreturbo.com/

13. http://superconductingsystems.com/engsol.htm

14. R. Holm, "Electric Contacts", Springer-Verlag, New York, 1967.

15. J.S. Walker, G.S. Erwin and N.A. Sondergaard, "Modeling of Metal Fiber Brushes Part I: The Electromechanics of Single Fibers", NSWCCD-98-TR-2009-02, 2009.

16. G. S. Erwin and N. A. Sondergaard, "Electromechanics of a Collection of Current Carrying Cantilevered Fibers Sliding against a Plane Moving Surface in a Three Dimensional Magnetic Field" 55th IEEE Holm Conference on Electrical Contacts, Vancouver, British Columbia Canada, September 14-16, 2009.

17. Philip Reichner," Metallic Brushes for Extreme High Current Applications", IEEE Trans. On Components, Hybrids and Manufacturing Technology, Vol.CHMT-3 No.1, March 1980, p 21-26.

18. "Evaluation of Platinum-Based Catalysts for Methanol Electrooxidation in Phosphoric Acid Electrolyte", by C. He., H.R. Kunz, and J.M. Fenton, J. of the Electrochemical Society, Vol. 144, pp. 970- 979 (1997).

19. N. Argibay, J.A. Bares, W.G. Sawyer. "Asymmetric wear behavior of self-mated copper fiber brush on disk sliding electrical contacts in a humid carbon dioxide environment", Wear (in review), (2009).

20. Philip Reichner, "High Current Tests of Metal Fiber Brushes" IEEE Transactions on Components, Hybrids and Manufacturing Technology, Vol. CHMT4 ,No. 1, pp. 2-4, March 1981.

21. Pourbaix, Marcel, Atlas of Electrochemical Equilibria in Aqueous Solutions, Oxford, NY, Pergamon Press, 1966, second edition, Houston TX, National Association of Corrosion Engineers, 1974.

22. Ignasi Puigdomenech "MEDUSA: (Make Equilibrium Diagrams Using Sophisticated Algorithms) program and Hydra: Hydrochemical Equilibrium Constant Database”, Inorganic Chemistry, Royal Institute of Technology, Stockholm Sweden, November Medusa http://w1.156.telia.com/ u15651596 http://www.kemi.kth.se/medusa

23. http://www.epa.gov/safewater/contaminants/index.html\#listsec

24. http://www.epa.gov/nrmrl/pubs/600R95085/effect.pdf

25. Xingyi Deng, Albert Verdaguer, Tirma Herranz, Christoph Weis, Hendrik Bluhm, Miquel Salmeron "The surface chemistry of $\mathrm{Cu}$ in the presence of $\mathrm{CO} 2$ and $\mathrm{H} 2 \mathrm{O}$ ", Lawrence Berkeley National Laboratory. Paper LBNL-1388E, Langmuir, American Chemical Society, 2008

26. David R. Lide, "CRC Handbook of Chemistry and Physics $87^{\text {th }}$ Edition ", CRC Press, 2006.

27. I.L. Singer, S.D. Dvorak, K.J. Wahl and T.W. Scharf, "Role of Third Bodies in Friction and Wear of Protective Coatings", J Vac Sci Technol A, Vol. 21, No.5, pp.232-240, 2003.

28. J. H. Payer, "Corrosion Processes in the Development of Thin Tarnish Films", Proceedings of the 36th IEEE Holm Conference on Electric Contacts, Montreal Canada, pp203-211, 1990.

29. W. A. Lynch, N.A. Sondergaard, "Liquid Additives to Improve Conductivity in Electric Contacts", 55th IEEE Holm Conference on Electrical Contacts, Vancouver, British Columbia Canada, September 14-16, 2009.

30. http://www.18carat.co.uk/hardnessofgoldalloys.html

\section{BIOGRAPHIES}

Dr. William A. Lynch was most recently at Raven Technology and has 20 years of experimentalist R\&D experience in the areas of Current Collector/Brush Technology, Energy Storage Device Characterization, and Power Electronic Electric Machinery Drive Systems. His graduate degree research focused on development of an Electric Vehicle Battery Evaluation Laboratory Facility and Photovoltaic System Design. He received his BSc, MSc and Doctorate degrees in Electrical Engineering from the University of Massachusetts Lowell in 1987, 1991, and 1997. He participated in Solar Race events including Sunrayce, the World Solar Challenge, and the American Tour De Sol. He is a member of IEEE. He has co-authored 24 research papers and DON reports and is a co-inventor on 9 U.S. Navy patents.

Dr. Mark J. Roberts received his Ph.D. in Electrical Engineering from Worcester Polytechnic Institute in 1991. He also holds a Masters of Science Degree in Electrical Engineering form Worcester Polytechnic Institute and a Bachelor of Science Degree in Physical Science from the United States Naval Academy. Since joining the Naval Surface Warfare Center in 2002 his research has focused on the use of finite \& boundary element analysis for the prediction of internal and external magnetic fields of electric motors. He has expertise in the areas of finite difference methods, finite and boundary element modeling, microwave and ultrasonic engineering, signal processing techniques, and linear and controls system theory. Prior to joining the NSWCCD, Dr. Roberts worked at the US Army Vehicle Technology Directorate at NASA Langley Research Center. $\mathrm{He}$ served five years as a commissioned Naval Surface Warfare Officer onboard Navy ships from 1978-1983.

Dr. Ronald P. Reitz retired after 29 years of service to the Navy. As the discoverer of the first and second Reitz effects, he investigated the phenomena associated with these effects while conducting research for NSWCCD in material science. During his service as a research physicist, he received numerous patent awards while working in the fields of acoustics, machinery silencing, naval auxiliary systems, magnetorheology as well as in the fields of electrorheology and electroset technology. Prior to joining NSWCCD, he attended the US Naval Academy and University of Maryland for his undergraduate work. He attended both University of Maryland and Catholic University for his graduate studies after joining the Center. Although currently in retirement, he has authored one book and is currently working on two more.

Dr. Neal Sondergaard is a senior scientist at NSWCCD with 29 years experience in $\mathrm{R}+\mathrm{D}$ including sliding electric contacts(brushes), Tribology, MHD, superconductivity and electric power production, generation, conversion, machinery and distribution and Propulsion System Modeling. He received his B.A. in Chemistry from Marist College, his M.Sc. and $\mathrm{Ph} . \mathrm{D}$. in Physical Chemistry from Brown University and was a Post Doctoral Fellow at the Johns Hopkins University. He is a member of Sigma Xi Scientific Research Society, and ASNE American Society of Naval Engineers. 


\title{
3-D Simulation of Open-Barrel Crimping Process
}

\author{
Study of the Effect of Serrations
}

\author{
Dmitry V. Zhmurkin \\ Interconnection \& Process Technology \\ Tyco Electronics \\ Harrisburg, USA \\ dzhmurkin@tycoelectronics.com
}

\begin{abstract}
A numeric simulation of an open-barrel crimping process of 7-stranded wire has been conducted using a full 3dimensional non-linear explicit dynamic finite element model. This work concentrated on studying the effect of serrations. Three types of open barrel crimps were compared one with 2 serrations and two with 3 serrations of different kinds. In addition, serration geometric parameters were varied. The model was validated using experimental results. The goal of the study was to investigate the effects of the number of serrations and their length, as well as serration width, depth, and the slope of serration sidewall on the quality of an open barrel crimp connection.
\end{abstract}

Keywords - Finite elements methods, numerical analysis, connectors, crimp, serrations.

\section{INTRODUCTION}

Crimping is considered one of the most reliable metal joining technologies in the connector industry. Although it is among the oldest methods of establishing a permanent wire-toterminal connection, the underlying mechanical principles of this process still become a source of heated debates within the industry's engineering community. Even a quick literature search is guaranteed to yield a list of publications that disagree with each other and state quite opposite points of view on 'what makes a good crimp'. Attempts to define the fundamental mechanics of crimped connections ranged from simplistic "back-of-the-envelope" derivations ([1], [2]) to extensive 3-dimensional dynamic Finite Element Analysis studies that take into account not only every detail of geometry of interacting parts but also the speed of the tool ([3]-[6]). However, a unified mechanical theory of a reliable crimped connection is yet to be established. In part, it is due to the complexity of the problem. As suggested by Whitley [1] and later confirmed by Mroczkowski et al. [7] and Zhmurkin et al. [6], a design task of creating a reliable crimped connection is not limited to providing significant plastic deformation to wire barrel and wire strands, but also focuses on minimizing effects of springback and maintaining reliable adhesion at the contact interfaces.

Another challenge in establishing an all-inclusive and well supported mechanical theory of reliable crimped connections is the difficulty of obtaining clear criteria that consistently identify good-quality crimp designs and weed out the faulty ones. Since the main function of crimped connections is to conduct current, it is a common practice to judge the quality of the connection by its electrical resistance. However, initial electrical resistance can hardly be chosen as a good indicator of future crimp reliability because of the simple fact that throughout their life cycle, crimps will be subjected to temperature swings, mechanical abuse, and/or harsh environments. All of these factors can potentially result in contact degradation and in increase of contact resistance. Therefore, it is the ability of the crimp to maintain low electrical resistance that is at the heart of a reliable crimped connection. As a result, proper testing of new crimp designs is very time consuming and expensive. This explains why companies (both manufacturers and customers of crimped connectors) are not very willing to share their empirical findings that took years to acquire. As a matter of fact, internal crimp design guides are among most guarded secrets within the companies that make crimped terminals.

Serrations in crimps are one of the examples of these well guarded secrets. It is now common knowledge that serrations are beneficial for crimp reliability but very little information is available on the specifics and the reasons for this recommendation. A few general connector publications [8][10] that do mention serrations provide absolutely no details on the recommended or preferred type of serrations, their number, width or depth, etc.

This paper provides some results of our ongoing Finite Element Analysis (FEA) study of the crimping process, and here we focused on effect of serrations. Advances in development of finite element code and computer processing power provide a tool that can be used effectively to study mechanics of crimped connections in spite of the complexity of the problem.

\section{OBJECTIVE AND METHOD DESCRIPTION}

Serrations are small impressions that are created either by removing or displacing material on the inside of the wire barrel as shown in Figure 1 and Figure 2.

The serrations in a crimp terminal are believed to serve 3 main purposes: 


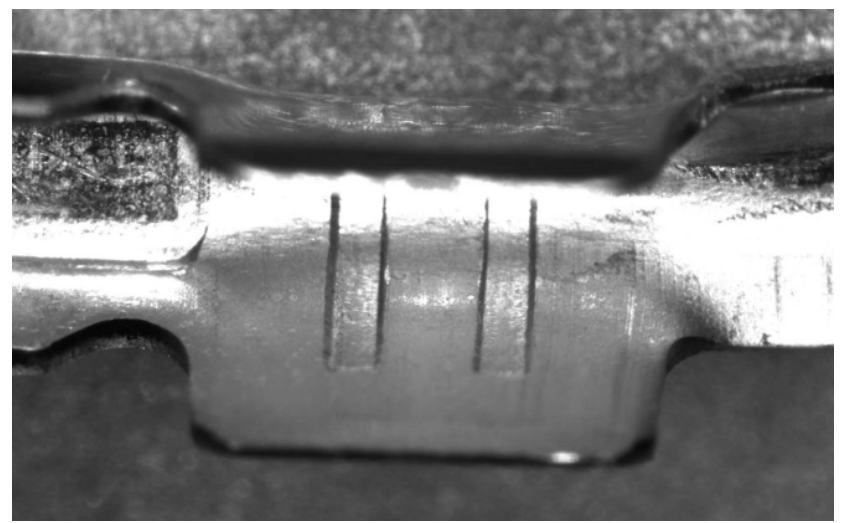

Figure 1. Wire barrel with 2 standard serrations

- High pressure of the crimping process deforms the conductor and forces it into the serration cavities and as it flows over the edge of serrations, the surface of the wire gets scraped and cleaned from oxides or organic films, thus providing a better electrical contact.

- Bringing clean metallic surfaces together with sufficient pressure allows "cold welding" to occur [1], [7].

- Deformation of conductor into the serrations provides a mechanical "lock" which improves mechanical stability of the crimp.

Recently we presented a robust and reliable 3-dimensional explicit FEA model of crimping process [6]. Now this model, modified by dimensions and materials of the parts being studied, was used to investigate the effect of serrations in crimped connections. Whereas the model does not account for "cold welding" at the contact interfaces, it is well suited for a study of mechanical effect of serrations on crimp quality, especially elongation of barrel and wire strands and quality of mechanical "lock" between the barrel and the wire.

A crimp terminal with two serrations was used as a benchmark for the study because of extensive test data that was available for this product. The wire barrel of this terminal is shown in Figure 1.

To study the effect of the number of serrations on crimp

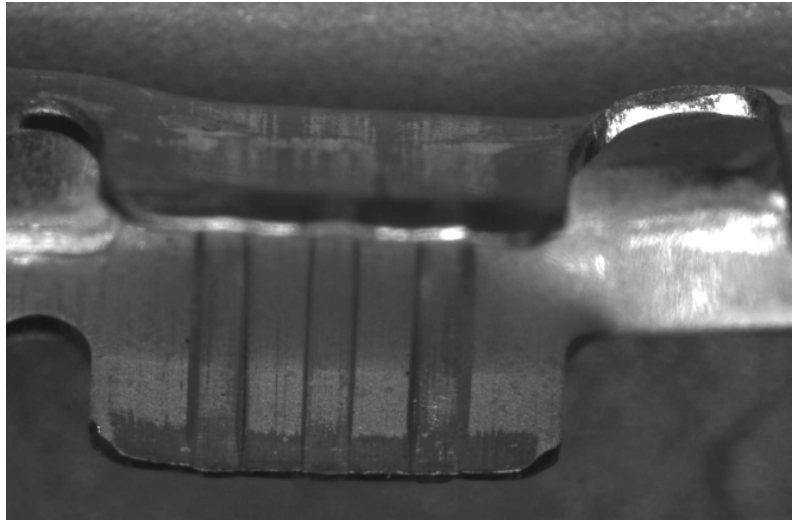

Figure 2. Wire barrel with 3 long serrations performance, a few new terminals were made. These terminals are identical to the benchmark terminals with only one difference - they have three serrations instead of the standard two and these serrations extend to the edges of the wire barrel, whereas in the standard terminals the serrations are shorter than the developed width of the barrels. These 3-serration terminals have also been subjected to extensive testing. The wire barrel of the terminals with 3 serrations is shown in Figure 2. The FEA models were validated using the test data for these two types of terminals.

In addition, a numerical study was conducted on a few other types of terminals. Among them was a crimp with a different type of a 3-serration wire barrel. Here the serrations did not extend to the edge of the wire barrel, but were the same length as those in the standard 2-serration terminal. A model of a crimp without any serrations was also created.

Also, we investigated the effect of variation of key serration parameters. Serration width was numerically varied $+/-20 \%$ from the nominal. Depth of serrations was varied $+/-$ $50 \%$ from the nominal. The sidewall angle of the serrations was varied $+/-10 \%$ from the nominal. These variations are summarized in a schematic in Figure 3.

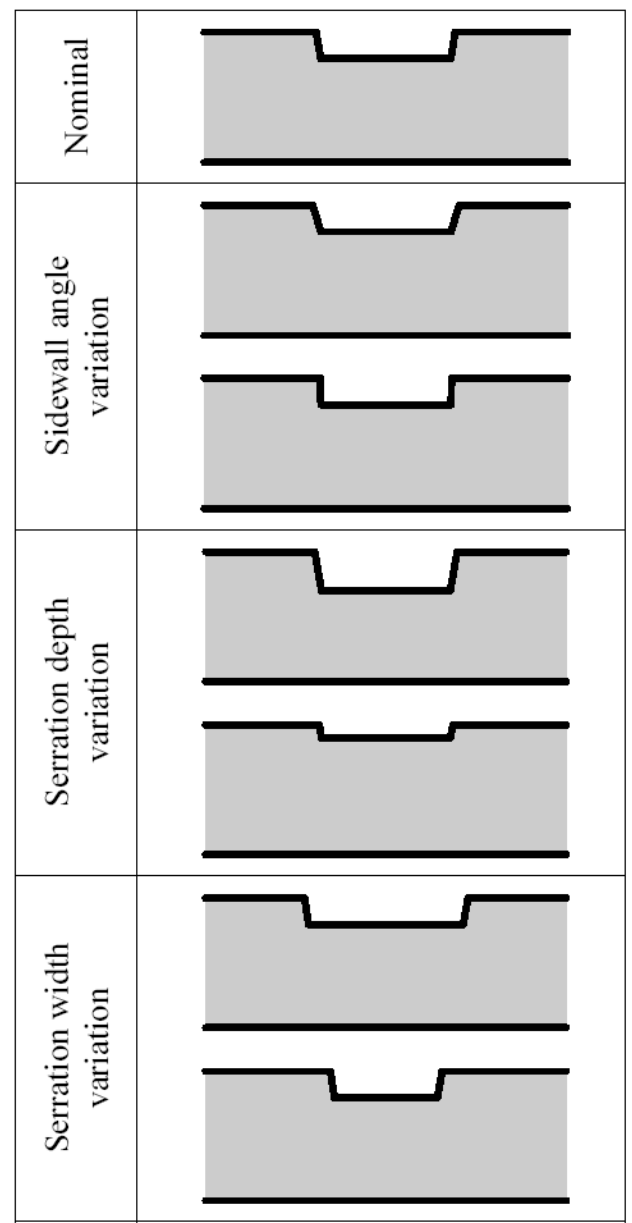

Figure 3. Variations of serration geometry 


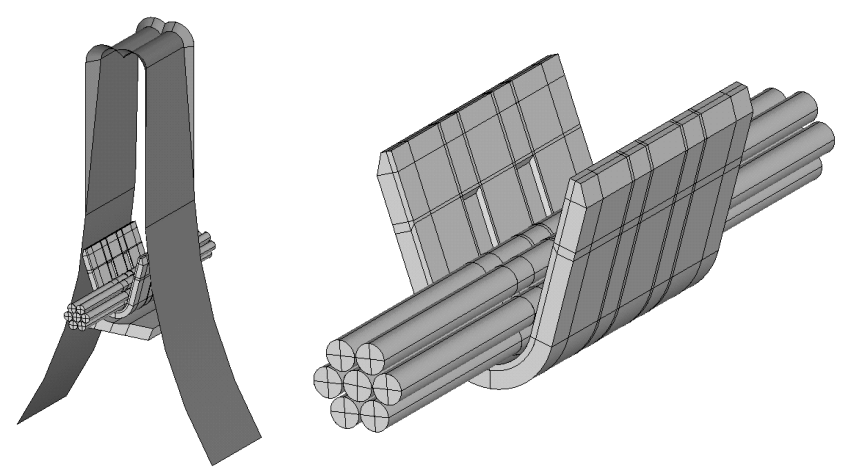

Figure 4. Geometry used in the FEA model

The details on general model setup and assumptions are discussed in our recent publication [6]. The wire barrel was modeled using 8-node brick elements with reduced integration. The barrel is $2.5 \mathrm{~mm}$ long and it is made out of 0.2 $\mathrm{mm}$ thick sheet of copper-nickel-silicon Wieland-K55 (C70250) alloy with TM00 temper. The crimping tool is assumed to be a non-deformable rigid body and is modeled with 4 and 3 node shell elements. The anvil was modeled using 8-node brick elements and it was also assumed to be a rigid body. Each strand of the 7-stranded copper wire was modeled separately using 8-node brick elements, and it has a diameter of $0.252 \mathrm{~mm}$, which corresponds to 22 AWG wire with a combined cross-sectional area of $0.35 \mathrm{~mm}^{2}$. Table 1 shows material properties of the terminal and the wire materials.

During the simulation the anvil is fixed and the crimper is moved downward via applied velocity. The sine wave shaped crimper velocity profile with the maximum velocity of 0.5 $\mathrm{m} / \mathrm{s}$, which is the same order of magnitude as the actual tool velocity during the crimping process, was chosen because it provided a good balance of model accuracy and computation time.

A total of 10 separate bodies can potentially come into contact with each other during the crimping simulation - the crimper, the anvil, the wire barrel, and the 7 wire strands. This resulted in 31 contact interfaces that needed to be independently defined during the analysis. A static coefficient of friction of 0.2 and a dynamic coefficient of friction of 0.1 were chosen for all contact interfaces. In our previous work [6] these values consistently resulted in satisfactory prediction of barrel and wire deformation, whereas higher values

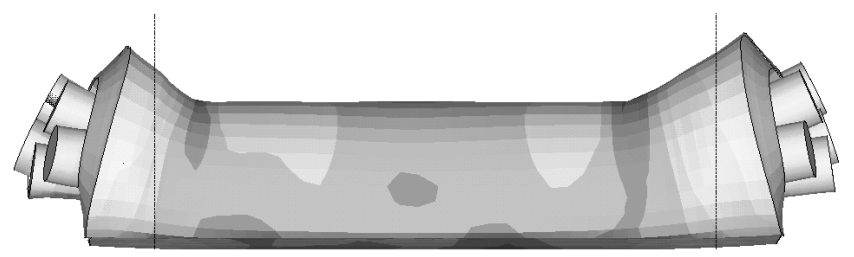

Figure 5. Extrusion of wire barrel and strands. Initial length of the terminal and displayed wire strands is indicated by vertical lines.
TABLE I

MATERIAL PROPERTIES OF MODEL COMPONENTS

\begin{tabular}{lcc}
\hline \hline Material Property & Wire Barrel & Conductor \\
\hline Modulus of elasticity, GPa & 124 & 109 \\
Yield Strength, MPa & 620 & 170 \\
Density, g/cm ${ }^{3}$ & 8.8 & 8.9 \\
Poisson's Ratio & 0.32 & 0.3 \\
\hline \hline
\end{tabular}

predicted significant bulging of the barrel walls and unsatisfactory "wing lock" and these predictions correlated well with experimental data.

\section{OBSERVATIONS AND RESULTS}

In addition to visual comparison of different cross-sections of the resultant crimps, predicted by the model, and subjective evaluation of the quality of the fill of the serrations by the wire strands, other metrics were used to compare the results. These metrics included: amount of longitudinal extrusion of the wire barrel, amount of extrusion of each wire strand, and relative extrusion of wire strands with respect to the wire barrel. Extrusion was measured by following the deformation of a few nodes selected at predefined and axially coincident locations in the wire barrel and each of the wire strands. This allowed consistent measurement of extrusion between different cases that were studied. Additional discussion on model extrusion predictions and their validation with experimental results is given in our previous publication [6].

Depending on their location in the wire barrel, the wire strands extrude a different amount, as can be seen from Figure 5. Wire strands on the outside of the wire bundle that were forced to fill the serrations generally extruded less than the wire strands that did not come into contact with serrations. For the cross-section shown in Figure 6, the strands numbered 2, 5,6 , and 7 extrude less than strands numbered 1,3 , and 4 .

To simplify the otherwise busy graphs in the figures that follow, only results for wire strands with maximal and minimal amount of extrusion were plotted. For example, graph (a) in Figure 7 shows the amount of extrusion of the wire barrel and the wire strands of the benchmark case - the wire barrel with 2 standard serrations. The solid line represents barrel extrusion, whereas the two dotted lines show the extrusion of two wires - with most and least amount of

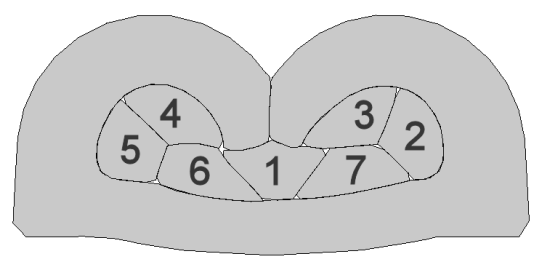

Figure 6. Cross-section of a crimp as predicted by the model 


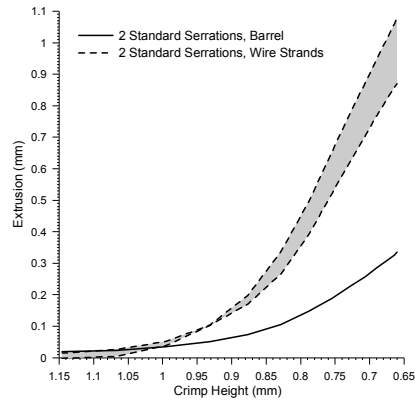
wire strands (a) Extrusion of wire barrel and

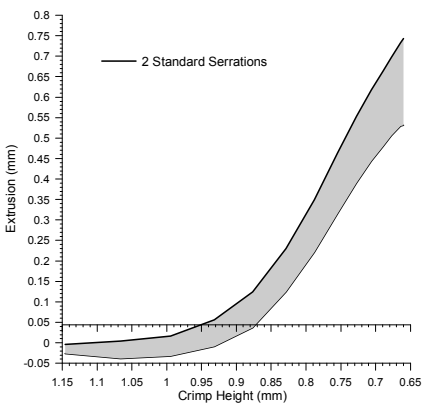

(b) Relative extrusion of wire strands with respect to wire barrel
Figure 7. Typical graphs used to present extrusion data predicted by the FEA models

extrusion. As noted above, the lower line represents a wire strand caught in the serrations, and the upper line - a strand either inside of the wire bundle or on top of the crimp and away from the serrations. If plotted, the curves representing the extrusion of all other strands would appear inside of the envelope defined by these dotted lines. Similar approach was

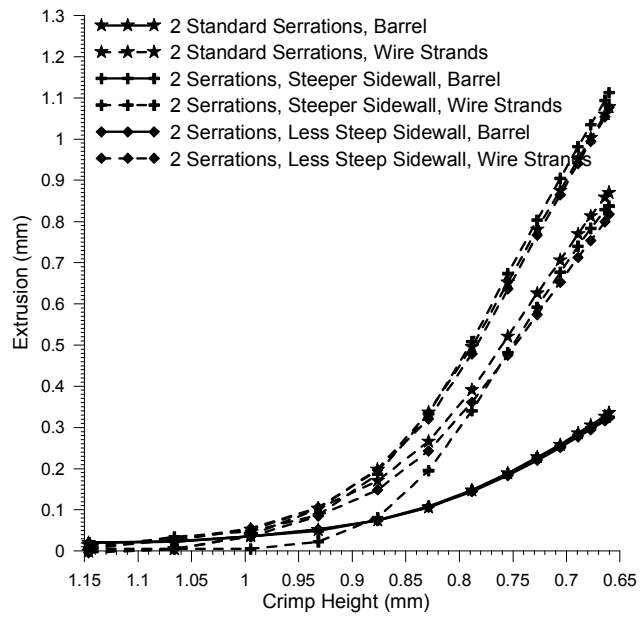

(a) Extrusion of wire barrel and wire strands

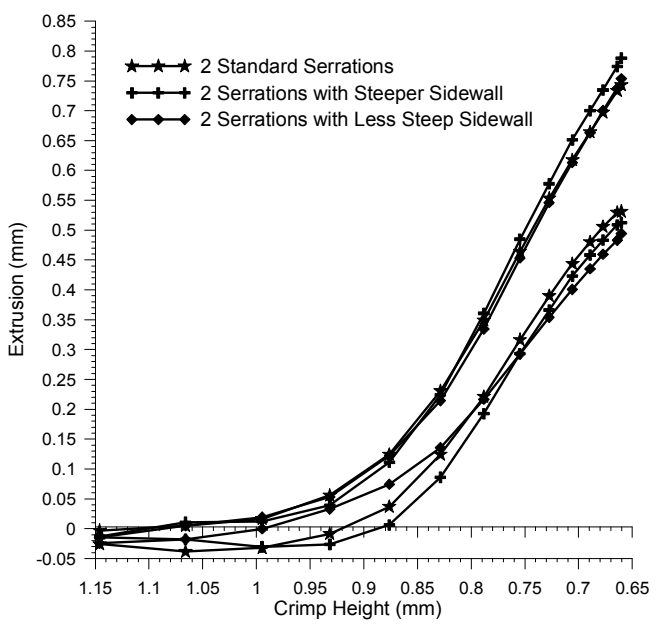

(b) Relative extrusion of wire strands with respect to wire barrel

Figure 8. Extrusion measurements for serrations with different wall slope taken in Figure 7 (b), showing relative extrusion of the wire strands with respect to the wire barrel. These curves were obtained by subtracting barrel extrusion from corresponding extrusion of the wire strands. This is why negative value is possible and it means that at that crimp height the barrel extruded more than the wire strand. Again, only the results for wire strands with minimal and maximal values were plotted results for other wire strands would be enclosed within the envelope defined by the shown curves. This method of presentation of results is maintained throughout this report to avoid unnecessarily busy graphs.

\section{A. Serration Sidewall Angle Variation}

In this group we modeled crimps with 2-serrations, and the slope of serration sidewalls was varied $+/-10 \%$ from the nominal angle. It can be argued that having a sharp edge (steeper slope of the serration sidewall) is beneficial for scraping off organic debris, films, and oxides from the wire surface, thus ensuring a better electrical contact of metallic interfaces. However, this behavior is beyond the scope of this study, and the metrics that were chosen for comparison did not

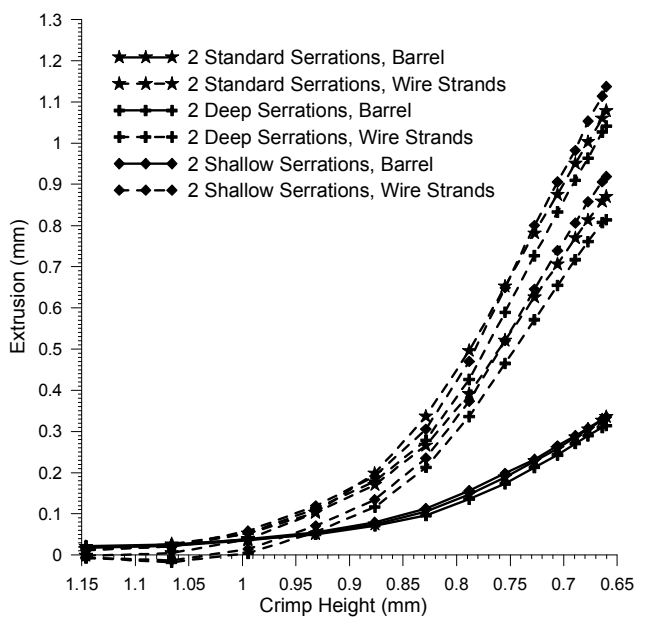

(a) Extrusion of wire barrel and wire strands

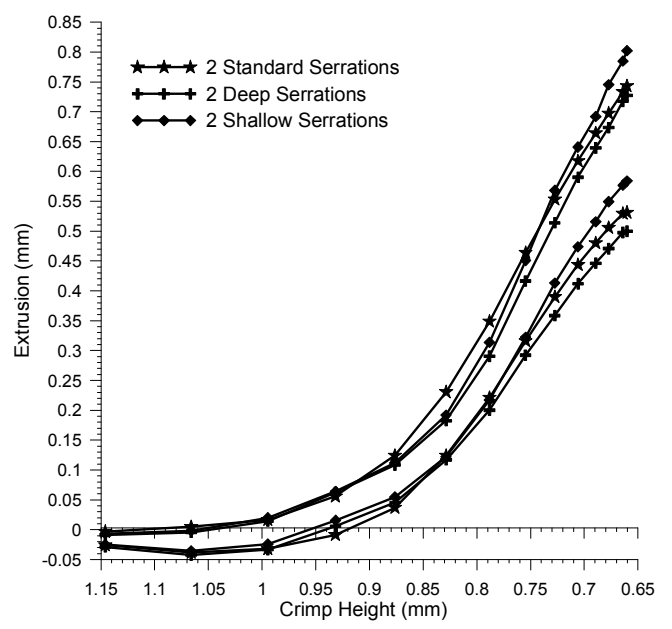

(b) Relative extrusion of wire strands with respect to wire barrel

Figure 9. Extrusion measurements for serrations of different depth 
show any significant difference between these wire barrels as seen in Figure 8. It does appear though that on the early stages of crimp formation, up to crimp height of approximately 0.85 $\mathrm{mm}$, in the case of steeper serration sidewalls, the wire strands that got caught in the serrations (lower dotted line on the graph) did not extrude as much as when the serrations have less steep sidewalls. The amount of wire barrel extrusion, on the other hand, was not affected by the variations in serration sidewall slope.

\section{B. Serration Depth Variation}

This study is based on FEA models of crimps with 2serrations and the depth of the serrations was varied $+/-50 \%$ from the nominal depth. Although counterintuitive, the measurements obtained by the model, showed very minor differences in overall amount of extrusion of wire barrel and wire strands between these cases. A slight deviation in extrusion of wire strands that were filling the serrations (lower dotted lines in (a)) can be seen at the later stages of crimp

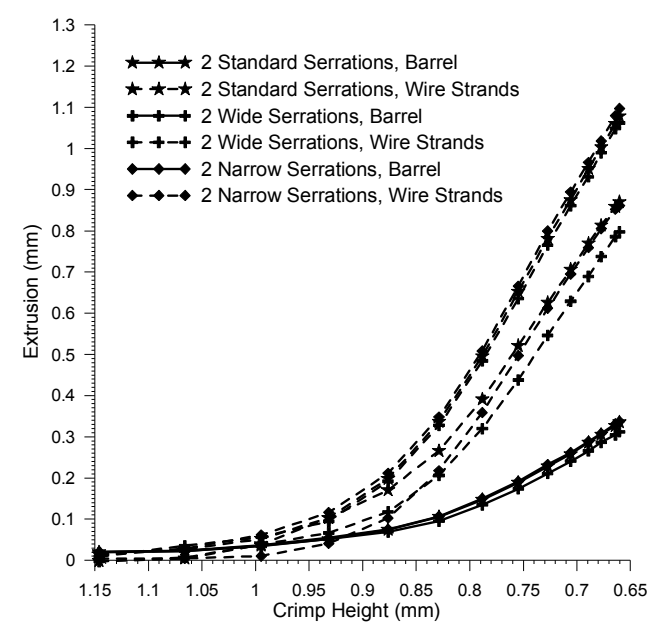

(a) Extrusion of wire barrel and wire strands

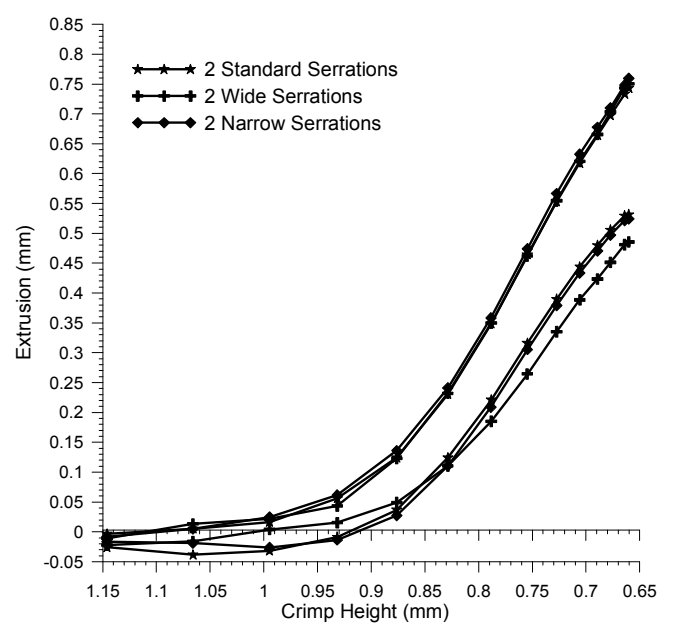

(b) Relative extrusion of wire strands with respect to wire barrel

Figure 10. Extrusion measurements for serrations of different width formation - wires extruded less when crimped to a barrel with deeper serrations. Comparison of these results is given in Figure 9.

\section{Serration Width Variation}

We compared models of crimps with 2 serrations and width of the serrations was varied $+/-20 \%$ from the nominal value. As shown in Figure 10, there is a slight reduction in amount of extrusion of wire strands that filled the serrations (lower dotted lines in (a)) in the case of a barrel with wider serrations. However, as in the previous cases, the amount of barrel extrusion did not get noticeably affected by the variations of serration width.

\section{Length of Serrations}

In this case we compared wire barrels with 3 serrations of different length - standard (as shown in Figure 1) and long, extending all the way to the edge of the wire barrel, as shown in Figure 2. Length of serrations had a direct effect on the amount of deformation of wire strands as can be seen in

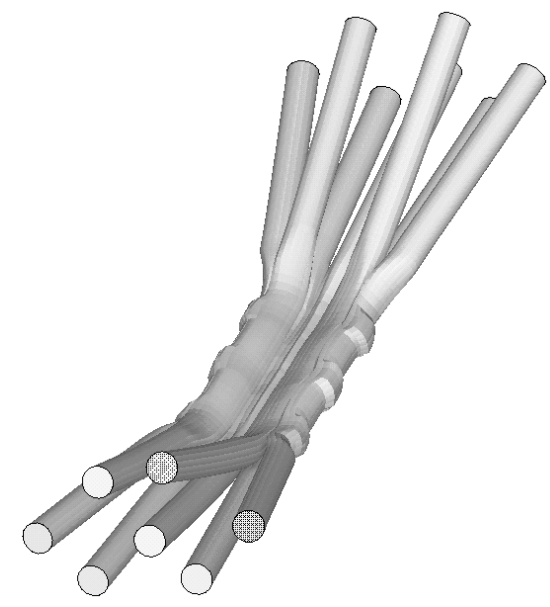

(a) Deformation of wire strands inside a barrel with standard serrations

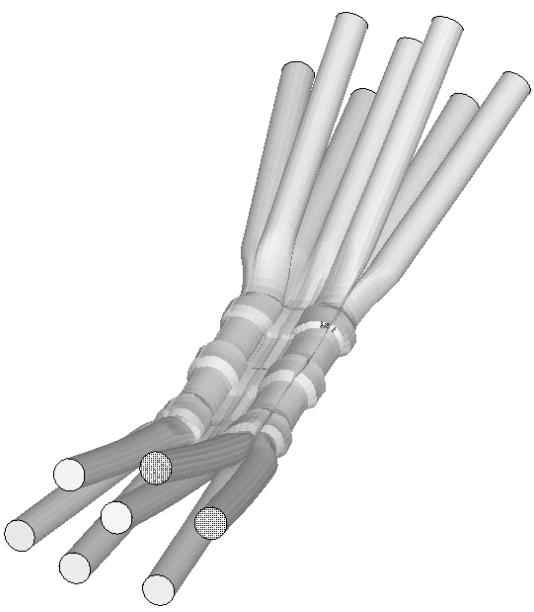

(b) Deformation of wire strands inside a barrel with long serrations 


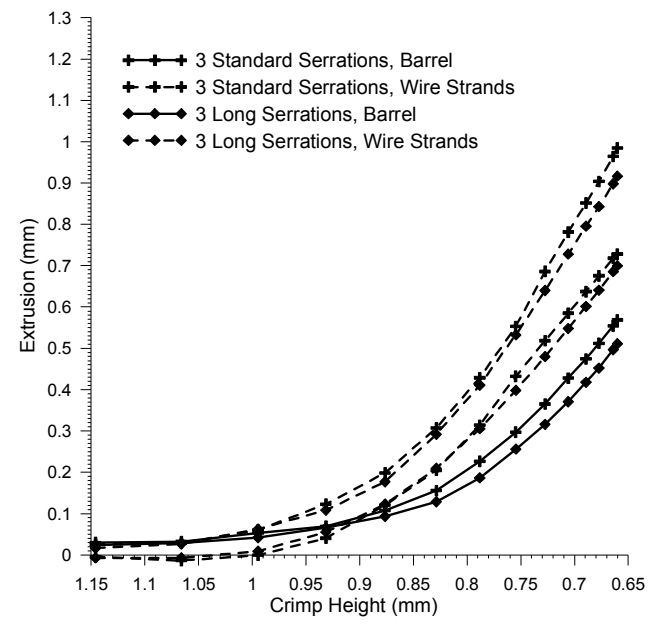

(a) Extrusion of wire barrel and wire strands

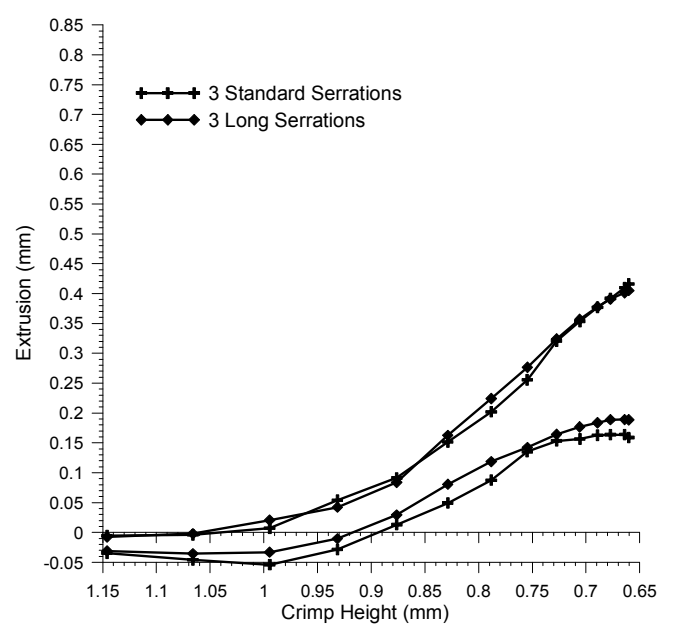

(b) Relative extrusion of wire strands with respect to wire barrel

Figure 12. Extrusion measurements for serrations of different length

Figure 11. Long serrations deformed the strands all the way around the wire bundle, effectively locking them and restricting their extrusion. Even the center strand experienced reduced extrusion. Graphs in Figure 12 confirm this observation, however, in spite of substantial wire deformation as a result of longer serrations, the change in amount of wire extrusion is not as dramatic as one might expect.

\section{E. Number of Serrations}

Finally, let us examine the effect serration count has on the amount of extrusion of the wire strands and the wire barrel. Here we compared 3 cases - the benchmark wire barrel with 2 standard serrations, a wire barrel without any serrations at all, and a wire barrel with 3 standard serrations. This property has had the most visible effect on the amount of extrusion compared to the geometric changes in serrations discussed above. There is no surprise that between these three cases the wire strands extruded the most in the terminal without any serrations, as can be seen in Figure 13(a). Surprisingly,

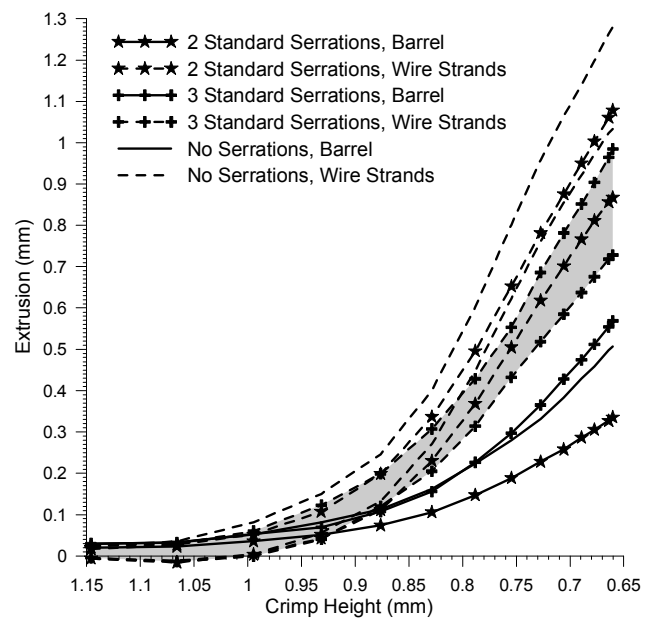

(a) Extrusion of wire barrel and wire strands

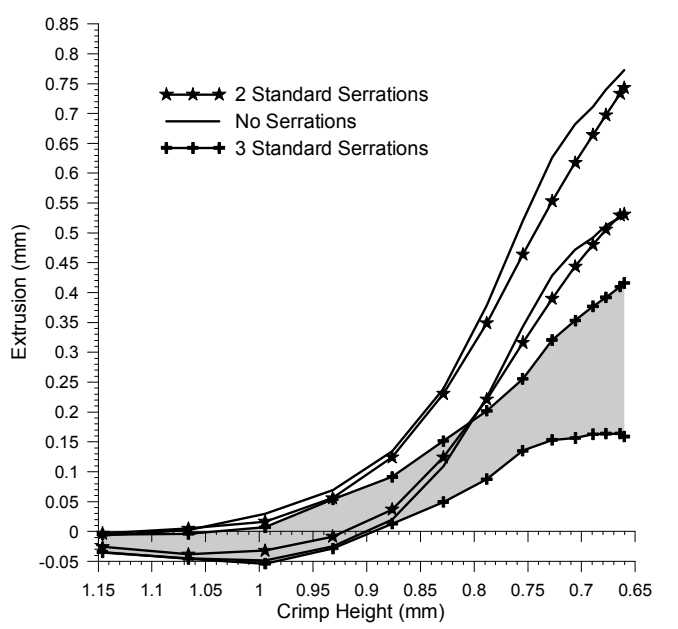

(b) Relative extrusion of wire strands with respect to wire barrel

Figure 13. Extrusion measurements for different serration count

although there are no serrations to provide the mechanical "lock", the wire extrusion is far from being uniform - the strands on the outside of the wire bundle extrude noticeably less than the core strand and the difference between the minimal and the maximal strand extrusion (the spread between two dotted lines in Figure 13(a)) is similar to that of any other case discussed in this report.

The least amount of extrusion was observed in the barrel with 3 serrations. Clearly, addition of the $3^{\text {rd }}$ serration resulted in a more noticeable "locking" of the wire strands. Another important observation is significantly reduced amount of relative extrusion between wire barrel and wire strands in the crimp with 3 serrations that is shown by the grayed out area in Figure 13(b).

\section{F. Reliability Testing}

Two types of crimps discussed above were also subjected to extensive testing per USCAR21 [11] and per European 
automaker group's standard LV 214-2 [12] - the one with 2 standard serrations (shown in Fig. 1) and the one with 3 long serrations (shown in Fig. 2). Both of these standards outline methods of accelerated electrical and mechanical testing of crimped connectors subjected to environmental and mechanical stress.

The testing revealed that addition of the $3^{\text {rd }}$ serration was beneficial for electrical and mechanical performance of the crimp. Samples with 3 long serrations consistently outperformed those with 2 standard serrations during the pullout force, thermal/humidity cycling, thermal shock, and mechanical flex testing.

It is too premature to make a direct correlation between the amount of barrel-wire relative extrusion and crimp reliability, however the comparison with test results indicate that there is a relationship between these effects. Crimps that showed lower amount of barrel-wire relative extrusion in the numerical model outperformed the other samples during accelerated testing.

\section{CONCLUSIONS}

This report presented some results of our ongoing Finite Element Analysis study of open-barrel crimping process. Advances in computing power and the level of development of the finite element codes allow engineers to address very complex problems such as the processes characterizing the mechanics of crimped connections. Although some complex attributes of the crimping process, such as adhesion at contact interfaces or micro-level surface interactions, are currently beyond the capabilities of standard FEA modeling techniques, numerical analysis can still be effectively used to study the mechanics of the crimping process, to optimize crimp designs, and to play "what if" scenarios.

Using the Finite Element Analysis we investigated the applicability of a metric that can potentially serve as one of the indicators of mechanical integrity of an open-barrel crimp amount of relative extrusion between wire barrel and wire strands. Although more work is necessary to make a direct correlation, the results of this study agreed well with accelerated crimp reliability test data available for some of the products that were modeled.

It was found that the single terminal modification that resulted in the most improvement of the amount of terminalwire relative extrusion was addition of the $3^{\text {rd }}$ serration. Testing data that exists on crimps with 2 and 3 serrations suggests that for this combination of materials and this geometry of the samples, crimps with 3 serrations outperformed those with 2 serrations in accelerated crimp reliability tests. Therefore, it is suggested that relative extrusion, as a measure of mechanical stability of crimps, can be used as one of the metrics in optimizing crimp designs.
Other modifications of the geometry of serrations in a 2serration terminal, such as increase of serration depth and width, as well as changes to serration sidewall angle, did not have such a pronounced effect on the amount of relative extrusion.

Increase in serration length and extension of serrations all the way to the edge of the terminal resulted in substantial deformation of the wire, however, the overall reduction in relative extrusion between the wire strands and the terminal was not very noticeable.

Although FEA is currently being successfully used for crimp design optimization, additional work is required to include more aspects of the mechanics of crimped connections for this technique to become an easily applied design tool that is capable of predicting performance of crimps in early stages of design process.

\section{ACKNOWLEDGMENT}

The author thanks C. D. Copper, N. E. Corman, and R. D. Hilty for continuous support and valuable discussions that defined the details of the study. The author acknowledges the help provided by T. Hauswald in the use of the new software and for her willingness to share results of her work.

\section{REFERENCES}

[1] J. H. Whitley, "The mechanics of pressure connections", presented at The EDN Regional Engineers Meeting, New York, 1964.

[2] R.S. Timsit, "Contact Properties of Tubular Crimp Connections: Elementary Considerations", in Proc. 54 $4^{\text {th }}$ IEEE Holm Conf. on Elec. Contacts, Orlando, 2008, pp. 161-167.

[3] T. Morita, K. Ohuchi, and M. Kaji, "Dynamic finite element analysis simulation of the terminal crimping process", in Proc. $42^{\text {nd }}$ IEEE Holm Conf. on Elec. Contacts, Chicago, 1996, pp. 151-155.

[4] G. Villeneuve, D. Kulkarni, P. Bastnagel, and D. Berry, "Dynamic finite element analysis simulation of the terminal crimping process", in Proc. $42^{\text {nd }}$ IEEE Holm Conf. on Elec. Contacts, Chicago, 1996, pp. 156-172.

[5] M. Abbas, J-L. Batoz, T. Courtin, and G. Dhatt, "Advanced numerical simulation of the crimping process of electric connectors", in Recent Advances in Integrated Design and Manufacturing in Mechanical Engineering, G.Gogu et al., Ed. Norwell, MA: Kluwer Academic Publishers, 2003, pp. 241-250.

[6] D. Z. Zhmurkin, N. E. Corman, C. D. Copper, and R. D. Hilty, "3Dimensional Numerical Simulation of Open-Barrel Crimping Process", in Proc. 54 $4^{\text {th }}$ IEEE Holm Conf. on Elec. Contacts, Orlando, 2008, pp. 178-184.

[7] R. S. Mroczkowski and R. J. Geckle, "Concerning "cold welding" in crimped connections", in Proc. $41^{\text {st }}$ IEEE Holm Conf. on Elec. Contacts, Montreal, Canada, 1995, pp. 154-164.

[8] R. S. Mroczkowski, Electronic Connector Handbook. New York, NY: McGraw-Hill, 1998.

[9] G. L. Ginsberg, Connectors and Interconnections Handbook, vol. 5, Terminations. Fort Washington, PA: The Electronic Connector Study Group, 1982.

[10] C. A. Harper, Handbook of Wiring, Cabling, and Interconnecting for Electronics. New York, NY: McGraw-Hill, 1972.

[11] SAE International USCAR21-1 Performance Specification For Cable-toTerminal Electrical Crimps: USCAR, 2004.

[12] AK Test Standard LV 214-2 „Kfz-Kontaktierungen; Slow-MotionPrufung“". AK group: Munich, 2007 


\title{
Use of Modeling Techniques to Optimize the Electrical Performance of a Novel RF Connector Design
}

\author{
Sean P. McCarthy \\ Technology \\ Tyco Electronics Corporation \\ Harrisburg, PA, USA
}

\begin{abstract}
Many RF connectors used in cable applications are coaxial in design with predictable performance, but require tedious assembly. Recently, a novel RF connector design with a new form factor was introduced by Tyco Electronics with improved assembly time and electrical performance. The unique connector structure and cable termination approach required careful modeling to predict and optimize the electrical performance.
\end{abstract}

At microwave frequencies, detailed features inside the connector structure begin to affect the smaller wavelength signals and multiple interactions occur. The impedance, VSWR, and shielding effectiveness of the connector design must be predicted during the design process to determine if the design will pass required specifications. Careful modeling of the critical parts of the connector can be used to predict performance and can lead to fewer prototypes and optimized designs.

The use of electrical modeling to accurately predict the interaction of multiple mechanical features inside the connector structure and their impact on VSWR and shielding effectiveness is presented. The visualization of currents and field quantities is used to optimize the design. In addition, the modeling of a hybrid shielding effectiveness measurement technique to isolate the connector behavior from the cable is shown with comparison to laboratory measurements.

Keywords-component; Connectors; Electrical Modeling, Shielding Effectiveness; Coaxial Cable

\section{INTRODUCTION}

The use of coaxially shaped connectors on coaxial cables is prevalent due to the matching structure and impedance as well as the complete shielding that they offer. Coaxial connectors, however, are not easy to manufacture, can be relatively expensive, and require a detailed and lengthy assembly process. Recently, Tyco Electronics set out to design a new coaxial cable connector that overcame the difficulties and cost associated with coaxial connectors and electromagnetic modeling played an important role in that development.
The initial target market for the connector was automotive telecommunications in which there is a need for multiple antennas to receive cell phone, Global Positioning System (GPS), and satellite radio signals at $860 \mathrm{MHz}, 1.575 \mathrm{GHz}$, and $2.3 \mathrm{GHz}$ respectively. The connector was therefore required to have acceptable performance to at least $3 \mathrm{GHz}$ to satisfy these markets. It was decided early on that the design would be pushed to perform to $6 \mathrm{GHz}$ to position the connector for future markets such as the Industrial, Scientific, and Medical (ISM) $5.8 \mathrm{GHz}$ band.

In order to create a connector design which was easier to manufacture, a planar geometry was selected. In particular, the stripline geometry was chosen as a reasonable transition from coaxial cable. A new cable attachment technique was also incorporated to decrease assembly time. This new technique involved the use of a coaxial cable displacement contact to attach the connector shield to the cable braid. This novel approach shortens the assembly time compared to tedious folding and crimping of the braid on traditional coaxial connectors. Cost to manufacture and assemble the connector was the primary constraint in the development process and this required multiple tradeoffs in the design. The new connector design is referred to as the RF stripline connector and is shown in Figure 1 [1].

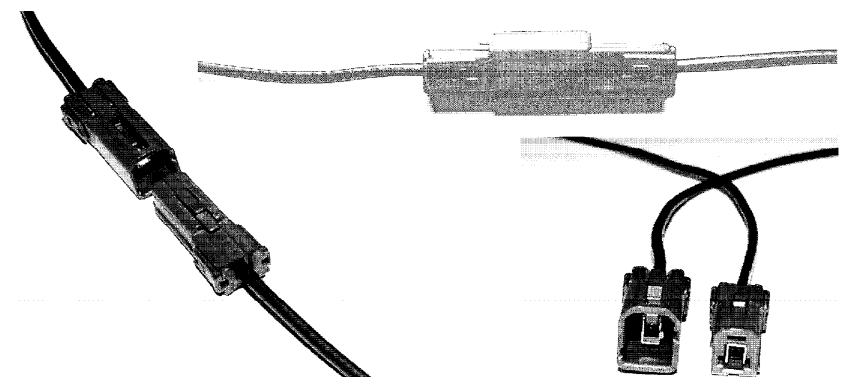

Figure 1: RF Stripline Connector 


\section{CONNECTOR ELECTRICAL MODEL}

The stripline geometry was chosen for the connector design to create a planar design for ease of manufacturing. The basic stripline geometry consists of two ground or return electrodes placed parallel to and on opposite sides of a center signal conductor as shown in Figure 2. The return spacing can be symmetric or asymmetric in form. An estimate of the impedance of an asymmetric stripline can be found with the formula:

$$
Z=\frac{80}{\sqrt{\varepsilon_{r}}} \ln \left[\frac{1.9(2 H+T)}{0.8 W+T}\right]\left[1-\frac{H}{4 H_{1}}\right]
$$

Where $\varepsilon_{r}$ is the relative dielectric constant of the material, $\mathrm{T}$ is the thickness of the signal conductor, $\mathrm{W}$ is the width of the signal conductor, and $\mathrm{H}$ and $\mathrm{H}_{1}$ are the distances between the center conductor and the return walls. The formula can be used to obtain an initial estimate of the design parameters; however finite element modeling was used to include the effects of variations from a perfect stripline geometry.

The basic connector system consists of two $50 \mathrm{Ohm}$ cables attached to a $50 \mathrm{Ohm}$ stripline geometry through two transition regions. The transition regions are located where the coaxial cable shield is attached to the connector shield and where the connector signal conductors are crimped onto the cable center conductors. The connector actually behaves as a system at high enough frequencies, rather than as an element with a single value of impedance. Each discontinuity in the connector design that is of a reasonable portion of the wavelength of the signal may affect its propagation. It is the overall effect of the multiple connector features and the signal interacting with them that determines its electrical performance. This is where the modeling of the connector design is mandatory to predict its high frequency performance.

The connector shields are attached to the coaxial cable braid with a cable displacement contact as shown in Figure 3. Here the shield is shown penetrating the outer jacket and dielectric of the cable and trapping sections of the braid in displacement slots designed into the shield. While this may appear to be a major disruption to the propagation of the electrical signal, the thin displacement contact in the direction of signal propagation has little effect on the signal integrity of the connector.

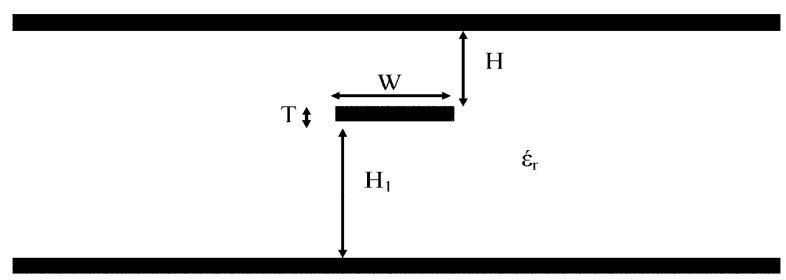

Figure 2: Asymmetric stripline geometry.

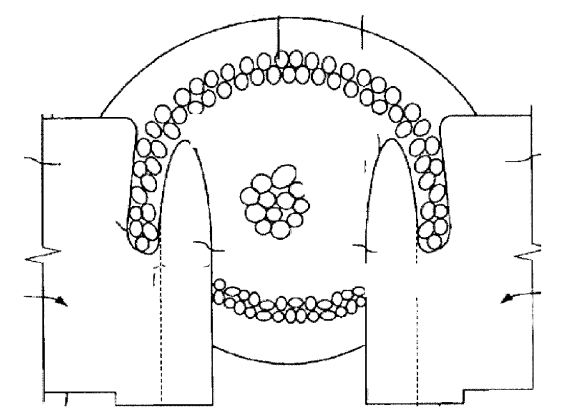

Figure 3: Coaxial cable displacement contact.

The electrical model of the RF stripline connector is shown in Figure 4. The model was created using Ansoft's HFSS finite element code [2]. While appearing quite simple, it contains the necessary detail to effectively model the performance of the connector.

What is shown is a mated pair with two U-shaped shields engaging to form shielding on all sides of the center conductors. The shields also facilitate the mounting of the connector onto the cable braid with a coaxial cable displacement contact. The width of the connector was determined by the width of the coaxial cable outer dielectric which guides the assembly and maintains straight walls for ease of manufacture. The variations in contact shape as well as the features molded into the dielectric for manufacturing and assembly ease are captured in the finite element model to ascertain their effects on the propagating signal.

Figure 5 shows a plot of surface current density from the model. The concentration of current near the inside center side indicates the return current for the stripline geometry. What is also apparent from the plot is the containment of current to the inside of the connector and cable structure that enhances shielding effectiveness.

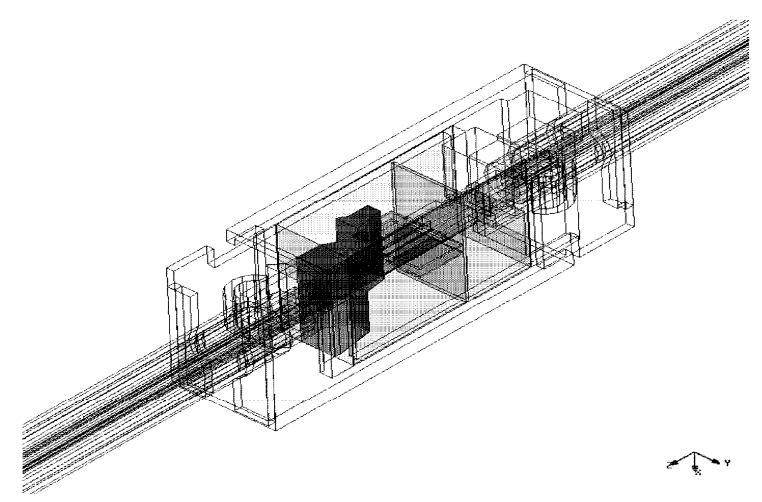

Figure 4: Electrical model of RF stripline connector. 


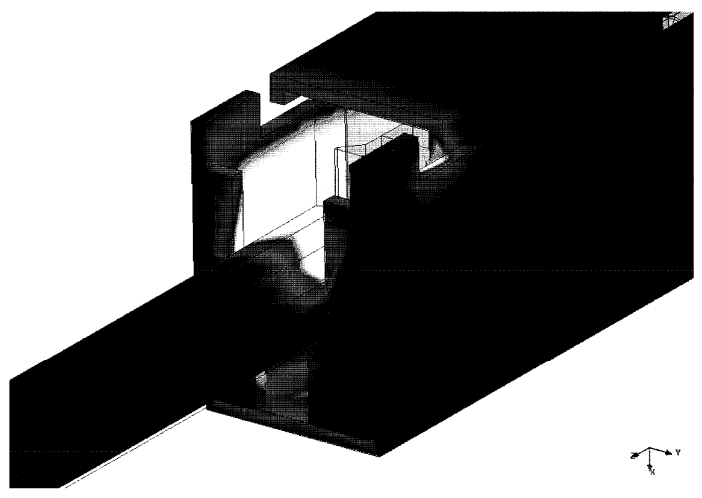

Figure 5: Surface Current Density on RF Stripline Connector.

The most important factor affecting the electrical performance of the connector is the high impedance of the transition regions. In the transition regions of the connector the dimensions of the geometry are dictated by the mechanical requirements of the crimp. The result is that the spacing between the crimp and the shield is too large to maintain a 50 Ohm impedance and a larger impedance results. The high impedance of the transition region causes a reflection of the propagating signal, as does the high impedance of the comparable crimp section of the mating connector.

To deal with these transitions, the outer shield dimension could be changed to reduce impedance, but not without adding manufacturing complexity and cost. Instead the high impedance sections were incorporated into the design. When the two transition regions are spaced at one fourth of a wavelength in the dielectric of the connector, the reflections interact and cancel each other giving a null in the VSWR. By controlling the length of the mated connector and the dielectric constant, the null was positioned to provide adequate performance of the connector. By using the modeling results, the dimensions of the connector were adjusted to make sure the null occurred at a location that was advantageous to the performance of the connector.

In Figure 6 is shown the VSWR calculation for the model as well as the VSWR measured from a connector. The null in the VSWR at $4.5 \mathrm{GHz}$ caused by the interaction of the high impedance transition regions is apparent. The null was positioned to maintain a low VSWR at the automotive telecommunication frequencies and to increase the bandwidth of the inexpensive connector design. The mechanical or molding changes that affected the component positions or effective dielectric constant were incorporated into the model so that modifications could be made to compensate for them prior to building the connector.

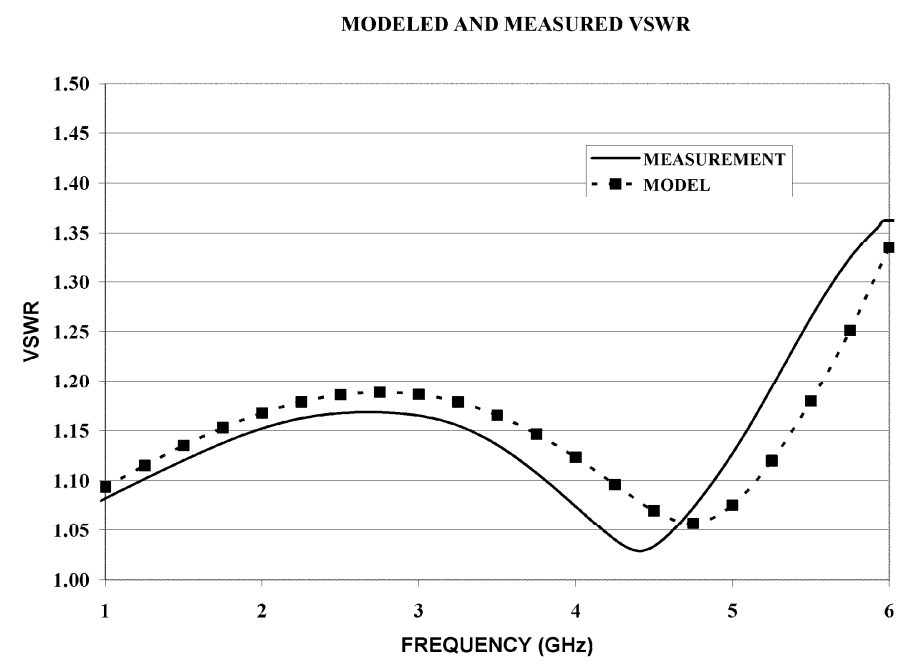

Figure 6: Voltage Standing Wave Ratio Comparison, Modeled and Measured.

A board-mount version of the RF stripline connector was designed to facilitate the connection of cables to radios as shown in Figure 7. In this application there is no crimp transition region with a high impedance region on the board side to compensate the high impedance of the crimp section on the incoming cable. The electrical model was used to design a high impedance section by notching the center conductor of the board connector. This assured that the connector pair performed with similar VSWR whether mated to a cable or board mount application. In addition the footprint of the connector was optimized in the modeling process.

\section{SHIELDING EFFECTIVENESS}

Because the stripline connector is a departure from traditional coaxial connector designs with 360 degrees of shielding, concern was raised over the shielding effectiveness performance of this new design. The shielding effectiveness is the ability of the connector shield to prevent the leakage of a signal. Any apertures in the connector can permit energy leakage which can cause interference with surrounding electrical equipment.

Shielding Effectiveness is defined as the power that leaks from a connector divided by that which is put in.

$$
\mathrm{SE}(\mathrm{dB})=10 * \log _{10}\left[\mathrm{P}_{\mathrm{o}} / \mathrm{P}_{\mathrm{i}}\right]
$$

If an RF connector is used on less than perfectly shielded coaxial cable its shielding effectiveness may not be an issue since the longer lengths of cable will most likely leak to a larger degree than the connector. Therefore a shielding effectiveness measurement approach that focuses on the connector and limits the effect of the cable is required. 


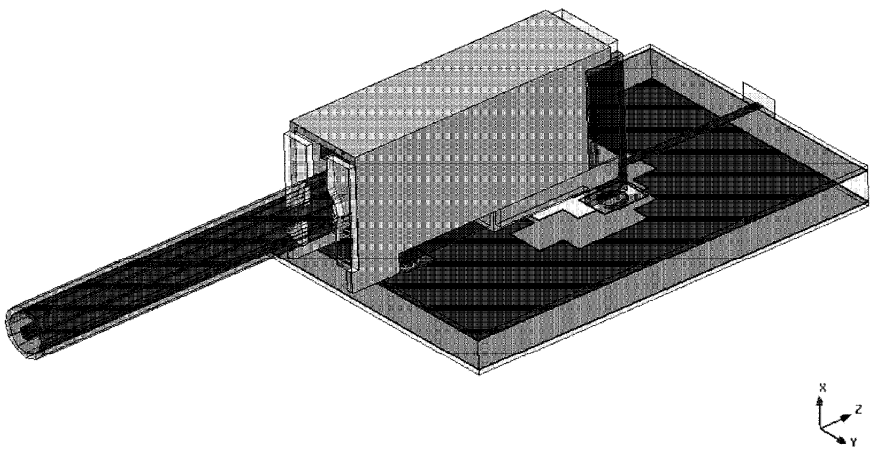

Figure 7: Board mount version of RF stripline connector.

A measurement structure was devised to isolate the connector from the cable as shown in Figure 8. This approach is a form of the current injection technique [3]. In this measurement, the connector on the cable is placed inside a conducting cylindrical chamber. The resulting structure is in essence a tri-axial cavity where the outer shield of the connector serves as the inner conductor of the outer coaxial geometry as shown in Figure 9.

Ports 1 and 2 are the ends of the cable which includes the connector under test. Ports 3 and 4 are at the ends of pieces of semi-rigid coaxial lines whose outer conductors are soldered to the coaxial cable braids and whose center conductors are soldered to the outer chamber wall. Any power that leaks from the connector will propagate on the outer transmission line of the tri-axial structure and present itself at ports 3 and 4 .

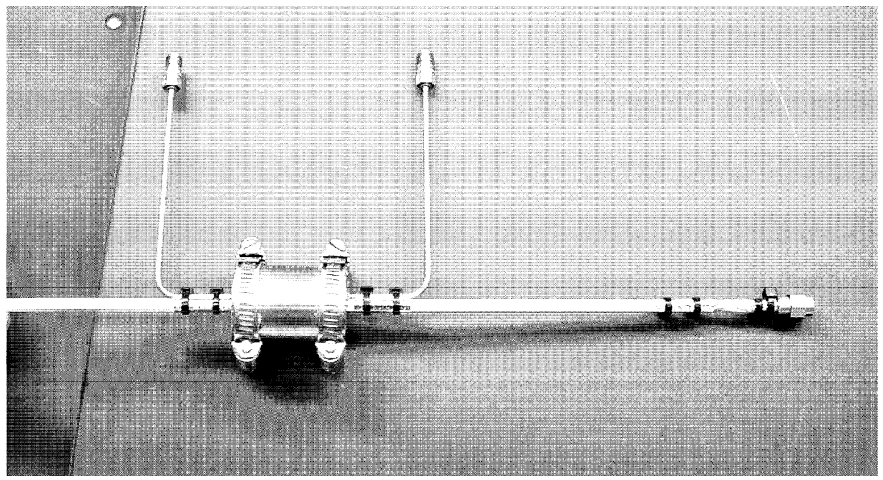

Figure 8: Shielding effectiveness measurement chamber.

If the impedance of the transmission line formed between the outer shield of the connector under test and the shielding chamber is kept the same as the $50 \mathrm{Ohm}$ connector, the shielding measurement becomes one of measuring the voltage at ports 3 and 4 . Therefore the chamber diameter is adjusted based on the outer diameter of the connector under test [4].

In the model a shielding chamber is easily formed as a coaxial cylinder over the connector model and the voltages at the ports are measured as the scattering parameters $S_{13}$ and $S_{14}$. In the case of the measurement, the scattering parameters are measured with a Network Analyzer. In both cases the scattering parameters can be used to obtain the SE for the connector with the formula:

$$
\mathrm{SE}(\mathrm{dB})=10 * \log _{10}\left[\mathrm{~S}^{2}{ }_{13}+\mathrm{S}^{2}{ }_{14}\right]
$$

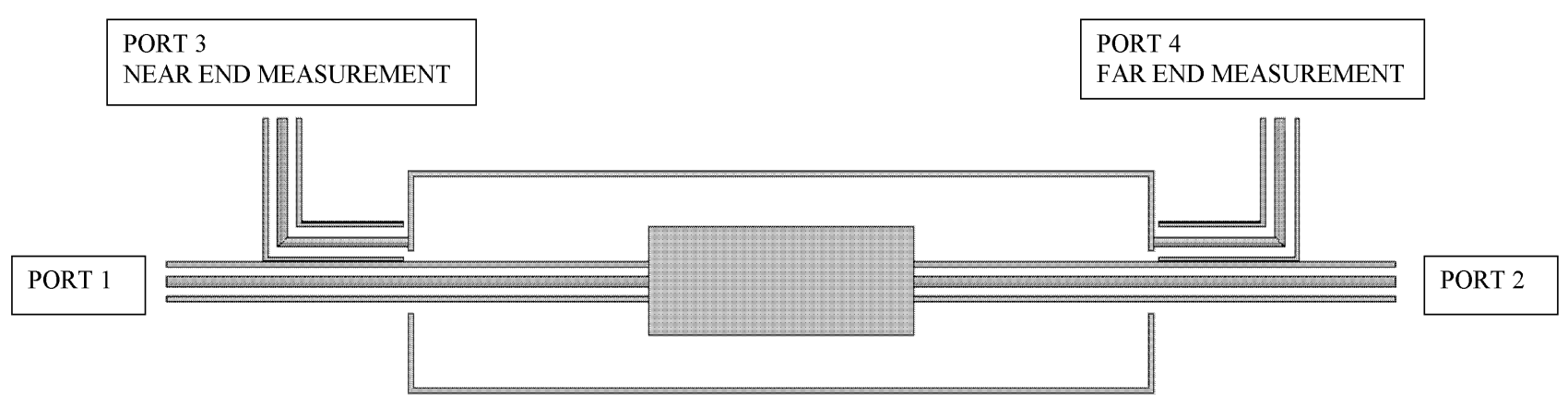

Figure 9: Shielding Effectiveness Fixture Cross-section. 
Figure 10 shows the modeled shielding effectiveness performance for the RF stripline connector. The measurement of $S_{13}$ is called the near end measurement due to its proximity to the input signal while $\mathrm{S}_{14}$ is termed the far end measurement due to its position near port 2. The plot shows each component as well as the total shielding effectiveness computed with equation (3). The two curves are quite different due to the interactions of the propagating signal and the various apertures in the connector.

A comparison between modeled and measured shielding effectiveness is shown in Figure 11. There is relatively good agreement between the two curves. In particular, the general level of shielding effectiveness, which is critical to pass specifications, is in good agreement. The measured SE has less of a slope at low frequencies. The actual measurement contained a portion of cable while the model did not. Therefore the inner and outer travel of current on the cable braid conductors, may have contributed to the less steep slope of the measured SE [5]. Nevertheless, the model provided sufficient accuracy to ascertain the effect on shielding effectiveness of changes in the connector shield and proved invaluable in the design process to make sure specifications would be met by successive designs.

\section{CONCLUSION}

The modeling of a novel RF connector design was presented with comparisons to measurements. The models were used to predict the electrical performance of the connector including VSWR and shielding effectiveness. At microwave frequencies, the connector must be viewed as a system rather than a single element where the features on the order of a fraction of a wavelength begin to interact with the signal. The finite element model permitted the prediction of performance variations due to changes such as mating distance and features in the molded parts. A relatively simple model was sufficient to accurately predict performance prior to building the connector.

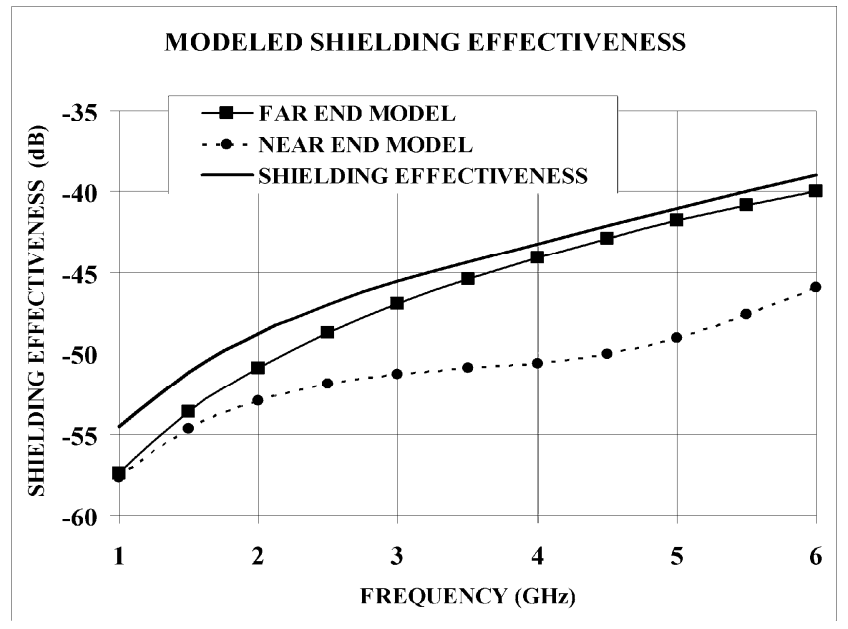

Figure 10: Modeled Shielding Effectiveness

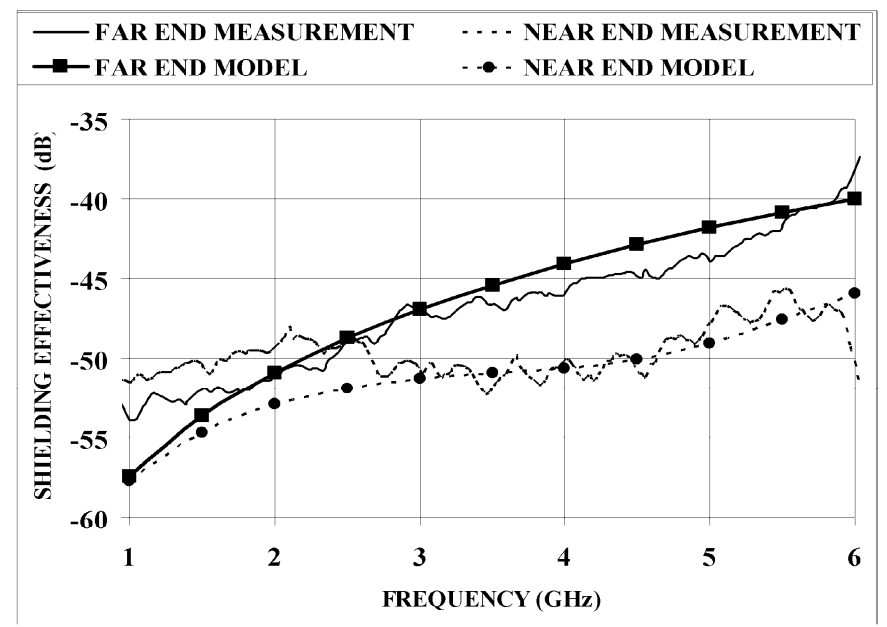

Figure 11: Comparison of Modeled and Measured Shielding Effectiveness.

A shielding effectiveness measurement approach that isolates a cable connector from the cable was presented. The model of the measurement approach proved accurate enough to predict that the required specifications would be achieved. The use of the model proved invaluable to assist in design variations prior to making the connector and the involved measurement.

\section{ACKNOWLEDGMENT}

The author thanks Steven A. Jarrett and Jerry H. Bogar for their measurements and guidance and Henry H. Herrmann and Michael F. Laub for their suggestions.

\section{REFERENCES}

[1] US Patent No. 6746277.

[2] Ansoft LLC, HFSS, Pittsburgh, PA, 2008.

[3] IEC 60512-23-3 "Electromechanical components for electronic equipment - Basic testing procedures and measuring methods - Part 233: Test 23c: Shielding effectiveness of connectors and accessories.

[4] S. McCarthy, J. Bogar, S. Jarrett, J. Raudenbush, "Shielding effectiveness measurements utilizing the Tyco Electronics hybrid current injection technique", Tyco Electronics Report, 2002.

[5] R. Tiedemann, "Current Flow in Coaxial Braided Cable Shields", IEEE Transactions on Electromagnetic Compatibility, Vol. 45, No. 3, August, 2003 


\title{
Coupled Thermal Electrical Finite Element Analysis of Power Contacts Used In High Speed Differential Connectors
}

\author{
Trent K. Do, Tom Cohen \\ Amphenol-TCS \\ Nashua, $\mathrm{NH}$
}

\begin{abstract}
This paper will present a coupled thermal electrical finite element analysis (FEA) of two different contact systems used for distributing power currently used in high speed differential connectors. The finite element models will be utilized to determine the temperature rise for each of the contact designs based on an applied current. Then the baseline models will be used to optimize the contact system designs by changing the current base material to a high performance copper alloy. Empirical data for each power contact design will be used to validate the FEA model results. The FEA models will be modeled utilizing the Abaqus/Standard finite element software.
\end{abstract}

Keywords-power connectors; high performance copper alloys, resistance, current.

\section{INTRODUCTION}

Packaging of today's advanced electronic systems such as switches, routers, mainframe computers, blade servers, mass storage devices, and various other equipment contain a common power distribution challenge.

These systems typically partition the box into subsystems on discreet plug in printed circuit boards (PCB's) or cards. These cards, from switch fabrics to CPU cards require increasing amounts of power in a very limited space. Cards are typically connected through a common PCB which is referred to as a backplane, midplane or motherboard. Regardless of the specific implementation, real estate is at a premium resulting in the need for increased power density on the plug in cards.

The scope of this paper will be to develop a finite element model (FEA) of two types of contacts utilized for power distribution. The FEA models will then be correlated to empirical data.

\section{BACKGROUND}

\section{A. Contact Designs}

Currently there are two types of contacts on the VHDM [2] product line that are utilized for power distribution. The first contact was designed as a power contact capable of distributing up to $10 \mathrm{amps}$ per contact. The second contact was designed as a signal contact but due to real estate on printed circuit boards the contacts are now also utilized for power. The signal contact is capable of distributing up to 1 amp per contact. Both of the contacts are stamped from C7025 TM02 high performance copper alloy material. The contacts are electro-plated with 0.762 microns thick of nickel hardened gold with a 1.27 microns thick nickel sulfamate under layer.

\section{B. Approach}

The approach to this study is to develop a finite element model using the Abaqus [6] finite element software and correlating the analysis data to empirical data. This study will only analyze the contact system and will not include the connector housings. The finite element models will be used to determine the temperature rise of the contacts at the mating interface.

\section{FINITE ELEMENT MODELS}

\section{A. Finite Element Model Set-up}

To develop the finite element models Abaqus/Standard finite element software was utilized to create the coupled thermal electrical models. Before the thermal electric models were created a static interference fit model was performed to include the normal force for thermal electric models. The interference analysis determines the normal force based on nominal interference between the male and female contacts. The nominal normal force for the contacts is 150 grams for the power contact and 60 grams for the signal contact. After the interference analysis the output database file is then imported into the thermal electric model. The material properties for the C7025 TM02 copper alloy material is given in table 1 . The models will also be analyzed with the 18080 Olin material. The material properties are shown in table 2 .

Table 1: C7025 Material Properties

\begin{tabular}{|l|l|}
\hline Properties & Values \\
\hline Elastic modulus & $13.1 \times 10^{4} \mathrm{MPa}$ \\
\hline Poisson's ratio & 0.30 \\
\hline Thermal conductivity & $14.4 \mathrm{~W} / \mathrm{mK}$ \\
\hline Electrical conductivity & $23.243 \times 10^{6} \mathrm{~S} / \mathrm{m}$ \\
\hline
\end{tabular}


Table 2: 18080 Material Properties

\begin{tabular}{|l|l|}
\hline Properties & Values \\
\hline Elastic modulus & $14.0 \times 10^{4} \mathrm{MPa}$ \\
\hline Poisson's ratio & 0.30 \\
\hline Thermal conductivity & $26.7 \mathrm{~W} / \mathrm{mK}$ \\
\hline Electrical conductivity & $46.486 \times 10^{6} \mathrm{~S} / \mathrm{m}$ \\
\hline
\end{tabular}

Once the interference model has been imported into the thermal electric model, the loads and boundary conditions were applied, see Fig. 1 and Fig. 2. Both contacts had an applied current at the backplane compliant pin and zero voltage boundary condition on the daughtercard compliant pin. The sink temperature (ambient temperature) was set to $20 \mathrm{C}$, this was determined based on the experimental set up. The models also assume all electrical energy is converted to thermal energy therefore the Joule heating fraction was set to 1.0. To reduce computation time, radiation affects will not be included in the thermal electric models. The temperature dependent convective heat transfer coefficient was assumed for each model based on the experimental data. The convective heat transfer coefficient is defined as a film coefficient in the Abaqus models and is applied on all surfaces of the model as shown in Fig. 3 and Fig. 4.

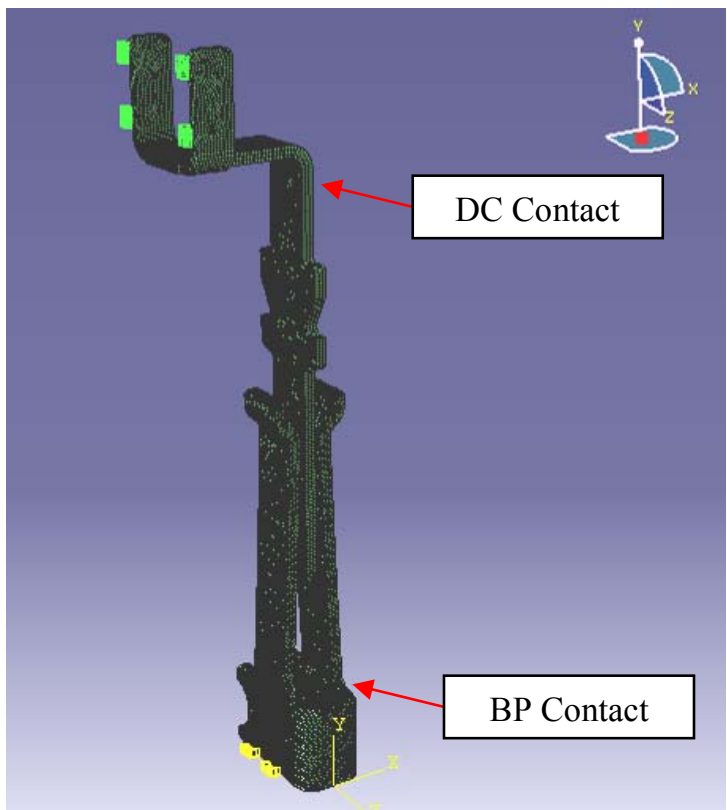

Figure 1: VHDM Power contact

The thermal and electrical conductance properties at the mating interface were applied within Abaqus' interaction module. The areas where the thermal and electrical conductances were applied were determined from micro cross sections of the mating interface.

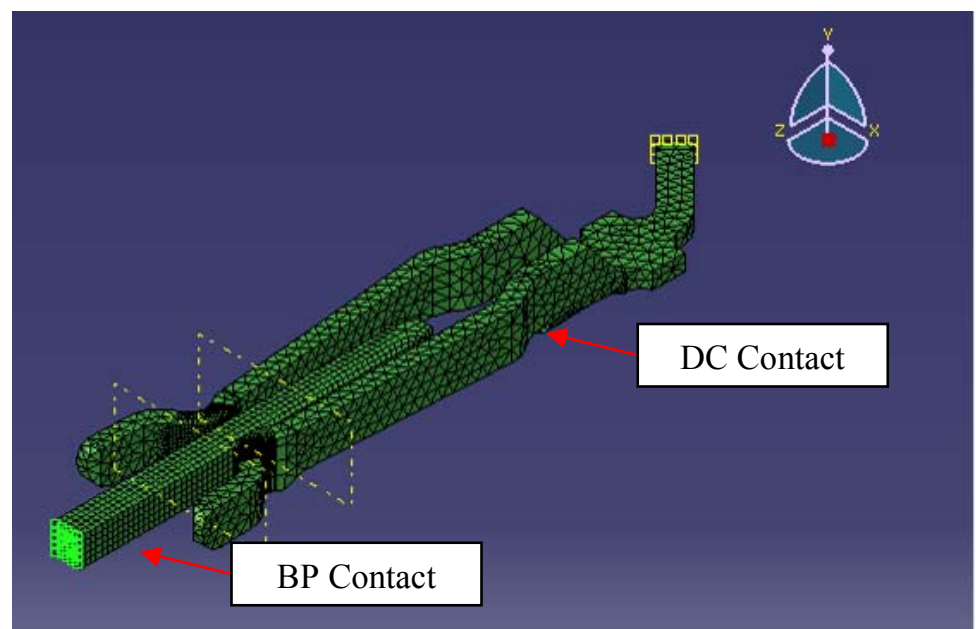

Figure 2: VHDM Signal contact

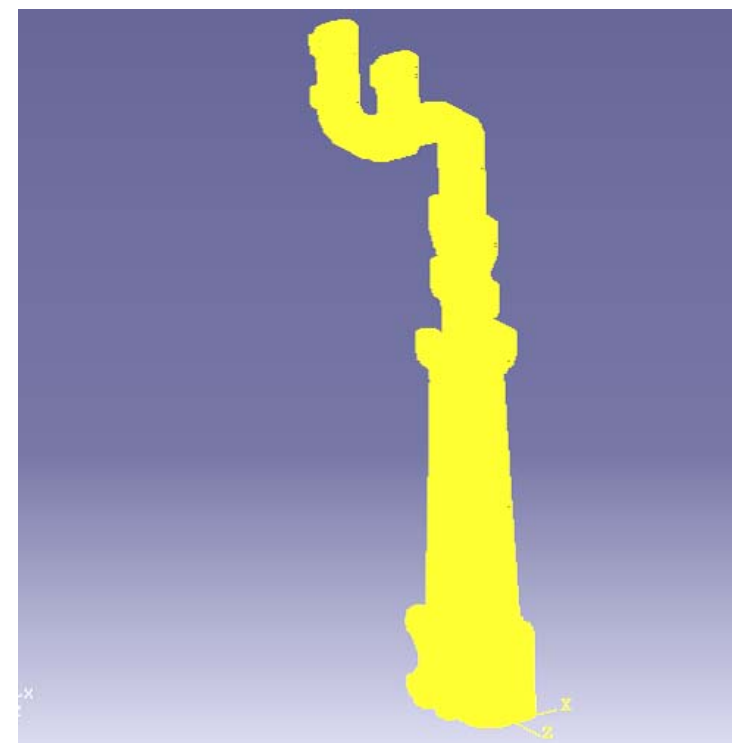

Figure 3: Film coefficient applied to all power model surfaces

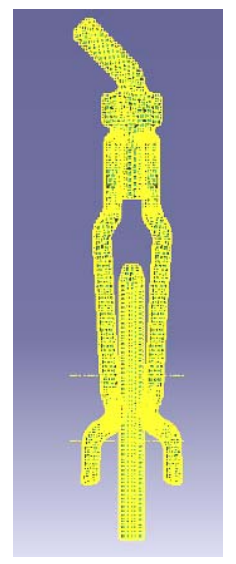

Figure 4: Film coefficient applied to all signal model surfaces 


\section{B. Finite Element Results}

Fig. 5 and Fig. 6 are the FEA results for the power and signal contacts for two different copper alloy materials. The FEA temperature rise results were taken at the mating interface of the thermal models. The FEA results show the 18080 copper alloy had better performance than the C7025 copper alloy for both the power and signal contact. At the maximum applied current of $15 \mathrm{amps}$ for the power contact there is a 9C difference between the two copper alloys. For the signal contact at the maximum applied current of 7 amps there is a $10 \mathrm{C}$ difference between the two copper alloys.

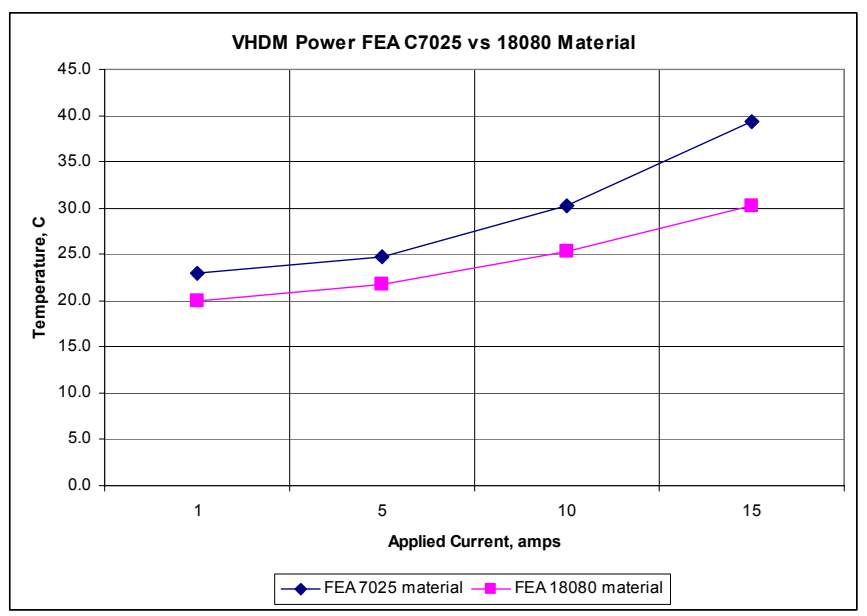

Figure 5: Power FEA temperature rise results at the mating interface

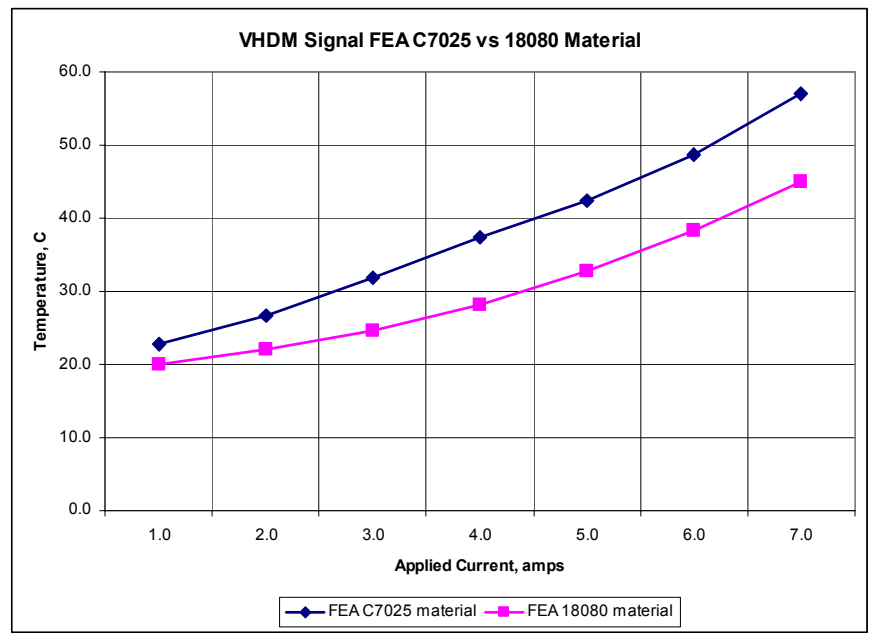

Figure 6: Signal FEA temperature rise results at the mating interface

\section{Experimental Set-up}

Both of the current contact system designs were experimentally tested to determine the temperature rise at the mating interface. The test setup schematics for each contact design are shown in Fig. 7 and Fig. 8. The contacts were tested per EIA-364-TP70 specification [7]. The contact systems were tested in a 24 "x24"x12" plexi glass box. Each contact had thermocouples placed at the mating interface $\mathrm{T} 1$, the current inlet $\mathrm{T} 2$, the current outlet $\mathrm{T} 3$, a thermocouple placed above the mating interface $\mathrm{T} 4$ and the ambient box temperature $\mathrm{T} 5$.

The applied current for each contact was applied at the backplane contact. For the power contact the applied current ranged from $1 \mathrm{amp}$ to $15 \mathrm{amps}$ and for the signal contact the applied current ranged from 1 amp to 7 amps. The applied current values were determined from the VHDM product specification and customer applications.

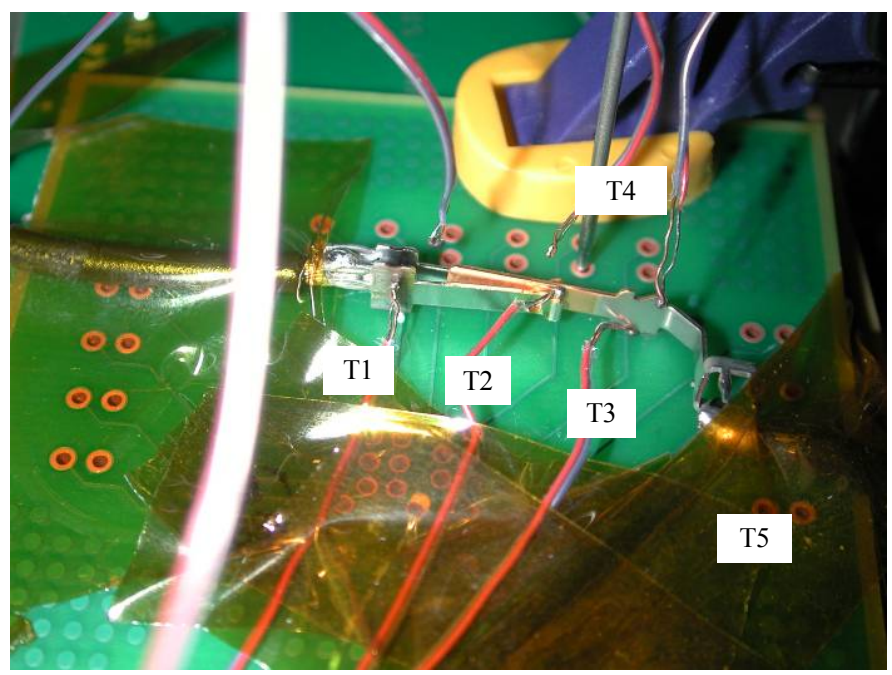

Figure 7: Temperature rise test setup - power contact

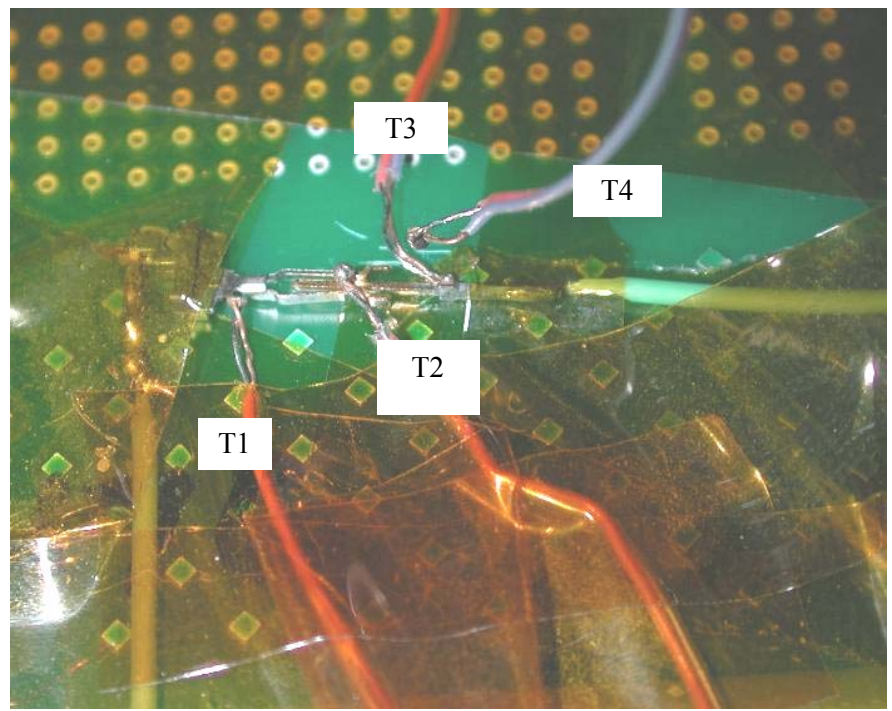

Figure 8: Temperature rise test setup - signal contact 


\section{RESULTS}

The experimental results for the power and signal contacts are shown in Fig. 9 and Fig. 10. The results for the power contact shows that at an applied current of 15 amps the maximum temperature at the mating interface measured $40 \mathrm{C}$ (temperature rise of 18C). The results for the signal contact shows that at an applied current of 7 amps the maximum temperature at the mating interface measured $53 \mathrm{C}$ (temperature rise of $30 \mathrm{C}$ ). Based on the experimental results the power and signal contacts meet the test specification EIA364-TP70 [7].

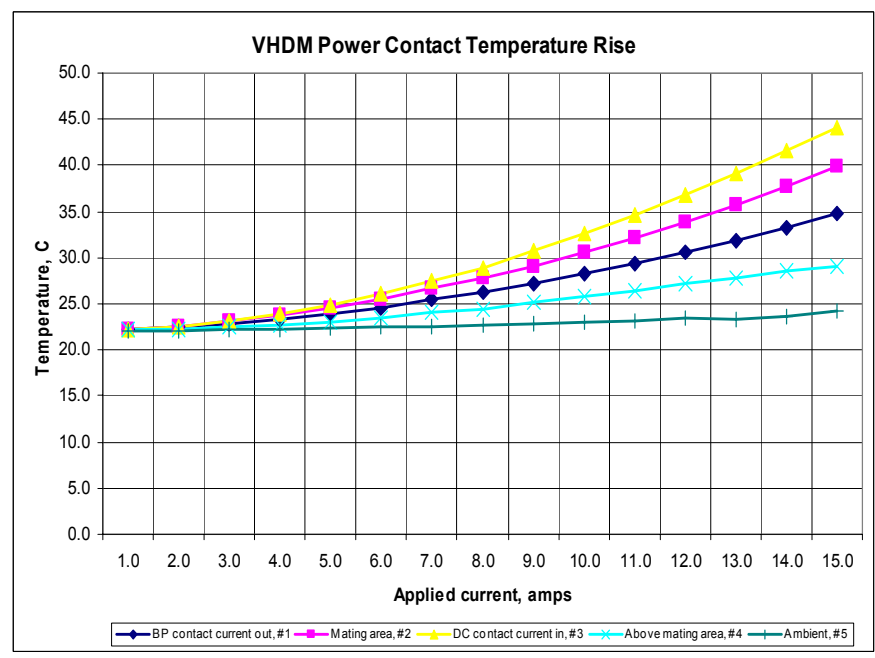

Figure 9: Power contact experimental temperature rise results

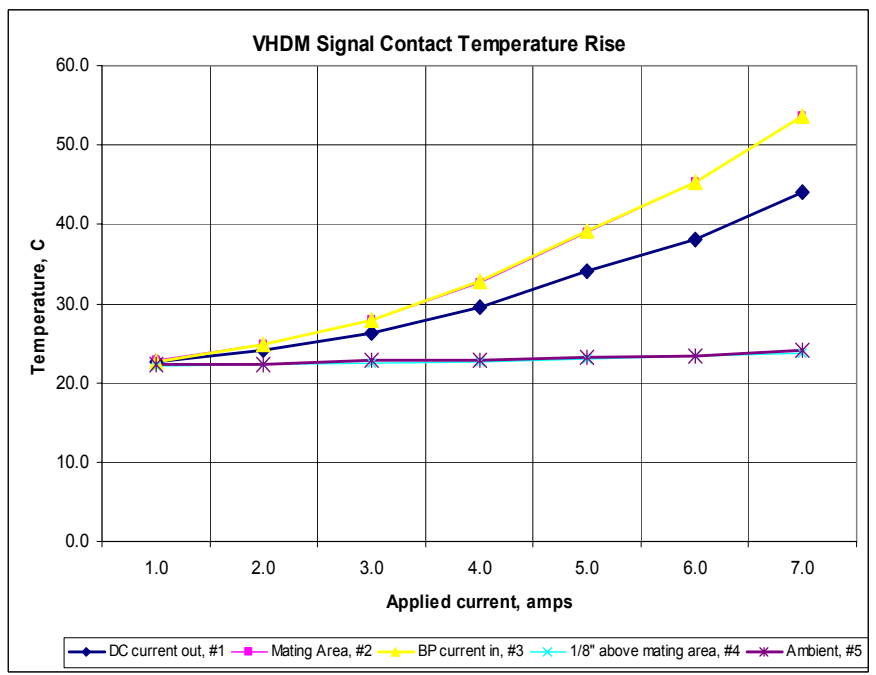

Figure 10: Signal contact experimental temperature rise results

\section{DISCUSSION}

The results of the power contact FEA model and the measured data correlated to within $2 \%$ as can be seen in Fig. 11. The signal contact FEA model correlated to within $10 \%$ as can be seen in Fig 12. The better correlation for the power contact could be attributed to using a better assumption for the convection coefficient (film coefficient) and using a more accurate contact area at the mating interface. The signal contact correlation could be improved by changing the convection coefficient (film coefficient) and changing the contact area at the mating interface.

With good model to measured data correlation with the C7025 copper alloy material, the material then was changed to a higher conductivity material 18080 . The results of the finite element models show that changing to the higher conductivity material reduced the average temperature rise of the power contact by $4 \mathrm{C}$ as shown in Fig. 5. And for the signal contact the average temperature rise reduced by $5 \mathrm{C}$ as shown in Fig. 6 .

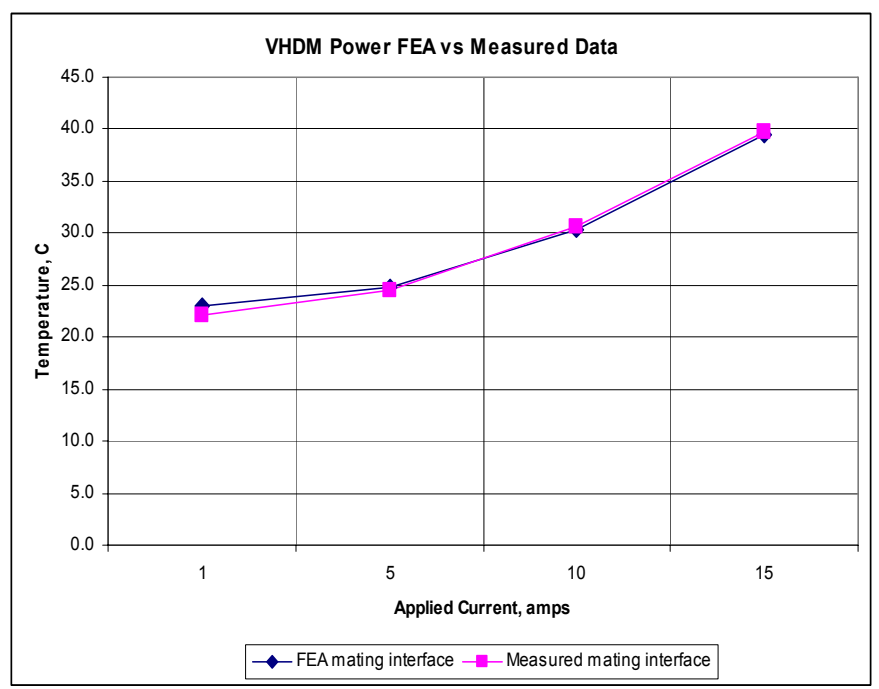

Figure 11: Power contact FEA vs. measured temperature rise at the mating interface

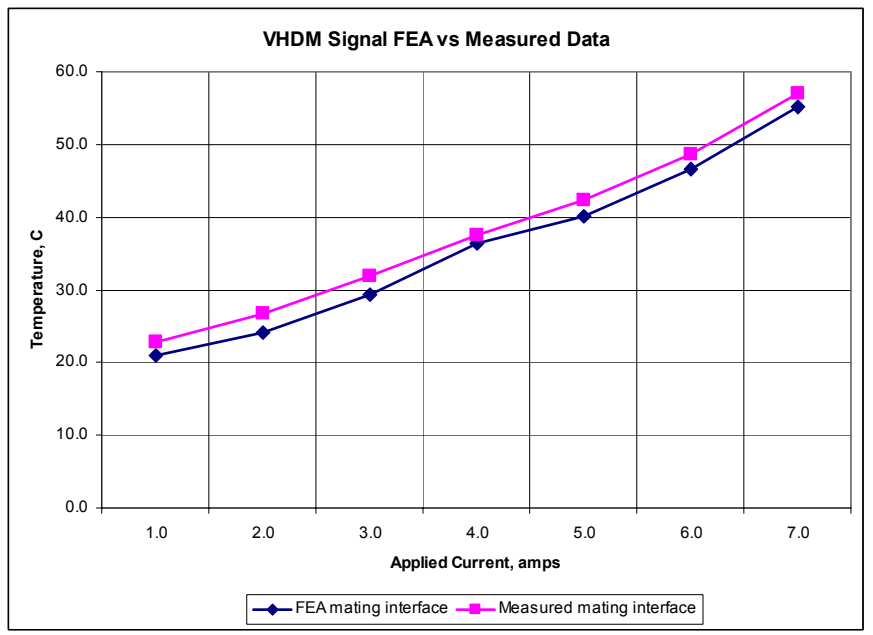

Figure 12: Signal contact FEA vs. measured temperature rise at the mating interface 


\section{CONCLUSION}

The purpose of this paper was to present a finite element model of two types of contacts used for power distribution in high speed interconnects. The results of the finite element model and the experimental data correlated to within less than $10 \%$. Although the models and the experimental data did not include the connector housings, the models can be used to analyze different materials. The model approach can also now be used to develop different power distribution contact designs. Several conclusions can be made from the finite element models:

1. The convective heat transfer coefficient (film coefficient) had a dramatic affect on determine the temperature distribution.

2. The models only represent one part of the entire power contact design, since it did not include the connector housings and the printed circuit board. Future work will have to include those variables.

3. Changing the material properties to a higher conductivity material can increase the current carrying capacity of the current contact designs.

\section{REFERENCES}

[1] R. Schmidt, "Crossroads in Thermal Management of Electronics from Chip to Datacom Room, 2005.

[2] VHDM is register trademark of Amphenol, Inc.

[3] R. Mroczkowski, "Electronic Connector Handbook", McGraw-Hill, 1998.

[4] R. Wabin, "Thermal Analysis of hermetically sealed electromagnetic relay in high and low temperature conditions", Proceedings of the $52^{\text {nd }}$ IEEE HOLM Conference, 2006.

[5] P.G. Slade, "Electrical Contacts, Principles and Applications", Marcel Dekker, Inc., New York, Basel, $1^{\text {st }}$ ed., 1999.

[6] Abaqus User's Manual Version 6.8.2009

[7] EIA Test Standards, EIA-364-TP70. 


\title{
Modeling and Analysis of a Connector System for the Prediction of Vibration-induced Fretting Degradation
}

\author{
Chen Chen, George T. Flowers, Michael Bozack, and Jeffrey Suhling \\ Center for Advanced Vehicle Electronics \\ Auburn University \\ Auburn, Alabama, USA \\ flowegt@auburn.edu
}

\begin{abstract}
-
It is generally recognized that vibration-induced fretting degradation can be a major problem for connector systems used in vehicle electronics. An earlier initial study about vibration-induced fretting degradation was conducted for a blade/receptacle connector pair. However, in real industry application of the connector systems in vehicles, not single but sixteen pairs of pin connectors are sealed and served as an entire connector system. Therefore, it would be of great value to develop and analyze the entire connector system to investigate the relative influence of connector system characters on fretting degradation problem. The present study is the next step in this effort. A simplified 2-D model and a detailed 3-D model were developed and compared using ABAQUS. A series of simulations were performed on the FEA model. Corresponding experiments were conducted to validate and test the simulation. It was demonstrated that, for this connector system, finite element modeling and analysis have great potential for the prediction of the influence of design variation on the fretting degradation of the connector system.
\end{abstract}

Keywords-connector system, fretting degradation, modeling, vibration

\section{INTRODUCTION}

Fretting degradation is generally recognized as one of the major failure mechanisms for electrical connector systems. One of the earliest studies in this area was presented in 1974 by Whitley and Bock [1]. Detailed surveys of work in the area are provided by Antler [2] (up to 1984) and by Malucci [3] for more recent work.

The major driver of fretting damage is relative motion at the contact interface, producing material displacement and transfer. For non-precious metal plated contact interfaces, this fretting damage serves to repeatedly expose fresh metal to atmospheric oxidation. The result is a substantial and rapid increase in contact resistance due to a localized buildup of an insulating layer [4]. Relative motion at the contact interface can be induced by thermal expansion/contraction, vibration, or by a combination of the two mechanisms. There has been considerable recent work on this topic, including experimental investigations (such as that of Maul, McBride, and Swingler [5]) and model development work (such as that of Bryant [6]). Previous investigations[10,13-14] on the topic of vibrationinduced fretting degradation have demonstrated some interesting behaviors, specifically a threshold vibration level for the onset of fretting and a strong relationship between vibration amplitude (beyond the threshold level) and the rate of resistance change. Currently, the basic mechanisms are not completely understood. So there is considerable interest in developing a better understanding of the basic physical causes of fretting degradation.

At present, much of the evaluation of fretting propensity for particular connector designs and the influence of variations in those designs on fretting performance is conducted through exhaustive experimental testing, which requires a major commitment of time and resources. Therefore, simulationbased method would be of great value to those working on connector design and applications.

In the authors' previous work, a simplified two dimensional finite element model and a highly detailed three dimensional finite element were developed and experimentally validated for the single Blade/Receptacle pair connector system. The $3 \mathrm{D}$ detailed model simulation results were compared with the 2D simplified model simulation results and with the physical experiments in many aspects. Both the 2D simplified model and 3D detailed model simulations were found to be in good agreement with the experiment. The 3-D simulation result shows a slight advantage than 2-D simulation in predicting overall shape of the transfer function but requires more computational cost.

However, in the industry application of the connector system in vehicles, not single but 16 pin connector pairs are sealed together and serve as one entire system. Therefore, it would be of great value to develop and analyze a connector system model to provide a reliable tool for the designers to evaluate the fretting characteristics of the connector designs. A two dimensional simplified model and detailed three dimensional detailed model were developed and compared for the connector system in ABAQUS. A series of simulations were performed on the model. Corresponding experiments were conducted to validate and test the performance of the system.

\section{EXPERIMENTAL CONFIGURATION}

In the authors' previous work [10], an experiments setup and test procedures for a connector system were developed and a series of experiments have been performed. A photograph showing the basic connector system configuration is shown in Figure 1. The connector consists of a 16 pin connector system for both the male and female terminals. These 16 wire leads were attached at each end of the connector and the other ends were clamped in the fixture. The 
connector was clamped at the fixture by the male terminal housing. In order to insure consistent fretting degradation in the frequency and amplitude ranges provided by the available laboratory shaker system, the supplementary inertial mass was clamped to the wire leads close to the connector housing, with a $2 \mathrm{~mm}$ gap between the connector housing and the mass. This inertial mass consisted of three plates, with one plate positioned between the two rows of wire leads and the remaining two plates on the outside of each row, with the resulting assembly clamped together with bolts.

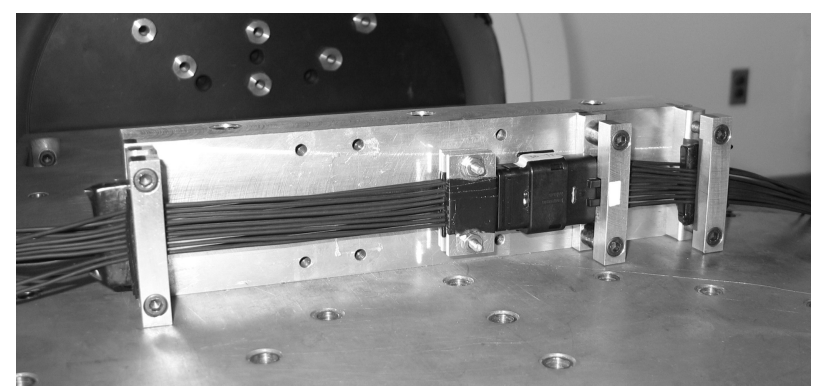

Figure 1: Photograph of the experimental setup

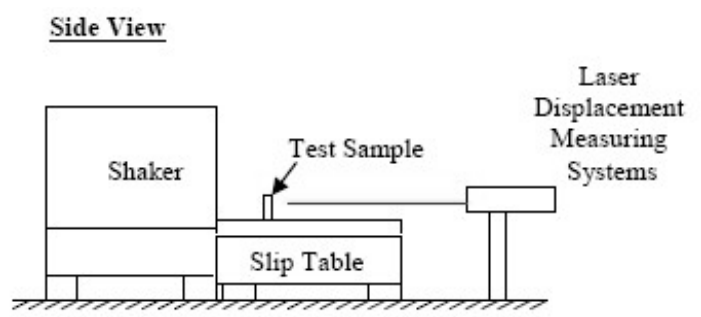

Front View

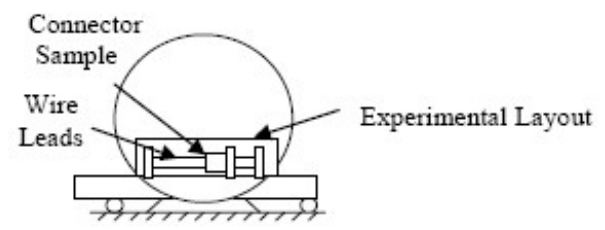

Figure 2: Photograph of the experimental setup

Figure 2 shows the schematic diagram of basic test setup. The entire assembly was bolted to a horizontal slip table, which was driven by a vibratory shaker. Flowers, et. al. [7-10] provided a description of the electrical measurement methods and test procedures for determining fretting thresholds and transfer functions of connector systems. The transfer function for each sample is determined by comparing the output vibration response at the connector interface to the input excitation at the vibratory shaker head. Both are measured using non-contacting laser displacement measuring systems as shown in Figure 2. The ratio of the steady-state output amplitude to the input amplitude for a given excitation frequency is the magnitude of the transfer function at that frequency. The phase difference between the steady-state output and input responses for a given frequency is the phase of the transfer function at that frequency.
The threshold fretting level for a given configuration and vibration frequency is determined by exposing samples to differing amplitudes of vibration and measuring the resistance for 10 minutes. For all electrical resistance measurements, a four-wire configuration was used. The resulting time trace is then visually inspected. If there is no net increase in resistance over the time period of the test, the sample is deemed to have not fretted. A fresh sample was then tested, with the vibration amplitude increased by an increment of $0.1 \mathrm{~g}$ and the inspection process repeated until a net increase in the resistance of greater than $5 \times 10^{-6} \mathrm{ohm} / \mathrm{sec}$ is observed. The lowest vibration amplitude for which such a net increase in resistance is observed is designated as the threshold g-level for that sample. For each configuration and frequency, a minimum of 5 samples are tested and the threshold g-levels averaged to obtain the nominal threshold g-level for that configuration and frequency.

\begin{tabular}{|c|c|c|}
\hline $\begin{array}{c}\text { Specimen } \\
\text { Type }\end{array}$ & Finish & $\begin{array}{c}\text { Normal } \\
\text { Force } \\
(\mathbf{N})\end{array}$ \\
\hline 1.a & 1 & 4.7 \\
\hline 1.b & 1 & 6.0 \\
\hline 1.c & 1 & 6.5 \\
\hline 2.a & 2 & 4.3 \\
\hline 2.b & 2 & 5.3 \\
\hline 2.c & 2 & 6.3 \\
\hline
\end{tabular}

Table 1: Types of specimens

The samples available for this connector system configuration included two different plating finish / base metal combinations and three contact normal force levels. Table 1 summarizes the basic sample descriptions below. Both finish Type 1 and finish Type 2 were based upon tin deposits, with the Type 2 finish having been modified to reduce the coefficient of friction and the corresponding insertion force.

\section{FINITE ELEMENT MODEL}

ABAQUS (SIMULIA, Inc., Providence, RI) finite element simulation models were developed from the CAD drawings for the sample configuration above. A simplified two dimensional (2-D) and a detailed three dimensional (3-D) model were developed and compared. The connector system model contains a large number of elements which results in long computational time for a single simulation run on a single-processor workstation. Therefore a workstation with multiple processors and great computational capability (2 Quad Core Xeon Proc 5320, 16GB ddr2 memory, 640GB hard drive) is used for the 3D simulation. Linux system which has proved capability of providing a much faster-running environment for ABAQUS was installed. Technology with parallel execution in ABAQUS is utilized to increase the 
computational speed. This technology allows the computer to run the complex model simulation in multiple processors ( 8 processors on this workstation) at the same time.

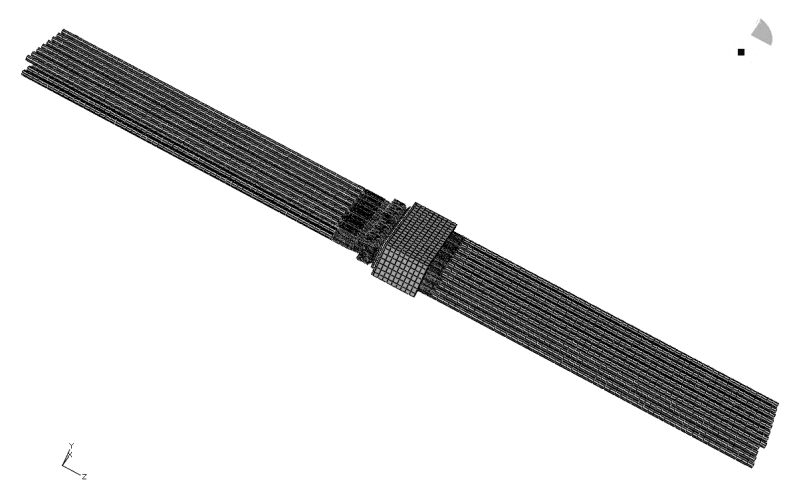

Figure 3: Diagram of 3-D Connector System Model

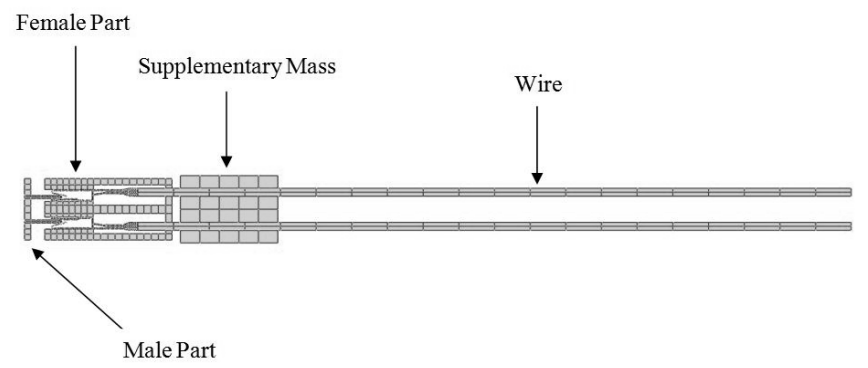

Figure 4: Overall View of 2-D Connector System

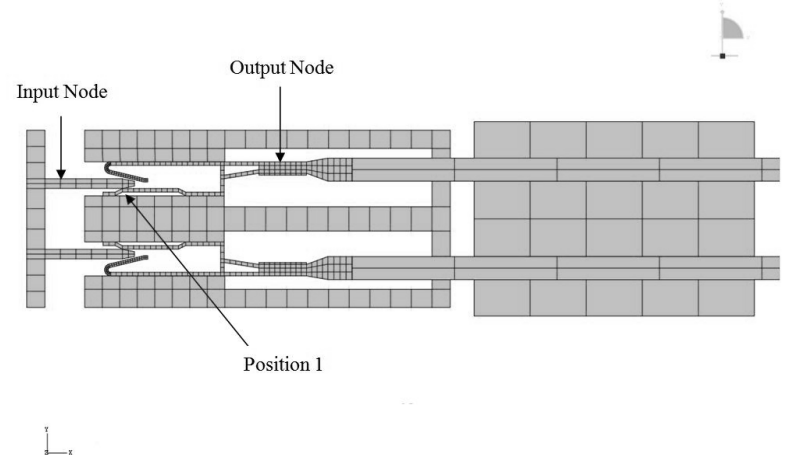

Figure 5: Close-up View of 2-D Connector System

The overall view of the 3-D detailed connector system model is shown in Figure 3. Sixteen pairs of blade/receptacle connectors with wire leads are sealed in housing in two rows and serve as an entire connector system. In comparison, Figure4 and Figure 5 show an overall view and close-up view for 2-D simplified connector system model respectively.

An initial move-in simulation (move the male part into female part to connect the entire system) run were performed on both 3-D and 2-D models to compare the computational cost. As shown in Figure 6, it takes about 3154 minutes to finish a single simulation run on the $3-\mathrm{D}$ model while the time required for the $2-\mathrm{D}$ model is only 30 seconds, a reduction of more than 6000 times. The large number of elements required for this detailed 3-D model tends to result in much more computational cost than 2-D simplified model. Therefore, the 2-D simplified model has been selected as a better tool to investigate the characteristics of the connector system.

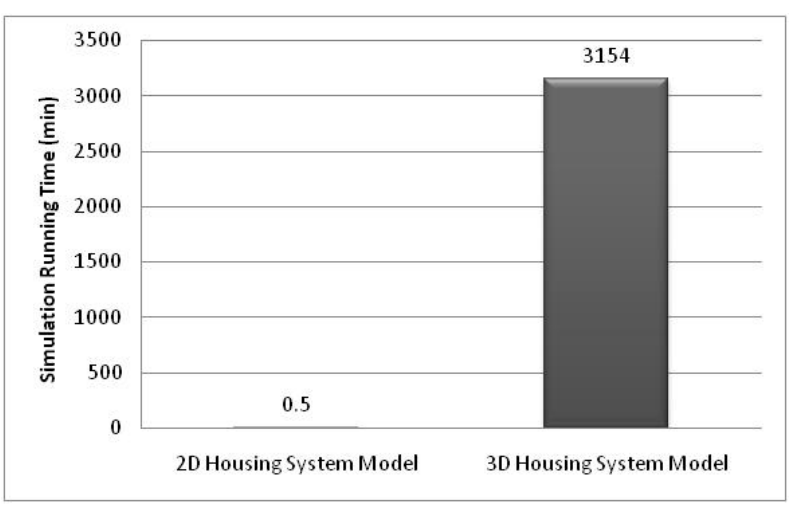

Figure 6: Comparison of Computational Cost for initial simulation between 2-D model and 3-D model

Material Properties

The material properties of each model component were selected to match as closely as possible those of the corresponding component from the actual blade/receptacle sample system. The mass values of the blade, receptacle, housing part, supplementary mass, and wiring used in the model were matched to those of the real components. Because some of the components, particularly the outer structure of the receptacle, the housing part and the wire, had their dimensions or thicknesses adjusted, the volume of these components had also changed. Hence, their densities also had to be adjusted to match the masses of the original 3-D samples in 2-D model. Additionally, the elastic modulus for the outer structure of the receptacle was also increased to stiffen the whole outer structure (excluding the $\mathrm{u}$-bend and beam spring inside the receptacle). The major material properties of each component in the model are listed in Table 2.

\begin{tabular}{|c|c|c|c|}
\hline Components & $\begin{array}{c}\text { Friction } \\
\text { Coefficient }\end{array}$ & Density & $\begin{array}{c}\text { Young's } \\
\text { Modulus }\end{array}$ \\
\hline Plating Type 1 & 0.55 & $\begin{array}{c}8910 \\
\mathrm{~kg} / \mathrm{m}^{3}\end{array}$ & $129.7 \mathrm{Gpa}$ \\
\hline Plating Type 2 & 0.33 & $\begin{array}{c}8910 \\
\mathrm{~kg} / \mathrm{m}^{3}\end{array}$ & $120.6 \mathrm{GPa}$ \\
\hline Wire portion & - & $\begin{array}{c}3423 \\
\mathrm{~kg} / \mathrm{m} 3\end{array}$ & $96.5 \mathrm{GPa}$ \\
\hline Housing Part & & 1130 & $18.1 \mathrm{Gpa}$ \\
& & $\mathrm{kg} / \mathrm{m} 3$ & \\
\hline Supplementary mass & - & 7793 & $195.2 \mathrm{GPa}$ \\
& & $\mathrm{kg} / \mathrm{m} 3$ & \\
\hline
\end{tabular}

Table 2 Material properties of the model components 


\section{RESULTS}

Based upon the system configuration described above, a series of simulation studies were performed to evaluate the dynamic behavior of the system and to validate the predictions of the simulation model to that of experimentally observed behaviors.

\section{Transfer Functions}

The relative motion between the contacting terminals is generally agreed to be the root cause of fretting degradation. From a dynamic modeling perspective, a transfer function is a functional relationship, in the frequency domain, between the magnitude (M) and phase $(\phi)$ of an input motion and the corresponding output motion. For example, a transfer function magnitude of 1 and zero phase shift indicates that there is no relative motion between the respective input and output measurement points. Similarly, a transfer function with a magnitude of 2 and a $45^{\circ}$ phase indicates that the output motion is twice as large as that of the input and that it lags the input response by $45^{\circ}$. For most dynamic systems, there is a peak magnitude for input excitations at one or more of the resonant frequencies. Typically, the phase shift is small for low frequency excitations and increases dramatically as the driving frequency approaches and exceeds the primary resonance value. Another way of thinking of the transfer function is in terms of a complex number where the real part is $M \cos (\phi)$ and the imaginary part is $M \sin (\phi)$.

The input and output measurement locations for both the simulation and experiments were the same for the transfer function simulation. The specific positions are shown as input and output node in Figure 5.

Example transfer functions for Type 1.b and Type 2b, obtained from both experimentally and using the simulation model, are shown and compared in Figure 7.It can be observed form the comparison that the simulation results generally matches overall shape of the experimentally measured results very well. The frequency where the highest peak occurs appears to be very similar between experiment and simulation results. For Type1, the frequency value generated from simulation is $125.1 \mathrm{~Hz}$ compared with $124 \mathrm{~Hz}$ from experiment; for Type2, the value decreased to $121.6 \mathrm{~Hz}$ and $123 \mathrm{~Hz}$. The magnitudes of the first resonant peak from the simulation results are only about $2.76 \%$ and $6.21 \%$ higher than those from the experiment for Type 1 and Type 2 respectively. Please note that there's a significant difference in magnitude between two different types.

Figure 8, 9, 10 and 11 show the transfer functions which were obtained from experimental testing and 2-D model simulations for different finish/base metal type and contact normal force levels respectively. As observed from the experiment results in Figure 8 and 10, the magnitude of the highest peak for Type 1 samples is about $20 \%$ higher than the Type 2 samples. So it appears that the resonant frequency and the associated amplitude peak for the higher friction finish (Type 1) were somewhat higher. Moreover, as the normal force is increased, the magnitude of the magnitude of the transfer function tends to be correspondingly increased. As shown in

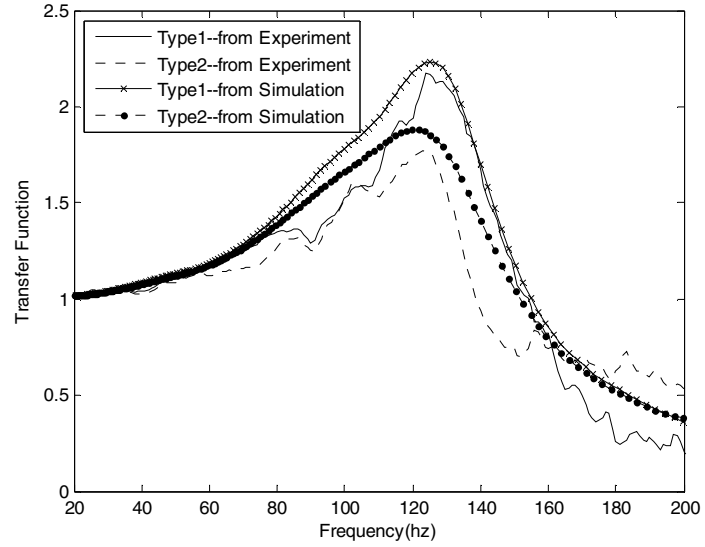

Figure 7: Comparison of Experimentally Determined Transfer Function and Simulation Model

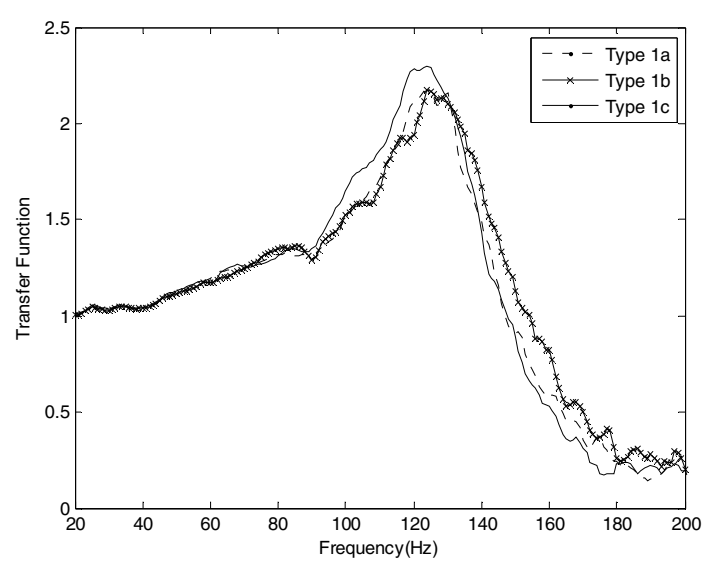

Figure 8: Experimental Results for Different Contact Normal Force Level (Specimen Type 1)

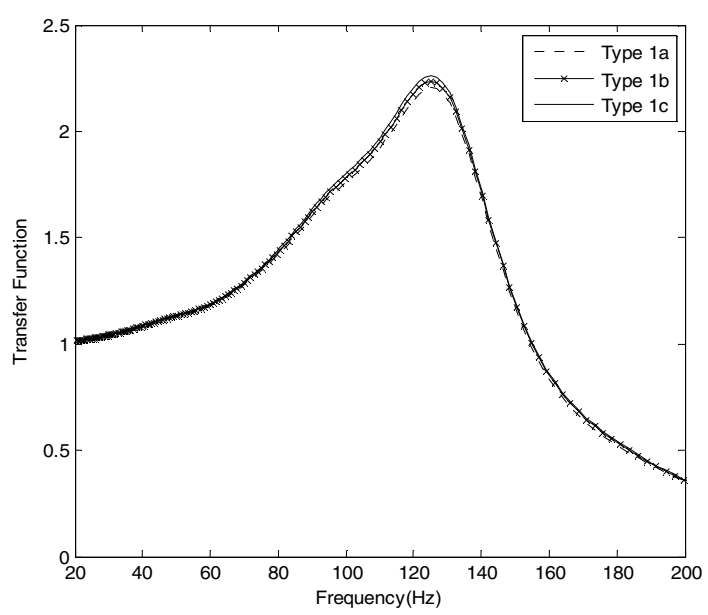

Figure 9: Simulation Results for Different Contact Normal Force Level (Specimen Type 1) 
Figure 9 and 11, the same phenomenon could be predicted by the 2-D model simulation very well. It could be observed from the simulation results that for both type of finish, the magnitude of Type $\mathrm{c}$ with the highest contact normal force is larger than Type b, while that of Type b is larger than Type a, which has the lowest contact normal force.

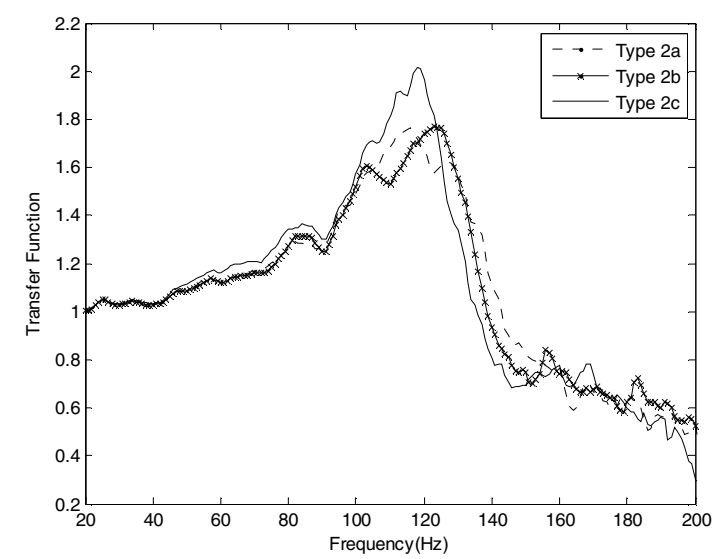

Figure 10: Experimental Results for Different Contact Normal Force Level (Specimen Type 2)

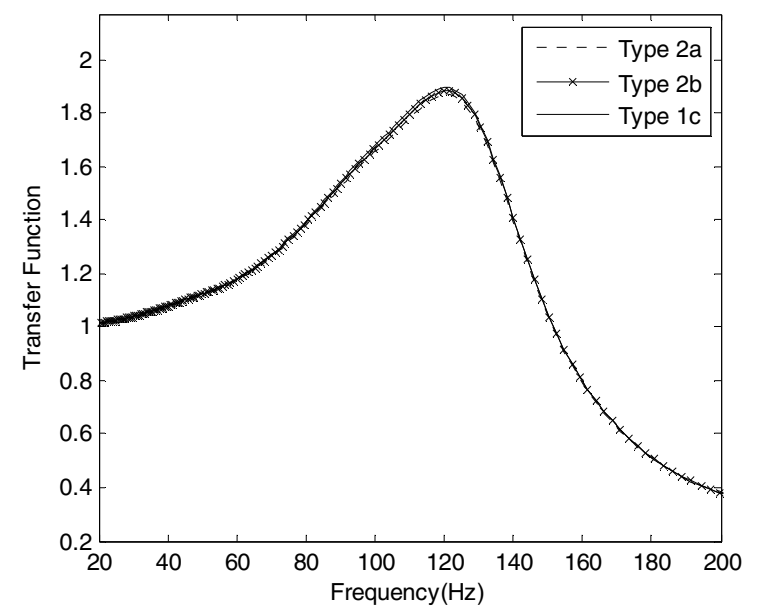

Figure 11: Simulation Results for Different Contact Normal Force Level (Specimen Type 2)

\section{Fretting Rate}

Fretting experiments were performed for each type of samples at $90 \mathrm{~Hz}$ In author's previous work [10]. The different g-levels (acceleration of the shaker head) used in the experiments were calculated and transferred to be the input excitation displacement for the vibration simulation. Moreover, the $\mathrm{x}$-axis relative motion at position 1 was selected as a great indicator for the onset of the fretting corrosion in simulation. This methodology was conducted and validated by the author before [13-14]. In this particular 2-D connector system model, position 1 was the point of the contact between the blade and the left inner bottom surface of the receptacle during the rocking motion as shown in Figure 5.

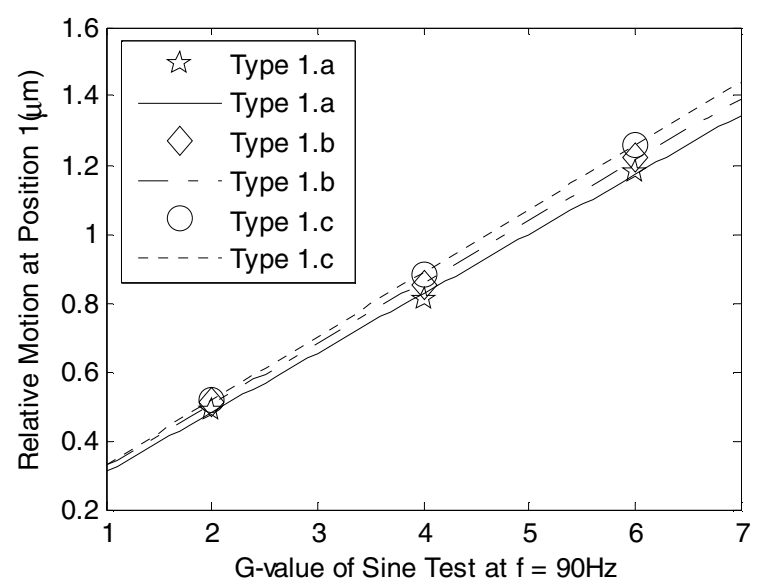

Figure 12: $\mathrm{X}$-axis Relative Motion for Different Contact Normal Force Level (Specimen Type 1)

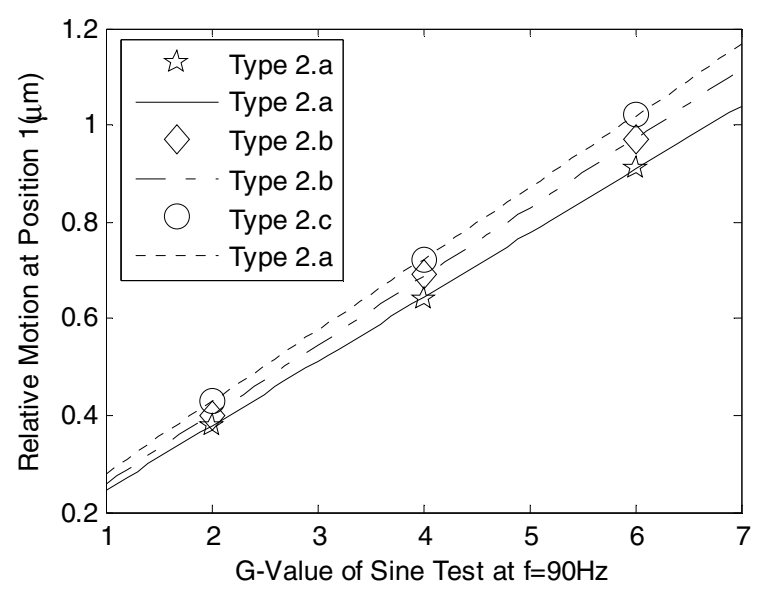

Figure 13: $\mathrm{X}$-axis Relative Motion for Different Contact Normal Force Level (Specimen Type 2)

From Figure 12 and 13, it could be observed that for each sample type, as the normal force increased, the $\mathrm{x}$-axis relative motion at position 1 generally correspondingly increased. In another word, the $\mathrm{x}$-axis relative motion amplitude of Type $\mathrm{c}$ is larger than that of Type $b$, while Type $b$ is larger than Type a. Moreover, the comparison of the results of the two finish types showed the significant differences between the relative motions, with the higher friction finish having markedly higher $\mathrm{x}$-axis relative motion amplitude. These results show a great agreement with the author's previous work of fretting rate in experiments [10] in Figure 14 and 15. The experimental results indicated that as the increase of the contact normal force, the fretting rate (rate of resistance changes) generally increased (expect type $2 \mathrm{~b}$ ) too. Meanwhile, the fretting rate results for Type 1 with higher friction finish is larger than Type 2, with the slopes of the linear regression line are approximately 0.12 and 0.06 respectively. These phenomenons are generally in good agreement with the transfer function results. The magnitude of the transfer 
function is increased as the increase of the contact normal force and the magnitude of Type 1 is larger than Type 2 .

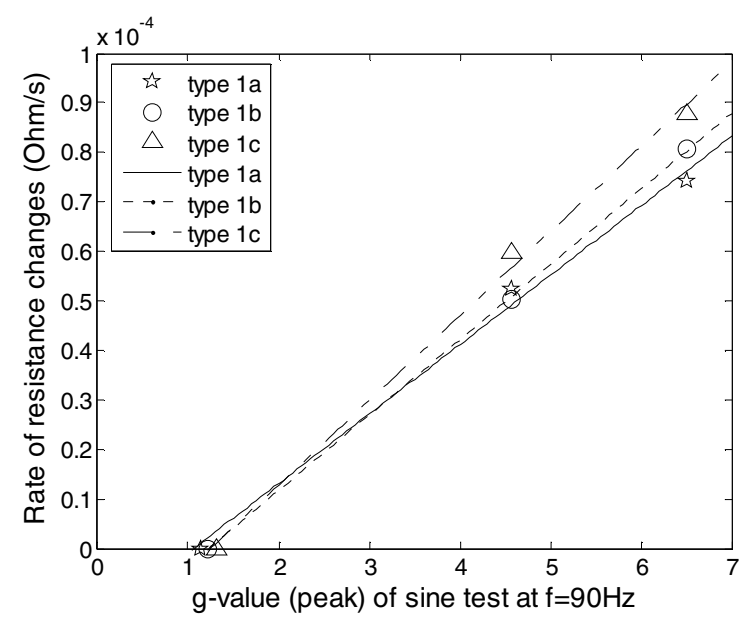

Figure 14: Fretting Rates results from Experiments with fit lines (Specimen Type 1)

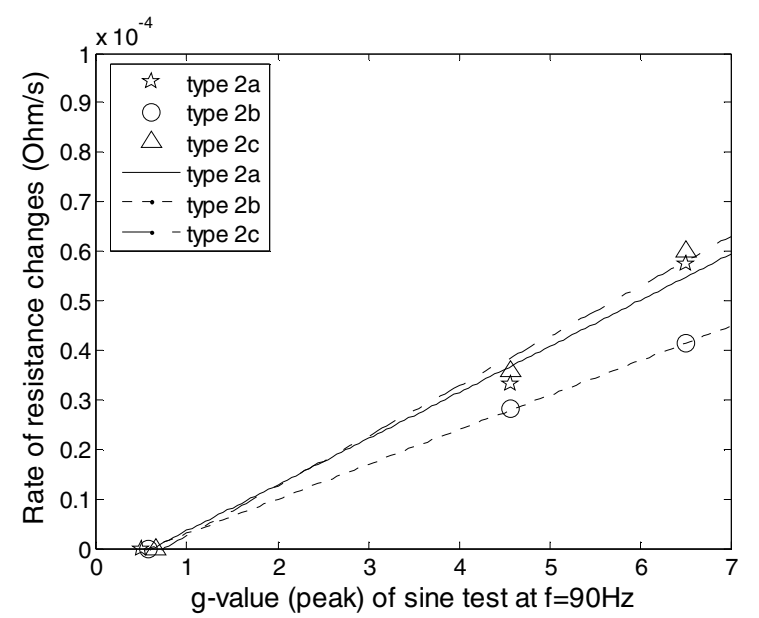

Figure 15: Fretting Rates results from Experiments with fit lines (Specimen Type 2)

Both results from experiment and simulation tend to follow the basic theory which was developed as part of earlier work on this topic [10-12]. In that work, it was observed that the fretting degradation rate satisfied the relationship:

$$
\frac{d \Delta R}{d t}=E \frac{Z_{F}}{f^{2}}\left(G_{f}-G_{f, \text { threshhold }}\right) f
$$

where $Z_{F}$ is the magnitude of the transfer function relating the relative motion between mating terminals (nominally equivalent to the motion of the "output" terminal relative to the "input" (shaker head), Gf is the G-level acceleration of the shaker head, G threshold is the threshold vibration of the shaker head before the onset of fretting, $f$ is the frequency of vibration, and $\mathrm{E}$ is a scaling factor. It is obviously from the relationship that as the increase of relative motion between mating terminals (E.g. position 1 in Figure 5 in simulation), the fretting rate should (rate of resistance changes) correspondingly increase. This relationship was proved by the trend shown in both simulation and experiment results. This value is in building a reasonable and consistent relationship between the experiment and simulation results.

\section{CONCLUSIONS AND SUMMARY}

A study of the vibration induced fretting degradation of a connector system was presented. Two variations of finish type were considered, with each finish type having three different contact normal force values. The transfer functions and the fretting rate changes at certain frequency were measured in the experiment. A detailed 3-D model and a simplified 2-D model were developed and compared using ABAQUS for the connector system. The geometric dimensions, material properties and boundary conditions of both models were generated based on the experimental values. The 2-D finite element model was selected to be a better simulation tool especially in the consideration of the computational cost. Parallel execution technology was utilized to enhance the simulation process. A series of simulations were performed on the 2-D housing connector model. Corresponding experiments were conducted to validate and test the performance of the system. The $\mathrm{x}$-axis relative motions for the contact interfaces and the transfer functions of all types of samples were simulated using this model. The $\mathrm{x}$-axis relative motion at the contact interfaces was observed when the experimental threshold displacements were used as the input vibration levels for this connector system. Finally, the results from the simulation were compared with the results from the experiment and generally found to be in good agreement. It was demonstrated that, for this limited system, finite element modeling and analysis are potentially valuable tools for the evaluation of the influence of design variations on the fretting behavior of the connector system. This study's findings can be summarized and concluded as follows:

- Threshold amplitudes and transfer function results were measured for all six types of samples in both experiment and simulation.

- The 2-D simplified model with proper assumption was proved to be a better tool than 3-D detailed model, especially in consideration of the huge computational cost of the 3-D simulation.

- The relationship between the relative motion function and the fretting rate for this connector system was found in good agreement with the fretting degradation relationship in previous study.

- The results from the simulation generally correlated well with that obtained from the experiment.

- Finite element modeling and analysis have great potential for evaluating the influence of design variations on fretting behavior of the connector system.

\section{ACKNOWLEDGMENT}

This research was sponsored by the NSF Center for Advanced Vehicle Electronics (CAVE). The authors would like to express our appreciation to Bretton I. Rickett, Robert D. 
Malucci, and Charlie Manlapaz of Molex, Inc. for their assistance and advice on this study.

\section{REFERENCES}

[1] E. M. Bock and J. H. Whitley, "Fretting Corrosion in Electric Contacts" Proceedings of $20^{\text {th }}$ Holm Conference on Electrical Contacts, 1974, p.128-138.

[2] M. Antler, "Survey of Contact Fretting in Electrical Contacts", Proceedings of the Twenty Ninth IEEE Holm Conference on Electrical Contacts, 1984, p.3.

[3] B. Malucci, "Fretting Corrosion Degradation, Threshold Behavior and Contact Instability", Ragnar Holm Scientific Achievement Award Address, Proceedings of the $44^{\text {th }}$ IEEE Holm Conference on Electrical Contacts, pp. 1-15, Washington, D.C., September 8-10, 2003.

[4] M. Braunovic, N. S.S. Mcintyre, W. J. Chauvin, and I. Aitchison, "Surface Analysis of Fretting Damage in Electrical Contact of Aluminum with Different Contact Materials," IEEE Transactions on Components, Hybrids, and Manufacturing Technology, Vol. CHMT-7, No.1, March 1984, pp. 96-106.

[5] J. Swingler and J. W. McBride, "Fretting Corrosion and the Reliability of Multicontact Connector Terminals," IEEE Transactions on Components and Packaging Technologies, Vol. 25, No. 4, December 2002, pp. 670-676.

[6] M. D. Bryant, "Resistance Buildup in Electrical Connectors Due to Fretting Corrosion of Rough Surfaces," IEEE Transactions on Components, Packaging, and Manufacturing Technology - Part A," Vol. 1, No. 1, March 1994, pp.86-95.

[7] G. T. Flowers, F. Xie, M. Bozack, and R.D. Malucci, "Vibration Thresholds for Fretting Corrosion in Electrical Connectors," Proceedings of the 48th IEEE Holm Conference on Electrical Connectors, pp.133-139, October 21-23, 2002.

[8] G. T. Flowers, F. Xie, M. Bozack, R. Horvath, R. D. Malucci,, and B. Rickett., "Vibration Testing of Fretting Corrosion in Electrical Connectors Subjected to Multi-Frequency and Random Spectral Profiles," Proceedings of the $49^{\text {th }}$ IEEE Holm Conference on Electrical Contacts, pp. 45-50, Washington, DC, September 8-10, 2003.

[9] G. T. Flowers, F. Xie, M. Bozack, X. Hai, B. Rickett and R. D. Malucci, "A Study of the Physical Characteristics of Vibration Induced Fretting Corrosion," Proceedings of the $50^{\text {th }}$ IEEE Holm Conference on Electrical Contacts," pp. 312-319, Washington, DC, September 20-23, 2004.

[10] G. T. Flowers, F. Xie, M. Bozack, R. Horvath, B. Rickett, and R. D. Malucci, "The Influence of Contact Interface Characteristics on Vibration-Induced Fretting Degradation," Proceedings of the $51^{\text {st }}$ IEEE Holm Conference on Electrical Contacts," pp. 312-319, Chicago, Illinois, September 23-25, 2005.

[11] F. Hubner-Obenland and J. Minuth, "A New Test Equipment for High Dynamic Real-Time Measuring of Contact Resistances," Proceedings of the $45^{\text {th }}$ IEEE Holm Conference, pp. 193-202, Oct. 4-6, 1999.

[12] Hammam, Tag, Kassman-Rudolphi, Asa, and Lundstrom, Per, "Vibration-induced deterioration of in-coated connectors studied by using a force controlled fretting bench-test," Proceedings of the FiftyFirst IEEE Holm Conference on Electrical Contacts, 2005, p 97-106, Sep 26-28 2005.

[13] Fei Xie, George T. Flowers, Chen Chen, Michael Bozack and Jeffrey Suhling, "Vibration-Induced Fretting Motion in a Blade/Receptacle Connector Pair", The 53rd IEEE Holm Conference on Electrical Contacts, pp.222-228,Sep.2007.

[14] C. Chen, Flowers, G.T., Bozack, M., Suhling, "Modeling and Analysis of a Blade/Receptacle Pair for the Prediction of Vibration-Induced Fretting Degradation", The 54th IEEE Holm Conference on Electrical Contacts, pp.276-283, Orlando, FL, Oct. 2008. 


\section{Micro Motion Characteristics of Carbon Pastes on}

\section{Rigid Circuit Boards}

Jin Chun Gao, Liang Jun $\mathrm{Xu} ， \quad$ Xiao Xiang Yu,

Lab of Electric Contacts,

Beijing University of Posts \& Telecommunications, Beijing 100876, China

gjc@bupt.edu.cn, ljxu@bupt.edu.cn,yxxbasketboy@gmail.com,

\author{
Guoping Luo \\ Technology Platform, \\ Nokia Corporation, \\ Salo, 24100 Finland \\ Guoping.Luo@nokia.com
}

\begin{abstract}
-
Carbon paste is commonly used in printed circuit boards as conductive pads and jumpers, or printed resistors etc. Recently, its application as conductive pads is expanding due to material cost competitiveness comparing with gold and other coating materials. This paper introduces the characteristics of carbon paste during micro motion. The micro motion test is carried out on a fretting test apparatus. Scanning Electron Microscope (SEM) and X-ray Energy Dispersive Spectroscopy (EDS), as well as 3-dimensional morphology analyzer are used in analysis of tested samples. It is found that the increase of contact resistance for carbon pastes during micro motion is a regular phenomenon and mainly caused by the accumulation of carbon particles and successive formation of carbon flakes on the surface, its mechanism is discussed. This research is helpful in understanding the tribology performance of carbon paste as conductive pads.
\end{abstract}

Key words: carbon paste, micro motion, circuit board, mechanism.

\section{INTRODUCTION}

Carbon paste is one commonly used coating material. Carbon paste on rigid PCB (Printed Circuit Board) is made from printed conductive carbonaceous materials by using screen printing methods $[1-2,11]$. Due to the inexpensive cost in production, less susceptive to chemical corrosion, carbon paste is a popular coating in many electronic products such as keyboards, calculators, remote control of TV, telephones [1]. Recently, its application is expanding due to material cost competitiveness comparing with gold and other coating materials. However, there is little research study reported on the behavior of carbon paste under micro motion when used for electrical contact purpose. For getting further understanding, our research is thus focused on the tribology behavior of carbon pasteboards. The results would be helpful for better seeking the application of carbon pastes in contact application.

It is shown in our previous study [3] that the contact resistance of carbon paste on test boards is increased during micro motion test. In order to find the reason why resistance keeps increasing, we investigated and analyzed the surface of carbon paste with the use of a Scanning Electron Microscope (SEM), an X-ray Energy Dispersive Spectroscopy (EDS), as well as a 3-dimensional morphology analyzer. This paper describes a possible mechanism involved in increase of contact resistance and attempts to give an explanation for the process.

\section{EXPERIMENT}

\section{A. Experimental Samples}

In the micro motion experiment, a flat specimen is carbon pasteboard. The carbon paste is $15 \mathrm{um}$ in thickness, screen printed over copper substrate of the rigid circuit board. The rider specimen is a gold plated probe with a thickness of $0.3 \mu \mathrm{m}$, its middle plating is $\mathrm{Ni}$ and the substrate is copper alloy, the diameter of this probe is $1 \mathrm{~mm}$ with spherical tip for electrical contact.

\section{B. Experimental Condition}

The specific parameters in micro motion experiment are as follows,

Cycle rate: $0.5 \mathrm{~Hz}$

Wipe distance: $200 \mu \mathrm{m}$

Force: $150 \mathrm{~g}$

DC current of contact resistance testing: $100 \mathrm{~mA}$

Voltage for controlling contact resistance testing: $2 \mathrm{~V}$

Number of cycles: 100,000

The samples including the carbon pasteboards and the probes are cleaned with ultrasonic cleaner for 20 minutes and are then tested in the fretting test apparatus.

\section{Experimental Equipment}

The micro motion experiment was carried out by a fretting test apparatus as illustrated in Fig 1. The equipment is designed for measuring the contact resistance during the period of fretting for 8 routes. Working plane is set up with only $\mathrm{X}$ coordinates. By using 4 points method for applying a given force to the probe on test coupon, contact resistance 
between probe and coupon is obtained. The fretting stage brings fretting between the coupons and probes, and then the test system can test the contact resistance.

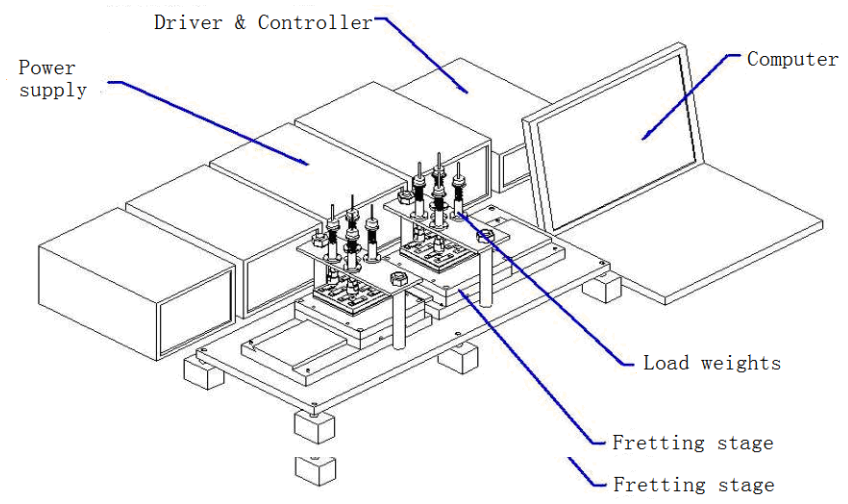

Fig. 1 Fretting test apparatus

The wear area on the carbon paste surface was then investigated and analyzed, using a Scanning Electron Microscope (SEM), an X-ray Energy Dispersive Spectroscopy (EDS), and a 3-dimensional morphology analyzer.

\section{RESULTS AND DISCUSSION}

\section{A. Testing of Contact Resistance During Micro Motion}

Four identical carbon pasteboards were tested by the fretting test apparatus. The results show the continuous increase of contact resistance during micro motion, same as we found in previous study. It indicates that the increase of contact resistance for carbon pastes during micro motion is a regular phenomenon. One of the results is illustrated in Fig 2. The incremental resistance value in the experiment is $1-1.5 \Omega$ after 100,000 cycles, $100-150 \%$ change in resistance.

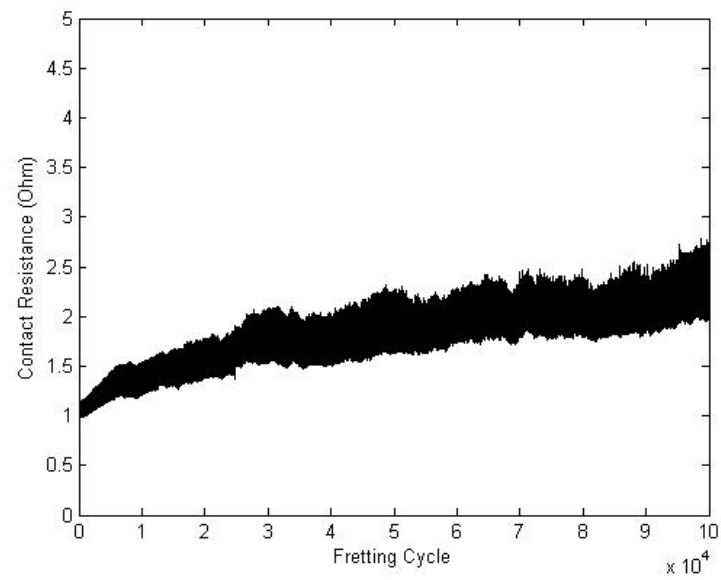

Fig. 2 Contact resistance of carbon pasteboards during micro motion, fretting cycles 100,000

\section{B. Morphology of Original Carbon Paste Surface}

The morphology of original carbon pasteboards was observed by SEM as illustrated in Fig 3a-3b. The composition of carbon paste was analyzed by EDS. It is shown that the atomic percentage of carbon is $91 \%$ whereas the atomic percentage of oxygen is $9 \%$. The main composition of carbon paste is the carbon element as mentioned also in material data sheet [11]. It consists of a large number of carbon particles with a small amount of polymer (such as epoxy resins) and organic additives [1-2, $11]$.

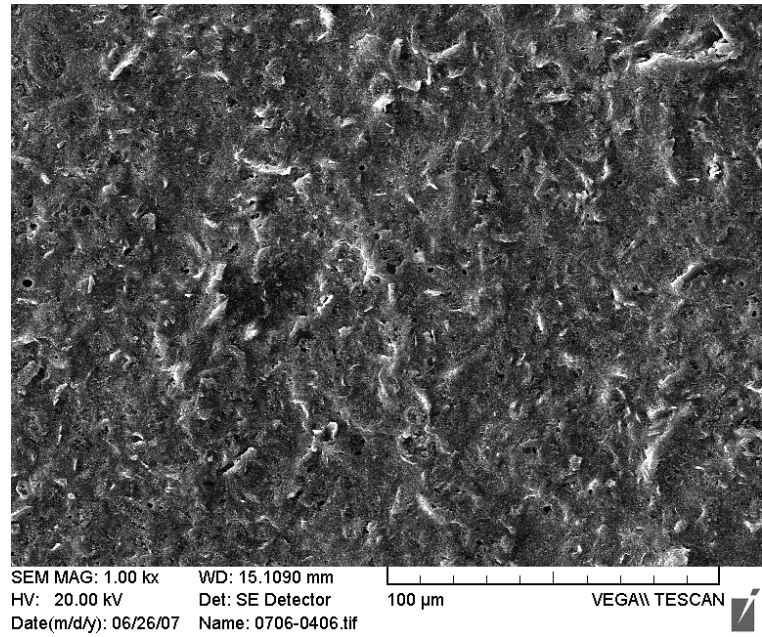

Fig. 3a Original carbon paste surface taken by SEM, magnification $1,000 \mathrm{X}$, gold coated.

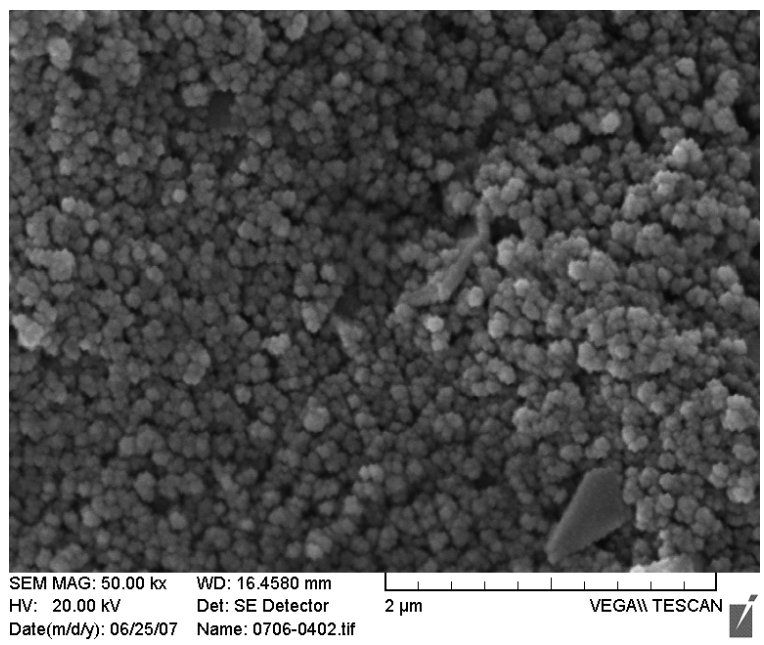

Fig. 3b Original carbon paste surface taken by SEM, magnification $50,000 \mathrm{X}$, gold coated

From Fig 3a-3b, it is found that carbon paste is composed of many spherical carbon particles with the diameter of $0.1 \mu \mathrm{m}$ approximately. Fig $3 \mathrm{a}$ shows the image of magnification 1,000. Fig $3 \mathrm{~b}$ is image of magnification 50,000 .

\section{Analysis of Micro Motion Area on Carbon Paste Surface}

The micro motion area of the carbon paste surface was inspected by SEM; a SEM image is illustrated in Fig $4 . \quad$ In order to investigate the carbon paste surface further, the points 
marked as A, B, C and D on Fig 4 were analyzed by SEM and EDS. Point $\mathrm{A}$ is located in the middle of the micro motion area where there are a lot of particles on the carbon paste surface. Point $\mathrm{B}$ is located upside one fourth of the micro motion area, point $\mathrm{C}$ is at the edge of the area and point $\mathrm{D}$ is in the zone where it seems to be a smooth surface. The SEM magnification images of point A, B, C, D are as shown in Fig $5 \mathrm{a}-5 \mathrm{~d}$.

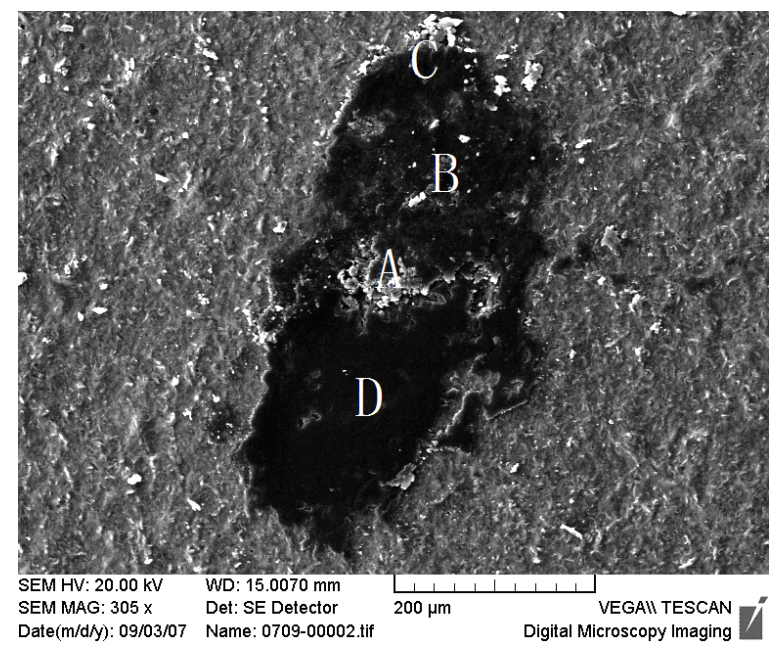

Fig. 4 Whole micro motion area of carbon paste surface, magnification $305 X$, gold coated, taken by SEM.

From Fig 5a-5c, it can be found that there are large numbers of accumulated particles in the wear area. The EDS results show that these substances are carbon particles and the atomic percentage of carbon is about $91 \%$ and that of oxygen, about 9\%. Carbon, being an inert element, is not easily oxidized in the air [4]. By analyzing several points in the wear area, it also validates the fact that the composition in micro motion area remains the same as the composition on the original carbon paste surface.

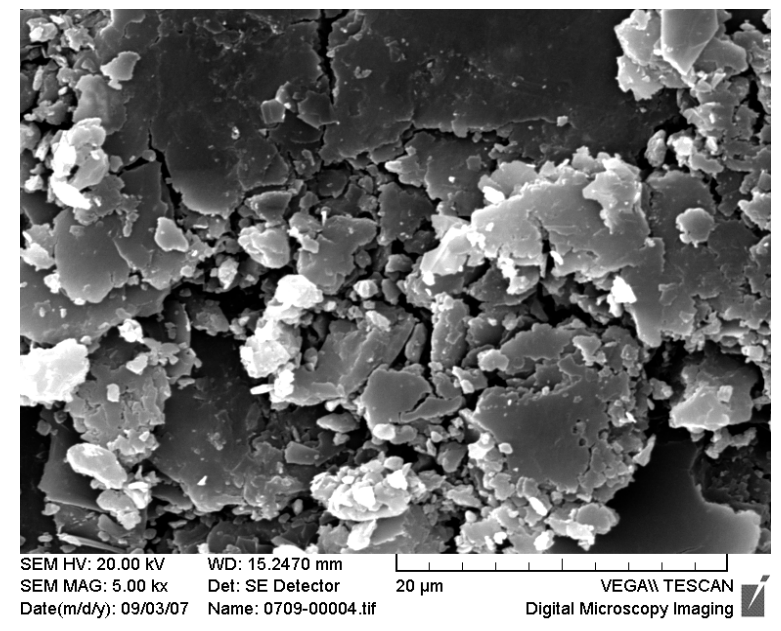

Fig. 5a Point A, SEM image, magnification 5,000X, gold coated.

During micro motion, these carbon particles on the surface were accumulated together into flakes. These flakes further formed the multi-layers carbon films on the surface, which can cause contact resistance to increase.

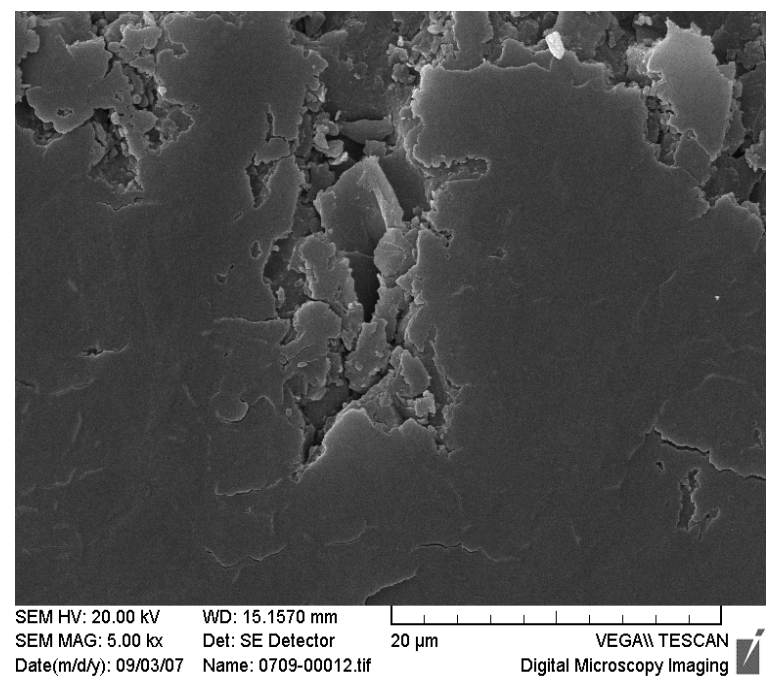

Fig. 5b Point B, SEM image, magnification 5,000X, gold coated.

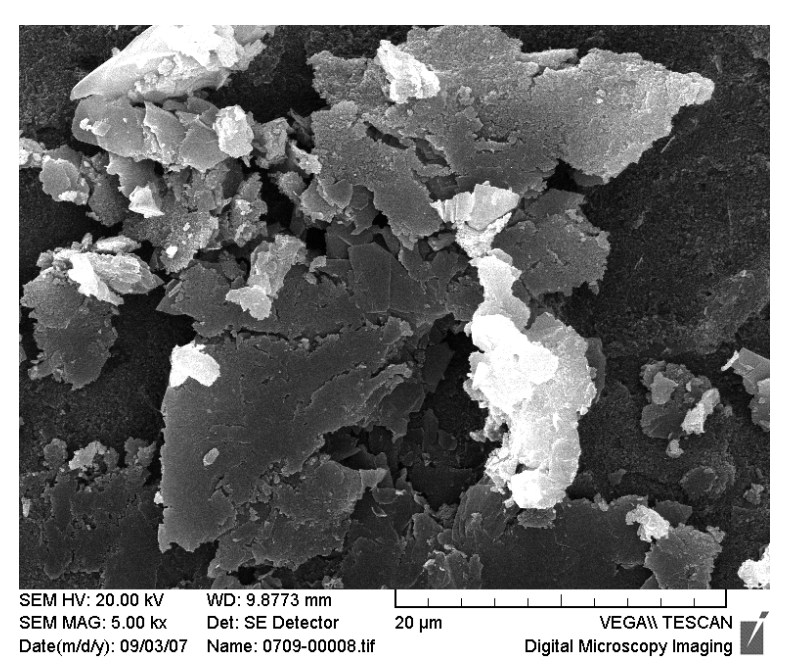

Fig. 5c Point C, SEM image, magnification 5,000X, gold coated.

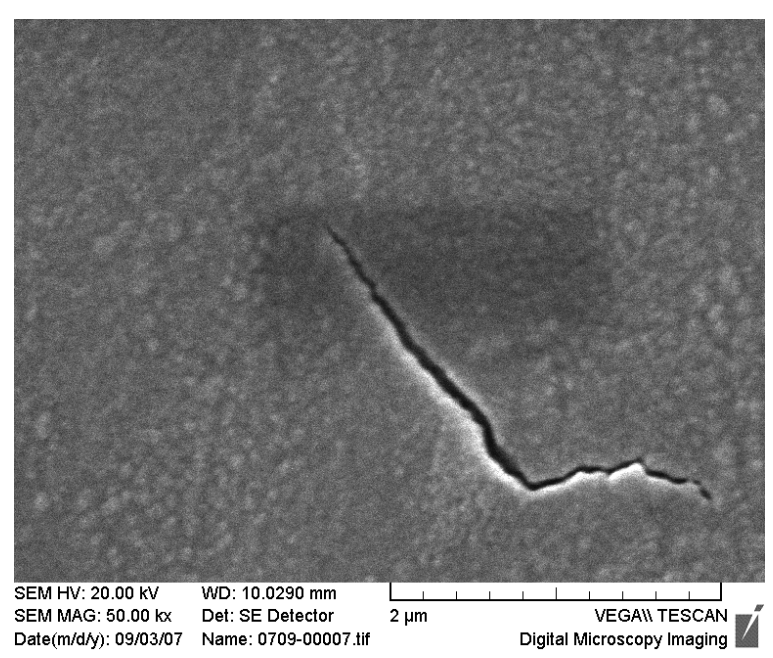

Fig. 5d Point D, SEM image, magnification 50,000X, gold coated. 
In order to investigate why there is a crack on the surface from Fig 5d, another sample was analyzed by SEM. The magnified image of the micro motion area in the carbon paste is illustrated in Fig 6. It shows that the surface is covered with a layer (or multi-layers) of carbon film formed from the accumulated carbon particles. Such carbon films newly formed by mechanical wear are loose and brittle. They can be broken under contact pressure. Hence the crack as seen in the above Figure (Fig 5d) is caused by the disruption of the accumulative formed carbon films in mechanical wear.

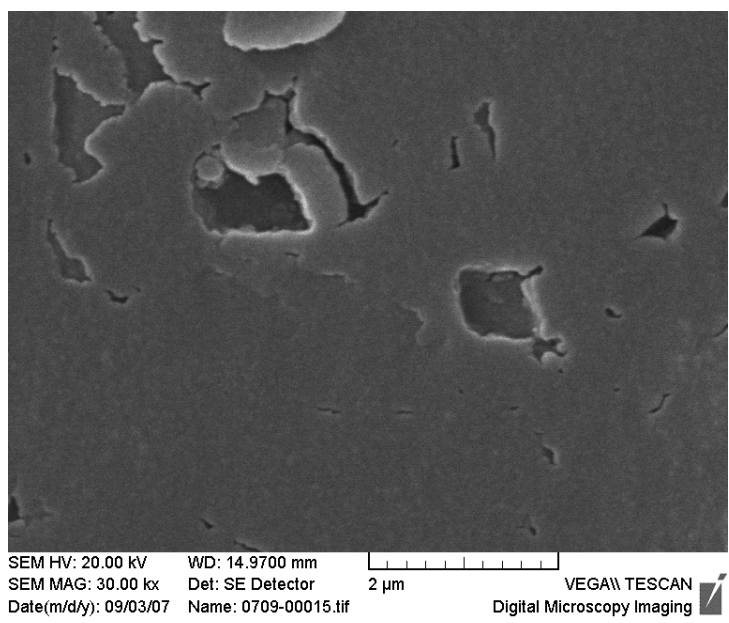

Fig. 6 SEM image of micro motion area in the carbon paste, magnification 30,000X, gold coated.

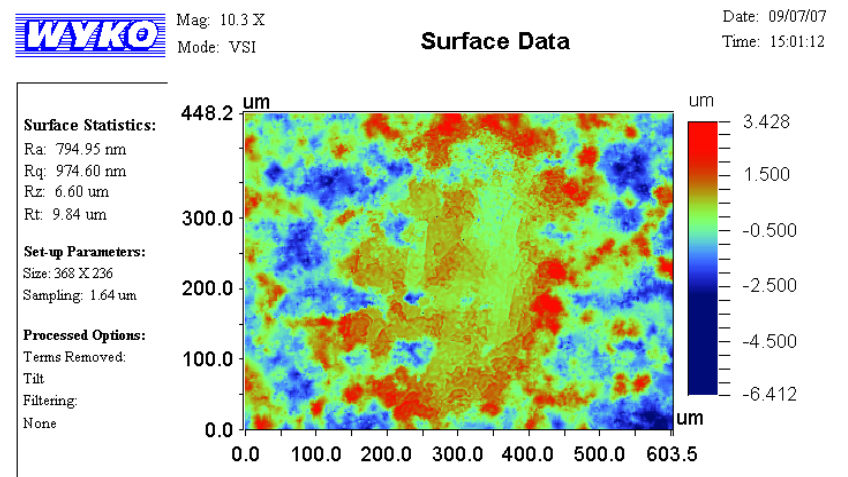

Fig. 7 Morphology of carbon paste surface after micro motion, gold coated, taken by 3-dimensional morphology analyzer. The contact force was $150 \mathrm{~g}$.

Fig 7 is a profile image of sample taken by a 3-dimensional morphology analyzer; it reveals that there is a high rise on the micro motion area, the height of accumulated flakes formed by the particles is about $1-1.5 \mu \mathrm{m}$, and the accumulative formations is higher at the edge of whole micro motion area, about $2-3.4 \mu \mathrm{m}$. Since the multi-layers carbon films are lying on the surface, whole fretting zone is higher than rest area of the image. Hence, this result also validates the accumulation of particles on the carbon paste surface.
D. Possible Mechanism of Causing Contact Resistance to Increase during Micro Motion

It has been known that carbon paste on PCB is made from carbon ink by using screen printing methods [1-2]. Carbon paste as a coating layer on metal is composed of carbon particles, the macromolecule resins and organic additives $[1-2,11]$. In our experiment, the spherical carbon particles had a diameter of about $0.1 \mu \mathrm{m}$. During the production process of the carbon paste, the organic solvents were evaporated, the resin was then solidified, and therefore the carbon particles were coated with a layer of organic materials on their surface. Conduction can occur between carbon particles when the organic materials are so thin. The mechanism of transmitting electron for composite conductive polymeric materials is reviewed in literature [5].

Fretting causes metal transfer and wear [6]. Fretting can also cause carbon paste to wear. Fretting debris on carbon pastes is composed of carbon particles coated with organic material, carbon particles, and a few other organic materials. This mixture of debris further accumulates to form multi-layers carbon films on the surface. These carbon films are laid on the carbon paste interface irregularly, and there are some gaps between the layers. Contact resistance may change when this occurs.

An experiment was conducted to validate the increase in contact resistance as a result of carbon particle accumulation. The process is as follows:

The experimental samples used are the wear debris from carbon paste, carbon paste sheet, and gold plated probe with a diameter $3 \mathrm{~mm}$.

The test equipment used is Precision Resistance Testing Apparatus.

The load forces are $50 \mathrm{~g}, 100 \mathrm{~g}$, and $150 \mathrm{~g}$, applied respectively.

The carbon particles were obtained from the carbon paste surface by grinding it against a hard object, and then these particles were put back on a fresh carbon paste sheet surface. If the contact resistances on both fresh carbon paste sheet and fresh carbon paste sheet plus accumulated carbon particles, is measured by the precision resistance tester separately, the resistance of carbon particles would be just the difference of these two contact resistance (total contact resistance minus contact resistance of carbon paste sheet).

The probe in the experiment is a gold plated probe.

We first prepared carbon particles to accumulate into one layer of carbon film on the carbon paste sheet surface, and measured the contact resistance under the different forces: $50 \mathrm{~g}$, $100 \mathrm{~g}$, and $150 \mathrm{~g}$, respectively. We followed to accumulate carbon particles into two layers, and similarly, into three layers, four layers. The contact resistances of carbon particles on the carbon paste sheet surface in each experiment were measured, as shown in Fig 8.

From Fig 8, it is found the contact resistance of carbon particles is increasing with the increment of the accumulation times. As the load forces rise from $50 \mathrm{~g}$ to $150 \mathrm{~g}$, the contact resistance is found to be decreasing accordingly. 
When the accumulation times are increased, the thickness of carbon firm layer formed is increased, and therefore the contact resistance will be raised. This result reveals that the carbon particles worn from carbon paste can cause a change in contact resistance.

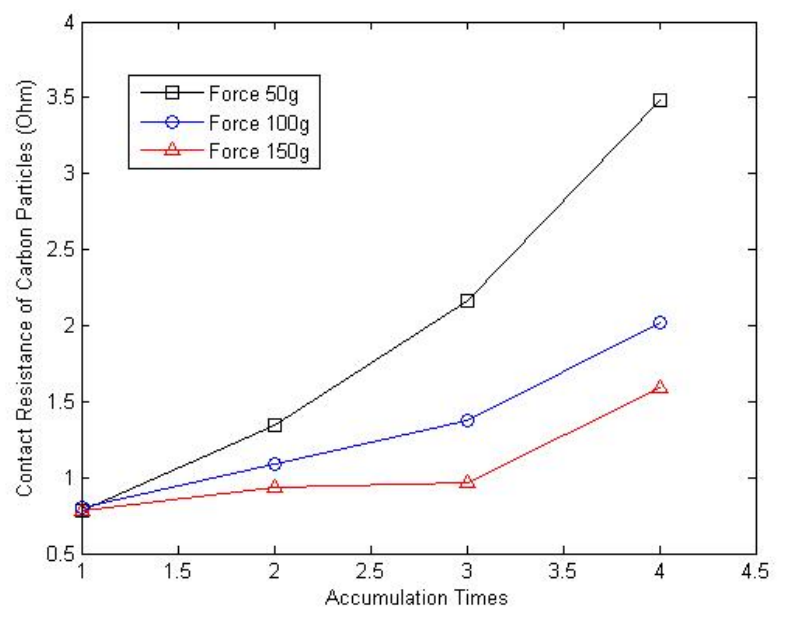

Fig. 8 Contact resistance of carbon particles

The probes were inspected by use of a BSE (Backscattered Electron) detector in SEM after the micro motion experiment. A BSE image of gold plated probe is illustrated in Fig 9. It is shown that a lot of carbon particles were also stuck on the probe. Carbon particles, as wear debris, can also be transferred to the surface of test probe.

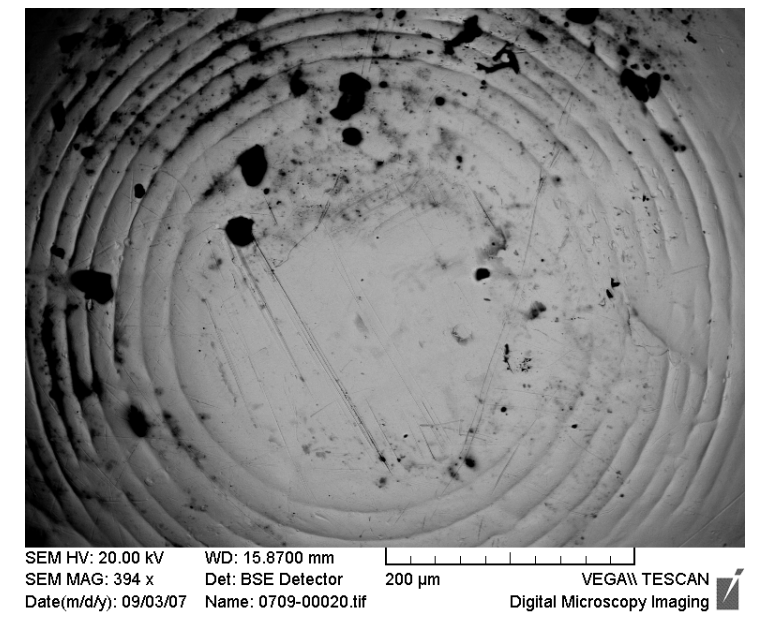

Fig. 9 BSE image of gold plated probe, magnification 394X. The black spots in the figure were carbon particles.

Micro motion plays an important role in the failure of movable contacts [7-10]. Micro motion can produce the wear particles from the interface. It may also collect and accumulate the particles on the surface [7]. In our experiments, there are large numbers of carbon particles worn out from the carbon paste in the wear area. These particles are accumulated to form multi-layers carbon films irregularly by the probe under micro motion, which can cause contact resistance to increase.

\section{CONCLUSION}

The contact resistances of carbon pasteboards were monitored by the fretting test apparatus and fretting behavior of carbon paste material was discussed. Our experiments show how contact resistance is increased during micro motion.

Meanwhile, the fretting wear surface of carbon paste was also investigated and analyzed by a Scanning Electron Microscope, an X-ray Energy Dispersive Spectroscopy, and a 3-dimensional morphology analyzer. It is found that there are a lot of carbon particles worn out on the carbon paste surface. These carbon particles are accumulated together into flakes, the flakes further form to multi-layers of carbon films on the surface, which can cause the contact resistance to increase. The result of our study is helpful for application of carbon paste as conductive pads.

\section{ACKNOWLEDGEMENT}

The authors would like to thank Prof. Ji Gao Zhang of Beijing University of Posts and Telecommunications for his helpful comments and suggestions and Prof. George T. Flowers of Auburn University for his substantial assistance. This research is supported by Nokia Co. of Finland and is gratefully acknowledged.

\section{REFERENCES}

[1] H. X. Ye, "Carbon Conductive Pastes and Carbon Film PCB." Screen Print", 2001.1

[2] B. Chen, Z. D. Huang, "Application of conductive adhesives on PCB", Information of PCB, No.4, pp.8-10, 2001

[3] Research Lab of Electric Contact, Internal report, 2008.

[4] South China University of Technology, Inorganic chemistry, People's Education Press, 1979.

[5] J.G. Yang, C.C. Liu, K. Shi, "Application of percolation theory for study of composite conductive polymeric materials", Chemical Intermediate, No.2, 2006, pp13-17

[6] P.G. Slade, Electrical Contacts: Principles and Application, Marcel Dekker, Inc. 1999, pp332-364

[7] J.G. Zhang. "Effect of Dust Contamination on Electrical Contact Failure", Proceedings of the 53rd IEEE Holm Conference on Electric Contacts, 2007, pp. xxi-xxx

[8] J.G. Zhang, J.C. Gao and C.F. Feng. "Adhesion and attaching of particles at the failed connector contacts." Proceedings of the $23^{\text {rd }}$ ICEC’ 2006, pp.192-196.

[9] Z.P. He and L.J. Xu, "Micro motion at the failed contact interfaces". Proc. 51 $1^{\text {st }}$ IEEE Holm Conference on Electrical Contacts. 2005. pp.180-185.

[10] J.G. Zhang, J.C. Gao and X.Y. Lin. "Analysis of connector contact failure." IEICE Trans. on Electronics, Vol.E86-C, No.6, 2003, pp.945-952.

[11] Carbon conductor pastes specification, internal report, 2007. 


\section{Computational Modeling for Fretting Simulation}

\author{
Yasuyuki Ishimaru \\ ES3 department \\ FITEC corporation \\ Tokyo, Japan
}

\author{
Keiji Mashimo and Kyota Susai* \\ $R \& D$ department \\ The Furukawa Electric co., ltd. \\ Yokohama and *Nikko, Japan
}

\author{
Fu Yingchao and Wang Anlin \\ College of Mechanical Engineering \\ TongJi University \\ China
}

\begin{abstract}
As the number of wire circuits used in automobiles increases, larger force is required to mate connectors. In order to reduce wire assembling cost, connectors with low insertion force are desired. This may, however, result in fretting problems in electric contacts of terminals. To understand the mechanism of this failure, computational modeling is believed to be beneficial. The authors have developed a computational model based on cellular automata to simulate the contact failure. One of the difficulties in computational models is to evaluate breakage of oxidized particles on the surface. This model can calculate both the behavior of moving particles and chemical reaction simultaneously, which is difficult by the conventional FEM model. The contact interfaces are modeled as two-dimensional rectangular cells. A state that represents the material properties is assigned to each cell. The rules of generation, movement and chemical reactions along with fretting progress are provided. The program can simultaneously analyze oxidization progress in areas near the surface and the variation of the contact resistance. The dependence of contact force, sliding distance, and material variety are evaluated. The calculated electric contact resistance profiles show good agreement with experimental results, proving the feasibility of the model.
\end{abstract}

Keywords-component; fretting, electric contact resistance, cellular automaton, computational model

\section{INTRODUCTION}

The material frequently used for connector terminals is tin plated copper alloy. High mechanical strength and conductivity are required for the substrate material. Desirable properties for the plated layer is low contact resistance, corrosion resistance, and ease of soldering. The oxidized layer on tin surface usually prevents electrical conduction because tin has low activation energy. Since the oxidized layer is thin and fragile, it is automatically removed after insertion of connectors and good electrical contact is established.

With the recent increase in the number of pins in plug socket pairs, a significant amount of force is required to fit a plug in its socket. It is difficult to assemble connectors in narrow workspaces such as engine compartments. However, when the insertion force decreases, the probability of fretting corrosion increases $[1,2,3,4,5,6]$.
Simulation tools that evaluate the influence of design parameters under several conditions would be helpful for the development of fretting-free connections. It has revealed that the behavior of the oxidized particles produced at the interface of the plugs and sockets is important.

Examples of FEM analysis of surface exfoliation have been reported. In the case of reference [7], mechanical, electrical, and thermal coupled-field analysis is performed. However, it is difficult to simulate fretting phenomenon on a realistic scale with FEM due to complexity and high amount of calculation.

An approach that uses molecular dynamics has also been applied for this purpose. An atom or a molecule is considered as one particle. This method has been used to conduct detailed research on phenomena such as the focused movement of atoms under fatigue of materials or frictional force. However, a large calculation time is required in this case as well. The analysis model must be limited to a small domain.

On the other hand, a fatigue prediction method using experimental data that describes the relationship between interfacial pressure and fretting fatigue has been proposed. Although accuracy level is high in some cases, as concluded from the experimental data, it is not easily applicable to general cases without any measurements.

As mentioned above, for abrasion, methods for evaluating interface phenomena can be classified into two major categories. The first is based on evaluation by experimental empirical equation; the other is based on evaluation by simulation after modeling the observed phenomena in detail. Each method has its advantages and disadvantages. It is difficult to evaluate interface phenomena that occur with changing abrasion conditions within a limited calculation time.

In this paper, a technique of third category is presented. The authors consider the generation of particles at an interface, the state of oxidization, and so forth, and simulate a relatively large domain in a short time. The domain of the interface between a plug and a socket, which is set as the object of analysis, is represented in a cell arranged in the state of an Euler lattice (shape of the lattice is stable), and computations of oxidization, generation of particles, movement of particles, 
resistance, etc. are performed according to the theory of cellular automaton. Cellular automaton computes the state of a cell in the next time in the local domain. Thus, computation times are much shorter than for the finite element method, which solves a rigid matrix. Moreover, since each particle position is not intricately treated as in molecular dynamics, memory is saved, and a large space can be treated [8].

The authors compare the simulation results with actual observations and evaluate the contact electrical resistance characteristic of fretting.

\section{ALGORITHM}

To simulate fretting phenomenon the algorithm in this paper is performed as following flow.

1: Initialize the simulation and set parameters

2: Set domain shape

3: for $\mathrm{i}=1$ to slip cycle do

4: for $\mathrm{j}=1$ to cell number do

5: Compute oxidization

6: Compute stress

7: Compute uniting power

8: if target cell represents a separated particle

9: Compute destination point

10: Update the state of the cell

11: end if

12: end for

13: Compute resistance

14: Output results

15: end for

The main rules of the cellular automata are the following: Oxidization rule, Exfoliation rule (generation of particles), and Movement (of particles) rule. The various stages are described in the following paragraphs.

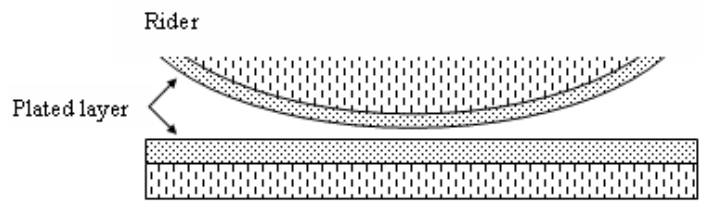

F1at

\section{A. Modeling of shape}

In No.2 of algorithm flow, the material interface is modeled. Although there are no restrictions as to the form of the material interface, the authors use the form of Fig. 1 as an example. The hemispherical object (the "rider") represents a socket terminal and the lower plate (the "flat") represents a plug terminal. The materials and properties that can be assigned concretely include the conductive substance and plated layer property of the material by the side of socket terminal, those of the material by the side of plug terminal, and air. For the initial model, only these material properties are set up; the states of the oxide, metal particles, and oxidization particles are treated as calculation progresses. Therefore, the shape of the model changes gradually by abrasion as vibration proceeds. Since both contact areas deform with contact pressure between the rider and flat, it is possible to set the deformed shape as forecasted before the analysis.

The model is divided into a lattice of cells and the neighborhood of the contact part of the socket terminal and the plug terminal made into analysis domains (Fig. 2). Material properties are assigned to each cell. Information on the allocated material is used at any time in considering the oxidization probability, bonding force, and so on. The cell size corresponds to the minimum particle size which generated by exfoliation. In setting the cell size, for the purposes of this simulation, it is not necessary to make the cell as small as an actual oxidization particle. For other purposes, such as investigating each moving state of a particle accurately, that may not be the case, and one would have to set a cell size in conjunction with computation time.

The material allocated to a cell changes as the calculation progresses due to oxidization, exfoliation, or movement. Thus, a cell that starts as a metal cell may change with oxidization to become an oxidized metal cell, or change with exfoliation (movement) to become an air cell. Similarly, when a particle forms, what was originally an air cell may change into a metal particle cell or an oxidized particle cell. Other types of change may occur when the rider moves to the flat side as the surface thins due to vibration.

One of the features of cellular automaton is that one can change the state of a cell quite easily. Thus a dynamic change such as fretting can be treated comparatively simply.

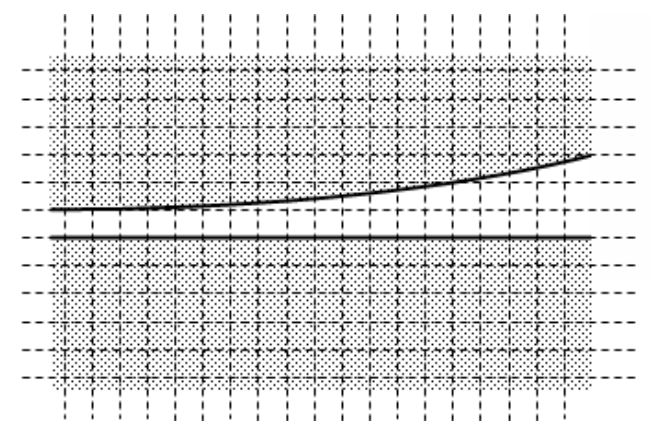

Figure 2. Mesh partition 


\section{B. Oxidization rule}

In No.5 of algorithm flow, the degree of oxidization of each cell is calculated. Each cell maintains a parameter called the oxidization ratio, which shows the progress of the oxidization. This ratio takes values from 0 (completely unoxidized) to 1 (completely oxidized). This ratio influences property values of the cell such as bonding force and volume resistivity. The increase in oxidization ratio of a cell per 1 cycle $(\mathrm{K})$ is computed by the following formulas:

$$
\begin{array}{r}
K=1-\prod\left(1-P_{i} \phi\right), \\
\phi=f(p) .
\end{array}
$$

$P_{i}$ indicates an oxidization probability of target cell which contact $\mathrm{i}$-th cell. $\mathrm{P}_{\mathrm{i}}$ is high if the surrounding cells are air, and low if they are metal (with a small oxidization ratio). The presence of air is assumed when a surrounding cell is a particle. Thus, $P_{i}$ is high if the surrounding cells are particle.

$\phi$ is a parameter for adjusting the oxidization probability. It is a function of contact pressure $p$. When contact pressure $p$ is large and adhesion between rider and flat is high, it becomes difficult for oxygen to reach the interface. When contact pressure is small, fretting appears notably. Oxygen advances easily and the particle changes readily to the oxidization particle with high specific resistance.

Using Fig. 3 as a concrete example, the increase in oxidization ratio $\mathrm{K}$ of the central cell is computed by the following expression:

$$
K=1-\left(1-P_{1}\right)^{3}\left(1-P_{2}\right)^{2}\left(1-P_{3}\right)^{3} .
$$

Here, the oxidization probability $\mathrm{P}_{\mathrm{i}}$ is set to $\mathrm{P}_{1}$ for the case in which the cell adjoins air, to $\mathrm{P}_{2}$ for the case in which it adjoins oxide, and to $\mathrm{P}_{3}$ for the case in which it adjoins metal. For simplicity, it is assumed that $\phi=1$.

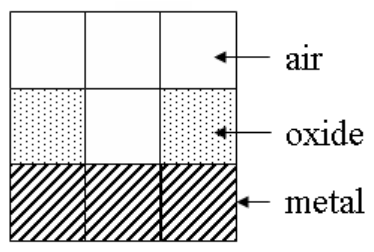

Figure 3. Example of composing cell surroundings

\section{Exfoliation rule}

In No.6 - 8 of algorithm flow, the exfoliation of each cell is determined. The researches for contact resistance have been reported $[9,10,11,12]$. In general, when the stress that is applied to the contact part of rider or flat surpasses the adhesive strength, exfoliation occurs. Here, the model computes the stress $\sigma$ applied to each cell and the adhesive strength of each cell (bonding force with the circumference) around the contact part, and determines whether the cell exfoliates.

The stress $\sigma$ that applies to each cell is calculated with No.6 of algorithm flow. Fig. 4 shows a closeup of the contact part; one lattice enclosed with a dotted line corresponds to the cell. First, one cell layer (Layer-1) corresponding to the contact side of the rider and the flat is considered as $\sigma=\mathrm{F} / \mathrm{S}_{1}$, where $\mathrm{F}$ is the contact force and $\mathrm{S}_{1}$ is the cross-sectional area of the contact surface (Layer-1). Next, the stress in the second layer (Layer-2) is requested from the flat side and the rider side with $\sigma=\mathrm{F} / \mathrm{S}_{2}$ where $S_{2}$ is the cross-sectional area of the second layer (Layer2 ). The cells in the second layer make it to the cells that are adjacent to the cell group in the first layer. Stress is calculated in the same way for all cells. The stress that applies to a cell near the surface is larger than that applied to cells farther from the surface $\left(\mathrm{S}_{\mathrm{i}}<\mathrm{S}_{\mathrm{i}+1}\right)$ and is easy to exfoliate. Moreover, the shape of the contact part changes because the position of rider and flat changes in one sliding. For this reason, the stress applying to a cell is computed in two or more states in one sliding. The stress finally used to determine whether exfoliation occurs is assumed to be a mean value of two or more stresses calculated in one sliding.

The adhesive strength $\kappa$ of each cell is calculated with No.7 of algorithm flow. The center cell in Fig. 3 is assumed to be $\kappa=$ $\Sigma \kappa_{\mathrm{i}}$, where $\kappa_{\mathrm{i}}$ is the bonding force corresponding to the material of the adjoining cell. The bonding force uses the shearing strength $\kappa_{\mathrm{a}}$ of each material. Eight cells exist in surrounding the cell of interest when the arrangement is a lattice as shown in Fig. 3, and it is assumed that $\kappa i=\kappa a / 8$. For an air cell, $\kappa i=0$. A surface cell has small adhesive strength and exfoliates easily compared with an inside cell. The bonding force of the particle is set to a relatively small value.

Therefore, the cumulative failure rate for the following Weibull statistics is used for determining exfoliation and for describing strength to the destruction of the material.

$$
L=1-\exp \left\{-\left(\frac{\sigma}{\kappa}\right)^{n}\right\}
$$

Here, $\mathrm{n}$ corresponds to ambiguity in the exfoliation probability. Ambiguity is large for small $\mathrm{n}$. The curve of probability density is sharp when $\mathrm{n}$ is large, and it is broad when $\mathrm{n}$ is small. L ranges from 0 to 1 , and the possibility of exfoliation is higher when $\mathrm{L}$ is large. 


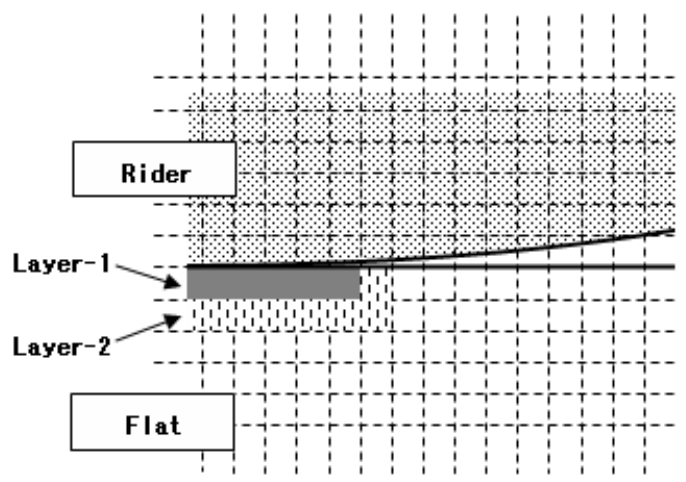

Figure 4. Diagram of stress calculation

\section{Movement rule}

In No.9 - 10 of algorithm flow, the movement of each cell is calculated. It is assumed that a particle is dragged by sliding and that it moves into an air cell within the sliding distance. And the air cell is adjacent to the surface of the rider or flat side.

The targets to move are metal, oxide, and particle cells. The move direction does not depend on the sliding direction in this setting. However, since the circumference has more air cells than does the contact part, the particles generated as the calculation progresses are deposited on the circumference.

\section{E. Calculation of electric contact resistance}

In No.13 of algorithm flow, the electric contact resistance is calculated. The resistance of tin in the plated layer and copper in the substance is small, whereas that of oxidized tin and exfoliating oxidization particles is large. Therefore the increase in resistance by fretting can be treated by evaluating the neighborhood of the interface. The interfacial geometry is divided into cells as shown in Fig. 2, and the volume resistivity of the material corresponding to each cell is set. A lumpedconstant network, connected vertically and horizontally, is built and the electric contact resistance between rider and flat is calculated.

\section{F. Output of result}

In No.14 of algorithm flow, the result is output. The state of each cell is output to screen or file. The generating situation of the particle and the deleted state of the plated layer are visualized. The value of the contact resistance for the previous and current sliding are presented.

\section{RESULTS}

Fretting corrosion in the interface was calculated by simulation and then compared with the results from experiment [2]. A hemisphere rider is pressed against a tabular flat as in Fig. 1, and they are made to slide. The radius of the rider is 1 $\mathrm{mm}$, and the thickness of the plating layer is $0.5 \mu \mathrm{m}$. Contact pressure is $3 \mathrm{~N}$, and sliding distance is $30 \mu \mathrm{m}$. Tin is used for the material of the plated layer, and copper-alloy is used for the material of the substrate. The molecular configuration of oxidized tin is assumed to be the mixture of $\mathrm{SnO}_{2}$ and $\mathrm{SnO}$. The volume resistivity is set between $1.9 \mathrm{E}-05 \Omega \mathrm{m}$ to $67 \Omega \mathrm{m}$. The mesh size is set to $50 \mathrm{~nm}$.

Fig. 5 shows how the electric contact resistance changes during sliding in this simulation. At stage A, resistance is small and rises gradually. At stage $\mathrm{B}$, resistance peaks, then decreases to stage C. Fig. 6 shows the experimental result. The first peak at around 100 cycles is a point of focus in this simulation. The behavior at this part is similar to that at the same part in Fig.5.

Fig. 7 shows the interface at each stage. The three crosssections correspond to the stages of Fig. 5 and are expansions of the contact part. In stage A, although particles are generated in the interface part, oxidization is not progressing for the main part and does not greatly influence the electric contact resistance. In stage B, many generated particles are in a state of advanced oxidization, and, as a result, the electric contact resistance rises. In stage $\mathrm{C}$, the particles are almost lost. Although the plated layer that exfoliates easily serves as a supply source of particles and the generated particles gradually move by sliding to the circumference of the contact region, in stage $C$, since the plated layer itself is lost, the amount of the particle in a contact with the interface keeps decreasing. In this state, since oxidized particles with large specific resistance hardly exist, the electric contact resistance decreases.

The authors used wavelength dispersive X-ray (WDX) spectroscopy to examine the distribution of elements on the surface of the contact point. The distribution of oxygen is shown in Fig. 8. To investigate the changes in resistance, two checking stages are set. Those are placed at A 52 cycles and B 90 cycles. Oxygen exists in stage A only sparsely at the point of contact, and in stage B all over the point of contact. This tendency corresponds to Fig. 7 very well.

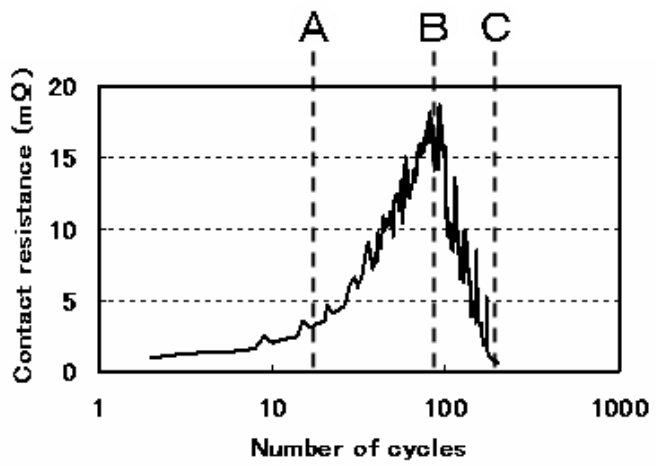

Figure 5. Contact resistance pattern ( calculated) 


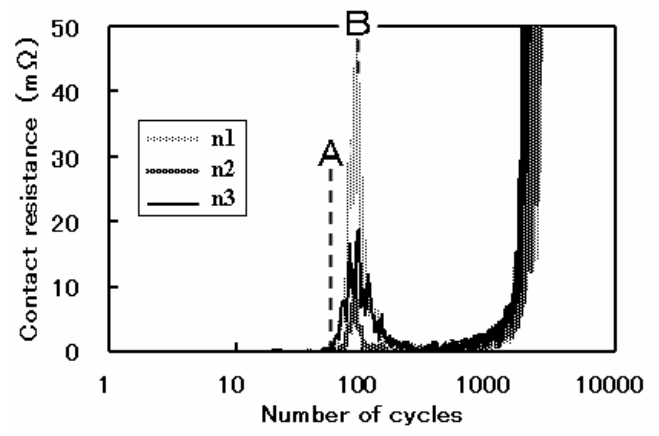

Figure 6. Contact resistance pattern ( obserbed)

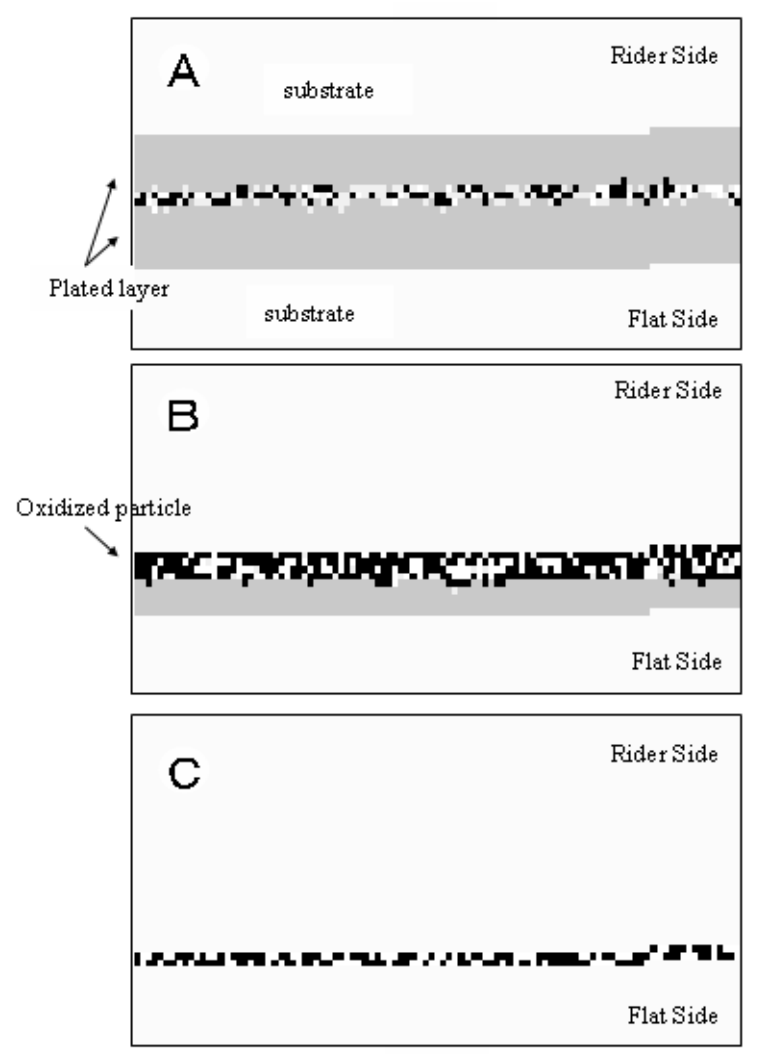

Figure 7. Transition of interfacial state by sliding
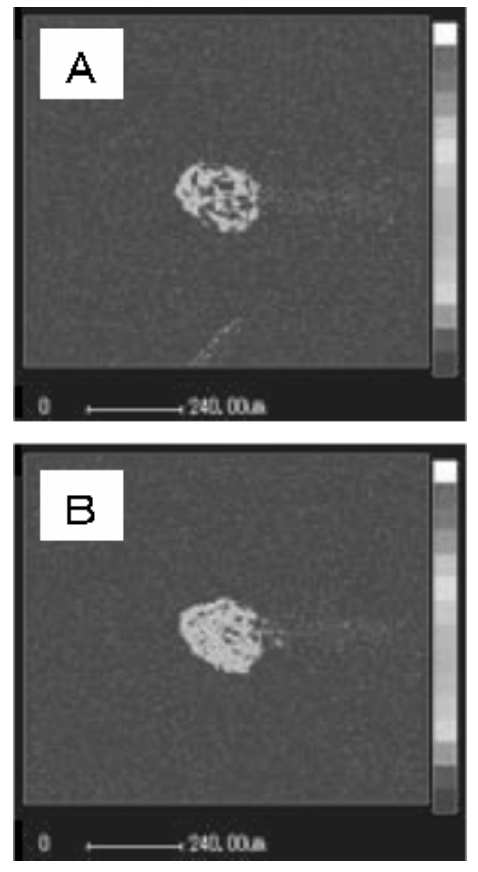

Figure 8. WDX mapping of flat side contact spot (1:After 50 cycles, 2 : After 92 cycles)

\section{CONCLUSIONS}

The behaviors of particles at an interface and changes in electric contact resistance accompanying fretting were simulated. Both simulations agree closely with observed values.

Although these simulations were specific to the problem of fretting, they can be applied to many abrasion phenomena. If the material of a plated layer or substrate changes due to a change in design requirements, one can evaluate the consequences of the change and determine the possibility of fretting. If the oscillating state changes due to changes in the surrounding composition, one can also determine the possibility of fretting. If the contact pressure on an interface decreases due to an increasing number of pins, one can evaluate whether problems might occur with the connector.

The authors will strive to improve the simulation's quantitative capability and to examine further applications to abrasion phenomenon in the future. 


\section{REFERENCES}

[1] T. Hammam: "Tin coating techniques for copper-base alloys the effects on friction, wear and electric properties", Proc. 43 IEEE Holm Conference on Electric Contacts, (1997), 201.

[2] K. Mitose: "Thickness Effect of Tin Layer on Initial Resistance Increase in Relatively Moving Contacts", Furukawa Electric Review, No.121, 2008

[3] C. E. Heaton and S. L. McCarthy: "High cycle fretting corrosion studies on tin-coated contact materials", Proc. 47 IEEE Holm Conference on Electric Contacts, (2001), 209.

[4] N. Aukland, C. Harrington and G. Drew: "A Preliminary Study on the Effect of Normal Force and Power on Fretted Surfaces", Proc. IICIT Connector and Interconnection Symposium and Tradeshow, p. 109, 2001

[5] G. T. Flowers, Fei Xie, M. Bozack and R. D. Malucci: "Vibration Thresholds for Fretting Corrosion in Electrical Connectors", Proceedings of the 48th IEEE Holm Conference on Electrical Contacts, p.133-139, 2002.

[6] G. T. Flowers, Fei Xie, M. Bozack, R. Horvath, B. I. Richett, and R. D. Malucci: "The Influence of Contact Interface Characteristics on Vibration-Induced Fretting Degradation", Proceedings of the 51th IEEE Holm Conference on Electrical Contacts, p.82-88, 2005

[7] A. Monnier, B. Froidurot, C. Jarrige, R. Meyer and P. Testé: “A mechanical, electrical, thermal coupled-field simulation of a sphereplane electrical contact", Proceedings of the Fifty First IEEE Holm Conference on Electrical Contacts, p.224-231, 2005.

[8] K. G. F. Janssens: "Computational Materials Engineering", ELSEVIER, 2001

[9] C. Maul and J. W. McBride: "A Model to Describe Intermittency Phenomena in Electrical Connectors", Proceedings of the 48th IEEE Holm Conference on Electrical Contacts, p.165-174, 2002.

[10] A. E. Manfalouti, N. Bejemaa, R. E. Abdi and T. Reiss: "Experimental and Theoretical Investigations on Connector Insertion Phase", Proceedings of the 49 IEEE Holm Conference on Electrical Contacts, p.17-22, 2003.

[11] Wen-hua Li, Guo-jin Liu and Zhi-gang Li: "Study and Reliability Analysis on Testing Instrument for Dynamic Contact Resistance on Contact", Proceedings of the 46th IEEE Holm Conference on Electrical Contacts, p.109-114, 2000.

[12] Kui Li, Fang Yao, Jianguo Lu and Zhigang Li: "Test and Analysis of Reliability for Electromagnetic Relay", Proceedings of the 46th IEEE Holm Conference on Electrical Contacts, p.79-82, 2000. 


\section{Fretting Analysis of Connector Terminals}

\author{
Keiji Mashimo and Kyota Susai* \\ R\&D department \\ The Furukawa Electric co., ltd. \\ Yokohama and *Nikko, Japan
}

\author{
Yasuyuki Ishimaru \\ ES3 Department \\ FITEC Corporation \\ Tokyo, Japan
}

\begin{abstract}
As the number of circuits used in automobiles increases, larger force is required to mate connectors. The larger insertion force necessitates the use of an assembling robot, otherwise increasing the cost due to complicated structure of connectors. In order to reduce the cost, connectors with low insertion force are required. This may cause an increase in the fretting failure in electric contacts of terminals. The authors have developed a computational model based on cellular automata to simulate the contact failure. This model can calculate both the behavior of moving particles and chemical reactions simultaneously, which is difficult by the conventional FEM model. In experiments, the fretting probability depends on the contact pressure, slip distance, and surface conditions. The dependence of electric contact resistance on the slip cycle is calculated by this method. When the contact pressure is very high or the slip distance is very small, fretting is absent. The effects of contact materials and the thickness of plated metals are evaluated. The calculated electric contact resistance profiles of the electric contacts agree well with the experimental results.
\end{abstract}

Keywords- fretting; connector; terminal; analysis; tin plating; contact resistance; cellular automaton

\section{INTRODUCTION}

In the automobile field, the need for low insertion force connectors has increased with the number of connecting pins. This increase is due to a trend toward an increasing number of electrical parts in the vehicle. Recent vehicles have many more functions driven with electric devices than before. For example, traction control systems, numerous types of sensors, and engine and transmission control systems are used in recent cars. The volume of circuits and wire harnesses increased five and four times, respectively, from 1980 to 1990. Typical connector terminals are made of a tin-plated copper alloy. The tin-plated layer is popular because it offers both low cost and low contact resistance. However, when there is a slip between the tin-plated plugs and sockets, fretting corrosion can occur $[1,2]$. The relationship between insertion force and fretting probability shows a trade-off. The fretting problem is not unique to the automobile field; it may exist in many fields [3]. Connectors for computer systems can also have fretting problems. Under repeated thermal stress, a slip between the plug and socket may appear even if vibration is absent. The most effective countermeasure for the fretting problem is creating designs with reduced slip distance while maintaining low insertion force. This means selecting the right combination of materials and connector structure.

To design connectors, computer simulation is generally useful; however, the fretting phenomena are too complex to simulate by conventional methods. It is possible to calculate stress distribution in connector pins by the finite element method (FEM), even if it is an integrated analysis of mechanical, thermal, and electrical coupled fields $[3,4]$. It may be difficult to calculate the transient profiles of electrical contact resistance at the contact leads using FEM. FEM is useful for limited parts of this problem. However, for simulating the whole phenomenon, one must simultaneously consider at least stress distribution, yield criteria, particle separation, particle transportation, oxidization, and temperature dependency, which is not easy for conventional methods. Therefore, the authors decided to construct a new modeling procedure.

In this paper, the authors propose an original modeling procedure based on cellular automata (CA) for fretting analysis of connector terminals. The transient profiles of contact resistance with several contact forces were investigated in experiments, and the results are compared with the analytical results.

\section{Methodology}

\section{A. Test Pieces}

Riders with a hemispherical convex portion and flats with a plain shape were prepared. Both were made of $\mathrm{Cu}-\mathrm{Ni}-\mathrm{Si}$ alloy. Table I shows the chemical composition of the substrate metal.

Tin was electroplated on the surface of copper alloy strips. The thickness of the tin layer was adjusted by changing the plating time. Ni undercoating was applied as a diffusion barrier, which minimizes copper-tin intermetallic growth during heat exposure over time, although the authors did not evaluate the aging effects in this study. The thickness of the tin layer comprising pure tin and tin in a copper-tin intermetallic compound was measured by a fluorescent $\mathrm{X}$-ray coating gauge. The Ni undercoating was evaluated in the same manner. A schematic view of the cross-sectional plating structure used for riders and flats is shown in Fig. 1. Table II shows the measured plating thickness of the test pieces.

TABLE I. Chemical Composition of Substrate Metal

\begin{tabular}{|c|c|c|c|c|c|}
\hline $\mathbf{N i}$ & $\mathbf{S i}$ & $\mathbf{Z n}$ & $\mathbf{S n}$ & $\mathbf{M g}$ & $\mathbf{C u}$ \\
\hline $2.0-2.8$ & $0.45-0.6$ & $0.4-0.55$ & $0.1-0.25$ & $0.05-0.2$ & bal. \\
\hline
\end{tabular}




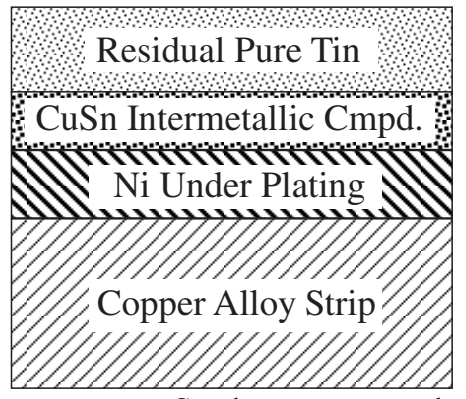

Cmpd means compound.

Figure 1. Schematic cross sectional view.

TABle II. Measured Plating Thickness of Test Pieces

\begin{tabular}{|c|c|c|}
\hline \multirow{2}{*}{ Flat } & Sn & Ni \\
\cline { 2 - 3 } & 0.25 & 0.6 \\
\hline \multirow{2}{*}{ Rider } & 0.5 & 0.6 \\
\hline
\end{tabular}

A rider with a hemispherical protrusion was created after cutting a 0.25 -mm-thick strip into a $10-\mathrm{mm}$-wide and $30-\mathrm{mm}$ long piece. The curvature radius of the dome was $1.0 \mathrm{~mm}$. A flat was prepared by cutting a strip into a $20-\mathrm{mm}$-wide and 50 mm-long piece.

The influence of the protrusion on the tin layer was examined. The plating thickness of a $50-\mu \mathrm{m}$ square at both the dome top and in an unprotruded region of the rider was measured by Auger electron spectroscopy with argon ion sputtering assist. This size is considered to be wide enough to measure the average thickness, even though the piece has uneven plating structure or varied plating crystal grains. Both the rider and flat were degreased by immersion in acetone in a beaker for 10 min under ultrasonic agitation.

\section{B. Experimental Procedure}

Fig. 2 shows a schematic diagram of this experiment. The rider was installed in a jig kept inside a constant temperature and humidity chamber in such a manner that the protruding section of the rider faced the flat side.

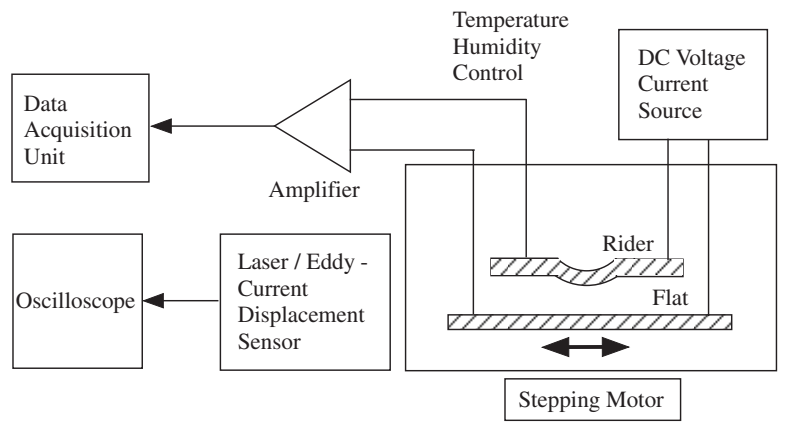

Figure 2. Schimatic diagram of fretting experiment setup.
The rider moved relative to the flat at a set distance with the help of a stepping motor. Here, the micro motion was set equal to a linear movement of $30 \mu \mathrm{m}$. Meanwhile, the relative displacement was measured using an eddy current displacement meter (NCDT3300 with an EC2 sensor, Micro Epsilon Corporation) or a laser displacement meter (LK-035, Keyence Corporation) and always monitored using an oscilloscope during the experiment. The movement frequency was about $3.3 \mathrm{~Hz}$.

Using a power supply, a constant current of $5 \mathrm{~mA}$ was supplied to the electric circuit. The rider was set to positive polarity and the flat to negative polarity. The open-circuit voltage was $20 \mathrm{mV}$, and the output current response of the power supply was set to the FAST mode; the current reached $\pm 0.05 \%$ of the set value within $3 \mathrm{~ms}$.

Contact resistance was calculated from the measured values of voltage drop using a four-terminal DC measurement technique. The potential difference was measured continuously during the test piece movement. The small potential was amplified using an amplifier and recorded with a data logger (NR250, Keyence Corporation). The amplifier had a cut-off frequency of $f_{c}=1 \mathrm{kHz}$, where the gain was less than $3 \mathrm{~dB}$. The data logger sampled four times per second, for $4 \mathrm{~ms}$ each time, and the average of those four values was recorded as the voltage drop per second.

The number of sliding movements was initially set to 5000 . The testing was terminated when the resistance had increased by $100 \mathrm{~m} \Omega$ from its initial value, and both the flat and rider were replaced for the next trial.

The test was carried out in a constant temperature and humidity chamber maintained at $20^{\circ} \mathrm{C}$ and $65 \%$ relative humidity. A set of three replications was conducted for each experimental condition.

\section{Modeling for Computer Simulation}

A computational model based on cellular automata (CA) was constructed. In typical CA, an Euler type lattice (cell) is used [8]. That means n-dimensional space is partitioned into a discrete subset of finite n-dimensional volumes. In this study, an Euler type lattice was used as well. In this paper, the case for two-dimensional cells is reported. A schematic image of the cells is shown in Fig. 3.

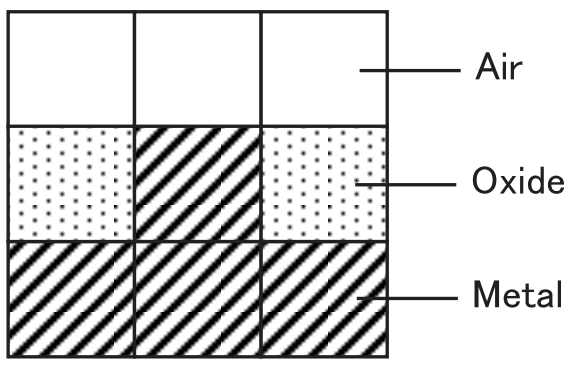

Figure 3. Schematic image of the cells 
A state that includes the material type, such as bulk or particle; the material phase, such as gas, liquid or solid; and the kind of material, such as copper, tin, or air, is assigned to each cell. The assigned state should not be stable if one wants to simulate alternating phenomena such as contact resistance values related to slip cycles.

The computer program for this analysis uses the following algorithm:

1: Set the domain shape

2: Set the plating thickness

3: Set the slip cycle

4: for $\mathrm{i}=1$ to slip cycle do

4: $\quad$ for $\mathrm{i}=1$ to $\mathrm{n}$ (cell number) do

5: Compute oxidization

6: Compute exfoliation

6: if target cell represents a separated particle

7: $\quad$ Compute destination point

8: $\quad$ end if

9: $\quad$ Copy new states of the cell (number i)

10: end for

\section{1: Compute resistance}

12: end for

The scope, relative to the target cell, can be set as desired. The surrounding eight cells are considered in this case. The following rules are applied for a target cell whose state is determined from the states of the surrounding eight cells.

1) Oxidization rule

Equation (1) and (2) describe the degree of oxidization K.

$$
K=1-\Pi\left(1-P_{i} \phi\right)
$$

$$
\phi=f(p)
$$

$\mathrm{P}_{\mathrm{i}}$ indicates the oxidization probability of a target cell which contacts the i-th cell. $\phi$ is a factor which is a function of the contact pressure $\mathrm{p}$.

\section{2) Exfoliation rule}

In general, exfoliation occurs when the stress applied to the contact part of the rider or flat surpasses the adhesive strength. Here, the model computes the stress $\sigma$ applied to each cell and the adhesive strength of each cell (bonding force with the circumference) around the contact part, and determines whether the cell exfoliate.

Fig. 4 shows a magnified image of the contact part. One lattice enclosed with a dotted line corresponds to a cell. One cell layer (Layer-1) corresponds to the contact side of the rider, and the flat is considered as $\sigma=\mathrm{F} / \mathrm{S}_{1}$, where $\mathrm{F}$ is the contact force and $S_{1}$ is the cross-sectional area of the contact surface (Layer-1). Next, the stress in the second layer (Layer-2) is requested from the flat and rider sides with $\sigma=F / S_{2}$, where $S_{2}$ is the cross-sectional area of the second layer (Layer-2). The cells in the second layer move to the cells that are adjacent to the cell group in the first layer. Stress is calculated in the same way for all cells. The stress applied to a cell near the surface is larger than that applied to cells farther from the surface $\left(\mathrm{S}_{\mathrm{i}}<\right.$ $\mathrm{S}_{\mathrm{i}+1}$ ), and therefore, the cells near the surface flake off easily. Moreover, the shape of the contact part changes because the positions of the rider and flat change during one slide. For this reason, the stress applied to a cell is computed in two or more states in one slide. The stress finally used to determine whether flaking occurs is assumed to be the mean value of two or more stresses calculated in one slide.

The center cell in Fig. 3 is assumed to be $\kappa=\Sigma \kappa_{i}$, where $\kappa_{i}$ is the bonding force corresponding to the material of an adjoining cell. The bonding force uses the shearing strength $\kappa_{a}$ for each material. Eight cells surround the cell of interest when the arrangement is a lattice, as shown in Fig. 3, and it is assumed that $\kappa_{\mathrm{i}}=\kappa_{\mathrm{a}} / 8$. For an air cell, $\kappa_{\mathrm{i}}=0$. A surface cell has small adhesive strength and flakes off easily compared with an inside cell. The bonding force of the powder is set to a relatively small value.

A cumulative failure rate following the Weibull statistics is used for determining exfoliation and for describing strength against the destruction of the material.

$$
L=1-\exp \left\{-\left(\frac{\sigma}{\kappa}\right)^{n}\right\}
$$

Here, $\mathrm{n}$ corresponds to ambiguity in the exfoliation probability. The ambiguity is large for small $\mathrm{n}$. The curve of probability density is sharp when $\mathrm{n}$ is large, and it is broad when $\mathrm{n}$ is small. $\mathrm{L}$ ranges from 0 to 1 , and the possibility of exfoliation is higher for large L.

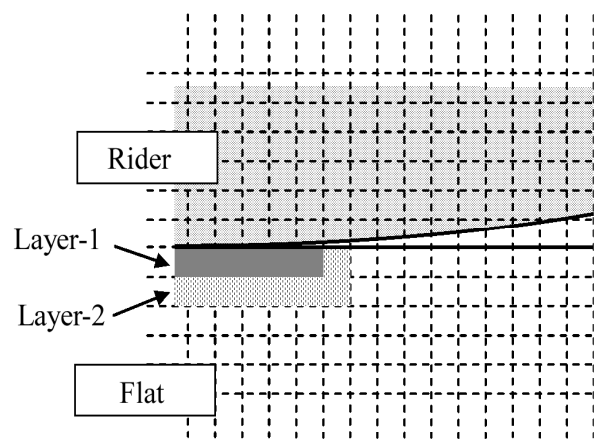

Figure 4. Magnified image of the contact part 


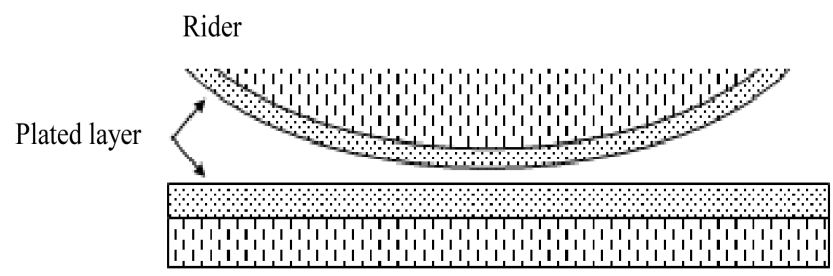

Flat

Figure 5. Computational domain

\section{3) Transportation rule}

The drive force of transportation is sliding move $[9,15]$. A virtual particle is dragged by sliding. Destination points for virtual particles are determined by the Monte Carlo method, which is based on probability theory. The destinations are selected from cells that have air as material property and are adjacent to the surface of the rider or flat side.

The movement probability for each cell is not uniform, and is small for large movement distances. The movement destination is decided by the abovementioned probability and random numbers.

The targets to move are metal, oxide, and particle cells. The move direction does not depend on the sliding direction in this setting. However, since the circumference has more air cells than does the contact part, the particles generated as the calculation progresses are deposited on the circumference.

\section{RESULTS AND DISCUSSION}
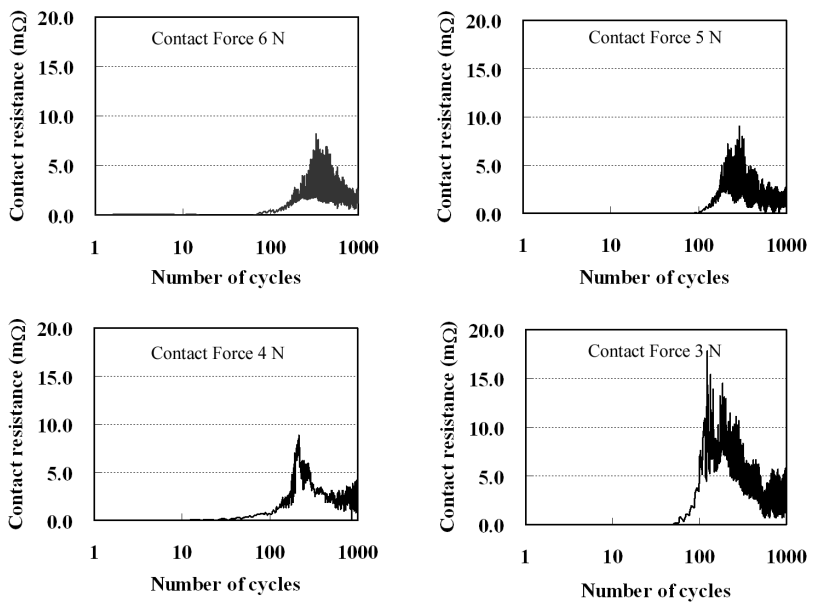

Figure 6. Resistance profiles in experiments

\section{A. Results from experiments}

Resistance profiles versus several contact forces are shown in Fig. 6. The range of contact force is 3 to $6 \mathrm{~N}$. The curve in Fig. 6 shows relative value while initial value is zero. The results indicate that fretting corrosion is absent when the contact force is large. When fretting corrosion exists, there is one peak of the resistance curve at about 100 cycles. In a typical case, the resistance decreases once during the fretting test and increases again at about several thousand cycles $[6,7]$.

\section{B. Comparison between Analysis and Experiment}

A computational domain is shown in Fig. 5. The size of cells is determined according to the size of oxidized particles. It is not necessary that the cell be smaller than the oxidized particles. The particles smaller than the cell size can be described as follows: If the total volume of four particles is the same as that of one cell, and one of the four is oxidized, then the target cell representing the four particles as a whole has a oxidization state of $25 \%$. It is evident that cells that are too large may cause a problem with the accuracy of the analysis. At the present stage, the strict conditions have not been found for determining cell size. As a result, when the cell size is on the same order as particle size, the analysis describes the experiments well. The sides of cells are set to $50 \mathrm{~nm}$ length in this study.

As shown in Fig. 7, the computational resistance profile agrees well with experimental values. The pattern of the curve is similar when the absolute value of resistance is calibrated by experimental data. The authors assumed that the oxide is a mixture of $\mathrm{SnO}$ and $\mathrm{SnO}_{2}$; however, the ratio of $\mathrm{SnO}$ and $\mathrm{SnO}_{2}$ is unknown and difficult to measure with high accuracy. Improvements are needed in this regard. In this paper, the resistivity of the oxide is taken to be intermediate of $\mathrm{SnO}$ and $\mathrm{SnO}_{2}$. Table III indicates the volume resistivity of materials in this paper.
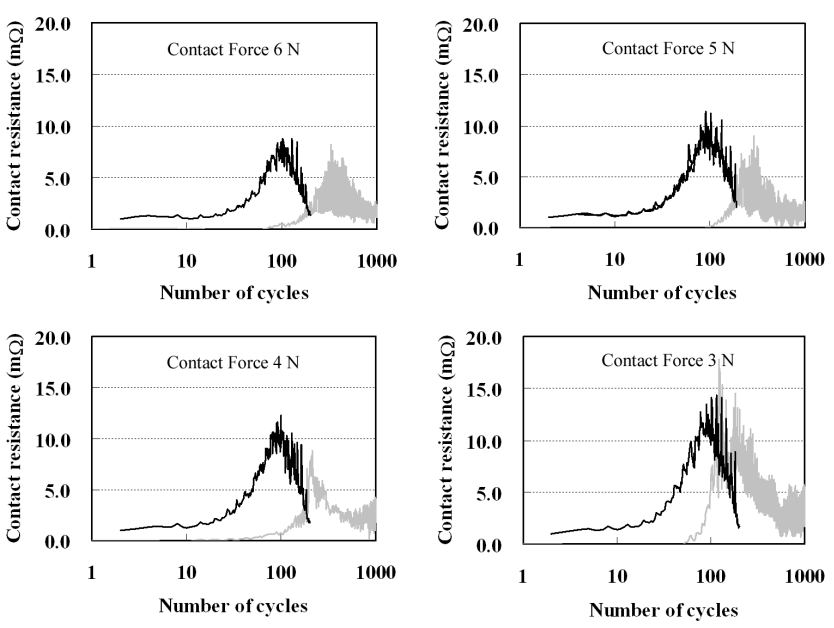

Figure 7. Result from CA analysis 

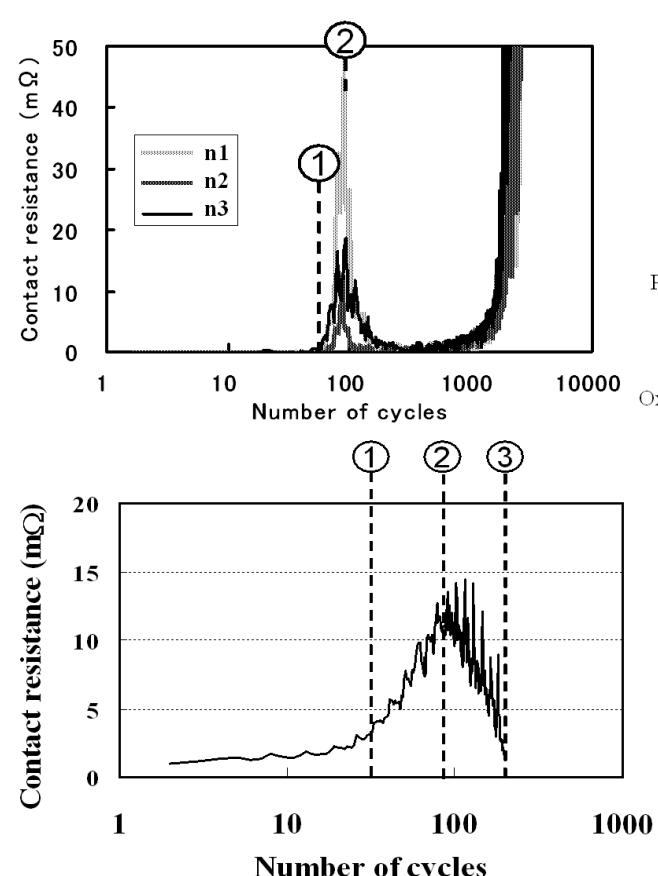

(a) Resistance profile $(\mathrm{Sn} 0.5 \mu \mathrm{m})$ Contact force: $3 \mathrm{~N}$

Sliding distance: $30 \mu \mathrm{m}$

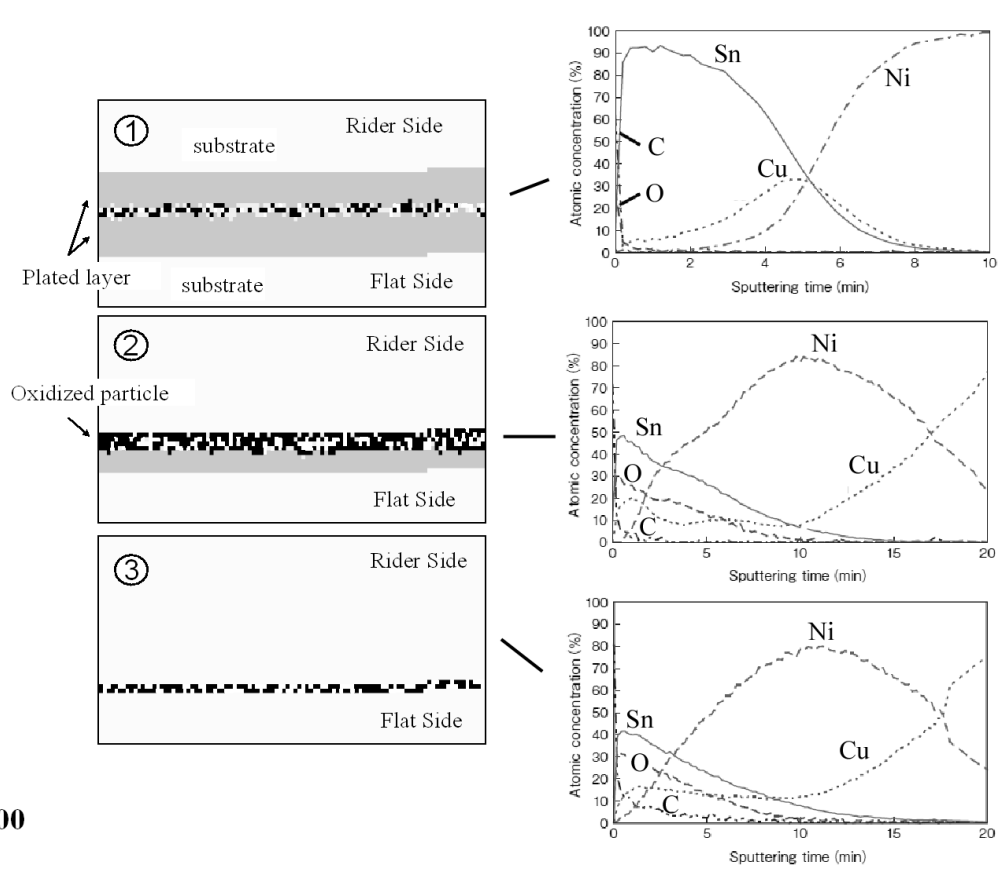

(b) Computed aspect of debris

Figure 8. Sn consumption, piling and a sweeping of debris

(c) Sn consumption (by AES)

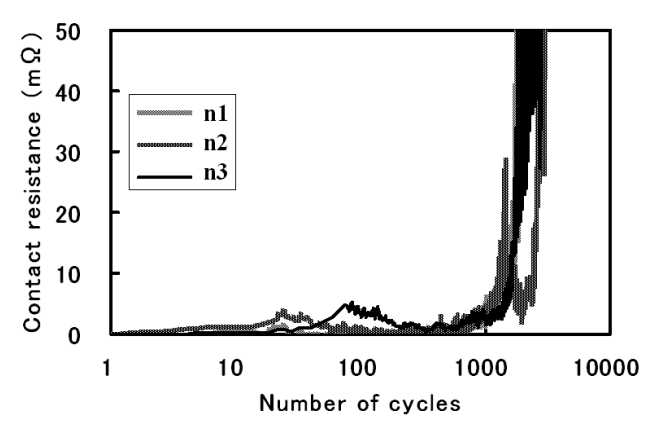

Figure 9. Measured resistance profile (Sn $0.25 \mu \mathrm{m})$

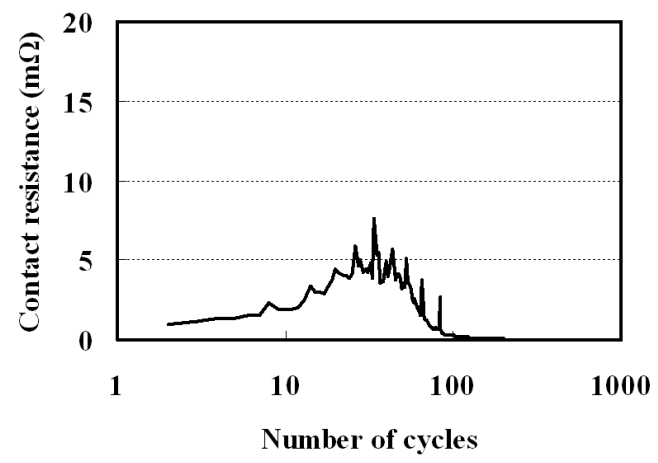

Figure 10. Computed resistance profile (Sn $0.25 \mu \mathrm{m})$
TABLE III. ELECTRICAL RESISTIVITY

\begin{tabular}{|c|c|}
\hline $\mathrm{Cu}$ & $1.55 \times 10^{-8}$ \\
\hline $\mathrm{Sn}$ & $1.15 \times 10^{-7}$ \\
\hline $\mathrm{SnO}_{2}$ & $1.9 \times 10^{-5}$ \\
\hline $\mathrm{SnO}$ & 67 \\
\hline
\end{tabular}

Unit: $\Omega \mathrm{m}$

Figure 11. Computed resistance profile (hard material) 


\section{ACKNOWLEDGMENT}

The mechanism of resistance rises and drops at around 100 cycles has been discussed by many researchers $[5,6,7,10,11,12,13,14]$. Unfortunately, the mechanism is not yet resolved adequately. Most researchers believe that the resistance changes are caused by piled debris. The volume of debris is the result of piling and sweeping, both of which occur simultaneously. If piling is greater than sweeping, then debris increases; otherwise, it decreases. The computational result of the alternating aspect of the debris in an interface between the rider and flat is shown in Fig. 8 (b). The numbers on the resistance curve in Fig. 8 (a) correspond to the numbers on the cross-sectional view of the interface. The upper side graph indicates experimental result while lower side graph was the result from computation in Fig. 8 (a). At stage 1, the resistance starts to increase and the debris volume also increases. Both the debris volume and resistance reach a maximum value at stage 2. At stage 3, both the debris volume and resistance decrease. In experiments, debris in the interface decreases once because of consumption of the Sn layer. In this case, resistance increases again as the layer of $\mathrm{Cu}$-alloy starts to be attacked. In corresponding experiments, Auger electron spectroscopy was used to investigate the consumption of the plated layer and its oxidization behavior, as shown in Fig. 8 (c).

As another example, the same calculation and experiment were done for a thinner plated layer $(0.25 \mu \mathrm{m})$. Fig. 10 shows how the electric contact resistance changes during sliding. The increase in electric contact resistance is small compared with that for the thicker layer (Fig. 8). The thinner layer supplies fewer particles, and moves quickly to stage 3. The experimental results are shown in Fig. 9. The initial increase is small, in agreement with the calculation.

These first two examples use material that exfoliates easily. In contrast, the case of hard material is shown in Fig. 11. The change in electric contact resistance is close to the experimental result for a thin plated layer (Fig. 10). However, the phenomenon at the interface is different. When a plated layer does not exfoliate easily, few particles are generated from the layer and many are executed to the surroundings by sliding. Therefore, particles do not accumulate significantly on the contact interface, and electric contact resistance does not rise.

\section{CONCLUSIONS}

An analytical model for fretting analysis was proposed. Experimental and computational analysis results were compared. The calculated electrical contact resistance profiles agreed well with the experimental results. The analysis can predict the slip cycle number where the first resistance peaks appear. The accuracy of the absolute value of resistance was insufficient. Further research is required into the computation of absolute resistance and the prediction of slip cycle numbers at which the second peaks appear.
The authors wish to thank Dr. Wang Anlin at Tongji University for excellent suggestions and fruitful discussions. They also thank Ms. Fu Yingchao for improving the algorithms.

\section{REFERENCES}

[1] G. T. Flowers, Fei Xie, M. Bozack, R. D. Malucci and B. I. Rickett, "Modeling early stage fretting of electrical connectors subjected random vibration," Proceedings of the Forty Ninth IEEE Holm Conference on Electrical Contacts, p.45-50, 2003.

[2] M. D. Bryant, "Resistance buildup in electrical connectors due to fretting corrosion of rough surfaces," Proceedings of the Thirty Ninth IEEE Holm Conference on Electrical Contacts, p.178-190, 1993.

[3] T. Hattori, M. Nakamura, H. Sakata and T. Watanabe, "Fretting fatigue analysis using fracture mechanics," JSME International Journal,vol. 31, No. 1, p100-107, 1988.

[4] A. Monnier, B. Froidurot, C. Jarrige, R. Meyer and P. Testé, "A mechanical, electrical, thermal coupled-field simulation of a sphereplane electrical contact", Proceedings of the Fifty First IEEE Holm Conference on Electrical Contacts, p.224-231, 2005.

[5] C. E. Heaton and S. I. McCrethy, "High cycle fretting corrosion studies on Tin-coated contact materials," Proceedings of the Forty Seventh IEEE Holm Conference on Electrical Contacts, p.209-214, 2001.

[6] N. Aukland, C Harrington and G. Drew, "A preliminary study on the effect of normal force and power on fretted surfaces," Proceedings of the IICIT Connector and Interconnection Symposium and Tradeshow, p.109-114, 2001

[7] T. Ito, M. Matsushima, K. Takata and Y. Hattori, "Factors influencing the fretting corrosion of tin plated contacts", Proceedings of the 23rd International Conference on Electrical Contacts (ICEC), p.76-80, 2006.

[8] K. G. F. Janssens, Computational Materials Engineering, Elsevier, 2001.

[9] L. Lam, J. W. McBride, C. Maul and J. K. Atkinson, "Displacement Measurements at the Connector Interface Employing a Novel Thick Sensor," Proceedings of the Fifty First IEEE Holm Conference on Electrical Contacts, p.89-96, 2005.

[10] G. T. Flowers, Fei Xie, M. Bozack, Xin Hai, B. I. Rickett and R. D. Malucci, "A study of the physical characteristics of vibration-induced fretting corrosion," Proceedings of the Fiftieth IEEE Holm Conference on Electrical Contacts and the Twenty Second International Conference on Electrical Contacts, p.312-319, 2004.

[11] G. T. Flowers, Fei Xie, M. Bozack, R. Horvath, B. I. Rickett and R. D. Malucci, "The influence of Contact Interface Characteristics on Vibration-Induced Fretting Degradation," Proceedings of the Fifty First IEEE Holm Conference on Electrical Contacts, p.82-88, 2005.

[12] T. Hammam, Å. K. Rudolphi and P. Lundström, "Vibration-Induced Deterioration of Tin-Coated Connectors Studied by Using a Force Controled Fretting Bench-Test," Proceedings of the Fifty First IEEE Holm Conference on Electrical Contacts, p.97-106, 2005.

[13] S. S. Babu, M. L. Santella, Z. Feng, B. W. Riemer and J. W. Cohron, "Empirical Model of Effects of Pressure and Temperature on Electrical Contact Resistance of Metals," Science and Technology of Welding and Joining, 2001,6(3), p.126-132.

[14] L. Tristani, E. M. Zindine,L. Boyer and G. Klimek, "Mechanical Modeling of Fretting Cycles of Electrical Contacts," Proceedings of the Forty Fourth IEEE Holm Conference on Electrical Contacts, p.44-52, 1998.

[15] G. T. Flowers, Fei Xie, M. Bozack and R. D. Malucci, "Vibration Thresholds for Fretting Corrosion in Electrical Connectors," Proceedings of the Forty Eighth IEEE Holm Conference on Electrical Contacts, p.133-139, 2002. 


\section{Study of Thin Underlayers to Hinder Contact Resistance Increase Due to Intermetallic Compound Formation}

S. Noël, D. Alamarguy, S. Correia

Laboratoire de Génie Electrique de Paris, Supélec, UMR

CNRS 8507, Plateau de Moulon, Gif sur Yvette, France

\author{
P. Gendre \\ PEM, Siaugues-Saint-Romain Siaugues-Sainte-Marie, France
}

\begin{abstract}
Many connectors are made of cuprous substrates with electroplated final surfaces. When failure of the devices occurs, fretting and intermetallic compound formation are very often involved. This paper describes the influence of different types of underlayers on tin intermetallic compound formation. The physical and chemical properties of the intermetallic compounds are described: various techniques are used to characterise their topography, structure and composition. These include: AFM, XPS, SEM, EDX. Glow discharge optical emission spectroscopy (GD-OES) is also used for quantitative depth profiling of the samples. A stripping method is used in order to evaluate the tribological and electrical properties of the intermetallic layers, independently of the tin particle formation occurring with tin layers. The analysis of the results is based on the various values of size and morphology of the grains. Very particular features are observed in the case of thin underlayers of cobalt, as the diffusion of copper into the tin layer seems to be minimised. The electrical and tribological properties of ball on plane contacts of tinned cuprous substrates with such underlayers are observed to be fairly stable even after periods of thermal heating.
\end{abstract}

Keywords-components; tin intermetallic compound, diffusion, fretting, contact resistance.

\section{INTRODUCTION}

The use of tin as a protecting coating for copper separable contacts is wide-spread in many electronic applications [1]. However, tin can form intermetallic phases with several metals such as $\mathrm{Ni}, \mathrm{Cu}, \mathrm{Co}, \mathrm{Au}$ and $\mathrm{Ag}$. Failure of aged tin electrical contacts has been attributed to this phenomenon in the numerous studies dedicated to electrical contact properties [2-4]. One of the major problems in choosing the thickness of tin coatings is to evaluate the best compromise between the initial thickness of the tin layer and the remaining one after intermetallic growth. Tin and copper are known to grow intermetallic compounds of $\mathrm{Cu}_{6} \mathrm{Sn}_{5}$ or $\mathrm{Cu}_{3} \mathrm{Sn}$ depending upon the temperature. Various underlayers have been deposited in this work, either in thick films of several microns or in thin films. Their compositions after ageing were compared to the composition of tin directly deposited on copper. The diffusion of the various species was measured. A technique involving dissolution of the free tin was used in order to analyze the surface of the intermetallic compounds. The microstructures of the various intermetallic compounds are also described.

The influence of different underlayers, such as nickel and cobalt, on the intermetallic formation was investigated. Cobalt interdiffusion with copper has been studied by some authors [5]. This study describes the mechanical and electrical properties of four different types of tin intermetallic layers in order to elaborate an optimized coating for low level electrical contacts. The influence of the underlayer thickness on the structure and composition of the intermetallic compounds was analyzed.

\section{SAMPLES AND EXPERIMENTAL SET-UPS}

A. Samples

a) Substrates

Samples were brass $(\mathrm{CuZn} 30)$ coupons with a $2 \mu \mathrm{m}$ layer of tin electrodeposited ( $1 \mu \mathrm{m}$ for the GD-OES profiles) from a matte tin bath. The arithmetic roughness substrate was $0.2 \mu \mathrm{m}$. Tin baths were based on MSA (methane sulfonic acid) chemistry. Underlayers of matte nickel or cobalt were deposited on some of the coupons with different thicknesses. Table 1 summarises the various samples of the study. Note that fretting experiments were performed immediately after the plating, thus avoiding the growth of an intermetallic layer.

\section{b) Ageing}

All the coatings were submitted to a heat treatment of $155{ }^{\circ} \mathrm{C}$ for $16 \mathrm{~h}$ in order to study the intermetallic formation.

TABLE 1: REFERENCE AND DESCRIPTION OF THE COATINGS DEPOSITED ON THE BRASS SUBSTRATES

\begin{tabular}{|c|c|c|c|c|}
\hline Sample & S 1 & S 2 & S 3 & S 4 \\
\hline Plating & \multicolumn{4}{|c|}{ Matte tin 2 $\mu \mathrm{m}$} \\
\hline $\begin{array}{c}\text { Underlayer } \\
(\mu \mathrm{m})\end{array}$ & None & Ni 2 & Ni 0.1 & Co 0.1 \\
\hline Substrate & \multicolumn{4}{|c|}{ Brass (CuZn30) } \\
\hline
\end{tabular}

After the thermal ageing, the remaining free tin was chemically removed in a stripping solution to reveal the 
intermetallic compound layers [6]. Different stripping solutions were used, depending on the underlayers [7]. The solution used for the copper underlayer was composed of sodium m-nitrobenzenesulfonate $(150 \mathrm{~g} / \mathrm{L})$ and fluoboric acid $(200 \mathrm{~g} / \mathrm{L})$. For nickel and cobalt underlayers, the solution was composed of sodium m-nitrobenzenesulfonate $(35 \mathrm{~g} / \mathrm{L})$ and sodium hydroxide $(50 \mathrm{~g} / \mathrm{L})$. In this case, the solution was heated at $70^{\circ} \mathrm{C}$. The different coatings were dipped into the stripping solution for a few seconds. Each solution was specially formulated in order to strip free tin without etching the intermetallic. Table 2 summarises the different samples after stripping.

TABLE 2: REFERENCE OF THE SAMPLES AFTER STRIPING OF THE FREE LAYER

\begin{tabular}{|c|c|c|c|c|}
\hline Samples & S 1 & S 2 & S 3 & S 4 \\
\hline $\begin{array}{c}\text { Label after } \\
\text { ageing and } \\
\text { stripping }\end{array}$ & IMC 1 & IMC 2 & IMC 3 & IMC 4 \\
\hline
\end{tabular}

For each type of coating, spherical shapes $(1.3 \mathrm{~mm}$ of radius) were stamped after the electrodeposition in order to study the fretting behaviour of sphere on plane contacts. The sphere and the plane undergoing a fretting test were always made out of the same coatings.

\section{B. Experimental set-ups}

a) Fretting test

A dedicated device comprising an electro-dynamic shaker [8] applied controlled cyclic movements $(50 \mu \mathrm{m}$ peak to peak, $1 \mathrm{~Hz}$ ) to the contact under a $2.5 \mathrm{~N}$ normal load. During the test, the voltage drop in the contact was measured with an acquisition card (333 $10^{3}$ samples per s) with the current value set at $20 \mathrm{~mA}$ and the voltage limit at $250 \mathrm{mV}$. The mean value of the 500 measurements per cycle was calculated every 10 cycles.

\section{b) Characterizations}

Atomic force microscopy (AFM) topographic and electrical images were performed with a Veeco multimode Nanoscope IIIa system equipped with a special device for the electrical measurements. Conducting tips were made out of doped silicon with a doped diamond coating. Scanning Electron microscopy (SEM) images were obtained with a Hitachi SEM microscope. Energy dispersive X-ray (EDX) spectroscopy was used to identify the intermetallic composition. X-ray photoelectron spectroscopy (XPS) spectra were recorded with a system using a non-monochromatized $\mathrm{MgK} \alpha$ radiation and a VG analyzer. Glow discharge optical emission spectroscopy was used in order to obtain composition profiles of the samples. Two techniques were used to measure the intermetallic layer thickness: coulometry and X-Ray Fluorescence Spectroscopy (XRF). First the thickness of the free tin (tin not combined with another element) was measured by integration of the current flow for the anodic dissolution of the tin. Then the thickness of identified intermetallic phases $\left(\mathrm{Cu}_{6} \mathrm{Sn}_{5}\right.$ or $\left.\mathrm{Ni}_{3} \mathrm{Sn}_{4}\right)$ was measured by XRF at the location of the coulometric spot. 3D images and profiles were measured by a contactless 3D Wyko profiler.

\section{RESULTS AND DISCUSSION}

\section{A. $\quad$ Fretting of tinned samples}

Cyclic fretting experiments simulating vibrations of electrical contacts were performed for 3500 cycles on all the samples described above. Some experiments were also performed for a smaller number of cycles. All the experiments were repeated at least 3 times; the results were perfectly reproducible (the curves could be superimposed).

Figure 1 shows the evolution of the mean contact resistance per cycle for the matte tin coatings on brass without underlayer before and after thermal ageing at $155^{\circ} \mathrm{C}$ for $16 \mathrm{~h}$.

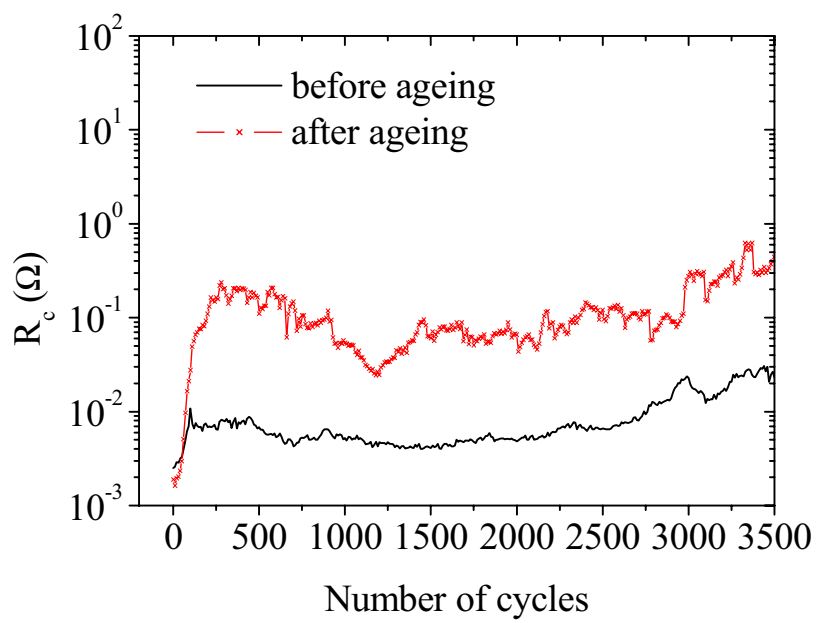

Figure 1: Mean value of the contact resistance $\mathrm{R}_{\mathrm{c}}$ per cycle during the fretting test for matte tin coating $(2 \mu \mathrm{m}$, no underlayer): before and after thermal ageing at $155^{\circ} \mathrm{C}$ for $16 \mathrm{~h}$.

For the as deposited sample, the contact resistance increases $\left(\mathrm{R}_{\mathrm{c}}=10 \mathrm{~m} \Omega\right)$ and then remains on a plateau around $4 \mathrm{~m} \Omega$ for 2000 cycles. After about 2500 cycles the contact resistance continually rises and reaches values above $10 \mathrm{~m} \Omega$ after 2740 cycles. The behaviour of the heat treated sample is very different: $R_{c}$ increases and reaches $238 \mathrm{~m} \Omega$ after a very small number (280) of fretting cycles. Then it decreases and stabilizes at about $100 \mathrm{~m} \Omega$. Thermal ageing creates the conditions for very fast and permanent fretting degradation.

The evolution of $R_{c}$ for the tin coating with a thick nickel underlayer of $2 \mu \mathrm{m}$ is shown on Figure 2. Before the heat treatment, three phases can be distinguished. First, $R_{c}$ increases $\left(\mathrm{R}_{\mathrm{c}}=6 \mathrm{~m} \Omega\right)$ and then it stabilizes around $4 \mathrm{~m} \Omega$ during a second stage of 200 cycles. Finally after 300 fretting cycles, the contact resistance continually raises; the value of $10 \mathrm{~m} \Omega$ is reached after 600 cycles (third stage). After thermal ageing $\left(155^{\circ} \mathrm{C}-16 \mathrm{~h}\right)$, three stages can also be distinguished. 
$\mathrm{R}_{\mathrm{c}}$ increases at the beginning of the test; it reaches $230 \mathrm{~m} \Omega$ after 200 fretting cycles. During the second stage the contact resistance values diminish and do not vary much around a value of $30-50 \mathrm{~m} \Omega$. After about 3000 cycles (third stage) a fast increase of $R_{c}$ is observed.

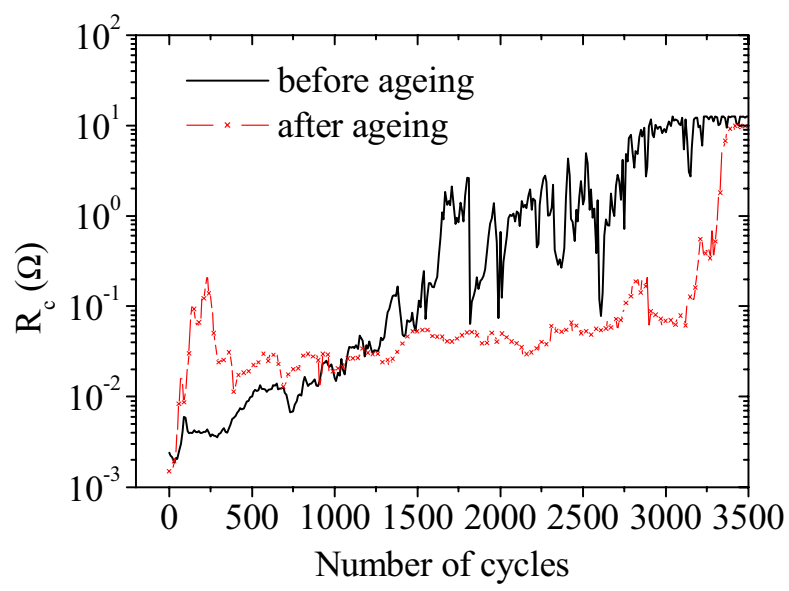

Figure 2: Mean value of the contact resistance $\mathrm{R}_{\mathrm{c}}$ per cycle during the fretting test for matte tin coating ( $2 \mu \mathrm{m}$ on $2 \mu \mathrm{m} \mathrm{Ni}$ underlayer): before and after thermal ageing at $155^{\circ} \mathrm{C}$ for $16 \mathrm{~h}$.

Figure 3 describes the behaviour of samples with a thin nickel underlayer of $0.1 \mu \mathrm{m}$. Before the thermal ageing, $\mathrm{R}_{\mathrm{c}}$ increases and reaches $5 \mathrm{~m} \Omega$ after 100 fretting cycles; it then remains almost constant around $4 \mathrm{~m} \Omega$ during 1500 cycles. After this, $R_{c}$ values continually increase. Optical observations of the wear tracks performed on samples submitted to only 1000 cycles (experiment stopped during the plateau of low $R_{c}$ values), showed that copper alloy was exposed (this was also the case for the previous sample of Figure 1). As was mentioned in the sample description experiments were done on fresh samples without any IMC. After the heat treatment, a resistance peak at $100 \mathrm{~m} \Omega$ is observed after 100 cycles; then $R_{c}$ decreases to about $4 \mathrm{~m} \Omega$ and remains almost constant for 2000 fretting cycles. Thus, after heat treatment of the samples, very different behaviours are observed when tin is deposited on a thick $2 \mu \mathrm{m}$ underlayer or on a thin $0.1 \mu \mathrm{m}$ one.

Figure 4 shows the behaviour of the samples with a thin underlayer of $0.1 \mu \mathrm{m}$ of cobalt. Before the thermal ageing, $R_{c}$ remains constant around a value between 2 and 3 $\mathrm{m} \Omega$ during 500 cycles. Then, the contact resistance values slowly rise. The value of $10 \mathrm{~m} \Omega$ is reached after 1250 cycles; $\mathrm{R}_{\mathrm{c}}$ remains below $100 \mathrm{~m} \Omega$ during the rest of the test. Comparing Figure 3 and Figure 4, shows that the contact resistance values with the thin Co underlayer are higher and less stable than those measured with the thin nickel underlayer.

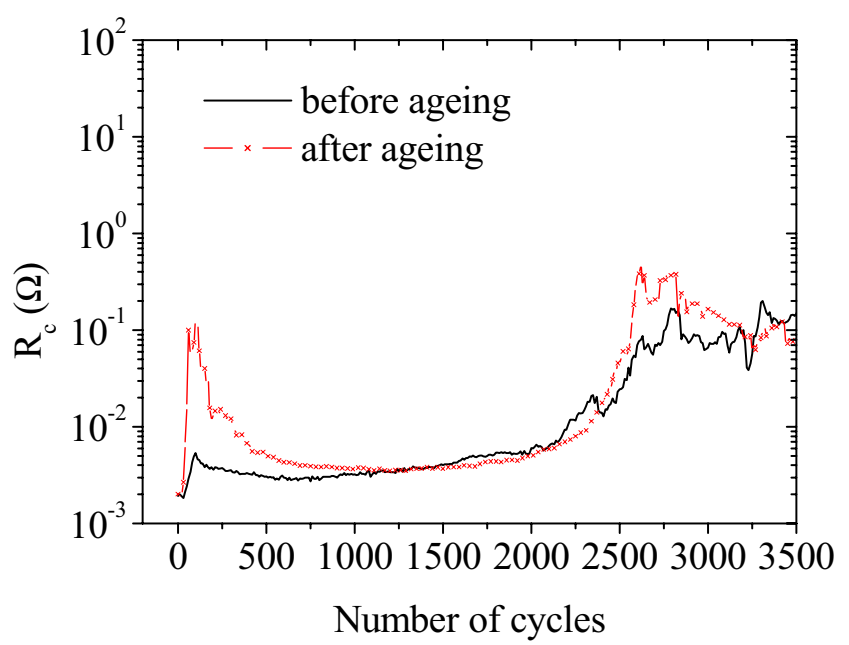

Figure 3: Mean value of the contact resistance $\mathrm{R}_{\mathrm{c}}$ per cycle during the fretting test for matte tin coating ( $2 \mu \mathrm{m}$ on $0.1 \mu \mathrm{m} \mathrm{Ni}$ underlayer): before and after thermal ageing at $155^{\circ} \mathrm{C}$ for $16 \mathrm{~h}$.

After the thermal ageing, Figure 4 shows that $R_{c}$ slightly increases to $5 \mathrm{~m} \Omega$, then remains constant around 4 $\mathrm{m} \Omega$ during 2000 fretting cycles. It reaches $10 \mathrm{~m} \Omega$ only after 2200 cycles. Thus, when a thin underlayer of Co is deposited before tin electrodeposition, the electrical behaviour of the contact during the fretting test is improved after the heat treatment. The reasons of such an improvement are analysed in terms of intermetallic compound formation in the sections that follow.

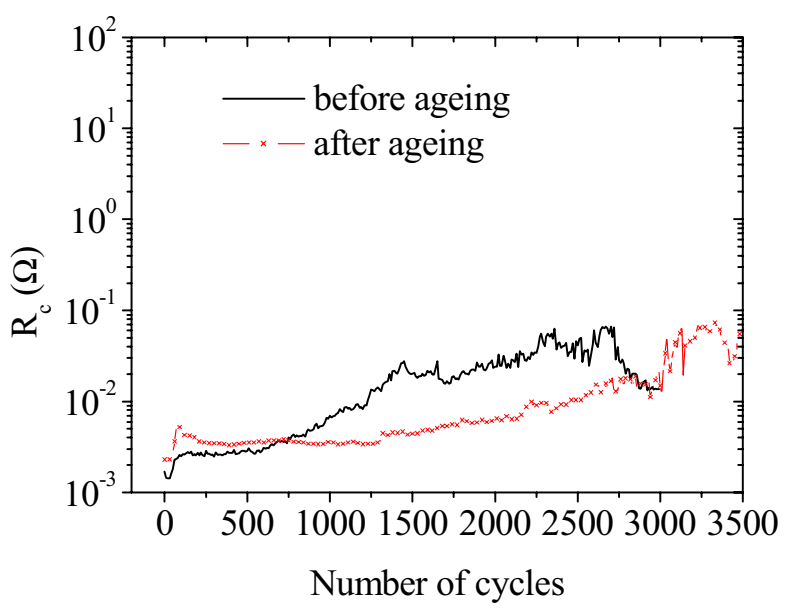

Figure 4: Mean value of the contact resistance $\mathrm{R}_{\mathrm{c}}$ per cycle during the fretting test for matte tin coating $(2 \mu \mathrm{m}$ on $0.1 \mu \mathrm{m}$ Co underlayer): before and after thermal ageing at $155^{\circ} \mathrm{C} 16 \mathrm{~h}$.

\section{B. Diffusion processes during heating and IMC formation}

The thickness of the intermetallic layers formed after the ageing treatment $\left(16 \mathrm{~h}\right.$ at $\left.155^{\circ} \mathrm{C}\right)$ was investigated as well as the composition of the compounds. Each heat treated coating was stripped with an appropriate solution. Then energy dispersive X-ray analyses were used to identify and/or 
estimate the intermetallic compositions. Care was taken to choose a rather low accelerating voltage value, $15 \mathrm{kV}$, in order to limit the analysis to the small thickness of the layers. Four different intermetallic layers were shown to form during the heat treatment of the samples, as summarized on table 3.

The analyses showed the presence of some zinc for IMC 1, IMC 3 and IMC 4 (5\%, $4 \%$ and $4 \%$ respectively).

\section{TABLE 3: INTERMETALLIC COMPOSITION}

\begin{tabular}{|c|c|c|c|}
\hline Samples & $\begin{array}{c}\text { Elements in } \\
\text { IMC }\end{array}$ & $\begin{array}{c}\text { IMC } \\
\text { compound }\end{array}$ & Label \\
\hline $\mathrm{M} 2$ & $\mathrm{Sn}, \mathrm{Cu}$ & $\mathrm{Cu}_{6} \mathrm{Sn}_{5}$ & IMC 1 \\
\hline $\mathrm{M} 2 \mathrm{Ni} 2$ & $\mathrm{Sn}, \mathrm{Ni}$ & $\mathrm{Ni}_{3} \mathrm{Sn}_{4}$ & IMC 2 \\
\hline $\mathrm{M} 2 \mathrm{Ni} 0.1$ & $\mathrm{Sn}, \mathrm{Ni}, \mathrm{Cu}$ & $(\mathrm{Sn}-\mathrm{Ni}-\mathrm{Cu})$ & IMC 3 \\
\hline $\mathrm{M} 2 \mathrm{Co} 0.1$ & $\mathrm{Sn}, \mathrm{Co}, \mathrm{Cu}$ & $(\mathrm{Sn}-\mathrm{Co}-\mathrm{Cu})$ & IMC 4 \\
\hline
\end{tabular}

No zinc was detected in the analyzed zone $\left(0.5 \mu \mathrm{m}^{3}\right)$ for the aged sample with the $2 \mu \mathrm{m}$ thick nickel underlayer which acted as an anti-diffusion barrier. For the thinner (0.1 $\mu \mathrm{m})$ underlayers, both of nickel and cobalt, diffusion of zinc was observed. The composition of IMC 4 was observed to be less homogeneous than the other ones (several spots were analyzed for each samples). Only very small quantities of oxygen were found which showed that intermetallic compounds oxidized very slowly during their exposure to the atmosphere. The intermetallic compound formed during thermal ageing of the tin coating on copper sample (sample 1) was identified as $\mathrm{Cu}_{6} \mathrm{Sn}_{5}$ in agreement with many authors. The one formed during thermal ageing of sample 2 (tin layer on the thick $2 \mu \mathrm{m}$ nickel underlayer) is also in agreement with many authors: it was clearly identified as $\mathrm{Ni}_{3} \mathrm{Sn}_{4}$. The analyses showed that diffusion of copper occurred for IMC 3 and IMC 4 and that the intermetallic compounds respectively involved $(\mathrm{Sn}-\mathrm{Ni}-\mathrm{Cu})$ and $(\mathrm{Sn}-\mathrm{Co}-\mathrm{Cu})$. Measurements could more likely be influenced by the substrate in the case of the thin $\mathrm{Ni}$ and Co underlayers but analysis show that the intermetallic layer is also due to the diffusion of $\mathrm{Cu}$.

In order to obtain the diffusion profiles of the various elements due to the thermal ageing, special samples with a thinner layer of tin $(1 \mu \mathrm{m}$ instead of $2 \mu \mathrm{m})$ were electrodeposited. Glow discharge optical spectroscopy was performed on the thermally aged samples to obtain composition profiles of the intermetallic compounds. The thinner layers of tin allowed quicker access to the copper interface and thus greater dimensional precision in the depth profile.

Figure 5 shows the composition profile for the tin coating without underlayer after the thermal ageing. The evolutions of concentrations of copper, tin, zinc and carbon were followed. The inflection points of all the curves were determined and allowed to delimit the various diffusion zones. Three different zones can be observed in figure 5: the substrate, the diffusion zone and the free tin (which in this case is extremely thin). It can be seen that zinc has diffused through the tin layer and accumulates at the tin surface. Copper also diffuses in the tin film and forms the intermetallic $\mathrm{Cu}_{6} \mathrm{Sn}_{5}$ (IMC 1) which corresponds to the diffusion zone. The intermetallic thickness can be estimated around $1 \mu \mathrm{m}$. Coulometry and X-ray fluorescence were also used to evaluate the IMC thickness which was also found to be around $1 \mu \mathrm{m}$.

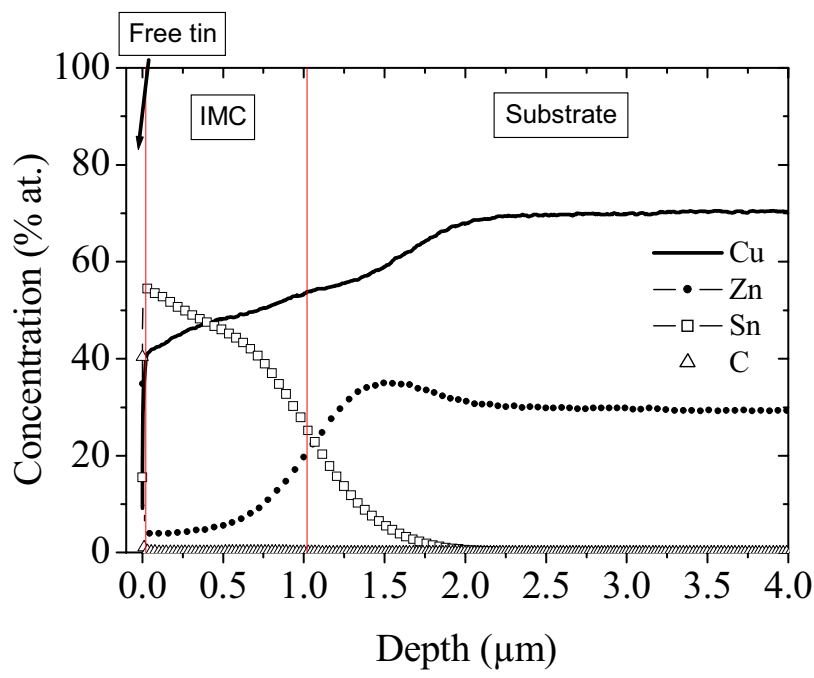

Figure 5: Composition profile for the matte tin coating (no underlayer) after thermal ageing at $155^{\circ} \mathrm{C}$ for $16 \mathrm{~h}$.

The composition profile for the aged tin coating with the thick $2 \mu \mathrm{m}$ underlayer of nickel is shown in Figure 6. It shows the evolution of copper, zinc, tin, nickel and carbon concentration with depth.

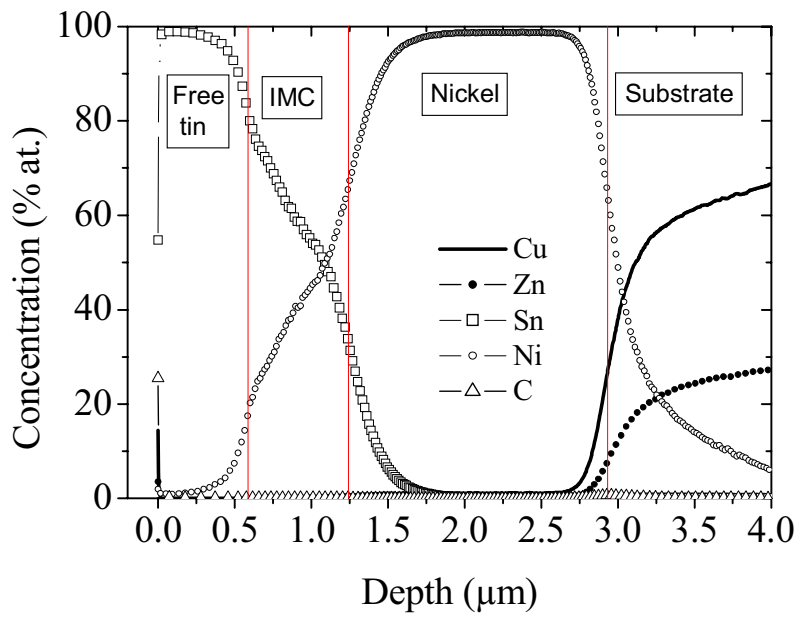

Figure 6: Composition profile for the matte tin coating (Ni $2 \mu \mathrm{m}$ underlayer) after thermal ageing at $155^{\circ} \mathrm{C}$ for $16 \mathrm{~h}$.

Four areas can be observed: the substrate, the nickel underlayer, the diffusion zone and the free tin. It shows that copper and zinc have not diffused through the nickel underlayer, consistent with the EDX analyses. Figure 6 also shows that nickel diffused in the tin layer and formed the intermetallic compound IMC 2 identified as $\mathrm{Ni}_{3} \mathrm{Sn}_{4}$ by EDX. The free tin remaining on the sample after the heat treatment is observed to be thicker than for the first sample involving IMC 1. This is in good agreement with the values of the 
diffusion coefficients and activation energies $\mathrm{D}_{0}$ and $\mathrm{Q}$ that were derived from a series of IMC thickness measurements collected after treatments of different durations and at different temperatures.

Diffusion occurs when two materials are in contact. For a diffusion pair composed of two metals $\mathrm{A}$ and $\mathrm{B}$, the mean free path of $A$ into $B$ is expressed as:

$$
X=c_{A \rightarrow B}\left(D_{A \rightarrow B} t\right)^{1 / 2}
$$

where $c_{A \rightarrow B}$ is the solubility of A into $\mathrm{B}, D_{A \rightarrow B}$ its diffusion coefficient. Diffusion coefficients can be expressed as a function of temperature in the following Arrhenius relationship:

$$
\begin{aligned}
& D=D_{0} \exp \left(-\frac{Q}{R T}\right) \\
& e=(D t)^{1 / 2}
\end{aligned}
$$

The activation energy $Q$ and the intrinsic diffusivity of the material $D_{0}$ can be calculated using equation 2 and equation 3 where $e$ is the IMC compound thickness. Table 4 reports the activation energy and $\mathrm{D}_{0}$ values calculated for $\mathrm{Cu}$ and Sn (IMC 1) and for Ni and Sn (IMC 2). These values are in very good agreement with Labie's work [4].

TABLE 4: ACTIVATION ENERGY AND DIFFUSION COEFFICIENTS FOR TIN AND COPPER, TIN AND NICKEL.

\begin{tabular}{|c|c|c|}
\hline IMC & $\begin{array}{c}\text { IMC 1 } \\
\left(\mathrm{Cu}_{6} \mathrm{Sn}_{5}\right)\end{array}$ & $\begin{array}{c}\mathrm{IMC} \mathrm{2} \\
\left(\mathrm{Ni}_{3} \mathrm{Sn}_{4}\right)\end{array}$ \\
\hline $\mathrm{Q}\left(\mathrm{kJ} \mathrm{mol}^{-1}\right)$ & 56.2 & 41.1 \\
\hline $\mathrm{D}_{0}\left(\mathrm{~m}^{2} \cdot \mathrm{s}^{-1}\right)$ & $6 \times 10^{-11}$ & $2 \times 10^{-13}$ \\
\hline $\mathrm{D}\left(25^{\circ} \mathrm{C}\right)\left(\mathrm{m}^{2} \cdot \mathrm{s}^{-1}\right)$ & $0.9 \times 10^{-20}$ & $1.2 \times 10^{-20}$ \\
\hline $\mathrm{D}\left(80^{\circ} \mathrm{C}\right)\left(\mathrm{m}^{2} \cdot \mathrm{s}^{-1}\right)$ & $2.2 \times 10^{-19}$ & $2.1 \times 10^{-19}$ \\
\hline $\mathrm{D}\left(155^{\circ} \mathrm{C}\right)\left(\mathrm{m}^{2} \cdot \mathrm{s}^{-1}\right)$ & $8.6 \times 10^{-18}$ & $3 \times 10^{-18}$ \\
\hline
\end{tabular}

The calculated values of Table 4 show that the growth kinetic of IMC 1 is faster than for IMC 2 for temperatures above $80^{\circ} \mathrm{C}$. Coulometry, X-ray fluorescence and GD-OES profiles gave an intermetallic thickness of 0.5 $\mu \mathrm{m}$ for IMC 2.

Figure 7 shows the composition profile for the tin coating with a $0.1 \mu \mathrm{m}$ nickel underlayer after the thermal ageing. The evolution of $\mathrm{Cu}, \mathrm{Zn}, \mathrm{Sn}, \mathrm{Ni}$ and $\mathrm{C}$ is plotted. Three main areas can be observed: the substrate, the diffusion zone and the free tin. The IMC formed was shown by EDX to be composed of $\mathrm{Ni}, \mathrm{Cu}$ and $\mathrm{Sn}$. $\mathrm{Cu}$ diffuses through the thin $\mathrm{Ni}$ layer and reacts with $\mathrm{Sn}$; $\mathrm{Ni}$ is also seen to diffuse slowly into tin layer. Both $\mathrm{Cu}$ and $\mathrm{Zn}$ can be observed in the entire tin layer. At the tin surface, carbon and zinc are present. The zinc and copper concentrations are lower than for the coating without underlayer (Figure 5) showing that the thin underlayer has some effect of hindrance on diffusion.

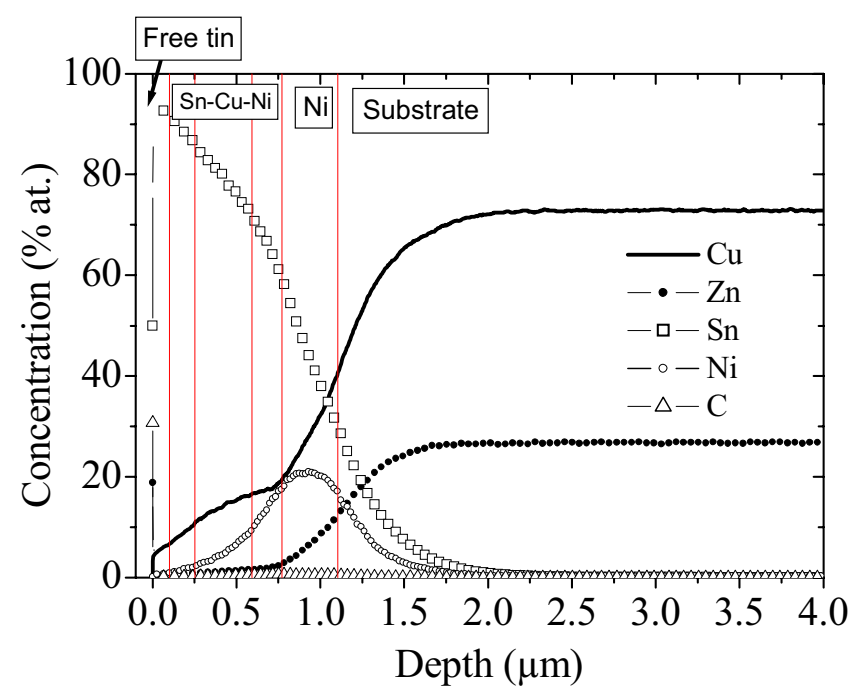

Figure 7: Composition profile for the matte tin coating ( $\mathrm{Ni} 0.1 \mu \mathrm{m}$ underlayer) after thermal ageing at $155^{\circ} \mathrm{C}$ for $16 \mathrm{~h}$.

Figure 8 displays the composition profile for the tin coating with a $0.1 \mu \mathrm{m}$ Co underlayer after the thermal ageing. The concentrations of $\mathrm{Cu}, \mathrm{Zn}, \mathrm{Co}, \mathrm{Sn}$ and $\mathrm{C}$ are plotted. Three areas can also be observed: the substrate, the diffusion zone and the free tin. In this case, the intermetallic was shown to be composed of $\mathrm{Cu}, \mathrm{Co}$ and $\mathrm{Sn}$. Figure 8 shows that the Co seems to be concentrated at the $\mathrm{Sn} / \mathrm{Cu}$ interface.

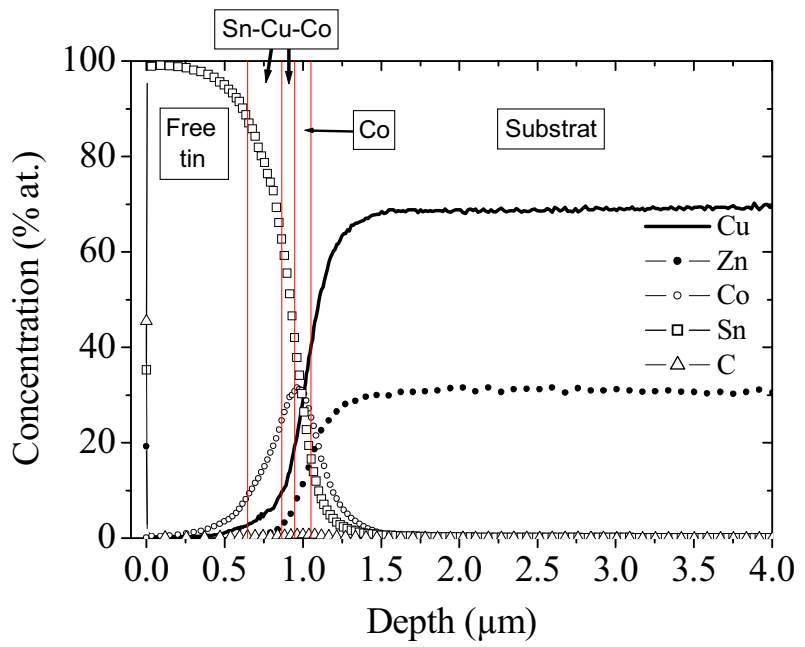

Figure 8: Composition profile for the matte tin coating (Co $0.1 \mu \mathrm{m}$ underlayer) after thermal ageing at $155^{\circ} \mathrm{C}$ for $16 \mathrm{~h}$.

It can be observed that the copper diffuses slower with a cobalt underlayer than a nickel one. Co also diffuses slower than $\mathrm{Ni}$ into the tin layer. This observation is in agreement with the energy dispersive analyses. $\mathrm{C}$ and $\mathrm{Zn}$ are also present on the tin surface. The thicknesses of IMC 3 and 4 could be obtained from the GD-OES profiles. The values deduced from the inflection points of different profiles were $0.7 \mu \mathrm{m}$ for IMC 3 and $0.3 \mu \mathrm{m}$ for IMC 4 . Thus, for the thermal ageing under study, the intermetallic layer, IMC 4 was observed to be the thinner one, indicating that the thin 0.1 
$\mu \mathrm{m}$ cobalt underlayer minimizes the diffusion process. Table 5 summarizes the values of the measured thickness of intermetallic compound formed.

TABLE 5: VALUES OF THE INTERMETALLIC COMPOUND FORMED AFTER HEATING AT $155^{\circ} \mathrm{C}$ FOR $16 \mathrm{H}$.

\begin{tabular}{|c|c|c|c|c|}
\hline & IMC 1 & IMC 2 & IMC 3 & IMC 4 \\
\hline $\mathrm{e}(\mu \mathrm{m})$ & 1 & 0.5 & 0.7 & 0.3 \\
\hline
\end{tabular}

The microstructure of the IMC compounds was observed by SEM after stripping the free tin. Figure 9 shows the microstructure of the four IMC samples. Very different grains sizes and shapes can be observed. The structure of IMC 1 is made of large grains of size 2 to $3 \mu \mathrm{m}$. The structure of IMC 2 shows grain smaller than $1 \mu \mathrm{m}$. For these two intermetallics, the grain growth is observed to be equiaxial (the grain growth occurs in all the direction). The structure of IMC 3 shows big grains of $1.5 \mu \mathrm{m}$. The grains size of IMC 3 is larger than the grain size of the IMC 2 but lower than the grain size of the IMC 1.
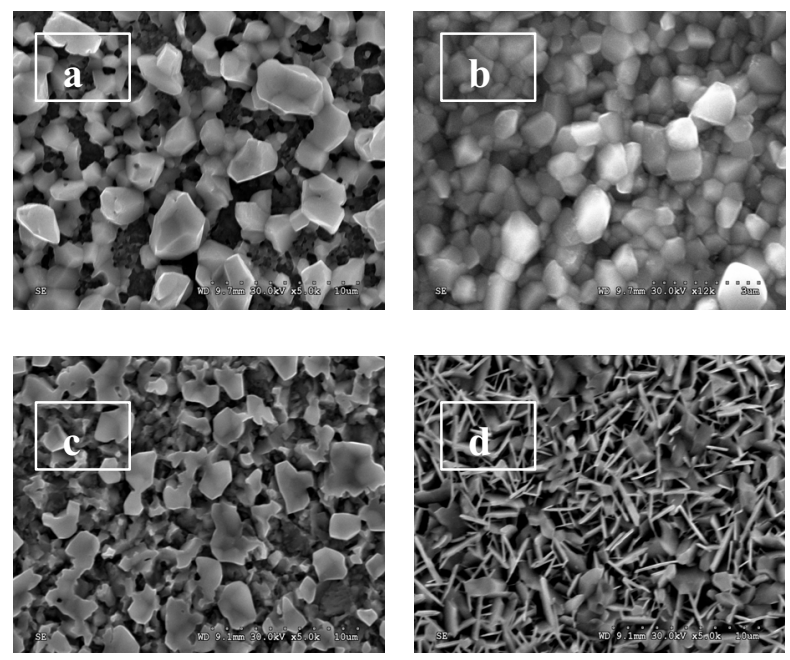

Figure 9: Scanning electron micrograph a) IMC 1 (magnification X 5000); b) IMC 2 (magnification X 12000); c) IMC 3 (magnification X 5000); d) IMC 4 (magnification X 5000)

The structure of IMC 4 is very different: it is composed both of "needles" of size 2 to $4 \mu \mathrm{m}$ by 0.3 to 0.5 $\mu \mathrm{m}$ and of grains. For the two last intermetallic compounds, the grain growth occurs in a specific direction. The roughness parameters $R_{a}$ was measured by AFM on $50 \mu \mathrm{m} \times 50 \mu \mathrm{m}$ surfaces. The roughness parameter $R_{a}$ was $460 \mathrm{~nm}$ for IMC 1 , $110 \mathrm{~nm}$ for IMC 2, $170 \mathrm{~nm}$ for IMC 3 and $130 \mathrm{~nm}$ for IMC 4. Therefore, IMC 1 is the roughest intermetallic. These results are in agreement with the grain sizes.

\section{Fretting of IMC compounds}

The tribological properties of the intermetallic compounds were investigated. Sphere and plane contacts made from stripped intermetallic coupons were submitted to the same fretting tests. Figure 10 shows the evolution of the mean contact resistance per cycle of the four compounds: IMC 1, IMC 2, IMC 3 and IMC 4. For IMC 1 and IMC 2, $R_{c}$ reaches $10 \mathrm{~m} \Omega$ after a few fretting cycle. For IMC 3 and 4 the increase of resistance occurs after a much longer period. The contact resistance value for IMC 4 is very low and stable for more than 1000 cycles.

In order to better understand the mechanisms, special fretting tests were stopped after 500 cycles and the wear scars were observed. After 500 fretting cycles, $R_{c}$ is $39 \mathrm{~m} \Omega$ for IMC $1,22.8 \mathrm{~m} \Omega$ for IMC $2,5.3 \mathrm{~m} \Omega$ for IMC 3 and $1.6 \mathrm{~m} \Omega$ for IMC 4 . It can be seen that a very low values of resistance are measured for IMC 4 after 500 fretting cycles; the value is lower than the value in static which was measured to be $5.8 \mathrm{~m} \Omega$. This indicates that a few friction cycles allowed an increase of the contact area without the formation of oxidized hardened debris and the onset of fretting degradation.

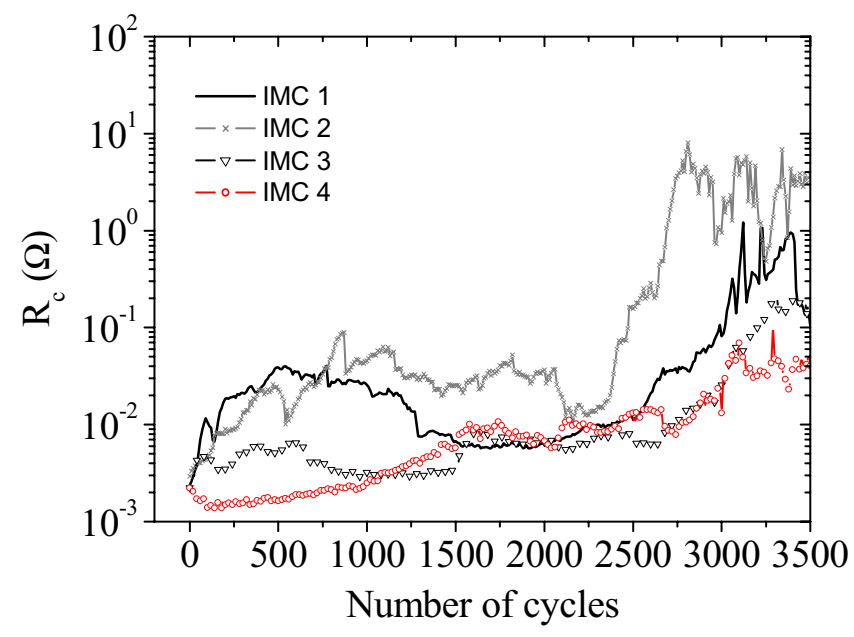

Figure 10: Mean value of the contact resistance $R_{c}$ per cycle during the fretting test for the IMC samples obtained after stripping the free tin.

For IMC 1 and IMC 2, the optical observations showed that friction occurred on the intermetallic layer and that some debris accumulated in the contact interface causing the contact resistance to increase. This was not the case for IMC 3 and IMC 4: large bumps due to material transfer were observed on the planes. A sticking phenomenon probably occurred between the ball and the plane [9]. This could be due to the mechanical properties of the intermetallic compounds involving $\mathrm{Sn}-\mathrm{Ni}-\mathrm{Cu}$ and $\mathrm{Sn}-\mathrm{Co}-\mathrm{Cu}$, changing the sliding conditions: the sticking of the surfaces prevents the movement (slip) to occur. For both IMC 3 and 4 the values of $R_{c}$ are lower $(5.3 \mathrm{~m} \Omega$ and $1.6 \mathrm{~m} \Omega$ ), being the lowest for IMC 4. This change in the sliding conditions [9] and the particular microstructure of IMC 4 observed in Figure 10 seem to lead to very low and stable values of $R_{c}$ for more than 1000 cycles of fretting.

\section{Discussion}

The electrical behaviour of IMC coatings obtained after stripping was analysed at a microscopic level with a 
customised conductive probe AFM (CP-AFM). CP-AFM images were performed on the four IMC layers. The equivalent "resistance" between the AFM tip and the surface was measured locally during the acquisition of the topographic images. The voltage bias during the measurement was set at $1 \mathrm{~V}$. The deflection set point is $1.5 \mathrm{~V}$ which, taking into account the cantilever stiffness, corresponds to an applied force between 50 and $100 \mathrm{nN}$. The electrical maps, not presented here, showed that the IMC surfaces are very conducting. Only small differences were observed for the different layers. The software developed in the laboratory treats the Log of these values because they are very high. The mean value of $(512 \times 512)$ values for each image, $\log (R)$, can be calculated. The values for the four intermetallic layers are summarized in table 6 . These values, compared to the value for the matte tin surface in the same conditions of measurement $(\log (R)=10.5)$, indicate that the intermetallic surfaces show little oxidization.

TABLE 6: MEAN LOG(R) (R IS THE "EQUIVALENT" RESISTANCE BETWEEN THE CANTILEVER TIP AND THE SURFACE IN THE CPAFM MEASUREMENT) FOR THE FOUR IMC COMPOUNDS.

\begin{tabular}{|c|c|c|c|c|c|}
\hline & IMC 1 & IMC 2 & IMC 3 & IMC 4 & Sn ref \\
\hline LogR & 6.6 & 7 & 8.1 & 6 & 10.5 \\
\hline
\end{tabular}

This is in agreement with the EDX measurements and the XPS ones which showed an oxide component and a metallic one for the tin $3 \mathrm{~d}$ core levels. This showed that only a very thin oxide layer $(d<5 \mathrm{~nm})$ formed on the IMC surfaces due to air exposure; this observation is in agreement with Tompkins's work showing that the IMC oxide films were thinner than the oxide films of metals (tin, copper) [10]. Using a thin cobalt film as an underlayer for tin coatings gives contact resistance values during fretting tests lower than those obtained with the usual thick nickel underlayer. This can be attributed to different adhesion and sliding conditions. When contacts are submitted to a heat treatment, comparing the thin $\mathrm{Co}$ and thin Ni layers, it can be observed that the Co layer hinders the diffusion of copper and that the formation of an intermetallic compound is slowed down. Moreover, the microstructure of the particular $\mathrm{Sn}-\mathrm{Co}-\mathrm{Cu}$ intermetallic comprising both grains and needles can contribute to a slower process of debris formation. The low oxidation of the intermetallic also prevents hard debris to form. This set of properties, both structural, mechanical and electrical allows to consider thin cobalt films as interesting candidate underlayers for tin coating less prone to fretting degradations.

\section{CONCLUSION}

IMC compounds, formed after tin layers electrodeposited on brass substrates were thermally aged at $155{ }^{\circ} \mathrm{C}$ for $16 \mathrm{~h}$, have been studied. The influence of depositing an underlayer of nickel or cobalt has been investigated; both thick $(2 \mu \mathrm{m})$ and thin $(0.1 \mu \mathrm{m})$ ones were analyzed. The diffusion of the various species was determined. A thick nickel underlayer of $2 \mu \mathrm{m}$ stops the diffusion of zinc to the surface. Thinner ones $(0.1 \mu \mathrm{m})$ did not stop zinc and copper. $\mathrm{Cu}$ was observed to diffuse through the nickel and cobalt thin underlayers and participate to the formation of intermetallic compounds. Different types of IMC were analysed according to the diffusing species. $\mathrm{Cu}_{6} \mathrm{Sn}_{5}$ and $\mathrm{Ni}_{3}, \mathrm{Sn}_{4}$ were observed, the former growing faster than the latter. Unidentified $(\mathrm{Sn}-\mathrm{Ni}-\mathrm{Cu})$ and $(\mathrm{Sn}-\mathrm{Co}-\mathrm{Cu})$ intermetallic compounds were formed by diffusion of species through the thin underlayers. The compositions and structures of the IMC compounds were characterised after stripping of the free tin layer. $\mathrm{Cu}_{6} \mathrm{Sn}_{5}$ showed the largest grains while $\mathrm{Ni}_{3} \mathrm{Sn}_{4}$ showed the smallest ones. The intermetallic compound involving Sn$\mathrm{Co}-\mathrm{Cu}$ (IMC 4) showed a typical structure displaying needles. The four types of intermetallic were analysed by CP-AFM and showed conducting surfaces. The electrical properties of these surfaces were investigated in low amplitude cycles aimed at causing fretting failure of materials. IMC 4 was observed to have a combination of properties leading to slow fretting degradation. It was shown that the use of a thin underlayer of Co allows to minimize the copper diffusion into the tin layer and to reduce the intermetallic layer thickness, which is consistent with other work. Further investigation on the ageing on longer periods of such coatings is necessary.

These results present an interesting line of investigation for the minimization of the effects of temperature on tinned electrical contacts reliability.

\section{REFERENCES}

[1] Y.W. Park, T.S.N. Sankara Narayanan, K.Y. Lee "Effect of temperature on the fretting corrosion of tin platted copper alloycontacts", Wear, vol. 262, 2007 , p. 320.

[2] U. Lindborg, B. Asthner, L. Lind, L.R. Reyan, "Intermetallic growth and contact resistance of tin contacts after ageing", Trans IEEE CMPT vol.12, $\mathrm{n}^{\circ} 1,1976, \mathrm{p} 33$.

[3] T. Hamman, "The impact of sliding motion and current load on the deterioration of tin-coated contact terminals", Trans IEEE CMPT vol.23, n², 2000, p.278.

[4] R. Labie, "Solid state diffusion in $\mathrm{Cu}-\mathrm{Sn}$ and Ni-Sn diffusion couples with flip-chip scale dimensions", J. of Intermetallics, vol.15, 2007, p. 396.

[5] C.L. Siu, H.C. Man, C.H. Yeung, "Interdiffusion coefficients of various cobalt base alloy coatings for Cu/Au systems", App. Surf. Sci, 245, 2005,7986.

[6] P.E. Davis, "Intermetallic compound growth and solderability", J. of Plating Surface Finishing, vol..70, 1983, p. 49.

[7] M. Jordan, "The electrodeposition of tin and its alloys", Eugen G Leuze Publishers, D-88348 Saulgau/Wurtt. Germany, 1995.

[8] S. Noël, N. Lecaudé, C. Bodin, L. Boyer, L. Tristani, E.M. Zindine, G. Gavazzi, I. Buresch, D. Siemers, "Electrical and tribological properties of hot-dipped tin separable contacts with fluorinated lubricant layers", $45^{\text {th }}$ IEEE Holm Conf. on Elec. Contacts, Pittsburg, USA, Oct. 1999, p. 225.

[9] S. Hannel, S. Fouvry, Ph. Kapsa, L. Vincent, "The fretting sliding transition as a criterion for electrical contact performance" Wear, vol. 249, 2001, p. 761

[10] H.G. Tompkins, J.E Bennett, J.A Augis, T.M Paskowski "Oxidation of $\mathrm{CuSn}$ and CuSn Films in Room Air from $175^{\circ}$ to $250^{\circ} \mathrm{C}$ ", J. of Electrochemical Soc. 1983, vol. 130, 1983, p. 1753. 


\title{
Deformation of Crystal Structure and Distribution of Mechanical Stress in Tin-Plated Layer under Contact Loading
}

\author{
Terutaka Tamai ${ }^{1}$, Shigeru Sawada ${ }^{1}$ and Yasuhiro Hattori ${ }^{2}$ \\ ${ }^{1}$ Mie University, Graduate School of Engineering, Laboratory of Vehicle Network Technology, Mie \\ 515-8507, Japan \\ ${ }^{2}$ AutoNetworks Technology, Ltd., Mie 513-8631, Japan
}

\begin{abstract}
Tin (Sn)-plated contacts are used in various electrical applications owing to their low cost and stability of contact resistance under a high contact load. At present, such contacts are widely applied in the automotive industry and the other fields. The surface of plated tin is covered with their oxide film such as $\mathrm{SnO}$ or $\mathrm{SnO}_{2}$, but it is possible to obtain low contact resistance by applying a high contact load. It is expected that downsized connectors will be developed in future. Therefore, it is important to study contact resistance behavior under a low contact load. In this study, we first review the mechanical deformation phenomena of the tin crystal structure in a contact area under contact loading with respect to the contact resistance behavior, which was presented at IEEE Holm Conference 2008. To clarify these important phenomena, the mechanical stress distribution in the tin-plated layer was numerically analyzed by FEM. It was found that the mechanical stress in the layer propagated to the periphery of the contact area along the radial direction during loading. Therefore, formation of the crystal of the contact area is forced outside of the contact area. Because of the peculiar distribution of the stress, the crystal grains are separated and piled up outside of the contact area. The mechanism of marked decrease in contact resistance during the piling up at the periphery with separation of crystal grains was clarified. This phenomenon is very different from commonly observed decrease in contact resistance due to elastic and plastic deformation.
\end{abstract}

Keywords- tin-plated contacts, contact resistance, contact load, contact trace, connector, FEM

\section{INTRODUCTION}

Tin (Sn)-plated contacts can be used to produce lower-cost connectors than other precious-metal-plated contacts. However, for tin-plated contacts, low and stable contact resistance behavior such as that of gold $(\mathrm{Au})$-plated contacts can be only obtained under a high contact pressure [1-3]. At present, tin-plated contacts are applied in various fields such as automotive industry and the other electrical fields [4-7]. Tin-plated surfaces are generally covered with an oxide film such as $\mathrm{SnO}$ or $\mathrm{SnO}_{2}$. This film acts as protective coating against corrosive gases in the atmosphere [8]. However, when an oxide film is interposed between contact interfaces, the contact resistance level increases. Therefore, to obtain low level contact resistance, it is necessary to mechanically break down the film under contact. The trend forward smaller connectors have recently been increased. Because it is difficult to achieve a high contact load in a small connector, low contact resistance cannot be obtained easily. Therefore, studies on contact resistance behavior under a low contact load are necessary. It was previously reported that to break down the film, a high contact load or sharp contact shape is essential for connector applications [1,2].

Previously, the authors reported $[9,10]$ that in the contact configuration of a platinum $(\mathrm{Pt})$ hemisphere and a tin-plated flat, crystal structures in the tin plated layer oriented perpendicular to a substrate of copper-zinc $(\mathrm{Cu}-\mathrm{Zn})$ alloy are markedly deformed by contact loading. This deformation was found by EBSP (Electron Back Scattered Diffraction Pattern) analysis. The following results have been obtained. At the periphery of the contact area, crystal grains piled up and separated from the surface. The oxide film interposed between contacts broke at the periphery with the separation of the tin crystal grains. During this film breakdown process, the high contact resistance markedly decreased.

This phenomenon was recognized to be the same as the piling up of the periphery of the contact area in the case of bulk tin contacts [10]. Previously, characteristic relationship between contact resistance and contact load for solid tin contacts was reported by the authors [10]. Namely, as the probe contact sinks into the soft solid tin surface, a periphery of the true contact area rises and piles up. During this process, a metallic contact formed between the hemisphere probe and are where crystal grains separated with piling up portion. During this process, contact resistance greatly decreases. Namely, in the case of bulk tin contacts, during the process of the piling up at the periphery, micro sliding between the side surface of the platinum hemisphere and the pilling up surface breaks down mechanically the oxide film, and remarkable decrease in contact resistance was appeared.

In this paper, we first reviewed the mechanical deformation of the tin crystal structure in the contact area under contact loading which was presented at IEEE Holm Conference 2008 [10]. Then, to clarify this mechanism, the distribution of the mechanical stress in the tin-plated layer was numerically analyzed by FEM (Finite Elemental 
Method). In results, because the center portion of the contact area is subjected to hydrostatic pressure, the crystal structure is not displaced owing to the pressure. Since it is widely recognized that the maximum stress occurred just below the center of the contact area, the breakdown of the film should occur at the center portion. However, the obtained results show that the film cannot be broken down at the center portion until the hydrostatic pressure is equal to the shear strength of the film. The crystal structure was compressed and broken down under contact loading. Since the mechanical stress in the plated layer propagated to its periphery along the radial direction, the formation of crystal inside the contact area are forced outside of the contact area. Owing to the distribution of stress, the crystal grains separated and piled up outside of the contact area. By studying these processes, the relationship between the crystal structure and the distribution of the stress in the tin plated layer was clarified. Furthermore, this process causes the mechanical breakdown of the film and contact resistance to markedly decrease.

\section{BACKGROUND}

We first review our previous study [10] on the singularity of contact resistance and contact trace. The specimens used in this study as one side contacts were tin- plated flats. This tin-plated specimen was composed of following structure. $\mathrm{Cu}+\mathrm{Zn}$ alloy was used as substrate, its surface was plated thinly with copper, then the copper surface was plated by the tin. After plating, the tin layer was subjected to a reflow process. The thickness of the plated tin-layer was between 1 and $5 \mu \mathrm{m}$. However, because an intermediate metal layer between the tin and the copper alloy forms during the reflow process, the actual thickness of the tin layer is less than the above apparent thickness. The surface of the tin layer was covered with an oxide film.

The contact between the tin-plated flat and the surface of a platinum hemisphere was investigated. The contact load was increased from 0 to $100 \mathrm{gf}(1 \mathrm{~N})$ then decreased from $100 \mathrm{gf}$ to $0 \mathrm{gf}$. During these changes in the contact load, contact resistance was measured, and after this measurement, the contact was separated to precisely observe contact traces by SEM and by using an optical microscope.

As a result, the following facts are clarified as peculiar phenomenon of tin plated contacts. Namely, the piling up phenomenon at the periphery of the contact trace was observed during contact loading. When this occurred, a sharp decrease in contact resistance was detected. Namely, during the process of the piling up at the periphery with the separation of tin crystal grains, the oxide film between piling up part of the periphery and the side surface of the platinum contact was subjected to micro sliding motion, which caused the film to break down mechanically, and a metallic contact to form. At the same time, separation of the crystal grains from the surface under piling up of the periphery is shown in Fig.1. In this figure, the separation of grains at the periphery and crushed grains inside the contact area can be seen.
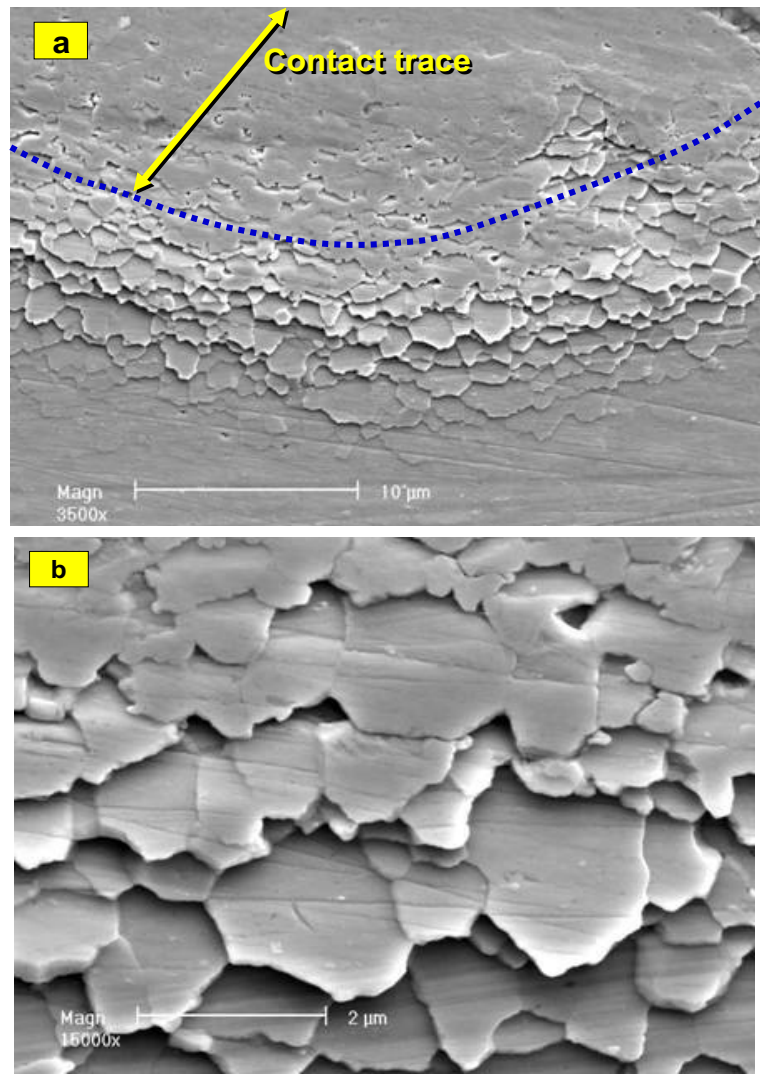

Fig.1 (a) Periphery of the contact trace showing piling up and separation of tin crystal grains. (b) Enlargement of separated grains at the periphery.

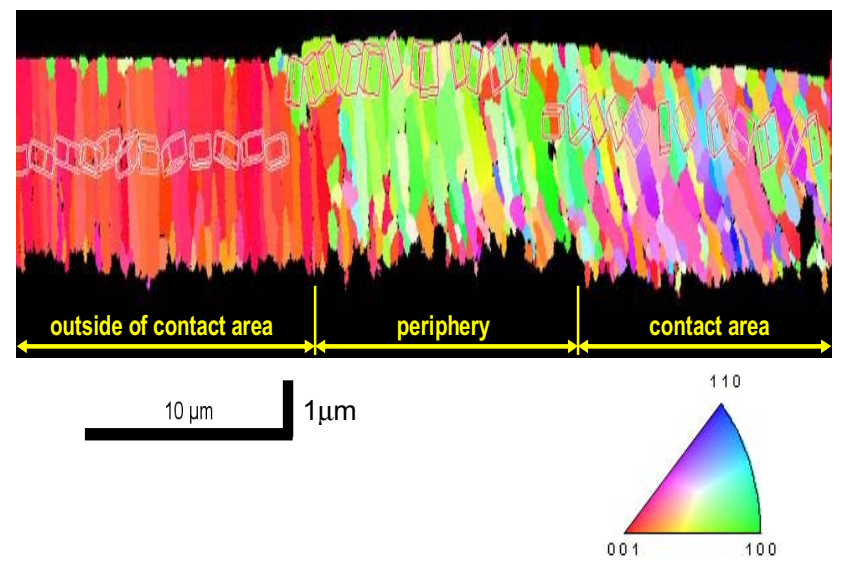

Fig.2 Results of EBSD analysis showing the crystal orientation of the cross section of the tin-plated layer, which is affected by the stress applied by the contact load.

Crystal structures of tin- plated layer obtained by EBSD are shown in Fig.2.

The relationship between contact resistance and contact load is shown in Fig.3. According to this figure, contact resistance sharply decreases when the in contact load increases from 1 to $2 \mathrm{gf}$. At this contact load, the separation 


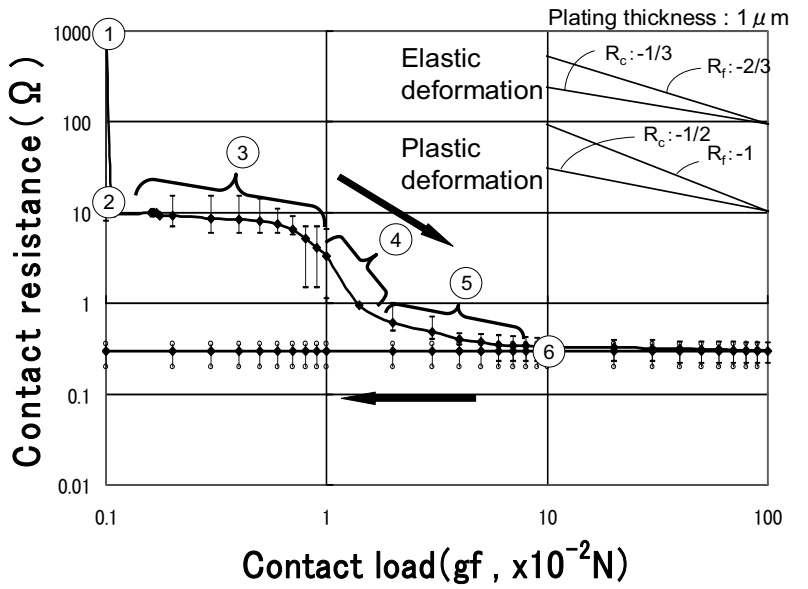

Fig.3 Relationship between contact resistance and contact load for contacts between the tin-plated flat and platinum hemisphere. Elastic and plastic deformations are indicated by the slope of the curve.

of the tin crystal grains with their piling up at the periphery starts to occur, and the film breaks down mechanically.

\section{FEM ANALYSIS OF STRESS IN TIN-PLATED LAYER}

\section{A. Application of FEM Analysis to the Stress Distribution}

The piling up at the periphery of the contact area in solid tin was studied by Tabor in detail [11]. The piling up at the periphery was shown to occur at a work-hardened metal surface. On the other hand, for an annealed metal, sinking down of the surface occurs at the periphery. Therefore, because occurrence of the piling up at the periphery of the tin contact trace, the tin-plated surface must have been workhardened by the reflow process involving the diffusion of the substrate metal.

These morphological changes of the contact traces are thought to be caused by the propagation of the mechanical stress in the tin plated layer under loading. To clarify this phenomenon, it is necessary to analyze the distribution of stress in the layer. In the present study, FEM was applied to study the distribution of stress in the tin-plated layer due to contact loading. The results of the FEM analysis are discussed in relation to crystal structure of the tin-plated cross section obtained by the results of EBSD analysis. The effects of the distribution of stress in the layer on the crystal structure upon loading were clarified.

In the present study, the distribution of stress in the tin-plated layer was analyzed by FEM for a platinum hemisphere pushing into a solid tin layer with different thicknesses. The calculation software used for FEM analysis was ABAQUS / Standard. The modeling of FEM analysis was as follows.

\section{B. Modeling for FEM Analysis.}

Modeling was performed by two dimensional axis symmetry for solid elements based on the symmetry of the

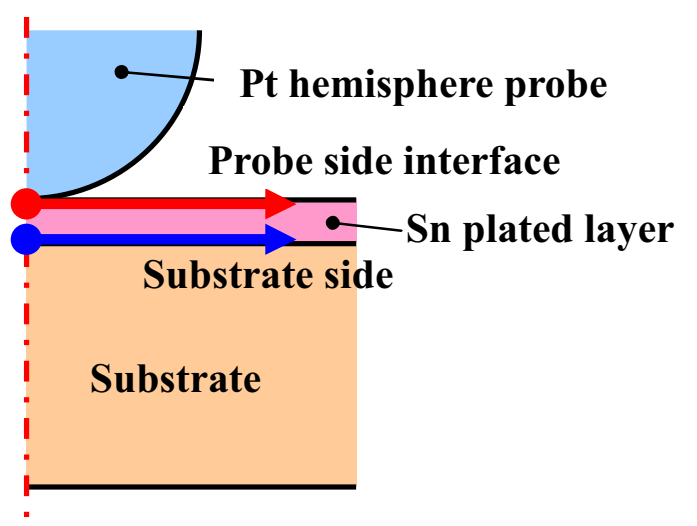

Fig.4 Model used for FEM analysis.

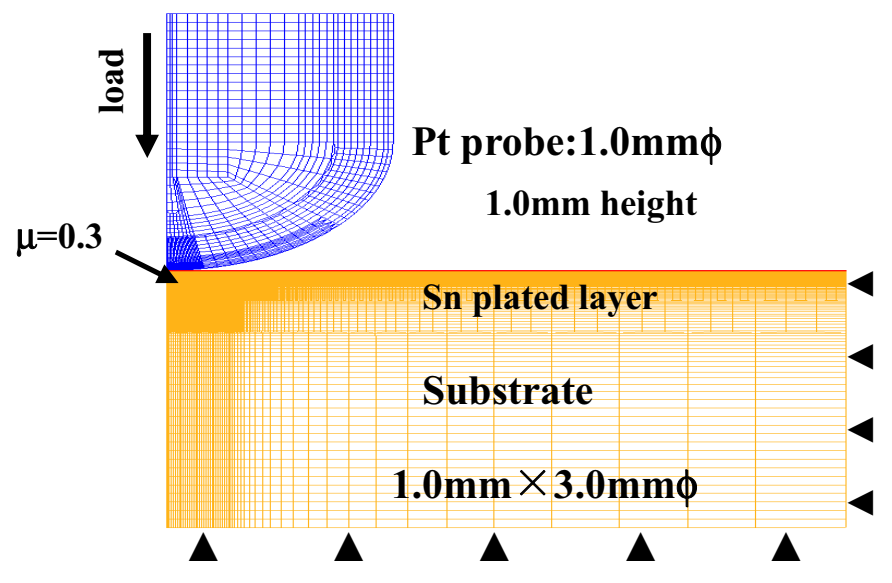

Fig.5 Elemental division of the contact model used for FEM.

Table 1 Material parameters for FEM

\begin{tabular}{|l||l|l|}
\hline & $\begin{array}{l}\text { Young's } \\
\text { modulus } \\
(\mathrm{MPa})\end{array}$ & $\begin{array}{l}\text { Poisson } \\
\text { ratio }\end{array}$ \\
\hline \hline Sn plated layer & 46000 & 0.34 \\
\hline $\begin{array}{l}\mathrm{Cu}-\mathrm{Zn} \text { alloy } \\
\text { substrate }\end{array}$ & 126000 & 0.34 \\
\hline Pt hemisphere probe & 168000 & 0.377 \\
\hline
\end{tabular}

contacts, which consist of contact between the platinum hemisphere and the tin plated surface. The followings are the modeling assumed. The center of the platinum hemisphere is taken as the axis of symmetry; the radial direction of the tin-plated flat and the thickness direction of plated layer do not effect the distribution of stress in the contact area. In the FEM model, the thin oxide film on the contact surface is not considered; because it is very thin, the film does not effect the stress distribution. Also, fine tin crystal grains were not considered in the model. The deformations of the tin crystal themselves in the tin-plated layer due to the contact of hemisphere surface is difficult to analyze by FEM, because the tin crystals in the layer consist of a set of pillar-shaped crystals. 
The configuration of the model of contact between the platinum hemisphere and the tin-plated flat is shown in Fig.4. Moreover, a typical example of the elemental division of the plated layer is shown in Fig.5. In the neighborhood of the contact interface, the elements used to represent the plated layer had a $0.4 \mu \mathrm{m}$, and a thickness of $0.25 \mu \mathrm{m}$. The coefficient of friction at the contact interface was assumed to be $\mu=0.3$. Physical values used in the model such as Young's modulus and Poisson ratio are shown in Table 1. In this analysis the tin-plated layer and substrate of copper-zinc alloy substrate were assumed to be deformed elastically, and the platinum probe contact was assumed to be plastic deformation owing to its high hardness.

\section{RESULTS OF FEM ANALYSIS AND DISCUSSION}

\section{A. Distribution of Stress in the Tin-Plated Layer.}

The results of FEM analysis indicated that the entire contact area of the tin-plated layer deforms plastically. Elastic deformation was over. The deformation due to contact loading is shown in Fig.6. As shown in Fig.7, the plated layer is deformed by the pushing out from the contact area. Namely, a large stress of breakdown of tin plated layer occurs at the periphery of the contact area causing tin crystal grains to separate with piling up at the periphery.

Next, the distribution of shear stress in the $\mathrm{z}$ axis along radial direction is shown in Fig.8. The shear stress in the tin-plated layer at the substrate side and the shear stress in the tin plated layer at the platinum hemisphere contact side are in opposite directions. Because the coefficient of friction between the platinum hemisphere surface and the tin-plated flat surface is assumed to be $\mu=0.3$, the sliding of each surface is possible. Therefore, the shear stress in the radial direction on the platinum side of the tin layer increases. In contrast, because the interface between the tin-plated layer and the substrate of copper alloy is restricted, the substrate side of the tin layer cannot move, stress which is forward to the center of the contact area in the radius direction is generated. Therefore, strong shear stress acts at the periphery of the contact area.

This finding convincingly explains the SEM images shown in Fig.1, which indicate the piling up and separation of tin crystal grains at the periphery of the contact trace. It shows also that the piled up at the periphery of solid tin contact occurred as well as at the tin plated contact surface. The stress distribution of the contact surface in the axial direction is shown in Fig.9. A change in the stress direction from the center of the contact to the periphery can be seen.. The direction of stress greatly changes, distribution of the stress at the periphery is irregular.

\section{B. Hydrostatic Pressure at the Center of the Contact Area.}

The hydrostatic pressure in the contact area obtained by FEM analysis is shown in Fig.9 and Fig.10. As shown in Fig.9, because hydrostatic pressure appears at the center

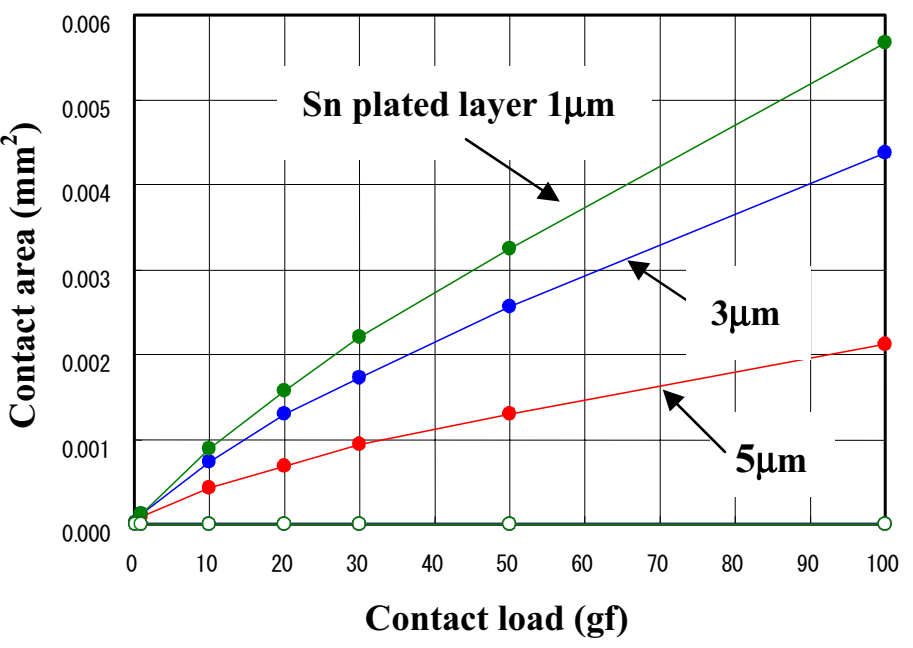

Fig.6 Relationship between contact area and contact load showing plastic deformation. •: plastic deformation, $\circ$ : elastic deformation for three tin plated thicknesses.

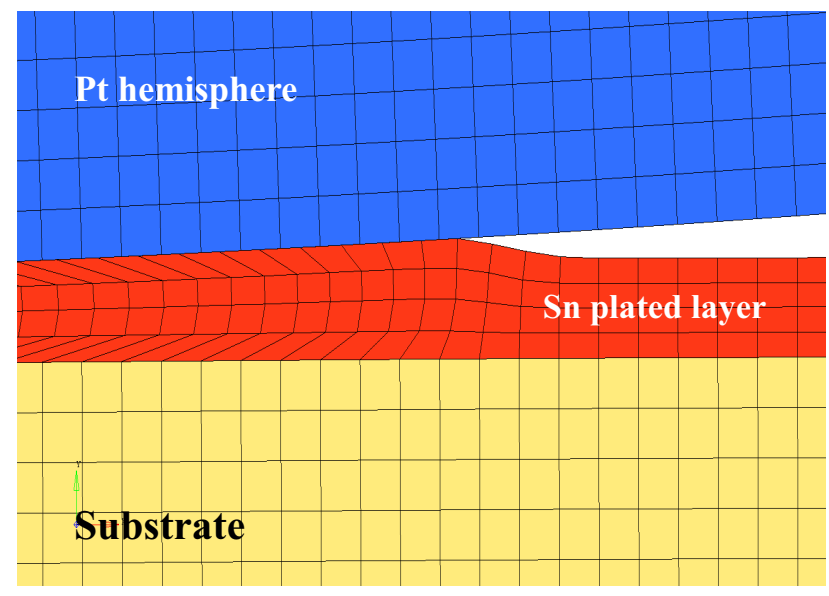

Fig.7 Deformation of the tin-plated layer under contact loading.

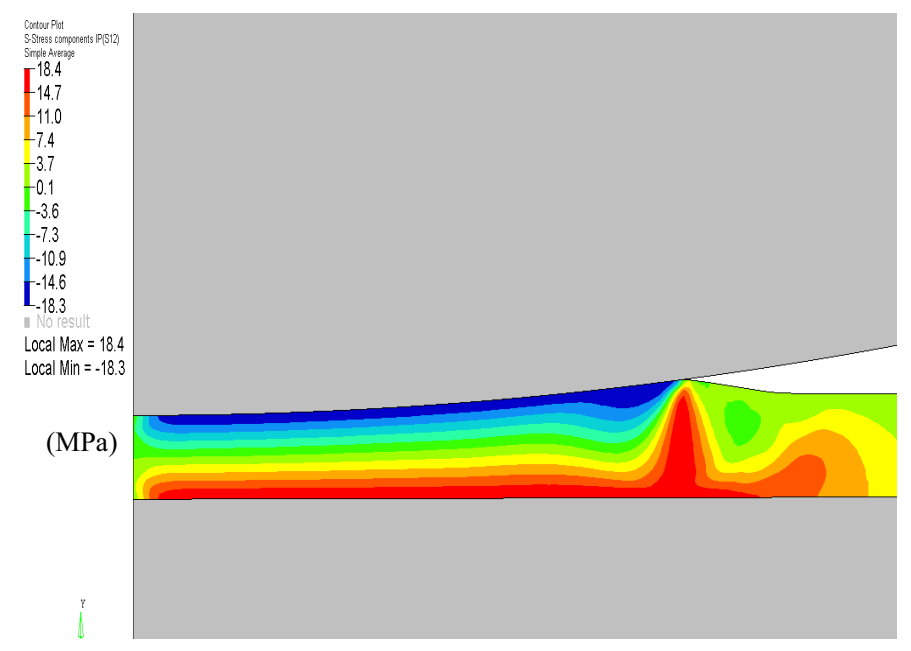

Fig.8 Distribution of shear stress in the tin-plated layer. 


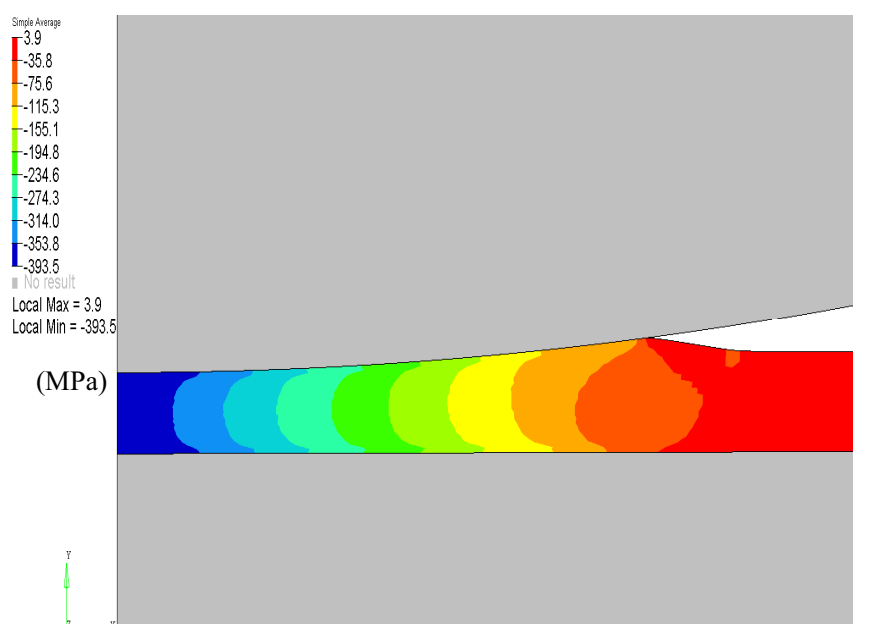

Fig.9 Distribution of stress along the circumference of the contact area. Hydrostatic pressure state appeared at the center of the area.

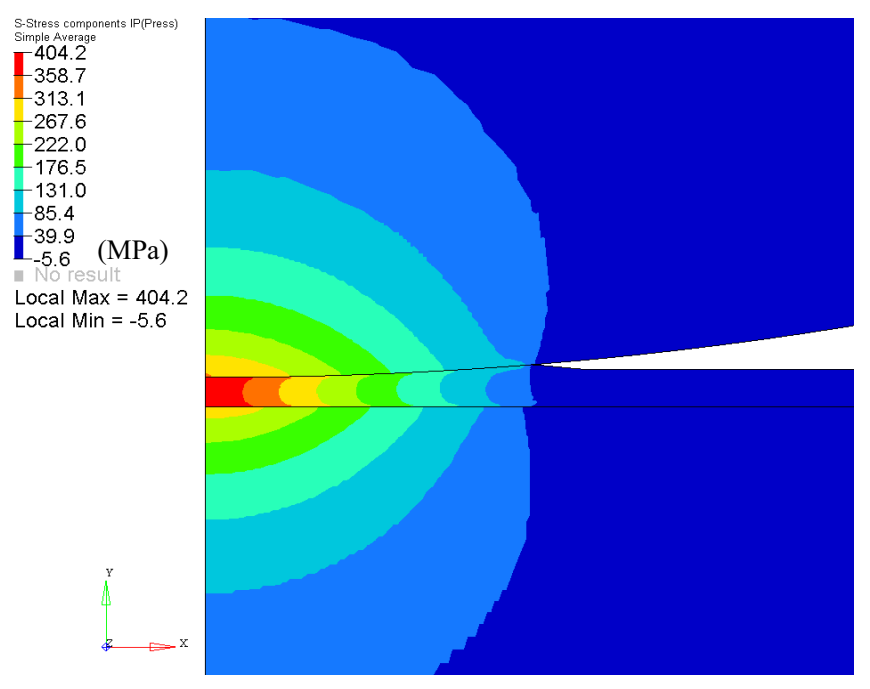

Fig.10 Distribution of hydrostatic pressure stress in the tin plated layer.

portion of the contact area, the tin-plated layer does not move in the radial direction of the contact area. Therefore, the mechanical breakdown of the oxide film interposed between the contacts does not occur until the hydrostatic pressure reaches the breakdown stress of the tin-plated layer. If the hydrostatic pressure becomes higher than breakdown stress of the tin-plated layer, the film between the contacts as above should be broken down.

For the tin-plated layer, a decrease in the contact resistance occurs upon the breakdown of the film due to the pile up at the periphery in load region (4) in Fig.3. With increasing contact load, the contact resistance converges to a constant level due to lining substrate with high hardness copper alloy in load region (6) as shown in Fig.3. However, a solid tin contact without a substrate metal lining such as plating undergoes a second decrease in contact resistance caused by the mechanical breakdown of the film at a higher contact load than that causing the first breakdown due to micro sliding at the contacts of the plied up periphery [10]. This second breakdown of the film is induced by the film being crushed by the higher hydrostatic pressure.

\section{CONCLUSION}

As previously reported, the peculiar contact phenomena occur at the contact between a platinum hemisphere and a tin plated contact $[9,10]$. It was found that when periphery of the contact area piles up and at the same time the separation of the tin crystal grains occurs with increasing contact load, the contact resistance decreases sharply at this changed contact area $[9,10]$. This decrease in contact resistance is caused by the formation of a metallic contact due to the mechanical breakdown of the films interposed between the contacts during this process.

To clarify the separation of the tin-plated crystal grains and piling up he periphery, the distribution of stress and strain in the plated layer caused by contact loading was analyzed by FEM.

As a result, it was found that when the shear pressure in the tin-plated contact area was increased to the shear stress of the contact area, the contact area deformed plastically in contact load region higher than $0.1 \mathrm{gf}$. Moreover, shear stress develops outside of the contact area. The tin- plated layer of the contact area is deformed in radial direction by stress such as push out from the contact area to outside. This deformation occurs from a point close to the center of the contact area to its periphery. The distribution of stress in the tin-plated layer on the contact interface side is generated along the radial direction when the layer is compressed the substrate side of the layer, the stress acts forward the center of the contact area owing to the restriction between the layer and the substrate. Therefore, inside of the tin-plated layer on the substrate side, where the surroundings are restricted, high vertical stress in the $\mathrm{z}$ direction extending to below the interface of the hemisphere was generated. It was found that piling up of the periphery with the separation of the tin crystal grains was induced by the distribution of this high stress at the periphery. Moreover, high hydrostatic pressure was generated at the contact area, where the tin layer was prevented from moving. Generally, because the highest stress in the contact area is just below the center point, mechanical breakdown should occur at the center portion. However, the initial mechanical break down of the film is caused by piling up at of the periphery with the separation of tin crystal grains. A low contact resistance was generated by this mechanism, and a metallic contact was found by these morphological changes.

\section{REFERENCES}

[1] M. Antler, W. F. Graddick, H. G. Tompkins, Base metal contacts: an exploratory study of separable 
connection to tin-lead, IEEE Trans. P.H.P., vol.PHP-11, no. $1,1975$.

[2] S. J. Krumbein, "Contact properties of tin plates", Proc. IEEE Holm Conference on Electrical Contacts, pp.38-49, October, 1974, Chicago.

[3] M. Antler, Survey of contact fretting in electrical connectors, Proc. $17^{\text {th }}$ International Conf. on Electrical Contacts, pp.3-22, 1984, Chicago.

[4] R. D. Malucci, "Characteristics of films developed in friction experiments on tin plated contacts", Proc. $45^{\text {th }}$ IEEE Holm Conference on Electrical Contacts, pp.175-185, 1999, Pittsburgh.

[5] R. D. Malucci, "Possible mechanism for observed dynamic resistance", Proc. IEEE-Holm Conf., pp.254-267, 2000, Chicago.

[6] T. Ito, S. Sawada, Y. Hattori, Y. Saitoh, T. Tamai, K. Iida, Microscopic study of fretting corrosion caused by tin plating thickness, Proc. IEEE Holm Conference, pp.294-300, 2008, Orland.
[7] S. Noël, N. Lècaudè, S. Correcia, P. Gengre, and A. Grosjean, Electrical and tribological properties of tin palted copper alloy for electrical contacts in relation to its intermetallic growth, Proc. IEEE Holm Conference, pp.1-10, 2006, Montreal, Canada.

[8] W. H. Abbott, J. H. Neer, H. J. Healey, Effects of test procedures and sequences on the performance of tin-plated connectors, Proc. IEEE Holm Conference, pp.191-204, 1993, Pittsburgh.

[9] T. Tamai, Y. Ito, K. Hotta, Y. Saitoh, K. Iida, and Y. Hattori, Singularity of tin contacts in relationship between contact resistance and contact load, Proc. ICEC 2008, Saint-Malo, France.

[10] T. Tamai, Y. Saitoh, S. Sawada, and Y. Hattori, Peculiarities in characteristics between contact trace and contact resistance of tin contacts. Proc. IEEE-Holm Conference. pp.337-343, 2008, Orland.

[11] D. Tabor, The Hardness of Metals, Oxford Clarendon Press, pp.64-65, 1951. 


\title{
Nanoindentation mapping of physical properties
}

\author{
Christian Holzapfel \\ Schleifring und Apparatebau GmbH \\ Fürstenfeldbruck, Germany \\ cholzapfel@schleifring.de
}

\begin{abstract}
With the help of modern nanoindentation instrumentation an automated measurement of local mechanical properties of materials such as hardness or elastic modulus becomes possible. Such studies can provide further insight in local variations of these properties along or across thin film layers used as functional layers in electrical applications (e.g. slip rings). In this paper a model multilayer system with typical geometries and varying layer thicknesses is studied for elucidating the resolution of nanoindentation measurements. First measurements on the sample surface were performed using the continuous stiffness measurement (CSM). Due to the fact that the plastic and elastic zone underneath the indenter extends to a significantly larger sample depth than the indentation depth, information of all layers is present in the result. However, the extent of this effect is different for plastic (hardness) and elastic (elastic modulus) interaction. Second, a cross section of the same sample was investigated by $1 \mathrm{D}$ and $2 \mathrm{D}$ measurements (profile and matrix measurement). For hardness it was found that for the investigated model system the lateral resolution is approximately 10-20 times the indentation depth. In addition sample preparation has to be taken into account when analyzing the values of hardness and elastic modulus.
\end{abstract}

Keywords-nanoindentation; hardness; elasticity; CSM; resolution limit

\section{INTRODUCTION}

The main motivation for this study comes from the application of thin functional layers for slip ring applications. Slip rings are machine components that transfer electrical energy, signals and data from a stationary to a rotating component. Both, contacting and non-contacting systems exist. In order to have a low power loss and high signal integrity the contact systems used for contacting slip rings must have a low static contact resistance as well as a low dynamic change in resistance (noise). In addition a long life time is a pre-requisite for the performance of slip ring technology. For achieving these requirements the tribological system must be characterized by low wear but allowing the passage of current at the same time. In order to optimize the slip ring with respect to long life time only selected materials can be successfully applied. As discussed in [1] galvanic coatings are often used as contact systems.

A variety of physical properties has to be considered when designing suitable contact pairs. For understanding friction and wear with respect to life time especially the mechanical properties are important. However, wear processes are complex including both elastic as well as plastic components $[2,3]$. As example Equation 1 describes the friction coefficient $\mu$ in the case of purely abrasive interaction [2]:

$$
\mu=\frac{F_{f}}{F_{n}}=c \frac{K_{I c}{ }^{2}}{E\left(H \times F_{n}\right)^{1 / 2}},
$$

Where $F_{f}$ is friction force, $F_{n}$ is the normal force, $c$ is a constant, $\mathrm{K}_{\mathrm{Ic}}$ is fracture toughness, $\mathrm{E}$ denotes the elastic modulus and $\mathrm{H}$ is the hardness. Equation 1 directly shows that friction is a complex interplay of elastic (elastic modulus or more generally elastic compliance) and plastic (hardness) properties as well as fracture mechanics $\left(\mathrm{K}_{\mathrm{Ic}}\right)$.

In this respect it becomes important to be able to characterize mechanical properties of thin film systems (more specifically: galvanic functional coatings). Conventional tests for determining mechanical properties are using a suitable indenter and measuring the size of the indentations (hardness measurement) as well as applying a tensile stress and measuring the strain response of the sample in order to deduce elastic and plastic properties such as elastic modulus, elastic limit or tensile strength. For most of these measurements either bulk samples or thick coatings are needed.

In order to be able to also characterize very thin coatings (down to nanometer scales) nanoindentation techniques have been developed over the past few years. A monograph of the experimental technique is given in [4]. Nanoindentation makes use of a force measurement as a function of indentation depth. From the force distance traces both the hardness as well as the elastic modulus can be derived. By using an additional forced oscillation of the sample both properties can be determined as a function of indentation depth (so-called continuous stiffness measurement, CSM, see Section II for further details).

The nanoindentation measurement itself is subject to a series of possible experimental challenges: In [1] the influence of the surface structure was investigated in detail. For precise measurements the indentation depth should be significantly larger then the length scale of the surface roughness. However, in order to avoid substrate effects, the indentation depth should normally be less than $10 \%$ of the layer thickness [4].

In many cases the distribution of hardness and elastic modulus on the surface (for example in the case of inhomogeneous coatings) as well as a function of depth (for example in the case of multilayered samples) is of interest. In these cases the lateral and vertical resolution as a function of the indentation depth becomes important. In order to investigate these effects experimentally in more detail, in this study, a multilayer model system will be used (Figure 1). 


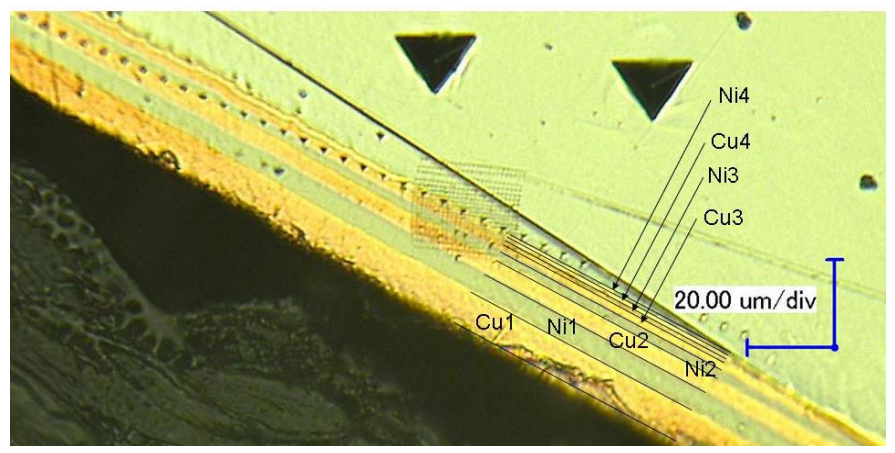

Figure 1. Multilayer model system used in this study. In addition some indents oriented along a line as well as in matrix form are shown. For further details see text.

This model system consists of a series of galvanically deposited $\mathrm{Cu}$ and chemically deposited $\mathrm{Ni}$ (with $\sim 8 \% \mathrm{P}$ ) layers on a steel substrate. The layer thickness gradually increases from the substrate to the surface as shown in Figure 1 by means of an optical micrograph of a cross section of the coating system together with some example indents.

Table 1 lists the individual layers of the model system together with thickness values derived as averages from repeated length measurements on optical micrographs similar to Figure 1.

TABLE I. MULTILAYER MODEL SYSTEM USED IN THIS STUDY: LAYER CONFIGURTION AND THICKNESSES; THE LAYERS ARE GIVEN FROM TOP (SURFACE) TO BOTTOM AND ARE NUMBERED FROM 1 TO 4 (FOR EXAMPLE CU1 AND NI1, SEE ALSO FIG. 1). ACCURACY OF THE THICKNESS MEASUREMENTS ARE ON THE ORDER OF $500 \mathrm{NM}$.

\begin{tabular}{|l|l|}
\hline \multicolumn{1}{|c|}{ Layer } & \multicolumn{1}{c|}{ Thickness, $\boldsymbol{\mu m}$} \\
\hline $\mathrm{Cu} 1$ & 8.3 \\
\hline $\mathrm{Ni} 1$ & 4.7 \\
\hline $\mathrm{Cu} 2$ & 4.2 \\
\hline $\mathrm{Ni} 2$ & 1.9 \\
\hline $\mathrm{Cu} 3$ & 1.8 \\
\hline $\mathrm{Ni} 3$ & 1.0 \\
\hline $\mathrm{Cu} 4$ & 0.8 \\
\hline $\mathrm{Ni} 4$ & 1.0 \\
\hline $\mathrm{Steel}$ & substrate \\
\hline
\end{tabular}

In the following, first the experimental technique used for nanoindentation will be briefly described. In section III a detailed description of nanoindentation into the original sample surface (without any sample preparation) is given with special emphasis on the observed substrate effect. Chapters IV and $\mathrm{V}$ further discuss nanoindentation results on a cross section of the multilayer ultimately leading to nanoindentation mapping (physical microscopy).

\section{EXPERIMENTAL}

For nanoindentation tests a MTS G200 (MTS) instrument was used. The system was tested for accuracy and precision in [1]. All measurements have been performed in the dynamic CSM mode using different indentation depths.
The analysis of the force distance curves during indentation uses the formulation of Oliver and Pharr [4], where the hardness is defined by (2):

$$
H=P / A \text {. }
$$

$P$ is the maximum load and $A$ is the projected area at that load. For further evaluation of the elastic properties of the sample, the reduced modulus of the system (taking both, indenter and sample, into account) can then be derived by using (3):

$$
E_{r}=\frac{\sqrt{\pi}}{2 \beta} \frac{1}{\sqrt{A}} S
$$

Here $S$ is the elastic stiffness of the contact (corresponding to the slope $\mathrm{dP} / \mathrm{dh}$ of the initial unloading curve) and $\beta$ denotes a constant which depends on the indenter geometry (1.034 for a Berkovich indenter as used in this study).

The elastic modulus of the sample is derived from the reduced modulus as defined in (3) and using the Poisson ratio of the indenter $v_{i}$ and sample $v$ (taken to be 0.3 for all materials investigated in this study) as well as the elastic modulus of the indenter, $\mathrm{E}_{\mathrm{i}}$, :

$$
\frac{1}{E_{r}}=\frac{\left(1-v^{2}\right)}{E}+\frac{\left(1-v_{i}^{2}\right)}{E_{i}} \text {. }
$$

From (2) and (4) only one value of the hardness and the elastic modulus at maximum indentation depth can be obtained. As stated above, in this study, the CSM mode is used which allows deriving hardness and elastic modulus as a continuous function of indentation depth. This can be achieved by superimposing a small oscillation onto the loading rate. The vibration response of the system is than analyzed in order to obtain a stiffness value as a continuous function of indentation depth. Hence, the elucidation of hardness and elastic modulus as a function of sample depth is possible. Of course the effects of interaction of the plastic and the elastic zone with any material change with increasing sample depth will affect the values obtained at each depth. This effect is more closely analyzed below.

The measurements performed in this study follow the same procedures as used in [1]. For all measurements a pre-defined depth of nanoindentation is defined (rather than a pre-defined force). A constant strain rate of $0.05 / \mathrm{s}$ is applied for the individual measurements. As indentation tip a Berkovich indenter was used. The frequency for the CSM measurements was $45 \mathrm{~Hz}$ with amplitude of $2 \mathrm{~nm}$. At maximum force a $10 \mathrm{~s}$ hold was performed. The measurement routine corrects the thermal drift by applying another hold at $10 \%$ maximum force.

\section{NANOINDENTATION RESULTS OBTAINED USING INDENTS INTO THE ORIGINAL SAMPLE SURFACE}

First the sample surface topography was characterized by nanoindentation microscopy and confocal microscopy. The term nanoindentation microscopy is used in this study for a constant force measurement combined with the stage 
movement in order to derive a surface structure as described in detail in [1]. Figure 2 compares results from the two different surface techniques. The measurement positions are not identical (hence a quantitative comparison is not possible). The lateral resolution of nanoindentation microscopy in this case is $500 \mathrm{~nm}$ (distance between different scans) whereas confocal microscopy possesses a lateral resolution on the order of 200 $\mathrm{nm}$ (depending on the objective used).
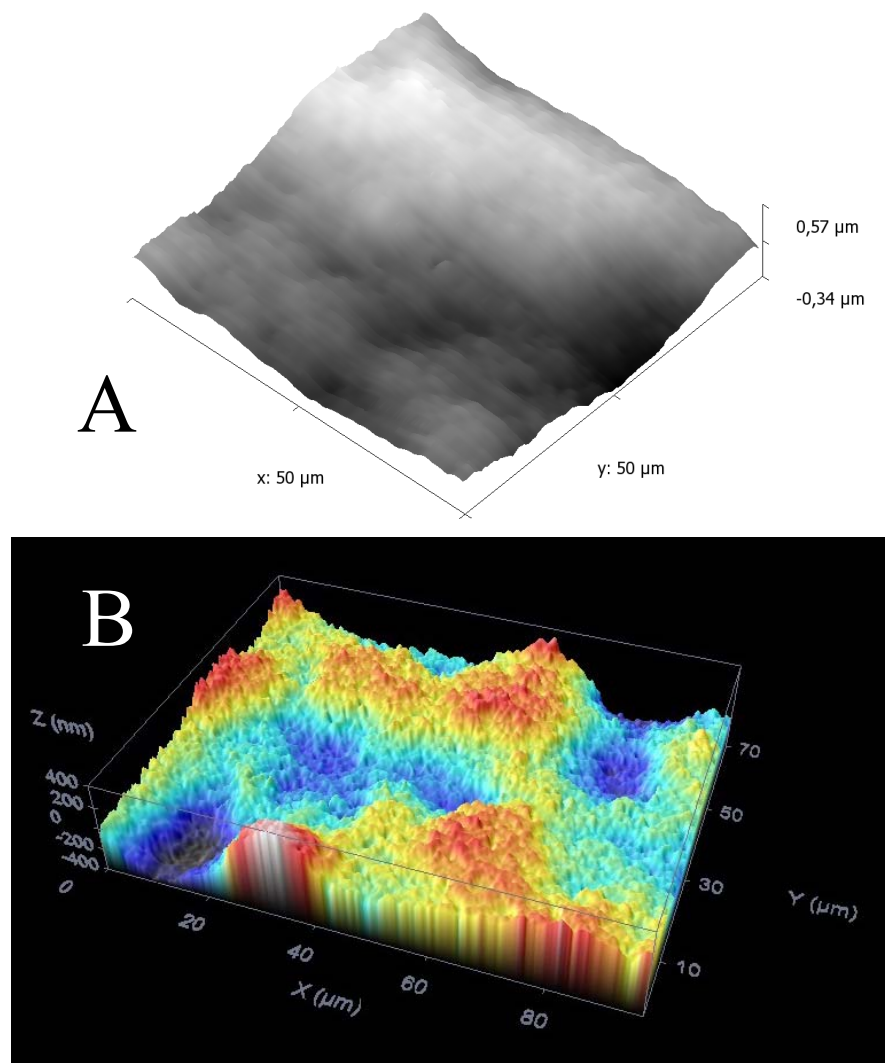

Figure 2. Surface topography of the multilayer sample measured with the nanoindenter itself (A) as well as by confocal microscopy (B). In both methods clearly a long range structure with a vertical variation on the order of $1 \mu \mathrm{m}$ can be depicted. In addition galvanic grains are present (diameters of several $100 \mathrm{~nm}$ and heights of $50-100 \mathrm{~nm}$ ).

The surface itself is relatively smooth. A galvanic structure is superimposed on a waviness with a wave length of approximately $25 \mu \mathrm{m}$ (as derived by Fourier analysis and not further discussed here). The waviness has structure heights on the order of $1 \mu \mathrm{m}$ whereas the galvanic structure height is much smaller $(\sim 50-100 \mathrm{~nm})$. Without further proof it is assumed that the galvanic structure results from the growth of individual crystals in the coating. Taking these values into account, it is not expected that the surface structure itself has a significant effect on nanoindention analysis on the original sample surface at least at indentation depths larger than 300$500 \mathrm{~nm}$.

A typical indentation experiment performed into the original sample surface is shown in Figure 3. Here in total 30 indentations have been performed to a nominal indentation depth of $4000 \mathrm{~nm}$. Figure 3A shows a typical force indentation depth curve whereas Fig. 3B shows the hardness and Fig. 3C shows the elastic modulus. The inset given in Figure $3 \mathrm{~A}$ shows an optical micrograph of a typical indentation. Here, a significant pile-up effect can be seen for this system. To achieve $4 \mu \mathrm{m}$ of indentation depth a force of approximately $580 \mathrm{mN}$ has to be applied. In addition to the individual hardness and elastic modulus traces, in Figures $3 \mathrm{~B}$ and $\mathrm{C}$, also the average value as a function of depth is shown.
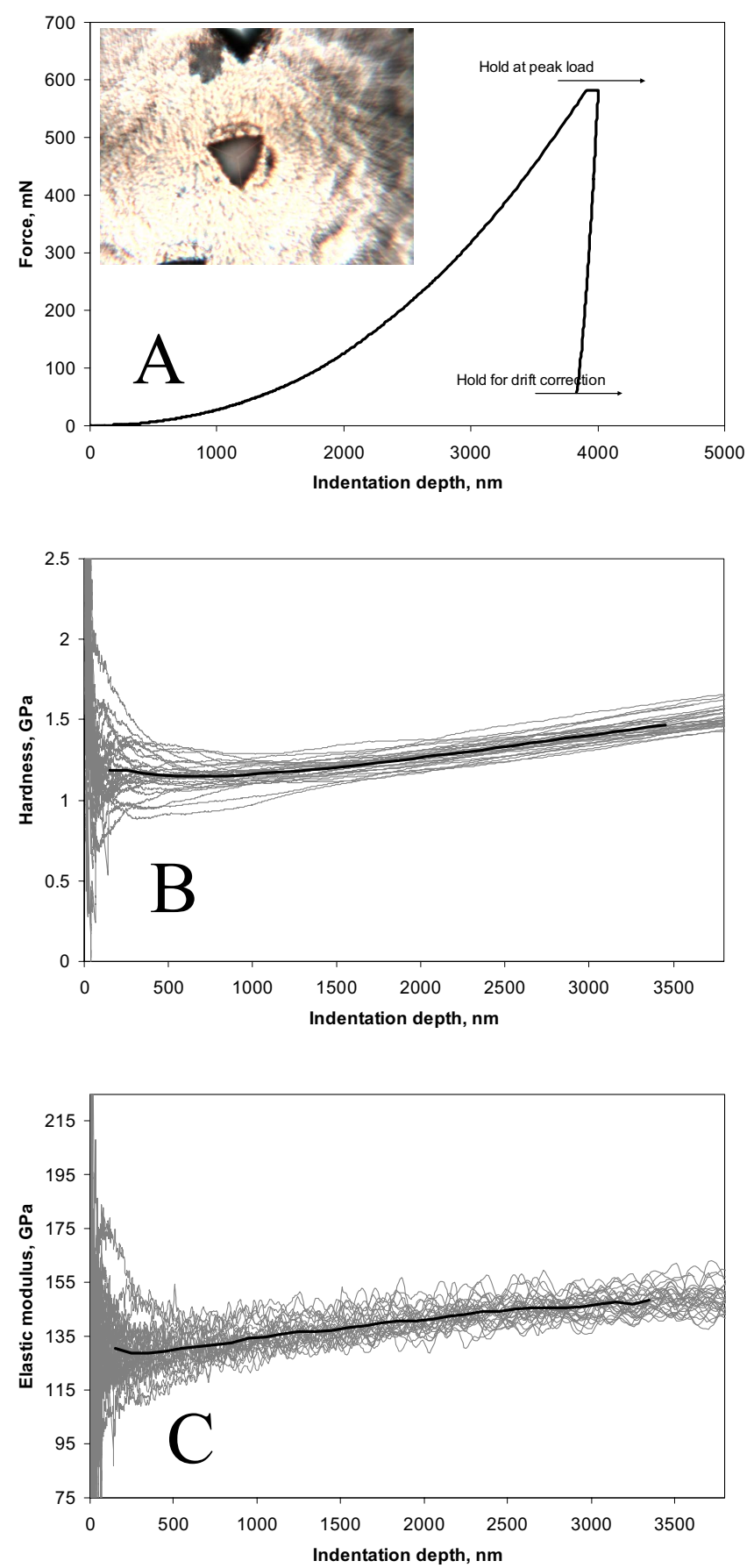

Figure 3. Indention into the model system on the original sample surface. A) Example of one indentation: Force as a function of indentation depth. An inset showing an example indent into the sample surface is also shown. B) Hardness as function of indentation depth (CSM values, average value is superimposed on the data), C) Elastic modulus as function of indentation depth (CSM values, average value is superimposed on the data). 
For hardness, apart from some indentation size effects at very low indentation depth (and probably some topography effects as well, see above) up to an indentation depth of roughly $1000 \mathrm{~nm}$ a flat hardness evolution with depth is observed. Above $1000 \mathrm{~nm}$ hardness increases monotonically from an average value of $1.15 \mathrm{GPa}$ (which is representative for the uppermost $\mathrm{Cu}$ layer) to $1.47 \mathrm{GPa}$ at $3500 \mathrm{~nm}$. This increase in hardness clearly is due to the substrate effect. As can be seen in Figure 1, below the first $\mathrm{Cu}$ layer a Ni layer is placed. The hardness of $\mathrm{Ni}$ is on the order of $8 \mathrm{GPa}$ (the exact value cannot be measured directly on the surface, see discussion below). Therefore at depths larger than $1000 \mathrm{~nm}$ a combined hardness depending on the size and shape of the plastic zone underneath the indenter is observed. If it is assumed that the plastic zone extends to approximately $10 \times$ the indentation depth then a layer thickness of the outermost $\mathrm{Cu}$ layer of $10 \mu \mathrm{m}$ would be deduced (which is fairly close and in agreement to the value of $8.3 \mu \mathrm{m}$ given in Table 1$)$.

For the elastic modulus a significant increase is already observed at $\sim 500 \mathrm{~nm}$ implying that the elastic interaction has a larger interaction volume than the plastic interaction characteristic for the hardness values discussed above. In addition at around $2500 \mathrm{~nm}$ the increase of the elastic modulus with depth becomes smaller. This effect might be explained by the fact that the elastic zone already extends into the second $\mathrm{Cu}$ layer $(\mathrm{Cu} 2$, Table 1, see also Figure 1).

In principle, the interaction of the plastic as well as the elastic zone with the different layers of the sample could be analyzed by FEM (finite element model) simulation [4]. Also theoretical approaches to hardness exist which relate the mean contact pressure to the development of a hydrostatic core directly underneath the indenter (expanding cavity models, [4]). This core is surrounded by a plastic zone (stress above the critical flow stress) and an elastic zone. However such a treatment is beyond the scope of the present study.

In [4] a summary of different equations trying to relate the effective hardness or elastic modulus of thin films to the individual properties of the film and substrate materials is given. However, these models depend on the relative ratio of the properties of the materials and no general theory exists.

For hardness, in the case of a soft film on a hard substrate (which is used as a realistic approximation for the present case with a soft $\mathrm{Cu}$ film, hardness $\sim 1.2 \mathrm{GPa}$, on a $\mathrm{Ni}(\mathrm{P})$ film, hardness $\sim 8 \mathrm{GPa}$, see below), (5) was proposed by [5]:

$$
\begin{aligned}
& H_{\text {eff }}=H_{s}+\left(H_{f}-H_{s}\right) \exp \left[-C\left(\frac{h}{t}\right)^{2}\right] \\
& C=\frac{Y_{f}}{Y_{S}} \frac{E_{S}}{E_{f}}
\end{aligned}
$$

In Equation 5 the subscripts $\mathrm{f}$ and $\mathrm{S}$ denote the properties of the film and substrate, respectively, $\mathrm{H}$ is hardness, $\mathrm{Y}$ is yield stress, $h$ is the indentation depth and $t$ is the thickness of the coating $(8.3 \mu \mathrm{m}$ in this case, see Table 1). For further calculation, for the hardness of $\mathrm{Cu}$ a value of $1.15 \mathrm{GPa}$ as deduced from the average hardness curve (excluding possible indentation size and surface effects, see above) and for $\mathrm{Ni}$ a value of $8 \mathrm{GPa}$ will be applied. Especially the latter value is subject to significant uncertainty because first it cannot be measured directly from the data presented to this point (although the value is representative for $\mathrm{Ni}$ as shown below) and second the approximation of [5] does not take the effect of a multilayer into account (hence any effect of the underlying soft $\mathrm{Cu}$ material is ignored). Figure 4 shows an overlay of the average hardness curve with results obtained using (5). In doing so, the value of $\mathrm{C}$ in (5) - ratios of yield stresses and elastic modules - was optimized in order to get the best fit. Taking all sources of possible deviations from the assumptions of the model into account the general form of (5) seems to reproduce the observed trend for hardness fairly well.

As further discussed in [4] the elastic modulus of a film substrate system can theoretically also be calculated. As a first order approximation, the elastic modules of the film and substrate are coupled by a series combination. Both, substrate and film contribute to the measured value at any indentation depth. [6] gives an approximation for the effective modulus of the total system in terms of the properties of the indenter, the substrate and the film. However, these equations incorporate an empirical constant which is unknown for the present system. In addition, values for the combined effective modulus would be required.

The model of [7] gives the effective modulus of the film substrate system in terms of the ratio of the film thickness and the contact radius. However, as discussed in [4] approximations of this kind are more suitable for hard films on soft substrates. In addition, as shown in the evolution of the elastic modulus as a function of depth and discussed above, the underlying layers (at least $\mathrm{Cu} 2$, see Table 1) play a significant role for the combined effect. Thus no further evaluations of the elastic modulus as a function of depth using analytical models are pursued in this study.

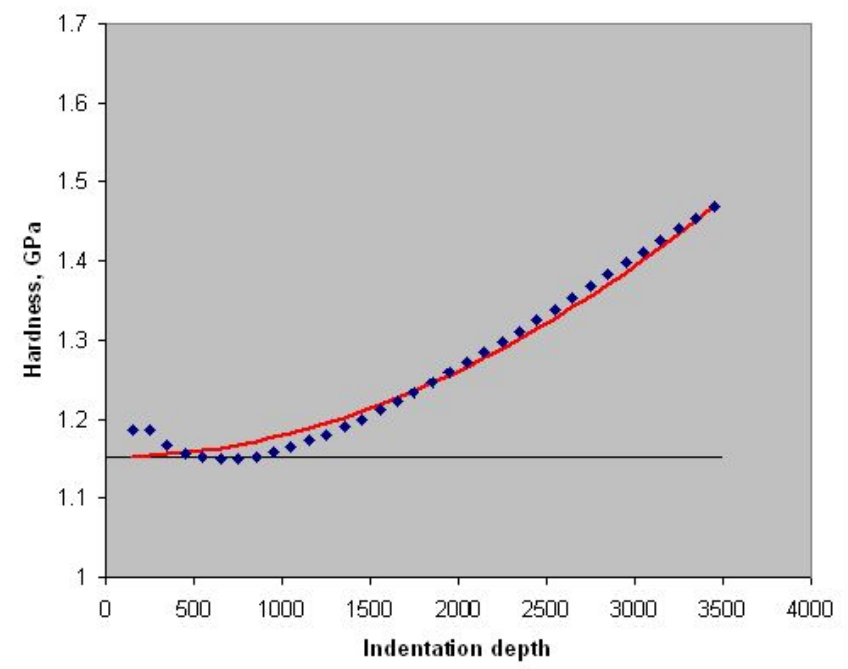

Figure 4. Average hardness as a function of depth for the data shown in Figure 3B. The average value representative of $\mathrm{Cu}$ is $1.15 \mathrm{GPa}$ (see text) and also shown as a horizontal black line. Superimposed onto the average hardness data is a calculation using the model of [5] as discussed in the text. 


\section{NANOINDENTATION PROFILES OBTAINED ON SAMPLE CROSS SECTIONS}

In the following, profile analyses of hardness measurements on a cross section of the model multilayer are described. Cross sections were prepared by fixing and positioning of the sample in embedding resin. The sample was then grinded with $\mathrm{SiC}$ paper and subsequently polished with a diamond emulsion down to $1 \mu \mathrm{m}$.

As a first step for indentation analysis, 1D-profiles were measured employing different indentation depths in order to be able to see any indentation size related resolution effects. For obtaining the largest possible resolution perpendicular to the individual layers, instead of orienting the profile directly perpendicular to the layers, the profiles were conducted at an oblique angle. In this case use is made of the fact that the layers themselves have to a first order approximation a planar geometry in space. Hence by using an oblique angle no interference of single indents is present (because a larger point distance can be used) at the same time ensuring a high resolution in the direction perpendicular to the layers (see also Figure 1 for an example). Table II lists the different profiles measured, the indentation depth, the angle between the profile and the interfaces $(\alpha)$ as well as the component of the displacement of the individual indents in the direction perpendicular to the interfaces. The last value will be used in subsequent plots of the profiles.

TABLE II. PARAMETERS FOR 1D-PROFILE ANALYSIS (PROFILES 1-3). THE ANGLE ALPHA IS BETWEEN THE PROFILE DIRECTION AND THE LAYER INTERFACE. THE POINT (PNT) DISTANCE IS RECALCULATED FOR THE COMPONENT PERPENDICULAR TO THE INTERFACES (YCOMP).

\begin{tabular}{|l|l|l|l|l|}
\hline $\begin{array}{l}\text { Profile } \\
\text { No. }\end{array}$ & $\begin{array}{l}\text { Indentation } \\
\text { depth, nm }\end{array}$ & Angle $\alpha$ & $\begin{array}{l}\text { Pnt- } \\
\text { Distance, } \\
\mu \mathrm{m}\end{array}$ & $\begin{array}{l}\text { Ycomp, } \\
\mathrm{nm}\end{array}$ \\
\hline 1 & 200 & 4.6 & 5 & 401 \\
\hline 2 & 100 & 4.7 & 5 & 410 \\
\hline 3 & 50 & 2.1 & 5 & 184 \\
\hline
\end{tabular}

The individual profiles are shown in Figure 5. In Figures $5 \mathrm{~A}$ and $5 \mathrm{~B}$ both, values determined from the unloading curve as well as from CSM measurements, are shown, whereas Figure 5C only shows values from the CSM measurements. The latter was chosen for data analysis reasons: In order to derive the initial slope of the unloading curve a suitable part has to be selected (normally $50 \%$ ). However, at such small indentation depths no single condition was found that gave a meaningful value for all measurements. In order not to introduce additional uncertainties, values from unloading measurements are not shown. Table III gives an overview of CSM values for the hardness as well as for elastic modulus from all layers down to $\mathrm{Cu} 4$ (if possible). The table lists average values in the case that a clear plateau can be identified in Fig. 5. If only a peak can be distinguished (meaning that the lateral resolution of the measurement is at least on the order of the thickness of the layer) the maximum value is used.
Several points can be made from a close inspection of the data given in Table III. If the layer thickness is sufficient to be resolved by all sizes of the indents (with indentation depths of $200 \mathrm{~nm}, 100 \mathrm{~nm}$ and $50 \mathrm{~nm}$ ) in general there appears to be an increase for both properties when the indentation depth decreases. This indentation size effect might be explained by sample preparation where the near-surface structure and hence physical properties are changed due to cold working. This observation is supported by the fact that the average hardness of $\mathrm{Cu}$ is $1.2 \mathrm{GPa}$ and the average elastic modulus is $129 \mathrm{GPa}$ deduced from the surface measurement whereas the lowest values for the same properties obtained using Table III are 1.6 $\mathrm{GPa}$ (hardness) and $136 \mathrm{GPa}$ (elastic modulus).
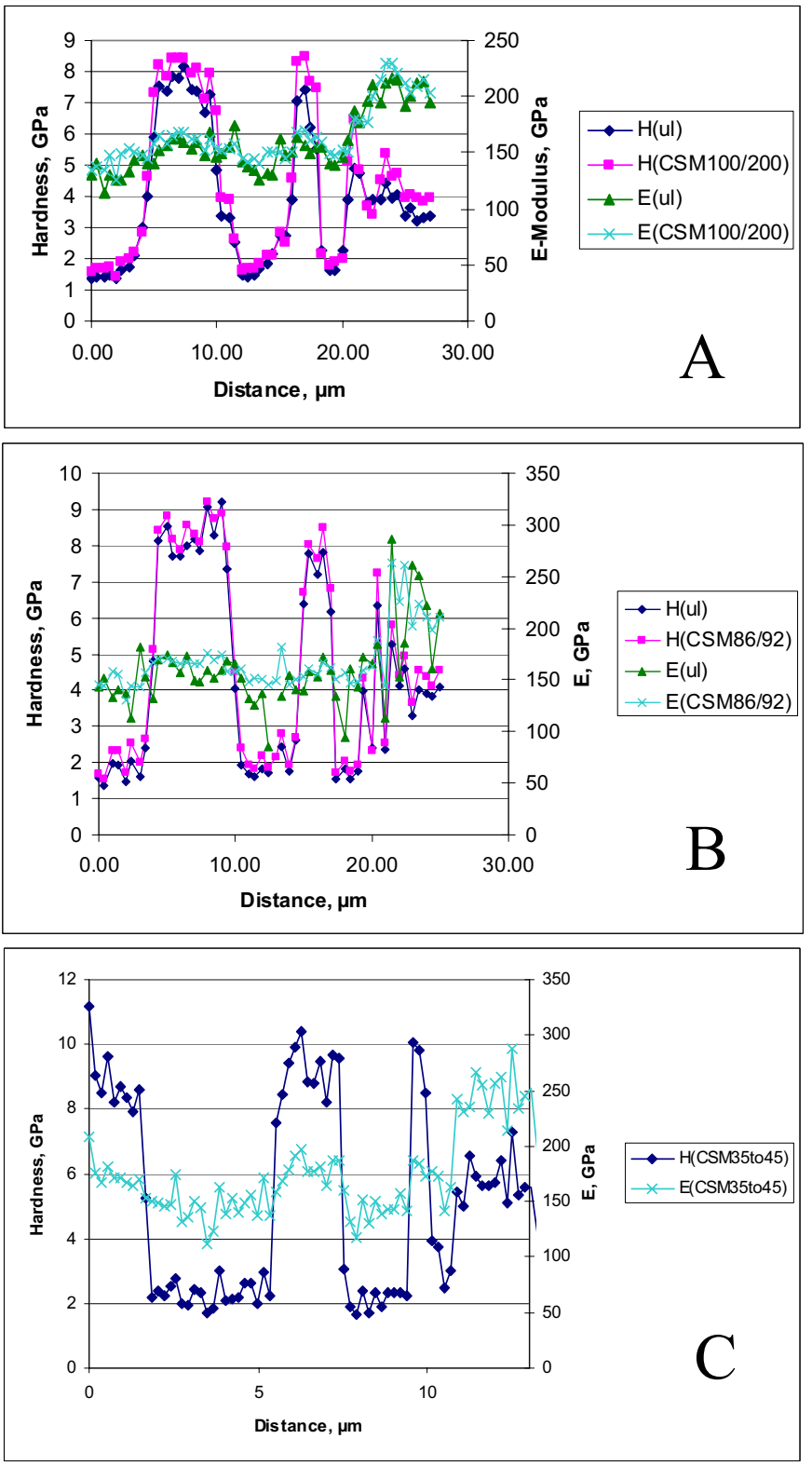

Figure 5. Profiles 1-3 as described in the text and in Table II. Profile 1 in A) and Profile 2 in B) show both CSM as well as values from the unloading curve, whereas in C), profile 3, only CSM values are given. Hardness values are shown on the primary axis and the elastic modulus is given on the secondary axis. 
TABLE III. OVERVIEW OF HARDNESS H CSM AND ELASTIC MODULUS E $_{\text {CSM }}$ VALUES EXTRACTED FROM THE PROFILES SHOWN IN FIGURE 5. ONLY VALUES OBTAINED BY THE CSM METHOD ARE LISTED. VALUES FOR ALL LAYERS UP TO CU4 (TABLE 1) EXTRACTED FROM ALL PROFILES (P1, P2, P3) ARE LISTED IF APPLICABLE. PROFILE 3 DOES NOT INCLUDE CU1. ALSO, IF THE RESOLUTION IS NOT SUFFICIENT TO RESOLVE A LAYER NO VALUES ARE GIVEN (N.D.). IN ADDITION THE NUMBER OF POINTS IS GIVEN THAT FORM A PLATEAU IN THE PROFILE AND ARE USED FOR AVERAGING.

\begin{tabular}{|c|c|c|c|}
\hline Layer & $\mathbf{H}_{\mathrm{CSM}}$ & $\mathbf{E}_{\mathrm{CSM}}$ & Points \\
\hline $\mathrm{Cu} 1, \mathrm{P} 1$ & $1.6(0.1)$ & $136(8)$ & 5 \\
\hline $\mathrm{Cu} 1, \mathrm{P} 2$ & $2.0(0.3)$ & $146(9)$ & 7 \\
\hline $\mathrm{Cu} 1, \mathrm{P} 3$ & n.d. & n.d. & n.d \\
\hline Ni1, P1 & $8.0(0.4)$ & $162(5)$ & 9 \\
\hline Ni1, P2 & $8.5(0.4)$ & $169(4)$ & 10 \\
\hline Ni1, P3 & $8.9(1.0)$ & $175(13)$ & 9 \\
\hline $\mathrm{Cu} 2, \mathrm{P} 1$ & $1.7(0.1)$ & $144(2)$ & 3 \\
\hline $\mathrm{Cu} 2, \mathrm{P} 2$ & $2.1(0.3)$ & $154(12)$ & $8(7)$ \\
\hline $\mathrm{Cu} 2, \mathrm{P} 3$ & $2.3(0.4)$ & $146(15)$ & 20 \\
\hline $\mathrm{Ni2}, \mathrm{P} 1$ & $8.4(0.1)$ & $169(2)$ & 2 \\
\hline $\mathrm{Ni} 2, \mathrm{P} 2$ & $8.0(0.4)$ & $161(6)$ & 3 \\
\hline $\mathrm{Ni2}, \mathrm{P} 3$ & $9.3(0.7)$ & 181(10) & 10 \\
\hline $\mathrm{Cu} 3, \mathrm{P} 1$ & $1.8(0.1)$ & $148(1)$ & 2 \\
\hline $\mathrm{Cu} 3, \mathrm{P} 2$ & $1.9(0.2)$ & $150(4)$ & 4 \\
\hline $\mathrm{Cu} 3, \mathrm{P} 3$ & $2.1(0.3)$ & $141(11)$ & 10 \\
\hline Ni3, P1 & n.d. & n.d & n.d. \\
\hline $\mathrm{Ni3}, \mathrm{P} 2$ & n.d. & n.d. & n.d. \\
\hline Ni3, P3 & $9.5(0.8)$ & 181(8) & 3 \\
\hline $\mathrm{Cu} 4, \mathrm{P} 1$ & n.d. & n.d. & n.d. \\
\hline $\mathrm{Cu} 4, \mathrm{P} 2$ & n.d. & n.d. & n.d. \\
\hline $\mathrm{Cu} 4, \mathrm{P} 3$ & $2.5(\mathrm{na})$ & 151(na) & 1 \\
\hline
\end{tabular}

The values observed in this study are significantly different from values for pure $\mathrm{Cu}$ and $\mathrm{Ni}: \mathrm{E}(\mathrm{Ni})=200 \mathrm{GPa}$, $\mathrm{H}(\mathrm{Cu}>99.95 \%)=0.5 \mathrm{GPa}$ and $\mathrm{E}(\mathrm{Cu}>99.95 \%)=110 \mathrm{GPa}[4]$ although it should be noted that the galvanic coatings investigated here are structurally and chemically very different (especially Ni with $\sim 8 \% \mathrm{P}$ ) than the bulk materials.

With respect to resolution, especially for hardness, there is a clear decrease in lateral resolution when decreasing the indentation depth. For example layers Ni3 and $\mathrm{Cu} 4$ can only be resolved in Profile 3 where an indentation depth of $50 \mathrm{~nm}$ is used. Hence, in this case the resolution of the measurement (and therefore the extension of the plastic zone) is on the order of $1 \mu \mathrm{m}$. On the other hand, Profile 1 with $200 \mathrm{~nm}$ indentation depth shows approximately 5 measurement points with mixed values going from one layer to the other (for example from $\mathrm{Cu} 1$ to Ni1). Thus the resolution in this case is $2 \mu \mathrm{m}$ at best (hence just sufficient to verify the presence of layer $\mathrm{Cu} 3$ ). These values imply that the lateral resolution is on the order of 10 to 20 times the indentation depth for hardness. For the elastic modulus the situation is not straightforward: The values obtained are relatively similar (but significantly different) for $\mathrm{Cu}$ and Ni. Therefore it is difficult to clearly distinguish if single measurements are affected by resolution or not. Hence the remarks given on resolution above are mainly valid only for hardness. In addition Profile 1 in Figure 5A also shows a marked asymmetry of the profiles. On the left hand side of a layer the values seem to increase "earlier" than on the right hand side. This observation might reflect an asymmetry of the plastic and elastic zone underneath the indenter resulting from the triangular indenter symmetry (compare also with Figure 1 where the line of large indents represents Profile 1).

As discussed above due to the fact that the line orientation is inclined with respect to the layer interfaces the spacing between different indents can be chosen relatively large while preserving a high resolution perpendicular to the layers without any interaction between individual indents. This fact is used for another approach for discussing resolution of indentation measurement of the model system used in this study: In Profile 4 (Figure 6) relatively large indents with a nominal indentation depth of $1500 \mathrm{~nm}$ are used. However, due to the CSM method used, hardness and elastic modulus can be calculated for any depth.

In Figure 7 the profile is shown in the form of hardness at given depth intervals as a function of distance (perpendicular to the interface of the layers). With increasing depth of evaluation of the CSM values the profiles tend to "smear out" meaning that more and more mixed analyses are obtained.

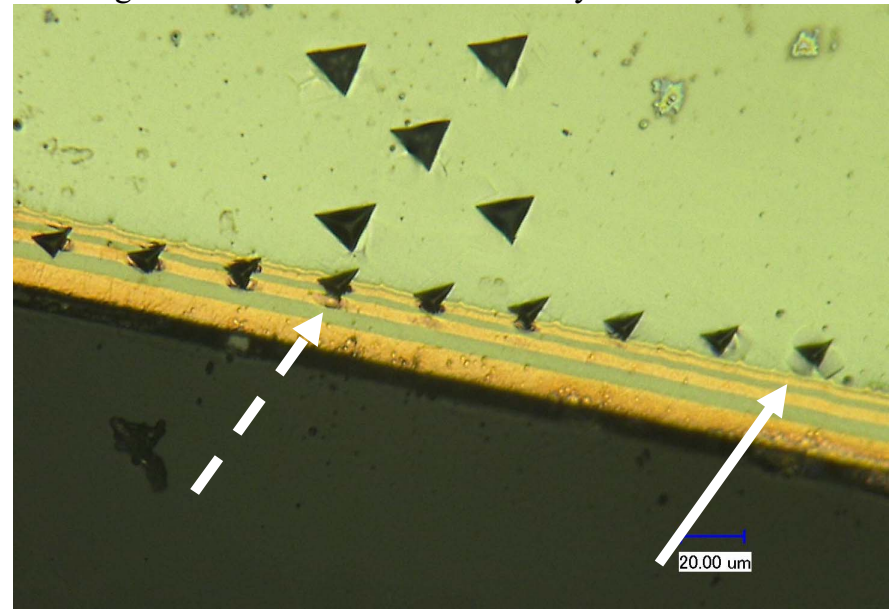

Figure 6. Profile 4. The regular array of 5 indents at the top was first used to calibrate the microscope to indenter position. The measured profile is at an angle of $3.4^{\circ}$, the distance between individual measurement points is $30 \mu \mathrm{m}$ and a nominal indentation depth of $1.5 \mu \mathrm{m}$ was used.

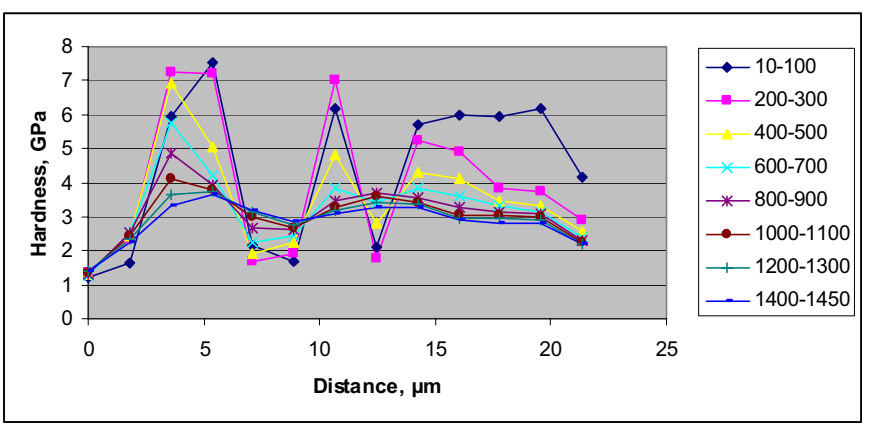

Figure 7. Hardness values calculated for Profile 4 (Figure 6). All values are CSM values for the depth intervals given in the figure legend in $\mathrm{nm}$. Note that the distance between different points perpendicular to the layer interfaces (1.8 $\mu \mathrm{m}$ ) is significantly larger than for the profiles 1-3 (Figure 5). Hence a sampling error is introduced and layers with thicknesses below that distance are not shown in the profile. However the resolution of individual points (extension of plastic zone) is not influenced. See text for further discussion. 


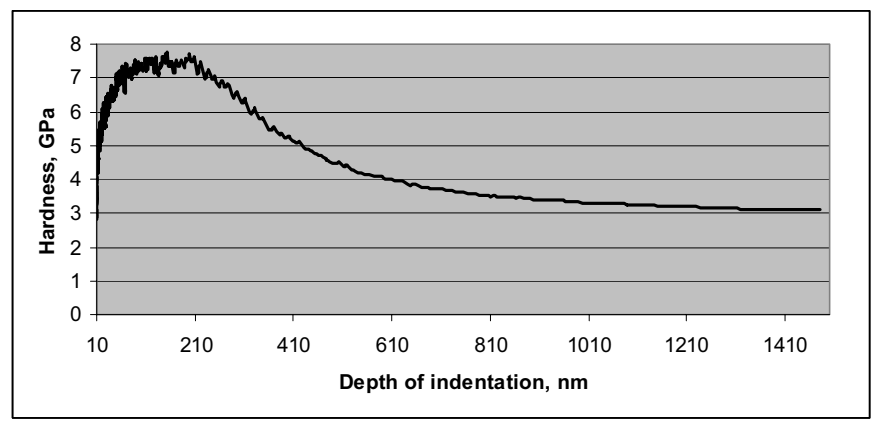

Figure 8. Hardness trace for the indent at $10.7 \mu \mathrm{m}$ distance in the profile shown in Figure 7. This indent is also marked in Figure 6 by a dashed arrow.

In Figure 8 hardness is shown as a function of depth of a selected indent (at position $10.7 \mu \mathrm{m}$ in Figure 7). The indent itself is marked by the dashed arrow in Figure 6. The indent is performed close to the border of $\mathrm{Ni} 2$ to $\mathrm{Cu} 3$ (Table 1). Hence initially at low indentation depth a mixed value of $\mathrm{Cu}$ and $\mathrm{Ni}$ is observed. With increasing depth the plastic zone mainly develops in the Ni layer and a maximum of hardness at values of $\sim 7.5 \mathrm{GPa}$ is reached. With further increase of the depth of indentation especially the layer $\mathrm{Cu} 2$ seems to contribute to an increasingly lower hardness value that at the maximum indentation depth is on the order of $3 \mathrm{GPa}$. A pronounced plastic deformation (pile-up effect) in $\mathrm{Cu} 2$ can be seen in Figure 6 after complete indentation.

More generally, for most of the indentations shown in Figure 6 a significant deformation difference between $\mathrm{Cu}$ and $\mathrm{Ni}$ exists: $\mathrm{Cu}$ shows clear indications of pile-up (restricted to the planar geometry of the layer) whereas this effect is much less clear in Ni. This finding implies a lower yield stress for the $\mathrm{Cu}$ layer than for $\mathrm{Ni}$ which is consistent with the constant $\mathrm{C}$ in (5). For the plot in Figure 4 a value for $\mathrm{C}$ of 0.4 was used. The ratio of the elastic modules $\mathrm{E}_{\mathrm{s}} / \mathrm{E}_{\mathrm{f}}$ in $\mathrm{C}$ is on the order of 1.2 (calculated using values from Table 1). Therefore a ratio $\mathrm{Y}_{\mathrm{f}} / \mathrm{Y}_{\mathrm{S}}$ of $\sim 0.3$ is derived using (5) for the different layers $(\mathrm{Ni}$ as substrate, $\mathrm{Cu}$ as film). The interaction of the plastic zone with the layer interfaces can also be seen in Figure 6 for the steel substrate (solid arrow in Fig. 6). The observation of strong interactions of the plastic zone with the individual layers implies also that models for bulk samples or thin films would have to be extended in the case of composite materials in cross section (e.g. calculation of the size of the plastic zone, radius of the hydrostatic core etc.). This finding also applies for small inclusions in a mechanically different matrix.

\section{2D MAPPING OF HARDNESS}

In the foregoing paragraphs the mechanical properties were measured ("mapped") in a 1-dimensional way (property as a function of a distance). If the 1-dimensional measurement is extended into two directions in a matrix fashion a 2D-map of hardness becomes possible. This section will be restricted towards hardness because as discussed above the observed differences in elastic modulus for the 2 layers $(\mathrm{Ni}$ and $\mathrm{Cu})$ are limited. Such a matrix of measurement points after measurement is shown in Figure 1. The positioning of the sample tray is achieved by mechanical movement. As can be seen in Figure 1, over the long period of the measurement, a deviation of the ideal positions arises. In principle this effect can be corrected by careful measurement of the positions after indentation for example by scanning electron microscopy (SEM) and application of a correction matrix for further evaluation. For the sake of the present discussion this procedure was not further pursued.

Unlike for 1-dimensional profiles an oblique orientation cannot be used in order to reduce the resolution with respect to positioning. Therefore the distance between individual indents in the 2D-matrix must be carefully chosen in order to avoid overlapping of the interaction volumes (plastic zone in the case of hardness). As shown above, at least a factor of 10 should be used between the depth of indentation and the distance between individual measurement points. The matrix shown in Figure 1 was performed using a nominal indentation depth of $100 \mathrm{~nm}$ and a distance of $1 \mu \mathrm{m}$ between individual measurement points. The hardness traces for all measurements are further analyzed in Figure 9: Here the distribution function of hardness is plotted by dividing the CSM data between 80 and $95 \mathrm{~nm}$ into 19 classes. The 3 different components, Ni-layer, $\mathrm{Cu}-$ layer and steel substrate, can be distinguished by the corresponding maxima of the distribution function (Ni: $\sim 8 \mathrm{GPa}$, steel $\sim$ $5 \mathrm{GPa}, \mathrm{Cu} \sim 2 \mathrm{GPa}$ ). In addition, one example trace of hardness as a function of indentation depth for $\mathrm{Cu}$ and for $\mathrm{Ni}$ is also shown in Figure 9. Especially traces corresponding to indentation into Ni show a relatively large number of so-called pop-in events. Such events are usually interpreted to be connected with sudden microstructure responses associated with movement of dislocations (e.g. [8]).

In Figure 10 a 3D representation of the whole dataset is given. The results are consistent with the 1D-profiles (Figure 4B): The layers can be clearly resolved up to the third $\mathrm{Cu}$ layer ( $\sim 2 \mu \mathrm{m}$ thickness, Table 1$)$ whereas the third Ni layer and the subsequent layers $(\sim 1 \mu \mathrm{m}$ thickness) cannot be identified separately. Thus, again, a lateral resolution of the indents of 1020 times the indentation depth is observed. Hence, the point distance in the matrix of $1 \mu \mathrm{m}$ for an indentation depth of $100 \mathrm{~nm}$ should be regarded as minimum point distance that could be used for the present configuration.

\section{CONCLUSIONS}

The current investigation was motivated by the necessity of understanding the mechanical properties of functional thin layers used in electrical sliding contacts. Due to wear the thin films may have thickness well into the nanometer scale and might be subjected to strong microstructural changes induced by cold-working (sliding), multiple cold-welding as well as high temperatures (frictional heating, constriction resistance, sparking). In this respect especially hardness and elastic modulus are fundamental mechanical properties. In this study, the investigation was focused onto an understanding to what resolution these properties can be investigated using modern nanoindentation equipment. It was found that apart from possible artifacts due to cross sectioning (sample preparation) the measurements can be used to determine the local mechanical properties down to a scale of approximately $1 \mu \mathrm{m}^{3}$. By using a stage automation also $1 \mathrm{D}$ (profiles) as well as 2D 
maps can be derived. With such application the nanoindenter might be called a physical microscope. If indentation depths of less than 50-100 nm are used even nanometer resolution might be possible. The results show that in the case of composite samples (here multilayers, but also inclusions etc.) the interaction are of a complex nature because of the difference in mechanical properties of the constituent phases. The plastic zone develops differently in the case of the $\mathrm{Ni}$ and $\mathrm{Cu}$ layers observed in this study (e.g. pile-up confined mainly to $\mathrm{Cu}$ ).
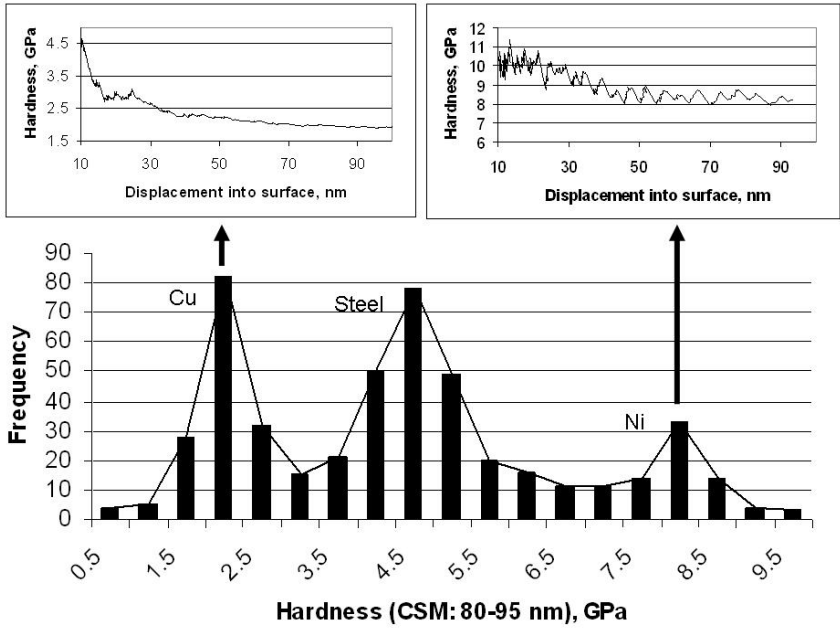

Figure 9. Distribution function of hardness values (CSM values between 80 and $95 \mathrm{~nm}$ ) for all measurements of the matrix shown in Figure 1 and described in Section V. Note clustering of values at approximately $2 \mathrm{GPa}, 5$ $\mathrm{GPa}$ and $8 \mathrm{GPa}$ corresponding to $\mathrm{Cu}$, steel and $\mathrm{Ni}$, respectively. In addition for $\mathrm{Cu}$ and $\mathrm{Ni}$ two example hardness traces are shown.

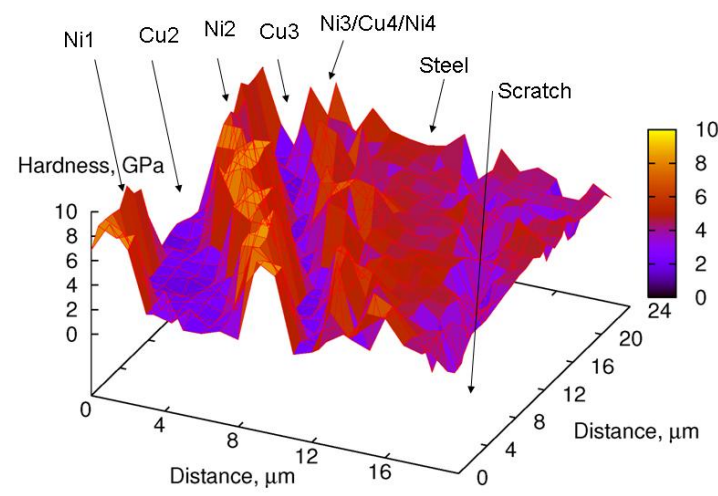

Figure 10. 3D representation of hardness values derived from a matrix measurement (see Fig. 1, 8, and Section III). In this case CSM values between 80 and $95 \mathrm{~nm}$ were used.

\section{ACKNOWLEDGMENT}

Sample preparation was kindly performed by $P$. Heinbuch. Confocal microscopy was performed at the Laboratory for Microsystems technology at the University of Applied Sciences, Munich. All help in 3D surface analysis by H. Herberg and M. Kaiser is gladly appreciated.

\section{REFERENCES}

[1] C. Holzapfel, "Nanoindentation studies of contact materials used for sliding electrical contacts," Proc. 54 ${ }^{\text {th }}$ IEEE Holm Conference, pp. 9097, 2008.

[2] H. Czichos and K.-H. Habig, Tribologie-Handbuch, $2^{\text {nd }}$ ed., Wiesbaden: Vieweg, 2003.

[3] A. G. Tangena, Tribology of Thin Film Systems, phd-thesis, Philips Natuurkundig Laboratorium, 1987.

[4] A. C. Fischer-Cripps, Nanoindentation, $2^{\text {nd }}$ ed., New York: Springer, 2004

[5] A. K. Bhattacharya and W. D. Nix, "Finite element simulation of indentation experiments," J. Mater. Res., vol. 18, pp. 1383-1391, 1988.

[6] R. B. King, "Elastic analysis of some punch problems for a layered medium," Int. J. Solids Structures, vol. 23, pp. 1657-1664, 1987.

[7] H. Gao, C.-H. Chiu, and J. Lee, "Elastic contact versus indentation modeling of multi-layered materials," Int. J. Solids Structures, vol 29, pp. 2471-2492, 1992.

[8] D. Lorenz, Untersuchungen zur homogenen Versetzungsnukleation mittels Nanoindentierung, phd-thesis, University of Halle, 2001.

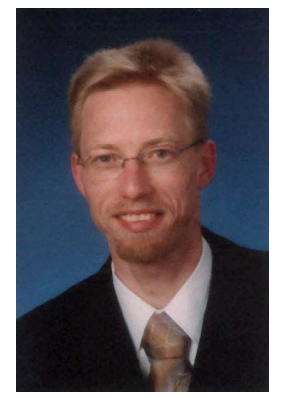

Christian Holzapfel received his diploma thesis and phd from the university of Cologne working in the field of thermodynamics and diffusion kinetics. During this time he also worked for 4 years at the Bayerisches Geoinstitut in Bayreuth. After finishing his phd he worked 3 years as laboratory supervisor for the Nanolab at the department of functional materials at the University of the Saarland in Saarbrücken. Here his main research work was in the field of $3 \mathrm{D}$ characterization of microstructures e.g. in plasma erosion phenomena as well as in the development of techniques using a focused ion beam. Since December 2006 he is working at Schleifring und Apparatebau GmbH in Germany in the development of contacting slip ring applications. Since 2008 he serves as group manager of the group development contact materials. 


\title{
Growth Law of the Oxide Film Formed on the Tin Plated Contact Surface and Its Contact Resistance Characteristic
}

\author{
Yuuya Nabeta $^{1}$, Yasushi Saitoh ${ }^{1}$, Shigeru Sawada ${ }^{1}$, Yasuhiro Hattori ${ }^{2}$ and Terutaka Tamai ${ }^{1}$ \\ ${ }^{1}$ Mie University, Graduate School of Engineering, Laboratory of Vehicle Network Technology \\ Laboratory \\ 1577 Kurimamachiya-cho, Tsu city, Mie 514-8507, Japan \\ Email:tamai@elec.mie-u.ac.jp \\ ${ }^{2}$ AutoNetworks Technologies, Ltd.
}

\begin{abstract}
Tin plating has been applied widely to electrical connectors to save the cost for electromechanical devices. However, the tin plated surface covered with oxide film is fundamentally different from gold plated surfaces. The oxide film prevents the surface from corrosion. When the film is interposed between contact interface, contact resistance increases. It is necessary to break down mechanically to obtain low contact resistance. However, detail study on the oxide film on the tin plated surfaces was not found in literatures.

On the other hand, fretting phenomena is very important for connector contacts, particularly, for automotive connectors under vibration. The failure to tin plate is many times from fretting and this is in the literature.

In the present study, growth law of the oxide film on the tin plated surface was found by measurement using an ellipsometry for exposure to the atmosphere. Moreover, the film thickness was identified by TEM (Transmission Electron Microscope). The results well agreed with results obtained by the ellipsometry. The initial oxide film formed at temperatures less than $120 \mathrm{C}^{\circ}$ is a mixture of crystalline $\mathrm{SnO}$ and an amorphous molecule form of tin oxide. However, the oxide formed on the surface exposed at $120 \mathrm{C}^{\circ}$ was identified as crystal $\mathrm{SnO}_{2}$.
\end{abstract}

As a result, growth of the oxide film showed linear law for the initial stage of the exposure. In the second stage, the oxide film indicated 1/4 law. After this stage, the film growth saturated. The contact resistance indicated low constant value until $10 \mathrm{~nm}$ in thickness. This low value is due to conduction mechanisms of thin film. For the thicker film than $10 \mathrm{~nm}$, contact resistance increased to $5 \Omega$.

Keywords- Tin plating, oxide film, contact electromechanical devices, contact resistance, ellipsometry

\section{INTRODUCTION}

Tin (Sn) plated surfaces are widely used with for certain type of connectors. Because contact resistance is increased when this film is interposed between contact interfaces, it is necessary to break down this film mechanically to obtain low contact resistance. Therefore, it is important to clarify oxide film growth [1], [2]. But detail study on the oxide film on the tin plated surface was not found previously.

In the present study, growth law of the oxide film and optical constants on the tin plated surface were clarified by using an ellipsometer for exposure to the indoor clean atmosphere. Moreover, the film thickness was identified by TEM. The results well agreed with results obtained by the ellipsometry. The initial oxide film formed at a temperature within a range between 25 and $80^{\circ} \mathrm{C}$ was identified by electron diffraction as a mixture of crystalline $\mathrm{SnO}$ and an amorphous molecular form of tin oxide, possibly $\mathrm{SnO}, \mathrm{SnO}_{2}$, and intermediate $\mathrm{Sn}_{3} \mathrm{O}_{4}$. For elevated temperature of $120^{\circ} \mathrm{C}$, oxide film was found to be crystal $\mathrm{SnO}_{2}$.

As a result, growth of the oxide film showed linear law for the initial stage of the exposure. In the later stage, the oxide film indicated 1/4 law. After this stage, growth of the film saturated for $15 \mathrm{~nm}$. The contact resistance indicated low constant value until $10 \mathrm{~nm}$ in thickness. This low value is due to conduction mechanisms of thin film. As the thickness grew higher, contact resistance increased to $5 \Omega$.

\section{EXPERIMENTAL PROCEDURE}

\section{A. Specimens}

Copper plated thinly on copper alloy with small additions of $\mathrm{Ni}$ and $\mathrm{Si}$ less than $0.1 \mathrm{wt} \%$ was used as a substrate. Tin was plated on the substrate surface, and the plated surface was subjected to reflow process. Three levels of tin plated thicknesses $(1,3,5 \mu \mathrm{m})$ were selected. Because these tin plated surfaces are covered with the oxide film and with contaminant film, the contaminations were removed by ultrasonic cleaning for five minutes in order of acetone, methanol, and distilled water. After this process, to remove the oxide film grown on the surface, the surfaces are subjected rapidly to the ion sputter in $\mathrm{N}_{2}$ gas. The ion sputter condition was selected as ion current of $3 \mathrm{~mA}$ for $30 \mathrm{sec}$. This ion sputter condition is suitable to remove oxide films and contaminations. Moreover, these values were selected from the change in the optical constants by measurement of the ellipsometry.

The clean specimen surfaces were exposed to indoor clean atmosphere of room temperature. After this exposure of indoor atmosphere, the specimens were set on the ellipsometer to measure film thickness. Furthermore, the other specimen surfaces were exposed to elevated temperature at each of 50,80 , and $120^{\circ} \mathrm{C}$ in an electrical 
furnace with clean air. After this high temperature exposure, the specimens were set on the ellipsometer to measure film thickness as same as indoor exposure.

\section{B. Measurement of thickness of surface film}

After sputtering, the cleaned surface specimen was set in the ellipsometer, and the thickness of the oxide film was measured. The ellipsometer used is single optical path method shown in Fig.1, the light source used is He-Ne laser with wavelength of $632.8 \mathrm{~nm}$, the angle of incidence to the specimen surface was set at $70^{\circ}$ [3], [7]. In addition, the clean surface was exposed in the atmosphere of clean laboratory at an elevated temperature, the growth of oxide films was measured for the oxidation time. As a result, the growth law of the oxide film on the specimen surface was judged from the data of the oxide film thickness. The optical constant was measured with the measurement of the oxide film thickness.

\section{Identification of the oxide film}

To confirm the oxide film thickness measured by the ellipsometry, the high magnification image of surface for the cross section of the tin plated layer was observed by TEM (Transmission Electron Microscope). The cross section of the specimen was prepared by FIM (Focused Ion Beam).

The surface state before and after the ion sputtering was examined in detail by observation of AFM (Atomic Force Microscope) and STM (Scanning Tunneling Microscope). Existence of the oxide film layer on the surface was identified by an electron diffraction method. Furthermore, in order to identity element of the surface formation, an element analysis of EDX (Energy Dispersion $\mathrm{X}$ ray Spectroscopy) was applied to the surface layer of the specimen. Both surfaces covered with the oxide film grown at a temperature less than $120^{\circ} \mathrm{C}$ and grown at a $120^{\circ} \mathrm{C}$ were identified.

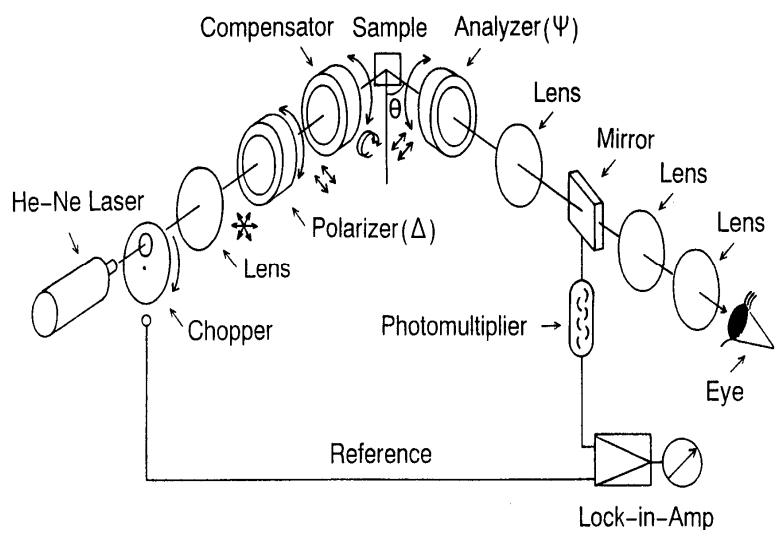

Fig.1 Ellipsometer of single optical path method used for film thickness measurement.

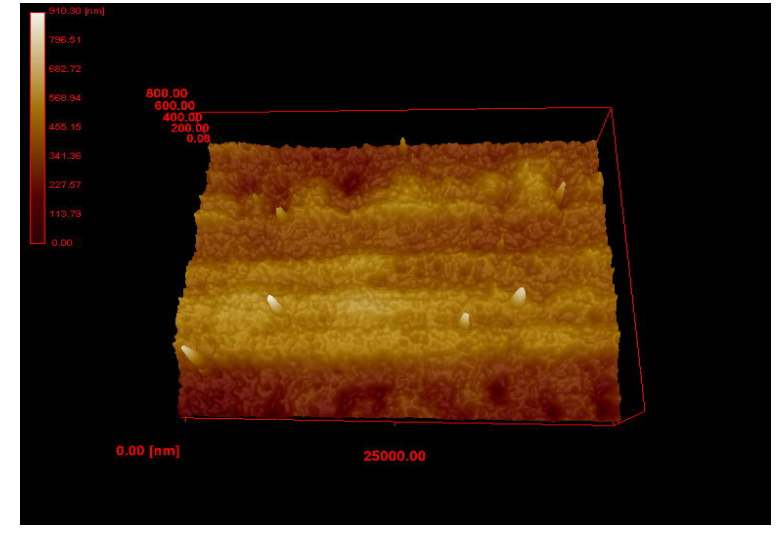

(1) Surface topography before the ion sputtering.

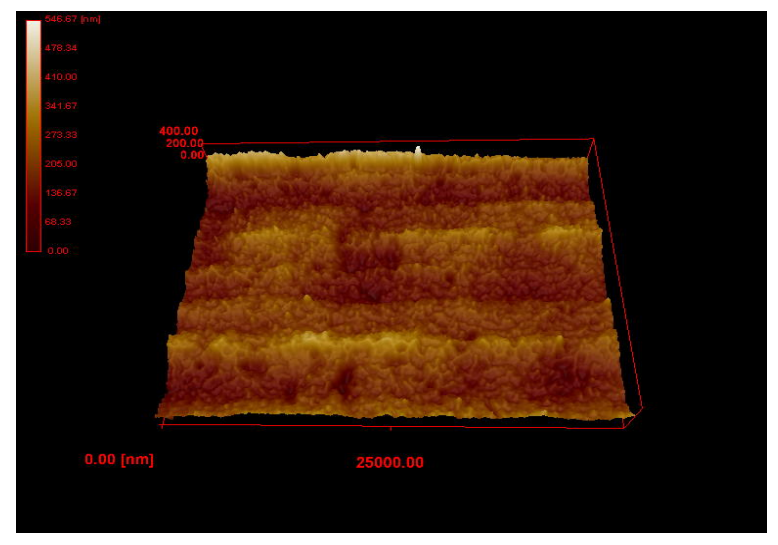

(2) Surface topography after the ion sputtering.

Fig.2 Change in surface topography by ion sputtering, thickness of the tin plated layer is $5 \mu \mathrm{m}$.

\section{Measurement of contact resistance}

For the exposed clean surfaces for both indoor atmosphere at room temperature and the elevated temperature, the contact resistance was measured at different exposure times, the influence of growth of the film on contact resistance was examined. In measurement of the contact resistance, a platinum $(\mathrm{Pt})$ hemisphere was used as one side contact member as a probe, and as opposite side contact member, tin plated flat specimen was used. The contact resistance was measured by four terminals potential drop method under $1 \mathrm{~mA}$ current.

\section{RESULTS AND DISCUSSION}

A. Topography of the surface before and after the ion sputtering and identification of the film

The changes in the AFM image topography on the surface before and after the ion sputtering are shown in Fig.2. As the copper base alloy of the substrate was made by rolling process, line asperities existed on the surface along rolling 
direction. It is expected that the surface roughness remains the same even after a thin outermost surface layer is removed by the ion sputtering. Influence of the line asperities on optical constant is measured by rotating the surface under the ellipsometer. The results are shown in Fig.3, 4.

\section{B. The anisotropy of the optical constants}

Because the tin plated substrate is the copper alloy with small additions of $\mathrm{Ni}$ and $\mathrm{Si}$ subjected to rolling process, the line asperities due to rolling is recognized. This influences the tin plated surface, and its surface topography is shown in the AFM image in Fig.2. The influence of the sputter on the occurrence of small roughness on the surface is shown. In the comparison of wavelength $(632.8 \mathrm{~nm})$ of light source with the surface roughness, it is understood that there is no influence in the optical constants.

The measurement result of the optical constant in the rotation of every $45^{\circ}$ on the specimen surface is shown in Fig.3, 4. It is shown that anisotropy on the surface exists as change of the optical constants. However, this tendency is not so much for the surface after the sputter. In this fact, the atomic structure of the surface depend on the roughness of the substrate exists weaker than before the sputter. Because

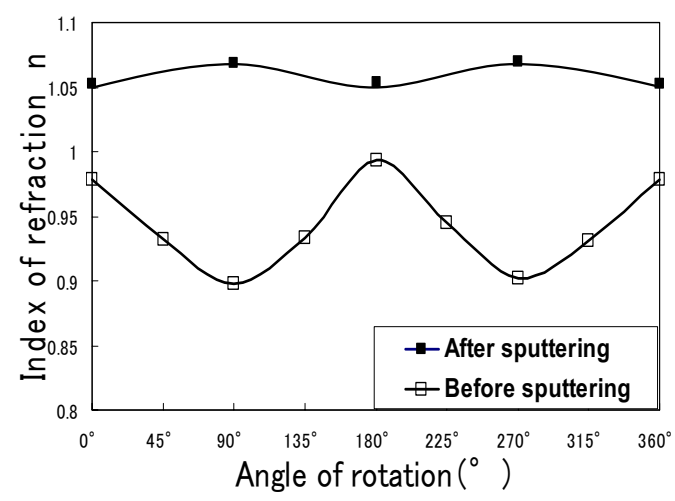

Fig.3 Change in index of refraction $\mathrm{n}$ for rotation angle of the surface (crystal anisotropy of the layer).

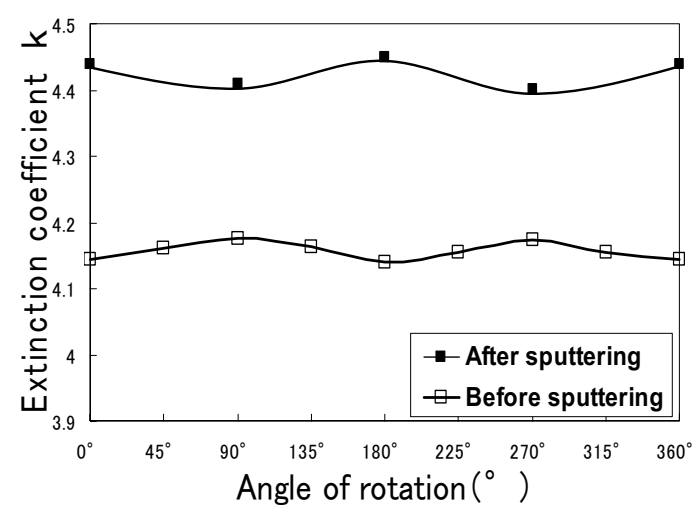

Fig.4 Change in extinction coefficient $\mathrm{k}$ for rotation angle of the surface (crystal anisotropy of the layer). the atomic structure is strongly affected by the sputter, it is expected that the anisotropy of the optical constants on the surface disappeared.

\section{Change in optical constants for oxidation time}

Changes in the optical constants for the growth of the oxide film are shown in Fig.5. This relationship shows the change of the optical constants from clean tin surface to the surface covered with oxide film. When the oxidation time in the atmosphere at room temperature is long (about $10^{3}$ hours), which corresponds to saturation of the film growth, the optical constants show as a $\mathrm{SnO}$ film.

\section{Identification of the formation of the oxide film}

The oxide film grown on the surface of specimen was examined by the electron beam diffraction method, and identified. The results are shown in Fig.6, 7. Obtained diffraction patter shown in Fig.6 (above) is not

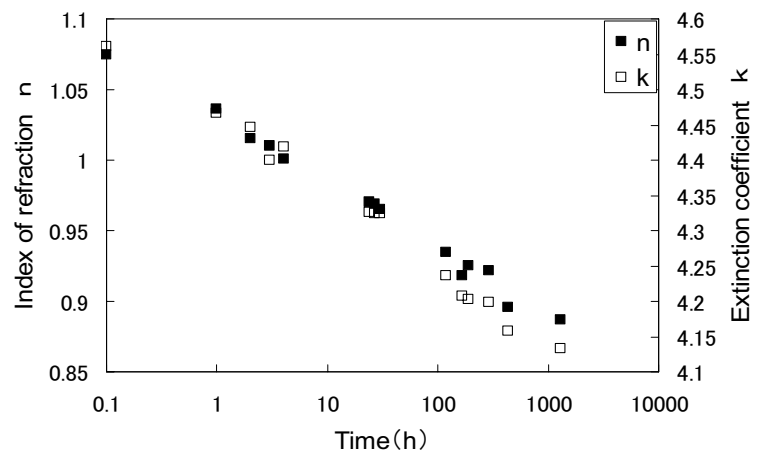

Fig.5 Change in optical constants with growth of $\mathrm{SnO}$ film.

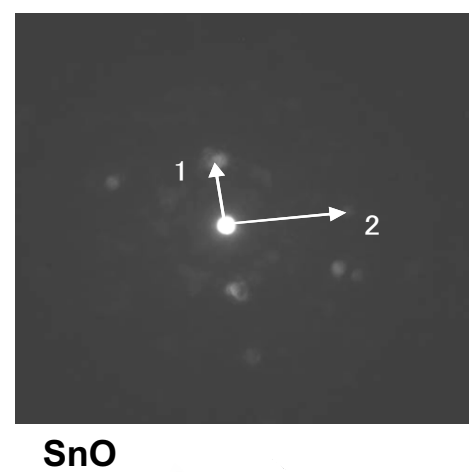

011

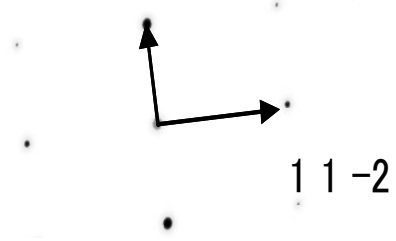

Fig.6 Above: Electron diffraction pattern of the tin plated layer surface at room temperature shows a mixture of crystalline $\mathrm{SnO}$ and an amorphous molecular form of tin oxide. Below: standard crystalline pattern of $\mathrm{SnO}$. 


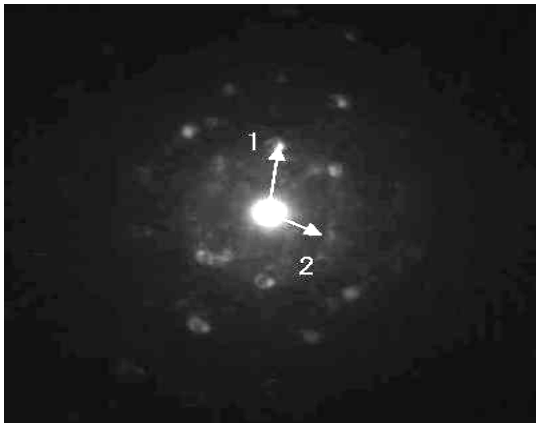

$\mathrm{SnO}_{2}$

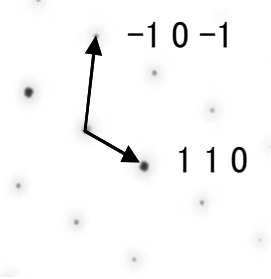

Fig.7 Above: Electron diffraction pattern of tin plated layer surface shows $\mathrm{SnO}_{2}$ film. Below: Standard pattern of $\mathrm{SnO}_{2}\left(120 \mathrm{C}^{\circ}\right) . \quad$ Thickness of plated layer : $3 \mu \mathrm{m}$

clear, but a halo and some unfocussed crystal lattice points can be seen. From standard halo pattern of amorphous $\mathrm{SnO}$ and standard crystalline lattice pattern, it is expected that the initial oxide layer grown at temperature less than $120^{\circ} \mathrm{C}$ is a mixture of crystalline $\mathrm{SnO}$ and an amorphous molecular form, possibly $\mathrm{SnO}, \mathrm{SnO}_{2}$, and $\mathrm{Sn}_{3} \mathrm{O}_{4}$ or a mixture these. $\mathrm{Sn}_{3} \mathrm{O}_{4}$ is an intermediate molecule that can form in the transition of $\mathrm{SnO}$ to $\mathrm{SnO}_{2}$. Recently, $\mathrm{Sn}_{2} \mathrm{O}_{3}$ is predicted as this type of tin oxide [8]. While, as in Fig.7, crystalline $\mathrm{SnO}_{2}$ was identified for the surface exposed at $120^{\circ} \mathrm{C}$.

\section{E. Growth of the oxide film}

The tin plated surface was set in the ellipsometer after the ion sputtering, the polarization state of the oxide film on the surface was measured by the polarization angle, and the film thickness is calculated by these angles. Relationship between coordinates of optical constants $\mathrm{n}, \mathrm{k}$ and film thickness $d$ were converted by coordinate with the polarization angle $\Delta$ and $\Psi$ which indicate polarization state of the oxide film for the exposure time in the indoor atmosphere. Relationship between polarization angle $\Delta$ and $\Psi$, and of film thickness for a typical example as $3 \mu \mathrm{m}$ thickness of the tin plated is shown in Fig.8. Increase in film thickness as the exposure time is shown in Fig.9. This relationship was obtained by rearrangement from the relationship shown in Fig.8.

As shown in Fig.9 (a), the oxidation film was rapidly grown until 5 hour after exposure to the clean surface in the indoor atmosphere, namely this is indicated as linear law. After this, the growth rate saturated gradually. This is a

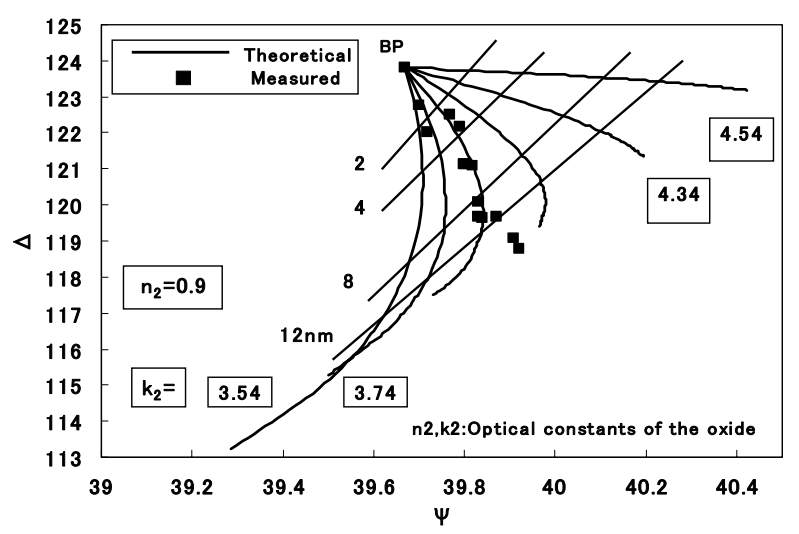

Fig.8 Typical example of the relationship between $\Delta$ and $\Psi$ for growth of the oxide film.

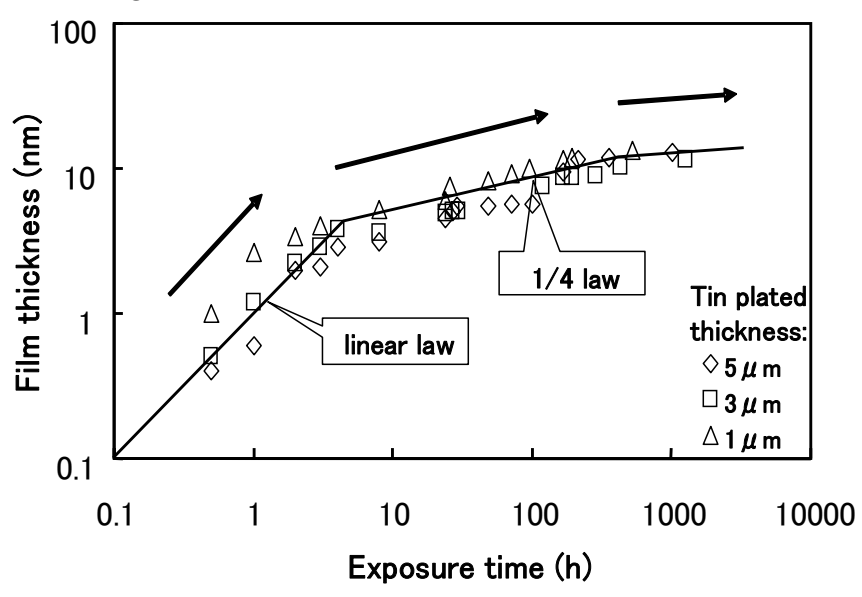

Initial stage: linear law, second stage: 1/4 law, last stage: saturation.

(a) Exposure in indoor environment. $\left(25^{\circ} \mathrm{C}, 30 \mathrm{RH}\right)$

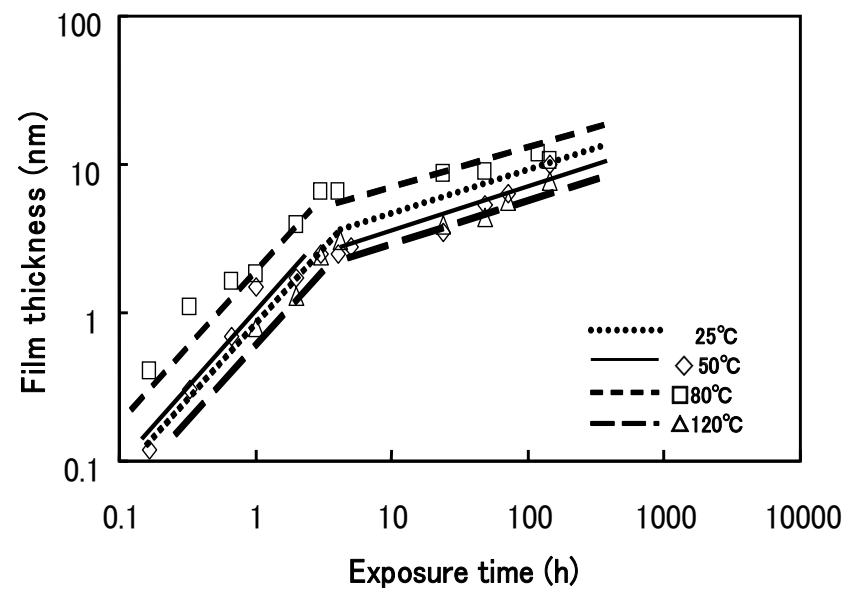

(b) Exposure for high temperature in indoor environment.

Fig.9 Growth of the oxide film for exposure time. 
typical oxidation feature in the initial oxidation process, it indicates basically a parabolic law.

Next, it is shown in Fig.9 (b), for at higher temperatures, $50^{\circ} \mathrm{C}$ and $80^{\circ} \mathrm{C}$, that increase in the growth rate was recognized in the first stage. This growth rate was linear law. In the second stage, growth rate shows same tendency for all temperatures. This fact shows that once growth of the film saturated, reaction of tin surface and $\mathrm{O}_{2}$ was separated by the grown film. They only react with diffusion through the film each other.

However, tendency of the growth of the film at the high temperature of $120^{\circ} \mathrm{C}$ is different from lower temperature. Namely, the growth rate of the film decreases to level of the film grown at the lower temperature as shown in Fig.5. One reason for this difference is that the temperature of $120^{\circ} \mathrm{C}$ is higher than recrystallization temperature of tin plated layer of $100^{\circ} \mathrm{C}$, the formation of the substrate may be changed.

Furthermore, formation of the grown oxide film changed from a mixture formation to crystal $\mathrm{SnO}_{2}$. The changes affect the growth rate of the oxide film. The diffraction pattern of $\mathrm{SnO}_{2}$ formed at $120^{\circ} \mathrm{C}$ is shown in Fig.7.

\section{F. Identification of the oxide film thickness by TEM}

The cross section of the tin plated specimen was prepared by FIB (Focused Ion Beam Method), the cross section of the film covered on the surface was observed TEM with 300,000 times of high magnification. The film on the surface could be identified and its thickness was $10-15 \mathrm{~nm}$. The TEM image of the cross section is shown in Fig.10. The thickness obtained by the TEM was coincided with the saturated film thickness measured by the ellipsometry shown in Fig.9.

To identify the film grown on the surface observed by the TEM image, points indicated by arrows shown in Fig.10 were subjected by element analysis of EDX. The table 1 shows the result of EDX element analysis. As oxygen $(\mathrm{O})$ and tin were detected in the 1st and 2nd arrows (EDX points) of the surface layers as shown in Fig.10 (a), they can be identified as tin oxides. Oxygen is not detected at the $3 \mathrm{rd}$ arrow (EDX point), copper of the substrate metallic elements and tin are only indicated. Therefore, these points coincided with the copper alloy substrate of tin plated layer. As the amount of additives in the substrate is very small, they are not detected in EDX analysis. Moreover, TEM image of cross section for the specimen surface exposed at $120^{\circ} \mathrm{C}$ is shown in Fig. 10 (b). Crystallized $\mathrm{SnO}_{2}$ in island states was found on the amorphous $\mathrm{SnO}$ film in this TEM image. In the case of $\mathrm{SnO}_{2}$, theoretical oxide (O) content of the film is 66 atomic \%. However, EDX analysis showed approximately 50 atomic $\%$ as shown in Table 2 . The difference between them should be induced by inhomogeneous formation of the island states $\mathrm{SiO}_{2}$. and accuracy of the EDX analysis for oxygen.

The crystallized $\mathrm{SnO}_{2}$ forms such lumps as islands at $120^{\circ} \mathrm{C}$ as shown in Fig. 10 (b). When the amorphous $\mathrm{SnO}$ layer is oxidized at $120^{\circ} \mathrm{C}$, crystallized $\mathrm{SnO}_{2}$ should be formed on this layer at a defect of outermost surface of the layer with a large density change.

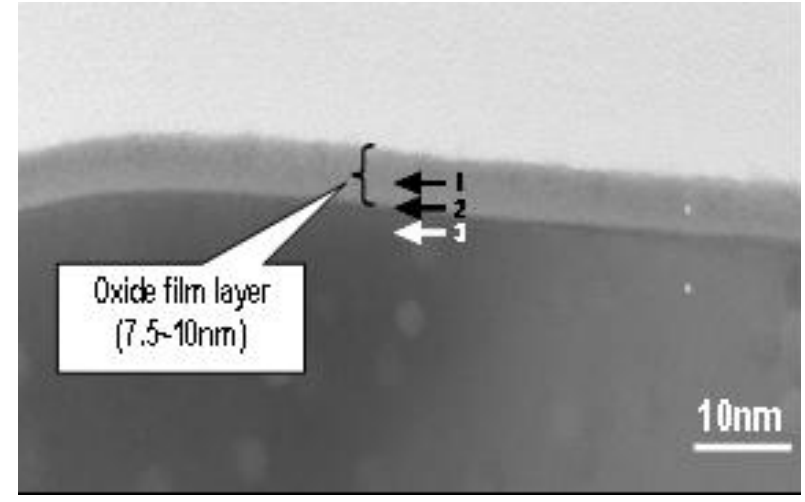

(a) Thickness of the film by TEM image shows amorphous $\mathrm{SnO}$ layer $\left(25^{\circ} \mathrm{C}, 30 \mathrm{RH}\right)$

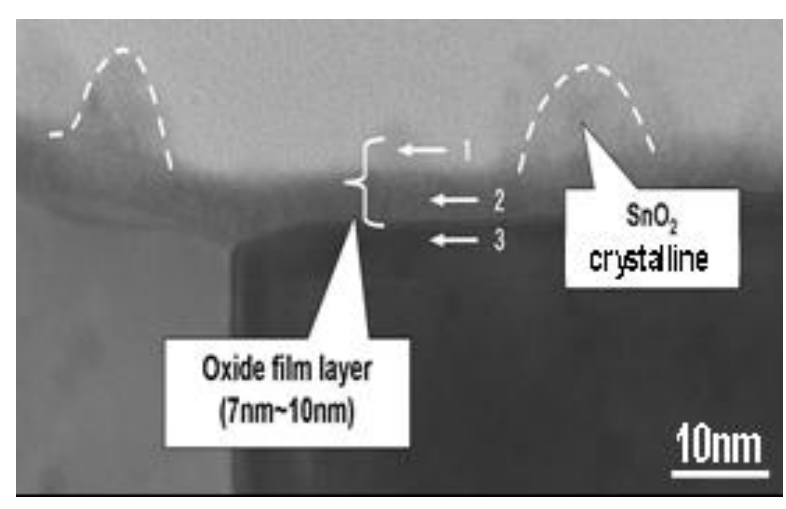

(b) Identification of thickness in TEM image shows crystal $\mathrm{SnO}_{2}$ on the amorphous $\mathrm{SnO}$ film $\left(120 \mathrm{C}^{\circ}\right)$

Fig.10 Identification of the film thickness by TEM images.

Table 1 Element analysis by EDX (room temperature) (atomic \%)

\begin{tabular}{|c|c|c|c|}
\hline EDX Point & $\mathrm{O}$ & $\mathrm{Cu}$ & $\mathrm{Sn}$ \\
\hline 1 & 43.7 & 2.4 & 53.9 \\
\hline 2 & 25.7 & 3.2 & 71.1 \\
\hline 3 & - & 2.5 & 97.5 \\
\hline
\end{tabular}

Table 2 Element analysis by EDM $\left(120 \mathrm{C}^{\circ}\right)$ (atomic \%)

\begin{tabular}{|c|c|c|c|}
\hline EDX Point & $\mathrm{O}$ & $\mathrm{Cu}$ & $\mathrm{Sn}$ \\
\hline 1 & 49.5 & 3.9 & 46.6 \\
\hline 2 & 44.1 & 2.6 & 53.3 \\
\hline 3 & - & 3.7 & 96.3 \\
\hline
\end{tabular}




\section{$\boldsymbol{G}$ Contact resistance characteristics}

First, the contact resistance of the tin plated surface was measured for both surfaces before and after the ion sputter. Effect of the ion sputter on static contact resistance characteristic is shown in Fig. 11 for $1 \mu \mathrm{m}$ of plated as an example. As the surface has been covered with the oxide film before ion sputter process, contact resistance showed high level of 60-90 $\Omega$ for low contact load. After ion sputter, contact resistance decreases to a lower level of $1 \Omega$ or less.

In addition, it is shown in Fig.11 that the film mechanically breaks down by the contact load of about $0.6 \mathrm{gf}$ in the specimen with the oxide film before sputtering. Whereas, such change in contact resistance is not recognized on the surface after ion sputtering. With increase in the load, a sudden decrease and the changing point of the contact resistance which indicates mechanical breakdown of the film at the changing from the elastic to the plastic deformation was not found. Therefore, it is indicated that the oxide film was clearly removed by the ion sputtering from changes in the contact resistance level.

Next, the change in the contact resistance for increase in film thickness is shown in Fig.12. It is clear that contact resistance depends on contact force and that contact resistance is low resistance when oxide thickness is less

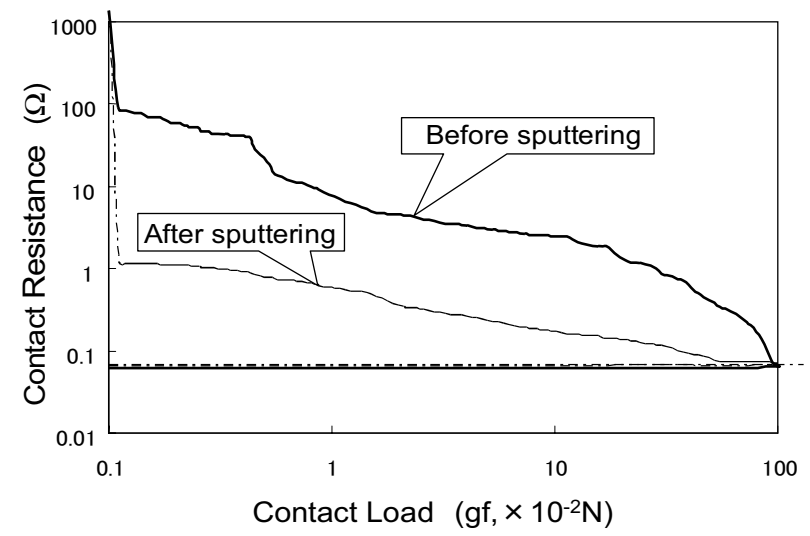

Fig.11 Relationship between contact resistance and contact load (Plated layer thickness: $1 \mu \mathrm{m}$ ).

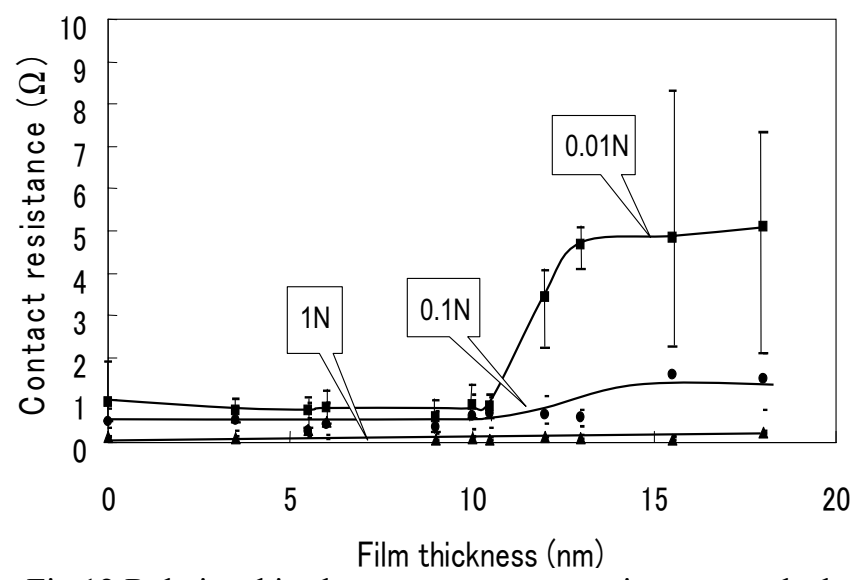

Fig.12 Relationship between contact resistance and the oxide film thickness. than $10 \mathrm{~nm}$ regardless of the load. For thin oxide film, low contact resistance should be induced by such conductive mechanism of the thin film as tunnel and schottky effect. It is recognized that mechanical breakdown of the film easily occurred by high contact load such as $100 \mathrm{gf}(1 \mathrm{~N})$.

\section{CONCLUSION}

The growth law of the oxide film formed on the tin plated surface was clarified by using the ellipsometry for surface exposed to an atmosphere at room temperature and some elevated temperatures.

As a result, the growth law of the oxide film showed linear law until $5 \mathrm{~nm}$ in its thickness at the initial stage of the exposure. After this stage, the growth of the oxide film indicated 1/4 law until $15 \mathrm{~nm}$ in thickness and the thickness saturates. The contact resistance indicated low constant level until $10 \mathrm{~nm}$ in thickness of the film. As film thickness increases, contact resistance can reach to $5 \Omega$. The oxide film thickness was identified by TEM observation. This result well agreed with results obtained by the ellipsometry. The oxide film formation also identified by electron diffraction. Obtained result shows the initial oxide film grown at the temperature less than $120^{\circ} \mathrm{C}$ is a mixture of crystalline $\mathrm{SnO}$ and an amorphous molecular form, possibly $\mathrm{SnO}, \mathrm{SnO}_{2}$ and $\mathrm{Sn}_{3} \mathrm{O}_{4}$ or a mixture of these. On the other hand, it was found that for the exposure at $120^{\circ} \mathrm{C}$ which is higher than recrystal temperature of tin, formation of oxide film changed to crystal $\mathrm{SnO}_{2}$.

\section{ACKNOWLEDGEMENT}

The authors acknowledge Dr. G. Witter for his valuable discussion, and Dr. Z. K. Chen for his kind cooperation.

\section{REFERENCES}

[1] T.Tamai, et al.,"Singularity of tin contacts in relationship between contact resistance and contact load", Proc. $24^{\text {th }}$ Internal. Conf. Electrical Contacts, pp.251-256, June 2008, Saint-Malo, France

[2] S. Sawada, et al., "The effect of plating and contact shape in elevtrical contact on contact resistance", Proc. $24^{\text {th }}$ Internal. Conf. Electrical Contacts, pp.257-261, June 2008, Saint-Malo, France.

[3] R.M.Azzam and N.M.Bashara, Ellipsometry and Polarized Light", Amsterdam, The Netherlands: North-Holland, 1987.

[4] R.J.Archer, "Manual of Ellipsometry", Chicago, Il.: Gaertner Sci. Corp., 1968.

[5] J.Bartels et al., Landolt-Bernstein Zahlenwerte und Funktionen, vol.2, $6^{\text {th }}$ ed. Berlin, FRG: Springer, 1962, sec. 8.

[6] T.Tamai, "Ellipsometric Analysis for Growth of $\mathrm{Ag}_{2} \mathrm{~S}$ Film and Effect of Oil Film on Corrosion Resistance of $\mathrm{Ag}$ Contact Surface", IEEE Trans. CHMT, Vol.12, No.1, March 1989.

[7] T. Tamai, "Growth of oxide films on the surface of $\mathrm{Cu}$ contact and its effect on the contact resistance property", Trans. Electronics and Communication in Japan, Part2, Vol.72, No.7, pp.87-93, 1989.

[8] A. Seko, A. Togo, F. Oba, and I. Tanaka, "Structure and stability of a homologous series of tin oxides", Physical Review Letters, PRL 100, 045702-1 - 4, 1 Feb., 2008. 


\title{
Comparison in Performance for Silver-Tin-Indium Oxide Materials Made by Internal Oxidation and Powder Metallurgy
}

\author{
Z.K. Chen and G.J. Witter \\ Chugai USA, LLC \\ Waukegan, IL
}

\begin{abstract}
Material transfer and material loss of two silver tin oxide materials made by internal oxidation (IO) and powder metallurgy (PM) have been investigated by using a model relay under automotive DC inductive load and lamp load conditions. The opening speed was chosen to insure transfer occurs. Break only testing was conducted using an inductive load and make and break testing was performed for a lamp load. Contact material transfer and loss due to arcing was measured. The results indicate that the IO material has a much stronger transfer tendency in lamp load than PM material. For inductive loads, both materials have a similar transfer and loss behavior. This testing method gave good insight to the material performance under both anodic and cathodic erosion and transfer conditions.
\end{abstract}

Keywords: Arc erosion; material transfer; silver metal oxides; inductive load, DC relay; contact material testing.

\section{INTRODUCTION}

The evaluation of electrical contact materials for medium switching devices in the range of 10 to 200 amperes normally involves testing under both tungsten lamp and inductive loads. These loads differ significantly for the type of stress that is applied to the contact material. By testing the material under both of these types of loads, a good understanding can be gained on the versatility of the material and expected performance versus the electrical application.

In the last 40 years there has been significant work for the development of silver metal oxide materials to substitute for silver cadmium oxide. By far most of this work has involved silver tin oxide type materials which have been successful in replacing most silver cadmium oxide applications. Basically two types of silver tin oxide materials were developed over this period of time. In Europe most of the development involved silver tin oxide made by powder metallurgy (PM). In Asia, mainly Japan, the materials were made by internal oxidation (IO) processes with the use of additives of indium or bismuth. For both of these types of processes there are many types of variations of the processes that have been developed to create new improved materials.

Even after this considerable time of development there are still many questions as to which type of material is best for an application. Since there are many variations of both types of processes it becomes difficult to make generalizations based on the basic process and one must take into account the actual process and additives used.

Chi Leung et al. [1, 2] did studies comparing silver tin oxide made with and without indium using powder metallurgy and also tested against internally oxidized silver tin indium oxide. For endurance testing in a small contactor they found only small differences in performance among the variables, indium level or processing, powder metallurgy versus internal oxidation. For this study, there was a large difference in the particle size distributions between the PM and IO materials.

Koffler et al. [3] did a similar study comparing only variations of powder metallurgical materials which varied in oxide particle size distribution and indium content. They found little difference among the materials for erosion under lamp load and a small difference under inductive loads with some high transfer for the very fine material. For AC testing they found a slightly longer life with the indium additive. With regard to metal oxide content this study claimed better performance for materials with higher oxide levels of $12 \%$ by weight compared to materials with 8 or $10 \%$ oxide content. As stated before this study only included PM materials and no comparisons were made to IO materials in terms of performance or microstructure. 
Chen and Witter $[4,5]$ have conducted several studies on both PM and IO materials at different oxide contents, gaps and opening speeds by using model switch. They found the opening speed has significant influence on material transfer. At the same testing condition, PM material has more material transfer and loss than IO material.

In the current study a comparison in performance is made for two silver tin oxide type materials with one made by IO and the other made by PM. Both materials have $12 \%$ by weight total oxide content which includes an indium oxide additive. In prior work most of the PM materials were much coarser in oxide particle size distributions than IO materials. In this case the PM material has a fine oxide particle size distribution and is similar in microstructure to the IO material.

The key to making a judgment about a material is to test it under different conditions to see how it reacts so that interpolation can be made for applying the material to new applications. In this case both lamp load and inductive load testing were performed. The materials were tested in a model switch which can duplicate electromechanical conditions seen in commercial relays. This model switch is described in two previous papers which studied the effects of contact opening and closing velocity variations on electrical erosion and anodic and cathodic material transfer that took place $[4,5]$.

In the present case the opening and closing speeds were adjusted to allow relatively sever transfer so that the reliable judgment can be made. In the case of lamp load testing, for contact closing the over travel was adjusted to allow only a single bounce at the start of the test and results in increased bounce severity as the test proceeds in endurance cycling. For the inductive load testing an opening speed of $0.75 \mathrm{~m} / \mathrm{sec}$ was used at two gaps.

The objective of this study is to compare the material transfer and material loss under inductive load and lamp load conditions for the two materials with same metal oxide content and microstructures but made by different methods.

\section{EXPERIMENTS}

Two $\mathrm{AgSnO}_{2}$ materials with the same $12 \%$ metal oxide content but different manufacturing methods were used in this study. One is made by PM method and the other is by IO method. The solid rivet contact samples with the same dimensions were made by both materials. The inductive load was conducted by using two contact gaps and break only. This allows a look at efficiency of material transfer for a material as the gap changes. The lamp load testing was for both make and break with a short amount of bounce erosion at the start of the testing and a contact gap of $0.51 \mathrm{~mm}$ on opening.

As described in our past papers $[4,5]$, it was found that the average opening speed for regular commercial relays is in the range of 0.6 to 1.3 meters per second, and also that the speed greatly affects the material transfer and loss. Since lower opening speeds were found to increase material transfer, an opening speed towards the lower end of the commercial relay range was picked for the current testing to better show the transfer characteristics.

The properties of the two materials tested are listed in table 1 and the electrical testing parameters are listed in table 2 .

Table 1. Contact material properties.

\begin{tabular}{|c|c|c|}
\hline Property & $\begin{array}{c}\text { PM } \\
\text { Silver metal } \\
\text { oxide }\end{array}$ & $\begin{array}{c}\text { IO } \\
\text { Silver metal } \\
\text { oxide }\end{array}$ \\
\hline Percent $\mathrm{Ag}$ & $88 \%$ & $88 \%$ \\
\hline Metal oxide & $\begin{array}{c}12 \% \\
\left(\mathrm{SnO}_{2} \& \mathrm{In}_{2} \mathrm{O}_{3}\right)\end{array}$ & $\begin{array}{c}12 \% \\
\left(\mathrm{SnO}_{2} \& \mathrm{In}_{2} \mathrm{O}_{3}\right)\end{array}$ \\
\hline $\begin{array}{c}\text { Conductivity } \\
\text { (\%IACS) }\end{array}$ & $62 \%$ & $62 \%$ \\
\hline Hardness & $123 \mathrm{~V} 200 \mathrm{G}$ & $110 \mathrm{~V} 200 \mathrm{G}$ \\
\hline
\end{tabular}

Table 2. Parameters used for the electrical endurance testing.

\begin{tabular}{|l|l|l|}
\hline \multicolumn{1}{|c|}{$\begin{array}{c}\text { Testing } \\
\text { Parameters }\end{array}$} & \multicolumn{1}{c|}{ Inductive } & \multicolumn{1}{c|}{ Lamp } \\
\hline Voltage & 13.5 volts DC & 13.5 volts DC \\
\hline Current & 20 A break & 20 A break, 120 A inrush \\
\hline Load Type & Inductive, $0.44 \mathrm{mH}$ & Tungsten lamp \\
\hline Contact Force & $0.8 \mathrm{~N}$ & $0.8 \mathrm{~N}$ \\
\hline Operations & 25,000 cycles & 25,000 cycles \\
\hline Switching Mode & Open only & Make \& break \\
\hline Contact Gaps & 0.31 and $0.51 \mathrm{~mm}$ & $0.51 \mathrm{~mm}$ \\
\hline Opening Speed & $0.75 \mathrm{~m} / \mathrm{sec}$ & $0.75 \mathrm{~m} / \mathrm{sec}$ \\
\hline
\end{tabular}

The microstructure of both materials is shown in Fig.1. The samples were chemically etched before SEM examination. The back scattered electron images were 
taken since it could clearly indentify the metal oxide particles. Except for several big metal oxide particles, it is clear that both materials have a similar metal oxide particle size distribution. Particle size analysis software was used to study the two materials and the results are shown in Table.3. It is clear that both materials have the very close particle size distribution.

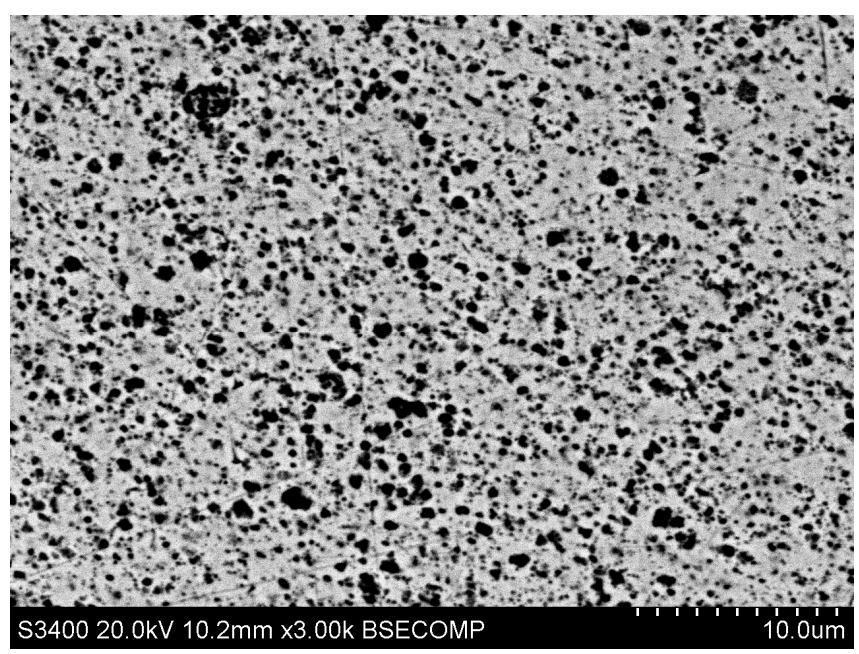

Fig.1 (a) Microstructure of PM material.

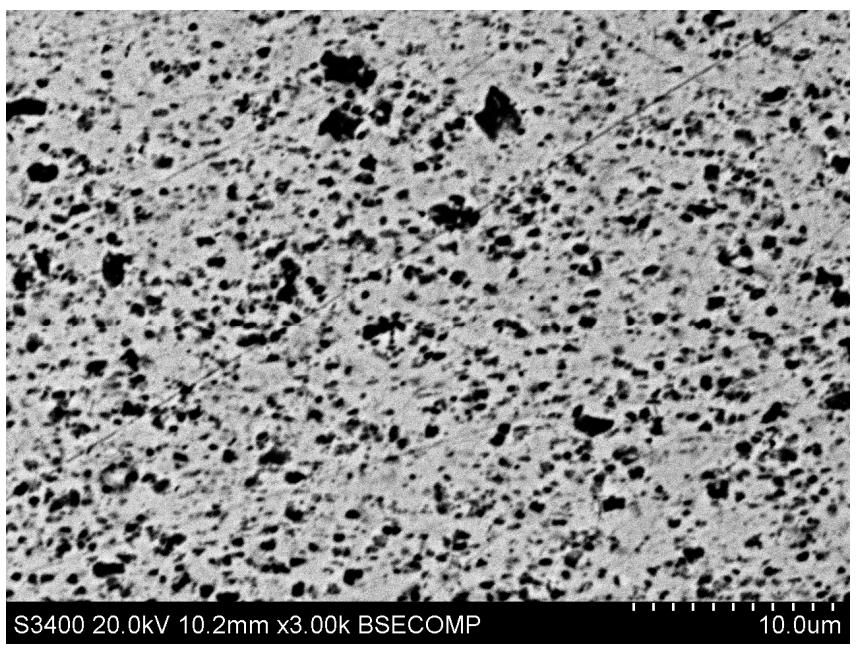

Fig.1 (b) Microstructure of IO material.

Fig.1 Microstructure of PM and IO materials.

Table 3. Particle size distribution comparisons of the metal oxides for the IO and PM contact materials.

\begin{tabular}{|l|c|c|}
\hline Material & IO & PM \\
\hline Mean Particle Size, $\mu \mathrm{m}$ & 0.61 & 0.60 \\
\hline Max Particle Size, $\mu \mathrm{m}$ & 3.1 & 3.0 \\
\hline Min Particle Size, $\mu \mathrm{m}$ & 0.2 & 0.2 \\
\hline Number of Counts & 805 & 850 \\
\hline
\end{tabular}

For studying the material transfer and loss due to arcing, the digital balance with about $10 \mu \mathrm{g}$ of resolution was used to weigh both the anode assembly and cathode assembly before and after testing. Ultrasonic cleaning was performed before weighing the samples to remove loose particulates both before and after testing. The weight loss was divided into three categories: (a) total net loss; (b) loose particle loss on both contacts; and (c) air loss.

\section{RESULTS}

\subsection{Arc Duration}

The typical break arc duration in inductive load testing is in the range of $850 \mu$ s to $950 \mu$ s during the whole 25,000 operations for both materials. As the number of operations goes up the break arc duration increases a little because the over-travel reduces due to the material loss.

For the lamp load testing the break arc in the whole test is in range of $180 \mu$ s to $210 \mu \mathrm{s}$. The contact bounce for the start of the testing is set to be one bounce. The inrush builds up to 120 amperes in several hundred of microseconds (typically 300 to $400 \mu \mathrm{s}$ ). Table 4 shows the typical range of inrush and bounce duration for a 25,000 operation lamp load test. Pip and crater formation increases the bounce duration significantly.

Table.4 Typical bounce data for lamp load testing

\begin{tabular}{|l|c|c|c|}
\hline & $\begin{array}{c}\text { Total } \\
\text { number }\end{array}$ & $\begin{array}{c}\text { Total } \\
\text { duration }\end{array}$ & $\begin{array}{c}\text { Highest } \\
\text { inrush }\end{array}$ \\
\hline At start & 1 & $134 \mu \mathrm{s}$ & $6 \mathrm{~A}$ \\
\hline At end & 3 & $307.6 \mu \mathrm{s}$ & $83.2 \mathrm{~A}$ \\
\hline
\end{tabular}

\subsection{Material Transfer}

\section{A. In inductive load testing}

Fig.2 shows the material transfer rate for PM and IO materials at the two contact gaps in break only inductive load testing. Each test is the average of two sets of testing samples and the $\mathrm{Y}$ axis is the average mass change in micrograms per 1000 cycles of operations. Since the break arc is long it is dominated by a gaseous phase arc for this test sequence. The transfer is from the 
cathode to the anode as expected for this type of arc. The PM and IO materials show similar amounts of material transfer for this testing. It can be seen from Fig.2, that the effect of contact gap on IO material transfer rate is also very small and can be neglected and that, for PM material, the transfer rate in short gap is a little bit more than that in large gap.

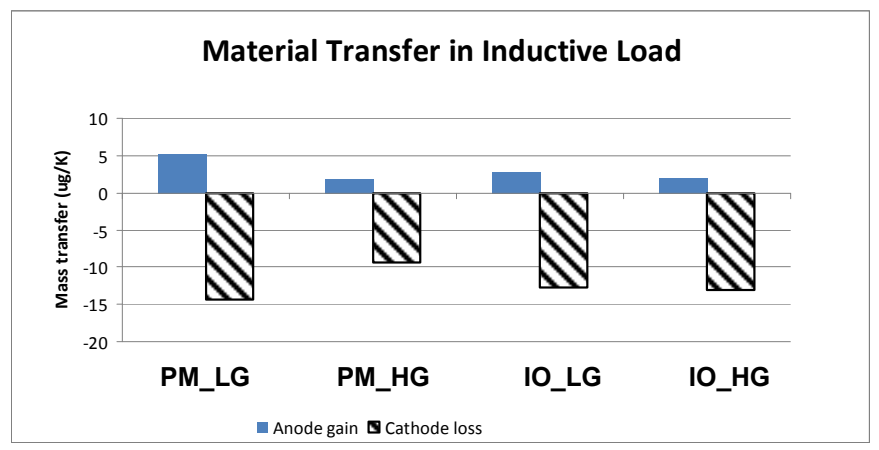

Fig.2. Results of material transfer rate for PM and IO materials at low gap (LG) and larger gap (HG) and anode (solid) and cathode (stripe).

\section{B. In lamp load testing}

In lamp load testing, the make bounce is the key parameter to determine the material transfer and loss behavior. The testing has been only conducted in large gap since the break arc is only in the $200 \mu$ s range, and is not significant for causing erosion compared to the make operation. Although the testing has been adjusted to keep the same make bounce at the start of the each test, there is a large difference in results for the IO and PM materials.

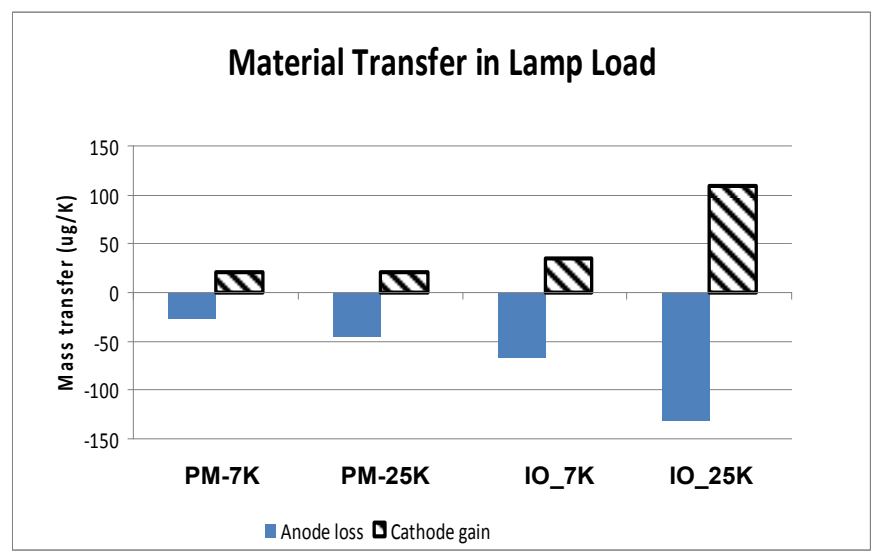

Fig.3 Material transfer rate in lamp load testing for PM and IO materials at two testing cycles $(7,000$ cycles and 25,000 cycles).
From Fig.3, it is obvious that both materials have anode to cathode material transfer as expected for the predominately metallic phase bounce arc. It is also clear that the PM material has a significantly lower material transfer rate compared with IO material for both the $7 \mathrm{~K}$ and $25 \mathrm{~K}$ test series. The IO material has greater tendency and rate of material transfer from the anode to the cathode, thus a higher probability of forming a pip on the cathode and a crater on the anode than PM material. This tendency was observed on the tested contacts by optical microscope.

During the lamp load testing, since the make bounces in high inrush current can produce a pip on the cathode and crater on the anode, once the pip and crater are formed, the number of bounces and the total duration of bounces increase, which in turn accelerate the material transfer from the anode to the cathode due to stronger electrical field on the pip, which increases the electron bombardment on the anode. This positive feedback can accelerate material transfer in bounce arcing. In order to demonstrate this effect, a set of separate tests has been conducted by using the same initial setting parameters, but only 7000 operations. By comparing the transfer rate for 7000 cycles with that in 25,000 cycles (Fig.3), it is obvious that the transfer rate in 7000 is less than that in 25000 for both materials. In lamp load testing the pip and crater grows as testing cycle goes up, thus leading to the increase in material transfer rate. It is clear that the rate of transfer from the anode to the cathode is about 2 to 3 times larger at the 25,000 operations than that for 7,000 operations for IO material. Also, the transfer rate for IO material is about 2 to 3 times larger than that in PM material under the same condition.

Comparing Fig. 2 to Fig.3, not only the transfer direction is different in lamp load, but the material transfer rate in lamp load is about 10 times higher than that in inductive load for IO material and about 2 to 3 times for PM material.

\subsection{Material Loss}

\section{A. Inductive load testing}

Another way to evaluate the arc erosion of contact material is to measure material loss in both electrodes, called total loss. The total loss in this study is measured by weighing both electrodes before and after electrical testing. Since ultrasonic cleaning is used to remove the loose particles on the contacts after testing the loss can be divided into air loss and loose particle loss. In order 
to indicate the effect of this ultrasonic cleaning, the total net material loss and anode and cathode loss due to cleaning are all given in Fig.4 for Inductive load. Fig.4 indicates that both PM and IO materials have similar material loss characteristics. From Fig.4, for total loss, the major loss is actually due to the loose particles generated on the contacts, which are removed by ultrasonic cleaning. Table 5 lists the quantity of the material loss due to the two factors in inductive load testing at two gaps. It is clear that PM material has higher (2 times in low gap and 4 times in larger gap) rate of material loss to air than that of IO material. The gap effect on material loss is relatively small.

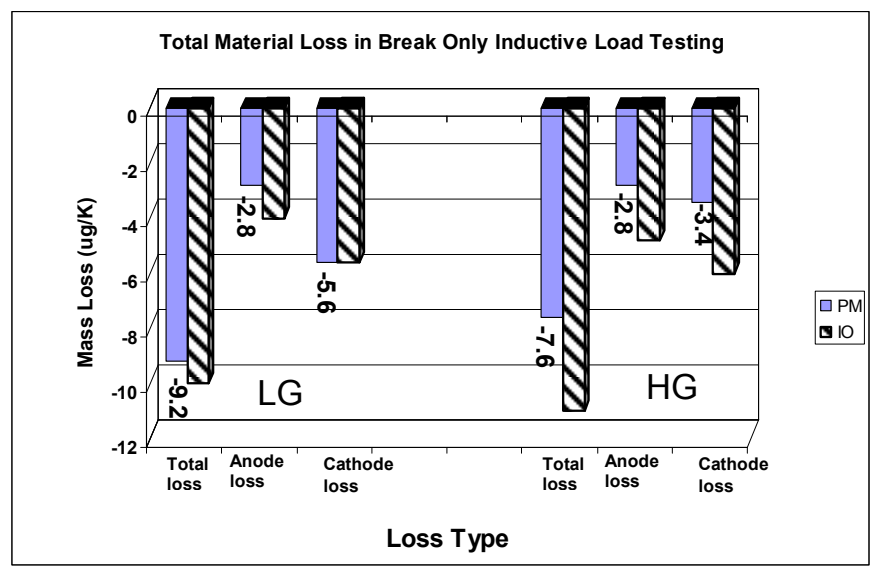

Fig.4 The rate of total material loss and loosing particle weight on both contacts in break only testing under inductive load condition at low gap (LG) and large gap (HG).

Table.5 Various material loss rates in inductive load testing at two gaps

\begin{tabular}{|l|l|l|l|c|}
\hline Gap & LG & LG & HG & HG \\
\hline Material & PM & IO & PM & IO \\
\hline Total net loss & -9.2 & -10 & -9.2 & -11 \\
\hline $\begin{array}{l}\text { Loose particle } \\
\text { weight }\end{array}$ & -8.4 & -9.6 & -8.4 & -10.8 \\
\hline Loss in air & -0.8 & -0.4 & -0.8 & -0.2 \\
\hline
\end{tabular}

\section{B. Lamp load Testing}

The total material loss rate in lamp load testing is shown in Fig.5. It is clear that both PM and IO materials have a similar quantity of loss. Compared with Fig.4, the total loss rate in lamp load is over 2 times larger than that in inductive load although the loose particle weight generated by arcing is in the same range. In other words, the predominated factor to cause material loss in lamp load testing is due to the loss of material to air during bounce arcing. Also the rate of "loss in air" for PM material is average at $12 \mu \mathrm{g}$ per 1000 cycles, which is larger than IO material (only $10.2 \mu \mathrm{g}$ per 1000 cycles).

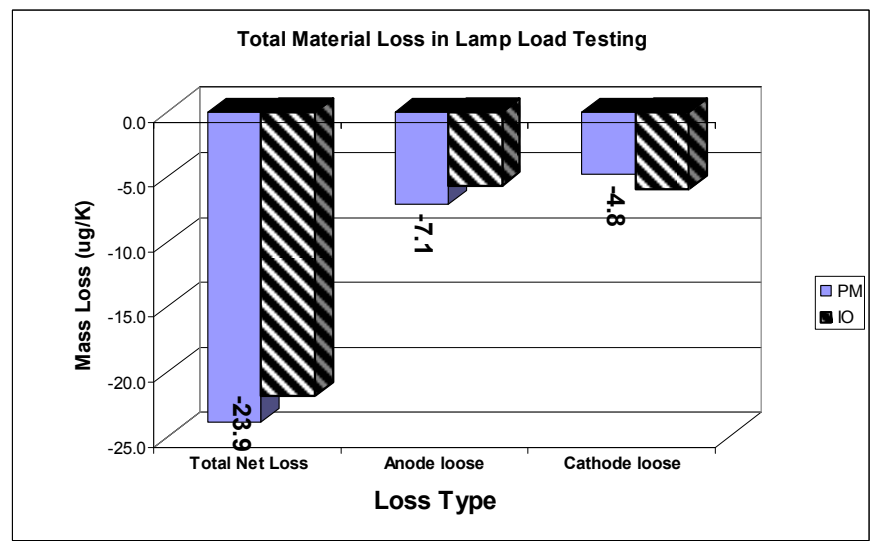

Fig.5 The rate of total material loss and loose particle weight on both contacts in lamp load testing.

\section{DISCUSSIONS}

For lamp load testing, Fig.3 indicates that the lamp load testing produces mainly anodic type erosion (i.e., material transfers from the anode to the cathode) since there is a large inrush current on closure and only normal load current on break. The severity of this type of erosion depends on the amount and frequency of the bounce on closure. A former paper by Witter and Polevoy [6] describes this type of erosion on automotive relays.

Based on the process of contact opening and arcing plasma physics, Chen and Sawa [7, 8] proposed the particle sputtering and deposition model (PSD model) explained how the material transfers during the early metallic phase arc and the later gaseous phase arc. From this PSD model, it is clear that if a pip is created on the cathode surface, the electrical field strength on the top of 
pip is much stronger than on the flat surface of the contact. This strong field not only can increase the electron field emission to provide more electrons in the plasma and keep the arc going but also tremendously enhances the electrical field strength in the arc plasma. The electrons are accelerated towards the anode. The dynamic energy of electrons is much higher at the same power voltage ( $13.5 \mathrm{~V}$ in this test) for a pip contact than that for a flat contact. These two factors: more electrons and high dynamic energy of electrons will lead to much higher electron sputtering efficiency with a pip present on the cathode, thus resulting in a much higher rate of material transfer from the anode to the cathode. As the size of the pip increases and a cascading feed back type effect takes place, more material will be transferred per operation. Figure 3 clearly shows a large increase (over 2 times) in the erosion rate per operation for 7,000 testing compared to longer 25,000 testing.

The inductive load testing shows the materials erosion and larger gap material transfer characteristics. In this case there is little difference between the IO and PM materials.

This combination of the two types of testing produces a picture of how the material reacts under both anodic and cathodic erosion conditions which differ significantly. These two materials with similar microstructures and composition react the same under inductive testing for cathodic type erosion but perform quite differently under make bounce anodic type erosion.

\section{CONCLUSIONS}

From the above testing results and discussions, we can conclude the following.

1. For inductive load testing, there is no significant difference between the PM and IO materials in material transfer and loss. If the metal oxide size distribution is close, PM and IO materials will have the same performance.

2. For the lamp load testing the PM material had much less erosion and material transfer compared to the IO material.

3. More work is needed to find the root cause for this large difference in performance.
4. It is a very effective way to evaluate the contact material performance by using the combination of the inductive load and the lamp load testing.

\section{References}

1. C. Leung, E. Streicher, D. Fitzgerald and J. Cook, "Contact Erosion of $\mathrm{Ag} / \mathrm{SnO} 2 / \mathrm{In} 2 \mathrm{O} 3$ Made by Internal Oxidation and Powder Metallurgy", 51 ${ }^{\text {st }}$ IEEE Holm Conf., Chicago, IL, Sept. 2005, pp22-27.

2. C. Leung, E. Streicher, D. Fitzgerald and J. Cook, "High Current Erosion of $\mathrm{Ag} / \mathrm{SnO} 2 / \mathrm{In} 2 \mathrm{O} 3$ Contacts and Evaluation of Indium Effects in Oxide Properties", 52nd IEEE Holm Conf., Montreal, Canada, Sept. 2006, pp143-150.

3. A. Koffler, P. Braumann and B. Kempf, "The Influence of Manufacturing Process, Metal Oxide Content, and Additives on Switching Behavior of $\mathrm{Ag} / \mathrm{SnO} 2$ in $\mathrm{DC}$ and AC Relays (2)", ICEC 2006, Sendai, Japan, June 2006, pp.288-293.

4. G. Witter and Z.K. Chen, "A Comparison for the Effects of Opening Speed, Contact Gap and Material Type on Electrical Erosion for Relays Interrupting Inductive Automotive Loads, “ ICEC 2006, June, Sendai, Japan

5. Z.K. Chen and G. Witter, "A Comparison of Contact Erosion for Opening Velocity Variations for 13 Volt Circuits", 52nd IEEE Holm Conf., Montreal, Canada, Sept. 2006, pp15-20.

6. G. Witter and I. Polevoy, "Contact Erosion and Material Transfer for Contacts in Automotive Relays", 42nd IEEE Holm Conf. \& $18^{\text {th }}$ ICEC, Chicago, IL, Sept. 1996, pp223-228.

7. Z. K. Chen and K. Sawa, "Particle Sputtering and Deposition Mechanism for Material Transfer in Breaking Arcs", J. Appl. Phys. Vol.76, pp.33263331, 1994

8. Chen Z.K. and K. Sawa, "Effect of Arc Behavior on Material Transfer: A Review," IEEE Trans. CPMT, Pat A V21, No 2, 1998, pp. 310-332.

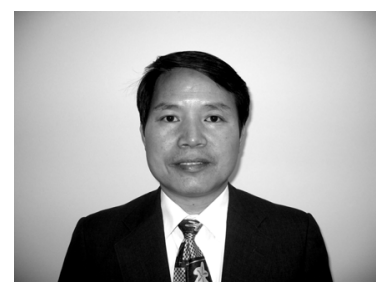

Zhuan-Ke Chen received the B.S. and M.S. degrees in Electronic Engineering from 
Xi'an Jiaotong University, China in 1982 and 1985, respectively, and $\mathrm{Ph}$. D degree in electrical engineering from Keio University, Japan, in 1995. From 1985 to 1991, he was with the department of electronic engineering of Xi'an Jiaotong University, where he taught several courses in electronic physics, gas discharge theory and cathode electronics. At that time, he was engaged in research on electromagnetic relay and numerical calculation of electromagnetic field in two and three dimensions using FEM. From 1991 to 1995, he received the Monbusho Scholarship from the Ministry of Education, Science, and Culture of Japan, and studied at the electrical engineering department of Sawa laboratory in Keio University, Japan, where he was involved in the investigation of electrical contact phenomena (including electrical arc erosion, mechanical wear, and contact resistance deterioration). Since 1996 he has been working in Chugai USA, Inc. as a Research Engineer mainly involving the silver metal oxide materials in automotive applications and ac relays and switches. He has published many papers in electrical contact studies. He is now the vice president of Chugai USA operations. Dr. Chen is a member of IEEE.

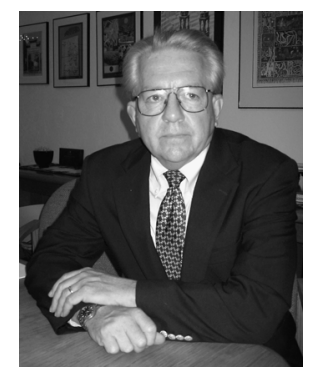

Gerald Witter has been the Chairman of IEEE CPMT Technical Committee\#1 for electrical contacts, connectors, and cable for over ten years. He is also President of Chugai USA, Inc., a manufacturer of electrical contacts and contact assemblies. He has an MS and a BS degree in Physical Metallurgy respectively from Illinois Institute of Technology and Michigan Tech. He has done over 30 years work in electrical contact technology including material development, process development, and development of electrical testing methods. He has published many papers on contact technology including several chapters in the book, "Electrical Contacts: Principles and Applications", Macel Dekker, NY, and is the editor of the IEEE CPTM Book, "Electrical Contacts An Introduction To Their Physics and Applications", By Prof. Werner Rieder. He has been active in the IEEE Holm Conference for over 25 years and has received the Prize Paper Award, The Ragnar Holm Scientific Achievement Award, and The Armington Recognition Award. 


\title{
Performance of Different Silver / Tin Oxide Contact Materials for Applications in Low Voltage Circuit Breakers
}

\author{
Jutta Röhberg, Thomas Honig, Norbert Witulski, Michael Finkbeiner and Volker Behrens \\ AMI DOCUCO GmbH, Pforzheim, Germany
}

\begin{abstract}
Typical contact material combinations in low voltage MCCB include silver/graphite and silver/nickel or frequently used with breakers designed to fulfill UL specifications refractory materials as tungsten/silver or tungsten carbide/silver.

The present paper compares different silver / tin oxide contact materials with oxide contents ranging from 12 to 22 weight \% to some standard contact material combinations. The $\mathrm{Ag} / \mathrm{SnO}_{2}$ manufacturing technologies are based on powder metallurgy applying press-sinter-repress as well as extrusion as further steps.

The electrical tests performed include contact erosion at $200 \mathrm{~A}$ and $20 \mathrm{kA}$, welding forces after electrodynamic lift off, contacts resistance and mechanical endurance.

The results are encouraging for a further development of silver / tin oxide materials with high oxide content for application in circuit breakers.
\end{abstract}

Keywords: circuit breaker, MCCB, contact material, silver/tin oxide, contact erosion, contact resistance, welding force.

\section{Introduction}

With the distribution of electrical power circuit breakers (CB) have to fulfill make and break operations under normal conditions and conduct steady state current for long periods without operation. In addition they have to be capable to manage any electrical load that can occur at their place in the electric grid including over currents and short circuit currents [1]. With their ability to break shorts the CB can be used in circuits without additional fuse and overtake in this case safety functions, typically including short circuit current limiting.

In the field of electric grids for alternating current (AC) the typical low voltage $C B$ for rated currents ranging from app. 20 to $1600 \mathrm{~A}$ is the MCCB (Molded Case $\mathrm{CB}$ ) with a plastic housing containing contact system, arc extinguishing chambers as well as all other functional parts.

The contact system for every pole of a MCCB is usually a single contact design. In other words there is no splitting of the different contact tasks to two contact systems working in parallel: one for the arcing task and the second for steady state current flow.

Thus the contact system and therefore the electrical contact material applied has to be suitable for any switching condition that occurs: it has to withstand the mechanical impact of the make operation and the contact erosion caused by the switching arc at make and break at rated, elevated or short circuit currents. In addition the contact resistance in new condition or after arcing has to stay at sufficient low values and the tendency of contact welding on make or after electrodynamic lift off has to be low.

These different and sometimes contradicting requirements for the contact material led in combination with the MCCB design details as contact force, opening velocities, magnetic blast field, wipe movements on make, and others to a variety of technical solutions for the same task.

In regards to the contact material the development and improvement is going on since many years and can be split up in two main approaches.

The first approach focused on low material erosion is based on silver plus refractory material as the second compound, which is in most cases tungsten (W), tungsten carbide (WC) or molybdenum (Mo). A disadvantage of this group of contact material is the elevated contact resistance. Thus some work is done to reduce high contact resistances $[2,3,4,5]$.

The second basic approach is focused on contact material combinations based on silver and graphite $(\mathrm{Ag} / \mathrm{C})$, which leads to excellent low contact resistances as well as to very low welding forces with the disadvantage of high contact erosion. These findings caused some work to understand the mechanisms occurring as well as to improve the $\mathrm{Ag} / \mathrm{C}$ material $[6,7,8]$.

Some successful work is done to see how far a combination of both approaches, thus materials as $\mathrm{Ag} / \mathrm{WC} / \mathrm{C}$ lead to a good balance between properties of these two groups $[9,10]$.

Up to now only little work is done to evaluate to what extend silver/tin oxide $\left(\mathrm{Ag} / \mathrm{SnO}_{2}\right)$ material can be used in CB $[11,12,13]$.

The present paper deals with $\mathrm{Ag} / \mathrm{SnO}_{2}$ materials with higher oxide contents and state of the art additives proven to have positive effects on the switching behavior in contactors. 


\section{Contact Materials}

Based on powder metallurgy three different ways of manufacturing techniques are used:

- Extrusion of sintered blocks

- Press, sinter and repress (PSR)

- Press, sinter and infiltration (PSI)

\subsection{Extruded contact material:}

$$
\mathrm{Ag} / \mathrm{SnO}_{2} 12 \text { and } \mathrm{Ag} / \mathrm{C} 4
$$

The way of production depends mainly on the composition of the contact material besides economical reasons. $\mathrm{Ag} / \mathrm{SnO}_{2}$ materials up to $14 \mathrm{wt} .-\%$ $\mathrm{SnO}_{2}$ and silver graphite $(\mathrm{Ag} / \mathrm{C})$ materials up to $5 \mathrm{wt}$.$\% \quad \mathrm{C}$ are typically manufactured by extrusion and subsequent rolling and drawing.

An advantage of extruded contact materials is the very low remaining porosity.

The $\mathrm{Ag} / \mathrm{SnO}_{2}$ materials used for the present evaluation have silver molybdate $\left(\mathrm{Ag}_{2} \mathrm{MoO}_{4}\right)$ as an additive, which is added by a coating of the $\mathrm{SnO}_{2}$ powder prior to blending with the $\mathrm{Ag}$ powder. The blended powders are then pressed to a block, followed by co-extrusion to manufacture a two-layer strip of $\mathrm{Ag} / \mathrm{SnO}_{2}$ with pure silver as a second layer to ensure good brazing. [14].

The production steps for $\mathrm{Ag} / \mathrm{C}$ are after blending of $\mathrm{Ag}$ and $\mathrm{C}$ powder block pressing with subsequent extrusion of a strip. A two layer strip $\mathrm{Ag} / \mathrm{C}$ with a silver back suitable for brazing is obtained by decarbonization of the $\mathrm{Ag} / \mathrm{C}$ surface on all sides, followed by machining on contact surface.

The tips used for switching tests are then cut out of the strip, thus the extrusion orientation of the material is parallel to the switching surface.

\subsection{Contact material by PSR:}

\section{$\mathrm{Ag} / \mathrm{SnO}_{2} 18, \mathrm{Ag} / \mathrm{SnO}_{2} 22, \mathrm{Ag} / \mathrm{WC} 27 / \mathrm{C} 3$}

The powder blend is filled into a die and compacted by two punches to a green. After sintering and repressing the contact tip is ready.

\subsection{PSI material: Ag/W50}

In case a contact green includes no compounds that hinder the infiltration with liquid silver this technique is to be preferred due to low final porosity and excellent mechanical stability of the contact tip.

\subsection{Material properties}

Typical material properties of all six contact materials are shown in table 1.

\subsection{Size of tips manufactured}

Table 2 gives sizes of tips used in the different switching tests. All tips are brazed with Cu80Ag15P5 solder to their carriers.

\subsection{Microstructure}

Fig. 1 shows metallographic crosssections of microstructure of the investigated contact materials.

Table 1: Properties of contact materials

\begin{tabular}{|c|c|c|c|c|c|c|}
\hline & A. & B. & C. & D. & E. & F. \\
\hline material & $\mathrm{Ag} / \mathrm{SnO}_{2} 12$ & $\mathrm{Ag} / \mathrm{SnO}_{2} 18$ & $\mathrm{Ag} / \mathrm{SnO}_{2} 22$ & $\mathrm{Ag} / \mathrm{C} 4$ & Ag/W50 & $\mathrm{Ag} / \mathrm{WC} 27 / \mathrm{C} 3$ \\
\hline main add. & $12 \% \mathrm{SnO}_{2}$ & $18 \% \mathrm{SnO}_{2}$ & $22 \% \mathrm{SnO}_{2}$ & $4 \% \mathrm{C}$ & $50 \% \mathrm{~W}$ & $27 \%$ WC, 3\% C \\
\hline technique & extrusion & PSR & PSR & extrusion & PSI & PSR \\
\hline el. cond. & $44 \mathrm{~m} / \Omega \mathrm{mm}^{2}$ & 33 & 28.5 & 47.5 & 35.5 & 28.5 \\
\hline hardness & 79 HV0,3 & 60 & 67 & 47 & 145 & 52 \\
\hline density & $9.85 \mathrm{~g} / \mathrm{cm}^{3}$ & 9.0 & 8.85 & 9.0 & 13.2 & 9.7 \\
\hline porosity & 0.4 vol. $\%$ & 6 & 6 & 2.0 & 2.5 & 6 \\
\hline
\end{tabular}

Table 2: Dimensions of contact tips for the tests

\begin{tabular}{|l|c||c|c|}
\hline & electr. service life & all other test: movable & fixed \\
\hline size $[\mathrm{mm}]$ & diameter $=7.0$ & $12 \times 7$ & $14 \times 8$ \\
\hline thickness $[\mathrm{mm}]$ & 2.5 & 2.4 & 2.4 \\
\hline contact radius $[\mathrm{mm}]$ & 30 & 70 & 70 \\
\hline brazed on & brass & copper & copper \\
\hline
\end{tabular}




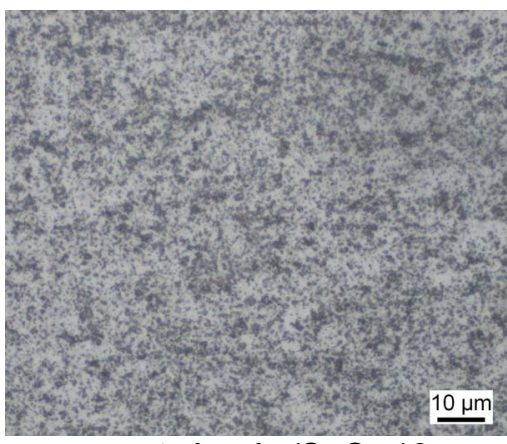

mat. A.: $\mathrm{Ag} / \mathrm{SnO}_{2} 12$

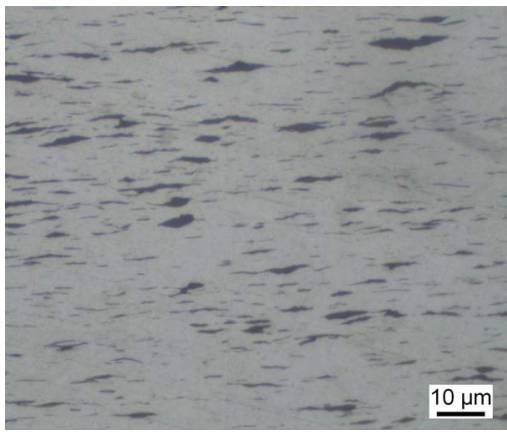

mat. D.: Ag/C4

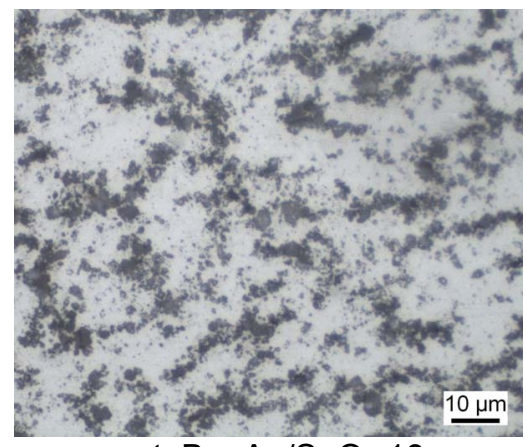

mat. B.: $\mathrm{Ag} / \mathrm{SnO}_{2} 18$

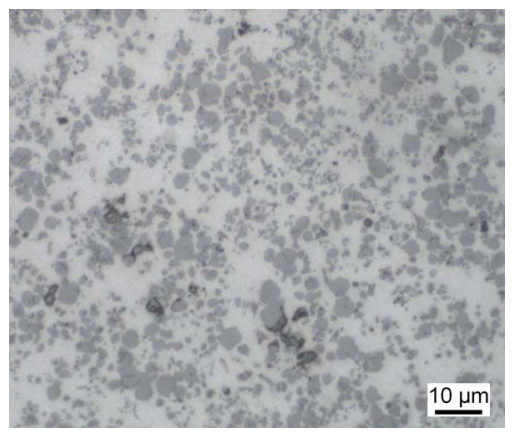

mat. E.: Ag/W50

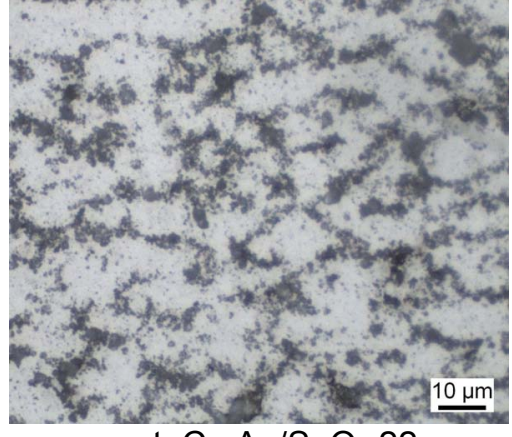

mat. C.: $\mathrm{Ag} / \mathrm{SnO}_{2} 22$

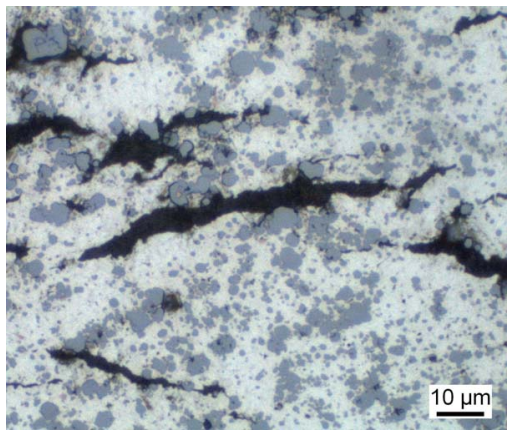

mat. F.: Ag/WC27/C3

Figure 1: Metallographic crosssections of the microstructure of the contact materials (s. Table 1)

\section{Test parameters}

All tests performed and test parameters chosen are paralleled to conditions typical for MCCB in the 100 to 200 A rated current range. All tests with all materials have been done at least with 3 pairs of contacts.

\subsection{Mechanical endurance test}

Test device: setup with stationary contact made of the material to be evaluated, brazed on a copper strip. A spring loaded impactor was designed to simulate the toggle closing; the part hitting the stationary contact is made of steel with radius $\mathrm{R} 70 \mathrm{~mm}$ and a width of app. $2 \mathrm{~mm}$ smaller than the stationary contact to be tested. The model switch is shown in figure 2 .

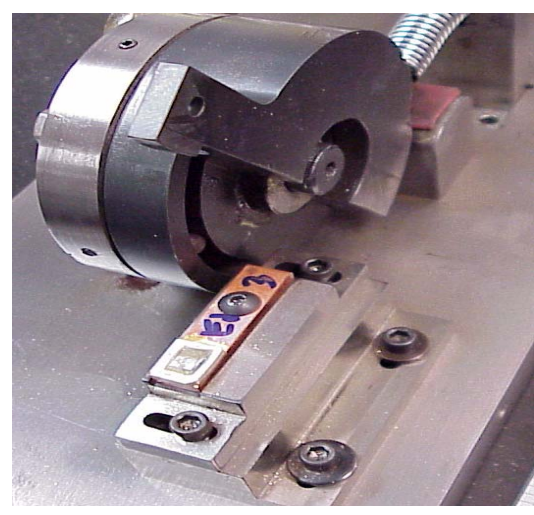

Figure 2:

Setup of mechanical endurance test

Test run: 20.000 operations at

$2.500 / \mathrm{h}$
Tests are performed in a one pole single break model switch.

- voltage:

$230 \mathrm{~V} \mathrm{AC}$ at $50 \mathrm{~Hz}$

- current: $200 \mathrm{~A}$, resistive load

- make and break at random timing

- contact gap: $7 \mathrm{~mm}$

- contact force: $11 \mathrm{~N}$

- operations: 1.000 to 7.000

- frequency: $1000 / \mathrm{h}$

Measured:

mass loss of stationary and movable contact.

\section{3 contact resistance}

Measured in the model switch of 3.2 after service life test, applying $40 \mathrm{~A} \mathrm{DC}$ for $10 \mathrm{~s}$.

\section{4 welding force}

Tests are performed in a setup with paralleled current flow paths of stationary and movable contacts resulting in magnetic repulsion force opposed to the contact force.

An 8 kA current sine half wave from a capacitor bank with a serial inductance applied to the closed contacts leads to an electrodynamic lift off and close again while current still flows. Fig. 3 is an oscillogram of a lift off.

Subsequently the force required to break the weld is measured by a piezoelectric measurement system with no break impact applied. 


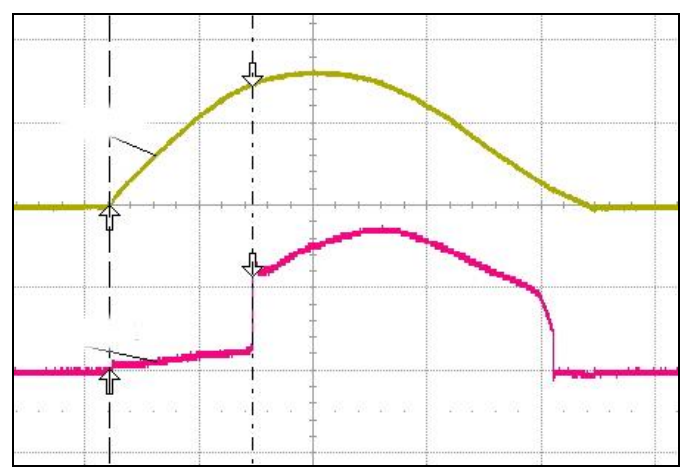

Figure 3: Oscillogram of electrodynamic lift off top curve: $\quad$ current (5 kA / div) lower curve: arc voltage (20 V/ div) time base: $2 \mathrm{~ms} / \mathrm{div}$

\subsection{Short circuit contact erosion test}

Tests are done in the setup used for test 3.4 with an additional commercial arc chute mounted directly in font of the contact gap.

Electric parameters of the test are:

- current (peak): $20 \mathrm{kA}$

- current flow time: $7 \mathrm{~ms}$

- arcing time: $\quad 6,4 \mathrm{~ms}$

- voltage (capacitor bank) $580 \mathrm{~V}$

- operations:

$5 \mathrm{x}$ open

- polarity: changed after every op.

Evaluated after test:

$>$ mass loss and size reduction of tips

$>$ microstructure of contact tips

Fig. 4 shows an oscillogram of this test. The DC voltage level of app. $150 \mathrm{~V}$ after current zero is the remaining voltage of the capacitor bank.

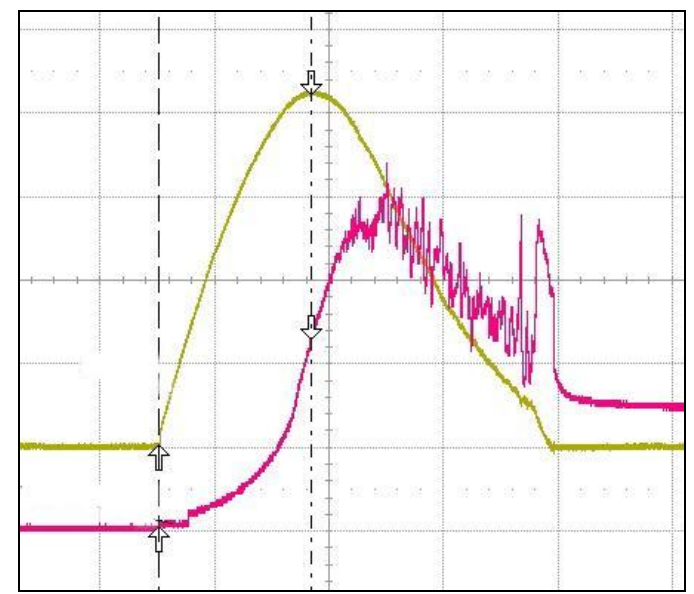

Figure 4: Oscillogram of a short circuit test top curve: current (5kA / div) lower curve: arc voltage (100V / div) time base: $2 \mathrm{~ms} /$ div

\section{Test results}

This chapter shows the test results obtained with the test setups described in chapter III.

The combinations of contact material tested are given in table 3.

Table 3: Contact material combinations

\begin{tabular}{|c|c|c|}
\hline & stationary & movable \\
\hline set 1 & $\mathrm{Ag} / \mathrm{WC} 27 / \mathrm{C} 3$ & Ag/W50 \\
\hline set 2 & $\mathrm{Ag} / \mathrm{C} 4$ & Ag/W50 \\
\hline set 3 & $\mathrm{Ag} / \mathrm{SnO}_{2} 12$ & $\mathrm{Ag} / \mathrm{SnO}_{2} 12$ \\
\hline set 4 & $\mathrm{Ag} / \mathrm{SnO}_{2} 18$ & $\mathrm{Ag} / \mathrm{SnO}_{2} 18$ \\
\hline set 5 & $\mathrm{Ag} / \mathrm{SnO}_{2} 22$ & $\mathrm{Ag} / \mathrm{SnO}_{2} 22$ \\
\hline set 6 & $\mathrm{Ag} / \mathrm{WC} 27 / \mathrm{C} 3$ & $\mathrm{Ag} / \mathrm{SnO}_{2} 18$ \\
\hline set 7 & $\mathrm{Ag} / \mathrm{SnO}_{2} 18$ & $\mathrm{Ag} / \mathrm{W} 50$ \\
\hline set 8 & $\mathrm{Ag} / \mathrm{W} 50$ & Ag/W50 \\
\hline
\end{tabular}

\subsection{Mechanical endurance test}

All material passed the endurance test without chipping or cracks occurring except for one $\mathrm{Ag} / \mathrm{SnO}_{2}$ 22 (mat. C): one tip of three showed chipping after 18500 operations. Fig. 5 shows 3

different contact materials after 20.000 endurance operations.

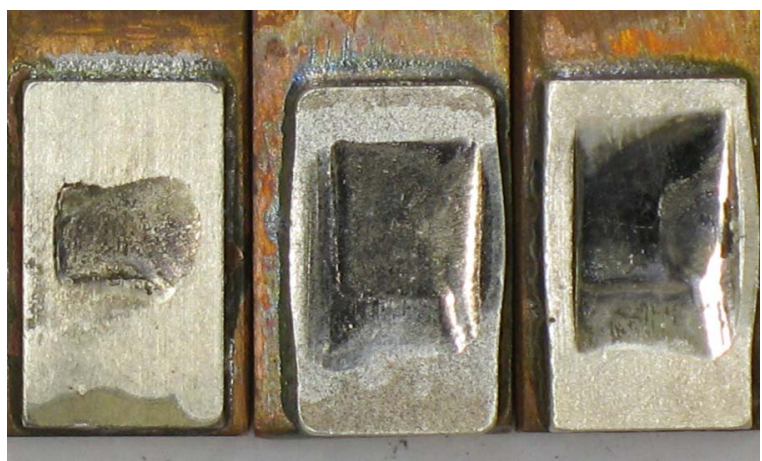

Figure 5: $\mathrm{Ag} / \mathrm{W} 50$ (mat. E), $\mathrm{Ag} / \mathrm{WC} 27 / \mathrm{C} 3$

(mat. F), and $\mathrm{Ag} / \mathrm{SnO}_{2} 18$ (mat. B.) (left to right) after 20.000 mechanical endurance operations: no chipping or cracks.

Gray rectangular area in the middle is deformed by the hammer impact.

\subsection{Electrical service life}

$\mathrm{Ag} / \mathrm{SnO}_{2}$ as well as $\mathrm{Ag} / \mathrm{W}$ material showed low erosion rates, and the differences in mass loss between stationary and movable contact are small. Lowest erosion was observed with $\mathrm{Ag} / \mathrm{SnO}_{2}$ 12. The erosion rates of materials with graphite were higher by a factor of 15 to 20 . 
Both materials of this group (Ag/WC27/C3 and Ag/C4) showed in addition to high material loss by arcing some material transfer to the mating contact Ag/W50. As a consequence service life tests had to be stopped after 1500 to 4000 cycles.

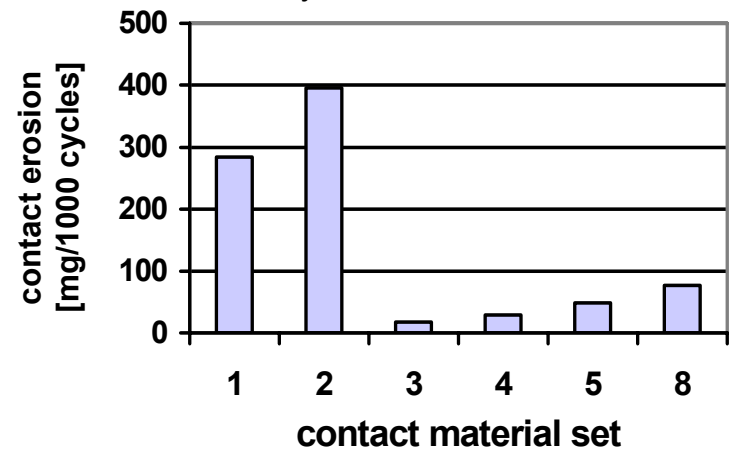

Figure 6: Average values of contact erosion rate in $\mathrm{mg}$ per 1000 cycles (stationary + moveable contact) with service life test for different contact material combinations, see tab. 3 .

\subsection{Contact resistance after service life}

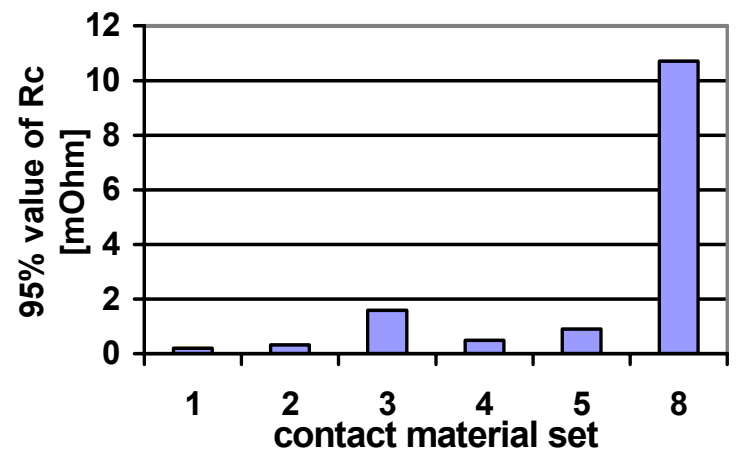

Figure 7: $95 \%$ values of contact resistance after service life test for different contact material combinations, see tab. 3

\subsection{Welding forces after lift off}

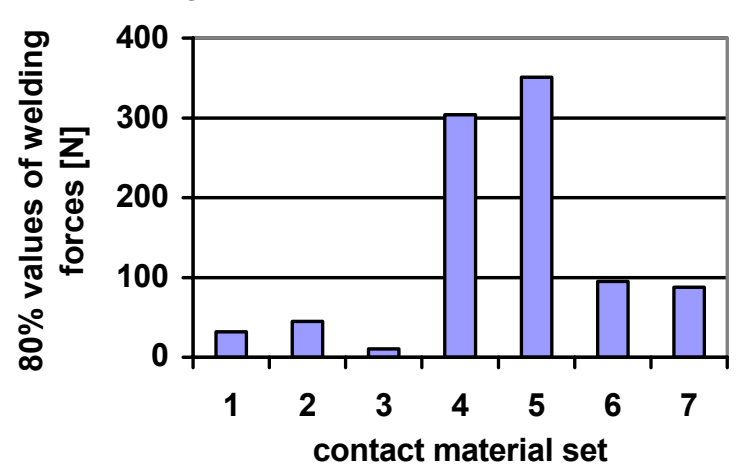

Figure 8: Welding forces after electrodynamic lift off at 8 kA for different contact material combinations, see tab. 3

\subsection{Contact erosion at $20 \mathrm{kA}$}

Fig. 9 a shows the contact erosion results of 5 break operations at $20 \mathrm{kA}$ peak for the stationary and movable contact tips respectively.

In fig. $9 \mathrm{~b}$. is presented the remaining contact volume after 5 break operations.
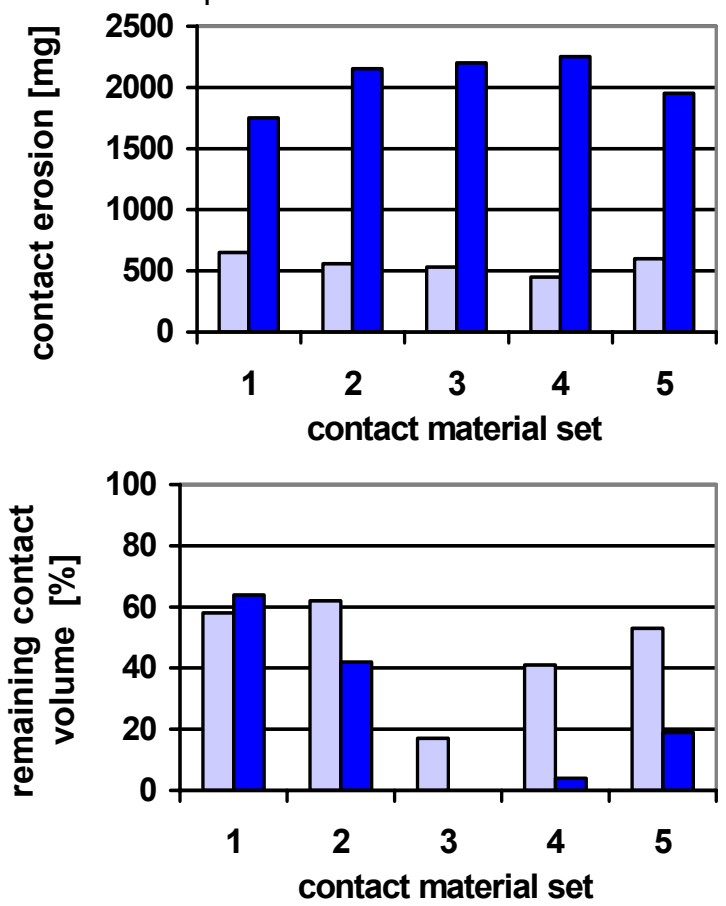

Figure 9 a.: Average values for contact erosion

b.: Average values for remaining contact volume after 5 O-operations at $20 \mathrm{kA}$ left column: stationary contact right column: moveable contact see table 3 for contact material sets

\section{Discussion}

\subsection{Electrical service life}

Results from electric service life showed the lowest erosion rate for the extruded $\mathrm{Ag} / \mathrm{SnO}_{2} 12$ material (mat. A). (fig. 6). The higher erosion rate of $\mathrm{Ag} / \mathrm{SnO}_{2}$ 18 and 22 compared to $\mathrm{Ag} / \mathrm{SnO}_{2} 12$ can be explained through the higher remaining porosity because of the manufacturing technique (s. tab. 1). The reason for the higher erosion rate of $\mathrm{Ag} / \mathrm{SnO}_{2} 22$ compared to $\mathrm{Ag} / \mathrm{SnO}_{2} 18$ is probably the higher electrical (s. tab. 1) and thermal conductivity of $\mathrm{Ag} / \mathrm{SnO}_{2} 18$.

Graphite bearing materials (mat. D. and F.) show substantial higher erosion. This can be attributed to the effect of graphite reactions with the ambient air during arcing and subsequent blow out of formed $\mathrm{CO}$ and $\mathrm{CN}$ gas compounds [8] through the molten contact surface. This effect prevents other contact material compounds for some time from oxidation, but causes high contact material losses as well as high porosity in 
the contact surface after arcing. Fig. 10 shows a microsection of $\mathrm{Ag} / \mathrm{SnO}_{2} 12$ after service life. The distribution of the oxide particle in the arc molten surfaces stays homogeneous with no formation of oxide layers. This can be attributed to the action of the wetting additive $\mathrm{Ag}_{2} \mathrm{MoO}_{4}$ used in this material [15]. Instead there can be very thin oxide layers which can explain the little bit higher observed contact resistance in compare to the silver tin oxide materials with higher oxide content. There the oxide layer formation seems to be further reduced because of the coarser $\mathrm{SnO}_{2}$ powder. With Ag/W50 material severe formation of tungsten oxide and silver tungstates [2. 4] occurs (fig. 11 ) and leads to high contact resistance observed (fig. 7)

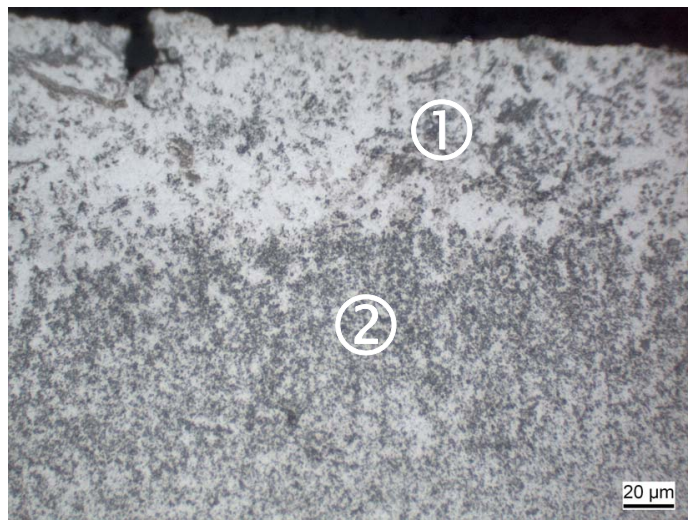

Figure 10: Microsection of $\mathrm{Ag} / \mathrm{SnO}_{2} 12$ (mat. A) after 7000 operations service life test. No oxide layer formation in the arc treated surface (region (1)) with similar oxide particle distribution as in the original material (region (2)).

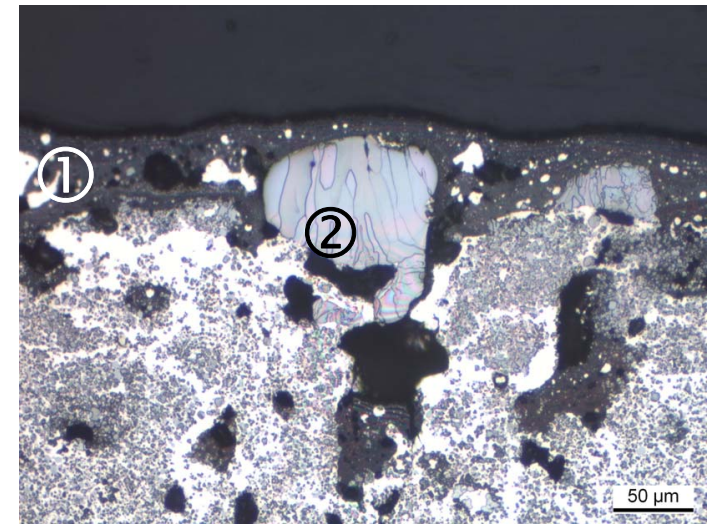

Figure 11: Microsection of Ag/W50 (mat. E) after 7000 operations service life test mating Ag/W50 (contact set 8). At the surface formation of oxide and tungstate layers (1) and coarse pieces of molten W (2)

Oxide layer formation on moveable Ag/W50 was substantially reduced with mating contacts $\mathrm{Ag} / \mathrm{C} 4$ or $\mathrm{Ag} / \mathrm{WC27/C3}$. Due to material transfer from $\mathrm{C}$ bearing contact tips to the mating $\mathrm{Ag} / \mathrm{W}$ the $\mathrm{Ag} / \mathrm{W}$ surface was protected from oxidation. Thus contact resistances stayed low with contact material combinations 1 and 2 .

\subsection{Welding test}

This transfer also occurred with the welding tests, compare fig. 12.

With the welding tests the high porosity of the arc treated contact surface of graphite bearing materials $D$ and $\mathrm{F}$ lead to low welding forces as shown in fig. 8.

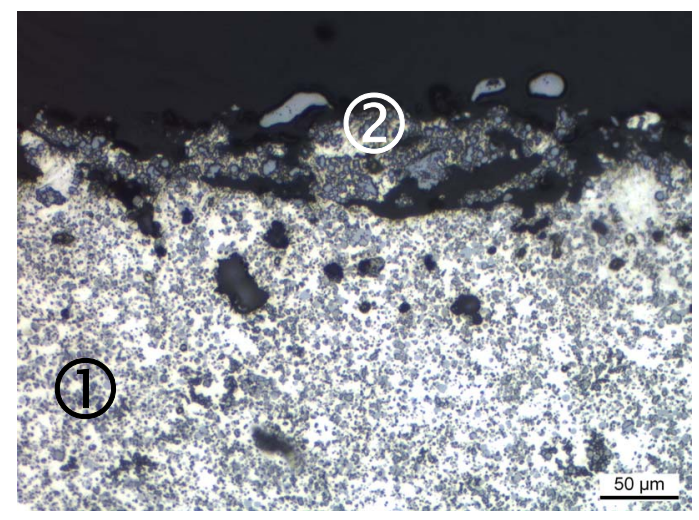

Figure 12: Microsection of Ag/W50 (mat. E) after welding test of contact set 1 . The $\mathrm{Ag} / \mathrm{W}$ material is not much effected by the arc (1) and covered by transferred material from mating Ag/WC/C (2)).

While $\mathrm{Ag} / \mathrm{SnO}_{2} 12$ (mat. A) showed low welding forces the materials with higher $\mathrm{SnO}_{2}$ content mat. $\mathrm{B}$ and $\mathrm{C}$ showed high values. Probably this is a consequence of different grain sizes of $\mathrm{SnO}_{2}$ powder used (compare fig. 1 mat. $A$ to $B$ and $C$ ) and better distribution of oxide particles in extruded material A. Fig. 13 shows a broken weld of $\mathrm{Ag} / \mathrm{SnO}_{2} 18$.

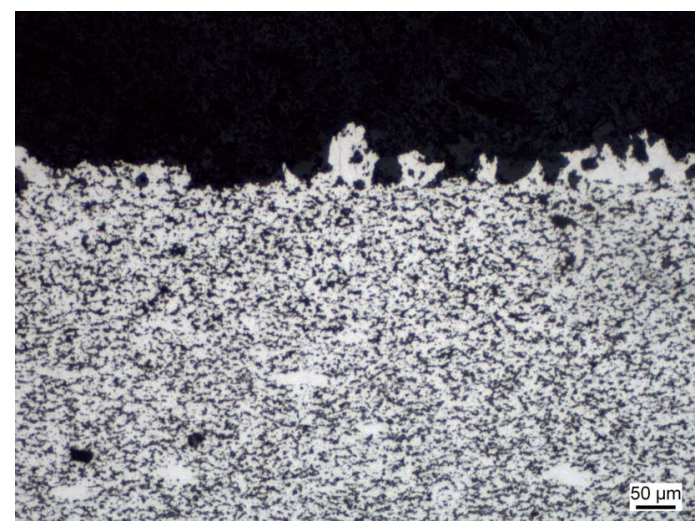

Figure 13: Microsection of $\mathrm{Ag} / \mathrm{SnO}_{2} 18$ (mat. B) after welding test. Rough surface structure results from broken weld.

In fig. 13 is also presented that the arc affected area is much smaller than after a service life test because of 
only two welding tests instead of 1000 to 7000 operations in the service life test.

\section{3 break operations at $20 \mathrm{kA}$ peak}

It seems to be, that contact erosion measurements (fig. 9 a.) are not reliable in this case because there is a lot of weld not only from contact materials formed upon the high current. And that can distort the measurement results. Because of that it will be more reliable to look on the remaining contact volume (fig 9 b).

The very high remaining contact volume of the $\mathrm{Ag} / \mathrm{WC} / \mathrm{C}$ and $\mathrm{Ag} / \mathrm{C}$ is astonishing. But it seems that between the break operations a coating of graphite is formed on the contact surface (fig. 14) which save the $\mathrm{Ag} / \mathrm{C} 4$ layer during the next break operations.

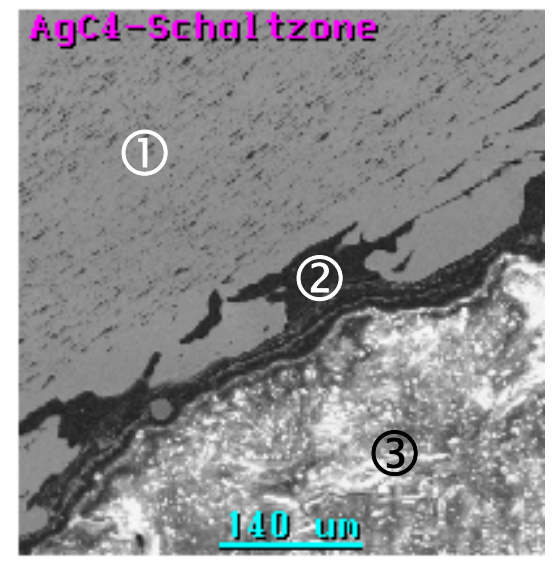

Figure 14: SEM micrograph of $\mathrm{Ag} / \mathrm{C} 4$ (mat. D) after after $5 \mathrm{O}$-operations at $20 \mathrm{kA}$ (Ag/C4 microstructure (1), C-layer (2) and mounting compound(3))

The comparison of the $\mathrm{Ag} / \mathrm{SnO}_{2}$ materials shows that at $20 \mathrm{kA}$ more coarse oxide particles and higher oxide contents are advantageous in regard to contact erosion under short circuit break arcs, as shown in fig. $9 \mathrm{~b}$. Especially the moving contact is subject to severe erosion with no or only little material left after 5 break operations performed. But for the stationary as well as the moveable contact the $\mathrm{Ag} / \mathrm{SnO}_{2}$ materials with rising $\mathrm{SnO}_{2}$ content show better results. As visible in fig. 15 the $\mathrm{Ag} / \mathrm{SnO}_{2}$ materials with high oxide content show after $20 \mathrm{kA}$ break operations a high porosity surface structure expected to be advantageous for low welding forces at higher short circuit currents.

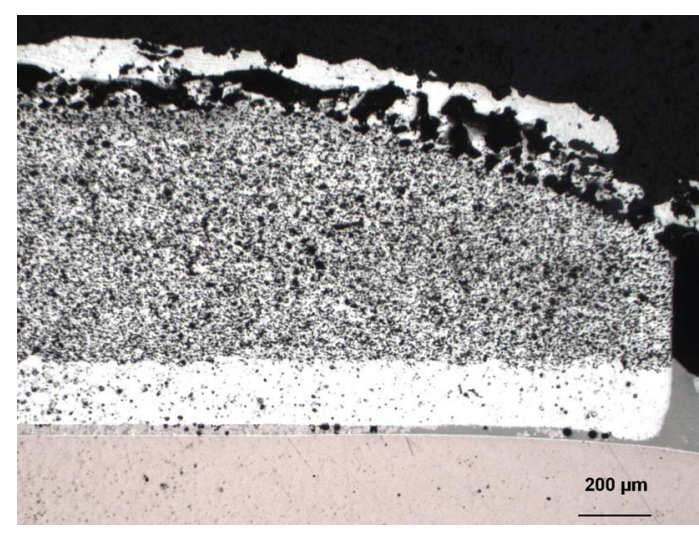

Figure 15: Microsection of $\mathrm{Ag} / \mathrm{SnO}_{2} 18$ (mat. B) 5 break operations at $20 \mathrm{kA}$, stationary contact.

\section{Conclusion}

Performance of different $\mathrm{Ag} / \mathrm{SnO}_{2}$ materials in switching tests paralleled to requirements of MCCBs show that oxide content, $\mathrm{SnO}_{2}$ particle size and manufacturing technology influences the results significantly. This in combination with the finding of excellent low contact erosion at service life and good contact resistance values gives indications for the further improvement of $\mathrm{Ag} / \mathrm{SnO}_{2}$ contact material for the use in circuit breakers.

\section{Acknowledgement}

The mechanical endurance tests have been performed by our colleagues Dr. Chi Leung, Dennis Fitzgerald, and Doug Harman from AMI Doduco Inc, Export (PA). Many thanks for excellent cooperation and fruitful discussions.

\section{References}

[ 1] H.Edmunds: Contacts in Low Voltage Circuit Breakers, Seminar on Electrical Contacts, The Pennsylvania State University (1954)

[ 2] G.Witter, V.Abele: The Change in Surface Resistance of Tungsten-Silver Contacts as a Function of Composition, Microstructure and Environment, Proc. $8^{\text {th }}$ ICEC (1976), Tokyo, pp. $445-451$.

[ 3] C.Leung, H.Kim, P.Wingert: Comparison of Reignition Properties of Serveral Ag/W, Ag/WC and Ag/Mo Electrical Contact Materials, IEEE CPMT Trans. 9, no. 1 (1986), pp. 86 - 91.

[ 4] P.Slade: Effects of the Electrical Arc and the Ambient Air on the Contact Resistance of Silver, Tungsten, and Silver-Tungsten Contacts, J. Appl. Phys., 47, no. 8 (1976), pp. $3438-3442$.

[ 5] E.Walczuk, S.Stolarz, K.Wojtasik: Experimental Study of $\mathrm{Ag}-\mathrm{W}-\mathrm{Re}$ Composite Materials under High Current 
Conditions, Proc. $32^{\text {nd }}$ IEEE Holm Conf. (1986), pp. 77 83.

[ 6] C.Lambert, G.Cambon: The Influence of Manufacturing Conditions and Metallurgical Characteristics on the Electrical Behaviour of Silver/Graphite Contact Materials, Proc. $24^{\text {th }}$ IEEE Holm Conf. (1978), pp. $401-$ 406.

[ 7] V.Behrens, T.Honig, A.Kraus, E.Mahle, R..Michal, K.Saeger: Test Results of Different Silver/Graphite Contact Materials in Regard to Applications in Circuit Breakers, Proc. $41^{\text {st }}$ IEEE Holm Conf. (1995), pp. 393 397.

[ 8] E.Vinaricky, V.Behrens: Switching Behavior of Silver / Graphite Contact Material in Different Atmospheres in Regard to Contact Erosion, Proc. $44^{\text {th }}$ IEEE Holm Conf. (1998), pp. $292-300$.

[ 9] S.Allen, E.Streicher, C.Leung: Electrical Performance of Ag-W-C and Ag-WC-C Contacts in Switching Tests, Proc. $20^{\text {th }}$ ICEC (2000), Stockholm, pp. $109-114$.
[10] C.Leung, E.Streicher, D.Fitzgerald: Weibull Analysis of Switching Contact Resistance in Laboratory and Commercial Circuit Breakers, Proc. $53^{\text {rd }}$ IEEE Holm Conf. (2007), pp. $56-62$.

[11] J.Ambier, M.J. Francillon, D.Jeannot, P.Ramoni, J.C. Ramseyer: AgMeO Contact Materials for Low Voltage Circuit Brekaers, Proc. $13^{\text {th }}$ ICEC (1986), Lausanne, pp. $206-210$.

[12] F.Hauner, J.Große, R.Herbst, R.Steger, G.Weidner:

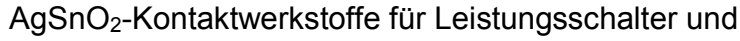
Leistungsschalter/Schütz-Kombinationen, VDEFachbericht 47 (1995), pp. 187 - 193.

[13] N.Behrens, W:Böhm; P.Braumann, G.Kepp: Experiences with the Contact Material $\mathrm{Ag} / \mathrm{SnO}_{2}$, Proc. $12^{\text {th }}$ ICEC (1984), Chicago, pp. $185-191$.

[14] C.Bernauer, T.Kuntze, V.Behrens, T.Honig: Substitution of Silver/Cadmium Oxide in High Voltage Disconnentors, Proc. $51^{\text {st }}$ IEEE Holm Conf. (2005), pp. $42-47$.

[15] European Patent EP 0660964 


\title{
Improvement of Percussion Welding Characteristics of $\mathrm{CuCr} 25$ Contact Material by Decreasing Tensile Strength
}

\author{
Li Yu ${ }^{1}$, Yingsan Geng ${ }^{1}$, Qian $\mathrm{Li}^{1}$, Jianhua Wang ${ }^{1}$, Zhiyuan Liu ${ }^{1}$, Dongwei $\mathrm{Li}^{1}$,Wenbin Wang ${ }^{2}$, and Xiaojun Wang ${ }^{2}$
}

1. State Key Laboratory of Electrical Insulation and Power Equipment, Xi' an Jiaotong University, Xi'an, China

2. Shaan'Xi Sirui Industries Co.,LTD,Xi'an, China

\begin{abstract}
Copper-Chromium contact material is widely used in vacuum interrupters. However, there are occasionally percussion welding phenomena when $\mathrm{CuCr} 25$ (25\% weight $\mathrm{Cr}$ ) contact material close electrical circuit, especially in the case of high rated short circuit making current tests. It was reported that the resistance welding performance of $\mathrm{CuCr}$ contact material can be improved by adding $0.1 \% \sim 4 \%$ Te to decrease the tensile strength. In order to investigate the influence of tensile strength of contact material on percussion welding performance by adding $\mathrm{Te}$ $(0.01 \%)$, a percussion welding experiment was designed to compare weld force between $\mathrm{CuCr} 25$ and $\mathrm{CuCr25}$-Te contact material. The percussion welding experiment simulated the contacts in a vacuum interrupter closed a DC circuit and bounced once. The DC currents were 300A and 400A. The bounce times were $2 \mathrm{~s}$ and $3 \mathrm{~s}$. With a lower tensile strength (220 240Mpa) than CuCr25 (335 345Mpa), the CuCr25-Te contact material was less proned to occur percussion weld than $\mathrm{CuCr} 25$ contact material. And the maximum percussion weld forces of $\mathrm{CuCr25}$-Te were also lower than $\mathrm{CuCr} 25$ under same arcing currents and arcing times. The percussion welding characteristic of $\mathrm{CuCr} 25$ contact material is proved to be superior by reducing its tensile strength.
\end{abstract}

Keywords-percussion weld; tensile strength; contact materials; vacuum interrupters.

\section{INTRODUCTION}

Contact welding can occur if a high enough current passes through closed contacts and causes the contact spots to melt. This phenomenon is known as resistance welding [1]. Another type of weld is the percussion welding. This weld occurs when contacts come together with a high voltage across them. If two contacts are close enough or bounce apart, an arc forms between them [2]. This arc will cause melting the contact surfaces. If this surface melting is severe enough, the contacts will weld together when they finally force together [3]. Under some conditions of current level and bounce duration, the percussion welding can produce stronger welds than can be experienced by resistance welding [2]. Once the strength of the weld prevents the proper operation of the vacuum interrupter, it could present a serious problem [1].Thus, it is necessary to investigate percussion weld force in high-current vacuum interrupters.

$\mathrm{Cu}-\mathrm{Cr}$-alloys were well known as a contact material with an outstanding capability for circuit interruption and dielectric recovery, endurance and resistance to erosion, high voltage withstand and current chop in vacuum interrupter [4], [5]. There have been a lot of studies that showed a $\mathrm{Cu}-\mathrm{Cr}$
(25\% 30\% wt) contact material worked better than $\mathrm{Cu}-\mathrm{Cr}(50$ wt \%) contact material in some conditions [6-11]. For example, Yanabu et al. [10] reported that the threshold interruption current at $25 \mathrm{wt} \% \mathrm{Cr}$ is higher than that at $50 \%$ wt $\mathrm{Cr}$. Recently, it was found occasionally in type tests that $\mathrm{CuCr} 25$ contact material made by a vacuum casting technology caused percussion welding when closing a high rated short circuit making current.

Slade [1] showed the maximum percussion weld force as a function of the arc energy both theoretically and experimentally. He used the following exponent relationship to calculate the maximum weld force $F w$ when percussion welding occurs,

$$
F_{w}=K W_{C}^{2 / 3}
$$

Where $K$ - coefficient of contact materials,

$$
W_{c} \text { - arcing energy. }
$$

If all the energy is used to melt the contacts, then

$$
W_{C}=\int_{t_{a}} U_{a} I d t
$$

Where $t_{a}$ - the arcing time,

$$
\begin{aligned}
& U_{a} \text { - the arc voltage, } \\
& I \text { - the arc current. }
\end{aligned}
$$

It is shown in equation (1) that the weld force is related with arc energy. Therefore, higher arc voltage and current level and longer arcing time can cause a higher weld force.

The coefficient $K$ is related with the contact materials, as equation (3) shows [1]

$$
K=\Gamma \pi\left[\frac{3}{4 \pi \delta\left[C_{V}\left(T_{m}-T_{b}\right)+C_{L}\right]}\right]^{2 / 3}
$$

Where $C_{v}$ - the specific heat,

$T_{m}$ - the melting temperature,

$T_{b}$ - the bulk temperature,

$C_{L}$ - the latent heat of fusion,

$\delta$ - the material density,

$\Gamma$ - the tensile strength of materials. 
That means, in general, the greater the tensile strength of materials $\Gamma$ of contact material is, the higher percussion weld force is.

Several researchers have proved that the effect of $\mathrm{Te}$ additive in $\mathrm{Cu}-\mathrm{Cr}$ contact materials can improve their anti-welding capability [12-14]. Ozawa et al. [12] showed that tensile strength reduced as a function of small addition $(0.1 \%$ to $4 \%$ ) of Te into sintered $\mathrm{Cu}-\mathrm{Cr}-\mathrm{Te}(50 \% \mathrm{wt} \mathrm{Cr})$, so that the weld force can be reduced with the lower tensile strength. However, only resistance welding was studied in the mentioned works [12-14]. The tensile strength $\Gamma$ of contact materials on percussion welding performance needs to be further investigated.

The objective of this paper is to investigate the influence of tensile strength on percussion welding characteristic of $\mathrm{CuCr} 25$ contact materials.

\section{EXPERIMENTAL SETUP}

In the experiments, $0.01 \%$ weight $\mathrm{Te}$ was added in the $\mathrm{CuCr} 25$ (25\% wt $\mathrm{Cr}$ ) contact material made by the vacuum casting technology. The effective of Te content in such low level was only to reduce the tensile strength of $\mathrm{CuCr} 25$. It measured that the $\mathrm{CuCr} 25$-Te contact materials had a lower tensile strength (220 240Mpa) than $\mathrm{CuCr} 25$ (335 345Mpa). The parameters of the test vacuum interrupters (TVIs) with butt-type contacts using the $\mathrm{CuCr} 25$ and the $\mathrm{CuCr} 25-\mathrm{Te}$ contact material were shown in table I.

TABLE I. PARAMETERS OF TEST VACUUM INTERRUPTER

\begin{tabular}{|c|c|c|c|c|}
\hline $\begin{array}{c}\text { Rating } \\
\text { voltage }\end{array}$ & $\begin{array}{c}\text { Rating } \\
\text { current }\end{array}$ & $\begin{array}{c}\text { short-time } \\
\text { withstand } \\
\text { current }\end{array}$ & $\begin{array}{c}\text { short-time } \\
\text { withstand } \\
\text { time }\end{array}$ & $\begin{array}{c}\text { Force from atmospheric } \\
\text { pressure on the bellows }\end{array}$ \\
\hline $1140 \mathrm{~V}$ & $250 \mathrm{~A}$ & $2 \mathrm{kA}$ & $10 \mathrm{~s}$ & $50 \pm 10 \mathrm{~N}$ \\
\hline
\end{tabular}

In order to obtain the percussion welding data for the two contact materials, we designed a percussion welding apparatus to simulate that the movable contact bounce once during closing operation of the vacuum interrupters. Fig. 1(a) shows the experimental circuit in the test. The welding current was supplied by a ZX7-500 DC current source, which can supply $0 \sim 500 \mathrm{~A}$ DC current. And the circuit current was measured by a current shunt $(150 \mu \Omega)$, which was in series with the TVI. The TVI was operated by an electromagnetism operation mechanism. A programmable logic controller (PLC) was used to control the operation mechanism. The PLC outputted a close signal to control the TVI close and an open signal to control the TVI open. In this way, the arcing time can be controlled.

The test procedure for the percussion welding simulation was as follows [see Fig. 1(b)]:

1) Close switch $S 1$ to by-pass the PLC.

2) The AC source supplied a $220 \mathrm{~V}$ voltage for the electromagnetism operation mechanism. Then, TVI was closed.

3) Open the S1. Thus the TVI was controlled by the PLC.

4) Switch on the S2. Hence, a DC current passed through the TVI.
5) As the PLC outputted an open signal, the TVI was opened. And a DC arc was burning between two contacts.

6) When the PLC outputted a close signal, the TVI was closed.

7) Immediately after the TVI was closed, S1 was closed again to lock the TVI in close position.

8) Switch off the S2. The DC current was shut down.

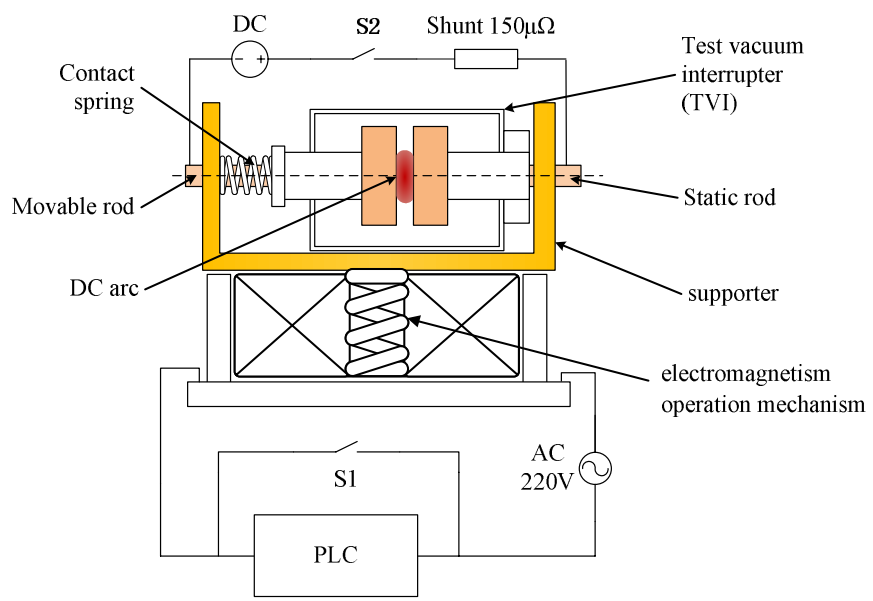

(a) Schematic of the test circuit for Percussion welding.

DC-ZX7-500 welding machine, can supply $0 \sim 500 \mathrm{~A}$ DC current, Shunt-shunt resistor of $150 \mu \Omega$, S1 - knife switch, S2 - switch of ZX7-500 welding machine, electromagnetism operation mechanism - operating voltage of $\mathrm{AC} 220 \mathrm{~V}$, voltage frequency of $50 \mathrm{~Hz}$

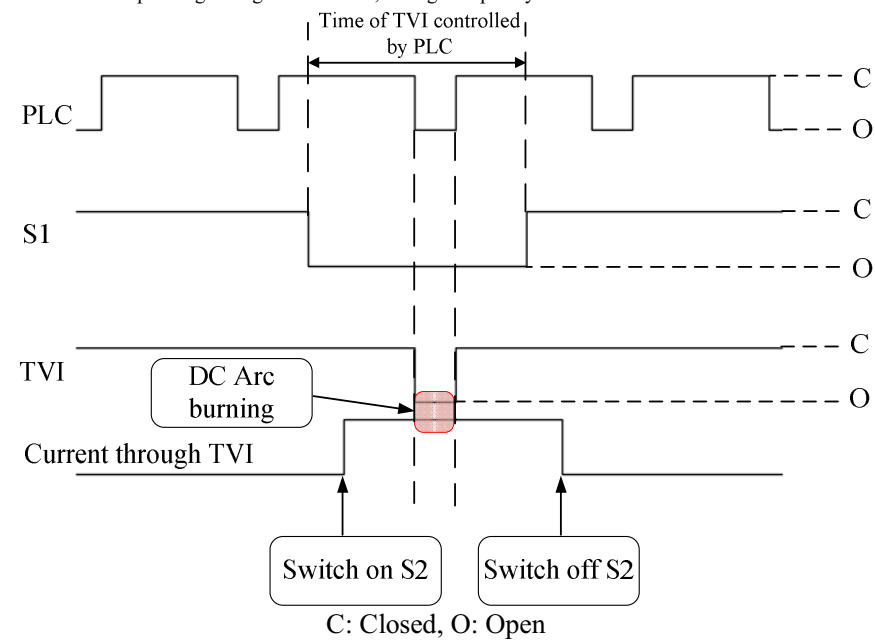

(b) Timing diagram of percussion welding test system

1) Close switch S1 to by-pass the PLC and close the TVI. 2) Open S1. Then the TVI was controlled by the PLC. 3) Switch on the S2. Hence, a DC current passed through the TVI.4) As the PLC outputted an open signal, the TVI was opened. And a DC arc was burning between two contacts. 5) When the PLC outputted a close signal, the TVI was closed again. 6) Immediately after the TVI was closed, S1 was closed again to lock the TVI in close position. 7) Switch off the S2. The DC current was shut down.

Fig. 1. Percussion welding test setup

This test procedure simulated a sequence of movable contact close-bounce-close once. And the time interval of the two contacts opening is the bouncing time, which is controlled by the PLC. After that, the TVI was taken off in its closed condition for a measurement of welding force.

Fig. 2 shows a setup for percussion weld force measurement. The stationary rod of the TVI was fixed with a transducer. And the movable rod was connected with a nut. When rotating the nut, the movable rod was pulled up slowly. And the force transducer outputted a force signal that was recorded by an oscillograph. The percussion weld force of the 
TVI was measured by a TJL-1S force-transducer. The full scale output of force transducer is $20 \mathrm{mV} / 100 \mathrm{~kg}$. An amplification circuit was used to amplify the output signal (about $2 \mathrm{mV}$ ) of the force transducer. In the experiment, we used an amplifier AD620. And the output of force transducer can be amplified by 97.655 times.

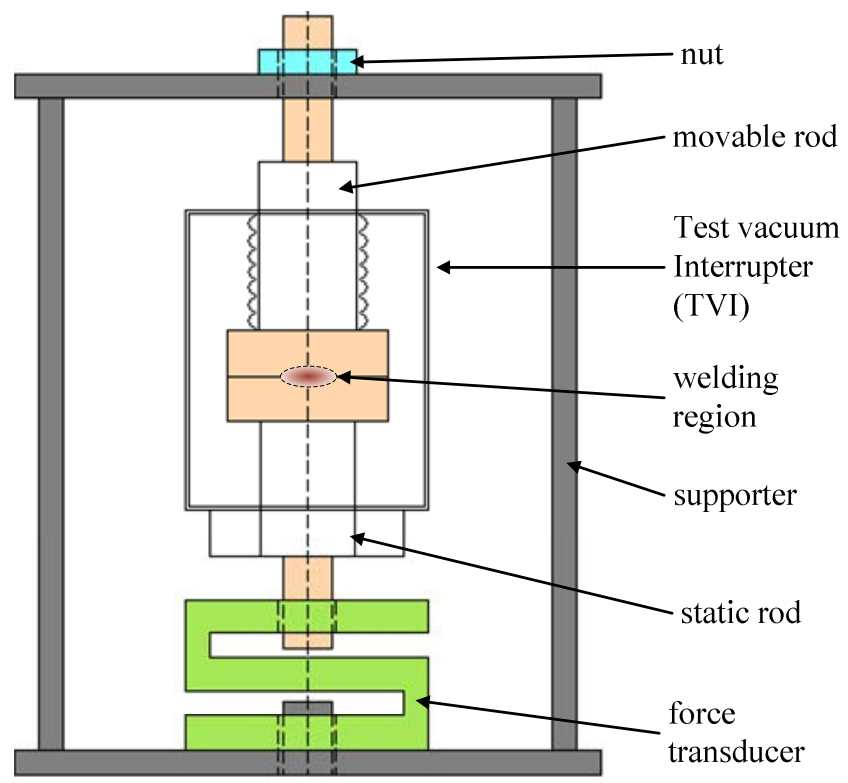

Fig. 2. Welding force measurement setup.

The stationary rod of the TVI is fixed with a transducer. And the movable rod is connected with a nut. When the movable rod is pulled up slowly, the force transducer outputted a force signal that was recorded by an oscillograph.

Force transducer - TJL-1S Force transducer, maximum output $20 \mathrm{mV} / 100 \mathrm{~kg}$

Before two contacts separated, the force was measured by the transducer followed equation (4). If we assumed the instant of contact separation to be zero, the force measured by transducer $F_{m}\left(0^{-}\right)$can be described as follows:

$F_{m}\left(0^{-}\right)=F_{w}+F_{\text {bellows }}-F_{g}+f$

Where $F_{w}$ - percussion weld force, $F_{\text {bellow }}$ - force from atmospheric pressure on the bellows, $F_{g}$ - gravity of TVI except movable rod, $f$ - friction force.

After the contacts separated, the force measured by the transducer $F_{m}\left(0^{+}\right)$can be described as follows:

$F_{m}\left(0^{+}\right)=F_{\text {bellows }}-F_{g}+f$

That means, the force falls sharply when two contacts separate.

Fig. 3 shows a typical oscillogram of percussion weld force measurement. When pulling up the movable role, the force measured by the transducer was increasing until the two contacts separated. The force signal fell sharply, because the welding region was broken. The difference between the maximum and minimum of the force signal at the instant of two contacts separation was the percussion weld force, which can be calculated by equation (6).

$$
F=\Delta U \times g \times a / k
$$

Where $\Delta U$ - the deviation of force signal $(\mathrm{mV}), g$ - gravity acceleration $\left(9.8 \mathrm{~m} / \mathrm{s}^{2}\right), k$ - magnification of signal (97.655), $a$ conversion ratio of the sensor $(5 \mathrm{~kg} / \mathrm{mV})$. In fig 3 , the deviation of force signal is $1080 \mathrm{mV}$. The maximum percussion weld force is $541.9 \mathrm{~N}$

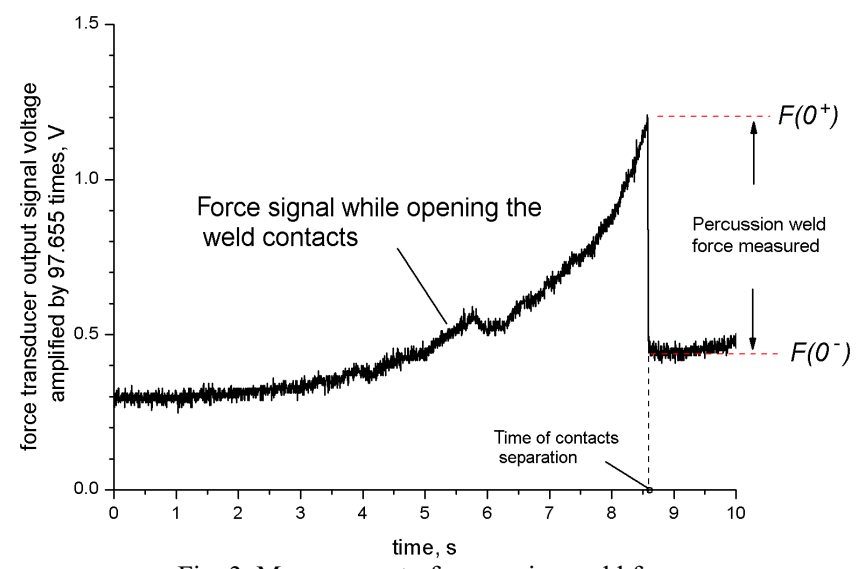

Fig. 3. Measurement of percussion weld force

The force signal falls sharply at the instant of contacts separation that means the welding region departure. The difference between the maximum and minimum of the force signal at the time of two contacts separation was the percussion weld force measured.

In the experiments, the arc current and bouncing (arcing) time varied as shown in Table II. Each contact materials was taken more than 15 operations under each arcing condition. And the contact gap distance between two contacts was $1 \pm$ $0.1 \mathrm{~mm}$.

TABLE II. PARAMETERS FOR THE EVALUATION OF THE WELDING CHARACTERISTICS

\begin{tabular}{|c|c|c|c|c|c|}
\hline \multirow{2}{*}{$\begin{array}{c}\text { Contact } \\
\text { materials }\end{array}$} & \multicolumn{4}{|c|}{$\begin{array}{c}\text { Number of operations under different DC } \\
\text { arc currents (A) and arcing times(s) }\end{array}$} & \multirow{2}{*}{$\begin{array}{c}\text { Contact } \\
\text { gap }\end{array}$} \\
\cline { 2 - 5 } & $\mathbf{3 0 0 A , 2 s}$ & $\mathbf{4 0 0 A , 2 s}$ & $\mathbf{3 0 0 A , 3 s}$ & $\mathbf{4 0 0 A , 3 \boldsymbol { s }}$ & \\
\hline $\mathrm{CuCr} 25$ & 21 & 18 & 21 & 24 & \multirow{2}{*}{$\begin{array}{c} \pm 0.1 \\
\mathrm{~mm}\end{array}$} \\
\hline $\mathrm{CuCr} 25-\mathrm{Te}$ & 20 & 16 & 21 & 17 & \multicolumn{2}{c}{} \\
\hline
\end{tabular}

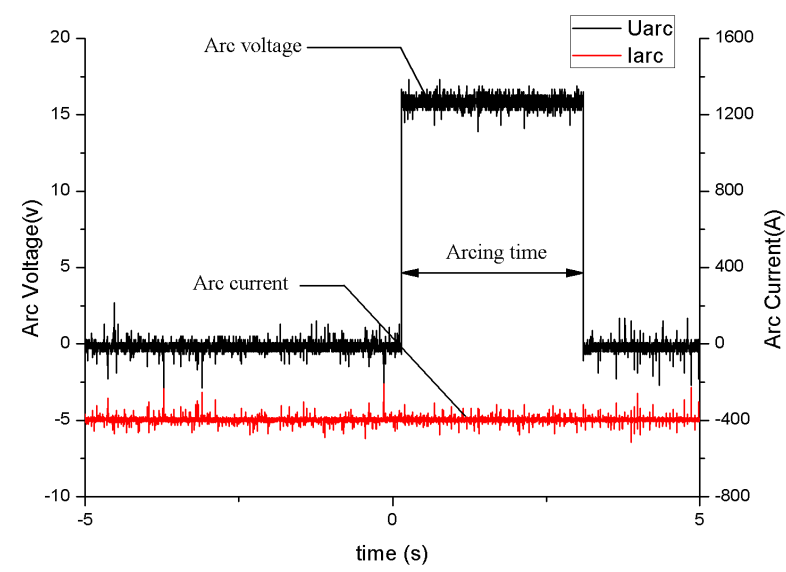

Fig. 4. Waveform of arc current and arc voltage

The upper curve is arc voltage. The lower curve is arc current. 


\section{RESULTS}

\section{A. Arc Energy}

Fig. 4 shows a typical waveform of arc current and arc voltage. The arc energy $W c$ was calculated by using equation (2). As the arcing currents were 300A and 400A and bouncing time were set as $3 \mathrm{~s}$ and $4 \mathrm{~s}$, four arcing energy were got as table III shows, which ranged from 9360J to 19680J.

TABLE III. ARC ENERGIES UNDER FOUR TEST CONDITIONS

\begin{tabular}{|c|c|c|c|}
\hline Time (s) & Current (A) & Voltage (V) & Energy (J) \\
\hline 2 & 300 & 15.6 & 9360 \\
\hline 2 & 400 & 16.4 & 13120 \\
\hline 3 & 300 & 15.6 & 14040 \\
\hline 3 & 400 & 16.4 & 19680 \\
\hline
\end{tabular}

\section{B. Percussion weld force of $\mathrm{CuCr} 25$ and $\mathrm{CuC25-Te}$}

Fig. 5 gives the cumulative distribution of percussion weld force for $\mathrm{CuCr} 25$ and $\mathrm{CuCr} 25-\mathrm{Te}$ contact materials at $2 \mathrm{~s}$ arcing time. The horizontal axis is the percussion weld force measured in the experiments. And the vertical axis is the cumulative percentage of weld forces that is less than a given value.

The $\mathrm{CuCr} 25-\mathrm{Te}$ contact material has a low percussion welding force than the $\mathrm{CuC} 25$ contact material under both $300 \mathrm{~A}$ and $400 \mathrm{~A}$ conditions. For example, at 300A there is a $94 \%$ probability that the percussion weld force is less than $90 \mathrm{~N}$ for $\mathrm{CuCr} 25-\mathrm{Te}$. $\mathrm{CuCr} 25$ contact material, on the other hand, has $71 \%$ probability that the weld force is less than $90 \mathrm{~N}$ at 300 A. And the maximum weld force measured of $\mathrm{CuCr} 25-\mathrm{Te}$ is $144 \mathrm{~N}$, while $\mathrm{CuCr} 25$ is $177 \mathrm{~N}$. At $400 \mathrm{~A}$, the probability of percussion welding of $\mathrm{CuCr} 25-\mathrm{Te}$ is much lower than the $\mathrm{CuCr} 25$ contact material. And the maximum weld force measured of $\mathrm{CuCr} 25-\mathrm{Te}$ is $181 \mathrm{~N}$, while $\mathrm{CuCr} 25$ is $357 \mathrm{~N}$.

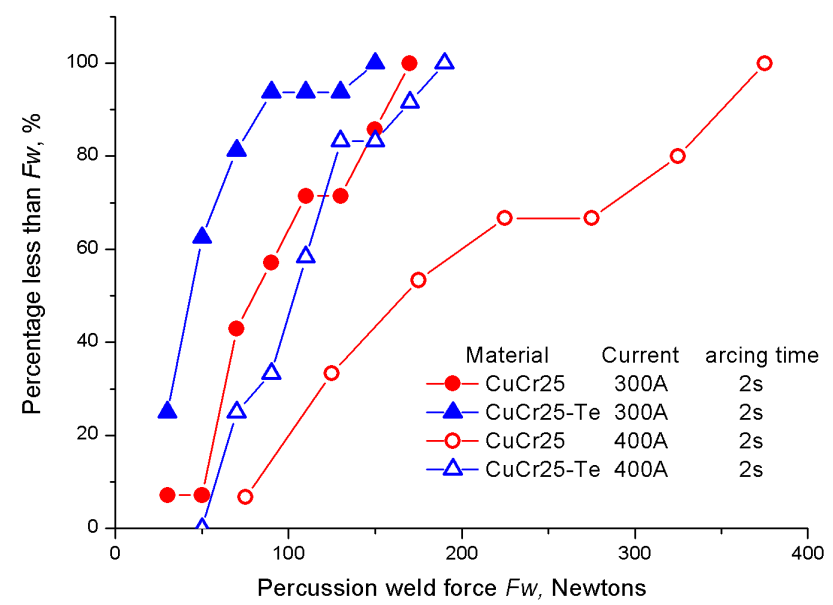

Fig. 5. Cumulative distribution of percussion weld force for $\mathrm{CuCr} 25$ and $\mathrm{CuCr} 25-\mathrm{Te}$ contact materials at $2 \mathrm{~s}$ arcing time

Fig. 6 shows the cumulative distribution of percussion weld force for $\mathrm{CuCr} 25$ and $\mathrm{CuCr} 25-\mathrm{Te}$ contact materials at $3 \mathrm{~s}$ arcing time. Again the probability for $\mathrm{CuCr} 25$-Te contact material that the percussion weld force less than a certain value is lower than for $\mathrm{CuCr} 25$ contact material at $300 \mathrm{~A}$. And the maximum weld force measured of $\mathrm{CuCr} 25-\mathrm{Te}$ contact material is $167 \mathrm{~N}$, which is lower than the $\mathrm{CuCr} 25(253 \mathrm{~N})$. However, when the data of the two contact materials are compared at $400 \mathrm{~A}$, it can be seen that the cumulative distribution of both contact materials were similar. The major difference between the $\mathrm{CuCr} 25$ and $\mathrm{CuCr} 25-\mathrm{Te}$ is evident in the maximum weld force measured. The maximum weld force of the $\mathrm{CuCr} 25$ contact material is nearly $800 \mathrm{~N}$, which is also larger than the $\mathrm{CuCr} 25$-Te contact materials $(491 \mathrm{~N})$.

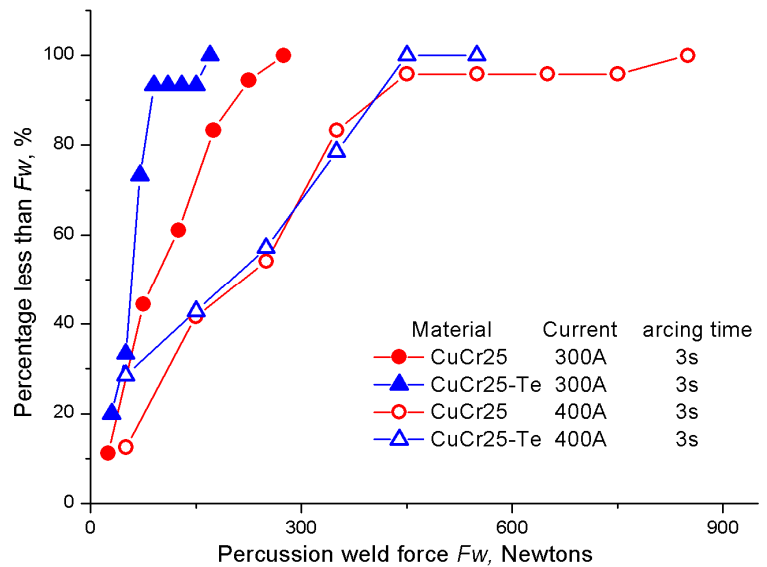

Fig. 6. Cumulative distribution of percussion weld force for $\mathrm{CuCr} 25$ and $\mathrm{CuCr} 25-\mathrm{Te}$ contact materials at $3 \mathrm{~s}$ arcing time

\section{CONCLUSION}

In order to investigate the influence of tensile strength on percussion welding characteristic of $\mathrm{CuCr} 25$ contact materials made by a vacuum casting technology, $0.01 \%$ Te was added in it so that the tensile strength was reduced from 335 345Mpa to 220 240Mpa. We designed a percussion welding apparatus that simulate a percussion weld phenomena in a vacuum interrupter. The simulation realized a sequence of a pair of contacts close-bounce-close once with controlled arc currents and bouncing duration. The results showed that the $\mathrm{CuCr} 25 \mathrm{Te}$ contact material was less proned to occur percussion weld than $\mathrm{CuCr} 25$ contact material. And the maximum percussion weld forces of $\mathrm{CuCr} 25 \mathrm{Te}$ were also lower than $\mathrm{CuCr} 25$ under same arcing currents and arcing times. The results supported that the percussion welding characteristic of $\mathrm{CuCr} 25$ contact material can be improved by reducing its tensile strength, such as adding Te.

\section{REFERENCES}

[1] P. G. Slade, The Vacuum Interrupter: Theory, Design, and Application, CRC Press, Taylor \& Francis Group, Boca Raton, Chapter 4, 2008, pp. 304-318.

[2] P. Kisliuk, "Arcing at electrical contacts on closure - part V the cathode mechanisms of extremely short arcs", Journal of Applied Physics, vol. 25, No 7, July 1954, pp. 897-900.

[3]P. P. Koren, P. G. Slade and C. Y. Lin, "Welding Characteristics of Ag-W Contacts Under High-Current Conditions", IEEE Trans. on Computer, hybrids, and Manufacturing Technology, vol.CHMT-3, No. 1, pp. 50-55, March 1980.

[4] H. Husler, H. Kippenberg and H. Schreiver, "Demands on contact materials for HV Vacuum Breakers", in Proc. for 10th International conf. on Electrical Contacts, Budapest, 1980, pp. 219-223. 
[5] P. G. Slade. "Advances in material development for high power, vacuum interrupter contacts", IEEE Trans. on Components Packaging and Manufacturing Technology, PART A, vol. 17. No. 1, pp. 96-106, March 1994.

[6] G. Filio, W. Rieder, M. Schussek, "Current chopping due to new and eroded vacuum contacts", in Proc. of IEEE XII International Symposium on Discharges and Electrical Insulation in Vacuum, Shoresh, Israel, Sep.1986, pp. 224-228.

[7] S. Temborius, M. Lindmayer, D. Gentsch, "Switching Behavior of Different Contact Materials for Vacuum Interrupters under Load Switching Conditions", in Proc. of IEEE 19th International Symposium on Discharges and Electrical Insulation in Vacuum, Xi'an, China, Sep. 2000, pp. 519-523.

[8] D. Heyn, M. Lindmayer, E.D Wilkening, "Effect of contact material on the extinction of vacuum arcs under line frequency and high frequency conditions, IEEE Trans. on Computer, Hybrids, and Manufacturing Technology, vol.14, pp. 65-70, March 1991.

[9] W. P. Li, R. Thomas, K. Smith, "Effects of Cr content on the interruption ability of Cu-Cr contacts", IEEE Trans. on Plasma Science, vol.29, pp. 744-748, Oct. 2001
[10] S. Yanabu, T. Tsutsumi, K. Yokokura, and E. Kaneko, "Recent technical developments in high-voltage and high-power vacuum circuit breakers", IEEE Trans. on Plasma Science, vol.17, pp. 717-723, Oct. 1989.

[11] J. Ballat, D. Konig, "Insulation characteristics and welding behavior of vacuum switch contacts made from various $\mathrm{Cu}-\mathrm{Cr}$ alloys", IEEE Trans. on Electrical Insulation, vol.28, pp. 628-634, 1993.

[12] K. Ozawa, K. Matsumoto, T. Nakanishi, K. Yoshigae, M. Yamazaki, and H. Shinohara, "Sintered $\mathrm{Cu}-\mathrm{Cr}$-Te alloy for the vacuum circuit breaker contact," in Proc. International Conf. on Electrical Contacts,

Electromechanical Components and their Applications, Nagoya, Japan, July 1986, pp. 823-830

[13] S. J. Fu, M. Fang, G. X. Miao. "Low Fusion Welding Contact Material CuCrTe for Vacuum Interrupter", High Voltage Apparatus, Vo1.33, 1997, pp $16-18$

[14] S.X. Xiu, J. M. Wang. "CuCrTe CONTACT MATERIAL PREPARED BY SMELTING IN VACUUM AND ITS PROPERTIES", in Proc. of IEEE 21 st International Symposium on Discharges and Electrical Insulation in Vacuum, vol. 21, pp. 322-324, Yalta, Ukraine, Sep. 27-Oct. 01, 2004 


\title{
Temperature Rise Behavior of $\mathrm{Ag} / \mathrm{SnO}_{2}$ Contact Materials for Contactor Applications
}

\author{
Timo Mützel, Peter Braumann, Ralf Niederreuther \\ Umicore AG \& Co. KG \\ Business Line Contact Materials, Applied Technology \\ 63457 Hanau-Wolfgang, Germany \\ timo.muetzel@eu.umicore.com
}

\begin{abstract}
Critical temperature rise can be a failure mechanism for contact materials in power applications. The use of contact materials with reduced silver content to be more cost effective and the smaller sizes of devices impact this failure mode. Furthermore this effect is enforced by reductions in the volume of contact material and lower contact forces, due to energy saving by less power consumption of the driving coil.
\end{abstract}

This paper presents influences of metal oxide content to contact resistance and temperature rise by experiments in a break-only model switch and in contactor applications. Temperature rise tests according to IEC standards show the influences of contact force on temperature rise of contactors and the effect of different switching conditions/loads on temperature rise. Therefore results of $\mathrm{AC}-3$ and $\mathrm{AC}-4$ standard contactor lifetime tests have been compared.

contact material; contactor; $\mathrm{Ag} / \mathrm{SnO}_{2}$; temperature rise; contact resistance

\section{INTRODUCTION}

Miniaturization, power consumption and of course cost efficiency have become the driving factors in modern power systems. This leads to switching device designs with smaller contact tips and/or usage of contact materials with reduced silver content. Furthermore devices and actuating mechanisms are miniaturized to the limit. In contactor design economizer circuits are used to reduce the power consumption while the contacts are closed. Consequently contact forces are reduced very often.

Some of the mentioned effects are influencing contact resistance and temperature rise behavior and therefore will be studied in this paper.

According to HoLM [1] the total contact resistance is made up of three parts. These are film, constriction and bulk material resistance. The area of true mechanical contact between two contact tips is called a-spot. The radius $\alpha$ of a circular a-spot can be estimated by applying HERTZIAN elastic contact mechanics:

$$
\alpha=\left(\frac{3 \cdot r \cdot F}{4 \cdot E}\right)^{1 / 3}
$$

where $r$ is the radius of the contact surface (so-called apparent contact area), $\mathrm{F}$ is the contact force and YounG's modulus E. Generally, any electrical contact is believed to contain some number of a-spots, which cover a very small fraction of the contact surface.

The resultant constriction resistance $R_{C}$ of a flat circular metallic contact area can be written as

$$
R_{C}=\frac{\rho}{2 \alpha}=\rho \sqrt{\frac{\pi \cdot H}{4 \cdot F}}
$$

where $\rho$ is the electrical resistivity and $H$ is the contact hardness.

A quadratic reciprocal dependency between contact resistance and contact force can be seen. The impact of a contact force reduction on switching and temperature rise behavior will be pointed out in chapter 2 . The resulting a-spot area $\mathrm{A}$ at given contact force and material hardness can be estimated by the following equation:

$$
\mathrm{A}=\alpha^{2} \cdot \pi=\frac{\mathrm{F}}{\mathrm{H}}
$$

Silver tin oxide materials with reduced silver content are requested by the market for realizing cost efficient switching devices. The a-spot ratio of mechanical contact comparing $\mathrm{Ag} / \mathrm{SnO}_{2} 14 \%$ to $12 \%$ metal oxide content at constant contact force results in:

$$
\frac{\mathrm{A}_{86 / 14}}{\mathrm{~A}_{88 / 12}}=\frac{\mathrm{H}_{88 / 12}}{\mathrm{H}_{86 / 14}} \approx 75 \%
$$

Here the influence of a higher metal oxide content on the constriction resistance of a new, unswitched contact surface can be clearly seen. Furthermore the material with higher oxide content will also provide a worse bulk resistance. The influence of these effects on contact resistance and temperature rise will be studied in chapter 3 . 


\section{IMPACT OF CONTACT FORCE}

Energy saving devices, providing minimum power consumption, have become very important today. Therefore economizer circuits are installed on the coils of industrial contactors. Furthermore optimized magnetic circuits and lower contact forces are possibilities to realize the demands for lower power consumption. Even latching contactors, which have two operating coils, momentarily energized to close and open the contacts, are available. The influence of this tendency towards reduced contact forces on the switching behavior has been studied. Therefore the contact force of a $160 \mathrm{~kW}$ standard contactor has been reduced by $30 \%$ and electrical lifetime tests were performed under the following test conditions:

- $\mathrm{V}=400 \mathrm{~V}$

- $\mathrm{I}_{\text {make/break }}=1,140 \mathrm{~A}$

- $\cos \varphi=1$

- $\mathrm{n}=1671 / \mathrm{h}$

During this test the contactor is loaded $6 \cdot \mathrm{I}_{\mathrm{r}}$ at make and break operation. Multiple experiments showed that these test conditions lead to results, which can be compared to a standard AC-4 test. A standard powder blended silver tin oxide material with $12 \mathrm{wt} \%$ total metal oxide content $\left(\mathrm{SnO}_{2}\right.$ doped with $\mathrm{Bi}_{2} \mathrm{O}_{3}$ and $\mathrm{WO}_{3}$ additives) has been used for performing these tests.

First of all the impact of contact force on the bouncing behavior of the contactor at make has been studied. Figure 1 shows the frequency of an open contact - at least one side of the contact bridge is open - and therefore arcing probability at time $t$ after first touching of the closing contacts at $t=0$. The possibility to differ between one- and two-sided bounces is given by analyzing the voltage drop across the contacts. All three phases of the contactor are analyzed separately.

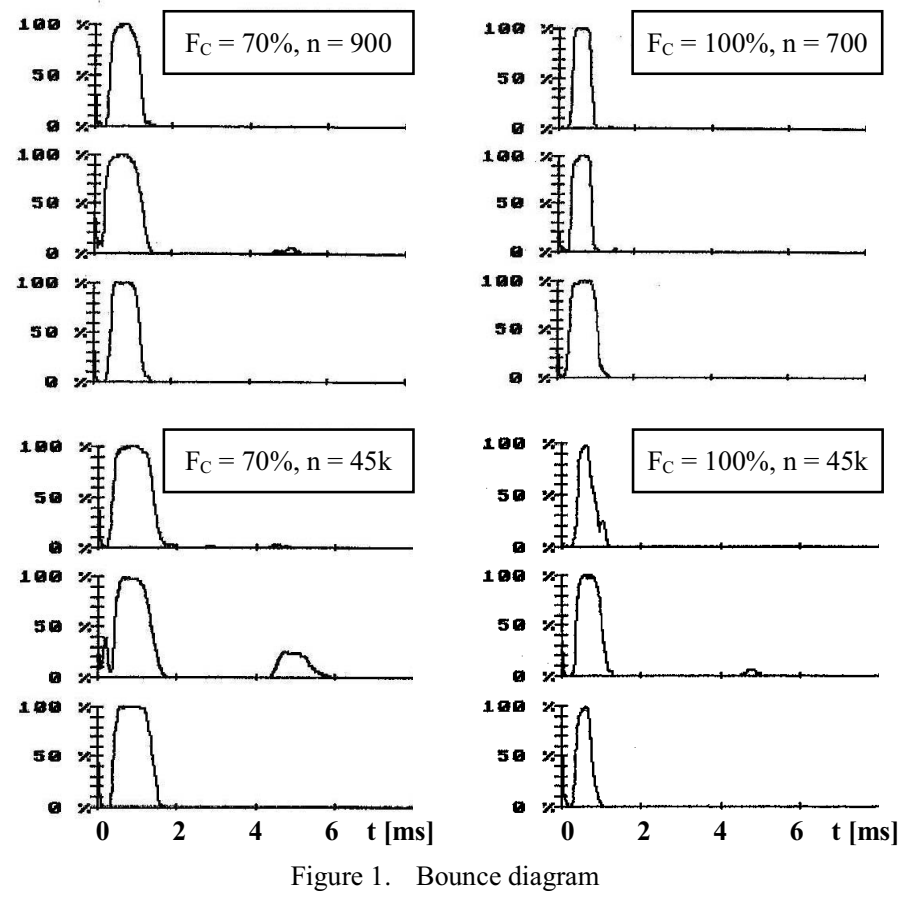

Bouncing behaviors seem to be similar for the new contactors $(n<1000)$. But, the observed primary bounces $(\mathrm{t}<2 \mathrm{~ms})$ can very often be stated as two-sided bounces for the reduced contact force contactor by further analysis of the voltage drop. In contrast to this behavior bouncing on the $100 \%$ contact force contactor is most often only one-sided. Furthermore a tendency towards secondary bounces ( $4 \mathrm{~ms}<\mathrm{t}<6 \mathrm{~ms}$ ) can be found in the second phase of the new contactor providing the reduced contact force. This effect will even grow during electrical lifetime test (see Fig. 1, $\mathrm{n}=45,000)$

Nevertheless comparable erosion rates (Fig. 2) can be observed for both contact forces due to comparable energies at break $\mathrm{W}_{\text {break }}$. This result shows that there is no significant influence of the contact spring on the opening mechanism for the tested contactor design.

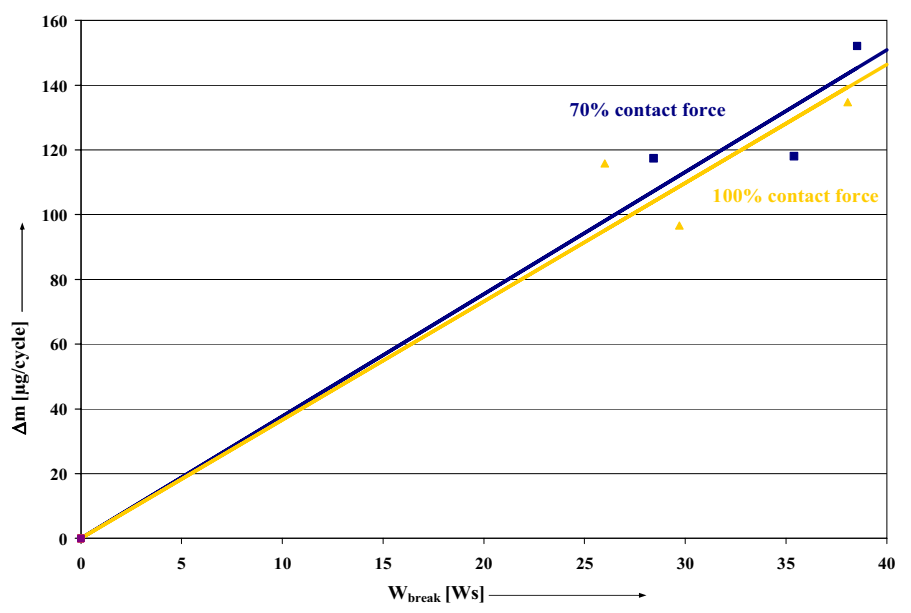

Figure 2. Erosion rates in contactor application

The calculation of energy at break for a double-breaking contactor is carried out by integrating the product of two times anode-cathode voltage drop $\mathrm{V}_{\mathrm{AC}}$ and current for each phase from contacts opening until $100 \mathrm{~V}$ are reached:

$$
\mathrm{W}_{\text {break }}=2 \cdot \mathrm{V}_{\mathrm{AC}} \int_{\mathrm{t}_{1}}^{\mathrm{t}_{1000 \mathrm{~V}}} \mathrm{i}(\mathrm{t}) \mathrm{dt}
$$

The mass loss $\Delta \mathrm{m}$ is determined by weighing the contacts and calculating the average for each phase. From the computed erosion rates it can be seen, that the higher tendency towards secondary bounces at reduced contact force didn't limit the electrical lifetime. But, this effect comprises a severe risk of contact welding for higher currents at make.

Furthermore temperature rise measurements on the moveable contacts were performed for several times during the electrical lifetime test. According to Eq. 2 a rise of $20 \%$ in the contact resistance is expected for a contact force reduction of $30 \%$. Figure 3 is showing the maximum temperature values for two different numbers of switching cycles of a $100 \%$ and $70 \%$ contact force contactor. During the test sequence the current has been increased from $1 \cdot I_{r}$ to $1.5 \cdot I_{r}$ to show the influence of 
decreased contact forces and increased rated currents on the temperature rise.

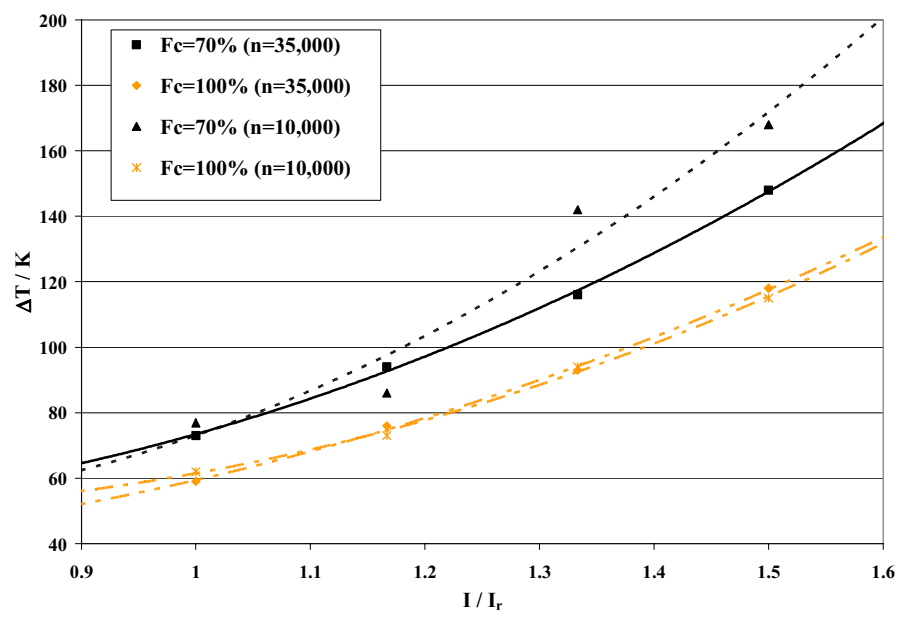

Figure 3. Temperature rise test results

Two different basic temperature levels for two different contact forces can be derived from the temperature rise tests. At rated current $I_{r}$ an increase of $15 \mathrm{~K}$ in the maximum temperature can be seen for a contactor with a contact force reduced by $30 \%$. Furthermore a greater variance and a tendency towards sharper temperature rise at increased currents can be stated for lower contact forces. Therefore contactor designers have to be careful by decreasing contact forces from cost saving aspects or increasing rated currents on existing contactors, if contact forces are already close to a lower limit.

\section{InFluence OF Metal OXIDE CONTENT}

Silver metal oxide contact materials with high oxide contents are desired to produce well-priced contactors. For the following tests the tin oxide content of a powder blended and extruded material $\left(\mathrm{Ag} / \mathrm{SnO}_{2} \mathrm{SP}\right)$ has been varied in a range from $4 \mathrm{wt} \%$ to $17 \mathrm{wt} \%$. The impact of the metal oxide content (moc) on the switching performance will be shown.

The influence of tin oxide content on contactor service life has already been studied in [2]. These studies were based on $\mathrm{Ag} / \mathrm{SnO}_{2}$ materials doped with different contents of $\mathrm{Bi}_{2} \mathrm{O}_{3}$. Therefore the achieved results do not only depend on the total metal oxide content. They are also influenced by using $\mathrm{Bi}_{2} \mathrm{O}_{3}$ as additive.

Tests utilizing a break-only model switch were performed to show the effect of different metal oxide contents on erosion rates, arc movement and contact resistance. The electrical parameters chosen for these tests can be summarized as follows:

$$
\begin{array}{ll}
\text { - } & \hat{\mathrm{i}}=350 \mathrm{~A} \text {, peak value } \\
\text { - } & \mathrm{B}=30 \mathrm{mT} / \mathrm{kA} \text { magnetic field } \\
\text { - } & \mathrm{n}=1,000 \text { cycles }
\end{array}
$$

The detailed kinetic parameters of the model switch have already been described in [3]. The break arc can be driven off the contact material and commutate onto arc runners by applying a magnetic field. From Fig. 4 it can be clearly seen that the lower the metal oxide content the better the arc root mobility. The dwell time of materials tested was defined by the time period from contacts opening - detected by the initial anode-cathode voltage drop - until a voltage drop of $60 \mathrm{~V}$ across the contacts is reached. This voltage drop is the typical value for the commutation of the breaking arc onto the arc runners taking place in the applied model switch.

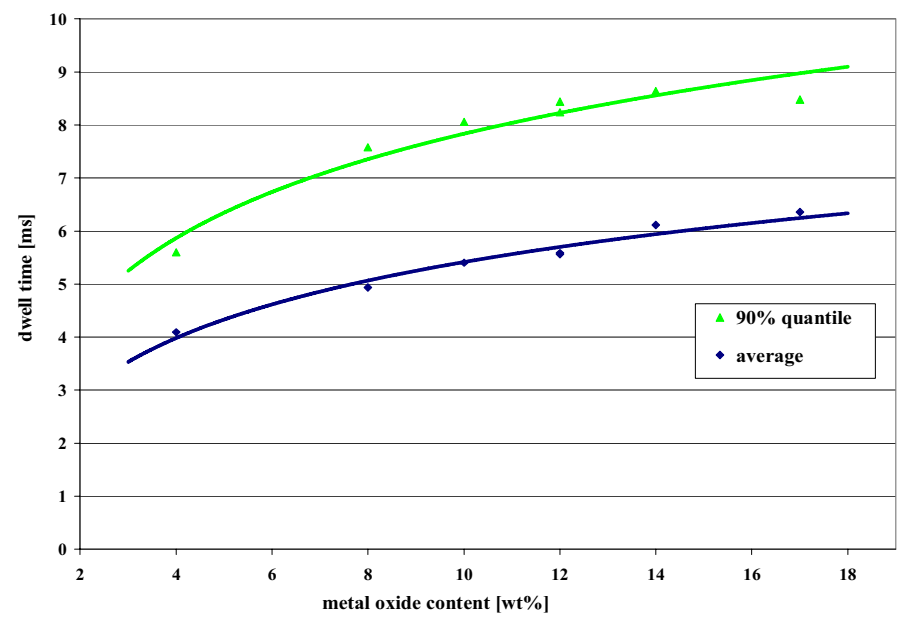

Figure 4. Dwell time against metal oxide content

Better arc root mobility and therefore shorter dwell times, consequently lead to lower energies at break stressing the contact material. Therefore material loss has to be divided by this energy for comparing the different materials. This specific erosion rate (Fig. 5) was investigated for metal oxide contents from $4 \%$ to $17 \%$. This dependency was already examined for automotive relay applications under resistive and lamp load in $[4,5]$ and therefore at significantly lower breaking energies. The impact of metal oxide content on weld break forces and erosion rates of sintered $\mathrm{Ag} / \mathrm{SnO}_{2}$ materials for contactor applications $(\mathrm{I}=375 \mathrm{~A}, \cos \varphi=0.35)$ was already studied in [6]. The performed lifetime tests showed a decreased contactor lifetime due to increasing erosion rates for a $20 \mathrm{wt} \%$ material in addition to the tendency of Fig. 5.

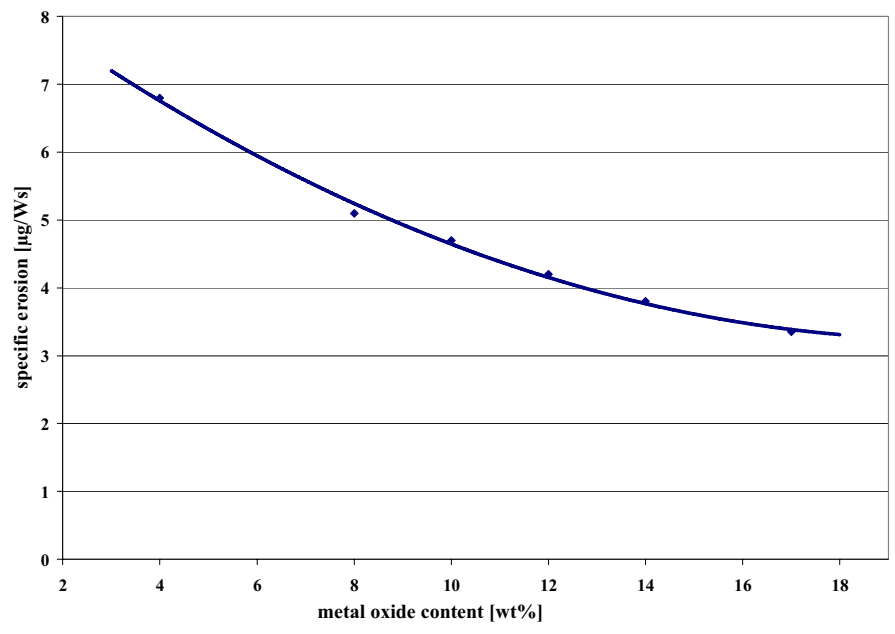

Figure 5. Specific erosion rate against metal oxide content 
Better specific erosion rates for higher metal oxide contents can be observed. This behavior can be described by the following equation:

$$
\frac{\Delta \mathrm{m}}{\mathrm{W}_{\text {break }}} \sim-\ln (\mathrm{moc})
$$

Because of longer dwell times and therefore higher breaking energies the significantly better specific erosion rates of materials providing high metal oxide contents shown in Fig. 5 must be put into perspective by the total mass loss during the experiment. The total erosion rate (Fig. 6) is only slightly decreasing with rising metal oxide content under the applied test conditions.

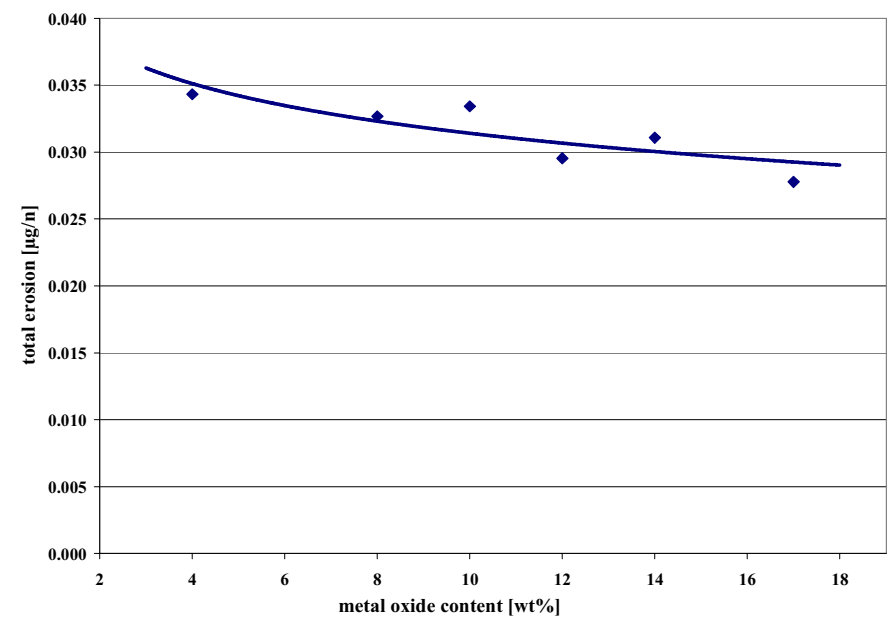

Figure 6. Total erosion rate against metal oxide content

Furthermore the advantages in erosion rates for high contents of metal oxide come along with a rise in contact resistance with increasing metal oxide content (Fig. 7). This rise in contact resistance $(99 \%$ quantile) may lead to temperature rise problems in the device, if contact forces are too low.

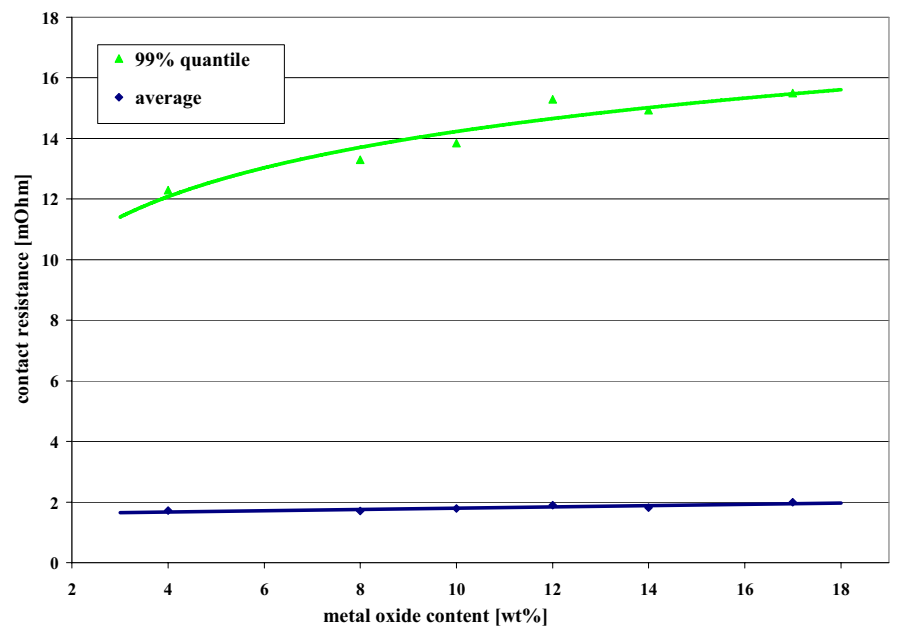

Figure 7. Contact resistance against metal oxide content
Of course the interaction of device design parameters with the material will influence these general results in a real contactor application. Therefore contactor lifetime tests have also been performed in addition to these model switch tests. The following test parameters have been chosen:

- $\mathrm{V}=400 \mathrm{~V}$

- $\quad \mathrm{I}_{\text {make/break }}=324 \mathrm{~A}$

- $\cos \varphi=1$

- $\mathrm{n}=2501 / \mathrm{h}$

Figure 8 shows the observed erosion rate per switching cycle during this test. Erosion behavior in contactor application shows $30 \%$ less erosion of a $14 \%$ material in comparison to a $12 \%$ material. Therefore the electrical lifetime, especially for contactors with high nominal current, can be increased by applying a contact material with higher metal oxide content (Ag/SnO ${ }_{2}$ 86/14 PMT3). Other experiments showed that the main influence of this effect can be found in the difference of the total metal oxide content of the materials. A slightly different composition of additives is needed to make the materials producible by extrusion process.

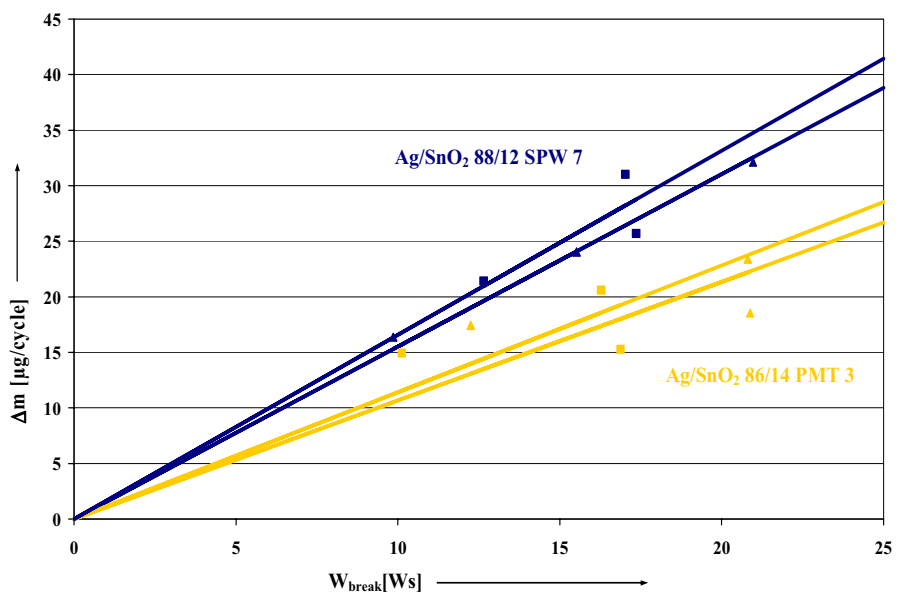

Figure 8. Erosion rates in contactor application

Furthermore temperature rise tests were performed during the electrical lifetime test. The temperature rise was measured on the contactor's contact bridges at a current $\mathrm{I}=100 \mathrm{~A}$. The contact bridge temperature is approximately two times the terminal temperature. After one hour constant current flow the temperature of the hottest bridge is memorized. After performing a dry switching the current is applied again. For statistics 24 cycles are recorded and analyzed. At least five such temperature rise tests are performed during the electrical lifetime test. Typical values of materials with different metal oxide contents, verified in several tests, are shown in Fig. 9. 


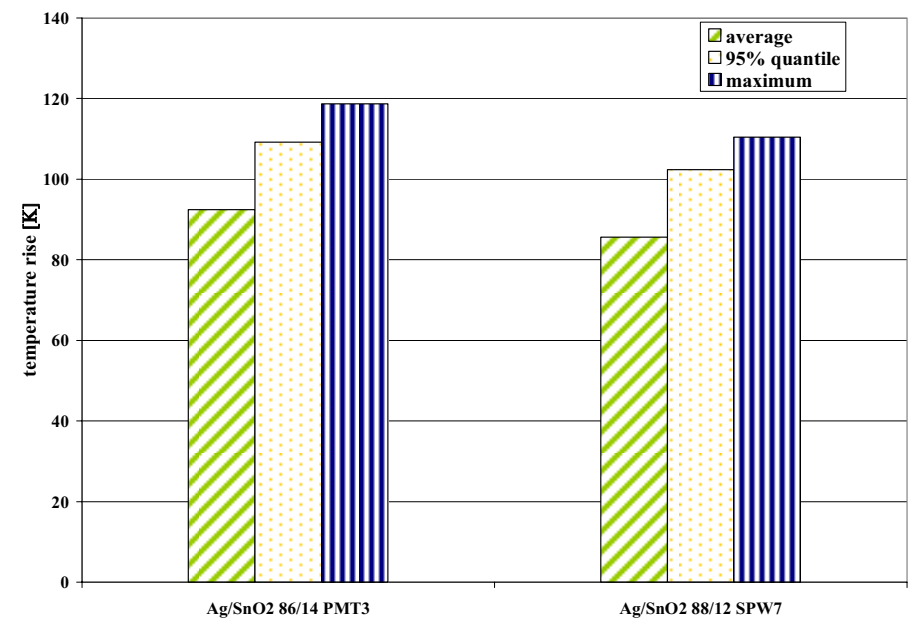

Figure 9. Temperature rise test results (contact bridge)

The temperatures on the contact bridge are approximately $7 \mathrm{~K}$ higher for a material containing $2 \%$ more metal oxide in this type of contactor. In this case a remarkable increase in electrical lifetime, coming along with moderate higher temperature rise values, can be achieved by applying an $\mathrm{Ag} / \mathrm{SnO}_{2} 86 / 14$ PMT3 material to the contactor.

\section{IMPACT OF UTILIZATION CATEGORY}

The influence of switching contactors at different utilization categories, AC-3 (normal switching duty of squirrelcage motors) and AC-4 (extreme switching duty of squirrelcage motors), on temperature rise tests has also been studied. A $30 \mathrm{~kW}$ contactor applying $\mathrm{Ag} / \mathrm{SnO}_{2}$ 88/12 SPW7 as contact material was chosen for this type of test. The temperature rise on moveable contact was measured at a tests current $\mathrm{I}=100 \mathrm{~A}$. Several temperature rise tests were performed during the electrical lifetime at different numbers of switching cycles.

During the lifetime test the contact material is stressed by bouncing arcs at make and by intensive break arcing at break. The energy levels of these effects depend on the load. Under normal switching duty (AC-3) arcing at make due to bouncing effects in combination with inrush currents dominate the material behavior. At extreme switching duty (AC-4) the high current (and therefore energy) at break dominates the contact material erosion behavior. This leads to different surface structures and material decompositions under different types of electrical loads. As a result different temperature levels can be seen for the different loads during the temperature rise test (Fig. 10).

The average temperatures of the two contactors tested at AC-3 load are $10 \mathrm{~K}$ higher than those switched under AC-4 load conditions. The $95 \%$ quantile of the maximum values is even $40 \mathrm{~K}$ higher. For contactors providing much higher/lower rated powers this effect can turn around, mainly depending on the ratio between energies at make and break - influenced by the contactor design - under the different types of load.

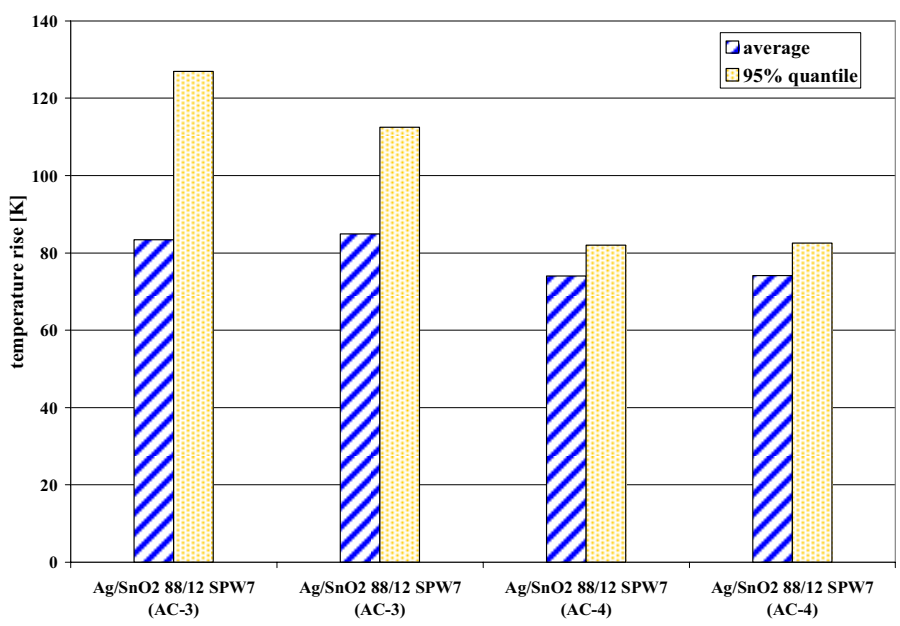

Figure 10. Temperature rise test results (contact bridge)

\section{CONCLUSIONS}

Silver tin oxide contact materials are widely used for industrial contactor applications. The technical requirements on these materials are influenced by actual trends in contactor design. Temperature rise is a critical failure mode for contactors in industrial application and the consequences of design changes on the contact resistance have to be considered carefully.

Therefore studies on switching behavior of contactors influenced by reduced contact forces, higher metal oxide contents and usage under different utilization categories have been performed.

Reduction of the contact force influences contactor kinematics and bouncing behavior. Though no large impact on erosion rates and lifetime under extreme switching duty (AC-4) could be seen, the influence on the bouncing behavior and the amount of energy converted in the contact material at make has to be considered at normal switching duty (AC-3) [7]. Experiments on these effects will be performed in future. Nevertheless, increasing temperatures in the device have to be considered already under AC-4 load. Critical temperature rises were observed, especially if reduced contact forces come along with increased rated currents or usage under overload.

Silver tin oxide materials with higher metal oxide content offer precious metal saving capacities to device manufacturers. Materials like $\mathrm{Ag} / \mathrm{SnO}_{2}$ 86/14 PMT3 provide better erosion rates and therefore increased electrical lifetimes in comparison to $88 / 12$ materials or possibilities for contact tip size reduction - approximately a $20 \%$ contact material volume reduction is possible - if slightly higher temperature rise values can be tolerated or eliminated by the design of the contactor.

Studying influences on contact resistance and temperature rise, the switching load and device kinematics have to be considered carefully. The energies converted during the make and break process by arcing have great impact on the contact material surface and therefore on the temperature rise. Reasonable tests have to include device, material and load parameters. 


\section{REFERENCES}

[1] R. Holm, "Electric Contacts: theory and application," Springer, Berlin/Heidelberg/New York, 2000

[2] F. Hauner, D. Jeannot, K. McNeilly, "Advanced $\mathrm{AgSnO}_{2}$ Contact Materials with High Total Oxide Content," $21^{\text {st }}$ International Conference on Electrical Contacts (ICEC), Zurich, Switzerland, 2002

[3] P. Braumann, "Auswirkungen von Verunreinigungen auf das Schaltverhalten von Kontakten der Energietechnik", 9. VDE Seminar Kontaktverhalten und Schalten, Karlsruhe, Germany, 1987

[4] P. Braumann, A. Koffler, "The Influence of Manufacturing Process, Metal Oxide Content, and Additives on the Switching Behaviour of $\mathrm{Ag} / \mathrm{SnO}_{2}$ in Relays," $50^{\text {th }}$ IEEE Holm Conference on Electrical Contacts, Seattle, WA, USA, 2004

[5] G. Witter, Z. Chen, "A Comparison of Silver Tin Indium Oxide Contact Materials Using A New Model Switch That Simulates Operation Of An Automotive Relay," $50^{\text {th }}$ IEEE Holm Conference on Electrical Contacts, Seattle, WA, USA, 2004

[6] H. Yamasaki, S. Oda, K. Miyakawa, "Sintered Silver Tin Oxide Materials for Electrical Contacts," $10^{\text {th }}$ International Conference on Electrical Contacts (ICEC), Budapest, Hungary, 1980

[7] E. Hetzmannseder, W. Rieder, "The Influence of Bounce Parameters on the Make Erosion of Silver/Metal-Oxide Contact Materials," $39^{\text {th }}$ IEEE Holm Conference on Electrical Contacts, Pittsburgh, PA, USA, 1993

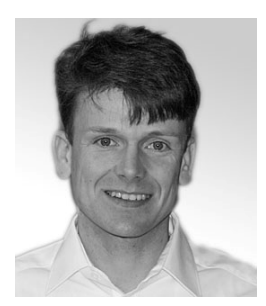

Timo Mützel received the Dipl.-Ing. (2003) and the Dr.-Ing. (2008) in Electrical Engineering and Information Technology from TU Ilmenau, Germany. From 2003 to 2007 he was with the department of Electrical Apparatus and Switchgear at TU Ilmenau. His research areas included switchgear technologies, numerical simulations and transients in power systems. Since 2008 he is with Umicore AG \& Co. KG, Hanau, as Manager Applied Technology in the business line Contact Materials. He is a recipient of the 2003 VDE Adam-Herbert-Prize. 


\title{
The Impact of Spot Size and Location on Current Density
}

\author{
By \\ Robert D. Malucci \\ RD Malucci Consulting \\ Naperville, Illinois
}

\begin{abstract}
The impact of spot size and location within a contact zone was evaluated using a formulation provided by Greenwood which utilizes the interaction of the current in each spot with all the other current carrying spots. The results indicate that both spot size and position affect the average current density through each spot. Moreover, a random variation in spot size appears to mask some of the effects from size and position. However, it was seen that significant variation in current density across the contact region suggests, that as a contact degrades, there will be a non uniform degradation and failure of the contact spots as aging progresses. This is thought to produce a cascade effect where the spots carrying the highest current density begin to fail causing the remaining spots to carry greater current loads and subsequently age faster towards failure.
\end{abstract}

\section{Introduction}

In previous papers $[1,2]$ the present author has promoted current density as a fundamental parameter in assessing the performance of power contacts. To this end it was shown how the change in contact resistance is directly related to a change in metallic contact area and thus a change in current density at the contact interface. It is believed that when the current density reaches critical levels, aging is greatly accelerated and contact failure may be eminent. It is difficult to assess at what current density this occurs, as different materials will be affected differently. It is believed that two mechanisms associated with high current density play a role in accelerated degradation, localized heating and electro-migration. Both are believed to drive diffusion of metal ions. This in turn can cause inter-metallic compounds and void formation at the contact interface. Other additional effects such as cracking and weakening of the joint can also occur. This subject has been discussed at some length by Timsit [7] and Braunovic [8]. Specifically, they addressed aluminum to copper contact systems but the phenomena may well affect other base metals such as tin. Braunovic comments that when current levels reach about $10^{5} \mathrm{~A} / \mathrm{cm}^{2}$, electromigration becomes a factor .Consequently, excessively high current densities at the asperity level can cause accelerated aging and subsequent contact failure.

Part of the problem, which was not addressed in the past, is how the current actually distributes over the contact spots. It is believed as a contact degrades that the current density in individual spots may reach critical levels according to the size and location of the spots within the contact zone. In previous discussions by the present author $[1,2]$ it was assumed that the current distributes uniformly over the metallic contact area at the interface. However, a simple analysis quickly shows that size is a factor according to the assumed relation of spot resistance to spot size. According to the Holm model [3] of multi spot contacts, each spot is assumed to contribute to the resistance according to its constriction resistance. In the case of a circular contact spot between similar metals the resistance is given by the simple expression $\rho / 2 a$, where $\rho$ is the resistivity and a the radius of the spot. This equation assumes the spot size is much smaller than the dimensions of the macroscopic contact. Consequently, the current through this spot is approximately given as $I=V /(\rho / 2 a)$, where $\mathrm{V}$ is the contact voltage. Moreover, the average current density through this spot is $\mathrm{J}=\mathrm{I} / \pi \mathrm{a}^{2}$. Combining these equations shows the average current density to be inversely proportional to spot size ( i.e. $\mathrm{J}=2 \mathrm{~V} / \pi \rho \mathrm{a}$ ). One may wonder why the term "average current density" is used in this discussion. The reason is that current density is thought to increase from the center of a spot as one moves towards the edge. Thus the current density is greater near the edge than the center. This is commonly known and will be discussed in a following section.

This simple analysis suggests that the smaller spots would reach the critical current density first and thus fail quicker than larger spots. Evidently, there would be a cascading effect as smaller spots drop out, larger spots would have to handle higher currents and thus eventually reach higher current densities. Consequently, the larger spots would in turn reach critical densities and subsequently fail as aging progressed. Once this sequence starts one might imagine the cascade to occur fairly quickly. This has been observed in tests of power contacts [1,2]. Typically failure seems to occur after a threshold in change in voltage drop (or contact resistance) has been reached. This threshold is generally quite small (tens of mv) and reveals that relatively small changes initiate the cascade effect.

In addition, the spot position in the contact region may also affect the amount of current in a spot as Greenwood suggested in a previous paper [4]. In this case it is 
recognized that the currents passing through each spot can interact, which results in an adjustment of all the currents to reach steady state equilibrium conditions. The model presented in reference 4 will be used to assess this factor.

To gain a better understanding of these phenomena, this paper addresses the impact that size and position have on current density. This is accomplished by evaluating various interface configurations that may affect current density in a multi-spot interface.

\section{Analysis}

Various interface configurations are used to conduct a multi spot analysis using the approach Greenwood presented in reference 4. In the latter method, Greenwood provides the basic equation used in this analysis (equation 3 in reference 4) as follows,

$$
\mathrm{U} / 2=(\rho / 4)\left\{\mathrm{I}_{\mathrm{i}} / \mathrm{a}_{\mathrm{i}}+(2 / \pi) \Sigma \mathrm{I}_{\mathrm{j}} / \mathrm{S}_{\mathrm{ij}}\right\}
$$

In Greenwoods notation, $U$ is the contact voltage, $\rho$ the resistivity of the contact metal, $I_{i}$ and $I_{j}$ the currents through the $i$ and $j$ spots respectively and $S_{i j}$ the distance between the $i$ and $j$ spots. Greenwood calls the first term in brackets the self resistance, which one can see is the current times the familiar expression for the half constriction resistance. The second term provides the interaction between the ith spot and the other n-1 spots. This term represents the influence of the other spots and depends on their separation and current. As pointed out by Greenwood, this phenomenon is the analog of an electrostatic system of $n$ charged discs which mutually induce charges and thus voltages on each other. As a result, one can apply electrostatic theory to this problem as Greenwood did to establish an equation such as given above. The second term also provides the effects from the size of the contact region and sometimes can be used to define the so called Holm radius. For a well defined circular region the Holm radius provides a circle that incorporates all of the spots. It should be noted that equation 1 is a first order approximation for the case where all spots have the same size. Consequently, higher order terms such as dipole and quadrupole moments are neglected. In effect higher powers of $\mathrm{a}_{\mathrm{i}} / \mathrm{S}_{\mathrm{ij}}$ are ignored. In the present analysis the errors caused by this approximation are thought to be small as the size of the spots $\left(a_{i}\right)$ are significantly less than the separation $\left(S_{i j}\right)$ of the spots.

In order to proceed, a contact configuration was defined that provided the size and location of the spots in the contact region. In this evaluation, the configuration shown in figure 1 was used for all simulations. A series of simulations were run by varying the spot sizes either uniformly or randomly to show the effects of this variable. In the case of using varying spot sizes, equation
1 was modified according to Greenwoods discussion in the appendix of reference 4 . The latter was done by substituting the following expression in equation 1 for $\left(\mathrm{S}_{\mathrm{ij}}\right)$,

$\left(\mathrm{S}_{\mathrm{ij}}\right) \rightarrow\left(\mathrm{S}_{\mathrm{ij}}\right)\left(\mathrm{a}_{\mathrm{i}} / \mathrm{a}_{\mathrm{j}}\right)\left\{\left(\pi \mathrm{S}_{\mathrm{ij}}-2 \mathrm{a}_{\mathrm{j}}\right) /\left(\pi \mathrm{S}_{\mathrm{ij}}-2 \mathrm{a}_{\mathrm{i}}\right)\right\}$

One problem that arises in using equation 2 is that negative currents can occur in some spots if the ratio of the spot sizes varies too much. This is not thought to be possible in reality. Consequently, the limitation in using equations 1 and 2 require not only restrictions on spot size and separation but also the ratio of spot sizes as well. These shortcomings are thought to occur as a result of the formulation and approximations used to develop these equations.

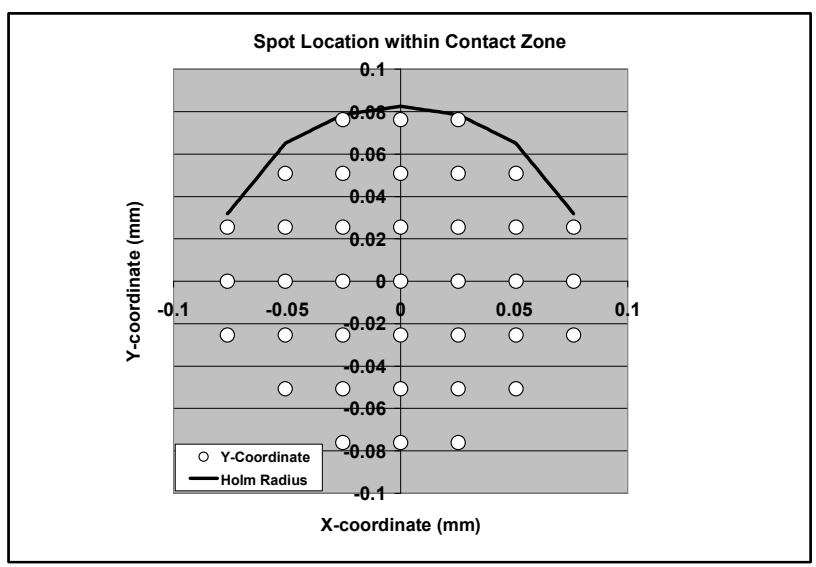

Figure 1

The method of calculation was to set up a system of simultaneous equations using equation 1 and solving for the current through each spot using matrix theory. As one can see equation 1 represents the ith spot. Thus, one gets $\mathrm{n}$ equations with $\mathrm{n}$ currents. This provides a $\mathrm{n}$ by $\mathrm{n}$ coefficient matrix with elements $\mathrm{Cij}$ that transforms an $\mathrm{n}$ dimensional current vector with components $\mathrm{Ai}$ into an ndimensional voltage vector with components $\mathrm{Vi}$ as shown symbolically below.

$\mathrm{C} \times \mathrm{A}=\mathrm{V}$

All that's needed to solve for the currents is to calculate the determinants of the appropriate $\mathrm{n}$ by $\mathrm{n}$ matrices according to the usual rules of matrix theory. This was done by keeping the voltage constant across all contact spots. Specifically, these matrices were set up in an excel spread sheet and the determinants calculated using the associated software tools. With this approach, the currents through each spot were determined as a function of position and size. Subsequently, current density is provided as a function of size and position as well. Moreover, the contact resistance can be calculated, as the 
total current at constant voltage is provided in this method. In addition, since the Holm model for multi-spot contacts is widely used, the contact resistance from the Greenwood method is compared to Holm predictions.

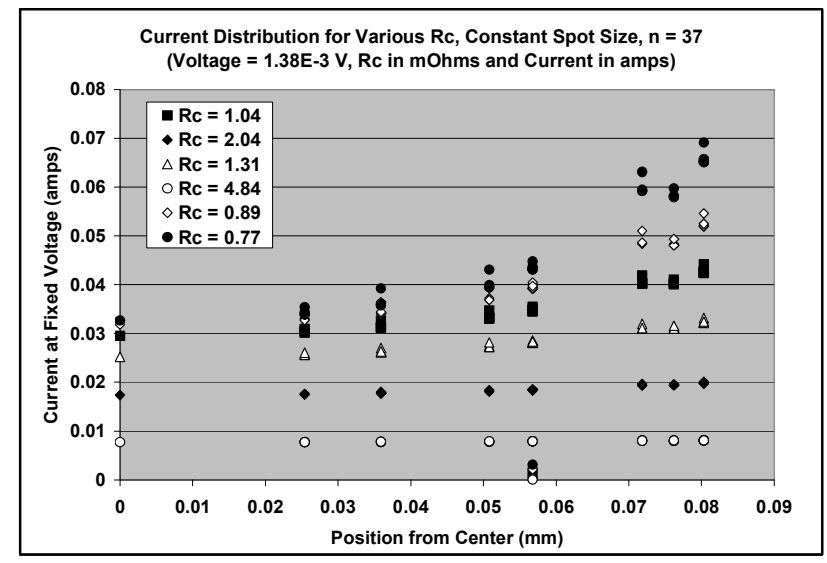

Figure 2a

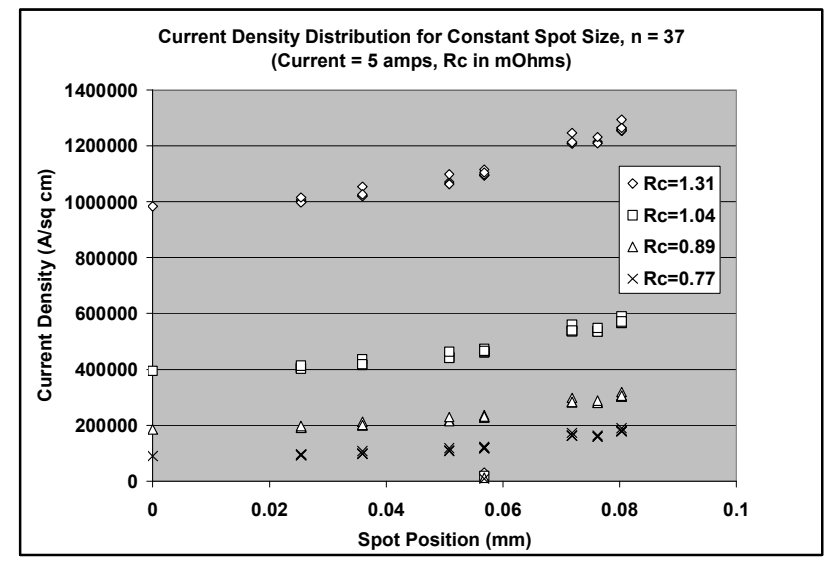

Figure 2b

\section{Results for Uniform Spot Size}

Figures $2 \mathrm{a}$ and $2 \mathrm{~b}$ provide results for the cases shown in the legend. Each case had uniform spot sizes which were distributed according to the pattern shown in figure 1 . Each case represents a different spot size and thus a different contact resistance as shown in the legend. As one can see in figure 1a, the current increases as the contact spot moves away from the center of the contact region. Since the contact size for each case was kept constant, one can say the current density increases as the spot moves out. However, since the data plotted in figure $2 \mathrm{a}$ is for a fixed voltage and may be somewhat misleading, a second plot of current density for these cases at fixed current $(5 \mathrm{~A})$ is provided in figure $2 \mathrm{~b}$. This provides a direct comparison of these cases. Clearly one can see that each case exhibits an increasing current density as the spots move away from the center. In addition, for the highest resistance case shown in figure $2 b$ it can be seen that the current densities exceed the level of $10^{5} \mathrm{~A} / \mathrm{cm}^{2}\left(10^{3} \mathrm{~A} / \mathrm{mm}^{2}\right)$ as discussed earlier. Consequently, if this contact represented a real interface, it may well experience electro-migration as a degradation mode.

Moreover, a comparison of the resistance found from Greenwoods method with the Holm model is shown below in table I. These results reveal good agreement between the two methods. One should note that the Holm model assumes a uniform current distribution across the contact spots and treats the individual spots as electrically in parallel. In addition, an envelop constriction is added to the multi-spot contribution as well. This model is represented as follows,

$\operatorname{Rc}=\rho / D+\rho / n d$

Where $\rho$ is the resistivity, $D$ the size of the circular contact region, $\mathrm{n}$ the number of contact spots in $\mathrm{D}$ and $\mathrm{d}$ the average spot size. In the present analysis, D is defined as the diameter that encloses the contact region as shown in figure 1 .

Table I: All dimensions in $\mathrm{mm}$ and resistances in mohms

Spot Size n Rc(GW) Rc(Holm) Ratio

$\begin{array}{lllll}3.56 \times 10^{-4} & 37 & 4.84 & 4.85 & 1.002 \\ 1.02 \times 10^{-3} & 37 & 2.04 & 2.13 & 1.044 \\ 1.97 \times 10^{-3} & 37 & 1.31 & 1.42 & 1.084 \\ 3.00 \times 10^{-3} & 37 & 1.04 & 1.16 & 1.115 \\ 4.19 \times 10^{-3} & 37 & 0.89 & 1.02 & 1.146 \\ 6.20 \times 10^{-3} & 37 & 0.77 & 0.91 & 1.182\end{array}$

Note: The contact is assumed to be made of Tin where $\rho=$ $11 \times 10^{-8}$ ohm-m

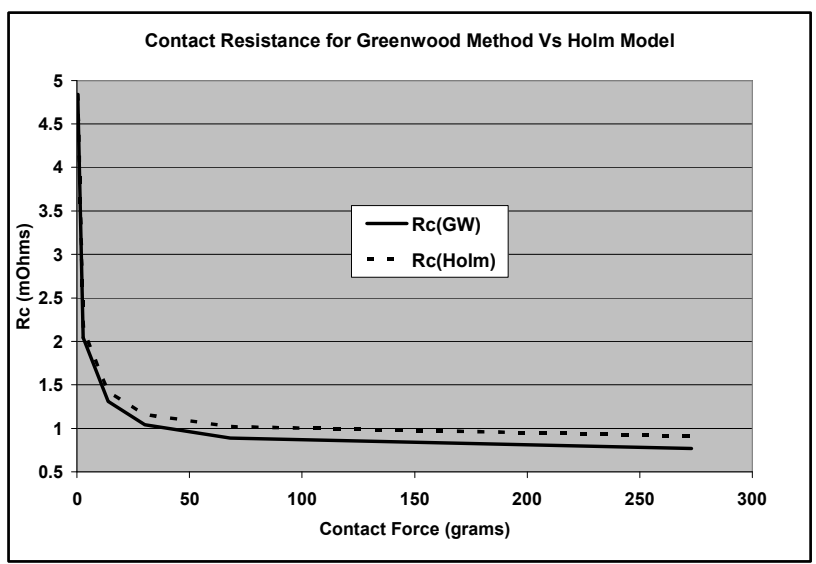

Figure 3

A graph of the data in table I is provided in figure 3 where one can see good agreement between the two independent 
methods. The force values used in this plot were calculated using the micro-hardness of tin and the contact area generated by the spot size and number of spots. The results for uniform spot size show that the Greenwood method and the Holm model are very comparable in predicting contact resistance even though the Greenwood method shows the current density to vary across the contact region. The least agreement seen is the case of lowest resistance where a greater variation in current is found. However, even though the current varies over a factor of about two the two methods are still within $20 \%$ of each other.

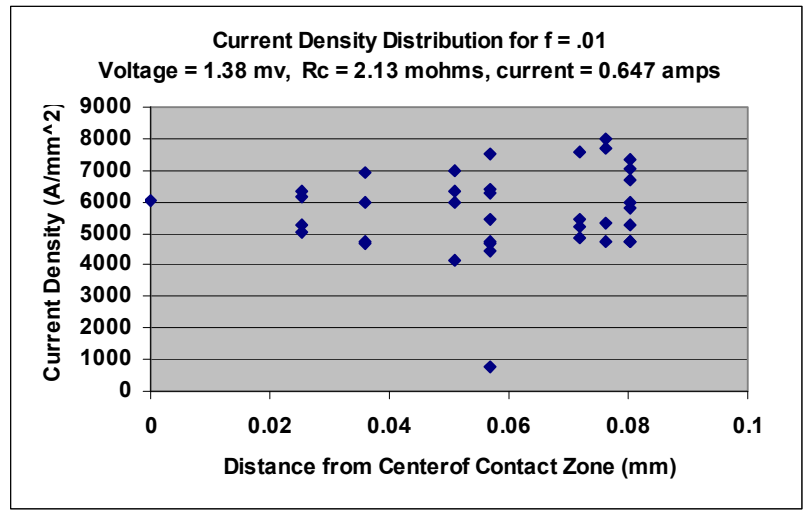

Figure 4

\section{Results for Varying Spot Size}

The results of typical cases where the spot sizes were varied randomly are shown in figures 4, 5 and 6 . Figure 4 reveals that as a function of position the results are less pronounced as in the case of uniform spot size. Clearly, spot size has a significant impact on current and current density as shown in figures 5 and 6 . In the case of current as a function of spot size, the results show a linearly increasing relation as seen in figure 5. However, since average current density is given by the current divided by area, figure 6 shows current density generally going down with increasing spot size. Also included in figure 6 is a plot of what one expects if the contact spots were isolated and didn't interact as discussed in the introduction. Clearly the interaction of the contact spots, as represented by Greenwoods method, can cause a significant reduction for the smaller spot sizes. It appears that the interaction of currents tends to reduce the effects of spot size.

Figures 7, 8 and 9 show the results from another case with varying spot sizes. Here the spot sizes are bigger than the previous case and the resistance is less as shown in the legends. Again one sees a significant effect from the current interactions. It is clear from these cases that the interaction between the micro-currents causes a masking of the effects of spot size and position on current density. However, it is also seen in these cases that current density varies by about a factor of two and thus one would expect the associated rates of degradation to vary as well.

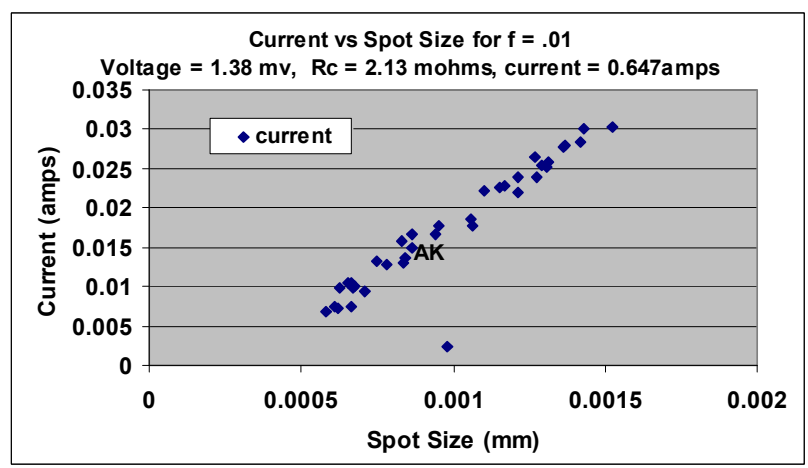

Figure 5

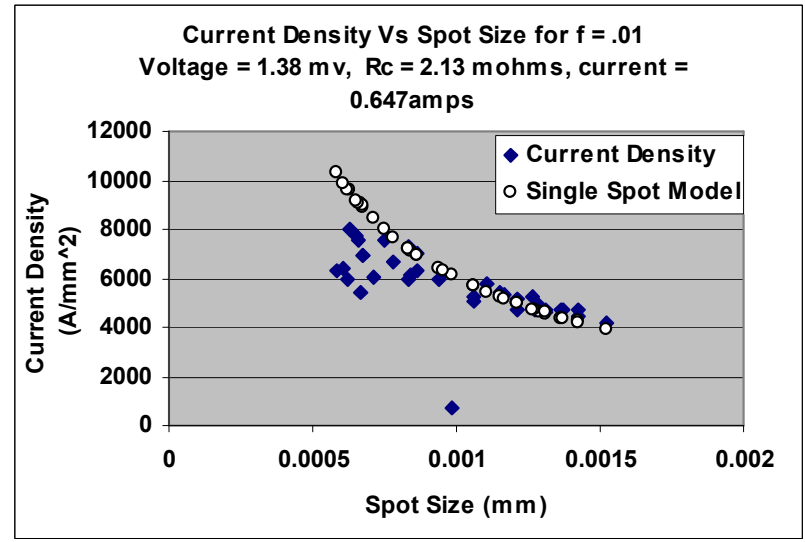

Figure 6

\section{Discussion and Conclusions}

One can see from these results that spot size, position and the interaction between neighboring currents have significant effects on the current density through each contact spot. It appears that those spots carrying the highest densities will most probably fail first. However, this does not mean the smallest spots will always fail first as some larger spots have been seen to exhibit higher densities. Generally smaller spots have higher current densities as do spots farther from the center. It was seen that the average current densities have a range of about two in typical cases. In addition, the level of current density in typical power applications appears to have increased to relatively high levels (on the order of $10^{6}$ $\mathrm{A} / \mathrm{cm}^{2}$, see figure 2) after only small increases in resistance (1 -2 mohms). This supports observations seen in tests of power contacts where very small changes in voltage drop signaled eminent failure. One however, should be cautious about generalizing from these results. It should be noted (as discussed earlier) that the method used is a first order approximation to very complex phenomena. Consequently, there may be artifacts associated with the method, approximations and limited 
number of cases used to demonstrate the results. Moreover, the present author had difficulty using too broad a range of spot sizes. When this occurred, a few of the spots exhibited cases with negative currents, which is thought to be unphysical in a case such as this where the voltage is kept constant. This may reflect inadequacies in the method that depend on the assumptions and formulations used.

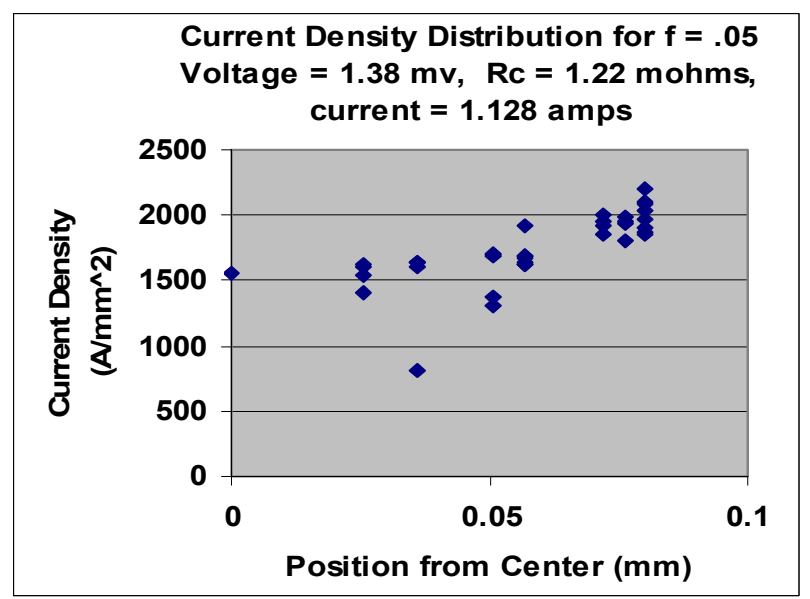

Figure 7

One other factor which was discussed briefly in the introduction is the expected current density distribution on the individual spots. For the case of an infinitely thin disc, the density distribution is given by the well known equation $[5,6]$, as follows,

$\mathrm{J}=(\mathrm{I} / 2 \pi \mathrm{a}) / \mathrm{SQRT}\left(\mathrm{a}^{2}-\mathrm{r}^{2}\right)$

This equation appears to produce an infinite current density at the edge of a circular spot. However, in the real world this will not happen as the detailed structure of the spots and the interaction between spots will change the situation. Although, one might expect the current density of each spot to increase towards the edges, it is not known by how much. All one can say is that individual spots may degrade from the outside towards the center as aging occurs. One should note that this factor was partially observed in this study as shown in figure 2 where it is seen that the current density increases as one moves towards the edge of the contact region.

Finally, if one wants to use a method such as this to do more detailed studies, it is recommended that the basis for this formulation be critically analyzed in terms of the assumptions made by the original author and the approximations made in the present work.

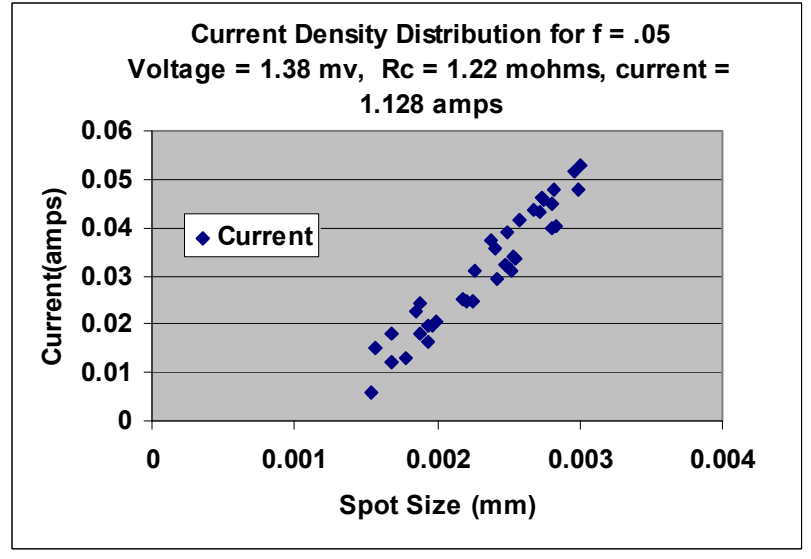

Figure 8

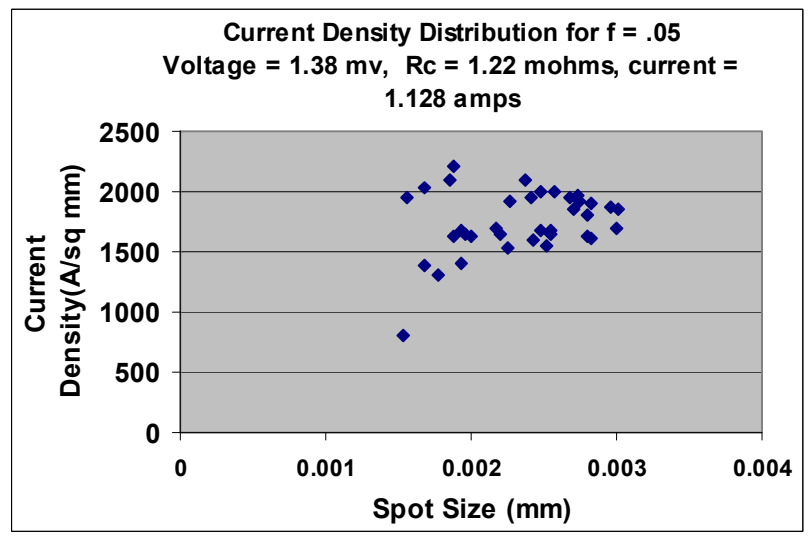

Figure 9

\section{References}

[1] R.D. Malucci and F.R. Ruffino, "A Method for Power Rating Contacts using Voltage Drop

", Proceedings of the Fifty-Third IEEE Holm Conference on Electrical Contacts, 2008

[2] R.D. Malucci and F.R. Ruffino, "Materials Considerations in Using Voltage Drop for Power Rating", Proceedings of the Fifty-Second IEEE Holm Conference on Electrical Contacts, 2007, pp 25-31

[3] Holm, "Electric Contacts" , Springer-Verlag, 1967, pp. 21

[4] J.A. Greenwood, "Constriction Resistance and the Real Area of Contact", British Journal of Applied Physics, 1966, Vol.17

[5] Jackson, "Classical Electrodynamics", Wiley 1963, pp89

[6] Roland S. Timsit, Chapter 1, Paul G. Slade, "Electrical Contacts", Marcel Dekker, 1999, pp4

[7] Roland S. Timsit, Chapter 1, Paul G. Slade, "Electrical Contacts", Marcel Dekker, 1999, pp34

[8] Milenko Braunovic, Chapter 4, Paul G. Slade, "Electrical Contacts", Marcel Dekker, 1999, pp210 


\section{Biography:}

Robert D. Malucci received his Ph.D. and B.A. Degrees in Physics from the State University of New York at Buffalo. He has held a number of positions involving technical management and applied research in the Commercial and Aerospace fields. Career engagements include Director of the Reliability Group at Molex Incorporated, Technical Manager on SDI projects at Ford Aerospace, Applied Research Manager at ITT Cannon and Research Scientist at AMP Incorporated. He has worked in the field of electrical contacts for 30 years on problems involving contact physics, product designs, reliability, materials properties and failure analysis and has published numerous papers in these subjects. He has recently taken early retirement from Molex and currently has started a consulting business in the field of electrical contacts. 


\section{Spring Stiffness Investigations for Long Lifetime Connectors}

\author{
H. Essone-Obame ${ }^{1,3}$ and L. Cretinon ${ }^{1}$ \\ ${ }^{1} \mathrm{EDF}$ R\&D \\ F-77818 Moret sur Loing Cedex-France
}

\author{
R. El Abdi ${ }^{2}$, N. Ben jemâa ${ }^{3}$ and E. Carvou ${ }^{3}$ \\ ${ }^{2}$ Larmaur laboratory, ${ }^{3}$ IPR - University of Rennes 1 \\ CS 74205 - 35042 Rennes Cedex - France
}

\begin{abstract}
Semi-permanent electrical contacts are found throughout the whole range of instrumentation and control product, connecting devices. The reliability of this kind of connector - designed for long lifetime service is highly dependant on mechanical and corrosion degradations. In this domain, corrosion is avoided by using a thick gold layer while the major cause of connector ageing remains the contact spring degradation (relaxation, loss of force...) leading subsequently to a change of contact resistance. In this paper, a non intrusive (laser) technique is used to view and measure both the deflection and its associated force. These techniques and tools are applied both to new springs and to degraded springs. In the absence of an analytical model due to the complex geometry of the spring, a numerical finite element model has been developed to compute the deflection versus force. Linear deflections are observed for various stiffnesses for different springs located in the same connector. The main explanation for this change is the variation of the embedding length of the spring inside the connector. In addition the stiffness dependence due to ageing by thermal cycling is discussed.
\end{abstract}

Keywords: connector, deflection, contact force, spring relaxation, finite element model.

\section{INTRODUCTION}

The reliability of semi-permanent connectors is related to the ageing of the different materials and component parts of the connector and the operating conditions [1]. Numerous papers have stated that during aging, the most deleterious influence comes from corrosive gases and organic pollutants in the surrounding atmosphere or out-gassed from various parts of the connector (housing, component, PCB, etc.) [2, 3]. Effective solutions to this problem have used gold coating layers to avoid oxidation and the formation of poor conducting, tarnished films. In addition to this, natural aging leading to spring relaxation and stiffness modification are expected to act as limitation factors on the connector working life. Indeed, the subsequent loss of contact force may have a direct effect on the contact interface and affect contact resistance stability.

For high reliability levels required for long lifetime service, (up to many decades), a thick gold film of $3 \mu \mathrm{m}$ has been used to protect the contact surface against well known corrosion phenomena. However, mechanical aging remains a possible root cause of contact failure and may reduce the expected durability of connectors. In this work, a low-signal connector HE9 (female part) commonly used in energy plant production or in electrical instrument composed by printed circuit plane boards (PCB - male part) has been studied.

The aim of this work is to qualitatively evaluate the role of extreme mechanical efforts caused by a high number of insertions/ extractions into/out of the connector and concurrent spring compressions both under ambient conditions and after thermal cycling. Two main tests are described examining the insertion and extraction forces during more than a few thousand matings as well as the compression force of the spring whether embedded or not in the housing. The experimental data of spring stiffness is compared to numerical calculations obtained by Ansys code.

\section{APPARATUS AND PROCEDURE}

\section{A. Connector and Test Description}

The male part of the connector consists of a printed circuit board (PCB) of $1.6 \mathrm{~mm}$ thickness, made of a fibre material (FR4); a strip of copper of $60 \mu \mathrm{m}$ coated with a second layer $(10 \mu \mathrm{m})$ of nickel with a thin cobalt-gold layer $(3 \mu \mathrm{m})$ on top. The female part of the connector is an HE9 (structure in copper and nickel) [2] (Fig. 1). Regarding the design, a stop was placed to maintain the initial gap at $600 \mu \mathrm{m}$ and to reduce the insertion peak. Consequently, the spring is pre-deflected by $\mathrm{D}_{0}$ and this induces the initial force $\mathrm{F}_{0}$. The insertion test was performed by inserting the PCB $3 \mathrm{~mm}$ into the connector slot while the compression test of the spring is made on the half connector by an indentation of $300 \mu \mathrm{m}$. A free spring was also used for the stiffness and relaxation tests. The connector springs are of Copper alloys $(\mathrm{CuBe})$ with a Young's modulus $\mathrm{E}=120 \mathrm{GPa}$ and a Poisson's ratio $v=0.3$ [4]. Direct investigation of this spring avoids taking into account the effect of the housing.

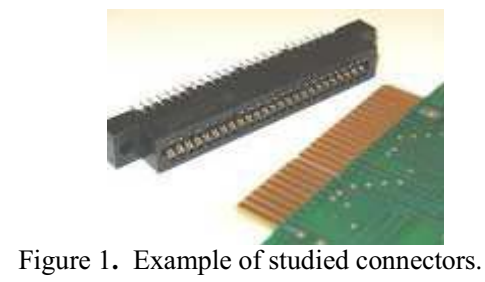




\section{B. Apparatus and Test Setup}

The connector female part (Fig. 2) is fixed onto the XYZ platform and two holes at left and right sides allow us to view and measure the spring deflection by means of two lasers spots $(.1 \mu \mathrm{m})$.

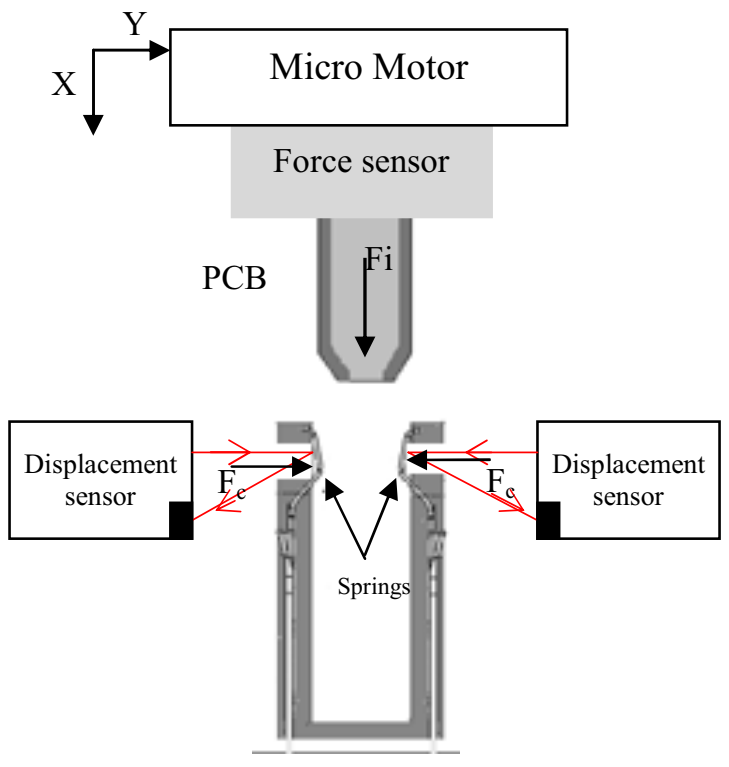

Figure 2. Connector test set up

The male part (PCB) is fixed onto a motorized plate in series with a strain gauge for measuring the insertion force $(.01 \mathrm{~N})$ during more than 3000 cycles; periodically we draw the curve of insertion force and spring deflection after each 100 mating operations. This test is carried out to determine the global connector behavior during insertion and mainly at the final stage of full insertion between 2 and $3 \mathrm{~mm}$. In this functioning zone, knowledge of the friction coefficient $\mu$ (from others experiments) and the insertion $\mathrm{F}_{\mathrm{i}}$ force allows us to calculate the contact force applied on each side $F_{c}$ (Fig. 2):

$$
\mathrm{F}_{\mathrm{i}}=2 \mu \mathrm{F}_{\mathrm{c}}
$$

Unfortunately this method may cause the friction coefficient to change significantly over the high number of mating cycles and may thus yield a false contact force exerted by the spring stiffness. At the same time, this contact force can be deduced from the deflection and spring stiffness.

A direct indentation of the spring has been developed to evaluate the stiffness and possible relaxation during severe stresses. As shown in Fig. 3, the spring is isolated and the deflection applied is $600 \mu \mathrm{m}$ and the subsequent force is measured. When the spring is pre-deflected (spring in connector) by $\mathrm{D}_{0}$ with an initial force $\mathrm{F}_{0}$, we apply a deflection of $300 \mu \mathrm{m}$ and measure the subsequent force. This test is made on a new spring embedded at different lengths $\ell(8-12 \mathrm{~mm})$

and submitted to a few thousands of indentation cycles under ambient conditions. In addition, following thermal cycling ($40^{\circ} \mathrm{C}$ to $120^{\circ} \mathrm{C}$ ) for 22 cycles each of 4 hour durations, the stiffness of the spring was evaluated.

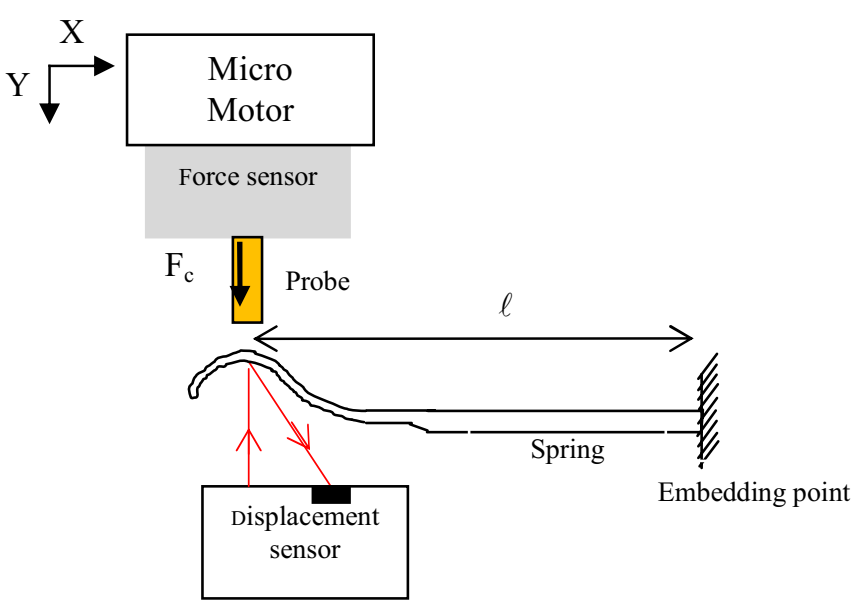

Figure 3. Spring under bending test

In addition to these measurements, an FEM analysis was performed with Multiphysics Ansys Software, the stiffness of the spring being computed and compared to the experimental data.

\section{RESULTS}

\section{A. Investigation of New Connectors and Springs}

1. Experimental results

Fig. 4 shows a typical HE9 insertion force and spring deflection along the $3 \mathrm{~mm}$ of insertion. We note that the initial deflection of $300 \mu \mathrm{m}$ induced by the pre-stress force must be added to the deflection. As related in a previous paper [5], we distinguish the insertion peak force followed by the stabilization of this force. In this final zone, the deflection of the spring is constant and ensures the contact force during the lifetime of the connector.

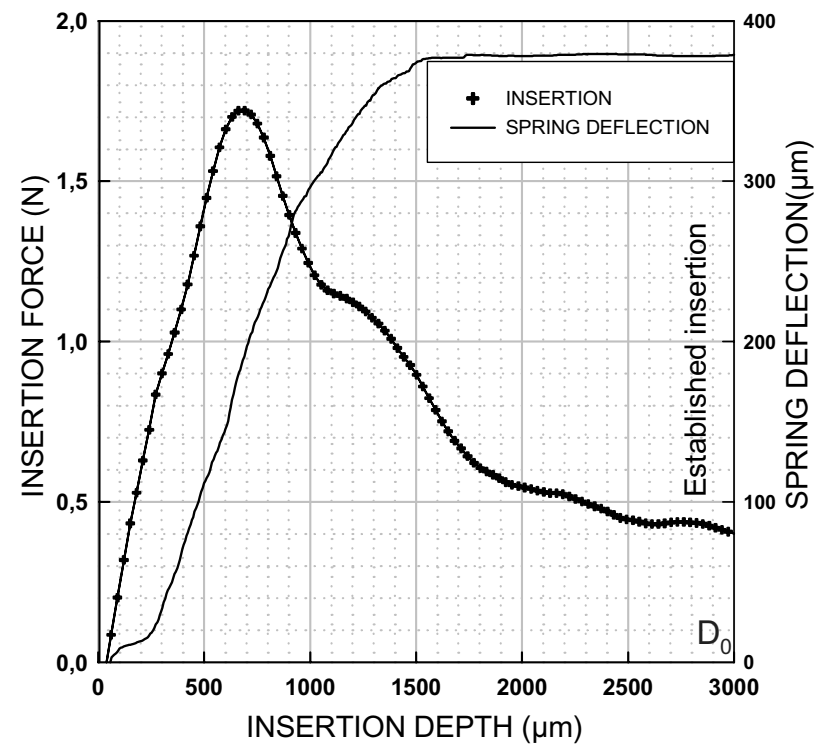

Figure 4. Insertion force and spring deflection (counted from initial deflection $D_{0}$ ) versus insertion depth for new connector 
However, the friction coefficient value is needed to evaluate the contact force at the final stage of insertion. So it was decided to determine this force using direct spring indentation either isolated or lodged in the housing.

For these two cases, the deflection and the resulting indentation force are shown in the Fig. 5. It is observed that the deflection $\Delta \mathrm{x}$ appears to be a linear function of the contact force $\mathrm{Fc}$ with $\mathrm{F}_{\mathrm{c}}=\mathrm{K} \Delta \mathrm{Y}$, the constant $\mathrm{K}$ is the stiffness of the spring and has the same value of $2.2 \mathrm{~N} / \mathrm{mm}$ for the two configurations. In addition, by extrapolation of the deflection law (dashed curve), we deduce that the pre-deflection is approximately $\mathrm{D}_{0}=300 \mu \mathrm{m}$ and the initial deflection force is $\mathrm{F}_{0}=0.65 \mathrm{~N}$. From 0 to $50 \mu \mathrm{m}$, the initial increase of the force up until $\mathrm{F}_{0}$ is due to spring detachment from the housing wall.

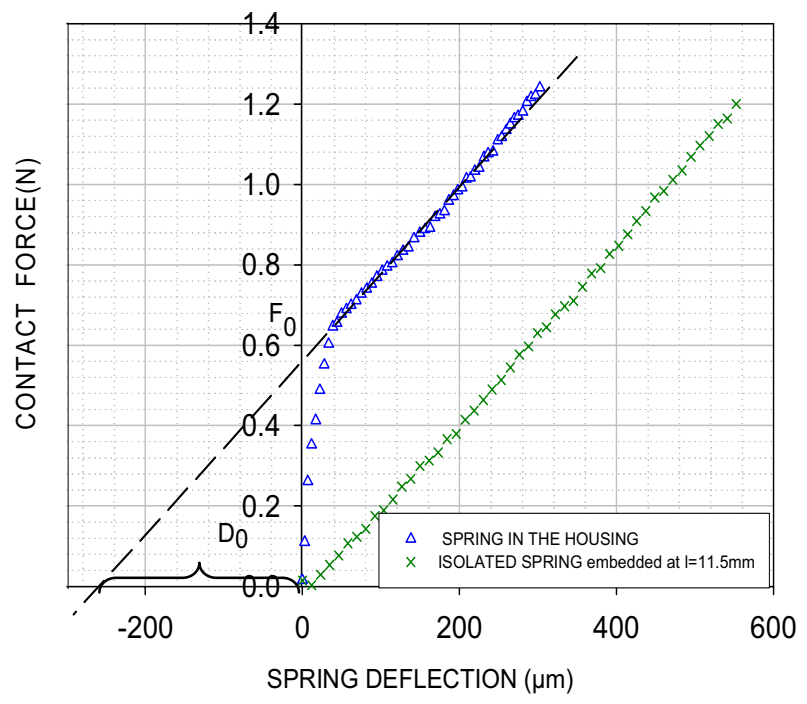

Figure 5. Comparison between contact forces for pre-stress and isolated springs vs. deflection

By analogy with the theoretical formula for girder deflection for a homogeneous beam, we may predict the deflection under normal applied forces $F_{c}$ as:

$$
\mathrm{F}_{\mathrm{c}}=\frac{3 \mathrm{EI}}{\ell^{3}} \Delta \mathrm{Y}
$$

where E is Young's modulus, I is the moment of inertia that depends on the spring's cross section. Comparing this formula with the experimental law, $\mathrm{F}_{\mathrm{c}}=\mathrm{K} \Delta \mathrm{Y}$ we can deduce the expression for the stiffness:

$$
\mathrm{K}=\frac{3 \mathrm{EI}}{\ell^{3}}
$$

This expression shows that the stiffness $\mathrm{K}$ is very sensitive to the embedding length $\ell$. Hence, during its lifetime, the changing of the embedding length $\ell$ will highly impact the stiffness and subsequent contact forces. Due to the complex geometry of the spring (spring shape, inhomogeneous cross section, etc.) the theoretical formula for the stiffness $\mathrm{K}$ is not valid and this value cannot be calculated, although this value is well determined by the experimental test.

These results are plotted in Fig. 6 for incremental values of the spring embedding range $\ell(8.5-12 \mathrm{~mm})$ by steps of $0.5 \mathrm{~mm}$.

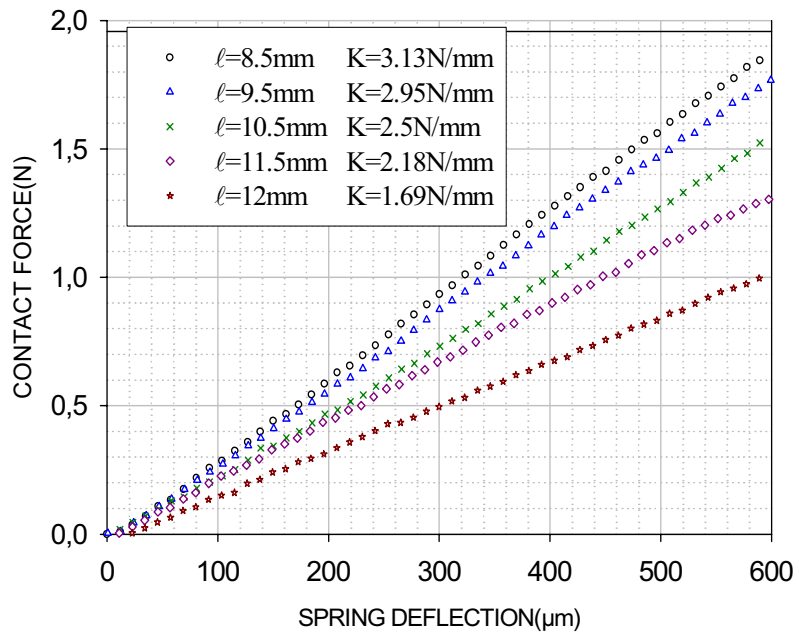

Figure 6. Contact force for different embedding lengths

As shown, the stiffness is roughly doubled when the embedding point is decreased to 9.5 from $12 \mathrm{~mm}$. Also by comparison of Fig. 5 and Fig. 6, it can be stated that the embedding point of the spring in the connector is approximately $11.5 \mathrm{~mm}$. So we confirm that any change of embedding during operation by mechanical or thermal constraints may greatly affect the stiffness. Consequently the contact force is varied and the contact resistance affected.

\section{Numerical results}

There is no analytical law that defines the contact force vs. spring deflection variation for a spring connector. A 2D model using the plane stress assumption has been constructed to show the influence of the embedding length variation on the contact force in order to obtain a numerical simulation of the indentation test with one imbedded spring and one moving pin part. Indeed, the HE9 connector (Fig.1) contains 26 springs. The various springs are not embedded exactly at the same embedding length. Thanks to the numerical simulation, the influence of the variation of embedding spring length on the spring deflection and thus on the contact force can be analyzed.

In addition to the experiment, the numerical simulation leads to the stresses and strains evolutions inside the used material and thus to obtain the zones which can be plasticized. The finite element analysis makes it possible to validate the proposed material behaviour law and to estimate the error between a simple numerical modelling of the connector compared to the real behaviour of this one.

For the contact surfaces between the spring and the pin plane part, the friction coefficient $\mu$ is equal to 0.2 and the Young's 
modulus is equal to $120 \mathrm{GPa}$ (spring and pin are together made of beryllium-copper alloys with $80 \%$ of copper).

The boundary conditions may be expressed as follows:

$$
\mathrm{U}_{\mathrm{x}}\left(\mathrm{Node}_{\mathrm{pin}}\right)=0 \text { and } \mathrm{U}_{\mathrm{y}}\left(\mathrm{Node}_{\mathrm{pin}}\right)=0.6 \mathrm{~mm}
$$

for all nodes of the pin (translation pin movement along the $\mathrm{Y}$ axis),

$$
\mathrm{U}_{\mathrm{x}}(\mathrm{x}=0)=0 \text { and } \mathrm{U}_{\mathrm{y}}(\mathrm{x}=0)=0
$$

for the nodes of the fixed section of the spring.

$\mathrm{U}_{\mathrm{y}}, \mathrm{U}_{\mathrm{x}}$ are respectively the displacement according to $\mathrm{Y}$ and $\mathrm{X}$ axis (Fig. 7).

Numerical simulations are made with an elastic spring behavior obtained with the Ansys finite element (Ansys [6]) code. Underlying the approach in this code is the discretization of the continuum involved. Little by little, as the pin part progresses during the indentation test, the program automatically establishes the junction between the contact surface nodes of the pin surface and those of the spring. For the mechanical calculation, different element types were used with 32,000 nodes. The surface-to-surface contact elements use Gauss integration points as a contact detection point. The contact algorithm used is the Augmented Lagrangian method. Contact, material and geometric non-linearities require a full Newton Raphson scheme to be used with the sparse matrix solver (direct solver). The program checks the convergence of the iterative solution by using a force criterion. Other smoothness meshes were tested. The final Ansys file obtained from the converged solution for the structural spring simulation is run in Ansys to obtain the load in the displacement direction.

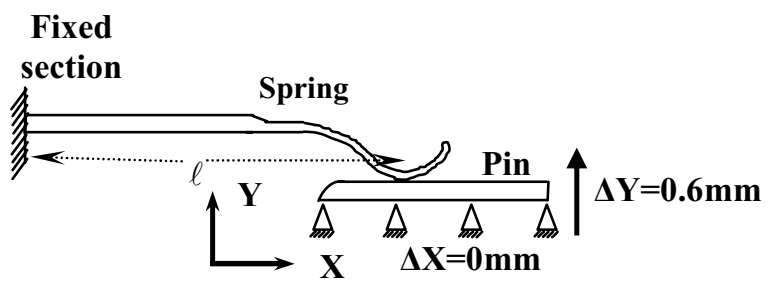

Figure 7. Boundary conditions

Fig. 8 gives the Von-Mises stress distribution. One can note that the maximum of the Von-Mises stress is located near the embedded zone which undergoes the greatest bending stresses.
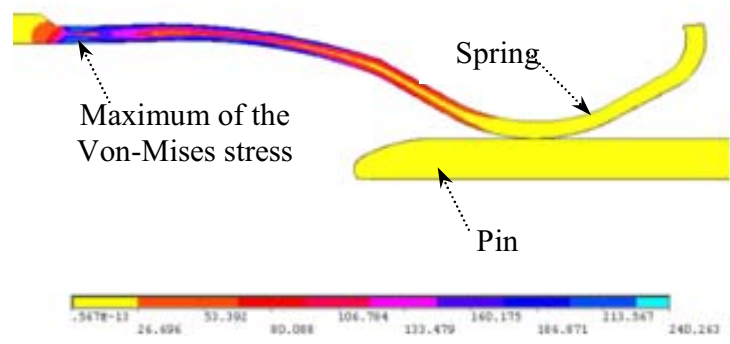

Figure 8. Von-Mises stress distribution
As found in the experimental results (Fig. 9), the contact force in the numerical results linearly increases with spring deflection up until $300 \mu \mathrm{m}$, and then it's also easy to determine spring stiffness and to compare experimental and numerical curves. Figure 9 also gives for four embedding lengths the contact force at a spring deflection of $600 \mu \mathrm{m}$. The simplified model used in the numerical simulation (the use of a simple geometrical model perfectly embedded and of an elastic material behaviour law) explains the differences between the numerical and the experimental results. Moreover, the spring contact force decreases with increasing embedding length for all results.

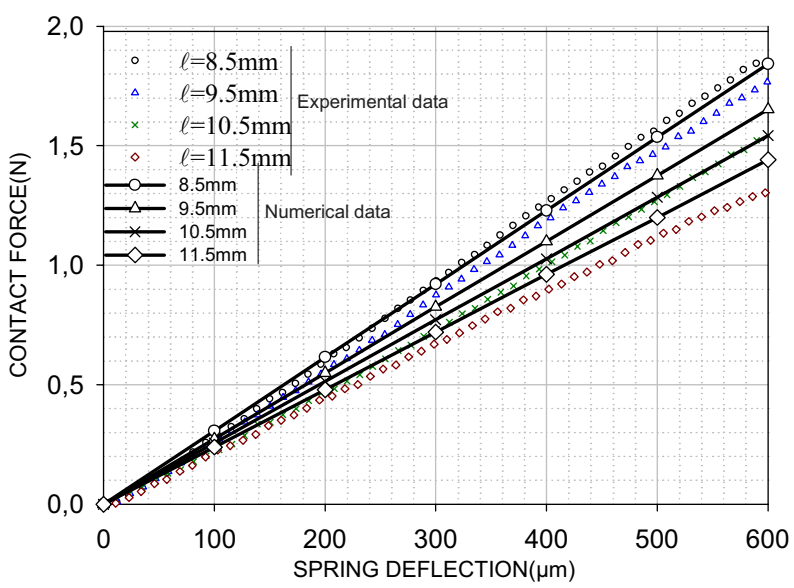

Figure 9. Numerical and experimental results for contact force versus spring deflection

\section{B. Investigations on an Aged Spring Connector}

1. Repetitive insertion cycles

In order to observe the impact of a large number of matings, higher than the servicing cycle number, we have made 2000 cycles of insertions-extractions. Insertion parameters are plotted in Fg.10 at the initial state and after 500, 1000, 1500 and 2000 insertion-extraction cycles. The main remark is that the insertion force rises to higher and higher peak values while the final value at the end of the insertion seems to be less affected. This increase of insertion force is attributed to the degradations of the male head (edge of the PCB) as can be seen by profile analysis. Furthermore, in the final stage i.e. permanent contact zone, a minor change of insertion force could be a combination of opposing effects such as modifications of the friction coefficient and the stiffness. The spring deflection remains roughly constant during the test. 


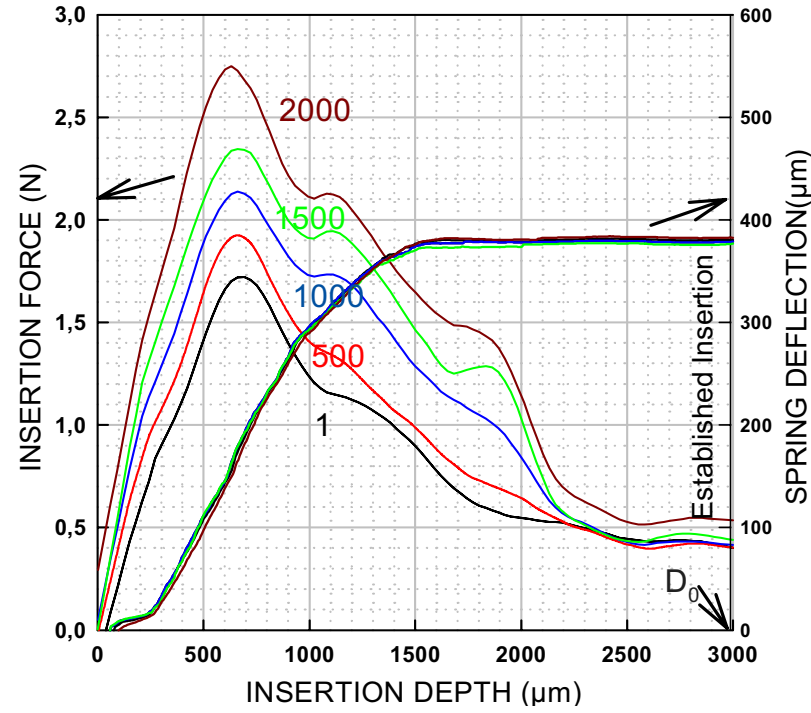

Figure 10. Insertion force and spring deflection (shifted by initial deflection $\mathrm{D}_{0}$ ) after mating cycles number 1, 500, 1000, 1500, 2000.

\section{Flexion-deflection cycles}

Aging the connector under a higher number of flexiondeflection degradations may affect the embedding length and stress relaxation [7] of the spring. In our test, the sample (spring connector and isolated spring) are submitted to 10,000 flexion-deflection cycles.

For the spring mounted in the housing, the initial force $\mathrm{F}_{0}$ of $0.55 \mathrm{~N}$ remains constant during 10000 flexion indentation cycles. The stiffness constant $\mathrm{K}$ deduced from the initial and the last indentation also remains the same $(2.2 \mathrm{~N} / \mathrm{mm})$. Consequently, flexion-deflection cycles have a negligible effect on the stiffness of the spring in the housing. In addition we conclude that the embedding point keeps the same position.

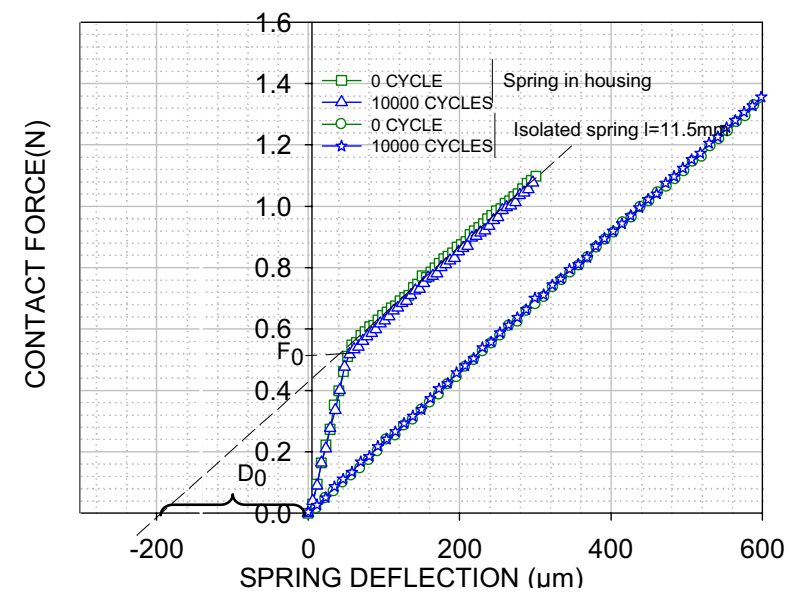

Figure 11. Contact force vs. spring deflection for spring in HE9 Housing

\section{Thermal cycles}

According to their datasheet, the connectors are designed for an operational temperature range of $-55^{\circ} \mathrm{C}$ to $125^{\circ} \mathrm{C}$. We assume that mechanical properties of the spring materials remain the same over this temperature range. However the insulating housing material suffers heat-hardening [6] which can change the embedding point of spring.

To check this assumption, thermal cycling from $-30^{\circ}$ to $120^{\circ} \mathrm{C}$ during four hours repeated 22 times was applied to the entire connector, the half connector and the isolated spring.

Insertion parameters (peak insertion force and final force) keep similar values after the thermal test since the connector gap didn't change because the housing materials remain unchanged (Fig .12).

Concerning the spring relaxation and embedding change after thermal test, we investigate the contact force vs. deflection of the embedded and the isolated spring. These results are given in Fig.13. Again, no changes are observed in the stiffness of the spring and the pre-stressed force $\mathrm{F}_{0}$.

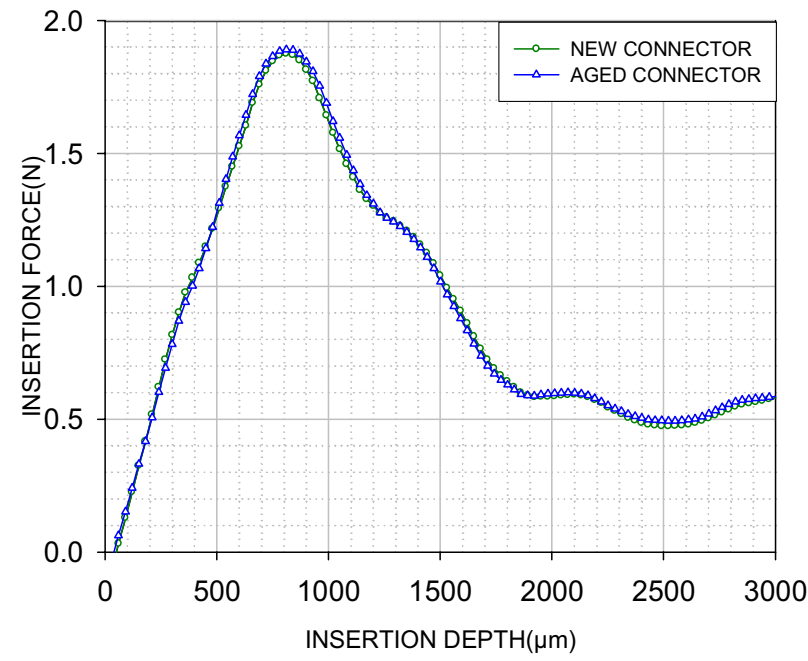

Figure 12. Insertion force comparison for new and aged springs

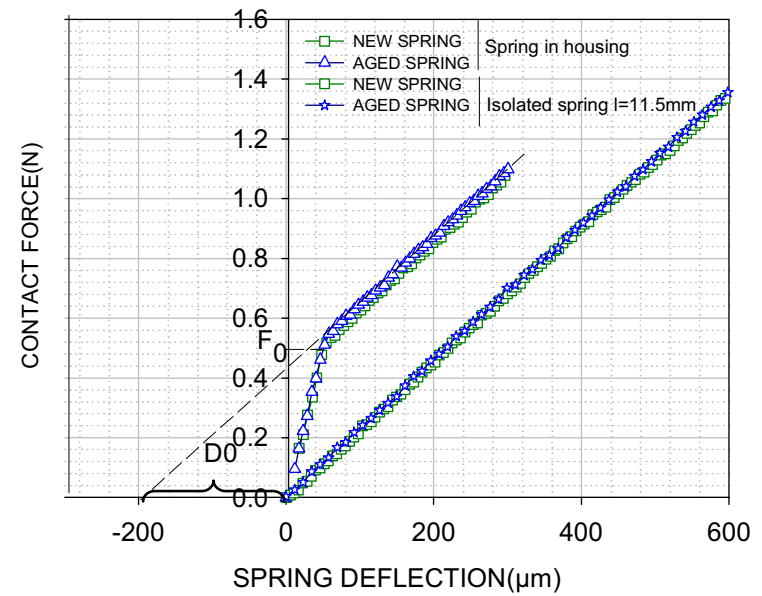

Figure 13. Contact force comparison for new and aged pre-stressed springs

As shown in Fig.13, there is no variation of the insertion force of the contact force values after aging. 


\section{CONCLUSION}

In spite of the embedding length variation has induced a variation of spring stiffness, no changes of stiffness are observed after large number of flexion and thermal cycling. This maximum stress level applied to the spring and made to simulate the long lifetime of the connector, does not lead to significant degradation of the stiffness nor of the relaxation stress. We can conclude that contact forces exerted by the spring will remain unchanged during its long lifetime and contact resistance stays stable value (exempting other parameters: pollution, corrosion, etc).

On the other hand, the insertion effort needed to ensure full insertion seems to become greater with increasing number of connector matings. As a consequence, some care has to be taken during operations, when replacing the PCB.

Numerical results give the same contact force progression for different embedding lengths and show that the maximum Von-Mises stress is located near the embedded zone.

It is planned to simulate a non-perfect embedding (a small torque can be introduced into the embedded zone) to approach the real embedding.

The potential mechanism causing the reduction of the contact force should be explored further in the future.

\section{ACKNOWLEDGMENT}

The authors wish to thank Professor Brian Mitchell for reviewing the manuscript. They also acknowledge the French Agence Nationale de la Recherche et de la Technologie (ANRT) for financial support.

\section{REFERENCES}

[1] S. Robert Mroczkowski, "A perspective on connector reliability", Holm2004, p.125, August 2004.

[2] L. Cretinon, M. El Hadachi, F. Augereau, G. Despaux, L. Doireau "Microelectronic reliability", ESREF, 48, 8, p. 1129, 2008.

[3] P. Chaudhuri "Contact reliability in dusty environments", $13^{\text {th }}$ ICEC Lausanne, p. 348, 1986.

[4] M. El Hadachi,"Diagnostic du vieillissement de la connectique encartable dorée bas-niveau", Thesis, Dec.2008, p.108.

[5] R. El Abdi and N. Benjemaa, "Experimental and analytical studies of the connector insertion phase", IEEE Transaction on Components and Packaging Technologies Volume 31, pp.751-758, Dec. 2008.

[6] Ansys website accessed at: http://www.ansys.com

[7] F. Blumenroth, H.LÖbl, S.Großmann and M. Kudoke, "Influence of stress relaxation and sliding on long time behavior of Plug-In power connectors with helical springs", ICEC2008, pp.368-373, June 2008 .

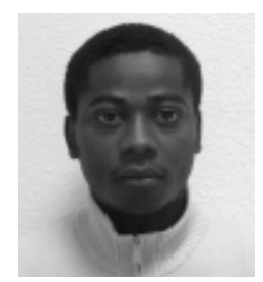

H. ESSONE OBAME was born in Libreville, Gabon in 1983. He received a Master Degrees in electrical engineering in Poitiers University, France. Actually, he is pursuing his Ph.D. Degree of Electrical Engineering at EDF R\&D (Moret sur Loing - France) and at the Electrical Contact Laboratory (Rennes -France). His research interests focus on connector reliability for long life time.

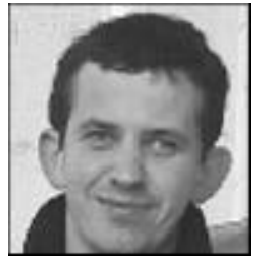

L. CRETINON received an engineer diploma from the Institut National Polytechnique de Grenoble (France) in the field of materials science in 2001 and a $\mathrm{PhD}$ in superconductivity from the Joseph Fourie University in 2004. He is now working as research engineer at Electricité de France (EDF). His domains of interest are the reliability and the ageing of materials used in electronic components.

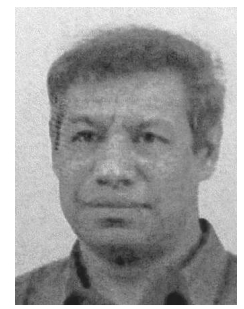

R. EL ABDI is a professor at Rennes1 University (Rennes Applied Mechanics Laboratory). $\mathrm{He}$ is specialized in the structure behavior analysis, in fracture fatigue, damage and mechanical engineering. His current research interests include analysis of numerical methods, finite-element methods, boundary integrals, nonlinear damage modeling and experimental optic fiber analysis. He manages a mechanical team which is interested in surfaces and contact problems.

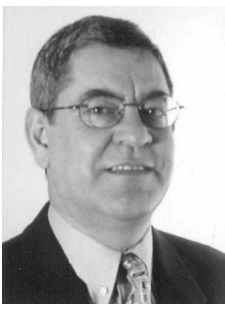

N. BENJEMAA (IEEE Member for 11 years NO 01629807) received the Doctorat és-sciences in physics from the University of Rennes I, France, in 1985. He has 25 years of research covering the physics and degradation encountered in electrical contacts. This research has been mainly concerned with low and medium electrical levels and has covered arc parameters and contact resistance. This work has been published in more than 80 papers mainly in ICEC, Holm, NARMS, IEEE journals and used in the telecommunication and automobile fields. He is currently professor of physics and electronics at the University of Rennes I where he directs and manages the electrical contacts group research.

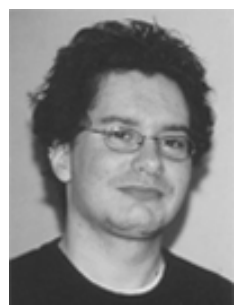

E. CARVOU received the PH.D. Degree in Electronics from the University of Rennes in 2003. $\mathrm{He}$ currently works on electrical contacts for automotive, power and domestic area. This research concerned the conduction and effect of arc disconnection 


\title{
Investigation of dust test and modeling of dusts ingression
}

\author{
Dong Wang, Liang Jun $\mathrm{Xu}, \mathrm{Na} \mathrm{Lu}$ \\ Research Lab of Electric Contacts \\ Beijing University of Post and Telecommunications \\ Beijing, China
}

\begin{abstract}
Dust is a causative factor in connector's failure. To evaluate dust influence on the reliability of electric/electronic devices and connectors, dust tests are required for both ingression and contact behavior. Dust influence involves two typical stages: deposition and redistribution. Many factors such as air flowing, particle size, connector structure, electromagnetic characteristic of electronic devices, electric charge of dusts and dynamic environments may affect the results. Normally, vibration including bouncing is used to simulate dust ingression in the test. However, the results are not quite consistent with real situation. In this paper, for improving dust test method, the study of some influencing factors in the ingress of dust into electronic devices is performed. Dynamic models of finite element method were established for the evaluation of these influences. Based on analysis, an improved test method is presented.
\end{abstract}

Keywords- dust bouncing test; dusts ingression; gas flowing; particle size; FEM

\section{INTRODUCTION}

It is known that contact failure is one of the main factors reducing electric system's reliability. Dust particle is a contact failure causative factor that cannot be ignored. Some theoretical and experimental studies on dust effects on electric contacts have been presented [1-4]. Although vibration and bouncing have been carried out in dust test, it should be noticed that this test could hardly reflect the contact failure caused by dust contamination. Both ingression and contact behavior should be involved in dust tests for evaluating the reliability of electric/electronic devices and connectors.

Dust ingression could be divided into two stages: deposition and redistribution. The factors such as air flowing, particle size, structure and electromagnetic characteristic of electronic devices, electric charge of dusts and dynamic environments are significantly influencing the results. For improving dust ingression test method, all these factors and the combined effects should be considered. Study in the ingress of dust into electronic devices was performed in this paper.

In this paper, the ingression simulating results of the vibration/bouncing test and the dust characteristics in same kind of mobile phones inside from the field were compared. The observation by optical microscope found that, after vibration/bouncing test, dust particles were seriously concentrated in the middle, which is not quite consistent with that in mobile phones from the field.

\section{Modeling OF DUSTS INGRESSION}

\section{A. Air Flowing Effects}

The movement of dust particle by air flowing and gravity [5] is expressed in ${ }^{(1 a, b)}$.

$$
\begin{aligned}
m_{p} \frac{d^{2} x}{d t^{2}}= & -\frac{\rho \pi D_{p}^{2}}{8}\left(\frac{24 v}{D_{p} \sqrt{(\dot{x}-u)^{2}+\dot{y}^{2}}}+\frac{6}{1+\sqrt{D_{p} \sqrt{(\dot{x}-u)^{2}+\dot{y}^{2} / v}}}+0.4\right) \\
& \times(\dot{x}-u) \sqrt{(\dot{x}-u)^{2}+\dot{y}^{2}} \\
m_{p} \frac{d^{2} y}{d t^{2}}= & -\frac{\rho \pi D_{p}^{2}}{8}\left(\frac{24 v}{D_{p} \sqrt{(\dot{x}-u)^{2}+\dot{y}^{2}}}+\frac{6}{1+\sqrt{D_{p} \sqrt{(\dot{x}-u)^{2}+\dot{y}^{2} / v}}}+0.4\right) \\
& \times \dot{y} \sqrt{(\dot{x}-u)^{2}+\dot{y}^{2}}-m_{p} g
\end{aligned}
$$

Where, $x y$ is the location of dust particle. $D_{p}$ is the diameter of dust particle. $\mathrm{m}_{\mathrm{p}}$ is the weight of dust particle. $\rho$ is the density of the air. $v$ is the viscosity of the air. $g$ is the gravity.

The inter particles collision is also an important effect [6], the probability of particles collision is expressed in ${ }^{(2)}$.

$$
\begin{aligned}
& p\left(T=\frac{ \pm\left(y_{i+1}-y_{i}\right)}{\dot{y}}\right) \\
& =e^{\mp a \int_{y_{i}}^{y_{i+1}} c(\zeta) d \zeta}=e^{\mp a \int_{y_{i}}^{y_{i+1}} c_{0} e^{-v y} d y}=e^{ \pm \gamma\left(e^{-v y_{i+1}}-e^{-v y_{i}}\right)} \\
& p(M)=1-p,\left(T=\frac{ \pm\left(y_{i+1}-y_{i}\right)}{\dot{y}}\right)
\end{aligned}
$$


Where, $a=\frac{\pi D_{p}^{2}}{\sin \varphi}, \quad \gamma=\frac{a c_{0}}{v}, \quad c(y)=c_{0} e^{-v y}$ is the density of dust particle in y direction. $\varphi$ is the angle between the velocity direction of dust particle and the $\mathrm{x}$ direction.

Combing all these effects, the dust transport rate which varies with height and air flowing is expressed in ${ }^{(3)}$. Fig.1 shows the calculated and experimental result [6].

$$
\begin{aligned}
& Q_{C}(y)=Q_{C 1}(y)+Q_{C 2}(y) \\
& =\int_{0_{\text {min }}}^{v_{\text {max }}} s m_{p} f\left(v_{0}\right)\left[\left(1-p_{\uparrow}\left(v_{0}, y\right)\right) \frac{\dot{x}_{\uparrow}\left(v_{0}\right)}{\dot{y}_{\uparrow}\left(v_{0}\right)}-\left(1-p_{\downarrow}\left(v_{0}, y\right)\right) \frac{\dot{x}_{\downarrow}\left(v_{0}\right)}{\dot{y}_{\downarrow}\left(v_{0}\right)}\right] d v_{0} \\
& +\int_{0_{\text {min }}}^{v_{\text {max }}} s m_{p} f\left(v_{0}\right)\left[p_{\uparrow}\left(v_{0}, y\right) \frac{\dot{x}_{\uparrow}\left(v_{0}\right)}{\dot{y}_{\uparrow}\left(v_{0}\right)}-p_{\downarrow}\left(v_{0}, y\right)\left(\frac{\dot{x}_{\downarrow}\left(v_{0}\right)}{\dot{y}_{\downarrow}\left(v_{0}\right)}\right)_{p}\right] d v_{0}
\end{aligned}
$$

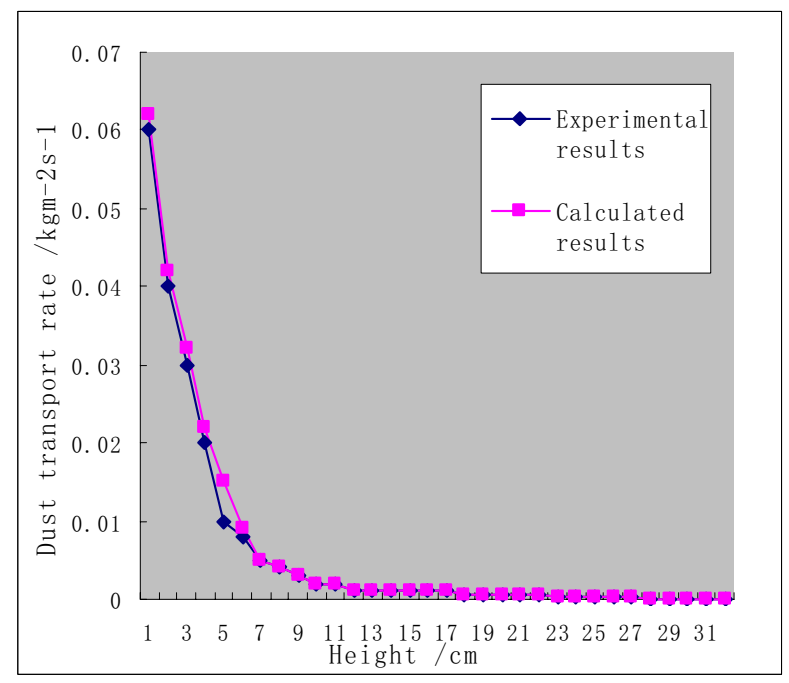

Figure 1. The dust transport rate varies with height (dust size: $0.228 \mu \mathrm{m}$, air velocity $=10 \mathrm{~m} / \mathrm{s}$ )

The force operating on the dust particle by air flowing [7] is expressed in ${ }^{(4)}$.

$$
F_{D}=\frac{1}{8} C_{D} \rho \pi D_{P}^{2} V_{\gamma}^{2}
$$

Where, $C_{D}=\frac{24}{R_{e}}+\frac{6}{1+\left(R_{e}\right)^{1 / 2}}+0.4 \approx 1, \mathrm{~V}_{\mathrm{r}}$ is related velocity between the dust particle and the air flowing.

When the size of particles varies from $0.01 \sim 1 \mathrm{~mm}, \mathrm{~F}_{\mathrm{D}}$ varies from $10^{-11} \sim 10^{-7} \mathrm{~N}$.

\section{B. Structure Densification Effects}

The air flowing is changed through the structure densification of electric/electronic devices. The fluid-dynamics part of the simulations was performed using ANSYS Multi-physics, ANSYS FLOTRAN. The model of air flow in a two-dimensional mobile phone was established. Basing on the experimental results (size range of particles is $0 \sim 200 \mu \mathrm{m}$, size of fibers is $0 \sim 1000 \mu \mathrm{m}$ ), two models of different dimensions (D1 and D2) were established. Table I gives the dimensions and properties.

Firstly, an inlet velocity was defined to simulate laminar flow. The resulting vector plot shows the trace of flow in the mobile phone, see Fig. 2. The resulting contour plot shows the pressures that occur in it, see Fig. 3.

Normally, wind speed varies from $0 \sim 30 \mathrm{~m} / \mathrm{s}$. Then, for two different hermetically-sealed constructions (D1 and D2), several inlet velocities were simulated. Fig. 4 shows the resulting maximum differential pressure in the mobile phone was lower than $920 \mathrm{~Pa}$.

\section{TABLE I. DIMENSIONS AND PROPERTIES}

Mobile phone length $\quad 0.01 \mathrm{~m}$

Mobile phone width $\quad 0.005 \mathrm{~m}$

Inlet length

$D 1: 0.001 \mathrm{~m} ; D 2: 0.0002 \mathrm{~m}$

Outlet length

$D 1: 0.001 \mathrm{~m} ; D 2: 0.0002 \mathrm{~m}$

Outlet pressure

$0 P a$

Air density

$1.205 \mathrm{~kg} / \mathrm{m}^{3}$

Air viscosity

$$
1.8135 \times 10^{-5} \mathrm{~kg} / \mathrm{m}-s
$$

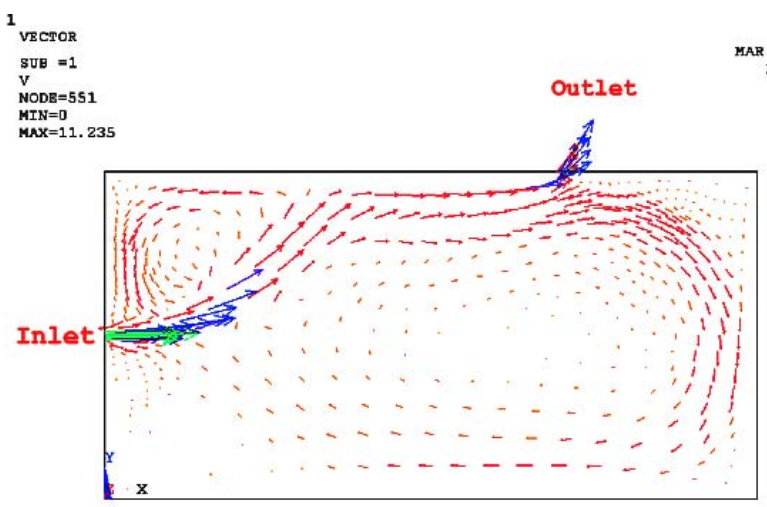

NNSYS 13:57:51

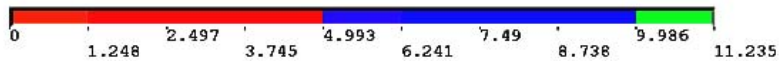

Figure 2. The resulting flow vector plot (air flowing effects) 


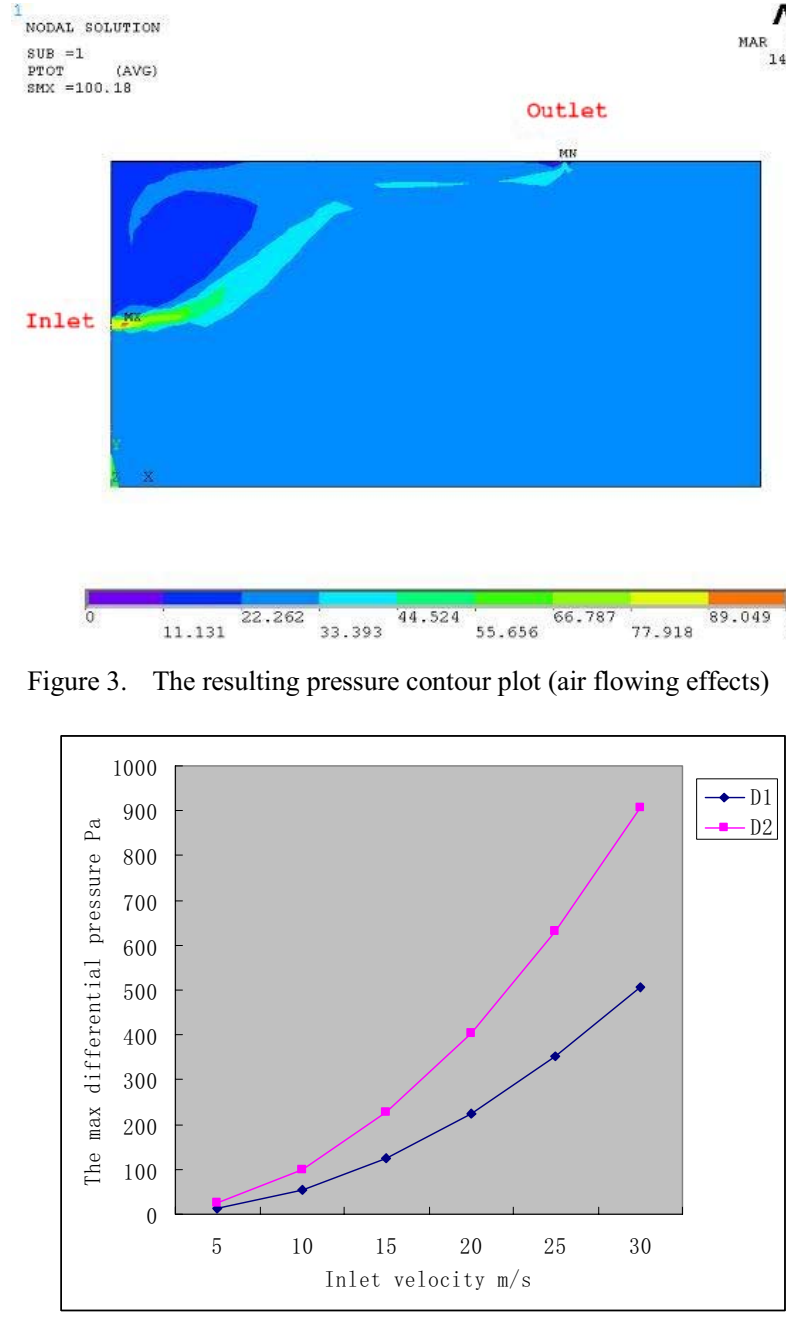

Figure 4. The resulting max pressure values for various inlet velocities in the mobile phone(air flowing effects)

Base on the surveying results, a 3-D model of finite element analysis was established (shown in Fig. 5), which is closer to real condition. The results (shown in Fig. 6 and Fig. 7) show that it is almost consistent with the D2 result in Fig. 4. Fig. 8 also presents an example which is the simulated result of the volume keys.

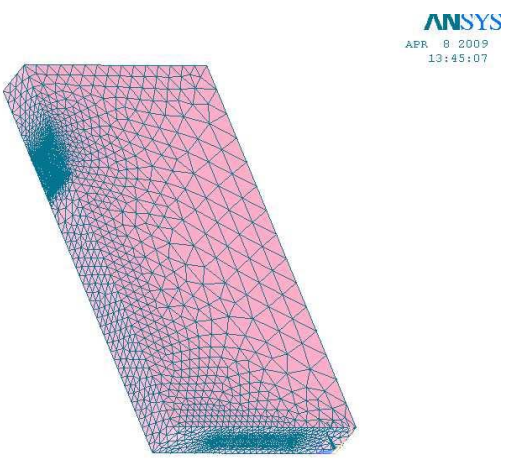

Figure 5. 3-D finite element modal for mobile phones

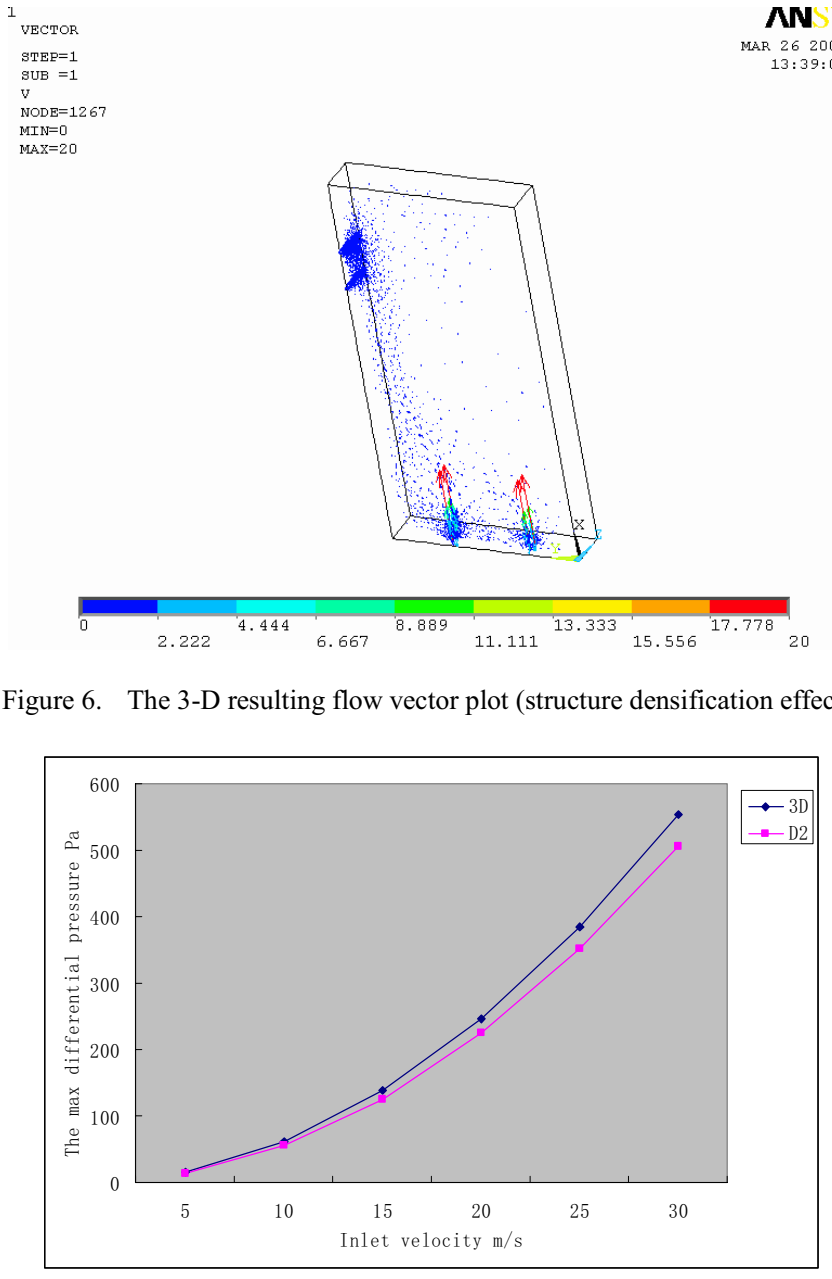

Figure 7. The $3 \mathrm{D}$ resulting max pressure values for various inlet velocities in the mobile phone(comparing with D2)
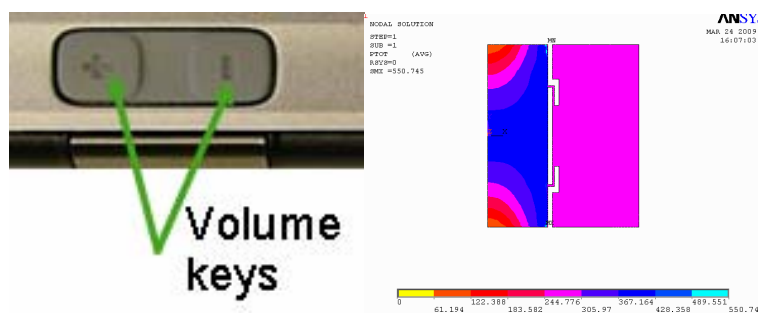

Figure 8. The resulting flow vector plot of volume keys

\section{Thermal Characteristic of Electronic Devices Effects}

When electron components is working, heat change the air flowing in the electric/electronic devices. For evaluating this effect, a coupled-field (fluid-thermal) analysis was established. The simulation was performed using ANSYS Multi-physics, ANSYS FLOTRAN CFD, and Thermo-dynamics. Based on the consistent results in Fig. 7, the geometric model of 2-D was established for convenience. The dimensions and properties are shown in Table I. Table II shows the temperature in the analysis. 
TABLE II. TEMPERATURE

\section{C1 $\mathrm{T} 1=293 \mathrm{~K}, \mathrm{~T} 2=303 \mathrm{~K}$ \\ C2 $\mathrm{T} 1=293 \mathrm{~K}, \mathrm{~T} 2=313 \mathrm{~K}$}

Although the thermal effect is opposite to the air flowing effect, it is weak. Fig. 9 shows the similar trace flow with the result in Fig. 2. The resulting points in Fig. 10 are coincident with the resulting point of D2 in Fig. 4. This coupled-field (fluid-thermal) analysis proved that the thermal characteristic of electronic devices effects is weak, which could be ignored compared with the air flowing effects here.

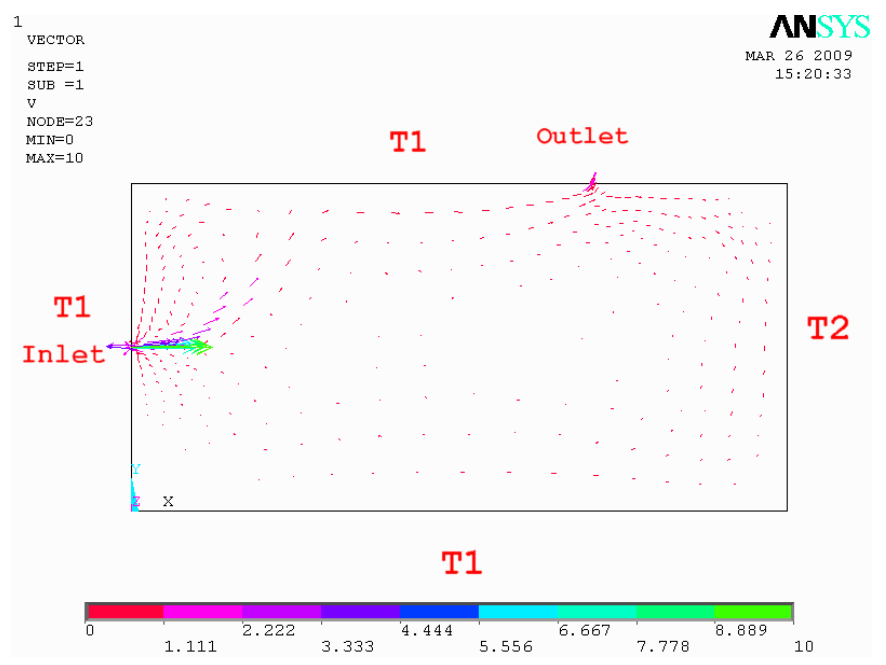

Figure 9. The resulting flow vector plot (effects of thermal characteristic of electronic devices )

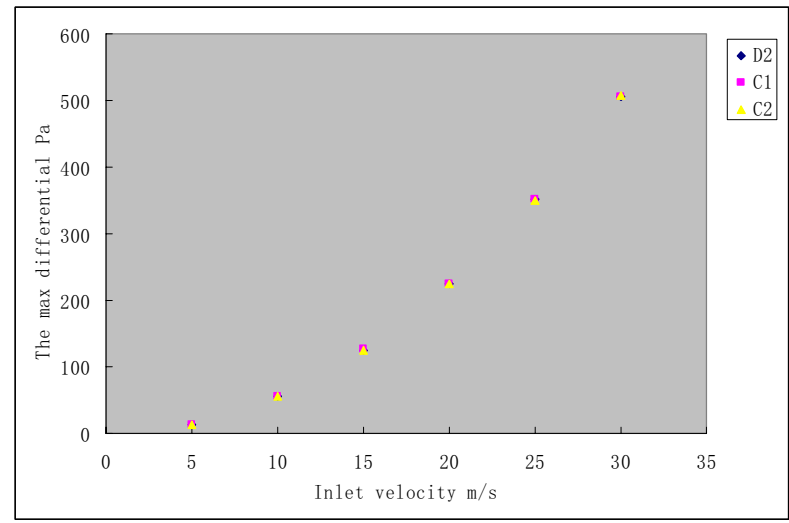

Figure 10. The resulting max pressure values for various inlet velocities in the mobile phone( $\mathrm{C} 1$ and $\mathrm{C} 2$ compared with $\mathrm{D} 2)$

\section{Electromagnetic Characteristic of Electronic Devices Effects}

Dust particle carrying an electric charge may build up a tiny static electric field at the metal surface [8,9]. Attracting force $\mathrm{F}_{\mathrm{e}}$ between particle and the metal surface is expressed in ${ }^{(5)}$.

$$
F_{e}=-Q^{2} / 16 \pi \varepsilon_{0} d_{0}^{2}
$$

Where, $\mathrm{Q}$ is the electric charge carried by particle. $\varepsilon$ is the permittivity of air, and $\mathrm{d}_{0}$ is the distance from the center of particle to the metal surface. When the distance is small, the attracting force can be very strong.

Consider two parallel vertical metal sheets with a DC potential between them. The electric attracting force $\mathrm{F}_{\mathrm{E}}$ is expressed as $F_{E}=Q E$, where $\mathrm{E}$ is the $\mathrm{DC}$ electric intensity.

When distance between probe and sheet varies from $0.01 \sim 1000 \mu \mathrm{m}, \mathrm{F}_{\mathrm{E}}$ varies from $10^{-8} \sim 10^{-13} \mathrm{~N}$.

Compared with the $F_{D}$ by air flowing varies from $10^{-7} \sim 10^{-11} \mathrm{~N}$, the electromagnetic characteristic of electronic devices effects can not be ignored.

\section{E. Dynamic Environment Effects}

Nowadays electric/electronic devices are widely used in various dynamic environments. When the frequencies of vibration and external disturbance coincide with the natural frequencies of the structure, the structure is very sensitive to disturbances. They cause electrical contact instability $[10,11]$. Normally, it is ignored that dust redistribution caused by this phenomena. In fact, this influence should be considered basing on our compared investigation.

\section{NEW DUST TEST PROPOSSING}

For improving dust ingression test method, all above factors and combined effects should be considered. Fig. 11 gives the possible layout of dust test. In further research, theoretical analysis and experiments will be performed for each part.

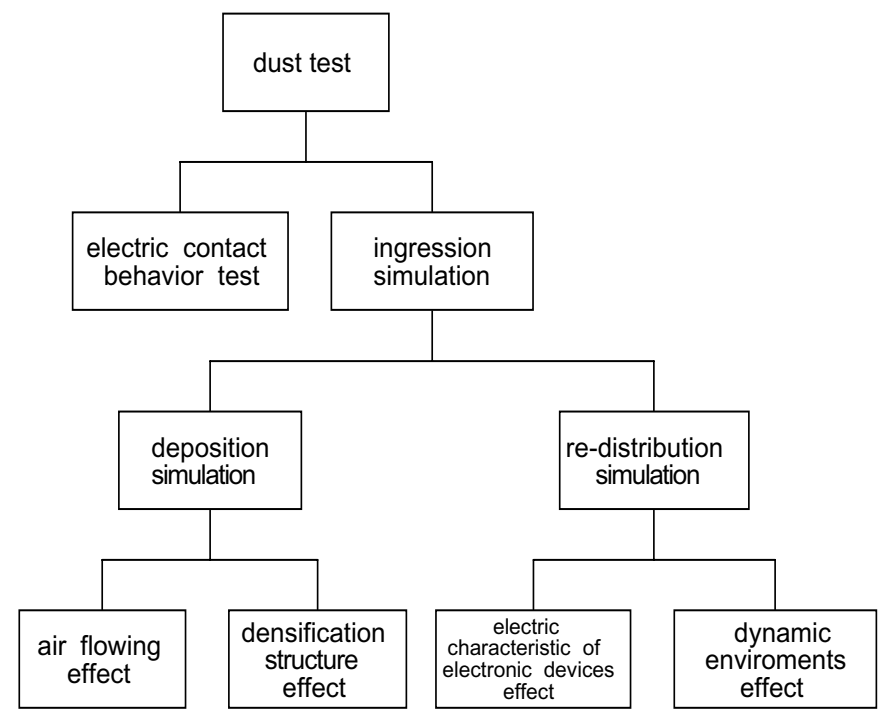

Figure 11. The layout of dust test

\section{CONCLUSIONS}

1 The normally used dust vibration/bouncing test was investigated. Dust test like that could hardly reflect the contact failure caused by dust contamination. For evaluating the 
reliability of electric/electronic devices and connectors, both ingression and contact behavior should be involved in dust test.

2 The experimental results show ingression simulation of vibration/bouncing test is not quite consistent with real situation. Air flowing, particle size, densification structure and electromagnetic characteristic of electronic devices electric charge of dusts and dynamic environments are important influencing factors. For improving dust ingression test method, all these factors and the combined effects should be considered.

3 Theoretical and finite element analysis were established for the evaluation of the influence of these effects. When the Size range of particles varies from $0.01 \sim 1 \mathrm{~mm}$, the force operating on the dust particle by air flowing $F_{D}$ varies from $10^{-11} \sim 10^{-7} \mathrm{~N}$. When distance between probe and sheet varies from $0.01 \sim 1000 \mu \mathrm{m}$, the electric attracting force $F_{E}$ varies from $10^{-8} \sim 10^{-13} \mathrm{~N}$. Based on structure densification structure of mobile phone FEM results, the max differential pressure is 920 $\mathrm{Pa}$, and the thermal characteristic of electronic devices effects can be ignored compared with other effects here. The concentrated phenomenon in the contrastive experiments proved that dynamic environments effects can not be ignored.

4 The layout of dust test was presented. Theoretical analysis and experiments as well as further research are still needed.

\section{REFERENCES}

[1] B.T. Reagor, C.A. Russel. "A survey of problems in telecommunication equipment resulting from chemical contamination." Proceedings of the 31th IEEE Holm Conference on Electrical Contacts, 1985, pp.157-162..

[2] K. Mano. Reliability of contact components (Japanese). Chapter 4. Contact failure by dust contamination. General Electronics Publisher, Japan, 3rd edition, 1981, pp.121-146.

[3] J.G. Zhang, "Effect of dust contamination on electrical contact failure." Proceedings of the 53th IEEE Holm Conference on Electric Contacts, 2007, pp.xxi-xxx.

[4] J.G. Zhang, L.J. Xu, and C.F. Feng, "Futher studies of the mechanical behavior of particles on electric contact failure." Proceedings of the 24th International Conference on Electrical Contacts, Saint-Malo, France, June.2008, pp.21-26.

[5] N. Huang, X.J. Zheng, Y.H. Zhou, et al, "Simulation of wind-blown sand movement and probabilty density function of lift-off velocities of sand particles," J Geophys Res, D20201, 2006, pp.111-117.
[6] N. Huang, Y.1. Zhang, D.R. Adamo, "A model of the trajectories and midair collision probablities of sand particles in a steady state saltation cloud," J Geophys Res, D08206, 2007, pp.112.

[7] R.S. Anderson, P.K. Haff. "Wind modification and bed response during saltation of sand in air," Acta Mech, (supp.)1, 1991, pp.21-25.

[8] J.G. Zhang, J.C. Gao, C.F. Feng. "The "selective" deposition of particles on electric contact and their effects on contact failure." Proceedings of the 51st IEEE Holm Conference on Electric Contacts, Chicago, IL, Sept.2005, pp.127-134.

[9] J.C. Gao, J.G. Zhang, "Measurement of electrical charges carried by dust particles." Proceedings of the 48st IEEE Holm Conference on Electric Contacts, Orando, FL, Oct.2002, pp.191-196.

[10] L.J. Xu. "Dynamic influence on contact failure." IEICE Trans. On Electronics, Vol.E86-C, No.6, June 2003, Japan, pp.963-967.

[11] Z.P. He and L.J. Xu. "Micro motion at the failed contact interfaces." Proceedings of the 51st IEEE Holm Conference on Electric Contacts, Chicago, IL, Sept. 2005, pp. 180-185.

Dong Wang received bachelor degree from Beijing University of Posts \& Telecommunications in 2005 . He is currently a PhD candidate working at the Lab of Electric Contacts in Beijing University of Posts and Telecommunications.

Liang-Jun Xu, was born in Aug. 1956. He is a professor of electrical-mechanical engineering, vice dean of Automation School, Beijing University of Posts and Telecommunications (BUPT), charted engineer of IET. He received Bachelor, Master degree of mechanical engineering from BUPT in 1982 and 1986 respectively. received $\mathrm{PhD}$ degree in Electrical Engineering institute, Technical University of Wroclaw, Poland in 1999. He fulfilled many scientific and technical projects including national scientific fund, ministry and university scientific fund, international cooperation (USA, Finland and Poland). Published more than 50 papers. His interested areas are dynamic analysis, computer simulation and design of electro-mechanical systems; theory and applications of vibration; theory and applications of electric contacts; design of electric-mechanical components etc.

$\mathrm{Na} \mathrm{Lu}$ received master degree from Beijing University of Posts $\&$ Telecommunications in 1999. She is now a senior engineer working at the Lab of Electric Contacts in Beijing University of Posts and Telecommunications. 


\title{
Reliability of Press Fit Contacts and adjoining SMD components on Printed Circuit Boards
}

\author{
Karl Ring, Thomas Schreier-Alt \\ Fraunhofer Institute for Reliability and Microintegration, \\ Oberpfaffenhofen / Munich, Germany \\ karl.ring@zve.izm.fraunhofer.de
}

\begin{abstract}
Reliability studies of press fit contacts have been performed during environmental stress tests as well as during the manufacturing process. We investigated several press fit pin geometries according to their reliability under thermal, mechanical and electrical load. Correlations with the push-out force have been performed. In this paper we also focus on the mechanical reliability of electronic components which are soldered nearby the press-in pins. During press-in of the contact pin, a strain affected zone is created in the printed circuit board (PCB) near the contacts. The reliability of electronic components soldered near the press fit pins can be reduced if the solder joints are bearing strains $>400 \mu \mathrm{m} / \mathrm{m}$. We investigated the strains on the surface of PCBs during press fit injection by two different sensor technologies and numerical simulations. Apart from standard electrical strain gauges we used fiber optic Bragg grating sensors. Their advantage is the ability to detect strain gradients, which are common in the sphere of influence of a press fit contact. In a distance of $3 \mathrm{~mm}$ away from the investigated contact pins the surface strain on the PCB is around $0.1 \%$; in a distance of $10 \mathrm{~mm}$ at least $500 \mu \mathrm{m} / \mathrm{m}$.
\end{abstract}

Keywords- Press fit, environmental tests, 3D FEA, strain measurement, soldered electronic components

\section{INTRODUCTION}

Press fit contacts have seen a comeback within electronics industry as a measure to fulfill the lead-free requirement (RoHS directive). Additionally the trend within automotive industry towards hybrid power trains has increased the demand for solderless contacts, especially if they can be used in high power / high voltage applications.

A press-fit contact is manufactured by injecting a pin into a plated-through hole within a printed circuit board (PCB). As the cross section of the pin is slightly larger than the plated PCB hole, the mechanical contact force generates frictional heating which leads to a low ohmic resistance connection between the metal surfaces. For measurement of friction between pin and hole see [1]. The elastic deformation of the pin and the circuit board have to ensure that the mechanical contact keeps long term stability under multiple physical loads such as vibration, thermal and mechanical shock as well as electro-chemical load.

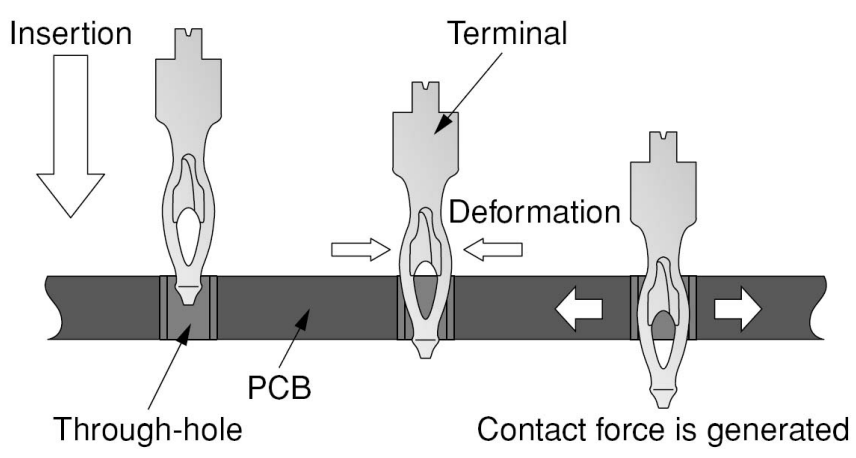

Figure 1. Principle of press fit connection [after 2]

Press fit contacts show several advantages compared to soldered contacts. As the press-fit process is conducted at room temperature, no components are subjected to thermal stress. The thermal resistance of a press fit connection is excellent and there is no danger that welding flux residues or solder bridges reduce reliability. Another advantage is the possibility to combine mechanical fixing and electrical contact without additional components like screws. Although not intended, press fit contacts mostly offer repairability. When the pin is pressed into the hole, excess material might be pressed either into the plating or into the pin which can reduce reliability of the contact. Figure 2 shows a connection where a metal sliver was pressed through the plating and into the FR-4 glass laminate.

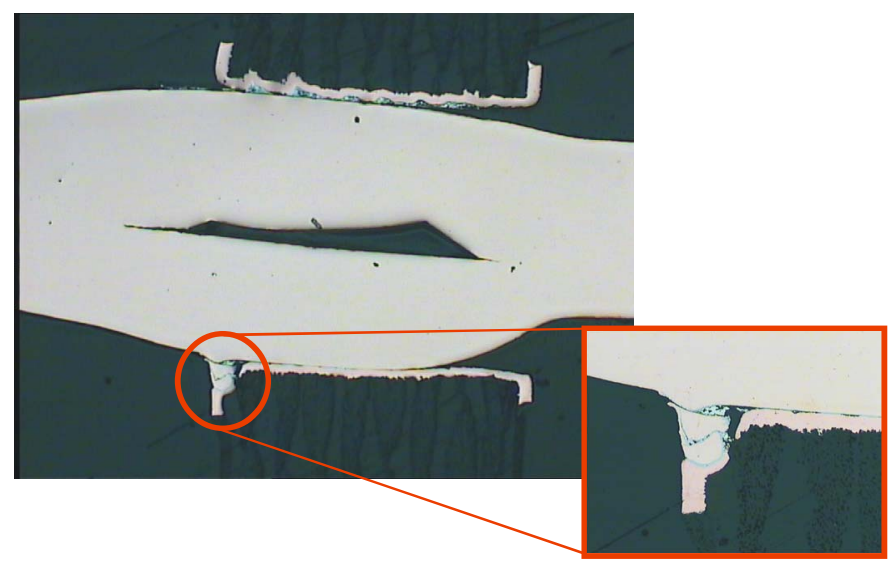

Figure 2. Press fit contact with metal sliver inside PCB 


\section{MECHANICAL LOAD ON SMD COMPONENTS}

\section{A. Press-in Forces}

A low ohmic and practically gas tight connection between the through-hole plating of the PCB and the contact pin can only be generated if both contact partners show a plastic deformation to a certain amount within the contact area ("shoulder"). During the press-in process, significant strains can be generated within the PCB near the pins. Assembly of SMD components and reflow soldering is done before press fitting in order to avoid thermal loads on the press fit contact and surrounding mechanic components. Consequently, mechanical stresses generated during press-in also affect all components and electrical circuits nearby.

The amount of stress mainly depends on the type of pin used. The press fit pin can be designed either in a rigid (solid pin) or flexible layout (compliant pin). While the early generations of press fit contacts mostly had been rigid pins, most engineers have switched to flexible pins in order to shift the deformation from the plastic, crack sensitive PCB into the elastic press-fit pin. Nowadays there exists a large variety of contact geometries, also with soldered pins.

The flexible design has several advantages, especially regarding its long time reliability:

- As the pin is applying a constant contact force on the plating, the electrical connection is kept also at elevated temperatures when the PCB shows creep behavior and the elastic forces might be reduced by plastic material rearrangement near the press-fit zone or in the pin.

- Another important advantage of flexible pins is their tolerance towards different through-hole diameters within the PCB. Especially the press-in force can be kept much more constant, even if the diameter of pin and hole show geometrical variations. The acceptability of larger tolerances simplifies production processes and reduces costs.

- Press-in forces of flexible pins are generally lower compared to rigid pins. This enables press-fitting of numerous contacts into the same PCB without a shattering impact of large areas of the board.

- Lower strains within the surrounding PCB allow reduction of distances between pins and between electrical layers near the press fit contacts without increasing the danger of microcracks.

- Smaller strain affected zones enables the usage of more PCB area by placement of components near the pins. If soldered parts are too close to the strain affected press fit zone, their reliability can be reduced. This is especially valid for SMD or CSP (chip scale package) components, because their mechanical coupling the PCB is much closer compared to components like DIP (dual inline package), QFP (quad flat package) or through hole mounted electronic parts [3].
The measurement of press-in force of a pin into the plated through-hole is a standard method in manufacturing for qualification of the press-fit process. The shape of the forceposition diagram during the press-in process mainly depends on the following physical parameters:

- Shape of pin (maximum diameter, increase of diameter...)

- Material of pin (core material, sometimes plating)

- Diameter and homogeneity of through hole

- Thickness of PCB and amount of laminate layers

- Material and thickness of plating

- Press-in speed and temperature

Figure 3 shows a typical force / path diagram of the pressfit process investigated where the pin has been inserted with constant speed. It is characterized by a steady increase of pressin force with increasing press-in depth. The slope does not show any spikes of the insertion force that could indicate a sticking of the pin during injection. The optimum slope further exhibits a plateau of the press-in force. Here, the pin is already completely compressed, but slides deeper into the throughhole. A small decrease of the force indicates the completion of plastic deformation within the materials, but should not be based on a beginning penetration of the pin through the hole.

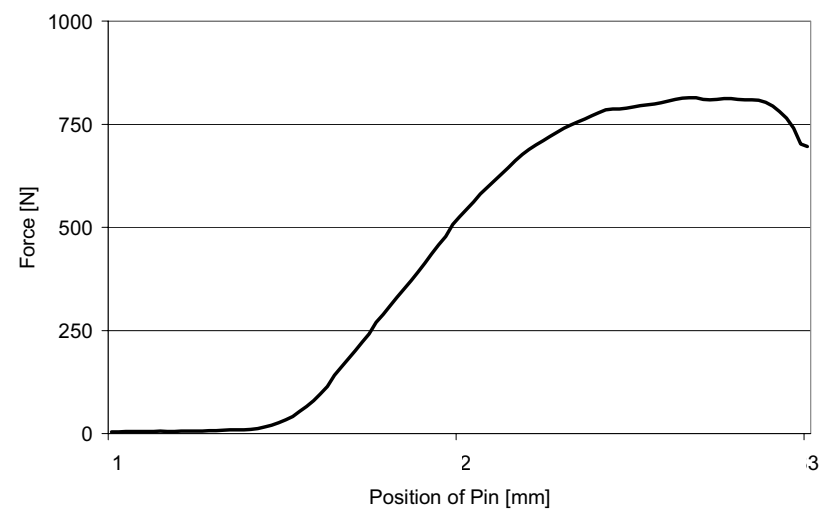

Fig. 3: Typical process monitoring of pressing-in. The force applied onto the pin is recorded depending on its position

A reliable press-in contact can be characterized by a stable and long lasting clamping force that the metal pin is exerting on the surrounding PCB plating. Therefore the elastic range of the pin should not be exceeded. A typical example of an over-pressed contact is displayed in Figure 4. The contact on the left side still has much resilience and can be characterized as acceptable; the contact on the right side is completely deformed. It can be expected that the deformation is highly plastic and has a reduced ability to keep up a constant pressure on the PCB. This contact should be rejected. A good press-in contact additionally appears with a close, low-ohmic connection between both plating surfaces, see Figure 5. Voids or contact less areas should also be avoided. 

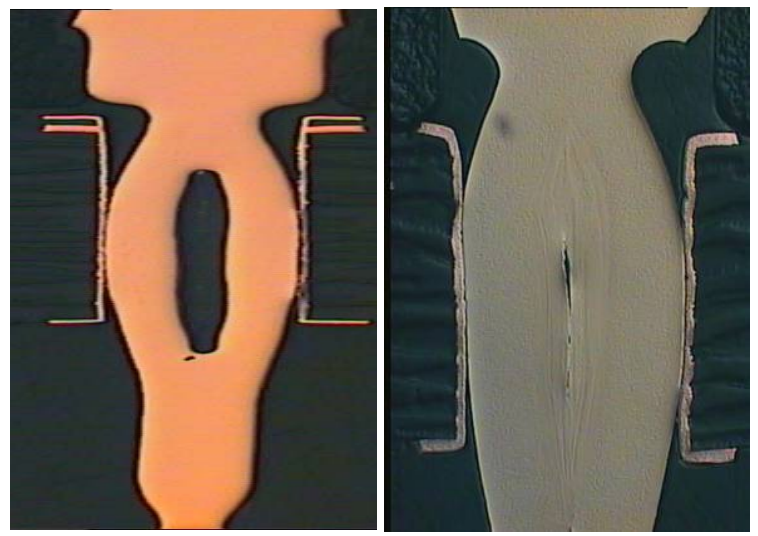

Fig. 4: Typical press-in contacts (left: good, right: bad)

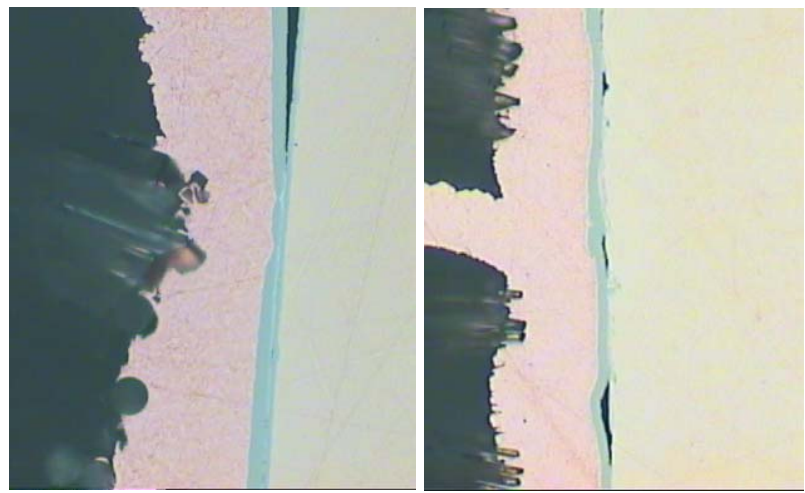

Fig. 5: Metallurgical interfaces between pin and plating

\section{B. Measurement principles for strain near press fit contacts}

The measurement of press-in forces is a generally accepted method for production controlling. For qualification of the process it can be helpful, to measure also the deformation in the printed circuit board according to a distinct press-in force. As a tight through-hole with thin plating can show a similar press-in force as a wide hole with thick plating, this method enables to distinguish force driven deformation of the pin (normally elastic) from deformation inside the PCB which is to a large amount plastic deformation.

A widespread method to measure deformations on the PCB surface is to glue electrical strain gauges (Fig. 6). They have the advantage of simplicity, but cover a certain area over which they sum up the resistance change. Therefore strain gradients or even alternating strain within the measurement area can not be depicted.

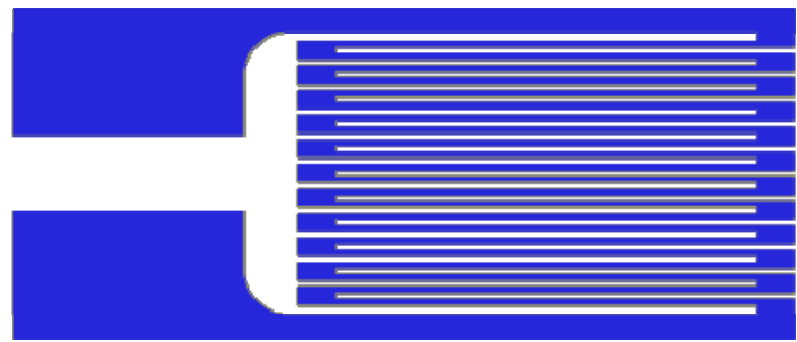

Fig. 6: Layout of electrical strain gauge
As printed circuit boards consist of a glass fiber laminate embedded into epoxy, the usage of a pure glass fiber sensor is regarded advantageous. Especially the mechanical matching between a glass fiber sensor and the glass fiber FR-4 laminate would be perfect, enabling a minimal invasive measurement system. Fiber Bragg Grating (FBG) sensors can be described as optical strain gauges within a glass fiber that can even be laminated into the PCB.

Unique advantages of FBG sensors can be summarized as following:

- The sensor can be embedded directly into the Printed Circuit Board without additional fixtures (Figure 4)

- The sensor's diameter of $125 \mu \mathrm{m}$ ensures minimal influence by the fiber sensor

- Multiplexing allows the readout of dozens of sensors within one fiber ("minimal invasive sensor network")

- Optical loss of $0,16 \mathrm{~dB} / \mathrm{km}$ enable signal propagation over long distances

- Optical sensors are insensitive towards external electromagnetic fields

The measurement unit at Fraunhofer IZM is build up by commercially available parts, schematically visualized in Fig. 7. It consists of a broadband light source (LED with $\lambda_{\max }=$ $1550 \mathrm{~nm}$ ) and several $3 \mathrm{~dB}$ couplers spreading out a sensor network with up to $\mathrm{i}=36$ gratings of period $\Lambda_{\mathrm{i}}$. The reflected light is analyzed by an optical spectrum analyzer, a CCD measurement unit or a pair of photodiodes behind complementary edge filters.

Light traveling through a uniform grating with grating spacing $\Lambda$ and mean ("effective") refractive indes $n_{\text {eff }}$ will be reflected according to the classical Bragg condition.

$$
\lambda_{\mathrm{B}}=2 \cdot \mathrm{n}_{\mathrm{eff}} \cdot \Lambda
$$

The gratings sensitivity against strain $\varepsilon=\Delta \mathrm{l} / \mathrm{l}$ and temperature changes $\Delta \mathrm{T}$ is given by the following formulas

$$
\begin{aligned}
\frac{\Delta \lambda}{\lambda_{B}} & =\Delta T\left(\alpha_{\Lambda}+\frac{1 d n_{\text {eff }}}{n_{\text {eff }} d T}\right) \\
& +\varepsilon\left(1-\frac{n_{\text {eff }}^{2}}{2}\left[p_{12}-v\left(p_{11}+p_{12}\right)\right]\right) \\
\approx & 11,8 \mathrm{pm} / \mathrm{K}+1,25 \mathrm{pm} / \mu \varepsilon
\end{aligned}
$$

We have $p_{i j}$ being the Pockel's coefficients of the strain optic tensor and $\alpha_{\Lambda}$ as the TCE of the silica glass fiber $\alpha_{\Lambda}=(1 / \Lambda) \quad(\partial \Lambda / \partial \mathrm{T})=0,55 \mathrm{ppm} / \mathrm{K}$ with the thermo-optic coefficients $\alpha_{\mathrm{n}}=\left(1 / \mathrm{n}_{\text {eff }}\right)\left(\partial \mathrm{n}_{\text {eff }} / \partial \mathrm{T}\right)=8,6 \mathrm{ppm} / \mathrm{K}$. All numerical data are valid for a central wavelength of $\lambda=1550 \mathrm{~nm}$. Maximum measurement rates used are $100 \mathrm{~Hz}$, but can go up to several $\mathrm{kHz}$. Resolution of the fiber optic sensor for mechanical strain is around $1 \mu \mathrm{m} / \mathrm{m}$ and for temperature around $0,1 \mathrm{~K}$. 


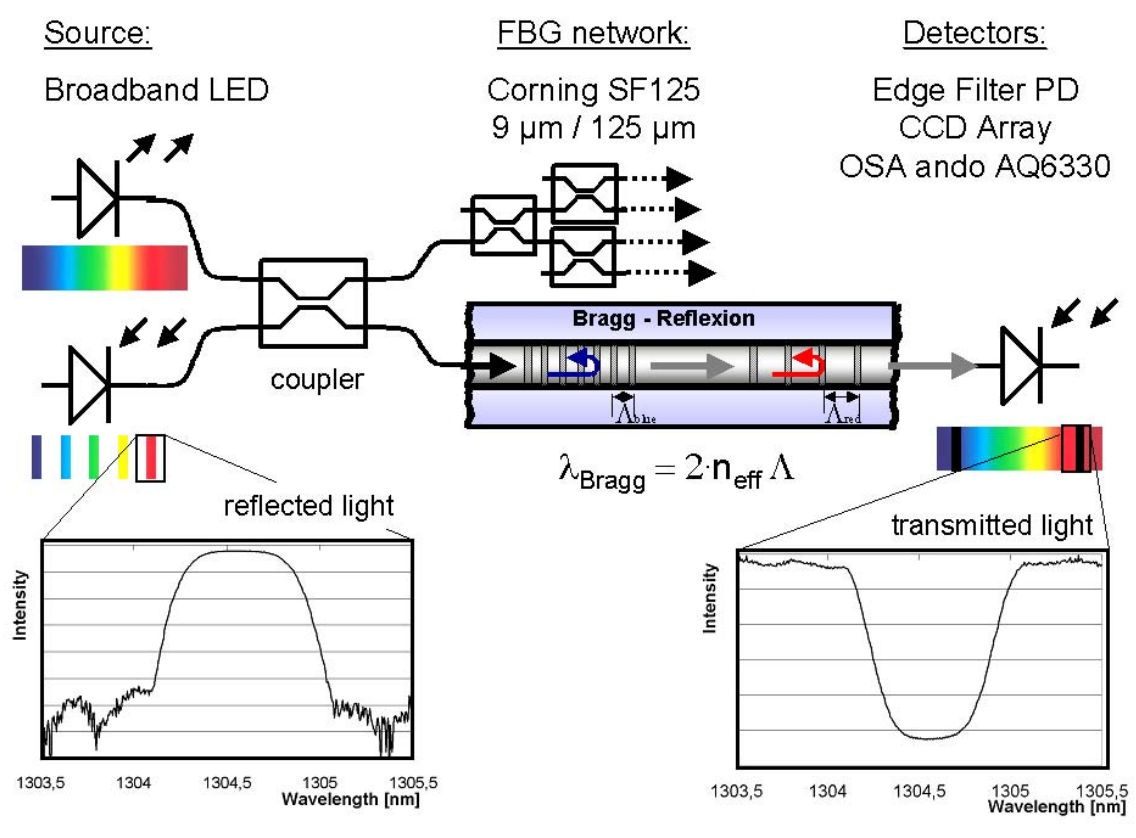

Fig. 7: Measurement setup

An important advantage of Bragg Gratings regarding press-fit characterization is their ability to detect strain gradients along the sensor's dimension. As each index change of the grating is the origin of a wavelet of distinct wavelength, the reflection spectrum is composed of the reflections at every position of the grating. If the grating's period $\Lambda$ varies along the sensitive length, the Bragg Wavelength $\lambda_{\mathrm{B}}$ also varies. This will broaden the shape of the reflected wavelength, the so called Full Width Half Maximum (FWHM). The low wavelength part of the FWHM is correlated with the compressed parts of the grating and the long wavelength part depends on the strained parts of the grating. If the strain along the sensor is alternating from compression to elongation, the mean reflected wavelength will keep constant, but the FWHM will increase. This characteristic of FBG sensors could be used advantageous in the characterization of press-fit processes.

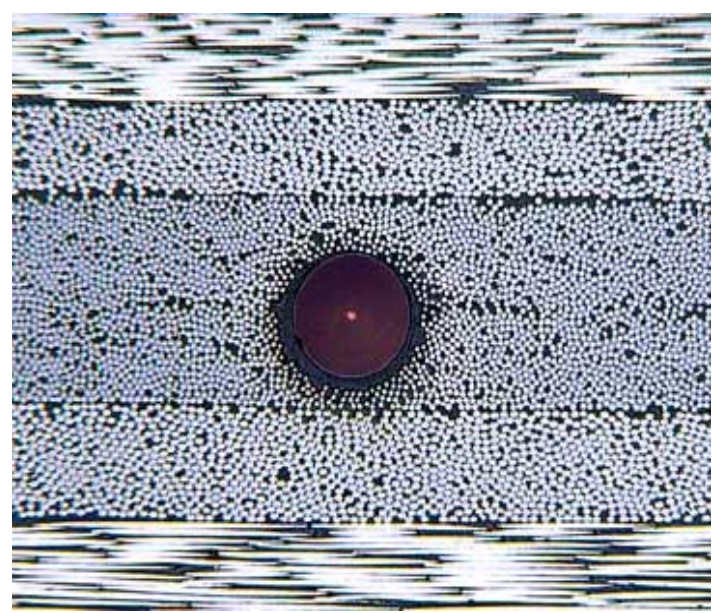

Fig. 8: FBG fiber sensor embedded into PCB [5]

\section{Simulation of strains on PCB surface near press fit contacts}

The press-fitting process to be monitored consists of the simultaneous press-in of six adjoining pins. Each of the pins is pressed into the plated through-hole with a maximum force of $100 \mathrm{~N}$. The detailed area around the through holes has been sketched in Figure 9.
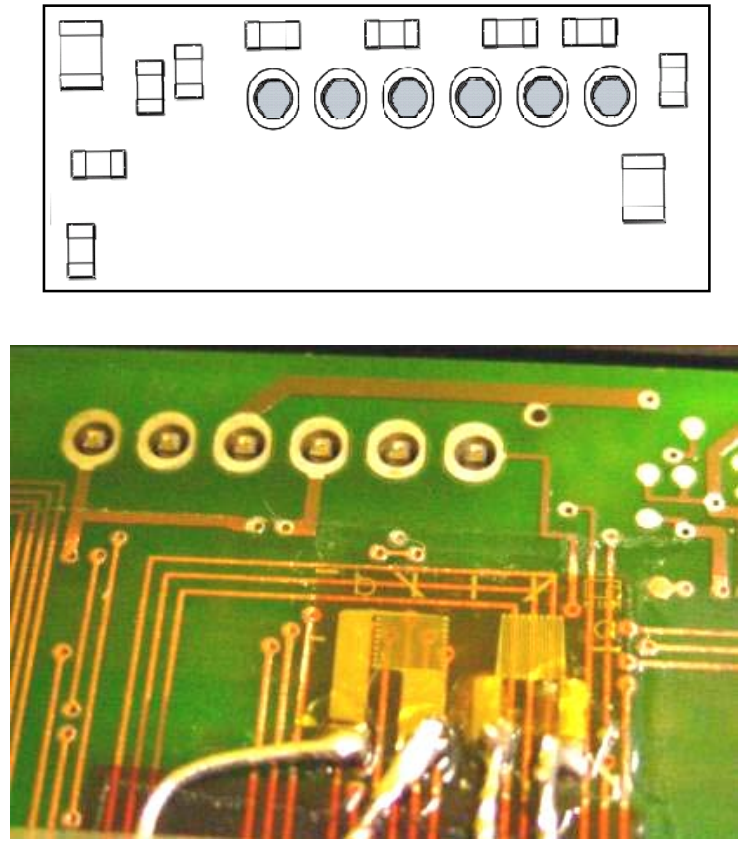

Fig. 9: Press-fit region on PCB (top: CAD of top side with SMD components, bottom: photography of bottom side with electrical strain gauges) 
The strain after press-fitting within the PCB has also been analyzed by FEA simulations. The thickness of the PCB is $1.6 \mathrm{~mm}$, lateral dimensions are $45 \mathrm{~mm} \times 30 \mathrm{~mm}$. We used the following material parameters:

\section{PCB: $\quad 24 / 20.7 \mathrm{GPa}$ in warp / fill direction (x /y) $1.5 \mathrm{GPa}$ in $\mathrm{z}$-direction (perpendicular to FR-4 plane with low density of $\mathrm{Cu}$ wiring) \\ Pins: $\quad 110 \mathrm{GPa}$ (Copper) \\ Electronics: $300 \mathrm{GPa}$ (Ceramic)}

The deformation of the through-hole plating was modelled by correlation of FEA material parameters with metallographic analysis. The strain capability of lead-free solder joints is rarely provided by the manufacturers and has to be determined by a 4-point bending test. In detailed simulations within other projects we could show that strains on the PCB surface $<400 \mu \mathrm{m} / \mathrm{m}$ could already be critical. The damage of the soldered contacts at these regions might not be visible, but already reduces the creep resistance of leadless solder. These different regions near the press fit location where no electronic part should be soldered is coloured and encircled within the following figure:

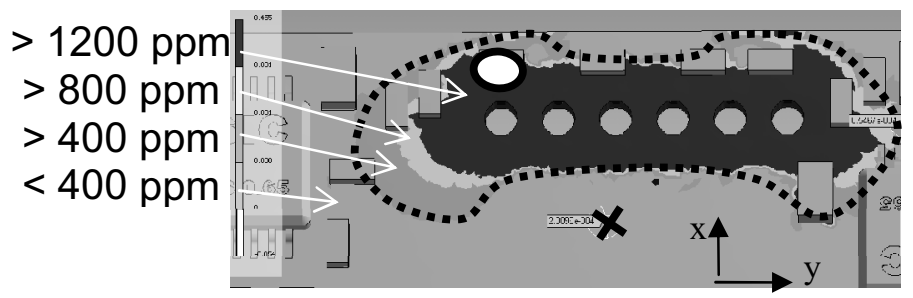

Fig. 10: FEA simulation of normed strain $\varepsilon$ on top side of PCB near press-fit region (black: strain $>1200 \mu \mathrm{m} / \mathrm{m}$, light grey: $800 \mu \mathrm{m} / \mathrm{m}<\varepsilon<1200 \mu \mathrm{m} / \mathrm{m}$, grey within black line: $400 \mu \mathrm{m} / \mathrm{m}$

$<\varepsilon<800 \mu \mathrm{m} / \mathrm{m}$, grey outside black line: $\varepsilon<400 \mu \mathrm{m} / \mathrm{m}$ )

In a distance of $3 \mathrm{~mm}$ away from the contact pins the surface strain on the $\mathrm{PCB}$ is around $0.1 \%$, in a distance of $10 \mathrm{~mm}$ at least $500 \mu \mathrm{m} / \mathrm{m}$. The shape of the highly strained PCB region not only depends on the factors summed in chapter II $\mathrm{A}$, but also on the pin orientation within the whole, the kind of mechanical fixation and tolerances inside the PCB that could affect the hole-to-hole distance.

\section{Measurements of strain affected zone near press fit contacts}

In order to monitor surface strains on PCBs near the pressin region glass fibers with Bragg Gratings have been mounted at the positions of the SMD components that should be soldered near the press-fit contacts. Positions where fiber optic gratings have been positioned are marked by a circle (position near pins) and a cross (position distant from pins) within Figure 10; the electrical strain gauges were glued only at the crossed position on both sides of the PCB.

The electrical strain gauge recorded strains on both sides of the board: elongation strains on the bottom side and compressive strains on the top side. The symmetrical behavior with different signs derives from a bending of the PCB during injection. The non symmetrical fraction of the strain derives from an elongation on both sides of the PCB. The strains after press fitting show a high variation, but are according to the FEA simulations. The strain along the press fit row (ydirection) is slightly higher than perpendicular to it ( $\mathrm{x}-$ direction).

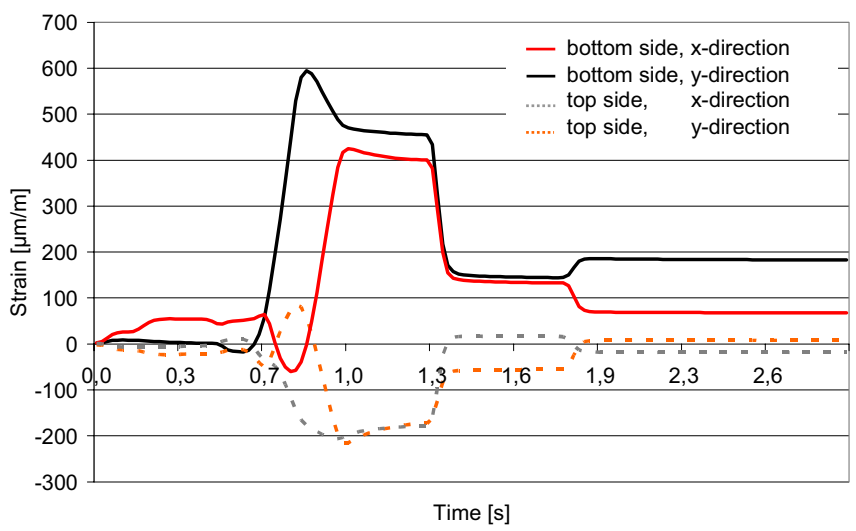

Fig. 11: Strain measured by electrical strain gauges

The first optical strain gauge recorded strains on the bottom side of the board along the x-direction: The strains differ from the electrical strain gauge, but show a good agreement with the electrical strain values. The maximum strains of $500 \mu \mathrm{m} / \mathrm{m}$ as well as the remaining strain after pressin of $100 \mu \mathrm{m} / \mathrm{m}$ are comparable to the electrical values of $400 \mu \mathrm{m} / \mathrm{m}$ and $80 \mu \mathrm{m} / \mathrm{m}$.

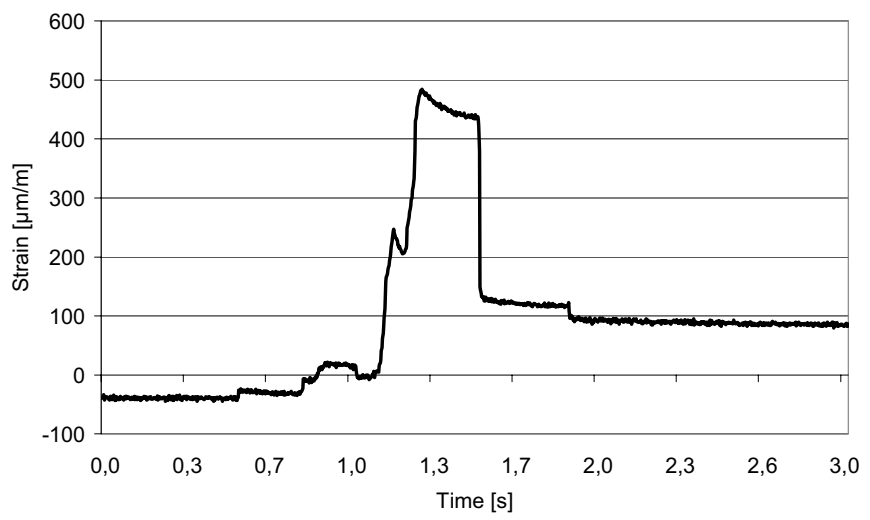

Fig. 12: Strain measured by optical strain gauges (bottom side, $\mathrm{x}$-direction)

If both sensors types are mounted near the press fit pins (marked with a circle in Fig. 10), the difference between electrical and optical strain gauge increases. Still the shapes of the strain signal remain comparable, but the maximum strain value of the FBG sensor (Fig. 14) is about three times higher than the electrical strain value (Fig. 13). 


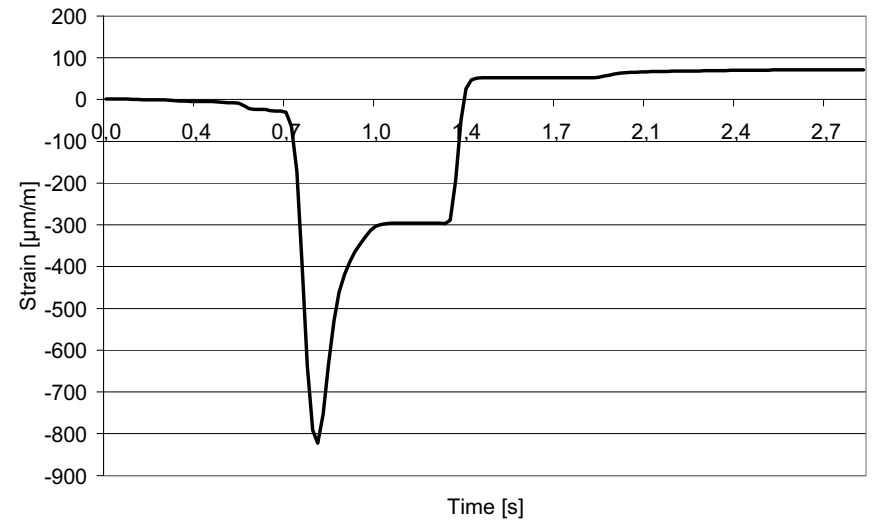

Fig. 13: Strain measured by electrical strain gauge on top side of the PCB (y-direction)

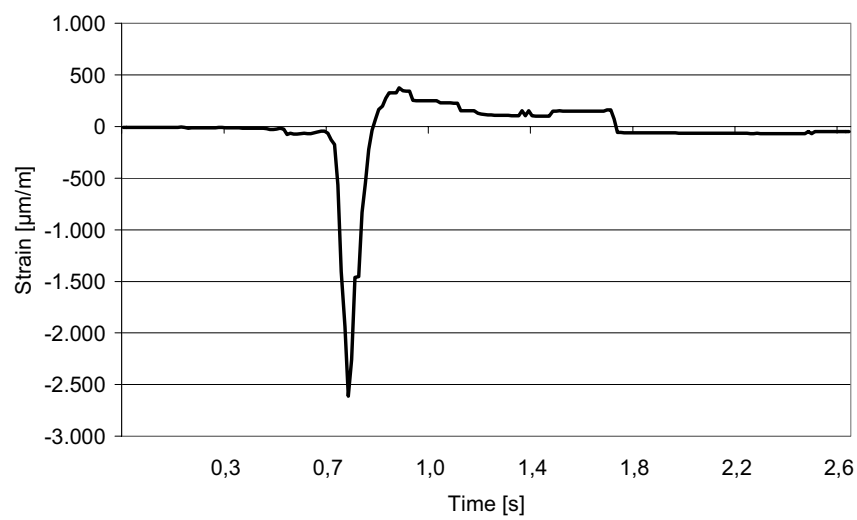

Fig. 14: Strain measured by optical strain gauge on top side of the PCB (y-direction)

It must be stressed that the electrical strain gauge should not be fixed near regions with strong strain gradients, because the measurement signal of a strain gauge is summing up all local electrical resistance values along the circuit path. Especially if compressive and tensile strains alternate, the integrated resistance change will be much lower than the extreme strain values below the sensor. The strain distribution near press-fit contacts is clearly visible in the next Figure 15. Tensile strained regions, indicated by red color, alternate with compressed regions that are highlighted in blue. An electrical strain gauge integrates over these strain values which explains the small strain value within Figure 13.

An optical grating within the glass fibre also covers a certain length with changing strain values. But in contrast to electrical gauges, FGB sensors show a significant broadening of their reflection spectrum in the presence of strain gradients: Each section of the grating reflects the wavelength according to its local strain and a variety of wavelengths is reflected along the whole grating. The minimum and maximum strain values along the grating can be derived by analyzing the FHWM values of the reflected spectrum. Consequently, Bragg gratings can be used advantageous in press-fit regions.

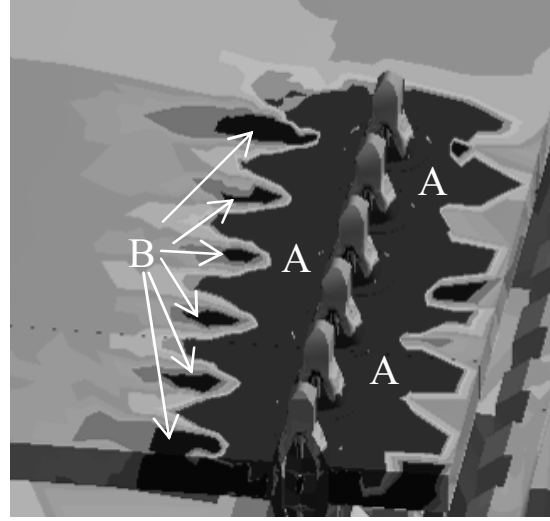

Fig. 15: FEA simulation of strain $\varepsilon$ on bottom side of PCB (black area A: $\varepsilon>+400 \mu \mathrm{m} / \mathrm{m}$, grey: $-400 \mu \mathrm{m} / \mathrm{m}$ $<\varepsilon<+400 \mu \mathrm{m} / \mathrm{m}$, black spots C: $\varepsilon<-400 \mu \mathrm{m} / \mathrm{m}$ )

Figure 16 shows the reflected wavelength spectra of two gratings before and after press fitting. Both do not show significant mean strain values after pin insertion, as can be expected from Figure 14. But it can be seen clearly, that highly compressed and elongated regions exist along the grating. Wavelength shifts of $2 \mathrm{~nm}$ correspond to strain values around $2500 \mu \mathrm{m} / \mathrm{m}$, which are exactly the maximum values during injection. It can be concluded that the maximum strain during insertion is still present at distinct regions of the PCB.

A comparison of optical and electrical strain gauge with FEA simulations is summarized in table 1.
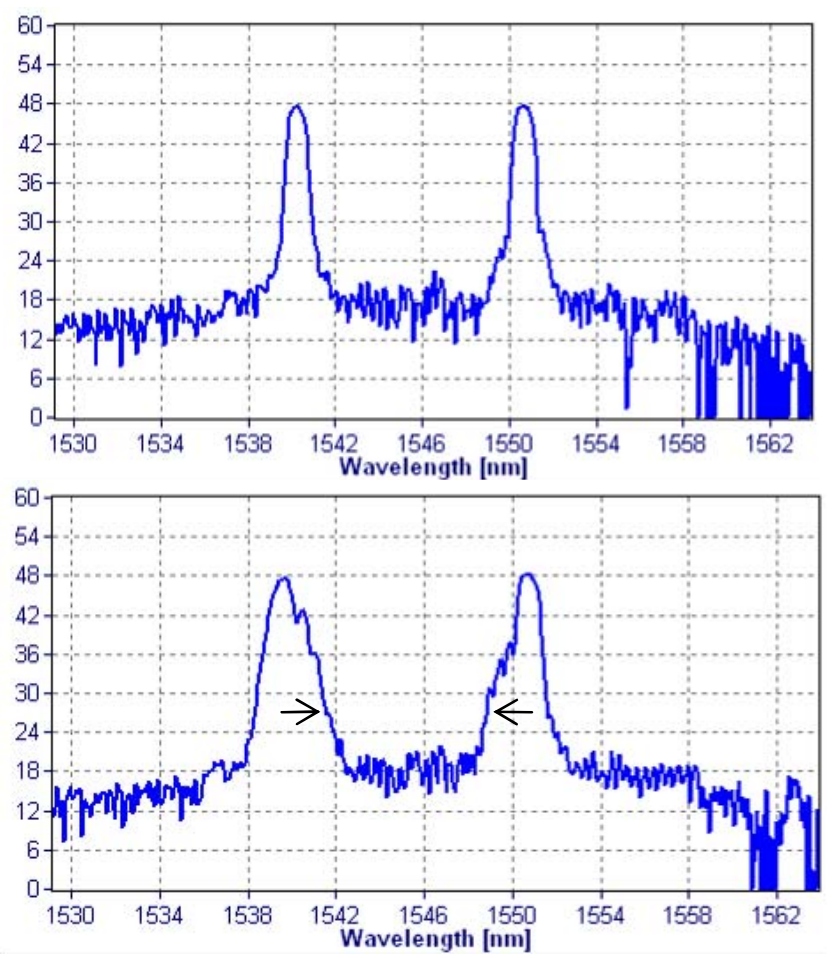

Fig. 16: Reflected wavelength spectra of two gratings mounted near the press fit positions. Top: before press-fitting; bottom: after press-fitting 


\begin{tabular}{|l|c|c|c|c|}
\hline \multicolumn{1}{|c|}{$\begin{array}{c}\text { all strain values } \\
\text { in } \mu \mathrm{m} / \mathrm{m}\end{array}$} & $\begin{array}{c}\text { Sensor } \\
\text { Maximum value }\end{array}$ & $\begin{array}{c}\text { Sensor } \\
\text { value after } \\
\text { press-fitting }\end{array}$ & $\begin{array}{c}\text { FEA } \\
\text { Maximum value }\end{array}$ & $\begin{array}{c}\text { FEA } \\
\text { value after press-fitting }\end{array}$ \\
\hline $\begin{array}{l}\text { Optical sensor (FBG) } \\
\left(\varepsilon_{\mathrm{x}}, \text { mounted distant from pins) }\right.\end{array}$ & $\begin{array}{c}+280 \\
\text { up to }+500\end{array}$ & $\begin{array}{c}100 \\
\text { up to } 200\end{array}$ & 600 & 100 \\
\hline $\begin{array}{l}\text { Electrical strain gauge } \\
\left(\varepsilon_{\mathrm{x}}, \text { mounted distant from pins) }\right.\end{array}$ & $\begin{array}{c}+320 \\
\text { up to }+430\end{array}$ & $\begin{array}{c}0 \\
\text { up to } 80\end{array}$ & 350 & -750 \\
\hline Optical sensor (FBG) \\
$\left(\varepsilon_{\mathrm{y}}\right.$, mounted near pins)
\end{tabular}

\section{QualificATION OF PRESS Fit CONTACTS}

The quality requirements of press fit contacts are described within the standard "Solderless connections Part 5: Press-in connections - General requirements, test methods and practical guidance (IEC 60352-5:2008)". The standard qualification tests of groups $\mathrm{A}, \mathrm{B}$ and $\mathrm{C}$ have been performed on the individual press-in connections, independently of the application. The thickness of the FR-4 boards was $1.6 \mathrm{~mm}$ with a copper plating of $35 \mu \mathrm{m}$. The PCB geometry differs from the boards tested with the strain sensors to test a higher number of electrical contacts: each test board contains 360 press fit contacts, each through-hole with a diameter of $1.3 \mathrm{~mm}$. We investigated motor and MQS contacts as well as Action Pin contacts.

\section{A. Qualification tests Group A: Inspection of the microsection}

The microsections have been made with two different plating types of the though-holes: chemical tin coating and hot air leveling (HAL); both used within mass production. Special attention was directed to display the contact zone of the pin. Figure 17 shows the cross section of a $25 \mathrm{~mm}$ Action $\mathrm{Pin}^{\mathrm{TM}}$. The plated via shows minimal deformation according to the specification: Deformation "a" of the drilled hole contour is smaller than $70 \mu \mathrm{m}$, the remaining thickness "b" of the plating is more than $8 \mu \mathrm{m}$.

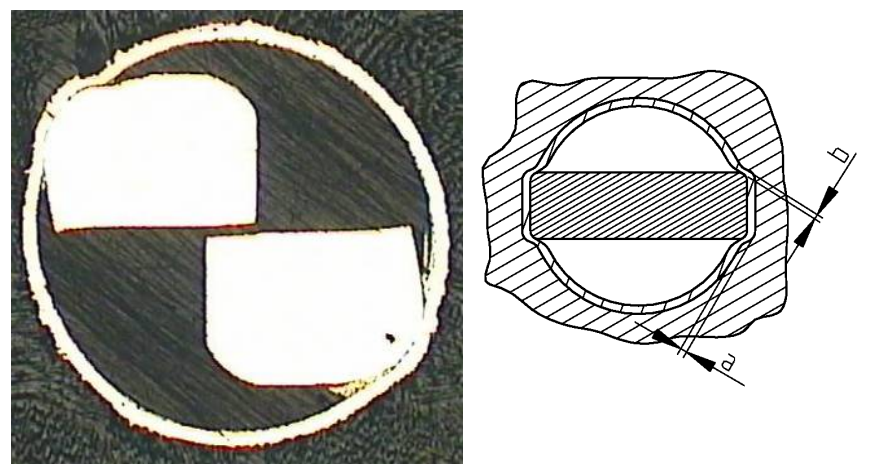

Fig. 17: Microsection and qualification of Action Pins
The contact still shows space for elastic deformation. Another "Group A" test demands a qualification of the pressin tooling. A typical failure mode is a geometrical misfit between the PCB and the tool, which gets especially critical, if press-in pins have to be manufactured at distant locations on the PCB board at the same time. Deformation of the pin and consequently of the PCB can be the result.

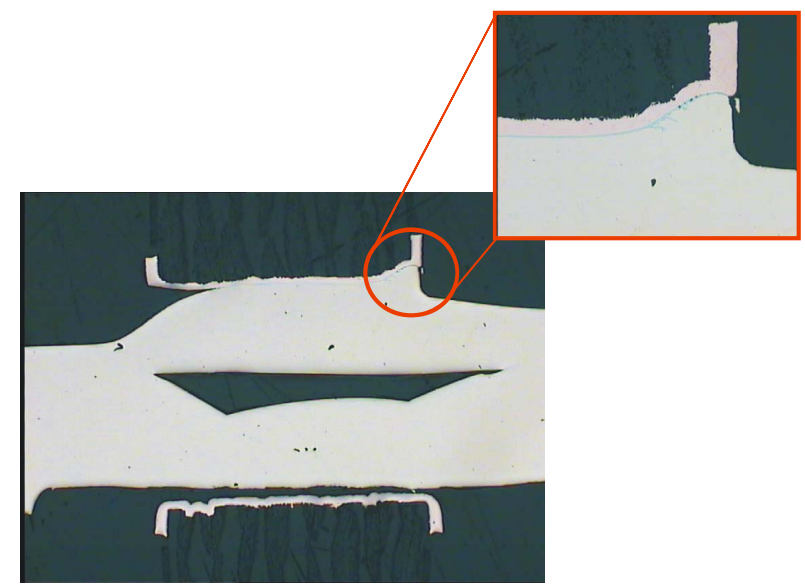

Fig. 18: Failure mode due to geometrical tolerance.

\section{B. Qualification tests Group B: Press-in and Push-out Forces}

Push-out forces have been measured according to IEC 352-5 / 3.2.2.3. This test shall show the withstand of the connection to mechanical stress caused by an axial force to the pin in the printed board holes. It can also be used as a simple quality indicator concerning the mechanical strength of the press fit connection. The test is performed with a fixed printed board. Then the pin is pressed against press-in direction with constant speed of $12 \mathrm{~mm} / \mathrm{min}$ until the pin moves in the plated-through hole. This force is recorded. Monitoring of press-in forces and accompanying strains within the PCB has already been described in section II. 


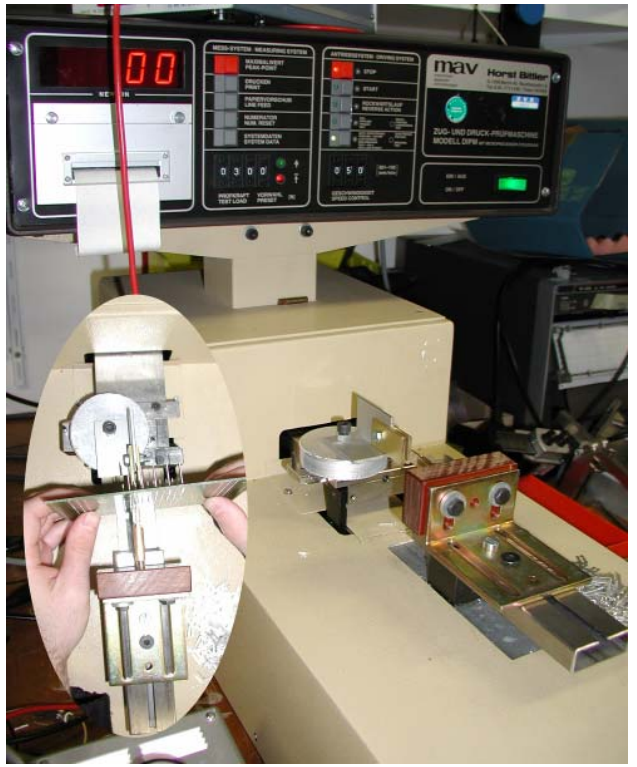

Fig. 19: Test environment for measurement of push-out forces

We could show a significant correlation between the diameter of the plated through-hole and the push-out force. A reduction of the hole diameter from $0.1 \mathrm{~mm}$ (HAL plating) to $0.05 \mathrm{~mm}$ (chem. Sn) increased the push-out forces significantly. The shape of the Press-fit pin also influences these forces. Flat contact pins are relatively insensitive against variations of the plated hole diameter; the push-out force increases only from $110 \mathrm{~N}$ to $130 \mathrm{~N}$ if the diameter is halved. Other contact geometries (e.g. Action Pin ${ }^{\mathrm{TM}}$, see Figure 18) show a much higher increase of push-out forces from $135 \mathrm{~N}$ to $335 \mathrm{~N}$.

\section{Qualification tests Group C: Electrical performance under durability test}

For qualification a test environment was built up in order to check the following quality indicators:

- Electrical tests: Contact resistance after press-in, voltage drop between reliability tests

- Durability during vibration

- Thermal shock and moisture / temperature endurance test

- Electrical load \& Temperature

The electrical tests of the contact resistance have been performed by 4-point-resistance measurements according to IEC 352-5 / 3.2.3.1. The contact resistance is the main characteristic of a connection to document the quality and durability. We tested a significant amount of contacts by a daisy chain layout. All pins had a contact resistance after press-fitting of $\leqslant 1 \mathrm{mOhm}$. The electrical test environment can be seen in Figure 20.
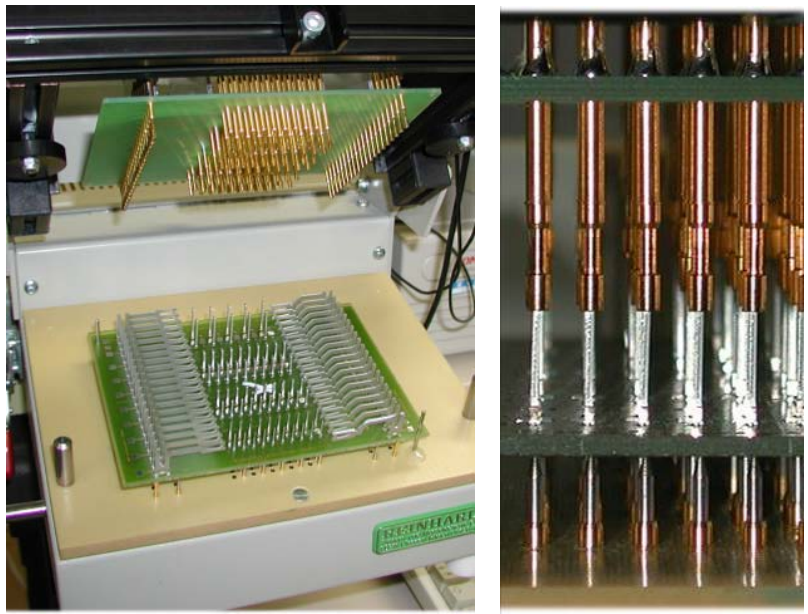

Fig. 20: Test environment for measurement of contact resistance

Thermal shock tests have been performed according to IEC 512-6 / 11d. Rapid temperature cycling at prevailing humidity was simulated by a two chamber temperature shock system from Weiss Inc., Germany. After storage at $-40^{\circ} \mathrm{C}$ for $30 \mathrm{~min}$ the contacts were transferred to the $+85^{\circ} \mathrm{C}$ hot chamber and stored for additional $30 \mathrm{~min}$. After 500 cycles the contact resistance was measured at 2 and 4 amperes.

Moisture/Temperature endurance has been measured according to PSA B $217050 / 11.3$. We performed 30 cycles with a constant current of 1,8 A (no current between change from $+55^{\circ} \mathrm{C}$ to $-40^{\circ} \mathrm{C}$ ) inside a climatic chamber. The temperature characteristic is displayed in the following figure. The moisture tests were followed by a dry heat endurance test $\left(1000 \mathrm{~h}\right.$ at $\left.85^{\circ} \mathrm{C}\right)$.

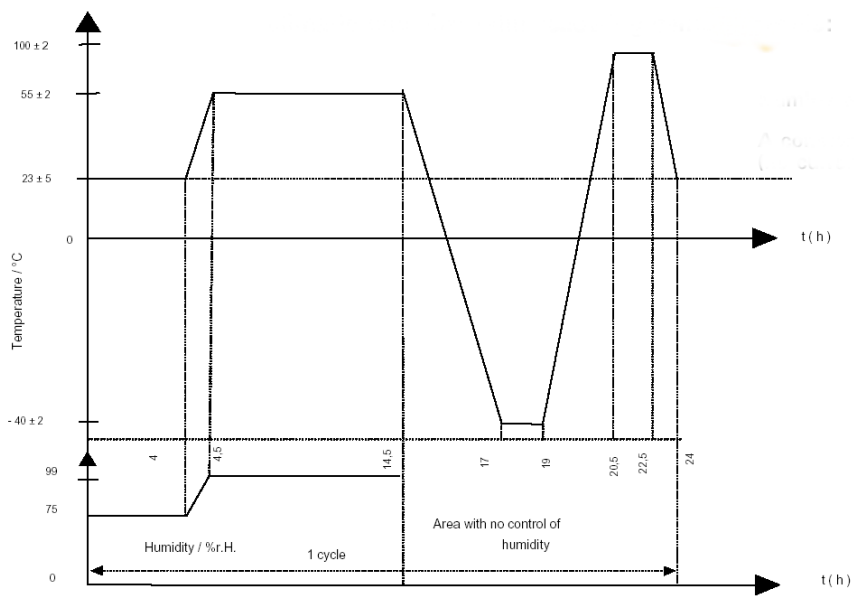

Fig. 21: Temperature characteristic during moisture test. 


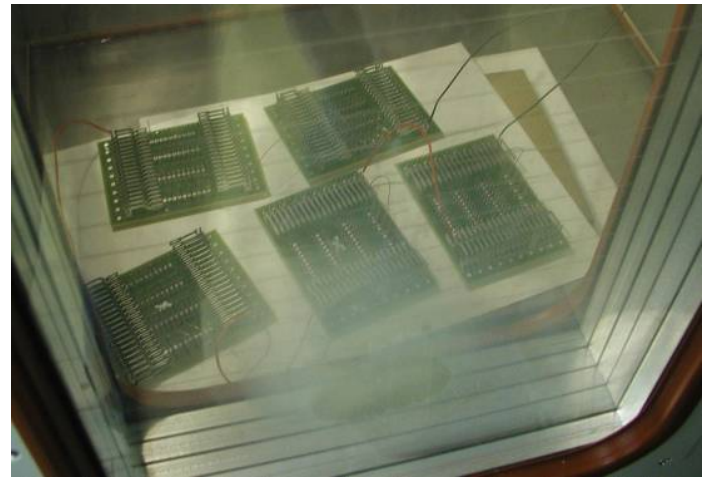

Fig. 22: PCB specimen during moisture test.

Vibration test have been performed according to PSA B $217050 / 11.4$. The purpose of this test is a simulation of sinus vibration occurring at a car. The test samples are preconditioned at dry heat of $85^{\circ} \mathrm{C}$ for 48 hours. For each axis the vibration test is combined with temperature cycles between $-30^{\circ} \mathrm{C}$ and $+85^{\circ} \mathrm{C}$. The test has been performed currentless as well as with a current of $100 \mathrm{~mA}$ set to the samples during the vibration test. At the end of vibration in each axis a sinus sweep given to the samples to check disconnection. The test equipment is shown in the following figure.

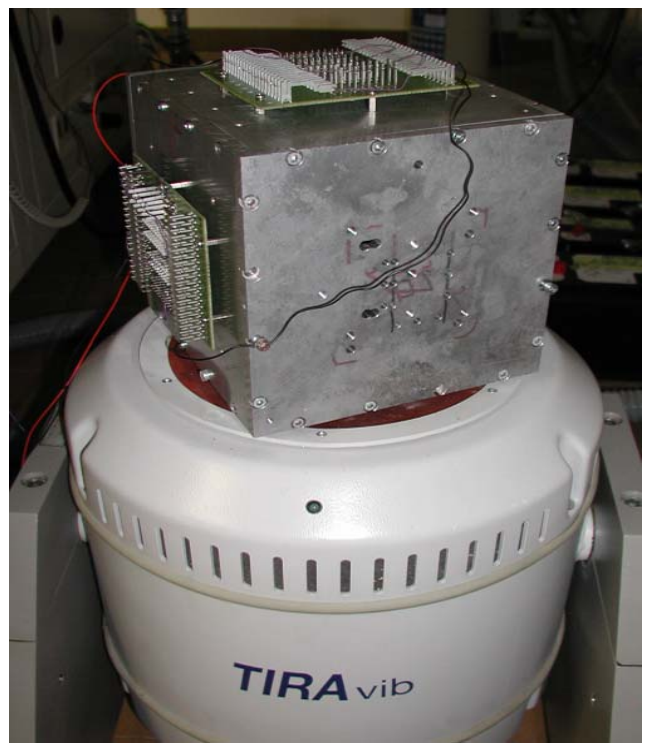

Figure 23: Test environment for vibration test

Electrical Load \& Temperature has been tested according to IEC 512-5/9b. The purpose of the test is to stress the connectors at a surrounding temperature of $60^{\circ} \mathrm{C}$ and to raise the current steadily until $85^{\circ} \mathrm{C}$ is reached within the contact. This temperature was achieved at $4.5 \mathrm{~A}$. Then the current is hold constant at $1.8 \mathrm{~A}$ over 500 hours. Afterwards additional current pulses up to $28 \mathrm{~A}$ and a duration of $260 \mathrm{~ms}$ have been applied to the contacts.

All tests were passed without any electrical failure. No increase of resistance could be measured after all tests. No damage of any kind could be detected (cracks or degradation of contact area by metallurgical phase separation or oxidation due to overload). The advantage of a chemical tin plating are tight tolerances and thin plating thicknesses that can be achieved. This is especially advantageous if small SMD components have to be soldered. HAL platings have the advantage of reduced press-in forces, if the diameter of the through-hole is tolerated correctly (easy sliding of pin because of higher plating thicknesses).

\section{CONCLUSIONS}

Fiber optic Bragg gratings have been proven to be appropriate sensors for strain measurement within the printed circuit board during press-fit processes. As the press-in forces depend on a large amount of influencing factors, the mechanical reaction of the $\mathrm{PCB}$ on the press-in process can enhance correct characterization of this manufacturing process. By comparison with electrical strain gauges the FBG measurement technique could be validated. Differences in strain values from both sensors have been discussed with the conclusion that electrical stain gauges are integrating over alternating strains and display a reduced maximum strain value. This is especially critical, if compressive and tensile strains are situated near each other. The methods and insights developed within this paper can be the basis for an enhanced reliability characterization of press-in processes regarding strains within SMD components nearby.

The qualification tests of the press-fit contacts indicate that the technology enables a reliable solderless electrical connection. Environmental stress tests like climatic sequences, temperature or vibration load do not increase the electrical resistance of the press fit connection. No differences in reliability between chemical tin plating and HAL platings have been found. Nevertheless, galvanic platings have a higher hardness and normally a smaller layer thickness than HAL platings and therefore show a bigger risk for cracks. When looking to the different press-in zones, the highest deformations can be found on the Action Pin ${ }^{\mathrm{TM}}$. These results are especially important for reliable operation within harsh environments.

\section{REFERENCES}

[1] N. Corman, M. Myers, C. Copper, "Friction Behavior of Press-Fit Applications: Test Apparatus and Methodology", Proceedings of the Forty-Ninth IEEE Holm Conference on Electrical Contacts, 2003. Page(s):38 - 44, 8-10 Sept. 2003.

[2] Y. Nomura, Y. Saitoh, K. Furukawa, Y. Minami, K. Horiuchi, Y. Hattori, "Development of Press-fit Connector for Automobile Applications", SEI Technical Review, vol. 63, pp. 33-39, December 2006.

[3] M. Roellig, R. Dudek, S. Wiese, B. Michel, „Fatigue analysis of miniaturized lead-free solder contacts based on a novel test concept", Microelectronics Reliability 47, pp. 187-195, 2007

[4] A. Othonos, K. Kalli "Fiber Bragg Gratings: "Fundamentals and Applications in Telecommunications and Sensing", Artech House (Boston, 1999)

[5] D. Betz, L. Staudigel, M. N. Trutzel, M. Kehlenbach: „Structural monitoring using fiber-optic Bragg grating sensors", Preceedings of the "Structural Health Monitoring Conference 2002", Cachan, France, 10-12 July, 2002 


\title{
Estimation of Remaining Lifetime of Power Connections using Infrared Thermography
}

\author{
M. Braunovic \\ MB Interface, 5975 Place de l'Authion, Suite 503, Montréal. QC, Canada, H1M 2W3 \\ E-mail: mbinterface@yahoo.com \\ V.V. Izmailov, A. E. Naumov and M.V. Novoselova \\ Tver State University of Technology, 22 A.Nikitin Emb., Tver, 170026, Russia
}

\begin{abstract}
One of the measures used to prevent the occurrence of power connection failures is to estimate the anomalous behavior of an electrical connection in the early stages of contact operation when the first signs of critical stage become apparent. This paper investigates the possibility of applying the statistical prognostic methods for determining the residual life of power connections.
\end{abstract}

\section{INTRODUCTION}

It is now well established that approximately one quarter of failures of power equipment is related to electrical contacts. Such accidents often results in fire and as a result serious consequences may occur. Hence, it is clear that electrical contacts are essential components of equipment whose reliability has to be ensured at high levels.

In spite of seeming simplicity of contact joint design, the reliability of electrical contact is determined by complicate physical processes occurring at contact spots (a-spots) under the influence of electrical current, temperature and other environmental factors.

There are two approaches to estimate the reliability of crucial equipment, including electrical contacts. The first one is based on the integrated average statistical data analysis of general population, such as reliability function, mean operating time between failures, gammapercentile operating time to failure, etc.

This approach is acceptable at the equipment designing stage, while the average lifetime for a large number of equipment (general population) has to be estimated. This approach is frequently used to schedule the preventive inspection and repair works. However, this approach is disadvantageous since it does not take into account different operating times of different contact joints under current load, different level of current loads and consequently degradation stages of different joints.

On the other hand, if the residual lifetime of a contact joint is predicted based on its actual state at the particular time instance, then its lifetime can be estimated exactly for the given joint in the given specific conditions.
The conventional methods of evaluating the states of power connections, including measurements of the contact resistance of a joint during the OFF periods and other measurement procedures, are not always as effective as expected. The problem of residual lifetime forecasting for the electrical connections may be solved if there is a capability to monitor continually the evolution of the key (resource) parameters until reaching their critical values considered as the failure criteria. However, continuous monitoring of the key parameters determining the power connection performance under operating conditions is not always possible.

The most effective approach from the economical and also practical viewpoints would be periodic monitoring of key parameters determining the quality of an electrical connection whose changes with the time can be used to forecast the occurrence of the critical stages of connection performance.

The above forecasting may be completed based on the methods of the statistical analysis of the time series considered as a powerful prognostic tool [1]. Statistical methods describe phenomena covering the incidental factors that cannot be described in purely deterministic terms. Typical examples of this type of prognostic models are time series used for economical prognosis, marketing, industry, financing, control.

The aim of this work is to examine the possibility of using the statistical prognostic methods to predict the residual lifetime of power connections.

\section{SELECTION OF KEY PARAMETERS}

Electrical contacts undergo to ageing failure. To predict the residual lifetime of a given contact joint, it is necessary to select the key parameter which determines the contact joint reliability. Exceeding the allowed limits of the key parameter is considered as a contact joint failure.

In consideration of electrical contact functions and the physical nature of degradation processes, the 
behavior of key parameters may be followed by measuring the contact resistance or contact temperature. For instance, Russian standards require that electrical resistance and temperature of the connection joint be monitored. This approach has advantages and disadvantages.

Electrical resistance seems to be more appropriate characteristic of contact joint than contact temperature, because it is directly defined by the contact conditions. On the other hand, contact resistance cannot be measured remotely. Therefore, the contact resistance measurements under tension are unsafe, especially under high voltage conditions. Since contact resistance is small as a rule, its measurement requires sufficiently precise measuring equipment.

Actually, temperature of the contact parts is indirect characteristic of the contact state, since it is an indication of the state of the contact outer surface, instead of the contact spots. However it is evident that the temperature of the contact outer surface is closely related to contact spots temperature. Hence, the temperature of the contact outer surface may be used as the key parameter since it permits noncontact measurements. The temperature may be measured using infrared thermography techniques. These techniques are absolutely safe since they do not involve any physical contact of the operator with the parts being under tension.

The forecasting of the residual lifetime of electrical contacts based on the electrical resistance as a key parameter is described in [2]. In the present paper, the use of temperature for estimating the residual lifetime of power connections is described.

\section{EXPERIMENTAL SETUP AND METHODS}

For the purpose of this study, a total of 3500 different types of joints were examined. Figure 1 show an example of such joints operating at $380 \mathrm{~V}$ and nominal current ranging from 80 to $4000 \mathrm{~A}$.

The joints were assembled using a combination of one or two bolts and lock-washers. The tests were carried out using the equipment situated in the industrial zone. All connections were installed indoors whereby the environmental temperature varied in the range $10-25^{\circ} \mathrm{C}$ and the relative humidity was $60-80 \%$. Operating current throughout the experiment was maintained at the $350 \mathrm{~A}$.

Contact resistance or temperatures of a joint are used as the key parameters of the performance or failure of a connection. Both of these parameters have their advantages and disadvantages as described in section 2 of the present paper.

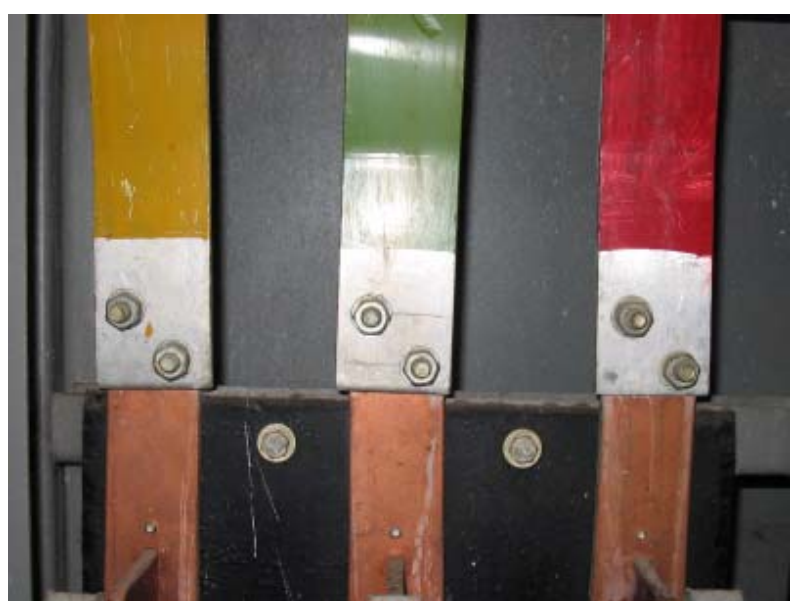

Fig. 1 Typical power connections used in this work

Clearly, periodic measurements of contact resistance and temperature of a connection during the actual operation are the manifestation of the ad hoc processes of $\mathrm{R}(\mathrm{t})$ and $\mathrm{T}(\mathrm{t})$ at a particular time instant. On Fig. 2 the examples of stochastic processes $\mathrm{R}(\mathrm{t})$ and $\Delta \mathrm{T}(\mathrm{t})$ are shown, where $\Delta \mathrm{T}(\mathrm{t})$ is the temperature of a hottest point of the outer surface of connection exceeding that of surrounding environment.
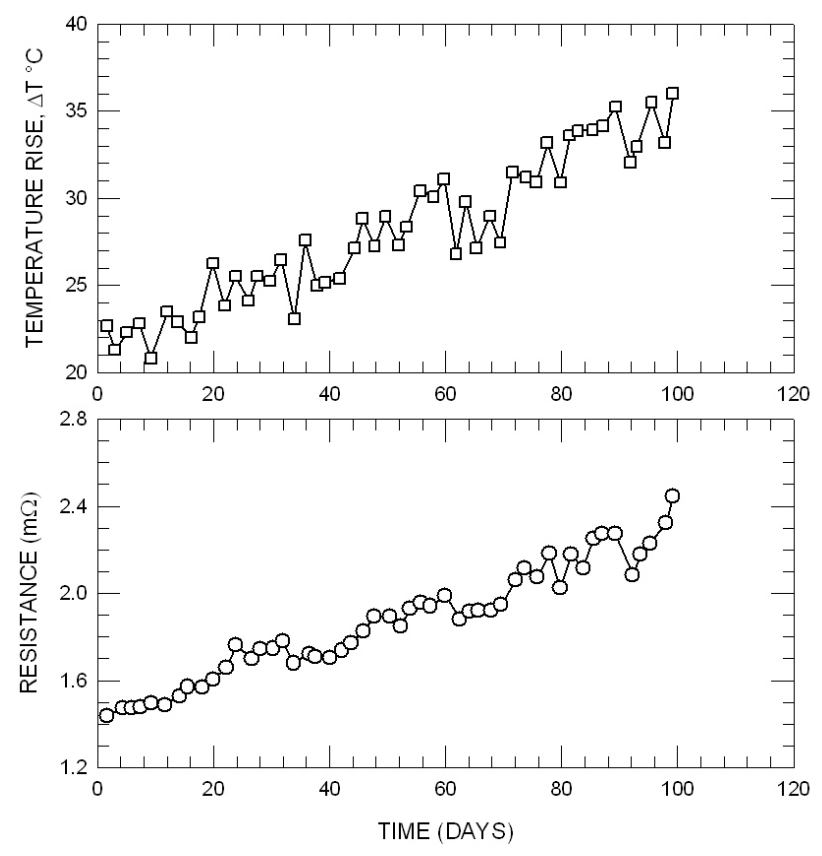

Fig. 2 Examples of stochastic processes $\Delta T(t)$ and $R(t)$

Based on the statistical analysis of the data it is possible to establish correlation between two key parameters of a contact - its resistance and temperature of the hottest point. Quantitative measure of the correlation between two stochastic variables is correlation factor. 
Table 1

\begin{tabular}{ccc}
\hline \hline $\begin{array}{c}\text { Connection } \\
\text { number }\end{array}$ & $\begin{array}{c}\text { Cross correlation } \\
\text { factors } r_{\mathrm{R}, \Delta \top}\end{array}$ & $\begin{array}{c}\text { Confidence interval at } \\
95 \% \text { confidence level }\end{array}$ \\
\hline \hline 1 & 0,964 & $0,937 \leq r_{\mathrm{R}, \Delta} \leq 0,979$ \\
\hline 2 & 0,891 & $0,782 \leq r_{\mathrm{R}, \Delta \top} \leq 0,947$ \\
\hline 3 & 0,983 & $0,970 \leq r_{\mathrm{R}, \Delta} \leq 0,990$ \\
\hline \hline
\end{tabular}

As an example, Table 1 contains the values of the cross correlation factors for the contact resistance and the temperature of the hottest point of three connections randomly selected from the investigated ones. The right column in Table 1 contains the confidence intervals for the cross correlation factors at $95 \%$ confidence level.

As seen, all correlation factors range within the confidence interval at $95 \%$ confidence level. In mathematical statistics, the correlation between two random variables is considered as strong or very strong when the correlation factor is about 0.9. Hence, from the statistical point of view of the validity of degradation characteristics and estimating the reliability of a joint, both parameters can be used.

Selecting either of these parameters in a particular case will depend on the economical limitations and also the actual measurement capabilities. In this paper, the temperature of the hottest contact point exceeding that of the surrounding environment $\Delta T$ was selected (for simplicity "temperature rise" term is used).

Thermography was used to measure the temperature of the joints tested. The obtained thermal images enabled to determine the surface temperature distribution of all parts and to control the local temperature rise of the hottest contact point. Thermographic measurements were carried out with a ThemoCAM P65 Flir System IR camera. The accuracy of temperature measurements in the range $-40^{\circ} \mathrm{C}$ to $+120^{\circ} \mathrm{C}$ was $\pm 2{ }^{\circ} \mathrm{C}$. Temperature measurements were carried out during the stabilized current $\mathrm{ON}$ time and under natural air convection conditions. In Fig. 3 the example of IR thermography measurement is shown with the indication of the hottest joint points.

The experimental results were used to determine the time dependences of the hottest contact point temperature, which appears to follow typical time series.

\section{ESTIMATION OF RESIDUAL LIFETIME OF POWER CONNECTIONS.}

As shown in [3], the behavior of a contact can be described using a statistical (probabilistic) model based on the Box-Jenkins time series forecasting method. In
[3], the contact resistance was selected as the key parameter. In this work, as mentioned above, temperature of the contact was as selected as the key factor.
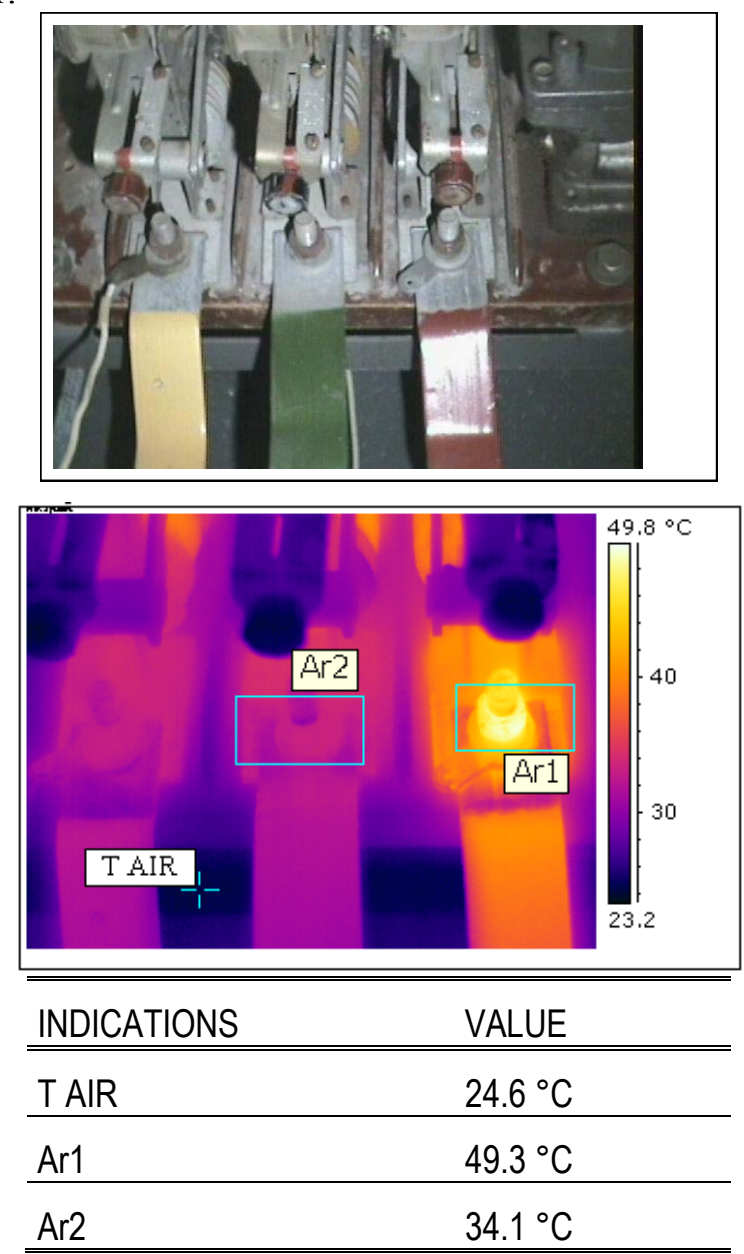

Fig. 3 Example of joint temperature measurement a) overall view of a joint, b) IR thermography image.

An example of the predicted temperature behavior of a permanent contact joint as a function of time is shown in Fig. 4. The lifetime estimates were based on the measurements of temperature rise during so called estimation period (it is time interval from the beginning of temperature monitoring till the moment of the forecasting).

Estimation was carried out within predetermined time interval following the last infrared thermography measurements. This time interval is called forecasting period. In this experiment the estimation period was 5 months and forecasting period was one month. On Fig. 4 the forecasted results based on the ARIMA $(2,2,2)$ model (Autoregressive Integrated Moving Average model) are shown. This model is similar to that used in [3] for forecasting the contact residual lifetime based on the contact resistance as the key parameter. 
The use of lower values for ARIMA parameters $p, d$, $q$ is reasonable only for sufficiently "simple" time series with insignificant fluctuations of measured parameters. These types of time series rarely occur under real monitoring conditions. Furthermore, the ARIMA $(p, d, q)$ model with lower values of parameters $p, d, q$ does not provide the universality that is required when analyzing complex functional relationships.

Parameters values of ARIMA model were estimated on the base of autocorrelation function (ACF) and partial autocorrelation function (PACF). Several reasonable values of parameters were chosen and then the reliability of the model was estimated. For the model reliability estimation residuals investigation was used. Residuals are the differences between observed and forecasted values of the key parameter.

The model is considered to be the adequate if the ACF and PACF reveal the white noise spectrum, i.e. the graphs of ACF and PACF do not reveal sharp peaks. In addition, some other statistical tests were used for the model reliability estimation, e.g. AIC (Akaike information criterion).

This procedure was realized as the original PC-based computer program. It estimates the ARIMA models with parameters varying in the range $0 \div 3$ and choose the adequate ones by the AIC test.

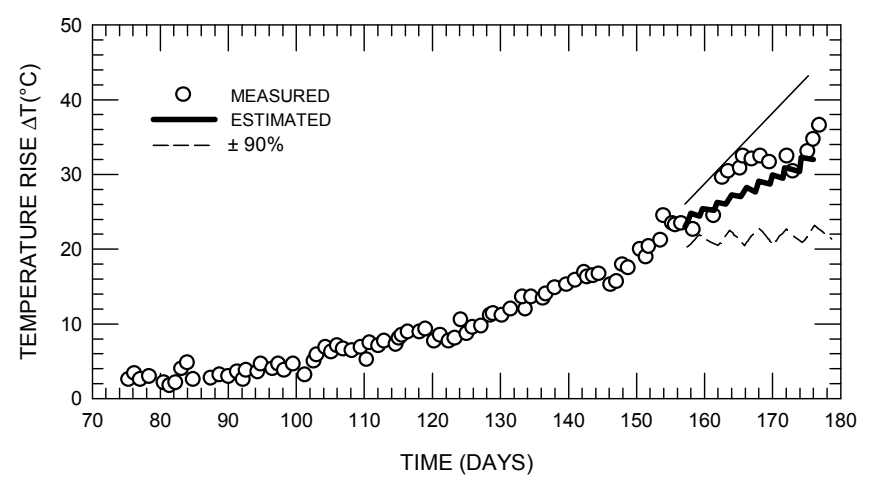

Fig. 4 Estimation of temperature rise of the joint hottest contact point based on periodical temperature measurements with 2 days interval

To verify the validity of the forecasting procedure, the experiment was prolonged and the obtained data were compared with the estimated ones (Fig. 4).

Analogous forecasting can be carried out using selective monitoring of the joint over longer time intervals, for instance, once per week or even once per month. In this case, the estimation period is about 12 months whereas the forecasting period is approximately 3 months.
Experiments carried out confirmed the validity of the results obtained thus allowing the practical use of the proposed method. The frequency of temperature measurements may be once a month and less since the economical efficiency of such approach directly depends on the frequency of data acquisition.

The critical parameter influencing the prognostic quality is the duration of estimation period. Fig. 5 shows as example how the duration of the estimation period influences prognostic quality in comparison with the experimental data. The confidence intervals for $90 \%$ confidence level are also shown in Fig. 5.

The estimation (observation) period was 40, 60, 80 and 100 days in consecutive order, whereas the forecasting (prognostic) period was 20 days. The estimation method was as follows. Following 40 days of exposure, the forecasting was made for the next 20 days that is up to 60 days. The estimated temperature values were compared with the measured ones. After 60 days a new estimate based on a larger number of measurements was made and compared with the experimentally measured data for the contact temperature, etc.

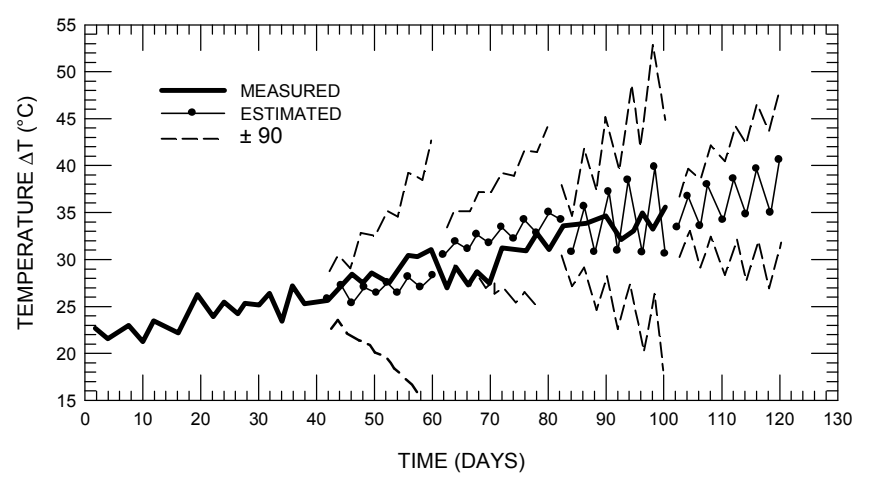

Fig. 5 Measured and estimated temperature rise of power connection.

As seen in Fig. 5, all experimental values fall within confidence intervals thus confirming the validity of this method. The confidence intervals and thus the estimation error increases with increasing the forecasted period for a given estimation time interval and decreases when increasing the estimation period for a given forecasting interval.

Based on this method, it is possible to predict the behavior of a power connection ahead of time when we know its "history" based on the results of measurements of the key resource parameters (in this case connection temperature rise). Forecasting of the temperature rise of the connection enables to predict the moment then the temperature will exceed the allowed limit. This fact is 
considered as a parametric failure of a connection. Thus, the residual lifetime (i.e. time interval from the moment of the forecasting to the moment of parametric failure) may be estimated. This estimation may be specified after the next IR inspection (if the temperature does not exceed the allowed limit before it).

\section{CONCLUSIONS}

- It is shown, that the temperature rise of the contact joint surface may be used as the key (resource) parameter. Periodical monitoring of the above parameter may be used for the forecasting of the residual lifetime of electrical contact joint. Periodical measurements of the temperature rise of the contact parts surface can be carried out using the infrared thermography technique. Temperature measurements may be performed monthly.

- Residual lifetime forecasting based on the current state of electrical contact joint (using the key monitoring parameter) enables setting deadline for the next infrared thermography control, scheduling and repair procedure, schedule replacement date and scope of failing joints. All these measures increase the reliability, safety and efficiency of electrical equipment.

- The successful application of the proposed method for forecasting the residual lifetime of electrical contacts requires suitable hardware and software for the processing a large volume of diagnostic information.

\section{REFERENCES}

[1]. Box, G. E. P., \& Jenkins, G. M. (1976). "Time series analysis: Forecasting and control". San Francisco: Holden-Day.

[2]. [Braunovic M., Izmailov V.V., Novoselova M.V. "A Model for Life Time Evaluation of Closed Electrical Contacts ", Proceedings of the 51 IEEE Holm Conference on Electrical Contacts. - Chicago, Sept. 2005. pp. 217-223.

[3]. Braunovic M., Izmailov V.V., Novoselova M.V. "Evaluation of the life-time of permanent electrical contacts" Proc. $23^{\text {rd }}$ International Conf. Electrical Contacts, ICEC 2006, pp. 4-50.

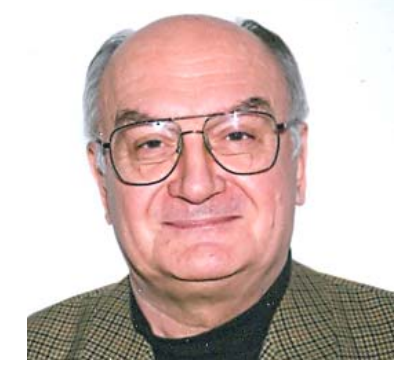

Dr. Milenko Braunovic received his Dipl. Ing Degree in Technical Physics from the University of Belgrade, Serbia, in 1962 and the M. Met. and Ph. D. degrees in Physical Metallurgy from the University of Sheffield, England in 1967 and 1969 respectively. From 1971 until 1997 he was working at IREQ, HydroQuebec Research Institute, Varennes, Quebec as a senior member of the scientific staff. He retired from IREQ in

1997 and established his own scientific consulting company, MB Interface.
During the last 35 years, he has been responsible for the development and management of a broad range of research projects for Hydro-Québec and the Canadian Electrical Association (now CEA Technology) in the areas of electrical power contacts, connector design and evaluation, tribology and accelerated test methodologies. He also initiated and supervised the R\&D activities in the field of shape-memory alloy applications in power systems. He is presently R\&D Manager with the AGS Taron, Technologies a company specializing in aluminum foam materials.

Dr. Braunovic is the co-author of the book of electrical contacts published by the Francis \& Taylor Group and author of more than 100 papers and technical reports, including contributions to encyclopaedias and books, in his particular areas of scientific interests. In addition, he frequently lectures at seminars worldwide and has presented a large number of papers at various international conferences. For his contributions to the science of electrical contacts, Dr. Braunovic is recipient of the Ragnar Holm Scientific Achievement Award, the Ralph Armington Recognition Award and the IEEE CPMT Best Paper Award. $\mathrm{He}$ successfully chaired the 15th International Conference on Electrical Contacts held in Montreal 1990 and was a Technical Program Chairman of the $18^{\text {th }}$ International Conference on Electrical Contacts held in Chicago 1996. He is a Senior Member of IEEE and a member of American Society for Metals (ASM), Materials Research Society (MRS), Planetary Society, American Society for Testing of Materials (ASTM) and The Minerals, Metals \& Materials Society (TMS).

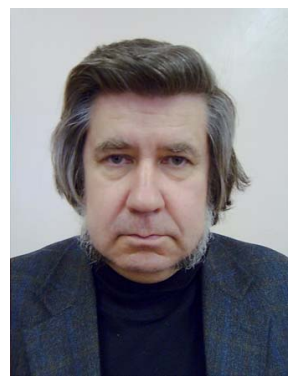

Dr. Vladimir V. Izmailov graduated from the State University of Technology in Tver, USSR, in 1971. He received his first scientific degree (Candidate of Sciences) in 1974 and Doctorate degree in tribology in 1995 from the Railway Research Institute, Moscow, Russia. He is now a Professor of Physics at Tver State University of Technology. He is the author of more than 150 scientific publications on electrical contacts, tribology, materials science, energy saving, including hand-book and standard.

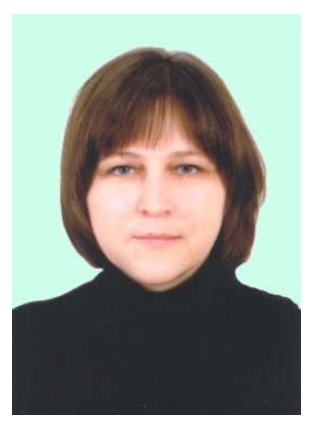

Marina V. Novoselova received the diploma degree in physics from the State University in Tver, USSR in 1986. From 1986 to 1996 she worked as a research engineer. She has been involved in the research and design of electronic devices. In 1996 she joined Tver State University of Technology. In 2003 she received the scientific degree - Candidate of Sciences- in materials science from Railway Research Institute in Moscow, Russia. She is now an assistant professor of Physics at Tver State University of Technology. Her scientific interests are in the field of design and tests of materials for electrical contacts. She is the author of more than 50 scientific publications.

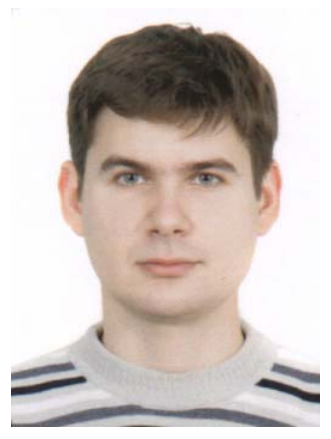

Alexander E. Naumov graduated with honors from the State University of Technology in Tver, Russian Federation, in 2004. From 2004 to 2007 he continued with his education as the postgraduate student at the same University. Now he is the senior engineer of the joint-stock company "Federal Grid Company of Unified Energy System" (FGC UES). He is engaged in service of high-voltage equipment, automatic devices and relay protection. He goes on with his scientific researches. His scientific interests are in the field of energy saving, emergency diagnostics in electric power network, reliability of electric contacts. He is the author of 18 scientific publications. 


\title{
Modelling of the Interruption Process in $\mathrm{SF}_{6} \mathrm{High}$ Voltage Circuit Breaker and Preliminary Study of the Arc Characteristics for Different Gases
}

\author{
Jian Zhang, Shenli Jia, Xingwen Li, Pengwei Liu, Zongqin Shi, Lijun Wang \\ State Key Laboratory of Electrical Insulation and Power Equipment \\ Xi'an Jiaotong University \\ Xi'an, China \\ xwli@mail.xjtu.edu.cn
}

\begin{abstract}
SF}_{6}$ is exclusively used as insulation and quenching medium for high voltage circuit breaker because of its high insulation strength and high interruption capability of fault current at present. Therefore it is very necessary to investigate $\mathrm{SF}_{6}$ arc interruption process in high voltage circuit breaker. On the other hand, its greenhouse effect has stimulated the studies for searching environmentally friendly substitute gas. In the paper, a 2-D arc magnetohydrodynamic (MHD) model is presented to one puffer type arc quenching chamber in high voltage circuit breaker firstly. Then, from the view of the difference of medium properties including thermodynamic properties, transport coefficients and radiation characteristic, the arc behavior of $\mathrm{SF}_{6}, \mathrm{~N}_{2}$ and $\mathrm{CO}_{2}$ gases is simulated based on the model, respectively, taking into account the influence of arc current, chamber pressure. From the preliminary study, good agreement has been achieved with the experimental result, and the validity of the proposed arc model is partially verified. In addition, the effects of medium properties on arc interruption parameters, such as arc radius, arc temperature and arc voltage, are also presented.
\end{abstract}

Keywords-arc model; circuit breaker; interruption process; arc behavior; quenching medium

\section{INTRODUCTION}

Due to its excellent dielectric strength and interruption performance, $\mathrm{SF}_{6}$ is used for GCB and GIS very widely, especially for the high voltage class above $110 \mathrm{kV}$. It is very necessary to investigate $\mathrm{SF}_{6}$ arc interruption process in high voltage circuit breaker, in order to aid the design of the products with higher performance and less development cost. Since the recent 20 years, many researchers relevant to $\mathrm{SF}_{6}$ high voltage circuit breaker have applied themselves to the arc magnetohydrodynamic (MHD) modeling studies [1][2].

However, the global warming potential (GWP) of $\mathrm{SF}_{6}$ is very high, it is about 23900 times that of $\mathrm{CO}_{2}$. It is regulated in the 3rd Conference of the Parties to the United Nations Framework Convention on Climate Change (COP3) as a greenhouse effect gas. Therefore, much research is now being conducted on a substitute gas for $\mathrm{SF}_{6}$. A range of pure gases and gas mixtures has been investigated [3][4][5], but at present there is no reliable alternative being manufactured.

This work is supported by National Natural Science Foundation of China, No. 50877059 .
This paper is devoted to present a 2-D arc magnetohydrodynamic (MHD) model for one puffer type arc quenching chamber firstly, and to investigate the arc characteristic influenced by $\mathrm{SF}_{6}, \mathrm{~N}_{2}$ and $\mathrm{CO}_{2}$ medium with the help of this model and an experimental quenching chamber model.

\section{THE ARC MODEL AND BOUNDARY CONDITIONS}

The arc plasma is assumed to be axisymmetric, turbulent and under local thermodynamic equilibrium (LTE). The governing equations can be written as follows.

Continuity equation

$$
\frac{\partial \rho}{\partial t}+\nabla \cdot(\rho \vec{V})=0
$$

Axial momentum conservation equations

$$
\begin{gathered}
\frac{\partial}{\partial t}(\rho w)+\nabla \cdot(\rho \vec{V} w)-\nabla \cdot\left(\left(\mu_{l}+\mu_{t}\right) \nabla w\right) \\
=-\frac{\partial P}{\partial z}+J_{r} B_{\theta}+\nabla \cdot(\overline{\bar{\tau}})
\end{gathered}
$$

Radial momentum conservation equations

$$
\begin{gathered}
\frac{\partial}{\partial t}(\rho v)+\nabla \cdot(\rho \vec{V} v)-\nabla \cdot\left(\left(\mu_{l}+\mu_{t}\right) \nabla v\right) \\
=-\frac{\partial P}{\partial r}+J_{z} B_{\theta}+\nabla \cdot(\overline{\bar{\tau}})
\end{gathered}
$$

Energy conservation equation

$$
\begin{aligned}
\frac{\partial}{\partial t}(\rho h) & +\nabla \cdot(\rho \vec{V} h)-\nabla \cdot\left(\left(\frac{k_{l}+k_{t}}{c_{p}}\right) \nabla h\right) \\
= & \sigma E^{2}-q+\nabla \cdot(\overline{\bar{\tau}} \cdot \vec{V})
\end{aligned}
$$

Here $P$ is the local pressure, $\rho$ the mass density, $\vec{V}$ the velocity vector of the plasma, $w$ and $v$ the axial and radial components of velocity, $\mu l$ and $\mu t$ the laminar and turbulent viscosity, $J_{r}$ and $J_{z}$ the radial and axial current density, $B_{\theta}$ the 
azimuthal component of the magnetic field, $h$ the enthalpy, $k$ the thermal conductivity, $\sigma$ the electrical conductivity, $c_{p}$ the specific heat at constant pressure, $E$ the electric field, $q$ the radiation loss, $\nabla \cdot(\overline{\bar{\tau}})$ the viscous terms and $\nabla \cdot(\overline{\bar{\tau}} \cdot \vec{V})$ the viscous dissipation.

Current continuity equation

$$
\nabla \cdot(\sigma \nabla \varphi)=0
$$

where $\varphi$ is the electrical potential.

Ampere's Law

$$
\frac{1}{r} \frac{\partial}{\partial r}\left(r B_{\theta}\right)=\mu_{0} J_{z}
$$

where $\mu_{0}=4 \pi \times 10-7 \mathrm{H} / \mathrm{m}$ is the permeability of free space.

The Spalart-Allmaras turbulence model is adopted in this paper since it is not necessary to calculate a length scale related to the local shear layer thickness [6]. The Prandtl mixing length model is used in [2], which is also known as the zero equation model. Although the computational cost of the zero equation model is economical, the turbulence parameter needs to be adjusted for a given geometry of the nozzle. However, for the Spalart-Allmaras model, was designed specifically for aerospace applications involving wall-bounded flows and has been shown to give good results for boundary layers subjected to adverse pressure gradients.

The transported variable in the Spalart-Allmaras model, $\tilde{v}$, is identical to the turbulent kinematic viscosity. The transport equation is

$$
\begin{gathered}
\frac{\partial}{\partial t}(\rho \tilde{v})+\frac{\partial}{\partial x_{i}}\left(\rho \tilde{v} u_{i}\right)=G_{v} \\
+\frac{1}{\sigma_{\tilde{v}}}\left[\frac{\partial}{\partial x_{j}}\left\{(\mu+\rho \tilde{v}) \frac{\partial \tilde{v}}{\partial x_{j}}\right\}+C_{b 2} \rho\left(\frac{\partial \tilde{v}}{\partial x_{j}}\right)^{2}\right]-Y_{v}
\end{gathered}
$$

where $G_{v}$ is the production of turbulent viscosity and $Y_{v}$ is the destruction of turbulent viscosity that occurs in the nearwall region due to wall blocking and viscous damping. $\sigma_{\tilde{v}}$ and $C_{b 2}$ are constants and $\mu$ is the molecular kinematic viscosity. All constants have the FLUENT 6.1 default values.

The Transport properties of the $\mathrm{SF}_{6}$ plasma are obtained from [7]. The NEC radiation model [8] has been used to calculate the radiation transport in the energy equation. The net emission coefficient from [9] is used.

The puffer type arc quenching chamber computational domain is shown in Fig. 1. The overall length of the nozzle is $68.75 \mathrm{~mm}$, with an inlet diameter of $25 \mathrm{~mm}$, an outlet diameter of $38.4 \mathrm{~mm}$ and a nozzle throat diameter of $12.50 \mathrm{~mm}$. The throat position is located $15.63 \mathrm{~mm}$ away from the inlet plane. There are two contacts with diameter of $4 \mathrm{~mm}$ at upstream (fixed contact) and $3 \mathrm{~mm}$ at downstream (movable contact).

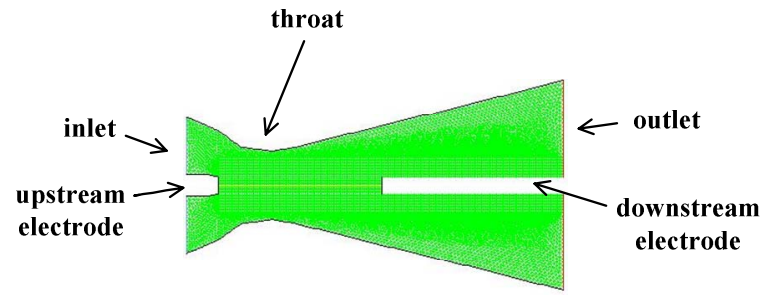

Figure 1. Nozzle geometry and the grid system used.

At the nozzle inlet, the mass, momentum and enthalpy fluxes are determined assuming isentropic flow of gas with a temperature of $300 \mathrm{~K}$ and stagnation pressure of $3.75 \mathrm{MPa}$. At the nozzle outlet, a static pressure of $0.3 \mathrm{MPa}$ is set. At downstream electrode, the electrical potential is set $0 \mathrm{~V}$. At upstream electrode, the current density is set by arc current is given [10]. The current density distribution $j_{i}$ must be satisfied

$$
\sum j_{i} \cdot s_{i}=I
$$

The general solution of this condition is given by

$$
j_{i}=I \cdot \frac{\alpha_{i}}{\sum_{k=1}^{N} \alpha_{k} s_{k}}
$$

where coefficients $\alpha$ is given by

$$
\alpha=T_{i}^{2} \exp \left(-\frac{W}{K T_{i}}\right) \sigma_{\text {in }}
$$

At outer boundary of the simulation domain, the gradient of the electrical potential is set to zero. The nozzle wall is assumed to be adiabatic with no ablation. The arcs and surrounding region should ensure that sufficient number of grids is used to compute the temperature profile.

\section{SimUlation RESUlts AND DiscusSION}

Based on the above model, the arc characteristics have been simulated by the half-sinusoid transient current. The peak value of current is $40 \mathrm{kA}$. The movement speed of movable contact is $3 \mathrm{~m} / \mathrm{s}$.

The temperature distribution of the arc plasma at different time is presented in Fig. 2. The pressure distribution and electric potential distribution of the $\mathrm{SF}_{6}$ arc at $0.6 \mathrm{~ms}$ is shown in Fig. 3 and Fig. 4. Before $2.5 \mathrm{~ms}$, the arc does not pass nozzle throat, the main part of the arc column has the tendency of limiting by nozzle throat. Moreover, arc current is not too big, so that arc radius is small and arc temperature is lower. At $2.5 \mathrm{~ms}$, the highest arc temperature is about $23000 \mathrm{~K}$. When $\mathrm{t}=5.0 \mathrm{~ms}$, the arc pass the nozzle throat, arc column begin to expand, at the same time, the arc current reach peak value 40 $\mathrm{kA}$, as a result, arc temperature reach the highest value 28800 $\mathrm{K}$. Hot gas which accelerated by nozzle throat flow to downstream, it appears high temperature region at downstream position. After $5.0 \mathrm{~ms}$, due to the arc current decrease and hotter gas sufficient flow, the arc temperature begins to drop and high 
temperature region become large. At $\mathrm{t}=8.5 \mathrm{~ms}$, the highest arc temperature is about $23000 \mathrm{~K}$. When arc current reach zero at $\mathrm{t}=10.0 \mathrm{~ms}$, arc column disconnect from the upstream contact, the highest arc temperature drop to $16000 \mathrm{~K}$. The comparison of arc voltage vs. arc current is shown in Fig. 5.

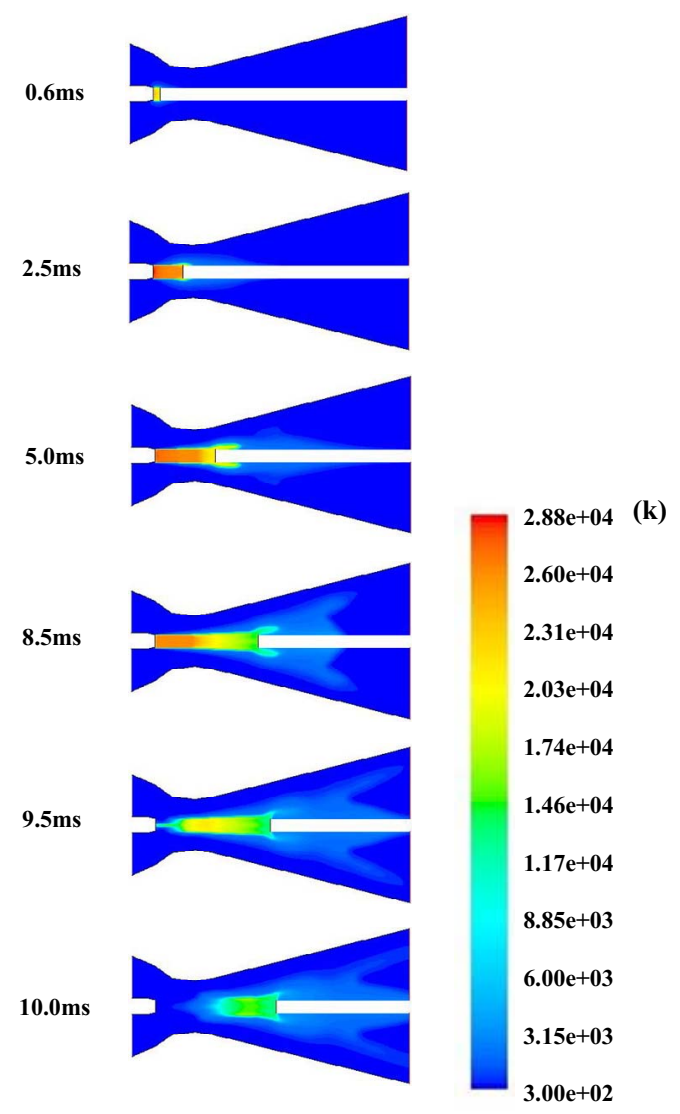

Figure 2. The temperature distribution of the $\mathrm{SF}_{6}$ arc at different time

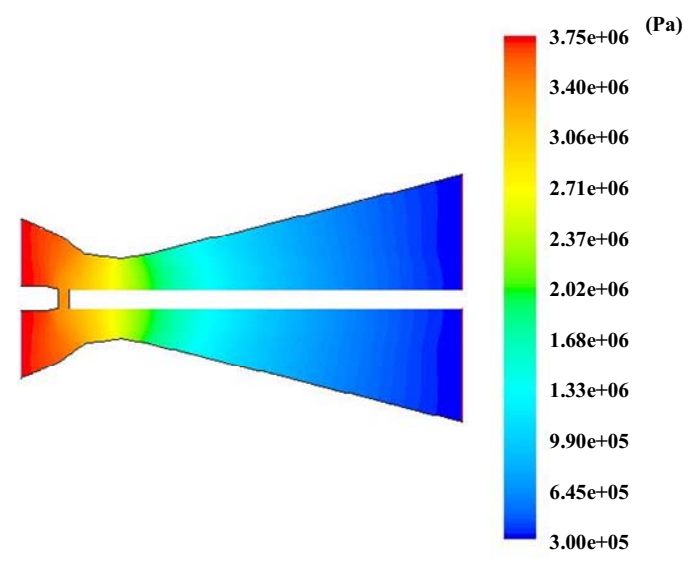

Figure 3. The pressure distribution of the $\mathrm{SF}_{6}$ arc at $0.6 \mathrm{~ms}$

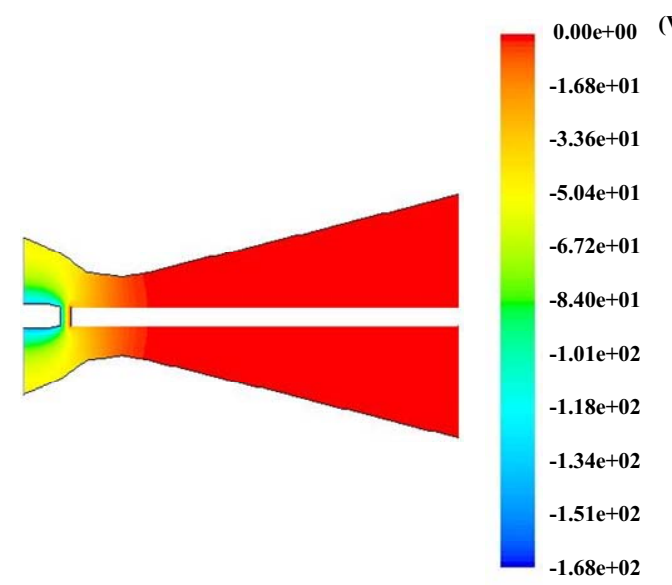

Figure 4. The electric potential distribution of the $\mathrm{SF}_{6}$ arc at $0.6 \mathrm{~ms}$

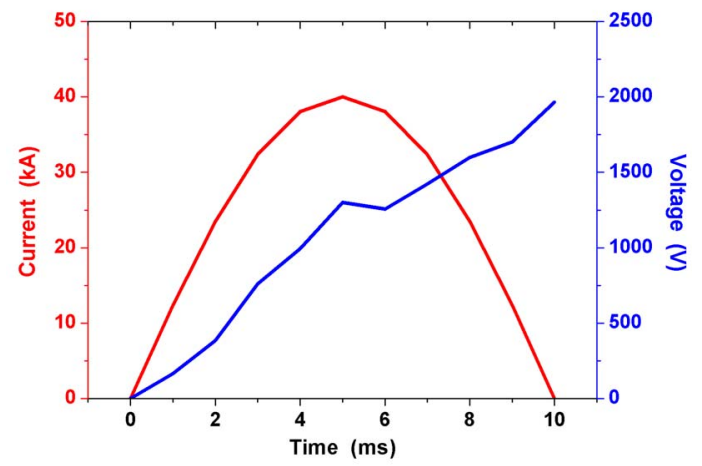

Figure 5. The comparison of arc voltage vs. arc current

\section{COMPARISON OF THE ARC CHARACTERISTICS IN SF $6 \mathrm{~N}_{2}$ AND $\mathrm{CO}_{2}$ GASES}

In order to investigate the influence of different quenching gases on arc characteristics, an arc quenching chamber model has been developed. In addition, the different gases arc characteristics have been simulated based on the above model under same experimental condition.

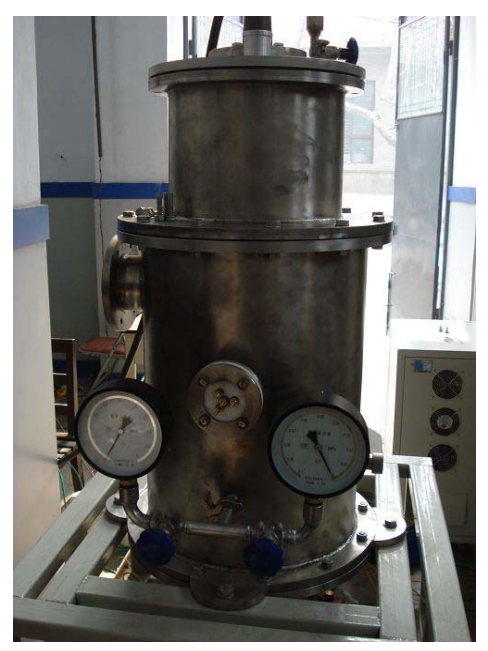

Figure 6. The photo of the experimental system. 


\section{A. Experimental setup}

The photo of the experimental system is shown in Fig. 6. The arc quenching chamber is divided into two parts. The heights of the upper and lower part are $300 \mathrm{~mm}$ and $600 \mathrm{~mm}$, and the diameters of the two parts are $305 \mathrm{~m}$ and $406 \mathrm{~mm}$, respectively.

The quenching gases are $\mathrm{SF}_{6}, \mathrm{~N}_{2}$ and $\mathrm{CO}_{2}$, respectively. The gas pressure may be 1 atm, 3 atm or 5 atm. The clearance between contacts is fixed to $8 \mathrm{~mm}$.

Capacitor bank circuit is used to provide short circuit current. The effective value of the prospective short circuit current is 3 $\mathrm{kA}$.

Shunt and voltage transducer are used to measure arc current and arc voltage respectively. And CCD (Phantom V10.0) is adopted to observe the arc motion, whose exposure of is 2 us, and frame speed is $17000 / \mathrm{s}$.

Fig. 7 shows one group of experimental results for arc voltage of the three kinds of gases with the gas pressure of 1 atm. The values from $0 \mathrm{~s}$ to $0.01 \mathrm{~s}$ have been as a result of measure value. It shows that the peak value arc voltage of $\mathrm{SF}_{6}$ is lowest and that of $\mathrm{N}_{2}$ is highest. In addition, because fuse is used to ignite, the impulse at the initial arcing is presented.

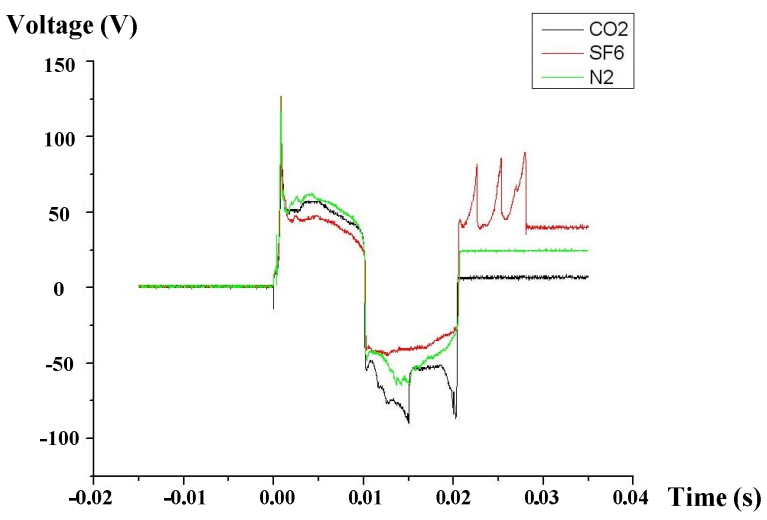

Figure 7. The arc voltage waveforms by three quenching gases at 1 atm

\section{B. Simulation comparison}

The computational domain is shown in Fig. 8. According to the experimental conditions, the diameter of the each contact is set to $18 \mathrm{~mm}$, and the clearance between contacts is set to 8 $\mathrm{mm}$. The Transport properties of the $\mathrm{N}_{2}$ and $\mathrm{CO}_{2}$ are obtained from [11]. The net emission coefficient from [12] is used.

In gas chamber, the mass, momentum and enthalpy fluxes are determined assuming isentropic flow of gas with a temperature of $300 \mathrm{~K}$ and stagnation pressure of $1 \mathrm{~atm}, 3 \mathrm{~atm}$ and 5 atm, respectively.

Based on the above experimental system and numerical model, the arc characteristics have been compared with $\mathrm{SF}_{6}, \mathrm{~N}_{2}$ and $\mathrm{CO}_{2}$, at $1 \mathrm{~atm}, 3 \mathrm{~atm}$ and $5 \mathrm{~atm}$. And the current is assumed to $3 \mathrm{kA}$.

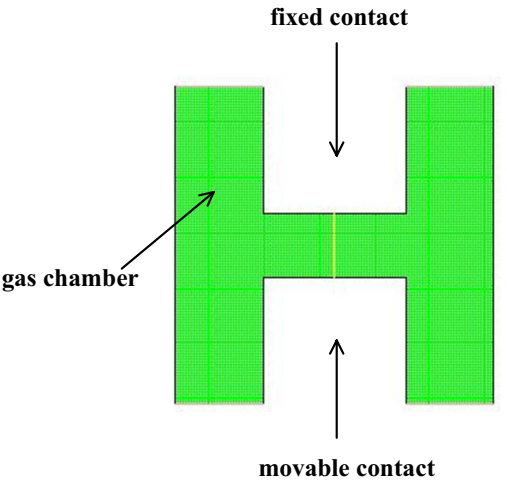

Figure 8. The computational domain and the grid system used.

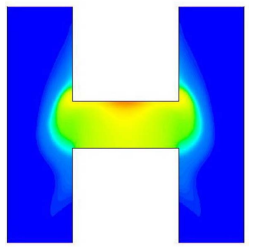

$\mathrm{SF}_{6}$

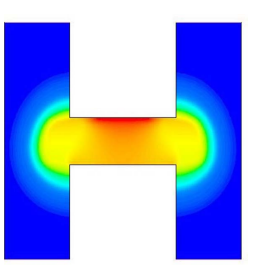

$2.60 \mathrm{e}+04$

(k)

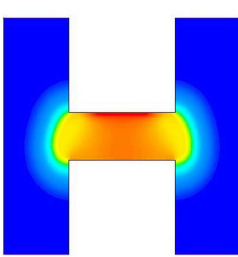

$2.09 \mathrm{e}+04$

$1.83 e+04$

$1.57 \mathrm{e}+04$

$1.31 \mathrm{e}+04$

$1.06 \mathrm{e}+04$

$8.01 \mathrm{e}+03$

$5.44 \mathrm{e}+03$

$2.87 \mathrm{e}+03$

$3.00 \mathrm{e}+02$

Figure 9. The simulation arc temperature distribution at 1 atm

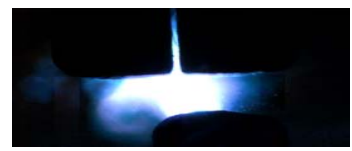

$\mathrm{SF}_{6}$

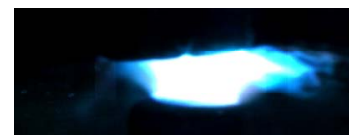

$\mathbf{N}_{2}$

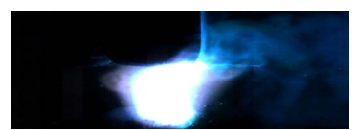

$\mathrm{CO}_{2}$

Figure 10. The high-speed movie of the arc at $1 \mathrm{~atm}$

In the first case, the chamber pressure is $1 \mathrm{~atm}$. The simulation arc temperature distribution by three quenching gases is shown in Fig. 9 and the corresponding high-speed 
movie of the arc is shown in Fig. 10. The highest $\mathrm{SF}_{6}$ arc temperature is about $23200 \mathrm{~K}$, the highest $\mathrm{N}_{2}$ and $\mathrm{CO}_{2}$ arc temperature is about $26000 \mathrm{~K}$. The simulation arc radius is defined as the region radius flowing $90 \%$ of the total arc current. $\mathrm{N}_{2}$ arc radius $13.71 \mathrm{~mm}$ is bigger than $\mathrm{CO}_{2}$ arc radius $10.34 \mathrm{~mm}$. The arc radius experimental result could be measured from the high-speed movie of the arc, $\mathrm{N}_{2}$ arc radius is about $11 \mathrm{~mm}$ and $\mathrm{CO}_{2}$ is about $8 \mathrm{~mm}$. The difference between simulation and experiment may partially derive from the simplification of the calculation model.

This can be attributed to constant-pressure specific heat of $\mathrm{SF}_{6}$ are higher than $\mathrm{N}_{2}$ and $\mathrm{CO}_{2}$, so $\mathrm{SF}_{6}$ arc temperature is lower than $\mathrm{N}_{2}$ and $\mathrm{CO}_{2}$. The net emission coefficient of $\mathrm{CO}_{2}$ is higher than $\mathrm{N}_{2}$, it leads to energy loss of $\mathrm{CO}_{2}$ is bigger $\mathrm{N}_{2}$, so $\mathrm{CO}_{2}$ arc radius are smaller than $\mathrm{N}_{2}$.

In the second case, the chamber pressure is $3 \mathrm{~atm}$. The simulation arc temperature distribution by three quenching gases is shown in Fig. 11 and the corresponding high-speed movie of the arc is shown in Fig. 12. The highest $\mathrm{SF}_{6}$ arc temperature is about $19500 \mathrm{~K}$, the highest $\mathrm{N}_{2}$ and $\mathrm{CO}_{2}$ arc temperature is about $23500 \mathrm{~K}$. The increased pressure leads to electrical conductivity and thermal conductivity increase, result in decrease of joule heat and increase of thermal transport, so that arc temperature drop obviously.

In the third case, the chamber pressure is $5 \mathrm{~atm}$. The simulation arc temperature distribution by three quenching gases is shown in Fig. 13 and the corresponding high-speed movie of the arc is shown in Fig. 14. The red line presents the position of the electrodes. The highest $\mathrm{SF}_{6}$ arc temperature is about $18300 \mathrm{~K}$, the highest $\mathrm{N}_{2}$ arc temperature is about 21600 $\mathrm{K}$, and the highest $\mathrm{CO}_{2}$ arc temperature is about $20500 \mathrm{~K}$.

Table 1 shows the comparison of the experimental results and simulation results for arc voltage of three quenching gases. It should be noted that $20 \mathrm{~V}$ electrode drop is added to the simulation results.
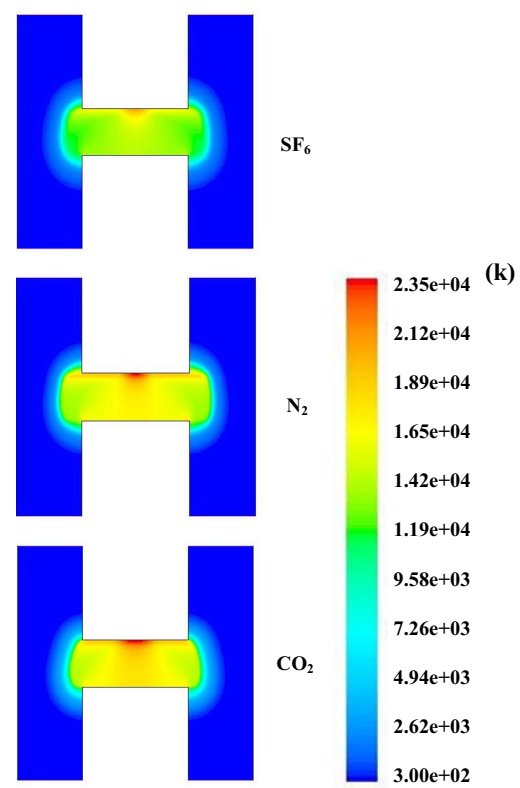

\section{(k)}

$12 \mathrm{e}+04$

$3.00 \mathrm{e}+02$

Figure 11. The simulation arc temperature distribution at $3 \mathrm{~atm}$

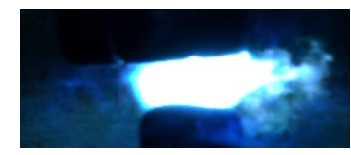

$\mathrm{SF}_{6}$

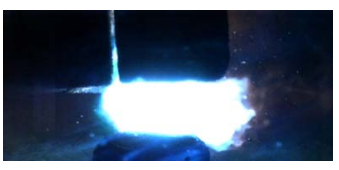

$\mathbf{N}_{2}$

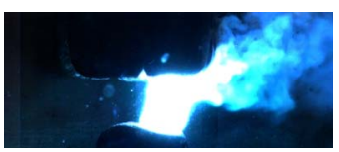

$\mathrm{CO}_{2}$

Figure 12. The high-speed movie of the arc at $3 \mathrm{~atm}$

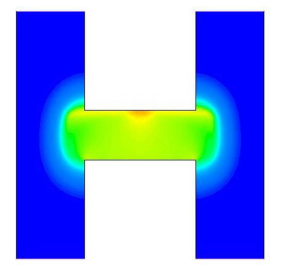

$\mathrm{SF}_{6}$

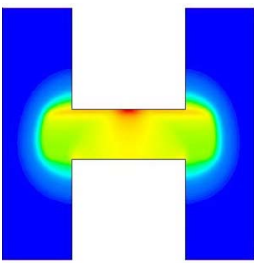

(k)

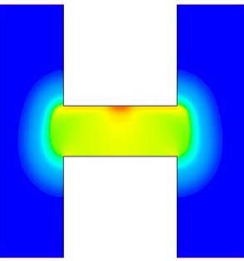

$2.16 \mathrm{e}+04$

$1.95 \mathrm{e}+04$

$1.73 \mathrm{e}+04$

$\mathbf{N}_{2}$

$1.52 \mathrm{e}+04$

$1.31 \mathrm{e}+04$

$1.10 \mathrm{e}+04$

$8.82 \mathrm{e}+03$

$6.69 \mathrm{e}+03$

$\mathrm{CO}_{2}$

$4.58 \mathrm{e}+03$

$2.43 e+03$

$3.00 \mathrm{e}+02$

Figure 13. The simulation arc temperature distribution at 5 atm

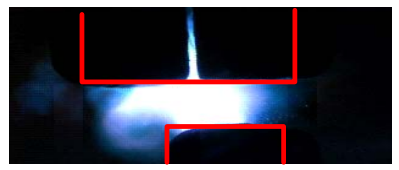

$\mathrm{SF}_{6}$

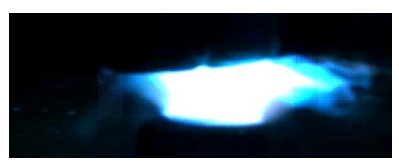

$\mathbf{N}_{2}$

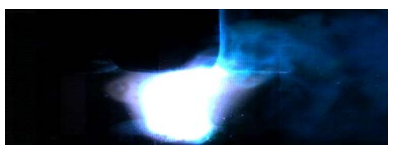

$\mathrm{CO}_{2}$

Figure 14. The high-speed movie of the arc at $5 \mathrm{~atm}$ 
TABLE I. THE COMPARISON OF THE EXPERIMENTAL AND SIMULATION RESULTS FOR ARC VOLTAGE (V)

\begin{tabular}{|c|c|c|c|c|}
\hline \multicolumn{2}{|c|}{ Pressure } & $\mathrm{SF}_{6}$ & $\mathrm{~N}_{2}$ & $\mathrm{CO}_{2}$ \\
\hline \multirow{2}{*}{1 atm } & experiment & 40 & 45 & 45 \\
\cline { 2 - 5 } & simulation & 34 & 39 & 38 \\
\hline \multirow{2}{*}{$3 \mathrm{~atm}$} & experiment & 47 & 53 & 53 \\
\cline { 2 - 5 } & simulation & 41 & 46 & 44 \\
\hline \multirow{2}{*}{$5 \mathrm{~atm}$} & experiment & 51 & 59 & 55 \\
\cline { 2 - 5 } & simulation & 44 & 51 & 49 \\
\hline
\end{tabular}

Table 2 shows the comparison of the experimental results and simulation results for arc radius of three quenching gases.

TABLE II. THE COMPARISON OF THE EXPERIMENTAL AND SIMULATION RESULTS FOR ARC RADIUS (MM)

\begin{tabular}{|c|c|c|c|c|}
\hline \multirow{2}{*}{ Pressure } & $\mathrm{SF}_{6}$ & $\mathrm{~N}_{2}$ & $\mathrm{CO}_{2}$ \\
\hline \multirow{2}{*}{ atm } & experiment & 8 & 11 & 8 \\
\cline { 2 - 5 } & simulation & 9.24 & 13.71 & 10.34 \\
\hline \multirow{2}{*}{ 5atm } & experiment & 9 & 12 & 9 \\
\cline { 2 - 5 } & simulation & 9.33 & 13.85 & 10.63 \\
\cline { 2 - 5 } & experiment & 10 & 13 & 12 \\
\cline { 2 - 5 } & simulation & 9.83 & 13.88 & 10.87 \\
\hline
\end{tabular}

\section{CONCLUSION}

A 2-D arc magnetohydrodynamic (MHD) model is presented to one puffer type arc quenching chamber in high voltage circuit breaker. The arc characteristics in a supersonic nozzle have been numerically investigated under the halfsinusoid transient current.

Investigate the arc characteristic influenced by $\mathrm{SF}_{6}, \mathrm{~N}_{2}$ and $\mathrm{CO}_{2}$ medium based on this model, respectively. An experimental system can change quenching gases is set up. From the preliminary study, good agreement has been achieved with the experimental result, it shows the effect of medium properties on arc interruption parameters, such as arc radius, arc temperature and arc voltage.
In the future work, the arc characteristic under dynamic gas blast condition for different quenching gases should be investigate, research dominant medium property that affects the results.

\section{REFERENCES}

[1] J. Zhang, SL. Jia, XW. Li, "Simulation of the Influences of the Pressure Ratio and $\mathrm{Cu}$ Vapour on $\mathrm{SF}_{6}$ Arc Characteristics" Plasma Science \& Technology, vol.11, 152-56, 2009

[2] V. K. Liau, B. Y. Lee, K. D. Song and K. Y. Park, "The influence of contacts erosion on the $\mathrm{SF}_{6}$ arc," J. Phys. D: Appl. Phys., vol. 39, pp. 2114-2123, 2006.

[3] D. T. Tuma, Brown, "A comparison of the behavior of $\mathrm{SF}_{6}$ and $\mathrm{N}_{2}$ blast arcs around current zero," IEEE Transactions on Power Apparatus and Systems, vol. PAS-99, No. 6 Nov/Dec 1980

[4] L. G. Christophorou, J. K. Olthoff and D. S. Green, "Gases for Electrical Insulation and Arc interruption: Possible Present and Future Alternatives to Pure $\mathrm{SF}_{6}$, National Institute of Standards and Technology Technical Note 1425, 1997

[5] Y. Qiu, E. Kuffel, "Comparison of $\mathrm{SF}_{6} / \mathrm{N}_{2}$ and $\mathrm{SF}_{6} / \mathrm{CO}_{2}$ gas mixtures as alternatives to $\mathrm{SF}_{6}$ gas," IEEE Transactions on Dielectrics and Electrical Insiulation, Vol. 6 No. 6, December 1999.

[6] P. Spalart, and S. Allmaras, "A one-equation turbulence model for aerodynamic flows," Technical Report AIAA-92-0439, American Institute of Aeronautics and Astronautics, 1992.

[7] L. S. Frost, and R. W. Liebermann, "Composition and Transport Properties of $\mathrm{SF}_{6}$ and Their Use in a Simplified Enthalpy Flow Arc Model," Proceedings of The IEEE, vol. 59, no. 4, 1971.

[8] J. F. Zhang, M. T. C. Fang, and D. B. Newland, "Theoretical investigation of a $2 \mathrm{kA}$ arc in a supersonic nozzle," J. Phys. D: Appl. Phys., vol. 20, pp. 368, 1987.

[9] A. Gleizes, J. J. Gonzalez, B. Liani, and G. Raynal, "Calculation of net emission coefficient of thermal plasmas in mixtures of gas with metallic vapours," J. Phys. D: Appl. Phys., vol. 26, pp. 1921, 1993.

[10] M. Lindmayer, "Complete simulation of moving arc in low-voltage switchgear," Proc. 14th Int. Conf. on Gas Discharges and their Applications (Liverpool) [C], vol.2:318, 2002.

[11] J F Zhang and M T C Fang, "A comparative study of $\mathrm{SF}_{6}$ and $\mathrm{N}_{2}$ arcs in accelerating flow,” J. Phys. D: Appl. Phys. vol.21,730-736, 1988.

[12] A Gleires, B Rahmani, J J Gonralez and B Liani, "Calculation of net emission coefficient in $\mathrm{N}_{2}, \mathrm{SF}_{6}$ and $\mathrm{SF}_{6}-\mathrm{N}_{2}$, arc plasmas," J. Phys. D: Appl. Phys. vol.24,1300-1309.1991. 


\title{
Research on Contact Force Pairing of MCCB with Double Repulsive Contact Structure
}

\author{
Liang Ji, Degui Chen, Yingyi Liu, and Weixiong Tong \\ State Key Laboratory of Electric Insulation and Power Equipment \\ Xi'an Jiaotong University \\ Xi'an, China \\ dgchen@mail.xjtu.edu.cn
}

\begin{abstract}
MCCBs with double repulsive contact structures (DRMCCBs for short) are helpful for improving their shortcircuit breaking capacities. A calculation model that enables us to simulate the interruption process of the DRMCCB is realized by carrying out second-development of ADAMS, in which a set of differential equations describing the coupling of complex mechanical movement, electric circuit, magnetic field and dynamic mathematic arc model is constructed. With this model, influence of contact force pairing on breaking capacity of DRMCCB is analyzed. Other factors affected by contact force pairing are analyzed as well.
\end{abstract}

Keywords- DRMCCB; dynamic mathematic arc model; multifield coupling; interruption process; ADAMS

\section{INTRODUCTION}

Double repulsive contact structure is widely used in Molded Case Circuit Breakers (DRMCCBs). Different from MCCBs with single repulsive contact structure (SRMCCBs), both of the upper and lower contacts (fixed contact) of DRMCCB are repulsive under short-circuit current, which makes it possible for DRMCCB to yield a better short-circuit breaking capacity than SRMCCB. For DRMCCB, the contact force pairing is one of the key factors affecting its short-circuit breaking capacity. Generally, in order to evaluate the influence of some factors on the breaking capacity of MCCB, shortcircuit interruption experiments are usually carried out, which are costly and time-consuming. So it is necessary to find methods to substitute the interruption experiment with computer simulation.

Various interruption models of low voltage circuit breakers have been established [1-6]. G.D.Gregory introduced the blackbox model, where the arc voltage and mechanical movements of the electromagnetic and operating mechanisms are predicted by experimental results and/or some empirical equations [7]. The USAN software constructed an integrated simulation model comprehensive of different physical fields, where every component field is calculated separately and the coupling of all the fields is realized by method of undetermined coefficients [8]. In [9], the author introduced the chain arc model and constructed a multi-field coupled interruption model with specified mechanical structures. In [10], the author improved the mechanical calculation function by carrying out second development of ADAMS, and described the arc model with an approximate curve typical of experimental arc voltage.

This paper proposes a method to model the interruption process of low voltage circuit breakers, especially to solve coupling of dynamic arc model and mechanical movements with other physical fields existing in the breaker's interruption process. The constructed model is capable of dynamically describing behavior and shapes of electric arc and calculating mechanical movements of MCCBs with complex mechanical structure. With this model, influence of contact force pairing on the DRMCCB's short-circuit breaking capacity is analyzed. Other factors affected by the contact force pairing, such as final contact force, contact over travel, etc. are analyzed as well.

\section{ANALYZED MODEL AND METHOD}

\section{A. Analyzed Model}

In this paper, a DRMCCB product with a rated current of $250 \mathrm{~A}$ is considered. Fig. 1 shows its virtual prototype model in ADAMS and its corresponding simplified representation.

When the fault current occurs, if the electromagnetic force exceeds the upper and lower contact spring forces, the two contacts will separate and arc will appear between them. At the same time, the blow open force appears and accelerates the two contacts apart from each other together with the electromagnetic force. During this process, the arc will elongate and enter the splitter plates with the help of magnetic force. Parallel to the above procedure, the mechanism will be triggered by the instantaneous release a short interval after the current comes. With the action of operating mechanism, the contact support starts its motion, then catches and stops the upper contact from re-closing. In addition, the latch structure in the mechanism also contributes to preventing re-closure of the upper contact. For the lower contact, however, once its contact spring force exceeds sum of the electromagnetic and blow open forces acting on it, it will return to its original position since there is no operating mechanism or latch structure to prevent its re-closure.

The motion status of upper and lower contacts, which is especially dependant on contact force paring of the two contacts as well as electromagnetic and blow open forces, affects the breaking capacity of DRMCCB to a great extent. In 
order to determine the influence of contact force pairing on the DRMCCB's breaking capacity, the interruption model of the DRMCCB has to be constructed.

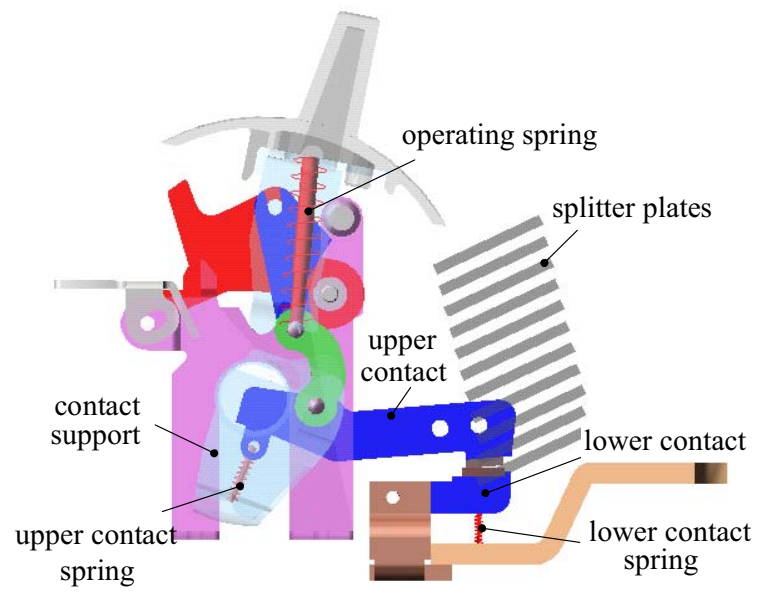

a) Virtual prototype model of DRMCCB

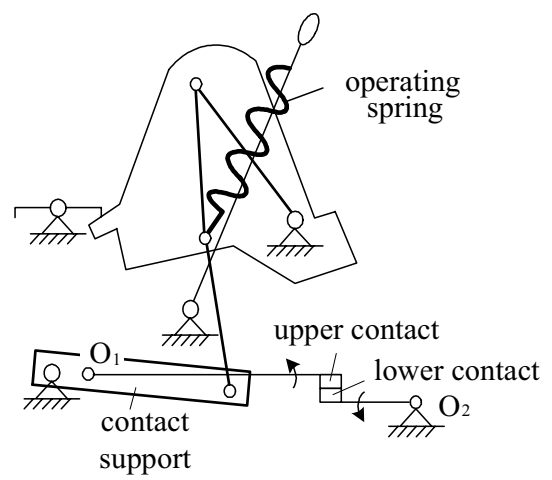

b) Simplified representation of DRMCCB's operating mechanism

Figure 1. Analyzed model

\section{B. Analyzed Method}

It is learned from the above description that the interruption process of DRMCCB is a very complicated and multi-field coupled phenomena comprehensive of electric circuit, magnetic field, complex mechanical movement and dynamical arc model. To construct its interruption model, modelling of each component field is the first step.

\section{1) Electric Circuit}

In this paper, the short-circuit current is provided by a capacitor bank circuit as shown in Fig.2a. Fig.2b shows its equivalent circuit diagram, in which capacitor bank $C$, resistor $\mathrm{R}_{0}$, inductance $\mathrm{L}$, and arc voltage $\mathrm{u}_{\text {arc }}$ are involved.

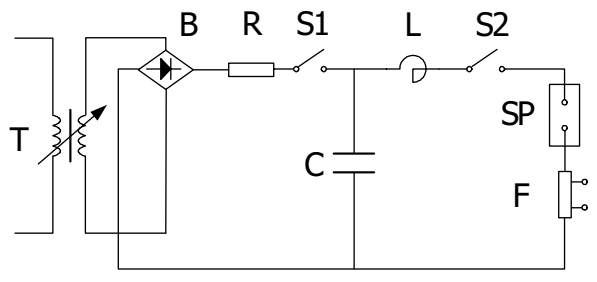

a) Capacitor bank circuit

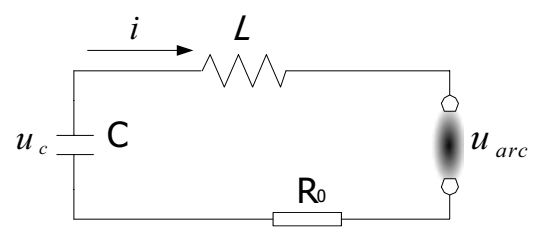

b) Equivalent circuit diagram

Figure 2. Short-circuit current test circuit

According to Kirchhoff's law, the differential equations describing the above electric circuit can be written as:

$$
\left\{\begin{array}{l}
\frac{\mathrm{d} i}{\mathrm{~d} t}=\frac{1}{L}\left(u_{\mathrm{c}}-R_{0} \cdot i-u_{\mathrm{arc}}\right) \\
\frac{\mathrm{d} u_{\mathrm{c}}}{\mathrm{d} t}=-\frac{1}{C} i
\end{array}\right.
$$

Where $i$ is the short-circuit current, and $u_{\mathrm{c}}$ is charging voltage of the capacitor bank $\mathrm{C}$.

\section{2) Magnetic Field}

The electromagnetic force acting on the contacts consists of Lorentz force and Holm's constriction force. The Holm force is calculated directly in the simulation model using the known equation [11]. The Lorentz force is evaluated beforehand with FEM program ANSYS from the current and position of the contacts. Besides the electromagnetic force, the contacts are also subject to the blow open force, which can be calculated according to $\mathrm{X}$ Zhou who calculates the blow open force with an algebraic equation [12].

Electric arc in arc chamber receives Lorentz force from the magnetic field generated by the electrified conductors, splitter plates and the arc itself. According to the method in [9], the magnetic intensity at any point of the arc chamber can be solved with Integral Equation Method.

\section{3) Mechanical Movement}

The mechanical movement of DRMCCB in its interruption process includes movements of instantaneous release, operating mechanism and contact system. The instantaneous release affects the delay time of the operating mechanism. In this paper, this delay time is experimentally determined and transferred to our model as a parameter. Movement of the contact system is affected by both short-circuit current and operating mechanism. For the operating mechanism, the influence can be automatically evaluated by ADAMS software. And for influence of forces generated by short-circuit current, a second development of ADAMS has to be carried out.

\section{4) Dynamic Arc Model}

Magnetically driven electric arc is a current path of electrons that deforms in various manners. To describe this feature, the chain arc model is employed ${ }^{[9]}$. It assumes the arc to be a chain of small cylindrical current elements (See Fig.3), each of which moves with a velocity decided by Lorentz force and fluid drag force and the current path is short-circuited if one element contacts with another. In this way the entire arc 
shows a particular behavior and shape similar with the actual electric arc.

According to the theory of shock wave [1], the current element moves with a velocity $\overrightarrow{v_{a}}$, whose magnitude is decided by:

$$
\left|v_{a}\right|=C_{0} \cdot \frac{5 \frac{B_{z} I}{2 P_{0} r}}{\sqrt{49+42 \frac{B_{z} I}{2 P_{0} r}}}
$$

Where $\mathrm{C}_{0}$ is the sonic speed in air, $B_{z}$ is z-direction component of the magnetic induction intensity $\vec{B}, I$ is magnitude of arc current $\vec{I}, P_{0}$ is atmosphere pressure, and r is radius of the current element. The direction of $\overline{v_{a}}$ is parallel to that of $\vec{I} \times \vec{B}$.

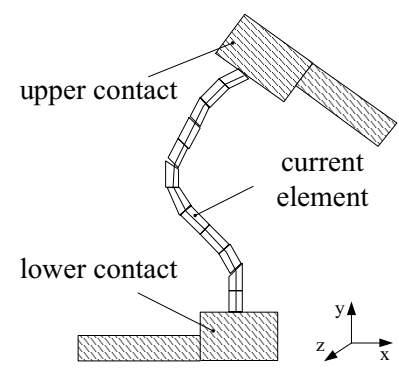

Figure 3. Current element chain

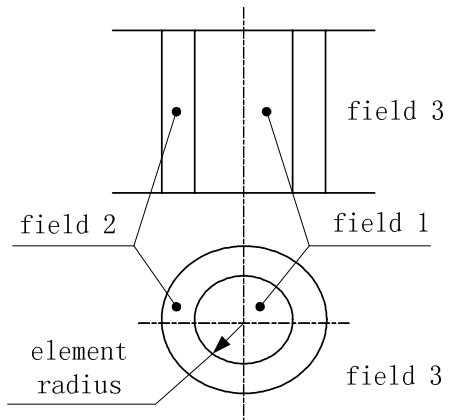

Figure 4. Field division of current element

Energy equilibrium of the current element exists throughout the breaker's interruption process. To describe this phenomenon, the current element is divided into 3 fields as shown in Fig.4. Where field 1 is an electric cylinder, field 2 is a high-temperature thermal boundary layer around field 1, and field 3 is an air layer whose physical parameter is constant. In field 1, the input energy of the external circuit is balanced by the temperature rise of field 1 as well as heat conduction and radiation. In field 2 , the thermal energy conducted from field 1 is balanced by the temperature rise of field 2 as well as heat convection and radiation. The energy conservation equations describing fields 1 and 2 can be written as:
Field 1:

$$
\rho c_{p} \frac{\partial T}{\partial t}=\sigma E^{2}-\frac{1}{r} \frac{\partial}{\partial r}\left(-r \lambda \frac{\partial T}{\partial r}\right)-u
$$

Field 2:

$$
\rho c_{p} \frac{\partial T}{\partial t}=-\frac{1}{r} \frac{\partial}{\partial r}\left(-r \lambda \frac{\partial T}{\partial r}\right)-u-\alpha\left(-\frac{\partial T}{\partial r}\right)
$$

with

$\rho c_{p} \frac{\partial T}{\partial t}:$ Temperature rise

$\sigma E^{2}$ : Input energy of the external circuit

$\frac{1}{r} \frac{\partial}{\partial r}\left(-r \lambda \frac{\partial T}{\partial r}\right):$ Heat conduction

$u$ : Heat radiation

$\alpha\left(-\frac{\partial T}{\partial r}\right):$ Heat convection

$T$ : Temperature of the current element

$\rho$ : Density

$C_{p}$ : Constant-pressure specific heat

$\sigma$ : Electric conductivity

$E$ : Electric-field intensity

$\lambda$ : Thermal-conductivity coefficient

$r$ : Radius of current element

$\alpha$ : Thermal-convection coefficient

For physical parameters of the electric arc, the values can be interpolated according to the arc temperature. Fig. 5 shows temperature variations of arc parameters $\rho, C_{p}, \sigma$, and $\lambda$. The radiation item $u$ of electric arc can also be interpolated according to Fig.6.

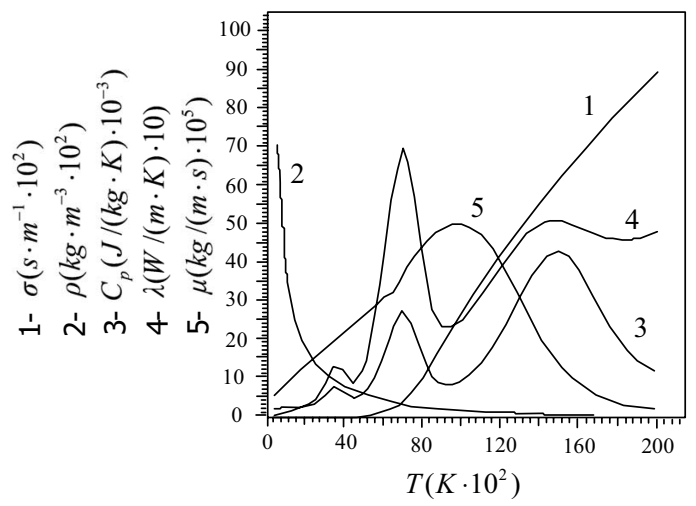

Figure 5. Physical parameters of electric arc 


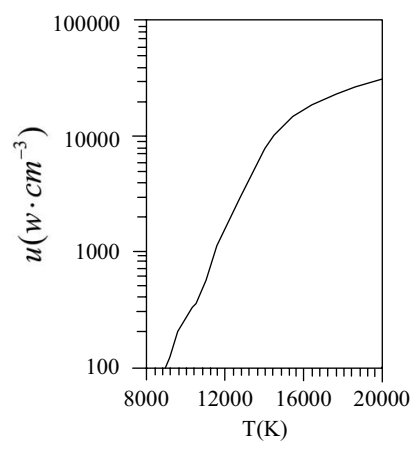

Figure 6. Radiation power of electric arc

Moreover, the electric-field intensity $E$ of the electric arc can be calculated according to the integral form of Ohm's law, we have:

$$
E=\frac{I}{\int_{0}^{r} 2 \pi r \sigma d r}
$$

Solving (2) to (5), the electric conductivity $\sigma_{\mathrm{i}}$ and electricfield intensity $E_{i}$ of every current element can be calculated. Consequently, the arc voltage can be determined by:

$$
u_{\mathrm{arc}}=M U_{0}+\sum_{i=1}^{N} E_{i} L_{i}
$$

Where $M$ is number of the short arcs cut by the splitter plates, $U_{0}$ is anode and cathode fall, $N$ is number of current element, and $L_{i}$ is length of every current element.

\section{5) Interruption Model of DRMCCB}

With the component fields described above, the interruption model of the DRMCCB can be set up. A complete simulation of the DRMCCB's interruption process includes calculations of every component field as well as interactions between them. In Fig.7, an integrated calculation model describing coupling of electric circuit, magnetic field, chain arc model, and complex mechanical movements is constructed.

In the model, the motion parameters, such as displacement, velocity and acceleration, etc. of every movable part are solved by ADAMS program. And the coupling of each component field is realized by carrying out second development of ADAMS. As shown in Fig.7, through the interface between ADAMS and user written subroutine, the open angles $\theta_{t(1)}$, $\theta_{\mathrm{t}(2)}$ of the upper and lower contacts at instant $t$ are transferred to the user program as two parameters. Together with shortcircuit current $i$ at the same instant, the electromagnetic forces $F_{t+\Delta t(1)}, F_{t+\Delta t(2)}$ acting on the upper and lower contacts can be calculated by the user program and returned to the ADAMS program.

By repeating above bilateral iterations continuously, the whole interruption process of DRMCCB can be calculated. In the user program, all the component fields except mechanical calculation are described by a set of differential equations. Where the electric circuit is solved by RG-KT method, the magnetic field distribution in arc chamber is solved by IEM method, the chain arc model is calculated by Control Volume Method [9], and the electromagnetic force is calculated by interpolation of pre-established data sheet according to current and contact position.

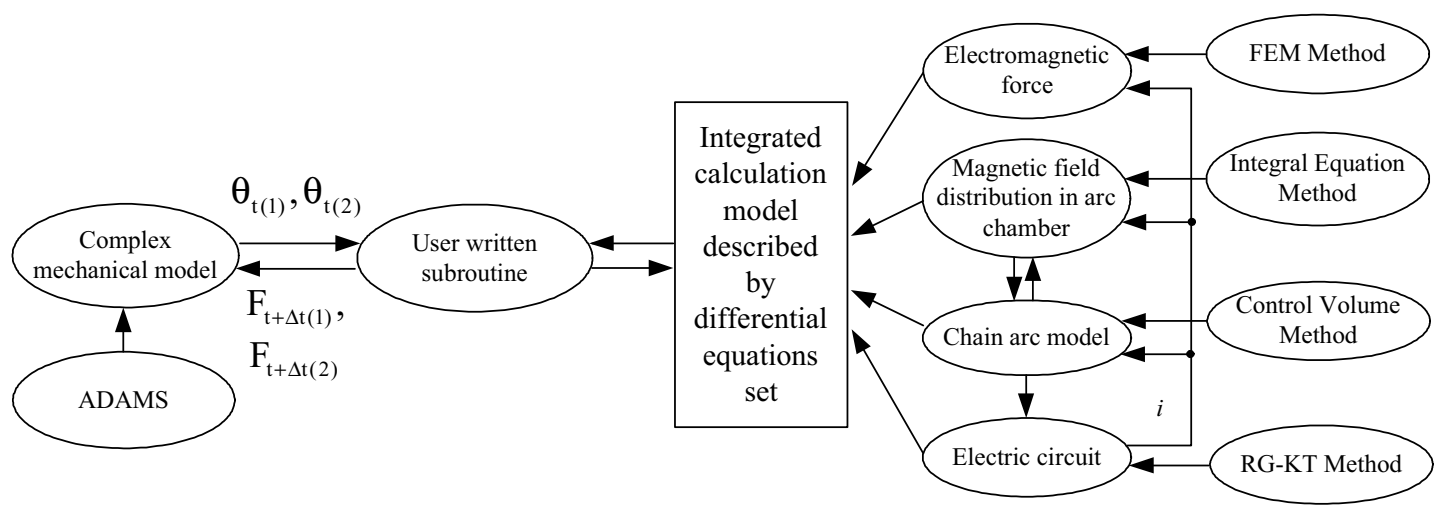

Figure 7. Calculation model of multi-field coupled interruption process of DRMCCB

\section{INFLUENCE OF FACTORS ON CONTACT FORCE PAIRING OF DRMCCB}

\section{A. Short-circuit breaking capacity}

1) Simulation Results and Analysis

In this section, the calculation model constructed above is applied to the analyzed DRMCCB to analyze influence of contact force pairing on its breaking capacity. In our calculation, the upper contact force is $14 \mathrm{~N}$, and the lower contact force is $14 \mathrm{~N}$ and $30 \mathrm{~N}$, respectively. Besides, the obtained experimental delay time of the mechanism is $6.5 \mathrm{~ms}$ and the prospective current is 10kA. Fig.8 shows calculation results of the DRMCCB's interruption waves under two different contact force pairings, i.e. contact parameter pairs of $14 \mathrm{~N} / 14 \mathrm{~N}$ and $14 \mathrm{~N} / 30 \mathrm{~N}$. 


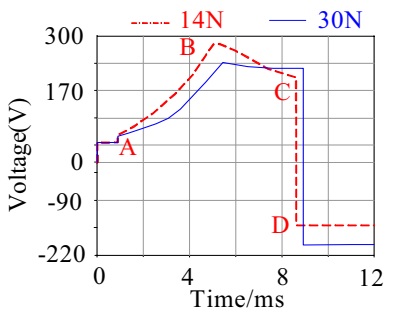

a) Arc voltage

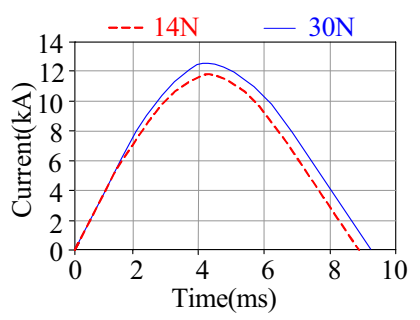

b) Arc current

Figure 8. Influence of contact force pairing on breaking capacity of DRMCCB

Take the interruption wave with contact force pairing of $14 \mathrm{~N} / 14 \mathrm{~N}$ for example: The electric arc generates at point $\mathrm{A}$ when the electromagnetic force is big enough to overcome the contact spring forces. After a short arc immobility stage, the breaker's upper and lower contacts rapidly repel off under the electromagnetic force and blow open force leading to a quick rise of the arc voltage. After the voltage reaches its peak value at point $\mathrm{B}$, it begins to fall down due to re-closure of lower contact. At the time the current goes to zero, the voltage falls directly from point $\mathrm{C}$ to point $\mathrm{D}$ to a extent decided by the opposite charging voltage of the capacitor bank.

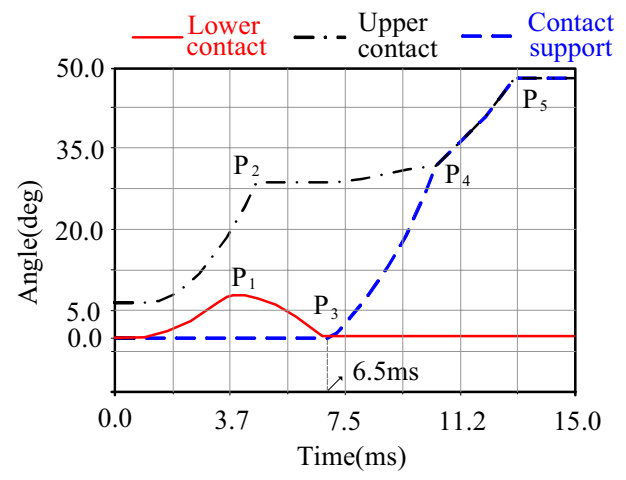

Figure 9. Angles of upper and lower contacts and contact support

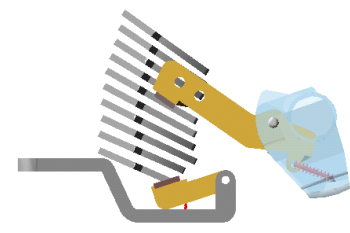

a) Point $P_{1}$

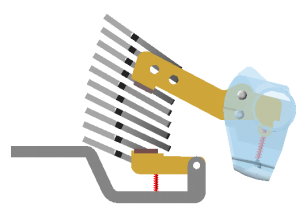

c) Point $\mathrm{P}_{4}$

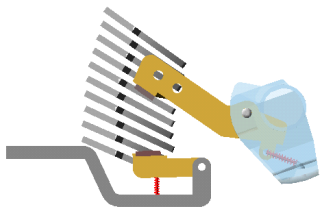

b) Point $\mathrm{P}_{3}$

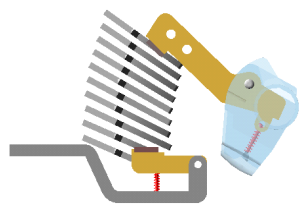

d) Point $\mathrm{P}_{5}$
Figure 10. Four typical motion states of the contact system

Fig.9 shows mechanical movements of the movable parts corresponding to the above interruption process. And Fig.10 shows 4 typical motion states of the contact system corresponding to points $\mathrm{P}_{1}, \mathrm{P}_{3}, \mathrm{P}_{4}$ and $\mathrm{P}_{5}$ in Fig.9: The upper and lower contacts open first (Point $\mathrm{P}_{1}$ represents the maximum open angle of lower contact) due to the high current while the contact support stays static until it is triggered by the instantaneous release at point $\mathrm{P}_{3}$. Almost at the same instant, the lower contact returns to its original position. Before the contact support moves, the upper contact has already opened to its latching position and is stopped by the latch structure of the mechanism from re-closing at point $\mathrm{P}_{2}$. The contact support starts its motion and catches the upper contact at point $\mathrm{P}_{4}$. After that, the upper contact is quickly lifted by the contact support to its maximum open angle at point $\mathrm{P}_{5}$.

Tab.1 gives the statistical result of the interruption calculation results shown in Fig.8. Where $i_{\mathrm{p}}$ and $u_{\mathrm{p}}$ are peak values of short-circuit current and arc voltage, $\frac{d u_{p}}{d t}$ is rising velocity of the arc voltage, and $t_{\mathrm{a}}$ is the arcing time.

TABLE I. STATISTIC OF THE CALCULATION RESULTS

\begin{tabular}{|l|c|c|c|c|}
\hline $\begin{array}{c}\text { Upper contact force } \\
\text { /Lower contact force }\end{array}$ & $\begin{array}{c}i_{p} \\
(\mathbf{k A})\end{array}$ & $\begin{array}{c}u_{p} \\
(\mathbf{V})\end{array}$ & $\begin{array}{c}\frac{d u_{p}}{d t} \\
(\mathbf{V} / \mathbf{m s})\end{array}$ & $\begin{array}{c}t_{a} \\
(\mathbf{s})\end{array}$ \\
\hline $14 / 14$ & 11.8 & 279 & 52.6 & 8.8 \\
\hline $14 / 30$ & 12.5 & 234 & 38.4 & 9.2 \\
\hline
\end{tabular}

Figure 11. Movements of lower contact under different contact force pairing

Fig.11 shows more information about the lower contact movements under different contact force pairing. Combining the calculation results shown in Fig.8 and Tab.1, the following conclusions can be reached:

a) When the upper contact force is fixed, the magnitude and rising velocity of arc voltage increases with the drop of lower contact force. As shown in Fig.11, the maximum opening angle and velocity of the lower contact increases with the drop of its contact force. So the magnitude and rising velocity of arc voltage increases accordingly. In Tab.1, when the lower contact force is $14 \mathrm{~N}$ and $30 \mathrm{~N}$, the peak value of arc voltage is $279 \mathrm{~V}$ and $234 \mathrm{~V}$, and the rising velocity is $52.6 \mathrm{~V} / \mathrm{s}$ and $38.4 \mathrm{~V} / \mathrm{s}$, respectively.

b) When the upper contact force is fixed, both the peak short-circuit current and arcing time of DRMCCB decrease with the drop of lower contact force. According to the calculation result in Tab.1, when the lower contact force is $14 \mathrm{~N}$ and $30 \mathrm{~N}$, the peak value of short-circuit current is $11.8 \mathrm{kA}$ and $12.5 \mathrm{kA}$, and the arcing time is $8.8 \mathrm{~ms}$ and $9.2 \mathrm{~ms}$ respectively. 
Consequently, if the upper contact force is determined, the breaking capacity of the DRMCCB increases with the drop of the lower contact force. In other words, in order to yield a better breaking capacity of the DRMCCB, the lower contact force should be as small as possible. However, an extremely small lower contact force may cause the lower contact to repel off in advance of upper contact and lead to subsequent welding of contacts due to re-closure of the lower contact. In order to prevent this phenomenon, the current disposal method is to endow the lower contact with a bigger contact force than the upper contact. Actually, the electromagnetic forces acting on the upper and lower contacts are not equal. Fig. 12 shows the comparison of calculated Lorentz forces acting on the two contacts with ANSYS software. Obviously, the Lorentz force acting on the upper contact is much bigger than that of the lower contact, which means the lower contact is unable to repel-off before the upper contact on the condition that the two contact forces are equal. Hence, in order to yield a maximum breaking capacity of the DRMCCB, it is not necessary for the lower contact force to be much bigger than upper contact force for the reason of preventing contact welding.

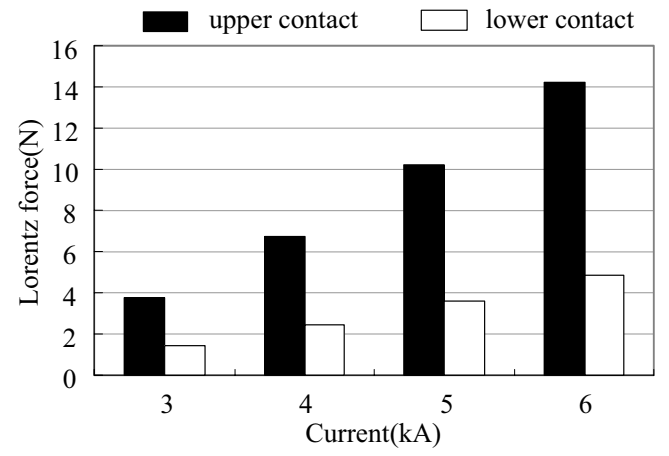

Figure 12. Comparison of forces acting on upper and lower contacts

\section{2) Experimental Verification}

In order to verify the calculation results, experiments are carried out for two DRMCCB samples in the capacitor bank circuit (see Fig.2). Tab.2 lists the experimental conditions.

TABLE II. EXPERIMENT CONDITION

\begin{tabular}{|l|l|}
\hline $\begin{array}{c}\text { Upper contact force } \\
\text { /Lower contact force }\end{array}$ & \multicolumn{1}{|c|}{$\begin{array}{c}\text { Prospective short-circuit current } \\
\text { (kA) }\end{array}$} \\
\hline $14 / 14$ & 10 \\
\hline $14 / 30$ & 10 \\
\hline
\end{tabular}

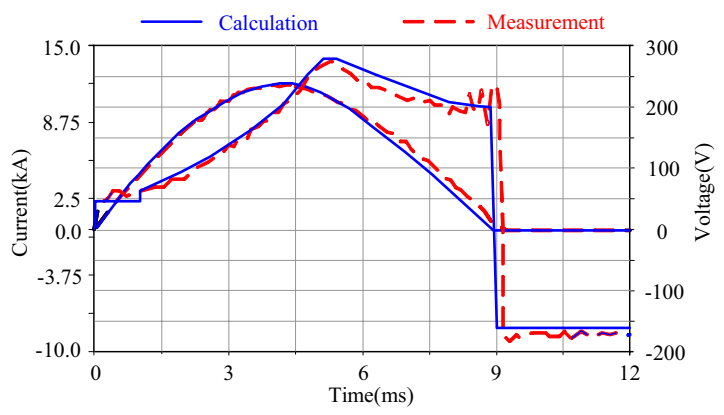

a) Contact force pairing: $14 \mathrm{~N} / 14 \mathrm{~N}$

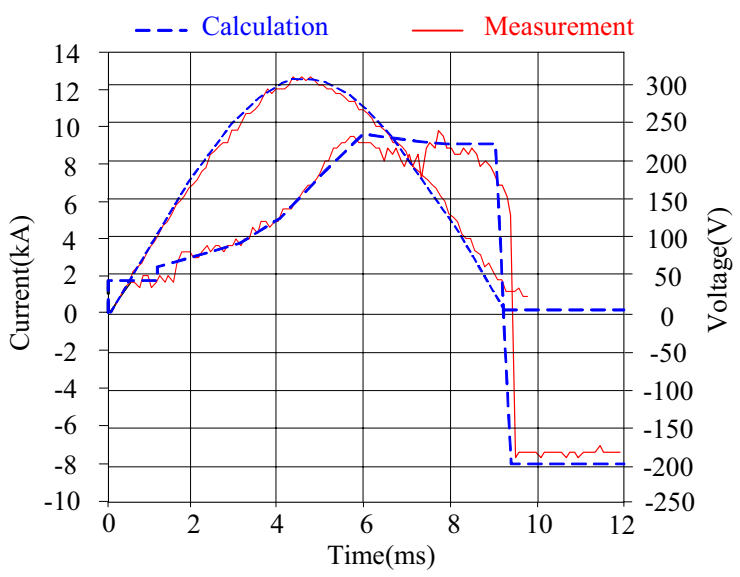

b) Contact force pairing: $14 \mathrm{~N} / 30 \mathrm{~N}$

Figure 13. Comparison between simulation and experimental results

Fig.13 shows the comparison between calculation and experimental results. The calculation values are in close agreement with the experimental results.

\section{B. Final Contact Force and Over Travel}

Determination of contact force pairing is also affected by the final contact force and over travel of the DRMCCB. According to the specification of the low voltage circuit breaker, the final contact force is designed to prevent the contact from repelling off before the instantaneous release triggers the operating mechanism, and also to concern with temperature rise of circuit breaker. And the over travel is designed to ensure a credible contact between the two contacts in case of contact abrasion. Both the values of the final contact force and over travel must satisfy this requirement.

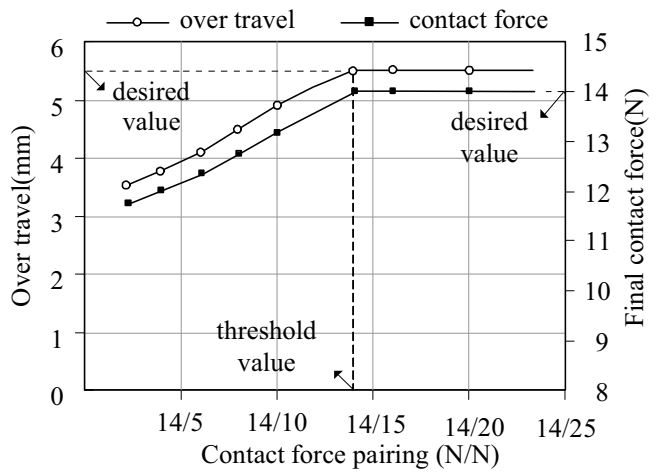

Figure 14. Influence of contact force pairing on final contact force and over travel of DRMCCB

For the DRMCCB, given a set of contact force pairing, the final contact force and over travel can be calculated by ADAMS according to the equilibrium positions of the two contacts. Fig. 14 shows variations of final contact force and over travel with contact force pairing of DRMCCB. It is learned from the figure that when the upper contact force is specified, both the final contact force and over travel decrease with drop of lower contact force. When the lower contact force becomes smaller than a threshold value, the breaker fails to promise its desired values of final contact force and over travel. The threshold value, however, is usually equal to the upper 
contact force. Therefore, in order to keep desired values of the final contact force and over travel, the lower contact force should not be less than the upper contact force.

\section{CONCLUSIONS}

a) A calculation model that enables us to predict breaking capacity of DRMCCB is realized by carrying out second-development of ADAMS, in which a set of differential equations describes the coupling of complex mechanical movement, electric circuit, magnetic field and dynamic mathematic arc model is set up. With this model, influence of different contact force pairing on breaking capacities of the DRMCCB can be analyzed.

b) When the upper contact force is determined, the breaking capacity of the DRMCCB increases with drop of the lower contact force.

c) In order to keep desired values of the final contact force and over travel, the lower contact force should not be less than the upper contact force.

d) Concerning the electromagnetic forces acting on the upper contact are much bigger than that of the lower contact, in order to yield a maximum breaking capacity of the DRMCCB, it is not necessary for the lower contact force to be much bigger than upper contact force for reason of preventing contact welding.

\section{REFERENCES}

[1] Meunier G, Abri A. Simulation of the arc interruption in circuit breaker. The 5th Int. Symp. On Switching Arc Phenomena, Lodz, Poland, 1985, pp.105-109.
[2] Belbel E M, Lauraire M. Behavior of switching arc in low-voltage limiter circuit breakers. IEEE Trans on CHMT. 1985, CHMT-8(1),pp. 312 .

[3] Smeets R P P, Sloot J G J, and R.D.Damstra. Computer simulation of the operation of a low_voltage miniature circuit breaker. In: Proc 1st Int Conf on ECAAA, Xi'an (China), 1989, pp.328-332.

[4] Sloot J G J, Smeets R P P. The simulation of a new type MCB in a lowvoltage network. In: Proc 6th Int Conf on Switching Arc Phenomena, Lodz (Poland), 1989, pp.62-66.

[5] Pohl F. Simulation of short circuit interruption with dynamic opening, low voltage power circuit breakers. In: Proc 6th Int Conf on Switching Arc Phenomena, Lodz (Poland), 1989.

[6] H. Stammberger, H. Pursch, A. Zacharias, and P. Terhoeven. Simulation of the temporal behavior of circuit breakers and motor start. Proc. $50^{\text {th }}$ IEEE Holm Conf. Elect. Contacts, Seattle, WA, Sep. 20-23,2004, pp.3540

[7] Gregory G D, Hall W M. Predicting molded-case circuit breaker let through characteristics in an electrical system under short-circuit conditions. IEEE Trans on Industry Applications. 1993, 29(3), pp.548555.

[8] Anheuser M. Simulation verification and validation of the short-circuit switching behavior of low voltage power circuit breakers. In: Proc 8th Int Conf on Switching Arc Phenomena, Lodz (Poland), 1997,pp. 38-42.

[9] J Zhang J, D G Chen, and J Fu. Mathematic model of magnetic field driven arc in arc chamber of low voltage circuit breaker. Proc ICECT'99, Nagoya（Japan）, 1999, pp.281-288.

[10] Xingwen Li, Degui Chen, Yunfeng Wang, Qian Wang, and Yingsan Geng. Analysis of the interruption process of Molded Case Circuit Breakers. IEEE Trans. on components and packaging technologies. Vol(30), 2007, pp. 375-382.

[11] Paul G. Slade (ed.): Electrical Contacts New York, Basel: Macel Dekker, 1999.

[12] X Zhou and $P$ Theisen. Investigation of arcing effects during contact blow open process. Proc $44^{\text {th }}$ IEEE Holm conf on Electrical Contacts [C], Arlington, 1998, pp.100-108. 


\title{
Rotational Drive of Break Arcs using a Permanent Magnet Embedded in an Electrical Contact
}

\author{
Junya Sekikawa and Takayoshi Kubono \\ Faculty of Engineering, Shizuoka University, \\ Hamamatsu, Japan. \\ E-Mail: tjsekik@ipc.shizuoka.ac.jp
}

\begin{abstract}
Break arcs are generated between silver electrical contacts in a DC $42 \mathrm{~V}$ resistive circuit. Circuit current when the contacts are closed is varied from $5 \mathrm{~A}$ to $21 \mathrm{~A}$. Break arcs are driven by the radial magnetic field that is formed between the electrical contacts with a permanent magnet embedded in the cathode. The arc motion is taken with a high-speed camera and contact surfaces are observed after break operations. Experimental results with the magnet are compared with those without the magnet to confirm the effect of the embedded magnet. For break operations with the magnet following results are shown for each circuit current. Break arcs are rotationally driven by the radial magnetic field in the direction according to Lorentz force. The shortening effect of the arc duration is confirmed. The traces of the arc spots on the contact surfaces are ring-shaped, wide and uniform. This result shows the prevention effect of local erosion of electrical contacts. The rotational frequency is increased by the increase of Lorentz force to drive the break arc rotationally with the increase of the arc current.
\end{abstract}

Keywords-break arc; magnetic drive; arc discharge; electrical contacts; permanent magnet; relay

\section{INTRODUCTION}

The range of use of the direct current circuit is extending in many fields, for example, renewable energy system, cars, and power supply. In those systems the higher voltage and diversification of the system voltage are developed to improve efficiency of electric power system. The generation of the arc discharge between the contacts is inevitable when the direct current is broken by the electrical contacts. Break arcs occurring between electrical contacts have been investigated [1, 2]. When the break arcs are generated at a same position, electrical contacts are eroded intensely and locally. Magnetic drive of break arcs by the transverse dc magnetic field was reported to investigate the dependence of the arc duration on the magnetic flux density for $\mathrm{Ag}$ and $\mathrm{Pd}$ contact pairs [3]. Rotational motion of the break arc was reported using the permanent magnet installed outside the electrical contacts in a circuit interrupter [4]. We have previously reported that the arc duration of the break arc was shortened by the transverse magnetic field to the arc current using a permanent magnet [5, $6]$.

Using these methods mentioned above to shorten the arc duration, however, a special space to form the magnetic field is required in the switching device. This is disadvantage for miniaturization of devices. Therefore a new structure of electrical contact in which a permanent magnet was embedded was proposed by authors [7]. Some effect of the magnet is confirmed for a DC $42 \mathrm{~V}-10 \mathrm{~A}$ resistive circuit. The break arcs were rotated with the radial magnetic field formed by the permanent magnet embedded in the cathode. In this paper the effect of the new contact structure is examined by breaking the DC $42 \mathrm{~V}$ resistive circuit for different circuit current when the contacts are closed. The circuit current is varied from $5 \mathrm{~A}$ to $21 \mathrm{~A}$.

\section{EXPERIMENTAL METHODS}

\section{A. Experimental circuit and conditions)}

The experimental circuit is shown in Fig.1. The circuit is composed of a DC $42 \mathrm{~V}$ power supply, a load resistor $R$, electrical contacts and a reference resistor $(1 \Omega)$ to measure the current. These resistors are non-inductive wire wound resistors. Circuit current when contacts are closed is varied from $5 \mathrm{~A}$ to 21A. The load resistor $R$ is changed to set the circuit current. Material of contacts is pure silver (99.99\%). As shown in Fig.2 the anode is a column whose diameter is $5.0 \mathrm{~mm}$ and the cathode is a pipe whose outside and inside diameters are $5.0 \mathrm{~mm}$ and $3.0 \mathrm{~mm}$ respectively. Photographs of contact surfaces before experiments are shown in Fig.3.

Contact surfaces are finished with a fine emery paper (\#3000) and the contacts are treated with ultrasonic cleaning in ethanol for 15 minutes and in pure water for 15 minutes before each series of experiments with and without the permanent magnet in the cathode. The contact force is controlled to $7.8 \mathrm{~N}$. Electrical contacts are separated at constant speed $10 \mathrm{~mm} / \mathrm{s}$. The cathode is fixed and the anode is movable. The contact opening apparatus is the same as described in our previous paper [8]. Time evolutions of the arc voltage and current are measured with $\mathrm{A} / \mathrm{D}$ converter boards (1MS/s, 12bit) and a personal computer.

\section{B. Permanent magnet}

The permanent magnet embedded in the cathode is a nickel-plated neodymium magnet. As shown in Fig.2 the diameter and length of the magnet are $3.0 \mathrm{~mm}$ and $5.0 \mathrm{~mm}$, respectively. The $\mathrm{N}$ pole of the magnet is faced to the anode. The gap between the surfaces of the cathode and magnet is $1.0 \mathrm{~mm}$. Surface photographs of the cathode and anode before experiments with the magnet are shown in Fig.3. Radial magnetic field is formed by the magnet embedded in the 
cathode between the contact surfaces. The magnetic flux density around the magnet is measured with a Gauss meter (MG-701, Magna). The magnetic flux density of radial component of the magnetic field is $64 \mathrm{mT}$ at $r=2.0 \mathrm{~mm}$ and $z$ $=1.0 \mathrm{~mm}$. The $r$ is radial position from the center axis of the magnet and the $z$ is the position from the magnet surface on the center axis of the magnet.

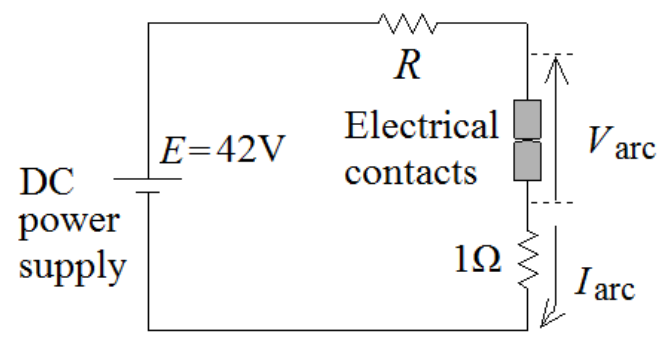

Figure 1. Experimental circuit.

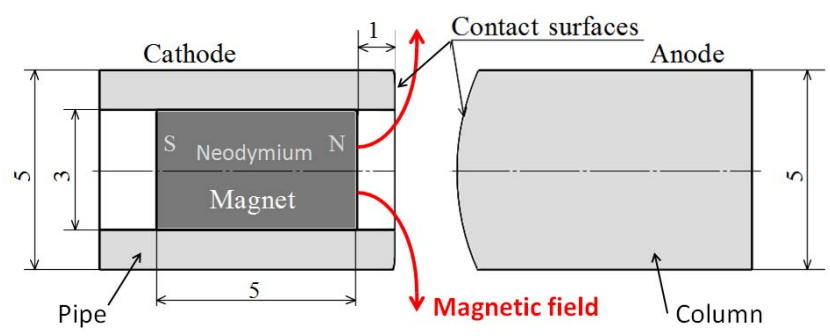

Figure 2. Cross section of contacts

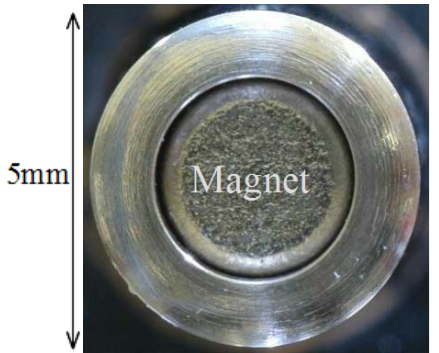

(a) Cathode

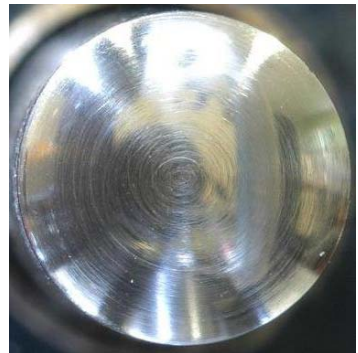

(b) Anode
Figure 3. Surface photographs of cathode and anode before experiments with the magnet.

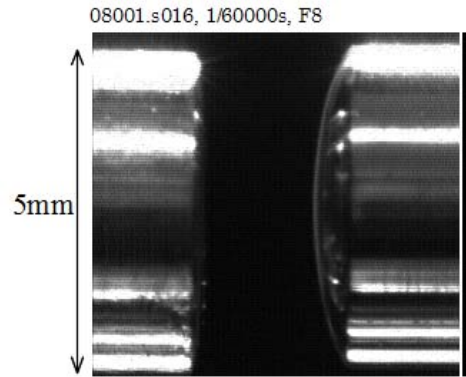

(a) Before experiments

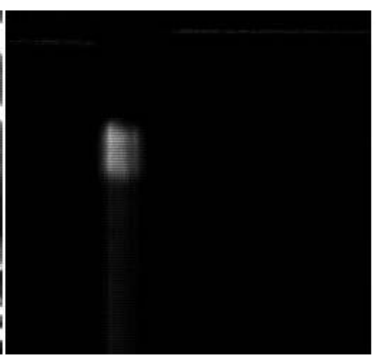

(b) Break arc
Figure 4. Images taken with high-speed camera.

(a) Before experiments, (b) An example of image of break arc with the magnet after $30 \mathrm{~ms}$ from start of contact opening $(I=10 \mathrm{~A})$.

\section{High-speed camera}

The high-speed camera (FASTCAM-PCI 1K, Photron) is set to take images from horizontal direction. The frame rate of the camera is 1000 frames/s. The image shown in Fig.4 (a) was obtained before experiments. The cathode is on the left side and the anode is on the right side of the image. An example of an image of a break arc with the magnet in the cathode is shown in Fig.4 (b). The break arc is observed between the contacts.

\section{EXPERIMENTAL RESULTS AND DISCUSSION}

\section{A. Arc duration and position of break arc on the contact surfaces without and with the magnet}

Typical time evolutions of the arc voltage and current are shown in Fig. 5. Figure 5 (a) and (b) indicate experimental results of $I=14$ A without and with the magnet in the cathode, respectively. As shown in Fig.5 the arc voltage increases linearly with time after arc ignition. Fluctuation level of the arc voltage and current with the magnet is higher than that without the magnet. The arc duration with the magnet is shorter than that without the magnet.

Typical time evolutions of the arc current for each circuit current $I$ are shown in Fig. 6. In addition, the dependence of arc duration $D$ on the circuit current when contacts are closed $I$ is shown in Fig.7 for cases without and with the magnet. Each plot in Fig. 7 indicates the averaged arc duration for 10 times break operations. As shown in Fig. 6 and 7 the arc duration with the magnet is shorter than that without the magnet for each circuit current $I$. The arc duration is shortened by the effect of the magnet in the cathode. This shortening effect may be caused by the increase of the arc voltage because of the cooling effect of the arc column with the rotational motion.

Photographs of contact surfaces of the cathode and anode

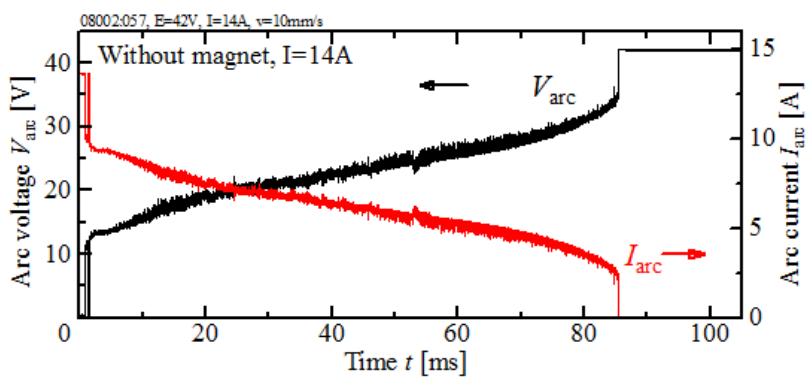

(a) Without the magnet

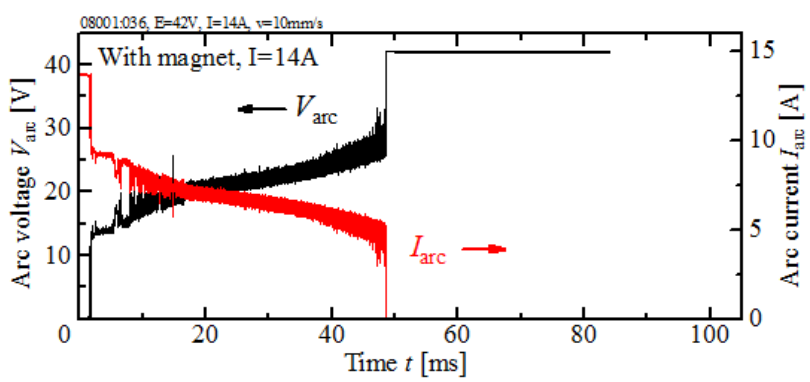

(b) With the magnet

Figure 5. Time evolutions of the arc voltage and current. $(I=14 \mathrm{~A})$ 


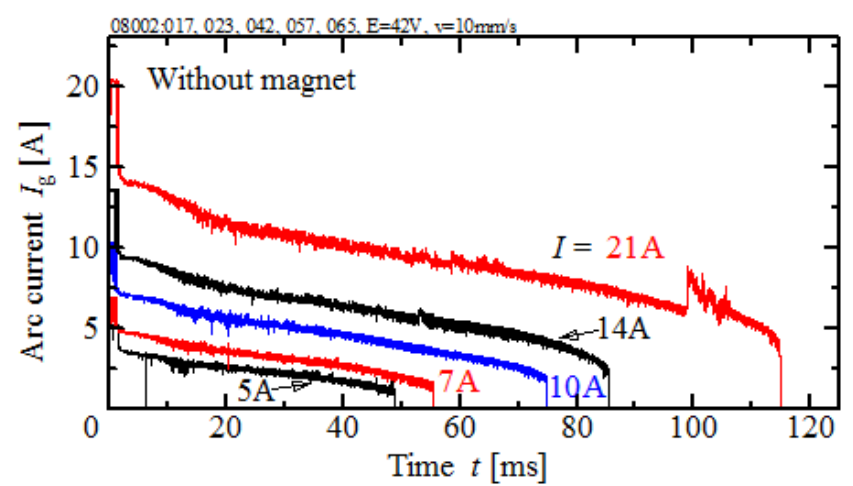

(a) Without the magnet

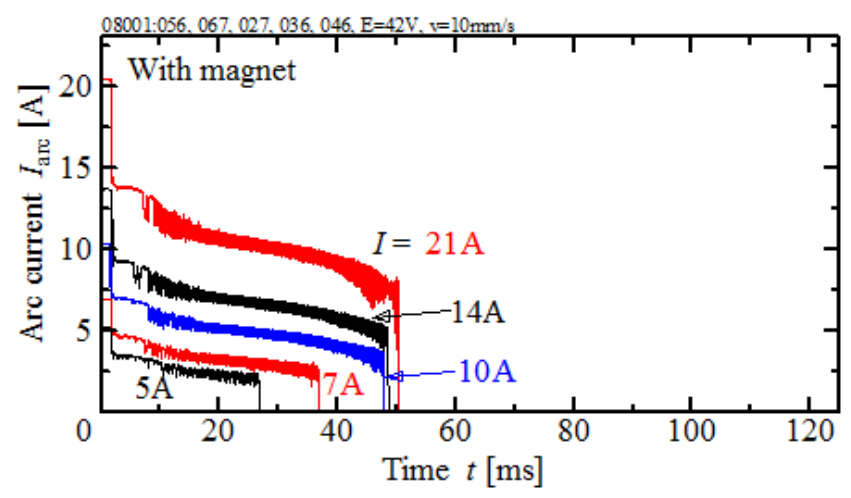

(b) With the magnet

Figure 6. Time evolutions of the arc current for each circuit current when the contacts are closed $I$.

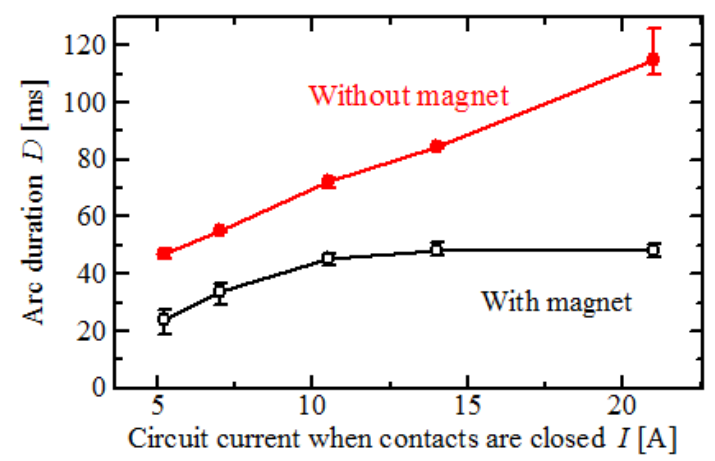

Figure 7. Dependence of arc duration $D$ on the circuit current when contacts are closed $I$.

without and with the magnet after break operations are shown in Fig. 8. Figure 8 (a) and (b) are photographs taken after each operation of Fig.5 (a) and (b), respectively. As shown in Fig. 8 (a) the trace of the break arc localize on the small area of contact surfaces without the magnet. The trace of the cathode spot is on the top left of the cathode surface and is arrowed in Fig. 8 (a). The images of the break arc observed with the highspeed camera are shown in Fig. 9 without the magnet. It is indicated that the break arc almost stays on the upper area between the contacts. The result corresponds to the traces on the contact surfaces shown in Fig. 8 (a).

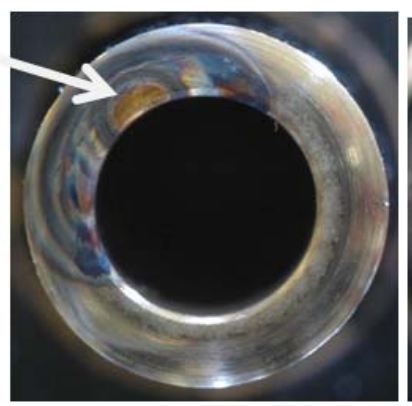

Cathode

(a) Without the magnet

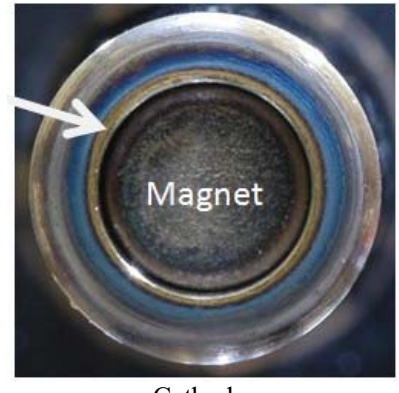

Cathode

(b) With the magnet

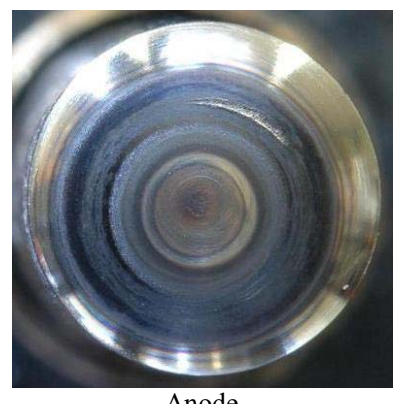

Anode

Figure 8. Surface photographs of cathode and anode after break operations $(\mathrm{I}=14 \mathrm{~A})$. Traces of arc root on the cathode surface are arrowed in images of cathode surfaces.
For the case with the magnet, the images of the break arc observed with the high-speed camera are shown in Fig. 10. The position of the break arc does not stay on a certain area between the electrical contacts. The break arc moves widely between the contacts during the exposure time of $1 / 20000 \mathrm{~s}$ $(=50 \mu \mathrm{s})$. As shown in Fig. 8 (b) the ring-shaped traces on the cathode surface exist near the inside of the cathode pipe. The trace of the cathode spot is arrowed in Fig. 8 (b). The traces on the cathode and anode surfaces are wide, uniform and ringshaped. Therefore it is predicted that the break arc is rotated by the radial magnetic field of the magnet. This result shows the prevention effect of local erosion of electrical contacts.

\section{B. Motion of break arc with the magnet}

To investigate the motion of the break arc with the magnet, the enlarged time evolution of the arc voltage and current of Fig. 5 (b) and the images of the break arc are shown in Fig. 11 (a) when $I=14 \mathrm{~A}$. As shown in Fig. 5 the fluctuation level of the arc voltage with the magnet is larger than that without the magnet. The fluctuation of the arc voltage and current with the magnet is periodic as shown in Fig. 11 (a). Examining the relation between Fig. 11 (a) and Fig. 11 (b) in detail, the arc current is lower level when the break arc is at lower position (at 22 and $24 \mathrm{~ms}$, labeled L), and the arc current is higher when the break arc is at upper position (at $23 \mathrm{~ms}$, labeled U). The break arc is in the middle position and at the backward of the anode when the arc current is decreasing (at 26ms, labeled B). And the break arc is in the middle position and at the forward of the 


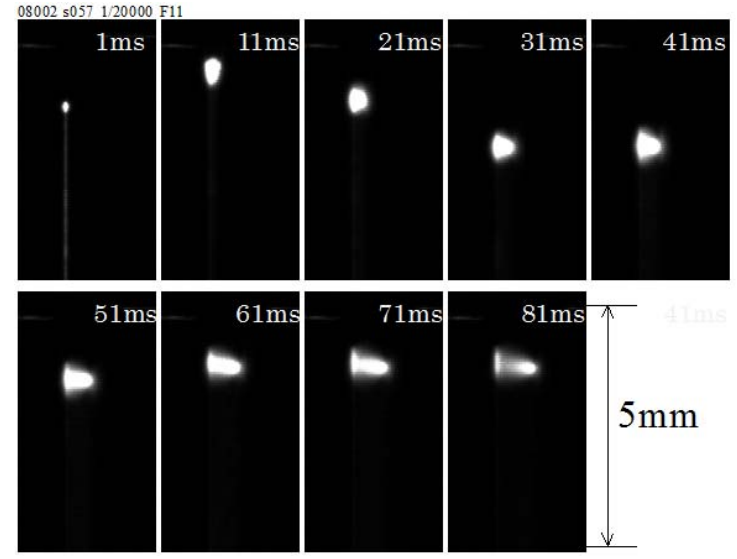

Figure 9. Images of break arc without the magnet observed by the highspeed camera with F11 and 1/20000s. The time indicated under each image corresponds to Fig. 5 (a) when $\mathrm{I}=14 \mathrm{~A}$.

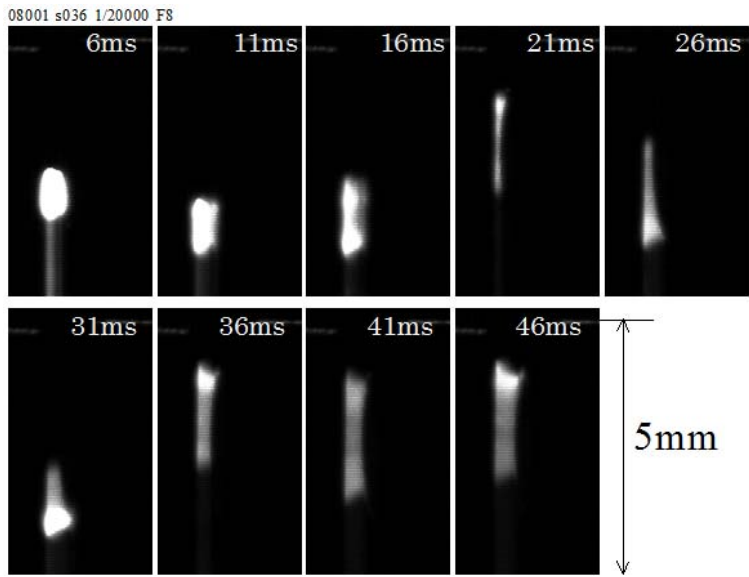

Figure 10. Images of break arc with the magnet observed by the high-speed camera with F8 and 1/20000s. The time indicated under each image corresponds to Fig. 5 (b) when I=14A.

anode when the arc current is increasing (at $25 \mathrm{~ms}$ and $27 \mathrm{~ms}$, labeled F). Schematic of the arc current is shown in Fig.12(a). Solid line in Fig.12(a) shows the periodic oscillation of arc current shown in Fig.11(a). Labels in Fig.11 is indicated in Fig.12(a). For example the arc current is decreasing when the break arc is in the middle position at the backward of the anode as labeled B. Therefore the order of motion is B, L, F and $\mathrm{U}$ as shown in Fig.12(a). The break arc rotates clockwise on the cathode surface when the cathode surface is seen as shown in Fig.12(b). The rotation is in the direction according to Lorentz force. Similar rotational motion is observed for each circuit current $I$.

As shown in Fig. 4(a) the position of the center axis of the anode is slightly higher than that of the cathode. The gap between the anode surface and the inner edge of the cathode pipe when the break arc exists at the lower position of the gap is slightly larger than that at the higher position of the gap. The voltage of longer break arc is higher. Therefore the arc voltage and current are periodically fluctuated by the change of arc length with the rotational motion of the break arc.

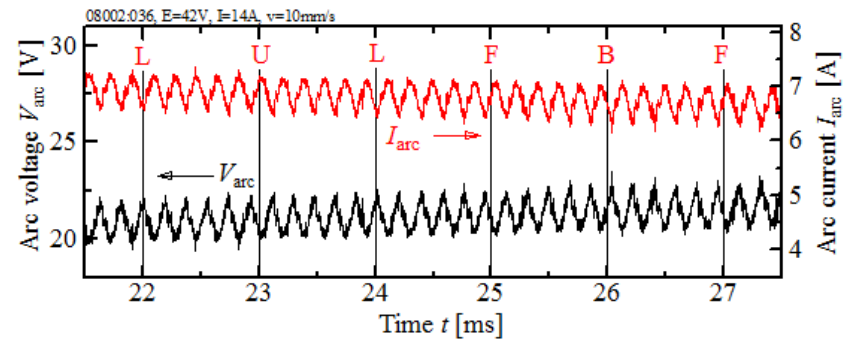

(a) Enlarged time evolution of arc voltage and current of Fig.5(b). Labels indicate the position of the break arc
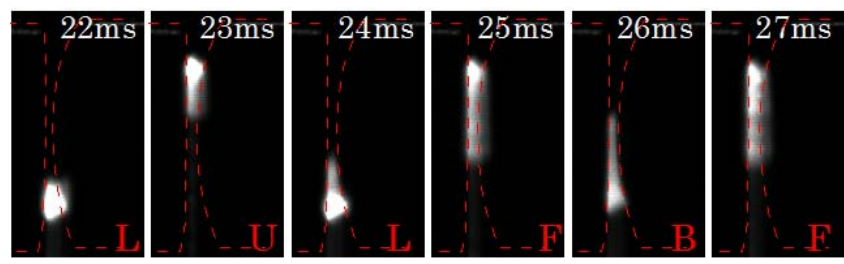

(b) Images of break arc with the magnet

Figure 11. (a) Enlarged time evolution of arc voltage of Fig. 5(b) with the magnet and (b) Images of break arc with the magnet observed by the highspeed camera with F8 and 1/20000s. (I=14A) Predicted positions of the cathode and anode are indicated as dashed red lines.

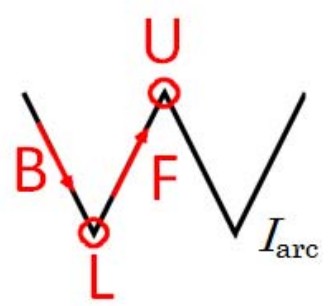

(a) Schematic of arc current and corresponding arc positions labeled in Fig.11.

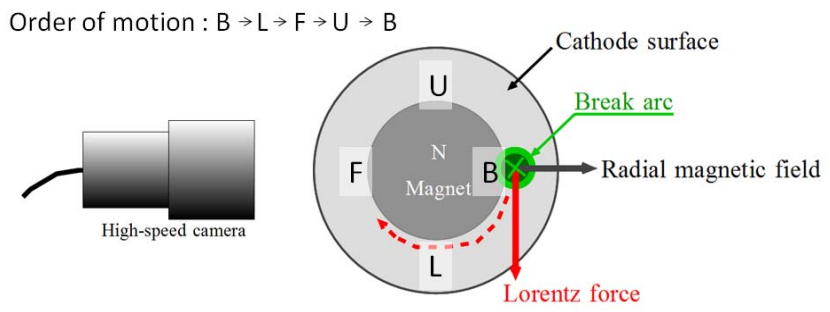

(b) Motion of break arc on the cathode surface

Figure 12. Rotational motion of break arc.

\section{Rotational frequency of break arc}

Enlarged time evolutions of arc current of Fig. 6(b) are shown in Fig. 13. As shown in Fig. 13 the arc current periodically fluctuates with the magnet for each circuit current when contacts are closed $I$. The period of the fluctuation becomes short when the current $I$ is increased. 


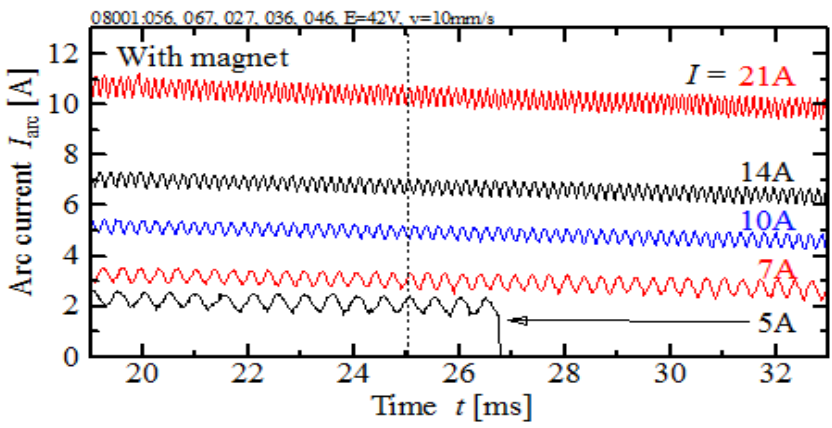

Figure 13 Enlarged time evolutions of arc current of Fig. 6(b) with the magnet for each circuit current when contacts are closed $I$.

Table 1. Rotational period and frequency of break arc when time $t$ is $25 \mathrm{~ms}$.

\begin{tabular}{|c|c|c|c|c|c|}
\hline$I[\mathrm{~A}]$ & 5 & 7 & 10 & 14 & 21 \\
\hline$I \operatorname{arc}[\mathrm{A}]$ & 2.1 & 3.0 & 4.9 & 6.7 & 10.3 \\
\hline$T[\mathrm{~ms}]$ & 0.49 & 0.33 & 0.23 & 0.18 & 0.15 \\
\hline$f[\mathrm{kHz}]$ & 2.0 & 3.0 & 4.3 & 5.6 & 6.7 \\
\hline
\end{tabular}

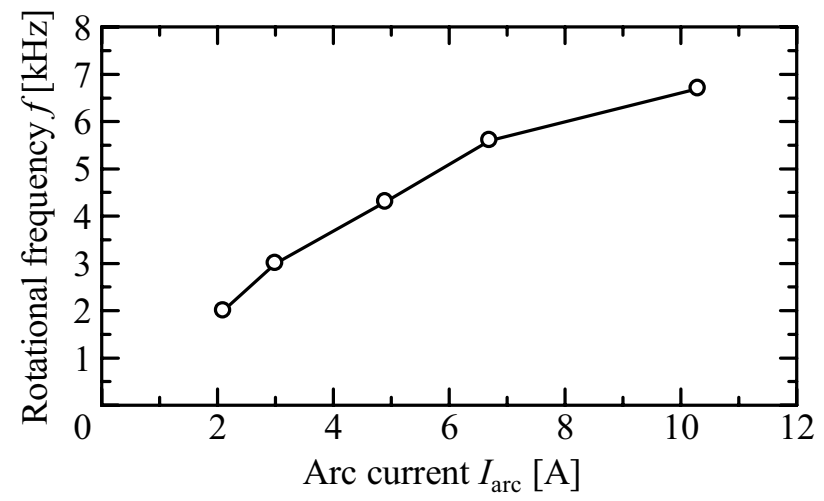

Figure 14 Dependence of the rotational frequency $f$ on the arc current $I_{\text {arc. }}$.

The rotational period $T$ and frequency $f$ of break arc are shown in Table 1 for each circuit current $I$. The period $T$ is analyzed when the time is $25 \mathrm{~ms}$ (shown as dotted line in Fig. 13). Dependence of the rotational frequency $f$ on the arc current $I_{\text {arc }}$ is shown in Fig. 14. The rotational frequency $f$ is increased by the increase of Lorentz force to drive the break arc rotationally with the increase of the arc current $I_{\text {arc }}$.

\section{Summary}

Break arcs were generated between silver electrical contacts in the DC $42 \mathrm{~V}$ resistive circuit. Circuit current when the contacts were closed was varied from 5A to $21 \mathrm{~A}$. Break arcs were driven by the radial magnetic field that was formed between the electrical contacts with the permanent magnet embedded in the cathode. The arc motion was taken with the high-speed camera and contact surfaces were observed after break operations. Experimental results with the magnet were compared with those without the magnet to confirm the effect of the embedded magnet. For break operations with the magnet following results were shown for each circuit current.

- Break arcs were rotationally driven by the radial magnetic field in the direction according to Lorentz force.

- The shortening effect of the arc duration was confirmed.

- The traces of the arc spots on the contact surfaces were ring-shaped, wide and uniform. This result showed the prevention effect of local erosion of electrical contacts.

- The rotational frequency $f$ was increased by the increase of Lorentz force to drive the break arc rotationally with the increase of the arc current $I_{\text {arc }}$.

\section{ACKNOWLEDGMENT}

This work was supported by KAKENHI (20760182).

\section{REFERENCES}

[1] R.Holm, Electric Contacts, 4th ed. New York: Springer, 1967, pp.275286.

[2] P.G. Slade, Electrical Contacts Principles and Applications, Marchel Dekker, New York, Basel, 1999, pp.433-486.

[3] Hisami Sasaki, Hiroshi Inoue, Daitaro Okuyama, "A Measurement of Contact Breaking Arc Duration with Transverse dc Magnetic Field," IECE Technocal report, Vol. 83, No. 166, EMC83-25, pp. 9-16, 1983.

[4] Shigeru Kasai, Tatsumi Ide, "Study of Arc Interruption Utilizing Arc Column Rotation Phenomena," Proceedings of the 23rd ICEC together with the 6th IS-EMD2006, Sendai, Japan, pp. 522-527, 2006.

[5] Junya Sekikawa, Takayoshi Kubono, "Motion of Break Arcs Driven by External Magnetic Field in a DC42 V Resistive Circuit," IEICE Trans. on Electronics, Vol. E91-C, No.8, pp.1255-1260, 2008.

[6] Junya Sekikawa, Takayoshi Kubono, "Magnetic drive by a small magnet of break arc occuring between electrical contacts in a DC42V/7-21A circuit," IEICE Technical Report Vol. 108, No. 145, EMD2008-31, pp. 49-54, 2008.

[7] Junya Sekikawa, Takayoshi Kubono, "Rotational Motion of Break Arcs Driven by Radial Magnetic Field in a DC Resistive Circuit," IEICE Technical Report, Vol.108, No.296, EMD2008-101, pp. 145-148, 2008.

[8] Junya Sekikawa, Takayoshi Kubono, "Observation of Breaking Arcs of $\mathrm{Ag}$ or $\mathrm{Cu}$ Electrical Contact Pairs with a High-speed Camera," IEICE Trans. Electron., Vol. E87-C, No. 8, pp. 1342-1347, 2004. 


\title{
Experimental study on arcing process and contact erosion under transverse magnetic field
}

\author{
Guofu ZHAI ${ }^{1}$, Xue ZHOU ${ }^{1}$, Xiaodong ZHAO ${ }^{1}$, Chengyan LIU $^{2}$, Fubiao LUO ${ }^{2}$ \\ 1. School of Electrical Engineering, Harbin Institute of Technology, Harbin, China, 150001 \\ 2. Guilin Aerospace Electronics Ltd., P.O. Box 58, Guilin, China, 541002 \\ Email: zhouxue296@163.com
}

\begin{abstract}
Arc characteristics and contact erosion of $\mathrm{Ag}, \mathrm{AgNi}$ and $\mathrm{Ag} / \mathrm{SnO}_{2}$ contacts were studied when the opening arcs were driven by transverse magnetic field. The experiments were carried out with a DC $28 \mathrm{~V} / 10 \mathrm{~A}$ pure resistive load. The opening velocity varies from $10 \mathrm{~mm} / \mathrm{s}$ to $50 \mathrm{~mm} / \mathrm{s}$. The transverse magnetic fields are provided by magnets and their flux density on the contact axes varies from 0 to $200 \mathrm{mT}$. The experiments were conducted in a hermetic chamber filled with pure nitrogen. Time variations of arc images were recorded by using a high speed camera. Relationships between flux density and arc parameters, such as arc durations, 2-dimensional distribution of arc positions and arc velocities, were obtained for those contacts. Contact erosion was observed and material transfer mass was measured after breaking operations. Decline ratio of the arc durations and alleviation of the mass transfer by the magnetic field no longer increase with the increment of the flux density, so the critical flux density for each contact material were finally obtained. The results can be used to design magnetic blow contacts in hermetic relays.
\end{abstract}

Key Words - Electrical arc; Transverse magnetic field; Mass transfer; Arc root distribution.

\section{INTRODUCTION}

$\mathrm{T}$ The material transfer during arcing process is a main factor which decides lifetime of a switch. Study on the methods that accelerate extinguishment of the arc and alleviate the material transfer plays a foundational role on improving lifetime of switches in future.

Transverse magnetic field (TMF) and axial magnetic field (AMF) were adopted as an effective way to blow the arc and alleviate the contact erosion in high voltage and large current circuit breakers [1]-[3]. Studies on the TMF were falling into two categories: the one was simulation by solving a MHD model [4]-[5] and the other was experiment by analyzing time evolutions of arc images obtained by using a high speed camera [3], [6]-[8]. Influence of TMF on arc durations and arc roots positions on a pair of silver contacts was studied by Sekikawa [6]. Rotational motion of the arc driven by a radial magnetic field was then observed [7]. Arc root distributions on contact surface and one-dimensional arc motion velocities when TMF is applied were investigated in previous study [8].

In this paper, two-dimensional motion of arcs driven by TMF is studied. Influence of TMF on arc durations, material transfer and contact erosion is also investigated. The arcing atmosphere is nitrogen and the source voltage is DC28V, which is a typical condition in a hermetical relay used in aerospace and military field.

\section{EXPERIMENT CONFIGURATIONS AND CODITIONS}

\section{A. EXPERIMENT EQUIPMENT}

Figure 1 shows the experiment equipment [9] used in this paper. A control unit generates pulses according to instructions from an industrial computer. The pulses are amplified by a motor driver and used to drive a stepping motor, so that a lead screw rotates and a sliding plate moves in a set velocity. The sliding plate collides with an electrode holder and moves with it together, which ensure the holder and the electrode move in a constant velocity. A spring and a screw nut are adopted to provide an adjustable contact force. The opening velocity can be set to be from $1 \mathrm{~mm} / \mathrm{s}$ to 500 $\mathrm{mm} / \mathrm{s}$. A permanent magnet is mounted on back of the electrodes and its $\mathrm{S}$ polar is faced to the electrodes. This equipment is sealed in a chamber which is filled with one atmosphere of nitrogen. A glass window on the chamber wall is necessary for taking photos.

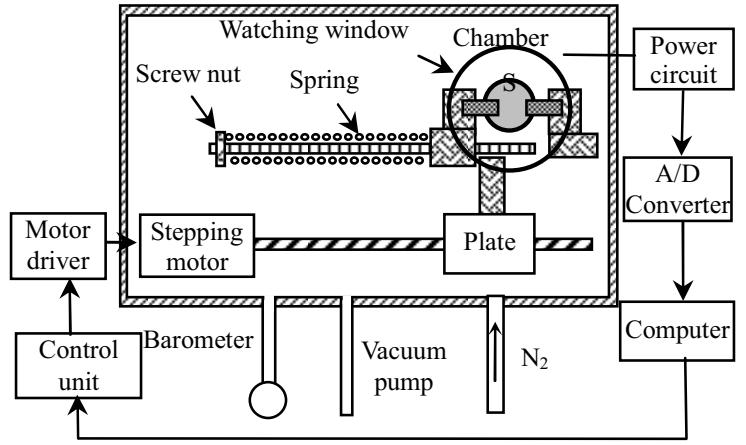

Fig. 1 Experiment equipment

The magnet, the contacts and a coordinate system employed are shown in Fig.2. The coordinate system is defined as follows: origin is the center of the stationary contact surface, $z$ is the normal direction of the contact surface, $x$ is faced to the glass window, and $y$ is vertical with the $x z$ plane. The center of the $\mathrm{S}$ polar of the magnet is $(-l, 0,0)$. Magnetic flux density on the origin is adjusted by varying $l$.

A high speed camera (Phantom V7.3) is used to take photos of arcs through the glass window, as shown in Fig.3. A mirror is mounted beside the contacts and its angle with both $x$ and $y$ axes is $\mathbf{4 5}$ degree, so that both front face and side face of the arc are recorded, and so does arc motion both in $x$ and $y$ direction. Focusing surface of the camera is set to middle position of the real contacts and their virtual images in the mirror, i.e., its coordinate is set to $(-\Delta x / 2,0,0)$. Arcs and their 
virtual images are projected onto CMOS sensor array of the camera. The upper part of the photo is the side face while the lower part is the front one.

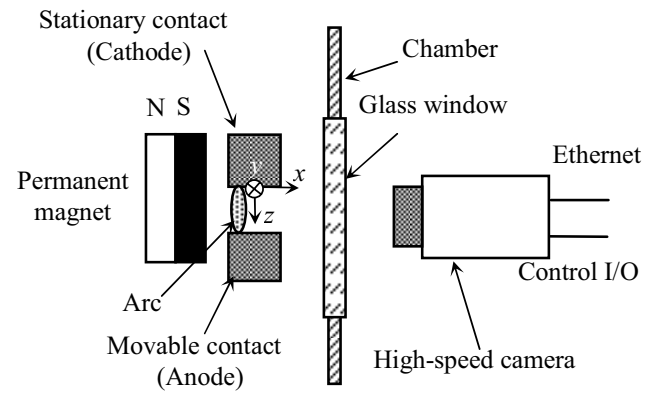

Fig. 2 Contacts and coordinate system

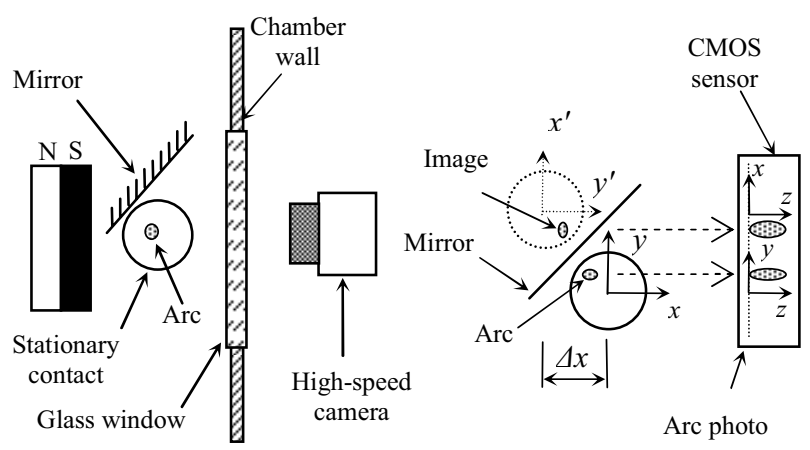

Fig. 3 Schematic figure of taking arc photos

\section{B. EXPERIMENT CONDITIONS}

Experiment circuit is shown in Fig.4. It consists of a $28 \mathrm{~V}$ DC power source, a load resistor $(2.7 \Omega / 350 \mathrm{~W})$, a sampling resistor $(0.1 \Omega / 50 \mathrm{~W})$ and a pair of contacts. The break current is $10 \mathrm{~A}$.

Parameters of the high speed camera are as follows: resolution is $256 \times 64$; frame rate is 100,000 frames per second; frame interval and exposure time are $10 \mu \mathrm{s}$ and $8 \mu \mathrm{s}$, respectively. Focus length of the lens is $90 \mathrm{~mm}$, object distance is about $252 \mathrm{~mm}$, and image distance is about 140 $\mathrm{mm}$. The difference between the object distances of the arc and its virtual image, which is within $6 \mathrm{~mm}$, may lead to magnification error of about $2 \%$. Ratio between pixels and millimeters is defined to be $P$ and its value is 25 pixels $/ \mathrm{mm}$.

A micro-weighing is adopted to measure contacts mass before and after 15,000 break only operations. Contact mass variations $\Delta m_{c}$ and $\Delta m_{a}$ are then determined. The accuracy is $10 \mu \mathrm{g}$.

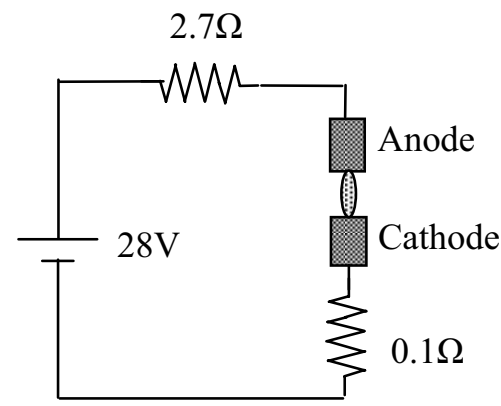

Fig. 4 Experiment circuit

\section{CONTACTS}

Contact materials are silver (99.9\%), AgNi alloy (Ni: 10\% $w t)$ and $\mathrm{Ag} / \mathrm{SnO}_{2}\left(\mathrm{SnO}_{2}: 12 \% w t\right)$. Diameter and length of the contacts are $3 \mathrm{~mm}$ and $10 \mathrm{~mm}$, respectively. Before the experiments, contact surfaces are firstly polished with a sand paper (\#2400). The polished contacts are cleaned with ultrasonic in ethanol for 15 minutes then in distilled water for 15 minutes, and finally dried in air.

A contact set mounted on the equipment is shown in Fig. 5. The camera is rotated for 90 degree to take photos of both the arcs and their virtual images. The rectangle zone in Fig. 5 shows an area of $256 \times 64$ pixels. The arcs and their virtual images are all within this area, and photos of this area are recorded during experiments.

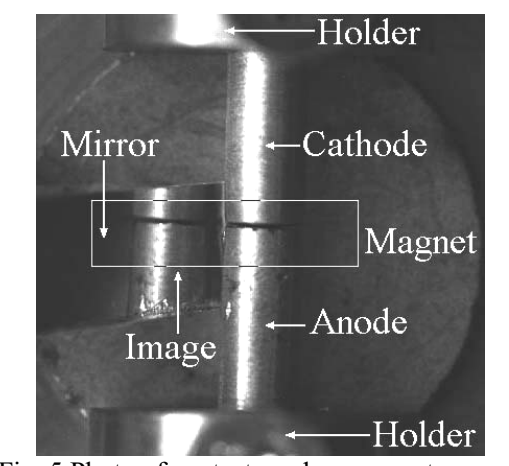

Fig. 5 Photo of contacts and permanent magnet

\section{OPENING VELOCITY AND MAGNETIC FIELD}

Opening velocity varies from $10 \mathrm{~mm} / \mathrm{s}$ to $50 \mathrm{~mm} / \mathrm{s}$ at a step of $10 \mathrm{~mm} / \mathrm{s}$. S pole of the magnet is faced to the contact set. Magnetic flux density is adjusted by changing the distance between the contacts and the magnet. Diameter of the magnet is $10 \mathrm{~mm}$, and material is neodymium iron boron $\left(\mathrm{Nd}_{2} \mathrm{Fe}_{14} \mathrm{~B}\right)$. The Lorentz force on the macro current is in $-y$ direction.

\section{E. IMAGE PROCESSING}

An image processing is necessary for obtaining anode arc roots and cathode arc roots from both the arc and its virtual image. An example of arc photo obtained by the camera is shown in Fig.6. As defined in Fig.6, arc root of the real arc is value of $y$, and arc root of the virtual one is value of $x$. Steps for processing the arc photos are as follows.

(1) Split an arc photo into two parts according to contact positions. One part is used for analyzing arc roots of the real arc. Another part is for arc roots of the virtual one.

(2) Get lengths of both the two arcs. The arc lengths are defined as $z_{r}$ and $z_{v}$ for the real arc and the virtual one, respectively.

(3) Calculate edge positions of both the two arcs by using edges detection arithmetic. A Canny operator is chosen for edges detection.

(4) If the arc length is smaller than 6 pixels, positions of the cathode and the anode arc roots are both mean position of the left and the right edges. In other words, the arc is treated as a line and there is no deviation between anode and cathode roots. 


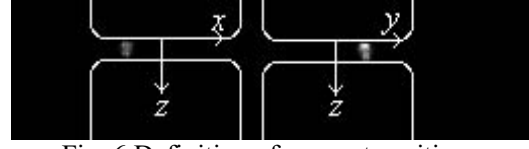

Fig. 6 Definition of arc root positions

(5) If the arc length is equal with or larger than 6 pixels, cathode and anode arc root is treated separately. The cathode arc root is mean position of the edges as $z$ varies from 1 to 3 , while the anode one is that of the edges as $z$ varies from $z_{r}$ to $z_{r}-2$ (or from $z_{v}$ to $z_{v}-2$ for the virtual arc).

Mean velocity of arc motion is calculated through using Eq (1) when time evolution of arc root positions on the $x y$ plane is determined.

$$
v_{\text {avg }}=\frac{1}{N-1} \sum_{n=1}^{N-1}|v(n)|=\frac{1}{N-1} \sum_{n=1}^{N-1} \frac{\left|l_{i}(n+1)-l_{i}(n)\right|}{P t}
$$

where, $v_{\text {avg }}$ is mean velocity, $N$ is frame number of arc photos, $v(n)$ is velocity at time of $(n \times 10) \mu \mathrm{s}, l_{i}$ is arc root position $(i=$ $x, y), P$ is ratio between pixels and millimeters and its value is 25 pixels $/ \mathrm{mm}$, and $t$ is frame interval and its value is $10 \mu \mathrm{s}$.

\section{RESULTS}

\section{A. ARC DURATIONS}

Figure 7 shows relationship between arc durations and opening velocities when magnetic is applied. It shows that the arc duration decreases as opening velocity increases no matter whether magnetic field is applied or not. Arc duration is shortened by the magnetic field. It can be easily noticed that arc duration decreases with the increment of flux density, except the Ag contact at the velocity of $30 \mathrm{~mm} / \mathrm{s}$. Excellent shortening ratio of arc duration is found at the $\mathrm{Ag}$ and $\mathrm{AgNi}$ contacts. Arc durations are decreased to $23 \%$ and $19 \%$ of that without magnetic field when the opening velocity is $10 \mathrm{~mm} / \mathrm{s}$. On the other hand, the ratio is only $82 \%$ on the occasion of $\mathrm{Ag} / \mathrm{SnO}_{2}$ under the same condition. Furthermore, Fig.7 (a) and (b) show that impact of the magnetic field on arc duration is not evident when the arc duration is shorter than 1 millisecond.

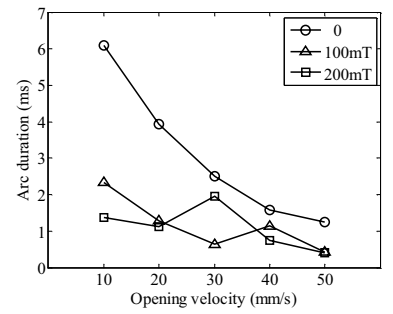

(a) $\mathrm{Ag}$

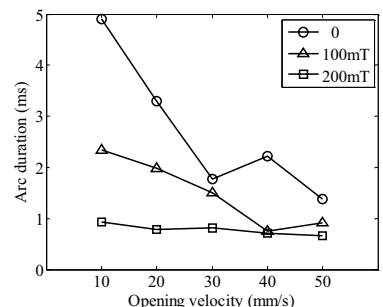

(b) $\mathrm{AgNi}$

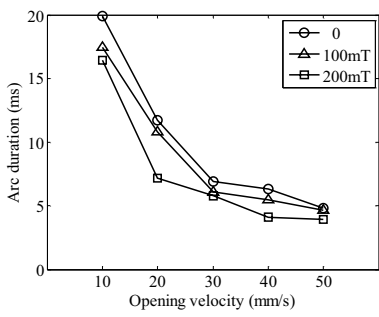

(c) $\mathrm{Ag} / \mathrm{SnO}_{2}$

Fig. 7 Relationship between arc durations and opening velocities

\section{B. ARC PHOTOS AND ARC POSITIONS}

It was found out that the most obvious shortening ratio of arc duration occurs at the opening velocity of $10 \mathrm{~mm} / \mathrm{s}$, so it is meaningful to analyze and discuss the arc photos at this velocity. Experiment data, whose arc duration is closest to the average arc duration of ten experiments, is selected for image analysis. The arc positions in those figures are mean positions of the cathode and anode roots.

Figure 8 shows arc voltages and their corresponding time evolutions of arc positions and photos when the contact material is $\mathrm{Ag}$. In these arc photos, dimension of the showing zone is $3 \mathrm{~mm} \times 0.8 \mathrm{~mm}$. Figure 8 (a) illustrates that when no
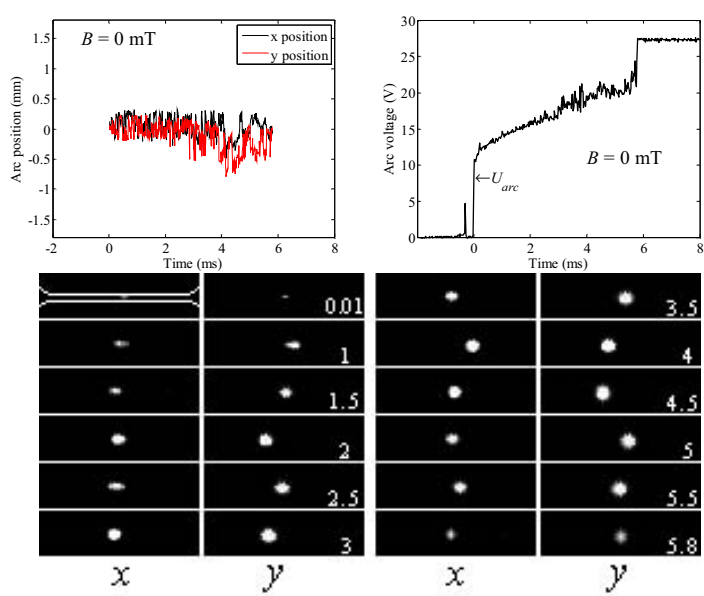

(a) $B=0 \mathrm{mT}$
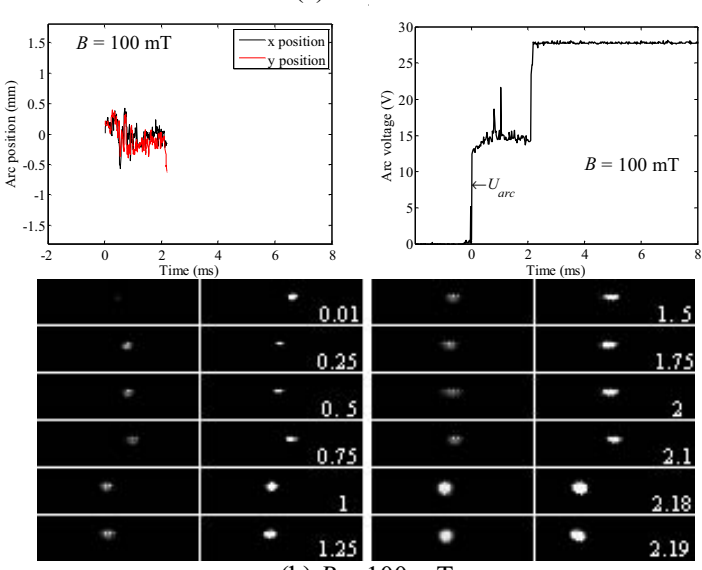

(b) $B=100 \mathrm{mT}$
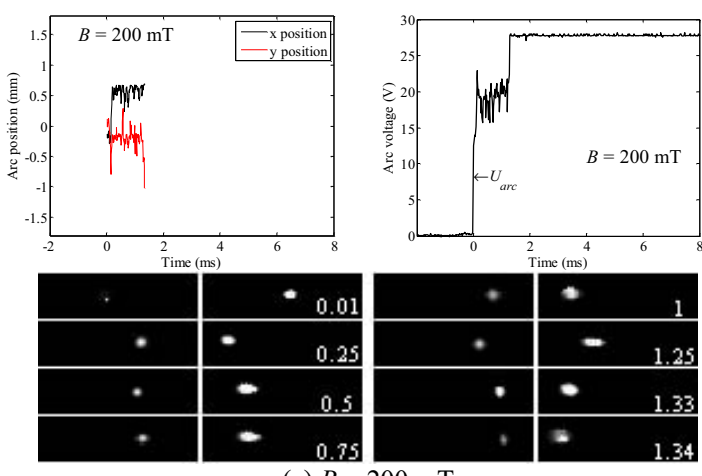

(c) $B=200 \mathrm{mT}$

Fig. 8 Time evolutions of arc images, positions and voltage (Ag) 

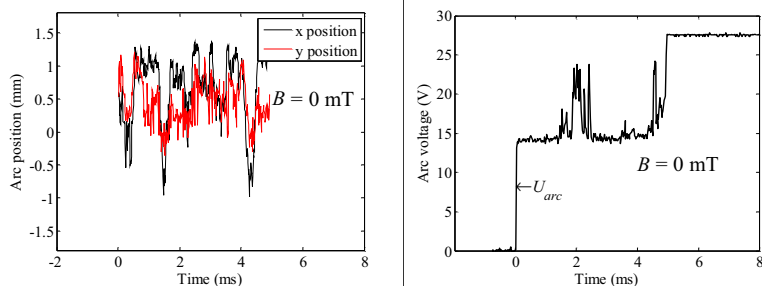

(a) $B=0 \mathrm{mT}$
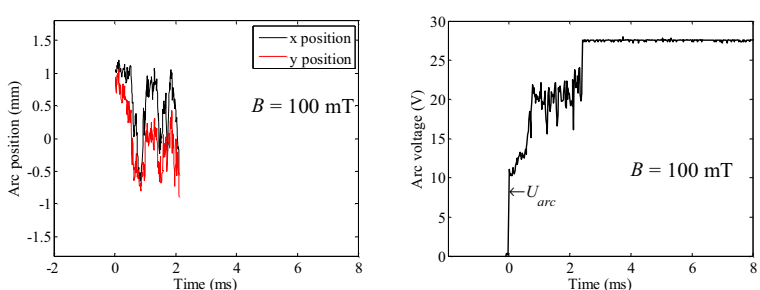

(b) $B=100 \mathrm{mT}$
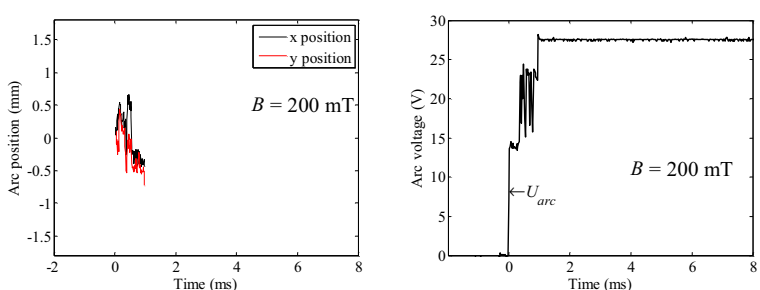

(c) $B=200 \mathrm{mT}$

Fig. 9 Time evolution of arc positions and voltage (AgNi)
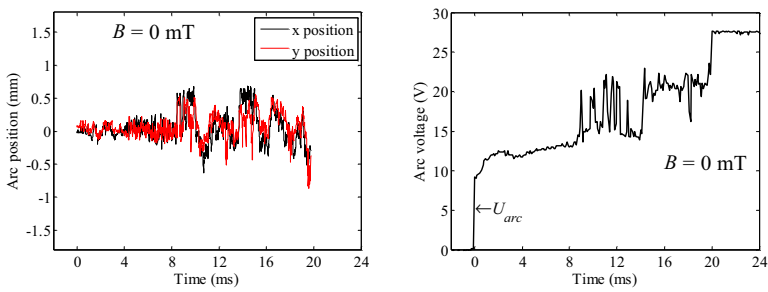

(a) $B=0 \mathrm{mT}$
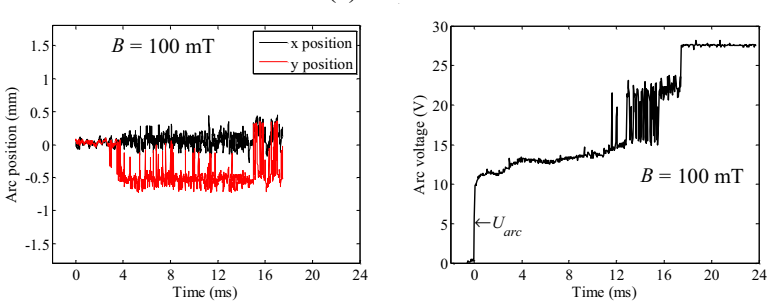

(b) $B=100 \mathrm{mT}$
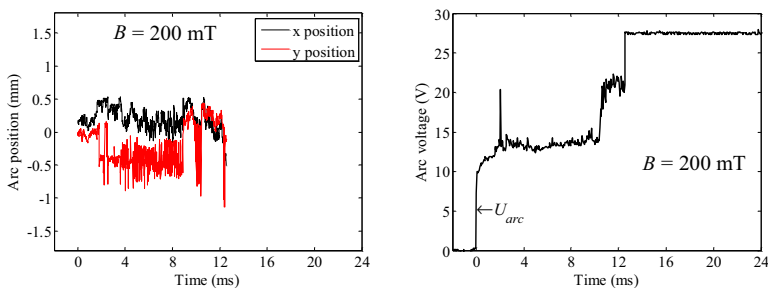

(c) $B=200 \mathrm{mT}$

Fig. 10 Time evolution of arc positions and voltage $\left(\mathrm{Ag} / \mathrm{SnO}_{2}\right)$

magnetic field is applied, the arc moves randomly and irregularly in both $x$ and $y$ directions in the contact gap. By comparing arc positions before and after $4 \mathrm{~ms}$, we know that amplitude and frequency of random motion become larger with arc lengthening. When the magnetic field is applied, arc motion in $x$ direction is not affected because no Lorentz force exists in this direction. Time evolution of $y$ positions shows that arc offsets to left side of the contacts and moves towards left with random fluctuations when the field is applied. At the time of $0.3 \mathrm{~ms}$ before arc extinguishment, the arc rapidly moves along the $-y$ direction to left side of the contacts then extinguishes.

Time evolutions of arc positions and voltage of $\mathrm{AgNi}$ and $\mathrm{Ag} / \mathrm{SnO}_{2}$ contacts are shown in Fig.9 and Fig.10. It can be known that $x$ positions of the arcs are still not affected by the magnetic field and offset of $y$ positions exists. It is obvious that the more acutely the arc voltage fluctuates, the more rapidly the arc positions moves, when the contacts material is $\mathrm{Ag} / \mathrm{SnO}_{2}$. On the other hand, the arc duration and arc length is so short on the occasion of $\mathrm{AgNi}$ contacts that the arc is directly driven to the contact edge and extinguished. Therefore, the arc position fluctuation cannot be clearly observed.

\section{TWO-DIMENSIONAL MOTION VELOCITY}

Figure 11 shows relationship between motion velocity and flux density. Figure 11 (a) indicates that arc motions in $x$ direction $\left(v_{x}\right)$ are not affected by the magnetic field. Figure 11 (b) reveals that arc motions in $y$ direction $\left(v_{y}\right)$ are affected by magnetic field, especially when the material is $\mathrm{AgNi}$ and $\mathrm{Ag} / \mathrm{SnO}_{2}$. When the flux density increases to $100 \mathrm{mT}, v_{y}$ of both $\mathrm{AgNi}$ and $\mathrm{Ag} / \mathrm{SnO}_{2}$ increases. When the flux density increases to $200 \mathrm{mT}, v_{y}$ of $\mathrm{Ag} / \mathrm{SnO}_{2}$ increases and that of $\mathrm{AgNi}$ decreases.

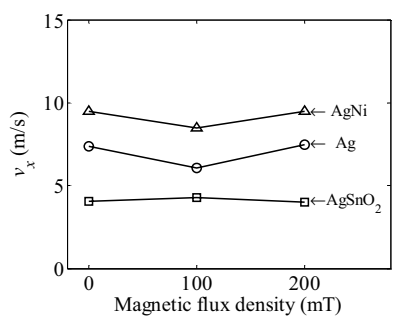

(a) $v_{x}$

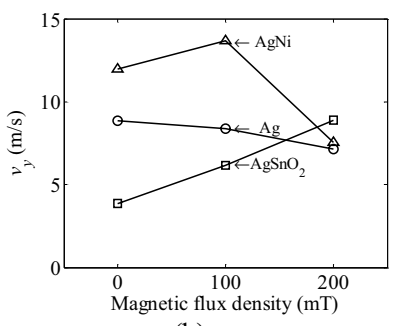

(b) $v_{y}$
Fig. 11 Relationship between motion velocities and flux density

\section{MATERIAL TRANSFER}

Figure 12 shows average mass variations of 15,000 break only operations at the velocity of $10 \mathrm{~mm} / \mathrm{s}$. The mass variations decrease with the increment of flux density for the $\mathrm{Ag}$ and $\mathrm{AgNi}$ contacts, as shown in Fig. 12 (a) and (b). Direction of material transfer is from cathode to anode when no magnetic field is applied. When flux density is $200 \mathrm{mT}$, arc length is shortened and material transfer direction is from anode to cathode. As the flux density increases, duration and length decrease so that transition of mass transfer direction from the cathodic loss stage to the compensation stage or, maybe, the anodic loss stage occurs [10], [11]. It can be known that when a certain flux density is applied, the mass accumulated during the anodic loss stage by the cathode is all transferred to the anode by the cathodic arc, i.e., the net zero erosion appears. This flux density for the $\mathrm{Ag}$ contact is between $100 \mathrm{mT}$ to $200 \mathrm{mT}$ under our experiment conditions, and it is about $200 \mathrm{mT}$ for AgNi. However, the duration 
(about $17 \mathrm{~ms}$ ) and length (about $170 \mu \mathrm{m}$ ) of $\mathrm{Ag} / \mathrm{SnO}_{2}$ contacts are not evidently shortened by the magnetic field, so no transition of transfer direction is observed and net zero erosion flux density cannot be determined at this velocity, as shown in Fig. 12 (c).

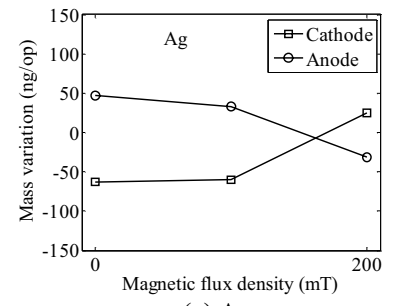

(a) $\mathrm{Ag}$

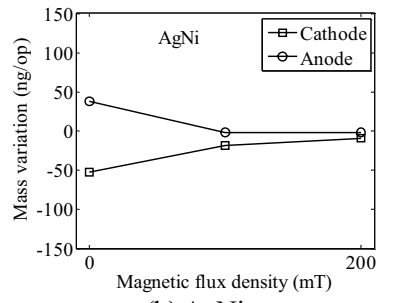

(b) $\mathrm{AgNi}$

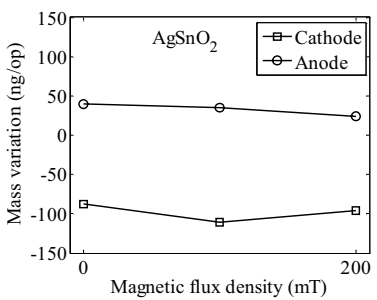

(c) $\mathrm{Ag} / \mathrm{SnO}_{2}$

Fig. 12 Relationship between mass variations and magnetic flux density

\section{E. CONTACT SURFACES}

Figure 13 shows photos of the contact surfaces after 15,000 break only operations when flux density is 0 and $200 \mathrm{mT}$. Craters appear on the cathode contacts of $\mathrm{Ag} / \mathrm{SnO}_{2}$ and tips are found on the anode ones, no matter whether magnetic field is applied or not. On the occasion of $\mathrm{Ag}$ and $\mathrm{AgNi}$ contacts, result changes. Craters also appear on the cathode and tips on the anode when no magnetic field is applied. On the contrary, craters are found on the Ag anode while tips on the cathode when magnetic field is applied. It is difficult to distinguish whether craters or tips appear on the AgNi contact when the field is applied. The craters and the tips on the contact surfaces also indicate the transition phenomena in Fig. 12. Moreover, Fig. 13 also shows that erosion zone deviates from work zone that carries current because of Lorentz force. This deviation may help decrease contact resistance when carrying current.

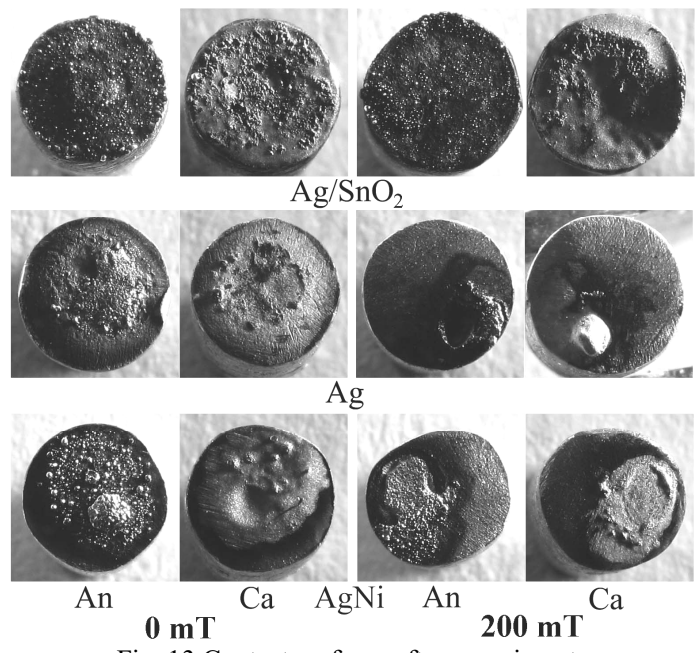

Fig. 13 Contact surfaces after experiments

\section{CONCLUSIONS}

(1) Magnetic field shortens arc durations. Shortening ratio of duration for $\mathrm{Ag}$ and $\mathrm{AgNi}$ is much better than that of duration for $\mathrm{Ag} / \mathrm{SnO}_{2}$.

(2) Arc motion can be divided to two components. One is in parallel with Lorentz force and the other is in vertical with it. Arcs move randomly in the vertical direction, and it moves along the Lorentz force with random fluctuations.

(3) Motion velocities in the vertical direction are not affected by magnetic field, while that of in the parallel direction are affected. The parallel velocities of arc on $\mathrm{Ag} / \mathrm{SnO}_{2}$ contacts are evidently accelerated by magnetic field.

(4) Magnetic field has greater influence on mass transfer of $\mathrm{Ag}$ and $\mathrm{AgNi}$ contacts than that of $\mathrm{Ag} / \mathrm{SnO}_{2}$ contacts. Transition of transfer direction is observed for $\mathrm{Ag}$ and $\mathrm{AgNi}$ contacts at the opening velocity of $10 \mathrm{~mm} / \mathrm{s}$. The craters and the tips on contact surfaces also indicate the transition. The flux density for net zero erosion is about $200 \mathrm{mT}$ for $\mathrm{AgNi}$ and $\mathrm{Ag}$, but it is not determined in this paper for $\mathrm{Ag} / \mathrm{SnO}_{2}$.

(5) A permanent magnet can be mounted beside contacts of switches to provide transverse magnetic field, which shortens arc duration and alleviates contact erosion, therefore, prolongs lifetime of the switches. Further studies will be focused on the arc blown by TMF at a relatively high opening velocity as in a practical relay.

\section{Reference}

[1] Z. Y. Liu, S. Y. Cheng, Y. S. Zheng, M. Z. Rong and J. M. Wang, "Comparison of vacuum arc behaviors between axial-magnetic-field contacts," IEEE Trans. Plasma Sci., vol.36, no.1, pp.200-207, Feb. 2008.

[2] Z. Q. Shi, S. L. Jia, J. Fu and Z. Wang, "Axial magnetic field contacts with nonuniform distributed axial magnetic fields," IEEE Trans. Plasma Sci., vol.31, no.2, pp.289-294, Apr. 2003.

[3] D. Gentsch and W. K. Shang, "High-speed observations of arc modes and material erosion on RMF- and AMF-contact electrodes," IEEE Trans. Plasma Sci., vol.33, no.5, pp.1605-1610, Oct. 2005.

[4] Q. Ma, M. Z. Rong, A. B. Murphy, Y. Wu, T. J. Xu and F. Yang, "Simulation and experimental study of arc motion in a low-voltage circuit breaker considering wall ablation," IEICE Trans. Electron., vol.E91-C, no.8, pp.1240-1247, Aug. 2008.

[5] Y. Wu, M. Z. Rong, Z. Q. Sun, X. H. Wang, F. Yang and X. W. Li, "Numerical analysis of arc plasma behavior during contact opening process in low-voltage switching device," J. Phys. D: Appl. Phys., vol.40, pp.795-802, 2007.

[6] J. Sekikawa and T. Kubono, "Motion of break arcs driven by external magnetic field in a DC42V resistive circuit," IEICE Trans. Electron., vol.E91-C, no.8, pp.1255-1259, Aug. 2008.

[7] J. Sekikawa and T. Kubono, "Rotational motion of break arcs driven by radial magnetic field in a DC resistive circuit," IEICE Technical Report, vol.108, no. 296, pp.145-148, Nov. 2008.

[8] G. F. Zhai, X. Zhou and W. Y. Yang, "Investigation on arc characteristics under axial and transverse magnetic fields," IEICE Technical Report, vol.108, no.296, pp.137-140, Nov. 2008.

[9] N. Ben Jemaa, J. L. Queffelec and D. Travers, "Some investigations on slow and fast arc voltage fluctuations for contact materials proceeding in various gases and direct current," IEEE Trans. Comp. Hybrid., Manufact. Technol., vol.14, no.1, pp.113-117, Mar. 1991.

[10] N. Ben Jemaa and L. Morin, "Transition from the anodic arc phase to the cathodic metallic arc phase in vacuum at low DC electrical level," IEEE Trans. Comp. Packag. Technol., vol.25, no.4, pp.651-655, 2002.

[11] N. Ben Jemaa, L. Morin, S. Benhenda and L. Nedelec, "Anodic to cathodic arc transition according to break arc lengthening," IEEE Trans. Comp. Packag., Manufact. Technol. - Part A, vol.21, no.4, pp.599-603, Dec. 1998 


\section{An experimental study on influences of silicon-type and non-silicone-type polymeric cured materials on contact resistance characteristics of relay contacts}

\author{
*Makoto Hasegawa \\ Chitose Institute of Science \& Technology \\ Chitose, Hokkaido, Japan \\ *hasegawa@photon.chitose.ac.jp
}

\author{
**Yoshiyuki Kohno and Hiroshi Ando \\ Kaneka Corporation \\ Osaka, Japan \\ **Yoshiyuki_Kohno@kn.kaneka.co.jp
}

\begin{abstract}
A new acryl-based polymeric material (containing no silicone components) was evaluated with respect to its effects on contact resistance characteristics of relay contacts. A commercially-available mechanical relay was sealed into a metal can with the acryl-based polymeric material, and placed in a heating chamber to operate 40,000 break operations of an inductive load current (DC 14V-1A and DC 20V-1.5A) under each of $80{ }^{\circ} \mathrm{C}$ and $120{ }^{\circ} \mathrm{C}$ conditions. Contact resistance was measured over the operations. The same experiments were also conducted with two types of specific silicone-containing polymeric materials, and the experimental results were compared. As a result, relays sealed with the acryl-based polymeric material showed no significant increases in contact resistances, while relays sealed with each of the siliconecontaining polymeric materials showed remarkable increases in contact resistances, especially under the $120^{\circ} \mathrm{C}$ condition, even in the case where the material is of a counter-measure type against contact failures.
\end{abstract}

Keywords - silicone; soloxane; contamination; contact failure; contact resistance; arc discharge; polyacrylate

\section{INTRODUCTION}

Contact failure caused by silicone contamination phenomenon was reported by Kitchen and Russell in 1975 in the presence of silicone possibly coming from siliconecontaining lubricants, greases, coatings, encapsulants, and potting compounds [1]. Ishino and Mitani pointed out that such contact failures caused by silicone were affected by silicone vapors coming from silicone-containing materials assembled in a relay [2]. Witter and Leiper reported that the particle size and form of silicone contamination, as well as the concentration, are the factors that influence contact behaviors, and further reported that the load current level in switching tests has large effect on build-up of contact resistance, where current levels around 0.5 A showed the most build-up [3]. Witter and Leiper later reported methods for measuring silicone contaminations on contact surfaces by use of EDXA, and presented semi-quantitative data showing the relationship between volume percentages of $\mathrm{Si}$ and contact resistances [4]. Tamai studied silicone contamination phenomena in detail and reported various acceleration and suppression factors for related contact failures as well as relationships with electrical load conditions [5-7]. Volm and Geisenberger reported that diffusion of silicone contaminants into sealed relays is possible, resulting in contact failures of the relays [8].

Based on such previous reports, various counter-measures for avoiding contact failures due to silicone contamination have been employed. However, it seems that we still have silicone contamination problems and degradation or failure of reliability of electrical contacts, for example, in automotive applications. Some of the reasons will be related to the fact that various design parameters (e.g., size) of mechanical relays and switches, as well as conditions where they are to be used, have changed. Silicone-containing materials are widely used in industrial apparatuses, and mechanical relays or switches may be unintentionally placed in the vicinity of such siliconecontaining materials.

Recently, an acryl-based non-silicone-type polymeric material has been developed, which is intended to be used as potting, coating and/or sealing materials in various industrial applications. The material was evaluated with respect to its influences on contact resistance characteristics of mechanical relays and the results were compared with those with siliconecontaining polymeric materials [9].

In this paper, further experiments were conducted in which a commercially-available mechanical relay with AgSnIn contacts was sealed into a metal can with the acryl-based nonsilicone-type polymeric cured product, and placed in a heating chamber to operate 40,000 break operations of an inductive load current (DC $14 \mathrm{~V}-1 \mathrm{~A}$ and DC $20 \mathrm{~V}-1.5 \mathrm{~A}$ ) at temperatures of $80{ }^{\circ} \mathrm{C}$ and $120^{\circ} \mathrm{C}$. Contact resistance was measured during the operations. The same experiments were also conducted with two types of specific silicone-containing polymeric cured products, and the experimental results were compared. As a result, relays sealed with the acryl-based polymeric cured product showed no significant increases in contact resistances, while relays sealed with each of the silicone-containing polymeric cured products showed remarkable increases in contact resistances, especially under the $120{ }^{\circ} \mathrm{C}$ condition, even in the case where the product was marketed as a type designed specifically to avoid contact failures. Thus, advantages of the newly-developed acryl-based non-silicone-type heat-resistive polymeric material have been confirmed with respect to its influences on reliability of electrical contacts [10-11]. 


\section{POLYMERIC MATERIAL UNDER TESTS}

The following three types of polymeric materials were under comparisons. Each of the three materials is of liquid type. For preparation of samples to be used for experiments, $100 \mathrm{~g}$ of each of the materials was put onto the bottom of a 1liter metal can and cured over seven days at $23{ }^{\circ} \mathrm{C}$ and $55 \% \mathrm{RH}$.

TABLE I. POLYMERIC MATERIALS TO BE COMPARED.

\begin{tabular}{|l|l|}
\hline Sample A & Non-silicone type material \\
\hline Sample B & $\begin{array}{l}\text { Silicone-containing type material } \\
\text { (counter-measure type for contact failure) }\end{array}$ \\
\hline Sample C & $\begin{array}{l}\text { Silicone-containing type material } \\
\text { (normal grade) }\end{array}$ \\
\hline
\end{tabular}

The non-silicone type material (sample A) is a newlydeveloped telechelic polyacrylate [12-14], containing no siloxane components therein, that can be cured at room temperature. The material can exhibit curability (productivity), endurances against heat, chemicals and oils, all of which are comparable to silicone. In the experiments, a commerciallyavailable polymeric product, based on acryl-based nonsilicone-type raw material, was used.

The two kinds of silicone-type polymeric materials (samples B and C) were tested for comparisons. Each of them was commercially-available product that contains siloxane components and can be cured at room temperature. The sample B was of counter-measure type material against contact failures (i.e., a certain countermeasure is employed so as not lead to contact failures due to silicone). The sample $\mathrm{C}$ was of non-counter-measure type (normal grade) material.

\section{EXPERIMENTAL CONDITIONS AND PROCEDURES}

Throughout this study, the same type of commerciallyavailable mechanical relays was used as the test device. Although the relay has a transfer-type electrode setting, only the normally-closed contact pair was used with an anode as a movable electrode and a cathode as a stationary electrode. No pre-treatment was done for contact surfaces.

The samples of the polymeric materials were cured in the metal can through the above-mentioned procedure. Thereafter, one commercially-available mechanical relay with AgSnIn alloy contacts (with its outer case removed) was sealed into the metal can (provided with one of the polymeric cured product on its bottom) through an opening in its lid by means of nonsilicone-type sealant. When the relay was sealed in the metal can, the mating electrodes were exposed to the surrounding atmosphere containing any possible evaporated components originated from the polymeric products in the can since the plastic outer case of the relay was removed. For the purpose of comparison, further samples were prepared in which the relays were sealed into the metal can without polymeric products.

The metal can with the relay was then placed into a heating chamber (ESPEC ST-120), and the relay was operated to conduct break operations of a DC inductive load current $(\mathrm{L}=20 \mathrm{mH})$ at $1 \mathrm{~Hz}$ up to 40,000 operations under either $80^{\circ} \mathrm{C}$ or $120^{\circ} \mathrm{C}$. The load condition was set at DC $14 \mathrm{~V}-1 \mathrm{~A}$ or DC $20 \mathrm{~V}-1.5 \mathrm{~A}$. A load current was controlled to flow only at the opening of test contact pair, so that only breaking arc discharges occurred during the operations.

During the operation tests, contact resistance was measured at every 50 operations with a 4-wire method by using a digital multi-meter Kenwood DL2051. A current level for the 4-wire resistance measurement was equal to or less than $0.5 \mathrm{~mA}$. It should be noted that measured data include resistances of wiring and terminals.

\section{EXPERIMENTAL RESULTS}

Figs.1-4 respectively show typical measured contact resistance characteristics under each test condition. Specifically, Figs. 1 and 2 indicate the measured contact resistance data under ambient temperature of $120^{\circ} \mathrm{C}$ at DC 20V-1.5A (Fig.1) and DC14V-1A (Fig.2), while Figs.3 and 4 indicate the measured contact resistance data under ambient temperature of $80^{\circ} \mathrm{C}$ at DC 20V-1.5A (Fig.3) and DC 14V-1A (Fig.4). In each of the figures, the graph in (a) indicates the result with the non-silicone type product, the graph in (b) indicates the result with the counter-measure type, and the graph in (c) indicates the result without no polymer products. Although the number of samples was five for each of the test conditions, only the typical examples were shown in the respective figures for the purpose of simple clarification.

From these figures, when the relays sealed with the nonsilicone type product were operated, contact resistance showed relatively stable characteristics, although they showed some fluctuations in the beginning of the operations. The period of fluctuations was shorter under $120^{\circ} \mathrm{C}$, shown in Figs.1(a) and 2(a), than those under $80^{\circ} \mathrm{C}$ shown in Figs.3(a) and 4(a), in both of the load conditions.

When the relays were sealed with the silicone-containing polymeric products of counter-measure type for contact failures and operated under $120{ }^{\circ} \mathrm{C}$, contact resistance showed some fluctuations at the beginning, then remained at a relatively stable level, but thereafter showed unstable fluctuations in each of the load conditions, as shown in Figs.1(b) and 2(b). Under the ambient temperature of $80^{\circ} \mathrm{C}$, the similar tendencies were observed at DC $14 \mathrm{~V}-1 \mathrm{~A}$ as shown in Fig.4(b), although the level of fluctuations seemed smaller than the results under $120^{\circ} \mathrm{C}$. At DC $20 \mathrm{~V}-1.5 \mathrm{~A}$ under $80^{\circ} \mathrm{C}$ shown in Fig.3(b), some fluctuations of contact resistances were observed over 40,000 operations.

For comparisons, the relays sealed in the metal can without polymeric cured products also showed certain fluctuations in the beginning of operations, and then stayed in the stable level, when operated under $120^{\circ} \mathrm{C}$ at DC 14V-1A, as in Fig.2(c). At DC $20 \mathrm{~V}-1.5 \mathrm{~A}$ under $120^{\circ} \mathrm{C}$, two out of five samples showed similar tendencies (i.e., some fluctuations followed by the stable data) as in Fig.1(c), while the remaining three samples showed continuous fluctuations in contact resistances up to the end of 40,000 operations. In the cases of operations under the ambient temperature of $80^{\circ} \mathrm{C}$, the similar tendencies of some fluctuations followed by the stable data were observed at DC $14 \mathrm{~V}-1 \mathrm{~A}$ with smaller level of fluctuations, as shown in Fig.4(c). At DC $20 \mathrm{~V}-1.5 \mathrm{~A}$, the fluctuations in contact resistances seemed to continue in a longer period of time (close to the end of operations) as shown in Fig.3(c). 


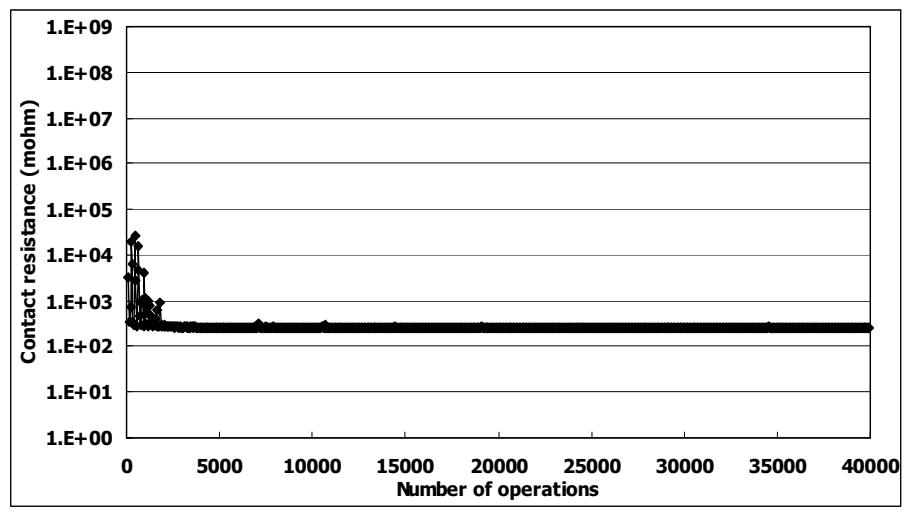

(a) Non-silicone-type product (sample A)

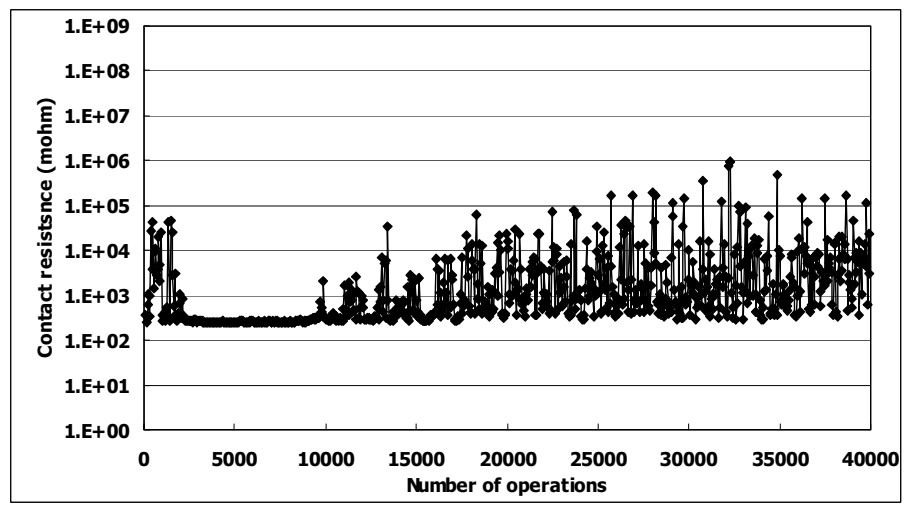

(b) Silicone-type product (sample B)

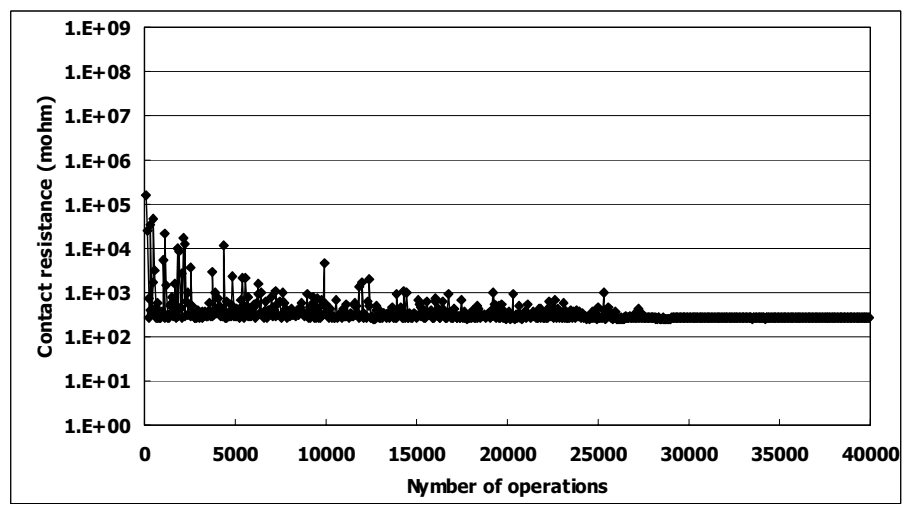

(c) Without polymeric products

Figure 1. Contact resistsnce characteristics with/without polymeric products at DC20V-1.5A under $120^{\circ} \mathrm{C}$.

Thus, the relays sealed with the acryl-based polymeric cured product (containing no silicone-components) showed no significant increases in contact resistances under ambient temperatures of $80^{\circ} \mathrm{C}$ and $120^{\circ} \mathrm{C}$, while relays sealed with the silicone-containing polymeric product showed certain fluctuations in contact resistances, even though the product was of a counter-measure type against contact failures. Such differences (disadvantages of the silicone-containing polymeric cured products) were especially remarkable in the relay operations under the ambient temperature of $120^{\circ} \mathrm{C}$.

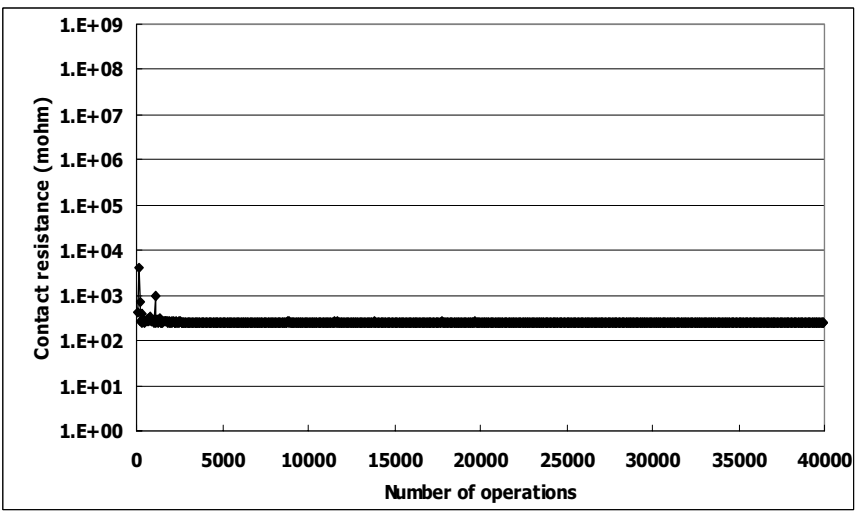

(a) Non-silicone-type product (sample A)

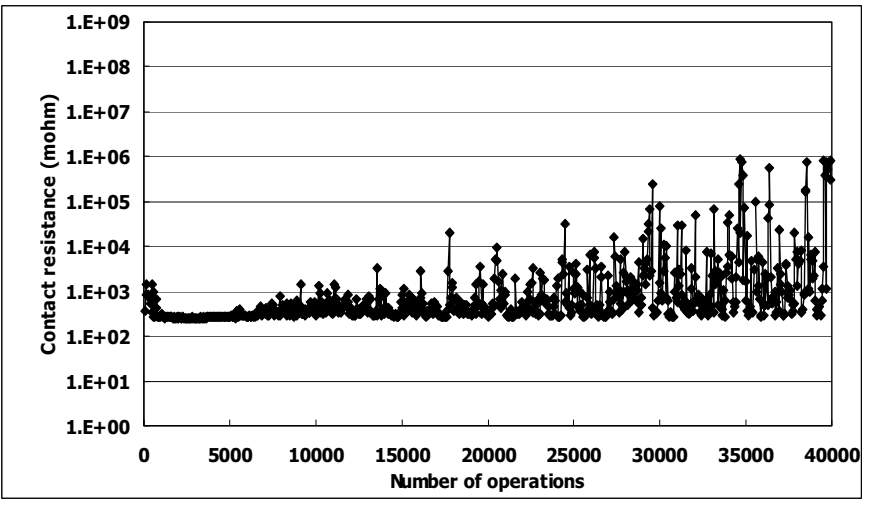

(b) Silicone-type product (sample B)

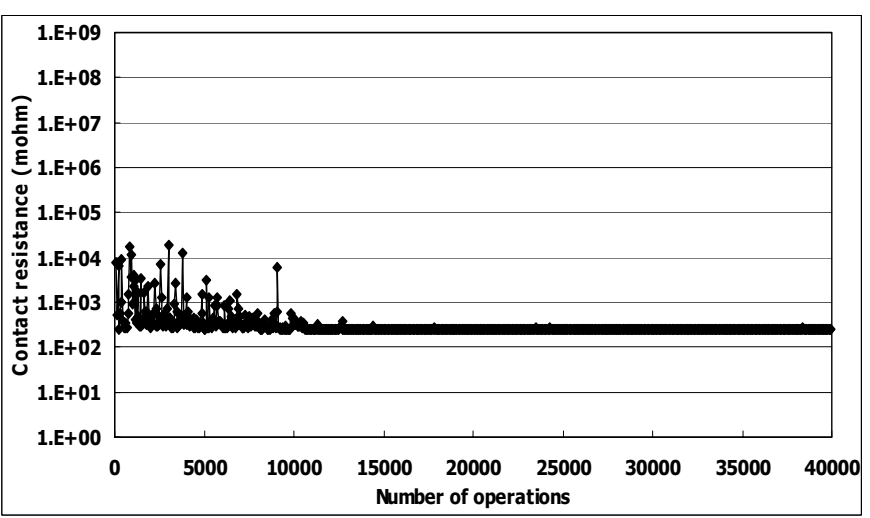

(c) Without polymeric products

Figure 2. Contact resistsnce characteristics with/without polymic products at DC14V-1A under $120^{\circ} \mathrm{C}$.

With respect to the contact resistance characteristics of the relays sealed into the metal can with the normal-grade siliconecontaining polymeric cured product, Fig.5 showed exemplary measured results when operated under $120^{\circ} \mathrm{C}$, at DC $20 \mathrm{~V}-1.5 \mathrm{~A}$ (Fig.5(a)) and at DC 14V-1A (Fig.5(b)). Again, although the number of samples in each condition was five, only the typical data were shown in these figures.

In each of the measured data, contact resistances first showed some fluctuations, and then their level remained at a relatively low range during a certain period of operations. 


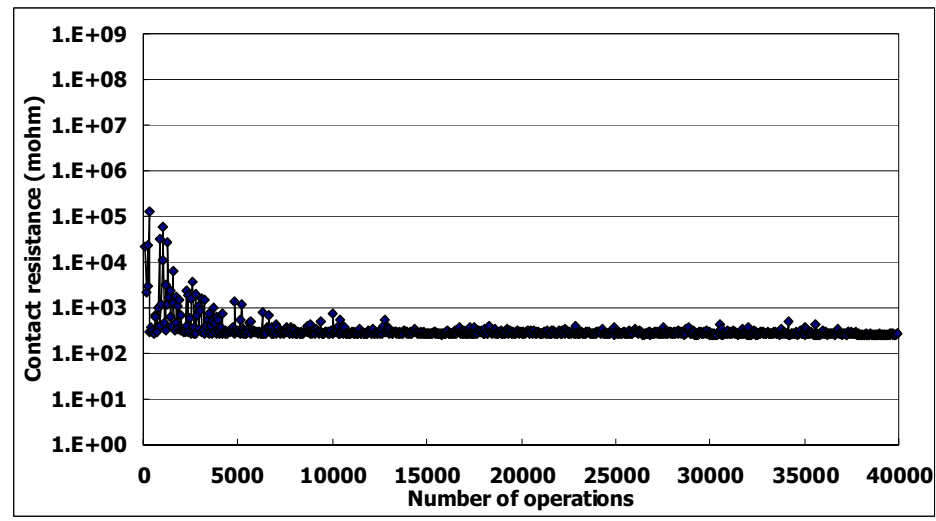

(a) Non-silicone-type product (sample A)

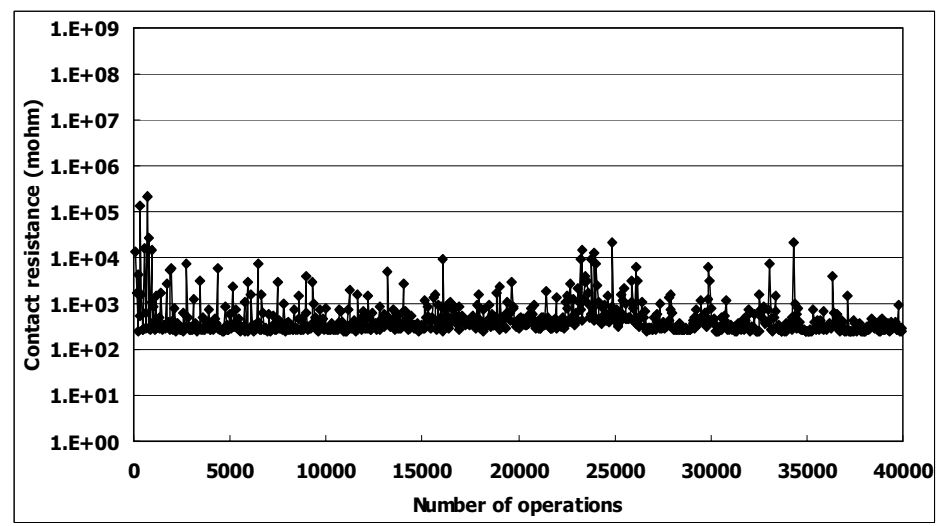

(b) Silicone-type product (sample B)

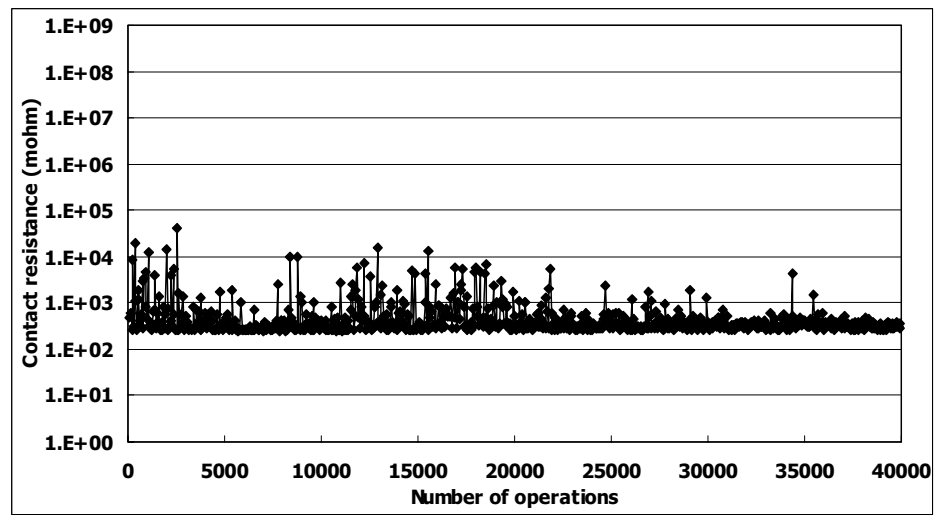

(c) Without polymeric products

Figure 3. Contact resistsnce characteristics with/without polymeric products at DC20V-1.5A under $80^{\circ} \mathrm{C}$.

Then, the contact resistances showed significant increases and had tendencies to stay in high levels (at the upper measurement limit of the digital multi-meter employed). When the relays were operated at DC $14 \mathrm{~V}-1 \mathrm{~A}$, such increase in contact resistances occurred at almost the same timing during the operations (at around 5,000 operations), while at DC $20 \mathrm{~V}$ $1.5 \mathrm{~A}$, the contact resistance values of the five samples increased at different timings from each other (in the earliest case, at around 8,000 operations as shown in Fig.5(a), while in the latest case, at around 30,000 operations).

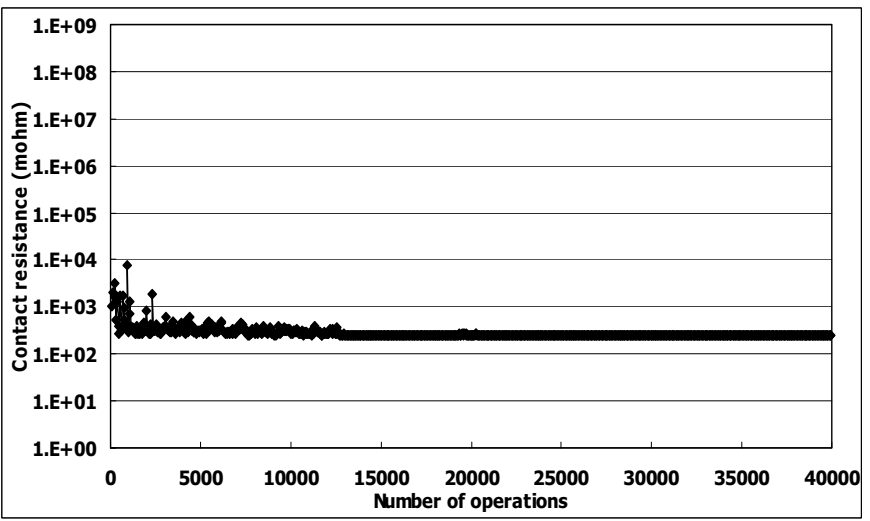

(a) Non-silicone-type product (sample B)

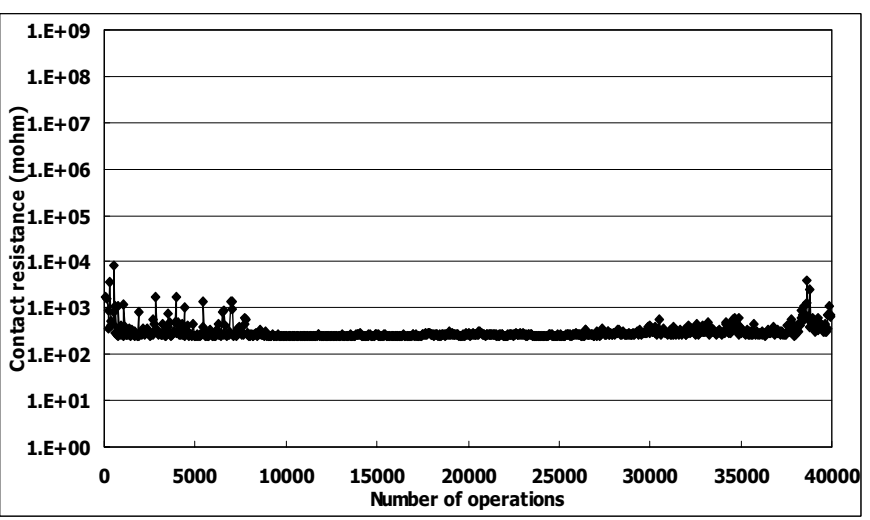

(b) Silicone-type product (sample B)

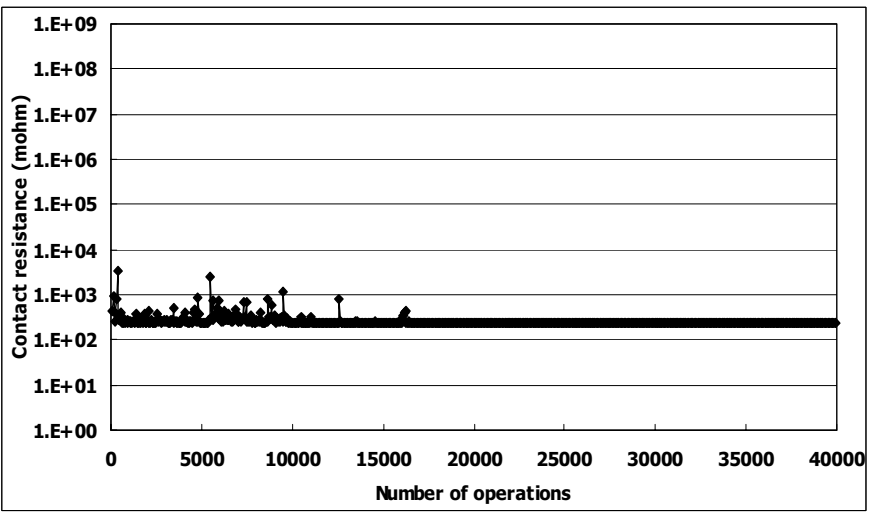

(c) Without polymeric products

Figure 4. Contact resistsnce characteristics with/without polymeric products at DC14V-1A under $80^{\circ} \mathrm{C}$.

When the relays were sealed with the same silicone-type polymeric cured product and operated under the ambient temperature of $80^{\circ} \mathrm{C}$, the measured contact resistances showed fluctuations up to the end of the 40,000 operations at DC $20 \mathrm{~V}$ $1.5 \mathrm{~A}$. At DC $14 \mathrm{~V}-1 \mathrm{~A}$, the four out of five samples showed the increasing tendencies of contact resistances.

Thus, it was confirmed, as expected, that the normal-grade silicone-type polymeric cured product disadvantageously influenced the contact resistance characteristics of relays operated in the raised ambient temperature. 


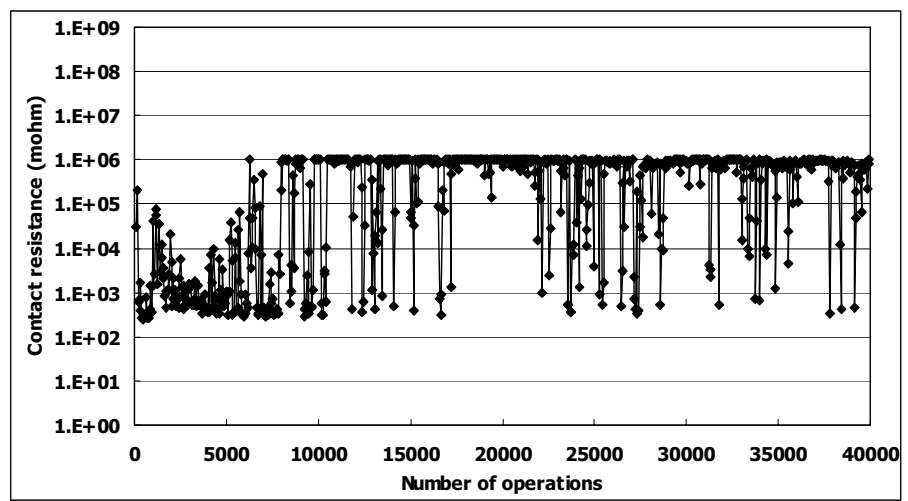

(a) DC20V-1.5A

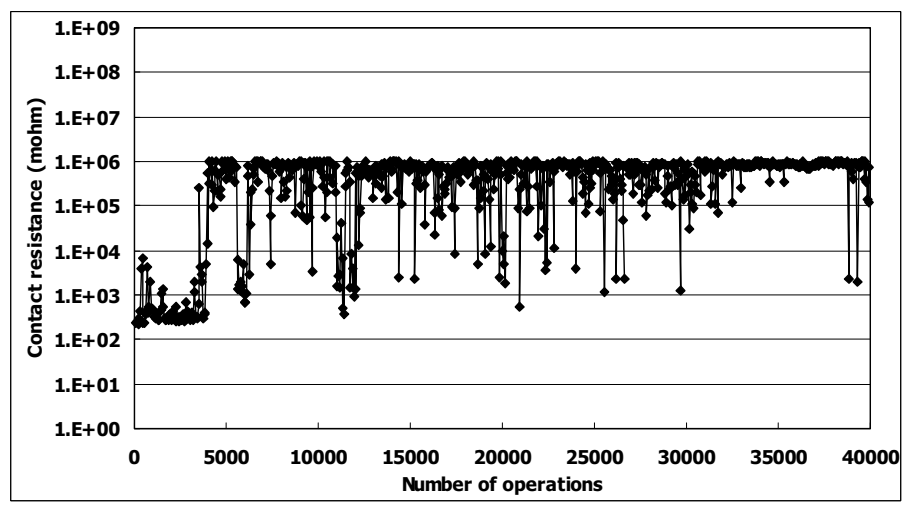

(b) DC14V-1A

Figure 5. Contact resistance characteristics with normal-grade silicone-type polymeric product (sample C) under $120^{\circ} \mathrm{C}$.

\section{DISCUSSIONS}

\section{A. Evaporations from the polymeric products}

In order to further study the influences of the compared polymeric cured products on contact resistance characteristics, the evaporation characteristics of the products were investigated.

Fig. 6 shows the amounts of evaporation from the respective polymeric cured products used in this study. More specifically, the respective products were heated $\left(10^{\circ} \mathrm{C} / \mathrm{min}\right)$ in TG-MS analysis and losses in weights of the respective products were measured, which indicated the amounts of evaporation.

From the measured data, the normal-grade silicone-type product showed larger amounts of loss in weight in accordance with elevation of temperature, indicating larger amounts of evaporation from the product. The other two products exhibited the similar evaporation tendencies, with the amounts of evaporation of about a half of those from the normal-grade silicone-type polymeric product.

From the qualitative analysis, the evaporated components from the normal-grade silicone-type polymeric product were found to be mainly low-molecular weight siloxane components (such as D3 and D4), which are well-known to cause contact failures due to silicone contamination.

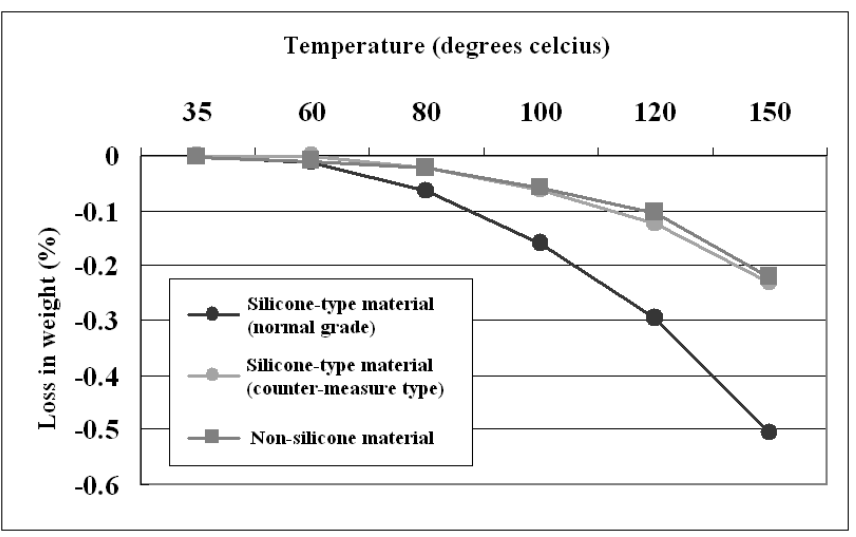

Figure 6. Evaporation characteristics of the polymaric cured products used in this study.

TABLE II. THE AMOUNTS OF D3 AND D4 COMPONENTS IN EVAPORATED GASES FROM THE SILICONE-TYPE POLYMERIC MATERIAL OF COUNTERMEASURE TYPE.

\begin{tabular}{|l|c|c|}
\hline$(\mu \mathrm{g} / \mathrm{g})$ & $\mathrm{D} 3$ & $\mathrm{D} 4$ \\
\hline $0-1.5 \mathrm{hrs}$ & 22 & 6 \\
\hline $1.5-3 \mathrm{hrs}$ & 9 & 3 \\
\hline $3-6 \mathrm{hrs}$ & 14 & 3 \\
\hline $6-9 \mathrm{hrs}$ & 12 & 3 \\
\hline $9-12 \mathrm{hrs}$ & 11 & 3 \\
\hline
\end{tabular}

The evaporated components from the counter-measure type silicone-type polymeric product were also analyzed. The main evaporated components were found to be the similar lowmolecular weight siloxane components (D3 and D4), although the material was of the counter-measure type for contact failures. Table II shows the quantitative analysis results of the amounts of D3 and D4 components included in the evaporation from the product per unit mass, indicating that these two components continued to be evaporated over several hours in heated condition.

In the case of the non-silicone type acryl-based polymeric cured product, a certain amounts of evaporation were found in the early stage of heating. They were not low-molecular weight siloxane components, but the components mainly originated from plasticizers contained in the product. Thus, although the amounts of evaporation from the non-silicone type acryl-based product were on the almost same level as those from the counter-measure type silicone-type product, main evaporated components were completely different from each other with respect to the presence of siloxane components.

As previously described with reference to the measured data shown in Figs.1-5, the polymeric cured products compared in this study exhibit different influencing effects on the contact resistance characteristics of relay contacts. The abovementioned evaporation characteristics are believed to have certain relationships with the contact resistance behaviors. 
B. Influences of polymeric products on contact resistsnce characteristics of relay contacts

The followings are tentative models explaining the experimental results obtained in this study.

1) During the early stage of operations;

At the electrical load conditions employed in this study, certain arc discharges occurred when breaking the load current, inevitably leading to depositions of arc products and/or contact surface damages. Thus, contact resistances exhibit certain fluctuations. The early fluctuations observed in each of the measured data were believed to be attributed to such usual arc discharge phenomena.

\section{2) During the following stage of operations;}

When electrical contacts are operated in open air (i.e., without significant existence of any evaporated components), fluctuations in contact resistances due to arc discharges are believed to continue. If some evaporated components exist in the ambient atmosphere, however, there is the possibility of realizing certain coating effects on the contact surfaces, possibly leading to stable contact resistances during a certain period of time.

The contact resistance characteristics with different polymeric products obtained in this study will be explained as follows.

a) With non-silicone type polymeric cured product;

The evaporated components from this newly-developed acryl-based non-silicone type product may have advantageous coating effects onto the relay contact surfaces, possibly suppressing early fluctuations in contact resistances due to arc discharges and resulting in stable characteristics. Since the evaporated amounts under $120^{\circ} \mathrm{C}$ are believed to be larger than those under $80{ }^{\circ} \mathrm{C}$, the early fluctuations in contact resistances are to be suppressed in the earlier stage under $120^{\circ} \mathrm{C}$ than under $80^{\circ} \mathrm{C}$, as can be seen by comparing Figs.1(a) and 2(a) with Figs.3(a) and 4(a).

Fig.7(a) shows the EPMA result on the contact surface of the relay after the 40,000 operations with the non-silicone type polymeric cured product, indicating that $\mathrm{Si}$ was not detected.

b) With silicone-type polymeric cured product (counter-measure type for contact failures);

The evaporated components from this silicone-type polymeric cured product of counter-measure type for contact failure are mainly low-molecular weight siloxane components such as D3 and D4. Although these components lead to serious silicone contamination phenomena, they may exhibit some coating effects on the contact surfaces providing a temporary stable contact resistance period, prior to decomposition of siloxane components into insulating $\mathrm{SiO}_{2}$ and their build-ups. Thus, as will be seen in the case with DC $14 \mathrm{~V}-1 \mathrm{~A}$ load condition under $80^{\circ} \mathrm{C}$ shown in Fig.4(b), there is the possibility that contact resistances appear to be stable. However, after a certain period of time, such coating effects seem to be lost and contact resistances tend to fluctuate. Fig.7(b) shows the EPMA result on the contact surface of the relay after the 40,000 operations with the silicone-type polymeric cured product, indicating that $\mathrm{Si}$ was detected.

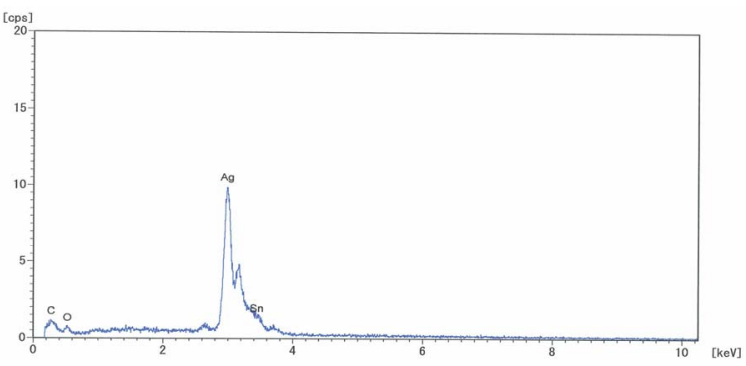

(a) with the non-silicone type acryl-based product

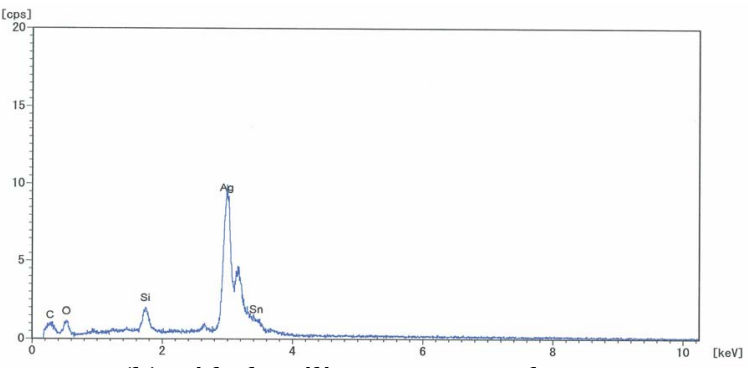

(b) with the silicone-type product

Figure 7. EPMA results of the contact surfaces after the 40,000 operations.

Under a higher temperature of $120^{\circ} \mathrm{C}$ as shown in Figs.1(b) and 2(b), such possible coating effects as well as fluctuations of contact resistances occur at earlier timings as compared to the results under $80^{\circ} \mathrm{C}$, probably because of the larger amounts of evaporation due to the elevated temperature.

c) With silicone-type polymeric cured product (normal grade);

The large amounts of low-molecular weight siloxane components are evaporated from this polymeric cured product. Thus, the coating effects on the contact surfaces are not clearly observed, and silicone contamination phenomena are dominant, leading to significant increases of contact resistances.

\section{d) Without polymeric cured products;}

Even in the comparison samples in which the mechanical relays were sealed into the metal can without specifically placing any polymeric cured products therein, some coating effects can be seen as in Figs.1(c) and 2(c). In this case, the evaporated components are coming from the sealant that is used for attaching the sample relay onto the lid of the metal can.

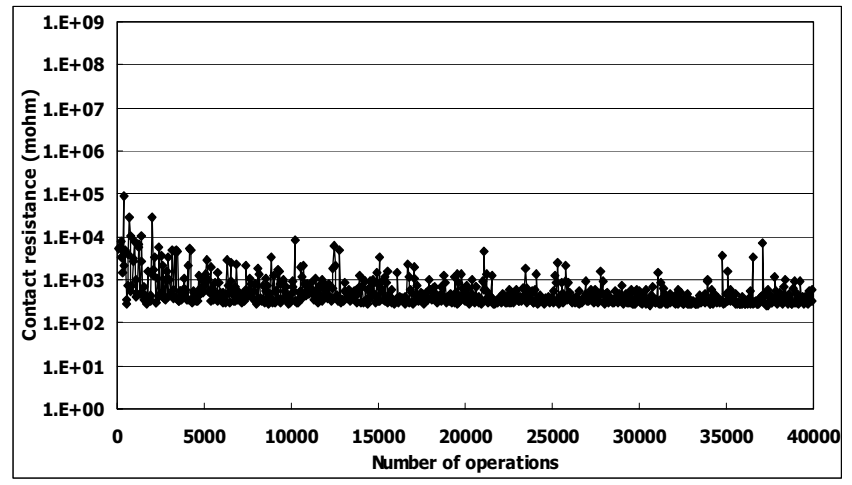

Figure 8. A typical contact resistance characteristic without any polymeric products nor sealant at DC $20 \mathrm{~V}-1.5 \mathrm{~A}$ under $120^{\circ} \mathrm{C}$. 
For the purpose of comparison, Fig. 8 shows the typical contact resistance characteristics of the relays operated at DC $20 \mathrm{~V}-1.5 \mathrm{~A}$ under $120^{\circ} \mathrm{C}$ for which any sealant was used for attaching the relay to the lid of the metal can. In this case, fluctuations in contact resistances, due to arc discharges and the resultant deposition of arc products on the contact surfaces, continued over the 40,000 operations. This can be explained by the fact that no evaporated components exist in the vicinity of the mated contacts, since neither sealant nor any polymeric cure products are provided in the metal can.

Thus, evaporated components originated from polymeric products may have both advantageous and disadvantageous effects on contact resistance characteristics. From the acrylbased non-silicone type polymeric product used in this study, some evaporated components seem to exhibit advantageous coating effects on contact surfaces, leading to relatively stable contact resistances. Further analysis will be done to find out specific component(s) introducing such advantageous effects.

\section{Influences of electrical load levels}

When comparing the experimental results, especially with the silicone-type polymeric cured products, at DC $20 \mathrm{~V}-1.5 \mathrm{~A}$ with those at DC $14 \mathrm{~V}-1 \mathrm{~A}$ in this study, there may be some slight differences in the contact resistance characteristics due to differences in the load conditions.

For example, when the relays were operated with the counter-measure type silicone-type product under $80^{\circ} \mathrm{C}$, the contact resistances exhibited more intensive fluctuations at $20 \mathrm{~V}-1.5 \mathrm{~A}$ than at $14 \mathrm{~V}-1 \mathrm{~A}$. This will be related to differences in arc discharge characteristics (e.g., average arc durations). On the other hand, when the relays were operated with the normal-grade silicone-type product under $120{ }^{\circ} \mathrm{C}$, significant increases in contact resistances were observed at earlier timings at $14 \mathrm{~V}-1 \mathrm{~A}$ than at $20 \mathrm{~V}-1.5 \mathrm{~A}$.

Contact resistance characteristics are certainly related to contact surface conditions. Even when contamination exists on the contact surfaces, low contact resistance can be obtained if metallic contact can be realized between the mating electrodes. On the contrary, contact resistance will increase remarkably when the contacting area is covered with thick depositions of arc products or contamination and no metallic contact can be realized, even if the covered are is relatively small.

Trade-off between build-ups of arc products or silicone compounds and their cleaning effects has to be considered to fully understand effects of silicone contaminations.

\section{CONCLUSIONS}

A new acryl-based non-silicone type polymeric material was evaluated with respect to its effects on contact resistance characteristics of relay contacts in 40,000 break operations of an inductive load current (DC 14V-1A and DC 20V-1.5A) under $80{ }^{\circ} \mathrm{C}$ and $120^{\circ} \mathrm{C}$ conditions. The experimental results were compared with those obtained with the siliconecontaining polymeric materials. As the results, advantages of the non-silicone type material have been confirmed with respect to its influences on reliability of electrical contacts.
Evaporated components from the polymeric cured products seem to have both advantageous and disadvantageous effects on contact resistance characteristics. In the case of the acrylbased non-silicone type polymeric product used in this study, their evaporated components seem to exhibit advantageous coating effects on the contact surfaces, leading to relatively stable contact resistances after the early fluctuations caused by arc discharges and depositions of arc products.

Further experiments are under way with different load conditions, especially lower load levels, and different types of relays. Observations of contact surfaces after the switching operations, as well as components analysis of the arc product deposition on the contact surfaces, are also being conducted to further understand the phenomena.

\section{ACKNOWLEDGMENT}

The authors thank undergraduate students at Chitose Institute of Science \& Technology for their helps in this study.

\section{REFERENCES}

[1] N.M.Kitchen and C.A.Russel, "Silicone oils on electrical contacts effects, sources, and countermeasures", Proc. 21st. Holm Seminar on Electrical Contacts, pp.79-84, 1975.

[2] M.Ishino and S.Mitani, "On contact failure caused by silicones and an accelerated life test method", Proc. 23rd. Holm Conf. on Electrical Contacts, pp.207-212, 1977.

[3] G.J.Witter and R.A.Leiper, "A comparison for the effects of various forms of silicon contamination on contact performance", Proc. 24th Holm Conf. on Electrical Contacts, pp.371-376, 1978.

[4] G.J.Witter and R.A.Leiper, "A study of contamination levels measurement techniques, testing methods, and switching results for silicon compounds on silver arcing contacts", Proc. 38th IEEE Holm Conf. on Electrical Contacts, pp.173-180, 1992.

[5] T.Tamai, "Effect of silicone vapour and humidity on contact reliability of micro relay contacts", Proc. 41st. IEEE Holm Conf. on Electrical Contacts, pp.252-259, 1995.

[6] T.Tamai, "Acceleration and suppression factors for contact failure due to silicone contamination", Proc. 52nd. IEEE Holm Conf. on Electrical Contacts, pp.26-31, 2006.

[7] T.Tamai, "Silicone contamination and contact failure of electromechanical devices", Proc. 54th Intl. Relay \& Switch technology Conf., pp.113-121, 2006

[8] D.Volm and P.Geisenberger, "Silicon contamination caused by diffusion of silicone into plastic sealed relays", Proc. 21st. Intl. Conf. on Electrical Contacts, pp.105-109, 2002.

[9] M.Hasegawa, Y.Kobayashi, Y.Kohno and H.Ando, "Evaluation of new polymeric material on performances of mechanical relays", Proc. 24th. Intl. Conf. on Electrical Contacts, pp.145-150, 2008.

[10] M.Hasegawa, T.Matsuto, Y.Kohno and H.Ando, "An experimental study on influences of silicone-type and non-silicone-type polymeric materials on contact resistance characteristics of relay contacts", IEICE Techenical Report, No.EMD2008-130, February 2009 (in Japanese).

[11] M.Hasegawa, T.Matsuto, and Y.Kohno, "An experimental study on influences of silicon-type and non-silicone-type polymeric cured materials on contact resistance characteristics of relay contacts", Proc. IEICE 2009 General Conf., No.SC-7-1, March 2009 (in Japanese).

[12] US Patent 6,441,101B1, "Processes for preparing (meth)acrylic polymers having functional groups at the chain ends".

[13] European Patent 0789 036B1, “(Meth)acrylic polymers having functional groups at chain ends".

[14] U.S. Patent 6,552,118B1, "Curable adhesive composition". 


\section{Optical Investigations of High-Current Vacuum Arc Behavior on Spiral-Shaped and Cup-Shaped RMF- Contacts}

\author{
Christian Wolf, Michael Kurrat, Manfred Lindmayer, \\ Ernst-Dieter Wilkening \\ Institute for High Voltage Technology \\ and Electric Power Systems \\ University of Technology Braunschweig \\ Schleinitzstr. 23, 38106 Braunschweig, Germany \\ chri.wolf@tu-bs.de, m.kurrat@tu-bs.de, \\ $\underline{\text { m.lindmayer@tu-bs.de, e.wilkening@tu-bs.de }}$
}

\author{
Dietmar Gentsch \\ ABB AG \\ Calor Emag Mittelspannungsprodukte \\ Oberhausener Straße 33, 40472 Ratingen, Germany \\ dietmar.gentsch@de.abb.com
}

\begin{abstract}
Vacuum circuit breakers are mostly equipped with radial magnetic field (RMF) contacts forcing the electric arc on a circular motion by a self-induced magnetic field. Using a high-speed-camera with a recording rate above 25,000 frames per second the appearance of vacuum arcs was investigated from two 90 degrees displaced views regarding three different contact designs with $\mathrm{CuCr} \mathbf{7 5 / 2 5}$ as contact material. Spiral shaped contacts and cup-shaped contacts with diameter of 68 $\mathrm{mm}$ and $65 \mathrm{~mm}$, respectively, and spiral shaped contacts with diameter of $90 \mathrm{~mm}$ were investigated with $45 \mathrm{~Hz}$ short-circuit currents of amplitudes up to $85 \mathrm{kA}$. From the high-speed films arc motion, shape and velocity as well as current density were determined in consideration of arc voltage and current strength. Results were compared to former publications.
\end{abstract}

Keywords: vacuum circuit breaker; interrupters; RMFcontacts; high-speed camera; optical analysis

\section{INTRODUCTION}

Meanwhile, vacuum interrupters are installed from low voltage to high voltage areas breaking currents up to several tens of kiloamperes. The lion's share of the electrode design is the radial magnetic field (RMF) contact.
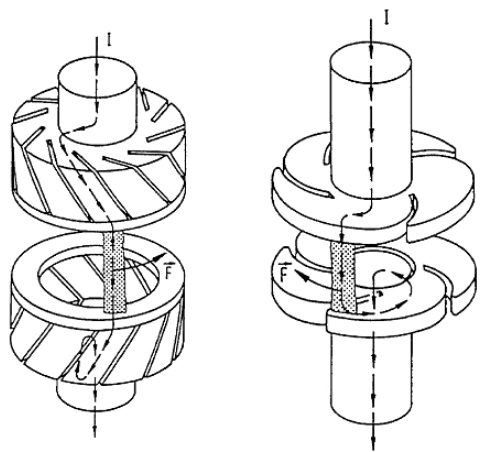

Figure 1. Cup-shaped (left) and spiral-shaped (right) RMF-contact systems

This work was supported by Deutsche Forschungsgemeinschaft DFG
It forces the electric arc for higher currents on a circular motion by a self-induced magnetic field created by the breaking current, i.e. to reduce local overheating and severe erosion of the contact surface. Two different executions of RMF-contacts are used, the cup-shaped and the spiral shaped contact shown in Fig. 1. First authors Heberlein, Paulus, Schulman, Miller and Zalucki already presented for spiral and plane contacts that the behavior of the developing arc shows strong dependence to various influences as gap distance and instantaneous current $[1-5,7]$, whereas Kimura took electrode shape as well as contact material into account for arcing behaviors [8]. Experimental conditions have to be differentiated between drawn arcs with separating contacts and fixed gaps using a pre-current to initiate the arc, which strongly affects the arc behavior as shown explicitly by Dullni for spiral contacts [9].

Analyses of the arc velocity on RMF-contacts were conducted in previous experiments by Paulus, Gentsch, Haas, Kimura, Dullni and Teichmann giving speed ranges from some tens to several hundreds of $\mathrm{m} / \mathrm{s}$ due to parameters mentioned above $[2,5,6,8-10]$. Several behavior patterns have been investigated for spiral and plane electrodes differing between the published papers, whereupon diffuse arcs, anode behaviors and columnar (or intense) arcs were differentiated by Heberlein, Paulus, Schulman, Miller and Zalucki $[1-4,7]$, whereas Kimura presented slow, acceleration and high-speed periods of arc movement [8]. Gentsch showed three modes called ignition, constricted and diffuse arc [5]. In almost every paper diffuse arcs are described, which were found at instantaneous currents of 30 kA ([10]) or below. Although several previous investigations of RMF-electrodes have been carried out no comparison between cup- and spiral-shaped contacts under same experimental conditions were conducted.

The objective of this research was the determination of behavior characteristics of drawn high-current vacuum arcs on cup-shaped and spiral-shaped electrodes and its comparison to known publications. 


\section{EXPRERIMENTAL SETUP}

The experiments were conducted in a demountable vacuum chamber with a diameter of $200 \mathrm{~mm}$. Both electrodes are insulated by glass cylinders from the middle part made of stainless steel on a floating potential. The lower contact is on ground potential and can be moved by a pneumatic drive with an average speed (15-75\% of maximum gap distance) of $1,4 \mathrm{~m} / \mathrm{s}$. The adjusted gap distance was between 13 and $14 \mathrm{~mm}$, the full stroke was approximately reached at current zero. For all tests a pressure below $1 \cdot 10^{-6}$ mbar was sustained by a turbomolecular pump connected to the test vessel. The vacuum chamber was equipped with two rectangular pipes with metal mirrors at the bend inside and view glasses at the end flanges permitting an optical analysis in three dimensions. A shield ( $\varnothing 123 \mathrm{~mm}$ ) with two viewing ports surrounded the contact system and was connected to floating potential. A schematic of the setup is shown in Fig. 2. The two perspectives from the tubes were recorded side by side in a digital 8 bit highspeed CCD camera providing a frame rate for all tests of above 25,000 frames per second with a resolution of $512 \times 80$ pixels. The spectral camera sensitivity ranged from 400 to $1000 \mathrm{~nm}$ with decreasing efficiency. Neutral density filters in front of the camera lens to avoid overexposure were used. Due to the positioning of the mirror in optical path (2) the resulting picture field is mirror-inverted, which was considered for the analyses.

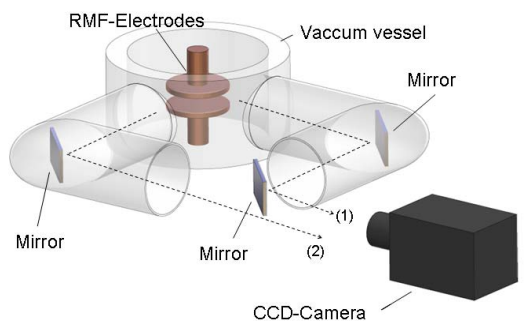

Figure 2. Schematic of optical setup; optical paths (1) and (2) are recorded side by side in one frame

A capacitor bank with a maximum charging voltage of $3 \mathrm{kV}$ supplying sinusoidal current half-wave duration of approximate $11 \mathrm{~ms}$ was connected to the electrodes in the vacuum chamber. The current range of the interrupting tests was 15-60 kA rms. A transient recorder in an isolated circuit recorded current and arc voltage.

Specimens for the investigations were cup-shaped and spiral-shaped contacts with an outer diameter of $65 \mathrm{~mm}$ and $68 \mathrm{~mm}$, respectively, and spiral contacts with $90 \mathrm{~mm}$ diameter. Both types of spiral-shaped contacts had four arms and were mounted slot-to-slot. Standard $\mathrm{CuCr} 75 / 25$ was used for contact material. Before mounting the electrodes in the test vessel ultrasonic cleaning was carried out.

\section{EXPERIMENTAL RESULTS AND DISCUSSION}

The velocity could be determined using the movement of the arc in the two perspectives. Only the second half of the current sine-wave was considered due to the acceleration period of the drive allowing only a gap distance of 2-3 mm until current peak. For almost all tests no movement could be found before the gap reached approximately $2 \mathrm{~mm}$ as also mentioned for the investigations by Paulus, Schulman and Gentsch [2, 3, 5]. Only with the $90 \mathrm{~mm}$ contacts at high momentary currents acceleration processes of the arc could be distinguished for gaps below $2 \mathrm{~mm}$ during the opening period. For the determination of the current density the arc root area at anode and cathode was approximated elliptically using the arc dimensions from the two $90^{\circ}$ displaced perspectives.

\section{A. Cup-shaped contacts $65 \mathrm{~mm}$}

Cup-shaped contacts were investigated for currents from 15 to $35 \mathrm{kA}$ rms and showed rather a reproducible behavior of the vacuum arcs. After the ignition one or two columnar arcs always developed and started to move at approximately $2 \mathrm{~mm}$ gap distance.

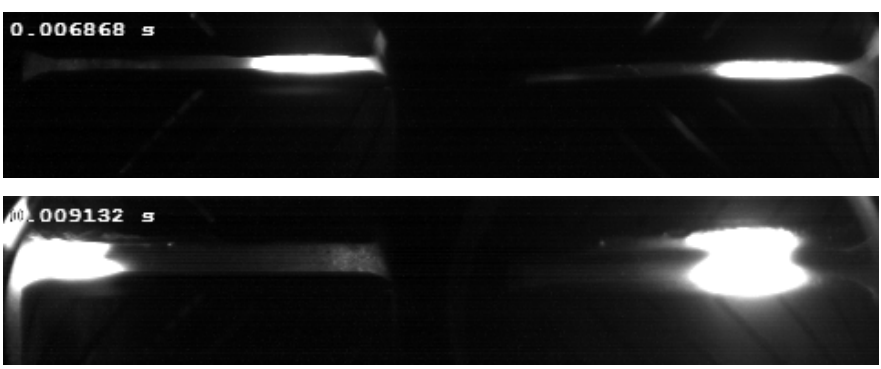

Figure 3. Arc shape at gap $\approx 2 \mathrm{~mm}$ (top) and at gap $\approx 10 \mathrm{~mm}$ (bottom) $\mathrm{I}_{\mathrm{RMS}}=25 \mathrm{kA}$
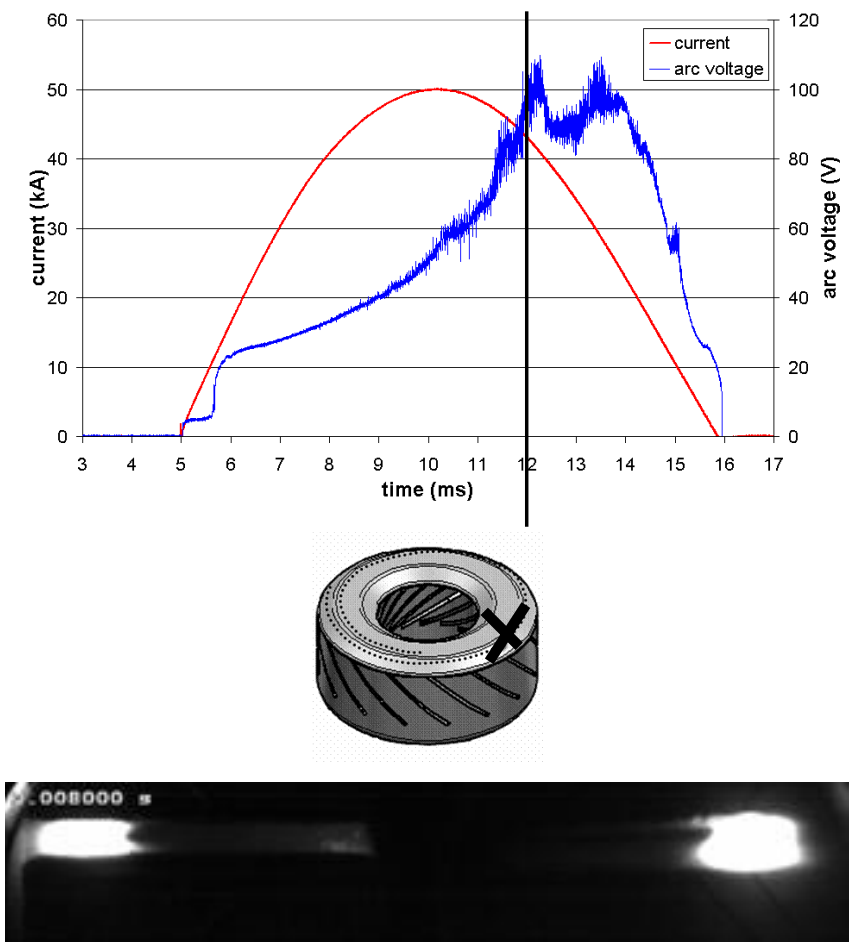

Figure 4. Top: Current and arc voltage against time for $\mathrm{I}_{\mathrm{RMS}}=35 \mathrm{kA}$ Middle: Arc trace on the cathode (narrow dotted line) and whereabout (black cross) at time specified by line in graph above

Bottom: Taken frame of the arc at time specified by line in graph above 
If two arcs occurred at the beginning after a short while they contracted to one, as also observed by Paulus [2]. The movement was always smooth and steady. No other patterns than columnar arcs and diffuse arcs were observed, comparable to investigations by Haas and Pavelescu $[6,11]$. The transformation from columnar to diffuse arc occurred at instantaneous currents of about $15 \mathrm{kA}$ at the falling edge of the half-wave. At gaps of around $6 \mathrm{~mm}$ the shape of the arc changed from a round to a hyperboloid shape, but with no clear dependence on the current (Fig. 3). In Fig. 4 one representative curve for the arc voltage of arcs on cup-shaped electrodes is given as well as a frame of a representative shape of the columnar arc. The lower picture of the contact indicates the whole trace of the arc (dotted line) and its momentary whereabout in comparison with the voltage trace. At the first contact separation always a voltage jump of 18$20 \mathrm{~V}$ was monitored independently from the instantaneous current. After a smooth rise of the voltage in the displayed curve one can find a drop in the trace resulting from the already heated surface by the previous rotation of the arc at this position of the contact. After the drop the voltage increases again for a short time, caused by the increasing gap distance. The arc voltage generally had low fluctuations in the measurements. Only for currents of $35 \mathrm{kA} \mathrm{rms} \mathrm{1-11/2}$ turns of the arc could be seen. If the arc rotated less than one time the voltage showed only one broad peak. For currents of $25 \mathrm{kA} \mathrm{rms} \mathrm{half} \mathrm{a} \mathrm{turn} \mathrm{was} \mathrm{observed} \mathrm{on} \mathrm{average.} \mathrm{The} \mathrm{mean}$ peak voltage was between 80 and $120 \mathrm{~V}$ without dependence on the rms current. Haas found in [6] for gaps of $5 \mathrm{~mm}$ no strong change of the arc voltage for peak currents from 20 to $100 \mathrm{kA}$ with an average of $90 \mathrm{~V}$, comparable to the conducted tests here considering the longer gap distances at the falling edge of the current.

The determined velocities of the arc are shown in Fig. 5 for the investigated rms currents. As the values for instantaneous currents showed a strong scatter this illustration is chosen.

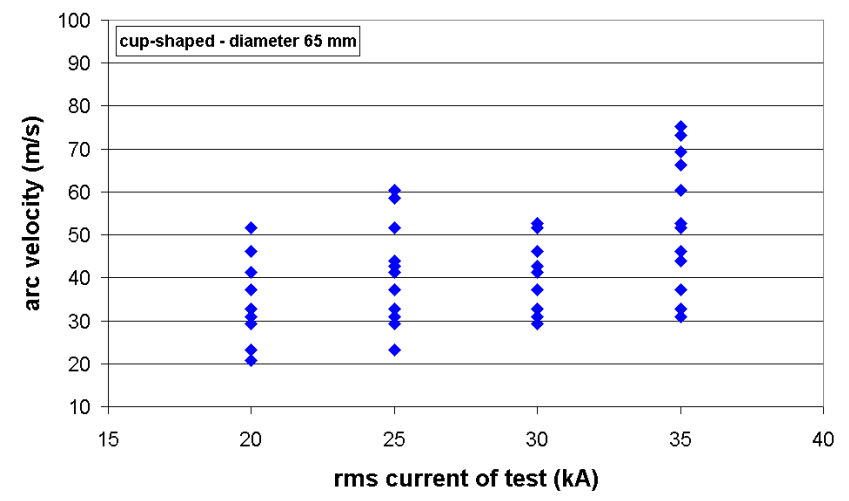

Figure 5. Arc velocity for cup-shaped contacts with diameter $65 \mathrm{~mm}$

For tests with $15 \mathrm{kA}$ no arc movement could be found. Measured velocities range from 20 to $75 \mathrm{~m} / \mathrm{s}$ with a slight increase of the average up to higher currents, but comparable values could be found for all rms currents. In [6] values from 25 to $250 \mathrm{~m} / \mathrm{s}$ were found for cup-shaped contacts and peak currents up to $100 \mathrm{kA}$. In the displayed chart a slight increase of the mean and maximum speed to higher currents can be identified, so that a further rise for higher currents to the values mentioned by Haas could be assumed.

The investigated current densities shown in Fig. 6 were between 10 and $45 \mathrm{kA} / \mathrm{cm}^{2}$ for the cathode and between 15 and $55 \mathrm{kA} / \mathrm{cm}^{2}$ for the anode with a noticeable scatter at similar current values. On average a slight increase of the densities with increasing instantaneous current can be determined indicated by the polynomial regression lines as well as a mean difference between anode and cathode of around $10 \mathrm{kA} / \mathrm{cm}^{2}$. In [6] differences between anode and cathode of $100 \%$ for cup-shaped electrodes were mentioned, this could not be found in that dimension in the tests carried out here. Several theories for cathode and anode plasma behavior have been developed in the past considering complex interactions of magnetic field and physical effects on the anode with varying approaches, e.g. shown in [12].

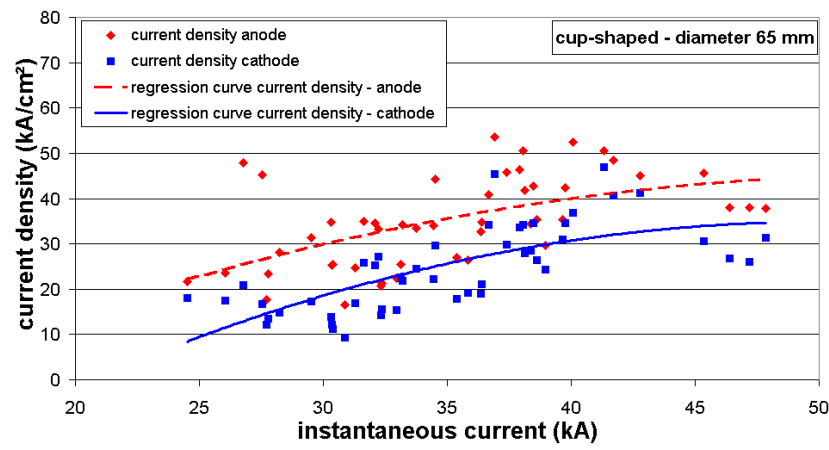

Figure 6. Current density on anode and cathode for cup-shaped contacts against instantaneous current; regression lines are polynomial

\section{B. Spiral contacts $68 \mathrm{~mm}$ and $90 \mathrm{~mm}$}

For spiral contacts many more behavior patterns were found. The electrodes with diameter of $68 \mathrm{~mm}$ were investigated for currents from 15 to $30 \mathrm{kA} \mathrm{rms}$ and those with diameter of $90 \mathrm{~mm}$ for currents from 20 to $60 \mathrm{kA} \mathrm{rms}$. Generally, the recorded shapes can be described as columnar and diffuse arc as with cup-shaped contacts and also as plasma jets as also found by Zalucki [7]. The behavior patterns did not differ significantly between the two electrode sizes in the investigated current range. As with the cup-shaped contacts right at the beginning of the opening process one or more constricted arcs appeared. But after forming one constricted arc at small gap distances sometimes more than one columnar arc appeared randomly again with increasing gap distances. Generally, after the first millimeters of gap distance columnar arcs only occurred for short periods of less than $1 \mathrm{~ms}$ just as plasma jets, comparable with [7]. Randomly wide illumination over half the electrode with several plasma jets on anode and cathode in parallel were recorded. Diffuse arcs with separated cathode spots could be seen occasionally for instantaneous currents above $20 \mathrm{kA}$ at switching tests up to $30 \mathrm{kA} \mathrm{rms}$. For higher rms currents no diffuse modes were noticed, presumably due to higher 
neutral density filters used for these tests. As then completely black frames were recorded sporadically with no change of the current curve, one can assume that diffuse modes occur for higher currents as well.

Mean dimensions of the arc kept the same range for varying exposure times of the camera. Generally, one can say that the mean intensity of the radiation increased with the rms current value, but the brightness of the arc and its dimensions can change significantly within microseconds at comparable current values. Fig. 7 gives an impression of the difference in the luminous appearance within $64 \mu \mathrm{s}$.
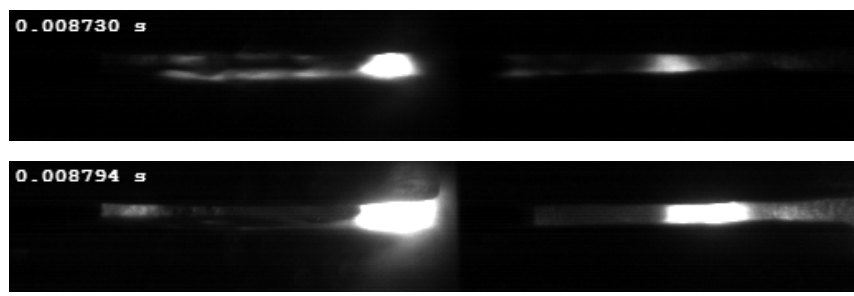

Figure 7. Luminous intensity change of arc at instantaneous current of $\mathrm{i} \approx 76 \mathrm{kA}$ for time step of $64 \mu \mathrm{s}$

Compared to cup-shaped contacts the arc voltage showed more fluctuations and higher peaks for similar currents. The voltage trace strongly depended on the instantaneous arc behavior. Alike the results in part $A$ the voltage was quiescent and comparably low for constricted columnar arcs, whereas for jet appearances high peaks occurred. Fig. 8 gives an impression of a representative curve for a test with $30 \mathrm{kA}$ rms and the appropriate optical recordings of the arc occurrences are shown in Fig. 9. There is a time offset between graph and frames of exactly $5 \mathrm{~ms}$ due to using pretrigger for current and voltage recordings. Occurrences of plasma jets were accompanied by an increase of the arc voltage, whereas columnar arcs showed a comparably lower voltage. Similar effects were presented by Zalucki for butt contacts of $\mathrm{CuCr} 40$ [7]. For instantaneous currents between 8 and $20 \mathrm{kA}$ he also showed the appearance of autonomous cathode spots, which can be distinguished in frame 8 in Fig. 9 as well for a current of $35 \mathrm{kA}$

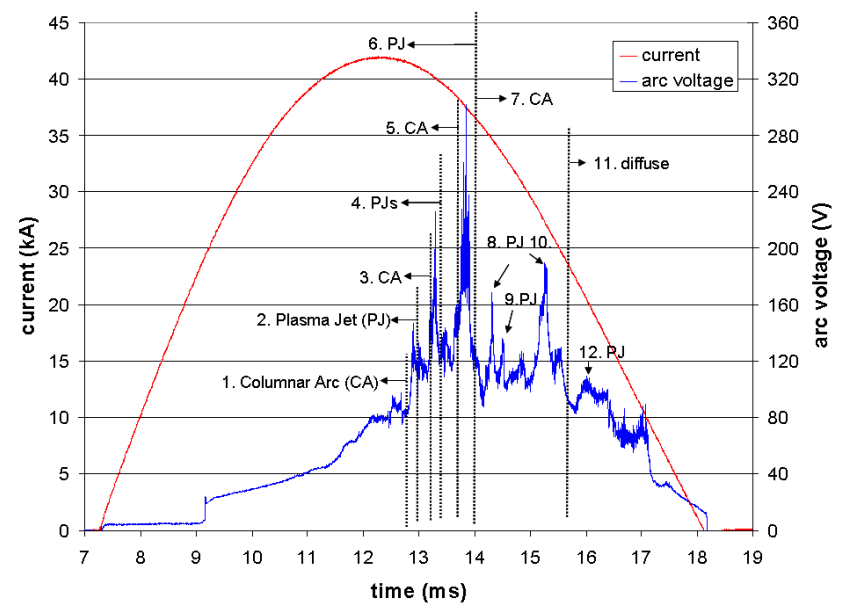

Figure 8. Current and arc voltage against time for $I_{R M S}=30 \mathrm{kA}$ for spiral contact $(90 \mathrm{~mm})$; indicated arc appearances (1.-12.) are specified in Fig 9
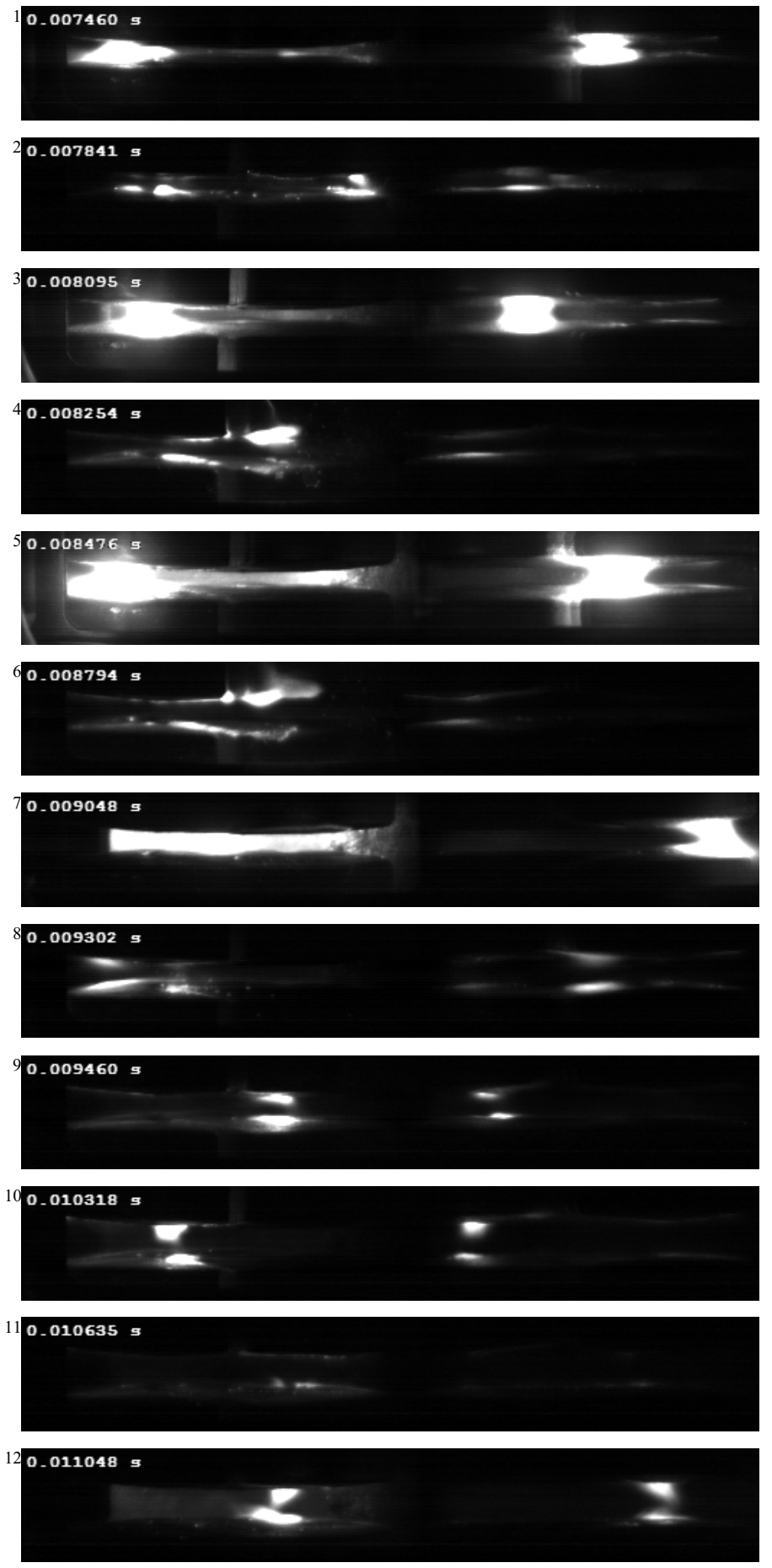

Figure 9. Arc appearances corresponding to Fig 8 with time offset of 5 ms; each frame shows two $90^{\circ}$ displaced perspectives divided in the middle

For all tests independent of the current a jump of the voltage of approximately $13 \mathrm{~V}$ was monitored at the first operating instant. The mean voltage for all rms currents was between 90 and $120 \mathrm{~V}$, but with peaks up to $300 \mathrm{~V}$. In several cases a deceleration of a constricted arc could be noticed in front of a slot between two spiral arms with a following transformation to plasma jets as shown in frames 5 and 6 in Fig. 9 accompanied by a fast rise in the voltage. 
Plasma jets on $68 \mathrm{~mm}$ contacts occasionally showed a movement to the outer edge of the contacts with directing to the shield. For the larger electrodes this was not detectable by reason of the size of the viewing ports. This behavior was not accompanied by significant voltage changes in comparison to plasma jets inside the contact gap.

Fig. 10 shows the determined velocities for the spiral contacts with $68 \mathrm{~mm}$ ranging from 10 up to $350 \mathrm{~m} / \mathrm{s}$. Compared to results in part $A$ considerably higher speeds were found resulting from a lower azimuthal force for cupshaped contacts due to the current path according to the electrode design.

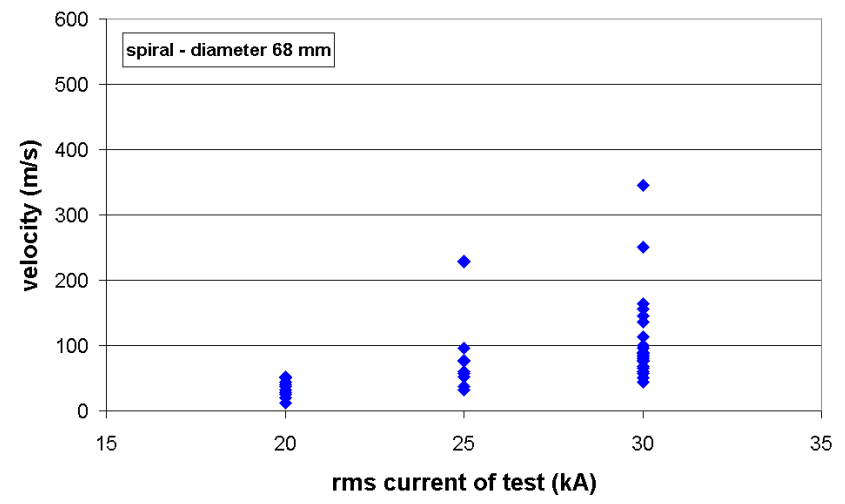

Figure 10. Arc velocity for spiral contacts with diameter $68 \mathrm{~mm}$

The determined speed range coincides with the values presented by Gentsch for the same electrode size [5]. Investigations by Dullni for spiral contacts of about half the size and peak currents of $20 \mathrm{kA}$ showed velocities of up to $400 \mathrm{~m} / \mathrm{s}$ [9], which here could no be found at all for this current range. The maximum velocities did not necessarily occur with the current peak but rather randomly. The highest as well as the instantaneous speed in the conducted tests did not seem to depend on the instantaneous current or gap length, but more on surface conditions, e.g. previous melting.

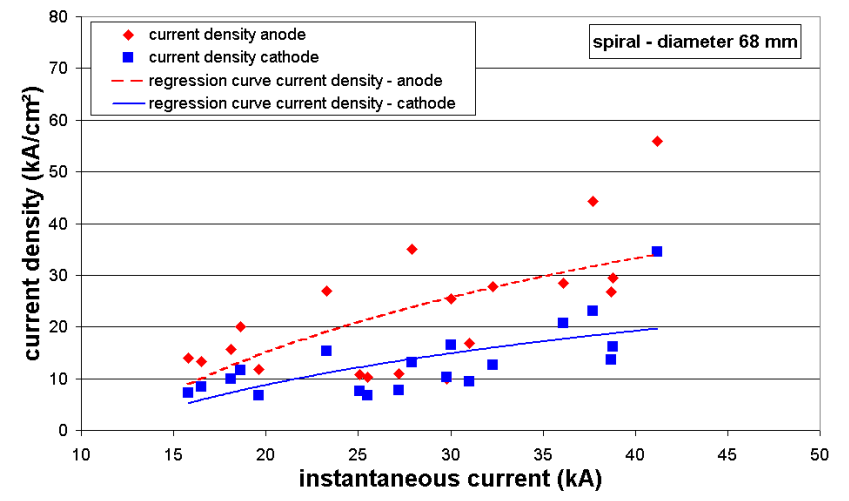

Figure 11. Current density on anode and cathode for spiral contacts with diameter $68 \mathrm{~mm}$ against instantaneous current; regression lines are logarithmical

In Fig. 11 current densities for $68 \mathrm{~mm}$ spiral contacts are displayed giving a range of values for the cathode between 7 and $31 \mathrm{kA} / \mathrm{cm}^{2}$ and for the anode between 10 and $55 \mathrm{kA} / \mathrm{cm}^{2}$. Dullni mentioned for smaller contacts a similar region of values for peak currents of $20 \mathrm{kA}$ [9]. The logarithmical regression line in the graph denotes a slight rise for higher currents within the determined values.

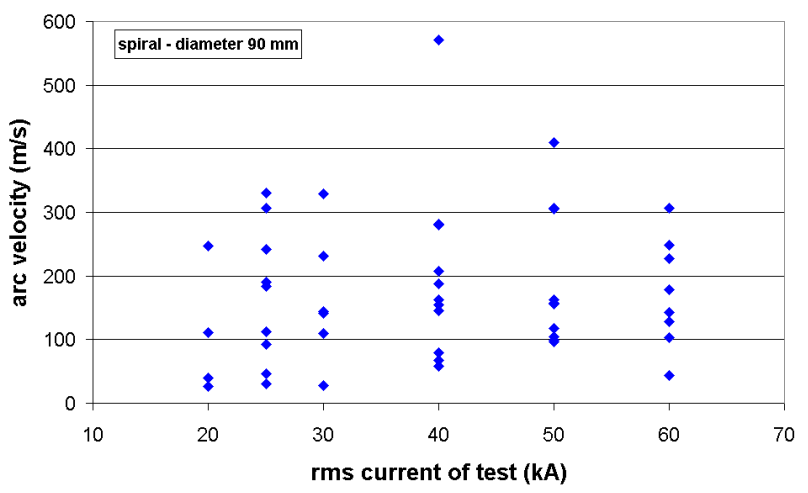

Figure 12. Arc velocity for spiral contacts with diameter $90 \mathrm{~mm}$

For spiral electrodes with diameter of $90 \mathrm{~mm}$ the carried out velocities showed a similar range for all investigated $\mathrm{rms}$ currents (Fig. 12). With no further detailed spiral $\mathrm{CuCr}$ contacts Kimura found arc speeds from 20 to $160 \mathrm{~m} / \mathrm{s}$ for rms currents up to $50 \mathrm{kA}$ [8]. These values lie significantly below those here, indicating that the spiral design and material have an important influence on occurring velocities. Alike the smaller electrodes discussed before no dependence neither on instantaneous current nor on gap length was clearly distinguishable. In his work Gentsch found a total number of nine arc turns for rms currents of $30 \mathrm{kA}$ [5]. In the entire tests for all currents and both diameters between three and seven turns were recorded. Several times no clear rotation could be seen, as the arc vanished at one spot and developed at a different spot again.

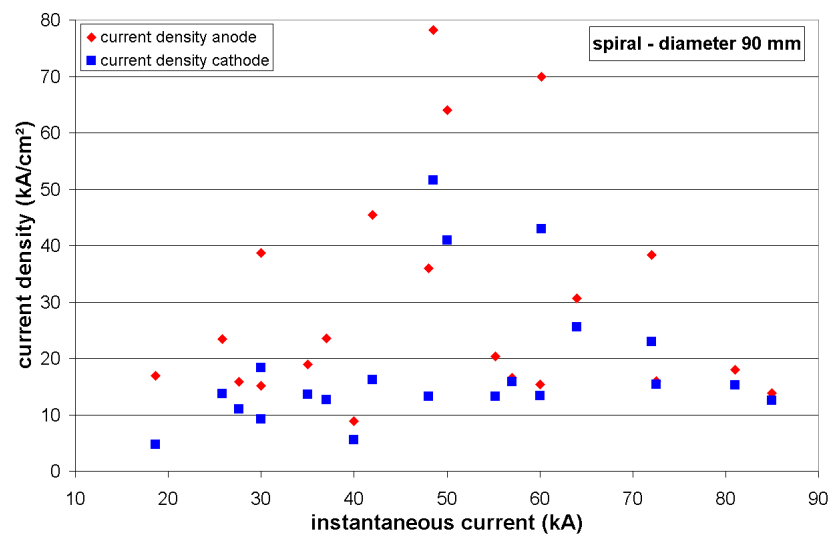

Figure 13. Current density on anode and cathode for spiral contacts with diameter $90 \mathrm{~mm}$ against instantaneous current

Determined current densities for spiral contacts with diameter $90 \mathrm{~mm}$ did not differ strongly from those of $68 \mathrm{~mm}$ electrodes. The highest values were slightly above those of the smaller contacts, caused by higher instantaneous currents with tests up to $60 \mathrm{kA} \mathrm{rms}$, although it is noticeable that the 
highest values did not necessarily occur with highest instantaneous currents. In Fig. 13 the maximum values were determined for $\mathrm{i} \approx 50 \mathrm{kA}$ and not for the values around $80 \mathrm{kA}$. One reason for that is the varying size of the columnar arc at similar currents. Teichmann mentioned for $75 \mathrm{~mm}$ diameter $\mathrm{CuCr}$ spiral contacts with different shapes and peak currents of 40-120 kA in [10] average velocities ranging from 80 to $400 \mathrm{~m} / \mathrm{s}$ basically coinciding with the presented results here, but current densities were specified between 50 and 100 $\mathrm{kA} / \mathrm{cm}^{2}$ for constricted arcs differing from the densities in Fig. 11 and 13. On the one hand this allows the speculation that velocities are in a similar scale for a wide range of currents, on the other hand it shows that current densities seem to depend more on electrode shape and material characteristics.

\section{CONCLUSION}

For cup-shaped electrodes the results showed a good reproducibility for shape and general behavior. Movements were always smooth and constant. Arc voltages had low fluctuations.

For spiral contacts many more behavior patterns were found accompanied by strong fluctuations in the arc voltage. Velocity and current density did not seem to depend clearly on instantaneous current, gap distance or contact size.

Generally, current density ranges coincide for both cupshaped and spiral-shaped contacts, but arc velocities differ for rms currents above $25 \mathrm{kA}$ due to lower azimuthal forces with cup-shaped contacts.

\section{REFERENCES}

[1] J. V. R. Heberlein, J. G. Gorman, "The high current metal vapor arc column between seperating electrodes," IEEE Trans. on Plasma Science, vol. PS-8, No. 4, pp. 283-288, December 1980

[2] I. Paulus, "The short vacuum arc - Part I: Experimental investigations", IEEE Trans. on Plasma Science, vol. 16, No. 3, pp. 342-347, June 1988

[3] M. B. Schulman, "Seperation of spiral contacts and the motion of vacuum arcs ant high AC currents", IEEE Trans. on Plasma Science, vol. 21, No. 15, pp. 484-488, October 1993

[4] H. C. Miller, "Anode Modes in Vacuum Arcs," IEEE Trans. on Dielectrics and Electrical Insulation, vol. 4, No. 4, August 1997

[5] D. Gentsch, W. Shang "High-speed observations of arc modes and material erosion on RMF- and AMF-contact electrodes," IEEE Trans. on Plasma Science, vol. 33, No. 5, pp. 1605-1610, October 2005

[6] W. Haas, W. Hartmann, "Investigation of arc roots of constricted high current vacuum arcs," IEEE Trans. on Plasma Science, vol. 27, No. 4, pp. 954-960, August 1999

[7] Z. Zalucki, J. Janiszewski, "Investigation of arc roots of constricted high current vacuum arcs," IEEE Trans. on Plasma Science, vol. 27, No. 4, pp. 991-1000, August 1999

[8] T. Kimura, A. Sawada, K. Koyama, H. Koga, T. Yano, "Influence of vacuum arc behavior on current interrupting limit of spiral contact," Proceedings of the $19^{\text {th }}$ IEEE Conference on Discharges and Electrical Insulation in Vacuum, pp. 443-446, Xi' an 2000

[9] E. Dullni, "Motion of high-current vavuum ars on spiral-type contacts," IEEE Trans. on Plasma Science, vol. 17, No. 6, pp. 875879, December 1989

[10] J. Teichmann, M. Römheld, W. Hartmann "Magnetically driven high current switching arcs in vacuum and low pressure gas," IEEE Trans. on Plasma Science, vol. 27, No. 4, pp. 1021-1025, August 1999

[11] D. Pavelescu et al. "Investigation of the rotating arc plasma generated in transverse magnetic field (TMF) contacts of vacuum interrupter," IEEE Trans. on Plasma Science, vol. 33, No. 5, pp. 1504-1510, October 2005

[12] R. L. Boxman, P. J. Martin, D. M. Sanders, Handbook of vacuum arc science and technology. Park Ridge, NJ: Noyes Publications, 1995 


\section{Effect of Different Venting Conditions on the Interruption Performance of Arc Chamber}

\author{
Ruicheng Dai, Degui Chen, Xingwen Li \\ State Key Laboratory of Electrical Insulation and Power \\ Equipment \\ Xi'an Jiaotong University \\ Xi'an, China \\ dgchen@mail.xjtu.edu.cn
}

\author{
Haitao Sun, Ensheng Xie \\ Schneider Electric China Investment Co. Ltd \\ Shanghai, China \\ HaiTao.Sun@cn.schneider-electric.com
}

\begin{abstract}
Gas flow in arc quenching chamber has an important effect on the interruption capability of low voltage circuit breakers. In this paper, based on a simplified model of arc chamber with a single break, which can be opened by the electrodynamics repulsion force automatically, the effect of different vent conditions including middle venting and side venting on the interruption performance is investigated. First, the effect of the different size of side vent type on the chamber pressure, and interruption capability were studied experimentally. It demonstrates that the smaller vent size is, the higher pressure and better the interruption performance will be, but when the vent size smaller than a certain proportion, the arc will re-ignite and when the vent size larger than a certain proportion, the arc will also re-ignite. Experiments are carried out to compare the different performance in the interruption process between middle vent to be conventional vent type and side vent type. In addition, according to the experimental model, a 3-D magnetohydrodynamic model was developed by adapting and modified the commercial computational fluid dynamics software FLUENT. The simulation results show the same trend in arc motion as explained in the experimental conclusions in theory.
\end{abstract}

Keywords- arc chamber; vent structure; low voltage swiching devices; MHD model

\section{INTRODUCTION}

Low voltage switching devices are widely used for the distribution of electrical energy and the control of electrical machines. Many investigations have reported studies on low voltage switching devices with the aim of improving the interruption capability and current-limiting performance.

McBride and others studied the effect of gas flow on the arc root mobility in the contact region of a miniature circuit breaker (MCB). The result indicated that the gas flow had a positive effect on improving the interruption capability [1], [2]. Tsukima and Mitsuhashi investigated and verified that a gas flow can directly drive the arc movement [3]. Our previous research work demonstrates adding arc runner in quenching chamber will benefits to the arc motion towards to splitter plates [4]. Up till now, there are many published papers discussion the arc simulation for low voltage switching devices [5]-[8]. In these simulations, MHD approach is widely used. They are set up the numerical model, and cutting phase.
This paper, based on the on a simplified arc model quenching chamber with single break, experimental investigates effect of the venting structure on the interruption performance. Then the arc simulation is carried out by 3-D MHD model to investigate the influence of two different venting structures on the arc column shape and arc voltage.

\section{EXPERIMENTAL METHOD AND MODEL}

\section{A. Experiment circuit and method}

In this paper, a short circuit current is provided by the capacitor banks, which is a typical single-frequency oscillation circuit. The oscillating frequency is $50 \mathrm{~Hz}$ and the ratio of the effective value between the charge voltage and the first wave of discharge current is $50: 1(\mathrm{~V} / \mathrm{kA})$. Therefore, if the desired effective current is $10 \mathrm{kA}$, then the charge voltage of the capacitor banks is $500 \mathrm{~V}$.

In the experiments, arc current and arc voltage are measured using a shunt, with resistance $90 \mu \Omega$ and a highvoltage transducer (Tektronix 6015A), respectively. The chamber pressure is measured by a PT-100 pressure sensor. In addition, to avoid electromagnetic interference, the pressure sensor is shielded.

\section{B. Experiment model}

Fig. 1 illustrates the model quenching chamber schematic in isometric view. The dimension of the model was $130 \mathrm{~mm} \times 90 \mathrm{~mm} \times 50 \mathrm{~mm}$. From Fig. 1 , it can be seen that a slip block part made of DMC material is mounted on the movable contact to well seal the back region of the model, which is used to prevent the gas flow opposite to the splitter plates. Recently, many methods are used to seal the back region of the arc chamber of MCCB products in order to improve the gas flow effect. In this paper, the model chamber has 9 splitter plates. The movable contact is opened by the electro-dynamics repulsion force automatically, when the test current coming.

Fig. 2 gives two different venting configurations. The first is the conventional type with several rectangles vents arranged also in parallel in the middle of the top barrier, as show in Fig2. (a). The second has two vent sides on the top barrier as show in Fig.2 (b). In this paper, the two type of venting are called middle venting and side venting, respectively. 


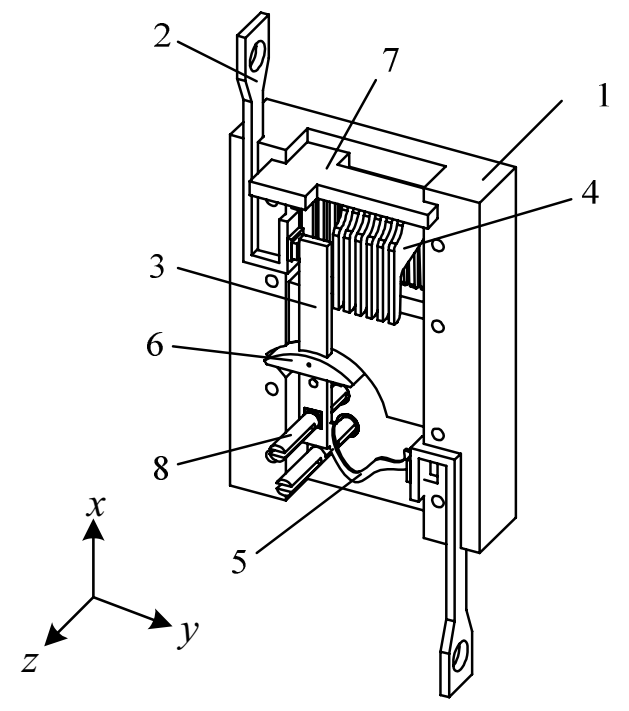

(a)

Fig. 1 model quenching chamber 1 chamber wall, 2 stationary contact, 3 movable contact, 4 splitter plates, 5 soft link, 6 slip barrier part, 7 top barrier with side venting, 8 rotating axis

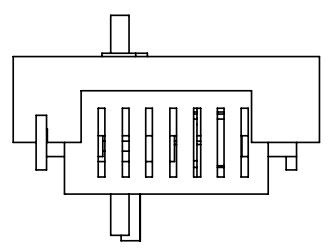

(a)

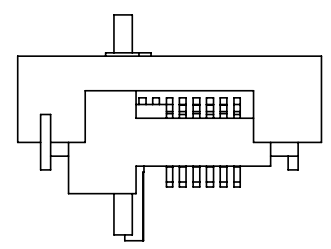

(b)
Fig.2 Two kind of venting structure

\section{EXPERIMENTAL RESULTS AND DISCUSSION}

\section{A. The effect of the venting size on the interruption}

According to side venting, Fig. 3 shows the structure of the venting. The hatched region indicates the venting. It can be seen the high temperature gas may escape from the arc quenching chamber through the two-side venting.

It should be noted that the venting size is defined by the ratio between $l \mathrm{v}$ and $l$. In the following experiments, we compared the influence of venting size of $100 \%, 90 \%, 80 \%$, $70 \%, 50 \%, 25 \%, 15 \%$ and $5 \%$ on interruption performance, under the same effective value of prospective short circuit current of $10 \mathrm{kA}$.

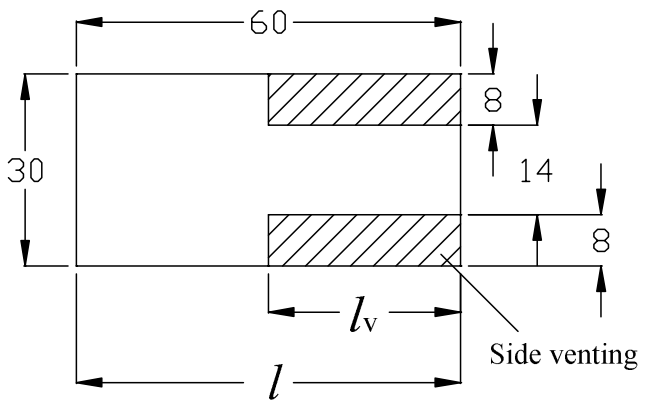

Fig.3 The structure of two-side venting (unit: $\mathrm{mm}$ )
Fig. 4 shows the experimental results of the arc current, arc voltage and gas pressure waveforms with the different venting sizes under the venting ratio of $100 \%, 80 \%, 50 \%$ and $15 \%$, respectively.

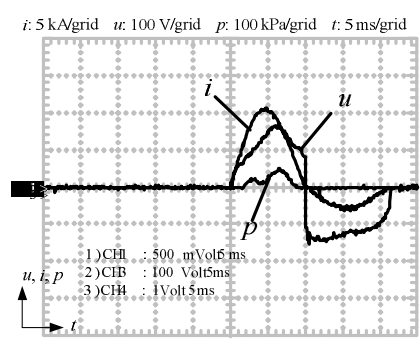

(a) $100 \%$

(c) $50 \%$

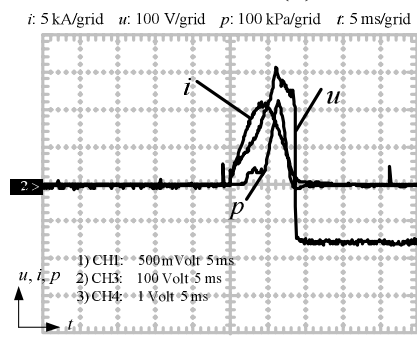

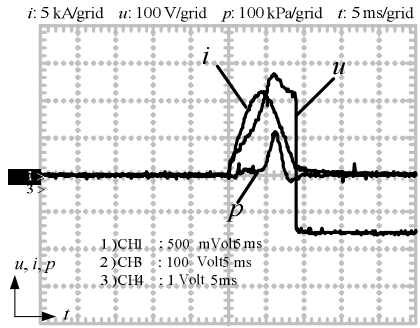

(b) $80 \%$

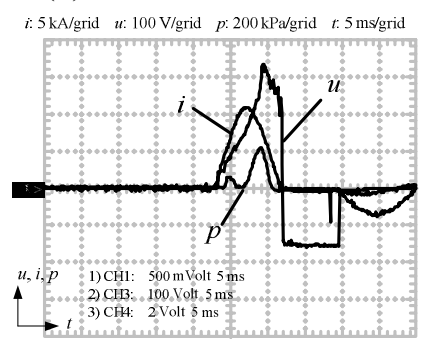

(d) $15 \%$
Fig.4 Experimental result of the waveforms of different venting size ( $t$ unit: $5 \mathrm{~ms} /$ grid)

Based on the results of Fig. 4, table 1 show the comparisons of the peak value of arc voltage $u_{\mathrm{m}}$, the peak value of arc current $i_{\mathrm{m}}$, the peak value of gas pressure pm, arcing duration time $t_{\mathrm{a}}$, and the time corresponding to the arc voltage peak value $t_{\mathrm{pv}}$.

Table 1 Experimental results for different venting size

\begin{tabular}{llllll}
\hline Venting size & $u_{m}(\mathrm{~V})$ & $i_{m}(\mathrm{kA})$ & $p_{m}(\mathrm{kPa})$ & $t_{a}(\mathrm{~ms})$ & $t_{p v}(\mathrm{~ms})$ \\
\hline $100 \%$ & 170 & 13.3 & 109.8 & 21.10 & 6.14 \\
$90 \%$ & 236 & 13.1 & 118.0 & 9.43 & 6.67 \\
$80 \%$ & 274 & 12.4 & 130.4 & 9.19 & 5.95 \\
$70 \%$ & 288 & 12.2 & 152.0 & 9.05 & 5.90 \\
$50 \%$ & 306 & 12.4 & 162.4 & 9.00 & 5.81 \\
$25 \%$ & 319 & 12.2 & 220.8 & 8.90 & 5.95 \\
$15 \%$ & 336 & 12.2 & 236.2 & $8.86^{*}$ & 5.93 \\
$5 \%$ & 358 & 12.0 & 277.5 & $8.62^{*}$ & 5.79 \\
\hline
\end{tabular}

* Due to the occurrence of dielectric reignition, the arcing duration time only includes the first half cycle under the venting size of $15 \%$ and $5 \%$.

From the above experimental results, it demonstrates that the venting size has evident effect on interruption performance.

To the experimental result of the venting size of $100 \%$, as shown in Fig. 4(a), the peak value of arc voltage is only $170 \mathrm{~V}$, which results in that dielectric recovery strength is not high enough, then re-ignition occurs just post arc current zero. In addition, the measured gas pressure rise inside the arc quenching chamber is only $109.8 \mathrm{kPa}$, so the action of gaspuffer effect is less.

According to the experimental results as shown in Table 1, when the venting size varies from $90 \%$ to $25 \%$, the interruption processes are all successful with the peak value of arc voltage rising from $236 \mathrm{~V}$ to $319 \mathrm{~V}$, and the gas pressure rising inside 
the arc quenching chamber from $118 \mathrm{kPa}$ to $220.8 \mathrm{kPa}$, which indicates that smaller venting size improves the interruption performance for a certain range of venting size.

When the venting size decreases to $15 \%$, arc re-ignites after the first current zero with the currentless period $7.7 \mathrm{~ms}$. A similar result can be drawn if the venting size is $5 \%$. In this case, the peak value of arc voltage is $358 \mathrm{~V}$, the arc also reignites after the first current zero, and the currentless period is $5.14 \mathrm{~ms}$. It should be noted that the type of reignition in these two cases the arc reignition after first current zero, but with currentless period lasting.

If the venting size is comparatively bigger, then the gas pressure rise inside the arc quenching chamber is lower, and the pressure gradient is less. In this paper, we define the vector $\frac{\overrightarrow{\Delta p}}{\Delta x}$ from arc igniting position to the vent in $x$ direction as the pressure gradient when $t$ is any constant during the interruption time. Therefore, the action of the gas-driven arc is unapparent, and the arc can not enter the splitter plates so the interruption performance is lower. In addition, the dielectric recovery strength is reduced and re-ignition occurs.

On the other hand, if the venting size is extremely small, then the gas pressure rise and the pressure gradient inside the arc quenching chamber are all higher. Therefore, the gas-driven force acting on arc is stronger, and the interruption performance is better during the first half cycle. However, due to the smaller venting size, it is difficult for the metallic vapor to escape from the arc quenching chamber, and the heat exchange between the high temperature gas and the environmental atmosphere is not sufficient. Therefore, the reignition occurs either. In addition, the smaller venting size is, the currentless period time is shorter. For example, to $15 \%$ and $5 \%$ venting size, the currentless period are $7.7 \mathrm{~ms}$ and $5.14 \mathrm{~ms}$, respectively.

In order to analyze the effect of the venting size on gasdriven arc performance, we define the arc voltage rise as $\triangle u$ / $\triangle t_{\mathrm{pv}}$ the results is shown in Table 2.

Table 2 Comparison of the rising ratio of arc voltage

\begin{tabular}{ccccccccc}
\hline Venting size & $100 \%$ & $90 \%$ & $80 \%$ & $70 \%$ & $50 \%$ & $25 \%$ & $15 \%$ & $5 \%$ \\
\hline$\triangle u(\mathrm{~V})$ & 170 & 263 & 224 & 288 & 306 & 319 & 336 & 358 \\
$\triangle t_{p v}(\mathrm{~ms})$ & 6.14 & 6.67 & 5.95 & 5.90 & 5.81 & 5.95 & 5.93 & 5.79 \\
$\triangle u / \triangle t_{p v}(\mathrm{~V} / \mathrm{ms})$ & 27.69 & 35.38 & 46.05 & 48.81 & 52.67 & 53.61 & 56.67 & 61.83 \\
\hline
\end{tabular}

The analysis demonstrates that with the reduction of venting size, both the arc voltage rising ratio and arc voltage increases significantly with the action of the gas-driven force.

\section{B. The effect of the two kind of venting structure on the interruption under higher voltage}

In the following experiments, the venting size of the two kind of venting structure is same and equal to $50 \%$, all of them the venting area is $480 \mathrm{~mm}^{2}$, and the effective value of prospective short circuit current is $10 \mathrm{kA}$, however the charging voltage of the capacitor bank is $750 \mathrm{~V}$ in the following experiments.
Fig. 5 (a) and (b) show the experimental results of waveforms according to the above two kind of venting structure. The detail results are shown in Table 3. Under 500V charging voltage of capacitor banks, the peak value of arc voltage in side venting stricture is $306 \mathrm{~V}$, which is $30 \mathrm{~V}$ higher than middle venting structure. The arcing time is $9.0 \mathrm{~ms}$, which is shorter than middle venting structure. When the charging voltage increasing to $750 \mathrm{~V}$, the peak value of arc voltage in side venting structure is $374 \mathrm{~V}$, which is $78 \mathrm{~V}$ higher than middle venting structure. The arcing time is $9.1 \mathrm{~ms}$, but the arc in middle venting structure reignites and the arcing time is $20.3 \mathrm{~ms}$.

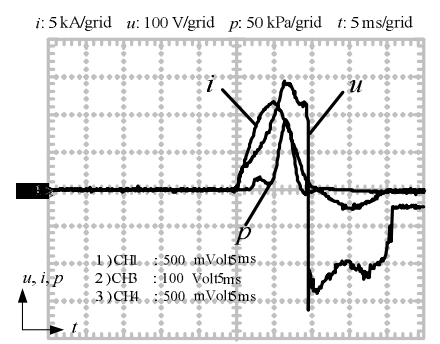

(a) Middle venting

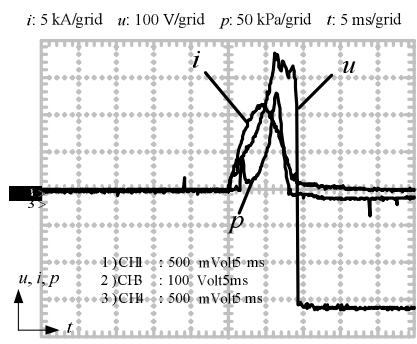

(b) Side venting
Fig.5 Experimental waveforms of the two kind of venting structure under $750 \mathrm{~V}$

Table 3 Experimental results of two kind of venting structure

\begin{tabular}{cccccc}
\hline $\begin{array}{c}\text { Venting } \\
\text { condition }\end{array}$ & $\begin{array}{c}\text { Changing } \\
\text { voltage(V) }\end{array}$ & $i_{\mathrm{m}}(\mathrm{kA})$ & $u_{\mathrm{m}}(\mathrm{V})$ & $p_{\mathrm{m}}(\mathrm{kPa})$ & $t_{\mathrm{a}}(\mathrm{ms})$ \\
\hline $\begin{array}{c}\text { Middle } \\
\text { venting }\end{array}$ & 750 & 13.1 & 296 & 99.2 & 20.3 \\
$\begin{array}{c}\text { Middle } \\
\text { venting }\end{array}$ & 500 & 13.3 & 276 & 106.8 & 9.48 \\
$\begin{array}{c}\text { Side } \\
\text { venting } \\
\text { Side } \\
\text { venting }\end{array}$ & 750 & 12.3 & 374 & 138.6 & 9.1 \\
\hline
\end{tabular}

From the results, the side venting structure gets higher arc voltage and pressure in chamber and shorter arcing time, therefore it gets better interruption performance under the condition of $500 \mathrm{~V}$ and $750 \mathrm{~V}$ charging voltage respectively. Especially, re-ignition occurs with the middle venting structure under $750 \mathrm{~V}$ charging voltage. It indicates that the post arc dielectric recovery strength is higher with the two-side venting structure under $750 \mathrm{~V}$. The side venting structure is benefit to improve the interruption performance, especially under the higher charging voltage of the capacitor bank.

\section{SimULATION RESULTS AND ANALYSIS}

In the paper, in order to investigate the arc behavior at the different venting structure, 3-D MHD model was developed by adapting and modifying the commercial computational fluid dynamics software FLUENT.

\section{A. Numerical model}

To the case of 3-D model, the governing equations are as follows, with $\mathrm{t}$ being time, $x_{\mathrm{k}}$, and $x_{\mathrm{n}}$ Cartesian coordinates, $\vec{v}$ flow velocity, $\vec{v}_{i}$ velocity in i-direction (i=x, y, z), $p$ pressure, $T$ temperature, $H$ dynamic enthalpy, $\vec{J}$ current 
density, $\vec{B}$ magnetic flux density, $v$ viscous dissipation function, $S_{\mathrm{R}}$ radiative cooling, $\rho$ density, $\eta$ viscosity, $\lambda$ thermal conductivity, $\sigma$ electrical conductivity[5],[8].

Mass balance:

$$
\frac{\partial \rho}{\partial t}+\operatorname{div}(\rho \vec{v})=0
$$

Momentum balance:

$$
\begin{aligned}
& \frac{\left(\partial \rho v_{i}\right)}{\partial t}+\operatorname{div}\left(\rho \vec{v} v_{i}\right) \\
& =-\frac{\partial p}{\partial x_{i}}+\sum_{k=1}^{3} \frac{\partial}{\partial x_{k}}\left[\eta\left(\frac{\partial v_{i}}{\partial x_{k}}+\frac{\partial v_{k}}{\partial v_{i}}\right]+(\vec{J} \times \vec{B})_{i}\right.
\end{aligned}
$$

Source term, $\vec{J} \times \vec{B}$, is the Lorentz force density, which describes the interaction between the electric current and the magnetic field.

Heat balance:

$$
\begin{aligned}
& \frac{\partial(\rho H)}{\partial t}+\operatorname{div}(\rho \vec{v} H) \\
& =\operatorname{div}(\lambda \operatorname{grad} T)+\frac{\partial p}{\partial t}+V-S_{R}+\frac{1}{\sigma} \vec{J}^{2}
\end{aligned}
$$

\section{Electromagnetic equation [7]:}

The magnetic field is obtained by calculating the potential vector $\vec{A}$.

$$
\nabla \cdot\left(\nabla A_{i}\right)=-\mu_{0} j_{i}
$$

The electrical potential is calculated by

$$
\nabla \cdot(\sigma \nabla \phi)=0
$$

Basing on Maxwell's equation, the following equations can be obtain

$$
\begin{aligned}
\vec{B} & =\nabla \times \vec{A} \\
\vec{E} & =-\nabla \phi \\
\vec{j}_{i} & =\sigma \vec{E}
\end{aligned}
$$

$S_{R}$ represents the radiation energy losses of per unit volume, which is the function of plasma temperature and pressure. In this section, the gray body radiation model is adopted as shown in equation 9.

$$
S_{R}=4 \alpha k\left(T^{4}-T_{0}^{4}\right)
$$

Where, $k=13 \frac{1}{m} \cdot \frac{p}{p_{0}}, \alpha=5.67057 \cdot 10^{-8} \mathrm{~W} / \mathrm{m} 2 \mathrm{~K}$

Fig.6 (a) shows the calculated geometry with the current of $400 \mathrm{~A}$, the geometry size is $20 \mathrm{~mm} \times 4 \mathrm{~mm} \times 9 \mathrm{~mm}$.

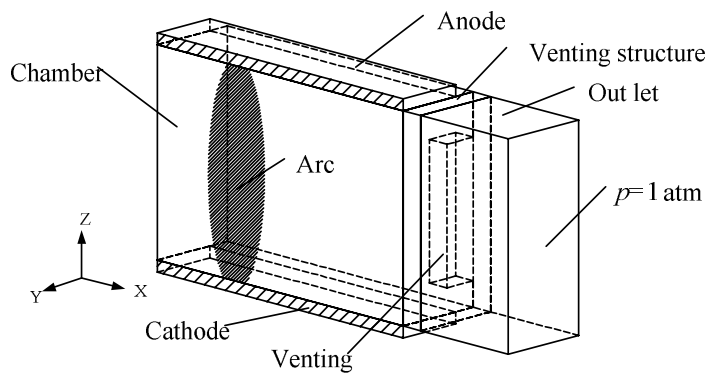

(a)

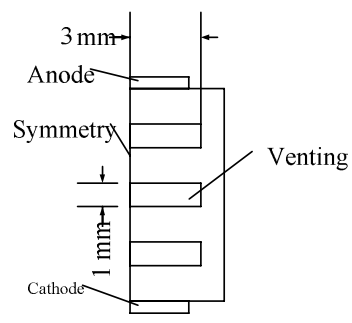

(b)

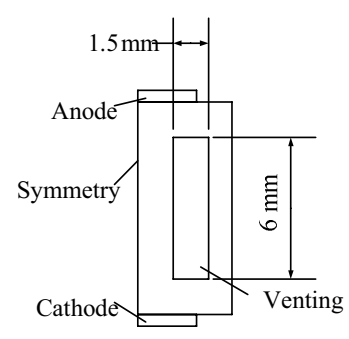

(c)
Figure 6 Calculation geometry

Fig.6 (b) and (c) gives the middle venting structure and side venting structure, respectively. The current flows through the anode, the arc column and cathode. The two structure vent area is same and equal to $6 \mathrm{~mm}^{2}$.

\section{B. Simulation results and analysis}

In this section, we use DC 400A current to simulate the arc motion in the model chamber. The temperature distribution at venting is shown in Fig.7 during the arc motion. It can be noticed that while reaching the venting barrier, the arc column's shape changes greatly, because the venting structure difference. In the conventional middle venting structure, the arc column concentrates in the center of the chamber. However, the arc column changes sector shape and moves alongside the two sides venting at the venting face in the side venting structure.

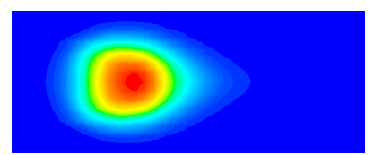

$$
t=0.2 \mathrm{~ms}
$$

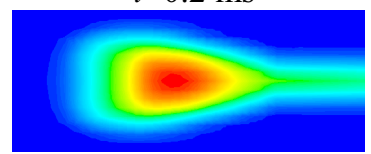

$t=0.3 \mathrm{~ms}$

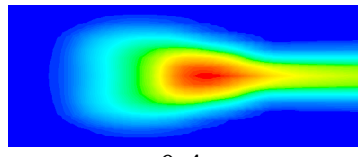

$t=0.4 \mathrm{~ms}$

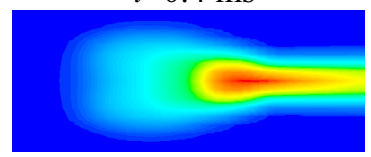

$t=0.5 \mathrm{~ms}$

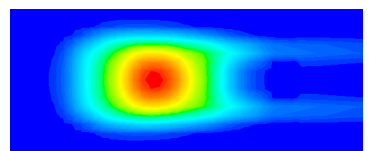
$t=0.2 \mathrm{~ms}$

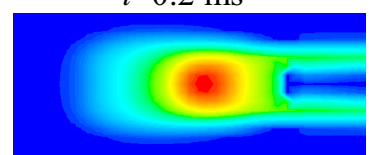

$t=0.3 \mathrm{~ms}$

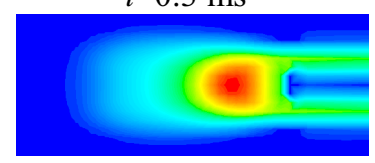

$t=0.4 \mathrm{~ms}$

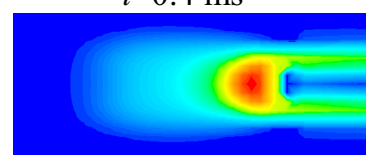

$t=0.5 \mathrm{~ms}$ 


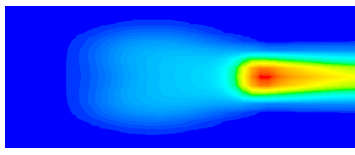

$t=0.6 \mathrm{~ms}$

(a)

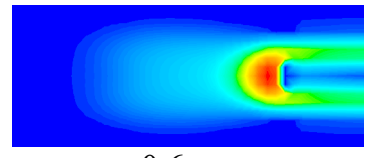

$=0.6 \mathrm{~ms}$

(b)
Figure 7 Temperature distributions in the $x-y$ plane located in the center of the geometry with different venting structure according to time

$\begin{array}{ll}\text { (a) Middle venting (b) Side venting } & \end{array}$

Fig.8 shows the time dependence of the calculated arc voltage between anode and cathode for the two venting structures. Clearly, side venting structure leads to an increasing in arc voltage at venting. Especially the arc voltage is magnificent difference when the arc column at the venting. It increases nearly $9 \mathrm{~V}$, about $25 \%$, more than middle venting.

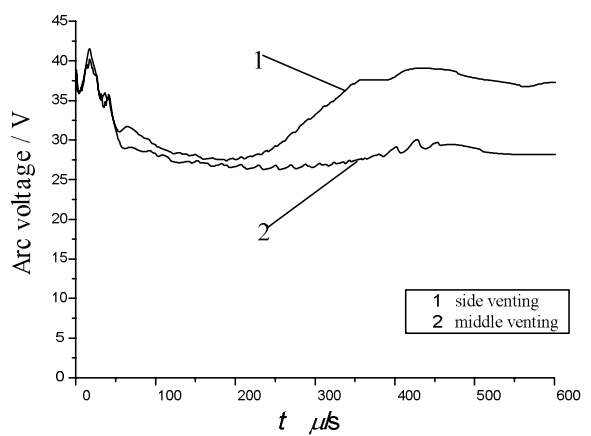

Figure 8 Arc voltages for different venting structure

The arc voltage increasing indicates the side venting structure gets better interruption performance than middle venting structure. This is benefit to dielectric recovery strength after current zero. Therefore, the side venting structure interrupts the test current successfully, when the charging voltage of the capacitor bank increases to $750 \mathrm{~V}$.

\section{SUMMARY}

From the study above, the following points can be obtained:
1) It is one certain venting size range to implement better interruption performance. If the venting size is comparatively bigger, the reignition may occur due to less gas flow effect. If the venting size is extremely small, the reignition may occur due to the escape difficulty of metallic vapor, and the insufficient of heat exchange.

2) The side venting structure benefits to improve the interruption performance, both under $500 \mathrm{~V}$ and $750 \mathrm{~V}$ charging voltage of capacitor bank conditions.

3) A 3-D MHD model is used to simulate the arc motion in the two different venting conditions. The results demonstrate the arc column has a better shape and gets a higher arc voltage in side venting structure.

\section{REFERENCES}

[1] J. W. McBride, and K. Pechrach, "Arc Motion and Gas Flow in Current Limiting Circuit Breakers Operating with a Low Contact Switching Velocity" IEEE Trans. Components and Packaging Technologies, vol.25, no.3, pp.427-433.

[2] J. W. McBride, K. Pechrach, and M. Paul "Weaver Arc Root Commutation from Moving Contacts in Low Voltage Devices. IEEE Trans. Components and Packaging technologies, vol.24, no.3, pp.331336, 2001.

[3] X. M. Tsukima, T. Mitsuhashi, "Low Voltage Circuit Breaker using Auto-puffer Interruption Technique," Transactions of the Institute Electrical Engineers of Japan (B), vol.122, no.9, pp.969-975, 2002.

[4] Chen D, Dai R and Li X "Experimental Investigation on the Arc Motion with Different Configurations of Quenching Chamber in AC Contactor".2006 IEICE Trans. on Electron. E89C 1201-1205.

[5] Frank Karetta and Manfred Lindmayer, "Simulation of the Gasdynamic ad Electromagnetic Processes in Low Voltage Switching Arcs." IEEE Trans on Components, Packaging, And Manufacturing Technology21, 1998, 96-103.

[6] Lindmayer M, Marzahn E, Mutzke A, Ruther T and Springstubbe M 2004 The process of arc-splitting between metal plates in low voltage arc chutes Proc. 50th IEEE Holm Conf. Electrical Contacts (Seattle) p 28-34.

[7] M. Rong, Y. Wu, Y. Fei, Z. Sun, W. Wang and X. Wang "the Reaearch of Electric Arc Simulation in Low Voltage Switching Devices." 17th Gas Discharge and Their Application Conf. (Cardiff) p 9-16.

[8] A Gleizes, J J Gonzalez and Freton, "Thermal Plasma Modelling." Journal of Physics D: Applied Physics 38, 2005 R153-183. 


\section{Gold coated Multi-Walled Carbon Nanotube Surfaces as Low Force Electrical Contacts for MEMS devices: Part 1.}

\author{
J.W.McBride \\ University of Southampton \\ School of Engineering Sciences \\ Southampton, UK, SO17 1BJ
}

\author{
E.M Yunus \\ University of Southampton \\ School of Engineering Sciences \\ Southampton, UK, SO17 1BJ
}

\author{
S.M Spearing \\ University of Southampton \\ School of Engineering Sciences \\ Southampton, UK, SO17 1BJ
}

\begin{abstract}
An experimental investigation of gold coated, multiwalled carbon nanotube (CNT) surfaces was conducted, to determine the limits of these surfaces as electrical contacts. The multi-walled CNT's (MWCNT's) were synthesized on a silicon planar and sputter coated with a gold film. The planar surfaces were mounted on the tip of a piezo-electric actuator and mated with a coated Au hemispherical probe. The load conditions were typical of MEMS relay applications; $(4 \mathrm{~V}, 1-10 \mathrm{~mA})$ with an applied force of $1 \mathrm{mN}$. For these conditions the surfaces were shown to maintain a stable contact resistance over $10^{6}$ switching cycles. To determine the limits, the contact force was increased to $3 \mathrm{mN}$ under dry circuit conditions and the current increased to $20 \mathrm{~mA}-50 \mathrm{~mA}$ for the $1 \mathrm{mN}$ contact force. The contact resistance and surface degradation were compared to a reference Au-Au contact surface. For the coated MWCNT surfaces investigated the current loading limit was determined to be $20 \mathrm{~mA}$ with the contacts failing with increased contact resistance after 50x10 cycles.
\end{abstract}

Keywords-component; Carbon nanotube surfaces, MEMS switching surfaces, Carbon nanatube composites.

\section{INTRODUCTION}

The application of $\mathrm{Si}$ based Micro-Electro-Mechanical (MEMS) switching devices is limited by the poor reliability of the electrical contact surfaces. Switching an electrical load is often referred to as "hot switching" when related to MEMS. A typical MEMS electrical load of $1 \mathrm{~mA}-10 \mathrm{~mA}, 4 \mathrm{~V}$, is below the minimum arcing voltage $(\sim 12 \mathrm{~V})$, however above the softening and melting voltages for metallic surfaces, leading to the degradation processes. When switching an electrical load, metallic surfaces such as gold degrade; as a result of localized thermal phenomena such as the molten metal bridge phenomena, [1]. The "dry circuit" condition is associated with mechanical switching where the applied voltage and current have no physical influence on the surface, [2]. To overcome the limitations of the metallic electrical contact surfaces, consideration is given to the development of a compliant substructure using multi-walled carbon nanotubes (MWCNT). This study focuses on experimental results, while in Part 2, [3], results are provided on the material and electrical properties of the surfaces.
A MEMS relay or switch requires a low stable contact resistance $(1 \sim 2 \Omega)$ over millions of switching cycles. This requires a contact material with low resistivity, high wear resistance and high resistance to oxidation [4-9]. To overcome the limitations of the metallic electrical contact surfaces, other materials have been investigated, including silicon carbide and diamond films. Both have high elastic moduli but also a high electrical resistivity. There have been attempts to lower the resistivity. When doping $\mathrm{SiC}$ film with $\mathrm{NH}_{3}$ the resistivity drops to $1 \times 10^{-4} \Omega \mathrm{m}[10]$ and doping DLC with ruthenium the resistivity drops to $1 \times 10^{-5} \Omega \mathrm{m}$ [11], however, both materials have a high resistivity compared to gold and gold alloys (for example Au-6.3\% Pt has a resistivity of $7.17 \times 10^{-8} \Omega \mathrm{m}$ ), [4].

Carbon nanotube (CNT) coated surfaces show potential as a MEMS electrical contact material, since the mechanical and electrical properties are potentially comparable to diamond and gold respectively [12-16]. In [17] the contact resistance against applied force relationship was investigated between two multi-walled CNT's (MWCNT's) surfaces. The contact resistance was measured in ambient air and in a vacuum. It was concluded that the contact resistance was lower in ambient air $(\sim 160 \Omega)$ than in vacuum $(>4 \mathrm{k} \Omega)$. In [18] a microtribometer was used to determine the contact resistance between electrodes where the upper and lower electrodes were coated with $\mathrm{Au}$ and CNT respectively. The CNT surface in this case was a tangled single walled carbon nanotubes structure, and the applied force $\sim 150 \mathrm{mN}$. The authors concluded that a tangled single-walled carbon nanotube (SWCNT) film in contact with a Au coated surface has a lower contact resistance than two contacting SWCNT films.

A potential solution to the MEMS electrical contact problem is to use a "forest" of vertically aligned, multi-walled carbon nanotubes (MWCNT), and to coat these with a conductive gold layer. This creates a CNT composite surface, with a high conductivity surface layer and a compliant under layer. In [19], a modified nano-indentation apparatus was used to determine the contact resistance as a function of contact force $(1 \mathrm{mN})$ with up to 10 "dry circuit" switching operations. In [20] a piezo-electric (Lead Zirconate Titanate), PZT, actuator was 
used to support planar coated surfaces for "dry circuit" switching up to 1000 cycles. It was shown that the mechanical performance and contact resistance of $\mathrm{Au}-\mathrm{Au} / \mathrm{MWCNT}$ contact pairs were comparable to Au-Au contact pairs. In [21] the PZT apparatus was used in an initial study of the "hot switching" condition $(1 \mathrm{~mA}-10 \mathrm{~mA}$ at $4 \mathrm{~V})$. The results showed that the $\mathrm{Au}-\mathrm{Au} / \mathrm{MWCNT}$ contact pair maintained a stable contact resistance over $2 \times 10^{6}$ switching cycles.

\section{METHODS}

\section{A. Material Preparation}

Two contact pairs were investigated; $\mathrm{Au}$ to $\mathrm{Au}$ and $\mathrm{Au}$ to a $\mathrm{Au} /$ multi walled carbon nanotube (MWCNTs) composite. The pairs consisted of a $2 \mathrm{~mm}$ diameter hemisphere probe sputter coated with $\mathrm{Au},(\sim 500 \mathrm{~nm})$, with a surface roughness $\mathrm{R}_{\mathrm{a}} \approx 400 \mathrm{~nm}$; contacting a flat or planar surface, [19-21]. The $\mathrm{Au}$ flat surface was a silicon $(\mathrm{Si})$ substrate, sputter coated with $\mathrm{Au}$ $(\sim 500 \mathrm{~nm})$, with surface roughness, $R_{\mathrm{a}} \approx 30 \mathrm{~nm}$.

The MWCNTs were grown on a $\mathrm{Si}$ wafer using thermal CVD. The catalyst used was $\mathrm{Fe}$ and the gaseous carbon source ethylene. The growth temperature and time, $875^{\circ} \mathrm{C}$ and 3 minutes respectively to produce a dense forest of vertically aligned MWCNT of an average length of $\sim 50 \mu \mathrm{m}$; as shown in Fig 1. The packing density of the structure has yet to be determined; however Fig.1 shows the uniformity of the structure and the apparent roughness of the top surface of the structure. The MWCNT surface was sputtered with $\mathrm{Au}$ to produce the Au/MWCNT composite, as shown in Fig 2, with surface roughness $\mathrm{R}_{\mathrm{a}} \approx 1.5 \mu \mathrm{m}$. Fig. 2 shows the $\mathrm{Au}$ film to have a number of sub-micron voids. The thickness of the Au has yet to be determined; however pervious SEMs studies suggest that the Au diffuses into the CNT structure, [19-21]

\section{B. Experimental Apparatus}

The apparatus used is shown in Fig 3, and described in detail in, [20]. The piezo-electric, PZT actuator (Lead Zirconate Titanate) was used to support the planar coated surfaces. The system has been designed to model a typical MEMS relay electrical contact. The contact force can be controlled and was set to $1 \mathrm{mN}$ or $3 \mathrm{mN}$. The apparatus was mounted in a sealed enclosure at room temperature. The performance of the Au-Au/MWCNT surfaces were compared to a reference $\mathrm{Au}-\mathrm{Au}$ contact pair under the same experimental conditions in order to assess the mechanical and electrical stability and reliability. Surface characterization methods were used, including SEM and 3D surface profiling (TaiCaan Technologies XYRIS 4000CL), to measure the surface degradation.

\section{Experiment 1: Determining the Mechanical Switching Limits of the Au to Au/MWCNT surface.}

In the previous mechanical studies the contact force was limited to $1 \mathrm{mN}$, [19]; in this study the static force is increased to $3 \mathrm{mN}$. With the increased static force the impact forces will be much higher. The aim is to determine the limits of the mechanical or "dry switching" characteristics.

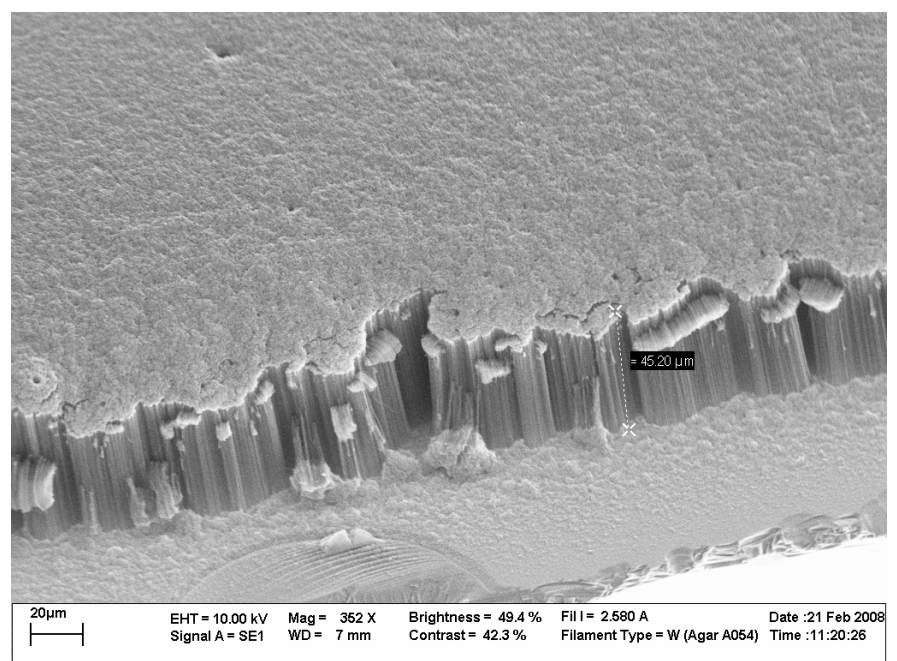

Figure 1. MWCNT with average length $\sim 50 \mu \mathrm{m}$.

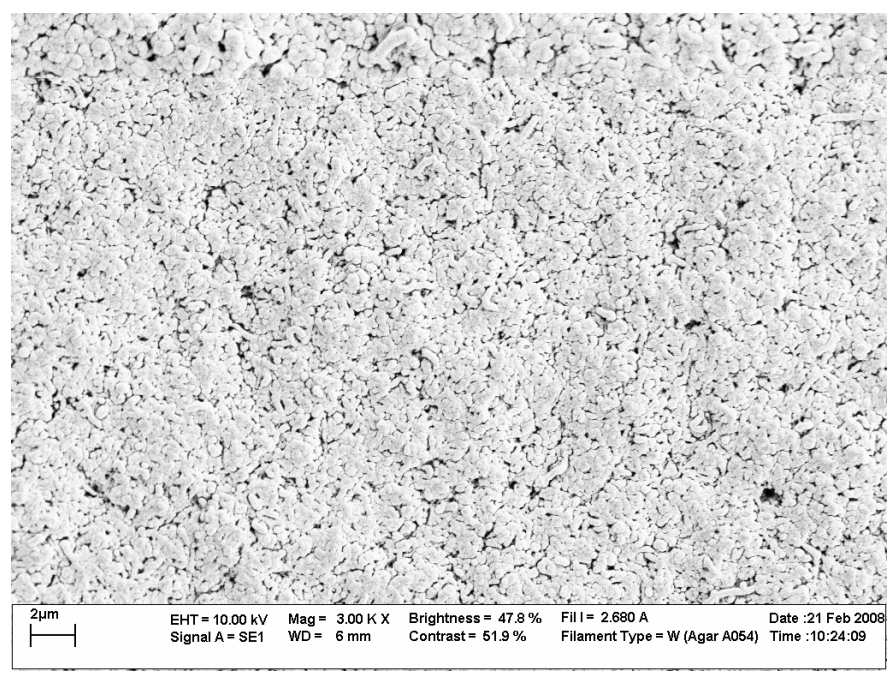

Figure 2. $\mathrm{Au} / \mathrm{MWCNT}$ composite contact surface.

\section{Experiment 2: Hot-switched condition with $1 \mathrm{~mA}$ and $10 \mathrm{~mA}$ at $4 \mathrm{~V}$}

Over the first 1000 cycles the PZT actuator was controlled at low frequency $(0.2 \mathrm{~Hz})$ to allow a quasi-static study of the contact surfaces. At $0.2 \mathrm{~Hz}$ the contact force is applied for $\sim 3$ seconds so that a representative average contact resistance value can be determined. To replicate the conditions of a MEMS relay the "hot-switching" loads used were $1 \mathrm{~mA}$ and 10 $\mathrm{mA}, 4 \mathrm{~V}$, [22-24]. Fig 4 shows the circuit arrangement for the hot-switching experiment. After every $10^{\text {th }}$ cycle the load current is switched off and the contact resistance, $\left(\mathrm{R}_{\mathrm{c}}\right)$ measured using the 4 wire-measurement method. To study the contact surfaces over a million cycles, the PZT actuator was actuated at a higher frequency $(10 \mathrm{~Hz})$. To determine the contact resistance the cycling frequency is reduced to the quasistatic frequency $(0.2 \mathrm{~Hz})$, at $3000,6000,9000,10000,300000$, 865000,1 and 2 million cycles. 


\section{E. Experiment 3: Hot-switched condition, 20mA-50mA, $4 \mathrm{~V}$.}

The current load was increased to $20 \mathrm{~mA}-50 \mathrm{~mA}$ to observe and benchmark the performance of these surfaces. For currents of $30 \mathrm{~mA}-50 \mathrm{~mA}$ the PZT actuator was actuated at $0.2 \mathrm{~Hz}$. After every 10 cycles the current and supply voltage was switched off and the contact resistance measured. For the current loading of $20 \mathrm{~mA}$ the sample was actuated at $0.2 \mathrm{~Hz}, 10 \mathrm{~Hz}$ and $20 \mathrm{~Hz}$. At $0.2 \mathrm{~Hz}$ every 10 cycles the $\mathrm{R}_{\mathrm{c}}$ was measured. After 1000 cycles the frequency was changed to $10 \mathrm{~Hz}$ and the contact resistance measured after 10000, 100000 and 1 million cycles. After 1 million cycles the frequency was changed to $20 \mathrm{~Hz}$ and the resistance monitored every 2 million cycles.

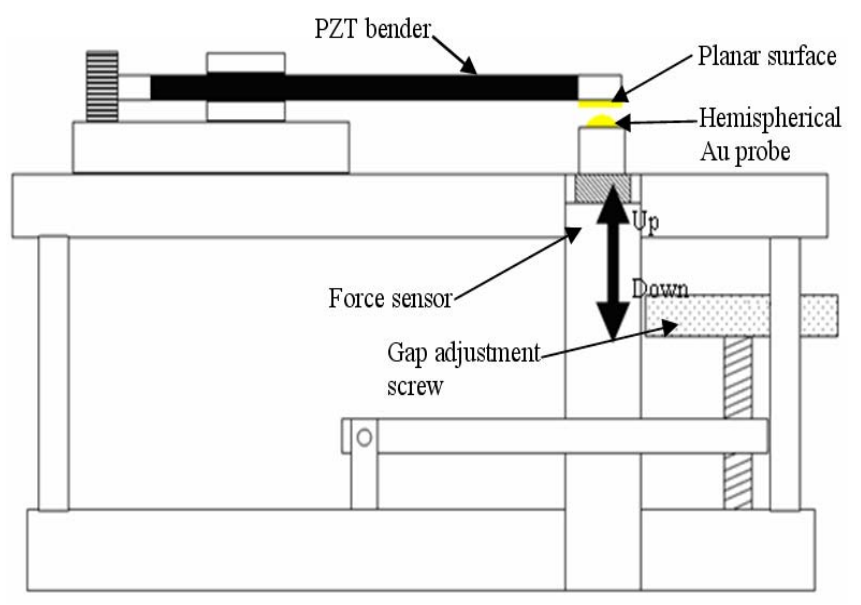

Figure 3. Schematic side view of the PZT test rig

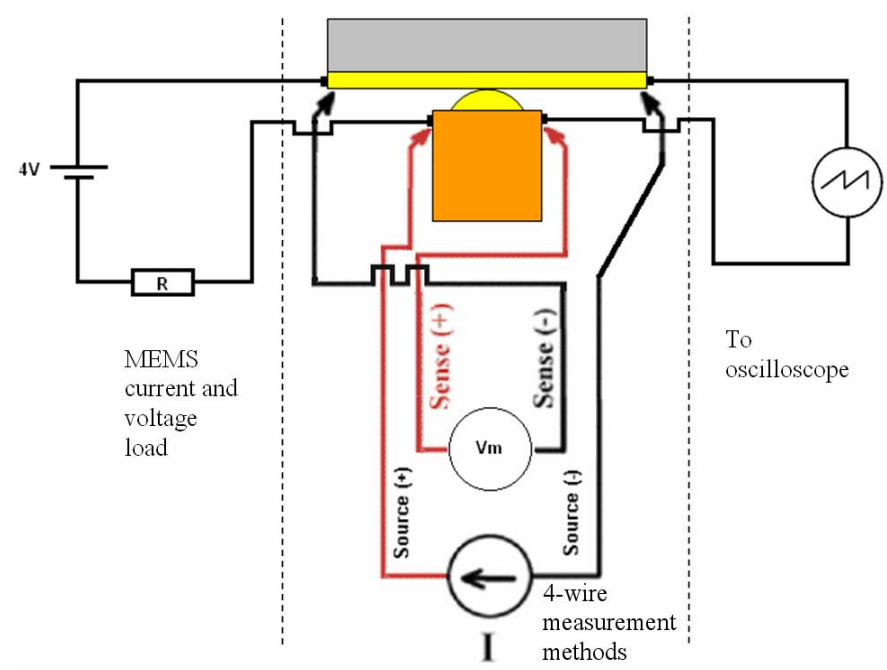

Figure 4. Schematic of contact zone with its electrode, current and supply load, 4 wire measurement and the monitoring.

\section{RESUlTS}

\section{A. Experiment 1: Determining the Mechanical Switching Limits of the Au to Au/MWCNT surface}

Fig. 5 shows the switching surfaces after $20 \times 10^{6}$ mechanical "dry circuit" switching cycles. There is limited damage to the ball surfaces in (a), while in (b) the Au/MWCNT surface shows evidence of cracking surrounding the impact area, further emphasized in the 3D data shown in (c). The depression in the surface (c) is $60 \mu \mathrm{m}$. This is a significant departure from results with $1 \mathrm{mN}$ where the deformation was typically $1-2 \mu \mathrm{m},[19]$. It is expected that the increase in the applied force has significantly increased the transient impact forces. There was no increase in the contact resistance during this study.

The depth of the crater $(60 \mu \mathrm{m})$ emphasizes the difficulty in determining the height of the CNT structure prior to testing. It was suggested in Fig. 1 that the height of the CNT structure is approximately $50 \mu \mathrm{m}$. It is expected that for the sample tested the $3 \mathrm{mN}$ force has deformed the surface to the base Si surface level. Fig. 5 also shows that the Au plating on the MWCNT structure is cracked. This is apparent in both SEM and 3D data images. It is expected that the cracking is associated with the fatigue performance of the Au plated structure.

\section{B. Experiment 2. Hot-switched condition with $1 \mathrm{~mA}$ and $10 \mathrm{~mA}$ at $4 \mathrm{~V}$}

Fig 6 shows the contact resistance of the Au-Au reference surface over 1000 cycles at a maximum (quasi static) applied load of $1 \mathrm{mN}$, with a $1 \mathrm{~mA}$ current. The contact resistance is initially $\sim 0.58 \Omega$ and increases rapidly to $4-10 \Omega$ at $\sim 220$ cycles. The increase in the contact resistance corresponds to the adhesion of the Au-Au pair and the resultant delamination of the Au coating on the ball, as shown in Fig 7. Fig.7 (a) shows the damaged $\mathrm{Au}$ hemispherical probe surface and the corresponding adhesion of the delaminated film to the $\mathrm{Au}$ planar surface. Fig 7 (c) shows 3D profile of the damaged $\mathrm{Au}$ hemispherical probe, to determine the thickness of the Au film. A is the top of the Au surface while B is the top of the underlying ball surface. This result confirms the thickness of the Au film on the ball to be $\sim 500 \mathrm{~nm}$. The peaks on the surface are caused by the tearing of the surface during the delamination process. The adhesion between the Au surfaces is a well know phenomena, and is in this case a combination of the softening and melting the Au layer, associated with the bridging phenomena resulting from the localized current density at the asperity contact peaks [1] and $\mathrm{Au}-\mathrm{Au}$ adhesion, [25]. Fig 6 also shows the contact resistance of the Au$\mathrm{Au} / \mathrm{MWCNT}$ contact pair at two different current loads (1mA and $10 \mathrm{~mA}, 4 \mathrm{~V})$. The contact resistance is higher than for the $\mathrm{Au}-\mathrm{Au}$ pair at the start of the cycling, $(\sim 0.68 \Omega)$ for both current load conditions and the resistance remains stable over the first 1000 cycles. This observation is consistent with previous force, contact resistance studies, [19]. 
Fig. 8 shows the contact resistance during extended cycling over 2 million cycles. The contact resistance is similar and stable for the current load levels, $1 \mathrm{~mA}$ and $10 \mathrm{~mA}$. Fig.9 shows the surfaces after the testing with a small amount of $\mathrm{Au}$ adhesion on the $\mathrm{Au}$ hemispherical probe in Fig. 9(a), at $1 \mathrm{~mA}$ and $10 \mathrm{~mA}$ in (b). This $\mathrm{Au}$ adhesion appears to be in one direction, from the Au/MWCNT planar surfaces to the Au ball. No observable damage on Au/MWCNT planar composite surfaces has been detected.

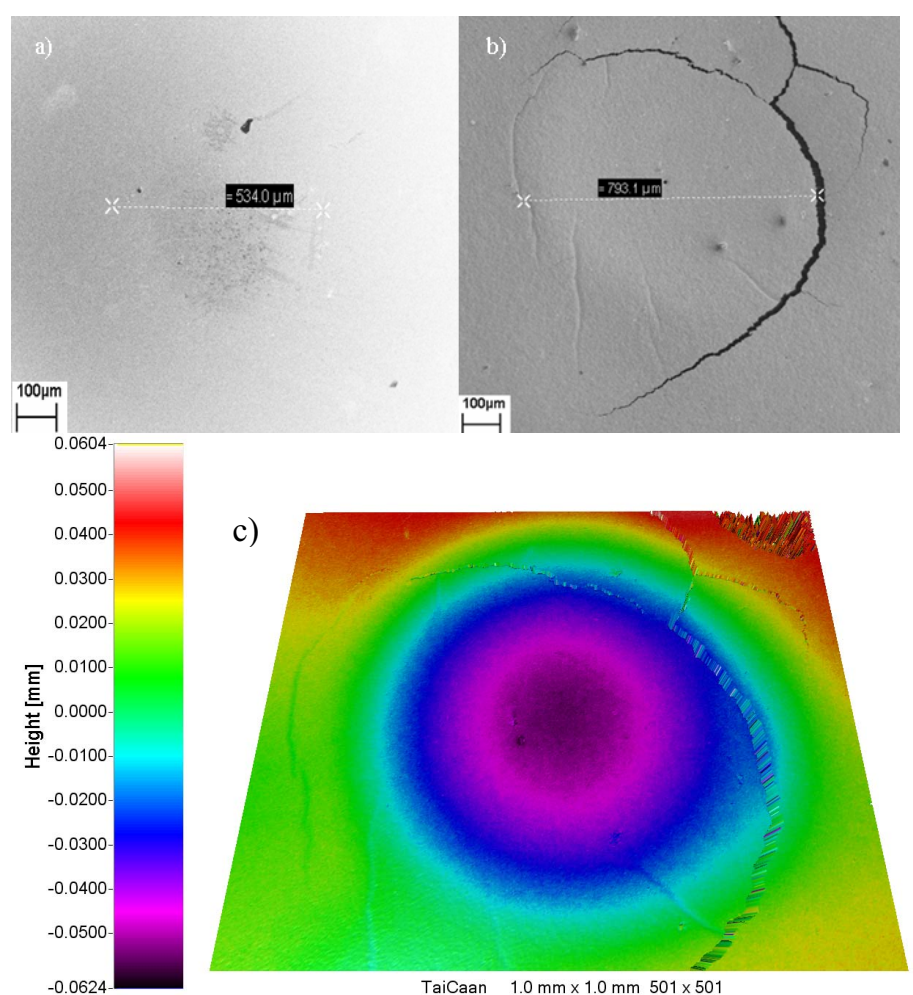

Figure 5. SEM images of a) Au hemispherical probe and b) Au/MWCNT surface after 20 million cycles at $3 \mathrm{mN}$, c) $501 \times 501(1 \mathrm{~mm} \times 1 \mathrm{~mm})$, 3D scan of $\mathrm{Au} / \mathrm{MWCNT}$ surface.

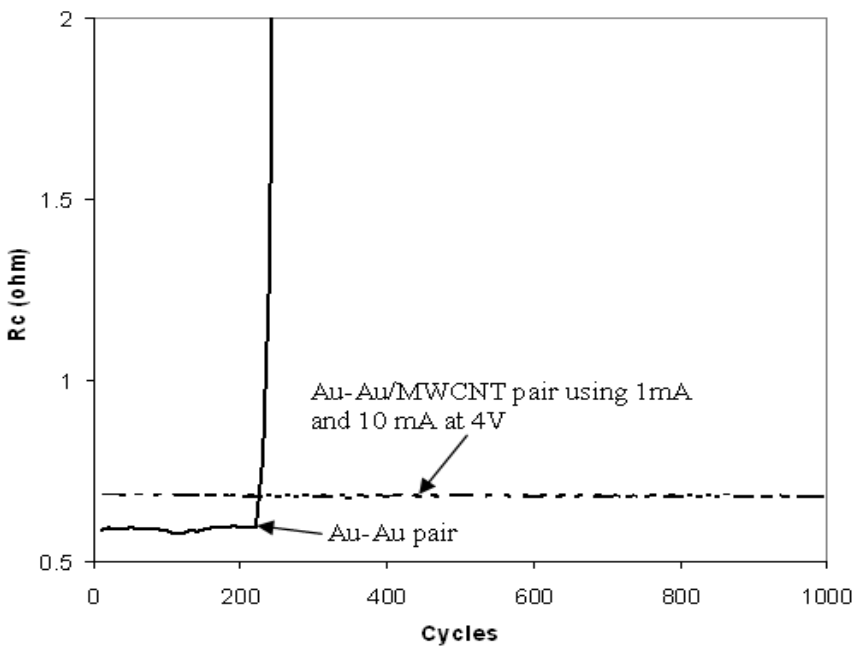

Figure 6: Contact resistance for $\mathrm{Au}-\mathrm{Au}$ and $\mathrm{Au}-\mathrm{Au} / \mathrm{MWCNT}$ contact pair.

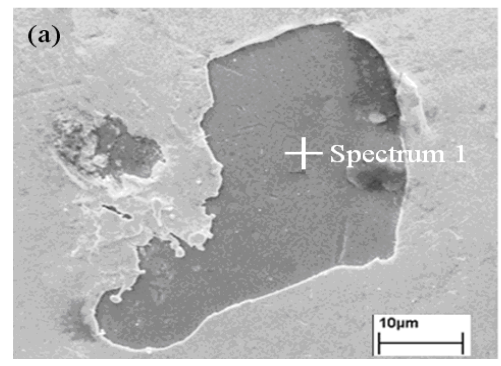

(b)

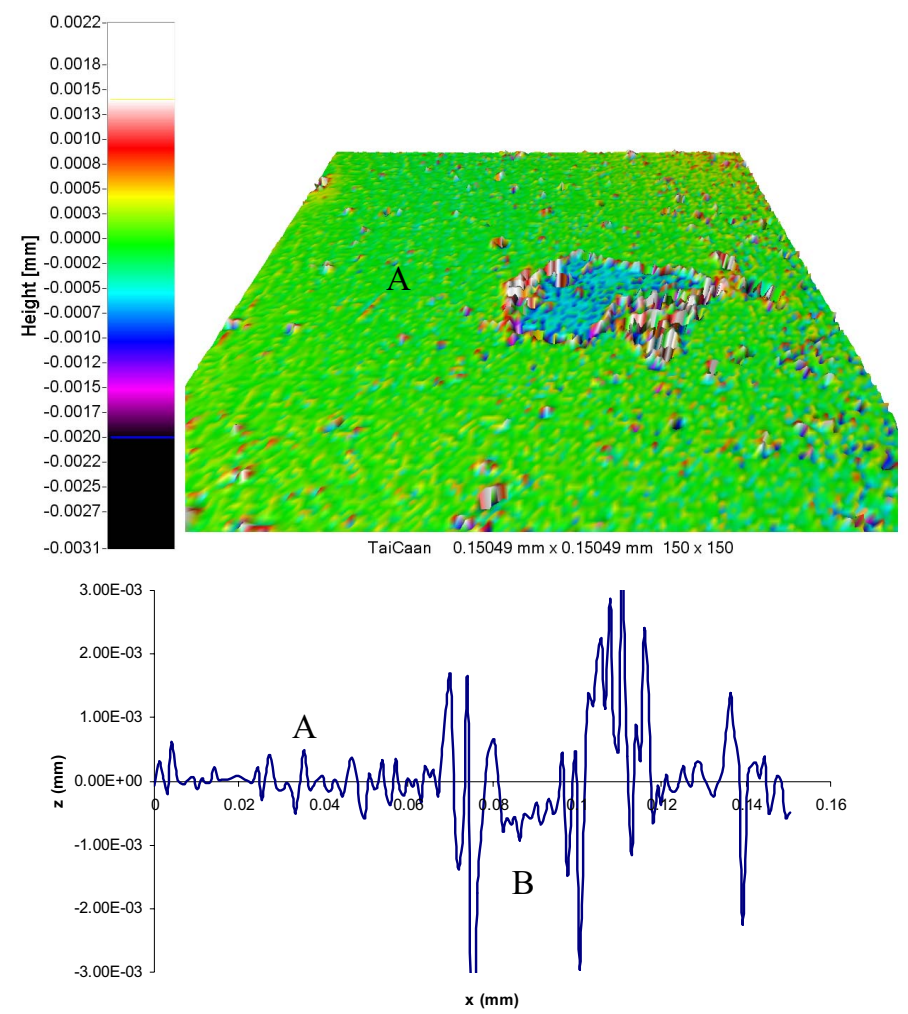

Figure 7: (a) SEM image of Au hemispherical probe degradation (b) SEM image of Au planar with Au debris (c) Scanned image of damaged Au ball for $\mathrm{Au}-\mathrm{Au}$ pair $(1 \mathrm{~mA}, 4 \mathrm{~V})$ with the sphere removed, contact pair $150 \times 150$ $(0.15 \mathrm{~mm} \times 0.15 \mathrm{~mm})$ using TaiCaan (Xyris $4000 \mathrm{CL}$ ).

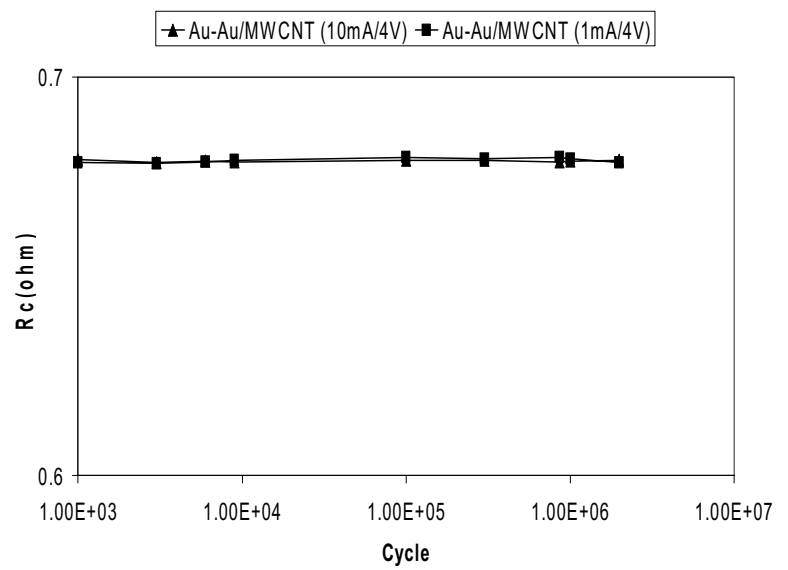

Figure 8: Contact resistance for $1 \mathrm{~mA}$ and $10 \mathrm{~mA}$ over 2 million cycles for the $\mathrm{Au}-\mathrm{Au} / \mathrm{MWCNT}$ contact. 


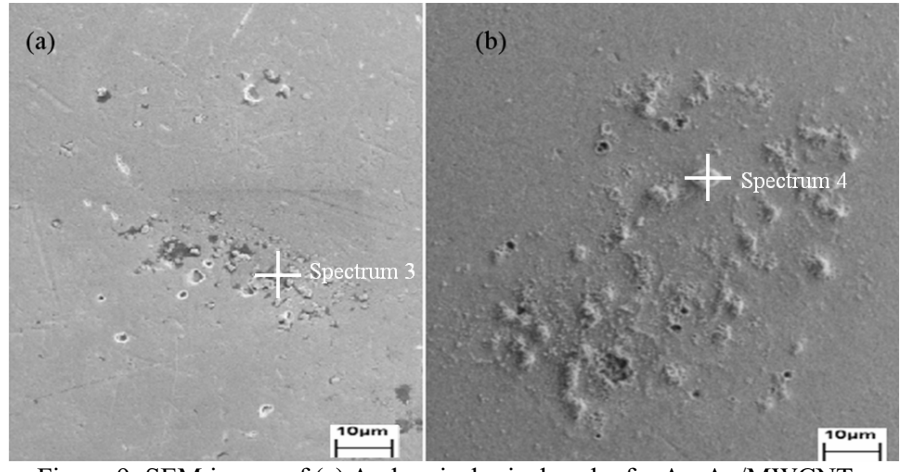

Figure 9: SEM image of (a) Au hemispherical probe for $\mathrm{Au}-\mathrm{Au} / \mathrm{MWCNT}$ contact pair after 2 million cycles at current load $1 \mathrm{~mA}, 4 \mathrm{~V}$ and (b) $\mathrm{Au}$ hemispherical probe at current load $10 \mathrm{~mA}, 4 \mathrm{~V}$.
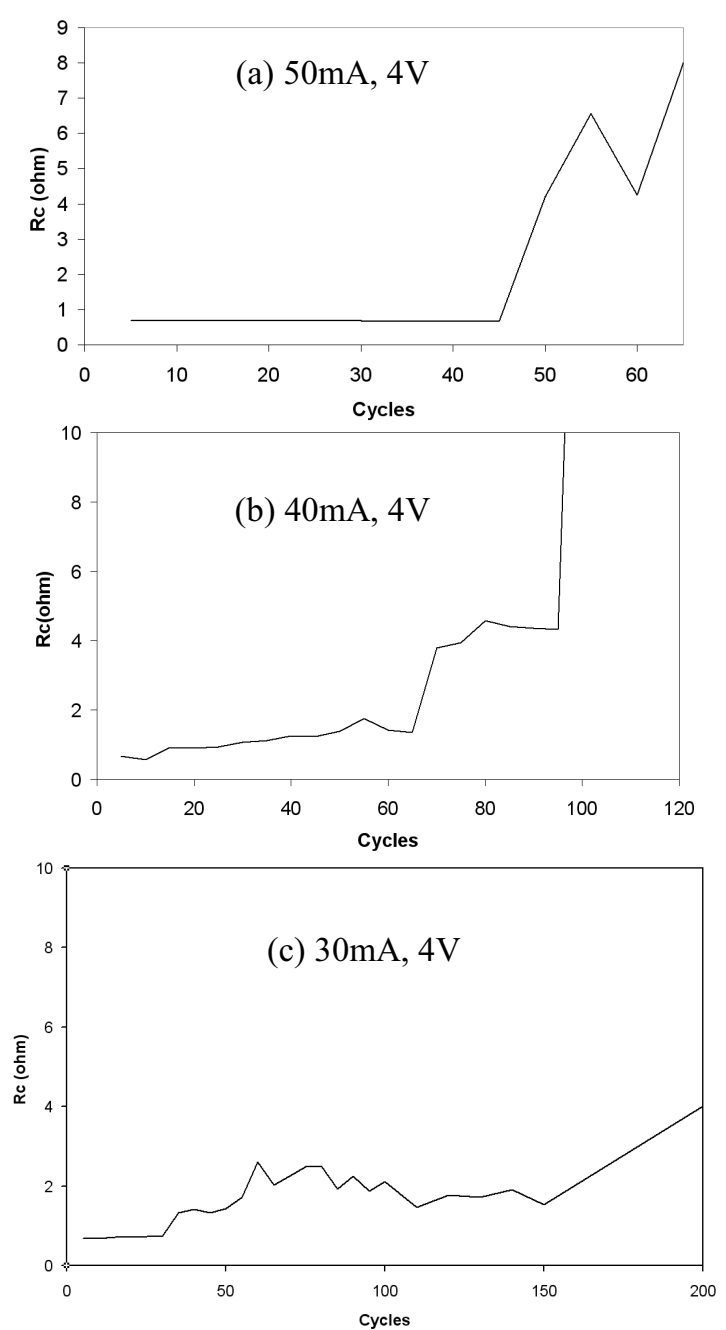

Figure 10: Graph of contact resistance against number of cycles for $\mathrm{Au}$ $\mathrm{Au} / \mathrm{MWCNT}$ contact pair with current load of (a) $50 \mathrm{~mA}$, and (b) $40 \mathrm{~mA}$ and (c) $30 \mathrm{~mA} 4 \mathrm{~V}$ (all $1 \mathrm{mN})$.

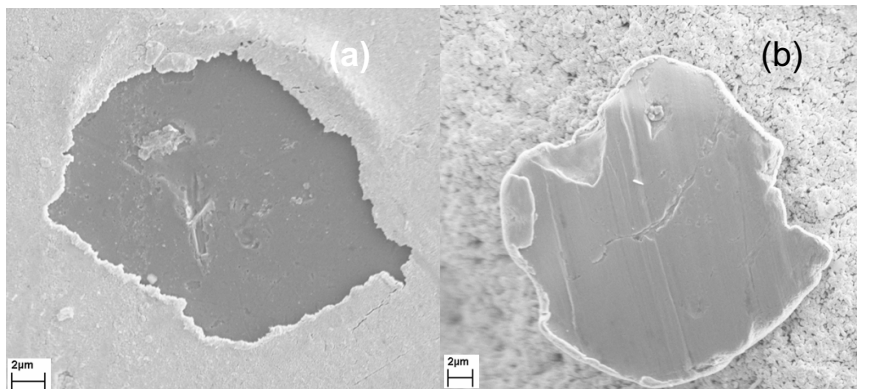

Figure 11: Damaged surface of Au hemispherical probe after using current load (a), and the Au adhered on the Au/MWCNT composite surface (b) for $50 \mathrm{~mA}, 4 \mathrm{~V}$

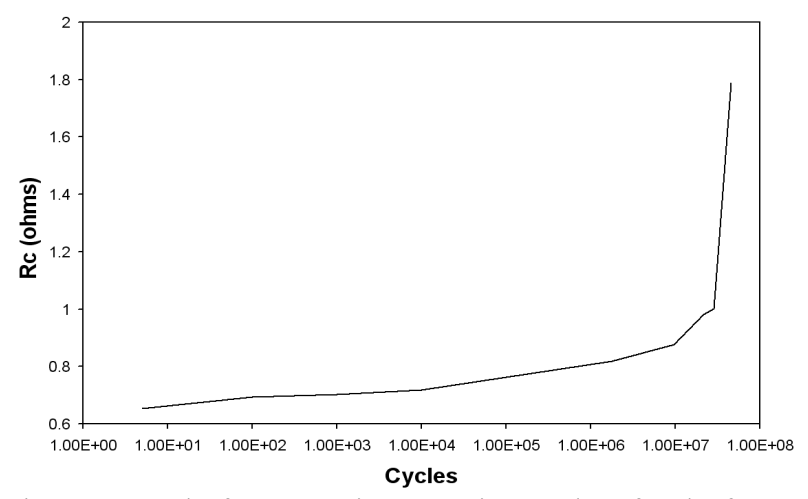

Figure 12: Graph of contact resistance against number of cycles for $\mathrm{Au}$ $\mathrm{Au} / \mathrm{MWCNT}$ contact pair with current load of $20 \mathrm{~mA}, 4 \mathrm{~V}(1 \mathrm{mN})$.

\section{Experiment 3: Testing the Limits; 20mA-50mA at $4 \mathrm{~V}$}

Fig 10 (a), (b) and (c) shows the contact resistance against number of cycles for the Au-Au/MWCNT contact pair using a current load between $30 \mathrm{~mA}-50 \mathrm{~mA}, 4 \mathrm{~V}$. In all cases it was observed that degradation has occurred between 45 and 150 cycles. As with the $1 \mathrm{~mA}$ test on Au-Au surfaces in Fig.7 the degradation has occurred on the surface of the Au coated ball shown in Fig 11, for the $50 \mathrm{~mA}$ test. The delamination corresponds to an increase in the contact resistance.

Fig 12 shows the contact resistance against number of cycles for Au-Au/MWCNT composite with a current load of $20 \mathrm{~mA}$. The contact resistance remains stable until $\sim 50$ million cycles. For this condition the degradation is no longer by the delamination process, but by the material transfer from the anode surface to the cathode, as shown in Fig 13 (a) and (b). $\mathrm{Au}$ from the top surface of the Au/MWCNT composite surface (anode) has transferred to the $\mathrm{Au}$ hemispherical probe (cathode).

\section{DISUSSION}

The results show there to be two degradation mechanism in the "hot switching" of the Au-Au/MWCNT structure. In the first, at higher current levels $>30 \mathrm{~mA}$ the process is consistent with the adhesion of the Au coating on the ball to the top of the $\mathrm{Au} / \mathrm{MWCNT}$ structure, as shown in Fig.11. This is a similar to that obtained on the reference Au-Au sample at a much lower current level of $1 \mathrm{~mA}$. Au-Au systems are known to exhibit cold welding even at very low contact forces, 
however in this case the phenomena observed is linked to the current density through the asperities of the surface and reflected in the current loading. The results presented here and in [19-21] show that the Au-MWCNT structure deforms to the applied force, increasing the apparent area between the contact surfaces, (see Fig.5).

The second degradation mechanism is consistent with bridge transfer between surfaces. This particular mechanism was widely studied in the 1950's and 1960's, for communication relays, which were super-seeded by electronic switching devices. The surface shown in Fig 13(a) on the Au ball, corresponds to the cathode surface, and shows a build up of material from the anode in Fig 13(b), for 20mA. The increase in the resistance is consistent with the removal of the $\mathrm{Au}$ conduction path on the top of the MWCNT surface. Fig. 9 shows a much lower level of transfer over the low number of cycles, and lower current levels, $1 \mathrm{~mA}$ and $10 \mathrm{~mA}$. It is interesting to note that although the conduction path has become depleted in Fig. 13, the contact resistance level is still relatively low $(<2 \Omega)$, showing the ability of the surface to also conduct through the CNT structure as observed in [19].

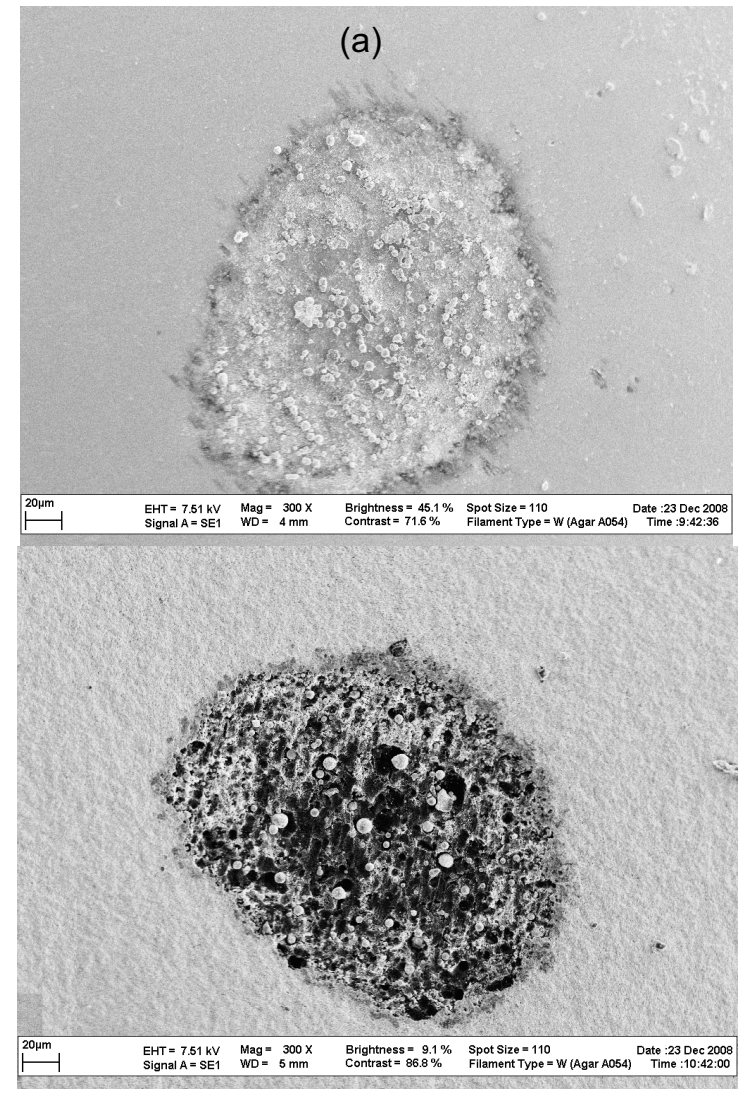

Figure 13: (a) Au adhering on the Au hemispherical probe (anode) and (b) damaged image on the Au/MWCNT composite surface (cathode).

\section{CONCLUSIONS}

It has been shown that a $\mathrm{Au}$ surface coupled with a $\mathrm{Au} / \mathrm{MWCNT}$ (gold coated multi-walled carbon nanotube structure), has the ability to sustain millions of switching cycles with a electrical load typical of current MEMS switch devices, typically $4 \mathrm{~V}, 1$ to $10 \mathrm{~mA}$, with a low contact force of $1 \mathrm{mN}$. This is an important observation, and lays the foundation for the application of carbon nanotube structures for electrical contact applications. The surfaces developed here use an approximate $500 \mathrm{~nm}$ covering of $\mathrm{Au}$ on a Stainless steel ball surface and a MWCNT surface. The contact resistance across the interface is used as the parameter for determining the failure modes of these surfaces.

For the mechanical cycling of the surfaces, two contact force levels have been used, $1 \mathrm{mN}$, and $3 \mathrm{mN}$. In both cases no failure mechanisms have yet been reported, however the contact force has been shown to have a significant influence of the cyclic deformation of the Au/MWCNT surface. Results show that the surface deforms to the base level of the CNT structure.

For the "hot switching" or electrically loaded surfaces, the contact force was maintained at $1 \mathrm{mN}$ with a $4 \mathrm{~V}$ supply, with a range of applied currents. An Au-Au reference surface was shown to fail after 250 switching cycles, due to adhesion of the Au surfaces. The Au-Au/MWCNT surfaces were subjected to current loads from $1 \mathrm{~mA}$ to $50 \mathrm{~mA}$. Failure was shown to occur in the 30-50mArange after a low number of cycles with a mechanism similar to that exhibited in the $\mathrm{Au}-\mathrm{Au}$ surface, with adhesion and delamination of the Au plating on the balled surface. For the Au-Au/MWCNT surfaces with current loads from $1 \mathrm{~mA}$ to $20 \mathrm{~mA}$, an apparent failure was observed in the $20 \mathrm{~mA}$ case after 50 Million cycles, as a result of a material transfer process associated with metallic bridge transfer. No failures were observed at the lower current levels; however these samples were only tested to 2 Million cycles.

\section{ACKNOWLEDGMENT}

The authors thank Dr. David Smith of the School of Physics and Astronomy, for providing the facilities for developing the MWCNT deposition, and to Mr. Tim Hartley for designing and fabricating the test apparatus.

\section{REFERENCES}

[1] Holm. R "Electrical Contacts: Theory and Application", $4^{\text {th }}$ Edition, Springer, Section 65, pp338

[2] ASTM B539-02(2008). Standard Test Methods for Measuring Resistance of Electrical Connections (Static Contacts)

[3] McBride. J.W. Gold coated Carbon Nanotube Surfaces as Low Force Electrical Contacts for MEMS devices:Part 2.

[4] Coutu, R. A., Kladitis, P. E., Leedy, K. D. and Crane, R. L., 2004, "Selecting metal alloy electric contact materials for MEMS switches," Journal of Micromechanics and Microengineering, (14) pp. 1157-1164.

[5] Lee, H., Coutu, R.A., Mall, S., and Leedy, K.D., 2006, "Characterization of metal and metal alloy films as contact materials in MEMS switches," Journal of Micromechanics and Microengineering, pp.557-563.

[6] Yao, J.J., 2000, "RF MEMS from a device perspective," Journal of Micromechanics and Microengineering, v 10, n 4, pp. R9-38.

[7] Schaffner, J.H., Schmitz, A.E., Tsung-Yuan Hsu, Chang, D.T., Loo, R.Y., and Sievenpiper, D.F., 2003, "Metal contact RF MEMS switch 
elements for ultra wideband RF front-end systems," IEEE Conference on Ultra Wideband Systems and Technologies, pp. 32-6.

[8] Rebeiz, G.M., 2003, "RF MEMS theory, design, and technology," New Jersey, Wiley.

[9] Varadan, V.K., Vinoy, K.J. and Jose, K.A., 2003, "RF MEMS and their Applications, "London, Wiley.

[10] Gao, D., Wijesundara, M. B. J., Carraro, C., Low, C. W., Howe, R. T. and Maboudian, R., 2003, "High modulus polycrystalline 3C-SiC technology for RF MEMS," The 2th International Conference on Solid State Sensors, Actuators and Mircosystems, 3D3.4, pp. 1160-1163.

[11] Lian, G. D., Dickey, E. C., Ueno, M. and Sunkara, M. K., 2002, "Rudoped nanostructured carbon films," Diamond and Related Materials, 11, pp. 1890-1896.

[12] Yu, M. F., Lourie, O., Dyer, M. J., Moloni, K., Kelly, T. F. and Ruoff, R.S., 2000, "Strength and breaking mechanism of multiwalled carbon nanotubes under tensile load," Science, 287(5453), pp. 637 - 640.

[13] Wong EW, Sheehan PE, Lieber CM, 1997 "Nanobeam mechanics: elasticity, strength, and toughness of nanorods and nanotubes," Science, 277, pp. 1971-5.

[14] Qi, H.J., Teo, K.B.K., Lau, K.K.S., Boyce, M.C., Milne, W.I., Robertson, J. and Gleason, K.K., 2003, "Determination of mechanical properties of carbon nanotubes and vertically aligned carbon nanotube forests using nanoindentation," Journal of Mechanics and Physics of Solids, 51, pp. 2213-2237.

[15] Thostenson, E. T., Ren, Z. and Chou, T.W., 2001, "Advances in the science and technology of carbon nanotubes and their composites: a review," Composites Science and Technology, 61, pp. 1899-1912.

[16] Hjortstam, O., Isberg, P., Söderholm, S. and Dai, H., 2004, "Can we achieve ultra-low resistivity in carbon nanotube-based metal composites?" Journal of Applied Physics A, Materials Science \& Processing, 78, pp. 1175-1179.

[17] Tzeng, Y., Chen, Y. and Liu, C., 2003, "Electrical contacts between carbon-nanotube coated electrodes," Diamond and Related Materials, 12, pp. 774-779.
[18] Yaglioglu, O., Hart, A. J., Martens, R. and Slocum, A. H., 2006, "Method of characterizing electrical contact properties of carbon nanotube coated surfaces," Review of Scientific Instruments, 77, pp 095105/1-3.

[19] Yunus, E.M., McBride, J.W. and Spearing, S.M. (2007) The relationship between contact resistance and contact force on $\mathrm{Au}$ coated carbon nanotube surfaces. Proceedings of The 53rd IEEE Holm Conference on Electrical Contacts., 167-174.

[20] Yunus, E.M., McBride, J.W. and Spearing, S.M. (2008) Improving the contact resistance at low force using gold coated carbon nanotube surfaces. Proceedings of the 24th International Conference on Electrical Contacts (ICEC 2008), St Malo, France, 9-12 Jun 2008. St Malo, France, pp, 507-513.

[21] Yunus, E.M., McBride, J.W. and Spearing, S.M. (2008) Low force electrical switching using gold coated vertically aligned multi-walled carbon nanotubes surfaces. IEICE Technical Report, 108, (296), 61-64.

[22] Patton, S.T. and Zabinski, J. S., 2005, "Fundamental studies of $A u$ contacts in MEMS RF switches," Tribology Letters, Vol 18, No.2, pp. 215-230.

[23] Dickrell III, D. J. and Dugger, M. T., 2005, "The effects of surface contamination on resistance degradation of hot-switched low-force MEMS electrical contacts," Electrical Contacts 2005 - Proceedings of the Fifty-First IEEE Holm Conference on Electrical Contacts, pp. 255258.

[24] Dickrell III, D.J. and Dugger, M. T., 2007, "Silicone oil contamination and electrical contact resistance degradation of low-force gold contacts, "Journal of Microelectromechanical Systems, Vol. 16, No. 1, pp. 24-28.

[25] Almeida, L., Ramadoss, R., Jackson, R., Ishikawa, K., and Yu, Q., 2006, "Study of the electrical contact resistance of multi-contact MEMS relays fabricated using the MetalMUMPs process," Journal of Micromechanics and Microengineering, v 16, n 7, pp. 1189-94. 


\title{
An original apparatus for endurance testing of MEMS electrical contact materials
}

\author{
Maxime Vincent $^{1,2}$, Laurent Chiesi ${ }^{1}$, Patrick Rousset ${ }^{1}$, Christophe Lapiere $^{1}$, Christophe Poulain ${ }^{2}$, Laurent Carbone ${ }^{1}$, \\ Frédéric Houzé ${ }^{3}$, Jérôme Delamare ${ }^{4}$ \\ ${ }^{1}$ Innovation Division, Schneider Electric Industries, Grenoble, France \\ ${ }^{2}$ Microsystems Characterization and Reliability Lab., CEA-Léti, Minatec, Grenoble, France \\ ${ }^{3}$ Laboratoire de Génie Electrique de Paris, UMR CNRS 8507, Gif sur Yvette, France \\ ${ }^{4}$ Grenoble Electrical Engineering Lab, UMR CNRS INPG/UJF 5269, Saint Martin d'Hères, France
}

e-mail: maxime.vincent@fr.schneider-electric.com

\begin{abstract}
This paper presents a new experimental setup dedicated to the evaluation of contact materials for MEMS switches. The setup is an alternative way to more complex equipment like modified SPM or nanoindenter [1-4]. It has been designed to test various contact materials upon electrical cycling, without requiring any specific micro-fabricated test vehicles. The setup is compact and housed in a vacuum chamber, which can be filled with gas mixtures to evaluate contact materials under various environmental conditions. The experimental contact is made of a commercial reed blade, contacting on a tip with a curvature radius of a few microns. It mimics a sphere/plane experimental contact, at a scale similar to MEMS switch microcontacts. The two contact parts are easily removable, and can be covered with the appropriate contact material. Preliminary tests focused on lifetime evaluation of gold contacts under nitrogen. To validate the relevance of this setup, contact degradation is compared to the degradation modes we studied previously in a magnetic MEMS switch [5, 6].
\end{abstract}

Keywords- MEMS switch, electrical contact, contact material, endurance test, contact resistance, lifetime evaluation

\section{INTRODUCTION}

The magnetic MEMS switch developed by Schneider Electric in collaboration with the CEA-Léti $[5,6]$ has now met the specifications and is ready for industrialization. One of the most important criteria for a commercial application is the product lifetime. In this field, this MEMS switch is able to hotswitch more than 100 millions times under $3 \mathrm{~V} / 10 \mu \mathrm{A}$, more than 1.5 millions times under $5 \mathrm{~V} / 1 \mathrm{~mA}$ and has almost no lifetime limit when it is mechanically cycled.

In order to improve the MEMS switch, especially in term of switched power capability, we are now investigating new contact materials. Until now, contacts were made of gold, the most studied material for MEMS switch contacts due to its insensitivity to oxidation and its low resistivity. However, we highlighted the apparition of an insulating carbon layer on the contacts surfaces while gold contacts are hot-switched under low voltages $(3 \mathrm{~V}$ and $5 \mathrm{~V})$ [5]. This carbon layer comes from the decomposition of surface contaminants during hot switching tests and leads to a high contact resistance. Under higher voltages $(14 \mathrm{~V})$, the degradation mechanism is different: gold is rapidly transferred from one contact to the other and this leads to a permanent sticking of the contacts.

To limit erosion of our contacts, we deposited a thin layer $(100 \mathrm{~nm})$ of ruthenium on top of gold. Ruthenium presents higher hardness and melting temperature, and we highlighted a clearly better resistance to erosion than gold when cycles under $14 \mathrm{~V}$. However, ruthenium is a catalytic material, and generates frictional polymers when cycled in presence of carbonaceous contamination [7]. This generated a large resistance increase when the switch is cycled under low electrical loads and even under pure mechanical cycling (without any electrical load applied to the contact).

As highlighted previously, none of the usual contact materials fully satisfies in regard to electrical endurance. It is necessary to investigate new materials and test them in an environment as close as possible to that of MEMS switches. However, integrating directly a new material into the whole process of fabrication of a MEMS switch is really tricky. Indeed, MEMS technology is a "planar technology", made by stacking many different structured layers of materials. Each deposited layer has to withstand the next process steps. Fabrication of our MEMS switch requires almost 100 process steps, and involves many different solvents, acids and etching steps. Integrating a new contact material implies modifying a large part of the process. It explains why all the investigations of new contact materials, except Coutu et al. [8], are performed on dedicated setups. However, these setups are always based on complex equipments: scanning probe microscope (SPM) $[1$, 2] or nanoindenters $[3,4]$ and require the use of microfabricated test vehicles.

In this paper, we present an alternative setup we developed dedicated to the evaluation of contact materials for MEMS switches. This setup is simple, inexpensive, compact and housed in a vacuum chamber, which can be filled with different gas mixtures in order to evaluate contact materials under various environmental conditions. 


\section{TEST SETUP CONCEPTION}

The whole conception of the test setup has been based on the MEMS switch we developed. The most important parameter is that its behavior has to be as close as possible to MEMS switch behavior. Indeed, to be pertinent we want to compare the reliability results we obtained on the switch with the results we will obtain on the test setup. Table 1 summarizes the parameters we took into account for this point. The second criterion which came into consideration for the conception of the setup is the simplicity. To perform well, the setup has to be robust and easy to use, in order to ensure maximal reproducibility of the tests. The last constraint we had to consider is the size of the setup: it had to be compact enough to be housed in a vacuum chamber in order to evaluate our contact materials under different atmospheres.

TABLE I. SPECIFICATIONS OF THE TEST SETUP COMPARED TO A MEMS SWITCH

\begin{tabular}{|c|c|c|}
\hline & MEMS switch & Test setup \\
\hline Contact force $(\mu \mathrm{N})$ & $100-500$ & $100-1000$ \\
\hline $\begin{array}{c}\text { Contact surface } \\
\left(\mu \mathrm{m}^{2}\right)\end{array}$ & $<1$ & $<1$ \\
\hline Opening force $(\mu N)$ & $\approx$ Contact force & $=$ Contact force \\
\hline $\begin{array}{l}\text { Surface roughness } \\
\text { RMS (nm) }\end{array}$ & $4-5$ & $\sim 200$ \\
\hline Atmosphere & $\mathrm{N}_{2}$ & $\begin{array}{l}\text { Primary vacuum (1- } \\
\left.10^{-2} \mathrm{mbar}\right), \mathrm{N}_{2}, \mathrm{Ar} \ldots\end{array}$ \\
\hline $\begin{array}{c}\text { Parasitic } \\
\text { capacitance (pF) }\end{array}$ & 30 & 0 - desired value \\
\hline Contact material & $\mathrm{Au}, \mathrm{Ru}$ & $\begin{array}{l}\mathrm{Au}, \mathrm{Ru}, \mathrm{W}, \mathrm{Rh}, \\
\text { alloys... }\end{array}$ \\
\hline
\end{tabular}

The starting point of our test setup is the reed switch. A reed switch is a tiny switch composed of two ferromagnetic blades hermetically sealed in a glass envelope [9]. Its working principle is similar to a magnetic MEMS switch [6], which gave us the idea to use a reed blade as the main component of our setup: it acts as the mobile part of the experimental contact we use in our test setup. The reed blade is directly removed from a commercial reed switch, which ensures a good reproducibility of the mechanical characteristics of the blade. Moreover the blade is made of permalloy (FeNi), which allows to use a magnetic actuation to perform the cycling. The fixed part of the experimental contact is a commercial probe tip, usually used in electrical test stations. This tip is made of tungsten carbide (WC) and the tip radius is a few microns, which can be easily modified by using different tip geometries. With this approach, no micro-fabrication steps are required to build the test vehicles, which is a serious advantage for the flexibility of the setup. When the reed blade is brought into contact with the probe tip, we obtain a sphere-plane contact with a well controlled contact surface. This surface is in the order of a few microns or less, representative of what we can find in a MEMS switch.

Reed switches are sealed under vacuum, encapsulated in glass, and contact surfaces are free from organic contamination. Probe tips are cleaned after fabrication and packaged under nitrogen to prevent contamination. So, using commercial parts to build the experimental contact ensures a rather good cleanliness of our contact surfaces. We performed surface analysis measurements on these parts and no major contamination was noticed. The only drawback is the surface roughness of these parts. We performed AFM measurements on the reed blades and noticed they have a typical surface roughness (RMS) of $200 \mathrm{~nm}, 50$ times higher than the roughness of MEMS contact surfaces. This problem will have to be solved in the future, knowing that roughness is a major parameter in contact physics [10].

Applying a contact force of a few hundreds of micronewtons with macroscopic objects is not trivial. After having explored many ideas, we finally opted for a "normally closed" contact configuration. The reed blade is brought into contact with the tip thanks to a piezoelectric actuator (Newport NanoPZ) with a resolution of $10 \mathrm{~nm}$. The tip is linked to a customized force sensor (SMD Sensors S251) which has a resolution of $10 \mu \mathrm{N}$. A calibration of the sensor with proof masses has been performed, to confirm the supplier's data in the range $100 \mu \mathrm{N}-10 \mathrm{mN}$. When the blade contacts on the tip, the force sensor indicates the load applied to the tip by the blade. Once this load is adjusted to the required value, it remains constant during the entire test.

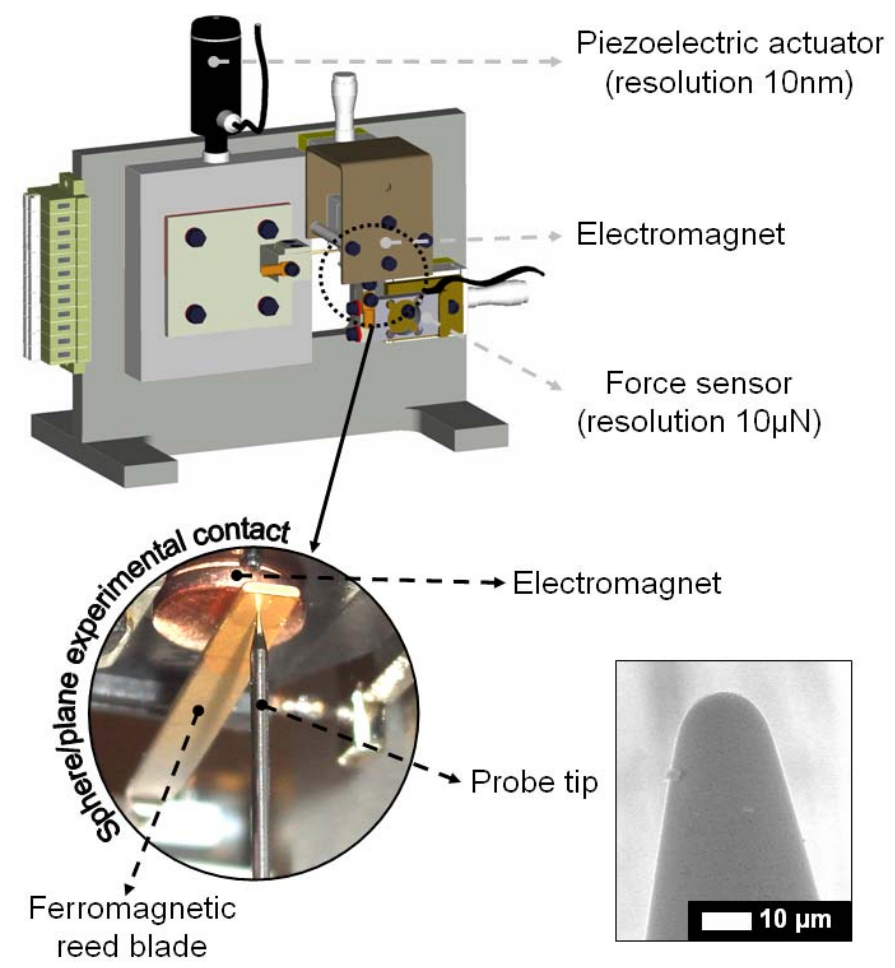

Figure 1. Conception of the test setup (top) and photography of the experimental contact with its cycling electromagnet (bottom). Bottom right inset is a SEM picture of the probe tip end

The cycling is performed using an electro-magnet, positioned above the mobile part of the contact (the ferromagnetic blade). The contact is normally closed, with a well controlled load; the electro-magnet only opens the contact 
by attracting the blade. A sinusoidal signal is sent to the electromagnet to provide a repetitive open/release movement to the contact. A sinusoidal signal has been chosen to optimize the response of the electromagnet, avoid bounces of the contact, and limit the impact force when the blade is released. Fig. 2 reports oscilloscope traces of the driving voltage applied to the electromagnet and the voltage across the $5 \mathrm{~V}$ biased electrical contact. Amplitude of the electromagnet driving voltage is set to provide an opening force as low as the opening force of a MEMS switch. This condition is necessary if we want to detect an eventual failure of the contact by sticking or welding. The conception result, summarizing the working principle of the setup is reported in Fig. 1. The mechanical conception has been performed using Pro/ENGINEER and the various parts of the setup are homemade, machined into aluminium alloy (AU4G).

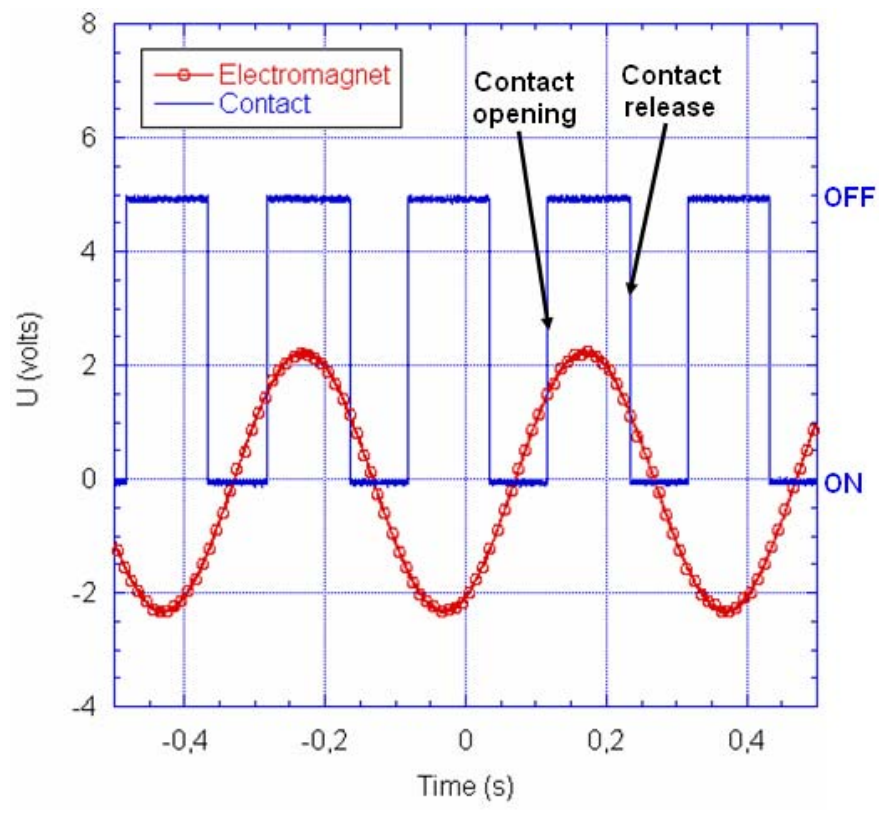

Figure 2. Oscilloscope traces of (a) the driving voltage applied to the electromagnet (circle line) and (b) voltage across the $5 \mathrm{~V}$ biased experimental contact

MEMS switches are ideally packaged with a hermetically sealed silicon packaging, like the MEMS switch we developed $[5,6]$. We noticed this packaging brings a parasitic capacitor of about $30 \mathrm{pF}$, in parallel to the switch. We showed this parasitic capacitor has detrimental effect on contact degradation. During switch opening, the capacitor charges but during switch closure, this capacitor discharges into the contact. This leads to current peaks, which can reach up to 1-2 Amperes during a few nanoseconds. In order to study this phenomenon with our setup, we ensured to have any parasitic capacitor and we can add an adjustable capacitor element in order to study its influence on contact lifetime.

The setup and all the instruments are piloted through Labview, schematic is presented in Fig. 3. Electrical contact resistance is measured thanks to a 4-wire measurement technique, using a Keithley 2400 SourceMeter. Contact resistance $\left(\mathrm{R}_{\text {on }}\right.$ and $\left.\mathrm{R}_{\text {off }}\right)$ and contact force can be recorded every 1 to 10,000 cycles, allowing monitoring the evolution of the contact resistance and the contact force as a function of the number of cycles performed.

The setup is housed in a vacuum chamber, which can be set under primary vacuum $\left(1-10^{-2} \mathrm{mbar}\right)$ or filled with different gases. Finding the best combination of contact material and atmosphere to enhance contact performance is one of the major goals of this study.

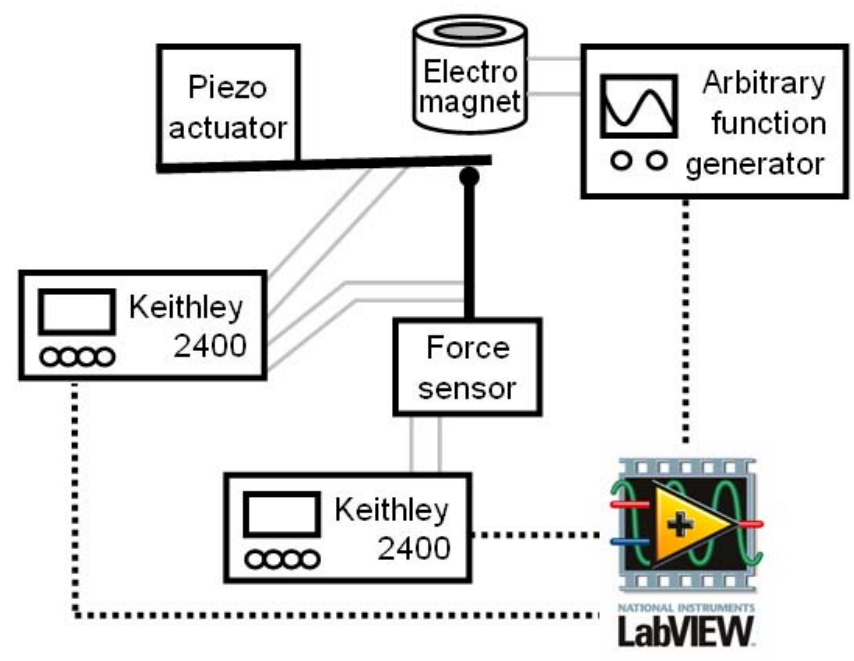

Figure 3. Schematic of the test setup

\section{EXPERIMENTAL PROCEDURE}

Contact materials are deposited by DC-magnetron sputtering using a Leybold Heraeus Z-400 with argon plasma. The sputtering system is equipped with two cathodes, allowing the deposition of binary alloys. Materials are deposited directly on the test vehicles and an annealing step can be performed. Samples are characterized after deposition by scanning electron microscopy and atomic force microscopy to evaluate their surface roughness. Electron diffusive X-Ray analysis (EDX) is performed to detect the presence of surface contamination. XRay diffraction is conducted on the materials to evaluate their crystalline structure and resistivity measurements are performed on monitor samples.

After contact material deposition, the test vehicles (tip probe and reed blade) are mounted into the test setup. All parts of the setup are easily removable, in order to permit an easy access to the test vehicles fasteners. Contact force is applied with the piezo-actuator and controlled thanks to the force sensor. A typical curve of contact resistance as a function of the applied load is reported on Fig. 4. Contact force is applied first and will not be modified during cycling. So, we have to ensure the force we applied at the beginning will remain constant during all the cycling. To do this we record the contact force every time we record the contact resistance. A typical curve representing the evolution of the contact force versus the number of cycles is presented in Fig. 5. Various parameters can be set to stop cycling including a specified number of cycles, $\mathrm{R}_{\text {on }}$ too high or $\mathrm{R}_{\text {off }}$ too low (contact sticking). 


\section{RESULTS AND DISCUSSION}

The first tests focused on the evaluation of gold under nitrogen with a contact force around $250 \mu \mathrm{N}$. Tests are performed in hot cycling conditions, the voltage is fixed to $5 \mathrm{~V}$ and the current is limited to $1 \mathrm{~mA}$ using a $5 \mathrm{k} \Omega$ resistor. These parameters have been chosen in order to directly compare the degradation we will observe with the results obtained with our MEMS switch. This first step will indicate if the setup is pertinent to simulate a MEMS switch. For these first tests, cycling has been performed at $2 \mathrm{~Hz}$ and $10 \mathrm{~Hz}$, but the setup can work at a frequency up to several tens of hertz.

Unfortunately, we met some adhesion issues when depositing gold on the tungsten carbide probe tip. The gold layer did not stick on the material, resulting in unusable tips. On reed blade, we did not meet this problem, gold adhesion is very good. Consequently, the test results we present here have been obtained with a contact made of $700 \mathrm{~nm}$ of gold on the mobile contact (the reed blade) and bulk tungsten carbide on the fixed contact (the probe tip). Gold deposition has been performed with a pressure in the chamber of $5^{*} 10^{-3}$ mbars with an Ar flux of $50 \mathrm{sccm}$, a cathode power set to $100 \mathrm{~W}$ and a deposition time of 10 minutes. No carbonaceous contamination has been detected by EDX analysis. AFM analysis indicates a RMS roughness around $250 \mathrm{~nm}$, the same order of magnitude than the original blade surface.

As we can notice on Fig. 4, with $100 \mu \mathrm{N}$ of contact force a stable contact is obtained and contact resistance is below $0.3 \Omega$. Experiment has been carried out three times, showing a good reproducibility of the results. A simple calculation, considering a single spot contact model and assuming a fully plastic deformation regime allows estimating the theoretical contact resistance of our contact:

$$
R_{C}=\frac{\rho^{\prime}}{2 a}
$$

with $\quad a=\sqrt{\frac{F_{C}}{\pi H}}$

and where $F c$ is the contact force and $a$ the resulting contact spot radius. $H$ is the hardness of the softer material (gold in our case) and $\rho$ ' is the equivalent resistivity of our dissymmetrical contact: gold $(\mathrm{Au})$ versus tungsten carbide (WC), which can be calculated using:

$$
\rho^{\prime}=\frac{\rho_{A u}+\rho_{W C}}{2}
$$

Considering Fig. 4, this simple model is a good estimation of our contact resistance, for contact pressures high enough to attain the plastic deformation regime. It is usually admitted this regime is attained when contact pressure is three times the yield strength value of the contact material (in this case we considered the yield strength value of gold: $205 \mathrm{MPa}$ ).

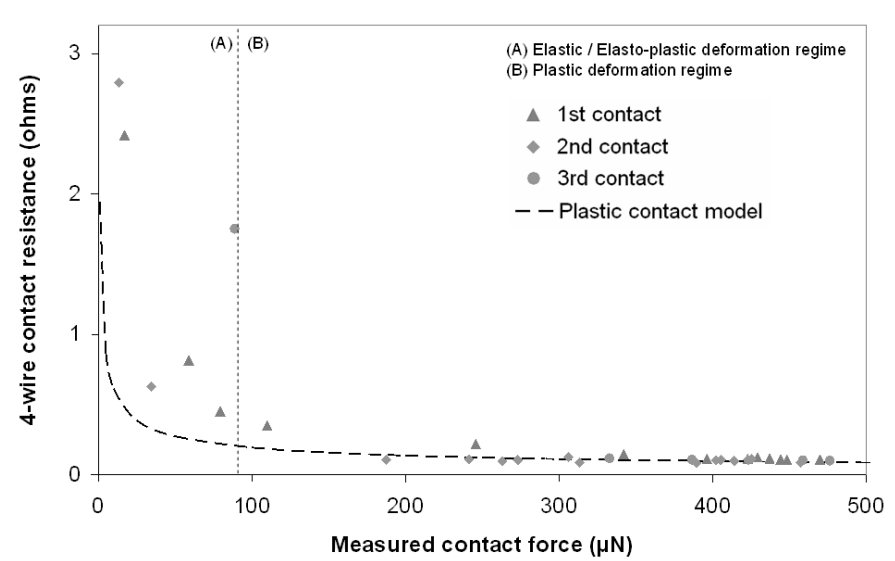

Figure 4. A typical plot of electrical contact resistance versus measured contact force for the gold / tungsten carbide experimental contact

A typical curve of electrical endurance cycling is reported on Fig. 5. Every test performed with these test parameters provides this type of result. Contact force and contact resistance have been recorded every 50 cycles during 20,000 cycles with a fixed current of $1 \mathrm{~mA}$. We notice that contact force is quite constant, with a medium value around $220 \mu \mathrm{N}$ and a standard deviation of $16 \mu \mathrm{N}$. However, it decreases slightly during the first $1000-2000$ cycles, probably due to the matting of the contact surface. Once contact surface is well stabilized, contact force becomes constant for the entire cycling. However, contact resistance increases to more than $1500 \Omega$ after only 20,000 cycles. Contact force remains constant and contact re-opens properly at each cycle, so this resistance increase is due to a degradation of the contact surfaces. In order to find the origin of this degradation, we performed some scanning electron microscopy (SEM) imaging on the contact surfaces.

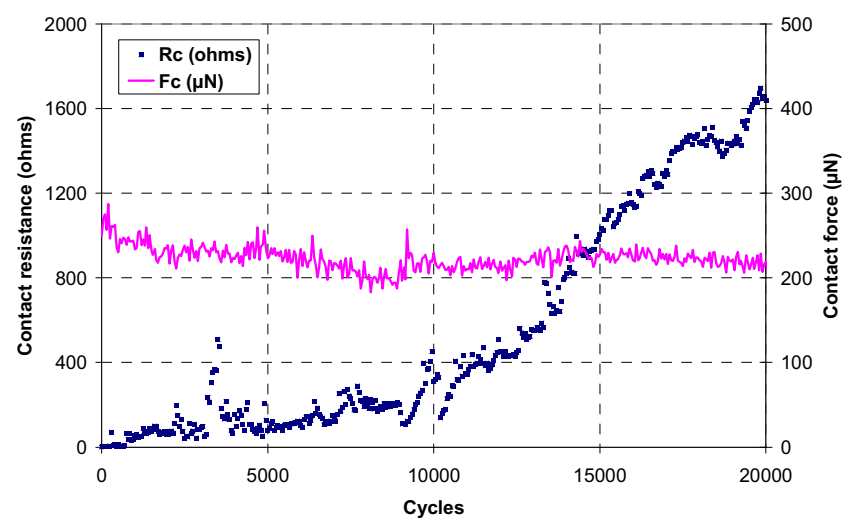

Figure 5. A typical plot of electrical cycling: contact resistance (dotted line) and contact force (continuous line) as a function of the number of cycles for a gold / tungsten carbide contact

SEM observation of the tip surface after cycling does not reveal any change, the surface is undamaged and no contamination can be detected. However, as noticed on Fig. 6, the blade surface has been damaged during cycling. A crater of about $6 \mu \mathrm{m}$ of diameter appeared, resulting from the repetitive contact of the tip onto the blade surface. The crater's bottom 
looks darker, revealing a probable contamination or oxidation of the contact surface, which explains the resistance increase we observe in Fig. 5.

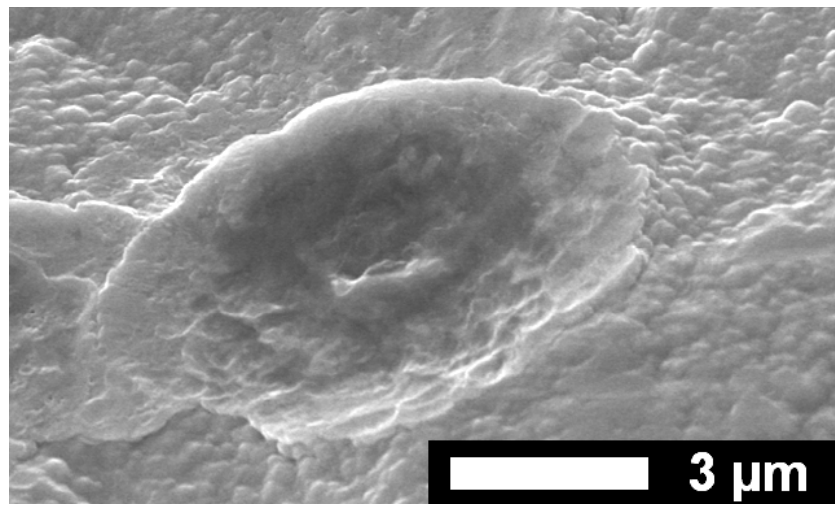

Figure 6. SEM picture of the reed blade surface after the endurance test presented in Fig. 5

Interpretation of the obtained results is not evident, since the contact materials we used in this set of experiments are not common. Indeed, here the experimental contact is made of tungsten carbide (tip material) contacting on the reed blade covered with $700 \mathrm{~nm}$ of gold. The reed blade base material is permalloy covered with rhodium.

So, we are in a bad configuration, where a very hard material (tungsten carbide, HV $\sim 1550$ ) contacts on a thin layer of soft material (gold, HV $\sim 50$ ). The tip penetrates easily in the gold layer and breaks through it, to contact on the rhodium layer. If a zoom is performed on the first 1000 cycles in Fig. 5, we notice that contact resistance is very low during the first 100 cycles $(0.3-0.5 \Omega)$, then increases to about $5 \Omega$ for 700 cycles and finally increases three orders of magnitude. This reveals the erosion of the gold layer during the first 100 cycles due to the tip penetration. It leads to a rhodium contact ( $\mathrm{Rc} \sim 5$ $\Omega$ ) and finally the rhodium surface probably oxidizes or is contaminated during cycling, explaining the one decade resistance increase.

Rhodium is a commonly used material in reed switches, and is known to produce frictional polymers on the contact surfaces, due to the mechanical shock between the two blades during cycling. This contamination layer leads to a contact resistance increase of 3 orders of magnitude higher than initial resistance and resistance increases progressively as soon as cycling begins [11]. The use of rhodium as contact material has been possible thanks to the development of an efficient surface deactivation process, which suppress the adsorbing and catalytic properties of the rhodium surface by covering it with an oxygen film. Our EDX analyses confirm the presence of this film. However, in our case, deposition of the gold layer on top of the rhodium probably destroyed this oxygen film. Thus, once the gold layer is eroded, rhodium acts as contact material and produces frictional polymers which lead to the contact resistance increase of three orders of magnitude that we observe in Fig. 5.

\section{CONCLUSION}

We developed an original and simple setup to easily evaluate new contact materials for MEMS switches. It allows testing the endurance of these materials upon electrical cycling without requiring the use of micro-fabrication steps. The setup is very flexible: any material can be integrated to the experimental contact and contact geometry can be modified easily. Cycling can be performed under a wide variety of adjustable parameters (including contact force, opening force, cycling and frequency) and environments (including primary vacuum and gas mixtures).

We showed the setup is reliable, offers stable test conditions and provides repeatable results. The only modification to be done is replacing the probe tip used in the setup, which is made of a too hard material. The use of beryllium copper tips (HV 325) will limit this issue. However, the first results we obtained are explainable and very close to previous studies performed on reed switches.

Unfortunately, due to materials deposition issues we could not evaluate a proper gold/gold contact. Future work will now focus on contact materials deposition, especially gold which will be our proof material and tungsten, a promising contact material for MEMS switches.

\section{REFERENCES}

[1] L. Chen, H. Lee, Z. Guo, N. McGruer, K. Gilbert, S. Mall, K. Leedy and G. Adams, "Contact resistance study of noble metals and alloy films using a scanning probe microscope test station", J. of Appl. Phys., 102, 074910, 2007

[2] Z. Yang, D. Lichtenwalner, A. Morris, S. Menzel, C. Nauenheim, A. Gruverman, J. Krim, and A. Kingon, "A new test facility for efficient evaluation of MEMS contact materials", J. Micromech. Microeng., 17, pp. $1788-1795,2007$

[3] K.W. Gilbert, S. Mall, K.D. Leedy and B. Crawford, "A nanoindenter based method for studying MEMS contact switch microcontacts", Proc. of the 54th IEEE Holm Conf., pp. 137-144, 2008

[4] D. Dickrell and M. Dugger, "The effect of surface contamination on resistance degradation of hot-switched low-force MEMS electrical contacts", Proc. of the 51st IEEE Holm Conf., pp. 255-258, 2005

[5] M. Vincent, L. Chiesi, J.-C. Fourrier, A. Garnier, B. Grappe, C. Lapiere, C. Coutier, A. Samperio, S. Paineau, F. Houzé and S. Noël, "Electrical contact reliability in a magnetic MEMS switch", Proc. of the 54th IEEE Holm Conf., pp. 145-150, 2008

[6] C. Coutier, L. Chiesi, A. Garnier, J.C. Fourrier, C. Lapière, M. Trouillon, B. Grappe, M. Vincent, A. Samperio, S. Borel, C. Dieppedale, E. Lorent and H. Sibuet, "A new magnetically actuated switch for precise position detection”, Proc. of Transducers'09, 2009

[7] H.W. Hermance and T.F. Egan, "Organic deposits on precious metal contacts", Bell Syst.Tech. J., 37, pp. 739-777, 1958

[8] R.A. Coutu Jr., P.E. Kladitis, R. Cortez, R.E. Strawser, R.L. Crane, "Micro-switches with sputtered Au, AuPd, Au-on-AuPt, and AuPtCu alloy electric contacts", IEEE Trans. Compon. Packag. Manuf. Technol., 29, vol. 2, pp. 341-349, 2006

[9] P.G. Slade, "Electrical Contacts: Principles and Applications", CRC Press, 1999

[10] J. A. Greenwood and J. B. P. Williamson, "Contact of Nominally Flat Surfaces", Proc. Roy. Soc. A, 295, pp. 300-319, 1966

[11] T. Yokokawa and C. Kawakita, "High reliability reed switches with rhodium plated contacts", Proc. of the 21st Relay Conf., pp. 131-137, 1973 


\title{
Experimental Validation of a 2-D Constriction Resistance Model at the Microscale
}

\author{
Lise Bilhaut $^{1}$, Christophe Poulain ${ }^{2}$, Romain Anciant ${ }^{2}$, and Laurent Duraffourg ${ }^{1}$ \\ ${ }^{1}$ Microsystems Components Lab., CEA-LETI MINATEC, Grenoble, France \\ ${ }^{2}$ Microsystems Characterization and Reliability Lab., CEA-LETI MINATEC, Grenoble, France
}

Corresponding author: christophe.poulain@cea.fr

\begin{abstract}
This work proposes and tests the limits of a simplified analytical model of an electrical micro-contact, which considers the resistance of a tapered line taking into account the current crowding effect at the constriction point. We have designed and fabricated micro-constriction patterns with different contact widths, aperture angles, and thicknesses, and correlated their constriction resistances with a 2-D model based on circular electric equipotential lines distributed along a triangle. We also propose an extension of the model, where the resistivity includes the electron loss at the surface of the film and at the grain boundaries. This allows validating the model for film as thin as $35 \mathrm{~nm}$.
\end{abstract}

Keywords - micro electrical contact, 2-D constriction resistance, thin metal film, conduction modes

\section{INTRODUCTION}

The recent development of MEMS switches requires a better understanding of electrical contact phenomena at the microscale, since it will determine the specification of the switch (loss, power handling, reliability...) MEMS designers need reliable models to predict electrical parameters of the systems they develop. Study of electrical contacts when one of the contacting bodies is a thin metal film is thus of great interest, and has been already considered by several authors [1] [2].

This work proposes an original model of a contact resistance, allowing studying the sole effect of the convergence of the current lines at the contact point. Thanks to microelectronics technology, metallic micro-constriction patterns corresponding to the proposed 2-D model have been fabricated. Because of the continuous metallic layer at the constriction point, we truly have a single contact orifice, avoiding any interface problems or roughness issues. We were thus able to measure only the constriction resistance and correlated it to the model.

After a section describing the theoretical model and another one describing sample processing and measurement set-up, we present plots of the measured constriction resistances along with the ones predicted by the model. We then discuss the possible use of the model for films as thin as $35 \mathrm{~nm}$, providing a modification of the resistivity of the film.

\section{2-D CONSTRICTION RESISTANCE MODEL}

The presented 2-D contact model describes the constriction resistance between two triangles facing each other and made of a continuous thin metallic layer, as shown on Figure 1. The size of the constriction is controlled by the width $w$. The model assumes a diffusive electrical conduction regime, which is adequate as long as the electron mean free path is much smaller than the characteristic geometric dimensions [3]. Considering classical metals used for MEMS development, the mean free path $l_{0}$ ranges between $15 \mathrm{~nm}$ and $40 \mathrm{~nm}$, which means the model is well adapted for films at least as thick as two hundreds nanometers (actually, it can also describe films as thin as $35 \mathrm{~nm}$ as it will be discussed in section V). Since equipotential lines are perpendicular to current lines, we consider them to be circular $\left(l_{1}(x)\right.$ and $l_{2}(x)$ on Figure 1$)$. This assumption, although still an approximation, seems more realistic for large apertures than a model proposed in the literature [4], based on straight and parallel equipotential lines $\left(l^{\prime}(x)\right.$ on Figure 1$)$.

The resistance between two equipotential lines $V_{1}$ and $V_{2}$ can be written as:

$$
d R_{V_{2}-V_{1}}=\frac{\rho_{f} d x}{t l(x)}
$$

The constriction resistance of the first body is the sum of those infinitesimal resistances along the full triangle:

$$
R_{\text {constriction_1 }}=\rho_{f} \int_{b 1}^{L} \frac{d x}{t l(x)}=\rho_{f} \int_{b 1}^{L} \frac{d x}{2 t \beta x}
$$

where $b 1=\frac{w / 2}{\tan (\beta)}$

If we develop the expression, we obtain:

$$
R_{\text {constriction }_{-} 1}=\frac{\rho_{f}}{2 t \beta} \ln \left(\frac{2 L \tan (\beta)}{w}\right)
$$

We can calculate the constriction resistance of the second body similarly:

$$
R_{\text {constriction_2 }}=\frac{\rho_{f}}{2 t \alpha} \ln \left(\frac{2 L \tan (\alpha)}{w}\right)
$$

The total constriction resistance can be written as the sum of $R_{\text {constriction_l }}$ and $R_{\text {constriction_2 }}$ : 


$$
\begin{array}{r}
R_{\text {constriction }}=\frac{\rho_{f}}{2 t}\left(\frac{1}{\beta} \ln \left(\frac{2 L \tan (\beta)}{w}\right)+\right. \\
\left.\frac{1}{\alpha} \ln \left(\frac{2 L \tan (\alpha)}{w}\right)\right)
\end{array}
$$

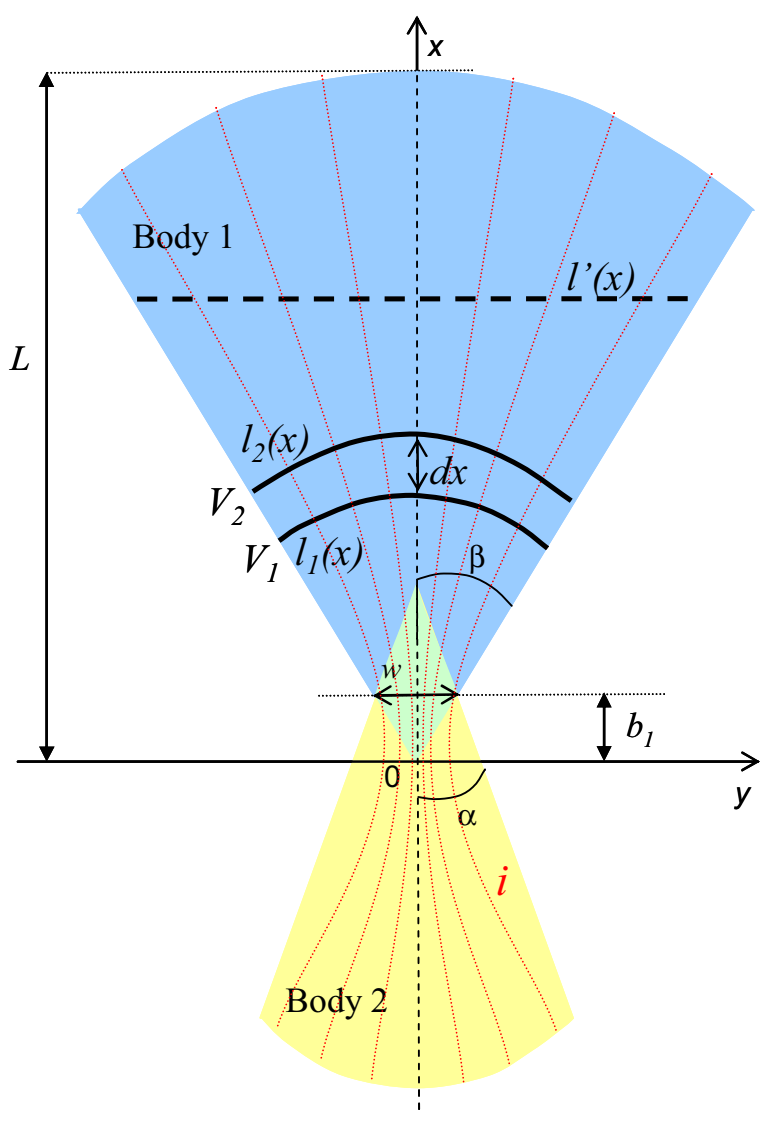

Figure 1: Representation of the 2-D contact model. In the proposed formula, the equipotential lines are assumed to be circular $\left(l_{1}(x)\right.$ and $\left.l_{2}(x)\right)$, when the previously proposed model is based on straight lines $\left(l^{\prime}(x)\right)$

\section{SAMPLES PROCESSING AND CHARACTERIZATION}

We have designed a set of constriction patterns varying regarding different parameters:

1. Aperture width $w(1.6 \mu \mathrm{m}$ to $100 \mu \mathrm{m})$

2. Aperture angles $\alpha$ and $\beta\left(2.5^{\circ}\right.$ to $\left.45^{\circ}\right)$

3. Thickness $t$ of the metallic layer (30 $\mathrm{nm}$ to 300 $\mathrm{nm})$

4. Length $L(100 \mu \mathrm{m}$ and $200 \mu \mathrm{m})$

Samples are manufactured thanks to a three steps process on oxidized Si wafer:

1. Photolithography of the design (AZ1512 resist);

2. Deposition of the metallic layers with an electron beam evaporator;

3. Lift-off of the resist in acetone.
A $5 \mathrm{~nm}$ Ti layer is deposited just before the metallic layer of interest in order to improve its adhesion on the $\mathrm{SiO}_{2}$ substrate. The full stack is presented on Figure 2. The metallic layers were chosen to be either gold (classical metal used for micro-contacts) or aluminum.

Specific patterns have been designed in order to evaluate the resistivity for each sample, taking into account the adhesion layer and the variation of the resistivity with the thickness.

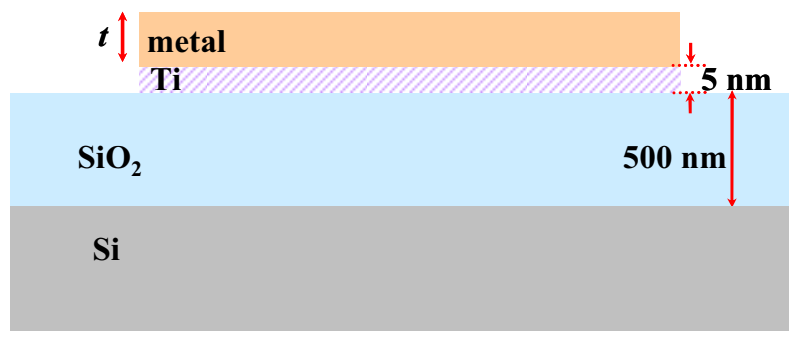

Figure 2: Sample stack: the metal of interest (Au or Al, thickness $t$ ) lies onto a Ti adhesion layer, evaporated onto a $\mathrm{SiO}_{2}$ insulating layer on a $\mathrm{Si}$ wafer

Due to the mask manufacturing process, there is an uncertainty on the geometrical parameters. Figure 3 shows a SEM picture of an Al 50-nm-thick pattern with aperture angles $\alpha=30^{\circ}$ and $\beta=45^{\circ}$ and $w=1.6 \mu \mathrm{m}$.

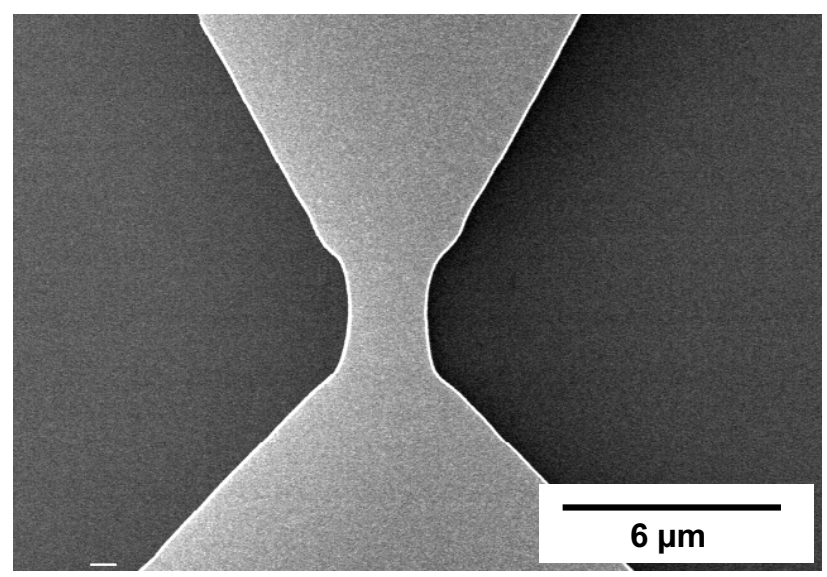

Figure 3: SEM picture of a device made of a $50 \mathrm{~nm}$-thick layer of $\mathrm{Al}$, with $\alpha=30^{\circ}$ (top triangle), $\beta=45^{\circ}$ (bottom triangle) and $w=1.6 \mu \mathrm{m}$

The electrical tests have been carried out with an Electroglas 2001X prober driven by GAMME V2, a software developed at CEA/LETI. The current source was a Keithley 2420 and the voltage measurement was made with a Keithley 2750 multimeter. The accuracy of the electrical voltage measurement is estimated to be about \pm 5 $\mu \mathrm{V}$. WRe probes were used to test the thick layers $(\geq 50$ $\mathrm{nm})$, and $\mathrm{CuBe}$ ones for very thin film $(30 \mathrm{~nm})$. 
The measurement set-up is shown on Figure 4: the current $i$ is injected via two contact pads and the voltage drop across the upper and lower triangles $\mathrm{V}$ is measured in two others. The value of the current was adjusted in order to have a meaningful value of the measured voltage. For instance, we did tests under $1 \mathrm{~mA}$ and $10 \mathrm{~mA}$ for the sample with 300-nm-thick Au layer. This gave a voltage between $50 \mu \mathrm{V}$ and $30 \mathrm{mV}$. This 4-points measurement method allows the value of the resistance to be independent of the contact resistance of the current injector probes. Furthermore, we did two runs of measurement for each device, with reverse current direction, in order to compensate for the effect of thermoelectric electromotive force (EMF). The measurements were averaged on at least two devices.

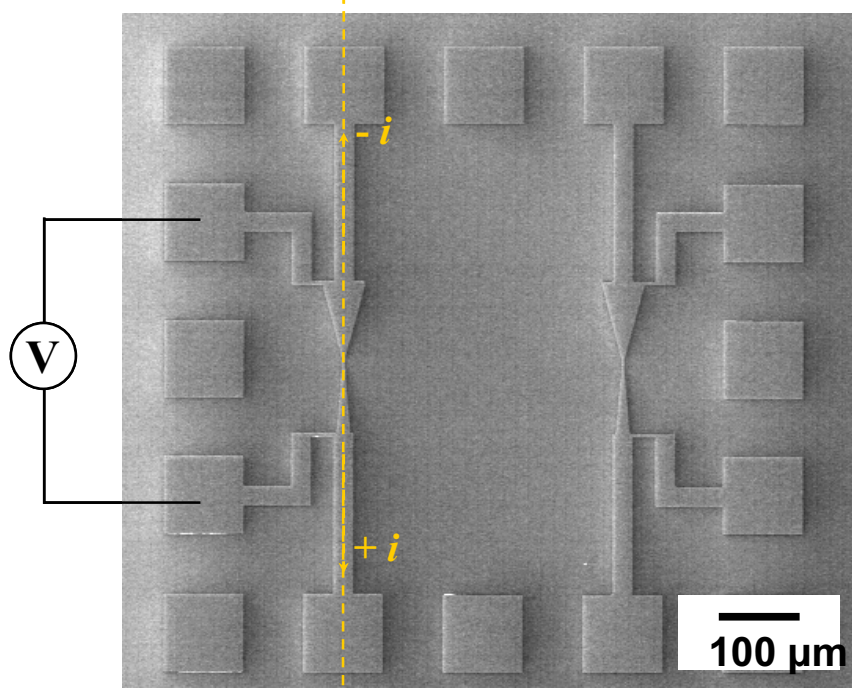

Figure 4: SEM picture of an Aluminum 50-nm-thick full pattern with two identical test devices

\section{RESUlTS AND ANALYSIS}

Automated tests were carried out onto samples of seven different thicknesses, on 62 different patterns, duplicated two or four times for each sample. The maximum current density that a gold sample could withstand reaches $10^{11}$ A.m ${ }^{-2}$. The resistivity of $\mathrm{Au}$ and $\mathrm{Al}$ has been measured on dedicated patterns for each layer thickness. This measured resistivity was then used in formula (6).

The variation of the constriction resistance with the aperture angle for a 300-nm-thick gold layer is plotted on Figure 5. On this figure as on all the subsequent ones, the full curves correspond to the experimental data, labeled as "sample", and the dotted ones to the analytical model, labeled as "formula". We can see that the model fits quite well the measurement: the smaller the aperture, the bigger the resistance. The analytical model indicated an overestimation of the resistance by less than $14 \%$, which may be explained by the uncertainty of the lateral dimension, the inhomogeneity of the layer thickness, the accuracy of the measurement set-up as well as the assumptions made by the model.

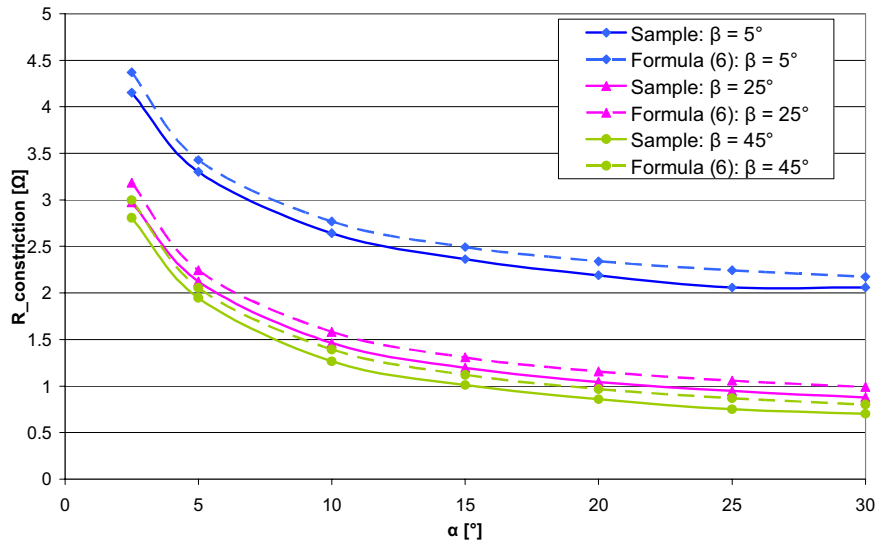

Figure 5: Variation of the constriction resistance with the aperture angle $\alpha$ (Au sample, $L=200 \mu \mathrm{m}, t=300 \mathrm{~nm}, w=1.6$ $\mu \mathrm{m}$ and $i=10 \mathrm{~mA}$ )

The variation of the constriction resistance of the same sample regarding the width of the constriction is plotted on Figure 6. Again, we can see that the model describes quite well experimental data. For very small constriction width $\left(w \sim l_{0}\right)$, one should expect to reach the ballistic regime of electrical conduction, therefore limiting the use of the formula. Unfortunately, because of the limited resolution of optical lithography, we could not obtain a sample with a width narrow enough.

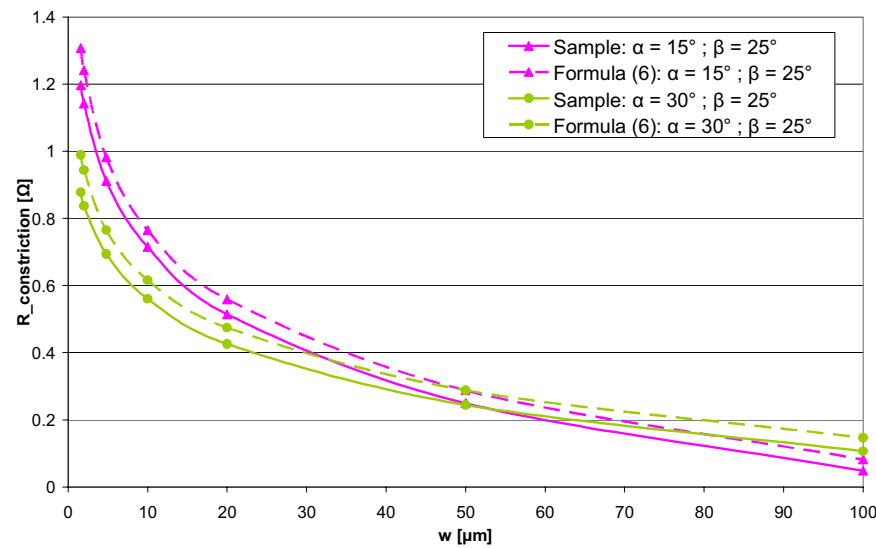

Figure 6: Variation of the constriction resistance with the constriction width $w$ (Au sample, $L=200 \mu \mathrm{m}, t=300 \mathrm{~nm}$ and $i=10 \mathrm{~mA}$ ) 
Figure 7 shows the resistance constriction of two different metals: gold $\left(l_{0}=36 \mathrm{~nm}\right)$ and aluminum $\left(l_{0}=15\right.$ $\mathrm{nm})$. Because the resistivity of both metal are close, the values of the constriction resistance are very similar. This figure shows that the model is robust when tested with different parameters, such as $L$ or $t$.

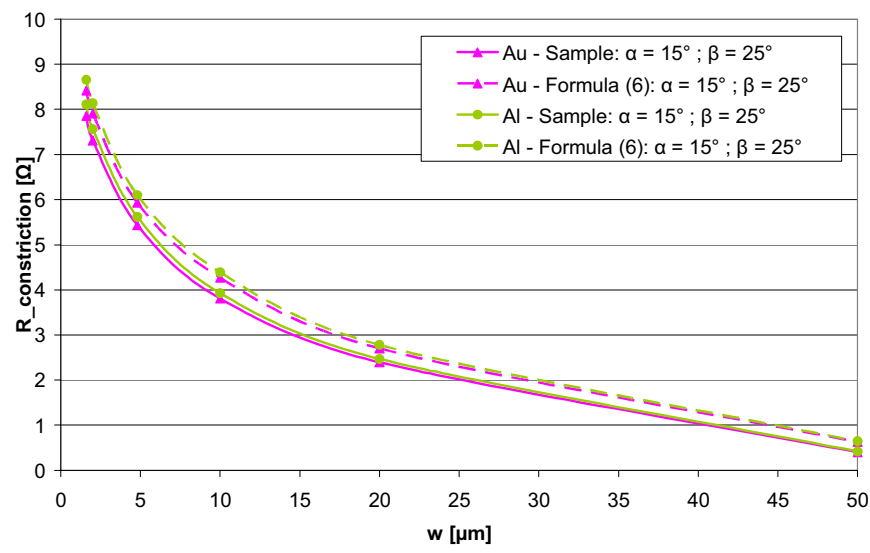

Figure 7: Variation of the resistance of $\mathrm{Au}$ and $\mathrm{Al}$ film with the constriction width $w(t=50 \mathrm{~nm}, L=100 \mu \mathrm{m}$ and $i=1 \mathrm{~mA})$

On Figure 8, we can see the evolution of the resistance regarding the layer thickness. When we use the resistivity associated with the layer thickness, a good agreement between the model and experimental data is observed. However, when we chose a resistivity value (e.g. the one measured for a 300 -nm-thick layer) and we keep it as constant, the resistance values given by the formula differs from the experimental curve more and more as the thickness decreases. This shows that our model remarkably fits experimental data even for very thin layer $(\leq 100 \mathrm{~nm})$, as long as the variation of resistivity with layer thickness is taken into account.

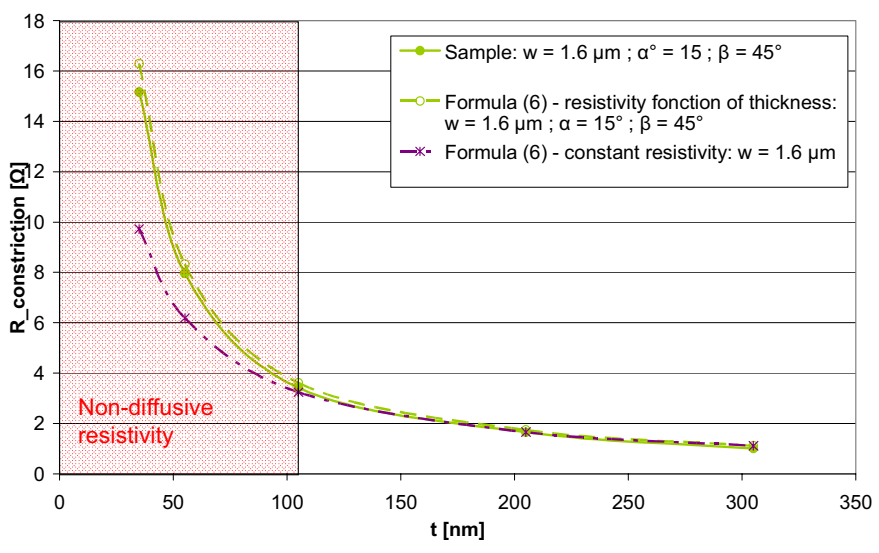

Figure 8: Variation of the constriction resistance with the layer thickness $t$ (Au sample, $L=200 \mu \mathrm{m}, i=1 \mathrm{~mA}$ )

\section{DISCUSSION}

According to Figure 8, resistivity is an essential parameter to consider when one wants to predict the resistance of a thin film. Indeed, it is modified from bulk resistivity due to the loss of electron energy at the surface and, in the case of a polycrystalline material, at the grain boundaries (Figure 9) [5] [6] [7] [8].

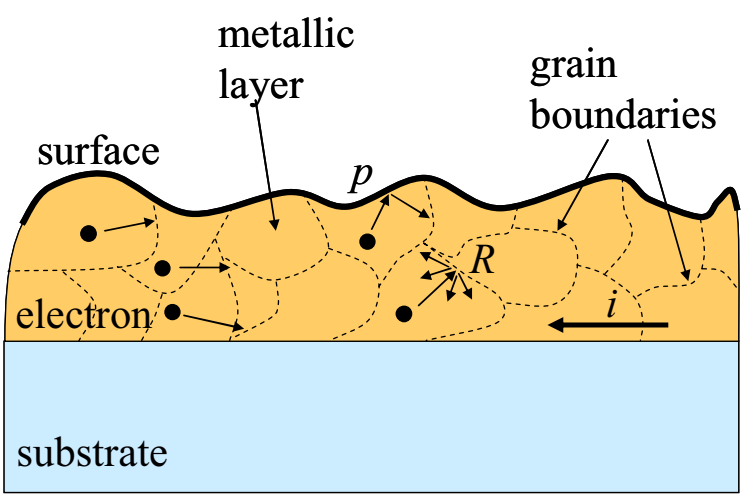

Figure 9: Electrical transport loss mechanisms in polycrystalline thin films: the electron can loose energy when reflected on the film surface, and when scattered on the grain boundaries

The resistivity of a thin film of thickness $t$ can be written as [9]:

$$
\rho_{f}=\rho_{b u l k}\left(1+\frac{3 l_{0}(1-p)}{8 t}+\frac{7 l_{0} R}{5 d(1-R)}\right)
$$

where $\rho_{\text {bulk }}$ is the resistivity of the bulk material, $l_{0}$ the mean free path of electron, $p$ the specular reflection parameter of electrons at the film surface $(0 \leq p \leq 1), R$ the electron reflection coefficient at the grain boundaries $(0 \leq$ $R \leq 1)$ and $d$ the mean grain size.

A plot of the theoretical resistivity of a gold layer versus its thickness is shown on Figure 10. We used $p=0$ [5], $R=$ $0.1[6], l_{0}=36 \mathrm{~nm}, d=27 \mathrm{~nm}$ (the grain size was estimated by an AFM image - see Figure 11), and $\rho_{b u l k}=2.3 .10^{-8}$ $\Omega$.m. We also plotted the measured resistivity of samples with different thicknesses. The curve shows the same tendency for the resistivity to increase as the thickness decreases. 


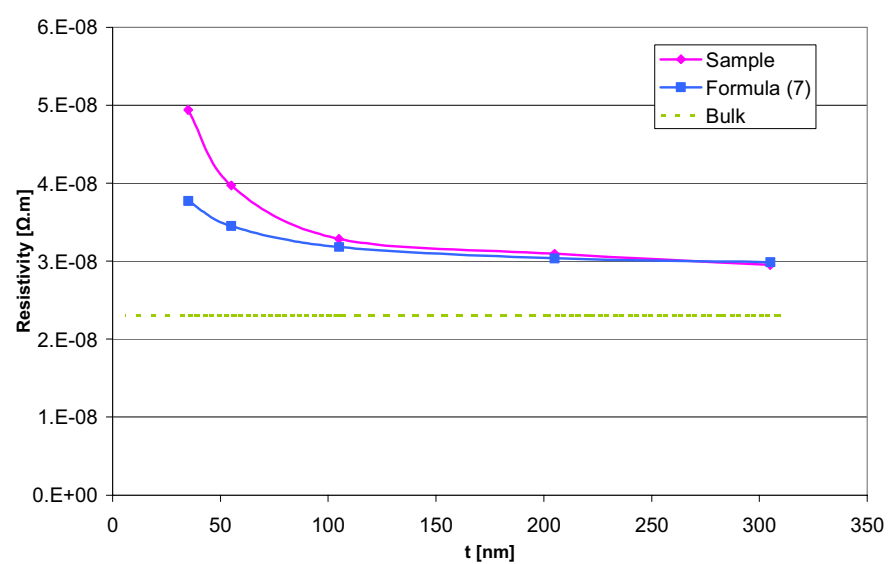

Figure 10: Variation of the resistivity with the thickness $t$ of the layer (Au samples, $L=200 \mu \mathrm{m}, i=1 \mathrm{~mA}$ )

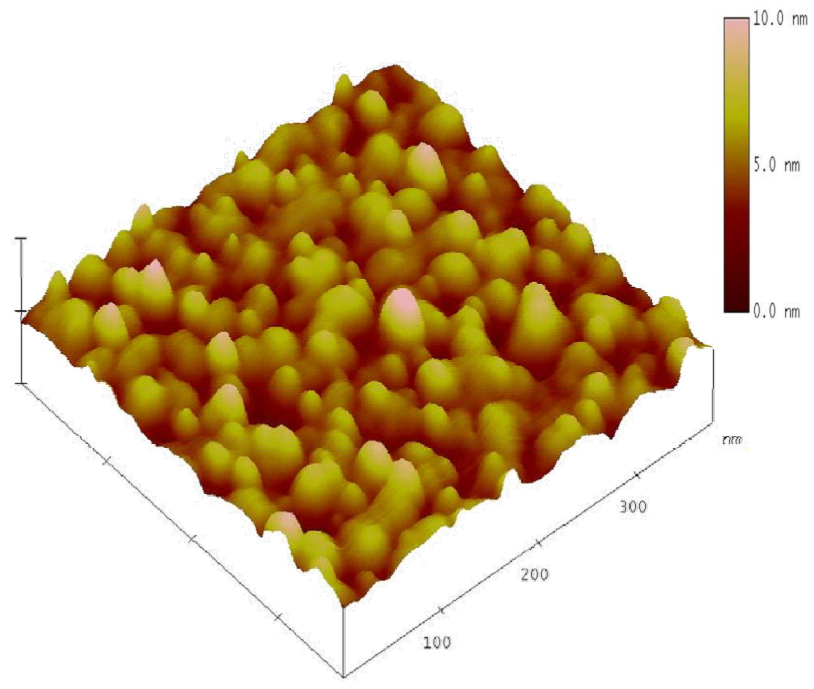

Figure 11: AFM picture showing the grains of a polycrystalline layer (made on the $50 \mathrm{~nm}$ thick Au sample)

\section{CONCLUSION}

We have presented a simple analytical expression giving the resistance of a 2-D constriction pattern. The model has been compared and validated with experimental data, on samples made of tapered lines with different geometrical parameters. Those samples correspond to ideal constrictions, excluding effect of contaminating film barriers, surface roughness (i.e. multiple contact spot distribution), and self resistance caused by heating and thermoelectric EMF.

We have also shown that the constriction resistance is no longer only dominated by the constriction effect as the film thickness is no longer large compared to the electron mean free path. We have taken into account this change of resistivity and shown that the theoretical formula can then be applied also in the case of very thin film. Many authors who studied thin metal contact model have not always distinguished the thin film resistivity from the bulk, whereas it actually evolves dramatically with the thickness.

The proposed model could be improved by considering ellipsoidal equipotential lines instead of circular one, and adding the resistance coming from the straight current line at the junction point.

\section{ACKNOWLEDGMENT}

This work was supported by the French National Research Agency (ANR) through Carnot funding. It also has been performed with the help of the "Plateforme technologique amont" de Grenoble, with the financial support of the "Nanosciences aux limites de la Nanoélectronique" Foundation.

\section{REFERENCES}

[1] G. Norberg et al., "Contact Resistance of Thin Metal Film Contacts", IEEE Transaction on Comp. and Packaging Tech., Vol. 29, No 2, pp 371-378, 2006

[2] R.S. Timsit, "Constriction Resistance of Thin Film Contacts", Proceedings of the 54th IEEE Holm Conference on Electrical contacts, 2008

[3] E. H. Sondheimer, "The mean free path of electrons in metals", Advances in Physics Vol 50, No 6, pp 499-537, 2001 (originally published in Vol. 1, 1952)

[4] N. García, et al., "Magnetoresistance in thin Permalloy film (10 nm thick and 30-200 nm wide) nanocontacts fabricated by e-beam lithography", Appl. Phys. Lett., Vol 89, 083112, 2006

[5] A.F. Mayadas, and M. Shatzkes, "Electrical-Resistivity Model for Polycrystalline Films: the Case of Arbitrary Reflection at External Surfaces", Phys. Rev. B, Vol 1, No 4, 1970

[6] W. Zhang, et al., "Influence of the electron mean free path on the resistivity of thin metal films", Microelectronic Eng., Vol 76, Issue $1-4,2004$

[7] Mayadas, "Intrinsic Resistivity and Electron Mean Free Path in Aluminum Films", J. Appl. Phys., Vol 39, No 9, pp. 4241-4245, 1968

[8] Mayadas, et al., "Electrical Resistivity Model for Polycrystalline Films: the Case of Specular Reflection at external Surfaces", Appl. Phys. Lett., Vo 14, No 11, pp345-347, 1969

[9] Q. G. Zhang, et al., "Influence of grain boundary scattering on the electrical and thermal conductivities of polycrystalline gold nanofilms", Phys. Rev. B, Vol 74, 134109, 2006 


\title{
Improved Micro-Contact Resistance Model that considers Material Deformation, Electron Transport and Thin Film Characteristics.
}

\author{
Ronald A.Coutu, Jr. ${ }^{1}$, John W. McBride ${ }^{2}$, LaVern A. Starman ${ }^{1}$ \\ ${ }^{1}$ Electrical and Computer Engineering \\ Air Force Institute of Technology \\ Wright-Patterson AFB, OH, USA \\ ${ }^{2}$ School of Engineering Sciences \\ University of Southampton \\ Southampton, UK
}

Ronald.Coutu@afit.edu

\begin{abstract}
This paper reports on an improved analytic model for predicting micro-contact resistance needed for designing microelectro-mechanical systems (MEMS) switches. The original model had two primary considerations: 1) contact material deformation (i.e. elastic, plastic, or elastic-plastic) and 2) effective contact area radius. The model also assumed that individual aspots were close together and that their interactions were dependent on each other which led to using the single effective aspot contact area model. This single effective area model was used to determine specific electron transport regions (i.e. ballistic, quasi-ballistic, or diffusive) by comparing the effective radius and the mean free path of an electron. Using this model required that micro-switch contact materials be deposited, during device fabrication, with processes ensuring low surface roughness values (i.e. sputtered films). Sputtered thin film electric contacts, however, do not behave like bulk materials and the effects of thin film contacts and spreading resistance must be considered. The improved micro-contact resistance model accounts for the two primary considerations above, as well as, using thin film, sputtered, electric contacts.
\end{abstract}

Keywords: micro-contacts, contact resistance, thin film

\section{INTRODUCTION}

Micro-switches exhibit superior performance and are expected to be direct replacements for field effect transistors (FET) and PIN diode switches for radio frequency (RF) applications. In order to accommodate this expectation, highperformance, high reliability, micro-mechanical devices, capable of billions of switching cycles, are needed for RF micro-electro-mechanical systems (MEMS) applications [1].

Predicting micro-switch performance (i.e. contact resistance) is an important design tool routinely used by macro switch engineers. Modeling contact resistance, using Holm's theory, is typically a first step when designing a macro relay [2]. For micro-switch engineers, however, the design task is more difficult because Holm's theory cannot be directly applied. This is primarily because the available contact force, when using micro-switches, is very low and cannot guarantee

The views expressed in this article are those of the authors and do not reflect the official policy or position of the United States Air Force, Department of Defense, or the U.S. Government. excellent performance when using traditional contact materials. In addition, micro-contact resistance theory is generally not well understood and therefore contact resistance predictions typically do not match measured data exactly [3]. This gap between modeled and measured data is often attributed to contaminant films being present on the microcontact's surface and not inadequacies in the theory.

Because of the contact force limitation, micro-switches are often fabricated using thin film gold contacts due to gold's relatively high conductivity and low material hardness. The low hardness value enables reasonable micro-switch performance despite the low contact force. Unfortunately, micro-switches with gold contacts also tend to fail either closed or by developing elevated contact resistance with increased switching cycles. The physics behind these two failure modes is also not well understood, but appears to be related to contact force and how the contact surface evolves. Attempts by designers to increase contact force (and lower contact resistance) by optimizing mechanical switch designs often leads to accelerated degradation and unacceptable device reliability. This is summarized by the observation that while higher contact force results in better performance, when using relatively soft contact materials, it also reduces device reliability due to adhesion or degraded performance.

In an attempt to improve micro-switch reliability, some research groups have investigated using harder contact materials such as platinum, "platinum group", gold alloys [46] and more compliant contact materials like CNTs [3]. Recently, it was reported that RF MEMS switches have been tested to more than 1.4 trillion cold-switched cycles [7]. The devices were tested in a packaged, hermetic environment to minimize contaminant films and the specific electric contact material was not discussed. Although, device reliability was excellent, micro-switch contact resistance performance predictions were not published hinting at a heuristic design approach. In other words, the mechanical design, extremely hard contact materials and parallel contacts were all used to "engineer away" the above failure modes and compensate for 
not fully understanding the micro-contact physics. Although this is a reasonable approach right now, improvements to micro-contact resistance modeling and micro-switch reliability theory will ensure future breakthroughs. Additionally, reliability models, based on accelerated test methods, will be extremely beneficial for predicting micro-switch lifetimes while minimizing valuable test time. The following section develops an improved micro-contact resistance model for better predicting micro-switch performance.

\section{IMPROVED MICRO-CONTACT RESISTANCE}

\section{A. The Baseline Analytic Model}

The baseline micro-contact resistance model used here was previously derived by the author and accounts for material deformation and electron transport [6]. An elastic-plastic (EP) material deformation model was used to account for the higher contact pressure developing at individual a-spot peaks despite the low contact forces being generated by typical microswitches (i.e. tens of $\mu \mathrm{N}$ 's). Fig. 1 depicts the hemispherical upper and planar lower contacts and the EP model used in this study.
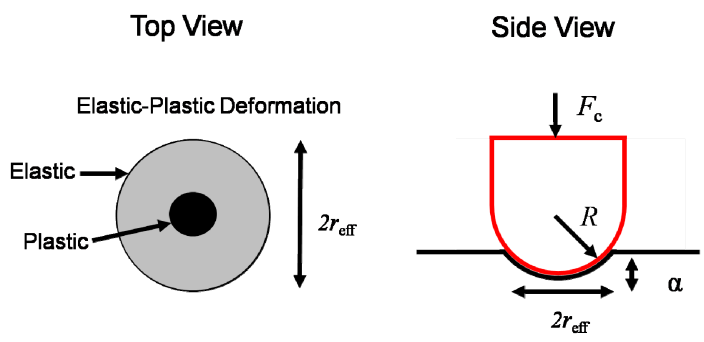

Figure 1. Elastic-plastic material deformation model: hemisphere and plate contact pair.

The baseline model also assumes that the contacting a-spots were sufficiently close together and dependent thereby justifying a single effective a-spot conducting area model shown in Fig. 2.

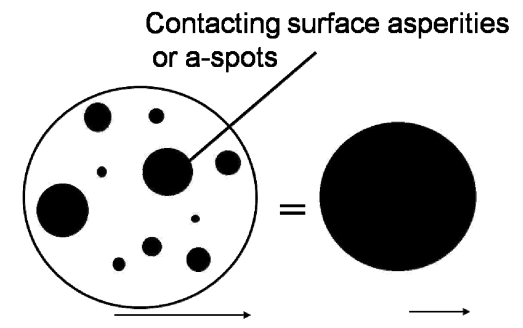

$$
\text { Apparent Radius }\left(r_{a}\right) \quad \text { Effective Radius }\left(r_{\text {eff }}\right)
$$

Figure 2. Single effective a-spot model.

Equation 1 is used to calculate the effective radius of an EP a-spot [6],

$$
r_{\text {eff }}=\sqrt{\frac{F_{c}}{H \Pi\left[1.062+0.354\left(\frac{2}{3} \mathrm{~K}_{\mathrm{Y}}-3\left(\frac{a_{\mathrm{c}}}{\alpha}\right)\right)\right]}}
$$

Where, $\mathrm{K}_{\mathrm{Y}}=1.1282+1.158 \mathrm{v}, \alpha$ is a-spot vertical deformation and $\alpha_{c}$ is the critical deformation where EP deformation begins.

This single effective a-spot assumption was further justified by using sputtered and co-sputtered thin film contacts with exceptionally low measured surface roughness values $(\sim 30-50$ $\AA)$ and tightly packed material grain structures $(\sim 50 \mathrm{~nm}$ in diameter). The low surface roughness was measured using AFM and is qualitatively observed visually in Fig. 3 which shows a fabricated micro-switch contact pair.
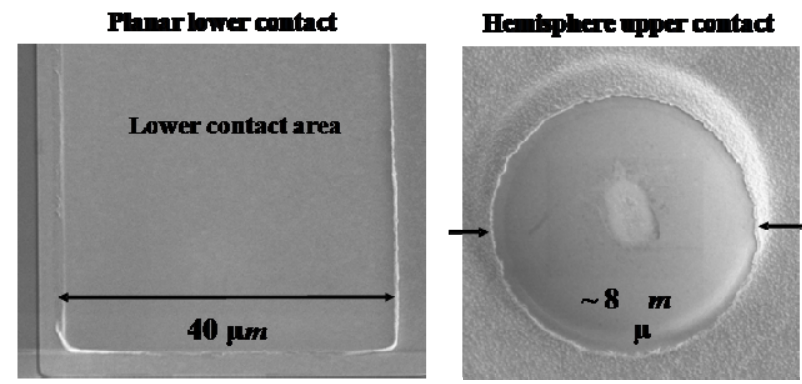

Figure 3. Hemisphere and planar micro-switch contact pairs with low surface roughness.

Based on the radius of the conducting area, and how it compares with the mean free path of an electron, current flow can be described as ballistic, quasi-ballistic or diffusive. Mikrajuddin, et al. derived a gamma function, shown in Fig.4, that is ideally suited for micro-contact resistance modeling [8].

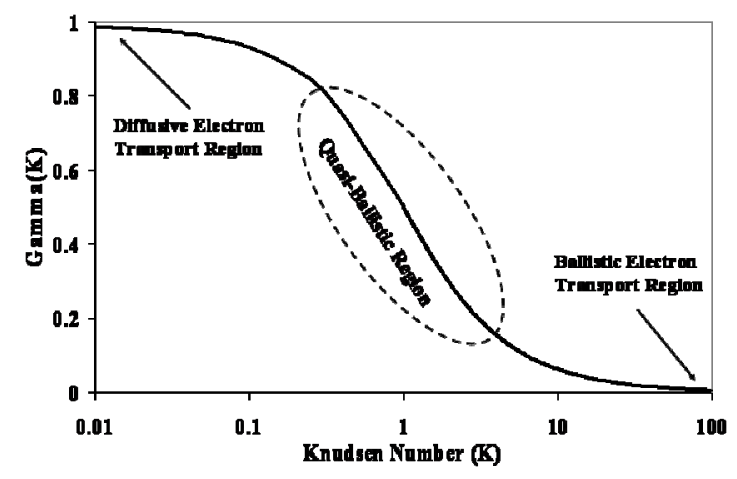

Figure 4. Plot of Mikrajuddin et al.'s derived Gamma function.

Holm's theory, based on diffusive electron flow, accurately predicts macro switch contact resistance but under predicts micro-switch contact resistance. Majumdar et al. addressed this by using Sharvin's equation (2) for resistance based on ballistic electron transport $[9,10]$.

$$
\mathrm{R}_{\mathrm{cBEP}}=\frac{4 \rho K}{3 \Pi r_{\mathrm{eff}}}
$$


where the Knuden, $K$, is defined by $K=\frac{l_{\text {eff }}}{r_{\text {eff }}}$.

The naming convention used in this paper describes the particular electron transport region and how the contact material is deforming. For example, (2) above is a contact resistance equation $\left(\mathrm{R}_{\mathrm{c}}\right)$ used to calculate ballistic transport $\left(\mathrm{R}_{\mathrm{cB}}\right)$ due to EP deforming contacts $\left(\mathrm{R}_{\mathrm{cBEP}}\right)$.

Wexler connected the diffusive and ballistic electron transport regions using a Gamma function to "toggle" between the two regions [11]. This model well represents micro-switch behavior, in that, micro-contacts are generally not large enough to support complete diffusive transport nor are they usually small enough to support only ballistic transport. This is true especially when using the single effective area model because of a-spot dependence. In other words, because the area is summed, the smaller portions of the overall area will support ballistic transport and the larger portions of the overall area will support diffusive transport. Clearly, Wexler's interpolation (3) is appropriate when using the single effective area model. The only question is how to set the Gamma function to accurately represent a real device. This will be estimated by considering measured surface roughness and actual contact area.

$$
R_{c W E P}=R_{c B E P}+\Gamma(K) R_{c D E P}
$$

The baseline EP model, summarized above, neglected the effects of using thin film electric contact materials and attributed differences between modeled and measure data entirely to contaminant films [6]. Yunus et al. identified this oversight and theorized that thin film effects were, in part, responsible for the differences [3]. Kwon et al., however, concluded that differences between modeled and measured data, when using soft thin film contacts, were due to a relatively hard underlying substrate that limited contact area growth with increased contact force [12]. This could be the case when high contact force (i.e. several $\mathrm{mN}$ 's) is used in conjunction with thin contact films. In a typical MEMS device, however, the available contact force is usually limited to 100 $200 \mu \mathrm{N}$. The difference between measured data and modeled predictions is most likely due to a combination of contaminant film resistance and distorted constrictions, due to thin film spreading resistance, not being considered $[2,13]$.

\section{B. Thin Film Improvements}

Often times, in micro-switches, the radius of the effective contact area is dimensionally similar to the actual electric contact film thickness. Fig. 5 depicts typical current flow lines that originate in a bulk region and flow into a thin film region [13].

The sharp curving of the current flow lines result in a "thin film" effect that is analogous to the well known "skin" effect observed in high frequency microwave systems [14]. In addition to theoretical consideration, increased contact resistance due to thin film spreading resistance has been experimentally verified [3]. Based on the discussion above, the baseline analytic model was modified to incorporate the effects of thin films, current crowding and distorted constrictions shown in Fig. 6 [14].

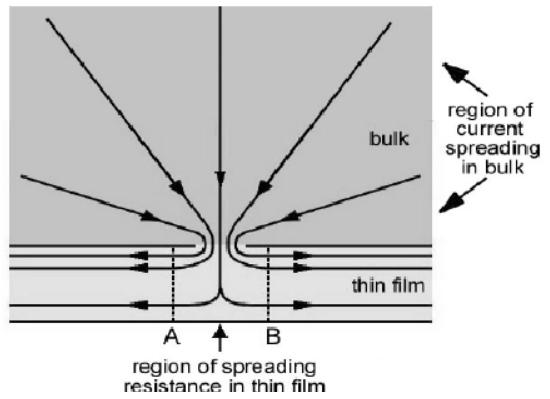

Figure 5. Schematic comparison of the region of spreading resistance in a solid conductor with the corresponding region in a thin film conductor [13].

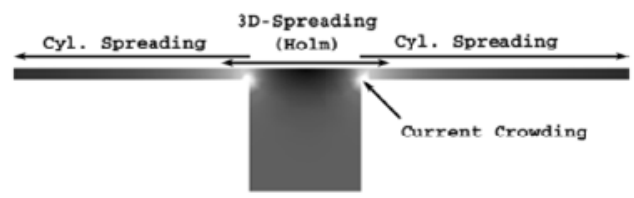

Figure 6. Regions of different spreading resistance contributions [14].

Norberg et al. analytically modeled Fig. 6 as the algebraic sum of the Maxwell-Rayleigh spreading resistance in a cylinder, corrected for non ideal current flow (4), and the resistance due to a radial current that is spread into a thin film cylinder (5). It was recognized, however, that this approach resulted in a spreading resistance overestimation due to both models making similar contributions [14]. To avoid this overestimation, each of the terms in (6) was weighted with a coefficient function that was determined using finite element analysis (FEA). The resulting equation (6) is an approximation for the spreading resistance exhibited by the model shown in Fig. 6 where the weighting coefficients, $\mathrm{k}_{1}$ and $\mathrm{k}_{2}$, are functions of both film thickness (d) and effective a-spot radius ( $\left.\mathrm{r}_{\text {eff }}\right)$. Equation (5) does not account for the contact film "thinning", observed by Kwon, et al., so an additional variable, $\alpha$ (i.e. vertical a-spot deformation), was subtracted from, $d$ (i.e. film thickness) in (5) resulting in (7).

$$
\begin{aligned}
& \mathrm{R}_{\text {max-Ray }}=\frac{\rho}{4 r_{\text {eff }}}(1.05) \\
& \mathrm{R}_{\mathrm{Scyl}}=\frac{\rho}{2 \Pi d} \ln \frac{r_{\mathrm{B}}}{r_{\mathrm{eff}}}
\end{aligned}
$$

where $r_{B}$ is the outer radius of modeled thin film cylinder shown in Fig. 6 and 7.

$$
\mathrm{R}_{\text {approx }}=\mathrm{k}_{1}\left(r_{\text {eff }}, \mathrm{d}\right) \cdot \mathrm{R}_{\max -\mathrm{Ray}}+\mathrm{k}_{2}\left(r_{\text {eff }}, \mathrm{d}\right) \cdot \mathrm{R}_{\text {Scyl }}
$$




$$
\mathrm{R}_{\mathrm{Scyl}}=\frac{\rho}{2 \Pi(d-\alpha)} \ln \frac{r_{\mathrm{B}}}{r_{\mathrm{eff}}}
$$

Equation (8) is used, in the model presented here, as the basis for the diffusive current flow in micro-contacts because it accounts for previously not considered "thin film" effects.

$$
\mathrm{R}_{\text {approx }}=\mathrm{R}_{\mathrm{cDEP}}
$$

In a typical micro-switch contact area pair, the upper contact can be considered a long constriction due to its overall thickness (i.e. 5-7 $\mu \mathrm{m}$ is typical) because it is physically part of the mechanical structure of the beam [2]. The lower contact, however, is best described as a distorted constriction due to its relative thickness (i.e. $500 \mathrm{~nm}$ ) [2]. In addition, RF MEMS switch lower contacts are usually deposited on highly resistive substrates to enhance RF performance which exacerbates the "thin film" effects exhibited by the lower contact. Fig. 7 depicts how the single effective area and Norberg's thin film effects models apply to micro-switch electrical contacts.

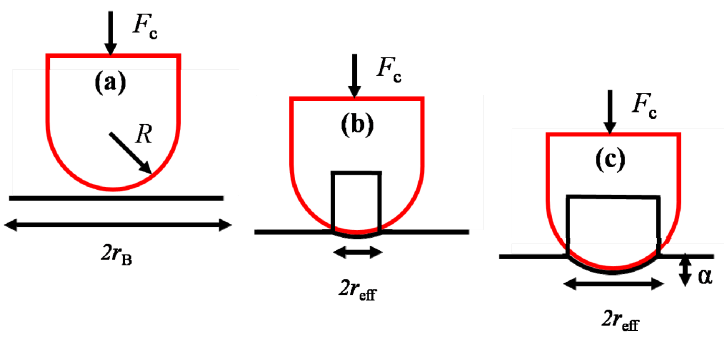

Figure 7. Norberg's thin film spreading resistance model applied to a microswitch contact pair. (a) prior to making contact (b) Initial contact, upper contact modeled as a cylinder (c) The upper contact cylinder's volume increases with increased contact force.

\section{The Improved Analytical Model}

The improved micro-contact resistance model is based on (3) that accounts for EP material deformation and for electron transport. The ballistic term is given by (2) and the diffusive term, which accounts for thin film effects, is given by (8) with (7) substituted for $R_{\text {Scyl }}$ and $r_{\text {eff }}$ (1) substituted for effective radius. The Gamma function is represented by Fig. 4 and the weighting coefficients, $\mathrm{k}_{1}$ and $\mathrm{k}_{2}$, are taken from FEA data plots provided in [14]. Modeled and measured data will be compared later.

\section{TEST SET-UP AND RESUltS}

A modified nano-indentation apparatus, shown in Fig. 8, was used to test micro-contacts by applying low contact force $(<1 \mathrm{mN})$ while simultaneously measuring contact resistance. A hemisphere-shaped upper and a planar lower contact pair were used during testing [3].

A Keithly 580 micro-ohmmeter was used to apply a constant DC current $(1 \mathrm{~mA})$ while measuring voltage in the four-wire contact configuration depicted in Fig. 9 [3].

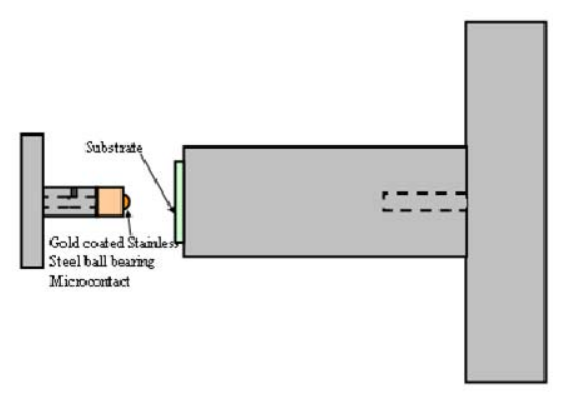

Figure 8. Schematic of Modified Nanoindentor [3].

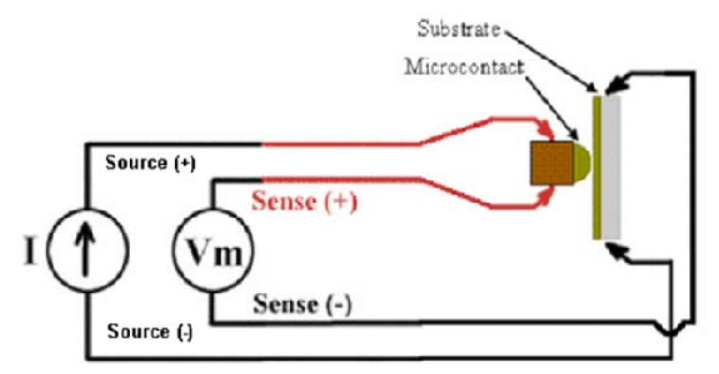

Figure 9. Schematic of contact zone with its electrode and CR measurement [3].

The micro-contacts were brought into contact at a controlled rate of $0.2 \mathrm{mN} / \mathrm{sec}$ until the maximum targeted load was achieved. The applied load was then held for $10 \mathrm{sec}$ to ensure that a peak load resistance was measured. The microcontacts were then unloaded at the same rate until separation was achieved [3]. Fig. 10 compares measured contact resistance data to predictions made using Holm's model for plastically deformed a-spots [2], the baseline analytical model [6] and the improved analytical models discussed previously. The baseline and improved analytical models were based on EP material deformation and assumed 2\% plastic deformation. The baseline model predictions assumed complete diffusive transport $(\Gamma=1)$ while the improved model used Mikrajuddin, et al.'s Gamma, shown in Fig. 4, to "toggle" between ballistic and diffusive electron transport [8]. In addition, the improved model implemented thin film considerations developed by Norberg, et al. [14]. At $0.5 \mathrm{mN}$, Fig. 10 shows a consistent $0.35 \Omega$ difference between the measured and modeled data. This anomaly was, most likely, due to a measurement bias error and was not attributed to contaminants on the gold electric contact films. This hypothesis is substantiated since all the analytical models converge at elevated contact force values.

Fig. 11 depicts the portion of Fig. 10 between $0.3 \mathrm{mN}$ and $0.9 \mathrm{mN}$ and reveals several key results. First the baseline model predicts contact resistance that is approximately $16 \mathrm{~m} \Omega$ lower than Holm's plastic deformation-based model. This is due to the baseline model's Gamma function being fixed to predict $100 \%$ diffusive electron transport. 


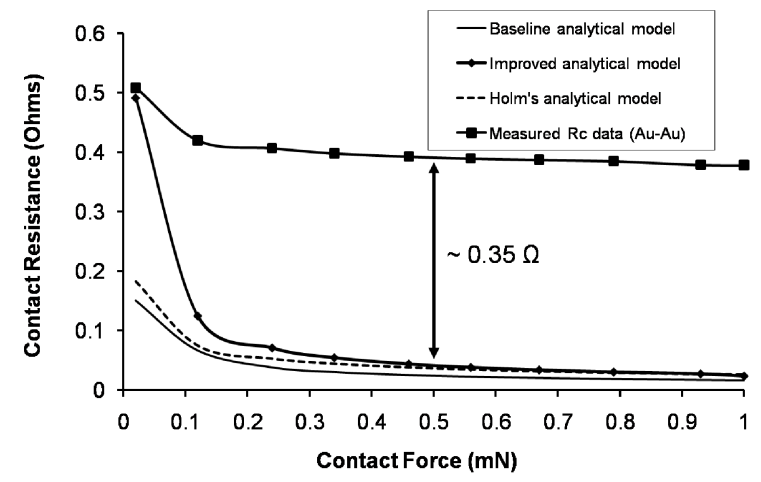

Figure 10. Measured and modeled contact resistance data for Au-Au microcontacts.

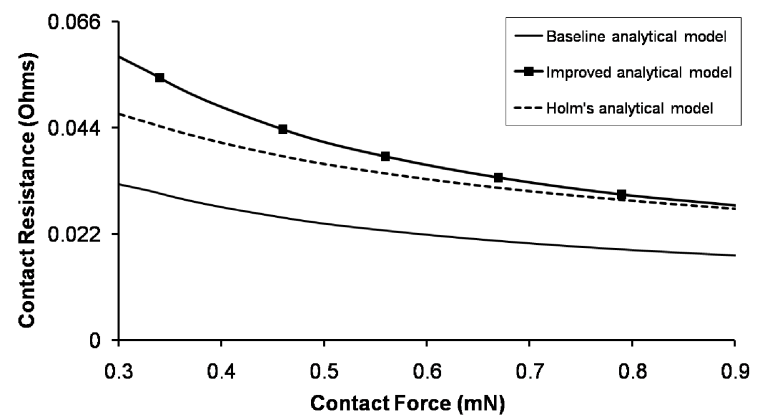

Figure 11. Comparison between the baseline, improved and Holm's contact resistance models.

Normally, the baseline model predicts micro-contact resistance that is "slightly" higher (1-2 $\mathrm{m} \Omega)$ than Holm's model when ballistic transport is included. Next, at approximately $0.5 \mathrm{mN}$ of applied contact force, the improved model predictions are approximately $6 \mathrm{~m} \Omega$ s higher than Holm and approximately $20 \mathrm{~m} \Omega$ higher than the baseline model (due to the Gamma function choice). Also, when the applied contact force is greater than $0.5 \mathrm{mN}$ the improved and Holm models converge by approximately $0.9 \mathrm{mN}$ while the baseline model converges with Holm at a much slower rate. The convergence of all these models at elevated contact forces is a positive indication that the lower contact force predictions (for which the new models were derived) are useful.

\section{CONCLUSIONS}

The purpose of this work was to improve a previously developed analytic micro-contact resistance model. Previous differences between modeled and measured data were attributed to contaminant films. In the improved model, thin film characteristics are included to improve modeling accuracy. Overall, the improved micro-contact resistance model closely predicts actual micro-switch behavior and the resulting modeled data matches the measured data.

\section{REFERENCES}

[1] Rebeiz, G., RF MEMS Theory, Design and Technology. New York: Wiley, 2003.

[2] Holm, R., Electric Contacts: Theory and Applications. Berlin, Germany, Springer, 1969.

[3] Yunus, E., McBride, J.W. and Spearing, S.M.., "The Relationship between Contact Resistance and Contact Force on Au coated Carbon Nanotube surfaces", in Proc. of the 53d IEEE Holm Conference, pp. 167-174, 2007.

[4] Duffy, et al., "MEMS Microswitches for reconfigurable microwave circuitry", IEEE Microwave and Wireless Component Letters, Vol. 11, No. 3, pp. 106-108, March, 2001.

[5] Majumdar, S, et al., IEEE Instrumention Measurement Mag. pp.12-15, March, 2003.

[6] Coutu, Jr., R.A., Reid, J.R., Cortez, R., Strawser, R.E. and Kladitis, P.E., "Microswitches with Sputtered $\mathrm{Au}, \mathrm{AuPd}, \mathrm{Au}-\mathrm{on}-\mathrm{AuPt}$, and $\mathrm{AuPtCu}$ Alloy Electric Contacts", IEEE Transactions on Components \& Packaging Technologies, Vol. 29, No. 2, pp. 341-349, 2006.

[7] Newman, S.H., Ebel, J.L., Judy, D. and Maciel, J., "Lifetime Measurements on a High-Reliability RF-MEMS Contact Switch", IEEE Microwave and Wireless Components Letters, Vol. 18, No. 2, pp. 100102, February 2008.

[8] Mikrajuddin, A., Shi, F., Kim, H. and Okuyama, K., "Size-dependent electrical constriction resistance for contacts of arbitrary size: from Sharvin to Holm limits", Materials Science in Semiconductor Processing, Vol. 2, No. 4, pp 321-327, 1999.

[9] Majumdar, S, et al., "Study of Microcontacts Tested with a Novel MEMS Device", Sensors and Actuators A., Vol. 93, No. 1, pp. 19-26, 2001.

[10] Sharvin, Y., "Sharvin Resistance Formula", Sov. Phys. JETP, Vol. 21, pp.655, 1965.

[11] Wexler G., "The size effect and the nonlocal Boltzmann Transport equation in orifice and disk geometry", in Proc. Phys. Soc., Vol. 89, pp. 927-941, 1966.

[12] Kwon, H., et al., "Investigation of the electrical contact behaviors in Auto-Au thin-film contacts for RF MEMS", Journal of Micromechanics and Microengineering, Vol. 18, pp. 1-9, September 2008.

[13] Timsit, R.S., "Constriction Resistance of Thin Film Contacts," Proceedings of The $54^{\text {th }}$ IEEE Holm Conference on Electrical Contacts, pp. 332-336, 2008.

[14] Norberg, G., Dejanovic, S., Hesselbom, H., "Contact Resistance of Thin Metal Film Contacts", IEEE Transactions on Components \& Packaging Technologies, Vol. 29, No. 2, pp. 371-378, 2006. 


\section{Contact Resistance in Flat Thin Films}

\author{
M.B. Read ${ }^{1}$, J.H. Lang ${ }^{2}$, A.H. Slocum ${ }^{1}$ \\ ${ }^{1}$ Department of Mechanical Engineering \\ ${ }^{2}$ Department of Electrical Engineering and Computer \\ Science \\ Massachusetts Institute of Technology \\ Cambridge, USA \\ melissar@mit.edu
}

\author{
R. Martens \\ FormFactor Inc. \\ Livermore, USA
}

\begin{abstract}
MEMS-fabricated electrical contacts are commonly used in MEMS relays. These electrical contacts can be as simple as two flat surfaces coming into contact [1]. Modeling their contact force/resistance relationship can be difficult because much of the theory on contact resistance was developed for macro-scale contacts [2], and contact properties for MEMS-scale contacts do not always agree with those predicted by this theory [3]. One contribution to this disagreement is that when the dimensions of the contact thickness are on the order of the a-spot dimensions, the spreading resistance is affected [4]. In order to determine the relationship between contact force and resistance for a wide range of parameters, we have developed a two-coupon test system which allows the properties of these contacts to be empirically determined. The design of the two-coupon system allows for the rapid fabrication of multiple contact materials and geometries. The two-coupon system was used to test the contact resistance properties of sputtered and electroplated Au films in thicknesses of $0.1 \mu \mathrm{m}, 0.3 \mu \mathrm{m}$, and $0.5 \mu \mathrm{m}$. Contact force was measured using a custom flexural force gauge and the 4-point contact resistance was measured using an integrated Kelvin Structure [5]. The results are compared to traditional Holm theory to determine the effects of film thickness on spreading resistance.
\end{abstract}

Keywords- thin films; contact resistance

\section{INTRODUCTION}

Traditional contact theory assumes that when two surfaces meet at a single contact spot, the electrical current flow lines spread in all directions upon exiting the contact spot, as shown in Fig. 1a. Holm theory holds that the spreading resistance between these two surfaces is [2]

$$
R_{S}=\frac{\rho}{4 a}+\frac{\rho}{4 a}
$$

where $\rho$ is electrical resistivity and $a$ is the contact spot radius. However, in thin films, where the film thickness $L$ is on the order of the contact spot radius $a$, the geometry of the contact limits the current lines from spreading in all directions. Instead, the current lines must curve fairly rapidly, as shown in Fig. 1b.

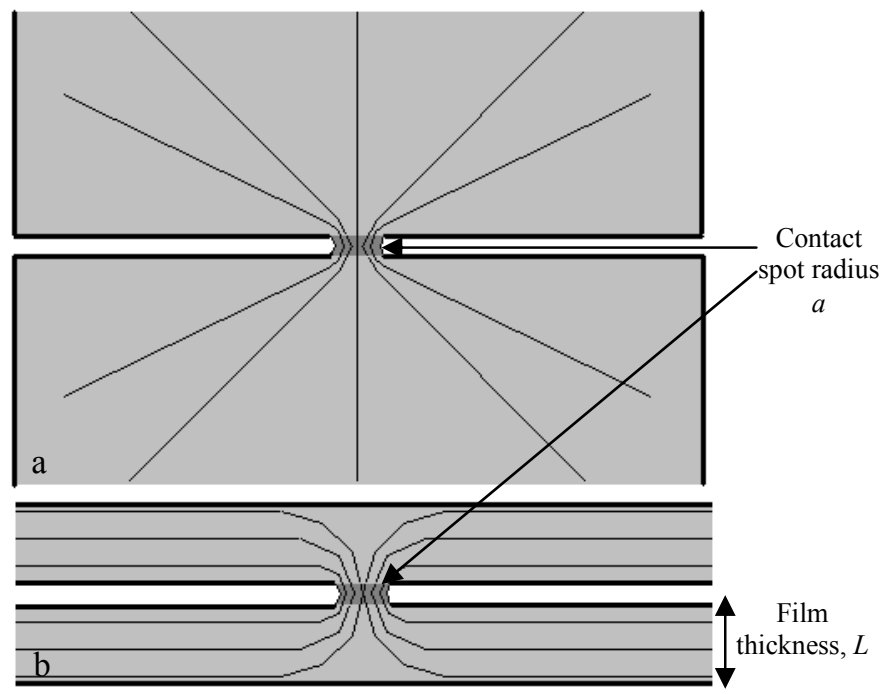

Figure 1. a) The current flow lines between two bulk marterials meeting at a contact spot as assumed in traditional contact theory. b) The current flow lines between two thin films meeting at a contact spot.

Several methods have been used to determine the effect of the ratio between contact radius $a$ and film thickness $L, a / L$, on contact resistance. One method uses a mathematical model to predict the dependence of normalized contact resistance (as defined as the ratio between actual contact resistance and that predicted by Eq. (1)) on the ratio $a / L$ [4]. This method theorizes that thin film geometry causes the spreading resistance to be constrained to a much smaller region than the region where the spreading resistance takes place in non-thin films. This method finds that the thin film affected normalized contact resistance is given by [4]

$$
R_{N}=\frac{4}{\pi} \sum_{n=0}^{n=\infty}\left(\frac{\operatorname{coth}\left(\lambda_{n} L / b\right) \sin \left(\lambda_{n} a / b\right)}{\left(J_{1}\left(\lambda_{n}\right) \lambda_{n}\right)^{2}}\right)-\frac{2(a / L)}{\pi} \ln (b / a)
$$

where $R_{N}$ is the normalized spreading resistance and $b$ is the outer film radius for a cylindrical contact. These geometries are defined in Fig. 2. In Eq. (2) the argument for the Bessel function of the first kind of order $1, \lambda_{n}$, is 


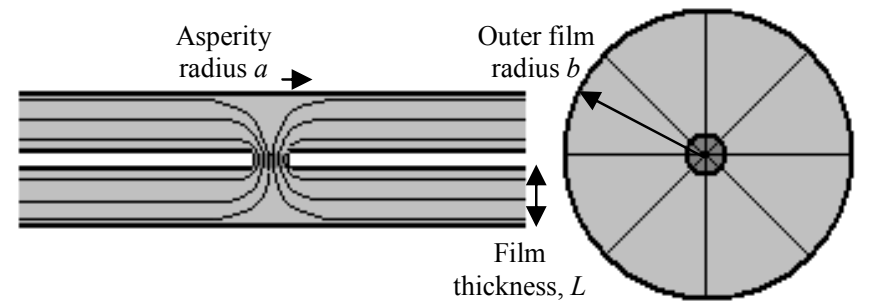

Figure 2. The contact spot radius $a$, the film outer radius $b$, and the film thickness $L$, for thin films meeting at a single asperity.

$$
\lambda_{n}=\frac{\beta}{4}\left[1+\frac{2}{\beta^{2}}-\frac{62}{3 \beta^{4}}+\frac{15116}{30 \beta^{6}}\right]
$$

where $\beta$ is

$$
\beta=\pi(4 n-1)
$$

These calculations were based on assumptions which were only valid where $0 \leq a / L \leq 0.5$ [4]. The effects of this on normalized contact resistance are shown in Fig. 3.

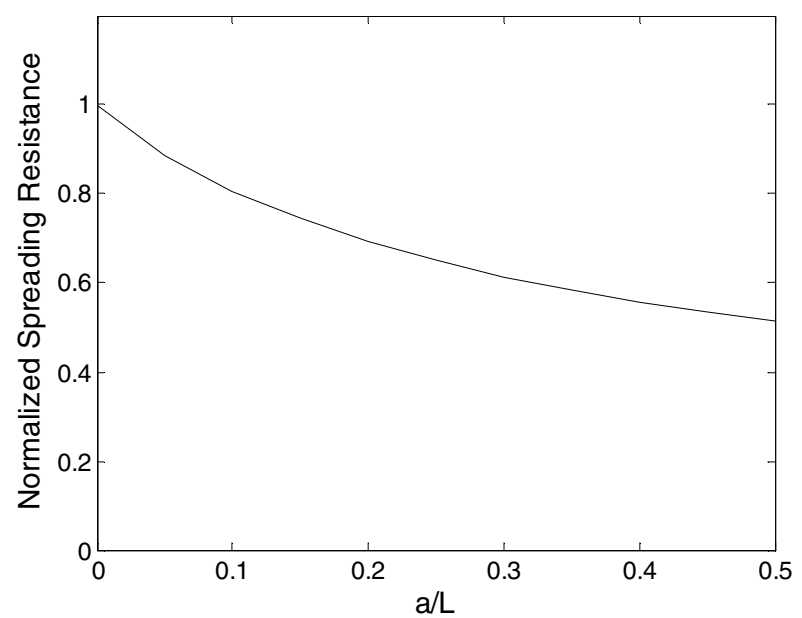

Figure 3. Normalized contact resistance as a function of the ratio $a / L$ as predicted by [4].

For very low values of $a / L$ there is no effect on contact resistance. As $a / L$ increases, the normalized contact resistance decreases. At $a / L=0.5$, the maximum valid value of these equations, the normalized contact resistance is approximately 0.52 . As is stated by [4], the ratio between film thickness and outer film radius, $L / b$, has an insignificant effect on contact resistance over the range $0.01 \leq L / b \leq 0.1$.
When a force is applied to two thin films in contact, the Holm radius $a_{H}$ is given by the classic formula [2]

$$
a_{H}=\sqrt{\frac{F}{\pi H}}
$$

where $F$ is force and $H$ is hardness. Film thickness is not a factor in this equation; therefore, films having identical compositions but different film thicknesses will have identical values of $a_{H}$ for a given applied force $F$. Assuming that as with traditional Holm theory, this value $a_{H}$ can be substituted for the constriction radius $a$ when determining contact resistance, then the $a / L$ values for any given applied force will decrease as film thickness increases. According to Fig. 3, when $a / L$ decreases, normalized contact resistance will increase. Therefore, in this model, normalized contact resistance increases as film thickness increases.

Other work suggests that Holm theory actually underestimates contact resistance for thin films. In [6], it is pointed out that in the derivation of Eq. (1), Holm assumes the constriction depth is equal to the constriction radius. However, this is not applicable in films where the Holm radius is on the order of the film thickness. In [6], an FEM model was developed to look at the contact geometry shown in Fig. 4.

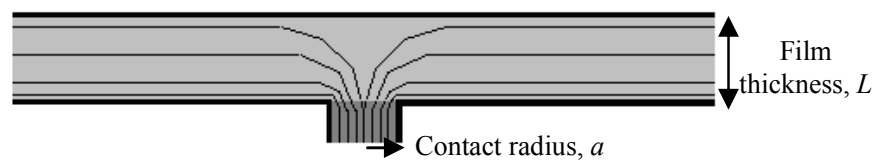

Figure 4. Geometry of contact modeled in [6].

The simulations in [6] found that contact resistance decreased as film thickness increased over the range of $L=1-50$. The $a / L$ values investigated went as high as 30 . Additionally, the contact resistance predicted for a film thickness of $1 \mu \mathrm{m}$ at $a / L=5$ was roughly 12 times that of Holm.

The mathematical model in [4] suggests that contact resistance increases with increasing film thickness, whereas the FEM in [6] suggests that contact resistance decreases as film thickness increases. Additionally, [4] predicts contact resistance below that predicted by Eq. (1), whereas [6] predicts contact resistance above that of Eq. (1). However, these two models looked at very different ranges. The model in [4] was limited to $a / L<0.5$ whereas the model in [6] looked at much higher $a / L$ values. However, the data shown in [6] only looked at film thicknesses where $L>1 \mu \mathrm{m}$.

It is not immediately obvious how either of these models would perform outside of the ranges presented in [4] and [6]. Therefore, measurements were performed on film thicknesses of $L=0.1 \mu \mathrm{m}, 0.3 \mu \mathrm{m}, 0.5 \mu \mathrm{m}$ and $a / L=0-17$. The results were evaluated on their own as well as compared to those predicted by [4] and [6] in their respective ranges. 


\section{SAMPLE PREPARATION}

A two-coupon system was used to measure the contact resistance between two films of multiple thicknesses of sputtered and electroplated $\mathrm{Au}$. The system consists of a bottom silicon coupon having 1) a metal trace and 2) three spherical contacts as well as a top silicon coupon having 1) a metal trace, 2) a flexible membrane with a stiff center cylinder, and 3) three $\mathrm{KOH}$ etched pits. The coupons are assembled by placing the $\mathrm{KOH}$ etched pits of the top coupon over the spherical contacts of the bottom coupon. This type of assembly creates a pseudo kinematic coupling, allowing for repeatable positioning [7]. This system is described in detail in [5]. The assembly leaves a gap between the top and bottom metal traces. When a force is applied to the stiff cylinder in the center of the membrane, the membrane strains which brings the two metal traces into contact [5]. The traces are each $1 \mathrm{~mm}$ wide. The diameter of the stiff cylinder in the center of the membrane is $4 \mathrm{~mm}$ making the overall apparent contact area $4 \mathrm{~mm}^{2}$. The metal traces create a Kelvin structure allowing for the isolation of the contact resistance. This assembly and Kelvin structure are shown in Fig 5.

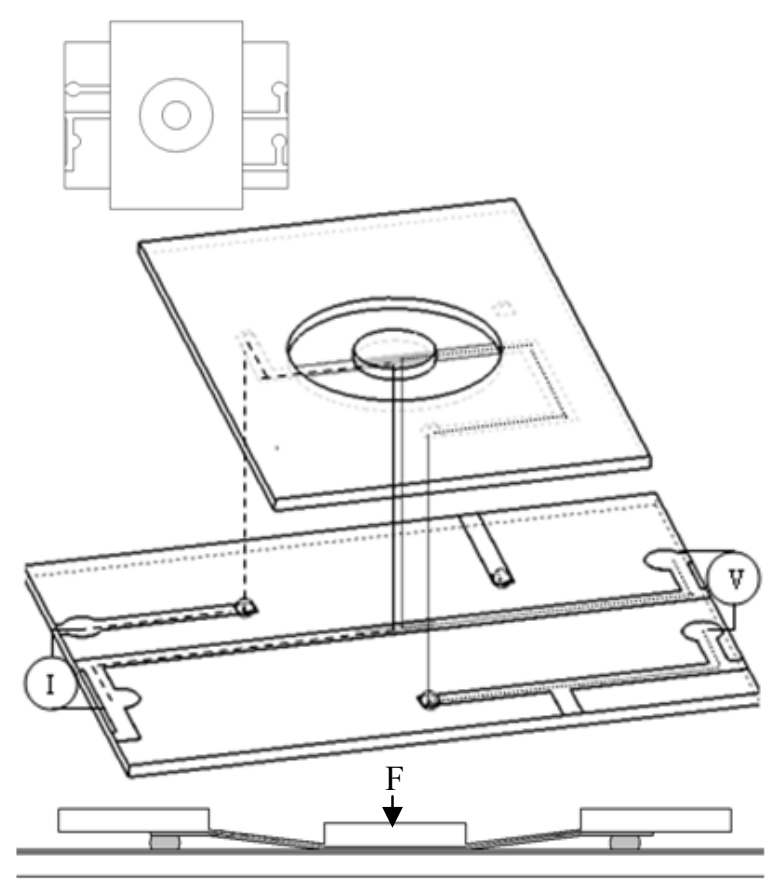

Figure 5. a) An image of the assembled coupons from above. b) An exploded image of the coupons, shown in transparency, displaying the integrated Kelvin structure. c) The membrane deflecting showing how the two metal traces come into contact (not-to-scale).

The contact area in this case is rectangular, unlike the circular contact discussed in [4] and [6]. However, it is reasonable to believe the individual contact spots are round. A not-to-scale drawing of a few individual contact spots in relation to the rest of the trace is shown in Fig. 6.

For the experimental set up, the contact length $l=4 \mathrm{~mm}$ and the contact width $w=1 \mathrm{~mm}$ making the apparent area of

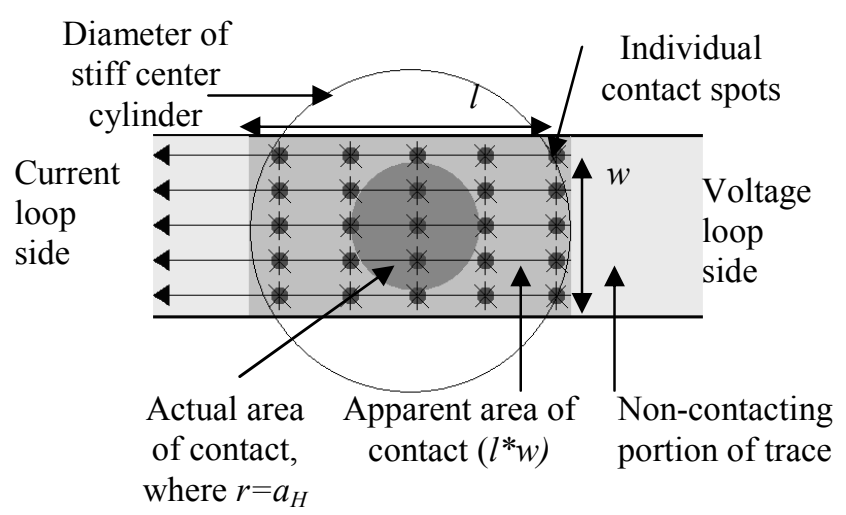

Figure 6. Apparent area of contact, actual area of contact, inidividual contact spots, and the diameter of the stiff center cylinder of the experimental setup. Drawing is not-to-scale and current flow lines are apprimiate and do not show how current recombines from the individual contact spots.

contact $4 \mathrm{~mm}^{2}$. However, the Holm radius corresponding to the actual area of contact is on the order of $1-10 \mu \mathrm{m}$. It is hypothesized that even though the macro-geometry is rectangular, the contact spots act locally much like those modeled in [4] and [6]. This is because $a_{H}<<w$ and $a_{H}<<l$.

Because of this inequality of scale, it is unlikely that the current flow lines immediately around the contact spots will be affected by the macro geometry. However, it is acknowledged that this is an assumption and that there might be a small effect to contact resistance caused by the macro geometry which this paper does not account for.

Also considered was the true flatness of the contact. In designing of this fixture, achieving flatness was a top priority. The actual flatness across the contact can vary by as much as $200 \mathrm{~nm}$. This means that there is a possibility of the contact rocking immediately after touchdown. No physical evidence of this rocking was seen under normal conditions; however, when a current greater than one Ampere was put through the contact, the contact did tend to fail along one edge, suggesting that the pressure may have been higher on that edge.

The sputtered coupons were prepared by sputtering a 0.03 $\mu \mathrm{m}$ Ti adhesion layer followed by $0.1 \mu \mathrm{m}, 0.3 \mu \mathrm{m}$, or $0.5 \mu \mathrm{m}$ of sputtered Au. An oxide layer insulated the Ti from the silicon substrate. Both the top and bottom coupons had identical films.

The electroplated coupons were prepared by sputtering $0.03 \mu \mathrm{m}$ Ti adhesion layer followed by a $0.1 \mu \mathrm{m} \mathrm{Au}$ seed layer. On top of this seed layer, $0.1 \mu \mathrm{m}, 0.3 \mu \mathrm{m}$, or $0.5 \mu \mathrm{m}$ of additional Au was plated. Again, an oxide layer isolated the traces from the silicon substrate and both the top and bottom coupons had identical films.

Due to the difficulty in measuring the hardness of films with thicknesses less than $1 \mu \mathrm{m}$, the hardness of the sputtered film was approximated as $3.5 \mathrm{GPa}$ and the electroplated films as $1 \mathrm{GPa}[8,9]$. The surface roughness of the sputtered films 
was found to be about $6 \mathrm{~nm}$ while the surface roughness of the electroplated films was found to be about $14 \mathrm{~nm}$.

The resistivity of the sputtered samples was measured as $\rho=4.27 \times 10^{-8} \Omega \mathrm{m}$. This value was consistent across all three thicknesses of the sputtered film samples. This is significantly higher than the bulk resistivity of Au. Sputtered films are known to have a higher than bulk contact resistivity [10]. Resistivity can be affected by various mechanisms such as temperature, electron surface scattering, impurities, intragranular defects, and scattering at grain boundaries [10]. It has also been shown that in thin sputtered Au films with an adhesion layer of $\mathrm{Ti}$, the $\mathrm{Au}$ and $\mathrm{Ti}$ can form an alloy of significantly higher resistivity [11]. The resistivity of the electroplated samples was also measured. Taking into account the resistivity of the seed layer as a parallel resistance, the resistivity of the bulk was found to be $2.18 \times 10^{-8} \Omega \mathrm{m}$, consistent with literature values for Au resistivity.

\section{TESTING AND RESULTS}

\section{A. Testing Procedure}

During the experiment, a force gauge compressed the top membrane bringing the two metal traces into contact, as shown in Fig. 5. The overall displacement of the membrane, the force, and the contact resistance were recorded throughout the test. When the membrane stopped moving, contact was made. This also corresponded to the first time finite contact resistance was seen since no oxides or films impeded current flow. This is the point where the contact sees zero force. After this point, force was further increased to $10 \mathrm{mN}$. The current was sourced at 5 $\mathrm{mA}$ and 4-wire resistance was measured using a Keithely 2420 source measure unit.

\section{B. Sputtered Film Results}

The contact resistance as a function of contact force was measured for the three thicknesses of sputtered Au films. The results along with the values calculated by traditional Holm theory using Eq. (1) and Eq. (5) are shown in Fig. 7. The resistivity used to calculate the Holm prediction was the measured resistivity of the sputtered film, $4.27 \times 10^{-8} \Omega \mathrm{m}$.

The results show contact resistance decreasing as film thickness increases. The $0.1 \mu \mathrm{m}$ and $0.3 \mu \mathrm{m}$ films both showed significantly higher contact resistance than that predeicted by Eq. (1). The $0.5 \mu \mathrm{m}$ film showed slightly lower contact resistance than the Holm theory. When contact force was increased significantly, contact resistance did drop below $1 \mathrm{~m} \Omega$, indicating that there is no significant bulk resistance in the test setup. The force gauge is accurate within $10 \mu \mathrm{N}$ and the resistance measurement is accurate to approximatley $20 \mu \Omega$ These results have a similar trend to the FEM presented in [6]. In both cases, the contact resistance decreases with increasing film thickness. However, the results of [6] showed a signficantly higher overall contact resistance than seen in these experimental results.

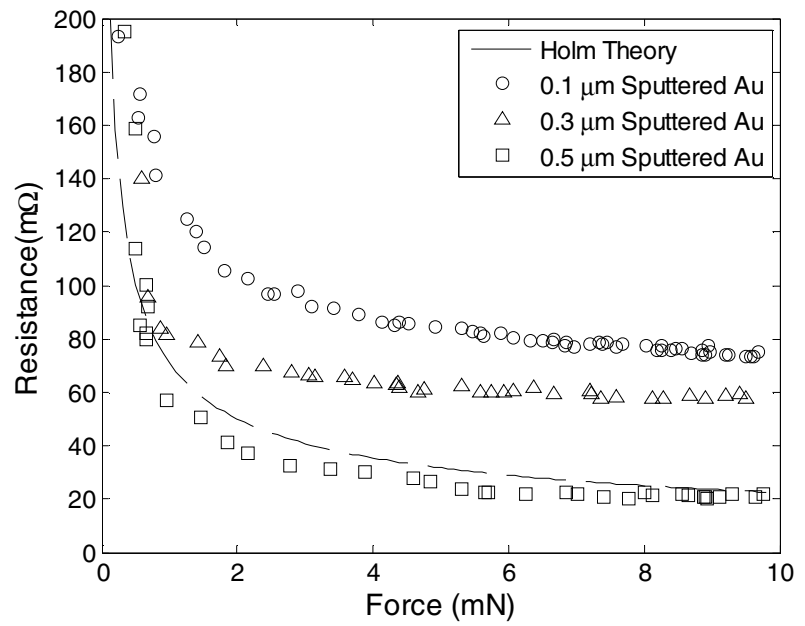

Figure 7. Contact resistance of sputtered thin $\mathrm{Au}$ films and the contact resistance predicted by Holm Theory.

These same results are shown plotted as normalized contact resistance $R_{N}$ vs. $a / L$ are shown in Fig. 7 . The normalized contact resistance is the measured resistance divided by the resistance predicted by Holm theory. The $a$ value is calculated from Eq. (5) using the approximated hardness of $3.5 \mathrm{GPa}$.

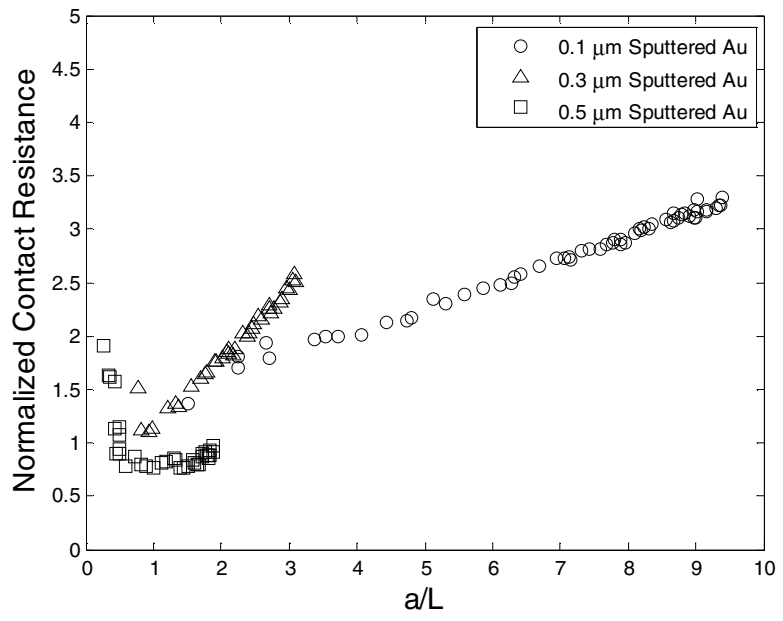

Figure 8. Normalized contact resisance of sputtered Au films.

At values of $a / L>1$, there is a clear trend of increased normalized contact resistance as $a / L$ is increased. This trend appears somewhat linear. However, at values of $a / L<1$, this trend does not exist. In this region, the normalized contact resistance drops below 1 . It makes sense that the relationship between normalized contact resistance and $a / L$ would be different in this range. At values of $a / L<1$, the Holm radius is less than the film thickness. However, at values of $a / L>1$, the Holm radius is greater than the film thickness.

One interesting note is that [4] shows a decreasing normalized contact resistance with increasing $a / L$ in the region of $a / L<0.5$, just as the experimental data shows a decreasing normalized contact resistance with increasing $a / L$ in the region 
of $a / L<1$. Unfortunately, the force gauge used was rather course; so, it is difficult to look at the experimental results where $a / L<0.5$ and, therefore, difficult to directly compare these results.

\section{Electroplated Seed Layer Correction Factor}

Determining the contact resistance for the plated $\mathrm{Au}$ was slightly more difficult because of the added effects of the seed layer. Traditional contact resistance theory models this added resistance as shown in Fig. 9a. However, this model assumes all of the current lines travel into the seed layer. In this test setup, as shown in Fig. 9b, only a portion of the current flow lines travel into the seed layer. In traditional modeling, the additional resistance $R_{a}$ of the transition from the electroplated $\mathrm{Au}$ into the sputtered seed layer would be [12]

$$
R_{a}=(8 / \pi)\left(\rho_{p} / \rho_{s}\right)(L / a)
$$

where $\rho_{p}$ is the resistivity in the plated region, $\rho_{s}$ is the resistivity in the sputtered region, $L$ is thickness of the electroplated region, and $a$ is the Holm radius. However, the fraction of current that actually travels in the bulk $R_{s \%}$ is defined as
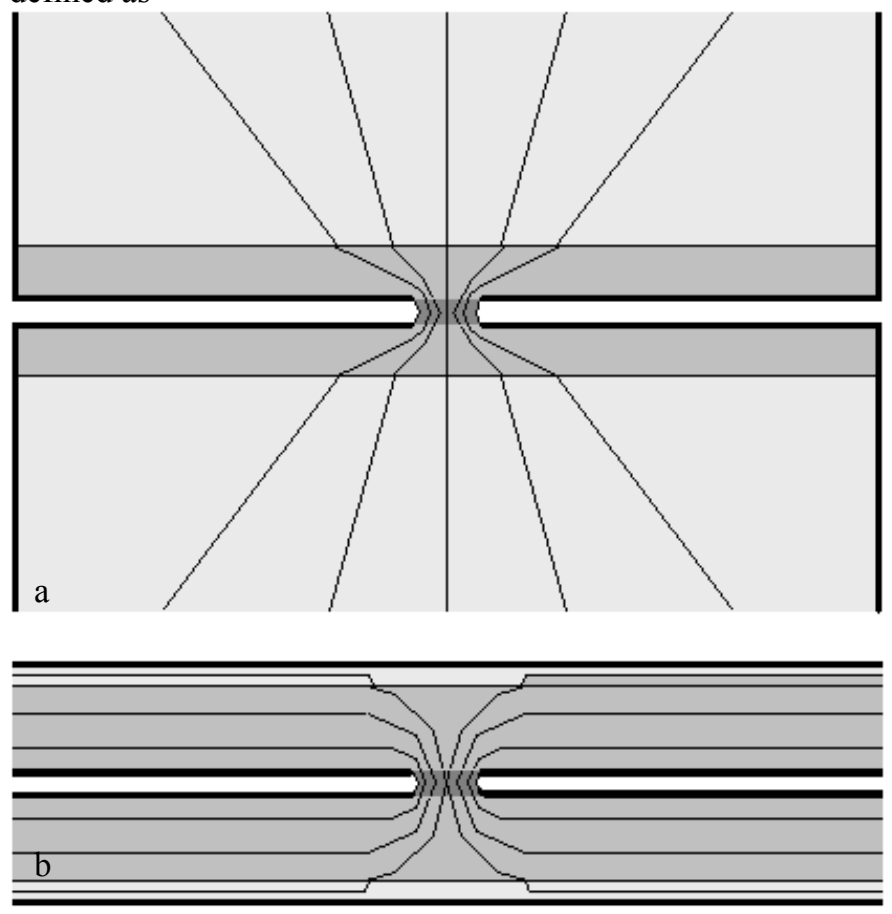

Figure 9. a) Current flow lines in traditional film theory. b) Current flow lines in a thin electroplated film on a sputtered seed layer.

$R_{s \%}=\frac{1 /\left(\rho_{p}\left(l / w L_{p}\right)\right)}{\rho_{p}\left(l / w L_{p}\right)+\rho_{s}\left(l / w L_{s}\right)}=\frac{\rho_{p} / L_{p}}{\rho_{p} / L_{p}+\rho_{s} / L_{s}}$

where $l$ and $w$ are the length and width of the bulk contact trace. Therefore, an approximation of the additional resistance added by the transition from the plated region to the sputtered region $R_{a c}$ is

$$
R_{a c}=R_{s \%} R_{a}
$$

Therefore, the contact resistance for the plated films $R_{c p}$ is

$$
R_{c p}=R_{m}-R_{a c}
$$

where $R_{m}$ is the resistance measured in the Kelvin structure. All of the results for the electroplated films have been corrected to remove the additional resistance added by the transition from from the plated region to the sputtered region.

\section{Electroplated Film Results}

Three thicknesses of plated Au films were tested. The results along with the values calculated by traditional Holm theory using Eq. (1) and Eq. (5) are shown in Fig. 10. The resistivity used to calculate the Holm predictions was the measured value of $2.18 \times 10^{-8} \Omega \mathrm{m}$. As with the sputtered films, contact resistance increased for thinner electroplated films. All three film thicknesses had contact resistances higher than those predicted by Holm theory. Once again, this increase in contact resistance with decrease in film thickness corresponds to the trend seen in [6]; however, the magnitudes of contact resistance are still lower than those seen in [6].

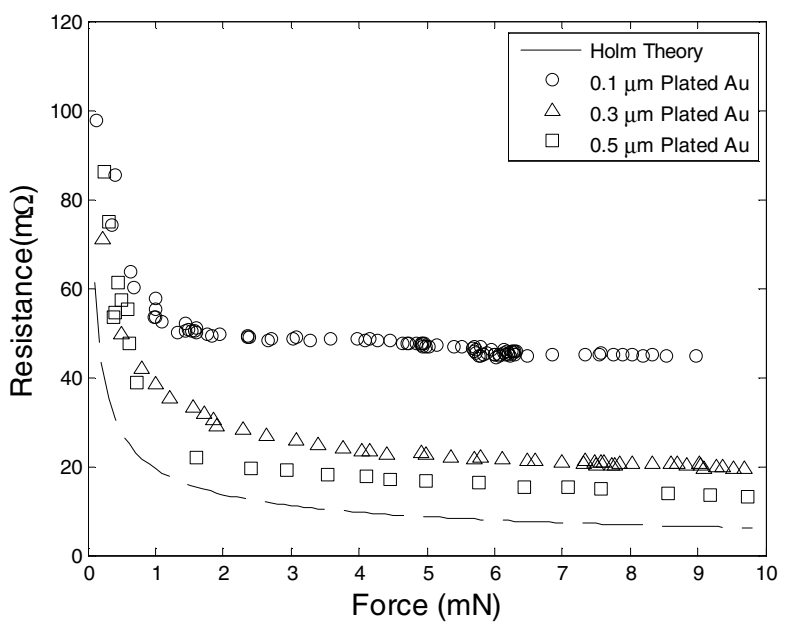

Figure 10. Contact resistance of plated thin $\mathrm{Au}$ films and the contact resistance predicted by Holm Theory.

The same results plotted as normalized contact resistance $R_{N}$ vs. $a / L$ are shown in Fig. 11. The $a$ values are calculated from Eq. (5). The hardness used to calculate $a$ was the approximated value of $1 \mathrm{GPa}$. 


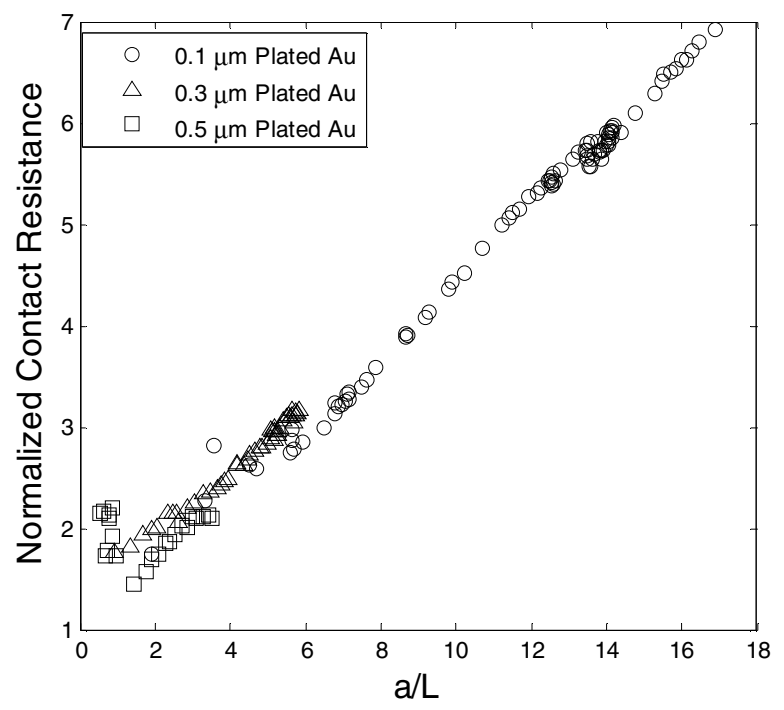

Figure 11. Normalized contact resisance of electroplated $\mathrm{Au}$ films and the normalized contact resistance predicted by (2).

This plot shows that normalized contact resistance tends to increase with $a / L$ for $a / L>1$. While the normalized contact resistance does not drop below 1 at low values of $a / L$, as it does in the sputtered films, it does exhibit a downward trend in the regoin $a / L<1$ similiary to how normalized contact resistance decreases with $a / L$ in the region $a / L<0.5$ in [4]. However, there is not enough experimental data in the range of $a / L<0.5$ to make a direct comparison to the theory presented in [4].

Finally, the relationships between normalized contact resistance and $a / L$ for both the sputtered films and the electroplated films were plotted together, which is shown in Fig. 12. In both cases, the trend in normalized contact resistance shifts around $a / L=1$. This may be because at this point, there is minimal spreading and constricting of the current flow lines.

\section{SUMMARY}

A two coupon system was used to measure the effect of the ratio between contact area and film thickness on contact resistance. The contact resistances between sputtered $\mathrm{Au}$ films as well as between electroplated $\mathrm{Au}$ films were measured. The resulting contact resistances were higher than those predicted by Holm theory. Their normalized contact resistances were compared to the ratio between the Holm radius and the film thickness, $a / L$. In high ranges of force, contact resistance increased with decreasing film thickness. The normalized contact resistance proved to be dependant on $a / L$. For high values of $a / L$, contact resistance increases with $a / L$, similarly to the trend seen in [6]. For low values of $a / L$ the contact resistance decreased with $a / L$, similar to the trend seen in [4]; however, there is insufficient data to make far reaching conclusions about this portion of the data. It is clear

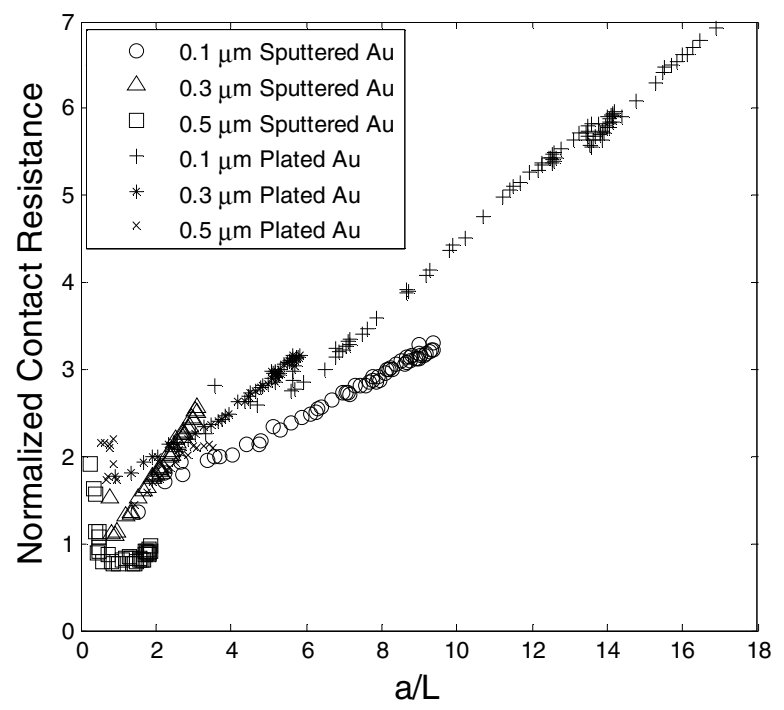

Figure 12. Normalized contact resisance of both the sputtered and electroplated films as a function of $a / L$.

that this relationship has two distinct regions. In the first region where $a / L<1$, normalized contact resistance decreases with increasing $a / L$. This is where the Holm radius is less than the film thickness. In the second region where $a / L>1$, normalized contact resistance increases with increasing $a / L$. This is where the Holm radius is greater than the film thickness. The exact mechanism for this transition is not completely understood. Future work will include looking at the region where $a / L<0.5$ with a finer force gauge to see how closely these results match those seen in [4] as well as looking closely at the region near $a / L=1$ to investigate the transition from the area where Holm radius is less than film thickness to the area where Holm radius is greater than film thickness.

\section{REFERENCES}

[1] A. Weber, J. Lang, and A Slocum, " $\{111\}$ Si etched planar electrical contacts for power MEMS-relays," Proceedings of the 53rd IEEE Holm Conference on Electrical Contacts, 2007, pp. 156-159.

[2] R. Holm, Electrical Contacts: Theory and Application, New York:Springer-Verlag 1967.

[3] R. Timsit, "Electrical conduction through small contact spots", IEEE Transactions on Components and Packaging Technologies, Vol. 26, No. 4, 2006, pp. 727-734.

[4] R. Timsit, "Constriction resistance of thin-film contacts," Electrical Contacts, Processdings of the $54^{\text {th }}$ IEEE Holm Conference on, pp. 332336, Oct. 2008..

[5] M. Read, A. Weber, O. Yaglioglu, R. Martens, J. Lang and A. Slocum, "A two-coupon system for the repeatable measurement of flat on flat micsroscale contact resistance," International Conference on Electrical Contacts, Proceedings of, Jun. 2008

[6] G. Norberg, S. Dejanovic, H. Hesselbom, "Contact resistance of thin metal film contacts," Components and Packaging Technologies, IEEE Transactions on, Vol. 29, No. 2, 2006, pp. 371-378.

[7] A. Slocum, "Degin of three-groove kinematic couplings," Precision Engineering, Vol. 14, No. 2, 1992, pp. 67-76. 
[8] D. Jang and D. Kim, "Tribological behavior of ultra-thin soft metallic deposits on hard substrates," Wear, Vol. 196, No. 1-2, 1996, pp. 171179.

[9] Y. Okinaka and M. Hoshino, "Some recent topics in gold plating for electronics applications," Gold Bulletin, Vol. 31, No. 1, 1998, pp 3-13.

[10] S. Riedel, J. Roeber, and T. Gessner, "Electrical properties of copper films produced by MOCVD," Microelectronic Engineering, Vol. 33, No. 1-4, 1997, pp 165-172.

[11] M. Gross, D. Altpeter, T. Stieglitz, M Schuettler and J. Meyer, "Micromachining of flexible neural implants with low-ohmic wire traces using electroplating," Sensors and Actuators, Vol. 96, No. 2-3, 2002, pp. 105-110.

[12] P. Slade, Electrical Contacts, Principles and Applications, New York: Marcel Dekker 1999. 


\title{
The Performance Implications of Silver as a Contact Finish in Traditionally Gold Finished Contact Applications
}

\author{
Marjorie Myers \\ Interconnection and Process Technology \\ Tyco Electronics \\ Harrisburg, USA
}

\begin{abstract}
Recent increases in gold metal prices have renewed interest in finding lower cost connector finishes. Silver is perceived as a possible alternative due to its 'near noble' nature, superior conductivity characteristics, and relatively low cost. The two finish types have very different performance characteristics and are typically not used in the same kinds of applications. Because silver is not 'as noble' as gold, there are some inherent risks for applying silver in typical gold finished contact interface applications. This paper will present relevant data to illustrate the mechanical and electrical performance implications of using silver at the performance levels typically required for gold finished connector applications - to improve understanding of the performance trade-offs associated with applying silver in historically gold finished applications.
\end{abstract}

Keywords-silver, tarnish, connector, contact resistance, coefficient of friction, durability

\section{INTRODUCTION}

Silver has a long history of performing well in the tarnished state in higher normal force/lower durability power applications and similar signal applications. Most signal connectors require significantly lower normal force and higher durability. The fact that silver will tarnish in most connector environments and is not a durable finish may present a problem for silver used in these lower normal force/higher durability signal connector applications. Increases in the cost of gold metal has provided motivation and interest in expanding the use of silver as an alternative signal separable contact interface finish in applications typically considered appropriate for hard gold finishes. There are potential risks associated with applying and testing silver finished contacts in applications appropriate for hard gold.

\section{A. Silver Finishes}

Silver has a unique combination of material properties such as the highest thermal and electrical conductivity of any metal and a relatively low hardness. Theory and experience show how these aspects lead to relatively low electrical contact resistance values for mated clean silver surfaces [1 - 4]. This unique combination of properties also leads to superior joule heating thermal performance. These performance attributes make it attractive for power applications.
The material characteristics that make silver contact finishes function well electrically also contribute to its inherently poor mechanical contact performance. Clean silver's high coefficient of friction characteristics can lead to relatively high insertion forces and relatively poor durability performance.

Silver also does not have the 'noble' character of gold. It will readily form surface tarnish films when exposed to some reducible sulfur bearing connector application atmospheres; as well as less common chloride bearing connector environments. Silver tarnish films can appear to be anywhere from tan, to more commonly blue, to black in more severe cases. Typical connector environment field exposure studies of silver plated connectors have shown that the tarnish films that form are predominantly covalently bonded semi-conducting $\alpha$ silver sulfide $\left(\mathrm{Ag}_{2} \mathrm{~S}\right)$ and to a lesser extent, small amounts of more insulating and potentially harder to displace silver chloride $(\mathrm{AgCl})[5-13]$. These field generated tarnish films were found to be semi-conductive at ambient temperatures, inherently soft, and relatively easily displaced with contact interface wipe at sufficient normal loads.

In addition to silver plated finishes being susceptible to tarnishing, they are also susceptible to the same substrate material corrosion migration as hard gold plated finishes. If excessive substrate material corrosion products (e.g. substrate diffusion and/or pore site migration) are incorporated into the silver tarnish film, contact resistance issues may occur $[14,15]$.

Environmental shielding can be an effective way to reduce or prevent silver tarnish films from forming. Examples of environmental shielding methods are the use of connector housings (open, closed, or actively sealed), equipment enclosures, environmental controls, greases or gels, reduced sulfur and/or sulfur absorbing packing material. They can all serve to limit the flow of corrosive elements to the silver contact surfaces. Surface treatments can also be used to improve the environmental resistance as well as improve the durability performance of silver finished contacts when appropriate. For a surface treatment to be effective, it has to perform without losing functionality or causing an unacceptable increase in contact resistance. They can be rendered ineffective if removed during assembly and use, or exposed to conditions outside of their proper operating range. 
The combination of these silver qualities leads to a set of conservative design recommendations for silver finished separable connector contacts. These would include the use of relatively high normal loads on the order of at least $250 \mathrm{cN}$, incorporation of wipe, and limitation of durability requirements to 10 or fewer wear cycles. Relative to hard gold applications, these higher force/wipe levels further limit the durability of silver finishes and contribute to the high insertion forces found with clean silver contacts. Silver is usually inappropriate for high durability applications. Finish recommendations would be to use at least 2 micrometers of silver along with a minimum 1.25 micrometer thick nickel underplate. Silver is commonly used without a nickel underplate; though with significantly thicker silver plating. Additionally, the use of some level of environmental shielding may be warranted.

\section{B. Environmental Testing: Mixed Flowing Gas Environment Exposure and Silver Finished Contact Surfaces}

Accelerated Environmental testing is done by exposing contact surfaces to elevated levels of single or multiple corrosive gases under specific temperature and humidity conditions. This type of exposure is usually done as part of a product qualification test sequence for specified time intervals.

Mixed Flowing Gas (MFG) accelerated environmental life test methods were developed after a long process of comparing laboratory to field trial data for a variety of connector finishes. In the end, no industry accepted accelerated MFG laboratory vs. field life correlations could be developed for silver. These MFG environments were determined to be excessive generating films with a variety of morphologies, chemistries, and properties which are not necessarily directly representative of field exposure generated films. This is attributed to factors such as the complexity of the silver corrosion process, the synergy between multiple gases, and the fact that silver is so sensitive to erratic fluctuations in hydrogen sulfide gas levels [5 - 9, 12, 16, 17]. MFG testing was ultimately developed primarily for gold finished nickel plated copper alloy substrates $[18,19]$. Alternate single gas testing environments (e.g. hydrogen sulfide, flowers of sulfur) were also determined to be misleadingly benign and also not representative of silver finished contact field exposures $[5,6,7,9]$.

The tarnishing of silver surfaces is driven by the presence of moisture, $\mathrm{H}_{2} \mathrm{~S}$ and $\mathrm{Cl}_{2}$ in these accelerated environments. The presence of moisture is needed for silver to tarnish in response to corrosive environments. This moisture allows for the dissolution of corrosive elements leading to dissolution of metallic silver. Surface water films can either form as monolayers generated by humidity, or in a condensed form [6, 8]. Silver tarnishing has been reported as both positively dependant on $[8,20]$, and independent of $[10,11]$, increasing relative humidity levels depending on the exposure environment. Silver sulfide $\left(\mathrm{Ag}_{2} \mathrm{~S}\right)$ forms when silver atoms react with reduced sulfur (HS-). The primary source of this reduced sulfur is dissolved gaseous hydrogen sulfide $\left(\mathrm{H}_{2} \mathrm{~S}\right)$, or to a lesser extent (less prevalent in the atmosphere) hydrolyzed carbonyl sulfide (COS) [7, 8, 16, 17]. Hydrogen sulfide comes from sources such as organic decay, combustion processes, volcanic activity, paper mills, sewage plants, and high sulfur packaging materials.
Silver is sensitive to the presence of chloride (Cl-) and will react to form silver chloride $(\mathrm{AgCl})[6,8,10,16]$. Chloride can come from species such as dissolved hydrochloric acid $(\mathrm{HCl})$ or other chloride containing particulates (e.g. $\mathrm{NaCl}$ ). Reports have shown that the higher the level of silver chloride in the tarnish film (relative to the level of softer semi-conductive silver sulfide), the more insulating and harder to displace the film becomes upon wipe $[6,7,9,10,12]$. This can potentially lead to contact resistance issues at thinner tarnish film levels.

Silver may not react directly with chlorine gas $\left(\mathrm{Cl}_{2}\right)$, but its presence is considered to have a synergistic effect on silver tarnish formation when combined with hydrogen sulfide gas. This is especially evident on silver surfaces exposed to accelerating environments containing both hydrogen sulfide and chlorine gas. Exposure of silver surfaces to MFG or single gas accelerated laboratory environments will generally lead to a greater rate of tarnish film formation with sometimes a greater level of silver chloride than is found on most field tarnished samples $[6,7,9,10,12,16]$. If the ratio of hydrogen sulfide to chlorine is great enough, the film growth rate has been reported to deviate from linear and approach parabolic.

Silver tarnish films don't develop with a uniform morphology and can be mixed with substrate corrosion products. This can make growth rate determination difficult and potentially misleading. The reality is that chlorine gas, commonly used in mixed flowing gas testing, is virtually nonexistent in the atmosphere. The $\mathrm{H}_{2} \mathrm{~S} / \mathrm{Cl}_{2}$ synergy may account for silver chloride level and contact resistance performance discrepancies between field and accelerated environment exposed silver surfaces.

\section{SAMPLES AND EQUIPMENT/TESTING PROCEDURES}

This testing was done to gain a better understanding of where and when silver can be applied in connector applications usually reserved for hard gold finishes. There are both electrical and mechanical performance risks, as well as potential issues with testing silver using methods deemed appropriate for gold applications.

\section{A. Silver Finished Samples}

Samples with silver plating thickness near the minimum of what is typically used in silver finished connectors with a nickel underplate ( $\sim 2$ micrometers) were used for this testing. Hemispherical cap (nominal radius $2.5 \mathrm{~mm}$ ) rider and flat coupon sample geometry combinations were used representing a less severe relatively smooth contact surface combination. All the samples were made from 0.4 mm thick H04 temper C51100 phosphor bronze $(\mathrm{CuSn} 4)$ rolled strip. These samples were made using standard connector industry high speed reel-to-reel production processes. Plating thickness measurements were done using X-ray Fluorescence (XRF) techniques. Table I lists the relevant sample parameters. The silver samples had been stored in a lab atmosphere for approximately one year before this testing was done and had a minor amount of tarnish on the surface at a level not quantifiable by X-ray Diffraction (XRD) techniques. 
TABLE I. TOPOGRAPHY ROUGHNESS PARAMETERS

\begin{tabular}{|c|c|c|c|}
\hline & \multicolumn{2}{|c|}{$\begin{array}{c}\text { Silver } \\
\text { Plating thicknesses } \\
\text { (micrometers) }\end{array}$} \\
\hline & $\begin{array}{c}\text { RMS Roughness } \\
\text { (micrometers) }\end{array}$ & $\mathrm{Ag}$ & Ni underplate \\
\hline cap & 0.25 & 2.39 & 1.63 \\
\hline flat & 0.47 & 2.38 & 1.60 \\
\hline \multicolumn{2}{|c|}{ Surface Condition } & \multicolumn{2}{|c|}{$\begin{array}{c}\text { 'as stored } \sim 1 \text { year' } \\
\text { (slight tarnishing) }\end{array}$} \\
\hline
\end{tabular}

\section{B. Test Environments}

Even though there is no established correlation between field exposure and laboratory Mixed Flowing Gas (MFG) exposures (film quality or functionality), these exposures will repeatably generate a significant level of silver sulfide and/or silver chloride containing tarnish films on silver finished contact surfaces. There are several versions of MFG in use for gold plated contacts, but the most common are the EIA 364-65 [19] four gas Class IIa (Indoor) and Class IIIa (Outdoor) (table II).

TABLE II. STANDARD MIXED FlOWING GAS ENVIRONMENTS EIA 364-65 (Electrical Connector/Socket Test Procedures Including Environmental Classifications) [19]

\begin{tabular}{|l|l|l|}
\hline & CIIa (Indoor) & CIIIa (Outdoor) \\
\hline $\mathbf{H}_{2} \mathbf{S}(\mathrm{ppb})$ & $\mathbf{1 0} \pm 5$ & $\mathbf{1 0 0} \pm 20$ \\
\hline $\mathbf{C l}_{2}(\mathrm{ppb})$ & $\mathbf{1 0} \pm 3$ & $\mathbf{2 0} \pm 5$ \\
\hline $\mathrm{SO}_{2}(\mathrm{ppb})$ & $100 \pm 20$ & $200 \pm 50$ \\
\hline $\mathrm{NO}_{2}(\mathrm{ppb})$ & $200 \pm 50$ & $200 \pm 50$ \\
\hline Temperature $\left({ }^{\circ} \mathrm{C}\right)$ & $30 \pm 1$ & $30 \pm 1$ \\
\hline Humidity $(\% \mathrm{RH})$ & $\mathbf{7 0} \pm 2$ & $\mathbf{7 0} \pm 2$ \\
\hline
\end{tabular}

Historically, the MFG environments used to generate silver tarnish involved a great variety of gas/humidity levels and combinations with varying results $[5,6,7,9-13,16,18]$. These MFG environments were different in one way or another than the current CIIa (Indoor) and CIIIa (Outdoor) environments. Since CIIa and CIIIa are routinely used to test hard gold plated contact surfaces, if silver is applied to such contacts, silver plated surfaces may be exposed to such testing.

Hard gold over nickel finished surfaces exposed to CIIa and CIIIA generate the same substrate material corrosion products at pore sites. Since the $\mathrm{H}_{2} \mathrm{~S}$ level of the CIIIa environment is 10 times that of the CIIa environment, the films that formed on silver finished surfaces should differ in quality due to the synergistic effect between the levels of $\mathrm{H}_{2} \mathrm{~S}$ and $\mathrm{Cl}_{2}$ described in the literature.

\section{Contact Resistance Probe (CRP)}

4-wire dry circuit contact resistance measurements were taken at variable loads, followed by wipe at load, using a computer controlled Contact Resistance Probe (CRP). Each individual contact resistance measurement is an average of a series of readings made under both forward and reverse current DC steady-state 4-wire dry circuit $(50 \mathrm{~mA} / 50 \mathrm{mV}$ limited) conditions. The maximum contact resistance the instrument is capable of reading under these dry circuit conditions is $1 \mathrm{k} \Omega$. All measurements are taken under lab atmosphere conditions.

The coupon of a mating half is mounted to the $\mathrm{X} / \mathrm{Y}$ table with an incorporated load cell. The current-out and voltage-out leads are attached to opposing sides of the mounted flat coupon. The hemispherical cap is mounted to the tip of the probe head which can move in the vertical $\mathrm{z}$ direction. The current-in connection is made at the outer rim of the cap and the voltage-in lead makes contact at the bottom of the back of the 'cup' of the cap. Further information and images of this instrument can be found in reference 4 .

\section{Friction Measurement Instrument}

For friction testing, the hemispherical rider and flat are mounted on the friction apparatus. The hemispherical cap is dead weight loaded against the flat which is horizontally moved with reciprocating simple harmonic motion. The reciprocating motion is produced using a micrometer adjustable eccentric cam driven DC motor. The reciprocating table holds a load cell attached to a precision slide which is used to make the lateral force measurements from which the coefficient of friction measurements are calculated. The sliding distance was set at $1 \mathrm{~cm}$. Images and more detailed information on this instrument can be found in reference 21 .

\section{E. Testing Conditions}

Multiple CIIa and CIIIa MFG exposures were used to generate samples for testing. Data is reported here for exposures done at 1 and 5 days where the samples were fully exposed with no form of shielding. Exposures of 10 and 20 days proved to be excessive for making dry circuit readings. As a result, a comparison of 1 and 5 days exposure data is reported in this study. Data reported here was drawn from multiple testing projects carried out over a period of time.

The resulting tarnish films were characterized by X-ray Diffraction (XRD) using a $\mathrm{Cu}-\mathrm{K} \alpha$ radiation source and a low incident grazing angle to improve the ability to resolve thin surface films.

The functional contact testing included both electrical contact resistance (CR) and mechanical Coefficient of Friction (COF) testing. The contact resistance and COF testing was done at both minimum silver normal loads $(250 \mathrm{cN}$ and 200 $\mathrm{cN})$ and at a normal load appropriate for hard gold $(50 \mathrm{cN})$. Dry circuit $(50 \mathrm{~mA} / 50 \mathrm{mV})$ contact resistance readings were taken at load, both before and 'after 'wipe' at load.

Additional testing was done on silver finished surfaces that had surface treatments applied prior to exposure. One capable of providing both environmental exposure resistance and lubricity (HM15 lubricant - a proprietary Tyco Electronics formulation) and one primarily designed to provide environmental exposure resistance (Evabrite $S$ ( Both were solvent based and applied via 'dip and dry' techniques. 


\section{RESULTS AND DISCUSSION}

A. Tarnish Film Characterisitics (CIIa vs. CIIIa vs.

\section{Field Exposure)}

There were two main differences between the 'less severe' CIIa (Indoor) and the 'more severe' CIIIa (Outdoor) class MFG environments pertaining to silver tarnishing: gas levels and the ratios. The two gases contained in these MFG's that most effect the level of silver tarnish are $\mathrm{H}_{2} \mathrm{~S}$ and $\mathrm{Cl}_{2}$. CIIIa has 10 times the amount of $\mathrm{H}_{2} \mathrm{~S}$ and twice the level of $\mathrm{Cl}_{2}$. Therefore, the less severe CIIa (Indoor) actually has a five times greater $\mathrm{Cl}_{2} / \mathrm{H}_{2} \mathrm{~S}$ ratio than the more severe CIIIa (Outdoor). This leads to the generation of two different tarnish films. The greater ratio of $\mathrm{Cl}_{2} / \mathrm{H}_{2} \mathrm{~S}$ in the $\mathrm{CII}$ environment generated a predominantly $\mathrm{AgCl}$ tarnish film where the lower ratio $\mathrm{Cl}_{2} / \mathrm{H}_{2} \mathrm{~S}$ in the CIIIa led to a predominantly $\mathrm{Ag}_{2} \mathrm{~S}$ tarnish film. XRD data in Figures 1 and 2 show the difference in tarnish film quality generated by CIIa and CIIIa exposures. These differences can also be seen in the light microscope images of the samples in figure 3, though there is some differences in describing what the 'eye can see' and what a light microscope will image. The 1 day CIIIa exposed silver have a distinct blue coloration where the original silver color cannot be seen. The 1 day CIIa exposed silver surfaces have a brown appearance where the visual characteristics of the metallic silver finish can still be seen. Both CIIa and CIIIa 5 day exposure samples have a more black appearance 'by eye.' As reported in the literature, field exposed silver surfaces form predominantly $\mathrm{Ag}_{2} \mathrm{~S}$ films. The predominantly $\mathrm{Ag}_{2} \mathrm{~S}$ nature and blue color of a representative field exposed sample (approximately 3 years in an unprotected storage environment) can be seen in both the XRD analysis and light microgaph in figure 4. Therefore, the CIIIa exposure may be more representative of field exposures in most cases, though there is no lifetime performance correlation to the test.

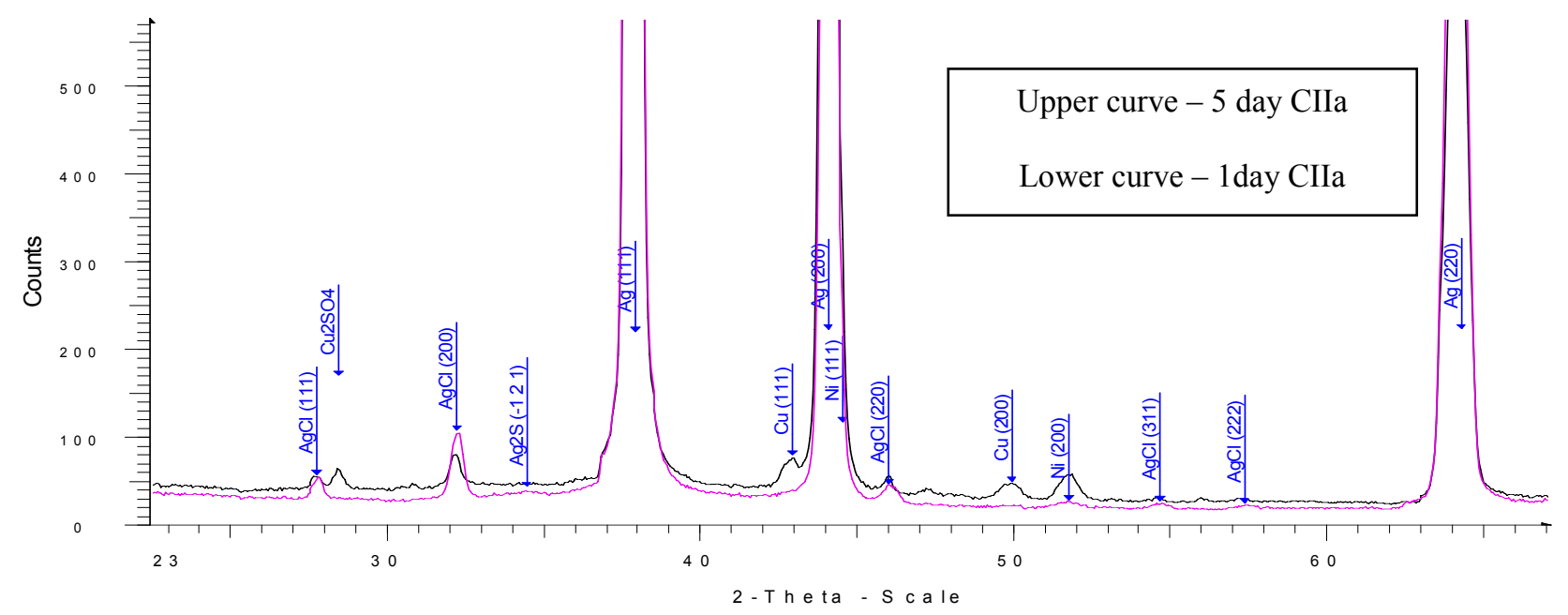

Figure 1. XRD spectrum taken from CIIa exposed silver plated surfaces (1 and 5 days)

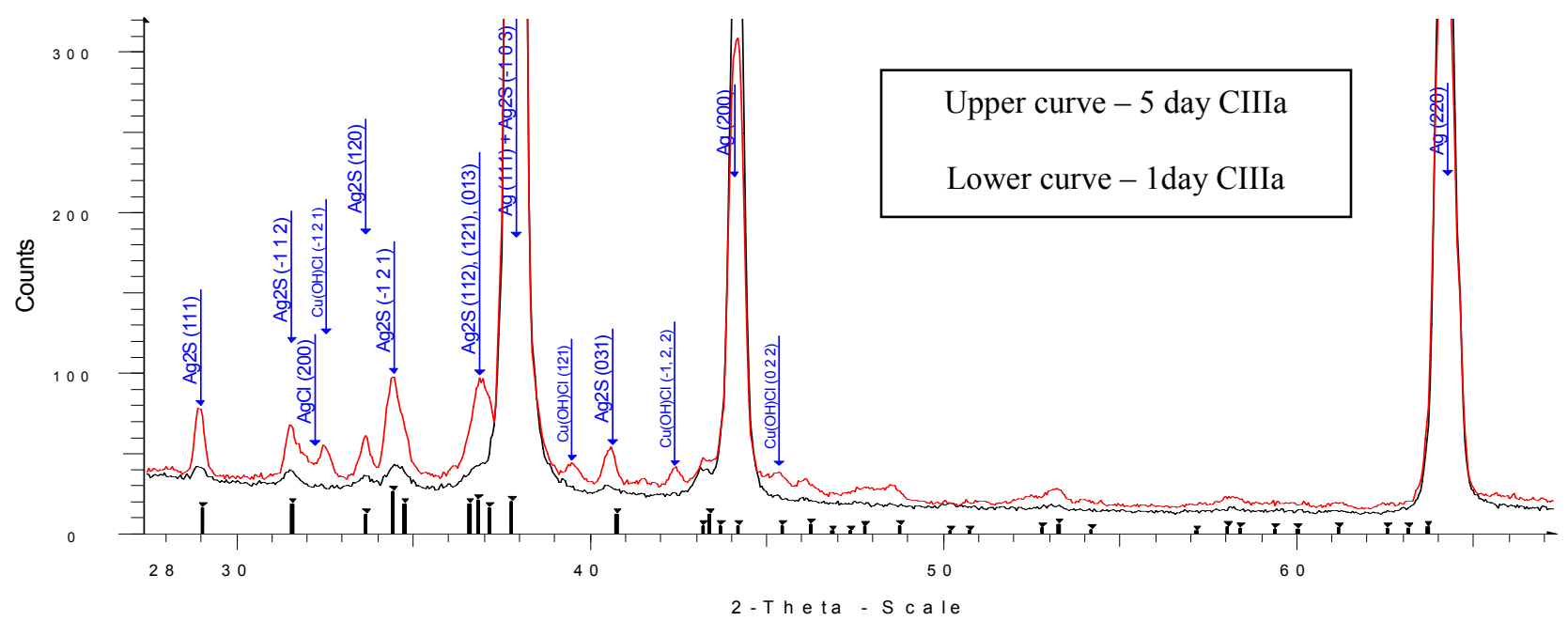

Figure 2. XRD spectrum taken from CIIIa exposed silver plated surfaces (1 and 5 days) $-\boldsymbol{\nabla}$ indicate $\mathrm{Ag}_{2} \mathrm{~S}$ peak locations 


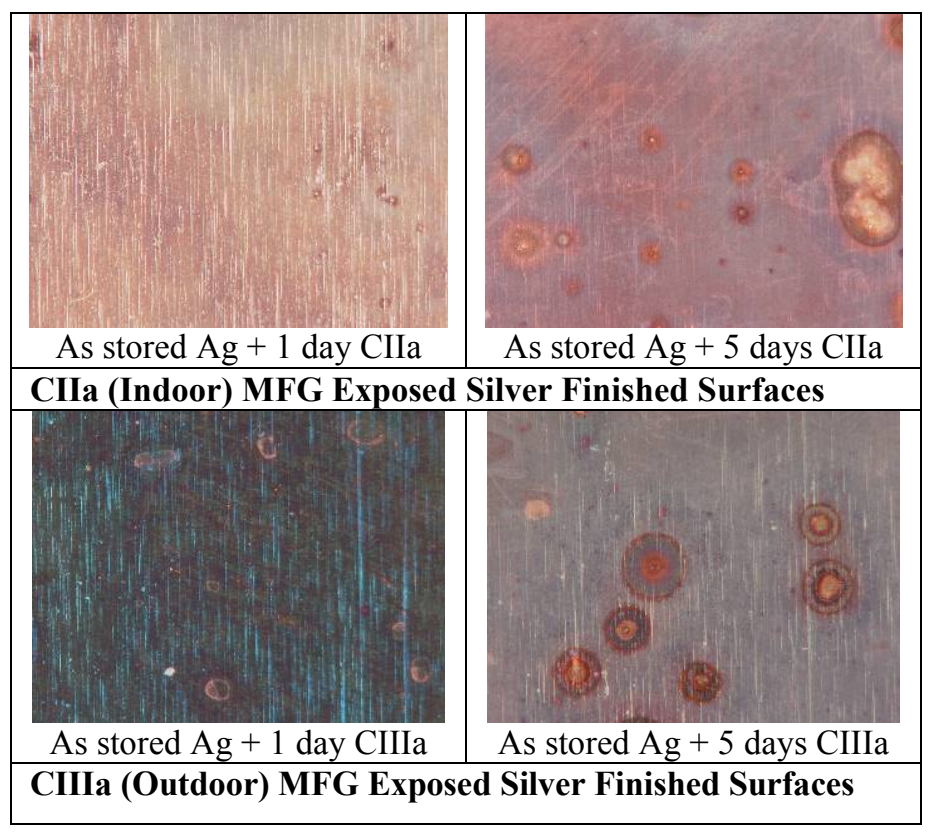

Figure 3. Optical micrographs of CIIa and CIIIa (Outdoor) MFG exposed silver finished surfaces taken at (100x)

\section{B. Contact Resistance Data Taken at 250cN Normal} Load for Tarnished Silver Finished Surfaces

Figure 5 shows contact resistance data from these silver finished samples measured at $250 \mathrm{cN}$ load (open symbol data points without center cross) along with data taken after 500 micrometers forward and back wipe (solid symbol data points with center cross). The contact resistance results for each state are represented by two data points: an average value plus one standard deviation (solid symbols) and a maximum value (open symbols). Each data point set represents at least 18 individual measurements (figures 5, 8, and 12). The sample conditions tested were pre-MFG exposed states (as-plated and as-stored) as well as after 1 day and 5 days of both CIIa and CIIIa exposure. The contact resistance axis is on a log scale.

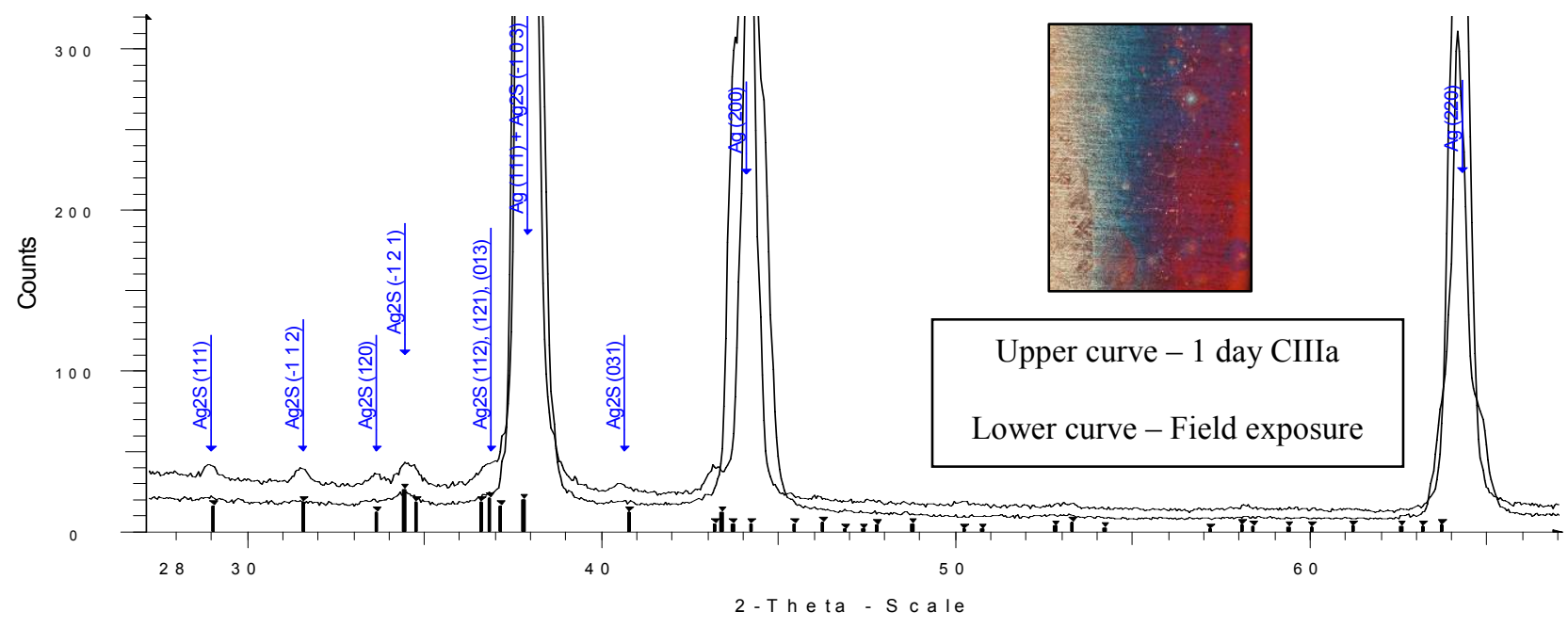

Figure 4. XRD spectrum taken from a field exposed silver plated surface (inset image of the surface) $-\boldsymbol{\nabla}$ indicate $\mathrm{Ag}_{2} \mathrm{~S}$ peak locations

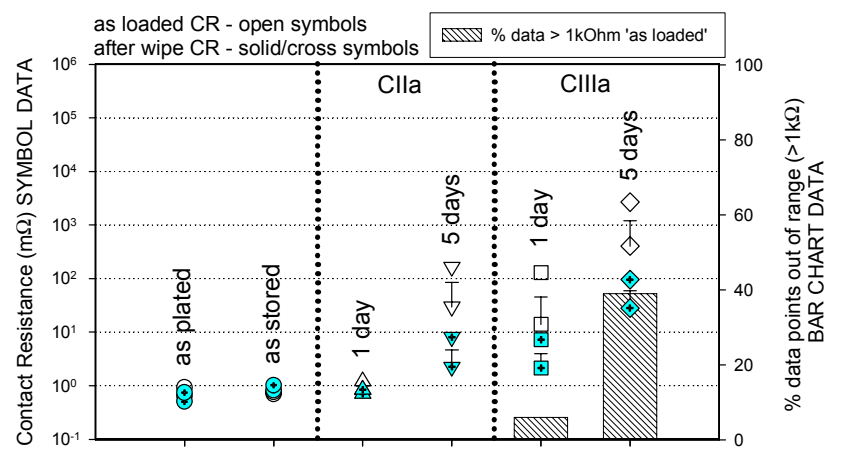

Figure 5. Contact resistance data taken at $250 \mathrm{cN}$ load in the 'as loaded' and the 'after wipe'at load state for various exposure states
Since the CRP does not resolve data greater than $1 \mathrm{k} \Omega$, the additional bar graph data is necessary to show what percentage of the data (right axis of the graph) is $1 \mathrm{k} \Omega$ or greater. The bars represent data from the 'as loaded' or the 'after wipe' states as designated on the graphs. These 'open circuit' readings $(\mathrm{R} \geq$ $1 \mathrm{k} \Omega$ ) could not be included in the average +1 standard deviation and maximum values reported. Therefore, the 'out of range' reading information needs to be included even though the resistance values were too high for the CRP to report an actual value.

When comparing the as-plated and the as-stored silver finished contact resistance data, the effect of any residual tarnish that formed during lab storage is neglible. All subsequent MFG exposures were done on as-stored silver finished samples. 
For many signal applications there is a limit of a change (delta) in contact resistance of $10 \mathrm{~m} \Omega$. With that metric, only the as-plated, as-stored, and 1 day CIIa MFG exposed samples 'passed' in the 'as loaded' state before wipe (figure 5). In the 'after wipe' state, only the 5 days CIIIa exposure, a relatively severe exposure, did not 'pass' this arbitrary $10 \mathrm{~m} \Omega$ delta contact resistance metric. Of all the sample conditions in the 'after wipe' group that 'passed', the highest values were an average of $1.40 \mathrm{~m} \Omega$, standard deviation of $1.76 \mathrm{~m} \Omega$, and a maximum of $7.9 \mathrm{~m} \Omega$ for the 1 day CIIIa exposed samples. This shows the benefit that wipe can have for a tarnished silver contact surface.

\section{C. $\quad$ Wipe and tarnished silver surfaces}

Wipe is a very effective way to remove silver tarnish from a contact interface (figures 6 and 7). Looking at the wipe marks left by the CRP, there were two varieties: where there was no pore site substrate corrosion and where there was pore site substrate corrosion. Figure 6 shows micrographs of such wipe marks from the 5 day CIIIa contact resistance samples $\left(\mathrm{H}_{2} \mathrm{~S}\right.$ films). The $\geq 1 \mathrm{k} \Omega$ readings represented by the 'after wipe' bar graph data in figure 8 were taken from sites as shown in figure 6 b) where the level of copper corrosion product (EDX analysis) within the film around a corrosion pore sites was too great to make good electrical contact; even after wipe.

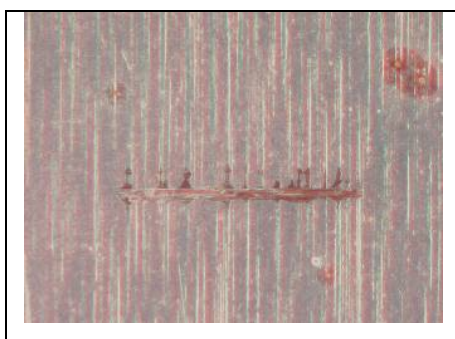

a) no pore site (CR measured)

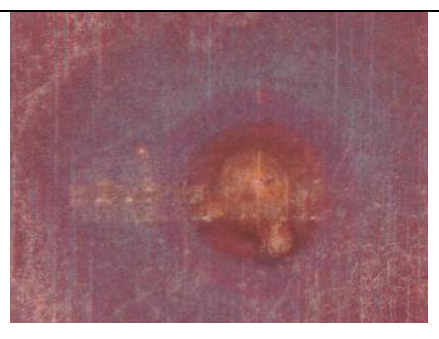

b)pore site $(\geq 1 \mathrm{k} \Omega \mathrm{CR})$
Figure 6. Representative contact resitance measurement wipe tracks (500 micrometers long) from a 5 day CIIIa exposed Ag finished surface $50 \mathrm{cN}$ load

Some limited testing was done where the spot on the cap rider used to make the readings was not changed between readings while the location on the flat coupon for each successive measurement was moved to a new location. This way, the cap surface experienced an increasing number of wipe cycles with each wipe cycle. Therefore, each successive flat surface measurement location had an undisturbed tarnish film prior to probing and wipe. Figure 7 shows two such flat wipe tracks taken using a 1 day CIIIa exposed silver finished cap rider and flat coupon pair: the $1^{\text {st }}$ flat wipe track (a) and the $9^{\text {th }}$ flat wipe track (b). The degree of silver metal exposure and track width on the flat increased with increasing number of wipe cycles. This illustrates both the value of wipe for tarnished silver samples as well as the potential increase in wear associated with increased wipe.

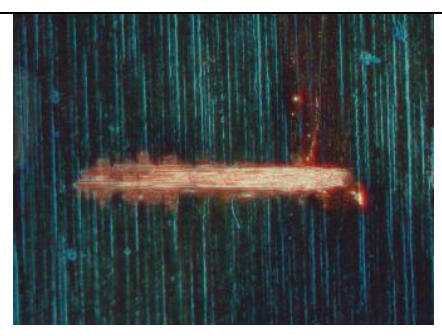

a) $1^{\text {st }}$ contact resistance wipe track - flat coupon surface

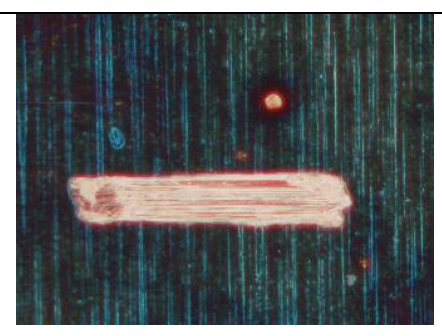

b) $9^{\text {th }}$ contact resistance wipe track - flat coupon surface
Figure 7. Representative contact resistance measurement forward and back wipe tracks (500 micrometers long)@250 cN load using a 1 day CIIIa exposed Ag finished surface. The cap rider spot was not changed between sucessive measurements while the flat coupon measurement location was changed betweeen readings

\section{Contact Resistance Data Taken at 50cN NormalLoad for Tarnished Silver Finished Surfaces}

If silver were to be applied in a typical hard gold finished application, the normal load may be more on the order of 50 $\mathrm{cN}$. The data in figure 8 is plotted in the same manner as in Figure 5; the difference being that a normal load of $50 \mathrm{cN}$ was used instead. Using the $10 \mathrm{~m} \Omega$ delta contact resistance metric, the risk of using such a low normal load for a silver finished surface is illustrated. Only the as-stored and the 1 day CIIa exposed samples were able to meet a $10 \mathrm{~m} \Omega$ delta contact resistance limit: with or without wipe. Even after wipe at 50 $\mathrm{cN}, 30 \%$ of the contact resistance readings taken on the 5 days CIIIa samples exceeded the $1 \mathrm{k} \Omega$ measurement capability of the instrument. If silver were to be used in a situation where this level of tarnish would occur, it is likely it would fail without some form of environmental shielding and possibly a more severe geometry. Any shielding used would have to be capable of preventing this level of corrosion in an application. More severe geometries may be better able to break through tarnish films; but could lead to further durability decreases for a finish that already has poor durability characteristics.

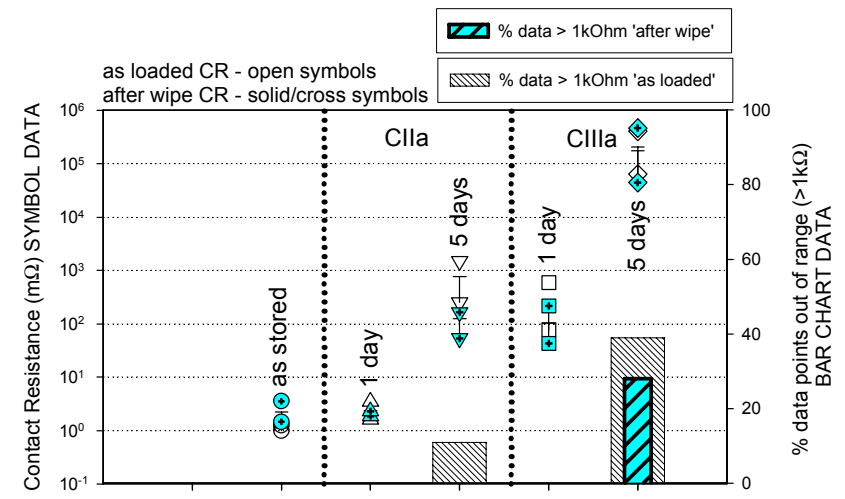

Figure 8. Contact resistance data taken at $50 \mathrm{cN}$ load in the 'as loaded' and the 'after wipe' at load state for various exposure states

\section{E. Coefficient of Friction/Durability Evaluation}

Clean hard gold has a coefficient of friction (COF) on the order of 0.3 to 0.4 . COF measurements were taken on silver 
and all data shown are the median and maximum value for a combination of at least three friction tests. The data in Figure 9 shows that as-stored silver has a significantly greater COF $(\sim$ 1.25 or greater) than hard gold. Hard gold contacts many times are specified for 50 durability cycles or greater. Silver finished contacts are usually specified for 10 or fewer durability cycles. The difference in COF illustrates the difference in the durability of the two finishes.

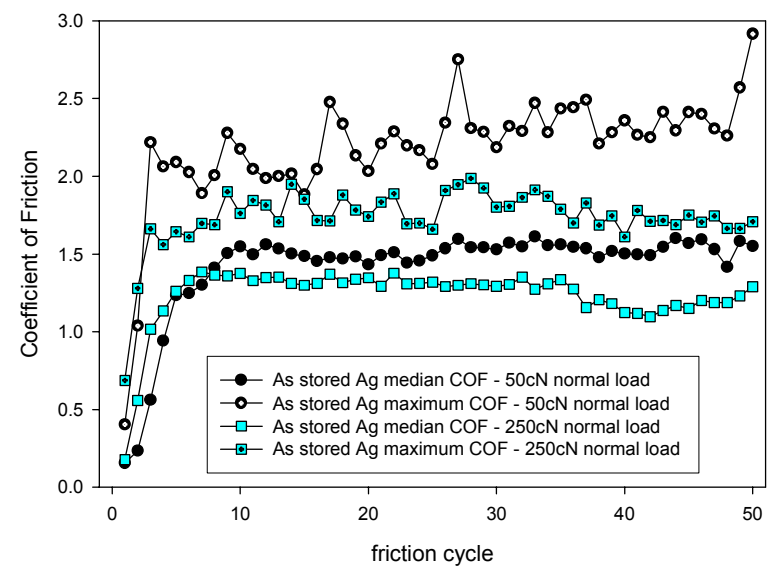

Figure 9. Median and Maximum Coefficient of Friction data for as-stored silver finished surfaces: data taken at $250 \mathrm{cN}, 1 \mathrm{~cm}$ wear track

The presence of even minor amounts of silver tarnish at the surface can weaken the adhesive metal-to-metal contact interface bonds by providing a shearable layer at the asperity junctions - leading to a lower COF. The minor amounts of storage tarnish found on these as stored silver surfaces reduces the COF for the initial cycles (1-4) only - even at the higher normal loads $(250 \mathrm{cN})$ more typically used with silver finished contacts. The COF values taken for the samples at $50 \mathrm{cN}$ (median and max) are higher than the ones taken at $250 \mathrm{cN}$. This has to do with the wear mechanism. The difference is evident when comparing the $250 \mathrm{cN}$ and $50 \mathrm{cN}$ (figure 10) cap rider wear marks. At the higher $250 \mathrm{cN}$ load, more of the harder nickel underplate is exposed which serves to make the sliding surface a composite structure of both the relatively soft silver and the relatively hard nickel - therefore the effective COF is lowered. This could lead to a greater risk of nickel corrosion in the contact area. This is why silver is not considered a durable finish. The wear marks for the testing done at $50 \mathrm{cN}$ to 50 cycles have no nickel exposure and are more representative of silver galling. This could lead to a risk of excessive insertion forces.

This combination of higher normal forces and silver's high coefficient of friction does have the benefit of promoting vibrational stability by promoting attenuation of the transfer of mechanically or thermally driven relative micro-motion (e.g. fretting) to a contact interface. Silver itself is not susceptible to fretting oxidation in typical connector environments but silver is susceptible to severe adhesive fretting motion wear. If mechanical cycling conditions are severe enough for fretting motion to occur, this could quickly lead to exposure of nickel and/or copper substrate materials which are susceptible to fretting oxidation failures and unacceptable contact resistance increases. How any fretting motion would or could be imparted to a contact interface is entirely dependant on the connector design and the application.

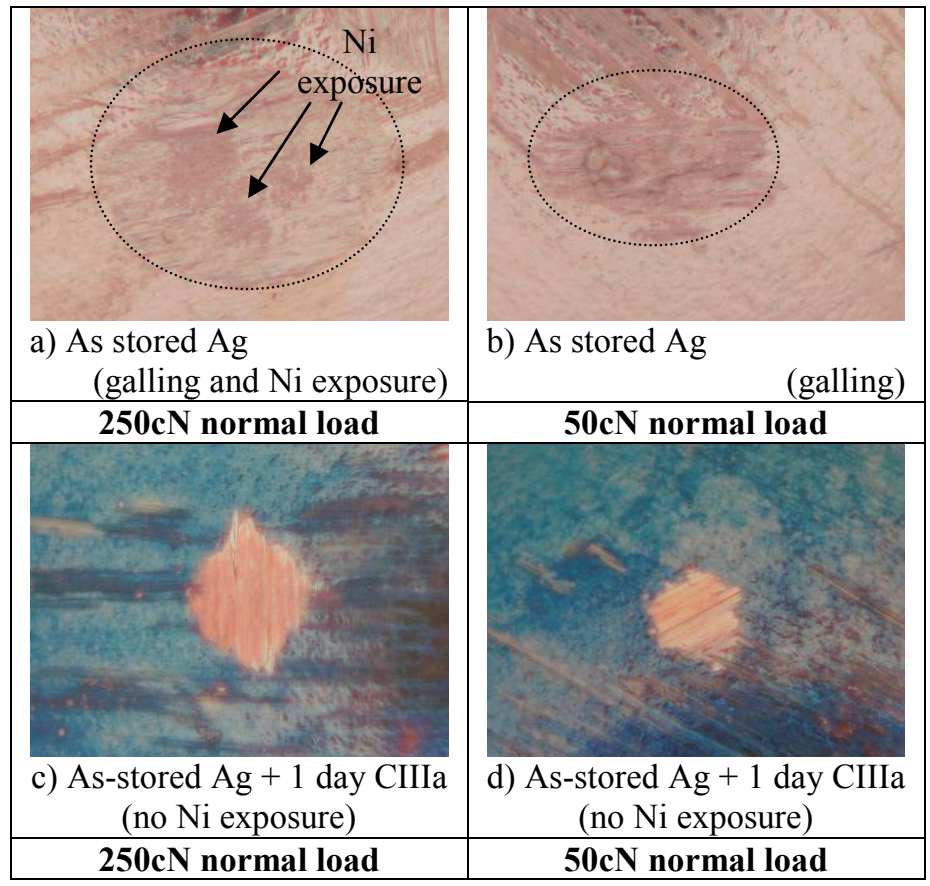

Figure 10. Representative optical micrographs of 50 cycle cap rider COF wear marks. One cm long wear track cycling (400x micrographs)

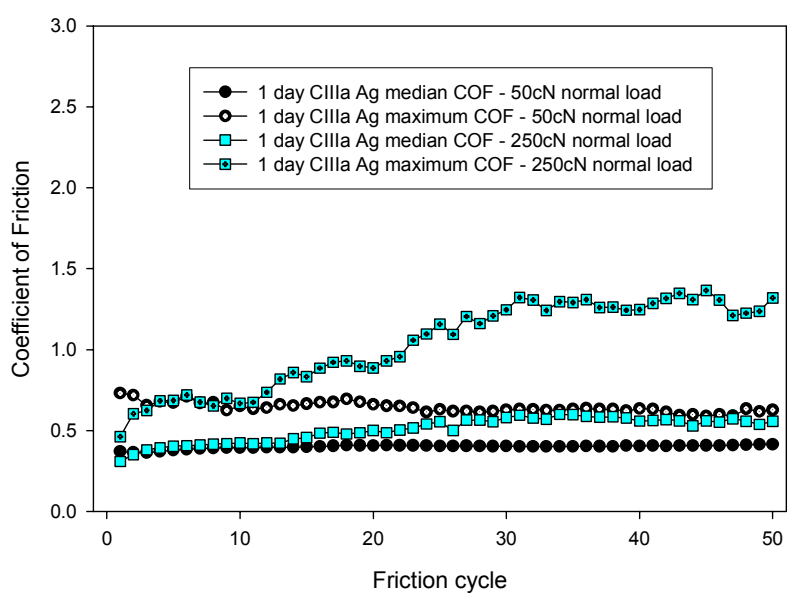

Figure 11. Median and Maximum Coefficient of Friction data for 1 day CIIIa exposed silver finished surfaces: data taken at $250 \mathrm{cN}$ and $50 \mathrm{cN}, 1 \mathrm{~cm}$ wear track

More extensive tarnish films can impart a significant level of COF reduction resulting in less wear. The difference in the cap rider wear area can be seen when comparing the as-stored and the 1 day CIIIa exposed wear marks shown in figure 10 . Figure 11 shows that 1 day CIIIa exposed tarnished silver surface can have a median COF less than 0.5. Even at 50 cycles where the maximum COF gets up to $\sim 1.25$, the reduction in COF imparted by the existence of the tarnish film can be seen when compared to the as-stored condition data shown in figure 9. Figure 11 also shows that at $250 \mathrm{cN}$ load, the COF reducing 
effect of this tarnish film starts to degrade after about 10 cycles of durability. The maximum COF steadily increases after about 10 cycles wear. At $50 \mathrm{cN}$ normal load, the tarnish film tends to stay effective even up to 50 cycles of durability. Field generated tarnish films tend to have a similar effect, but it would be as variable as the field tarnish films themselves.

\section{F. $\quad$ The Use of Surface Treatments on Silver Finished Surfaces}

Not only is silver susceptible to tarnishing itself, figures 3 and 6 shows that silver finished surfaces are also susceptible to the same pore site substrate corrosion migration as hard gold finishes when exposed to these types of MFG corrosive atmospheres. Many silver surface treatments are intended to prevent tarnishing of the silver itself. They also may need to mitigate corrosion at pore sites and/or impart lubricity to a contact interface like hard gold surface treatments.

Figure 12 shows comparative before and after CIIIa MFG exposure contact resistance data for treated silver finished surfaces, as well as after exposure data for silver with no surface treatment. Such treatments can be quite effective at attenuating silver tarnishing and/or pore site corrosion if used under appropriate conditions.

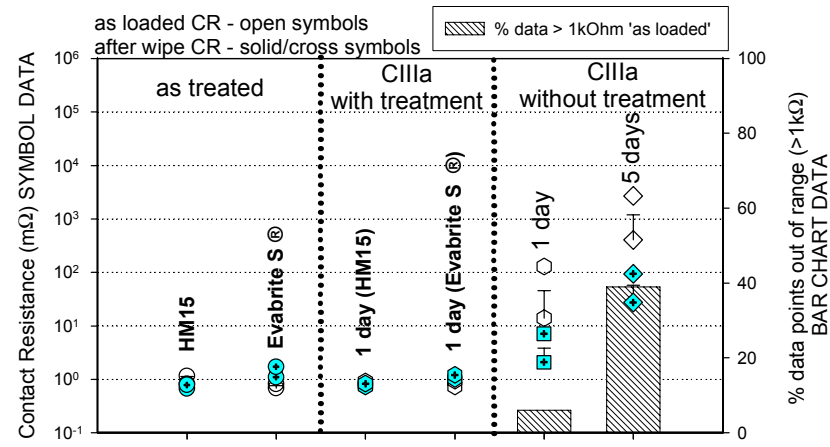

Figure 12. Contact resistance data taken at $250 \mathrm{cN}$ load in the 'as loaded' and the 'after wipe'at load state for various exposure and surface treatments

Figure 13 shows similar comparative COF data. Not only do surface treatments attenuate tarnish film formation, they also provide COF reduction. The presence of tarnish films can also have a significant COF reducing effect. Since tarnish film formation processes are not self limiting and are so unpredictable, they cannot necessarily be expected to consistently provide $\mathrm{COF}$ and/or wear reduction.

Figure 13 also shows the potential risks associated with using a surface treatment outside of its proper operating range. If the surface treatment is exposed to a temperature at which it is not stable, it may cease to function depending on the duration of the excessive thermal exposure. In this case, surface treated samples were exposed to $125^{\circ} \mathrm{C}$ for 100 hours. Both surface treatments provided some level of COF reduction as-applied, but the effect of the Evabrite $\mathrm{S}{ }^{\circledR}$ was eliminated when the surfaces were exposed to 100 hours at $125^{\circ} \mathrm{C}$ prior to friction testing.

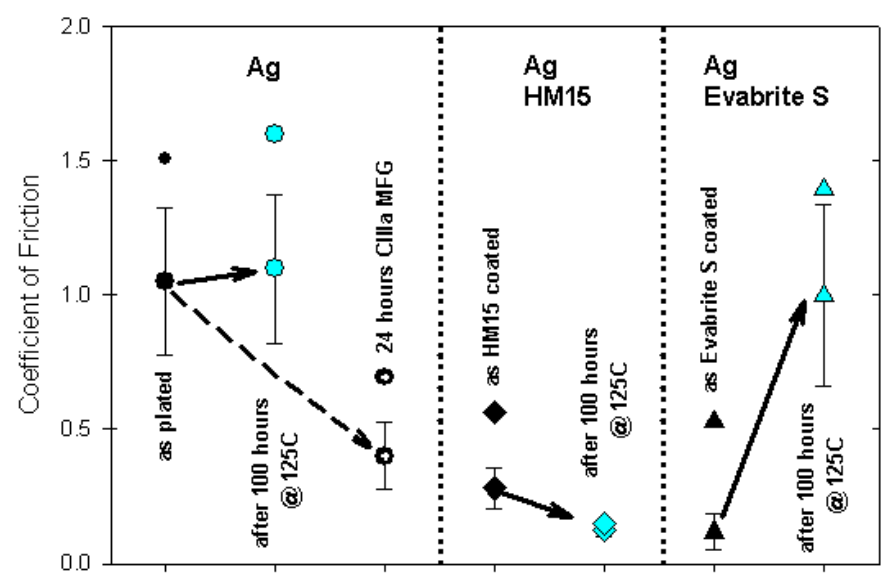

Figure 13. $5^{\text {th }}$ cycle Coefficient of Friction data taken at $200 \mathrm{cN}$ load with a 1 $\mathrm{cm}$ wear track length - median \pm 1 standard deviation and maximum COF values for silver finished surfaces with designated levels of treatment and/or exposure

\section{CONCLUSIONS}

What a connector designer needs to achieve is sufficient wear resistance, durability levels, insertion force, and stable contact resistance performance at the lowest cost possible for a given application. Applying silver in a hard gold finished connector design/application will most likely degrade these performance factors, though it may be a viable cost effective finish for some situations. Determining the 'appropriateness' of switching hard gold to silver would be very connector design, performance requirement, testing and application condition dependant.

- It is recommended to use an appropriate silver surface treatment. Surface treatments can be used to attenuate corrosion processes as well improve wear, durability, insertion force performance. Any selected surface treatment would have to be appropriate for the 'lifetime' exposure of the intended application.

- The use of environmental shielding is recommended for silver finished contacts. What level of shielding is entirely dependant on performance requirements and the environmental conditions in which the connector design will be used. At the very least it is recommended to use a closed housing design. In more severe applications, or situations where a surface treatment cannot be effectively used, a sealed connector design may be needed. There are environments where the use of silver may not be appropriate. Commonly cited application environments where the use of silver finished contact is not recommended are paper mills (large amount of ambient $\mathrm{H}_{2} \mathrm{~S}$ ) and marine environments (chloride ions from $\mathrm{NaCl}$ ). Silver is used in these situations if sufficiently sealed, but testing may need to be done to evaluate the ability of the design to exclude corrosive elements.

- The use of reduced sulfur, sulfur absorbing, and or closed bag packaging materials is recommended to reduce the risk of excessive tarnishing that may occur in storage and shipping environments. 
- Incorporation of wipe into a silver finished contact design is recommended to facilitate the removal of any tarnish films that may form in the contact interface. Wipe effectiveness might be improved through the use of higher normal forces and/or more severe contact geometries, but these approaches may lead to degraded wear, insertion force and durability performance.

- Another method used with some silver contacts is multiple contact redundant designs. There are limited situations where this method can be employed.

- The use of a lower normal force can lead to improved wear and durability performance, but also a reduction in wiping effectiveness. If the level of tarnish formed and retained in the contact area is excessive, it could lead to contact resistance issues - depending on the application requirements and conditions.

- Adjusting performance requirements of a connector to levels more appropriate for a silver finish (e.g. lower mating cycles) may be a way to allow silver to be a suitable finish for a particular hard gold design. For example, many times the required number of mating cycles for a connector design is functionally excessive. Reducing the number of required cycles may make silver a viable lower cost alternative finish.

- If switching finish of a design from gold to silver, one cannot ignore the qualification testing requirements. A test appropriate for gold will likely not be appropriate for silver. Testing concerns cannot be ignored.

- One other concern not addressed in this paper is silver electromigration failures. This may be a risk for designs with smaller conductor spacing under susceptible conditions [22 - 25].

It is all a balance between performance levels and cost. The data shows that silver may be a viable finish for some lower normal force/durability hard gold connector applications. That is if the potential for tarnish formation, increased COF and reduced wear performance are properly managed within a connector design.

\section{ACKNOWLEDGMENTS}

I would like to thank Ned Corman (Technology), William Newell (CIS), Larry Novotny (GAD), and Jackie Everidge (GAD) for their invaluable assistance with testing, samples, and discussion in the development of this work.

${ }^{1}$ Evabrite $\mathrm{S}$ is a registered trademark of Enthone Inc.

\section{REFERENCES}

[1] R. Holm, 'Electric Contacts, Theory and Application,' Springer Verlag, (1967/1999).

[2] J. A. Greenwood, 'Constriction Resistance and the Area of Real Contact,' British J. Appl. Phys., Vol. 17, (1966), pp 1621 - 1632.

[3] M. Leidner, M. Myers, H. Schmidt, H. Schlaak, 'A New Simulation Approach to Characterizing the Mechanical and Electrical Qualities of a Connector Contact,' Proceedings of the $24^{\text {th }}$ ICEC Conference, (2008), pp $165-170$.

[4] M. Myers, M. Leidner, H. Schmidt, Helmut Schlaak, 'Extension and Experimental Verification of a New 'First Contact' Method to Model
Performance of Multilayer Contact Interfaces', Proceedings of the $54^{\text {th }}$ Holm Conference, (2008), pp $66-73$.

[5] R. V. Chiarenzelli, 'Tarnishing Studies on Contact Materials,' IEEE Transactions on Parts, Materials and Packaging, Vol. PMP-3, \#3, (1967) (IBM).

[6] J. Guinement, C Fiaud, 'Laboratory Study of the Reaction of Silver and Copper with Some Atmospheric Pollutants,' Proceedings of the $13^{\text {th }}$ ICEC Conference, (1986), pp. 383 - 390.

[7] W. H. Abbott, 'The Development and Performance Characteristics of Mixed Flowing Gas Test Environments,' Proceedings of the 33rd IEEE Holm Conference, (1987), pp 63-78.

[8] T. E. Graedel, 'Corrosion Mechanisms for Silver Exposed to the Atmosphere,' Journal of the Electrochemical Society, V 139, \#7, (July 1992), pp 1963 - 1970 (Bell labs).

[9] W. H. Abbott, 'The Influence of Environment on Tarnishing Reactions,' Proceedings of the $4^{\text {th }}$ ICEC Conference, (1968), pp $35-39$.

[10] D. Rice, P. Peterson, E. Rigby, P. Phipps, R. Cappell, R. Tremoureaux, 'Atmospheric Corrosion of Copper and Silver,' Journal of the Electrochemical Society, V 128, \#2, (1981), pp 275 - 284 (IBM).

[11] M. Antler, 'Field Studies of Contact Materials: Contact Resistance Behavior of some Base and Noble Metals,' IEEE Trans. On Components, Hybrids, and Manufacturing Technology, Vol. CHMT-5, No. 3, (Sept. 1982), pp $301-307$.

[12] W. H. Abbott, 'The Effects of Test Atmosphere Conditions on the Contact Resistance of Surface Films on Silver,' Proceedings of the $11^{\text {th }}$ ICEC Conference, Berlin, Germany, (1982), pp 294 - 296.

[13] W. H. Abbott, 'Materials, Environment, Motion, and Electrical Contact Failure Mechanisms,' Proceedings of the 35th IEEE Holm Conference, (1989), pp 3 - 11 (1989).

[14] T. Imrell, 'The Importance of the Thickness of Silver Coating in the Corrosion Behavior of Copper Contacts,' Proceedings of the $37^{\text {th }}$ IEEE Holm Conference, (1991), pp 237 - 243 (ABB).

[15] T. Imrell, R. Sjovall, A. Kassman, 'The Composition of the Silver Coating Strongly Influences the Stability of Stationary Electrical Contacts,' Proceedings of the $17^{\text {th }}$ ICEC Conference, (1994), pp 447 454 (ABB).

[16] W. H. Abbott, 'Effects of Industrial Air Pollutants on Electrical Contact Materials,' IEEE Trans. on Parts, Hybrids, and Packaging Vol. PHP-10, \#1, (1974), pp $24-27$.

[17] Craig Hillman, Joelle Arnold, Seth Binfield, Jeremy Seppi, 'Silver and Sulfur: Case Studies, Physics, and Possible Solutions,' SMTA International, (2007), (DfR Solutions, College Park, MD) http://www.dfrsolutions.com/uploads/publications/2007_1 0 silver and sulfur.pdf - downloaded September 15, 2008.

[18] W. H. Abbott, 'The Corrosion of Porous Gold Plating in Field and Laboratory Experiments,' Proceedings of the $13^{\text {th }}$ ICEC Conference, (1986), p $343-347$.

[19] EIA 364-65 (Electrical Connector/Socket Test Procedures Including Environmental Classifications).

[20] S. P. Sharma, 'Atmospheric Corrosion of Silver, Copper, and Nickel Environmental Test,' Journal of the Electrochemical Society, V 125, \#12, (1978), pp 2005 - 2011 (Bell labs).

[21] N. Corman, M. Myers, C. Copper, 'Friction Behavior of Press-Fit Applications: Test Apparatus and Methodology', of the $49^{\text {th }}$ Holm Conference, (2003), pp 38 - 44.

[22] D. Cullen, 'Surface Tarnish and Creeping Corrosion on Pb-Free Circuit Board Surface Finishes,' IPC Review, V 47, \#1, (2006), pp 7.

[23] Simeon Krumbein, 'Metallic Electromigration Phenomena,' Proceedings of the 33rd HOLM Conference, (1987), pp 15-26.

[24] IPC-TR-476A (Electrochemical Migration: Electrically Induced Failures in Printed Wiring Assemblies).

[25] Donald Cullen, Gerard O'Brien, 'Implementation of Immersion Silver PCB Surface Finish in Compliance With Underwriters Laboratories,' Presented at IPC Printed Circuits Expo, SMEMA Council APEX, Designers Summit 04, pp S10-5-1 - S10-5-10 http://www.ul.com/pwb/ImmersionSilver_Cullen.pdf - downloaded $\underline{\text { Jan2008. }}$. 


\title{
Three-Dimensional Structural Study of Tin Plated Fretting Contacts
}

\author{
Tetsuya Ito, Yoshiyuki Nomura and Yasuhiro Hattori \\ Circuits and Connection R\&D Division \\ AutoNetworks Technologies, Ltd. \\ Suzuka, Japan
}

\begin{abstract}
In recent years, there has been increasing demand to miniaturize wiring harness connectors in automobiles due to the increasing volume of electronic equipment and the reduction of the installation space allocated for the electronic equipment in automobiles for the comfort of the passengers. With this demand, contact failure caused by the fretting corrosion seems to become a serious problem in the future.

In our previous study, 2-dimensional observations of tin plated fretting contacts using SEM (Scanning Electron Microscope) and TEM (Transmission Electron Microscopy) methods were made by changing the tin plating thickness as a parameter. However, these conclusions were made only for the cross-sectional observations. Recently, SEM combined with FIB (Focused Ion Beam) has been developed, but there are few reports about the observations of tin plated fretting contacts. In this report, 3dimensional SEM observations of tin plated fretting contacts using FIB - SEM method have been conducted. Spatial distributions of tin, tin oxide and so on have been confirmed quantitatively in two plating thicknesses of 1 and $5 \mu \mathrm{m}$.
\end{abstract}

Keywords- Fretting, FIB-SEM,Tin Plating Thickness

\section{INTRODUCTION}

In recent years, there has been increasing demand for wiring harness connectors in automobiles, due to the increasing volume of electronic equipment. On the other hand, for the comfort and improved convenience of the passengers, the installation space allocated for in-vehicle electronic equipment has tended to be reduced, hence the ever increasing demand for the miniaturization and the weight reduction of automotive wire harnesses, including automotive connectors.

In addition, a decrease in terminal insertion force is desirable, for improvement in automobile assembly work. Based on these external factors, there will be ever increasing demands for reduced contact force in the future.

However, it is well known that fretting corrosion, which is a typical problem for low-force electric contacts, is influenced by contact force [1-2]. Moreover, unique for the automotive connector is that the in-vehicle environment provides more severe conditions such as vibration stresses, thermal changes than that of the consumer connector and the tin plating is mainly used due to a severe cost constraint. Fretting corrosion is generated by external conditions on the contact interface, such as mechanical vibrations and temperature changes especially in the tin plating contacts. Therefore, it will become a serious problem in the future, due to the decrease in contact force. However, we do not have sufficient knowledge of the connector designs at low-level contact force. The aim of our study is to draft a design guideline for low force automotive connector contacts by the micro -structural observations of the fretting contacts leading to the investigation of fretting corrosion mechanism.

\section{PRevious Study}

Illustrating the typical fretting corrosion characteristics of the tin plated contact in our research, an example of contact resistance as a function of the number of fretting cycles is given in Fig.1.

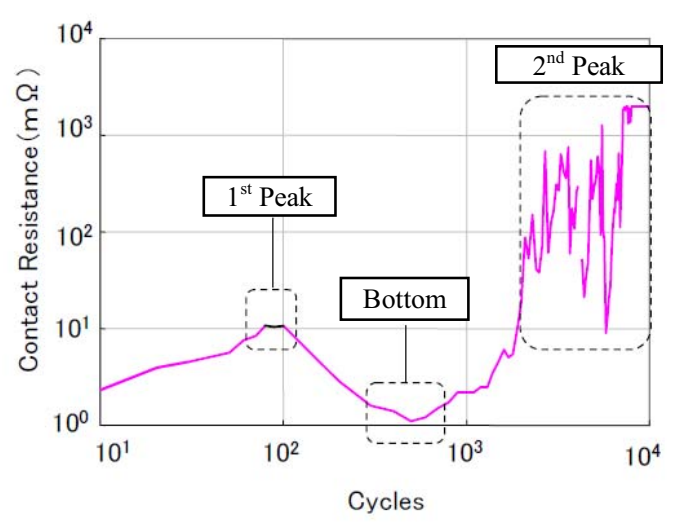

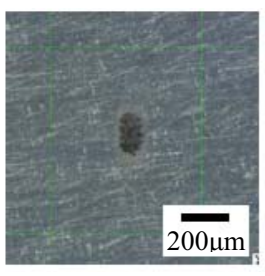

(a) $1^{\text {st }}$ Peak

(Oxidized tin)

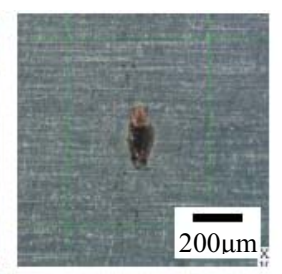

(b) Bottom (Exposed copper)

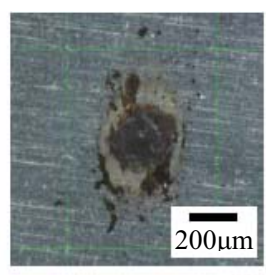

(c) $2^{\text {nd }}$ Peak

(Oxidized copper)
Figure 1. Typical fretting corrosion characteristic of tin plated contact

The initial level of contact resistance is low as seen in Fig.1. Next, it increases steadily to the $1^{\text {st }}$ high contact resistance peak at about 100 cycles. At this $1^{\text {st }}$ peak, contact resistance increases as a result of a tin oxidation film placed between the entire contact area. Next, contact resistance quickly decreases as a result of the removal of tin oxide and exposure of base metal (copper alloy). And then the contact 
resistance increases rapidly to the $2^{\text {nd }}$ high contact resistance peak as a result of a base metal (copper alloy) oxidation.

There are many reports about contact resistance transitions from the low contact resistance level to the high contact resistance level. Antler [3] surveyed the contact fretting phenomena, including mechanisms of transfer, wear, oxidation and the effects of parameters such as cycle rate, force, materials and lubricants. Heaton et al. [4] examined the fretting corrosion of tin-tin junctions after a high number of cycles (above 20,000) as a function of current. These papers show schematic view of formation and accumulation of fretting corrosion. Schneegans et al. [5] observed fretting contact surfaces using atomic force microscopy (AFM), but they do not identify the nature of the tin oxide $\left(\mathrm{SnO}\right.$ or $\left.\mathrm{SnO}_{2}\right)$. Malucci [6] tried to examine oxide films that developed during fretting. He identified the tin oxide is $\mathrm{SnO}$ using XPS analysis.

In our previous papers, 2-dimensional observations of the microstructure transition using Scanning Electron Microscopy (SEM) and Transmission Electron Microscopy (TEM) have been reported on low and high contact resistance test samples of tin plating thickness 1 and $5 \mu \mathrm{m}[7,8]$ and it has been made clear that the micro crystal structures of the fretting contacts. However, these conclusions were made only from the crosssectional observations.

Recently, SEM combined with FIB (Focused Ion Beam) has been developed and there are some reports using this technique $[9,10]$. However, there are few reports about the observations of tin plated fretting contacts. So in this report, 3-dimensional SEM observations of the tin plated fretting contacts using FIB - SEM method have been conducted. Spatial distributions of tin, tin oxide and so on have been confirmed quantitatively in two plating thicknesses of 1 and $5 \mu \mathrm{m}$.

\section{EXPERIMENTAL METHOD}

\section{A. Fretting test samples}

The fretting apparatus and the test samples were similar to that described in detail in our previous paper $[7,8]$. Briefly, the apparatus provided a cyclic motion with precise amplitude and frequency. It also measured the contact resistance according to the 4-wire method (current $10 \mathrm{~mA}$, maximum voltage $1 \mathrm{~V})$.

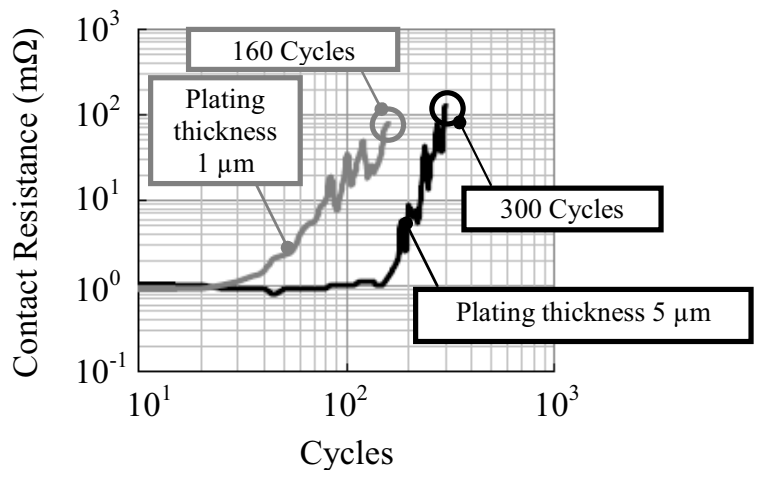

Figure 2. Contact resistance versus fretting cycles of the test samples
Fig. 2 shows the measured contact resistance versus fretting cycles of the test samples with different tin plating thickness of 1 and $5 \mu \mathrm{m}$. Three test samples of each tin plating thickness were made under the fixed conditions shown in Table 1.

TABLE $1 . \quad$ TEST CONDITIONS

\begin{tabular}{c|c|c}
\hline Tin Plating Thickness & $1 \mu \mathrm{m}$ & $5 \mu \mathrm{m}$ \\
\hline \hline Load $(\mathrm{N})$ & 1 & $\leftarrow$ \\
Amplitude $(\mu \mathrm{m})$ & 50 & $\leftarrow$ \\
Frequency $(\mathrm{Hz})$ & 1 & $\leftarrow$ \\
Cycles & 160 & 300 \\
\hline
\end{tabular}

These conditions are typical for low-level contact force designs and are most likely to produce the increase in contact resistance in the 1st peak in our previous study. All the tests were carried out under room temperature and humidity.

\section{B. FIB-SEM method}

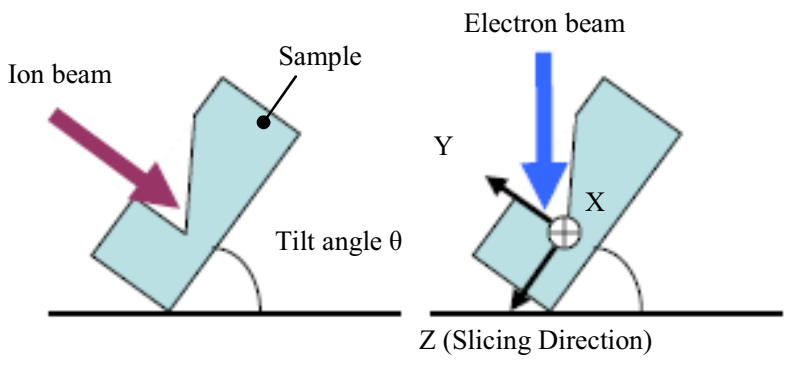

Figure 3. Schematics of the FIB-SEM method

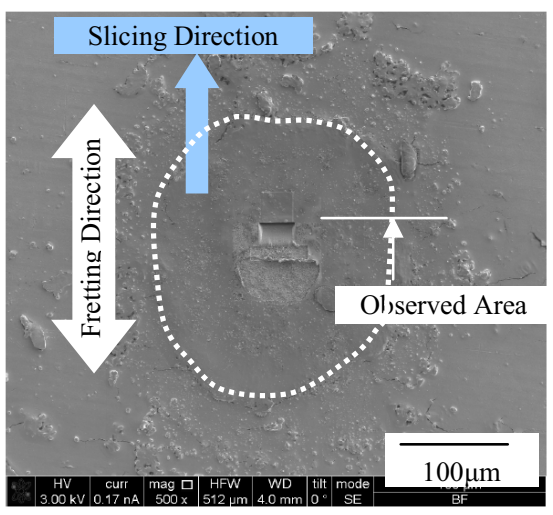

Figure 4. Fretting test sample (tin plating thickness $5 \mu \mathrm{m}$ )

Figure 3 shows the schematics of the FIB-SEM method. $[9,10]$ The method consists of performing serial FIB through the surface of a test sample. Each sliced surface is imaged using SEM. By the repetition of the FIB fabrication and SEM observations, the series of cross-sectional images can be acquired through the sample to be investigated. Figure 4 shows the fretting test sample of the tin plating thickness 5 $\mu \mathrm{m}$. In this study, the centre part of the fretting contacts area was investigated as shown in Figure 4. 

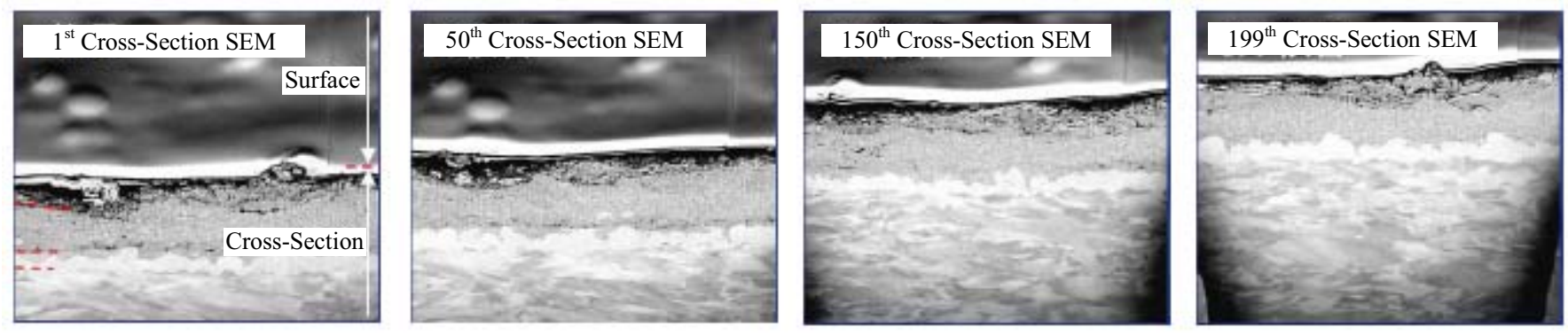

Figure 5. Cross-sectional SEM images (tin plating thickness $5 \mu \mathrm{m}$ )

Figure 5 shows the chosen cross-sectional SEM images of the test sample of the tin plating thickness $5 \mu \mathrm{m}$, which are acquired during the serial slicing. Only 4 of all 199 images are chosen for the better overview. In this study, the crosssectional SEM images at $50 \mathrm{~nm}$ intervals up to $10 \mu \mathrm{m}$ in slicing direction (axis $Z$ ) were acquired. Then 3D image was reconstructed from this cross-sectional SEM images. Table 2 shows the 3D-SEM observation conditions.

TABLE 2. 3D-SEM OBSERVATION CONDITIONS

\begin{tabular}{c|c}
\hline Item & SPEC \\
\hline \hline FIB-SEM & FEI Helios Nano Lab 600 \\
\hline acceleration voltage of FIB & $30 \mathrm{kV}$ \\
\hline acceleration voltage of SEM & $3 \mathrm{kV}$ \\
\hline 3D reconstruction software & Mercury Computer Amira \\
\hline
\end{tabular}
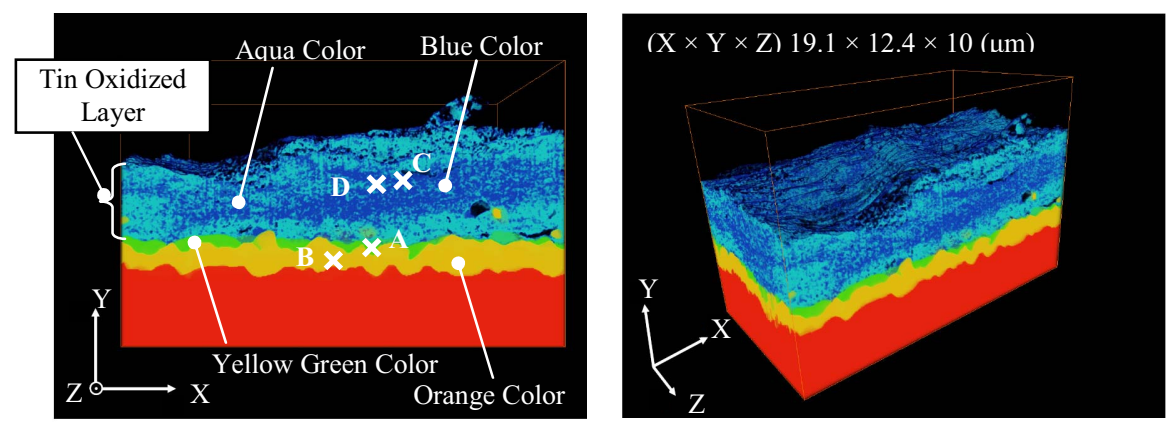

\section{RESULTS AND DISCUSSIONS}

\section{A. 3 D-SEM observation results}

Figure 6 shows the digitizing image of the test sample of the tin plating thickness $5 \mu \mathrm{m}$. Figure 6 shows that both oxidized layer and intermetallic compound layer are separated into two layers, respectively (Tin oxidized layer is separated into blue and aqua colors and intermetallic compound layer is separated into yellow green and orange colors). It is likely that blue and aqua colors consist of tin and tin oxide mixture and yellow green and orange colors consist of intermetallic compounds that differ in composition. Moreover, there are many voids (black color) in the oxidized layer.

Meanwhile, in the tin plating thickness $1 \mu \mathrm{m}$ (see Figure 7), both oxidized layer and intermetallic compound layer consist of one layer, respectively (Tin oxidized layer is aqua color and intermetallic compound layer is orange colors). And there are also some voids in the oxidized layer.

Table 3 and 4 show average 3-dimensional film thickness and the volume fraction of each color layer of tin plating thickness 1 and $5 \mu \mathrm{m}$, which are calculated from the quantitative analysis. It is clear that there are many

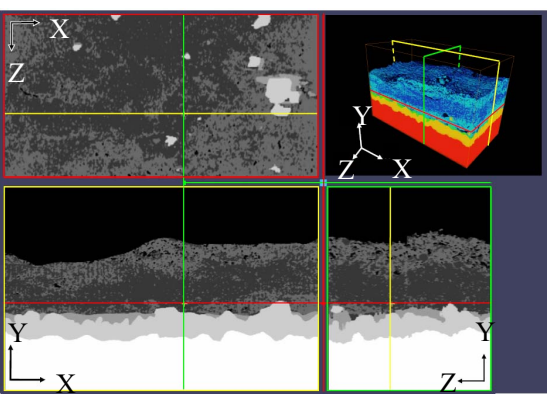

Figure 6. Digitizing image (tin plating thickness $5 \mu \mathrm{m}$ )
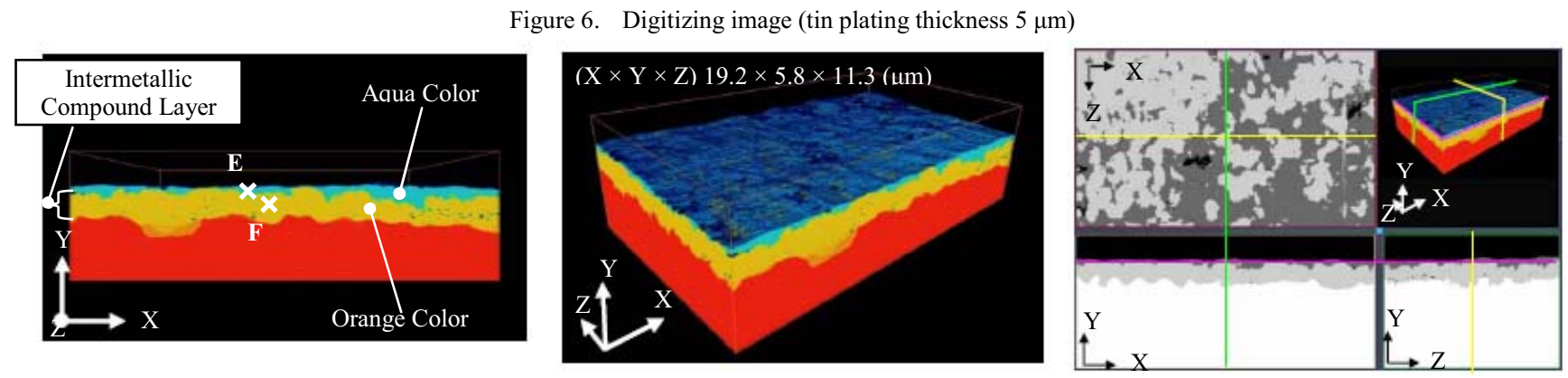

Figure 7. Digitizing image (tin plating thickness $1 \mu \mathrm{m}$ ) 
differences in composition between tin plating thickness 1 and $5 \mu \mathrm{m}$. However, in these observations, the composition of each color layer can not be identified. Therefore, AES (Auger electron spectroscopy) analyses to identify the compositions are conducted.

TABLE $3 . \quad$ 3-DIMENSIONAL FILM THICKNESS

\begin{tabular}{c|cc}
\hline \multirow{2}{*}{ Layer } & \multicolumn{2}{|c}{ Average Film Thickness $(\mu \mathrm{m})$} \\
\cline { 2 - 3 } & $1 \mu \mathrm{m}$ & $5 \mu \mathrm{m}$ \\
\hline \hline Tin Oxidized & 0.25 & 4.0 \\
\hline Intermetallic & 1.18 & 1.5 \\
\hline
\end{tabular}

TABLE 4. VOLUME FRACTION OF EACH COLOR LAYER

\begin{tabular}{c|cc}
\hline \multirow{2}{*}{ Layer Color } & \multicolumn{2}{|c}{ Volume Fraction $(\%)$} \\
\cline { 2 - 3 } & $5 \mu \mathrm{m}$ & $1 \mu \mathrm{m}$ \\
\hline \hline Orange & 24 & 81.5 \\
\hline Yellow-Green & 4 & - \\
\hline Aqua & 27.4 & 17.5 \\
\hline Blue & 41.5 & - \\
\hline Black & 3.1 & 1 \\
\hline
\end{tabular}

\section{B. AES elemental analysis results}

Figure 8 shows the elemental mapping results of tin plating thicknesses of 1 and $5 \mu \mathrm{m}$ and Table 5 shows the

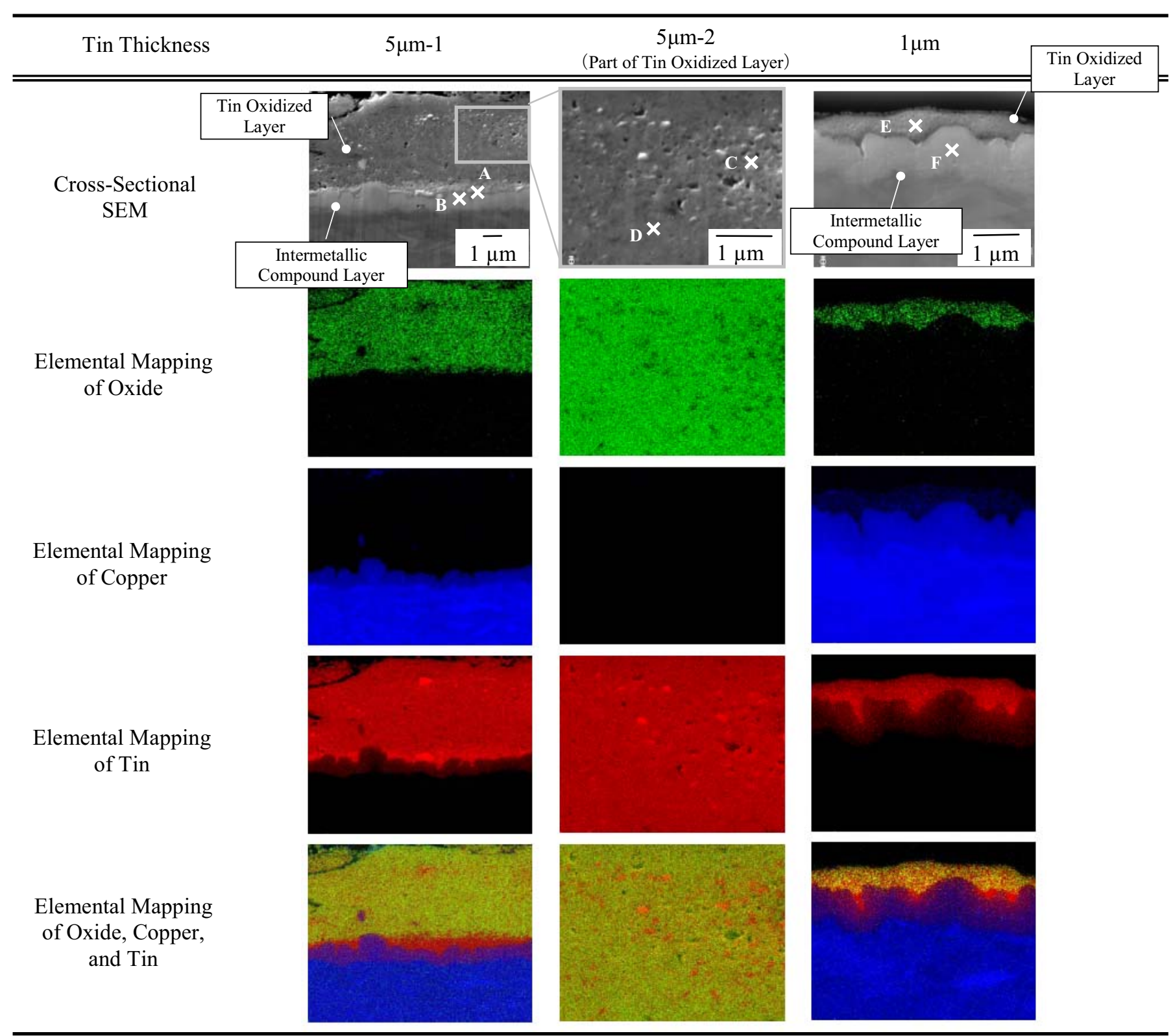

Figure 8. Elemental mapping results 
elemental ratio of each color layer of tin plating thicknesses of 1 and $5 \mu \mathrm{m}$. From these analysis results, it was found as follows;

(1) For tin plating thickness $5 \mu \mathrm{m}$, it was found that both blue and aqua color layers in Figure 6 mainly consisted of tin oxide. However, oxidation level of blue color layer was lower than that of aqua color layer.

(2) For tin plating thickness $5 \mu \mathrm{m}$, it was found that yellow green color layer in Figure 6 mainly consisted of tin. The reason why tin exists between oxide layer and intermetallic layer is thought to be caused by a rough surface of intermetallic compounds, in which tin remains without any wear.

(3) For tin plating thickness $1 \mu \mathrm{m}$, it was found that aqua color layers in Figure 7 mainly consisted of tin oxide. However, tin oxide contained not less than $10 \%$ copper component.

(4) In both tin plating thickness, it was found orange color layer mainly consisted of intermetallic compounds.

TABLE 5. ElEMENTAL RATIO (ATOMIC \%)

\begin{tabular}{c|c|c|c|c|c}
\hline $\begin{array}{c}\text { Tin plating } \\
\text { Thickness }\end{array}$ & Point & $\begin{array}{c}\text { Layer } \\
\text { color }\end{array}$ & $\mathrm{O}$ & $\mathrm{Cu}$ & $\mathrm{Sn}$ \\
\hline \multirow{5}{*}{$5 \mu \mathrm{m}$} & $\mathrm{A}$ & $\begin{array}{c}\text { Yellow } \\
\text { Green }\end{array}$ & - & - & 100 \\
\cline { 2 - 6 } & $\mathrm{B}$ & Orange & - & 78.5 & 21.5 \\
\cline { 2 - 6 } & $\mathrm{C}$ & Blue & 27.7 & - & 72.3 \\
\cline { 2 - 6 } & $\mathrm{D}$ & Aqua & 54.4 & - & 45.6 \\
\hline \multirow{4}{*}{$1 \mu \mathrm{m}$} & $\mathrm{E}$ & Aqua & 46.0 & 10.5 & 43.5 \\
\cline { 2 - 6 } & $\mathrm{F}$ & Orange & - & 77.6 & 22.4 \\
\hline
\end{tabular}

\section{CONCLUSIONS}

3-dimensional observations of tin plated fretting contacts using FIB-SEM technique have been presented and spatial distributions of tin, tin oxide and intermetallic compounds are confirmed quantitatively. However, this study is the beginning of our work using 3D-SEM method. In this study, the effectiveness of this method is confirmed, although this method has some weakness that only a part of the fretting contact area is able to observe.

The future direction of this work will be to consider the conductive mechanism of tin plated fretting contacts and the dependence of the tin plating thickness on the mixture structures of tin, tin oxide, etc using this 3D-SEM method.

\section{REFERENCES}

[1] J. M. Hooyer, and K. Peekstok. "The Influence of Practical Contact Parameters of Fretting Corrosion of Tin-Bass Low-Level Connector Contacts", Proc. Holm Conf on Electrical Contacts, 1987, p. 43.

[2] A. Lee, and M. Mamrick. "Fretting Corrosion of Tin Plated Copper Alloy", Proc. Holm Conf on Electrical Contacts, 1986, p. 45.

[3] M. Antler. "Survey of Contact Fretting in Electrical Connectors", Proc. Holm Conf on Electrical Contacts, 1984, P3.

[4] C. E. Heaton, and S. L. McCarthy. "High Cycle Fretting Corrosion Studies on Tin-Coated Contact Materials", Proc. Holm Conf on Electrical Contacts, 2001, p. 209.

[5] O. Schneegans, et al. "Fretting Degradation of Tin-Plated Contacts Studied by Means of a New Electrical Cartography Technique Based on Atomic Force Microscopy", Proc. International Conf on Electrical Contact Phenomena, 1998, p. 187.

[6] R. D. Malucci, "Characteristics of Films Developed in Fretting Experiments on Tin Plated Contacts", Proc. Holm Conf on Electrical Contacts, 1999, p. 175.

[7] T. Ito, K. Takata, Y. Hattori, K.Iida and Y. Saitoh. "Microscopy Study of Fretting Corrosion of Tin Plated Contacts", Proc. Holm Conf on Electrical Contacts, 2007, p. 216.

[8] T. Ito, S. Sawada, Y. Hattori, Y. Saitoh, T. Tamai, and K.Iida. "Microscopy Study of Fretting Corrosion Caused by the Tin Plating Thickness", Proc. Holm Conf on Electrical Contacts, 2008, p. ???.

[9] M. Kato, T. Ito, Y. Aoyama, K. Sawa, T. Kaneko, N. Kawase, H. Jinnai. "Three-Dimensional Structural Analysis of a Block Copolymer by Scanning Electron Microscopy Combined with a Focused Ion Beam”, J. of Polymer Science vol. 45, 2007, p. 677.

[10] F. Mucklich, et al. "Nanotomography of electrical contacts-new insights by high resolution $3 \mathrm{D}$ analysis of local material degradation", Proc. ICEC2008 , 2008, p. 31. 


\section{Investigation on Mechanism of Creep Corrosion of Immersion Silver Finished Printed Circuit Board by Clay Tests}

\author{
Yilin Zhou \\ Research Lab of Electric Contacts, Automation School \\ Beijing University of Posts and Telecommunications \\ Beijing, 100876, P. R. China, \\ E-mail: ylzhou@bupt.edu.cn
}

\author{
Michael Pecht \\ CALCE Electronic Products and Systems Center \\ University of Maryland, College Park, \\ 20742, USA \\ E-mail: pecht@calce.umd.edu
}

\begin{abstract}
This paper studies the creep corrosion of immersion silver (ImAg) finished printed circuit board (PCB) under high sulfur environments. Clays containing sulfur were heated with water to simulate high sulfur environment and to drive creep corrosion on ImAg finished pads. Except the silver sulfide film formed on ImAg finish, creep corrosion began by growth of dendrites on pad edges, which was caused by galvanic corrosion. Copper sulfide and silver sulfide were the main components of creep corrosion products. The different coverage extent of ImAg finish on pad edges caused different reaction speed, the morphology and the creep distance of corrosion products, and different content of copper sulfide and silver sulfide in corrosion products. The impact of creep corrosion products on surface insulation resistance (SIR) was measured. Weibull distribution with two parameters was used to analyze the length of dendrites and to quantitatively evaluate the corrosion resistance of $\operatorname{ImAg}$ finish. The methods to improve the reliability of $\operatorname{ImAg}$ finished PCB was also discussed in the paper.
\end{abstract}

Key words: immersion silver, creep corrosion, sulfur, surface insulation resistance, Weibull distribution

\section{INTRODUCTION}

Surface finishes are commonly used to protect the copper metallization of a PCB from oxidation to enable good solderability of the PCB. With lead-free legislation impacting PCB manufacturing on a global scale, the ImAg finish is rapidly gaining popularity as the lead-free surface finish of choice [1]. ImAg serves as a protective finish, thereby ensuring the solderability of the underlying copper on PCB for up to 12 months prior to assembly [2], [3], [4]. When exposed to the atmosphere both during shelf storage and under operating conditions, silver tends to form a layer of tarnish on the surface, which mostly includes $\mathrm{Ag}_{2} \mathrm{~S}$ and $\mathrm{AgCl}$ [5], [6]. To prevent tarnishing and minimize the associated loss of solderability, ImAg coatings now contain co-deposited organic inhibitors that limit the formation of tarnish [7]. Silver is compatible with most assembly processes and is becoming more commonly available in the electronics industry [6].

With the introduction of $\operatorname{ImAg}$ as the finish of choice for PC based technology, failures relating to high sulfur levels have increased dramatically. It was reported when $\operatorname{ImAg}$ finished PCs worked in a clay modeling center to design transportation products, PC technology failed within weeks [9]. The corrosion products on ImAg finishes appeared to begin with the growth of dendrites that took place equally in all directions, unlike electric potential-driven dendrite growth [10]. The explanation said copper exposed inside the VIA holes due to an incomplete silver finish covering was corroded in the presence of a suitable electrolyte [9]. As the corrosion products increased in thickness, the resistance decreased until functional shorting occurred [10]. It was suggested that the creep corrosion was driven by concentration gradients of 
chemical species of the corrosion products, so they moved from the sources with a higher concentration to those with a lower concentration [11], [12], [13]. Depending on the environmental conditions, corrosion products maybe continuously generate from the exposed sites, then diffuse over the surface [14]. To study corrosion resistance of ImAg finish, various industry groups tried MFG (Mixed Flowing Gases) testing to develop creep corrosion testing [15] [16]. DELL selected clays containing $30-50 \%$ elemental sulfur to simulate the actual sulfur environment [10].

However, the structure characteristics and formation mechanism of creep corrosion products on ImAg finish and their influence on the SIR of PCB were not discussed in details. In addition, how to assess the corrosion resistance of ImAg finish quantitatively needed to be studied. This paper uses clays to drive the creep corrosion on the $\operatorname{ImAg}$ surface finishes and explains the test failure phenomenon and the failure mechanisms of $\mathrm{ImAg}$ finished PCBs in high sulfur environments. The impact of creep corrosion products on SIR is tested. The length of creep corrosion products are measured and analyzed by Weibull distribution with two parameters.

\section{EXPERIMENTAL METHODS}

\section{A. Corrosion Method}

To study creep corrosion on ImAg finishes and assess PCBs, it is necessary to devise an easily applied testing method to simulate creep corrosion in high sulfur environment. Referred on Schuller's studies [10], a test method utilizing high sulfur clay (J-525, made by the Chavant Company) used in modeling studios was set up. The density of the clay (J-525) is 90 pounds per cubic foot, with a softening point between $57^{\circ} \mathrm{C} \sim 66^{\circ} \mathrm{C}$ and a working temperature between room temperature to $63^{\circ} \mathrm{C}$. The main element compositions of this clay detected by an X-ray energy dispersive spectroscope (XEDS) are listed in Table I. The weight percentage of sulfur is about $17 \%$.

In the experiment, to simulate the industrial environment and avoid too harsh corrosion, 1.5 pounds of clay was put in a plastic container, and $10 \mathrm{ml}$ of water was added to increase humidity and activate the galvanic corrosion. The corrosion test container is shown in Figure 1. The clay was put into container and heated for five minutes in a microwave oven. Then, the PCBs to be tested, which had been stored in a refrigerator for 10 minutes to enhance condensation, were put into the container. The relative humidity in the container was close to $100 \%$ and the temperature was $29.8^{\circ} \mathrm{C}$ detected by a hygrometer with a probe. The lid was closed finally. All of the samples were held by clamps, and none directly contacted the clay. The corrosion experiment lasted for three days uninterrupted at room temperature. After three days, the relative humidity in the container was $75.2 \%$, and the temperature was $26.2^{\circ} \mathrm{C}$.

TABLE I. ELEMENT COMPOSITIONS IN CLAY J-525

\begin{tabular}{|c|c|}
\hline Elements & Weight \% \\
\hline $\boldsymbol{C}$ & 64.46 \\
\hline $\boldsymbol{O}$ & 13.66 \\
\hline $\boldsymbol{A l}$ & 1.37 \\
\hline $\boldsymbol{S i}$ & 1.32 \\
\hline $\boldsymbol{S}$ & 17.27 \\
\hline $\boldsymbol{F e}$ & 0.23 \\
\hline $\boldsymbol{Z n}$ & 1.56 \\
\hline $\boldsymbol{B a}$ & 0.14 \\
\hline
\end{tabular}

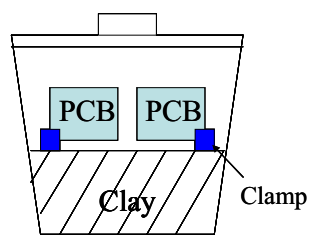

Figure 1. Corrosion test fixture

After corrosion test, the corrosion products were observed by environmental scanning electronic microscope (ESEM) and their compositions were detected by XEDS.

\section{B. Testing Method of SIR}

To investigate the impact of creep corrosion products on the degradation of SIR of PCB, a testing circuit was built up, shown in Figure 2. There are two gold plated probes, the radius of the hemisphere head of probes is $25 \mu \mathrm{m}$. Each probe was controlled by a manipulator in $x-y-z$ directions. One probe was pressed on the surface of ImAg finished pad, the other was pressed on the solder mask and moved to the creep corrosion products slowly by screwing the knob on the manipulator. A semiconductor parameter analyzer (Agilent 
$4155 \mathrm{C}$ ) was used to supply a voltage from 0 to $10 \mathrm{~V}$ with a step $50 \mathrm{mV}$ and monitor the current change at each step, so that the I-V curve can be achieved to calculate the SIR. If the creep corrosion products could be conductive, the SIR will drop dramatically comparing with that of PCB.

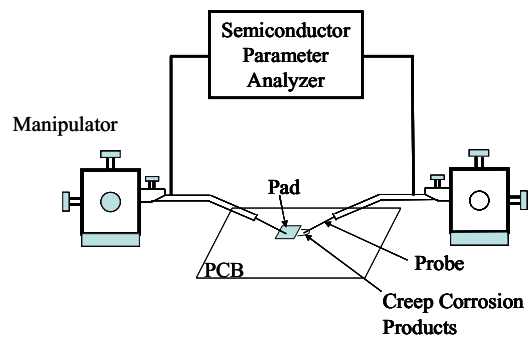

Figure 2. Testing circuit for SIR

\section{Experimental Samples}

Two ImAg finished PCBs were taken as testing samples. The thickness of the ImAg finish was detected by an X-Ray Fluorescent Spectroscopy. Five points ( $\Phi 0.3 \mathrm{~mm}$ collimator) on the pads were detected randomly on each PCB. The average thickness of the ImAg finish on PCB No.1 was $0.36 \mu \mathrm{m}, 60 \%$ thicker than that on PCBs No. $2,0.22 \mu \mathrm{m}$. Their standard deviations were 0.036 and $0.037 \mu \mathrm{m}$ respectively, as listed in Table II.

TABLE II. THICKNESS OF IMAG FINISH ON TWO PCBS $(\mu \mathrm{m})$

\begin{tabular}{|l|c|c|}
\hline Series No. of PCB & $\mathbf{1}$ & $\mathbf{2}$ \\
\hline Test point 1 & 0.305 & 0.188 \\
\hline Test point 2 & 0.350 & 0.261 \\
\hline Test point 3 & 0.341 & 0.202 \\
\hline Test point 4 & 0.382 & 0.260 \\
\hline Test point 5 & 0.401 & 0.198 \\
\hline Average & 0.356 & 0.222 \\
\hline Standard Deviation & 0.037 & 0.036 \\
\hline
\end{tabular}

III. CORROSION MECHANISMS

The corrosion of ImAg finished PCB in a high sulfur environment involves both direct chemical corrosion and galvanic corrosion. A silver finish will be corroded by direct chemical corrosion in a sulfur environment to form silver sulfide:

Galvanic corrosion occurs if the ImAg finish is not complete, underlying copper on PCB pads are exposed. The standard electrode potential of $\mathrm{Ag}$ and $\mathrm{Cu}$ is $+0.800 \mathrm{~V}$ and $+0.340 \mathrm{~V}$ respectively. Then, the standard Electromotive Force (EMF) for $\mathrm{Cu}-\mathrm{Ag}$ cell is

$$
\Delta \mathrm{E}_{0}=0.800-0.340=0.460(\mathrm{~V})
$$

Galvanic corrosion would occur on ImAg finished PCB if water from the atmosphere is present in monolayer quantities, which is enough to promote the reactions [9]. Ag becomes the cathode and $\mathrm{Cu}$ becomes the anode. $\mathrm{Cu}$ lost electrons and was sulfurized to form copper sulfide at last.

\section{CORROSION ON AN ImAg FINISHED PCB BY CLAY TESTS}

Taken PCB No.2 as an example, creep corrosion characteristics were analyzed in details. All the ImAg finished pads changed color to dark red after three days of corrosion. Creep corrosion occurred on the pad edges of the $\operatorname{ImAg}$ finished PCB, and corrosion products crept outward as shown in Figure 3. Two kinds of creep corrosion products were found. The first kind grew from the ImAg pad edges, where the pad edges were not covered by the solder mask. The second kind of corrosion products grew from the boundary between the $\operatorname{ImAg}$ pads and solder mask, where the pad edges were covered by the solder mask.

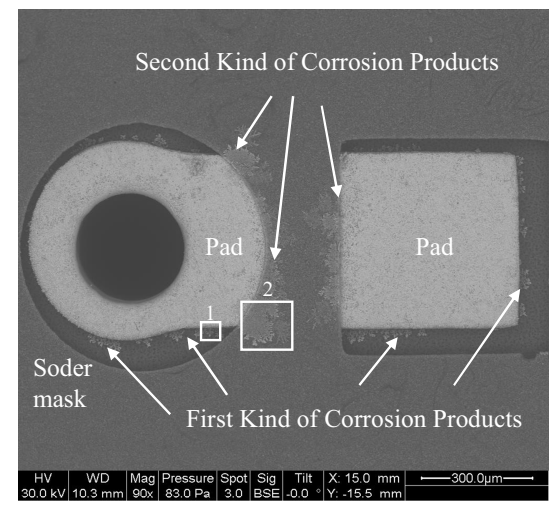

Figure 3. Creep corrosion on the pad edges of ImAg finished PCB after three days of clay corrosion.

\section{A. The Characteristics of the First Kind of Corrosion} Products

The first kind of corrosion products, No.1 square zone in Figure 3, is magnified and shown in Figure 4. Corrosion products formed from the substrate of the pad edges, appearing 
as dendrite growth. The "roots" of the dendrites were not continuous, their widths were usually less than $5 \mu \mathrm{m}$. The "stems" of the dendrites were piled up with small crystals and appeared to be compact in structure.

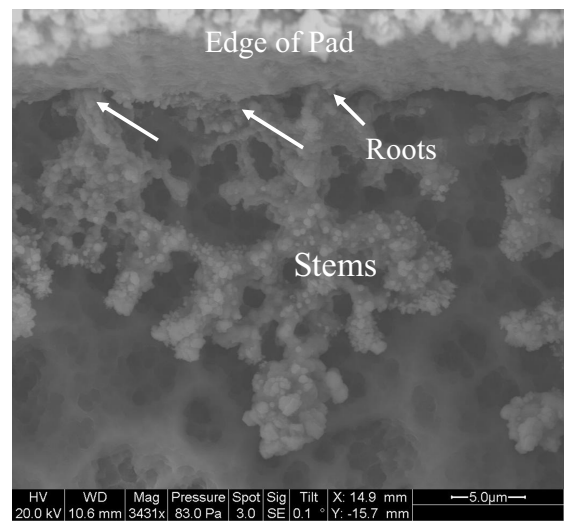

Figure 4. One dendrite of the first kind of creep corrosion products The square zone labeled by 1 in Figure 3 .

The element compositions of the pad and the creep corrosion products were detected by the line scan function of the XEDS, as shown in Figure 5.

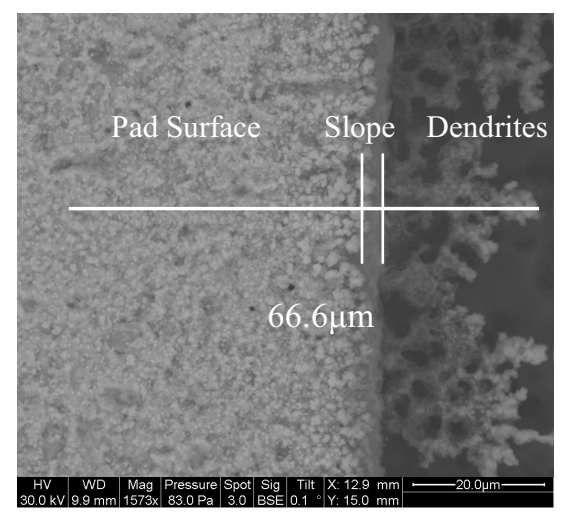

(a) ImAg finished pad and the first kind of corrosion products

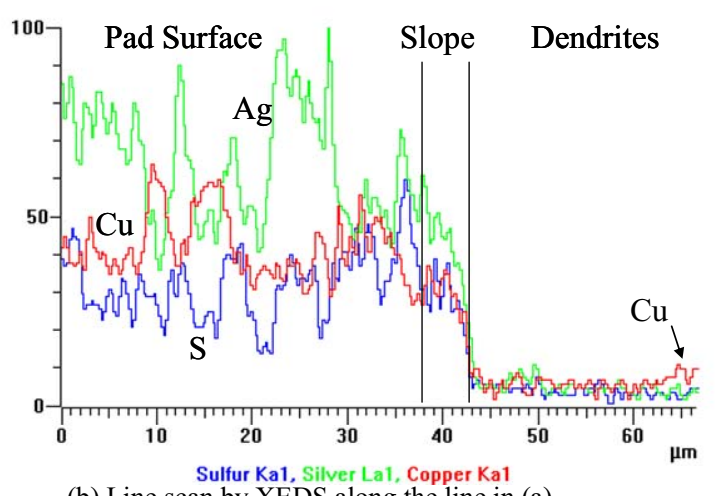

(b) Line scan by XEDS along the line in (a).

Figure 5. A line scan of the element compositions of the pad and the first kind of creep corrosion products
On the pad surface, the main elements were $\mathrm{Ag}, \mathrm{Cu}$ and $\mathrm{S}$. The change of the $\mathrm{S}$ content correlated with that of the $\mathrm{Ag}$, which means the dark red corrosion products on the pad surface were mainly silver sulfide. Since the Ag finish was very thin, the underlying copper was also detected. The corrosion products with dendrite shape were made up mainly of $\mathrm{Cu}$ and $\mathrm{S}$, which means the creep corrosion products were mainly copper sulfide. Little Ag on the pad migrated into the creep corrosion products.

\section{B. The Characteristics of the Second Kind of Corrosion Products}

The second kind of creep corrosion products, No.2 square zone in Figure 3, is shown in Figure 6a. Corrosion products formed from the boundary between the ImAg finished pad and the solder mask and crept outward to appear as various structures, shown in Figure $6 \mathrm{~b}$ and $6 \mathrm{c}$. On zone 1 close to the pad edge in Figure 6a, the structure of creep corrosion products appeared to be discrete crystals, which was not like the "roots" in the first kind of creep corrosion products, as shown in Figure $6 \mathrm{~b}$. The amount of crystals flooded from the boundary between the ImAg finished pad and solder mask, the width along the pad edge was more than several tens of micrometers, even hundreds of micrometers. As the corrosion products crept outward, the crystals accumulated and oriented to form compact dendrites, as shown in Figure 6c.

The element compositions of the pad and the second kind of creep corroded products were determined by the line scan function of the XEDS, as shown in Figure 7. It was clear that the element Ag decreased from the inside of the pad to the edge of the pad, while $\mathrm{Cu}$ changed inversely, but $\mathrm{S}$ almost remained stable. On the creep corrosion products, the $\mathrm{Cu}, \mathrm{Ag}$ and $\mathrm{S}$ were all detected, shown in Figure $7 \mathrm{~b}$. The edge of the pad was magnified as shown in Figure 8. The element compositions on two areas on the pad edge were listed in Table III. Ag content on area 2 was much lower than that on area 1 , and the $\mathrm{Cu}$ content changed inversely, which was consistent with the results of line scan on the pad edge in Figure 7b. The Ag on the edge of pad was consumed heavily and the substrate $\mathrm{Cu}$ was exposed.

\section{SIR TESTING}

The failure reason of ImAg finished PCBs under high sulfur 


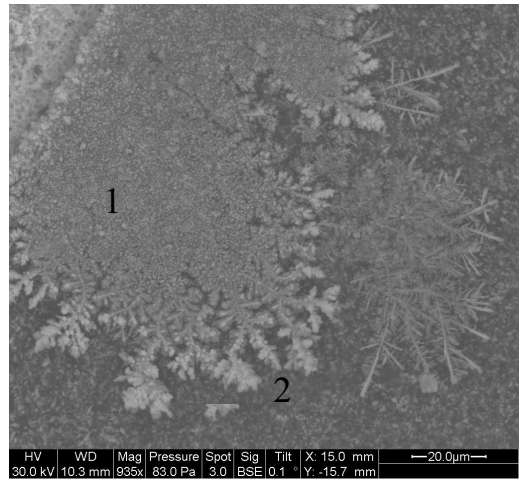

(a) The second kind of creep corrosion products, the square zone labeled by 2 in Figure 3, which grew on the boundary between the pad and solder mask.

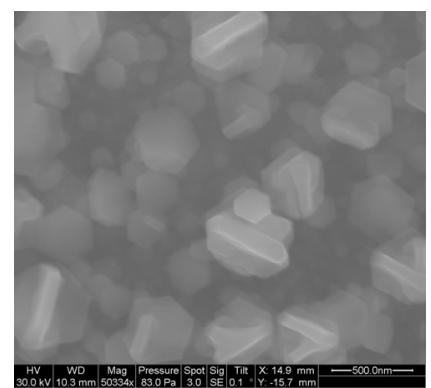

(b) Zone 1 in (a), the discrete crystals

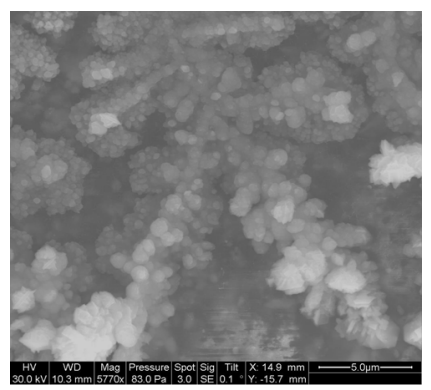

(c) Zone 2 in (a), the dendrites
Figure 6. The structures of the second kind of corrosion products

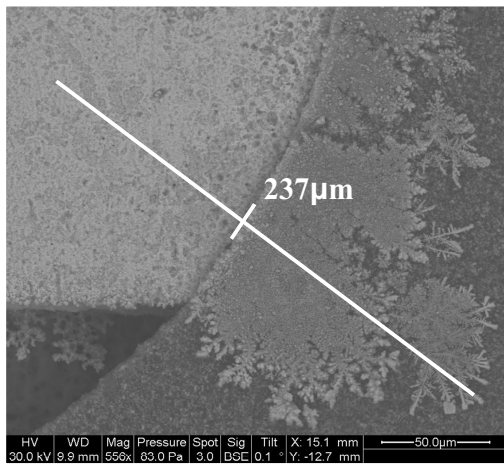

(a) ImAg finished pad and the second kind of corrosion products

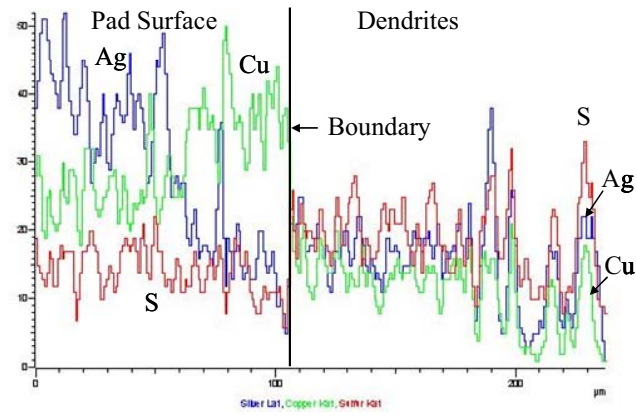

b. Line scan by XEDS along the line in (a).

Figure 7. A line scan of element compositions of the pad and the second kind of creep corrosion products

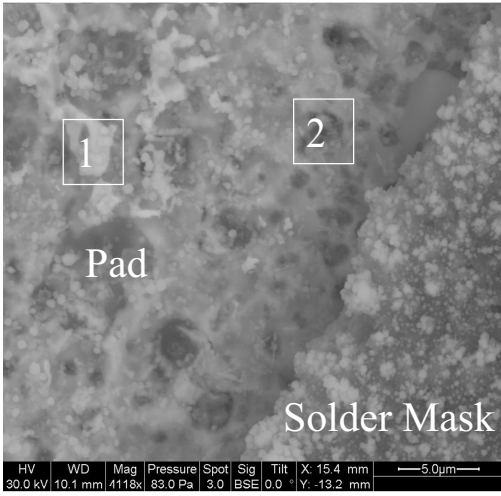

Figure 8. Magnification of the pad edge in Figure 7a.

TABLE III. Atomic Percentage OF THE ELEMENTS ON AREA 1 AND AREA 2 ON PAD EDGE IN FIGURE 8

\begin{tabular}{|l|c|c|}
\hline Elements & Area 1 & Area 2 \\
\hline $\boldsymbol{C}$ & 44.19 & 43.10 \\
\hline $\boldsymbol{O}$ & 12.91 & 9.28 \\
\hline $\boldsymbol{S}$ & 4.79 & 3.35 \\
\hline $\boldsymbol{C u}$ & 28.74 & 42.05 \\
\hline $\boldsymbol{A g}$ & 9.37 & 2.22 \\
\hline $\boldsymbol{C u}: \mathbf{A g}$ & 3.07 & 18.94 \\
\hline
\end{tabular}

environment was deduced that the corrosion products crept over solder mask to cause shorting among adjacent circuits, so a fixture was set up to measure the impact of creep corrosion products on SIR of PCB, shown in Figure 2.

Firstly, the two testing probes were pressed on a gold plated surface as a shorting state to get a resistance of the whole circuit, which was $2.72 \Omega$ averagely by eight tests. Secondly, one of the probes was pressed on an ImAg finished pad surface with creep corrosion products, the other probe was pressed on the solder mask to measure the SIR of PCB, where the SIR was higher than Mega-Ohms. Then, the right probe contacted on the solder mask was moved gradually by controlling the manipulator to touch the creep corrosion products, shown in Figure 9.

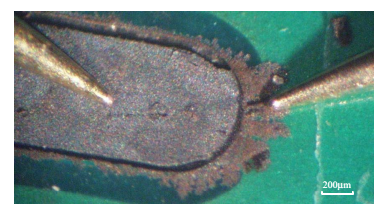

Figure 9. The testing of SIR on creep corrosion products 
The SIR was tested on 22 creep corrosion products, five out of them degraded, the others kept higher than Mega-Ohms. This may be caused by the different compact extent of creep corrosion products. Only those corrosion products that had enough thickness and compactness could degrade the SIR of PCB.

The current-voltage (I-V) curve tested on one creep corrosion product where SIR degraded is shown in Figure 10a. Correspondingly, the resistance-voltage curve is shown in Figure 10b. It was shown that after the right probe moved to touch the creep corrosion products, the current nonlinearly increased with the testing voltage increased, so the resistance of creep corrosion products dropped as the voltage increased, appearing as the characteristics of semiconductors. When voltage increased to $10 \mathrm{~V}$, the SIR on PCB dropped lower than $45 \mathrm{~K} \Omega$, which would seriously reduce the surface insulation if they crept over adjacent pads, even to shorting the circuits. Therefore, the length of creep corrosion products as dendrites growth was a key parameter to influence the PCB reliability. The statistics of length of creep corrosion products needed to be carried out.

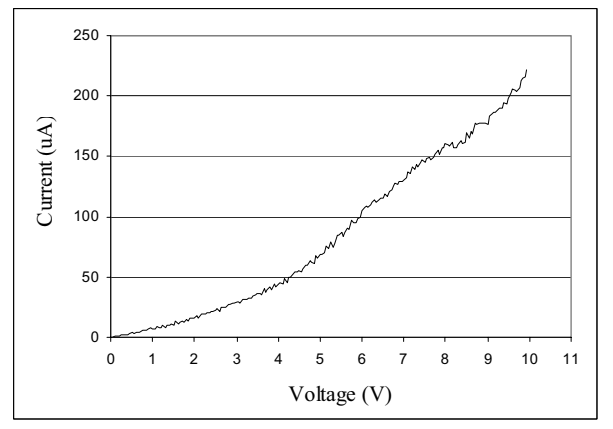

(a) The I-V curve of one creep corrosion product on PCB

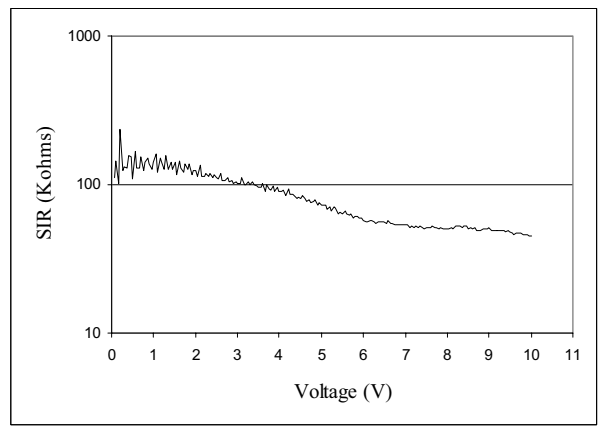

(b) The R-V curve of one creep corrosion product on PCB

Figure 10. The impact of creep corrosion products on SIR of PCB

\section{StATISTICS OF THE LENGTH OF CREEP CORROSION PRODUCTS}

It is well known that Weibull distribution can be applied on the statistics in many fields, especially life prediction, accelerated life testing, dielectric breakdown [17], [18], etc. The two-parameter Weibull distribution was used in the statistics of length of creep corrosion products, given by the expression:

$$
F(t)=1-\exp \left(-\frac{t}{\eta}\right)^{\beta}
$$

where $\mathrm{F}$ is the cumulative occurrence probability of creep corrosion products with certain length. $t$ is the random variable for length of creep corrosion products. $\eta$ is the mean length of corrosion products at the cumulative probability of $63.2 \%$ and $\beta$ is the shape factor of Weibull distribution, also known as Weibull slope. Plotting the Weibull distribution in the following form,

$$
W \equiv \ln \{-\ln [1-F(t)]\}
$$

Versus $\ln (\mathrm{t})$ yields a straight line with slope of $\beta$. $W$ is the quantity called Weibit.

The length of 255 creep corrosion products on 18 pads of PCB No.2 was measured from the root to the end of the dendrite. Among them, there were 148 of the first kinds of corrosion products from 12 pads and 107 of the second kind of corrosion products from 15 pads. Two examples of pads with the first kind and second kind of creep corrosion products are shown in Figure 11a and $11 \mathrm{~b}$ respectively. The Weibull distribution plots of these two kinds of creep corrosion products are shown in Figure 12. The confidence level $\rho$ of two fitting curves was higher than $98 \%$. The shape parameters $\beta$ of two kinds of corrosion products were 1.95 and 2.08 respectively. They were similar because their corrosion mechanism was same, galvanic corrosion, which was not dependent on the relative location between pads and solder mask. The mean length $\eta$ of the first kind of corrosion products was $21.2 \mu \mathrm{m}$, but that of the second kind of corrosion products, $98.1 \mu \mathrm{m}$, was 4.6 times of the first kind of products. Therefore, the second kinds of creep corrosion products would be detrimental for degradation of ImAg finished PCB. 


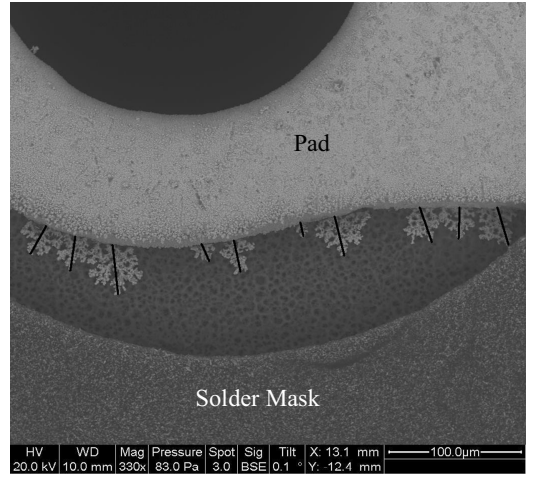

(a) Ten dendrites of the first kind of creep corrosion products

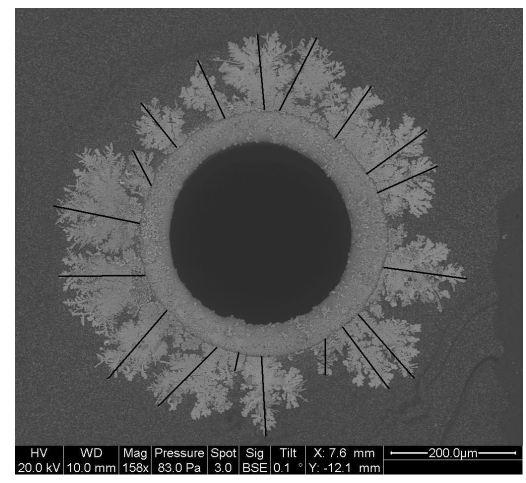

(b) Eighteen dendrites of the second kind of creep corrosion products

Figure 11. Measurement of the length of two kinds of creep corrosion products on PCB No. 2

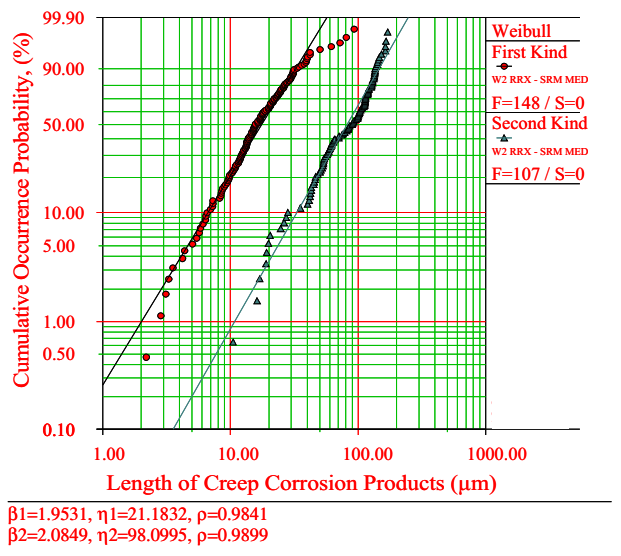

Figure 12. Statistic of lengths of two kinds of creep corrosion products on

PCB No.2

\section{ASSESSMENT OF THE IMAG FINISHED PCBS}

Since the creep corrosion products can cause degradation of the surface insulation of pads in vicinity on PCB, the length of the second kind of creep corrosion products would be concerned as a key assessment parameter for corrosion resistance of $\operatorname{ImAg}$ finished PCB. Therefore, the length statistics of creep corrosion products was used to evaluate the corrosion resistance of two ImAg finished PCBs with different finish thickness.

Both of the two PCB samples (No.1 and No.2) were corroded for uninterrupted three days. Then the length of the second kind of creep corrosion products on the two PCBs was measured, the results are shown in Figure 13. The main information is listed in Table IV.

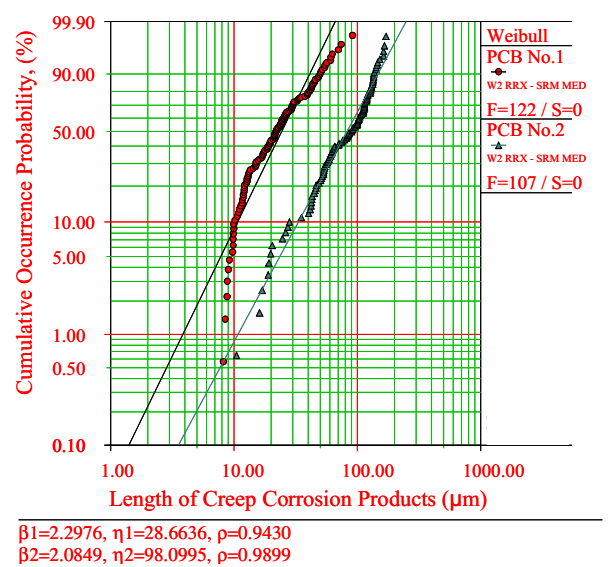

Figure 13. Weibull distribution of the length of the second kind of the creep corrosion products on two PCBs with different finish thickness

TABLE IV. THE STATISTICS OF THE LENGTH OF CREEP CORROSION PRODUCTS ON TWO PCBS

\begin{tabular}{|l|c|c|}
\hline \multicolumn{1}{|c|}{ Series No. of PCB } & $\mathbf{1}$ & $\mathbf{2}$ \\
\hline Number of detected Pads & 13 & 15 \\
\hline Numner of detected dendrites & 122 & 107 \\
\hline Max. length of dendrite $(\boldsymbol{\mu m})$ & 91.57 & 170.65 \\
\hline Min. length of dendrite $(\boldsymbol{\mu m})$ & 8.24 & 10.50 \\
\hline Mean length of dendrite $\boldsymbol{\eta}(\boldsymbol{\mu m})$ & 28.66 & 98.10 \\
\hline Shape factor $\boldsymbol{\beta}$ & 2.30 & 2.08 \\
\hline Confidence Level $\boldsymbol{\rho}$ & 0.94 & 0.99 \\
\hline
\end{tabular}

The shape factor of the Weibull distribution plot on PCB No.1 was 2.30, similar to that on PCB No.2, 2.08, because they had same corrosion mechanism. The mean length of the second kind of creep corrosion products on PCB No. 2 was $98.1 \mu \mathrm{m}, 3.4$ times of that on PCB No.1, 28.66 $\mu \mathrm{m}$. The longest dendrite was $170.65 \mu \mathrm{m}$ on PCB No.2. This was caused by the different 
thickness of ImAg finish. If the ImAg finish thickness increased, the opportunity and areas of $\mathrm{Cu}$ exposed would decrease, then the length of creep corrosion products would reduce. It showed that the PCB No.1 has the higher corrosion resistance since the thickness of ImAg finish on PCB No.1 was 1.6 times of that on PCB No.2.

\section{DISCUSSIONS}

\section{A. Analysis of the Corrosion Mechanisms}

Chemical corrosion occurred on the ImAg finish surface in a high sulfur environment. Silver sulfide was formed, which caused the color on the pad surfaces to change to dark red, but this corrosion product did not creep. Both the first and the second kind of the creep corrosion products with dendrite shape were formed by galvanic corrosion, mainly copper sulfide and a little silver sulfide. The differences between the first and the second creep corrosion products were the reaction speeds, the length and compactness of corrosion products, which depended on the relative location between ImAg finished pads and solder mask.

During the manufacturing process of PCBs, the PCBs with copper metallization are covered by solder mask, and then ImAg process is followed. The ImAg process involves cleaning of the bare copper, microetching, predipping, immersion silver deposition and a drying process. The silver bath selectively deposits a layer of silver of a certain thickness onto all of the exposed copper surfaces of the PCB. The plating process will cease automatically when the entire copper surface has been plated.

If the pads on the PCB were not covered by the solder mask, the slopes of the pad edges were also coated by ImAg finish. Only when some small pores and defects of substrate $\mathrm{Cu}$ along the slopes of the pad edges were exposed to the high sulfur environment, the substrate $\mathrm{Cu}$ could form copper sulfide dendrites by galvanic corrosion. These corrosion products were named as the first kind of creep corrosion products. The width of the exposed $\mathrm{Cu}$ along the pad edges was usually less than 5 $\mu \mathrm{m}$ individually and appeared as discontinuous states, which led to a slow reaction speed, so that the dendrites appeared compact. The mean creep distance of this kind of corrosion products was small, $21.2 \mu \mathrm{m}$ on PCB No. 2 after three days of corrosion. Since the $\mathrm{Cu}$ substrate was slightly consumed and the creep speed of the copper sulfide was slow, only a little $\mathrm{Ag}$ migrated from the pads into the creep corrosion products.

On the other hand, if the pad edges on the PCBs were fully covered by the solder mask, it was possible for narrow but long (tens of micrometers to hundreds of micrometers) crevices between the solder mask and the substrate $\mathrm{Cu}$ to exist, which led to a quick reaction speed of galvanic corrosion. These creep corrosion products were named by the second kind. The shapes of the second kind of creep corrosion products changed from the discrete crystal particles close to the pad edges to the compact dendrites far away from the pads. It can be deduced that the corrosion reaction was very strong and quick, so that it did not have enough time to form the "roots" and "stems" of dendrites close to the pad edges. As the corrosion products crept outward, the moving speed decreased, then the dendrites grew up from the discrete crystals and were piled up by many small crystals. Copper sulfide was the main composition. The Ag migrated towards the creep corrosion products due to the diffusion of $\mathrm{Cu}$. The $\mathrm{Ag}$ was also corroded in the high sulfur environment to form silver sulfide. The mean creep distance of the second kind of corrosion products was 4.6 times of the first kind of corrosion products on PCB No.2.

\section{B. The methods to improve the corrosion resistance of $\operatorname{ImAg}$ finished PCBS}

By comparing the mean lengths of the creep corrosion products on PCB No. 1 and No.2, it showed that the lengths of dendrites on PCB No.1 with $0.36 \mu \mathrm{m}$ thick ImAg finish was much shorter than those on PCB No.2 with $0.22 \mu \mathrm{m}$ thick ImAg finish. The reason is that the quantity and the size of the pores and defects on ImAg finish will reduce as the thickness of ImAg finish increases, so the extent of galvanic corrosion will drop at the same time. Therefore, increasing the thickness of ImAg finish can improve the creep corrosion resistance of ImAg finished PCBs.

Since the mean length of the first kind of creep corrosion products was much shorter than the second kind, reducing the occurrence probability of the second kind of creep corrosion products can improve the creep corrosion resistance of $\operatorname{ImAg}$ finished PCBs. That means the solder mask should be controlled to separate from pad edges, so that the slopes of pad 


\section{ACKNOWLEDGEMENT}

edges can be covered by $\operatorname{ImAg}$ finish.

\section{Corrosion method using clays}

Industrial clay was adopted as sulfur source to drive creep corrosion, which comes from one of real industrial environment where ImAg finished PC technology applies. The corrosion levels can be adjusted by changing the weight of clay, heating times and water to simulate harsh or mild environments. This method is not only valid to drive creep corrosion on ImAg finish and assess corrosion resistance for ImAg finish in high sulfur environment, but also much easier to operate and more cost effective than MFG test system. Since the sulfur released from clays could not be strictly controlled, it is recommended that all the comparison of various samples should be based on the same corrosion test.

\section{CONCLUSIONS}

Heating the clays with water can be used to drive creep corrosion on ImAg finished PCB. Silver sulfide film formed on ImAg finish by chemical corrosion. The creep corrosion products with dendrite shape formed on pad edges, which were caused by galvanic corrosion. The substrate copper as the anode lost electrons to form copper cations, which diffused in water film condensed on PCB surface and formed copper sulfide dendrites by reacting with sulfur evaporated from clays. The dendrites contained a little silver sulfide since part of silver finish close to the pad edges migrated to the dendrites as the copper diffused, and formed silver sulfide with sulfur finally. The characteristics of the two kinds of creep corrosion products were dependent on the relative location of the pad edges and the solder mask, which caused the different coverage extent of ImAg finish on copper substrate. The second kind of creep corrosion products, much longer than the first kind, was determinative to the shorting risk. The creep corrosion products can degrade the SIR of PCB to tens of Kilo-Ohms. Weibull distribution with two parameters was used to analyze the length of dendrites and to quantitatively evaluate the corrosion resistance of $\operatorname{ImAg}$ finish. The creep corrosion resistance of ImAg finish can be improved by increasing the thickness of the ImAg finish and by separating the solder mask from the pad edges.
The authors are very grateful to Prof. Jigao Zhang's discussion and review of this paper.

\section{REFERENCES}

[1] Ganesan S. and Pecht M., "Lead-Free Electronics", John Wiley and Sons, Inc., New York, 2006.

[2] Vianco P., "An Overview of Surface Finishes and Their Role in Printed Circuit Board Solderability and Solder Joint Performance", Circuit World, Vol. 25, No. 1, pp. 6-24, 1998.

[3] Lopez E., Vianco P., Buttry R., Lucero S., and Rejent J., "Effect of Storage Environments on the Solderability of Immersion Silver Board Finishes with Pb-Based and Pb-Free Solders", SMTA Journal, Vol. 18, Iss. 4, pp. 19-28, Oct., 2005.

[4] Vianco P., Lopez E., Buttry R., Kilgo A., and Lucero S., "Effects of Accelerated Storage Environments on the Solderability of Immersion Silver-Coated Printed Circuit Boards", Pan Pacific Symposium, Hawaii, Jan. 17, 2006.

[5] Graedel T.E., "Corrosion Mechanisms for Silver Exposed to the Atmosphere", Journal of the Electrochemical Society, Vol. 139, No. 7, pp. 1963-1970, July 1992.

[6] Cheung Y.M., Or S.W., Sze A. and Chung K., "Revisit of Wirebonding on Immersion Silver-Finish Board", International Symposium on Electronic Materials and Packaging, 2000, Hong Kong, China, pp. 444-448, Nov., 2000

[7] Norwood E. and Swanson J., "Enhancing the Tarnish Performance of Immersion Silver Finishes", SMTA International, Chicago, Illinois, Sep. $25,2005$.

[8] United States Environmental Protection Agency, "Alternative Technologies for Surface Finishing: Cleaner Technologies for Printed Wiring Board Manufacturers", Technical report, EPA-744-R-01-001, pp. 1-42, June 2001.

[9] Mazurkiewicz P., "Accelerated Corrosion of Printed Circuit Boards due to High Levels of Reduced Sulfur Gasses in Industrial Environments", Proceedings of the 32nd International Symposium for Testing and Failure Analysis, pp. 469-473, Austin, TX, Nov. 12-16, 2006.

[10] Schueller R., "Creep Corrosion on Lead-free Printed Circuit Boards in High Sulfur Environments", SMTA International Conference, Orlando, Fl, pp. 643-654, Oct. 11, 2007.

[11] Zhao P., Pecht M., "Field Failure Due to Creep Corrosion on Components with Palladium Pre-plated Leadframes", Microeletronics Reliability, Vol. 43, No. 5, pp. 775-783, 2003.

[12] Zhao P., Pecht M., "Mixed Flowing Gas Studies of Creep Corrosion on Plastic Encapsulated Microcircuit Packages with Noble Metal Pre-plated Leadframes", IEEE Transactions on Device and Materials Reliability, Vol. 5, No. 2, pp. 268-276, June 2005.

[13] Zhao P., Pecht M., "Assessment of $\mathrm{Ni} / \mathrm{Pd} / \mathrm{Au}-\mathrm{Pd}$ and $\mathrm{Ni} / \mathrm{Pd} / \mathrm{Au}-\mathrm{Ag}$ Preplated Leadframe Packages Subject to Electrochemical Migration and Mixed Flowing Gas Tests", IEEE Transactions on Components and Pachaging Technologies, Vol. 29, No. 4, pp. 818-826, Dec., 2006.

[14] Conrad, L.R., Pike-Biequnski, M.J., and Freed, R.L., "Creep Corrosion over Gold, Palladium and Tin-Lead Electroplate," 15th Annual Connectors and Interconnection Technology Symposium Proceedings, Philadelphia, PA, pp. 401-414, Nov., 1982.

[15] Cullen D., "Surface Tarnish and Creeping Corrosion on Pb-free Circuit Board Surface Finishes", IPC Works, 2005.

[16] Xu C., Flemming D., Demerkin K., "Corrosion resistance of PWB Surface Finishes”, Apex, Los Angeles, CA, Feb. 20-22, 2007.

[17] Wu, E.Y.; Vollertsen, R.-P., "On the Weibull shape factor of intrinsic breakdown of dielectric films and its accurate experimental determination. Part I: theory, methodology, experimental techniques", IEEE Transactions on Electron Devices, Volume 49, Issue 12, pp.2131-2140, Dec. 2002

[18] Wu, E.Y.; Sune, J.; Lai, W.; "On the Weibull shape factor of intrinsic breakdown of dielectric films and its accurate experimental determination. Part II: experimental results and the effects of stress 
conditions", IEEE Transactions on Electron Devices, Volume 49, Issue 12, pp.2141 -2150, Dec. 2002

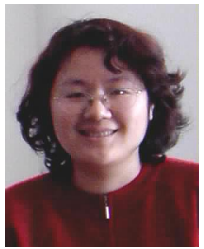

Yilin Zhou, Automation School, Beijing University of Posts and Telecommunications, internal box 71, Beijing, 100876, P. R. China, Emai: ylzhou@bupt.edu.cn , Tel/Fax : 86-10-62283173

She received the B. S. degree in mechanical and electrical engineering and $\mathrm{Ph}$. D. degree in circuit and system from Beijing University of Posts \& Telecommunications, China, in 1994 and 1999 respectively. She is now an associated professor in Automation School, Beijing University of Posts \& Telecommunications. She worked in the Center for Advanced Life Cycle Engineering (CALCE), University of Maryland, College Park as a visiting scholar from Aug., 2007 to Aug., 2008. She is interested in the study of contact failure caused by contamination and fretting, quality analysis of gold plating for connectors, the mechanism and application of lubricants for electric contacts. She has published more than 30 papers.

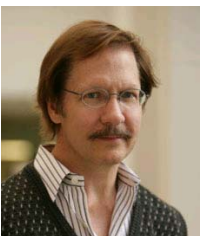

Michael Pecht, Visiting Professor in Electrical Engineering City University of Hong Kong, and Director CALCE Electronics Products and Systems Center University of Maryland - USA

Prof Michael Pecht is currently visiting Professor in Electrical Engineering at City University of Hong Kong. He has an MS in Electrical Engineering and an MS and PhD in Engineering Mechanics from the University of Wisconsin at Madison. He is a Professional Engineer, an IEEE Fellow, an ASME Fellow and an IMAPS Fellow. He was awarded the highest reliability honor, the IEEE Reliability Society's Lifetime Achievement Award in 2008. He served as chief editor of the IEEE Transactions on Reliability for eight years and on the advisory board of IEEE Spectrum. He is chief editor for Microelectronics Reliability and an associate editor for the IEEE Transactions on Components and Packaging Technology. He is the founder of CALCE (Center for Advanced Life Cycle Engineering) at the University of Maryland, College Park, where he is also the George Dieter Chair Professor in Mechanical Engineering and a Professor in Applied Mathematics. He has written more than twenty books on electronic products development, use and supply chain management and over 400 technical articles. He has been leading a research team in the area of prognostics for the past ten years. He has consulted for over 100 major international electronics companies, providing expertise in strategic planning, design, test, prognostics, IP and risk assessment of electronic products and systems. He has previously received the European Micro and Nano-Reliability Award for outstanding contributions to reliability research, 3M Research Award for electronics packaging, and the IMAPS William D. Ashman Memorial Achievement Award for his contributions in electronics reliability analysis. 


\title{
Electrical Resistance of Connectors with Different Contact Finishes under Low Level Conditions
}

\author{
J. Song and H. Schmidt* \\ University of Applied Sciences \\ Liebigstr. 87, 32657 Lemgo, Germany \\ Email: jian.song@hs-owl.de \\ *Tyco Electronics AMP GmbH, Bensheim, Germany
}

\begin{abstract}
In order to find out the behaviors of the contact resistances in the low current and low voltage range before physically starting the fritting phenomenon, several connector designs and contact finishes were investigated. The used finishes were hard-gold, silver, hot dip tin and electroplated tin. Similar coating combinations, in addition to different coating combinations, are used for these investigations. The environmental degradation was conducted by applying sequentially high temperature storage, mixed flowing gas and high humidity storage. Our investigations with new and degraded samples show that neither measurement methods nor low level current used for the measurements result in a difference in electrical resistance of new or environmentally degraded connectors. No fritting process was observed at relevant current levels. A measurement of contact resistance at the $100 \mathrm{~mA}$ level should adequately ensure performance at any lower level of current. Many metrological and instrumental efforts due to fritting can therefore be reduced.
\end{abstract}

Keywords - Electrical resistance, Low Level Conditions

\section{INTRODUCTION}

The electrical breakdown of the high resistant film on the electrical contacts is called fritting [1-4]. A high resistant film in the contact interface, as result of contaminations and several chemical processes, can cause variant contact resistances. A sign of the presence of these effects is the fritting phenomenon. There is a lot of discussion in the application field that the fritting process is not only an effect of switching contacts but also an issue of connector contacts. In case of a high resistant film existing on the contact surface, this can be broken down by an electrical potential gradient of $>10^{8} \mathrm{~V} / \mathrm{m}$, which means a voltage of more than $100 \mathrm{mV}$. In order to avoid the influence of fritting, caused by field emission through the thin layers, measurements are conducted with the maximum open circuit voltage of $20 \mathrm{mV}$. This requires the application of special instruments. Connector users, on the other hand, raise the question, under which conditions and how the contact resistances change due to fritting. Earlier investigations showed that measurements conducted with currents as high as $100 \mathrm{~mA}$ but below a critical voltage accurately indicated the quality of the contact at any lower value of currents. However, environmentally degraded samples were not included in the investigations [5]. Another study showed that this conclusion is also true for environmentally degraded samples with tin plates [6]. The limitation of the open circuit voltage of current source increases requirements and cost for test equipment.

This paper deals with the following issues:

- The behavior of connectors with different combinations of plates and different designs before and after environmental degradation at low level conditions

- The influences of test methodology and test equipment on the results

\section{INVESTIGATED CONNECTORS}

Two different connectors (Fig. 1a-b) with different plates (hard-gold, silver, hot-dip tin and electroplated tin) were used for the investigation (Tab. 1). The width of the JPT connectors (Junior Power Timer) is $2.8 \mathrm{~mm}$ and that of MT connectors (Micro Timer) is $0.6 \mathrm{~mm}$.
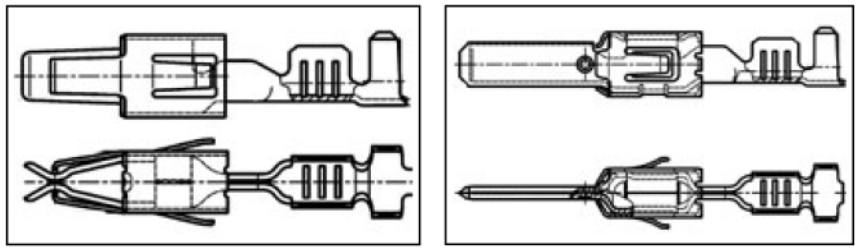

Fig. 1a: Junior Power Timer (JPT)
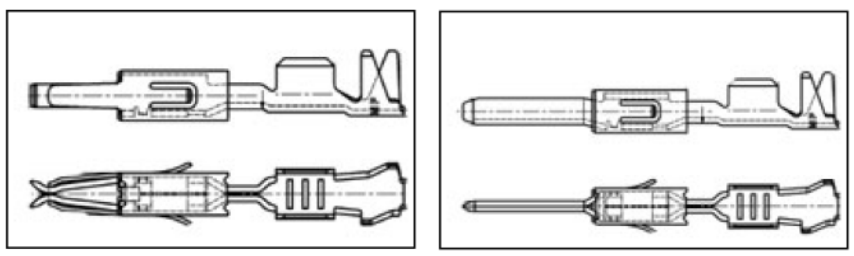

Fig. 1b: Micro Timer (MT) 
Tab. 1a, b: JPT and MT connectors of different plates

\begin{tabular}{|c|c|c|c|c|}
\hline male & $\mathrm{Au}$ & $\mathrm{Ag}$ & Sn hot & Sn gal \\
\hline $\mathrm{Au}$ & $\mathrm{x}$ & & & \\
\hline $\mathrm{Ag}$ & & $\mathrm{x}$ & $\mathrm{x}$ & \\
\hline Sn hot & & & $\mathrm{x}$ & \\
\hline Sn gal & & $\mathrm{x}$ & $\mathrm{x}$ & $\mathrm{x}$ \\
\hline
\end{tabular}

\begin{tabular}{|c|c|}
\hline $\mathrm{Au}$ & Sn hot \\
\hline $\mathrm{x}$ & \\
\hline
\end{tabular}

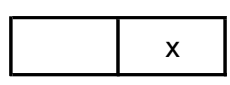

\section{Test Methodology AND Test EQUIPMENT}

Three different combinations of test equipments were used for the investigation [7]. Device combination A with a battery and a potential divider is the most time-consuming method, which eliminates all the possible influences of an electronic power source.

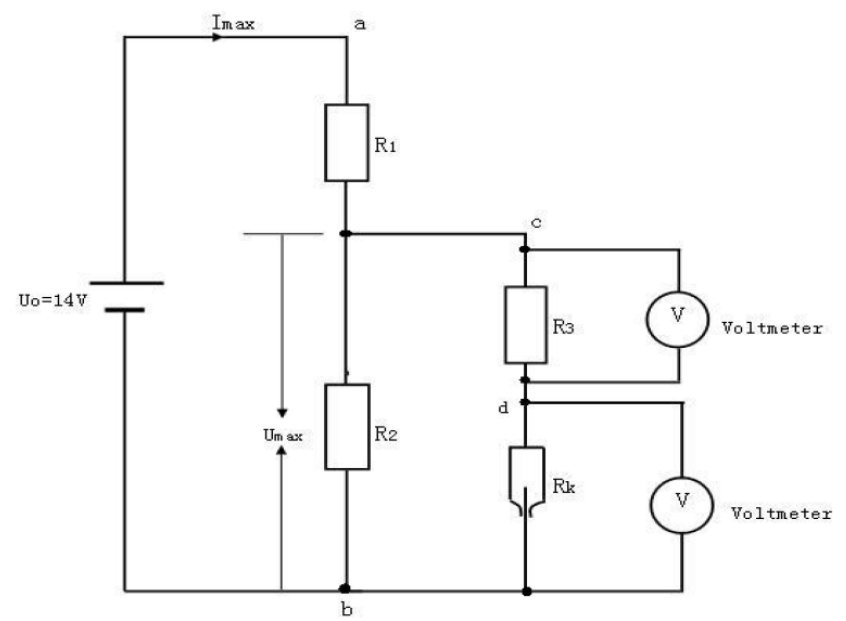

Fig. 2: Circuit of Method "Potential Divider"

Device combination B with a power source without a limitation of open circuit voltage is often not applied due to concerns that possible voltage peaks could hide a high resistant film between the electrical contacts.

This could be theoretically avoided by using device combination $\mathrm{C}$ with a power source with a limitation of open circuit voltage.

All the measurements were conducted with current in both the forward $(+)$ and reverse (-) direction in order to avoid influences due to different standard electrode potentials on the contact surface.

\section{A. Potential DIVIDER}

Measuring currents of $3 \mathrm{~mA}, 10 \mathrm{~mA}$ and $100 \mathrm{~mA}$ combined with $20 \mathrm{mV}$ and $100 \mathrm{mV}$ respectively were realized by different combinations of resistances. The circuit of this measurement method is shown in Fig. 2. The advantage of this method is that all the possible influences due to the behavior of electronics, which could lead to voltage or current peaks, are eliminated. Any difference between contact resistance at high open source voltage and at low open source voltage would be observed.

\section{B. ELECTRONIC CURRENT SOURCE WITHOUT LIMITATION OF OPEN-CIRCUIT-VOLTAGE}

The dry-circuit method (four-point probe resistivity measurement) was used for the measurements of the contact resistance. An oscilloscope was used for observing the transient voltage. The trigger voltage was $20 \mathrm{mV}$. The voltmeter used was a Keithley Nanovoltmeter 2182A. The power source was a Keithley 6220. The currents for the measurements were $3 \mathrm{~mA}, 10 \mathrm{~mA}$ and $100 \mathrm{~mA}$. All connectors were mounted without housing on the test board so that micro-motion could be minimized (Fig. 3).

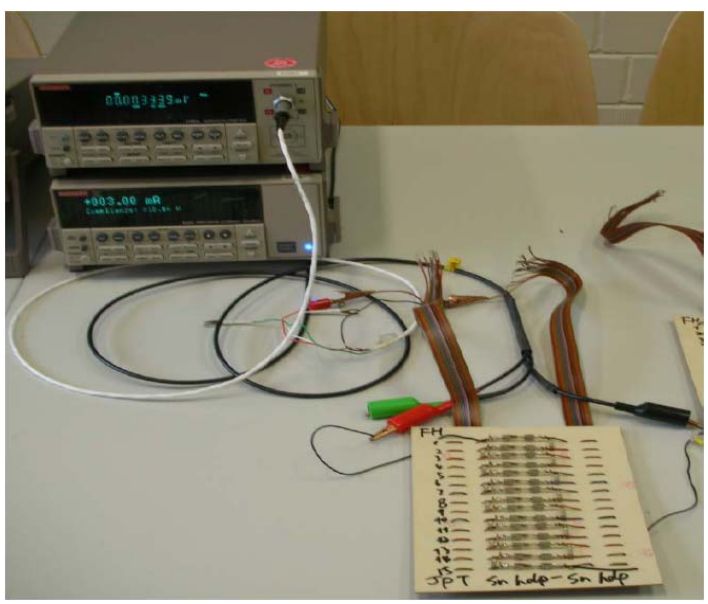

Fig. 3: Testing with electronic current source and nanovoltmeter

\section{ELECTRONIC CURRENT SOURCE WITH LIMITATION OF OPEN- CIRCUIT-VOLTAGE}

A multimeter (Keithley 3706) with an integrated current source was used for this measurement method. The opencircuit-voltage was limited to $27 \mathrm{mV}$. In case that any fritting effect exists, this limit should guarantee that the tarnish film will not be broken down because of the voltage peak of the current source.

\section{ENVIRONMENTAL DEGRADATION}

The investigated connectors are environmentally degraded in three steps between the initial measurements and final measurements. 


\section{Step I: High Temperature Storage [8]}

At this step the connectors were put into a chamber at $120^{\circ} \mathrm{C}$ for 120 hours.

\section{Step II: Mix Flowing Gas}

A mixture of gases $\left(\mathrm{H}_{2} \mathrm{~S}, \mathrm{Cl}_{2}, \mathrm{SO}_{2}\right.$ and $\left.\mathrm{NO}_{2}\right)$ with corrosive effects on contact surfaces was flowing into the chamber of the connectors. The chamber temperature was $25^{\circ} \mathrm{C}$ and the duration of the storage was 21 days.

\section{Step III: High Humidity Storage}

A climatic chamber was used for this step. The relative humidity was $95 \%$ and the duration of the storage was 21 days. The temperature was changed cyclically. The temperature cycle is shown in Fig. 4.

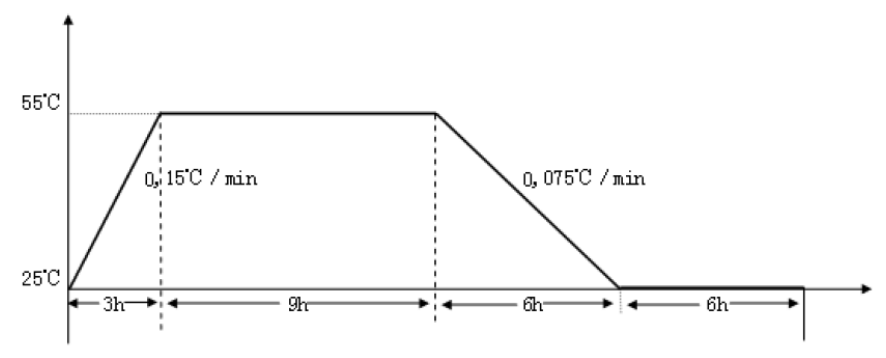

Fig. 4: Temperature cycle

\section{RESULTS AND DISCUSSION}

\section{A. INFLUENCE OF TEST METHOD}

Fig. 5.1a-g compare the contact resistances measured with different device combinations, at different currents with different open circuit voltage limits for gold, silver and tin. Every bar in the graphs shows a plus and minus one-standard deviation value.

The results of the final measurements with different devices are shown in Fig. 5.1a. There is obviously no relevant difference between the results from different combinations of measurement devices.

This is also true for the measuring current of $3 \mathrm{~mA}, 100$ $\mathrm{mA}$ (Fig. 5.1b-e) and for the open circuit voltage limit of 100 $\mathrm{mV}$ (Fig. 5.1f and g). Data are shown for current in both the forward (+) and reverse (-) direction. One notices that the contact resistance does not depend on current polarity.

These behaviors can be verified also for connectors with different plates (Fig. 5.2) and a different design of connectors (Fig. 5.3).

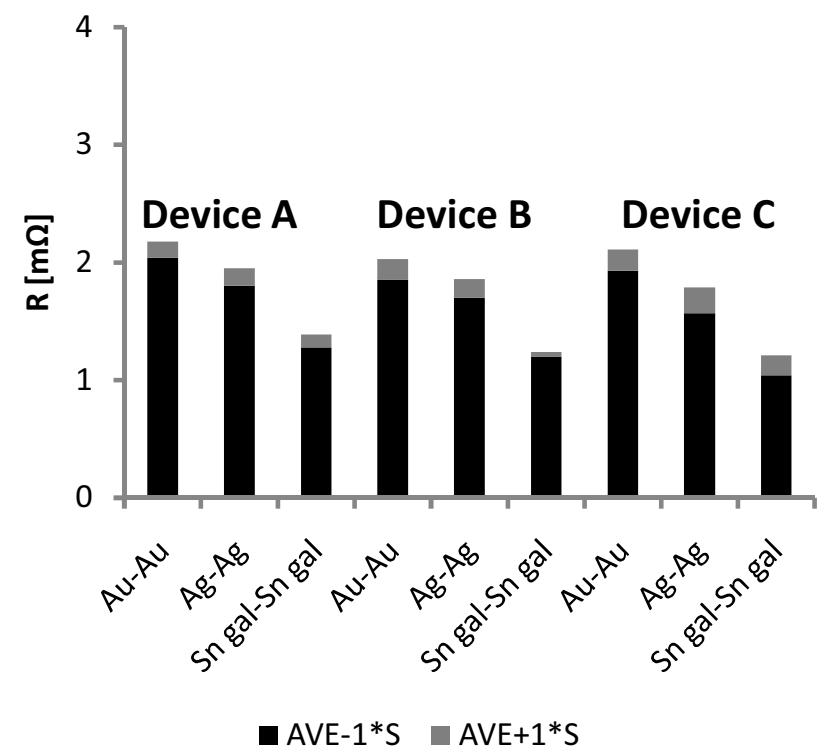

Fig. 5.1a: Influence of test methods for connectors with same plates (JPT, final measurement, $10 \mathrm{~mA}, 20 \mathrm{mV}$ )

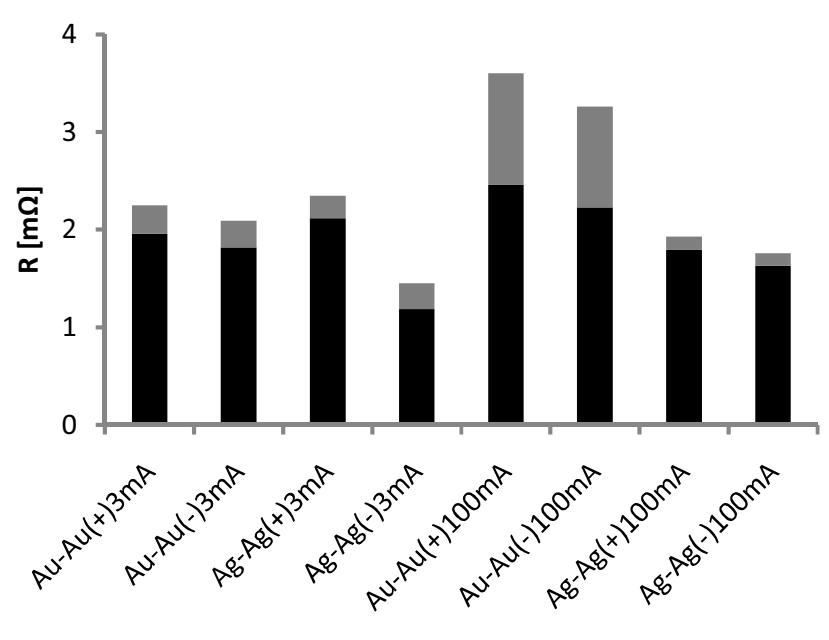

AVE-1*S AVE $+1 * S$

Fig. 5.1b: Influence of measuring current for connectors with $\mathrm{Au}$ or Ag plates (JPT, final measurement, Device A, $20 \mathrm{mV}$ ) 


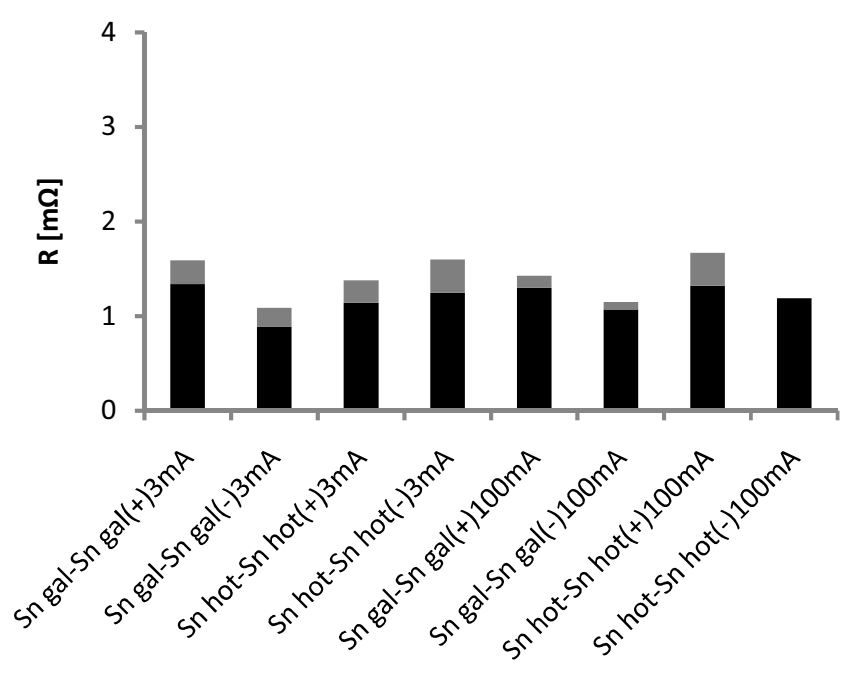

- AVE-1*S $\quad \mathrm{AVE}+1 * \mathrm{~S}$

Fig. 5.1c: Influence of measuring current for connectors with Sn plates (JPT, final measurement, Device A, $20 \mathrm{mV}$ )

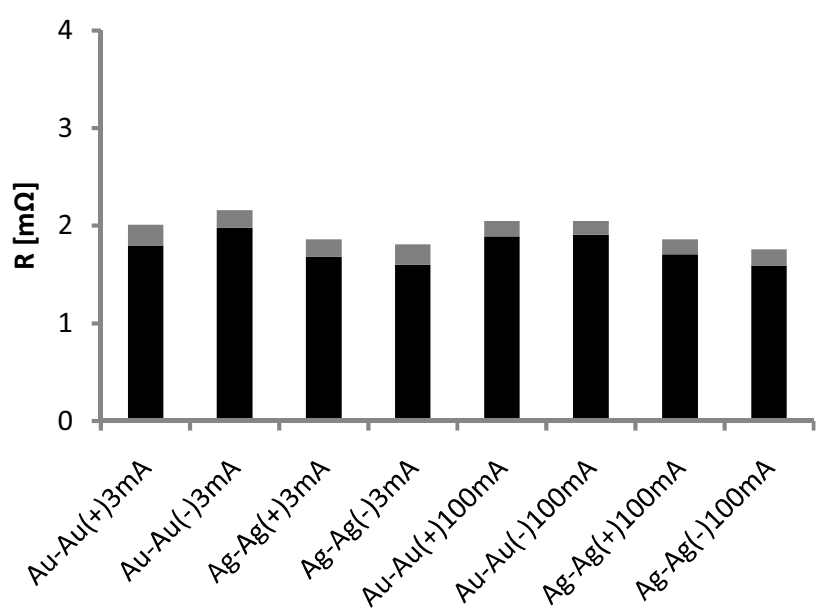

- AVE-1*S AVE+1*S

Fig. 5.1d: Influence of measuring current for connectors with $\mathrm{Au}$ or Ag plates (JPT, final measurement, Device B, $20 \mathrm{mV}$ )

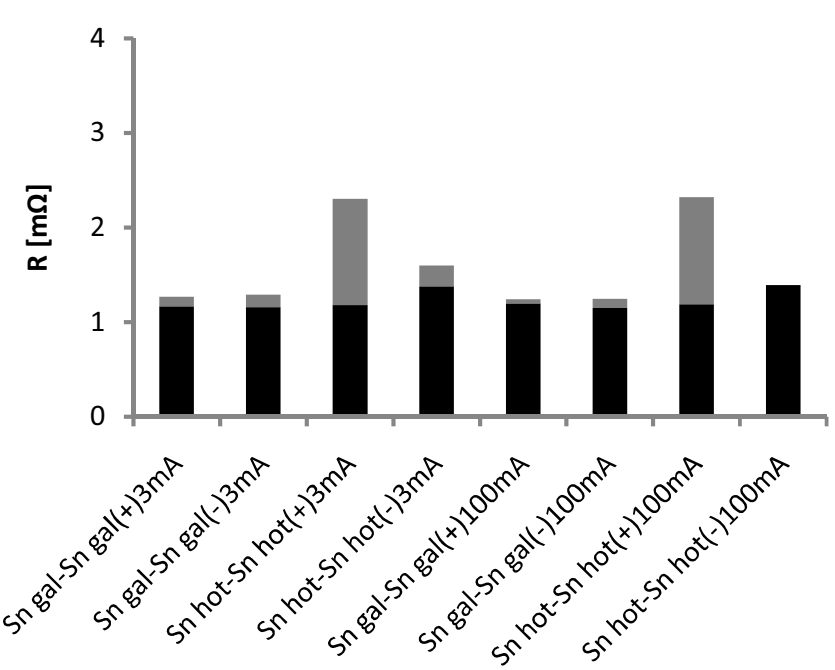

- AVE-1*S AVE $+1 * S$

Fig. 5.1e: Influence of measuring current for connectors with tin plates (JPT, final measurement, Device B, $20 \mathrm{mV}$ )

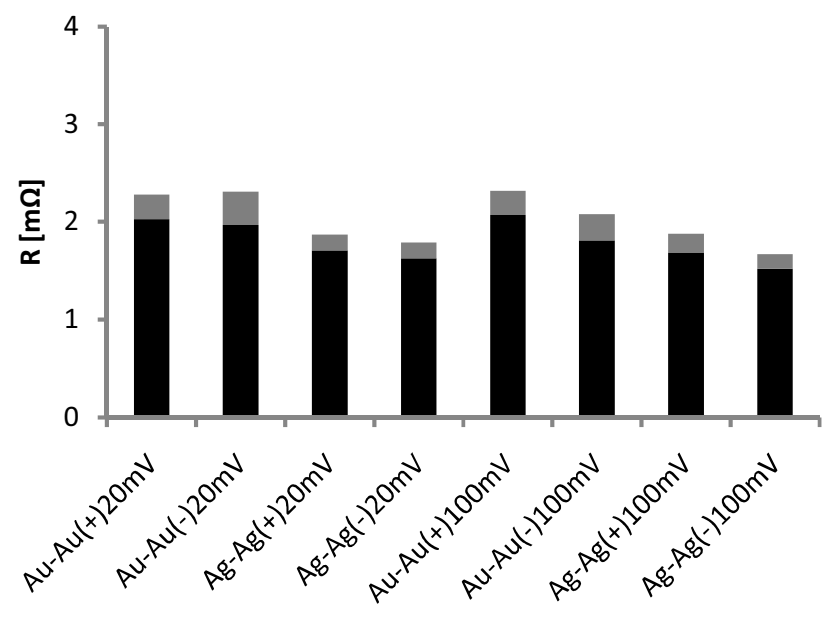

- AVE-1*S AVE+1*S

Fig. 5.1f: Influence of open circuit voltage limit for connectors with $\mathrm{Au}$ or Ag plates (JPT, final measurement, Device A, $10 \mathrm{~mA}$ ) 


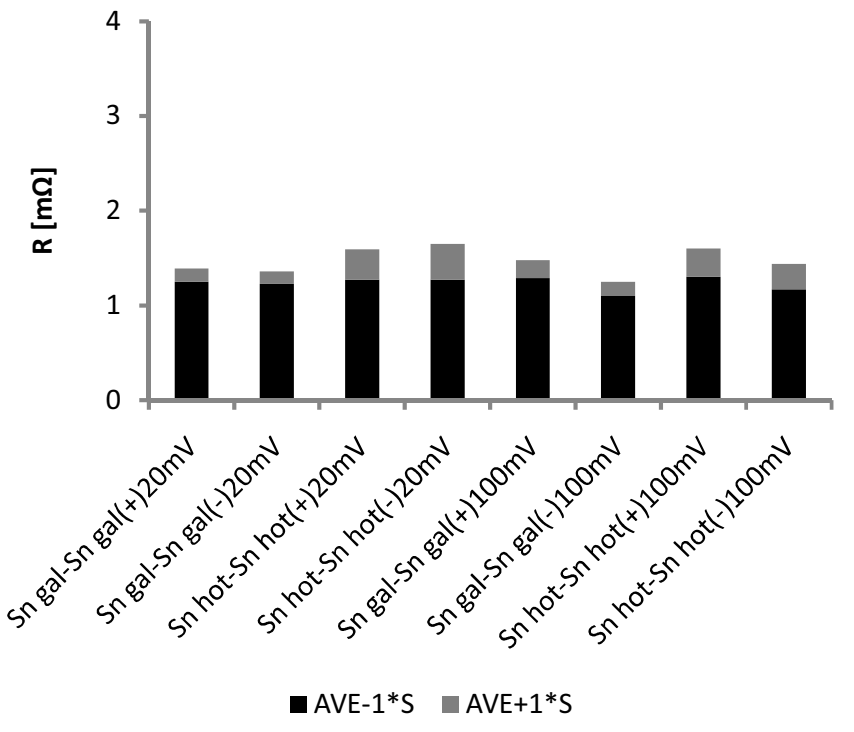

Fig. 5.1g: Influence of open circuit voltage limit for connectors with Sn plates (JPT, final measurement, Device A, $10 \mathrm{~mA}$ )

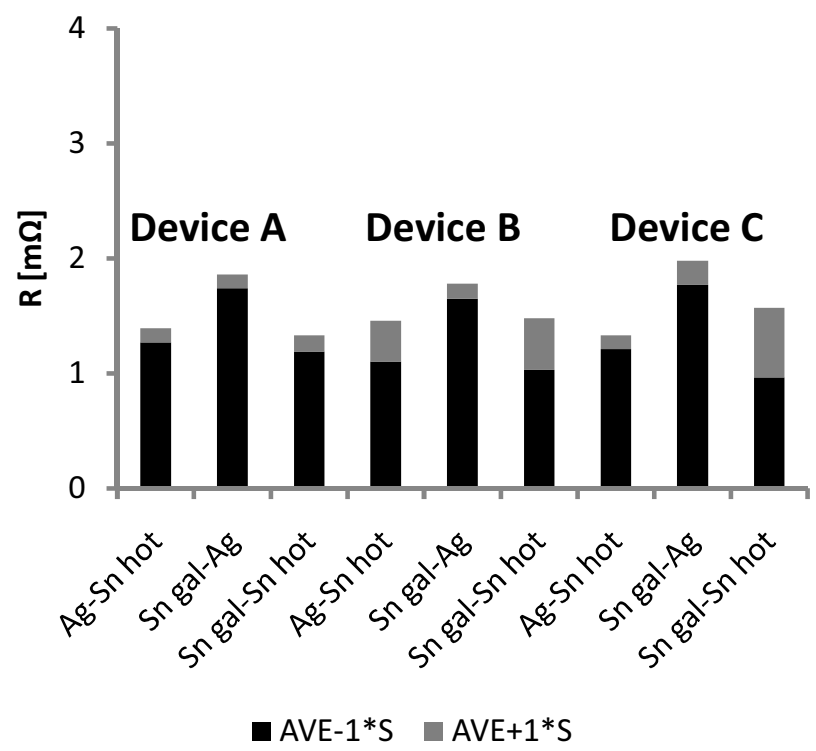

Fig. 5.2: Influence of test methods for connectors with different plates (JPT, final measurement, $10 \mathrm{~mA}, 20 \mathrm{mV}$ )

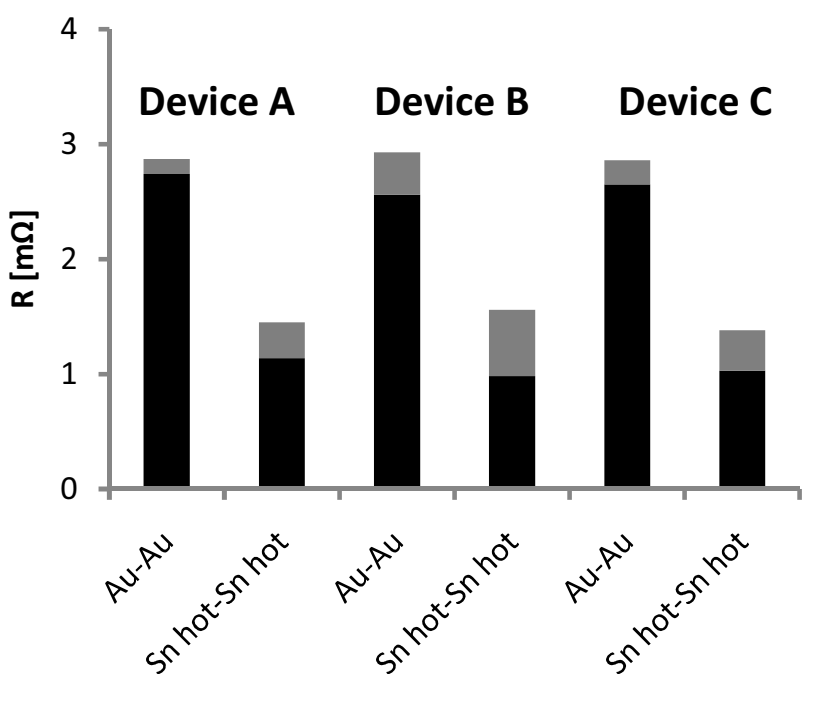

AVE-1*S AVE $+1 * S$

Fig. 5.3: Influence of test methods for connector with same plates (MT, final measurement, $10 \mathrm{~mA}, 20 \mathrm{mV}$ )

\section{B. INFLUENCE OF ENVIRONMENTAL DEGRADATION}

The following diagrams (Fig. 5.4a-d) show the contact resistance at the beginning of the investigation and after the different degradation steps. A relevant increase of contact resistance through the degradation cannot be observed. The initials in the diagrams indicate the stage, at which the measurements were conducted:
$\mathrm{V}$ : initial measurement
$\mathrm{T}$ : measurement after high temperature storage
S: measurement after mixed flowing gas degradation
F: final measurement after high humidity storage 


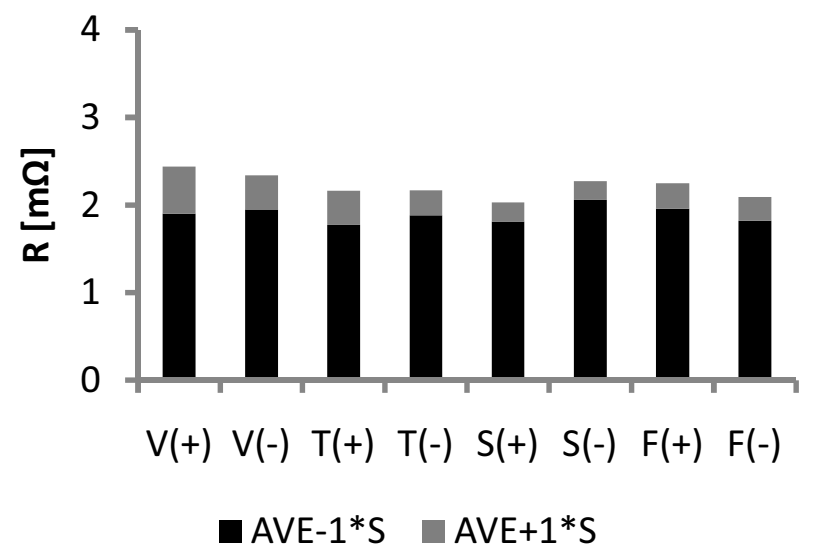

Fig. 5.4a: Contact resistance at different stage of degradation (JPT, Au-Au, $3 \mathrm{~mA}, 20 \mathrm{mV}$ )

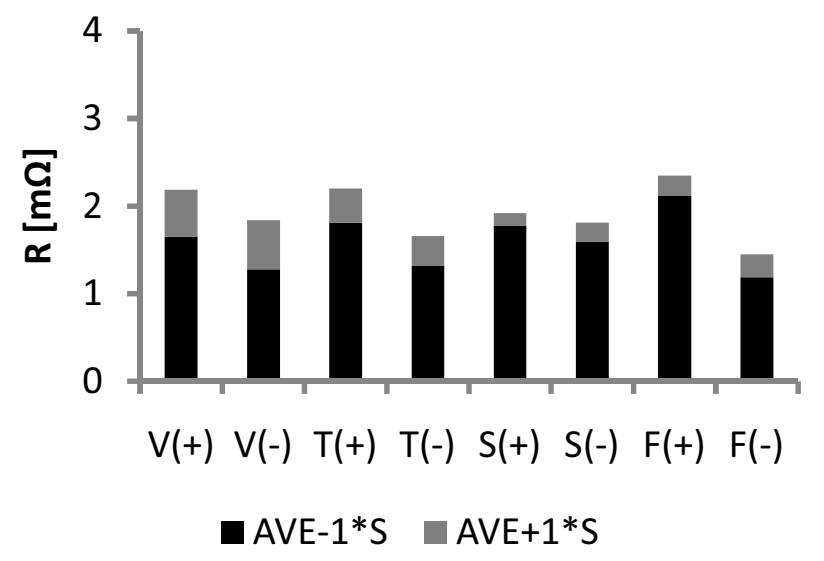

Fig. 5.4b: Contact resistance at different stage of degradation (JPT, Ag-Ag, $3 \mathrm{~mA}, 20 \mathrm{mV}$ )

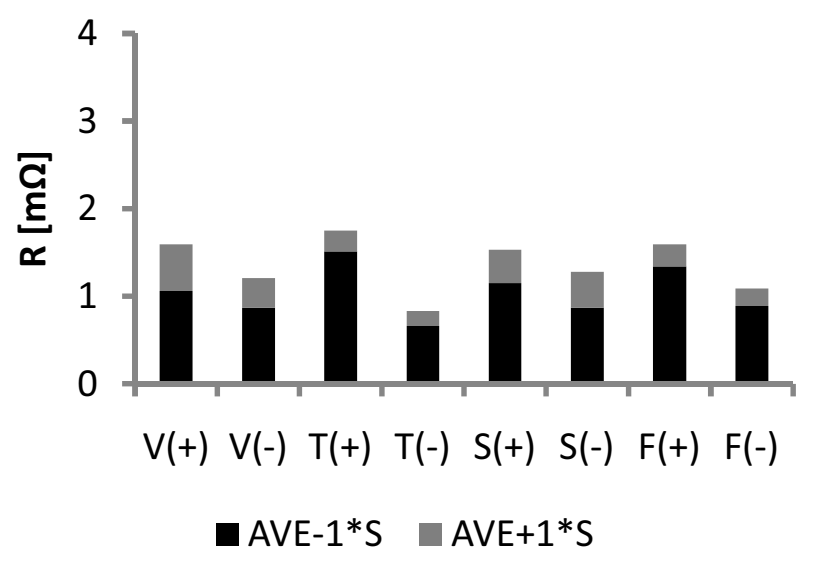

Fig. 5.4c: Contact resistance at different stage of degradation (JPT, Sn gal-Sn gal, $3 \mathrm{~mA}, 20 \mathrm{mV}$ )

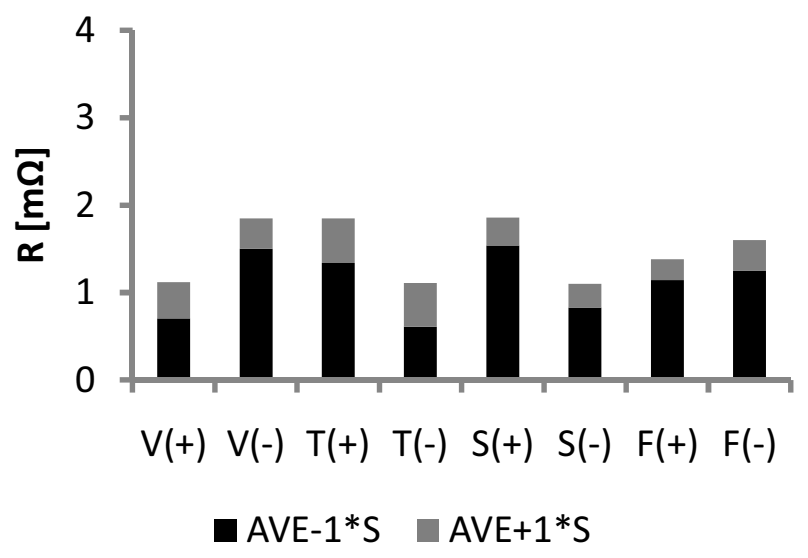

Fig. 5.4d: Contact resistance at different stage of degradation (JPT, Sn hot-Sn hot, $3 \mathrm{~mA}, 20 \mathrm{mV}$ )

\section{INFLUENCE OF PLATES}

As expected, slight differences between different plates could be observed (Fig. 5.5a and b). Gold-Gold and SilverSilver combinations show a higher resistance because of their higher hardness, which leads to smaller contact area at the same contact force.

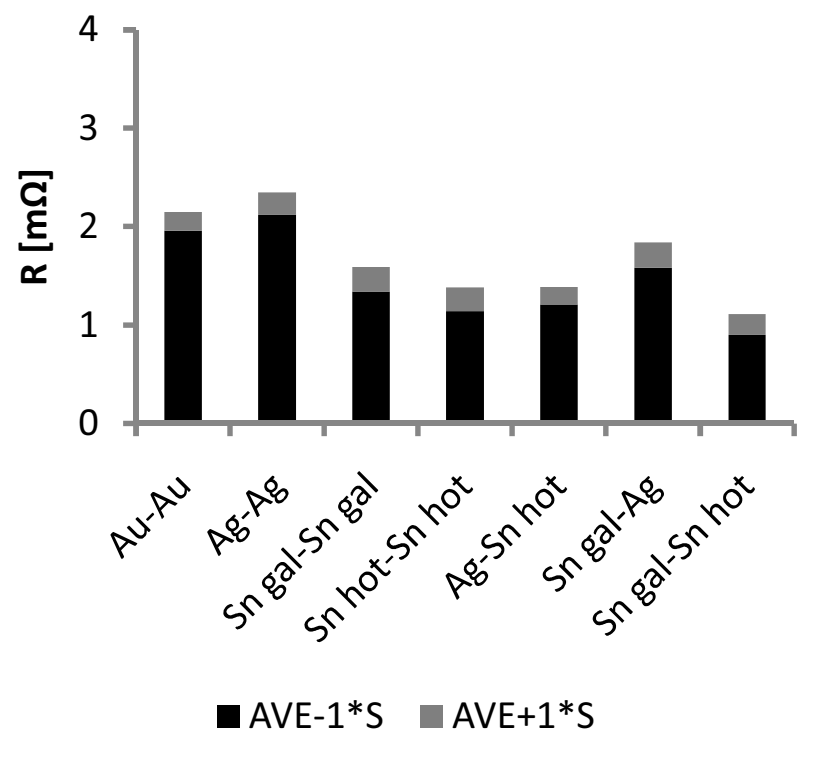

Fig. 5.5a: Contact resistance of different combinations of plates (JPT, final measurement, Device A, $3 \mathrm{~mA}, 20 \mathrm{mV}$ ) 


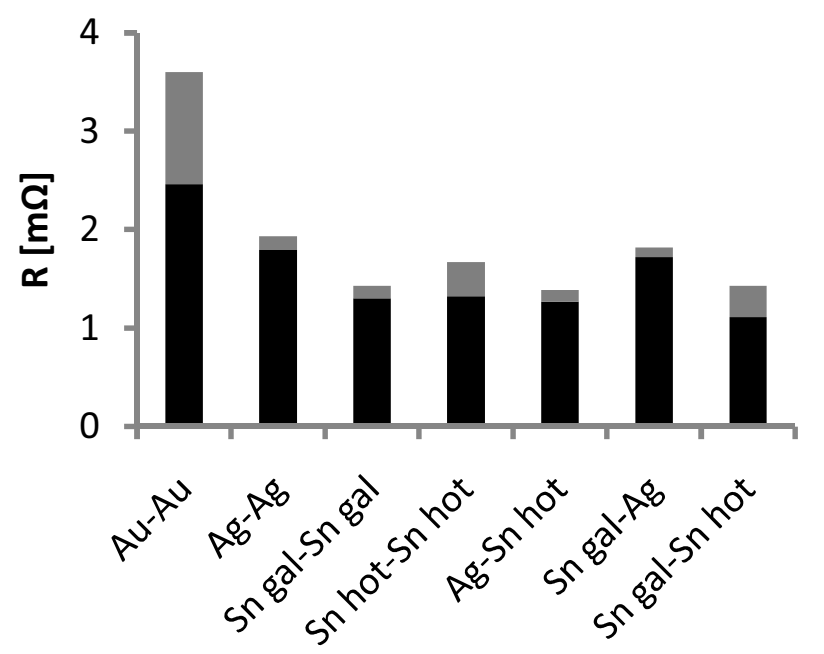

AVE-1*S $\quad A V E+1 * S$

Fig. 5.5b: Contact resistance of different combinations of plates (JPT, final measurement, Device A, $100 \mathrm{~mA}, 20 \mathrm{mV}$ )

\section{INFLUENCE OF CONNECTOR DESIGN}

The influence of design on the contact resistance is shown in Fig. 5.6.
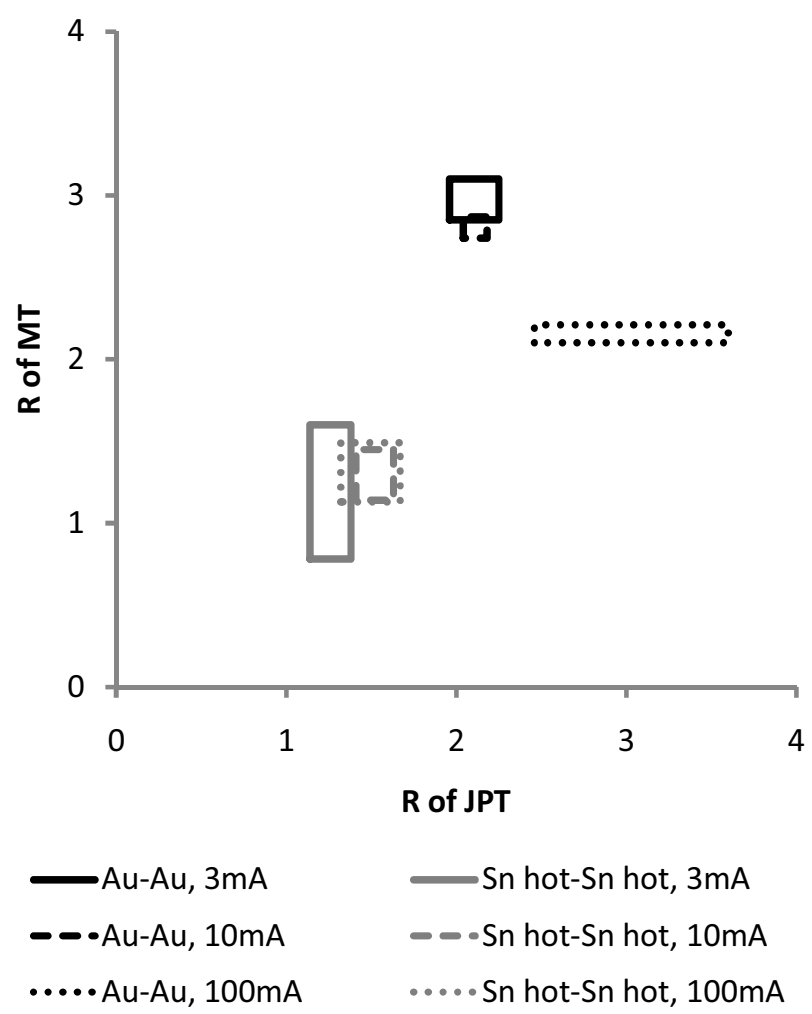

- - Sn hot-Sn hot, 10mA

....Sn hot-Sn hot, $100 \mathrm{~mA}$

Fig. 5.6: Contact resistance of different contact size
The design of Junior Power Timer and Micro Timer are similar. The main difference between them is the width, which is $2,8 \mathrm{~mm}$ for JPT and $0,8 \mathrm{~mm}$ for MT, respectively. It could be clearly observed that the influence of plates is much stronger than that of the contact size.

\section{CONCLUSION}

The electrical behavior observed for new and degraded connectors with gold, silver, hot-dip tin and electroplated tin and with different test methods shows that measurement at the upper region of the current range ( $3 \mathrm{~mA}$ to $100 \mathrm{~mA}$ ) completely characterizes contact resistance. Contact resistance is invariant with current. The requirement for the limitation of open circuit voltage of current source turns out to be overrated. Any influence of high resistance film could not be observed during the complete investigation.

\section{ACKNOWLEDGMENT}

Mrs. X. Yang conducted most of the tests. The investigation was partly supported by the Federal Ministry for Education and Research of Germany (BMBF).

\section{REFERENCES}

[1] R. Holm, Electrical Contacts. Springer, Berlin, 1996, pp. 135-152

[2] R.S. Timsit, "Electrical Contact Resistance: Fundamental Principles," in P.G. Slade (Ed.), Electrical Contacts - Principles and Applications. Marcell Dekker, New York, 1999, pp. 43-45

[3] E. Vinaricky, (Ed.), Elektrische Kontakte, Werkstoffe und Anwendungen. Springer, Berlin, 2002, pp. 38-44

[4] J. Song (Ed.), Elektrische Steckverbindungen, University OWL, Lemgo, 2006, pp. 12-16

[5] E.M. Bock, "Low Level Contact Resistance Characterization," AMP Journal of Technology, vol. 3, Nov. 1993, pp. 64-68

[6] J. Song and A. Alberani, "Fritting and Electrical Resistance of Tin Coated Contacts under Low Level Conditions," Proc. of ICEC 2008, St. Malo, pp. 262265.

[7] X. Yang, Kontaktverhalten von Steckverbindungen bei kleinen Strömen und Spannungen, Diploma thesis at University OWL, Lemgo, 2008

[8] N.N.: Environmental testing - Part 2-2: Tests - Test B: Dry heat, IEC 60068-2-2, 2007 
Jian Song received a Dipl.-Ing. (M.Sc.) degree in Precision Engineering from the Technical University in Braunschweig, and a Dr.-Ing. (PhD) degree from the University of Kassel in 1985 and 1990 respectively.

He was head of a research group at the University ErlangenNuremberg from 1990 to 1993 and R\&D Manager with firms in the electrical, electronic and automotive industry from 1993 to 2003.

Since 2003, he has been a Professor and Head of the Precision Engineering Laboratory at the University of OstwestfalenLippe in Lemgo, Germany. He is Chair of the Technical Committee for Optical and Electronic Interconnection of VDE/VDI-GMM (Microelectronics, Mechatronics and Precision Engineering Society of the German Association of Electrical and Electronic Engineers and the German Association of Engineers).

Helge Schmidt received a Dipl.-Ing. (M.Sc.) degree in 1983 and a Dr.-Ing. (PhD) degree in 1988 in Electrical Engineering, Electrochemistry and Electroplating Technologies at the University in Ilmenau.

$\mathrm{He}$ was responsible for various relays and connector manufacturing departments with Siemens AG in Speyer from 1990 to 1996 and Manager for Basic Engineering and New Technologies at the Siemens Electromechanical Business Unit until 1999. From 2000 to 2005 he worked as a manager Marketing Advanced Technology of Tyco Electronics AMP GmbH, Industrial and Commercial EMEA. Since 2006 he has been a Manager and Principal Engineer for Advanced Technology, Terminals and Connectors in the Global Automotive Division, Product Engineering EMEA, Germany. 


\section{A}

\begin{tabular}{|c|}
\hline Alamarguy, D. \\
\hline Anciant, R............. \\
\hline Ando, H. ............... \\
\hline 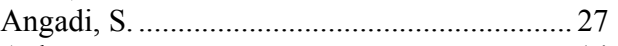 \\
\hline 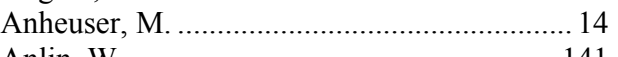 \\
\hline
\end{tabular}

\section{B}

Behrens, V. 187

Belhaja, Z. ................................................... 21

Ben jemâa, N. ................................................. 212

Bilhaut, L.................................................... 290

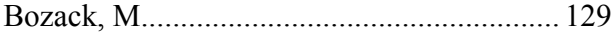

Braumann, P. .............................................. 200

Braunovic, M................................................. 232

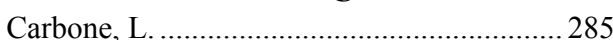

Carvou, E........................................ 21, 212

Chen, C..................................................... 129

Chen, D................................................ 243, 273

Chen, H...................................................... 7

Chen, Y............................................................. 41

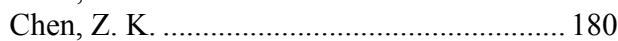

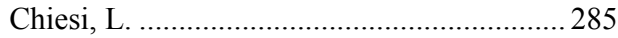

Ciocirlan, B. O............................................... 56

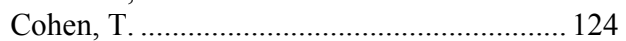

Correia, S................................................. 153

Coutu, R. A. Jr............................................... 295

Cretinon, L........................................... 212

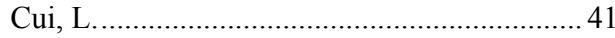

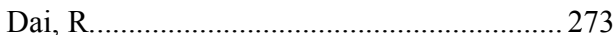

Delamare, J. ................................................ 285

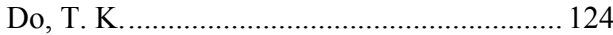

Duraffourg, L............................................... 290

\section{$\mathbf{E}$}

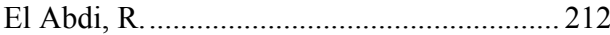

Erwin, G. S. .............................................. 94

Essone-Obame, H. ........................................ 212

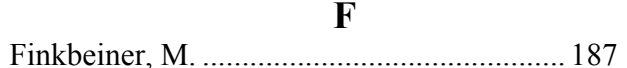

Flowers, G. T .............................................. 129

Gao, J. C. .............................................. 136

Gendre, P................................................. 153

Geng, Y. ............................................. 7, 195

Gentsch, D. ................................................. 267

\section{$\mathbf{H}$}

Hasegawa, M. ............................................... 260

Hastings, J........................................................... 1

Hattori, Y................................ 160, 174, 316

Herrmann, H................................................... 56

Hiramatsu, D............................................... 87
Holzapfel, C. .................................................. 166

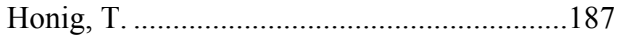

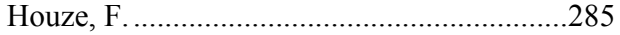

I

Ishimaru, Y......................................141, 147

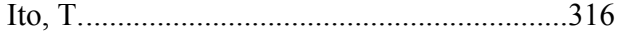

Izmailov, V. V.................................................232

Jackson, R. L....................................227, 48

Jemaa, N. B. ....................................................21

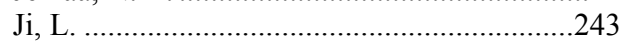

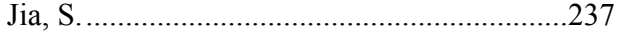

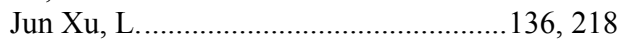

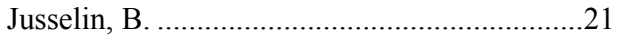

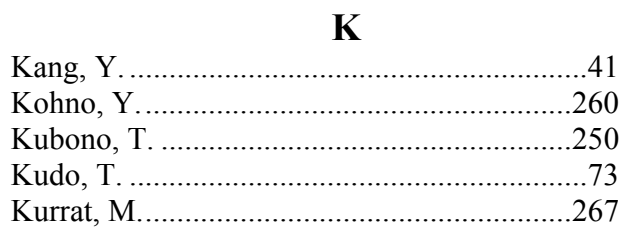

$\mathbf{L}$

Lang, J. H. ..................................................... 300

Lapiere, C.................................................285

Leidner, M..........................................................35

Li, D. .................................................. 7, 195

Li, Q............................................................195

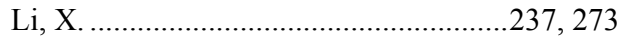

Liang, H. .................................................... 41

Lindmayer, M............................................267

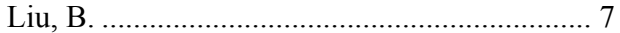

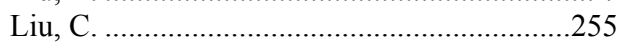

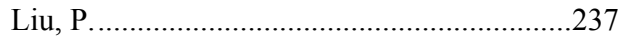

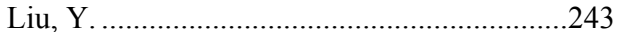

Liu, Z ......................................................... 195

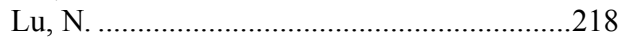

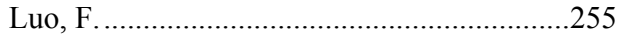

Luo, G. ...................................................... 136

Lynch, W. A.......................................78, 103

Maeda, T. ..................................................... 73

Maier, R. ......................................................... 14

Malucci, R. D.....................................27, 206

Martens, R ….................................................300

Mashimo, K.......................................141, 147

McBride, J. W.....................................278, 295

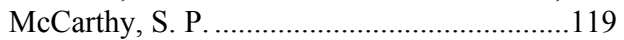

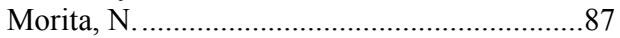

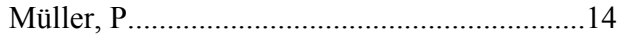

Mützel, T...................................................200

Myers, M.............................................35, 307

Nabeta, Y. .....................................................174

Naumov, A. E................................................232

Niederreuther, R...........................................200

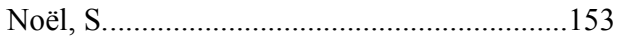




\section{Author Index}

Nomura, Y.

316

Novoselova, M. V

232

\section{$\mathbf{O}$}

Otaka, T

$\mathbf{P}$

Pecht, M....................................................... 321

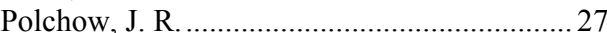

Poulain, C. ......................................... 285, 290

$\mathbf{R}$

Read, M. B............................................. 300

Reitz, R. P...................................................... 103

Ren, W............................................... 41

Ring, K. ......................................................... 223

Roberts, M. J..................................................... 103

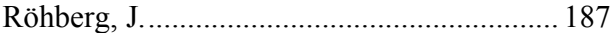

Rousset, P. .................................................. 285

\section{S}

Saitoh, Y.................................................... 174

Sawada, S. ...................................... 160, 174

Schmidt, H........................................... 35, 331

Schoepf, T. J. ................................................... 1

Schreier-Alt, T. .............................................. 223

Sekikawa, J............................................... 250

Shi, Z. ........................................................ 237

Slocum, A. H. ................................................. 300

Sondergaard, N. A. ........................... 78, 94, 103

Song, J........................................................ 331

Song, Z. ............................................................ 7

Spearing, S. M. ........................................... 278

Starman, L. A.................................................. 295

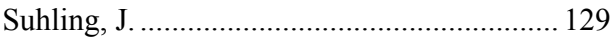

Sun, H.......................................................... 273

Susai, K. ............................................ 141, 147

$\mathbf{T}$

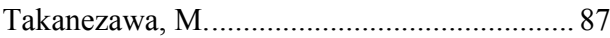

Takatsu, N.
Tamai, T...........................................160, 174

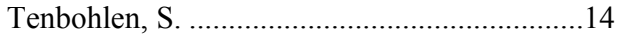

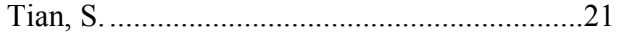

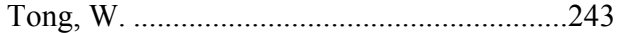

$\mathbf{U}$

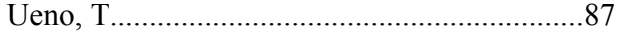

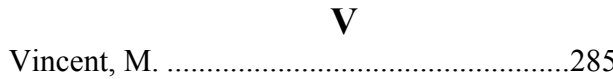

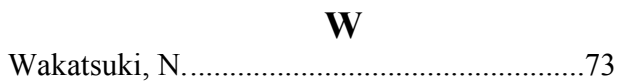

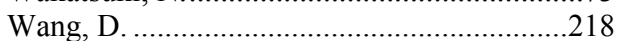

Wang, J................................................... 7, 195

Wang, L................................................41, 237

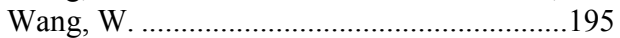

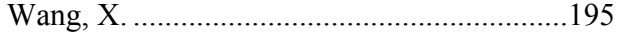

Wilkening, E.-D. ............................................267

Witter, G. J. ...................................................180

Witulski, N................................................187

Wolf, C.........................................................267

X

$\mathbf{Y}$

Yingchao, F...............................................141

Yu, L. .................................................. 7, 195

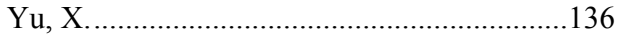

Yunus, E. M. ...............................................278

Zeller, P.....................................................65

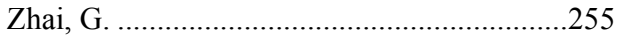

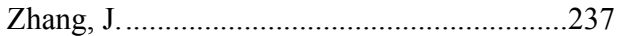

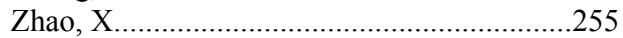

Zhmurkin, D. V.........................................112

Zhou, X..................................................1, 255

Zhou, Y....................................................... 321 